\title{
Hanford Site Ground-Water Monitoring for July Through December 1987
}

John C. Evans

Douglas I. Dennison

Robert W. Bryce

Pamela J. Mitchell

Douglas R. Sherwood
Kenneth M. Krupka

Nancy W. Hinman

Elizabeth A. Jacobson

Mark D. Freshley

December 1988

Prepared for

the U.S. Department of Energy

under Contract DE-AC06-76RLO 1830

Pacific Northwest Laboratory

Operated for the U.S. Department of Energy

by Battelle Memorial Institute 


\title{
DISCLAIMER
}

This report was prepared as an account of work sponsored by an agency of the United States Government. Neither the United States Government nor any agency thereof, nor Battelle Memorial institute, nor any or their employees, makes any warranty, expressed or implied, or assumes any legal liability or responsibility for the accuracy, completeness, or usefulness of any information, apparatus, product, or process disclosed, or represents that its use would not infringe privately owned rights. Reference herein to any specific commercial product, process, or service by trade name, trademark, manufacturer, or otherwise does not necessarily constitute or imply its endorsement, recommendation, or favoring by the United States Government or any agency thereof, or Battelle Memorial Institute. The views and opinions of authors expressed herein do not necessarily state or reflect those of the United States Government or any agency thereof.

\author{
PACIFIC NORTHWEST LABORATORY \\ operated by \\ BATTELLE MEMORIAL INSTITUTE \\ for the \\ UNITED STATES DEPARTMENT OF ENERGY \\ under Contract DE-AC06-76RLO 1830
}

\begin{tabular}{|c|c|}
\hline \multicolumn{2}{|c|}{ Printed in the United States of America } \\
\hline & \\
\hline \multicolumn{2}{|c|}{$\begin{array}{l}\text { Avallable from } \\
\text { National Technical Information Service }\end{array}$} \\
\hline \multicolumn{2}{|c|}{ United States Departinent of Commerce } \\
\hline 528 & Road \\
\hline \multicolumn{2}{|c|}{ Springfield, Virginia 22161} \\
\hline \multirow{2}{*}{\multicolumn{2}{|c|}{$\begin{array}{l}\text { NTIS Price Codes } \\
\text { Microfiche A0t }\end{array}$}} \\
\hline & \\
\hline \multicolumn{2}{|c|}{ Printed Copy } \\
\hline & Price \\
\hline Pages & Codes \\
\hline $001-025$ & $\mathrm{~A} 02$ \\
\hline $026-050$ & $\mathrm{~A} 03$ \\
\hline $051-075$ & A04 \\
\hline $076-100$ & A05 \\
\hline $101-125$ & A06 \\
\hline $126-150$ & $\mathrm{~A} 07$ \\
\hline $151-175$ & A08 \\
\hline $176-200$ & A09 \\
\hline $201-225$ & A10 \\
\hline $226-250$ & A11 \\
\hline $251-275$ & A12 \\
\hline $276-300$ & A13 \\
\hline
\end{tabular}


HANFORD SITE GROUND-WATER MONITORING

FOR JULY THROUGH DECEMBER 1987

John C. Evans

Douglas 1. Dennison

Robert W. Bryce

Pamela J. Mitchell

Douglas R. Sherwood

Kenneth M. Krupka

Nancy W. Hinman

Elizabeth $A$. Jacobson

Mark D. Freshley

December 1988

Prepared for

the U.S. Department of Energy

under Contract DE-ACO6-76RLO 1830

Pacific Northwest Laboratory

Richland, Washington 99352 



\section{SUMAARY}

The Pacific Northwest Laboratory monitors ground-water quality at the Hanford Site for the U.S. Department of Energy to assess the impact of Site operations on the environment. Work undertaken between July and December 1987 included monitoring ground-water elevations across the Site, monitoring hazardous chemicals and radionuclides in ground water, geochemical evaluations of unconfined ground-water data, and caltbration of ground-water flow and transport models.

Water levels continued to rise in areas receiving increased recharge (e.9., beneath B Pond) and decline in areas where the release of water to disposal facilities has been terminated (e.g., U Pond). The major areas of ground-water contamination defined by monitoring activities are 1) carbon tetrachloride in the 200-West Area; 2) cyanide in and north of the 200-East and 200-West Areas; 3 ) hexavalent chromium contamination in the 100-B, 100-D, $100-\mathrm{F}, 100-\mathrm{H}, 100-\mathrm{K}$, and $200-$ West Areas; 4) chlorinated hydrocarbons in the vicinity of the Central Landfill and 300 Area; 5) uranium in the 100-F, 100-H, 200-West, and 300 Areas; and 6) tritium and nitrate across the Site.

The MINTEQ geochemical code was used to identify chemical reactions that may be affecting the concentrations of dissolved hazardous chemicals in the unconfined ground water. Results indicate that many cations are present mainly as dissolved carbonate complexes and that a majority of the groundwater samples are in near equilibrium with carbonate minerals (e.g., calcite, dolomite, otavite). The influence of silicate minerals on ground-water composition could not be evaluated because of a lack of analyses for dissolved silicon in the ground water. Modeling results indicate that lower detection limits are required for iron and phosphate before an analysis of their solubility controls can be made. Additional information on redox conditions is also required before the impact of many of the reactions controlling contaminant concentrations can be assessed.

A preliminary calibration of a ground-water flow model for the unconfined aquifer at the Hanford Site was conducted using a parameter estimation method. The calibration of the unconfined aquifer assumed steady-state conditions and was based on the December 1979 water-level data. Four 
applications of the parameter estimation method were investigated. The applications differed in the treatment of the boundary condition along the cold Creek valley (i.e., either prescribed head or prescribed flux) and of areal recharge. Water levels calculated with the prescribed flux boundary condition were unreasonably high in the cold creek valley, indicating that these applications do not yield good calibrations for the water levels in this region. The two applications with prescribed heads along the cold creek valley yield the best overall fit to the measured water levels. Of these two applications, the application that included areal recharge produced the best match with the measured water levels. 


\section{ACKNOWL EDGMENTS}

The authors wish to thank A. G. Law and J. A. Serkowski (Westinghouse Hanford Company) for carefully reviewing this report, D. J. Bates for preparation of tables and report review, S. B. Moore for map preparation,

B. V. Johnson and R. E. Brown for technical editing, and P. Young,

C. Baldwin, L. Valdez, and D. Kennedy for text processing. 



\section{CONTENTS}

SUMMARY ...................................... i

ACKNOWLEDGMENTS. . . . . . . . . . . . . . . . . v

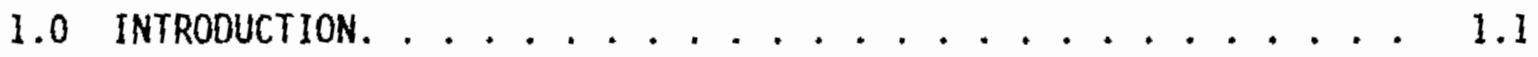

2.0 WATER-LEVEL ELEVATION MONITORING. . . . .......... 2.1

2.1 DATA COLLECTION. ................. 2.1

2.2 RESULTS. . . . . . . . . . . . . . 2.2

3.0 RADIOLOGICAL AND CHEMICAL GROUND-WATER HONITORING . . . . . 3.1

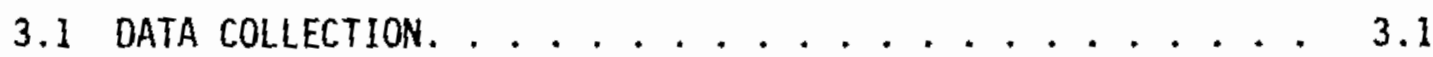

3.1.1 Facility-Specific Monitoring. . . . . . . . . 3.1

3.1.2 Operational Monitoring. . . . . . . . . . 3.2

3.1.3 Site-Wide Monitoring. . . . . . . . . . . 3.2

3.1.4 Sample Collection July through December 1987. . . 3.4

3.1.5 Sample Collection Planned for 1988. . . . . . 3.5

3.1.6 Monitoring Well Design. . . . . . . . . . . 3.6

3.1.7 Sampling Methods. . . . . . . . . . . . . . 3.6

3.2 RESULTS. . . . . . . . . . . . . . 3.20

3.2.1 Carbon Tetrachloride in the 200-West Area... . . 3.29

3.2.2 Cyanide Contamination in and North of the 200-East Area. . . . . . . . . . . . . . 3.31

3.2.3 Hexavalent Chromium in the 100-B, 100-D, 100- $\mathrm{H}$, and 100-K Areas.............. . . 3.34

3.2.4 Chlorindated Hydrocarbons Near the Central Landfill ............... 3.35

3.2.5 Volatile Organic Compounds in the 300 Area . . . 3.35

3.2.6 Nitrate Across the Site............. 3.39

3.2.7 Other Observations ............. 3. . 34 


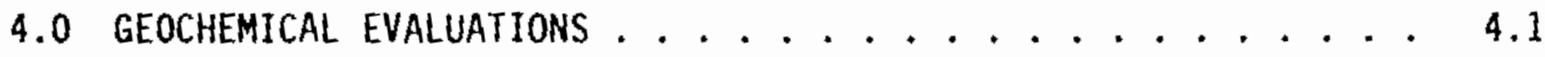

4.1 MINTEQ CODE. . . . . . . . . . . . . 4.1

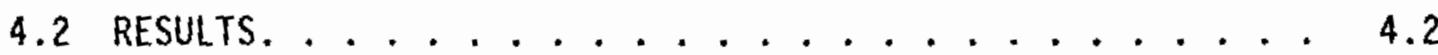

4.2.1 Aqueous Speciation. ............ 4.4

4.2 .2 Solubility Controls . . . . . . . . . 4.8

4.2.3 Sensitivity Analyses. . . . . . . . . 4.10

5.0 GROUND-WATER FLOW MODELING. . . . . . . .... 5.1

5.1 DESCRIPTION OF PREVIOUS WORK . .......... 5.1

5.2 CURRENT WORK . . . . . . . . . . . . 5.4

5.2.1 Parameter Estimation Method ......... 5.4

5.2.2 Data Preparation for the Inverse Model .... 5.5

5.3 APPLICATION AND RESULTS OF THE INVERSE MODEL $\ldots \ldots . .5 .8$

5.3.1 Comparison of Inverse Results for Varying Cold Creek Valley Boundary Conditions with No

Areal Recharge. ............. 5.9

5.3.2 Comparison of Inverse Results for Varying Cold Creek Valley Boundary Conditions with Areal

Recharge. ............ 5.9

5.3.3 Comparison of the Inverse Results for Varying Areal Recharge with Fixed Boundary Conditions

Along the cold Creek Valley......... 5.10

5.3.4 Comparison of the "Best" Results of the Inverse Applications to the Hand-Contoured Water Levels . . 5.10

5.4 PRELIMINARY TRITIUM TRANSPORT MODEL. . . . . . 5.12

5.4.1 Application of the Tritium Transport Model. . . . 5.19

5.4.2 Limitations of the Tritium Transport Model. . . . 5.19

6.0 REFERENCES. . . . . . . . . . . . . . 6.1

APPENDIX A - WATER LEVELS MEASURED OURING OECEMBER $1987 \ldots \ldots . \ldots$ A. $\ldots$

APPENDIX B - SAMPLING SCHEDULE .............. 8.1

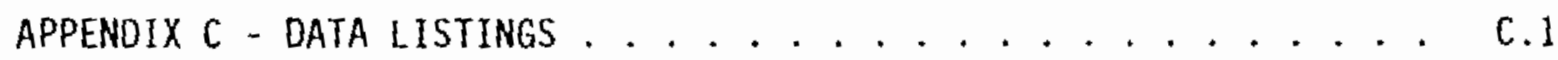


APPENDIX D - MAXIMUM CONTAMINANT LEVELS AND DERIVED CONCENTRATION

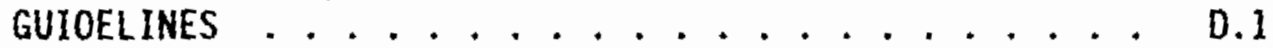

PLATE 1 - Hanford Site Water-Table Map, December 1987. . . . . . back pocket 


\section{FIGURES}

1.1 Hanford Site Location Map. . . . . . . . . . . . . 1.2

3.1 Location of Hanford Site Ground-Water Monitoring

Wells. . . . . . . . . . . . . . . . 3.7

3.2 Location of 100-B Area Ground-Water Monitoring Wells Sampled During Juty Through December 1987. . . . . . . . . 3.9

3.3 Location of 100-D Area Ground-Water Monitoring WeTls Sampled During July Through December 1987. . . . . . . . . . 3.11

3.4 Location of 100-F Area Ground-Water Monitoring Wells Sampled During July Through December 1987. . . . . . . . . 3.13

3.5 Location of 100-H Area Ground-Water Monitoring Wells Sampled During JuTy Through December 1987. . . . . . . . . . 3.15

3.6 Location of 100-K Area Ground-Water Monitoring We1ls Sampled During July Through December 1987. . . . . . . . . . 3.17

3.7 Location of 100-N Area Ground-Water Monitoring Wells

Sampled During July Through December 1987. . . . . . . . . 3.19

3.B Location of 200-East Area Ground-Water Monitoring Wells Sampled During July Through December 1987. . . . . . . . 3.21

3.9 Location of 200-West Ground-Water Monitoring Wells Sampled During July Through December 1987. . . . . . . . 3.23

3.10 Location of 300 Area Ground-Water Monitoring Wells Sampled During July Through December 1987. . . . . . . . . . 3.25

3.11 Location of 400 Area Ground-Water Monitoring Wells Sampled During July Through December 1987. . . . . . . . . . 3.27

3.12 Maximum Carbon Tetrachloride Concentrations in 200-West Area Unconfined Aquifer Wells in 1987. . . . . . . . . . . .

3.13 Cobalt-60, Cyanide, Calcium, Sulfate, Tritium, Nitrate, and Gross Beta Concentrations in Ground-Water Samples Collected from Well 699-50-53............ 3.33

3.14 Maximum Cyanide Concentrations in 200 Areas' Unconfined

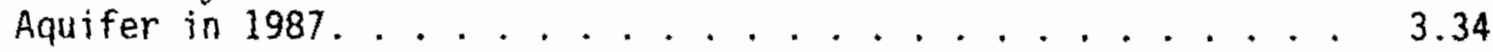

3.15 Maimum Chromium Concentrations in Unconfined Aquifer

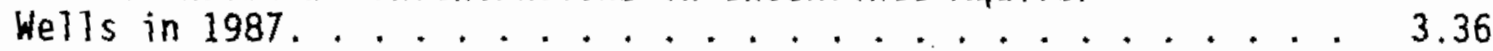


3.16 Maximum Nitrate Concentrations in Unconfined Aquifer Wells in 1987. ......................... 30

3.17 Maximum Fluoride Concentrations in Unconfined Aquifer Wells in 1987........................... 36

3.18 Maximum Sulfate Concentrations in Unconfined Aquifer

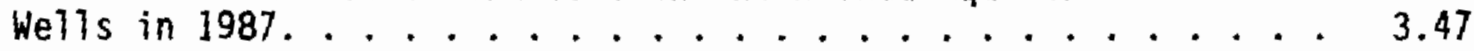

3.19 Maximum Tritium Concentrations in Unconfined Aquifer

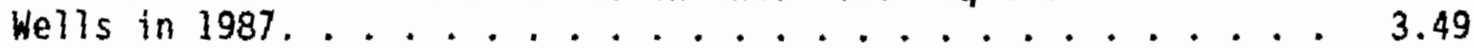

3.20 Tritium Concentrations in Ground Water Sampled from Wells 699-32-43 and 699-33-42..................... 32

3.21 Tritium Concentrations in Ground Water Sampled from Wellis 699-35-66, 699-35-70, and 699-38-65 ......... 3.53

3.22 Tritium Concentrations in Ground Water Sampled from

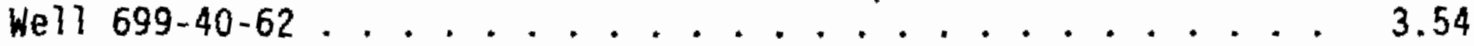

3.23 Gross Alpha Concentrations in Wells Near the 216-U-1

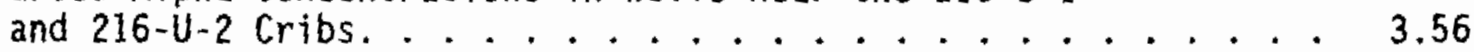

3.24 Uranium Concentrations in Wells Near the 216-U -1

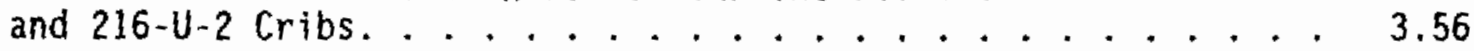

3.25 Gross Aipha Concentrations in Well 299-W19-2 . . . . . . . 3.57

3.26 Uranium Concentrations in Well 299-W19-2 ........ 3.57

3.27 Maximum Uranium Concentrations in Unconfined Aquifer Wells in 1987. . . . . . . . . . . . 3.58

3.28 Maximum Cobalt-60 Concentrations in Unconfined Aquifer Wells in 1987............... 3.62

3.29 Maximum Strontium-90 Concentrations in Unconfined Aquifer Wells in 1987. ..................... 3.64

3.30 Maximum Technetium-99 Concentrations in Unconfined Aquifer wells in 1987................ 3.66

4.1 Distribution of $\mathrm{pH}$ Values for Ground-Water Samples from the Individual 100, 200, and 600 Areas During the Second Quarter of 1987 and from Spring and Surface Waters Reported by Early, Spice, and Mitchen .......... 4.3

4.2 Distribution of A\}kalinity Values for Ground-Water Samples from the 100, 200, and 600 Areas and Springs and Surface Waters During the Second Quarter of 1987. 
4.3 Distribution of Aqueous Zn Complexes Calculated for a Water Sample from the Second Quarter of 1987 from Well 199-K-19. . . .

4.4 Distribution of $\mathrm{Zn}^{2+}, \mathrm{Zn}-\mathrm{CO}_{3}$, and $\mathrm{Zn}-\mathrm{OH}$ Complexes for Aqueous In Complexes Plotted as Function $\mathrm{pH}$ and Alkalinity for Water Samples from the Second Quarter of 1987 from Five Wells in the 100 Areas. . .

4.5 Saturation Indices for Calcite, Barite, $\mathrm{Fe}(\mathrm{OH})_{3}$, and Gypsum, Plotted as Function of pH for Compositions of hater Samples Taken During the Second Quarter of 1987. . . . . . . . . . .

5.1 Finite Element Grid and Boundary Conditions for the Model Based on the CFEST Code.

5.2 Contour Map of Initial Transmissivities for Inverse Calibration. . . . . . . . . . . . . . .

5.3 Comparison of Water Levels Computed by the Case 2 Inverse Model and Hand-Contoured Water Levels. . . . . . . ...............

5.4 Comparison of Water Levers Computed by the Case 4 Inverse Model and Hand-Contoured Water Levels. . . . . . . . . . . . .

5.5 Contour Map of the Transmissivity Estimates for the Case 2 Inverse Model . . . . . . . . . . . . . .

5.6 Contour Map of the Coefficients of Variation for the Case 2 Inverse Mode1.................

5.7 Contour Map of the Transmissivity Estimates for the Case 4 Inverse Model . . . . . . . . . . . . .

5.8 Contour Map of the Coefficients of Variation for the

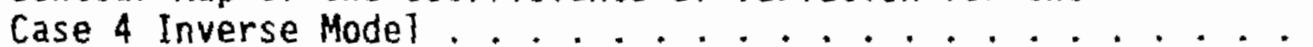

5.9 Initial Distribution of Tritium in the Unconfined Aquifer During 1983. . . . . . . . . . . . . . .

5.10 Predicted Distribution of Tritium in the Unconfined Aquifer at the End of 1985 


\section{IABLES}

3.1 Major Chemical Ground-Water Contaminants and Their Link to Site Operations................. 3.5

3.2 Estimated Background Levels for Selected Constituents in Hanford Ground Water . . . . . . . . . . . . . . . . 3.30

3.3 Iodine-129 Data for Samples Collected July 1 Through September 30, 1987. . . . . . . . . . . . . . 3.70

4.1 Major Chemical Constituents Dissolved in Unconfined Aquifer Ground Water at Hanford . . . . . . . . . . . . . . . 4.4

4.2 Chemical Constituents That May Be Present in Minor Amounts in Unconfined Aquifer Ground Water at Hanford . . . . . . . . 4.5 



\subsection{INTRODUCTION}

The purpose of the Hanford Site ground-water monitoring program is to monitor the distribution of radionuclides and other hazardous materials in ground water at the Hanford Site (Figure 1.1) and to evaluate the impact of past and present Site operations on the environment. The specific objectives of the program are to 1) determine the distribution of radionuclides and nitrate ion to define the extent of impacted ground water, 2) relate the distribution of these constituents to Site operations, 3 ) establish background concentrations for naturally occurring regulated hazardous materials, and 4) identify in the ground water those hazardous chemicals that resulted from Site operations. The Hanford Site ground-water monitoring program conducted by the Pacific Northwest Laboratory (PNL) involves the measurement of water-table elevations, collection and analys is of ground-water samples, computer modeling of contaminant transport, and use of geochemical models to meet these objectives.

The Hanford Site monitoring well network is designed to meet the intent

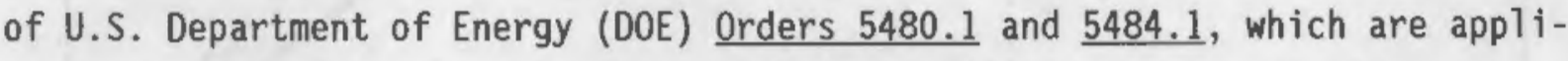
cable to environmental monitoring. DOE Order 5480.1, Chapter XI, lists the permissible concentrations of radionuclides applicable to the discharge of liquid effluent in controlled and uncontrolled areas. This order also requires that the environment be monitored sufficiently to ensure that the radiation doses to which the public is exposed are as low as reasonably achievable. DOE Order 5480.1 , Chapter XII, requires that DOE cooperate with the U.S. Environmental Protection Agency (EPA) and state, interstate, and local agencies in the prevention, control, and abatement of environmental pollution. Annual reports (e.g., Jaquish and Mitchell 1988) are published by PNL to document all surface and subsurface monitoring activities at the Site, as well as measured and calculated doses to the public.

In addition to the Hanford Site ground-water monitoring program, two ground-water monitoring activities are being conducted by PNL at the Hanford Site for Westinghouse Hanford Company (WHC). Ground-water samples are collected by PNL for operational monitoring in and around the 200 Areas 


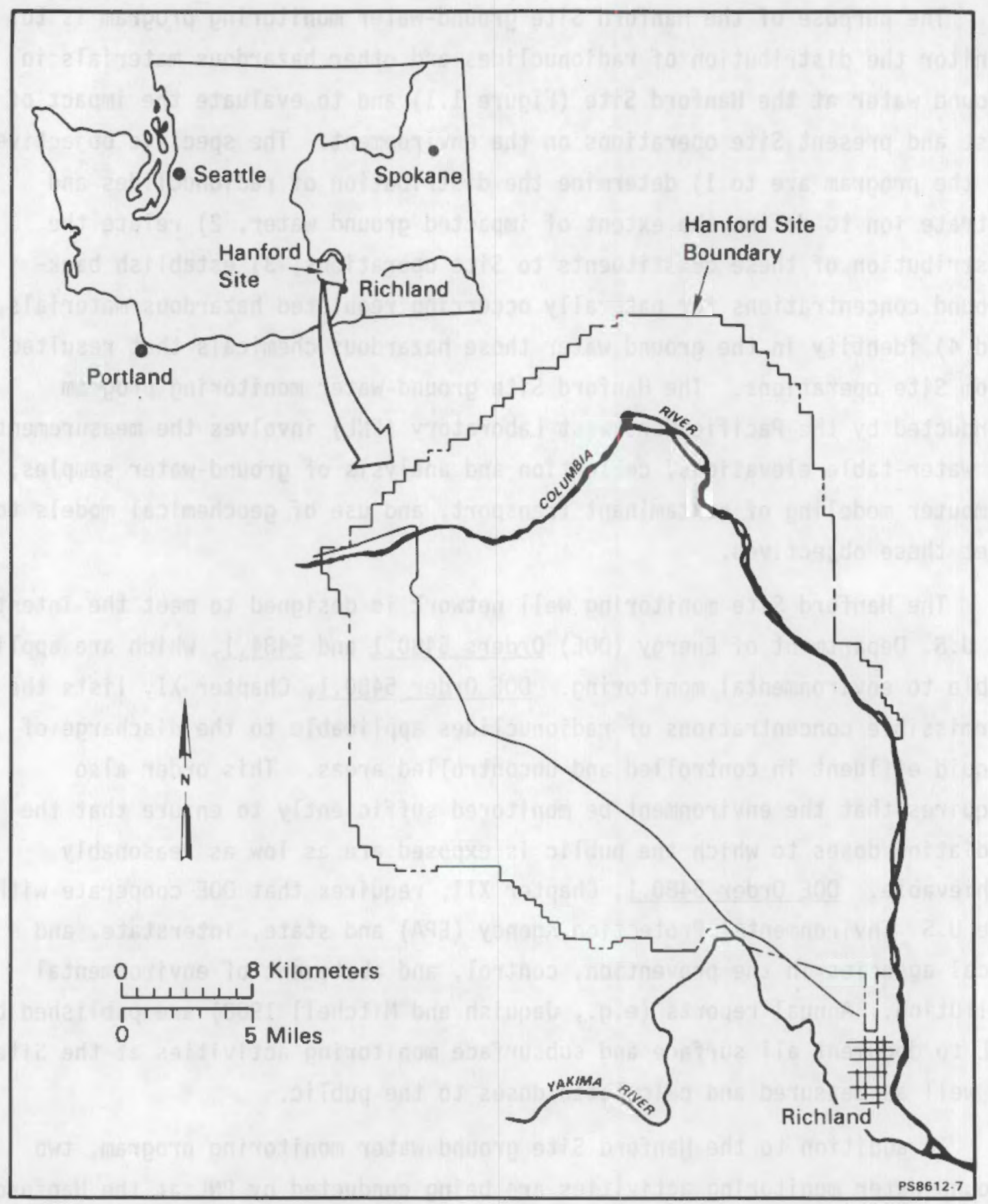

FIGURE 1.1. Hanford Site Location Map 
for compliance with DOE orders and for facility-specific monitoring for compliance with the Resource Conservation and Recovery Act (RCRA) and Washington Administrative Code (WAC) 173-303 and 304. The facility-specific activities include sampling programs at the 183-H Solar Evaporation Basins in the 100-H Area, 1301-N/1325-N Cribs and 1324-N/NA Ponds in the 100-N Area, Transportable Grout Facility, 300 Area Process Trenches, Solid Waste Landfill (SWL), and Nonradioactive Dangerous Waste Landfill (NRDW). (The SWL and the NRDW together will be referred to as the Central Landfi11.) The results of some of these activities are discussed briefly in this report and will be reported in more detail elsewhere. The compliance monitoring results (primarily for chemicals) are valuable in determining the total impact of Site operations on ground water and, therefore, are utilized by the Hanford Site ground-water monitoring program to meet its objectives.

This semiannual report provides brief discussions and detailed data listings of results for ground-water monitoring at the Hanford Site during July through December 1987 . This report presents and discusses the watertable elevation map (Plate 1 , in pocket) for the Hanford Site for December 1987 and results of ground-water sampling and analysis. Because of their high concentrations or widespread distribution, the following contaminants are discussed in detai1: 1) carbon tetrachloride in the 200-West Areas; 2) cyanide in and north of the 200-East and the 200-West Areas; 3) hexavalent chromium contamination in the 100-B, 100-D, 100-F, 100- H, 100-K, and the 200-West Areas; 4) chlorinated hydrocarbons in the vicinity of the Central Landfill and 300 Area; 5) uranium in the 100-F, 100- $\mathrm{H}$, 200-West, and 300 Areas; 6) nitrate across the Site; and 7) tritium across the Site. The implications of several other observations are also discussed briefly. Also presented is an update on ground-water flow and geochemical modeling for the period covered by this report.

More detailed discussions of the hydrology and geology of the Site, operational activities, sampling, analysis, and distributions of average constituent concentrations during 1986 are included in the most recent annual report by PNL (Jaquish and Mitchel1 1988). In addition, Serkowski et al. (1988) reported 1987 radiological monitoring results for the 200 Areas and some of the surrounding 600 Area. Also Resource Conservation and Recovery 
Act (RCRA) monitoring results are documented in quarterly reports entitled Ground-Water Monitoring Compliance Projects for Hanford Site Facilities

(e.g., PNL 1987). 


\subsection{HATER-LEVEL ELEVATION MONITORING}

Water levels in selected wells open to the unconfined aquifer on the Hanford Site are measured in June and December of each year to determine the configuration of the water table and to assess the impact of waste disposal on that configuration. Water-level elevations for June 1987 were presented by Schatz, Anmerman, and Serkowski (1987). Historical water-level data and an evaluation of past water-level changes are presented by Zimmerman et a1. (1986). Water-level elevations for this report were measured in approximately 240 wells open to the unconfined aquifer during a 2-week period in December 1987.

Water-table maps can be used to infer directions of ground-water flow, with ground water moving from regions of higher water levels to regions of lower water levels at right angles to contours in systems with isotropic hydraulic conductivities. Water-table configurations can be used to identify recharge and discharge areas, and to determine the potential for water movement between adjacent ground-water and surface water bodies. Further utilization of water-level elevations is discussed in the section entitled, "Ground-Water Flow Modeling."

\subsection{DATA COLLECTION}

Water levels are measured in wells and piezometers following written procedures. Most monitoring wells at the Hanford Site used for water-level measurement are 6 or $8 \mathrm{in}$. (15 or $20 \mathrm{~cm}$ ) in diameter and are constructed of steel casing. Several small-diameter [2-in. $(5-\mathrm{cm})]$ piezometers and some larger diameter wells are also used. Monitoring wells used to measure water levels for the unconfined aquifer are completed with well screens or perforated casing generally open to the upper 10 to $20 \mathrm{ft}(3$ to $6 \mathrm{~m}$ ) of the aquifer. This type of completion allows measurements to be representative of the water-table elevation. Well locations for water-level monitoring are shown in Plate 1.

A written procedure adapted from the U.S. Geological Survey (USGS 1977) was followed to measure water levels in wells. A chalked steel tape was used to measure the depth to water. These measurements were repeated to confirm 
initial value. If the second measurement differed by more than $0.05 \mathrm{ft}$ from the first, the measurement was repeated until two measurements within the proper tolerance were obtained. Measurements were compared to previous water levels to further reduce operator error. Water-level elevations for each well were determined by subtracting the depth to water from the surveyed measuring point elevation on the well casing. All water levels are measured within a 2-week period to reduce the effects of time-variant water-level changes on the water-table map.

\subsection{RESULTS}

Water Tevels measured during December 1987 are Tisted in Appendix A. The water-table map constructed by contouring these water-ievel elevations is presented in Plate 1 . The following are important features of the watertable map:

- water-table elevations generally decrease from west to east across the Hanford Site south of Gable Mountain and Gable Butte

- water-table elevations decrease northward through the gap between Umtanum Ridge and Gable Butte and between Gable Butte and Gable Mountain

- ground-water mounds are present beneath B Pond and the location of decommissioned $U$ Pond

- the water level in the Yakima River is of higher elevation than that of the water table in the adjacent region of the Hanford Site. Elevated water levels in the western region of the Hanford Site may be attributable to ground-water recharge in Cold Creek and Dry Creek valleys and on the adjacent ridges (i.e., Yakima, Umtanum, and Rattlesnake Ridges). Recharge is most likely caused by rain and snow at the higher elevations and by irrigation of agricultural land at the lower elevations. Water-table elevation generally decreases toward the Columbia River at the eastern edge of the Hanford Site and north of the Gable Mountain-Gable Butte anticline, implying discharge of unconfined ground water to the Columbia River along the Hanford reach. Ground-water mounds beneath B Pond and the location of the decommissioned $U$ Pond have been caused by process cooling water and other 
liquid wastes recharging the ground water at those locations. A summary of waste discharges to disposal facilities in the 200 Areas is presented by Serkowski et al. (1988).

$U$ Pond was decommissioned in 1984, and the elevation of the ground-water mound at that location continues to decline. The elevation of the groundwater mound beneath $B$ Pond, which receives process cooling water from the plutonium-uranium extraction (PUREX) plant, has increased approximately $\mathrm{l} f \mathrm{ft}$ $(30 \mathrm{~cm}$ ) since June 1987 (as reflected in water levels in we11 699-43-42). A water-table elevation lower than the Yakima River in the region between the Horn Rapids Dam and the city of Richland implies that the ground water is being recharged by the Yakima River. Water levels measured in this region also indicate that ground water recharged by the Yakima River flows to the east beneath the Hanford Site and discharges to the Columbia River. 


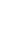




\subsection{RADIOLOGICAL AND CHEMICAL GROUND-WATER MONITORING}

\subsection{DATA COLLECTION}

The network of wells used for the Hanford Site ground-water monitoring program is a combination of several networks that have been designed for facility-specific, operational, and Site-wide monitoring activities. The basis for selecting wells, sampling frequencies, and constituents to be analyzed is different for each of these programs based on the program objectives.

\subsubsection{Facility-Specific Monitoring}

Well networks have been estab7ished around specific waste disposal facilities for WHC to comply with RCRA requirements. Facility-specific activities include sampling programs at the 183-H Solar Evaporation Basins in the 100-H Area, 1301-N/1325-N Cribs and 1324-N/NA Ponds in the 100-N Area, Transportable Grout Facility, 300 Area Process Trenches, SWL, and the NRDW.

The requirements for monitoring well design and location, constituents to be sampled for, and sampling frequencies are specified in RCRA regulations and by Washington Administration Code (WAC 173-303 and 304). Ground-water monitoring systems at each site must consist of at least one monitoring well hydraulically upgradient and at least three monitoring wells downgradient of the facility. The location, depth, and number of wells included in the network must ensure that results obtained to evaluate the migration of contaminants to the uppermost aquifer are statistically significant. The RCRA regulations require that ground water be sampled and analyzed for 1) drinking water parameters, 2) parameters that establish ground-water quality, and 3) parameters used as indicators of ground-water contamination. Samples are also analyzed for contaminants known to have been disposed of at the facility being monitored. The frequency of sampling for each parameter is also specified in the RCRA regulations, based on the status of permitting of the facility (e.g., interim status, permitted status). 
Quarterly reports entitled Ground-Water Monitoring Compliance Projects for Hanford Site Facilities document monitoring networks and analytical plans for these RCRA sites. One such report covers the period July 1 to September 30, 1987 (PNL 1987).

\subsubsection{Operationai Monitoring}

Operational monitoring near waste facilities in the 200 Areas is conducted for WHC to allow them to evaluate the performance of waste disposal and storage sites and assess the impact of specific sites on ground water (Serkowski et al. 1988). Well location, monitoring frequency, and constituents for which samples are analyzed are selected to meet this objective. The sampling network design, analytical plans, and results are presented each year in an annual report (e.g., Serkowski et al. 1988). Changes in the monitoring network are described in that report. The planned network and schedule for calendar year 1988 are listed in Appendix $C$ of Serkowski et al. (1988).

\subsubsection{Site-Wide Monitoring}

The objective of the Site-wide Ground-Water Monitoring Program is to monitor the distribution of radionuclides and other hazardous materials in ground water at the Hanford Site. The selection of wells, constituents for which samples are collected and analyzed, and sampling frequency are based on knowledge of waste disposal practices and inventories, regulatory requirements, contaminant mobility, and the site hydrogeology. The Site-wide monitoring network is designed as a network for radiological monitoring and a network for chemical monitoring.

\subsubsection{Radiological Monitoring}

The radiological monitoring network was developed to monitor the extent of contamination, identify new instances of contaminant release to the ground water, and sample for selected radionuclides that may contribute to radiation dose. Wells and constituents near operational and facility-specific networks were selected to complement monitoring under these programs. For example, some wells in the 200 Areas monitored by WHC to evaluate facility operation are sampled for additional constituents to meet the objectives of Site-wide monitoring. 
The primary analyses done on samples collected for radiological analysis are tritium and gross alpha, beta, and gamma scans. The maximum extent of radionuclide contamination in the ground water beneath the Hanford site is defined using tritium because nearly all radioactive waste disposed of at Hanford contains tritium. Tritium exists as part of the water molecule and as such moves with the ground water unretarded by chemical and physical interaction with dissolved constituents and aquifer materials. Tritium was also concentrated in certain large-volume wastes, such as reactor coolant in the 100 Areas and process condensates in the 200 Areas.

Gross alpha and beta analyses and gamma scans are used to identify potential new releases of radionuclides in a cost-effective manner at certain locations. These techniques are used to survey wells throughout the Site for a wide variety of alpha-, beta-, and gamma-emitting radionuclides. If results of these survey techniques indicate it is warranted, samples are collected and analyzed for individual radionuclides. Subsequent analyses are chosen on the basis of radionuclide inventories, radionuclide mobilities, and potential dose concerns.

Gross alpha concentrations above background may indicate the sample contains uranium or plutonium. Uranium is an alpha-emitting radionuclide that is mobile in the Hanford ground water. It is commonly the radionuclide responsible for elevated gross alpha concentrations. Uranium is also a potential concern in terms of its dose to man. Plutonium is another alpha emitter that could contribute to gross alpha activity. Past monitoring for plutonium suggests that it is immobile in the ground water and hence is monitored in only a few wells near facilities suspected of receiving plutonium. These wells are all within the 200 Areas.

Elevated gross beta concentrations are more difficult to associate with a single radionuclide because of the potentially large number of betaemitting radionuclides discharged in Hanford liquid wastes. Strontium-90 has been a common contributor to elevated gross beta concentrations in ground water. Strontium-90 is monitored in ground-water samples collected throughout the Hanford Site, with emphasis on the operating areas. Another beta emitter of potential dose concern that is relatively mobile in the ground water is technetium-99. Technetium-99 is not efficiently detected using 
gross beta analysis because it is a low-energy beta emitter. Few technetium-99 measurements have been made in the past because of the costly analytical method; however, a less costly technique was developed in 1987. Using this new technique, technetium-99 concentrations will be determined for ground-water samples collected from wells in many areas during 1988, both to gather baseline data and to investigate elevated gross beta concentrations previously observed. Three other beta emitters will be selectively analyzed in a limited number of wells. These are carbon-14, nickel-63, and iodine-129. Isolated measurements of these radionuclides will be done to further assess elevated gross beta measurements previously observed and to investigate selected wells near possible sources of these radionuclides.

\subsubsection{Chemical Monitoring}

A subset of both the PNL Site-wide and the WHC operational radiological monitoring networks is used for Site-wide chemical sampling by PNL. Chemical sampling wells were selected primarily for their proximity to known active and inactive chemical disposal areas in the 100, 200, and 600 Areas, and on the basis of the compiled waste inventories (DOE 1986).

Nitrate is monitored in most of the wells sampled. Nitrate, which is mobile in ground water, was present in many of the waste streams disposed of to the ground and, like tritium, can be used to help define the extent of contamination in Hanford aquifers. A large historical data base also exists for nitrate. Other chemicals related to Site operations are listed in Table 3.1. Ground-water samples collected from wells in areas where these chemicals have been discharged are analyzed for the appropriate contaminant.

\subsubsection{Sample Collection July through December 1987}

The 1987 PNL Site-wide radiological sampling network consisted of 391 we1ls that were monitored quarterly. In the 200 Areas and surrounding 600 Area, PNL sampled 153 wells in WHC's operational monitoring network.

During the third quarter of 1987, 121 wells were monitored for chemical constituents; 102 wells were sampled during the fourth quarter. The wells sampled for chemicals in the fourth quarter included some new wells primarily 
TABLE 3.1. Major Chemical Ground-Hater Contaminants and Their Link to Site Operations

\begin{tabular}{|c|c|c|}
\hline Facilities Type & Area & Constituents \\
\hline Reactor Operations & 100 & $\mathrm{Cr}^{6+}$ \\
\hline Irradiated Fuel Processing & 200 & $\mathrm{NO}_{3}^{-}, \mathrm{CN}^{-}$, Uranium \\
\hline Plutonium Purification & 200 & $\mathrm{CCl}_{4}$ and other chlorinated hydrocarbons \\
\hline Fuel Fabrication & 300 & Uranium, $\mathrm{Cr}^{6+}$ \\
\hline
\end{tabular}

in the 600 Area, while a number of wells showing no significant contamination after three sampling periods were dropped from the network. Chemical data are available for an additional 93 compliance wells, as shown in Appendix A.

The Site-wide monitoring network for 1987 is shown in Figure 3.1. Detailed maps of operational and facility-specific monitoring well networks for the 100-B, 100-D, 100-F, 100-H, 100-K, 100-N, 200-East, 200-West, 300, and 400 Areas are included in Figures 3.2 through 3.11, respectively.

\subsubsection{Sample Collection Planned for 1988}

The wells to be sampled for radionuclide and nitrate analysis during 1988 are listed in Appendix B, Table B.1. The table includes planned sampling frequency. Nitrate is included in this table because historically most samples collected for radionuclide analysis have been analyzed for nitrate.

Seventy-seven wells will be sampled during the first quarter and 66 wells will be sampled during the second quarter of 1988 for the chemicals listed in Appendix C (Table C.1). The number of wells to be sampled in subsequent quarters will be reduced as wells showing no detection of contaminants on three or more successive samplings are eliminated from the schedule. Wells to be sampled during 1988 include some new wells, primarily in the 600 Area, that were sampled for the first time in the last quarter of 1987.

A group of 55 wells has been selected by the Site-wide program in conjunction with WHC personnel for operational chemical monitoring. These 
wells will be initially sampled during the first or second quarter of 1988. The decision to continue monitoring these wells will be made by WHC staff based on the results of the initial sampling.

\subsubsection{Monitoring Well Design}

Most monitoring wells at the Hanford Site are 6 or 8 in. $(15$ or $20 \mathrm{~cm}$ ) in diameter and are constructed of steel casing. Several small-diameter [2-in. (5-cm)] piezometers are sampled for radionuclides on]y. Monitoring wells for the unconfined aquifer are completed with well screens or perforated casing in the upper 10 to $20 \mathrm{ft}(3$ to $6 \mathrm{~m})$ of the aquifer. Completion at the water table allows samples to be collected near the top of the aquifer where maximum concentrations for some radionuclides were measured at a few locations at the Hanford Site (Eddy, Myers, and Raymond 1978). Confined aquifer monitoring wells have screens, perforated casing, or an open hole within the monitored horizon. Only wells containing submersible pumps were chosen for chemical sampling to allow sufficient purging of wells before sampling.

\subsubsection{Sampling Methods}

Samples were collected using documented sampling procedures that follow formal, established guidelines (EPA 1986). Wells fitted with submersible pumps were sampled after pumping for a sufficient time (at least 20 min) to allow temperature, $\mathrm{pH}$, and specific conductivity to stabilize. The purging process ensured that any stagnant water in the well was removed, allowing collection of a sample that was representative of the ground water near the we11. A stainless steel sampling tee was then connected to the pump discharge line. One side of the tee consisted of a $3 / 16-\mathrm{in} .(0.476-\mathrm{cm})$ critical orifice discharging to a $1 / 4-i n .(0.635-\mathrm{cm})$ Teflon (a) sampling line. Excess water was discharged through a ball valve on the other branch of the tee. This arrangement allowed the pump discharge to be throttled back sufficiently to provide a continuous water column, while providing some pressure relief to avoid damage to the header pipe. Samples for volatile organic analyses were taken with zero head space and sealed immediately with a

(a) Teflon is the registered tradename for a fluorocarbon resin product of E. I. du Pont de Nemours and Company, Wilmington, Delaware. 


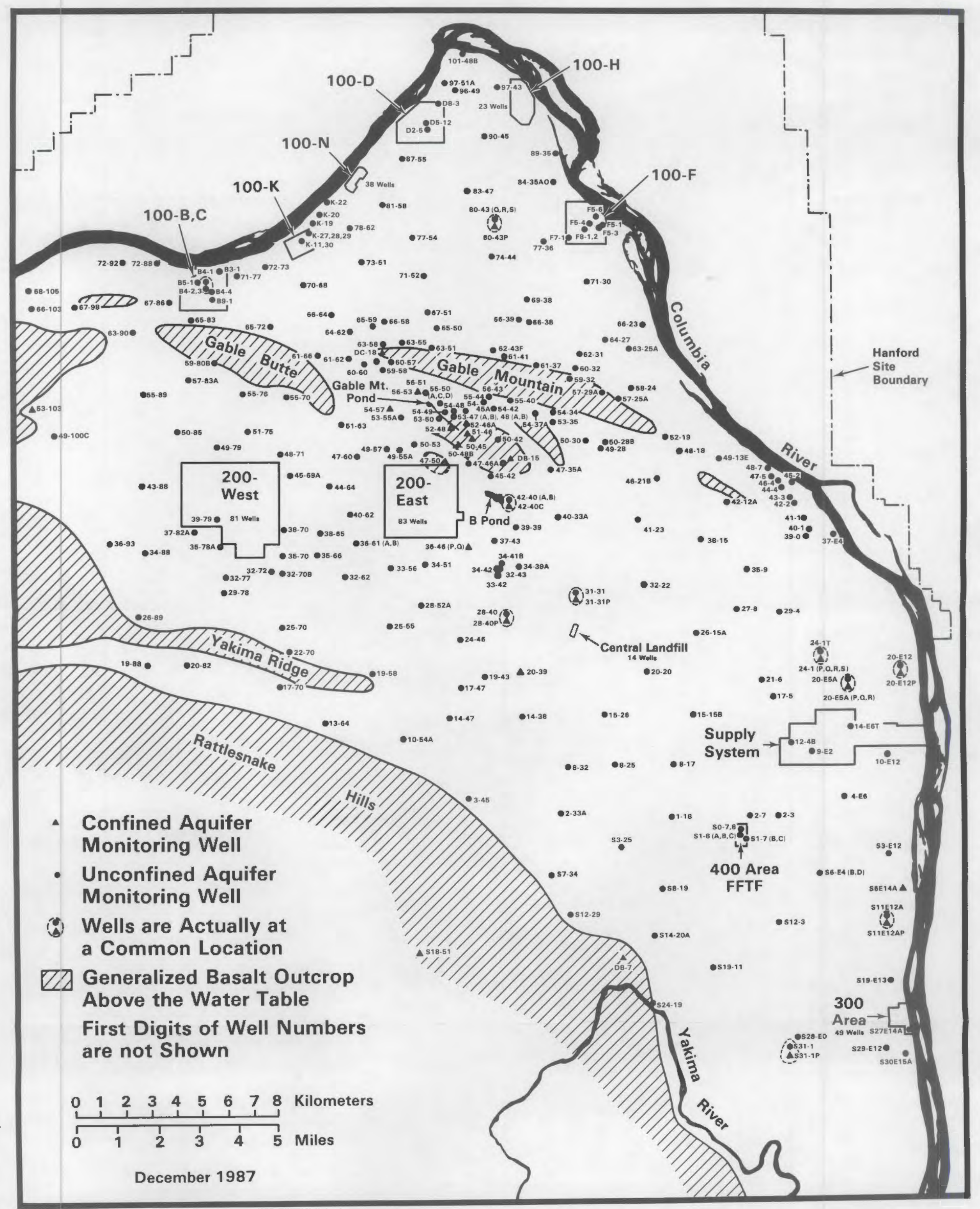

FIGURE 3.1. Location of Hanford Site Ground-Water Monitoring Wells 


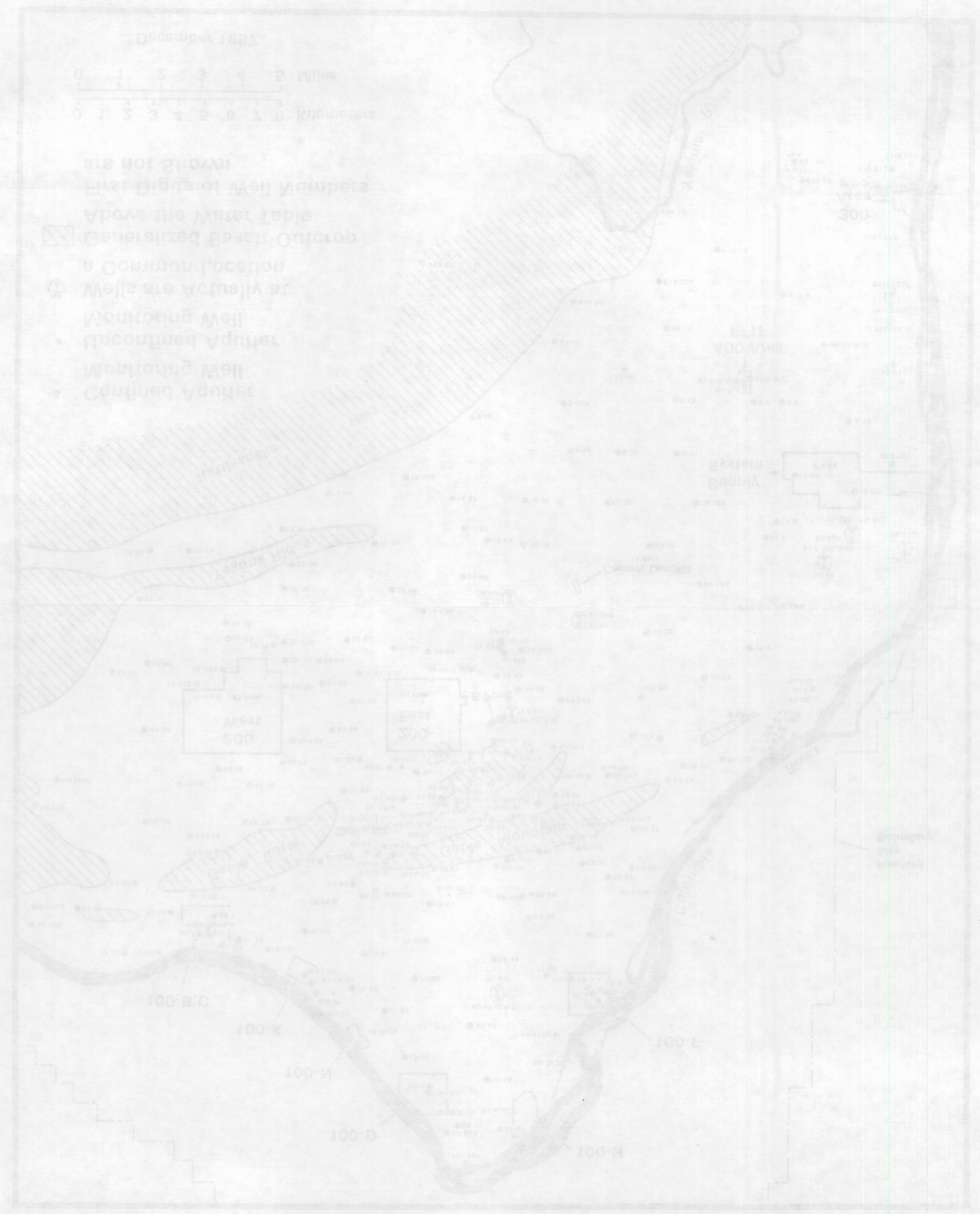

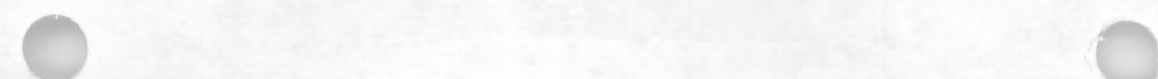




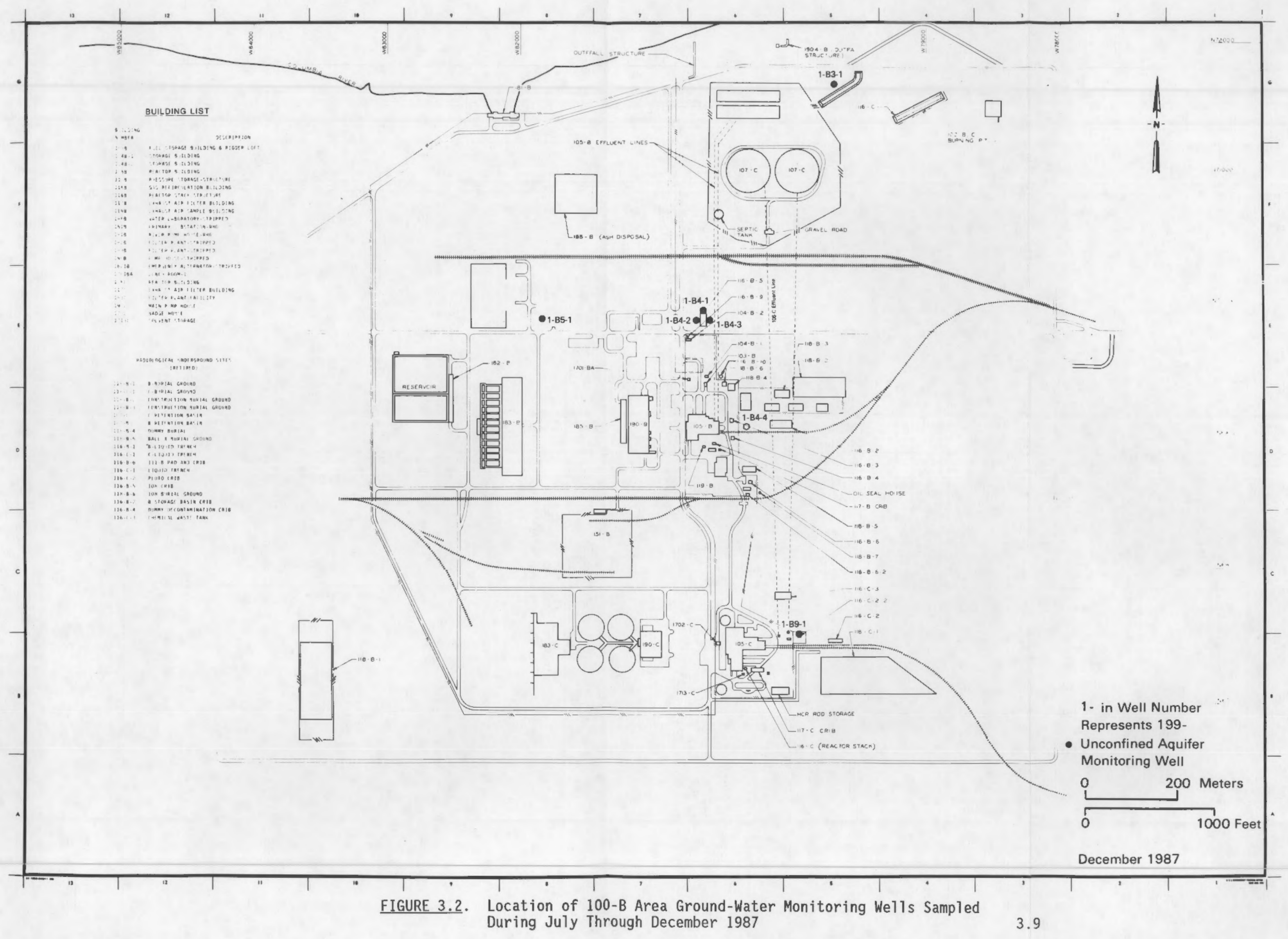




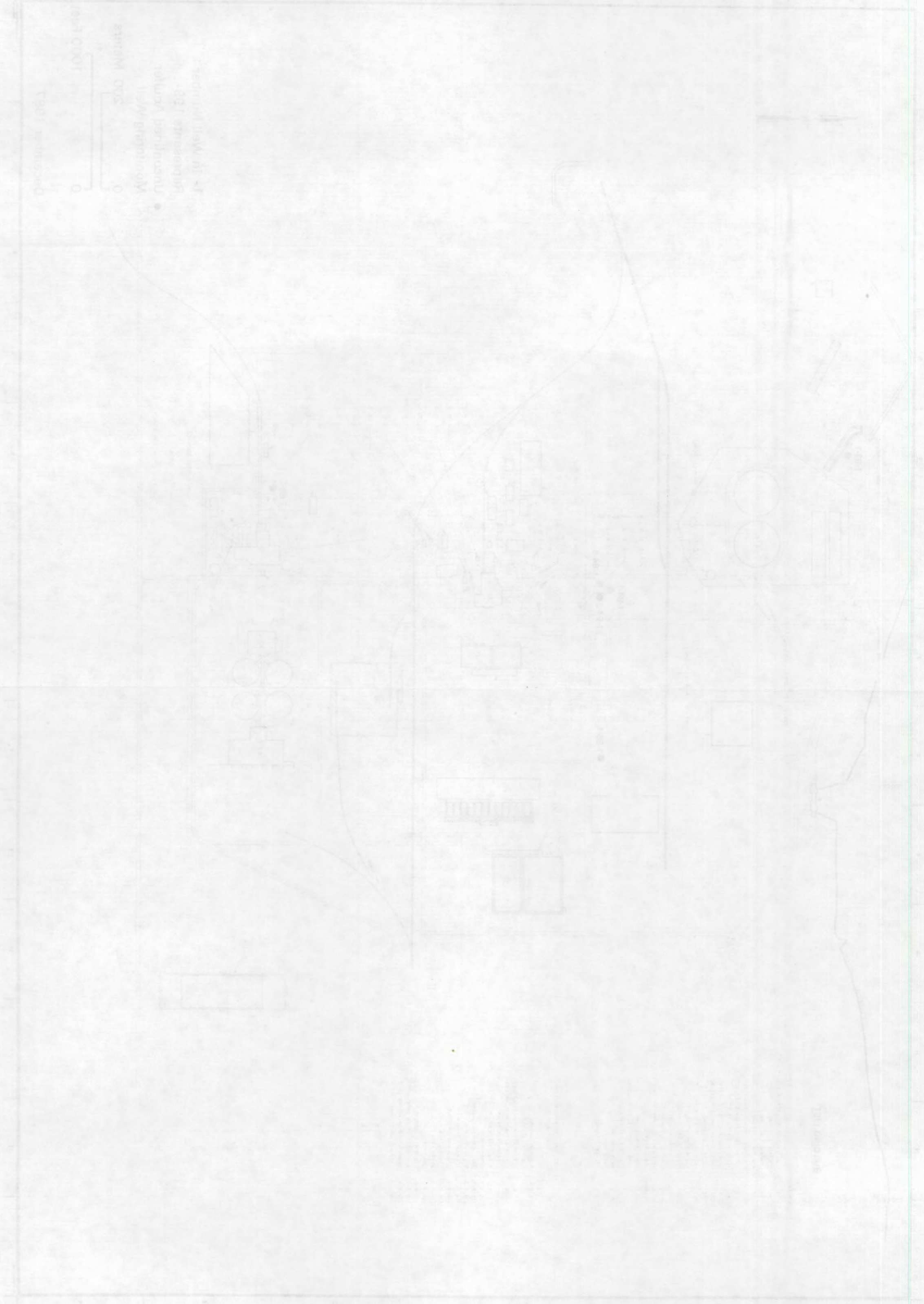




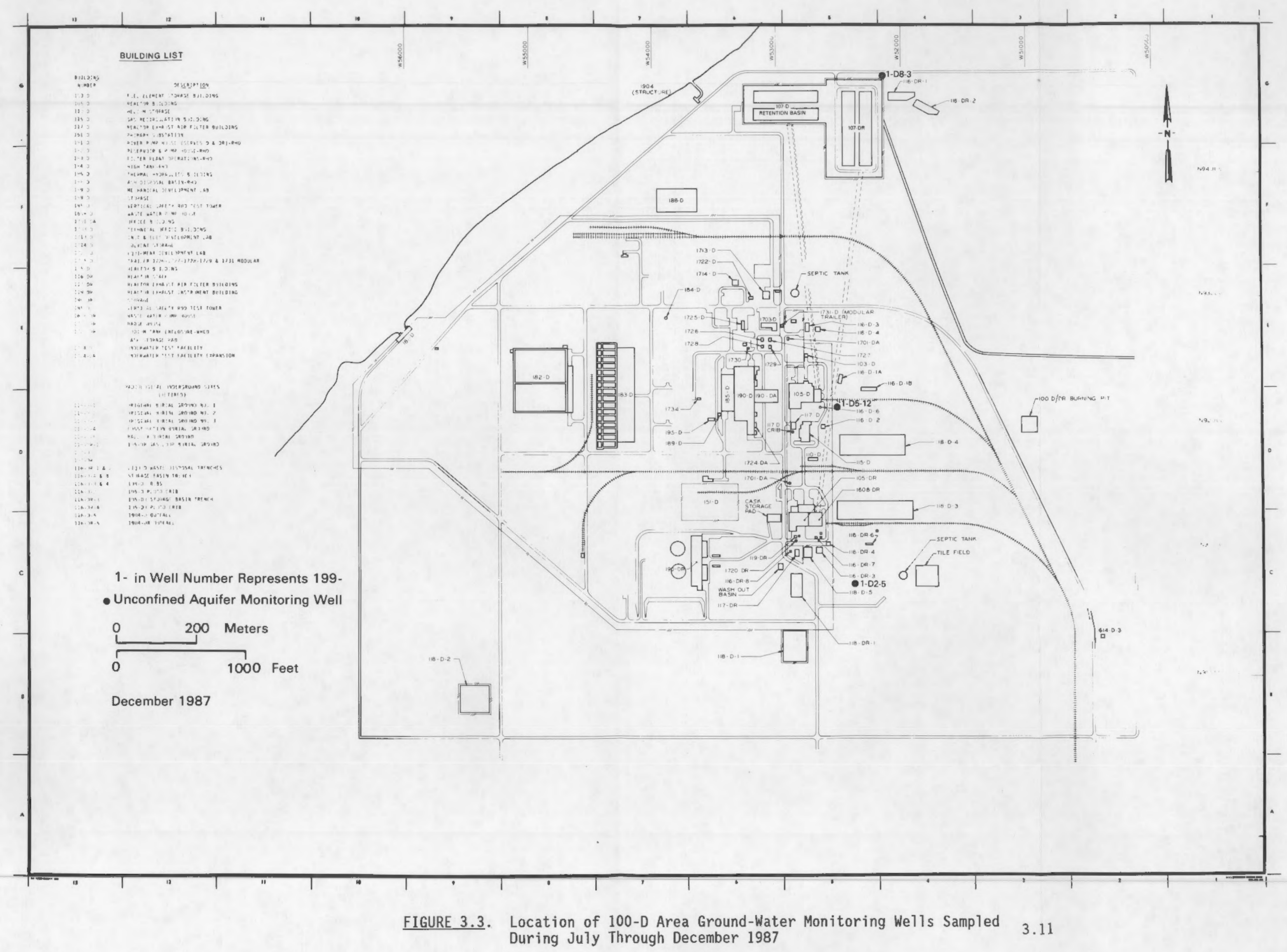




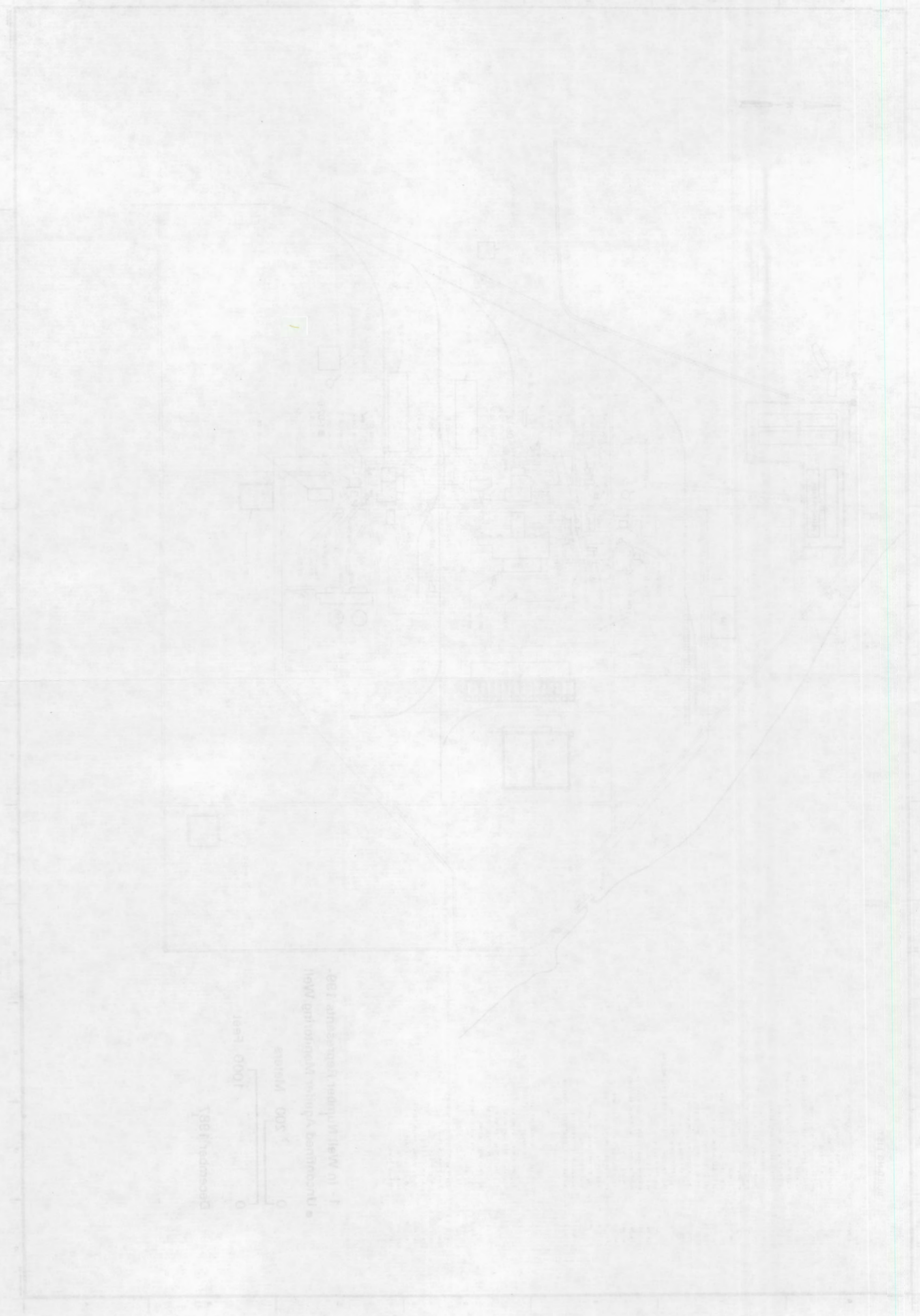

ค 


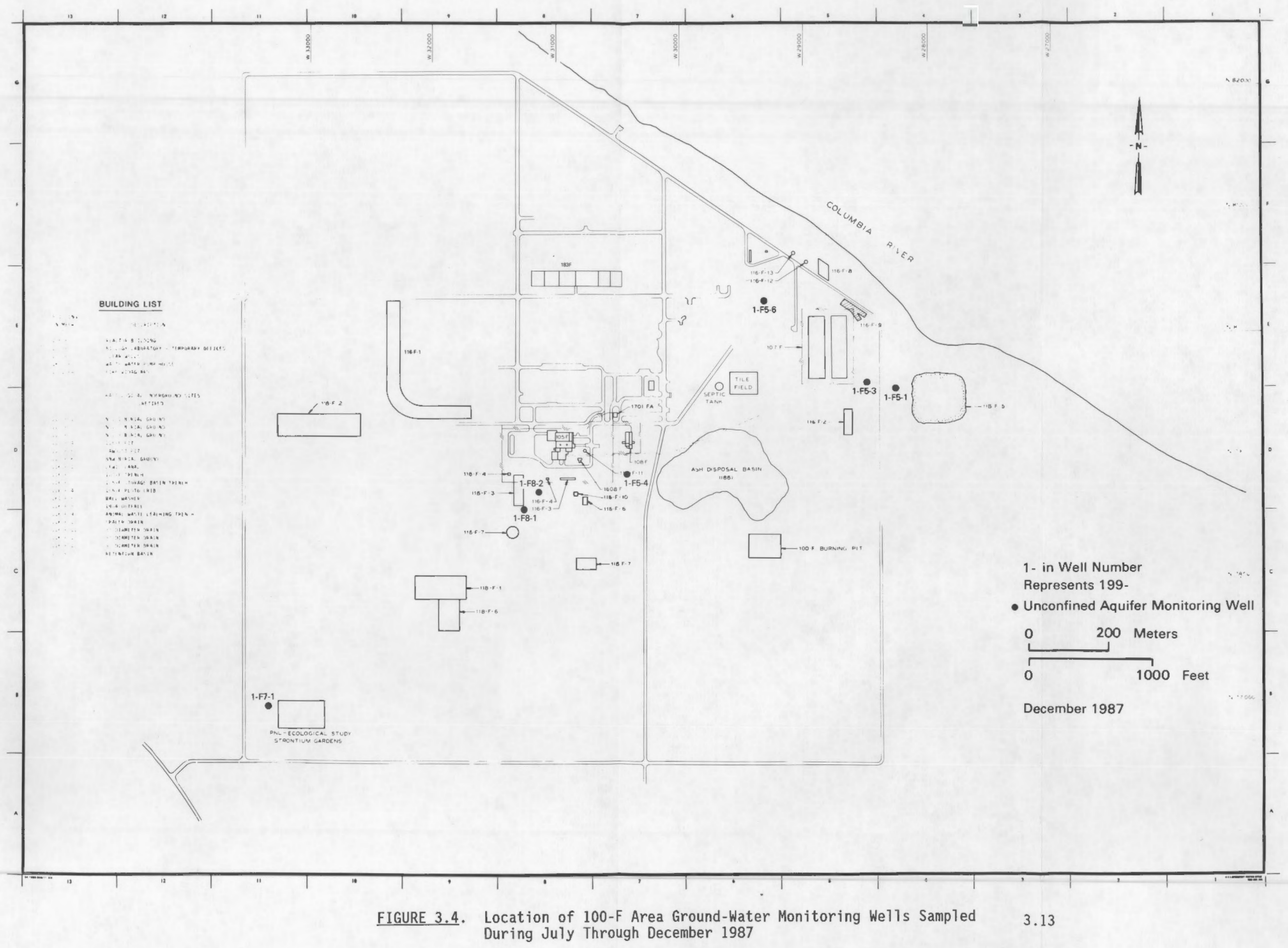





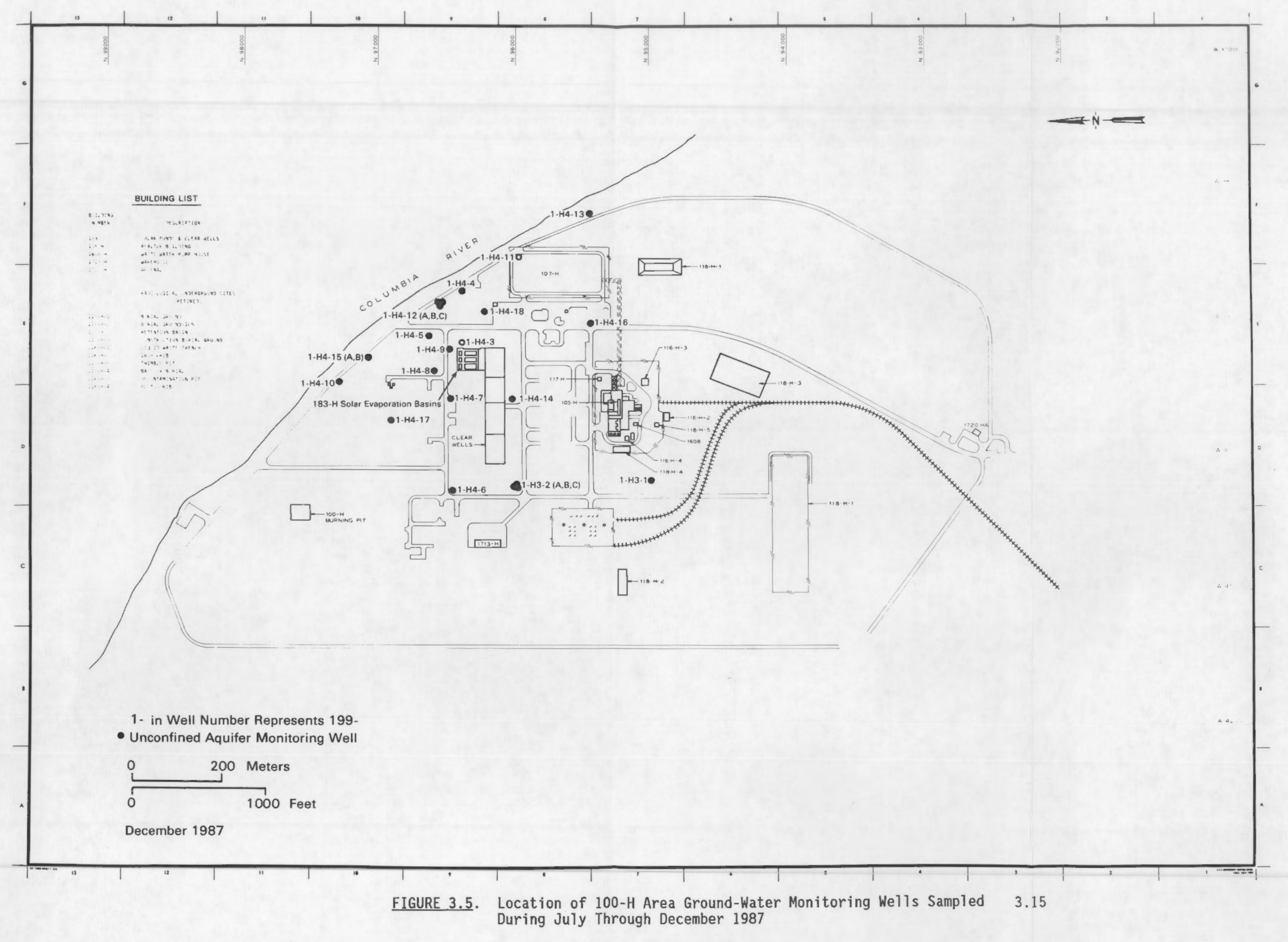





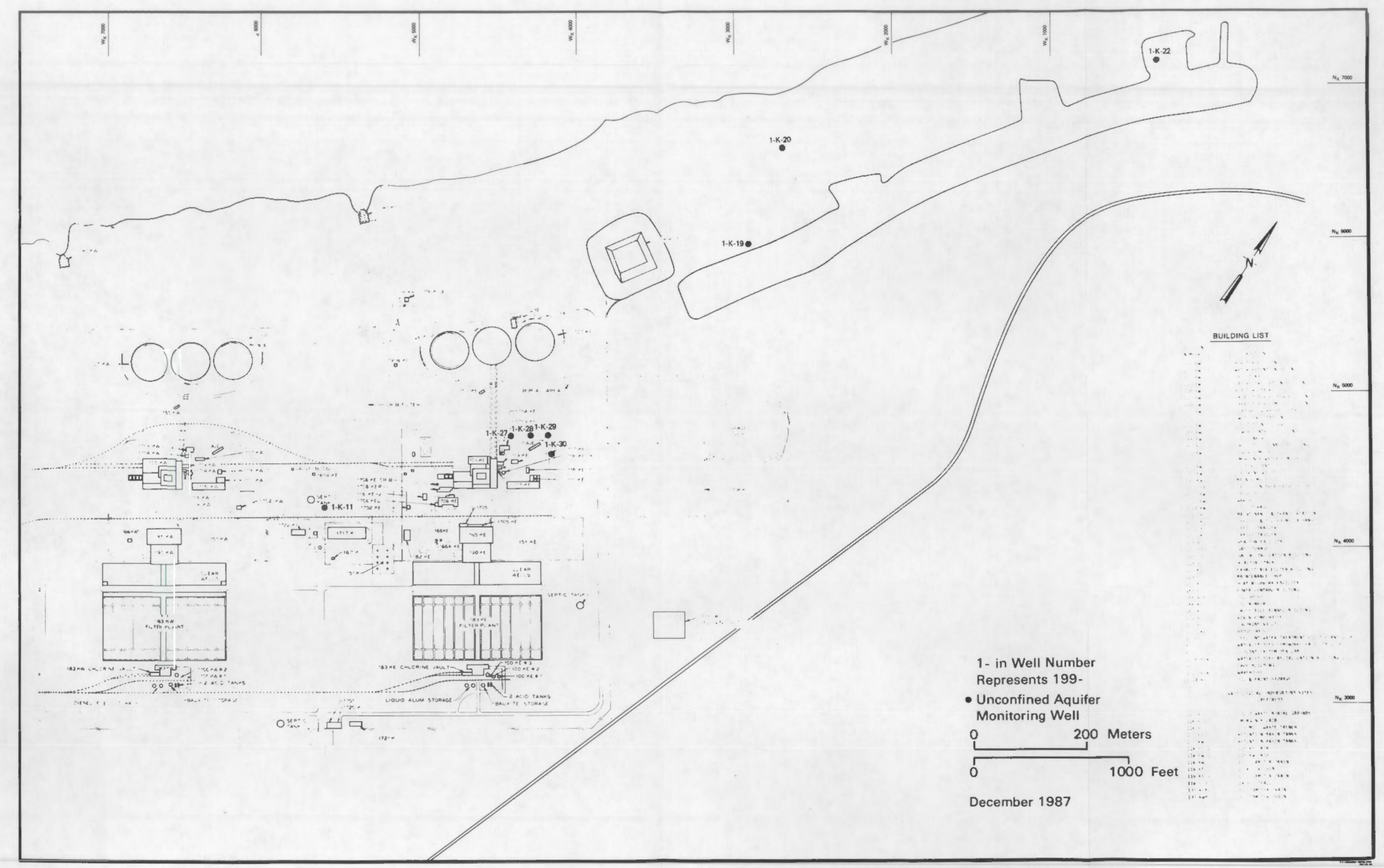

FIGURE 3.6. Location of 100-K Area Ground-Water Monitoring Wells Sampled During July Through December 1987 



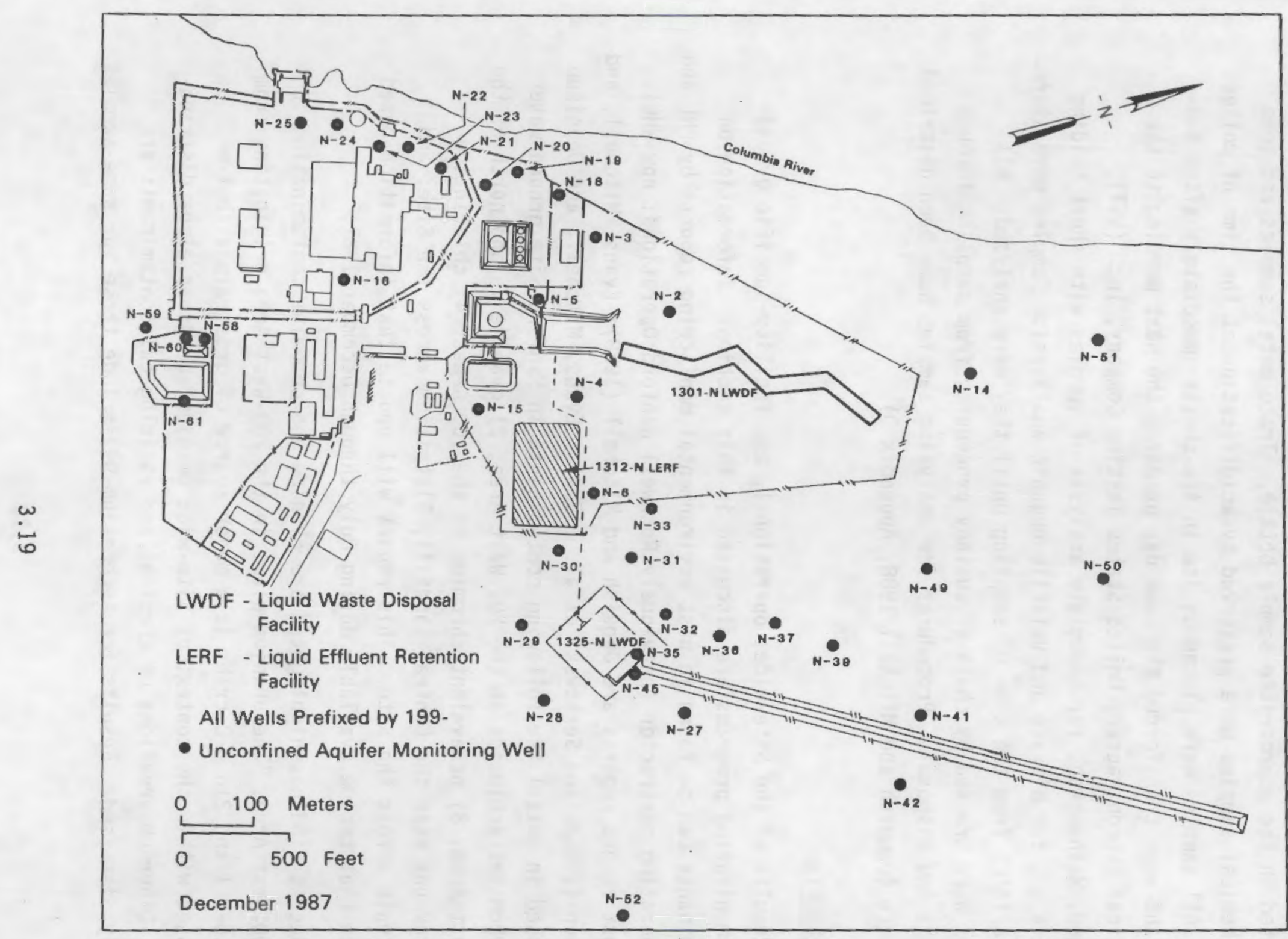

FIGURE 3.7. Location of 100-N Area Ground-Water Monitoring Wells Sampled During July Through December 1987 
septum-sealed cap. When sampling for filtered trace metals, a disposable, 0.45 -micron pore-sized filter pack was connected to the Teflon sampling line. The filter was purged with 0.13 gal $(500 \mathrm{~mL})$ of well water, then a sample was collected in the appropriate sample bottle. Trace metal samples and some radiochemical samples were preserved by acidification at the time of collection. All samples were placed on ice in ice chests immediately after sampling and were transferred the same day or early the next morning to the analytical subcontractor, United States Testing Company, Inc. (UST),

Richland, Washington, for immediate analys is of species with short holding times (e.g., for nitrate and volatile organic analyses). Samples were stored at $39^{\circ} \mathrm{F}\left(4^{\circ} \mathrm{C}\right)$ from the time of sampling until they were analyzed. All samples were tracked by chain-of-custody procedures from sampling through analysis and disposal. Procedures for analyzing samples have been described elsewhere (Jaquish and Mitchell 1988, Appendix D).

\subsection{RESULTS}

Results of the Site-wide, operational, and facility-specific groundwater monitoring programs are discussed in this section. Information on contaminants can be found in past environmental monitoring reports by PNL and the operating contractor (previousiy Rockwell Hanford Operations; now WHC). The most recent reports are Jaquish and Mitchell (1988); Evans, Mitche11, and Dennison (1988); and Serkowski et al. (1988). Evans, Mitche1l, and Dennison discussed in detail the following contaminants in Hanford Site ground water: 1) carbon tetrachloride in the 200-West Area, 2) cyanide in and north of the 200-East Area, 3) hexavalent chromium in the 100 Areas, 4) chlorinated hydrocarbons near the Central Landfi11, 5) tritium across the Site; and 6) nitrate across the Site. This report will update that information based on data that became available during July through December 1987.

Recent findings highlighted are cyanide and chromium contamination in the 200-West Area. The uranium plume in the 200-West Area originating from the 216-U-1 and 216-U-2 cribs is a major source of gross alpha that will be discussed within the context of Site-wide uranium and gross alpha distribution. Other observations of chemical and radiological contaminants are briefly discussed. Results for technetium-99 include those for some samples 


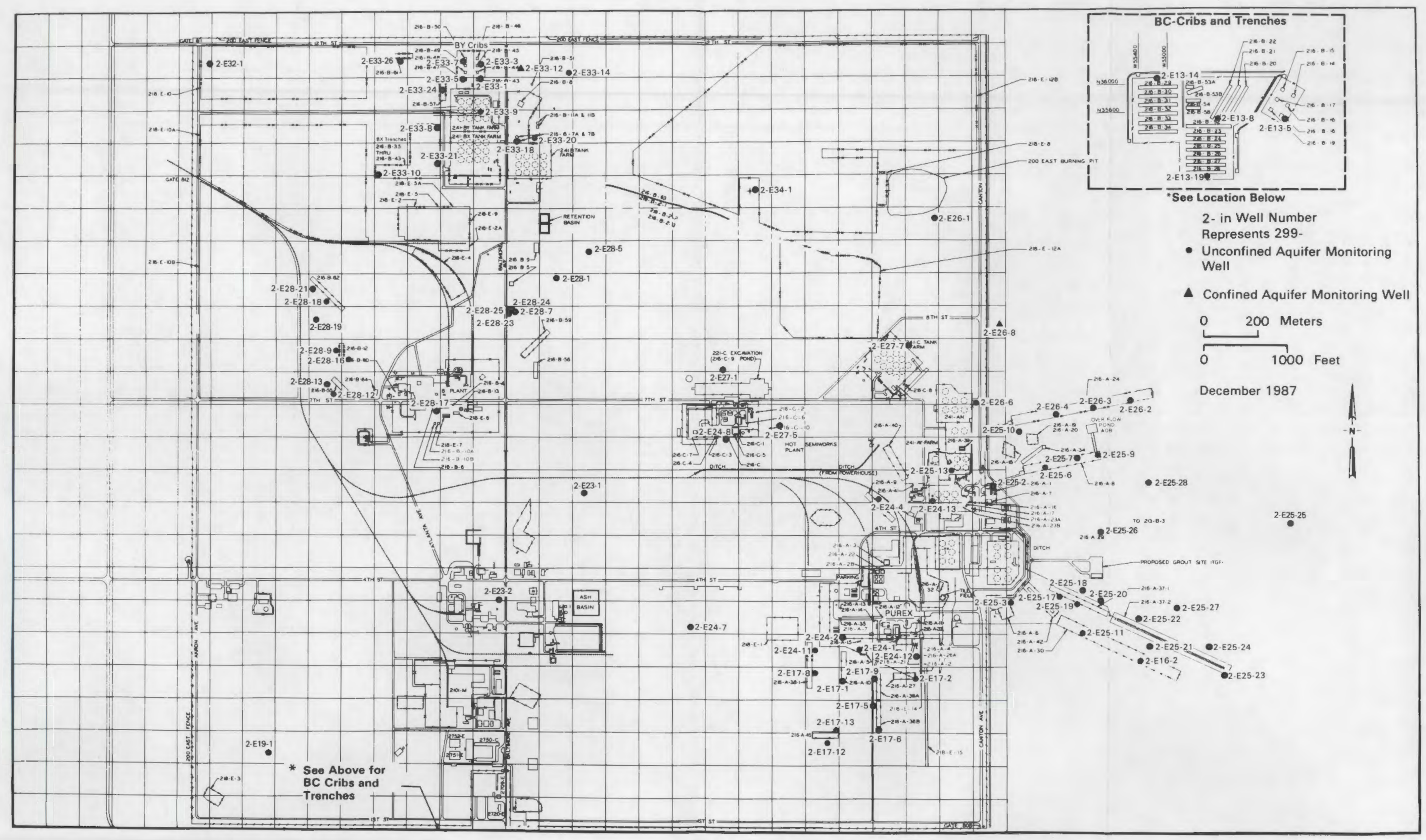
FIGURE 3.8. Location of 200-East Area Ground-Water Monitoring Wells Sampled 3.21
During July Through December 1987 



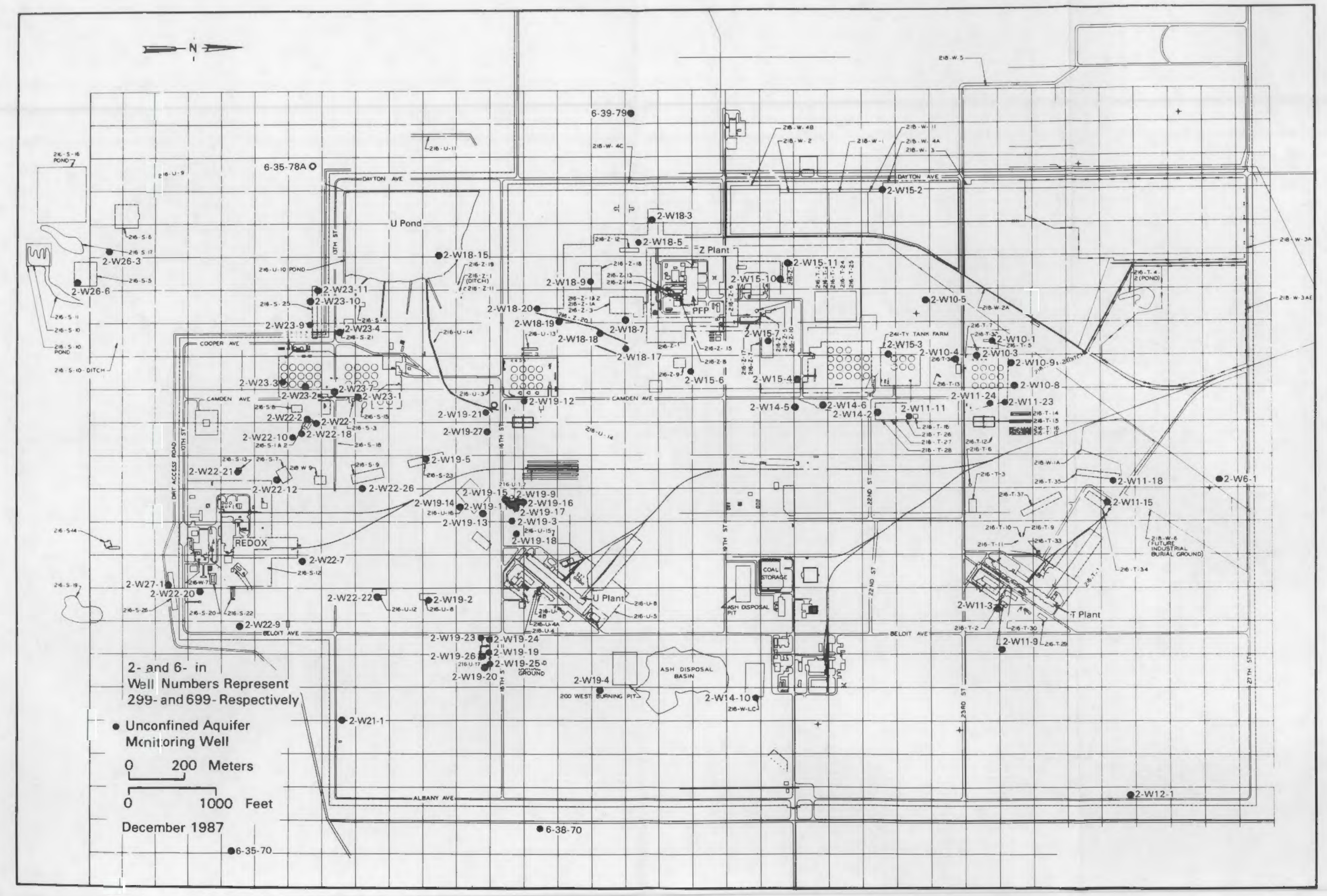

FIGURE 3.9. Location of 200-West Area Ground-Water Monitoring Wells Sampled 3.23 During July Through December 1987 



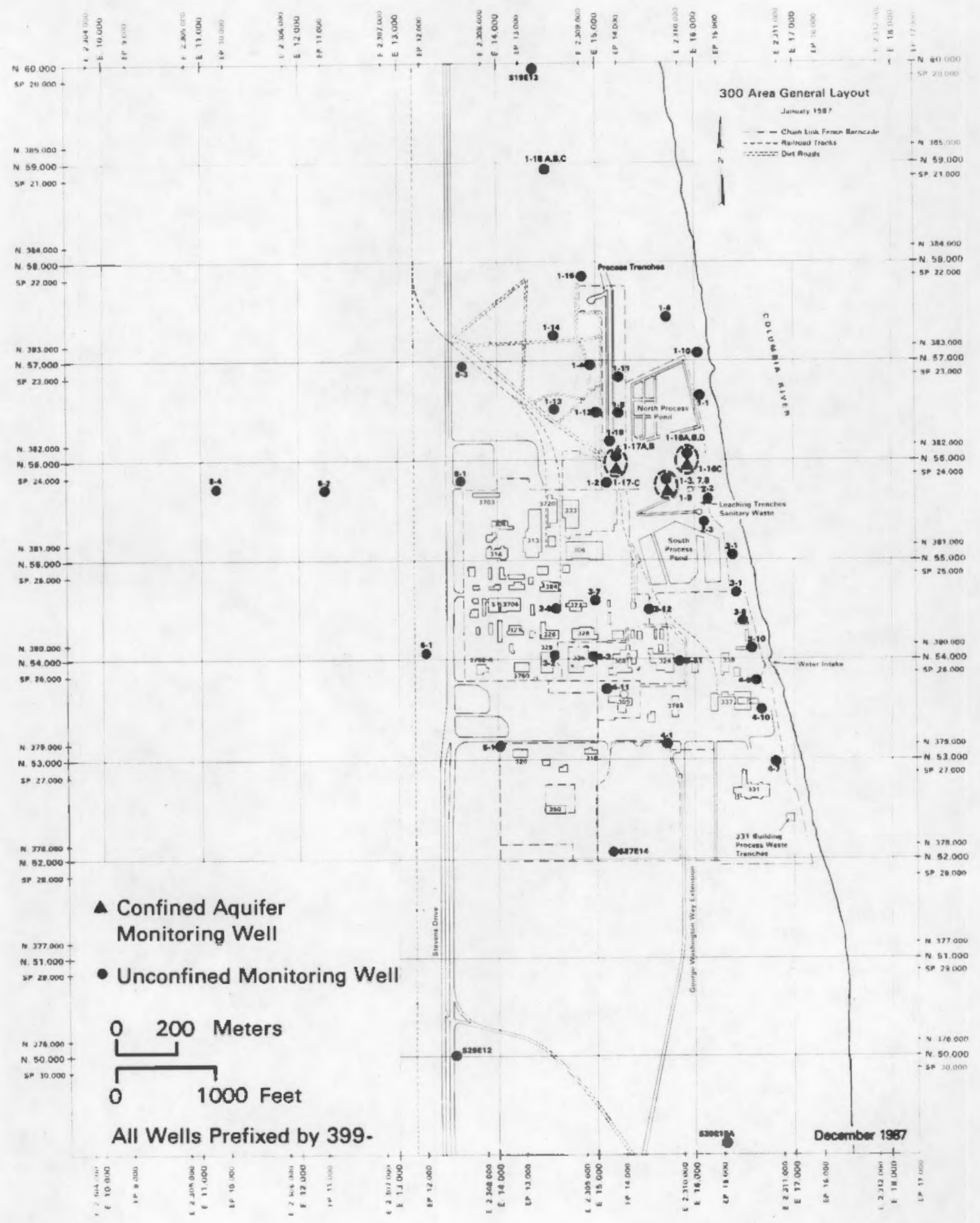

FIGURE 3.10. Location of 300 Area Ground-Water Monitoring Wells Sampled During July Through December 1987 


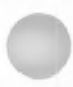

0

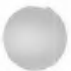




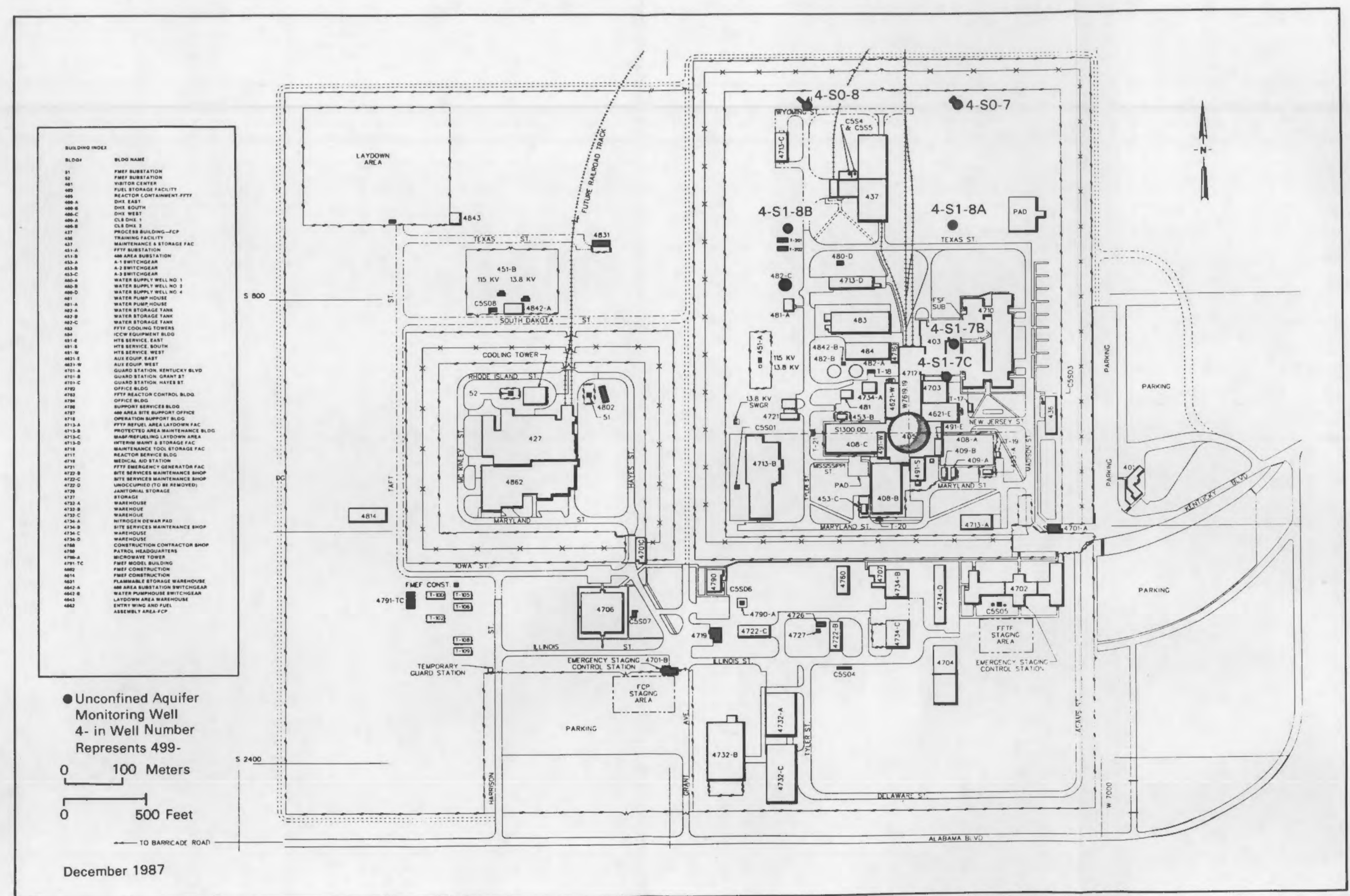

FIGURE 3.11. Location of 400 Area Ground-Water Monitoring Wells Sampled 3.27 During July Through December 1987 

collected in the previous quarter. This report also includes the neutron activation analysis results of annual iodine-129 samples.

Results are discussed relative to the EPA maximum contaminant level (MCL), to the DOE derived concentration guide (DCG) appropriate for each constituent (Appendix C), and to background concentrations. The MCLs for radionuclides are more restrictive than the DCGs because the MCLs are based on an annual dose to the affected organ of 4 mrem per year, while the DCGs are based on an effective dose of 100 mrem per year. DCGs are only relevant to radionuclides. Background concentrations of contaminants have been estimated from ground-water samples collected in areas not affected by Hanford operations. Estimates of background concentrations for selected constituents are listed in Table 3.2. Radionuclide concentrations in Hanford Site ground water are under administrative control by DOE Order 5480.1 , which contains the DCGs.

\subsubsection{Carbon Tetrachloride in the 200-West Area}

Carbon tetrachloride $\left(\mathrm{CCl}_{4}\right)$ contamination has been found beneath much of the 200-West Area. The contamination is believed to be from waste disposal operations associated with the Plutonium Finishing Plant (PFP) before 1973. Maximum concentrations detected in each well during 1987 are shown in Figure 3.12. The $\mathrm{MCL}$ for $\mathrm{CCl}_{4}$ is $5 \mathrm{ppb}$. The detection limit of the gas chromatography/mass spectrometry method used for analysis is also $5 \mathrm{ppb}$. A maximum concentration of $4520 \mathrm{ppb}$ (wel1 299-W15-11, Figure 3.12) was found near the PFP in December 1987 (10cation of the PFP is shown in Figure 3.9). The plot shows high concentrations of $\mathrm{CCl}_{4}$ near $\mathrm{T} \mathrm{Plant}$ and the PFP. The plume includes the area beneath the low-level burial ground. Carbon tetrachloride contamination from earlier Hanford Site operations in the 200-West Area is thus expected to cause ambiguities in the interpretation of monitoring data from the RCRA project for the low-level burial ground waste management units. There appears to be a plume of 1 imited areal extent across the southern end of the 200-West Area. It is difficult to define completely the $\mathrm{CCl}_{4}$ plume or plumes because of the lack of usable wells in some critical areas. A large fraction of the 200 -West Area has no wells usable for chemical monitoring, and the wells to the north are sparsely distributed. With 
IABLE 3.2. Estimated Background Levels for Selected Constituents in Hanford Ground Water

\section{Constituent}

Aluminum

Ammonia

Arsenic

Barium

Cadmium

Calcium

Chloride

Chromium

Copper

Cyanide

Fluoride

Lead

Magnesium

Manganese

Mercury

Nickel

Phosphate

Potassium

Selenium

Silver

Sodium

Strontium

Sulfate

Vanadium

Zinc

Alkalinity

$\mathrm{pH}$

Conductivity

Gross Alpha

Gross Beta

Radium

\section{Detectjon
Limit}

150

50

5

6

2

50

500

10

10

10

500

5

10

5

0.1

10

1000

100

5

10

10

300

500

5

5

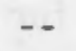

--

${ }_{1}^{200}$ (c)

$0.5^{(d)}$
$4^{(d)}$
$0.2^{(d)}$
Background Concentration $(a, b)$

$<150$

$60 \pm 47$

$<5$

$43 \pm 21$

$<2$

$43,000 \pm 14,000$

$9,430 \pm 5,530$

$<10$

$<10$

$<10$

$630 \pm 240$

$<5$

$11,700 \pm 2,750$

$16 \pm 25$

$<0.1$

$<10$

$<1000$

$5,835 \pm 1,378$

$<5$

$<10$

$20,540 \pm 6,690$

$320 \pm 86$

$40,100 \pm 13,200$

$17 \pm 7$

$10 \pm 11$

$123,000 \pm 21,000$

$7.64 \pm 0.16$

$586 \pm 347$

$380 \pm 82$

$2.5 \pm 1.4$

$19 \pm 12$

$<0.2$

(a) Units in ppb unless otherwise noted.

(b) Data printed is as received; does not indicate significant figures.

(c) Units in $\mu \mathrm{mho} / \mathrm{cm}$.

(d) Units in $\mathrm{pCi} / \mathrm{L}$. 


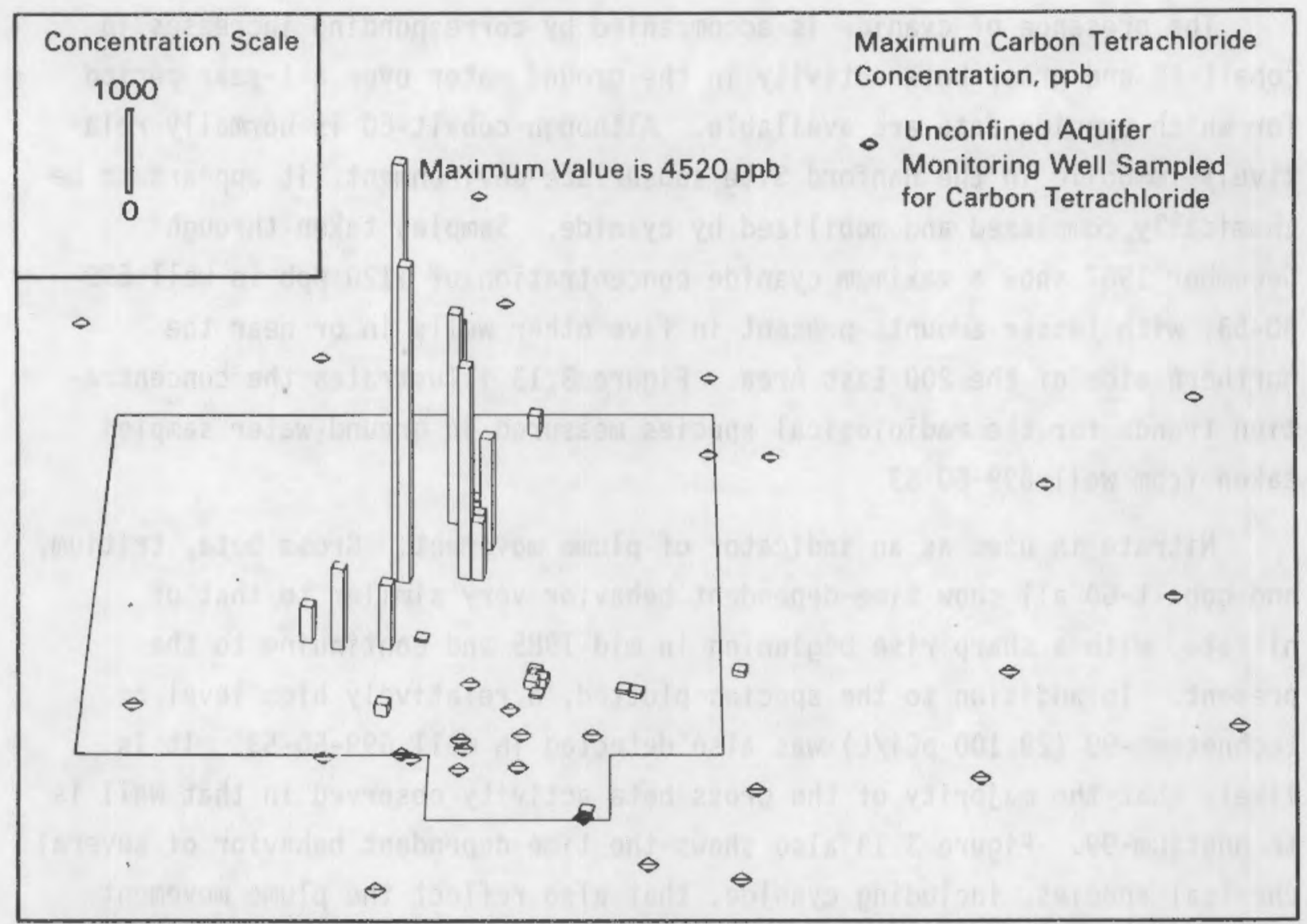

FIGURE 3.12. Maximum Carbon Tetrachloride Concentrations in 200-West Area Unconfined Aquifer Wells in 1987

the exception of trace amounts found at the Central Landfill, no significant $\mathrm{CCl}_{4}$ contamination was found outside the 200-West Area and immediate environs.

\subsubsection{Cyanide Contamination in and North of the 200-East Area}

Sampling for chemicals in March 1987 included several 600 Area wells directly north of the 200-East Area. Cyanide was detected in ground-water samples from two wells (699-49-57 and 699-50-53). More extensive sampling during the third quarter (April through June) confirmed those preliminary results. Ground water from a third well (299-E33-5) inside the 200-East Area was also found to contain cyanide. The cyanide source is believed to be wastes containing ferricyanide disposed of in the BY cribs in the 1950 s. 
The presence of cyanide is accompanied by corresponding increases in cobalt-60 and gross beta activity in the ground water over a 1-year period for which cyanide data are available. Although cobalt-60 is normally relatively immobile in the Hanford Site subsurface environment, it appears to be chemically complexed and mobilized by cyanide. Samples taken through December 1987 show a maximum cyanide concentration of $1120 \mathrm{ppb}$ in well 69950-53, with lesser amounts present in five other wells in or near the northern side of the 200-East Area. Figure 3.13 illustrates the concentration trends for the radiological species measured in ground-water samples taken from well 699-50-53.

Nitrate is used as an indicator of plume movement. Gross beta, tritium, and cobalt-60 all show time-dependent behavior very similar to that of nitrate, with a sharp rise beginning in mid-1985 and continuing to the present. In addition to the species plotted, a relatively high level of technetium-99 $(29,100 \mathrm{pCi} / \mathrm{L})$ was also detected in well 699-50-53. It is likely that the majority of the gross beta activity observed in that well is technetium-99. Figure 3.13 also shows the time-dependent behavior of several chemical species, including cyanide, that also reflect the plume movement. The extent of the contamination is currently limited to approximately $1 \mathrm{mi}^{2}$ $\left(2.5 \mathrm{~km}^{2}\right)$; however, the actual extent of the plume is difficult to determine because there are few wells in the area. No MCL for cyanide exists. The regulations specify detection-level monitoring for cyanide. The detection limit for cyanide is typically 5 to $10 \mathrm{ppb}$, using EPA's standard analytical method (SW-846 Method 9010, EPA 1982).

Low-level cyanide contamination has also been detected in three widely spaced wells in the 200-West Area (299-W12-1, 299-W14-2, and 299-W18-7) and one well (699-38-70) immediately east of the 200-West Area. The significance of these observations has not been fully assessed. Solutions containing ferrocyanide that have been disposed of in the 200-West and 200-East Areas are likely the source. Figure 3.14 illustrates the distribution of cyanide. Positive detection of cyanide is limited to the 200 Areas and immediate environs. 

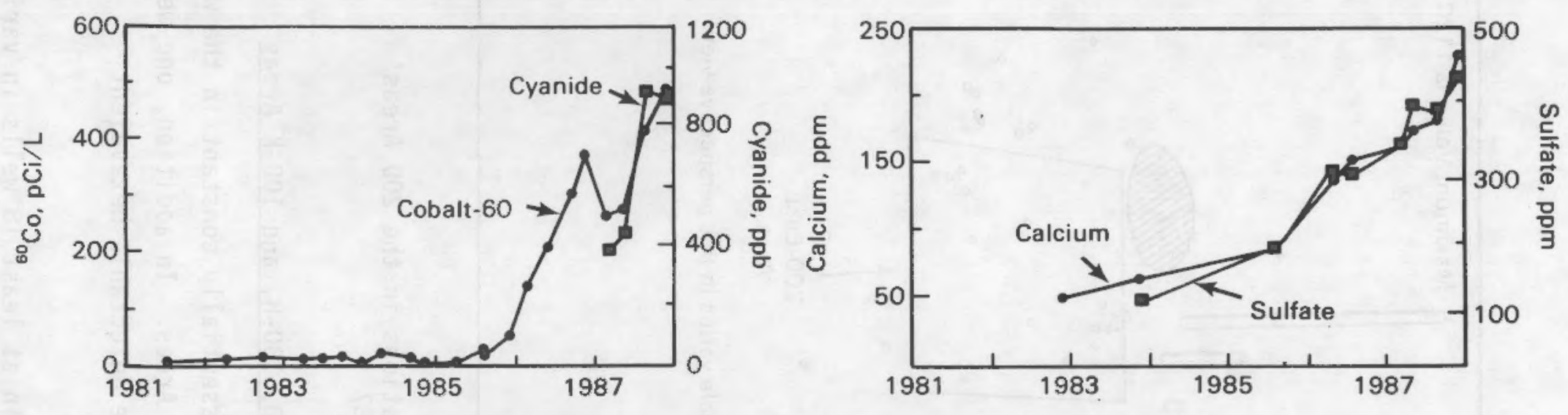

$\dot{\omega}$
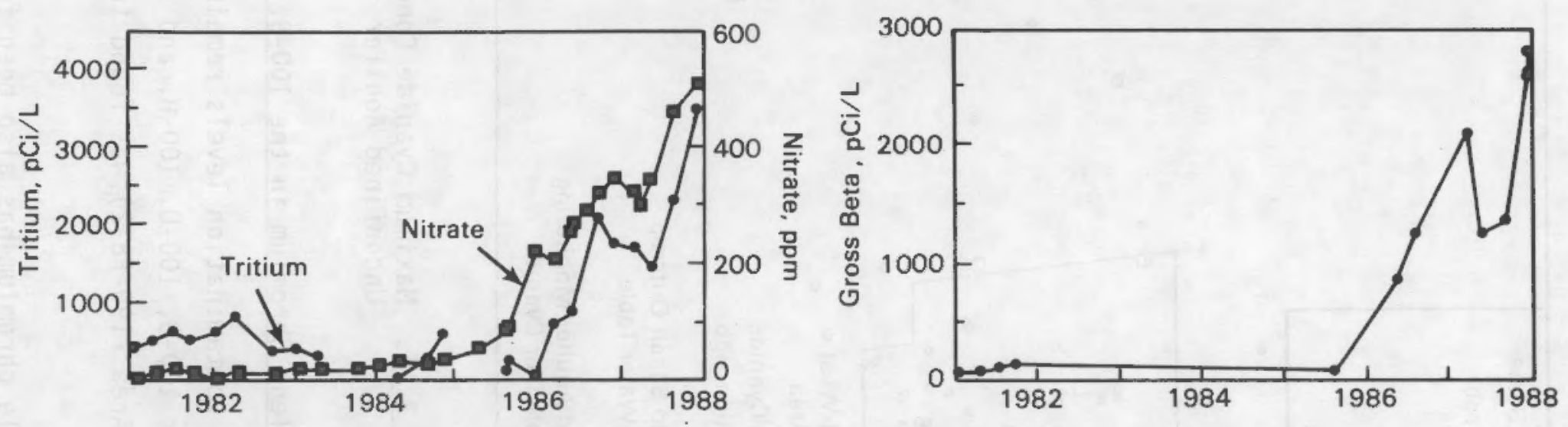

FIGURE 3.13. Cobalt-60, Cyanide, Calcium, Sulfate, Tritium, Nitrate, and Gross Beta Concentrations in Ground-Water Samples Collected from Wel1 699-50-53 


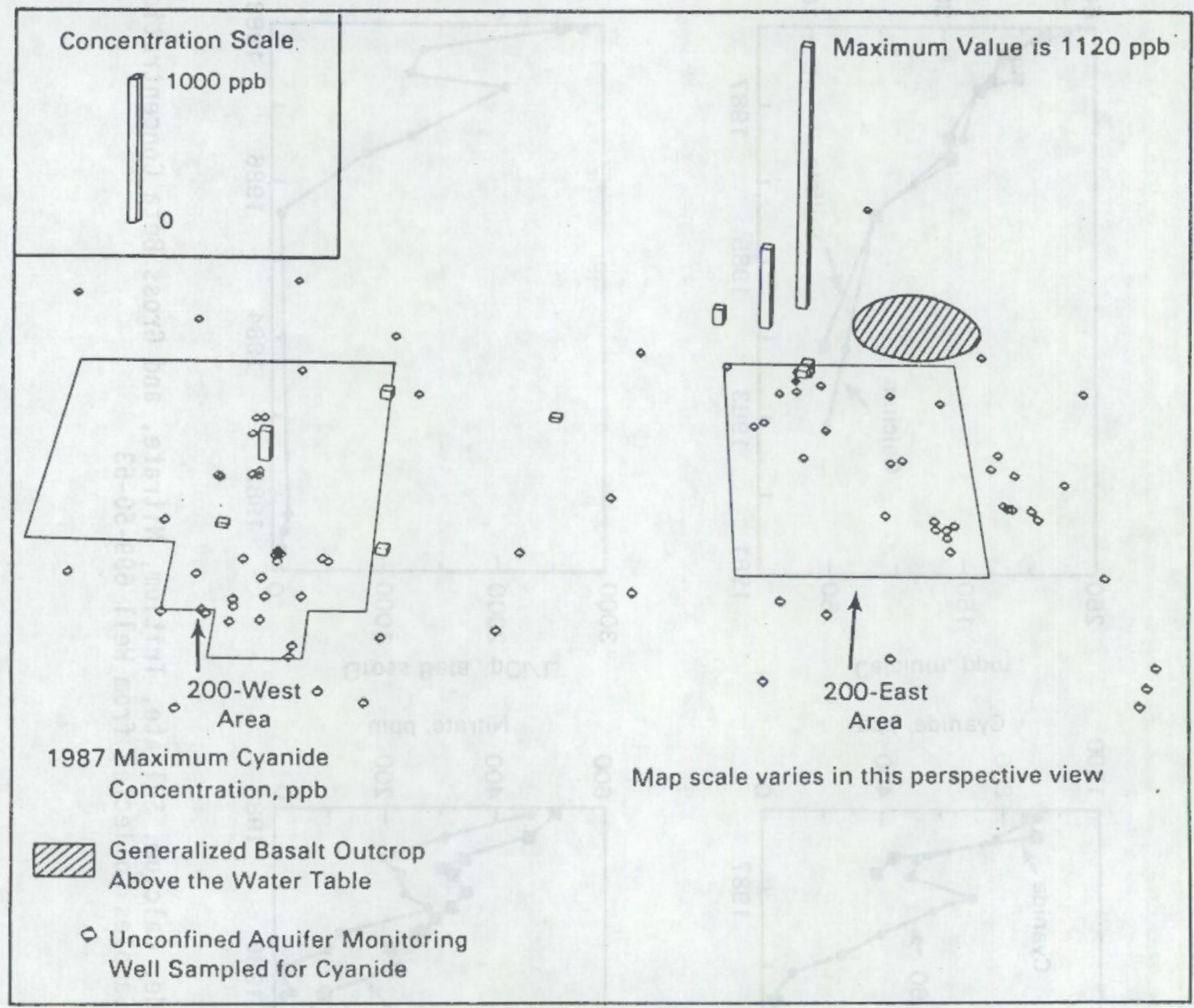

FIGURE 3.14. Maximum Cyanide Concentrations in the 200 Areas' $^{\prime}$ Unconfined Aquifer in 1987

\subsubsection{Hexavalent Chromium in the 100-B, 100-D, 100- $\mathrm{H}$, and 100-K Areas}

Chromium contamination levels remained essentially constant in the wells sampled in the 100-B, 100-D, 100- H, and 100-K Areas. In addition, one well in the 100-F Area (199-F8-2) was found to have detectable hexavalent chromium.

Detectable chromium has also been found in at least 18 wells in various parts of the 600 Area. Particularly noteworthy are three wells near the 100-D Area (699-83-47, 699-96-49, and 699-97-51A) that show chromium 
concentrations near or above the MCL. We11 699-97-43, which is approximately $0.6 \mathrm{mi}(1 \mathrm{~km})$ west of the $100-\mathrm{H}$ Area boundary, was found to have a hexavalent chromium level of $191 \mathrm{ppb}$, which is four times the MCL.

Chromium contamination also appears to be widely distributed in the 200West Area, with eight wells showing detectable chromium. Three wells in the 200-West Area (299-W10-4, 299-W10-9, and 299-W12-1) showed chromium concentrations above the $50-\mathrm{ppb}$ MCL. The distribution of chromium contamination appears to be random and may represent several independent, localized sources. A detailed delineation of the shape of the chromium plumes is not possible at this time because of the aforementioned problem of inadequate well coverage for much of the affected area.

A few wells in the 200-East Area also show evidence of minor chromium contamination. The highest level found is in well 299-E13-14, which showed a chromium concentration of $42 \mathrm{ppb}$ in December 1987.

Figure 3.15 shows the Site-wide distribution of dissolved chromium in ground water. Diamonds shown on the plot represent measurements below the detection limit of $10 \mathrm{ppb}$ for the inductively coupled-plasma spectrometry method.

\subsubsection{Chlorinated Hydrocarbons Near the Central Landfill}

Chlorinated hydrocarbon contamination levels at the Central Landfill remained essentially constant in the affected wells for three sampling periods approximately 1 month apart. In addition to the four species previously reported (1,1,1-trichloroethane, trichloroethylene, perchloroethylene, and 1,1-dichloroethane), minor amounts of $\mathrm{CCl}_{4}$ were also seen in ground-water samples from three of the wells in July. The origin of the contamination is believed to be steam stripper waste containing degreasing solvents originating from the 1100 Area motor pool maintenance operations and disposed of in the Central Landfill.

\subsubsection{Volatile Organic Compounds in the 300 Area}

Several volatile organic compounds have been detected in ground-water samples from wells in and around the 300 Area. Compounds detected during July through December 1987 include chloroform, methylene chloride, methyl ethyl ketone (MEK), 1,1,1-trichloroethane (TCA), perchloroethene (PCE), 


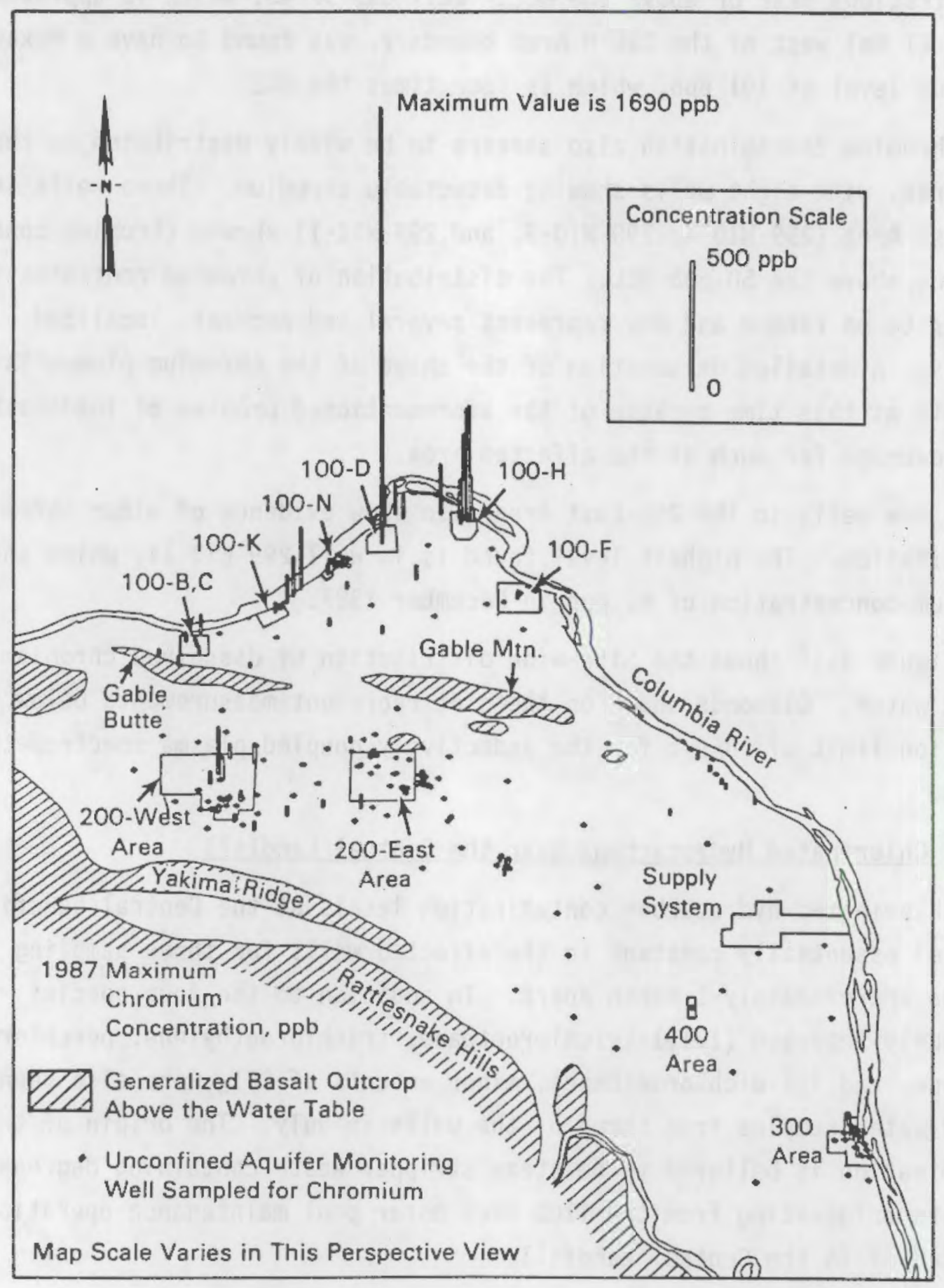

FIGURE 3.15. Maximum Chromium Concentrations in Unconfined Aquifer Wells in 1987 
trichloroethene (TCE), and 1,2-dichloroethene (1,2-DCE). Samples were collected for the RCRA Ground-Water Compliance Monitoring Project investigating the 300 Area Process Trenches.

Chloroform was detected in concentrations of 10 to $20 \mathrm{ppb}$ in a number of wells completed in the upper portion of the unconfined aquifer. Concentrations greater than the MCL $(100 \mathrm{ppb})$ were not observed in the sampled wells. Methylene chloride has been reported for samples from wells throughout the RCRA monitoring network. No pattern as to the occurrence of this constituent has been identified.

Samples collected in November from several wells (399-1-13, 399-1-16A, 399-1-16B, 399-1-18A, 399-1-18B, and 399-1-18C) contained MEK in concentrations ranging from $11 \mathrm{ppb}$ in well 399-1-16A to $45 \mathrm{ppb}$ in well 399-1-18A. This was the first observation of MEK in samples from more than one 300 Area we11. The detection of MEK in the samples may not be attributable to a ground-water source. The analytical laboratory reported MEK present in distilled water blanks analyzed during the same period as these ground-water samples. As a result of this finding, the analyst has stated that there is no conclusive evidence for MEK in the ground water. Additional samples will be collected and analyzed to confirm or refute the presence of MEK in the ground water.

The chlorinated hydrocarbon TCA was present in a least one ground-water sample collected from each of the following wells: 399-1-4, 399-1-11, 399-1-19, 399-8-2, and 699-S19-E13. Wel1 399-8-2 contained the highest concentration of TCA observed during the reporting period (19 ppb in November). A sample from this well contained $83 \mathrm{ppb}$ of TCA in March; however, the compound was not detected in samples taken during August or June. The MCL for TCA is $200 \mathrm{ppb}$.

Perchloroethene (PCE) and TCE have been detected in ground-water samples from 300 Area wells. Perchloroethene has generally been detected only in wells completed in the upper portion of the unconfined aquifer, while TCE is detected in both shallow and intermediate-depth wells. Concentrations of PCE in samples collected from shallow wells ranged from 1 to $6 \mathrm{ppb}$ between July 
and December. Well 399-8-2 contained $42 \mathrm{ppb}$ of PCE in March, but the concentration declined to $4 \mathrm{ppb}$ in August and $6 \mathrm{ppb}$ in November. No MCL is in effect for PCE.

Several wells (399-1-16B, 399-1-16C, 399-4-1, and 399-4-11) contained concentrations of TCE greater than the MCL (5 ppb) in at least one sample. The highest concentrations of TCE were found in well 399-1-16B, which had an average concentration of $21 \mathrm{ppb}$ from June to November (unchanged from previous measurements). Well 399-1-16C is completed in the confined aquifer and contained $10 \mathrm{ppb}$ of TCE in June and $2 \mathrm{ppb}$ in November.

A degradation isomer of TCE, 1,2-DCE, has been detected consistently in samples from intermediate-depth wells 399-1-16B and 399-1-17B. The recommended MCL for 1,2-DCE is $70 \mathrm{ppb}$. The average concentrations in samples taken in June, August, and November were $66 \mathrm{ppb}$ in well 399-1-16B and $25 \mathrm{ppb}$ in well 399-1-17B. These concentrations were essentially unchanged from concentrations measured previously in 1987. The only shallow wells that contained 1,2-DCE were 399-1-17A and 399-1-19, which contained concentrations ranging from 1 to $3 \mathrm{ppb}$. Well 399-1-16C contained $20 \mathrm{ppb}$ of 1,2-DCE in June, but the compound was not detected in subsequent samples.

The detection of organic compounds in we11 399-1-16C appears to be related to inadequate sealing of well 399-1-16D. Water levels in well 399-1-16C declined $20 \mathrm{ft}$ from February (the month in which the well was installed) to May 1987. No similar decline in water levels has been noted in other deep wells in the vicinity. The ground-water chemistry data from the June sampling of well 399-1-16C showed the presence of TCE, 1,2-DCE, and low concentrations of chloroform. Previously these compounds had only been detected in wells completed in the unconfined aquifer. Apparently all intervals of the local unconfined aquifer have been hydraulically interconnected as a result of inadequate sealing of well 399-1-16D. As a temporary measure to prevent the movement of these compounds from the unconfined aquifer into the confined aquifer, purging of well 399-1-16C to obtain a sample of the water in the confined aquifer has been limited.

The source of the organic compounds detected in 300 Area wells is unclear. With the exception of chloroform, which is normally only detected in wells adjacent to or downgradient from the process trenches, most of the 
organic compounds have been detected in wells upgradient from the process trenches (e.g., wells 399-8-2, 699-S19-E13, and wells in the 399-1-18 cluster) in addition to wells downgradient from the trenches. The widespread occurrence of the compounds suggests that sources other than, or in addition to, the process trenches may be involved.

\subsubsection{Nitrate Across the Site}

Nitrate is associated with process condensate and other liquid wastes discharged to the ground (PNL 1987). The extent of nitrate contamination in the unconfined aquifer reflects the extensive use of nitrogen-bearing compounds (e.g., nitric acid and ammonia) in chemical reprocessing and decontamination operations.

Nitrate is analyzed for in most wells in all monitoring networks and was measured at concentrations greater than the MCL [45 ppm(a) as nitrate ion] in wells in all operational areas, except for the 100-B and 400 Areas. A plot of the maximum nitrate concentration in ground water from each well sampled throughout the Site is presented in Figure 3.16.

\subsubsection{Nitrate in the 100 Areas}

In general, nitrate concentrations in wells in the 100-D, 100-F, and 100-K Areas were relatively unchanged from previous measurements. Concentrations in wells 199-D2-5 and 199-D5-12 remained above the MCL, with concentrations in July of 82 and $67 \mathrm{ppm}$, respectively. Concentrations in well 199-F8-1 continued to increase, rising from a concentration of $184 \mathrm{ppm}$ in July to $218 \mathrm{ppm}$ in 0ctober. Levels in the $100-\mathrm{F}$ Area that remained above the MCL were those in wells 199-F5-4, 199-F7-1, and 199-F8-2, with average concentrations of 67,100 , and $93 \mathrm{ppm}$, respectively. Nitrate concentrations in well 699-77-26, located west of the 100-F Area, remained above the MCL, averaging $60 \mathrm{ppm}$. Levels in wells 199-K-11, 199-K-19, and 199-K-30 remained above the MCL, with respective concentrations of 48,69 , and $49 \mathrm{ppm}$ in July.

Nitrate concentrations remained greater than the $\mathrm{MCL}$ in six $100-\mathrm{H}$ Area wells (with average concentration): 199-H3-1 (67 ppm), 199-H4-3 (473 ppm), 199-H4-4 (310 ppm), 199-H4-9 (179 ppm), and 199-H4-2A (78 ppm). In addition,

(a) $\mathrm{ppm}=\mathrm{ppb} / 1000$. 


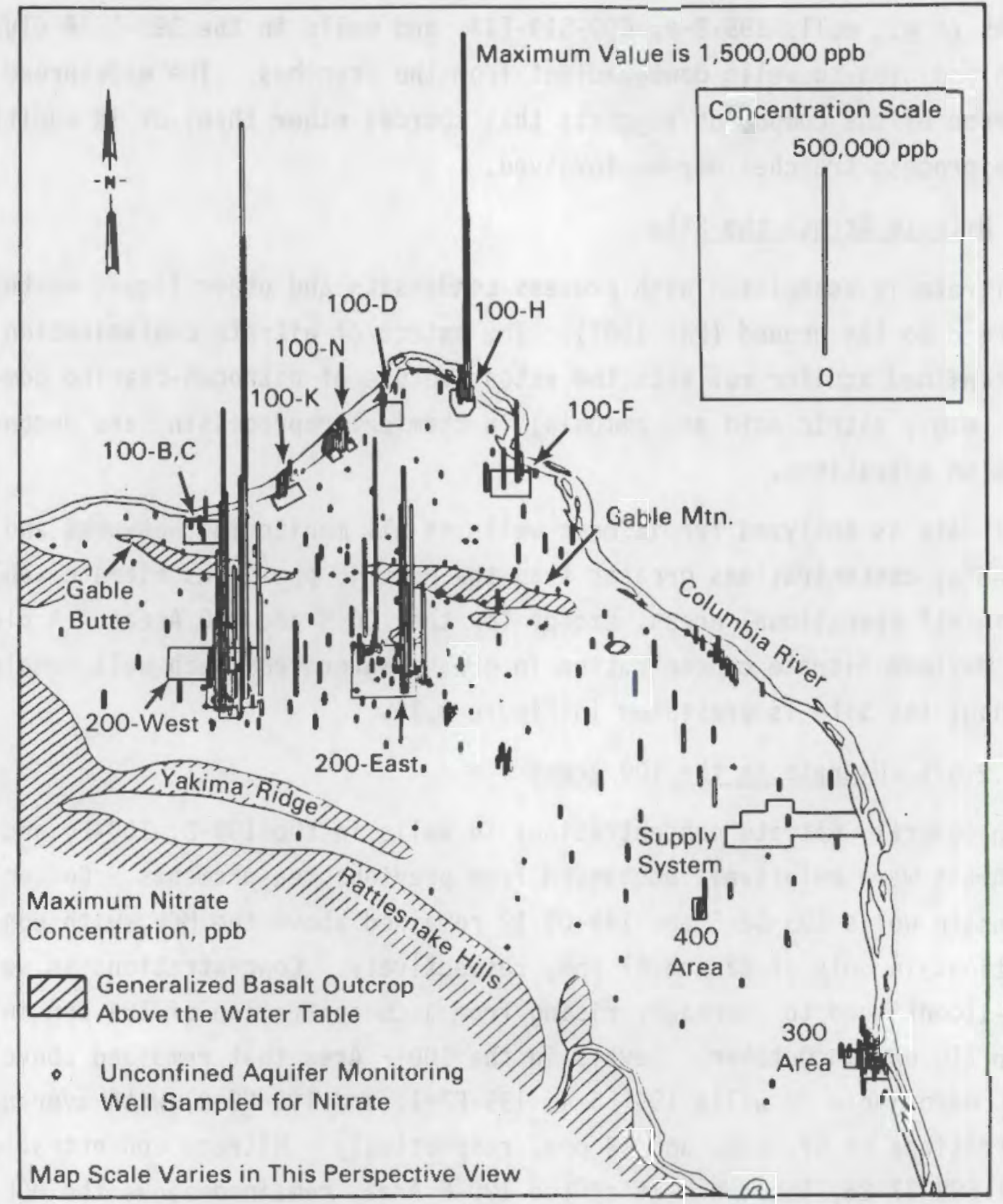

FIGURE 3.16. Maximum Nitrate Concentration in Unconfined Aquifer Wells in 1987 
concentrations in wells $199-\mathrm{H} 4-12 \mathrm{~A}$ and 199-H4-17 remained at or slightly above the MCL. With the exception of wells 199-H3-1 and 199-H4-17, all of these wells are located between the 183-H Solar Evaporation Basins and the Columbia River.

Nitrate concentrations in most $100-\mathrm{N}$ Area wells continued to decrease or stabilized at levels less than the MCL. Concentrations of 10 to $20 \mathrm{ppm}$ greater than the MCL were measured in ground-water samples from wells 199$\mathrm{N}-28,199-\mathrm{N}-32,199-\mathrm{N}-33,199-\mathrm{N}-39$, and 199-N-49. Wells 199-N-36, 199-N-37, and 199-N-45 previously contained nitrate in concentrations above the MCL, but concentrations fell below that level during this reporting period.

\subsubsection{Nitrate in the 200-East Area}

The highest nitrate concentrations in the 200-East Area continued to be found near liquid waste disposal facilities (LWDFs) that receive effluent from PUREX plant operations. Well 299-E25-13 had the highest nitrate concentration measured in the 200-East Area, with an average concentration of $385 \mathrm{ppm}$. Although recent changes in the disposal of liquid waste from the PUREX Plant will modify the distribution and concentration of nitrate and other ground-water constituents in wells near PUREX LWDFs, insufficient data are available for the identification of trends resulting from these changes. Nitrate concentrations in wells 299-E17-1 and 299-E24-2, located near the 216-A-10 crib, which was removed from use in early 1987, remained above the $\mathrm{MCL}$, with average concentrations of 370 and $180 \mathrm{ppm}$, respectively. Wells 299-E17-12 and 299-E17-13, located near the 216-A-45 crib, which replaced the 216-A-10 crib in receiving process condensate from the PUREX plant, contained average nitrate concentrations of 60 and $50 \mathrm{ppm}$, respectively. Discharge of ammonia scrubber waste from PUREX to the 216-A-36B crib was discontinued in 1987. Nitrate concentrations in wel1s 299-E17-5 and 299-E17-9, which monitor the 216-A-368 crib, remained above the $\mathrm{MCL}$, with average concentrations of 180 and $130 \mathrm{ppm}$, respectively. Other wells, located south of PUREX in the vicinity of the $216-\mathrm{A}-10,216-\mathrm{A}-36 \mathrm{~B}$, and $216-\mathrm{A}-45$ cribs, that contained nitrate in concentrations greater than the $\mathrm{MCL}$ include (with average concentration): 299-E17-8 (260 ppm), 299-E24-1 (310 ppm), 299-E24-11 (280 ppm), and 299-E24-12 (110 ppm). 
Other 200-East Area wells with nitrate concentrations greater than the $M C L$ are found near the following cribs: 216-A-30, 216-A-37-1, 216-B-62, and the $B Y$ cribs (i.e., cribs 216-B-43 through 216-B-50). Nitrate concentrations in wel1 299-E25-11 near the 216-A-30 crib, a PUREX steam-condensate-disposal crib, were above the MCL in June and July, but fell below that level during the remainder of 1987. Although the concentrations were fluctuating, nitrate in we11s 299-E25-19 and 299-E25-20, located near the 216-A-37-1 crib that receives process condensate from the $242-A$ evaporator, remained greater than the MCL. Average concentrations in we1ls 299-E25-19 and 299-E25-20 were 106 and $174 \mathrm{ppm}$, respectively.

The existence of a nitrate plume emanating from the southeastern corner of the 200-East Area, as indicated by increasing nitrate concentrations in wells 699-32-43, 699-33-42, and 699-37-43, has been reported previously (Evans, Mitche11, and Dennison 1987). Nitrate concentrations in wells 699-32-43 and 699-33-42 continued to increase and approached the MCL, with each well containing $41 \mathrm{ppm}$ in samples taken in August. The concentration in well 699-37-43 also continued to increase, but remained approximately four times less than the MCL.

Adjacent to the 216-B-62 crib, which receives $B$ Plant process condensate, well 299-E28-18 contained 73 ppm nitrate in July, but the concentration had declined to $36 \mathrm{ppm}$ by December. Five wells in the northern part of the 200-East Area and the adjacent 600 Area also contained nitrate in concentrations greater than the MCL. We11s 299-E33-1 and 299-E33-3, located near the BY cribs, contained concentrations of 54 and $61 \mathrm{ppm}$, respectively, in August, but these concentrations had fallen to 14 and $38 \mathrm{ppm}$ in December. Immediately north of the BY cribs, wells 699-49-55A, 699-49-57, and 699-50-53 had average nitrate concentrations of 245,65 , and 495 , respectively, which were relatively unchanged from the previous quarter.

\subsubsection{Nitrate in the 200-West Area}

Nitrate concentrations greater than the $M C L$ are widespread in the ground water beneath the 200-West Area. The highest concentrations appeared to be centered in three locations: 1) wells near U Plant, 2) wells in the northwestern part of the 200-West Area, and 3) wells near the 216-S-25 crib. 
The highest nitrate concentrations measured across the Site continued to be found in wells east of U Plant near the 216-U-17 crib, which has never been used. The source of nitrate is believed to be wastes disposed of in the 216-U-1 and 216-U-2 cribs. These cribs received over 1 million $\mathrm{kg}$ of nitrate during their operation from 1951 to 1967 (DOE 1986). Wells 299-W19-19, 299-W19-20, 299-W19-23, 299-W19-24, 299-W19-25, and 299-W19-26 had average nitrate concentrations of $1360,950,530,1330,680$, and $870 \mathrm{ppm}$, respectively. With the exception of well 299-W19-26, in which nitrate concentrations increased, concentrations in these wells remained constant throughout 1987. Nitrate concentrations in several wells located near the 216-U-1 and 216-U-2 cribs west of U Plant also remained greater than the MCL, but were generally decreasing. Concentrations in wells 299-W19-3, 299-W19-9, 299-W19-11, 299-W19-15, and 299-W19-18 decreased from 105, 129, 118, 98, and $253 \mathrm{ppm}$ in June, respectively, to $71,48,102,61$, and $167 \mathrm{ppm}$ in December. Other wells in the vicinity of $U \mathrm{Plant}$ with nitrate concentrations greater than the MCL were well 299-W19-2, near the inactive 216-U-8 crib, with an average concentration of 455 ppm, and wells 299-W19-13 and 299-W19-14, near the inactive $216-\mathrm{U}-16 \mathrm{crib}$, with average concentrations of 256 and $298 \mathrm{ppm}$, respectively. Nitrate concentrations in well 299-W19-2 fluctuated throughout 1987, while the concentrations in wells 299-W19-13 and 299-W19-14 remained relatively constant, except for large nitrate increases in samples from both wells taken in December.

Several wells in the northwestern part of the 200-West Area continued to contain nitrate in concentrations greater than the MCL. These wells (299-W10-4, 299-W10-9, 299-W11-23, 299-W11-24, 299-W14-2, 299-W14-5, 299-W15-3, and 299-W15-4) are located near several inactive LWDFs that received waste from early $\mathrm{T} P \mathrm{Pl}$ ant operations. Average concentrations in these wells ranged from 46 ppm in well 299-W14-5 to 540 ppm in well 299-W15-4 and were equal to or lower than those previously measured.

Wells 299-W23-9 and 299-W23-10 monitor the 216-S-25 crib, which received waste from the 242-S evaporator, and contained average nitrate concentrations of 210 and $194 \mathrm{ppm}$, respectively. Nitrate concentrations in these we1ls decreased throughout the reporting period. 
Other 200-West Area wells that contained nitrate concentrations greater than the MCL were 299-W6-1, 299-W14-10, 299-W15-10, 299-W15-11, 299-W22-20, and 299-W27-1, with average concentrations of 223,80,103,136, 131, and 95 ppm, respectively. Concentrations in 600 Area wells 699-38-70, 699-40-62, and 699-44-64, located in the nitrate plume between the 200-East and 200-West Areas, remained near or greater than the $\mathrm{MCL}$, with average concentrations of 245, 46, and $49 \mathrm{ppm}$, respectively. Well 699-38-65, also located within the plume, contained $189 \mathrm{ppm}$ nitrate in August, but the concentration had declined to $54 \mathrm{ppm}$ in November.

\subsubsection{Nitrate in Other Areas}

Nitrate concentrations in 300 Area wells remained relatively unchanged, with those in well 399-5-1 remaining above the $\mathrm{MCL}$, with an average concentration of $53 \mathrm{ppm}$. Concentrations in wells in the 400 Area were unchanged, with no concentrations above the MCL. Wells in the 600 Area not already mentioned that had concentrations greater than the MCL include 699-17-5, 69937-82A, 699-38-15, and 699-61-62, with average concentrations of $66,47,54$, and $49 \mathrm{ppm}$, respectively.

\subsubsection{0ther Observations}

Several other chemical and radiological species detected at levels that may be related to Hanford Site operations are discussed below.

\subsubsection{Ammonia}

Numerous wells (59) show evidence of the presence of ammonia. Ammonia and ammonium salts have been widely used in a variety of applications on the Site. Two locations are worth noting because both are in operating areas. Wells 199-N-28 and 199-N-29 have average ammonia levels of 1480 and $2570 \mathrm{ppb}$, respectively. The same two wells also showed positive detection of phosphate. It is likely that the material originated as a decontamination solution containing ammonium phosphate. Wells 299-E28-18 and 299-E28-21 show maximum ammonia levels of 890 and $234 \mathrm{ppb}$, respectively, but these concentrations are widely variable. Both wells are near the 216-B-62 crib that receives $\mathrm{B} P \mathrm{Pl}$ ant process condensate. Ammonia is also used in the process to remove the zircaloy cladding from $\mathrm{N}$ Reactor fuel. 


\subsubsection{Fluoride}

A number of closely spaced wells in the 200-West Area show elevated levels of fluoride. Affected wells include 299-W10-4, 299-W10-9, 299-W14-5, 299-W14-6, and 299-W15-4, with maximum concentrations of 3420, 5380, 3520, 3410 , and $12,700 \mathrm{ppb}$, respectively. The wells are all downgradient from LWDFs that received aluminum fluoride nitrate from $\mathrm{Z} \mathrm{plant}$. Fluoride levels outside the 200-West Area are uniformly low. A plot of the Site-wide distribution of fluoride is given in Figure 3.17.

\subsubsection{Sulfate}

Elevated levels of sulfate have been detected at several locations. We11s 199-N-58, 199-N-59, 199-N-60, and 199-N-61 contain very high concentrations of sulfate, ranging up to $819,000 \mathrm{ppb}$. The sulfate in these wells results from the disposal of ion-exchange-filter backwash. Wells north of the BY cribs also show elevated sulfate, with well 699-50-53 reaching a maximum of $474,000 \mathrm{ppb}$ in December (see Figure 3.13). The MCL for sulfate is $250,000 \mathrm{ppb}$. A plot of the Site-wide distribution of sulfate is given in Figure 3.18 .

\subsubsection{Selenium}

We11 299-W27-1 showed the presence of selenium at five times the MCL on four successive measurements. The explanation is unknown because there is no documented use of selenium at Hanford. Selenium levels are low or below detection in all other wells sampled.

\subsubsection{Acetone}

Acetone has been detected in ground-water samples from seven wells that are widely distributed throughout the Site. No discernible pattern exists, and the observations have not recurred on resampling of these wells. This observation is probably a result of sampling or laboratory contamination.

\subsubsection{Chlorinated Hydrocarbons}

Methyl ethyl ketone was detected in one well (299-E13-5) at a maximum level of $50 \mathrm{ppb}$. The significance of the observation is unknown; however, it 


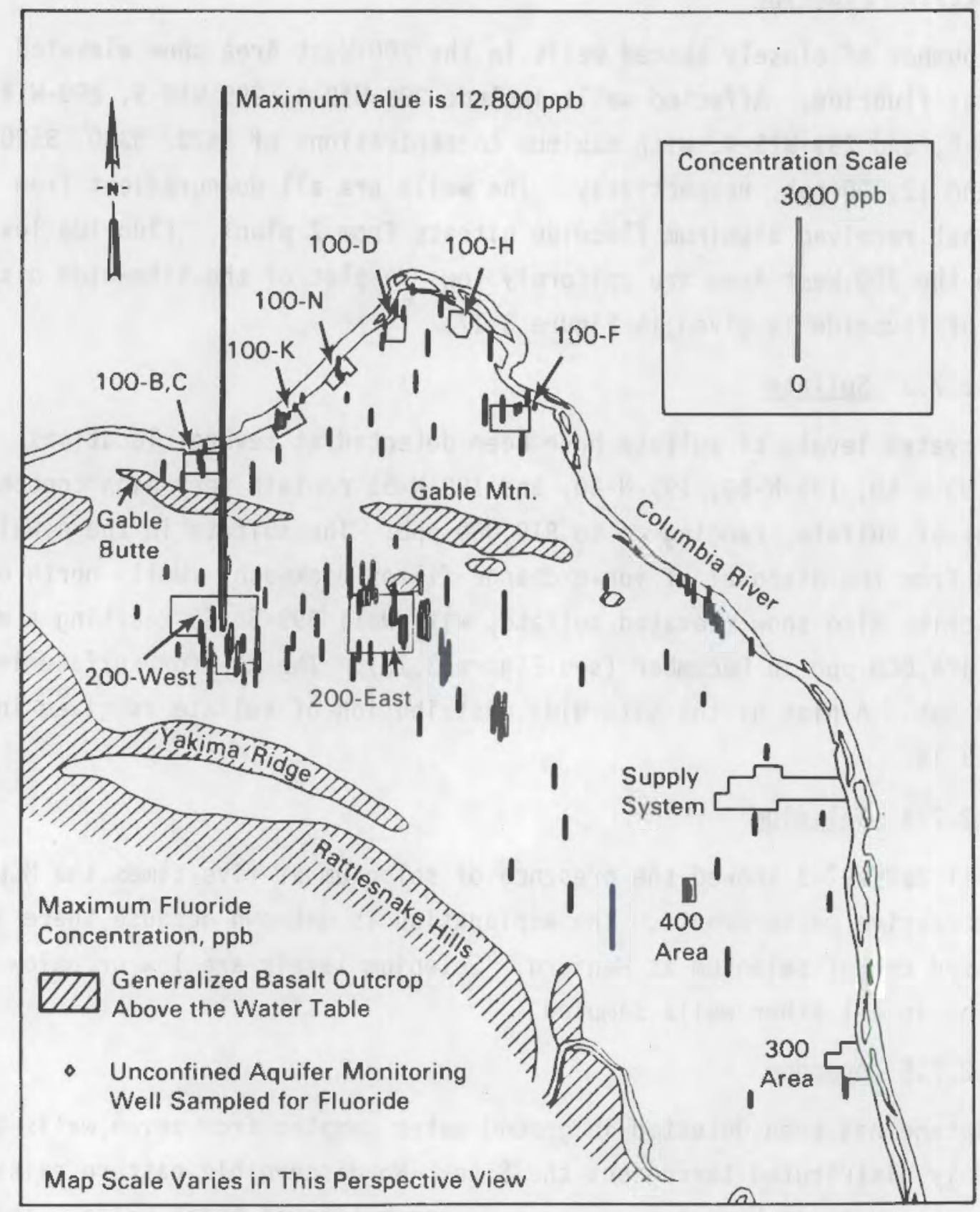

FIGURE 3.17. Maximum Fluoride Concentrations in Unconfined Aquifer Wells in 1987 


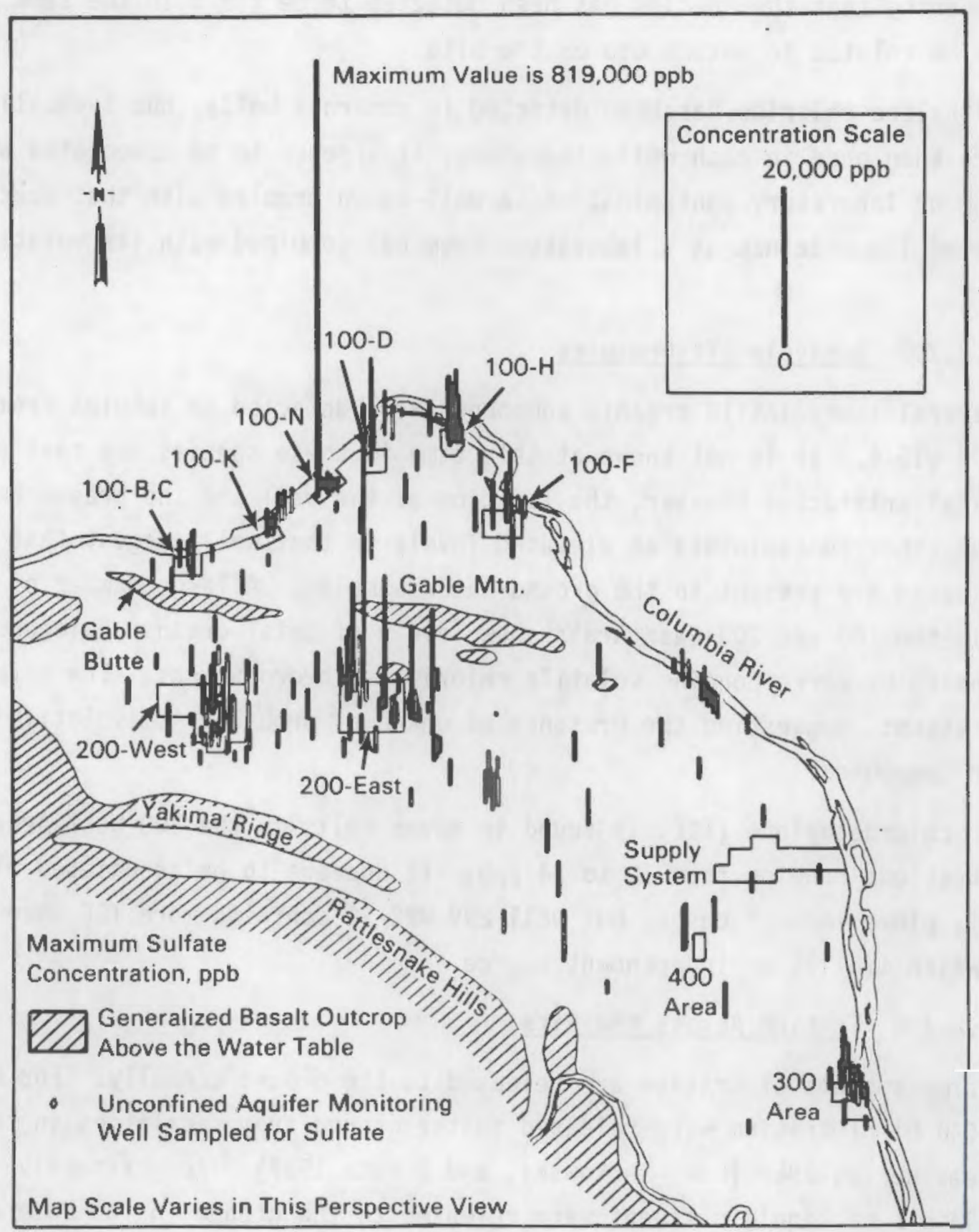

FIGURE 3.18. Maximum Sulfate Concentrations in Unconfined Aquifer Wells in 1987 
is noteworthy that the species has been detected three times in the same well and may be related to hexone use on the site.

Methylene chloride has been detected in numerous wells, but typically not more than once in each well; therefore, it appears to be associated with sampling or laboratory contamination, a well-known problem with that species because of its wide use as a laboratory chemical combined with its volatile nature.

\subsubsection{Semivolatile Organics}

Several semivolatile organic compounds were detected in samples from well 299-W15-4. It is not known at this time if these species are real or analytical artifacts; however, the location of the well and the presence of numerous other contaminants at elevated levels in that well suggest that contaminants are present in the ground-water samples. A large number of wells in the 100 and 200-East Areas show levels of total organic halogens not accompanied by corresponding volatile chlorinated hydrocarbons. The effect is persistent, suggesting the presence of unknown, probably semivolatile organic compounds.

Trichloroethylene (TCE) is found in seven wells in the 200-West Area at concentrations ranging from 10 to $34 \mathrm{ppb}$. It appears to be associated with the $\mathrm{CCl}_{4}$ plume in most cases, but we11 299-W22-20 contains more TCE than $\mathrm{CCl}_{4}$, which implies an independent source.

\subsubsection{Iritium Across the Site}

Large amounts of tritium are released to the ground annually. For example, $7040 \mathrm{Ci}$ of tritium were released to the ground from facilities in the 200 Areas during 1986 (Law, Serkowski, and Schatz 1987). Approximately 25 billion $L$ of liquid effluent were released to the ground in the same area during 1986 (PNL 1987).

Figure 3.19 shows the maximum tritium concentrations found at each well sampled during 1987. Tritium concentrations greater than the $20,000-p C i / L$ MCL were detected in portions of the 100-D, 100-K, 100-N, 200-East, 200-West, 400 , and 600 Areas. Concentrations greater than the 2,000,000-pCi/L DCG were detected only in wells in the 200-East Area and in one well in the 200-West Area. 


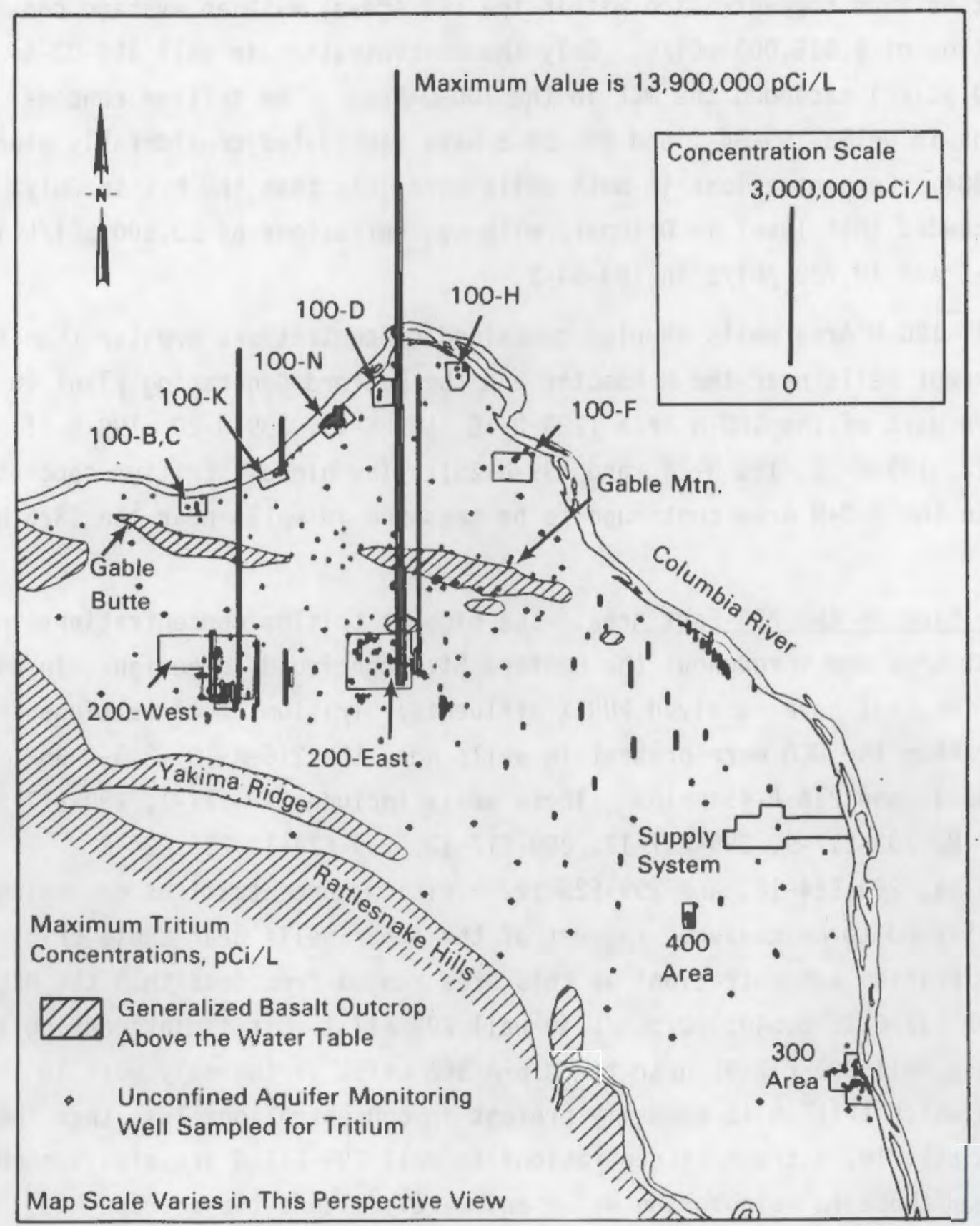

FIGURE 3.19. Maximum Tritium Concentrations in Unconfined Aquifer Wells in 1987 
Tritium in the 100 Areas. Well 199-K-30 continued to contain the highest tritium concentration within the 100 Areas, with an average concentration of $1,015,000 \mathrm{pCi} / \mathrm{L}$. Only the concentration in well 199-D2-5 $(31,450 \mathrm{pCi} / L)$ exceeded the $\mathrm{MCL}$ in the $100-\mathrm{D}$ Area. The tritium concentrations in wells 199-B4-1 and 199-B4-3 have fluctuated considerably since late 1984. Concentrations in both wells were less than the MCL in July, but had exceeded that level in October, with concentrations of $38,600 \mathrm{pCi} / \mathrm{L}$ in 199-B4-1 and 40,700 pCi/L in 199-B4-3.

All 100-N Area wells sampled contained concentrations greater than the $\mathrm{MCL}$, except wells near the $\mathrm{N}$ Reactor and the Hanford Generating Plant in the northern part of the 100-N Area (199-N-16, 199-N-19, 199-N-20, 199-N-21, $199-\mathrm{N}-22,199-\mathrm{N}-23,199-\mathrm{N}-24$, and $199-\mathrm{N}-25)$. The highest tritium concentrations in the $100-\mathrm{N}$ Area continued to be measured in wells near the $1325-\mathrm{N}$ LWDF.

Iritium in the 200-East Area. The highest tritium concentrations in the 200-East Area and throughout the Hanford Site continued to be found in wells near cribs that have received PUREX effluents. Tritium concentrations greater than the DCG were present in wells near the 216-A-10, 216-A-36B, 216-A-37-1, and 216-A-45 cribs. These wells include 299-E17-1, 299-E17-5, 299-E17-8, 299-E17-9, 299-E17-12, 299-E17-13, 299-E24-1, 299-E24-2, 299-E24-11, 299-E24-12, and 299-E25-19. Tritium concentrations exceeding the MCL continued to be measured in most of the other wells near these cribs. Average tritium concentrations in this area ranged from less than the MCL in well 299-E17-6 to 8,540,000 pCi/L in well 299-E17-1. It is interesting to note that well 299-E17-6, near the 216-A-36B crib, is the only well in this area in which tritium is normally present in concentrations less than the MCL. Similarly, nitrate concentrations in well 299-E17-6 are always much less than those in neighboring we1ls and rarely exceed the nitrate MCL. The cause of this discrepancy is unclear, but may be because the well has a perforated interval approximately $150 \mathrm{ft}$ below the water table. Water samples from this well are a composite of water taken from the entire perforated interval, while neighboring wells sample from the top of the aquifer where contaminant concentrations are suspected of being higher. Monitoring is 
planned for several depths within the aquifer at 216-A-36B, which may show more clearly the distribution of contamination within the aquifer.

Tritium continued to be detected in concentrations greater than the $\mathrm{MCL}$ in wel1s 299-E25-11 and 299-E25-22, located near the LWDF east of the PUREX plant. As with the distribution of nitrate near PUREX, insufficient data are available for conclusions to be drawn regarding changes in the distribution of tritium resulting from changes in PUREX waste disposal.

Other wells in the 200-East Area that contained tritium in concentrations greater than the MCL include wells 299-E24-7, 299-E26-4, 299-E28-12, and 299-E28-18. Average concentrations in these wells ranged from $37,450 \mathrm{pCi} / \mathrm{L}$ at well 299-E26-4 to $108,400 \mathrm{pCi} / \mathrm{L}$ at well 299-E28-12.

The movement of the widespread tritium plume that extends from the southeastern portion of the 200-East Area to the Columbia River was consistent with the patterns noted earlier (PNL 1987; Evans, Mitche11, and Dennison 1988). Separate tritium pulses associated with the two episodes of PUREX operations can be distinguished in the tritium plume. The 200,0002,000,000-pCi/L lobe east of the 200-East Area near the Columbia River is a result of discharges to ground water during the operation of PUREX from 1956 to 1972. Following an 11-year shutdown, operation of PUREX began again in 1983. Elevated tritium concentrations have been measured in several wells (e.g., wells 699-32-43 and 699-33-42), as shown in Figure 3.20. Concentrations downgradient from the 200-East Area represent the initial stages of the formation of a new pulse of tritium moving away from PUREX waste disposal facilities.

The general direction of movement of the eastern portion of the plume continues to be east-southeast toward the Columbia River. The migration of the plume to the south is indicated by increased tritium concentrations in wel1s 699-S3-E12, 699-S11-E12A, and 699-S19-E13. The confirmation of the western portion of the plume appears to closely match previous predictions of the direction of contaminant movement from the 200-East Area (Freshley and Graham 1988). Movement to the south may be enhanced by the spreading groundwater mound beneath $B$ Pond. This mound is spreading as a result of increased discharge of process cooling water to B Pond since 1984 . 


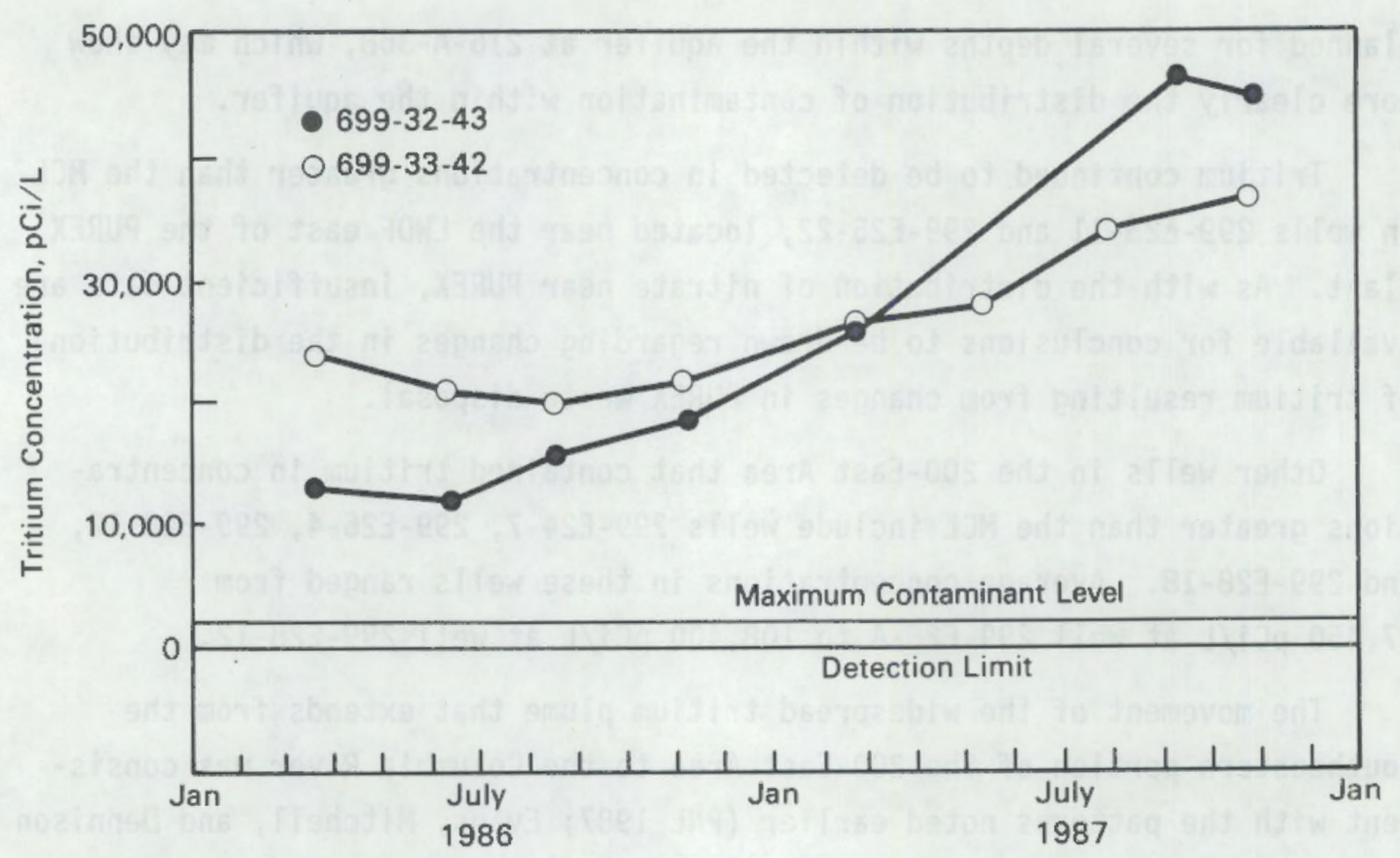

FIGURE 3.20. Tritium Concentrations in Ground Water Sampled from Wells $699-32-43$ and 699-33-42

Tritium in the 200-West Area. The movement of tritium plumes in the 200-West Area was also consistent with previous observations. The plume that extends from near the reduction-oxidation (REDOX) plant in the southern part of the 200-West Area continued to move slowly to the east and north. Well 299-W22-9 continued to be the only well in the 200-West Area with tritium concentrations greater than the DCG. The concentration in that well in September was $7,730,000 \mathrm{pCi} / \mathrm{L}$, which is unchanged from the previous quarter.

Tritium concentrations in nearby wells in the 200-West Area (299-W19-2, 299-W21-1, 299-W22-7, 299-W22-12, 299-W22-20, and 200-W22-26) and in the adjacent 600 Area (699-32-70B and 699-32-72) remained above the MCL and relatively constant throughout 1987 . Movement of a pulse of high tritium concentrations within the plume that extends north and east from REDOX was indicated by changes in the tritium concentrations in several 600 Area wells in the plume (Figures 3.21 and 3.22). Concentrations in well 699-35-70 continued to decrease, indicating that the pulse has moved beyond this well. Concentrations in wells 699-35-66 and 699-38-65, near the center of the 
plume, remained relatively constant, while concentrations in well 699-40-62 continued to increase as the plume moved northward (Figure 3.22 ). The northernmost extent of the plume appeared to be in the vicinity of well 699-40-62. Well 699-44-64, north of well 699-40-62, continued to contain concentrations of tritium near the detection limit ( $300 \mathrm{pCi} / \mathrm{L})$.

Tritium concentrations greater than the MCL continued to be measured in several wells within the plume in the northern part of the 200-West Area. Concentrations in wells 299-W14-2, 299-W14-6, and 299-W15-4 continued to decrease, presumably because of movement of the plume to the north. The concentration in well 299-W14-6 fell below the MCL in December. In general, tritium concentrations in wells within the plume between Gable Butte and Gable Mountain remained unchanged.

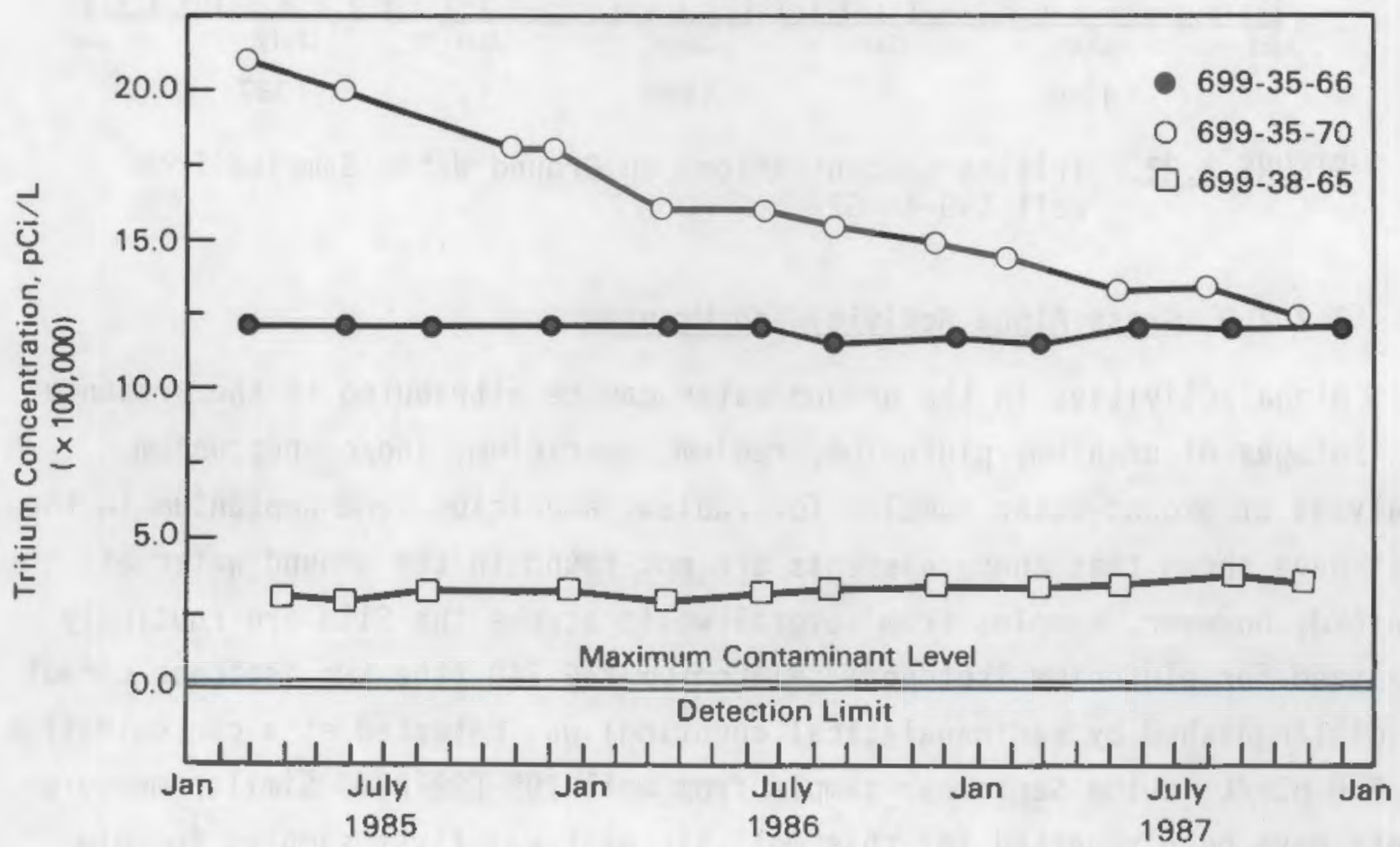

FIGURE 3.21. Tritium Concentrations in Ground Water Sampled from Wells 699-35-66, 699-35-70, and 699-38-65 


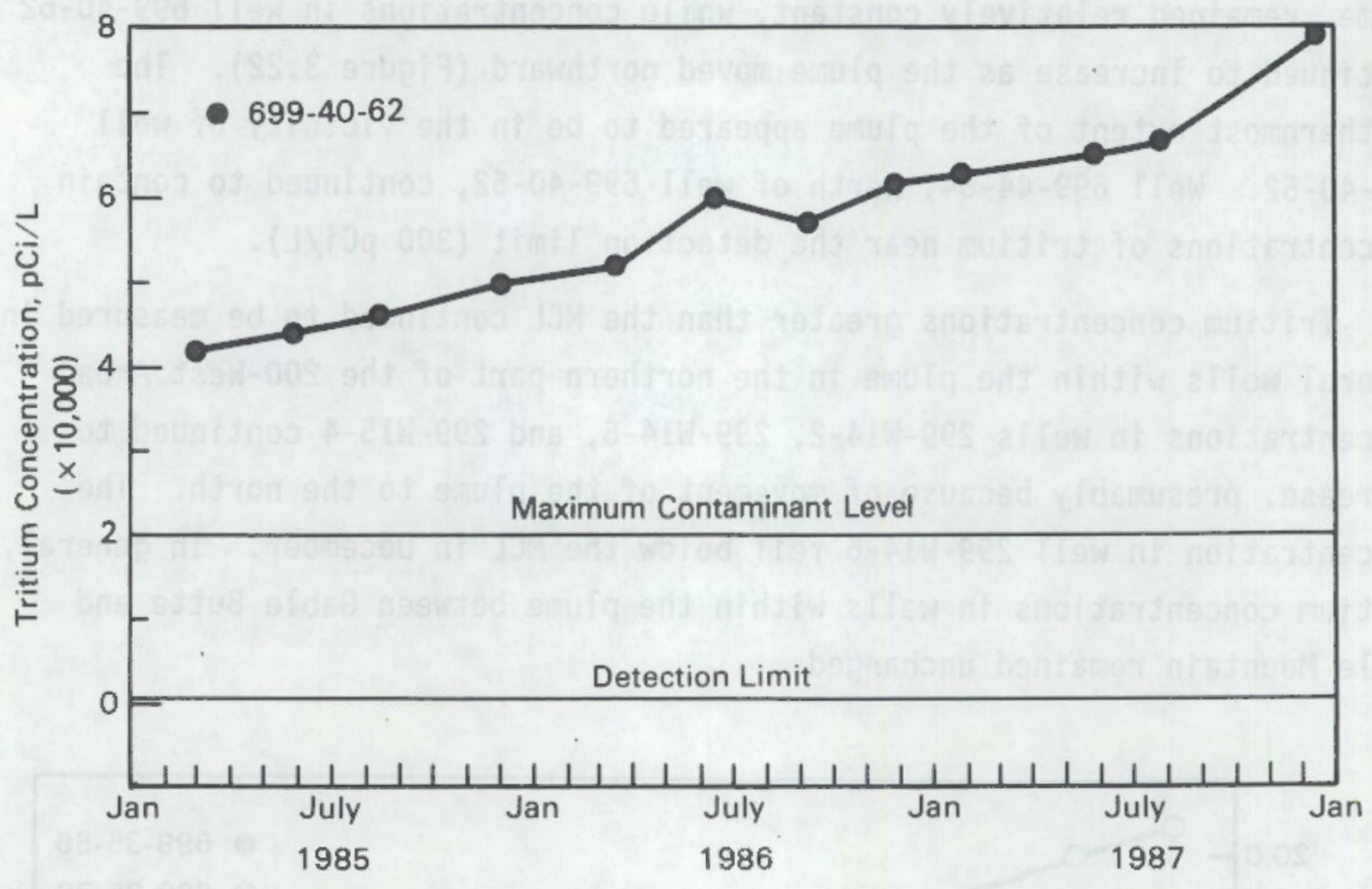

FIGURE 3.22. Tritium Concentrations in Ground Water Sampled from Well 699-40-62

\subsubsection{Gross Alpha Activity and Uranium}

Alpha activities in the ground water can be attributed to the presence of isotopes of uranium, plutonium, radium, americium, and/or neptunium. Analyses of ground-water samples for radium, americium, and neptunium in the past have shown that these elements are not found in the ground water at Hanford; however, samples from several wells across the Site are routinely analyzed for plutonium isotopes. Plutonium-239,240 (the two isotopes cannot be distinguished by radioanalytical counting) was detected at a concentration of $8.0 \mathrm{pCi} / \mathrm{L}$ in the September sample from we11 299-E28-23. Similar measurements have been reported for this well since it was first sampled for plutonium analyses in April 1986. Well 299-E28-23 is located near the 216-B-5 reverse we11, which received an estimated $244 \mathrm{Ci}$ of plutonium-239 and $65.7 \mathrm{Ci}$ of plutonium-240 (both values decayed through April 1, 1986) during its operation from 1945 to 1947 (DOE 1986). Wells in the 100-F, 100-H, 200, and 300 Areas in which the gross alpha concentration exceeded the MCL ( $15 \mathrm{pCi} / \mathrm{L}$, excluding uranium) all contained uranium in concentrations that would account 
for the gross alpha activities detected. Several wells in the 100-N Area also contained gross alpha activities exceeding the $\mathrm{MCL}$, but no analyses for alpha-emitting radionuclides were performed on samples from these wells.

Wells 299-E28-18, 299-E28-21, and 299-E28-23 continued to be the only 200-East Area wells with gross alpha activities greater than the MCL. Activities in these wells continued to decrease, as did the uranium concentrations. Gross alpha activities in wells 299-E28-18 and 299-E28-21, located near the 216-B-62 crib, averaged 47 and $41 \mathrm{pCi} / \mathrm{L}$, respectively. The average uranium concentrations in those wells were 68 and $55 \mathrm{ppb}(\mathrm{a})$, respectively. The average gross alpha activity and uranium concentration in well 299-E28-23 were $18 \mathrm{pCi} / \mathrm{L}$ and $35 \mathrm{ppb}$, respectively.

The highest gross alpha activities measured on the Site continued to be in wells adjacent to the inactive 216-U-1 and 216-U-2 cribs. Activities in wells 299-W19-3, 299-W19-9, 299-W19-11, 299-W19-15, 299-W19-16, and 299-W19-18 continued to decrease (Figure 3.23). All these wells contained uranium concentrations that would account for the gross alpha activities detected (Figure 3.24). Wells 299-W19-19, 299-W19-20, 299-W19-23, 299-W19-24, 299-W19-25, and 299-W19-26, located east of U P1 ant near the inactive 216-U-17 crib, contained gross alpha activities and uranium concentrations relatively unchanged from the previous quarter. Other 200-West Area wells that contained gross alpha activities greater than the MCL were wells 299-W18-15, 299-W19-2, 299-W22-21, 299-W23-4, 299-W23-9, and 299-W23-10. All of these wells also had uranium concentrations that would account for the gross alpha activity. The gross alpha activity and the uranium concentration in well 299-W19-2 have both steadily increased since mid-1986 (Figures 3.25 and 3.26).

Figure 3.27 illustrates the Site-wide distribution of uranium, showing the large concentrations found in the 200-West Area near the 216-U-1 and 216U-2 cribs.

(a) The conversion from $\mathrm{ppb}$ uranium to $\mathrm{pCi} / \mathrm{L}$ uranium is approximately 0.699 for natural uranium. Other conversion factors may be calculated where the isotopic composition has been measured [e.g., Law, Serkowski, and Schatz (1987) report a conversion factor of 0.679 for their data]. 


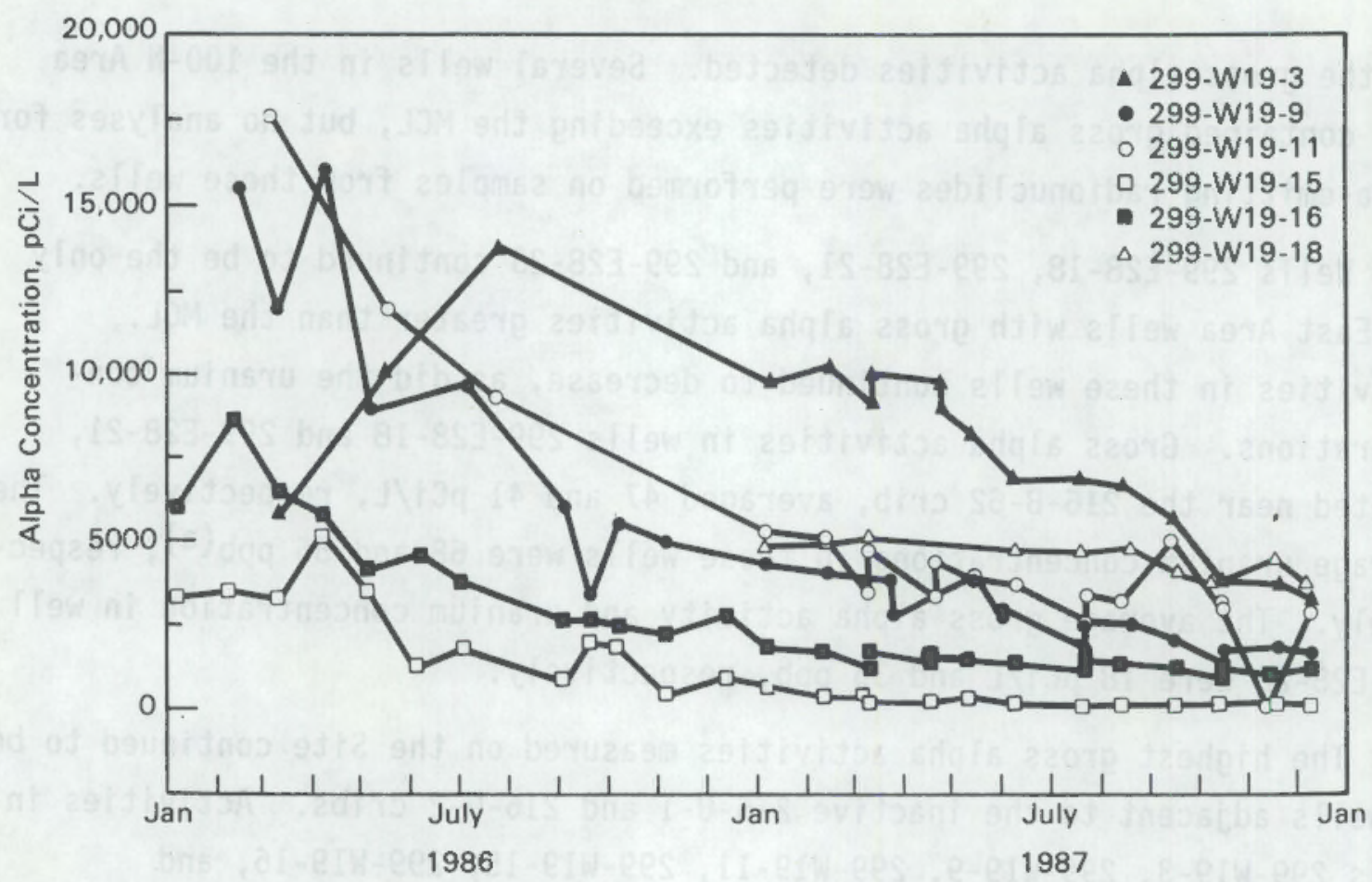

FIGURE 3.23. Gross Alpha Concentrations in Wells Near the 216-U-1 and 216-U-2 Cribs

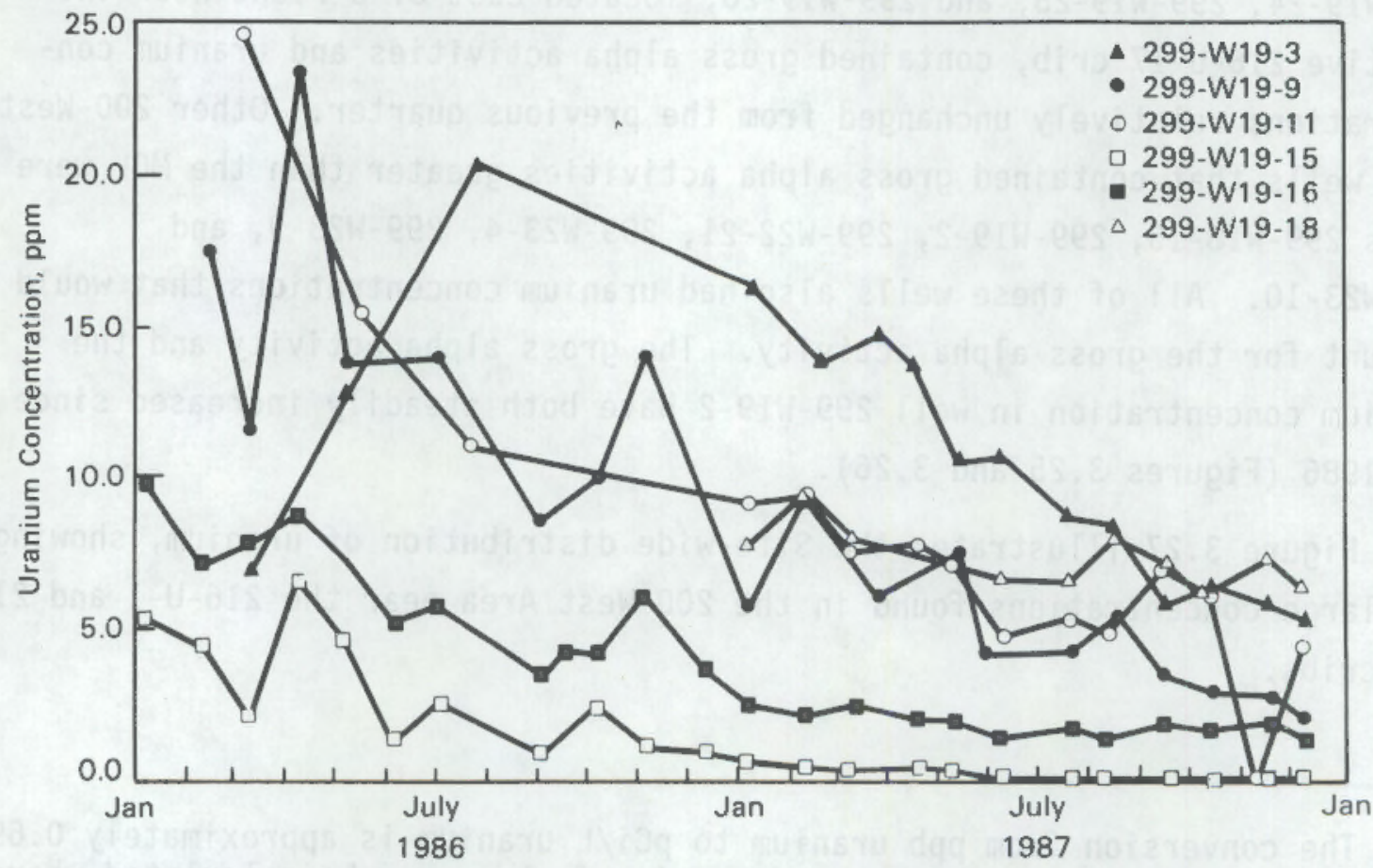

FIGURE 3.24. Uranium Concentrations in Wells Near the 216-U-1 and 216-U-2 Cribs 


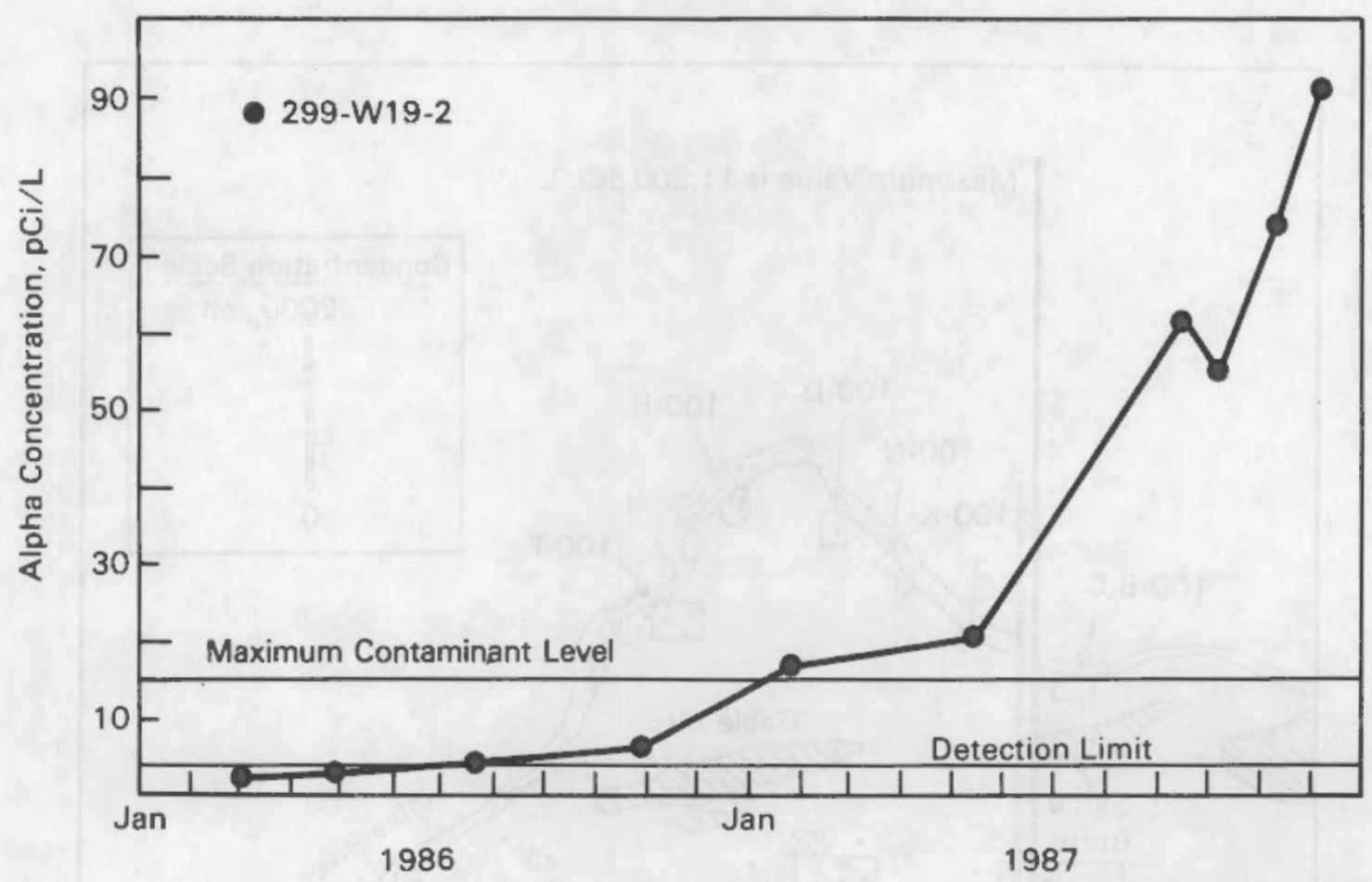

FIGURE 3.25. Gross Alpha Concentrations in Well 299-W19-2

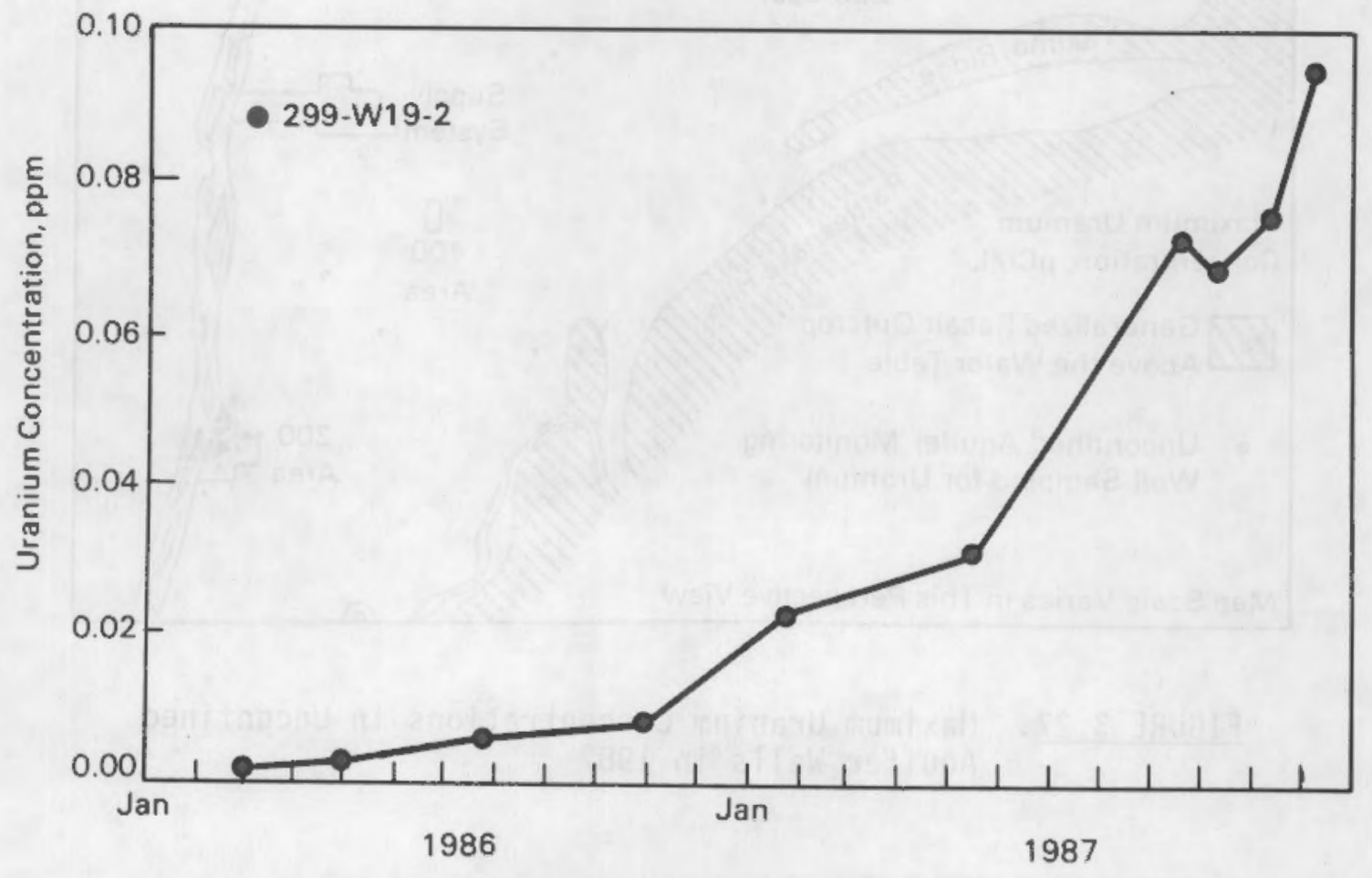

FIGURE 3.26. Uranium Concentrations in Well 299-W19-2 


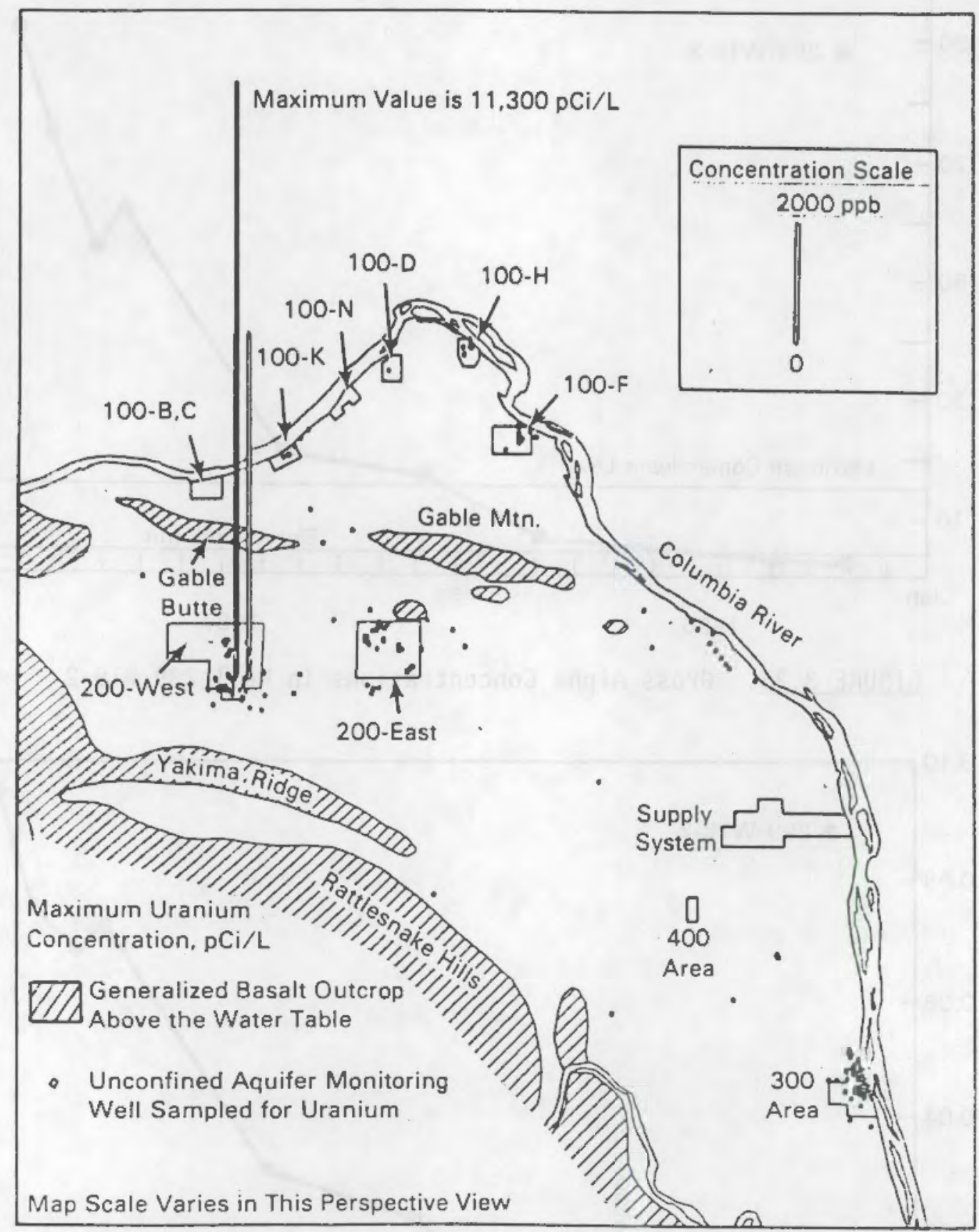

FIGURE 3.27. Maximum Uranium Concentrations in Unconfined Aquifer Wells in 1987 


\subsubsection{Gross Beta}

Gross beta concentrations greater than the $50-\mathrm{pCi} / \mathrm{L} \mathrm{MCL}$ were detected in wells from all operational areas, except the $100-\mathrm{K}$ and 400 Areas. Although gross beta concentrations greater than the $\mathrm{MCL}$ were widespread, the highest concentrations were found in wells near several waste disposal facilities in the 100-N, 200-East, and 200-West Areas and in the 600 Area adjacent to the 200 Areas. Gross beta activity can commonly be attributed to the presence of one or more of the following radionuclides in the ground water: carbon-14, cobalt-60, nickel-63, strontium-90, technetium-99, iodine-129, cesium-137, and uranium daughters. It is believed that in most cases throughout the Site, except for the 100-N Area and areas with high uranium concentrations, technetium- 99 accounts for much of the gross beta activity(a).

Gross Beta in the 100 Areas. Gross beta activities exceeding the MCL continued to be measured in several wells in the 100-N Area. The highest activities were found in wells $199-\mathrm{N}-2$ (1130 pCi/L), 199-N-3 $(4380 \mathrm{pCi} / \mathrm{L})$, 199-N-14 (2580 pCi/L), 199-N-29 (1465 pCi/L), and 199-N-39 (1770 pCi/L). Al1 of these wells contained elevated concentrations of cobalt-60 and strontium-90. In addition, carbon-14 was detected in we11s $199-\mathrm{N}-14$ and 199-N-29; wel1 199-N-29 also contained antimoney-125. Analyses of carbon-14 and antimony-125 were not performed on samples from wells $199-\mathrm{N}-2,199-\mathrm{N}-3$, and $199-\mathrm{N}-39$.

Gross Beta in the 200-East Area. Wells in the 200-East Area that contained high gross beta activities are related to past disposal of material to the inactive 216-B-5 reverse well and the inactive BY, 216-A-10, and 216A-36B cribs. Wells 299-E28-7, 299-E28-24, 299-E28-25, and 299-E28-23 near the 216-B-5 crib had average gross beta activities of 216,307,6975, and $10,370 \mathrm{pCi} / \mathrm{L}$, respectively. The gross beta activity in well 299-E28-23 continued to be the highest measured on the Site. These wells all contained elevated strontium-90 concentrations, and we11s 299-E28-23 and 299-E28-25 also contained cesium-137. The 216-B-5 reverse well received an estimated

(a) The detection efficiency of technetium-99 by the gross beta method is poor. Calibration experiments recently carried out by UST showed that samples dominated by technetium-99 activity contain 5 to 10 times the technetium-99 activity reported by the gross beta method. 
$27.9 \mathrm{Ci}$ strontium-90 and $31.8 \mathrm{Ci}$ cesium-137 (both values decayed through April 1, 1986) during its operation from 1945 to 1947 (DOE 1986). The BY cribs, located near the northern boundary of the 200-East Area, received U Plant waste produced from operations that scavenged uranium from bismuth phosphate waste stored in single-shell tanks. Wells 299-E33-1, 299-E33-3, 299-E33-5, 299-E33-7, 299-E33-8, 299-E33-20, 299-E33-24, and 299-E33-26 showed average gross beta activities greater than the MCL, ranging from $60 \mathrm{pCi} / \mathrm{L}$ at well 299-E33-8 to $795 \mathrm{pCi} / \mathrm{L}$ at well 299-E33-7. All these wells, with the exception of 299-E33-20, contained high concentrations of that radionuclide. The presence or absence of technetium-99 in well 299-E33-20 cannot be ruled out because samples from the well were not analyzed for that radionuclide. Wells 299-E33-1, 299-E33-3, 299-E33-5, and 299-E33-7 also contained cobalt-60. Three 600 Area wells, located immediately north of the BY cribs, continued to contain gross beta activities greater than the MCL. Activities in wel1s 699-49-55A, 699-49-57, and 699-50-53 averaged 1243, 267, and $2323 \mathrm{pCi} / \mathrm{L}$, respectively. All three wells contained detectable quantities of cobalt-60. We17s 299-E17-2, 299-E17-5, and 299-E24-12, near the 216A-10 and $216-A-36 \mathrm{~B}$ cribs, contained gross beta activities greater than the MCL. Activities in wells 299-E17-5 and 299-E24-12 were increasing and peaked in october, with activities of 590 and $270 \mathrm{pCi} / \mathrm{L}$, respectively. The activities decreased to $143 \mathrm{pCi} / \mathrm{L}$ in well 299-E17-5 and $50 \mathrm{pCi} / \mathrm{L}$ in well 299E24-12. The fluctuation in the gross beta activity in well 299-E17-5 corresponded to changes in the concentrations of cobalt-60 and ruthenium-106, while the changes in gross beta in well 299-E24-12 were related to changes in ruthenium-106 concentrations.

Gross Beta in the 200 -West Area. The highest gross beta activities in the 200-West Area were in wells near U Plant (i.e., wells with the 299-W19 prefix). Gross beta activities in wells near the 216-U-1 and 216-U-2 cribs remained above the MCL, but continued to decrease. Technetium-99 was detected in the wells surrounding these cribs. In addition, wells 299-W19-3, 299-W19-11, 299-W19-15, and 299-W19-16 contained carbon-14 and iodine-129. Well 299-W19-2, near the inactive 216-U-8 crib, had an average gross beta activity of $109 \mathrm{pCi} / \mathrm{L}$ and a technetium-99 activity of $581 \mathrm{pCi} / \mathrm{L}$ in September. Gross beta activities greater than the MCL continued to be measured in wells 299-W19-19, 299-W19-20, 299-W19-23, 299-W19-24, 299-W19-25, and 299-W19-26. 
Activities were equal to or slightly higher than those measured previously and ranged from 230 to $3090 \mathrm{pCi} / \mathrm{L}$. Wells 299-W19-23, 299-W19-24, and 299W19-25 contained high concentrations of technetium-99. Other 200-West Area wells that contained elevated gross beta activities included wells 299-W14-2, 299-W15-3, 299-W22-10, 299-W22-21, 299-W23-2, and 299-W23-7, with respective average activities of $79,81,74,192,1157$, and $808 \mathrm{pCi} / \mathrm{L}$. Technetium-99 was detected in we11s 299-W22-21 and 299-W23-7, but was not analyzed for in the other wells. Well 299-W22-10 had a high strontium-90 concentration, and well 299-W22-21 contained detectable quantities of carbon-14 and iodine-129.

Gross Beta in Other Areas. Gross beta activity remained above the MCL in several wells near Gable Mountain Pond (wells 699-53-47A, 699-53-47B, and 699-53-48B), ranging from 96 to $652 \mathrm{pCi} / \mathrm{L}$. These wells contained relatively high concentrations of strontium- 90 which would account for the gross beta activity measured. Gross beta activities in we11s 699-54-48 and 699-54-49 remained slightly above the $\mathrm{MCL}$; these wells also contained strontium- 90 .

\subsubsection{Carbon-14}

Samples from several wells in the 100-N, 200-East, 200-West, and 600 Areas were analyzed for carbon-14. None of the wells contained carbon-14 activities greater than the 2000-pCi/L MCL. Wells 199-N-14, 199-N-29, and 199-N-33 had carbon-14 activities of 232,41 , and $49 \mathrm{pCi} / \mathrm{L}$, respectively. We11s 299-E17-5 and 299-E17-8, located near PUREX, contained respective carbon-14 activities of 24 and 33 pCi/L. Wells 299-W19-3, 299-W19-5, 299W19-11, 299-W19-16, 299-W22-20, 299-W22-21, 699-32-43, 699-35-70, 699-37-43, and $699-38-70$ also contained detectable carbon-14 in concentrations ranging from 4 to $9 \mathrm{pCi} / \mathrm{L}$.

\subsubsection{Cobalt-60}

Al1 cobalt-60 results were at levels that cannot be accurately detected except in the 100-N Area and in isolated portions of the 200-East Area and adjacent 600 Area. Figure 3.28 summarizes the Site-wide cobalt-60 distribution in 1987. Concentrations of cobalt-60 were greater than the $100-\mathrm{pC} \mathrm{i} / \mathrm{L}$ MCL in several wells near the 1325-N LWDF in the 100-N Area; none exceeded the $5000-p C i / L D C G$. Concentrations in most of these wells continued to 


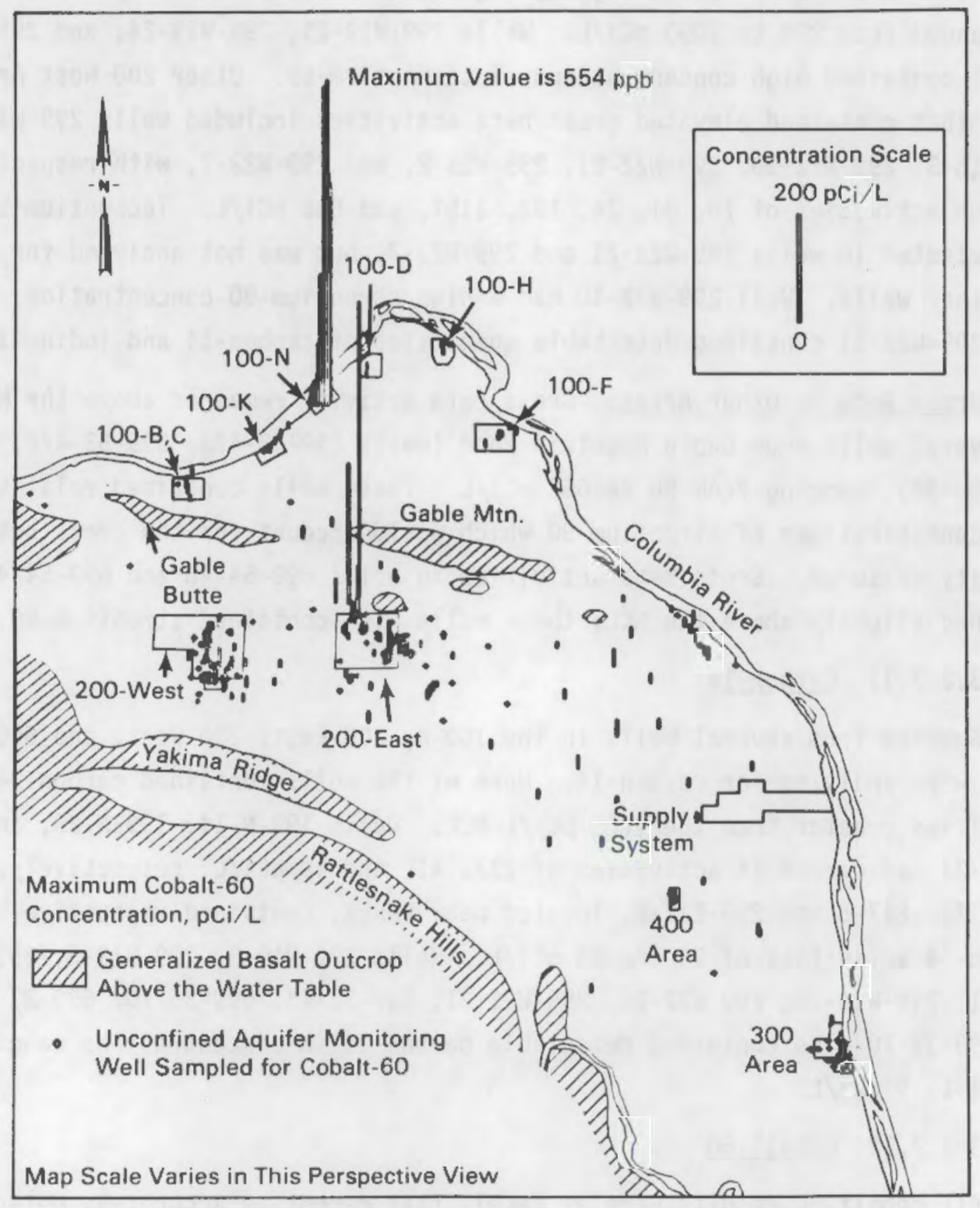

FIGURE 3.28. Maximum Cobalt-60 Concentrations in Unconfined Aquifer Wells in 1987 
fluctuate and ranged from an average of $181 \mathrm{pCi} / \mathrm{L}$ in well $199-\mathrm{N}-39$ to $389 \mathrm{pCi} / \mathrm{L}$ in well 199-N-37. The 200-East Area wells 299-E33-3, 299-E33-5, and 299-E33-7, located near the BY cribs, contained respective concentrations of 24,31 , and $77 \mathrm{pCi} / \mathrm{L}$ in August. The concentrations in all three wells decreased, with those in well 299-E33-7 containing $40 \mathrm{pCi} / \mathrm{L}$ in 0ctober and wells 299-E33-3 and 299-E33-5 falling to levels that cannot be accurately measured. Three wells in the 600 Area immediately north of the BY cribs continued to contain detectable concentrations of cobalt-60 (see related discussion in the section entitled "Cyanide Concentration in and North of the 200-East Area"). Well 699-49-57 had an average concentration of $27 \mathrm{pCi} / \mathrm{L}$, unchanged from previous measurements in 1987. Concentrations in wells 699-49-55A and 699-50-53 remained above the MCL. The concentration in wel1 699-50-53 increased from $275 \mathrm{pCi} / \mathrm{L}$ (previous reporting period) to $487 \mathrm{pCi} / \mathrm{L}$ in December, while the concentration in well 699-49-55A remained relatively unchanged, with an average of $190 \mathrm{pCi} / \mathrm{L}$.

\subsubsection{Nickel-63}

Samples from wells $199-\mathrm{N}-14,199-\mathrm{N}-29$, and $199-\mathrm{N}-33$ in the $100-\mathrm{N}$ Area and we11 699-38-70 in the 600 Area adjacent to the 200-West Area were analyzed for nicke1-63. All results for these wells were below the levels that can be accurately measured.

\subsubsection{Strontium-90}

Concentrations of strontium-90 were above the $8-\mathrm{pCi} / \mathrm{L} \mathrm{MCL}$ in wells in the 100-B, 100-N, 200-East, 200-West, 300, and 600 Areas. The Site-wide distribution of strontium-90 is illustrated in Figure 3.29.

Strontium-90 in the 100 Areas. Concentrations in well 199-H4-4 in the 100-H Area had risen above the MCL during the previous reporting period but fell below that level between July and December 1987. Concentrations in wells 199-B3-1, 199-84-1, and 199-B4-4 in the 100-B Area averaged 55, 27, and $33 \mathrm{pCi} / \mathrm{L}$, respectively. Strontium-90 analyses have not been performed on samples from these wells since 1982, and the concentrations were relatively unchanged from those measured at that time. Concentrations above the MCL were also present in $18100-\mathrm{N}$ Area wells. Most of these wells were located 


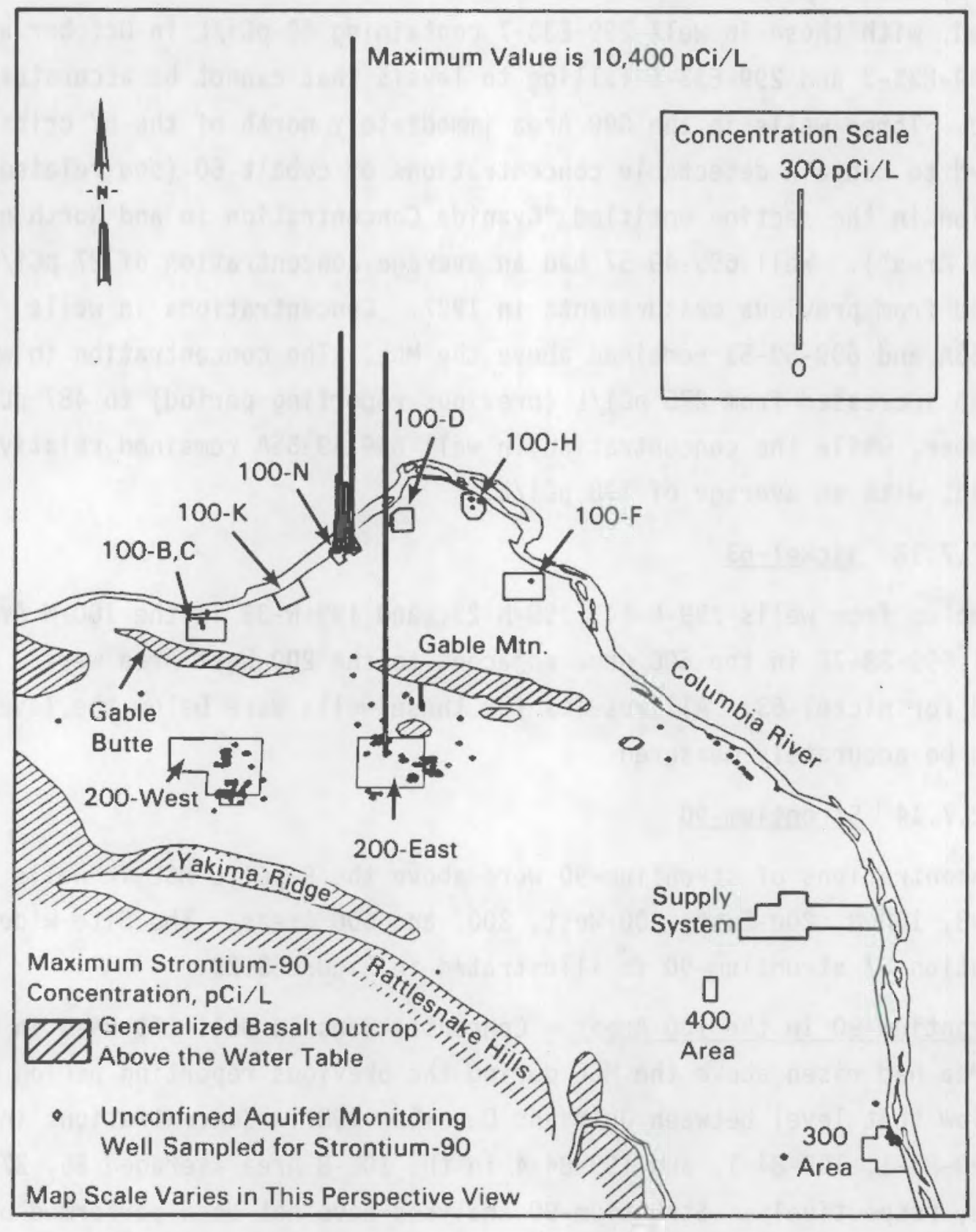

FIGURE 3.29. Maximum Strontium-90 Concentrations in Unconfined Aquifer Wells in 1987 
near the inactive 1301-N LWDF and the active 1325-N LWDF. Four wells (199-N-18, 199-N-19, 199-N-20, and 199-N-21) located in the northern part of the 100-N Area also contained concentrations greater than the MCL. Several of the wells near the 1301-N and 1325-N LWDFs contained concentrations greater than the $1000-\mathrm{pCi} / \mathrm{L} \mathrm{DCG}$. These wells (and their concentrations) included 199-N-2 (1270 pCi/L), 199-N-3 (1405 pCi/L), 199-N-14 (1170 pCi/L), and $199-\mathrm{N}-45(3160 \mathrm{pCi} / \mathrm{L})$.

Strontium-90 in the 200-East Area. Three wells in the 200-East Area contained strontium-90 in concentrations greater than the MCL. Concentrations in wells 299-E28-7 and 299-E28-23 averaged 108 and $4855 \mathrm{pCi} / \mathrm{L}$, respectively. These concentrations were similar to those measured previously and can be attributed to wastes discharged to the 216-B-5 reverse well during 1945 to 1947 (Smith 1980). Strontium-90 concentrations in well 299-E24-1 have risen since early 1985 and reached $16 \mathrm{pCi} / \mathrm{L}$ in September 1987.

Strontium-90 in the 200-West Area. Wells 299-W19-2 and 299-W22-10 continued to be the only 200 -West Area wells with strontium-90 concentrations greater than the MCL, with average concentrations of 14 and $30 \mathrm{pCi} / \mathrm{L}$, respectively. Strontium-90 in concentrations above the MCL has been detected for several years in five 600 Area wells near Gable Mountain Pond (699-53-47A, 699-53-47B, 699-53-48B, 699-54-48, and 699-54-49). The concentrations in wells 699-54-48 and 699-54-59 fluctuated but generally continued to increase, while the concentrations in the other wells have remained relatively constant.

\subsubsection{Technetium-99}

An extensive program to analyze ground-water samples for technetium-99 was initiated in May 1987. Results for samples taken in May and June were not included in the monitoring report for that quarter (Evans, Mitche11, and Dennison 1987). The following discussion summarizes all results obtained in 1987. A plot of the measured technetium-99 distribution in 1987 is shown in Figure 3.30. Technetium-99 concentrations above the $900-\mathrm{pCi} / \mathrm{L} \mathrm{MCL}$ were detected in wells in the 100-H, 200-East, 200-West, and portions of the 600 Area adjacent to the 200 Areas. None of the wells had concentrations exceeding the 100,000-pCi/L DCG. Wells 199-H4-3 and 199-H4-4 in the 100-H Area had average technetium-99 concentrations of 3093 and $1170 \mathrm{pCi} / \mathrm{L}$, respectively. 


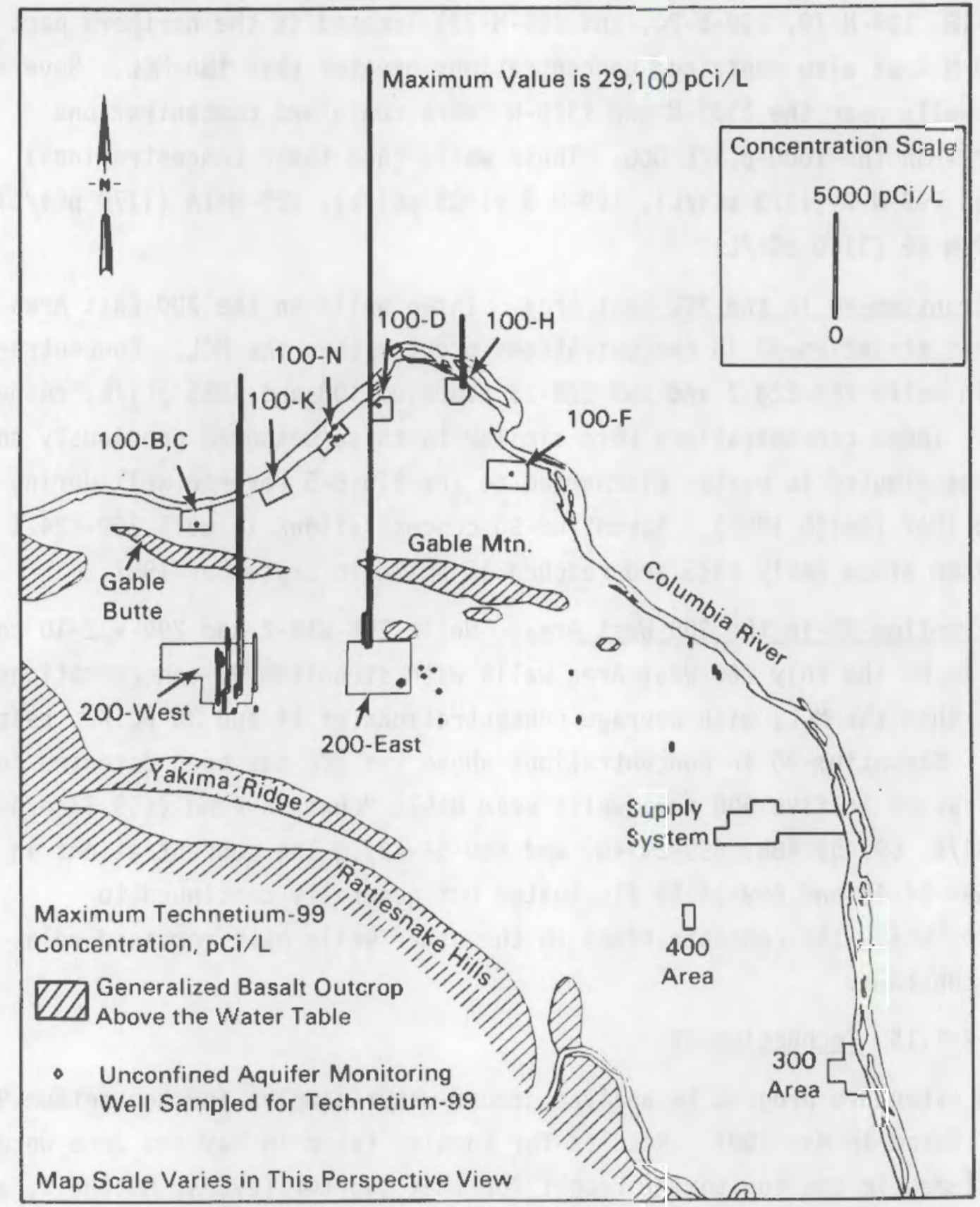

FIGURE 3.30. Maximum Technetium-99 Concentrations in Unconfined Aquifer Wells in 1987 
Technetium-99 in the 200-West Area. The highest concentrations of technetium-99 appear to be related to waste-disposal facilities that currently receive or have in the past received waste produced by U Plant and $\mathrm{UO}_{3} \mathrm{Pl}$ ant operations. Several wells located near LWDFs adjacent to the plants contained high concentrations of technetium-99. It is unclear if one large technetium-99 plume exists in this area or if several smaller plumes are emanating from individual facilities. Wells located near the inactive 216-U-1 and 216-U-2 cribs that contained technetium-99 concentrations greater than the MCL include (with average concentration) 299-W19-3 (2356 pCi/L), 299-W19-9 (1670 pCi/L), 299-W19-11 (2810 pCi/L), 299-W19-16 (1410 pCi/L), and 299-W19-18 (8160 PCi/L). Wells 299-W19-20, 299-W19-23, 299-W19-24, and 299W19-25, located near the 216-U-17 crib that has never been used, contained respective average concentrations of $11,600,1330,13,350$, and $9440 \mathrm{pCi} / \mathrm{L}$. In the 600 Area directly east of U Plant, well 699-38-70 had an average concentration of $2270 \mathrm{pCi} / \mathrm{L}$. Other 200-West Area wells that had concentrations greater than the MCL were wells 299-W22-21 and 299-W23-7, with concentrations of 906 and $2480 \mathrm{pCi} / \mathrm{L}$, respectively.

Technetium-99 in the 200-East Area. Several wells in the 200-East Area also contained technetium-99 in concentrations greater than the MCL. These wells are located near the inactive BY cribs. These cribs received waste produced from U Plant operations that scavenged uranium from bismuth phosphate waste stored in single-shell tanks. Wells 299-E33-1, 299-E33-3, 299-E33-5, 299-E33-7, 299-E33-24, and 299-E33-26 had concentrations of 960, $1400,1980,4700,1580$, and $1420 \mathrm{pCi} / \mathrm{L}$, respectively. Well 699-50-53, located in the 600 Area north of the BY cribs, had a maximum technetium-99 concentration of $29,100 \mathrm{pCi} / \mathrm{L}$.

Technetium-99 in 0ther Areas. A number of other wells across the Site contained detectable concentrations of technetium-99. of particular interest was the presence of technetium-99 in several wells located within the tritium plume that extends from the 200-East Area to the Columbia River. Wells 69927-8, 699-32-43, 699-40-1, 699-41-1, 699-41-23, and 699-46-4 had concentrations of $319,66,278,308,69$, and $192 \mathrm{pCi} / \mathrm{L}$, respectively. 


\subsubsection{Ruthenium-106}

Comparison of analytical results for ruthenium-106 to the $30 \mathrm{pCi} / \mathrm{L} \mathrm{MCL}$ is difficult because the analytical technique used can only accurately measure concentrations 4 to 5 times higher than the MCL. Although concentrations of ruthenium-106 above the MCL were reported in wells in all operational areas, the concentrations in all wells, except 299-E17-5 and 299-E24-12, have decreased to levels that cannot be accurately detected. Concentrations in wells 299-E17-5 and 299-E24-12, near PUREX in the 200-East Area, exceeded the MCL, with peak concentrations in September of 557 and $420 \mathrm{pCi} / \mathrm{L}$, respectively. Concentrations in both wells subsequently decreased to below the detection 1 imit. The DCG for ruthenium-106 is $6000 \mathrm{pCi} / \mathrm{L}$.

\subsubsection{Antimony-125}

Several wells near the 1325-N LWDF in the 100-N Area continued to contain detectable quantities of antimony-125, but none had concentrations exceeding the $3000-\mathrm{pCi} / \mathrm{L} \mathrm{MCL}$. Concentrations ranged from $99 \mathrm{pCi} / \mathrm{L}$ in well $199-\mathrm{N}-33$ to $224 \mathrm{pCi} / \mathrm{L}$ in well 199-N-36.

\subsubsection{Iodine-129}

The presence of iodine-129 in ground water is significant, primarily because of its relatively long half-life ( 16 million years), its potential for accumulation in the environment as a result of long-term releases from nuclear fuel reprocessing facilities (Soldat 1976), and its relatively low MCL $(1 \mathrm{pC} i / L)$. On the Hanford Site, the main contributor of iodine-129 to the ground water has been liquid discharges to cribs in the 200 Areas. Samples from wells in the 100-N, 200-East, 200-West, and 600 Areas were analyzed for iodine-129. Results are shown in Table 3.3, as well as in Appendix B. Samples from wells $199-\mathrm{N}-14,199-\mathrm{N}-29$, and $199-\mathrm{N}-33$ in the $100-\mathrm{N}$ Area a11 contained iodine- 129 concentrations at least 50 times less than the MCL. Several wells near PUREX in the 200-East Area showed concentrations greater than the MCL but less than the $500-\mathrm{pCi} / \mathrm{L}$ DCG. Seven we11s located within the tritium plume, which extends from the 200-East Area east to the Columbia River, were analyzed for iodine-129, and five of these wells (699-27-8, 699-32-22, 699-32-43, 699-37-43, and 699-41-23) contained concentrations greater than the MCL. North of the 200-East Area, we11s 
699-49-55A, 699-49-57, and 699-50-53 contained concentrations at least five times less than the MCL. Wells 299-W19-3, 299-W19-9, 299-W19-11, 299-W19-15, and 299-W19-16, located near the 216-U-1 and 216-U-2 cribs in the 200-West Area, had iodine-129 concentrations greater than the MCL, ranging from 9 to $33 \mathrm{pCi} / \mathrm{L}$. Wel1 299-W22-21, located near REDOX in the 200-West Area, contained an iodine-129 concentration of $3 \mathrm{pCi} / \mathrm{L}$. In the 600 Area adjacent to the 200-West Area, the iodine-129 concentration in we11 699-35-70 was $47 \mathrm{pCi} / \mathrm{L}$. A sample from well 699-47-50, which is screened in the confined aquifer north of the 200-East Area, was analyzed for iodine-129 and contained $0.006 \mathrm{pCi} / \mathrm{L}$.

In addition to the data obtained from samples analyzed during 1987, available data on iodine-129 in Hanford confined aquifer ground water were assembled and published by an intercontractor working group. The document (IWG 1987) discussed background levels of iodine-129 in ground water and identified locations where iodine-129 was observed at concentrations exceeding background. Analys is of ground water that was thought to have not been contaminated by iodine-129 generated by the nuclear industry and nuclear weapons testing since 1944 has indicated that background concentrations of iodine-129 are near the detection 7 imit $\left(1 \times 10^{-6} \mathrm{pCi} / \mathrm{L}\right)$ for available techniques.

The working group identified two boreholes (DB-7 and DB-15) where iodine-129 was observed to be above background levels in confined aquifer ground water. Iodine-129 was observed in samples collected from the Mabton Interbed at DB-7. The Mabton Interbed is a sedimentary layer between the lowermost basalt flow in the Saddle Mountains Formation and the uppermost basalt flow in the Wanapum Formation. The average iodine-129 concentration for 12 samples collected at DB-7 between 1976 and 1985 was $3.15 \times 10^{-4} \mathrm{pCi} / \mathrm{L}$. The presence of iodine-129 at this location was attributed to a source other than waste disposal on the Hanford Site. A possible source is drilling fluid contamination of the borehole.

The presence of iodine-129 in ground-water samples from basalt flowtops (zones of relatively higher transmissivity near the top of basalt flows) and sedimentary interbeds in the Saddle Mountains and Wanapum Formations at borehole DB-15 was attributed to contamination from 100 Areas waste disposal 
TABLE 3.3. Iodine-129 Data for Samples Collected July 1 Through September 30, 1987

\begin{tabular}{|c|c|c|c|}
\hline $\begin{array}{c}\text { We11 } \\
\text { Number }\end{array}$ & $\begin{array}{c}\text { Collection } \\
\text { Date } \\
\end{array}$ & $\begin{array}{l}{ }^{129} \mathrm{I}, \\
\mathrm{pCi} / \mathrm{L}\end{array}$ & $\begin{array}{l}\text { Counting } \\
\text { Error }\end{array}$ \\
\hline $199-N-14$ (a) & $9 / 10 / 87$ & 0.0077 & 0.0007 \\
\hline $199-N-29(a)$ & $9 / 10 / 87$ & 0.0062 & 0.0005 \\
\hline $199-N-33$ (a) & $9 / 10 / 87$ & 0.021 & 0.002 \\
\hline 299-E17-1 & $8 / 10 / 87$ & 47.3 & 5.5 \\
\hline 299-E17-5 & $8 / 10 / 87$ & 25.0 & 3.2 \\
\hline 299-E17-6 & $8 / 10 / 87$ & 1.9 & 1.1 \\
\hline 299-E17-8 & $9 / 02 / 87$ & 29.2 & 3.5 \\
\hline 299-E17-9 & $8 / 10 / 87$ & 27.0 & 3.4 \\
\hline 299-E24-1 & $7 / 13 / 87$ & 44.6 & 5.1 \\
\hline $299-E 25-25$ & $7 / 01 / 87$ & 1.1 & 0.4 \\
\hline 299-W19-11 & $8 / 20 / 87$ & 31.6 & 3.8 \\
\hline 299-W19-15 & $8 / 18 / 87$ & 9.9 & 1.8 \\
\hline 299-W19-16 & $8 / 18 / 87$ & 9.3 & 1.8 \\
\hline 299-W19-3 & $8 / 20 / 87$ & 32.9 & 3.9 \\
\hline 299-W19-9 & $8 / 20 / 87$ & 21.4 & 2.8 \\
\hline 299-W22-20 & $8 / 18 / 87$ & 1.6 & 1.0 \\
\hline 299-W22-21 & $9 / 17 / 87$ & 2.7 & 1.1 \\
\hline $699-27-8(a)$ & $8 / 20 / 87$ & 1.8 & 0.2 \\
\hline $699-32-22$ (a) & $9 / 04 / 87$ & 3.9 & 0.3 \\
\hline $699-32-43(a)$ & $9 / 04 / 87$ & 5.5 & 0.4 \\
\hline $699-35-70$ & $7 / 09 / 87$ & 47.2 & 5.4 \\
\hline $699-37-43(a)$ & $9 / 10 / 87$ & 10.7 & 0.9 \\
\hline $38-70$ & $8 / 25 / 87$ & 0.95 & 0.98 \\
\hline $699-41-1$ (a) & $8 / 07 / 87$ & 0.17 & 0.015 \\
\hline $699-41-23$ (a) & $8 / 20 / 87$ & 3.8 & 0.3 \\
\hline$-46-4(a)$ & $7 / 31 / 87$ & 0.15 & 0.012 \\
\hline $699-47-50$ (a) & $8 / 04 / 87$ & 0.0061 & 0.0004 \\
\hline $699-49-55 A(a)$ & $9 / 04 / 87$ & 0.048 & 0.004 \\
\hline $699-49-57$ (a) & $9 / 04 / 87$ & 0.20 & 0.02 \\
\hline$-50-53(a)$ & $9 / 04 / 87$ & 0.044 & 0.0038 \\
\hline $699-50-53$ (a) & $9 / 04 / 87$ & 0.042 & 0.0035 \\
\hline
\end{tabular}

(a) Analyzed by neutron activation. A11 others analyzed by low-energy photo detector.

facilities. An iodine- 129 concentration of $2.7 \times 10^{-2} \mathrm{pCi} / \mathrm{L}$ was observed in ground water sampled from the Rattlesnake Ridge Interbed. Concentrations of $10^{-3}$ to $10^{-5} \mathrm{pCi} / \mathrm{L}$ iodine- 129 were observed in samples collected from eight horizons from the lower Saddle Mountains and Wanapum Formations. The report further concluded that the presence of iodine-129 in the confined ground waters was a result of transport from disposal sites along natural pathways 
and not a result of DB-15 drilling activities. All iodine-129 concentrations reported for confined aquifer ground waters were below the MCL of $1 \mathrm{pCi} / \mathrm{L}$.

Ground water from Wanapum horizons sampled in DC-18 also contained iodine-129 in concentrations above background. Early, Hall, and Johnson (1988) reported iodine-129 concentrations of approximately $10^{-2} \mathrm{pCi} / \mathrm{L}$ for four flowtops within the Wanapum basalt at $D C-18$. They concluded that iodine-129 (and tritium and carbon-14) is present in the ground water at concentrations above background as a result of downward migration of unconfined ground water containing contamination from 200 Areas disposal facilities.

\subsubsection{Cesium-137}

Concentrations of cesium-137 were below levels that can be accurately measured in all wells, except 299-E28-23 and 299-E28-25. The average concentration in wel1 299-E28-23 was $1595 \mathrm{pCi} / \mathrm{L}$, which was down from an average concentration of $2490 \mathrm{pCi} / \mathrm{L}$ during the last quarter. The MCL for cesium-137 is $200 \mathrm{pCi} / \mathrm{L}$, and the $\mathrm{DCG}$ is $3000 \mathrm{pCi} / \mathrm{L}$. The concentration in we11 299-E2825 remained unchanged with an average of $34 \mathrm{pCi} / \mathrm{L}$. Both wells are near the 216-B-5 reverse we11. A concentration of $69 \mathrm{pCi} / \mathrm{L}$ was reported for a sample collected in August from well 299-W19-20. This measurement appears to be an analytical artifact in that cesium- 137 had not previously been detected in we11 299-W19-20 or any adjacent wells and was not detected in subsequent sampling. 


\subsection{GEOCHEMICAL EVALUATIONS}

Use of the MINTEQ geochemical reaction code (Felmy, Girvin, and Jenne 1984) was initiated during April 1987 to identify the chemical reactions that might be affecting the concentrations of dissolved hazardous chemicals and radionuclides in ground water from the unconfined aquifer on the Hanford Site. This section summarizes the geochemical code used for these calculations and the results of the geochemical modeling studies completed during the second half of 1987. This information was presented at the Fall Meeting of the American Geophysical Union in San Francisco (Hinman, Krupka, and Criscenti 1987).

The MINTEQ code was used to calculate the aqueous speciation (i.e., complexation), charge imbalance, and possible mineral solubility controls for the compositions of water samples taken from the unconfined aquifer during the first three quarters of 1987. Information regarding the types and degree of complexation of trace metals by dissolved ligands (e.g., hydroxyl, carbonate, and sulfate) and the identification of possible solubility controls for these constituents will aid in predicting the transport and attenuation of trace metals in the Hanford Site ground-water system. Calculation of the charge imbalance for the speciated water compositions also provides an additional check of the completeness and quality of the inorganic chemical analyses and of the accuracy of data entry into the computerized ground-water data base for the Hanford Site ground-water monitoring project.

\subsection{MINTEQ CODE}

The MINTEQ geochemical code (Felmy et al. 1984; Felmy, Girvin, and Jenne 1984; Peterson et al. 1987) was developed at PNL by combining the best features of the extant geochemical codes MINEQL (Westa11, Zachary, and Morel 1976) and WATEQ3 (Ba11, Jenne, and Cantrell 1981). The MINTEQ code may be used to calculate aqueous speciation, solid phase saturation states, precipitation/dissolution reactions, and adsorption at equilibrium conditions. The general mathematical approaches used in MINTEQ to solve these chemical equilibria problems are described in Felmy, Girvin, and Jenne (1984). 
The speciation submodel first computes the activities for the uncomplexed and complexed aqueous species from an initial water composition and from the equilibrium constants $\left(\log \mathrm{K}_{r}^{\circ}, 298\right)$ and enthalpies of reaction $\left(\Delta \mathrm{H}_{r}^{\circ}, 298\right)$ at $298 \mathrm{~K}\left(25.00^{\circ} \mathrm{C}\right)$ contained in the thermodynamic data base associated with the geochemical code. The thermodynamic data base for the MINTEQ code was taken from the earlier codes WATEQ (Truesdell and Jones 1974), WATEQ2 (Bal1, Nordstrom, and Jenne 1980), and WATEQ3 (Ba11, Jenne, and Cantrell 1981), as well as the data base supplements completed at PNL (e.g., Krupka and Jenne 1982; Peterson et a1. 1987). The charge imbalance is then determined from the summation of the charge equivalents for the cations and anions calculated to be present for the speciated water composition.

After aqueous speciation for a given water is determined, solubility equilibrium hypotheses are tested. Ion activity products are calculated from the activities for the species, using the stoichiometries of the solubility reactions for minerals in the thermodynamic data base of the geochemical code. These activity products are then compared to the equilibrium solubility products $\left(\log \mathrm{K}_{\mathrm{r}, \mathrm{T}}\right)$ stored in the MINTEQ data base and corrected for temperature and ionic strength to determine if the water is at equilibrium $\left[\log \left(\mathrm{IAP} / \mathrm{K}_{r}, \mathrm{~T}\right)=0\right]$, oversaturated $\left[\log \left(\mathrm{IAP} / \mathrm{K}_{r}, \mathrm{~T}\right)>0\right]$, or undersaturated $\left[\log \left(\mathrm{IAP} / \mathrm{K}_{\mathrm{r}, \mathrm{T}}\right)<0\right]$ with respect to the solid phases in the data base. These calculated saturation indexes $\left[\log \left(\mathrm{IAP} / \mathrm{K}_{r}, \mathrm{~T}\right)\right]$ make it possible to ascertain permissible equilibrium solubility controls for that water.

\subsection{RESULTS}

Ground waters from the unconfined aquifer are generally low-ionic strength, high-alkalinity solutions at near neutral pH. Figure 4.1 shows the distribution of $\mathrm{pH}$ values for ground-water samples taken from the unconfined aquifer in the 100, 200, and 600 Areas during the second quarter of 1987 and for comparison from Hanford Site spring and surface waters reported in 1979 through 1987 by Early, Spice, and Mitchell (1986). Figure 4.2 shows the range of alkalinity values for ground-water samples taken from the 100, 200, and 600 Areas and springs and surface waters. The ground water in the unconfined aquifer consists of the major constituents listed in Table 4.1, with or without minor amounts of constituents listed in Table 4.2 and several 


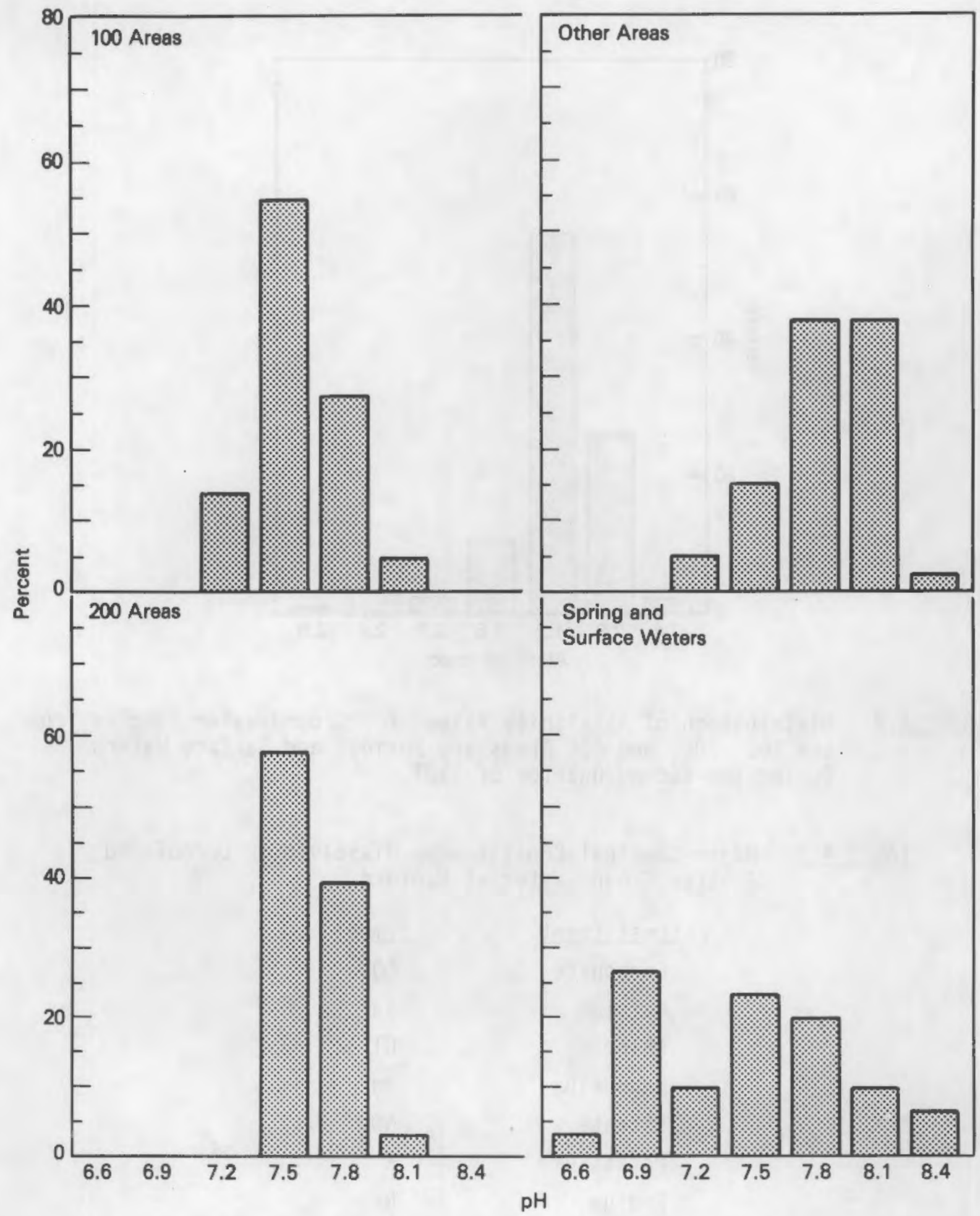

FIGURE 4.1. Distribution of $\mathrm{pH}$ Values for Ground-Water Samples from the Individual 100, 200, and 600 Areas During the Second Quarter of 1987 and from Spring and Surface Waters Reported by Early, Spice, and Mitchell (1986) 


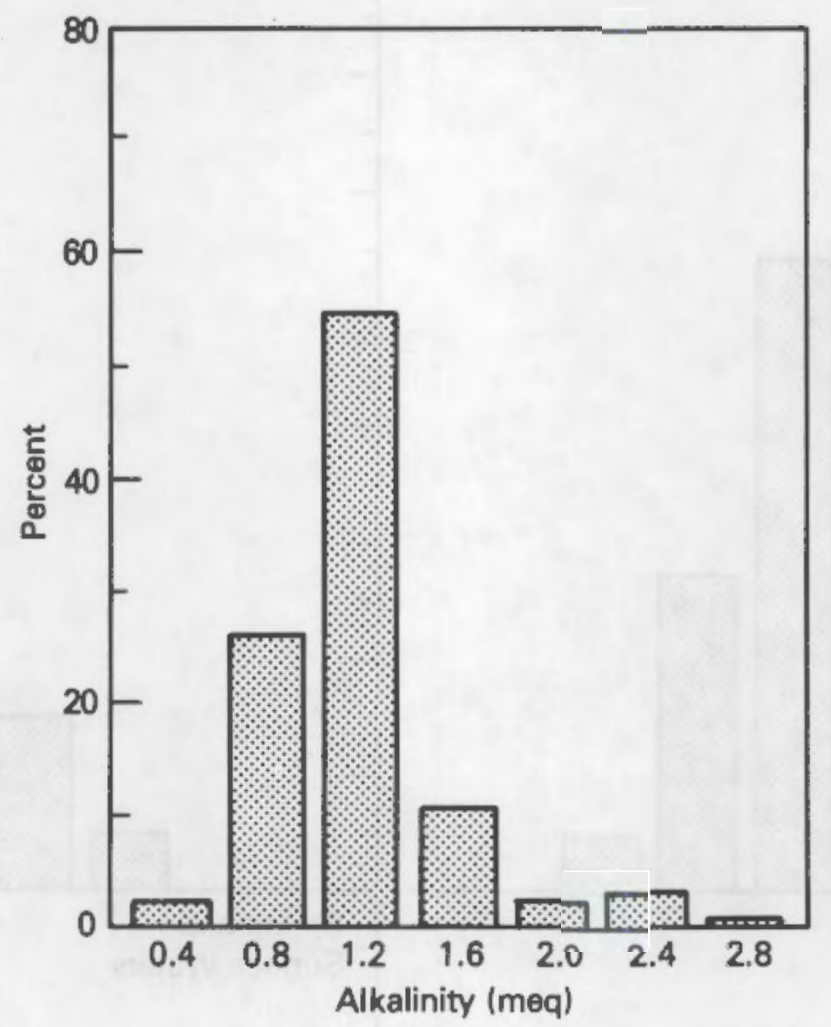

FIGURE 4.2. Distribution of Alkalinity Values for Ground-Water Samples from the 100, 200, and 600 Areas and Springs and Surface Waters During the Second Quarter of 1987

TABLE 4.1. Major Chemical Constituents Dissolved in Unconfined Aquifer Ground Water at Hanford

\begin{tabular}{ll} 
Constituent & Symbol \\
\cline { 2 - 3 } Carbonate & $\mathrm{CO}_{3}^{2-}$ \\
Calcium & $\mathrm{Ca}^{-}$ \\
Chloride & $\mathrm{Cl}^{-}$ \\
Magnesium & $\mathrm{Mg}$ \\
Nitrate & $\mathrm{NO}_{3}^{-}$ \\
Potassium & $\mathrm{K}$ \\
Sodium & $\mathrm{Na}$ \\
Sulfate & $\mathrm{SO}_{4}^{2-}$
\end{tabular}


TABLE 4.2. Chemical Constituents That May Be Present in Minor Amounts in Unconfined Aquifer Ground Water at Hanford

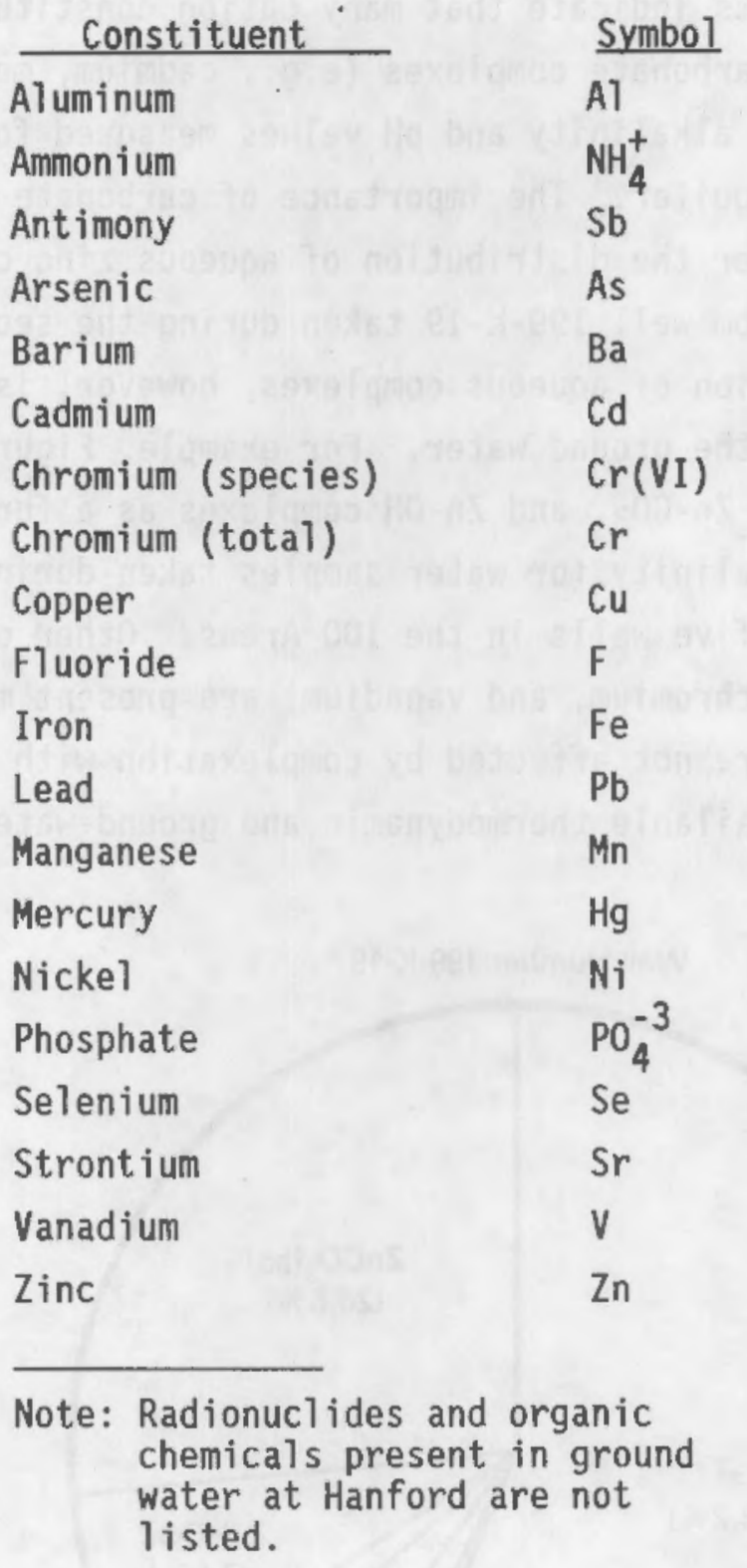

organic compounds. The MINTEQ results indicate that the general completeness and quality of the inorganic chemical analyses for the ground-water samples from the first three quarters of 1987 are adequate based on the low charge imbalances. If the calculated charge imbalance exceeded $15 \%$ for any of the modeled water compositions, the water sample was identified for reexamination. 


\subsubsection{Aqueous Speciation}

The MINTEQ results indicate that many cation constituents are present mainly as dissolved carbonate complexes (e.g., cadmium, mercury, lead, uranium, and zinc) at the alkalinity and $\mathrm{pH}$ values measured for the ground water from the unconfined aquifer. The importance of carbonate complexation is shown in Figure 4.3 for the distribution of aqueous zinc complexes calculated for a water sample from well 199-K-19 taken during the second quarter of 1987. This distribution of aqueous complexes, however, is dependent on the $\mathrm{pH}$ and alkalinity of the ground water. For example, Figure 4.4 shows the

distribution of $\mathrm{Zn}^{2+}, \mathrm{Zn}-\mathrm{CO}_{3}$, and $\mathrm{Zn}-\mathrm{OH}$ complexes as a function of increasing $\mathrm{pH}$ and decreasing alkalinity for water samples taken during the second quarter of 1987 from five wells in the 100 Areas. Other dissolved constituents (e.g., arsenic, chromium, and vanadium) are present mainly as hydrated aqueous species and are not affected by complexation with dissolved carbonate, based on the available thermodynamic and ground-water data. The

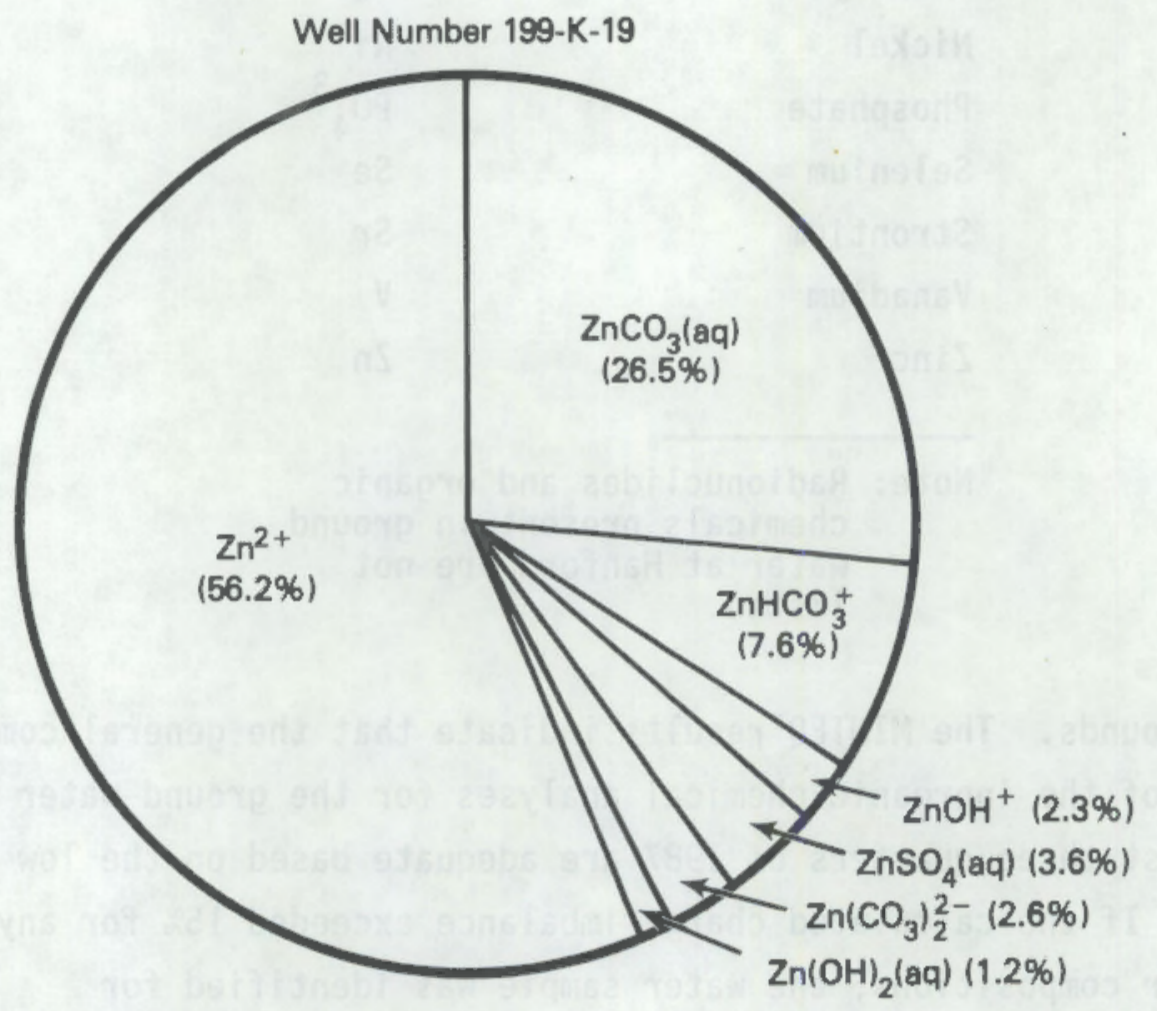

FIGURE 4.3. Distribution of Aqueous $\mathrm{Zn}$ Complexes Calculated for a Water Sample from the Second Quarter of 1987 from Well 199-K-19 

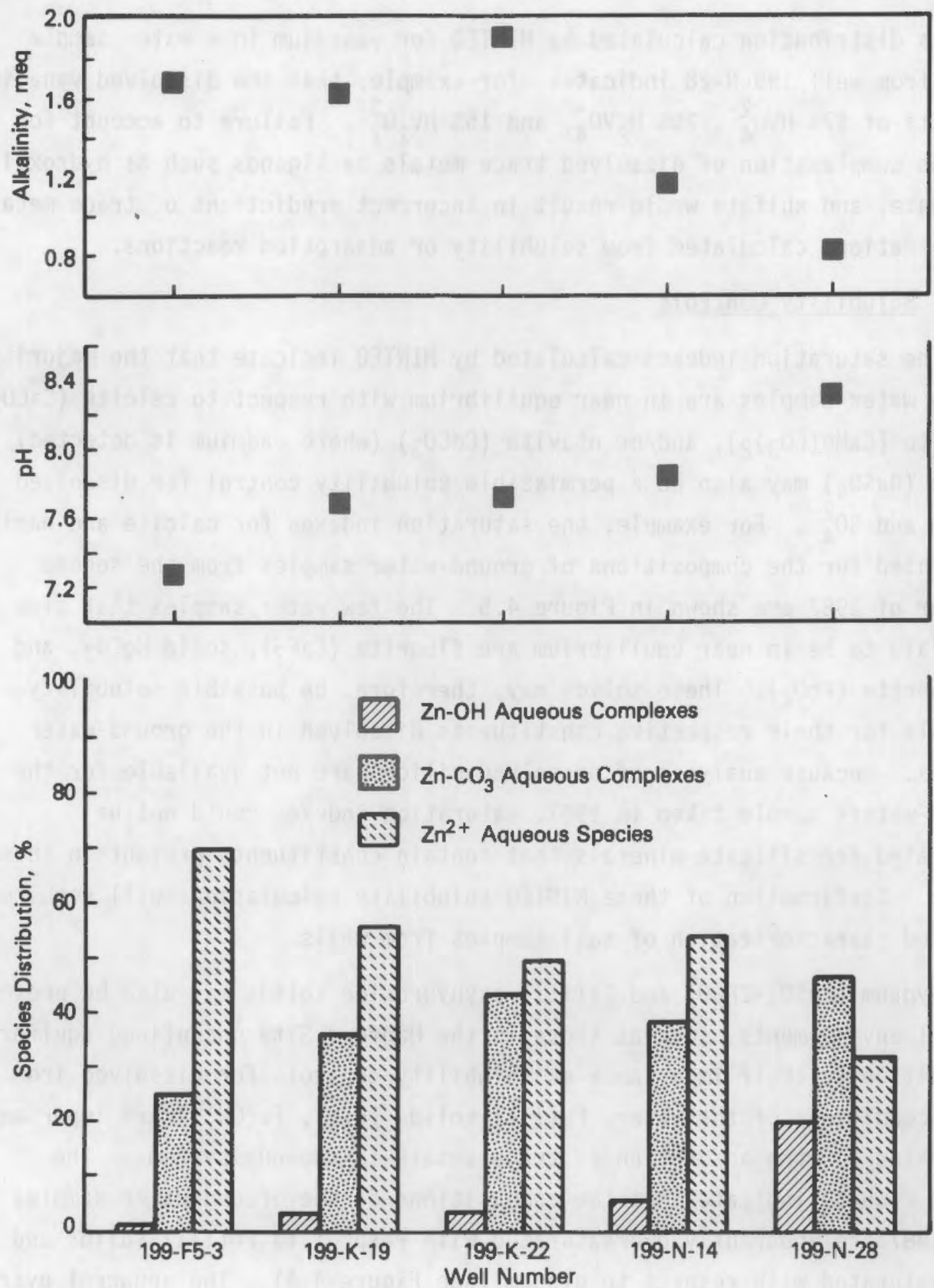

FIGURE 4.4. Distribution of $\mathrm{Zn}^{2+}, \mathrm{Zn}-\mathrm{CO}_{3}$, and $\mathrm{Zn}-\mathrm{OH}$ Complexes for Aqueous Zn Complexes Plotted as a Function of $\mathrm{pH}$ and Alkal inity for Water Samples from the Second Quarter of 1987 from Five Wells in the 100 Areas 
species distribution calculated by MINTEQ for vanadium in a water sample taken from well 199-N-28 indicates, for example, that the dissolved vanadium consists of $57 \% \mathrm{HVO}_{4}^{2-}, 28 \% \mathrm{H}_{2} \mathrm{VO}_{4}^{-}$, and $15 \% \mathrm{HV}_{2} \mathrm{O}_{7}^{3-}$. Failure to account for aqueous complexation of dissolved trace metals by ligands such as hydroxyl, carbonate, and sulfate would result in incorrect predictions of trace metal concentrations calculated from solubility or adsorption reactions.

\subsubsection{Solubility Controls}

The saturation indexes calculated by MINTEQ indicate that the majority of the water samples are in near equilibrium with respect to calcite $\left(\mathrm{CaCO}_{3}\right)$, dolomite $\left[\mathrm{CaMg}\left(\mathrm{CO}_{3}\right)_{2}\right]$, and/or otavite $\left(\mathrm{CdCO}_{3}\right)$ (where cadmium is detected). Barite $\left(\mathrm{BaSO}_{4}\right)$ may also be a permissible solubility control for dissolved barium and $\mathrm{SO}_{4}^{2-}$. For example, the saturation indexes for calcite and barite calculated for the compositions of ground-water samples from the second quarter of 1987 are shown in Figure 4.5. The few water samples that also calculate to be in near equilibrium are fluorite $\left(\mathrm{CaF}_{2}\right)$, solid $\mathrm{HgCO}_{3}$, and plattnerite $\left(\mathrm{PbO}_{2}\right)$. These solids may, therefore, be possible solubility controls for their respective constituents dissolved in the ground-water samples. Because analyses of dissolved silicon are not available for the ground-waters sample taken in 1987, saturation indexes could not be calculated for silicate minerals that contain constituents present in these waters. Confirmation of these MINTEQ solubility calculations will require detailed characterization of soil samples from wells.

Gypsum $\left(\mathrm{CaSO}_{4} \cdot 2 \mathrm{H}_{2} \mathrm{O}\right)$ and $\mathrm{Fe}$ (III) oxyhydroxide solids may also be present in soil environments, such as those in the Hanford Site unconfined aquifer. In addition to their importance as solubility controls for dissolved iron and redox conditions of the water, $\mathrm{Fe}(\mathrm{III})$ solids [e.g., $\left.\mathrm{Fe}(\mathrm{OH})_{3}\right]$ are important substrates for the adsorption of trace metals in aqueous systems. The MINTEQ results indicate that the compositions of the ground-water samples from 1987 are apparently oversaturated with respect to $\mathrm{Fe}$ (III) solids and undersaturated with respect to gypsum (see Figure 4.5). The apparent oversaturation of these waters with respect to $\mathrm{Fe}$ (III) solids requires further investigation because $\mathrm{Fe}(\mathrm{III})$ solids [e.g., $\mathrm{Fe}(\mathrm{OH})_{3}$ ] are commonly found to 

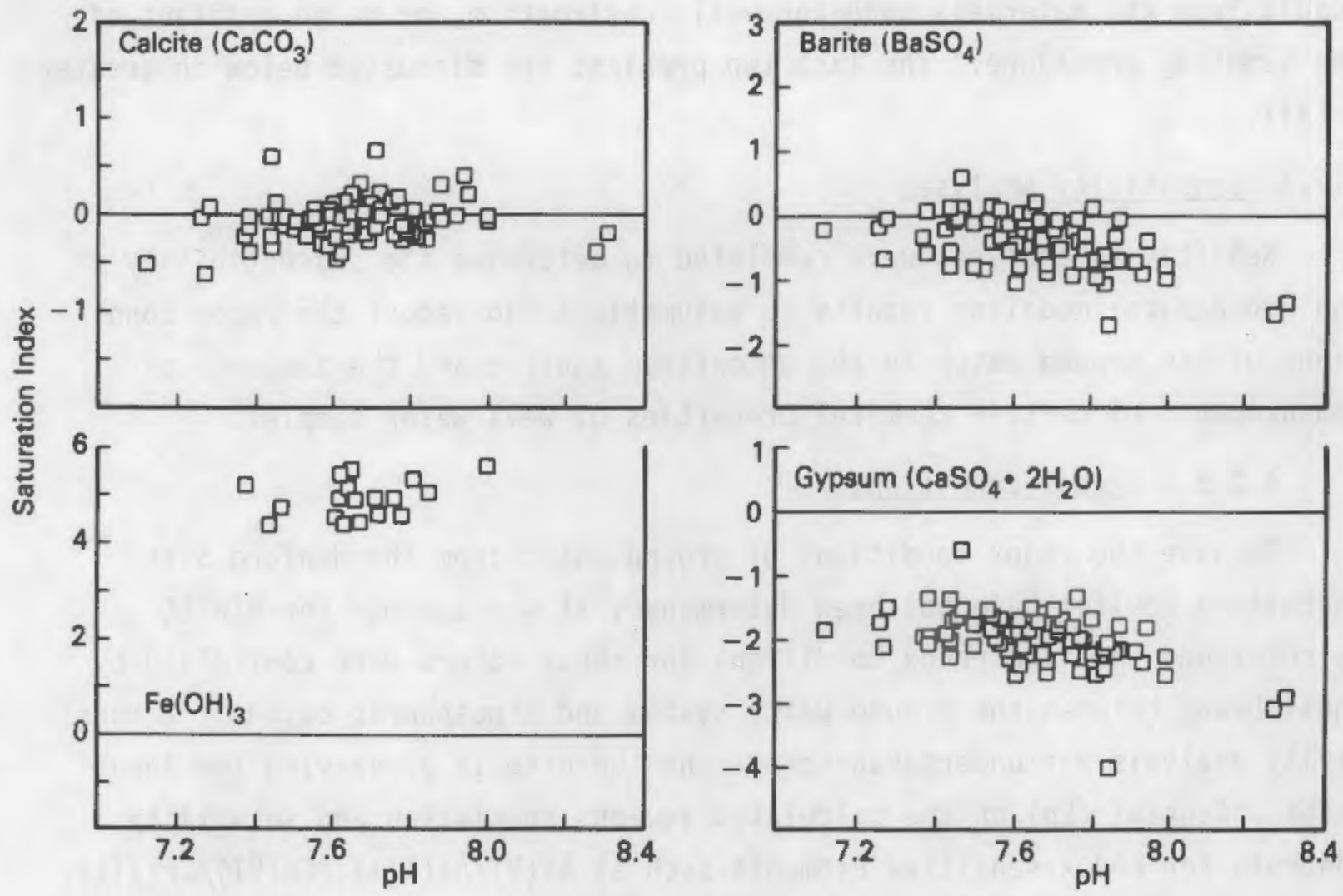

FIGURE 4.5. Saturation Indices for Calcite, Barite, $\mathrm{Fe}(\mathrm{OH})_{3}$, and Gypsum, Plotted as a Function of $\mathrm{pH}$ for Compositions of Water Samples Taken During the Second Quarter of 1987

control iron concentrations in oxidizing soil/water environments [e.g., Lindsay's (1979) discussion of Fe(III) in soil systems]. However, it is not possible at this time to determine the adequacy of the MINTEQ calculations for redox-sensitive elements such as dissolved iron because of the undetermined effect of colloidal iron, the lack of chemical and mineralogical characterization for soils from these wells, the lack of measurements of the redox state for ground water in the unconfined aquifer, and the lack of adequate sensitivity for solubility calculations of the analytical method used to measure dissolved iron in these ground-water samples. Filtration tests can be done on ground-water samples collected from wells that have shown elevated concentrations of dissolved iron in the past. The filtration tests will indicate if any of the detected iron is present as suspended, colloidal ferric hydroxide. The iron colloid may originate in the aquifer, 
result from the materials used for well construction, or be an artifact of the sampling procedure. The last two problems are discussed below in greater detail.

\subsubsection{Sensitivity Analyses}

Sensitivity analyses were completed to determine the susceptibility of the geochemical modeling results to assumptions made about the redox conditions of the ground water in the unconfined aquifer and the adequacy of measurements of certain chemical properties of well water samples.

\subsubsection{Redox Conditions}

Because the redox conditions of ground water from the Hanford Site unconfined aquifer have not been determined, it was assumed for MINTEQ calculations that the redox conditions for these waters were controlled by equilibrium between the ground-water system and atmospheric oxygen. A sensitivity analysis was undertaken to examine the effects of varying the input redox potential (Eh) on the calculated aqueous speciation and solubility controls for redox-sensitive elements such as $\mathrm{As}$ (V)/As(III), $\mathrm{Cr}$ (VI)/Cr(III), $\mathrm{Fe}(\mathrm{III}) / \mathrm{Fe}(\mathrm{II})$, and $\mathrm{Mn}(\mathrm{III}) / \mathrm{Mn}$ (II). Four ground-water compositions with $\mathrm{pH}$ between 7.4 and 7.7 were selected for the calculations.

The modeling results indicate that a decrease of $500 \mathrm{mV}$ from a starting Eh of $774.8 \mathrm{mV}$ at constant $\mathrm{pH}$ does not markedly affect the calculated concentrations of $\mathrm{As}$ (V), $\mathrm{Fe}$ (III), and $\mathrm{Mn}$ (II) that constitute the dominant redox valence states of these elements over this $\mathrm{pH}$-Eh range. The saturation indexes calculated for minerals containing these redox-sensitive elements, however, do change over this Eh range in relation to changes in the dissolved concentrations of their constituent elements. Measurements of Eh, dissolved $0_{2}$, and/or analytical redox couples will be needed to determine the validity of the assumption that these waters are in equilibrium with atmospheric oxygen.

\subsubsection{Detection-Level Sensitivity Analysis}

A series of MINTEQ calculations was also completed to test the sensitivity of the geochemical modeling results to the minimum detection levels reported for the analytical methods used to measure dissolved constituents such as iron and $\mathrm{PO}_{4}^{3-}$. Concentrations at the minimum detection limits for 
these constituents were entered into the MINTEQ input files to determine whether these levels were adequately sensitive to predict possible solubility controls for these constituents.

The minimum detection level reported for the analysis of dissolved iron is $50 \mu \mathrm{g} / \mathrm{L}$. Several wells from the 100,200 , and 600 Areas sampled during the first quarter of 1987 were analyzed using the MINTEQ geochemical code with and without the minimum detection levels of iron. In all cases where detection-level iron was used, the results indicated that the solutions are oversaturated with respect to all $\mathrm{Fe}$ (III) solids [e.g., $\left.\mathrm{Fe}(\mathrm{OH})_{3}\right]$ in the MINTEQ thermodynamic data base. These results indicate that the analytical method currently used to measure dissolved iron is not sufficiently sensitive to allow adequate prediction of possible solubility controls for iron. Because of the importance of iron solids as possible controls of redox conditions and as adsorption substrates for trace metals, analyses of dissolved iron with an analytical method having a lower detection level are required to accurately calculate the aqueous speciation and solubility controls for dissolved iron.

The minimum detection level reported for the analysis of dissolved $\mathrm{PO}_{4}^{3-}$ is $1000 \mu \mathrm{g} / \mathrm{L}$. Several wells from the 200 -West Area sampled during the first quarter of 1987 were modeled using the MINTEQ geochemical code with and without the minimum detection levels for $\mathrm{PO}_{4}^{3-}$. In all cases where detectionlevel $\mathrm{PO}_{4}^{3-}$ was used, the results indicated that the solutions were near equilibrium with respect to hydroxyapatite $\left[\mathrm{Ca}_{5}\left(\mathrm{PO}_{4}\right)_{3}(\mathrm{OH})\right]$ and slightiy undersaturated to slightly oversaturated with respect to solid $\mathrm{MnHPO}_{4}$ and strengite $\left(\mathrm{FePO}_{4} \cdot 2 \mathrm{H}_{2} \mathrm{O}\right)$. These results indicate that the detection level for dissolved $\mathrm{PO}_{4}^{3-}$ is not sufficiently sensitive to adequately predict solubility controls for $\mathrm{PO}_{4}^{3-}$. Analyses of dissolved $\mathrm{PO}_{4}^{3-}$ with an analytical method having a lower detection level are required to accurately calculate the aqueous speciation and solubility controls for this constituent. 


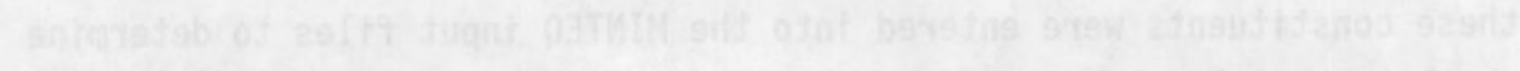

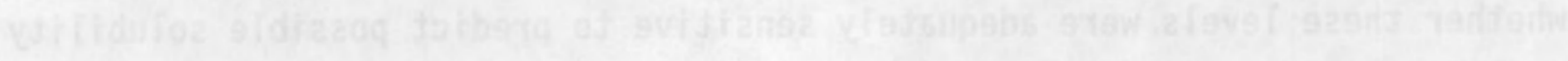

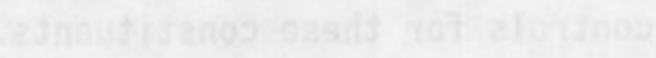

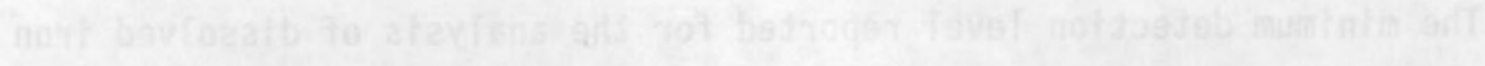

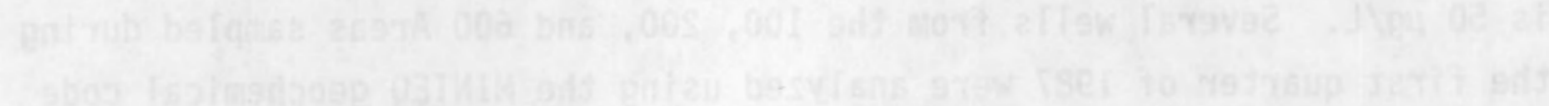

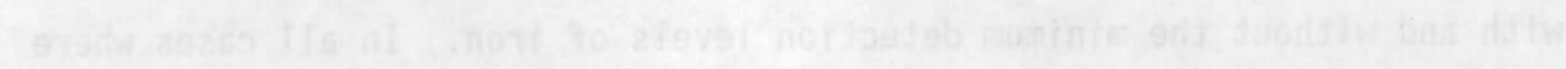

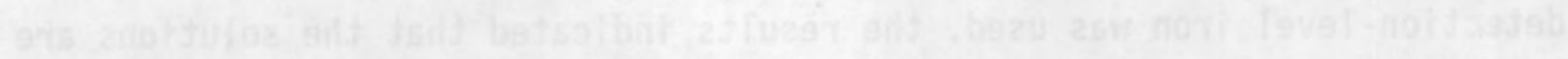

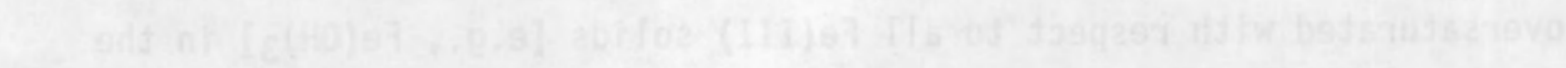

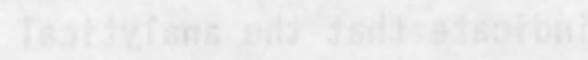

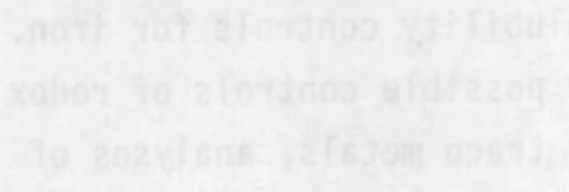

政 


\subsection{GROUNO-WATER FLOW MODELING}

Large volumes of process cooling water are discharged to the ground from operations in the central portion of the Hanford Site. Over the years, these large volumes of waste water have reached the water table and created groundwater mounds near major disposal facilities and have impacted ground-water and contaminant movement in the unconfined aquifer. The unconfined aquifer is located in a sequence of fluvial, lacustrine, and glaciofluvial sediments that were deposited on top of the Columbia River Basalt Group.

Ground-water flow and contaminant transport models developed for the unconfined aquifer during the 1970s have been applied to assess the impacts of operations on the rate and direction of ground-water flow and contaminant transport. Currently, these modeling capabilities for the unconfined aquifer are being improved by adapting new computer codes and calibrating models to more current Hanford Site data.

\subsection{DESCRIPTION OF PREVIOUS WORK}

Ground-water flow and contaminant transport models were developed for the unconfined aquifer and applied during the 1970s. The Variable Thickness Transient (VTT) finite difference flow code (Kipp et al. 1972) was calibrated to existing data and was used to predict ground-water flow in the unconfined aquifer. The Multicomponent Mass Transport (MMT) code (Ahlstrom et a1. 1977) was applied to simulate movement of the tritium plume between the 200-East Area and the Columbia River. The Hanford Pathline Calculational code (Friedrichs, Cole, and Arnett 1977) was applied to predict advective transport of contaminants along pathlines in the unconfined aquifer predicted with the VTT code. A later modification of the streamline approach, the TRANSS code, was developed by Simmons, Kincaid, and Reisenauer (1986). Specific applications of these codes for estimating ground-water and contaminant travel times at the Hanford Site are described in Freshley and Graham (1988).

The ground-water flow model based on the VTT code was calibrated with a Transmissivity Iterative Calculation Routine (Cearlock, Kipp, and Friedrichs 1975) to estimate the hydraulic conductivity distribution for the unconfined 
aquifer. The technique applied by Cearlock, Kipp, and Friedrichs (1975) is based on an equation obtained by numerical integration of the Boussinesq equation along instantaneous stream tubes of flow. The Boussinesq equation (Fetter 1980) describes ground-water flow in unconfined aquifers. The constructed stream tubes were based on a hand-contoured water-level map for 1973. A transmissivity value obtained from aquifer test data was needed in each stream tube as an initial value to calculate all other transmissivities along the stream tube. In portions of the study area that had stream tubes for which no transmissivity data were available, the spatial distribution of transmissivity could not be directly calculated. Thus, in these portions of the study area, the transmissivity values were estimated by interpolation.

The calibrated distribution of hydraulic conductivity was used in the VTT code along with estimates of the storage coefficient, recharge to and discharge from the aquifer, and boundary conditions to predict transient water levels in the unconfined aquifer from 1968 to 1973 (Cearlock, Kipp, and Friedrichs 1975). The calibrated ground-water flow model yielded reasonable predicted water levels over most of the unconfined aquifer but did not directly include recharge and flux boundary conditions in the calibration procedure.

In 1984, work was initiated to develop a two-dimensional model of ground-water flow and contaminant transport in the unconfined aquifer based on the Coupled Fluid, Energy, and Solute Transport (CFEST) code (Gupta et a). 1982). The CFEST finite element code was selected because it can be applied to simulate ground-water flow and contaminant transport in two or three dimensions under steady-state or transient conditions, it can account for radionuclide decay, and it allows recharge to or discharge from the aquifer.

The hydraulic conductivity distribution and other input data for the previously calibrated ground-water flow model based on the VTT code were adapted to the CFEST code. The finite element grid for the ground-water flow model based on CFEST (Figure 5.1) was designed to provide detail at locations of waste-water discharge and in areas with rapid changes in hydraulic conductivity. Results from the ground-water flow model based on CFEST were 


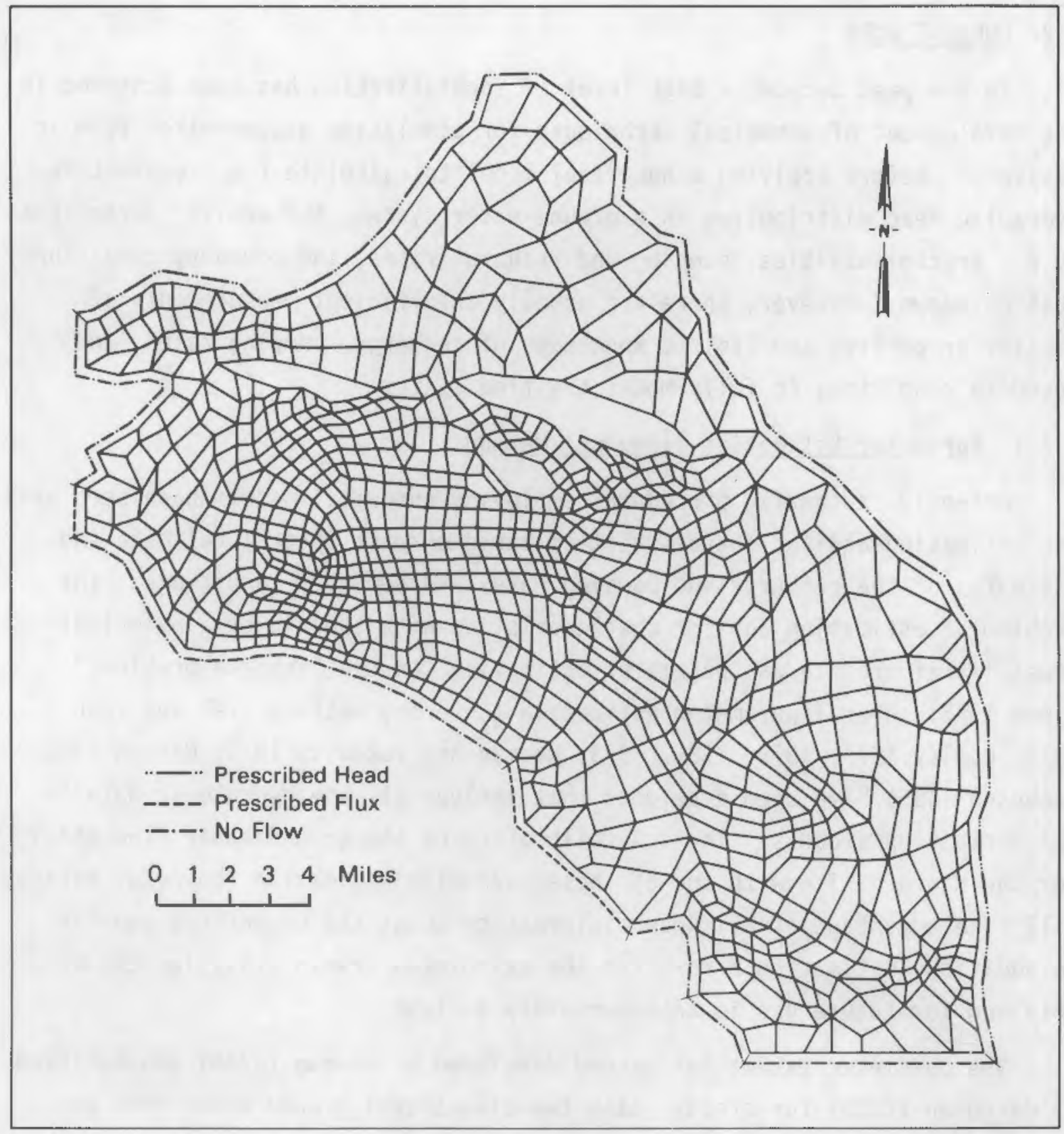

FIGURE 5.1. Finite Element Grid and Boundary Conditions for the Model Based on the CFEST Code

compared with those from the VTT model. For steady-state conditions, the results from the model based on CFEST reproduced those from the model based on VTT. 


\subsection{CURRENT WORK}

In the past decade, a high level of sophistication has been achieved in the development of numerical techniques for simulating ground-water flow in aquifers. Before applying a numerical model to calculate the steady-state hydraulic head distribution in a ground-water system, the aquifer parameters (e.g., transmissivities, pumping and recharge rates, and boundary conditions) must be known. However, there are usually insufficient measurements of aquifer properties and limited knowledge of recharge, pumping rates, and boundary conditions to fully model the flow system.

\subsubsection{Parameter Estimation (Inverse) Method}

Recently, automatic procedures employing numerical models have been used for estimating aquifer characteristics based on past water-level data and estimates of the recharge and pumping rates and boundary conditions. The problem of estimating aquifer characteristics with the aid of a numericalmodel is referred to as "parameter estimation" or the "inverse problem." Since 1975, several parameter estimation (inverse) methods (Yeh and Yoon 1976; Cooley 1977, 1979, 1982, 1983; Neuman and Yakowitz 1979; Neuman 1980; Jacobson 1985) have been developed that include all the hydrologic data in the estimation process. Thus, a calibration of the ground-water flow model for the Hanford Site with one of these parameter estimation (inverse) methods will take advantage of all known information about the unconfined aquifer. In addition, estimation errors for the calculated transmissivities can be obtained for future use in an uncertainty analysis.

The parameter estimation method developed by Neuman (1980) and modified by Jacobson (1985) for steady-state two-dimensional ground-water flow problems was applied to the data from the unconfined aquifer at Hanford. This research contributes to modeling capabilities at Hanford by providing an improved method for calibrating the current two-dimensional ground-water flow model. This improved method includes all available information about estimates of transmissivities, hydraulic heads, boundary conditions, and discharges to and withdrawals from the aquifer to obtain a preliminary inverse calibration of the ground-water flow model. 


\subsubsection{Data Preparation for the Inverse Model}

Preparation of transmissivity and hydraulic head data, as well as information on boundary conditions and discharges to the unconfined aquifer, is required before these data may be used in the inverse model of Neuman (1980). These data must represent a consistent set of information from the same period of time for the inverse calibration. Because the inverse model requires steady-state conditions, available data were reviewed to select a period when water levels and discharges to ground were relatively constant. Based on the water level and discharge information, 1979 was selected as the most appropriate year for use in the inverse calibration. Ground-water levels and discharges to ground remained constant beginning in 1976 and continuing through 1979. As a result, 1979 represented the closest approximation to steady-state conditions within recent Hanford Site operations.

Ground-water levels for June and December 1979 were reviewed. The December measurements were selected for the inverse calibration because these data are closer to the end of the steady-state period. In addition, the influence of changing river level is less in December because the Columbia River is typically lower and more constant than in June.

Water-level data collected in December 1979 were reviewed for consistency. Water-level measurements from wells that were obvious outliers or from wells strongly influenced by changes in river stage were not included in data used for the inverse calibration. A few wells were not included because their screened intervals are open to a large portion of the unconfined aquifer and may not reflect the water table. Of the 278 wells included in the original list of hydraulic head measurements for December 1979, 214 were used for the inverse calibration.

Neuman's (1980) inverse method employs a finite element representation that assumes transmissivity is constant in each element. If several elements have the same transmissivity, they can be treated as a zone of constant transmissivity. Transmissivity values obtained from aquifer tests are generally viewed as point measurements because they represent an average value over the aquifer close to the well. Thus, aquifer test data must be modified to obtain estimates of the spatial scale of elements or zones for input into the inverse model. 
Estimates of transmissivity have been made from aquifer tests conducted on the Hanford Site since 1945 (Bierschenk 1959). The transmissivity data through 1972 were included in the calibration of the VTT ground-water flow model for the unconfined aquifer at Hanford (Cearlock, Kipp, and Friedrichs 1975).

Results from tests on wells completed in the Hanford Site unconfined aquifer (Bierschenk 1959; Kipp and Mudd 1973; Deju and Summers 1975; Graham et al. 1981) were reviewed for the inverse calibration. In addition to the reported aquifer tests in the published documents, unpublished aquifer test data stored at PNL and WHC were reviewed and reanalyzed where required.

Although a number of additional published and unpublished aquifer tests for the unconfined aquifer are available, tests from 52 wells provided data that were suitable for interpretation and analysis. If a sufficient number of data points were available, a geostatistical technique (called kriging) could have been applied to interpolate and average the transmissivity data to obtain estimates for each zone. Because the transmissivity data could not be kriged, the distribution of transmissivity obtained from calibration of the VTT model (Cearlock, Kipp, and Friedrichs 1975) was adapted as an initial estimate for the inverse calibration.

A zonation pattern and prior estimates of transmissivities were developed based on the distribution of transmissivities obtained in the VTT model calibration (Cearlock, Kipp, and Friedrichs 1975). The prior estimates of transmissivity for the inverse method were calculated for each zone by taking the arithmetic average of the logarithm of the transmissivity values for all elements in the zone. No statistical information was available about the prior estimates of transmissivity; thus, only the prior estimates themselves were included in the inverse calibration. A contour map of transmissivities calculated by assigning the prior estimates of transmissivity to the center of each element in the zones illustrates the overall spatial variation of prior transmissivities (Figure 5.2). In actuality, these transmissivity values are treated in the ground-water flow model as constant in each zone. 


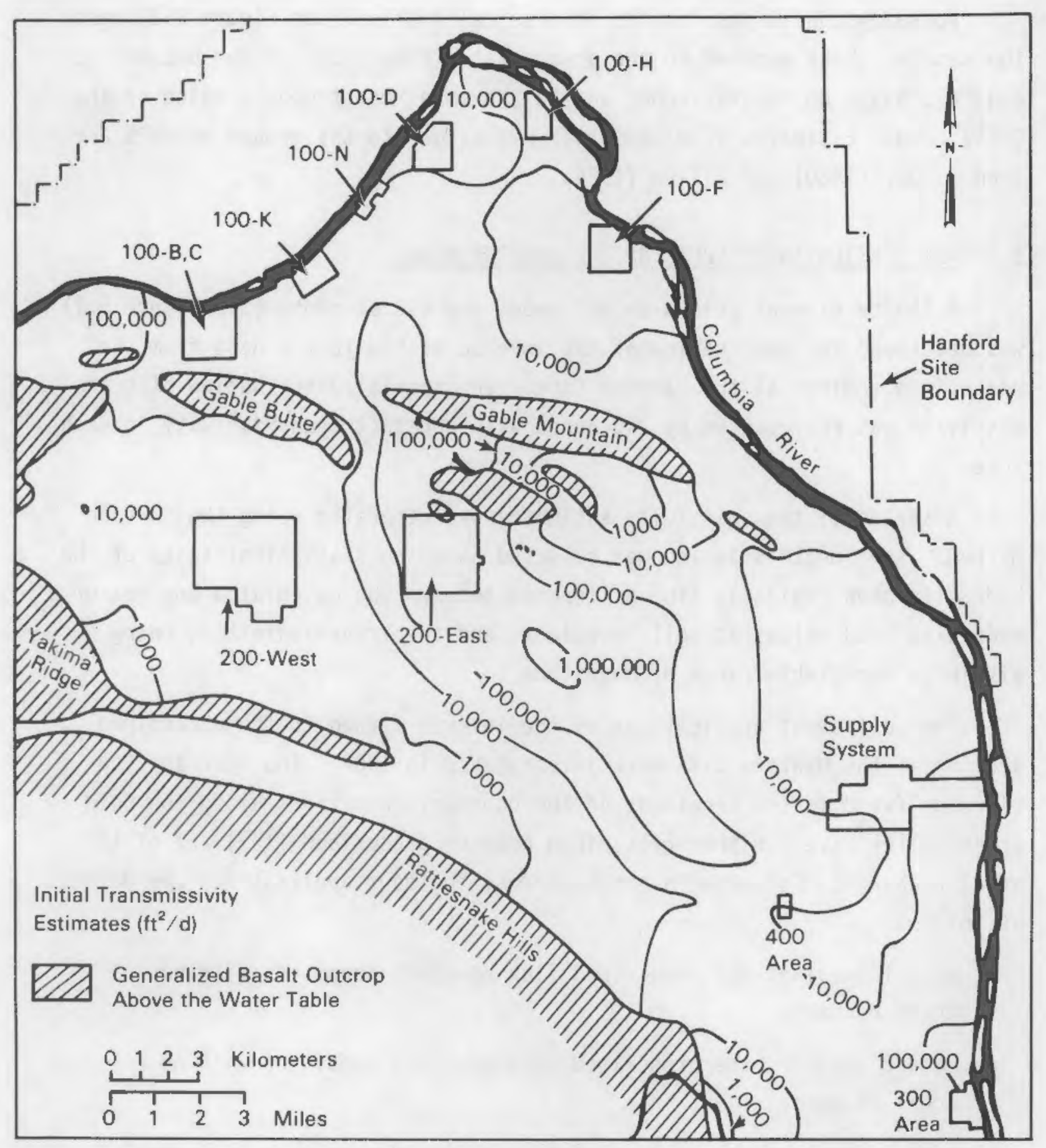

FIGURE 5.2. Contour Map of Initial Transmissivities for Inverse Calibration 
Boundary conditions for the inverse calibration (see Figure 5.2) were the same as those applied to the ground-water flow model of the unconfined aquifer, based on the VTT code, and to the more recent model, based on the CFEST code. Estimates of waste water discharged to the ground in 1979 are from Sliger (1980) and Dillon (1985).

\subsection{APPLICATION AND RESULTS OF THE INVERSE MODEL}

A finite element grid with 966 nodes and 878 elements (see Figure 5.1) was developed for application of the inverse method to the data from the unconfined aquifer at the Hanford Site. The spatial distribution of transmissivity was represented by 240 zones with constant transmissivity in each zone.

A series of transmissivity estimates was generated using the inverse method. The "best" solution was selected based on statistical tests of the hydraulic head residuals (the difference between the calculated and measured hydraulic head values at well locations) and the transmissivities being within an acceptable range of magnitude.

Four different applications of the inverse method to the unconfined aquifer at the Hanford Site were investigated in 1987. The four applications differed in the treatment of the boundary conditions along the cold Creek valley (i.e., either prescribed head or prescribed flux) and of the areal recharge. For ease in presentation, the four applications are defined as follows:

- case 1 involves the prescribed flux boundary condition with no areal recharge

- case 2 involves the prescribed head boundary condition with no areal recharge

- case 3 involves the prescribed flux boundary condition with areal recharge

- case 4 involves the prescribed head boundary condition with areal recharge. 
The objectives of the applications were to investigate how changes in the cold Creek valley boundary conditions and the addition of areal recharge affected the calibration of the ground-water flow model for the unconfined aquifer at the Hanford Site. Thus, three types of comparisons of the results of the different applications were made: 1) variations caused by differing boundary conditions, 2) variations caused by including areal recharge, and 3) differences between the "best" calibrations and the hand-contoured water levels.

\subsubsection{Comparison of Inverse Results for Varying Cold Creek Valley Boundary Conditions with No Areal Recharge}

The water levels calculated by case 1 with prescribed flux at the cold Creek valley boundary and no areal recharge are unreasonably high in the cold Creek valley and indicate that this inverse application does not yield a good calibration to the expected water levels in this region. The water levels calculated by case 2 with prescribed head boundary condition and no areal recharge in the cold Creek valley are reasonable because of the prescribed head conditions. A comparison of the case 1 and case 2 water levels, however, indicates that, in most of the study area, the water levels are very similar.

\subsubsection{Comparison of Inverse Results for Varying Cold Creek Valley Boundary Conditions with Areal Recharge}

The recharge estimates used in the inverse applications represent one possible spatial distribution based on knowledge of the soil and vegetation types on the Hanford Site. The goal in the inverse applications is to investigate the effect on the estimates of transmissivity of including areal recharge. The recharge values vary from 12 to $0.5 \mathrm{~cm} / \mathrm{yr}$, where the 7 arger value is $75 \%$ of the precipitation.

The water levels computed from case 3 with prescribed flux boundary condition along the cold Creek valley and areal recharge are unreasonable in the region of the cold Creek valley. The water levels computed from case 4 with prescribed head boundary conditions along the Cold Creek valley and areal recharge are reasonable along the cold Creek valley, and the values in 
other portions of the study area do not differ significantly from those obtained by using case 3 .

\subsubsection{Comparison of the Inverse Results for Varying Areal Recharge with Fixed Boundary Conditions Along the Cold Creek Valley}

Water levels produced by case 1 with no recharge and case 3 with recharge were compared. For both of these inverse applications, the boundary condition along the Cold Creek valley was prescribed flux. In general, the water-level patterns are similar for the two inverse applications. Although both applications produced water levels that were unreasonably high in the Cold Creek valley, the inverse results that included areal recharge (i.e., case 3 ) yielded water levels in this area that were $40 \mathrm{ft}$ smaller than the case 1 levels.

Water levels produced by case 2 with no areal recharge and case 4 with areal recharge were also compared. For both these inverse applications, the boundary condition along the Cold Creek valley was prescribed head. The water-level patterns are very similar for the two inverse applications. The water-1evel contours in the eastern portion of the study area have been shifted to the east when areal recharge is included (i.e., case 4), and this position is closer to the hand-contoured water levels.

\subsubsection{Comparison of the "Best". Results of the Inverse Applications to the Hand-Contoured Water Levels}

The water levels computed by the inverse applications with the prescribed head boundary condition along the cold Creek valley were considered to be the most representative of the observed water levels. For these inverse applications, the average residuals are smaller and the water levels along the cold Creek valley were reasonable.

The inverse applications used water levels at well locations in the fitting procedure; thus, a direct comparison to hand-contoured water levels may be misleading. However, the comparison gives an overall indication of the fit of calculated to measured values. The water levels for case 2 with no areal recharge are compared to the hand-contoured water levels in Figure 5.3. The general trends (e.g., steep gradient to the east of the 200-West Area) have been reproduced by the inverse results. The water levels 


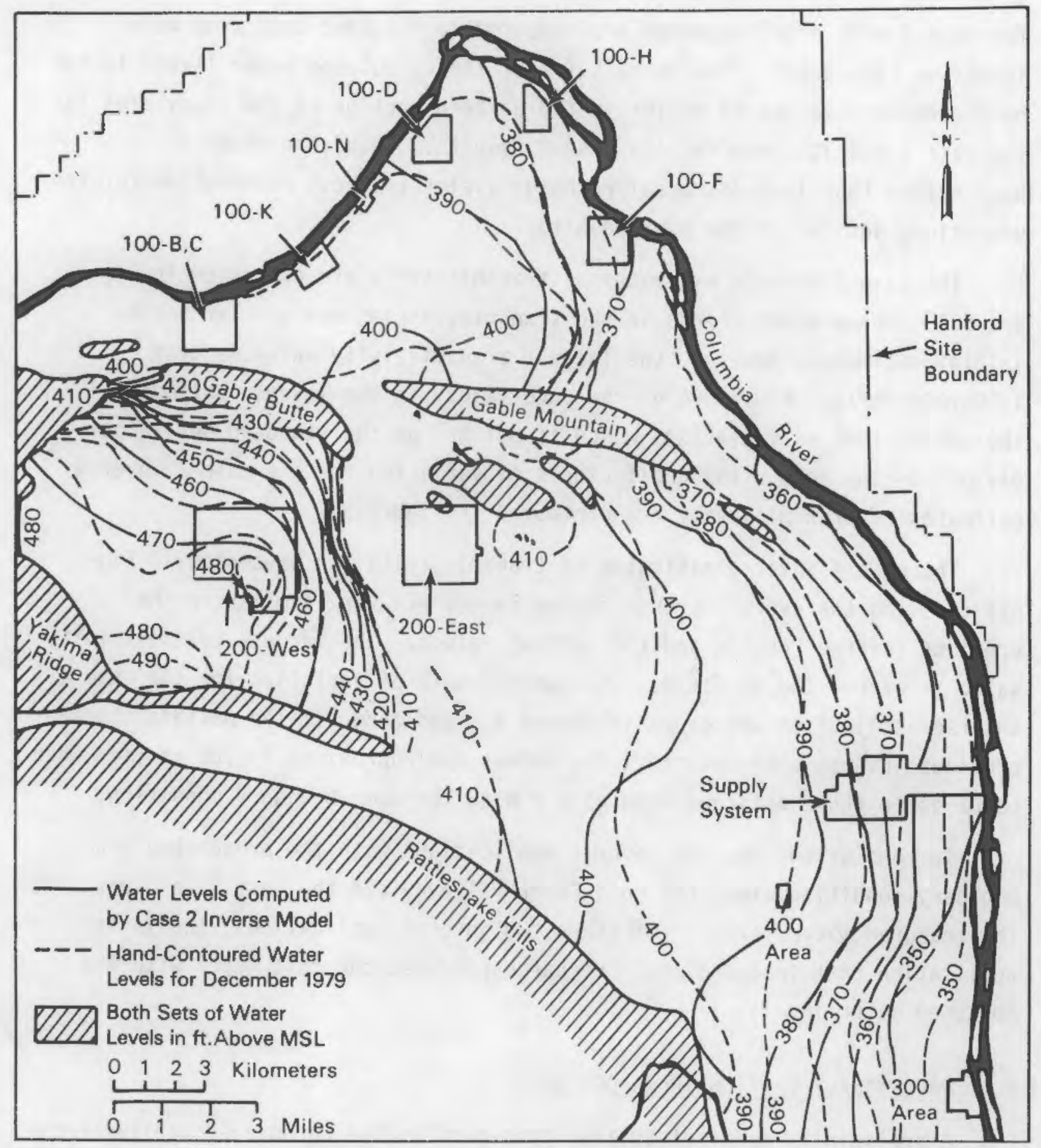

FIGURE 5.3. Comparison of Water Levels Computed by the Case 2 Inverse Model and Hand-Contoured Water Levels 
for case 4 with areal recharge are compared to the hand-contoured water levels in Figure 5.4. The overall fit of the calculated water levels to the hand-contoured values is better in the eastern portion of the study area for the case 4 results than for the case 2 results. Thus, the inverse application that includes areal recharge yields the best calibration for the unconfined aquifer at the Hanford Site.

The case 2 inverse estimates of transmissivity are presented in Figure 5.5. The overall trends in the transmissivities are similar to the initial estimates; however, the largest transmissivity value is over $1,500,000 \mathrm{ft}^{2} / \mathrm{d}$. A measure of the uncertainty in the inverse estimates is the coefficient of variation, which is defined as the standard deviation divided by the mean. The coefficients of variation for the case 2 inverse estimates of transmissivity are contoured in Figure 5.6.

The case 4 inverse estimates of transmissivity are presented in Figure 5.7, and the overall trends in the values are very similar to the previous inverse results and the initial values. The largest transmissivity value is over $1,200,00 \mathrm{ft}^{2} / \mathrm{d}$. The coefficients of variation for the case 4 inverse application are given in Figure 5.8 and show similar patterns to the previous inverse applications. The values are approximately $10 \%$ smaller when compared to those obtained from case 2 with the same boundary condition.

In conclusion, the two inverse applications with the prescribed head boundary condition along the Cold Creek valley yield the best fits overall to the measured water levels. Of these two inverse applications, the inverse application that included areal recharge produced the best match with the measured water levels.

\subsection{PRELIMINARY TRITIUM TRANSPORT MODEL}

A preliminary model of tritium transport in the unconfined aquifer from the 200-East Area was developed. The finite element grid for the tritium transport model was the same as that developed for the ground-water flow model (see Figure 5.1). The distribution of tritium in the aquifer for 1983 (Prater et al. 1984) was input to the model as an initial condition (Figure 5.9). Sources of tritium in the 200-West Area were not included in this modeling effort. 


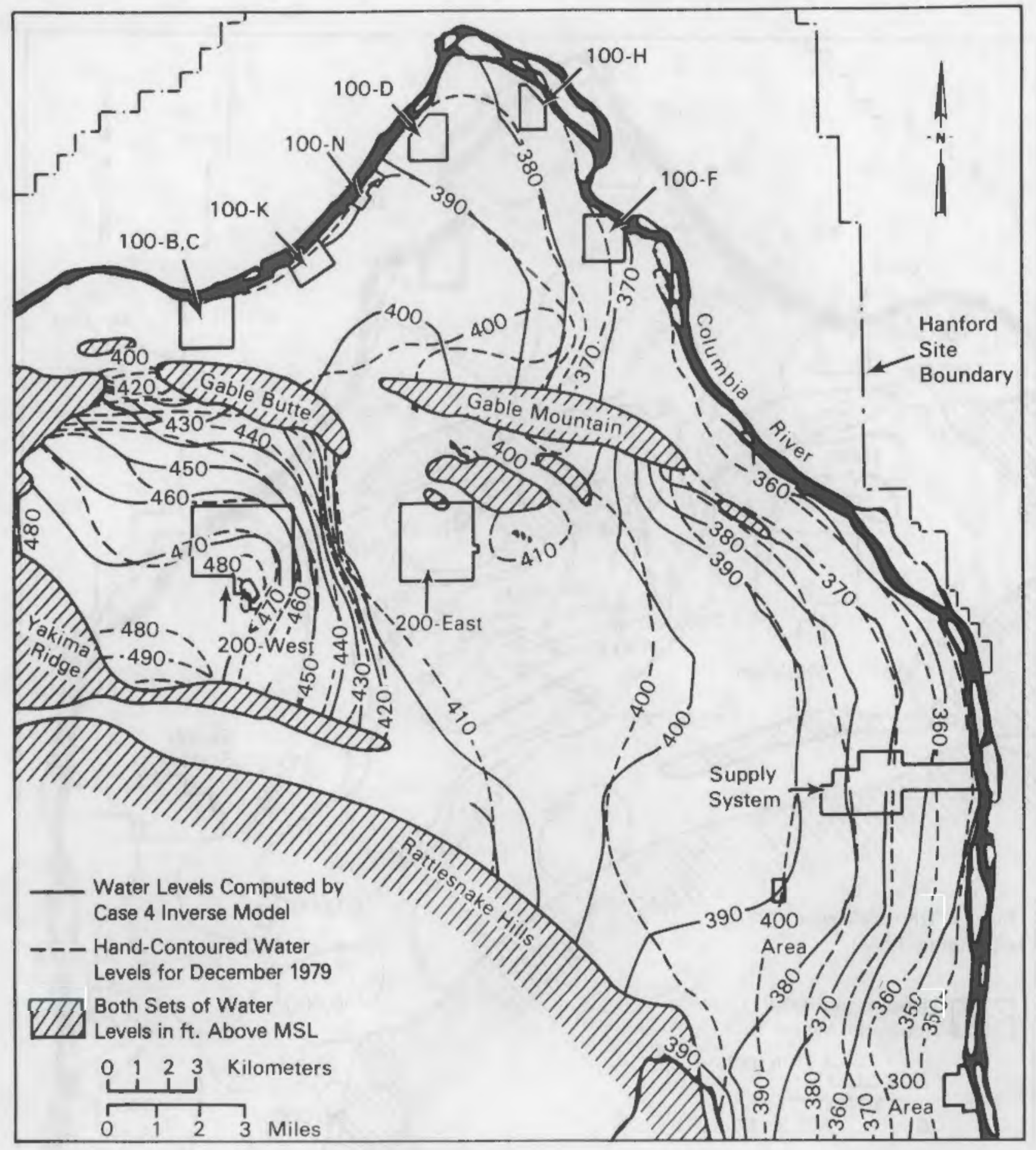

FIGURE 5.4. Comparison of Water Levels Computed by the Case 4 Inverse Model and Hand-Contoured Water Levels 


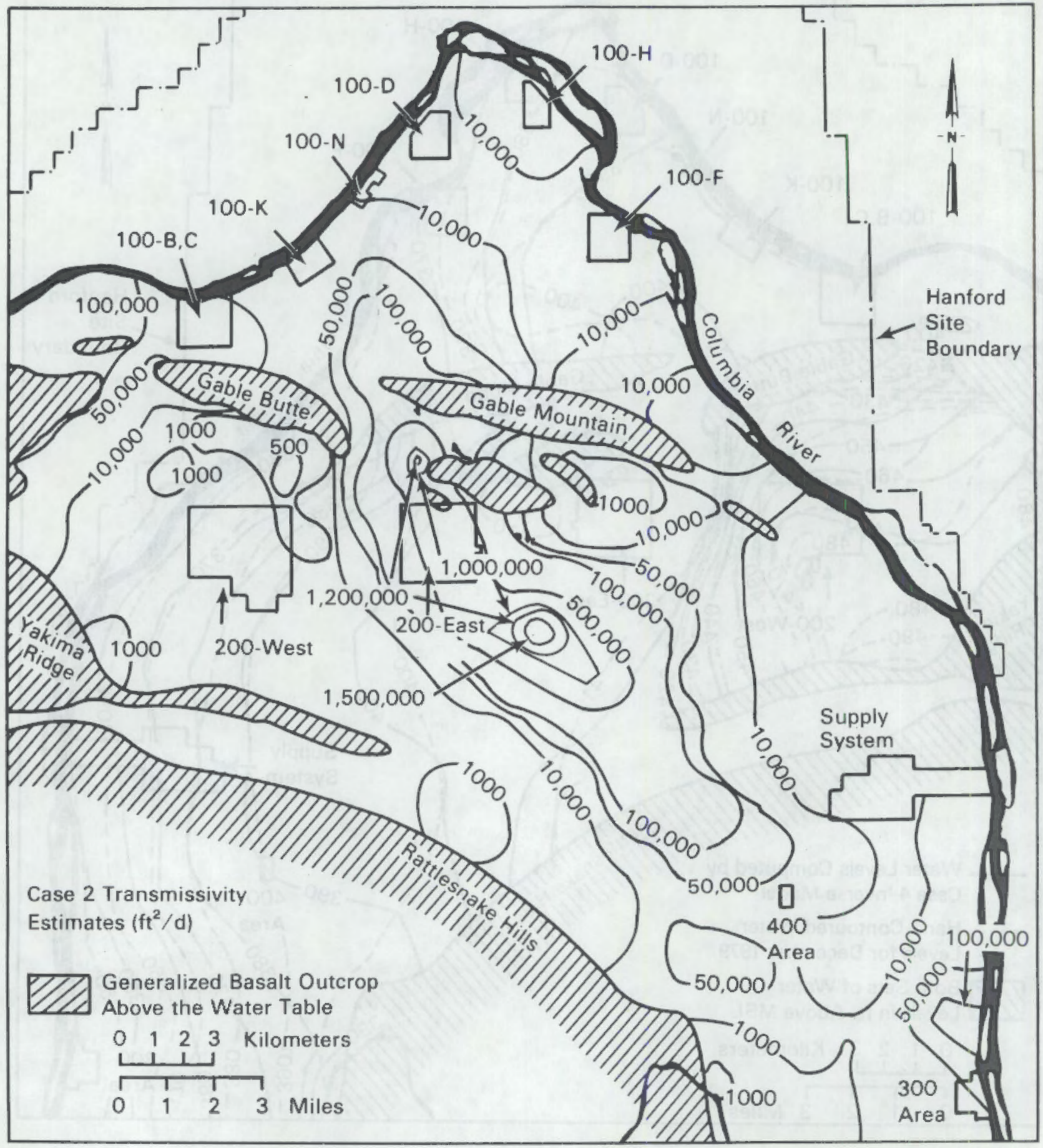

FIGURE 5.5. Contour Map of the Transmissivity Estimates for the Case 2 Inverse Model 


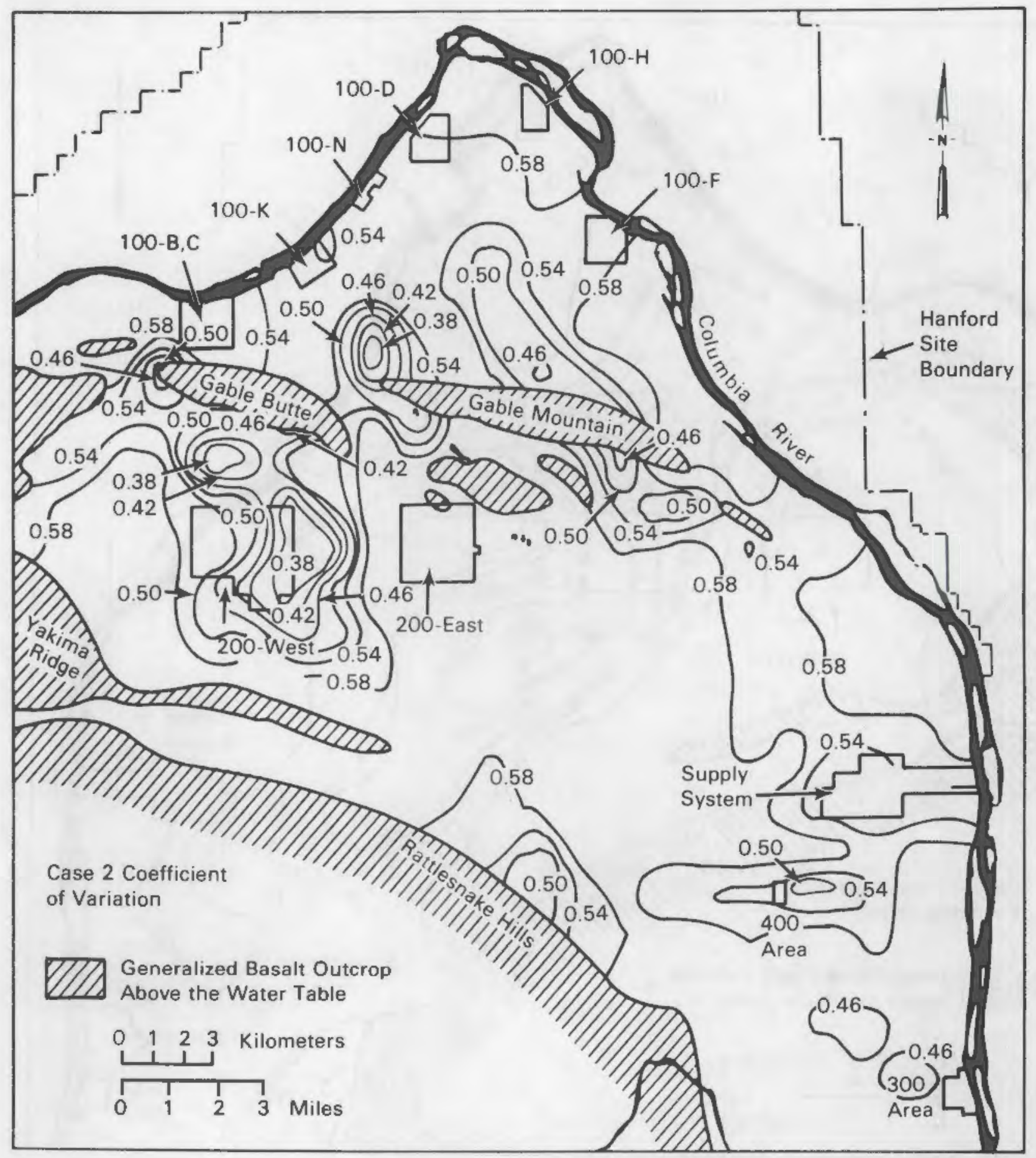

FIGURE 5.6. Contour Map of the Coefficients of Variation for the Case 2 Inverse Model 


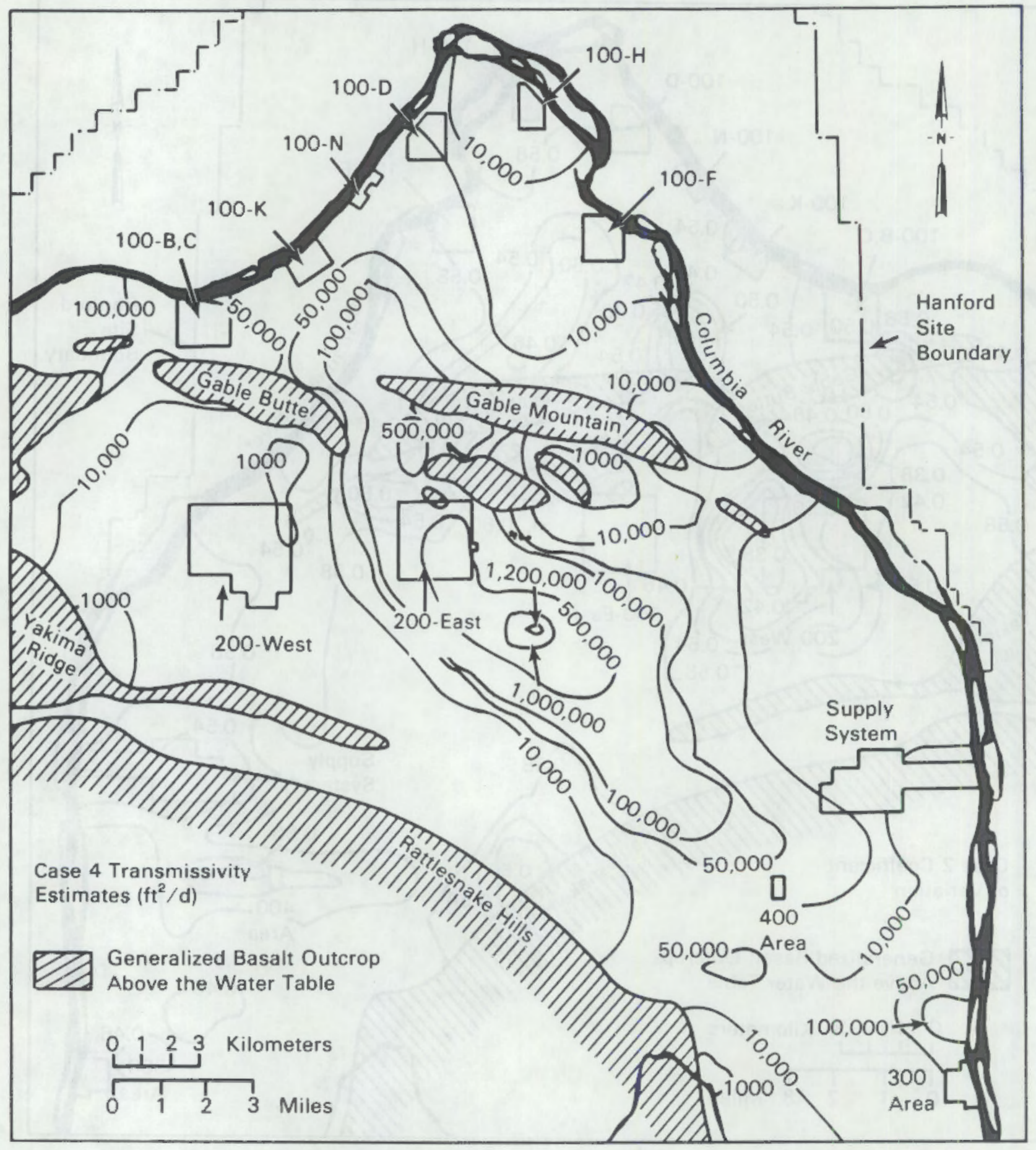

FIGURE 5.7. Contour Map of the Transmissivity Estimates for the Case 4 Inverse Model 


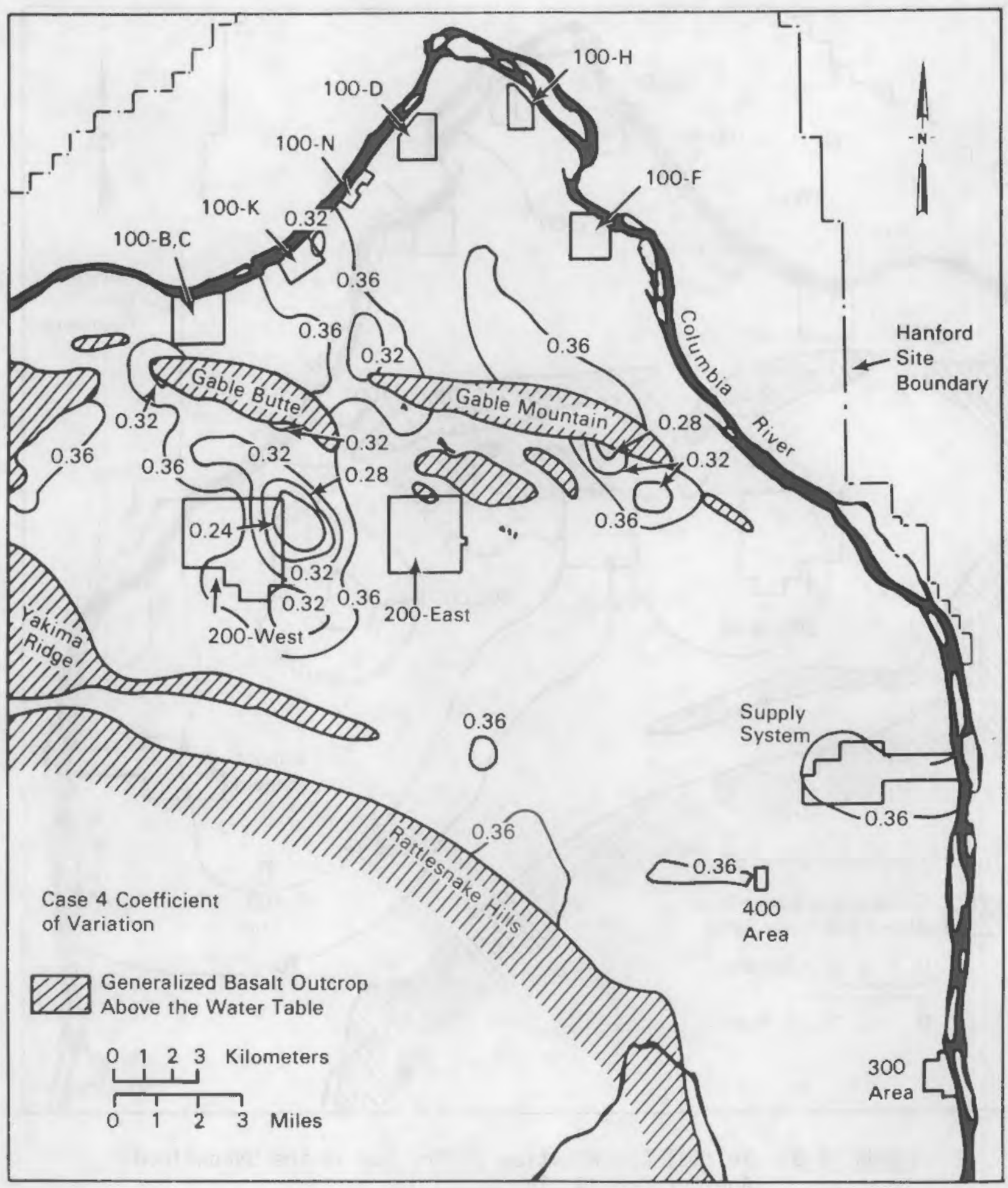

FIGURE 5.8. Contour Map of the Coefficients of Variation for the Case 4 Inverse Model 


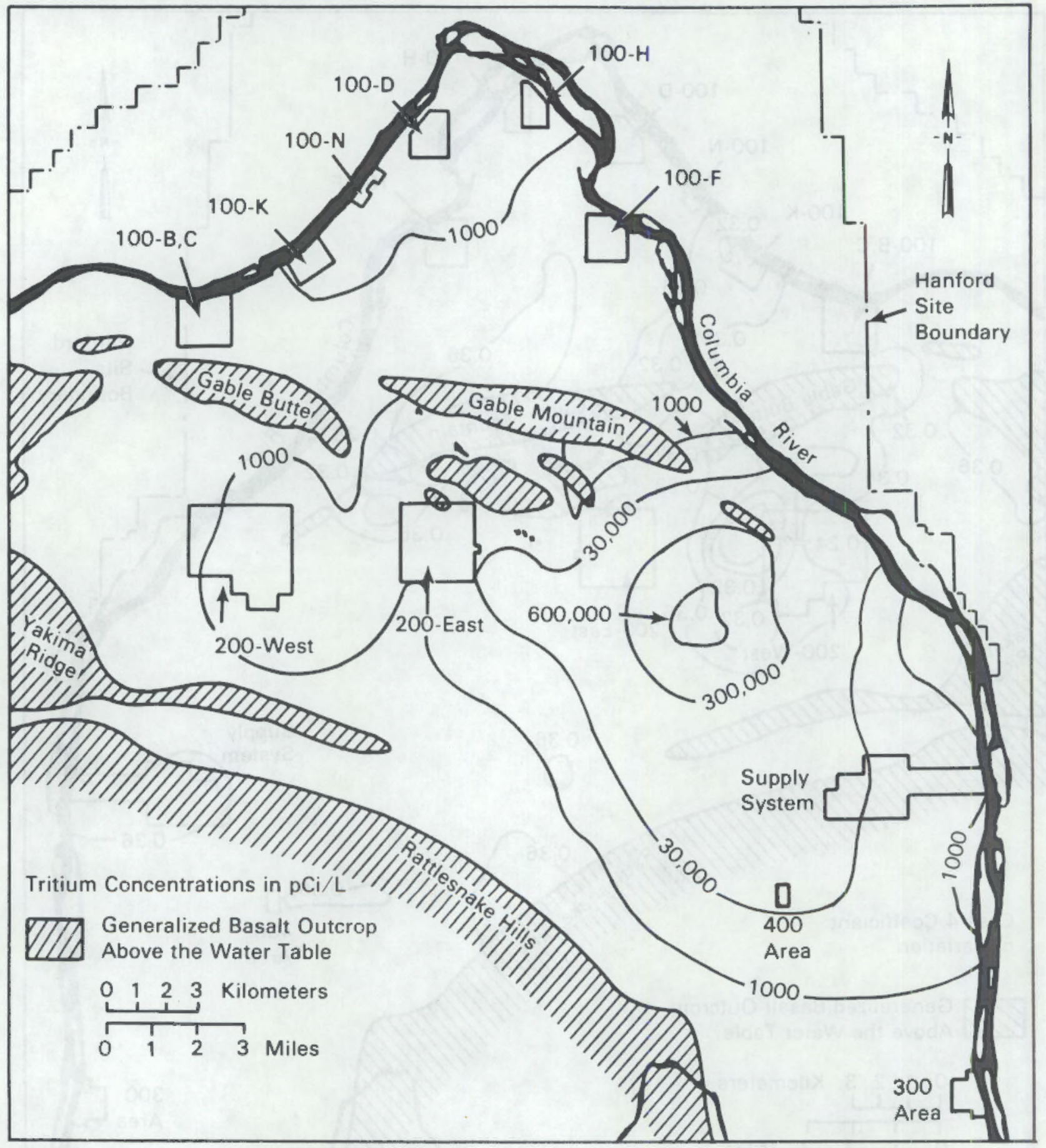

FIGURE 5.9. Initial Distribution of Tritium in the Unconfined Aquifer During 1983 
Parameters that were entered into the tritium transport model from the 200-East Area included the flow velocity field, effective porosity, and coefficients of dispersion. The flow velocity field for the tritium transport model was predicted with the model, based on the CFEST code and calibrated with the inverse procedure. A uniform effective porosity of 0.10 was assumed for the tritium transport model, based on estimates made for the unconfined aquifer (Bierschenk 1959). A longitudinal dispersivity of $100 \mathrm{ft}$ $(30 \mathrm{~m})$ and transverse dispersivity of $10 \mathrm{ft}(3 \mathrm{~m})$ resulted in a predicted distribution of tritium that agreed with the observed distribution. These values are similar to those reported by Ahlstrom et a1. (1977) as most suitable from previous calibration of a tritium transport model based on the MMT code.

\subsubsection{Application of the Tritium Transport Model}

The tritium transport model was applied to simulate the distribution of tritium in the unconfined aquifer during 1984 and 1985. An average steadystate flow velocity field and average constant tritium source concentrations were assumed for each year. The transport predictions were compared with the available ground-water monitoring data.

The predicted distribution of tritium in the unconfined aquifer at the end of 1985 is illustrated in Figure 5.10. Figure 5.9 illustrates the distribution of tritium in the aquifer with contour intervals that can be compared directly with those in Price (1986). The general shape of the tritium distribution in Figure 5.9 agrees with that reported by Price (1986). However, the predicted concentrations are higher than those observed at wells southeast and downgradient of the 200-East Area. The higher predicted concentrations may result from the assumptions or the average concentrations used in the transport model.

\subsubsection{Limitations of the Tritium Transport Model}

The tritium transport model was calibrated in two dimensions with ground-water monitoring data from the upper portion of the unconfined aquifer. An earlier study (Eddy, Myers, and Raymond 1978) indicated that tritium concentrations are higher near the upper portion of the aquifer. By simulating tritium transport in two dimensions, the tritium concentrations are 


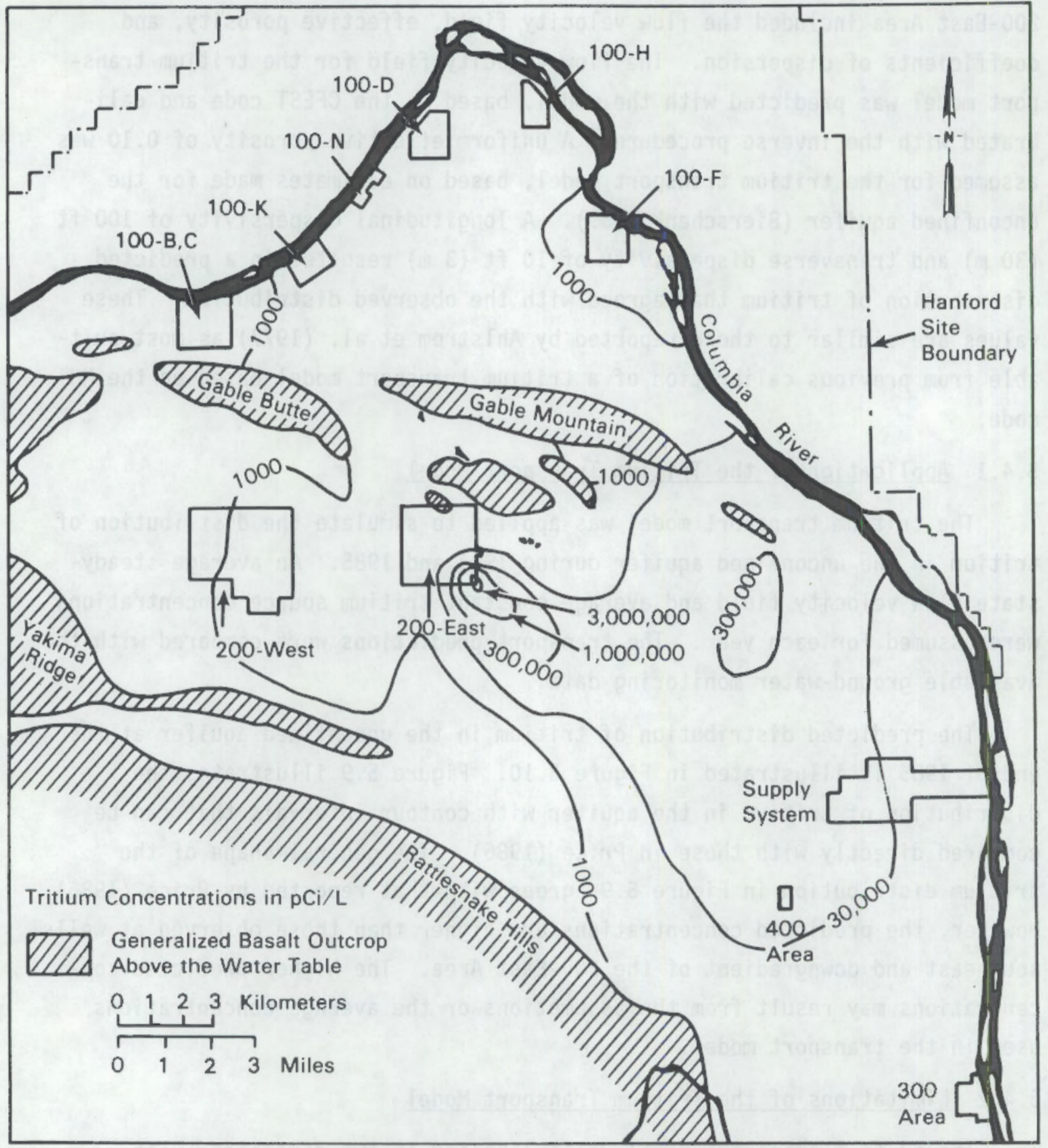

FIGURE 5.10. Predicted Distribution of Tritium in the Unconfined Aquifer at the End of 1985 
assumed to be uniform over the entire thickness of the unconfined aquifer. While the distribution of tritium concentrations may match the concentrations observed near the top of the aquifer, the amount of tritium in the model is overestimated. 



\subsection{REFERENCES}

Ahlstrom, S. W., H. P. Foote, R. C. Arnett, C. R. Cole, and R. J. Serne. 1977. Multicomponent Mass Transport Model: Theory and Numerical Implementation (Discrete-Parcel-Random-Walk Version). BNWL-2127, Pacific Northwest Laboratory, Richland, Washington.

Bal1, J. W., E. A. Jenne, and M. W. Cantrel1. 1981. WATEQ3 A Geochemical Model with Uranium Added. Open-File Report 81-1183, U.S. Geological Survey, Menlo Park, California.

Ball, J. W., D. K. Nordstrom, and E. A. Jenne. 1980. Additional and Revised Thermochemical Data and Computer Code for WATEQ2-A Computerized Chemical Model for Trace and Major Element Speciation and Mineral Equilibria of Natural Waters. Water Resources Investigations, WRI 78-116, U.S. Geological Survey, Menlo Park, California.

Bierschenk, W. H. 1959. Aquifer Characteristics and Ground-Water Movement at Hanford. HW-60601, Hanford Atomic Products Operation, General Electric Company, Richland, Washington.

Cearlock, D. B., K. L. Kipp, and D. R. Friedrichs. 1975. The Transmissivity Iterative Calculation Routine - Theory and Numerical Implementation.

BNWL-1706, Pacific Northwest Laboratory, Richland, Washington.

Comprehensive Environmental Response, Compensation, and Liability Act (CERCLA). 1980. Public Law 96-150, December 11, 1980, 94 Stat 2767, Titie 26.

Cooley, R. L. 1977. "A Method of Estimating Parameters and Assessing Re]iability for Models of Steady State Groundwater Flow, 1, Theory and Numerical Properties." Water Resour. Res. 13(2):318-324.

Cooley, R. L. 1979. "A Method of Estimating Parameters and Assessing Re1iability for Models of Steady State Groundwater Flow, 2, Application of Statistical Analysis." Water Resour. Res. 15(3):603-617.

Cooley, R. L. 1982. "Incorporation of Prior Information on Parameters Into Nonl inear Regression Groundwater Flow Models, 1, Theory." Water Resour. Res. 18(4):965-976.

Cooley, R. L. 1983. "Incorporation of Prior Information on Parameters Into Nonlinear Groundwater Flow Models, 2, Applications." Water Resour. Res. $19(3): 662-676$.

Deju, R. A., and W. K. Summers. 1975. Iransmissivity and Hydraulic Conductivity of Saturated Sedimentary Rocks in the Hanford Reservation. CA-168RAD-5, Atlantic Richfield Hanford Company, Richland, Washington. 
Dillon, K. L. 1985. Separations Area Water-Use Balance, 1977 Through 1984. RHO-RE-SR-50, Rockwe11 Hanford Operations, Richland, Washington.

Early, T. 0., S. H. Hall, and V. G. Johnson. 1988. "Tritium, Carbon-14 and Iodine-129 as Indicators for Localized Vertical Recharge Along an Anticline in the Columbia River Basalts Using a Decay-Corrected Mixing Mode1." Proceedings of Ground Water, Geochemistry Conference, pp, 597-620. National Water Well Association, Dublin, Ohio.

Early, T. 0., G. D. Spice, and M. D. Mitche11. 1986. A Hydrochemical Data Base for the Hanford Site, Washington. SD-BWI-DP-061, Rockwell Hanford Operations, Richland, Washington.

Eddy, P. A., D. A. Myers, and J. R. Raymond. 1978. Vertical Contamination in the Unconfined Groundwater at the Hanford Site, Washington. PNL-2724, Pacific Northwest Laboratory, Richland, Washington.

EPA. 1982. Test Methods for Evaluating Solid Waste: Physical/Chemical Methods. 2nd ed., SW-846, Washington, D.C.

EPA. 1986. Ground-Water Monitoring Technical Enforcement Guidance Document. OWSER 9950.1, U.S. Environmental Protection Agency, Washington, D.C.

Evans, J. C., P. J. Mitche11, and D. I. Dennison. 198B. Hanford Site Ground-Water Monitoring for Apri] Through June 1987. PNL-6315-1, Pacific Northwest Laboratory, Richland, Washington.

Felmy, A. R., S. M. Brown, Y. Onishi, S. B. Yabusaki, and R. S. Argo. 1984. MEXAMS--The Metals Exposure Analys is Modeling System. EPA-600/ 3-84-031 (PB84-157155), Prepared by Battelle, Pacific Northwest Laboratories for the U.S. Environmental Protection Agency, Athens, Georgia.

Felmy, A. R., D. C. Girvin, and E. A. Jenne. 1984. MINTEQ-A Computer Program for Calculating Aqueous Geochemical Equilibria. EPA-600/3-84-032 (PB84-157148), Prepared by Battelle, Pacific Northwest Laboratories for the U.S. Environmental Protection Agency, Athens, Georgia.

Fetter, C. W., Jr. 1980. Applied Hydrology. Charles E. Merrill Publishing Company, Columbus, Ohio.

Freshley, M. D., and M. J. Graham. 1988. Estimation of Ground-Water Travel Time at the Hanford Site: Description, Past Work, and Future Needs.

PNL-6328, Pacific Northwest Laboratory, Richland, Washington.

Friedrichs, D. R., C. R. Cole, and R. C. Arnett. 1977. Hanford Pathline Calculational Program - Theory, Error Analysis and Applications. ARH-ST-149, Atlantic Richfield Hanford Company, Richland, Washington.

Graham, M. J., M. D. Hal1, S. R. Strait, and W. R. Brown. 1981. Hydrology of the Separations Area. RHO-ST-42, Rockwell Hanford Operations, Richland, Washington. 
Gupta, S. K., C. T. Kincaid, P. R. Meyer, C. A. Newbill, and C. R. Cole. 1982. A Multi-Dimensional Finite Element Code for the Analysis of Coupled Fluid, Energy and Solute Transport (CFEST). PNL 4260 , Pacific Northwest Laboratory, Richland, Washington.

Hinman, N. W., K. M. Krupka, and L. J. Criscenti. 1987. "Chemical Speciation and Mineral Solubility Controls in Hanford Site Ground Water." Eos Transactions, American Geophysical Union 68(44):1291.

Interagency Working Group. 1987. Data Compilation: Iodine-129 in Hanford Groundwater. WHC-EP-0037, Westinghouse Hanford Company, Richland, Washington.

Jacobson, E. A. 1985. A Statistical Parameter Estimation Method Using Singular Value Decomposition With Application to Avra Valley Aquifer in Southern Arizona. Dissertation, Department of Hydrology and Water Resources, University of Arizona, Tucson, Arizona.

Jaquish, R. E., and P. J. Mitchell, eds. 1988. Environmental Monitoring at Hanford for 1987. PNL-6464, Pacific Northwest Laboratory, Richland, Washington.

Kipp, K. L., and R. D. Mudd. 1973. Collection and Analys is of Pump Test Data for Transmissivity Values. BNWL-1709, Pacific Northwest Laboratory, Richland, Washington.

Kipp, K. L., A. E. Reisenauer, C. R. Cole, and C. A. Bryan. 1972. Variable Thickness Transient Groundwater Flow Model. Theory and Numerical Implementation. BNWL-1703, Pacific Northwest Laboratory, Richland, Washington.

Krupka, K. M., and E. A. Jenne. 1982. WATEQ3 Geochemical Model: Thermodynamic Data for Several Additional Solids. PNL-4276, Pacific Northwest Laboratory, Richland, Washington.

Law, A. G., J. A. Serkowski, and A. L. Schatz. 1987. Results of the Separations Area Ground-Water Monitoring Network for 1986. RHO-RE-SR-87-24P, Rockwell Hanford Operations, Richland, Washington.

Lindsay, W. L. 1979. Chemical Equilibria in Soils. John Wiley \& Sons, New York.

Neuman, S. P. 1980. "A Statistical Approach to the Inverse Problem of Aquifer Hydrology: 3. Improved Solution Method and Added Perspective." Water Resour. Res. 16(2):331-346.

Neuman, S. P., and S. Yakowitz. 1979. "A Statistical Approach to the Inverse Probiem of Aquifer Hydrology, 1, Theory." Water Resour. Res., I5(4):845-860. 
Pacific Northwest Laboratory. 1987. Ground-Water Monitoring Compliance Projects for Hanford Site Facilities: Progress Report for the Period July 1 to September 30, 1987. PNL-6469, Pacific Northwest Laboratory, Richland, Washington.

Peterson, S. R., C. J. Hostetler, W. J. Deutsch, and C. E. Cowan. 1987. MINTEQ User's Manual. NUREG/CR-4808 (PNL-6106), Prepared for the U.S. Nuclear Regulatory Commission by the Pacific Northwest Laboratory, Richland, Washington.

Prater, L. S., J. T. Rieger, C. S. CTine, E. J. Jensen, T. L. Liikala, K. R. Oster, and P. A. Eddy. 1984. Ground-Water Surveillance at the Hanford Site for CY 1983. PNL-5041, Pacific Northwest Laboratory, Richland, Washington.

Price, K. R. 1986. Environmental Monitoring at Hanford for 1985. PNL-5817, Pacific Northwest Laboratory, Richland, Washington.

Resource Conservation and Recovery Act of 1976 (RCRA). 1976. Pub7ic Law 94-580, October 21, 1976, 90 Stat. 2795, Title 42.

Schatz, A. L., J. J. Ammerman, and J. A. Serkowski. 1987. Hanford Site Hater Table Map June 1987. WHC-EP-0054, Westinghouse Hanford Company, Richland, Washington.

Serkowski, J. A., A. G. Law, J. J. Ammerman, and A. L. Schatz. 1988. Results of Ground-Water Monitoring for Radionuclides in the Separations Area 1987. WHC-EP-0152, Westinghouse Hanford Company, Richland, Washington.

Simmons, C. S., C. T. Kincaid, and A. E. Reisenauer. 1986. A Simplified Model for Radioactive Contaminant Transport: The Transs Code. PNL-6029, Pacific Northwest Laboratory, Richland, Washington.

Sliger, G. J. 1980. Radioactive Liquid Waste Discharged to Ground in the 200 Areas During 1979. RH0-CD-79-34 40, Rockwell Hanford Operations, Richland, Washington.

Smith, R. M. 1980. 216-B-5 Reverse Well Characterization Study. RHO-ST-37, Rockwell Hanford operations, Richland, Washington.

Soldat, J. K. 1976. "Radiation Doses from Iodine-129 in the Environment." Heaith Physics 30:61-70.

Truesde11, A. H., and B. F. Jones. 1974. "WATEQ, A Computer Program for Calculating Chemical Equilibria of Natural Waters." J. Res. U.S. Geol. Survey 2(2):233-248.

DOE. 1981a. "Environmental Protection, Safety, and Health Protection Program for DOE Operations." DOE Order 5480.1A, U.S. Department of Energy, Washington, D.C. 
DOE. 1981b. "Environmental Protection, Safety, and Health Protection Information Reporting Requirements." DOE Order 5484.1, Department of Energy Washington, D.C.

D0E. 1986. Draft Phase I Insta11ation Assessment of Inactive Waste-Disposal Sites At Hanford. Vol 1. Prepared for the U.S. Department of Energy by the Pacific Northwest Laboratory, Richland, Washington.

USGS. 1977. National Handbook of Recommended Methods for Water-Data Acquisition. Office of Water Data Coordination, U.S. Geological Survey, Reston, Virginia.

Washington State Department of Ecology. 1986. "Dangerous Waste Regulations." Washington Administrative Code, Chapter 173-303, 01ympia, Washington.

Washington State Department of Ecology. 1986. "Minimum Functional Standards for Solid Waste Handling." Washington Administrative Code, Chapter 173304 , 01 ympia, Washington.

Westall J. C., J. L. Zachary, and F. M. M. Morel. 1976. MINEQL, A Computer Program for the Calculation of Chemical Equilibrium Composition of Aqueous Systems. Technical Note 18, Department of Civil Engineering, Massachusetts Institute of Technology, Cambridge, Massachusetts.

Yeh, W. W-G., and Y. S. Yoon. 1976. "A Systematic 0ptimization Procedure for the Identification of Inhomogeneous Aquifer Parameters." In Advances in Groundwater Hydrology, pp. 72-82, American Water Resources Association, Minneapolis, Minnesota.

Zimmerman, D. A., A. E. Reisenauer, G. D. Black, and M. A. Young. 1986. Hanford Site Water Table Changes 1950 Through 1980-Data Observations and Evaluation. PNL-5506, Prepared for Rockwell Hanford Operations by Pacific Northwest Laboratory, Richland, Washington. 
. 
APPENDIX A

WATER LEVELS MEASURED. DURING DECEMBER 1987 
APPENDIX A

WATER LEVELS MEASURED DURING DECEMBER 1987

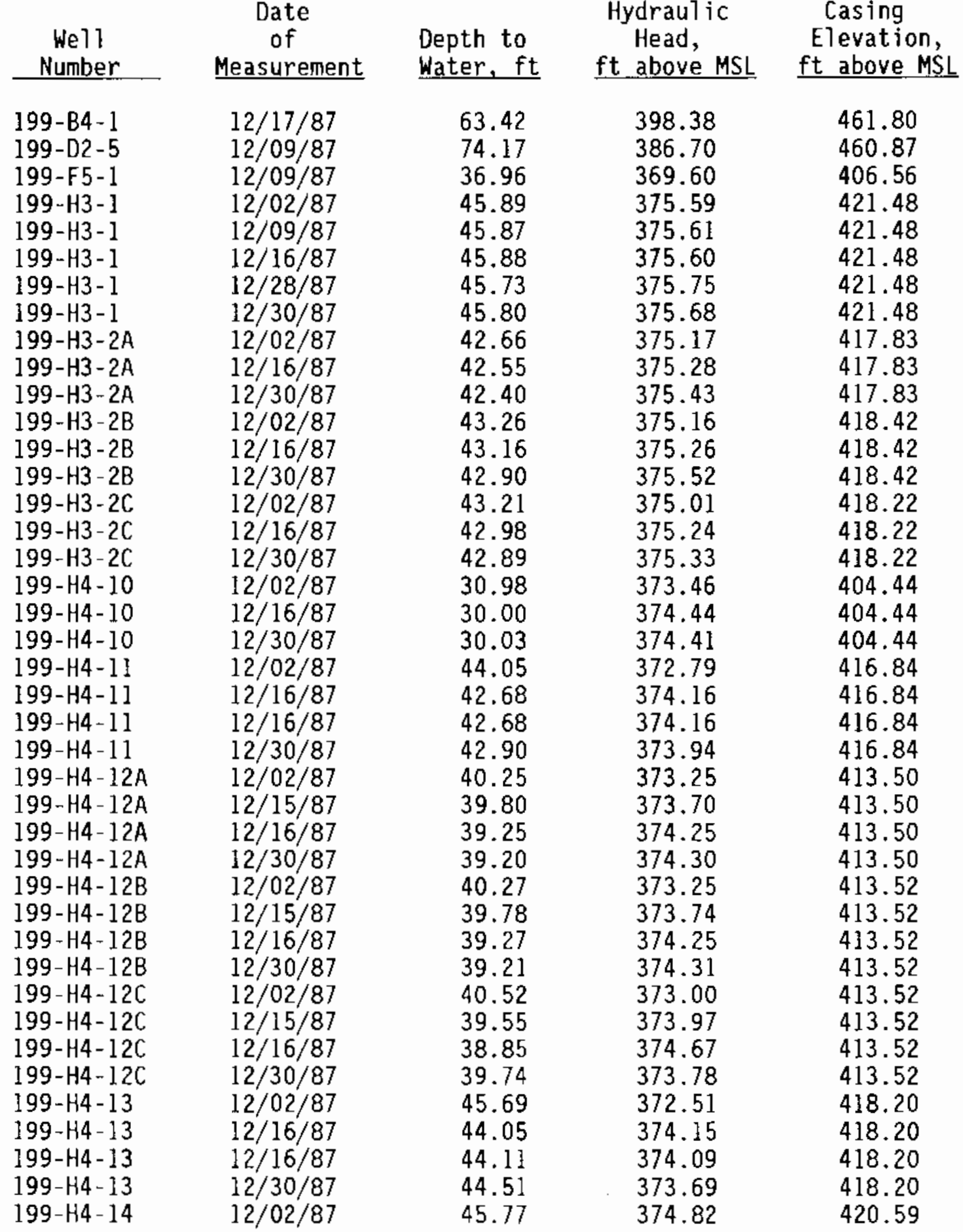




\begin{tabular}{|c|c|c|c|c|}
\hline $\begin{array}{c}\text { Well } \\
\text { Number }\end{array}$ & $\begin{array}{c}\text { Date } \\
\text { of } \\
\text { Measurement }\end{array}$ & $\begin{array}{l}\text { Depth to } \\
\text { Water, ft }\end{array}$ & $\begin{array}{c}\text { Hydraulic } \\
\text { Head, } \\
\text { ft above MSL }\end{array}$ & $\begin{array}{c}\text { Casing } \\
\text { Elevation, } \\
\mathrm{ft} \text { above MSL }\end{array}$ \\
\hline $\begin{array}{l}199-H 4-14 \\
199-H 4-14 \\
199-H 4-15 A \\
199-H 4-15 A \\
199-H 4-15 A \\
199-H 4-15 A \\
199-H 4-15 B \\
199-H 4-15 B \\
199-H 4-15 B \\
199-H 4-15 B \\
199-H 4-15 C Q \\
199-H 4-15 C Q \\
199-H 4-15 C Q \\
199-H 4-15 C R \\
199-H 4-15 C R \\
199-H 4-15 C R \\
199-H 4-15 C S \\
199-H 4-15 C S \\
199-H 4-15 C S \\
199-H 4-16 \\
199-H 4-16 \\
199-H 4-16 \\
199-H 4-16 \\
199-H 4-17 \\
199-H 4-17 \\
199-H 4-17 \\
199-H 4-17 \\
199-H 4-18 \\
199-H 4-18 \\
199-H 4-18 \\
199-H 4-18 \\
199-H 4-3 \\
199-H 4-3 \\
199-H 4-3 \\
199-H 4-4 \\
199-H 4-4 \\
199-H 4-4 \\
199-H 4-5 \\
199-H 4-5 \\
199-H 4-5 \\
199-H 4-6 \\
199-H 4-6 \\
199-H 4-6 \\
199-H 4-7 \\
199-H 4-7 \\
199-H 4-7 \\
199-H 4-8 \\
199-H 4-8\end{array}$ & $\begin{array}{l}12 / 16 / 87 \\
12 / 30 / 87 \\
12 / 02 / 87 \\
12 / 14 / 87 \\
12 / 16 / 87 \\
12 / 30 / 87 \\
12 / 02 / 87 \\
12 / 14 / 87 \\
12 / 16 / 87 \\
12 / 30 / 87 \\
12 / 02 / 87 \\
12 / 16 / 87 \\
12 / 30 / 87 \\
12 / 02 / 87 \\
12 / 16 / 87 \\
12 / 30 / 87 \\
12 / 02 / 87 \\
12 / 16 / 87 \\
12 / 30 / 87 \\
12 / 02 / 87 \\
12 / 16 / 87 \\
12 / 23 / 87 \\
12 / 30 / 87 \\
12 / 02 / 87 \\
12 / 16 / 87 \\
12 / 28 / 87 \\
12 / 30 / 87 \\
12 / 02 / 87 \\
12 / 16 / 87 \\
12 / 23 / 87 \\
12 / 30 / 87 \\
12 / 02 / 87 \\
12 / 16 / 87 \\
12 / 30 / 87 \\
12 / 02 / 87 \\
12 / 16 / 87 \\
12 / 30 / 87 \\
12 / 02 / 87 \\
12 / 16 / 87 \\
12 / 17 / 87 \\
12 / 02 / 87 \\
12 / 16 / 87 \\
12 / 30 / 87 \\
12 / 02 / 87 \\
12 / 16 / 87 \\
12 / 30 / 87 \\
12 / 02 / 87 \\
12 / 16 / 87\end{array}$ & $\begin{array}{l}45.61 \\
45.44 \\
33.82 \\
33.28 \\
32.74 \\
32.86 \\
33.52 \\
32.94 \\
32.45 \\
32.58 \\
18.46 \\
17.81 \\
18.15 \\
34.00 \\
33.79 \\
33.46 \\
34.20 \\
32.61 \\
33.42 \\
50.00 \\
49.79 \\
49.64 \\
49.59 \\
45.28 \\
44.89 \\
44.73 \\
44.53 \\
48.03 \\
47.65 \\
47.35 \\
47.37 \\
46.65 \\
46.21 \\
46.93 \\
40.61 \\
39.58 \\
39.51 \\
42.82 \\
42.28 \\
42.01 \\
44.41 \\
44.30 \\
44.14 \\
46.31 \\
46.06 \\
45.81 \\
46.13 \\
45.77\end{array}$ & $\begin{array}{l}374.98 \\
375.15 \\
373.39 \\
373.93 \\
374.47 \\
374.35 \\
373.40 \\
373.98 \\
374.47 \\
374.34 \\
388.81 \\
389.46 \\
389.12 \\
373.37 \\
373.58 \\
373.91 \\
373.24 \\
374.83 \\
374.02 \\
374.23 \\
374.44 \\
374.59 \\
374.64 \\
373.81 \\
374.20 \\
374.36 \\
374.56 \\
373.79 \\
374.17 \\
374.47 \\
374.45 \\
373.70 \\
374.14 \\
373.42 \\
373.15 \\
374.18 \\
374.25 \\
373.44 \\
373.98 \\
374.25 \\
375.17 \\
375.28 \\
375.44 \\
374.28 \\
374.53 \\
374.78 \\
373.87 \\
374.23\end{array}$ & $\begin{array}{l}420.59 \\
420.59 \\
407.21 \\
407.21 \\
407.21 \\
407.21 \\
406.92 \\
406.92 \\
406.92 \\
406.92 \\
407.27 \\
407.27 \\
407.27 \\
407.37 \\
407.37 \\
407.37 \\
407.44 \\
407.44 \\
407.44 \\
424.23 \\
424.23 \\
424.23 \\
424.23 \\
419.09 \\
419.09 \\
419.09 \\
419.09 \\
421.82 \\
421.82 \\
421.82 \\
421.82 \\
420.35 \\
420.35 \\
420.35 \\
413.76 \\
413.76 \\
413.76 \\
416.26 \\
416.26 \\
416.26 \\
419.58 \\
419.58 \\
419.58 \\
420.59 \\
420.59 \\
420.59 \\
420.00 \\
420.00\end{array}$ \\
\hline
\end{tabular}




\begin{tabular}{|c|c|c|c|c|}
\hline $\begin{array}{l}\text { Wel1 } 1 \\
\text { Number }\end{array}$ & $\begin{array}{c}\text { Date } \\
\text { of } \\
\text { Measurement }\end{array}$ & $\begin{array}{l}\text { Depth to } \\
\text { Water, } \mathrm{ft}\end{array}$ & $\begin{array}{c}\text { Hydraulic } \\
\text { Head, } \\
\text { ft above MSL }\end{array}$ & $\begin{array}{c}\text { Casing } \\
\text { Elevation, } \\
\text { ft above MSL }\end{array}$ \\
\hline $\begin{array}{l}199-H 4-8 \\
199-H 4-9 \\
199-H 4-9 \\
199-H 4-9 \\
199-H 4-9 \\
199-\mathrm{K}-11 \\
199-N-14 \\
199-N-27 \\
199-\mathrm{N}-4 \\
299-\mathrm{E} 13-10 \\
299-\mathrm{E} 13-12 \\
299-\mathrm{E} 13-14 \\
299-\mathrm{E} 17-5 \\
299-\mathrm{E} 23-1 \\
299-\mathrm{E} 23-20 \\
299-\mathrm{E} 24-2 \\
299-\mathrm{E} 24-4 \\
299-\mathrm{E} 25-11 \\
299-\mathrm{E} 25-19 \\
299-\mathrm{E} 25-21 \\
299-\mathrm{E} 25-25 \\
299-\mathrm{E} 25-26 \\
299-\mathrm{E} 25-27 \\
299-\mathrm{E} 25-4 \\
299-\mathrm{E} 26-1 \\
299-\mathrm{E} 26-2 \\
299-\mathrm{E} 26-4 \\
299-\mathrm{E} 27-1 \\
299-\mathrm{E} 27-10 \\
299-\mathrm{E} 27-10 \\
299-\mathrm{E} 27-8 \\
299-\mathrm{E} 27-8 \\
299-\mathrm{E} 27-9 \\
299-\mathrm{E} 27-9 \\
299-\mathrm{E} 28-12 \\
299-\mathrm{E} 28-18 \\
299-\mathrm{E} 28-26 \\
299-\mathrm{E} 28-26 \\
299-\mathrm{E} 28-27 \\
299-\mathrm{E} 28-27 \\
299-\mathrm{E} 28-7 \\
299-\mathrm{E} 32-1 \\
299-\mathrm{E} 32-2 \\
299-\mathrm{E} 32-2 \\
299-\mathrm{E} 32-3 \\
299-\mathrm{E} 32-3 \\
299-\mathrm{E} 32-4 \\
299-\mathrm{E} 32-4\end{array}$ & $\begin{array}{l}12 / 30 / 87 \\
12 / 02 / 87 \\
12 / 16 / 87 \\
12 / 28 / 87 \\
12 / 30 / 87 \\
12 / 17 / 87 \\
12 / 16 / 87 \\
12 / 14 / 87 \\
12 / 16 / 87 \\
12 / 11 / 87 \\
12 / 12 / 87 \\
12 / 11 / 87 \\
12 / 28 / 87 \\
12 / 28 / 87 \\
12 / 28 / 87 \\
12 / 28 / 87 \\
12 / 09 / 87 \\
12 / 14 / 87 \\
12 / 14 / 87 \\
12 / 14 / 87 \\
12 / 10 / 87 \\
12 / 04 / 87 \\
12 / 14 / 87 \\
12 / 11 / 87 \\
12 / 08 / 87 \\
12 / 11 / 87 \\
12 / 11 / 87 \\
12 / 08 / 87 \\
12 / 02 / 87 \\
12 / 11 / 87 \\
12 / 02 / 87 \\
12 / 11 / 87 \\
12 / 02 / 87 \\
12 / 11 / 87 \\
12 / 28 / 87 \\
12 / 08 / 87 \\
12 / 02 / 87 \\
12 / 11 / 87 \\
12 / 02 / 87 \\
12 / 11 / 87 \\
12 / 08 / 87 \\
12 / 08 / 87 \\
12 / 02 / 87 \\
12 / 11 / 87 \\
12 / 02 / 87 \\
12 / 11 / 87 \\
12 / 02 / 87 \\
12 / 11 / 87\end{array}$ & $\begin{array}{l}45.45 \\
44.50 \\
44.13 \\
43.85 \\
43.70 \\
74.13 \\
65.94 \\
54.00 \\
64.38 \\
333.91 \\
328.10 \\
340.09 \\
314.09 \\
304.28 \\
315.82 \\
312.59 \\
291.55 \\
275.76 \\
272.03 \\
271.61 \\
262.78 \\
262.38 \\
269.98 \\
252.99 \\
211.18 \\
227.64 \\
240.15 \\
275.52 \\
218.75 \\
218.84 \\
232.66 \\
232.78 \\
223.90 \\
223.98 \\
302.91 \\
287.38 \\
282.39 \\
282.39 \\
275.47 \\
275.61 \\
281.30 \\
251.07 \\
265.39 \\
265.45 \\
271.76 \\
271.84 \\
281.24 \\
281.33\end{array}$ & $\begin{array}{l}374.55 \\
373.58 \\
373.95 \\
374.23 \\
374.38 \\
393.53 \\
387.21 \\
395.08 \\
394.35 \\
399.84 \\
403.24 \\
402.76 \\
404.56 \\
405.37 \\
405.44 \\
404.60 \\
405.45 \\
405.52 \\
405.17 \\
405.66 \\
406.64 \\
406.17 \\
404.08 \\
406.40 \\
406.07 \\
405.96 \\
405.67 \\
405.53 \\
405.72 \\
405.63 \\
405.17 \\
405.05 \\
405.31 \\
405.23 \\
405.69 \\
405.20 \\
404.87 \\
404.87 \\
404.90 \\
404.76 \\
404.61 \\
405.10 \\
404.67 \\
404.61 \\
404.75 \\
404.67 \\
404.64 \\
404.55\end{array}$ & $\begin{array}{l}420.00 \\
418.08 \\
418.08 \\
418.08 \\
418.08 \\
467.66 \\
453.15 \\
449.08 \\
458.73 \\
733.75 \\
731.34 \\
742.85 \\
718.65 \\
709.65 \\
721.26 \\
717.19 \\
697.00 \\
681.28 \\
677.20 \\
677.27 \\
669.42 \\
668.55 \\
674.06 \\
659.39 \\
617.25 \\
633.60 \\
645.82 \\
681.05 \\
624.47 \\
624.47 \\
637.83 \\
637.83 \\
629.21 \\
629.21 \\
708.60 \\
692.58 \\
687.26 \\
687.26 \\
680.37 \\
680.37 \\
685.91 \\
656.17 \\
670.06 \\
670.06 \\
676.51 \\
676.51 \\
685.88 \\
685.88\end{array}$ \\
\hline
\end{tabular}


We1 Number

299-E33-14

299-E33-17

299-E33-21

299-E33-28

299-E33-28

299-E33-29

299-E33-29

299-E33-30

299-E33-30

299-E33-7

299-E33-8

299-E34-1

299-E34-3

299-E34-3

299-E34-5

299-E34-5

299-E34-5

299-E34-6

299-E34-6

299-E34-6

299-W10-13

299-W10-14

299-W10-2

299-W10-5

299-W10-8

299-W11-10

299-W11-12

299-W11-13

299-W]1-23

299-W11-7

299-W1I-9

299-W12-1

299-W14-1

299-W15-15

299-W15-16

299-W15-17

299-W15-18

299-W15-2

299-W15-4

299-W15-5

299-W18-15

299-W18-2I

299-W18-22

299-W18-23

299-W18-24

299-W19-1

299-W19-13

299-W19-14
Date

$\begin{array}{ll}\text { of } & \text { Depth to } \\ \text { Measurement } & \text { Water, ft }\end{array}$

$12 / 08 / 87$

$12 / 11 / 87$

$12 / 08 / 87$

$12 / 02 / 87$

$12 / 11 / 87$

$12 / 02 / 87$

$12 / 11 / 87$

$12 / 02 / 87$

$12 / 11 / 87$

$12 / 11 / 87$

$12 / 08 / 87$

$12 / 08 / 87$

$12 / 02 / 87$

$12 / 11 / 87$

$12 / 02 / 87$

$12 / 11 / 87$

$12 / 11 / 87$

$12 / 02 / 87$

$12 / 11 / 87$

$12 / 11 / 87$

$12 / 10 / 87$

$12 / 10 / 87$

$12 / 11 / 87$

$12 / 11 / 87$

$12 / 15 / 87$

$12 / 11 / 87$

$12 / 11 / 87$

$12 / 11 / 87$

$12 / 15 / 87$

$12 / 15 / 87$

$12 / 11 / 87$

$12 / 11 / 87$

$12 / 11 / 87$

$12 / 10 / 87$

$12 / 10 / 87$

$12 / 10 / 87$

$12 / 10 / 87$

$12 / 15 / 87$

$12 / 11 / 87$

$12 / 11 / 87$

$12 / 14 / 87$

$12 / 10 / 87$

$12 / 10 / 87$

$12 / 10 / 87$

$12 / 10 / 87$

$12 / 14 / 87$

$12 / 14 / 87$

$12 / 14 / 87$
214.98

226.65

263.13

259.30

259.36

268.98

268.99

258.87

258.89

221.82

245.76

224.00

205.73

205.80

184.60

184.66

184.67

192.71

192.80

192.80

232.11

232.90

207.63

205.02

213.39

270.77

213.03

224.96

219.52

243.64

262.22

273.43

200.94

228.32

214.51

214.71

214.81

225.07

192.25

197.68

188.44

198.54

199.17

226.74

213.14

199.33

225.50
227.58
Hydraulic

Head,

ft above MSL

407.14

405.00

400.30

404.93

404.87

404.79

404.78

404.83

404.81

404.76

402.27

405.42

405.79

405.72

406.19

406.13

406.12

405.12

405.03

405.03

466.93

466.53

466.70

467.29

466.94

458.12

466.55

467.05

466.34

465.47

460.72

453.03

464.89

469.64

470.38

469.93

470.90

465.64

469.75

473.00

472.32

470.08

469.32

470.07

471.21

474.44

243.69

246.03
Casing

Elevation,

ft above MSL

622.12

631.65

663.43

664.23

664.23

673.77

673.77

663.70

663.70

626.58

648.03

629.42

611.52

611.52

590.79

590.79

590.79

597.83

597.83

597.83

699.04

699.43

674.33

672.31

680.33

728.89

679.58

692.01

685.86

709.11

722.94

726.46

665.83

697.96

684.89

684.64

685.71

690.71

662.00

670.68

660.76

668.62

668.49

696.81

684.35

673.77

471.27

471.53 


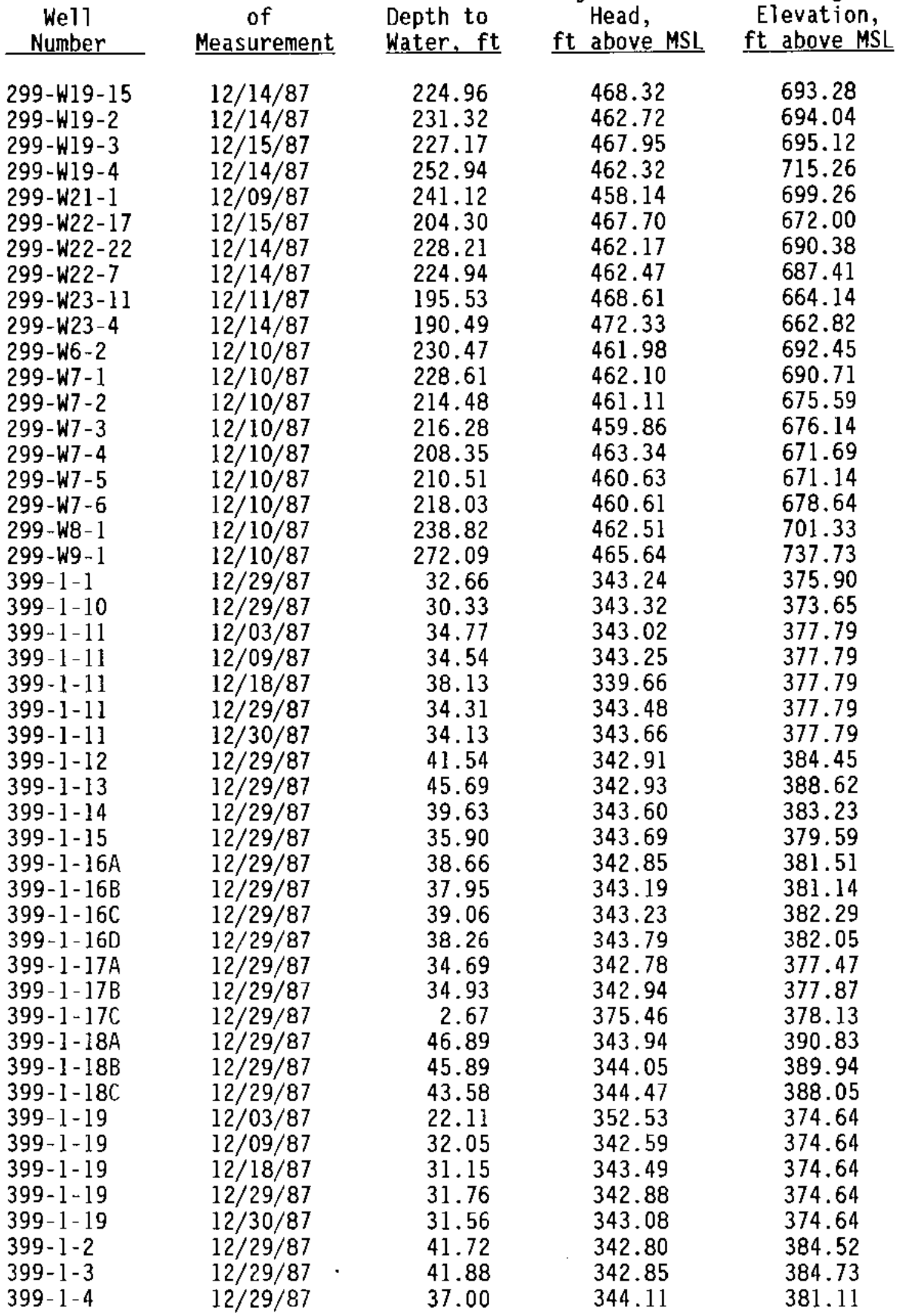




\begin{tabular}{|c|c|c|c|c|}
\hline $\begin{array}{c}\text { Wel1 } \\
\text { Number }\end{array}$ & $\begin{array}{c}\text { Date } \\
\text { of } \\
\text { Measurement }\end{array}$ & $\begin{array}{l}\text { Depth to } \\
\text { Water, ft }\end{array}$ & $\begin{array}{c}\text { Hydraulic } \\
\text { Head, } \\
\text { ft above MSL }\end{array}$ & $\begin{array}{c}\text { Casing } \\
\text { Elevation, } \\
\mathrm{ft} \text { above MSL } \\
\end{array}$ \\
\hline $\begin{array}{l}399-1-5 \\
399-1-6 \\
399-1-7 \\
399-1-8 \\
399-1-9 \\
399-2-1 \\
399-2-2 \\
399-2-3 \\
399-3-1 \\
399-3-10 \\
399-3-12 \\
399-3-2 \\
399-3-6 \\
399-3-7 \\
399-3-9 \\
399-4-1 \\
399-4-10 \\
399-4-11 \\
399-4-7 \\
399-4-9 \\
399-5-1 \\
399-6-1 \\
399-8-1 \\
399-8-1 \\
399-8-2 \\
399-8-3 \\
699-10-54 A \\
699-10-\mathrm{E} 12 \\
699-101-48 \mathrm{~B} \\
699-11-45 A \\
699-14-38 \\
699-14-47 \\
699-14-\mathrm{E} 6 \mathrm{~T} \\
699-15-15 A \\
699-15-26 \\
699-17-5 \\
699-17-70 \\
699-19-43 \\
699-2-3 \\
699-20-20 \\
699-20-39 \\
699-20-\mathrm{E} 12 \\
699-24-1 \mathrm{~T} \\
699-24-33 \\
699-25-55 \\
699-25-70 \\
699-25-70 \\
699-25-70\end{array}$ & $\begin{array}{l}12 / 29 / 87 \\
12 / 29 / 87 \\
12 / 29 / 87 \\
12 / 29 / 87 \\
12 / 29 / 87 \\
12 / 29 / 87 \\
12 / 29 / 87 \\
12 / 29 / 87 \\
12 / 29 / 87 \\
12 / 29 / 87 \\
12 / 29 / 87 \\
12 / 29 / 87 \\
12 / 29 / 87 \\
12 / 29 / 87 \\
12 / 29 / 87 \\
12 / 29 / 87 \\
12 / 29 / 87 \\
12 / 29 / 87 \\
12 / 29 / 87 \\
12 / 29 / 87 \\
12 / 29 / 87 \\
12 / 29 / 87 \\
12 / 04 / 87 \\
12 / 29 / 87 \\
12 / 29 / 87 \\
12 / 29 / 87 \\
12 / 16 / 87 \\
12 / 07 / 87 \\
12 / 21 / 87 \\
12 / 16 / 87 \\
12 / 15 / 87 \\
12 / 16 / 87 \\
12 / 07 / 87 \\
12 / 11 / 87 \\
12 / 16 / 87 \\
12 / 08 / 87 \\
12 / 21 / 87 \\
12 / 16 / 87 \\
12 / 04 / 87 \\
12 / 16 / 87 \\
12 / 15 / 87 \\
12 / 07 / 87 \\
12 / 07 / 87 \\
12 / 07 / 87 \\
12 / 11 / 87 \\
12 / 11 / 87 \\
12 / 14 / 87 \\
12 / 21 / 87\end{array}$ & $\begin{array}{r}36.69 \\
30.25 \\
42.71 \\
42.11 \\
10.26 \\
32.53 \\
32.72 \\
32.52 \\
41.80 \\
42.83 \\
43.70 \\
49.87 \\
50.31 \\
51.66 \\
44.64 \\
52.60 \\
35.00 \\
62.08 \\
34.46 \\
38.80 \\
53.12 \\
44.17 \\
52.39 \\
52.00 \\
52.75 \\
49.91 \\
103.42 \\
73.65 \\
42.59 \\
167.44 \\
110.96 \\
175.73 \\
93.09 \\
148.63 \\
121.65 \\
45.18 \\
89.04 \\
146.59 \\
87.55 \\
103.17 \\
135.30 \\
79.71 \\
99.29 \\
119.95 \\
263.38 \\
180.61 \\
179.79 \\
179.91\end{array}$ & $\begin{array}{l}343.13 \\
343.56 \\
342.92 \\
342.80 \\
374.54 \\
342.73 \\
342.81 \\
342.92 \\
342.63 \\
342.57 \\
342.68 \\
342.49 \\
342.54 \\
342.56 \\
342.67 \\
342.40 \\
342.59 \\
342.45 \\
342.53 \\
342.60 \\
342.48 \\
342.76 \\
342.49 \\
342.88 \\
343.31 \\
343.20 \\
412.98 \\
357.21 \\
347.56 \\
411.14 \\
403.93 \\
411.50 \\
365.29 \\
398.51 \\
402.18 \\
388.01 \\
474.14 \\
404.99 \\
389.59 \\
402.41 \\
404.68 \\
357.54 \\
376.25 \\
404.26 \\
413.17 \\
449.17 \\
449.99 \\
449.87\end{array}$ & $\begin{array}{l}379.82 \\
373.81 \\
385.63 \\
384.91 \\
384.80 \\
375.26 \\
375.53 \\
375.44 \\
384.43 \\
385.40 \\
386.38 \\
392.36 \\
392.85 \\
394.22 \\
387.31 \\
395.00 \\
377.59 \\
404.53 \\
376.99 \\
381.40 \\
395.60 \\
386.93 \\
394.88 \\
394.88 \\
396.06 \\
393.11 \\
516.40 \\
430.86 \\
390.15 \\
578.58 \\
514.89 \\
587.23 \\
458.38 \\
547.14 \\
523.83 \\
433.19 \\
563.18 \\
551.58 \\
477.14 \\
505.58 \\
539.98 \\
437.25 \\
475.54 \\
524.21 \\
676.55 \\
629.78 \\
629.78 \\
629.78\end{array}$ \\
\hline
\end{tabular}




\begin{tabular}{|c|c|c|c|c|}
\hline $\begin{array}{c}\text { We11 } \\
\text { Number }\end{array}$ & $\begin{array}{c}\text { Date } \\
\text { of } \\
\text { Measurement }\end{array}$ & $\begin{array}{l}\text { Depth to } \\
\text { Water, ft }\end{array}$ & $\begin{array}{c}\text { Hydraul ic } \\
\text { Head, } \\
\mathrm{ft} \text { above MSL }\end{array}$ & $\begin{array}{c}\text { Casing } \\
\text { Elevation, } \\
\mathrm{ft} \text { above MSL } \\
\end{array}$ \\
\hline $\begin{array}{l}699-26-15 A \\
699-27-8 \\
699-28-40 \\
699-28-52 A \\
699-29-78 \\
699-29-78 \\
699-29-78 \\
699-3-45 \\
699-31-31 \\
699-32-22 \\
699-32-43 \\
699-32-43 \\
699-32-62 \\
699-32-70 B \\
699-32-70 B \\
699-32-72 \\
699-32-72 \\
699-32-77 \\
699-32-77 \\
699-32-77 \\
699-33-42 \\
699-33-42 \\
699-33-56 \\
699-34-39 A \\
699-34-39 A \\
699-34-41 B \\
699-34-41 B \\
699-34-42 \\
699-34-42 \\
699-34-51 \\
699-34-88 \\
699-35-66 \\
699-35-66 \\
699-35-70 \\
699-35-70 \\
699-35-78 A \\
699-35-78 A \\
699-35-78 A \\
699-35-9 \\
699-36-61 A \\
699-36-61 A \\
699-36-93 \\
699-37-43 \\
699-37-82 A \\
699-37-82 A \\
699-37-82 A \\
699-38-65 \\
699-38-70\end{array}$ & $\begin{array}{l}12 / 07 / 87 \\
12 / 08 / 87 \\
12 / 07 / 87 \\
12 / 11 / 87 \\
12 / 11 / 87 \\
12 / 14 / 87 \\
12 / 21 / 87 \\
12 / 21 / 87 \\
12 / 11 / 87 \\
12 / 11 / 87 \\
12 / 07 / 87 \\
12 / 11 / 87 \\
12 / 11 / 87 \\
12 / 10 / 87 \\
12 / 14 / 87 \\
12 / 10 / 87 \\
12 / 14 / 87 \\
12 / 11 / 87 \\
12 / 15 / 87 \\
12 / 21 / 87 \\
12 / 07 / 87 \\
12 / 11 / 87 \\
12 / 11 / 87 \\
12 / 07 / 87 \\
12 / 11 / 87 \\
12 / 07 / 87 \\
12 / 11 / 87 \\
12 / 07 / 87 \\
12 / 11 / 87 \\
12 / 11 / 87 \\
12 / 15 / 87 \\
12 / 01 / 87 \\
12 / 03 / 87 \\
12 / 10 / 87 \\
12 / 14 / 87 \\
12 / 11 / 87 \\
12 / 15 / 87 \\
12 / 21 / 87 \\
12 / 07 / 87 \\
12 / 10 / 87 \\
12 / 28 / 87 \\
12 / 21 / 87 \\
12 / 11 / 87 \\
12 / 11 / 87 \\
12 / 15 / 87 \\
12 / 21 / 87 \\
12 / 03 / 87 \\
12 / 01 / 87\end{array}$ & $\begin{array}{r}43.12 \\
70.64 \\
154.69 \\
278.76 \\
182.01 \\
181.07 \\
181.41 \\
93.46 \\
125.04 \\
115.45 \\
111.68 \\
111.68 \\
276.95 \\
213.66 \\
213.45 \\
212.69 \\
212.50 \\
190.16 \\
189.27 \\
189.44 \\
110.99 \\
110.99 \\
311.33 \\
132.15 \\
132.18 \\
165.88 \\
165.88 \\
135.18 \\
135.18 \\
331.38 \\
161.49 \\
284.13 \\
285.23 \\
240.77 \\
240.54 \\
192.29 \\
191.49 \\
191.63 \\
113.45 \\
339.07 \\
338.53 \\
171.31 \\
284.54 \\
168.09 \\
167.37 \\
167.52 \\
322.10 \\
253.47\end{array}$ & $\begin{array}{l}399.52 \\
395.03 \\
404.75 \\
405.91 \\
465.04 \\
465.98 \\
465.64 \\
411.08 \\
404.28 \\
402.10 \\
404.94 \\
404.94 \\
430.14 \\
453.02 \\
453.23 \\
455.47 \\
455.66 \\
463.58 \\
464.47 \\
464.30 \\
405.01 \\
405.01 \\
405.70 \\
404.92 \\
404.89 \\
405.01 \\
405.01 \\
405.02 \\
405.02 \\
405.38 \\
471.33 \\
441.52 \\
440.42 \\
452.95 \\
453.18 \\
468.36 \\
469.16 \\
469.02 \\
386.38 \\
409.04 \\
409.58 \\
473.46 \\
405.63 \\
468.66 \\
469.38 \\
469.23 \\
431.23 \\
457.20\end{array}$ & $\begin{array}{l}442.64 \\
465.67 \\
559.44 \\
684.67 \\
647.05 \\
647.05 \\
647.05 \\
504.54 \\
529.32 \\
517.55 \\
516.62 \\
516.62 \\
707.09 \\
666.68 \\
666.68 \\
668.16 \\
668.16 \\
653.74 \\
653.74 \\
653.74 \\
516.00 \\
516.00 \\
717.03 \\
537.07 \\
537.07 \\
570.89 \\
570.89 \\
540.20 \\
540.20 \\
736.76 \\
632.82 \\
725.65 \\
725.65 \\
693.72 \\
693.72 \\
660.65 \\
660.65 \\
660.65 \\
499.83 \\
748.11 \\
748.11 \\
644.77 \\
690.17 \\
636.75 \\
636.75 \\
636.75 \\
753.33 \\
710.67\end{array}$ \\
\hline
\end{tabular}




\begin{tabular}{|c|c|c|c|c|}
\hline $\begin{array}{c}\text { Well } \\
\text { Number }\end{array}$ & $\begin{array}{c}\text { Date } \\
\text { of } \\
\text { Measurement }\end{array}$ & $\begin{array}{l}\text { Depth to } \\
\text { Water, ft }\end{array}$ & $\begin{array}{c}\text { Hydraulic } \\
\text { Head, } \\
\text { ft above MSL } \\
\end{array}$ & $\begin{array}{c}\text { Casing } \\
\text { Elevation, } \\
\text { ft above MSL }\end{array}$ \\
\hline $\begin{array}{l}699-38-70 \\
699-38-70 \\
699-39-39 \\
699-39-79 \\
699-40-1 \\
699-40-33 A \\
699-40-62 \\
699-41-23 \\
699-42-12 A \\
699-42-40 B \\
699-43-104 \\
699-43-42 A \\
699-43-89 \\
699-44-64 \\
699-45-42 \\
699-45-69 A \\
699-45-69 A \\
699-46-21 B \\
699-47-35 B \\
699-47-46 A \\
699-47-60 \\
699-47-60 \\
699-48-7 \\
699-48-71 \\
699-48-71 \\
699-49-13 E \\
699-49-28 \\
699-49-55 A \\
699-49-55 A \\
699-49-57 \\
699-49-57 \\
699-49-79 \\
699-49-79 \\
699-50-28 B \\
699-50-30 \\
699-50-42 \\
699-50-53 \\
699-50-53 \\
699-50-53 \\
699-50-85 \\
699-50-85 \\
699-51-63 \\
699-51-63 \\
699-51-75 \\
699-51-75 \\
699-52-19 \\
699-53-35 \\
699-53-35\end{array}$ & $\begin{array}{l}12 / 10 / 87 \\
12 / 14 / 87 \\
12 / 08 / 87 \\
12 / 14 / 87 \\
12 / 07 / 87 \\
12 / 07 / 87 \\
12 / 08 / 87 \\
12 / 18 / 87 \\
12 / 07 / 87 \\
12 / 10 / 87 \\
12 / 18 / 87 \\
12 / 10 / 87 \\
12 / 15 / 87 \\
12 / 10 / 87 \\
12 / 08 / 87 \\
12 / 08 / 87 \\
12 / 15 / 87 \\
12 / 17 / 87 \\
12 / 08 / 87 \\
12 / 08 / 87 \\
12 / 08 / 87 \\
12 / 10 / 87 \\
12 / 07 / 87 \\
12 / 08 / 87 \\
12 / 15 / 87 \\
12 / 07 / 87 \\
12 / 07 / 87 \\
12 / 07 / 87 \\
12 / 10 / 87 \\
12 / 07 / 87 \\
12 / 10 / 87 \\
12 / 08 / 87 \\
12 / 14 / 87 \\
12 / 07 / 87 \\
12 / 07 / 87 \\
12 / 07 / 87 \\
12 / 07 / 87 \\
12 / 07 / 87 \\
12 / 09 / 87 \\
12 / 08 / 87 \\
12 / 14 / 87 \\
12 / 08 / 87 \\
12 / 10 / 87 \\
12 / 08 / 87 \\
12 / 14 / 87 \\
12 / 07 / 87 \\
12 / 07 / 87 \\
12 / 10 / 87\end{array}$ & $\begin{array}{r}255.73 \\
255.51 \\
124.03 \\
203.70 \\
75.62 \\
107.03 \\
340.30 \\
67.51 \\
138.15 \\
123.40 \\
262.91 \\
144.54 \\
176.09 \\
317.69 \\
158.76 \\
275.69 \\
275.61 \\
130.73 \\
63.11 \\
173.56 \\
245.21 \\
245.21 \\
30.42 \\
240.59 \\
240.56 \\
51.52 \\
141.72 \\
125.24 \\
125.25 \\
148.05 \\
148.05 \\
230.75 \\
230.65 \\
144.03 \\
135.41 \\
55.67 \\
151.63 \\
151.50 \\
151.45 \\
282.56 \\
282.36 \\
165.16 \\
165.16 \\
190.63 \\
190.55 \\
49.62 \\
134.44 \\
134.47\end{array}$ & $\begin{array}{l}454.94 \\
455.16 \\
412.62 \\
469.82 \\
363.09 \\
411.02 \\
407.48 \\
398.99 \\
376.12 \\
423.06 \\
503.16 \\
419.94 \\
468.06 \\
407.91 \\
418.57 \\
449.77 \\
449.85 \\
391.29 \\
413.54 \\
406.58 \\
404.63 \\
404.63 \\
354.30 \\
447.56 \\
447.59 \\
361.20 \\
393.68 \\
404.90 \\
404.89 \\
404.76 \\
404.76 \\
458.45 \\
458.55 \\
393.27 \\
393.43 \\
411.17 \\
404.67 \\
404.80 \\
404.85 \\
456.79 \\
456.99 \\
406.68 \\
406.68 \\
450.88 \\
450.96 \\
361.46 \\
396.55 \\
396.52\end{array}$ & $\begin{array}{l}710.67 \\
710.67 \\
536.65 \\
673.52 \\
438.71 \\
518.05 \\
747.78 \\
466.50 \\
514.27 \\
546.46 \\
766.07 \\
564.48 \\
644.15 \\
725.60 \\
577.33 \\
725.46 \\
725.46 \\
522.02 \\
476.65 \\
580.14 \\
649.84 \\
649.84 \\
384.72 \\
688.15 \\
688.15 \\
412.72 \\
535.40 \\
530.14 \\
530.14 \\
552.81 \\
552.81 \\
689.20 \\
689.20 \\
537.30 \\
528.84 \\
466.84 \\
556.30 \\
556.30 \\
556.30 \\
739.35 \\
739.35 \\
571.84 \\
571.84 \\
641.51 \\
641.51 \\
411.08 \\
530.99 \\
530.99\end{array}$ \\
\hline
\end{tabular}




\begin{tabular}{|c|c|c|c|c|}
\hline $\begin{array}{c}\text { Well } \\
\text { Number }\end{array}$ & $\begin{array}{c}\text { Date } \\
\text { of } \\
\text { Measurement }\end{array}$ & $\begin{array}{l}\text { Depth to } \\
\text { Water, ft }\end{array}$ & $\begin{array}{c}\text { Hydraul ic } \\
\text { Head, } \\
\text { ft above MSL }\end{array}$ & $\begin{array}{c}\text { Casing } \\
\text { Elevation, } \\
\mathrm{ft} \text { above MSL }\end{array}$ \\
\hline $\begin{array}{l}699-53-47 A \\
699-53-47 A \\
699-53-478 \\
699-53-47 B \\
699-53-48 A \\
699-53-48 A \\
699-53-48 B \\
699-53-48 B \\
699-53-55 B \\
699-53-55 B \\
699-53-55 C \\
699-53-55 C \\
699-54-19 \\
699-54-34 \\
699-54-48 \\
699-54-48 \\
699-55-21 \\
699-55-40 \\
699-55-40 \\
699-55-50 C \\
699-55-50 C \\
699-55-70 \\
699-55-70 \\
699-55-76 \\
699-55-76 \\
699-55-76 \\
699-55-89 \\
699-55-95 \\
699-57-25 A \\
699-57-29 B \\
699-57-83 A \\
699-57-83 A \\
699-58-24 \\
699-59-32 \\
699-59-32 \\
699-59-58 \\
699-59-58 \\
699-59-80 B \\
699-59-80 B \\
699-60-32 \\
699-60-32 \\
699-60-60 \\
699-60-60 \\
699-61-37 \\
699-61-37 \\
699-61-41 \\
699-61-41 \\
699-61-62\end{array}$ & $\begin{array}{l}12 / 07 / 87 \\
12 / 10 / 87 \\
12 / 07 / 87 \\
12 / 10 / 87 \\
12 / 07 / 87 \\
12 / 10 / 87 \\
12 / 07 / 87 \\
12 / 10 / 87 \\
12 / 07 / 87 \\
12 / 10 / 87 \\
12 / 07 / 87 \\
12 / 10 / 87 \\
12 / 07 / 87 \\
12 / 10 / 87 \\
12 / 07 / 87 \\
12 / 10 / 87 \\
12 / 07 / 87 \\
12 / 07 / 87 \\
12 / 10 / 87 \\
12 / 07 / 87 \\
12 / 10 / 87 \\
12 / 08 / 87 \\
12 / 14 / 87 \\
12 / 02 / 87 \\
12 / 08 / 87 \\
12 / 14 / 87 \\
12 / 14 / 87 \\
12 / 14 / 87 \\
12 / 08 / 87 \\
12 / 08 / 87 \\
12 / 08 / 87 \\
12 / 14 / 87 \\
12 / 08 / 87 \\
12 / 07 / 87 \\
12 / 08 / 87 \\
12 / 04 / 87 \\
12 / 10 / 87 \\
12 / 08 / 87 \\
12 / 11 / 87 \\
12 / 07 / 87 \\
12 / 08 / 87 \\
12 / 04 / 87 \\
12 / 09 / 87 \\
12 / 07 / 87 \\
12 / 08 / 87 \\
12 / 07 / 87 \\
12 / 08 / 87 \\
12 / 04 / 87\end{array}$ & $\begin{array}{r}31.71 \\
31.73 \\
32.02 \\
32.04 \\
37.48 \\
37.54 \\
39.21 \\
38.17 \\
172.39 \\
172.42 \\
172.07 \\
172.14 \\
22.08 \\
141.97 \\
53.04 \\
53.06 \\
35.67 \\
133.39 \\
133.41 \\
40.46 \\
40.48 \\
136.14 \\
136.33 \\
142.86 \\
138.29 \\
138.20 \\
162.29 \\
310.64 \\
50.68 \\
53.84 \\
144.85 \\
144.73 \\
57.26 \\
62.34 \\
62.34 \\
92.62 \\
94.64 \\
156.19 \\
156.34 \\
63.54 \\
63.54 \\
108.24 \\
108.25 \\
60.67 \\
60.67 \\
32.40 \\
32.41 \\
93.81\end{array}$ & $\begin{array}{l}406.57 \\
406.55 \\
406.56 \\
406.54 \\
404.97 \\
404.91 \\
403.50 \\
404.54 \\
403.77 \\
403.74 \\
404.01 \\
403.94 \\
361.52 \\
408.27 \\
403.98 \\
403.96 \\
360.29 \\
409.74 \\
409.72 \\
403.97 \\
403.95 \\
432.89 \\
432.70 \\
440.38 \\
444.95 \\
445.04 \\
455.14 \\
466.41 \\
363.89 \\
362.34 \\
433.11 \\
433.23 \\
361.54 \\
361.95 \\
361.95 \\
405.15 \\
403.13 \\
427.06 \\
426.91 \\
361.76 \\
361.76 \\
403.79 \\
403.78 \\
382.27 \\
382.27 \\
396.52 \\
396.51 \\
403.70\end{array}$ & $\begin{array}{l}438.28 \\
438.28 \\
438.58 \\
438.58 \\
442.45 \\
442.45 \\
442.71 \\
442.71 \\
576.16 \\
576.16 \\
576.08 \\
576.08 \\
383.60 \\
550.24 \\
457.02 \\
457.02 \\
395.96 \\
543.13 \\
543.13 \\
444.43 \\
444.43 \\
569.03 \\
569.03 \\
583.24 \\
583.24 \\
583.24 \\
617.43 \\
777.05 \\
414.57 \\
416.18 \\
577.96 \\
577.96 \\
418.80 \\
424.29 \\
424.29 \\
497.77 \\
497.77 \\
583.25 \\
583.25 \\
425.30 \\
425.30 \\
512.03 \\
512.03 \\
442.94 \\
442.94 \\
428.92 \\
428.92 \\
497.51\end{array}$ \\
\hline
\end{tabular}




\begin{tabular}{|c|c|c|c|c|}
\hline $\begin{array}{c}\text { Well } \\
\text { Number }\end{array}$ & $\begin{array}{c}\text { Date } \\
\text { of } \\
\text { Measurement } \\
\end{array}$ & $\begin{array}{l}\text { Depth to } \\
\text { Water, ft }\end{array}$ & $\begin{array}{c}\text { Hydraulic } \\
\text { Head, } \\
\text { ft above MSL }\end{array}$ & $\begin{array}{c}\text { Casing } \\
\text { Elevation, } \\
\mathrm{ft} \text { above MSL }\end{array}$ \\
\hline $\begin{array}{l}699-61-62 \\
699-61-66 \\
699-61-66 \\
699-62-31 \\
699-62-31 \\
699-62-43 A \\
699-62-43 A \\
699-63-25 A \\
699-63-51 \\
699-63-51 \\
699-63-58 \\
699-63-58 \\
699-63-90 \\
699-63-92 \\
699-64-27 \\
699-64-62 \\
699-64-62 \\
699-65-50 \\
699-65-50 \\
699-65-59 A \\
699-65-59 A \\
699-65-72 \\
699-65-72 \\
699-65-72 \\
699-65-83 \\
699-65-83 \\
699-65-95 \\
699-66-103 \\
699-66-23 \\
699-66-38 \\
699-66-39 \\
699-66-58 \\
699-66-64 \\
699-66-91 \\
699-67-51 \\
699-67-86 \\
699-67-86 \\
699-67-98 \\
699-68-105 \\
699-69-38 \\
699-69-450 \\
699-70-23 \\
699-70-68 \\
699-70-68 \\
699-71-30 \\
699-71-30 \\
699-71-52 \\
699-71-52\end{array}$ & $\begin{array}{l}12 / 09 / 87 \\
12 / 04 / 87 \\
12 / 10 / 87 \\
12 / 07 / 87 \\
12 / 08 / 87 \\
12 / 07 / 87 \\
12 / 08 / 87 \\
12 / 08 / 87 \\
12 / 07 / 87 \\
12 / 08 / 87 \\
12 / 04 / 87 \\
12 / 09 / 87 \\
12 / 11 / 87 \\
12 / 11 / 87 \\
12 / 08 / 87 \\
12 / 04 / 87 \\
12 / 10 / 87 \\
12 / 07 / 87 \\
12 / 08 / 87 \\
12 / 04 / 87 \\
12 / 09 / 87 \\
12 / 04 / 87 \\
12 / 11 / 87 \\
12 / 21 / 87 \\
12 / 04 / 87 \\
12 / 11 / 87 \\
12 / 11 / 87 \\
12 / 14 / 87 \\
12 / 08 / 87 \\
12 / 08 / 87 \\
12 / 08 / 87 \\
12 / 09 / 87 \\
12 / 10 / 87 \\
12 / 11 / 87 \\
12 / 08 / 87 \\
12 / 11 / 87 \\
12 / 22 / 87 \\
12 / 11 / 87 \\
12 / 14 / 87 \\
12 / 08 / 87 \\
12 / 08 / 87 \\
12 / 08 / 87 \\
12 / 11 / 87 \\
12 / 21 / 87 \\
12 / 08 / 87 \\
12 / 13 / 87 \\
12 / 08 / 87 \\
12 / 13 / 87\end{array}$ & $\begin{array}{r}93.82 \\
119.52 \\
119.54 \\
72.41 \\
72.41 \\
35.41 \\
35.40 \\
33.91 \\
23.69 \\
23.70 \\
89.61 \\
89.61 \\
113.24 \\
100.19 \\
52.62 \\
98.33 \\
98.36 \\
66.24 \\
66.20 \\
105.11 \\
105.12 \\
140.85 \\
140.86 \\
145.27 \\
87.49 \\
87.49 \\
55.49 \\
66.15 \\
28.76 \\
32.74 \\
47.17 \\
101.64 \\
104.79 \\
69.57 \\
123.67 \\
74.65 \\
74.48 \\
58.50 \\
56.56 \\
20.95 \\
86.75 \\
30.38 \\
126.36 \\
137.53 \\
29.82 \\
29.83 \\
122.69 \\
122.55\end{array}$ & $\begin{array}{l}403.69 \\
402.66 \\
402.64 \\
361.71 \\
361.71 \\
396.89 \\
396.90 \\
361.24 \\
400.85 \\
400.84 \\
402.29 \\
402.29 \\
396.49 \\
397.31 \\
361.67 \\
401.92 \\
401.89 \\
400.82 \\
400.86 \\
401.85 \\
401.84 \\
399.43 \\
399.42 \\
395.01 \\
398.14 \\
398.14 \\
396.77 \\
396.86 \\
360.25 \\
403.46 \\
406.53 \\
401.69 \\
401.13 \\
398.18 \\
400.92 \\
397.74 \\
397.91 \\
396.97 \\
395.29 \\
401.98 \\
400.43 \\
361.33 \\
399.85 \\
388.68 \\
370.86 \\
370.85 \\
400.31 \\
400.45\end{array}$ & $\begin{array}{l}497.51 \\
522.18 \\
522.18 \\
434.12 \\
434.12 \\
432.30 \\
432.30 \\
395.15 \\
424.54 \\
424.54 \\
491.90 \\
491.90 \\
509.73 \\
497.50 \\
414.29 \\
500.25 \\
500.25 \\
467.06 \\
467.06 \\
506.96 \\
506.96 \\
540.28 \\
540.28 \\
540.28 \\
485.63 \\
485.63 \\
452.26 \\
463.01 \\
389.01 \\
436.20 \\
453.70 \\
503.33 \\
505.92 \\
467.75 \\
524.59 \\
472.39 \\
472.39 \\
455.47 \\
451.85 \\
422.93 \\
487.18 \\
391.71 \\
526.21 \\
526.21 \\
400.68 \\
400.68 \\
523.00 \\
523.00\end{array}$ \\
\hline
\end{tabular}




\begin{tabular}{|c|c|c|c|c|}
\hline $\begin{array}{c}\text { We]1 } \\
\text { Number }\end{array}$ & $\begin{array}{c}\text { Date } \\
\text { of } \\
\text { Measurement } \\
\end{array}$ & $\begin{array}{l}\text { Depth to } \\
\text { Water, ft }\end{array}$ & $\begin{array}{c}\text { Hydraulic } \\
\text { Head, } \\
\text { ft above MSL }\end{array}$ & $\begin{array}{c}\text { Casing } \\
\text { Elevation, } \\
\mathrm{ft} \text { above MSL }\end{array}$ \\
\hline $\begin{array}{l}699-71-77 \\
699-71-77 \\
699-72-73 \\
699-72-88 \\
699-72-92 \\
699-73-61 \\
699-74-44 \\
699-74-44 \\
699-74-48 \\
699-77-36 \\
699-77-36 \\
699-77-54 \\
699-78-62 \\
699-8-17 \\
699-8-25 \\
699-81-38 \\
699-82-45 A \\
699-83-47 \\
699-86-42 \\
699-87-55 \\
699-88-41 \\
699-88-41 \\
699-88-41 \\
699-89-35 \\
699-89-35 \\
699-89-35 \\
699-89-35 \\
699-9-\mathrm{E} 2 \\
699-90-34 \\
699-90-45 \\
699-90-45 \\
699-90-45 \\
699-90-45 \\
699-91-37 \\
699-91-37 \\
699-91-37 \\
699-91-37 \\
699-92-49 \\
699-96-49 \\
699-97-43 \\
699-97-43 \\
699-97-43 \\
699-97-43 \\
699-97-43 \\
699-97-51 A \\
699-512-29 \\
699-512-3 \\
699-514-20 A\end{array}$ & $\begin{array}{l}12 / 11 / 87 \\
12 / 13 / 87 \\
12 / 11 / 87 \\
12 / 11 / 87 \\
12 / 11 / 87 \\
12 / 13 / 87 \\
12 / 08 / 87 \\
12 / 13 / 87 \\
12 / 08 / 87 \\
12 / 09 / 87 \\
12 / 13 / 87 \\
12 / 09 / 87 \\
12 / 09 / 87 \\
12 / 15 / 87 \\
12 / 15 / 87 \\
12 / 09 / 87 \\
12 / 09 / 87 \\
12 / 09 / 87 \\
12 / 09 / 87 \\
12 / 09 / 87 \\
12 / 02 / 87 \\
12 / 16 / 87 \\
12 / 30 / 87 \\
12 / 02 / 87 \\
12 / 09 / 87 \\
12 / 16 / 87 \\
12 / 30 / 87 \\
12 / 08 / 87 \\
12 / 30 / 87 \\
12 / 02 / 87 \\
12 / 09 / 87 \\
12 / 16 / 87 \\
12 / 30 / 87 \\
12 / 02 / 87 \\
12 / 09 / 87 \\
12 / 16 / 87 \\
12 / 30 / 87 \\
12 / 09 / 87 \\
12 / 13 / 87 \\
12 / 02 / 87 \\
12 / 09 / 87 \\
12 / 13 / 87 \\
12 / 16 / 87 \\
12 / 30 / 87 \\
12 / 13 / 87 \\
12 / 18 / 87 \\
12 / 04 / 87 \\
12 / 04 / 87\end{array}$ & $\begin{array}{r}75.74 \\
75.80 \\
85.13 \\
38.01 \\
54.29 \\
131.43 \\
48.07 \\
48.11 \\
87.98 \\
35.62 \\
35.71 \\
81.92 \\
73.16 \\
123.74 \\
109.04 \\
26.87 \\
24.23 \\
45.72 \\
24.91 \\
70.42 \\
33.54 \\
33.51 \\
33.54 \\
27.11 \\
26.75 \\
26.51 \\
26.44 \\
46.18 \\
21.69 \\
37.23 \\
37.22 \\
37.33 \\
37.34 \\
50.22 \\
50.19 \\
50.17 \\
50.00 \\
48.37 \\
37.65 \\
43.06 \\
43.02 \\
43.05 \\
43.06 \\
43.06 \\
21.15 \\
83.17 \\
55.72 \\
92.10\end{array}$ & $\begin{array}{l}396.54 \\
396.48 \\
397.44 \\
399.36 \\
397.93 \\
400.10 \\
397.11 \\
397.07 \\
399.20 \\
376.66 \\
376.57 \\
398.67 \\
396.72 \\
398.70 \\
400.26 \\
379.60 \\
389.50 \\
389.55 \\
385.01 \\
388.21 \\
382.50 \\
382.53 \\
382.50 \\
370.35 \\
370.71 \\
370.95 \\
371.02 \\
371.91 \\
370.70 \\
384.92 \\
384.93 \\
384.82 \\
384.81 \\
372.71 \\
372.74 \\
372.76 \\
372.93 \\
383.63 \\
381.64 \\
378.75 \\
378.79 \\
378.76 \\
378.75 \\
378.75 \\
381.34 \\
404.51 \\
379.80 \\
400.64\end{array}$ & $\begin{array}{l}472.28 \\
472.28 \\
482.57 \\
437.37 \\
452.22 \\
531.53 \\
445.18 \\
445.18 \\
487.18 \\
412.28 \\
412.28 \\
480.59 \\
469.88 \\
522.44 \\
509.30 \\
406.47 \\
413.73 \\
435.27 \\
409.92 \\
458.63 \\
416.04 \\
416.04 \\
416.04 \\
397.46 \\
397.46 \\
397.46 \\
397.46 \\
418.09 \\
392.39 \\
422.15 \\
422.15 \\
422.15 \\
422.15 \\
422.93 \\
422.93 \\
422.93 \\
422.93 \\
432.00 \\
419.29 \\
421.81 \\
421.81 \\
421.81 \\
421.81 \\
421.81 \\
402.49 \\
487.68 \\
435.52 \\
492.74\end{array}$ \\
\hline
\end{tabular}




\begin{tabular}{|c|c|c|c|c|}
\hline $\begin{array}{c}\text { We11 } \\
\text { Number }\end{array}$ & $\begin{array}{c}\text { Date } \\
\text { of } \\
\text { Measurement } \\
\end{array}$ & $\begin{array}{l}\text { Depth to } \\
\text { Water, } \mathrm{ft}\end{array}$ & $\begin{array}{c}\begin{array}{c}\text { Hydraul ic } \\
\text { Head, }\end{array} \\
\text { ft above MSL } \\
\end{array}$ & $\begin{array}{c}\text { Casing } \\
\text { Elevation, } \\
\mathrm{ft} \text { above MSL } \\
\end{array}$ \\
\hline $\begin{array}{l}699-S 18-E 2 A \\
699-S 19-E 13 \\
699-S 19-E 13 \\
699-S 27-E 14 \\
699-S 29-E 12 \\
699-S 29-E 12 \\
699-S 3-25 \\
699-S 3-E 12 \\
699-S 30 E 15 A \\
699-S 30 E 15 A \\
699-S 31-1 \\
699-S 6-E 4 D \\
699-S 6 E 14 A \\
699-S 7-34 \\
699-S 8-19\end{array}$ & $\begin{array}{l}12 / 04 / 87 \\
12 / 04 / 87 \\
12 / 29 / 87 \\
12 / 29 / 87 \\
12 / 04 / 87 \\
12 / 29 / 87 \\
12 / 15 / 87 \\
12 / 04 / 87 \\
12 / 04 / 87 \\
12 / 29 / 87 \\
12 / 04 / 87 \\
12 / 04 / 87 \\
12 / 04 / 87 \\
12 / 18 / 87 \\
12 / 04 / 87\end{array}$ & $\begin{array}{r}75.58 \\
50.77 \\
50.23 \\
57.40 \\
42.24 \\
42.26 \\
124.27 \\
43.74 \\
58.26 \\
57.73 \\
81.23 \\
58.55 \\
27.97 \\
119.59 \\
107.95\end{array}$ & $\begin{array}{l}359.27 \\
343.74 \\
344.28 \\
342.36 \\
345.71 \\
345.69 \\
399.23 \\
354.16 \\
341.88 \\
342.41 \\
378.88 \\
371.92 \\
350.32 \\
407.53 \\
395.86\end{array}$ & $\begin{array}{l}434.85 \\
394.51 \\
394.51 \\
399.76 \\
387.95 \\
387.95 \\
523.50 \\
397.90 \\
400.14 \\
400.14 \\
460.11 \\
430.47 \\
378.29 \\
527.12 \\
503.81\end{array}$ \\
\hline
\end{tabular}


APPENDIX B

SAMPLING SCHEDULE 
APPENDIX B

\section{SAMPLING SCHEDULE}

This appendix lists those wells to be sampled in 1988, sampling frequency, and sampled constituents (Table B.1). It also lists those wells scheduled to be sampled for hazardous chemicals during the first two quarters of 1988 (Table B.2).

B. 1 
TABLE B.l. Wells to be Sampled for Radionuclide and Nitrate Analysis During 1988

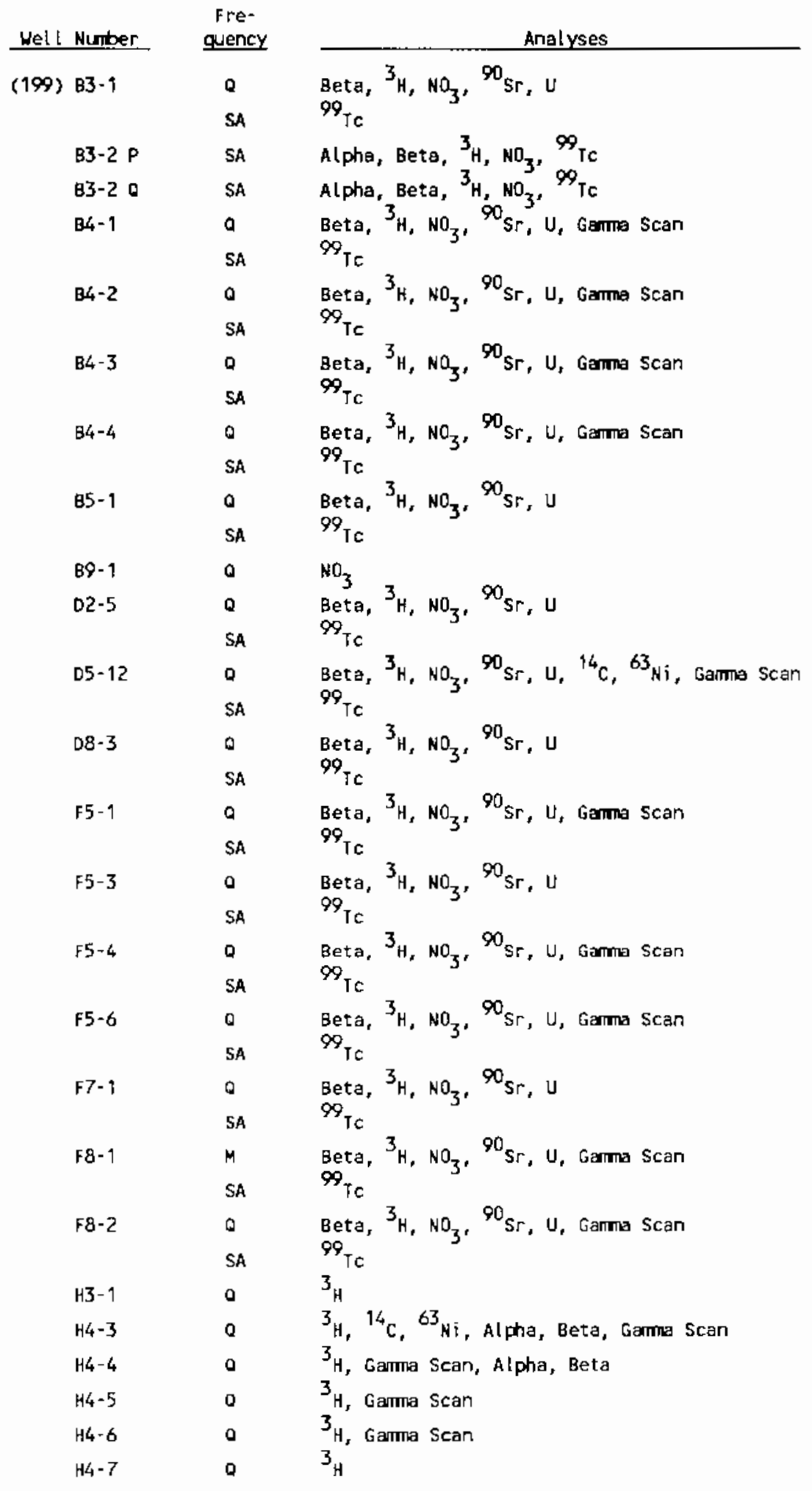


TABLE B.1. (contd)

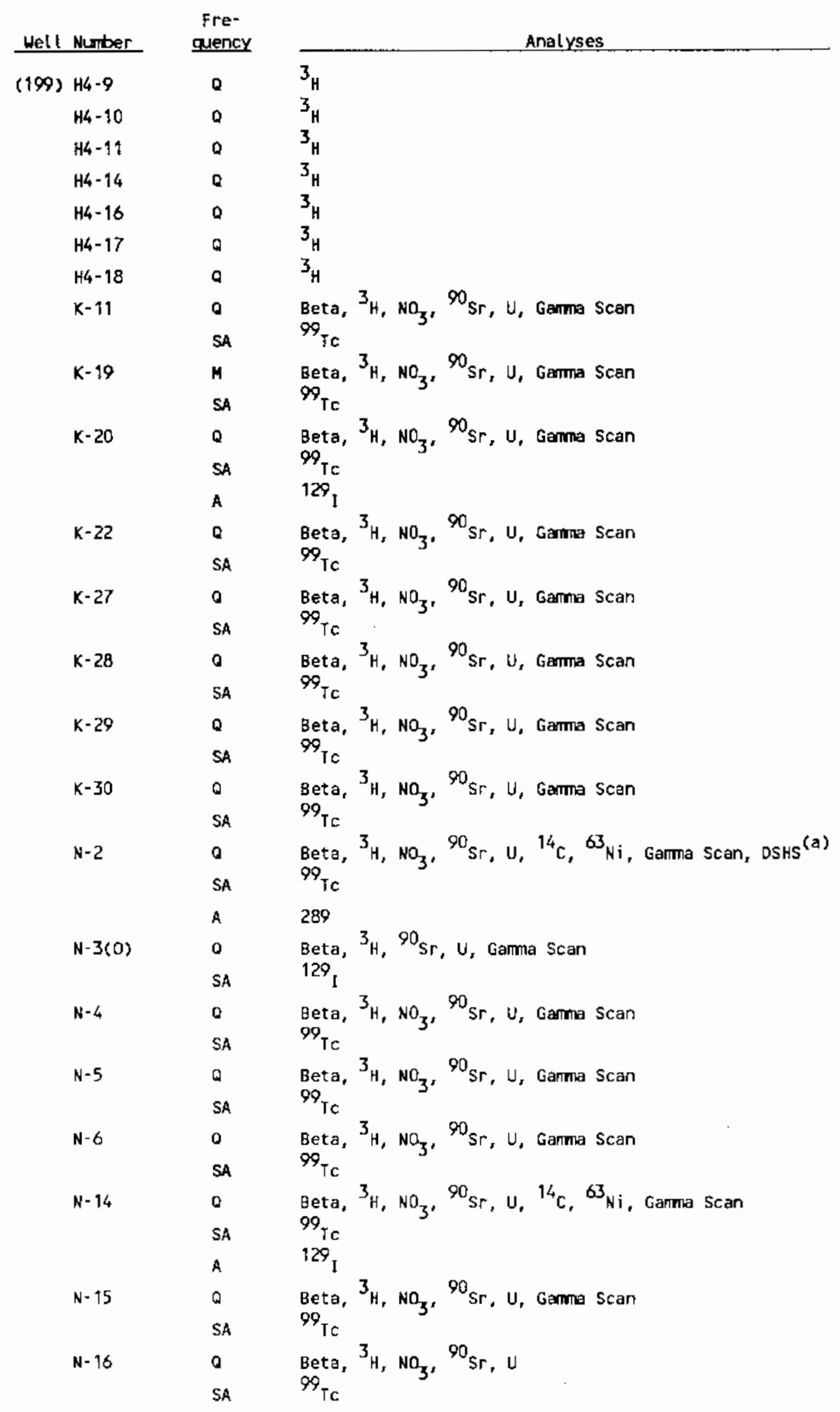


IABLE B.1. (contd)

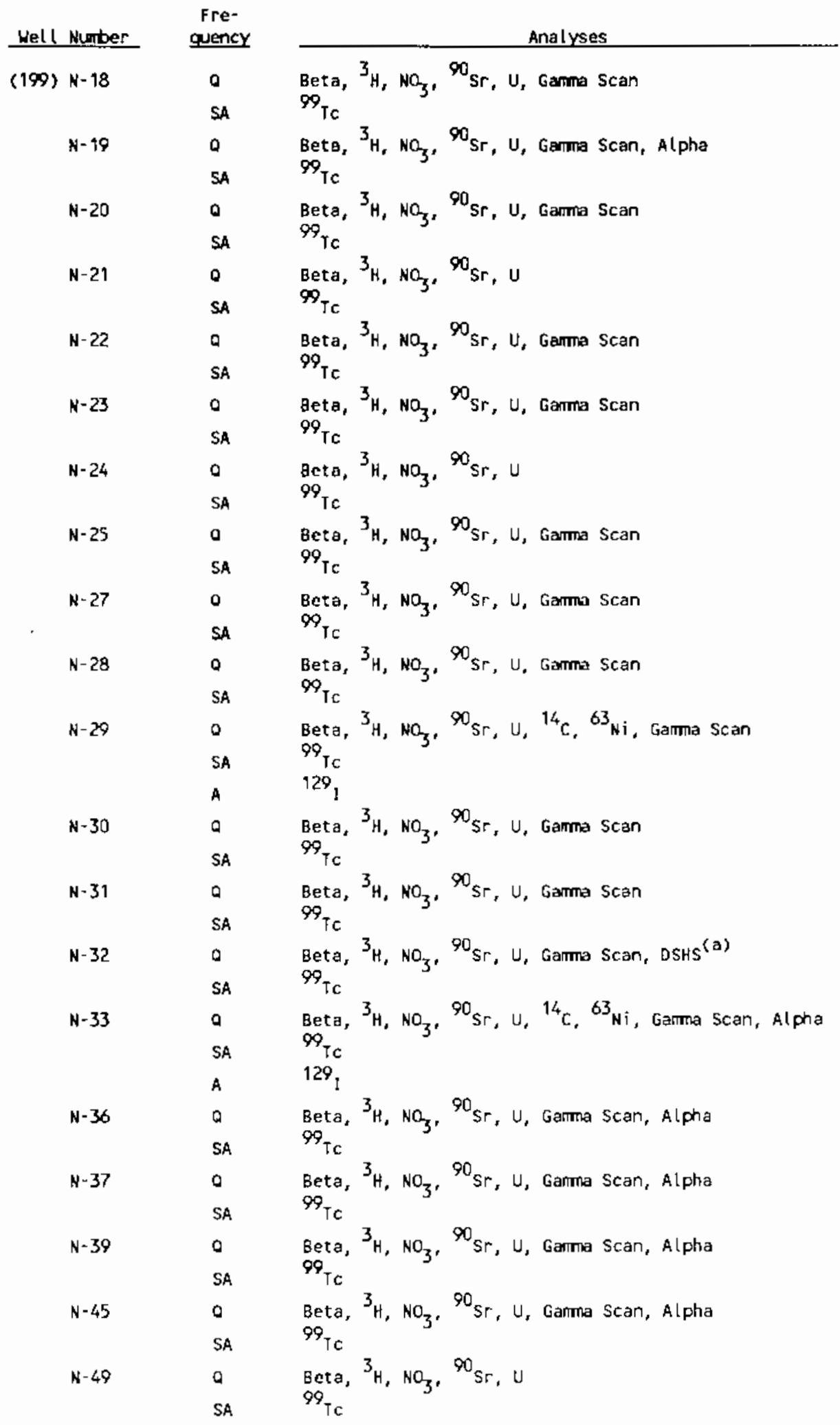


IABLE B.1. (contd)

\begin{tabular}{|c|c|c|}
\hline Hetl Number & $\begin{array}{c}\text { Fre- } \\
\text { guency }\end{array}$ & Analyses \\
\hline (199) N-50 & & Beta, ${ }^{3} \mathrm{H}, \mathrm{NO}_{3},{ }^{90} \mathrm{Sr}, \mathrm{U}$ \\
\hline $\mathrm{N}-51$ & Q & Beta, ${ }^{3} \mathrm{H}, \mathrm{NO}_{3},{ }^{90} \mathrm{Sr}, \mathrm{U}$ \\
\hline & SA & \\
\hline N- 52 & $a$ & Beta, ${ }^{3} \mathrm{H}, \mathrm{NO}_{3},{ }^{90} \mathrm{Sr}, \mathrm{U}$ \\
\hline & SA & ${ }^{99} \mathrm{TC}$ \\
\hline & & 2.0 200-AREA WELLS \\
\hline Hell Nunber & $\begin{array}{l}\text { Fre- } \\
\text { guency }\end{array}$ & Analyses \\
\hline
\end{tabular}

Sampled for PNL

(209) E13-5

$\mathrm{NO}_{3},{ }^{90} \mathrm{Sr}, \mathrm{U},{ }^{99} \mathrm{Ic}$, Gamma scan

E13-8

SA

${ }^{3}{ }^{3}, \mathrm{NO}_{3},{ }^{90} \mathrm{Sr}, \mathrm{U},{ }^{99} \mathrm{TC}$

129

E13-14

A

$3_{\mathrm{H},} \mathrm{NO}_{3},{ }^{90} \mathrm{Sr}, \mathrm{U},{ }^{99} \mathrm{Tc}$, Gamm Scan

A

129

E13-19 SA ${ }^{3} \mathrm{H}_{1} \mathrm{NO}_{3},{ }^{90} \mathrm{Sr}, \mathrm{U},{ }^{99} \mathrm{TC}$, Gamma Scan

E16-2 A $\quad 129_{1}$

E17-1 SA U

E17-5 Q $14 \mathrm{C}$

E17-6 $\quad$ SA $\quad{ }^{129}{ }_{\mathrm{I}}{ }_{\mathrm{Sr},}{ }^{14} \mathrm{C}$

$A \quad 129$ I

E17-8 a $\quad{ }^{14} \mathrm{C}$

129 ,

E17-12 SA 99 TC

E23-1 SA $3_{\mathrm{H}_{1}} \mathrm{NO}_{3}$

A $\quad 129 \mathrm{t}$

E23-2 SA U

E24-1 a Alpha, ${ }^{14} \mathrm{C}$

E24-2 $\quad$ SA $\quad 129$

E24-7 SA $\quad 3_{H_{1}} \mathrm{NO}_{3}$

E24-8 $\quad$ SA $\quad{ }^{129} \mathrm{I}{ }_{\mathrm{Sr}, U,}{ }^{99} \mathrm{TC}$

E24-13 SA ${ }^{3} \mathrm{H},{ }^{90} \mathrm{Sr}$, Gamma Scan

$\begin{array}{lll} & \text { A } & { }^{129}{ }_{1} \\ \text { E25-2 } & \text { SA } & 90_{S r} \text {, Gamma Scan }\end{array}$

(a) Duplicate samples sent to the Washington State Department of Social and Heal th Services. 
IABLE B.1. (contd)

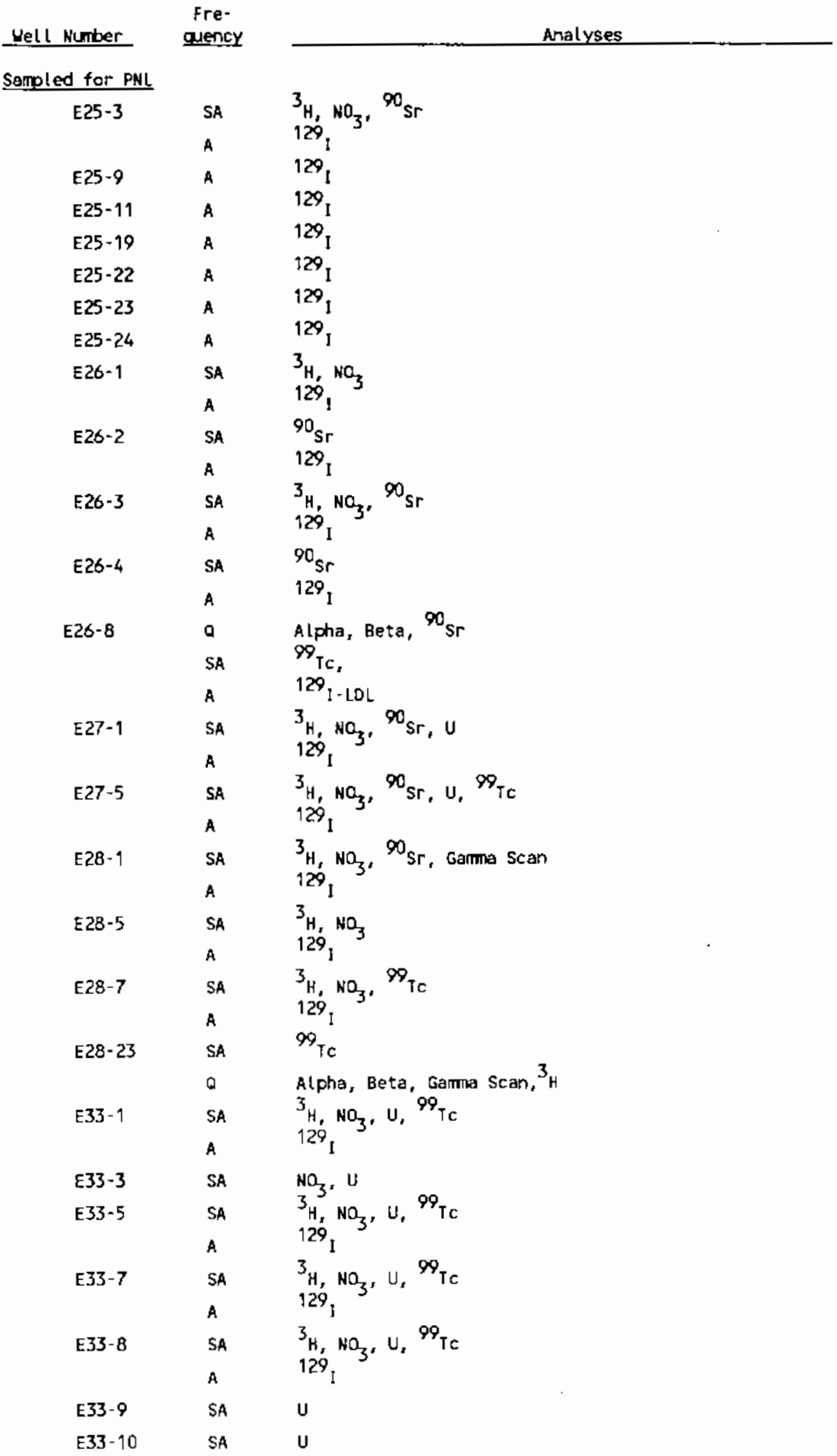


IABLE B.1. (contd)

Helt Nimer

Sempled for PNL

quency

Analyses

\begin{tabular}{|c|c|c|c|}
\hline (299) & $E 33-12$ & $\begin{array}{l}S A \\
A\end{array}$ & $\begin{array}{l}\text { Beta, }{ }^{90} \mathrm{Sr}, U,{ }^{99} \mathrm{Tc} \text {, Gamma Scan } \\
129_{\mathrm{I}-\mathrm{LDL}}\end{array}$ \\
\hline & E33-14 & SA & ${ }_{3}^{3} \mathrm{H}, \mathrm{NO}_{3},{ }^{90} \mathrm{Sr}, \mathrm{U}$ \\
\hline & E33-18 & $\begin{array}{l}\text { SA } \\
\text { A }\end{array}$ & ${ }_{129}^{3},{ }^{3} \mathrm{NO}_{3}, \mathrm{U},{ }^{99} \mathrm{TC}$ \\
\hline & E33-20 & $\begin{array}{l}\text { SA } \\
A\end{array}$ & ${ }^{3}{ }^{3}, 4,9_{1}^{99}$ \\
\hline & E33-27 & $\begin{array}{l}\text { SA } \\
\text { A }\end{array}$ & ${ }_{129}^{3}{ }_{1}{ }^{\mathrm{NO}_{3}},{ }^{90} \mathrm{Sr}, \mathrm{U},{ }^{99} \mathrm{Tc}$ \\
\hline & $E 33-24$ & SA & ${ }_{129}^{3} 9_{1} \mathrm{NO}_{3}, \mathrm{U}$ \\
\hline & E33-27 & SA & ${ }_{3}^{3}, \mathrm{U}$ \\
\hline & W-1 & $S A$ & ${ }_{129}{ }_{1}{ }_{3}$, Gamma Scan \\
\hline & $w 10-1$ & $S A$ & ${ }_{{ }_{3} \mathrm{H}_{1}, \mathrm{NO}_{3}, \mathrm{U},{ }^{\mathrm{SP}} \mathrm{TC}}$ \\
\hline & $W 10-3$ & SA & 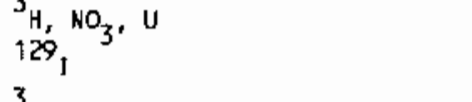 \\
\hline & $w 10-4$ & SA & ${ }_{2}^{2} 9_{1} \mathrm{NO}_{3} \cdot \mathrm{U}$ \\
\hline & W10-5 & SA & $129{ }_{3} \mathrm{NO}_{3}$ \\
\hline & $+10-8$ & $S A$ & $\begin{array}{l}3^{3}{ }^{H}, U \\
129 \\
I\end{array}$ \\
\hline & H10-9 & SA & $129^{\prime}$ \\
\hline & -111-3 & SA & ${ }_{3}^{3}, \mathrm{NO}_{3}, 90$ \\
\hline & $\begin{array}{l}-11-9 \\
+11-18\end{array}$ & SA & 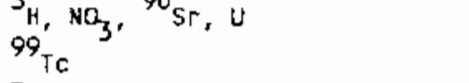 \\
\hline & 山12-1 & SA & ${ }_{129}^{3}{ }_{1} \mathrm{NO}_{3}$ \\
\hline & H14-2 & SA & $\mathrm{NO}_{3}, \mathrm{U},{ }^{99} \mathrm{TC}$ \\
\hline & $w 14-5$ & SA & ${ }_{90}^{90} \mathrm{Sr}, \mathrm{U}$, Garma Scan \\
\hline & $W 14-6$ & $S A$ & Sr, U, Gamma Scan \\
\hline & $415-2$ & SA & $\begin{array}{l}3_{H}, \mathrm{NO}_{3} \text {, Gamma Scan } \\
129_{1}\end{array}$ \\
\hline & $\begin{array}{l}H 15-4 \\
H 15-6\end{array}$ & SA & $\begin{array}{l}{ }_{90} \mathrm{Sr}, U,{ }^{\circ} \mathrm{TC} \\
\mathrm{TC}\end{array}$ \\
\hline & W15-7 & $\mathrm{SA}$ & $\begin{array}{l}3_{H} \\
\uparrow 29_{1}\end{array}$ \\
\hline & $W 15-10$ & $S A$ & ${ }_{129}^{3}$ \\
\hline
\end{tabular}

B. 7 
TABLE B.l. (contd)

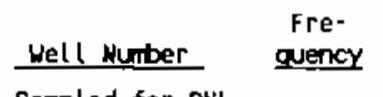

Sampled for PNL

(299) W15-11

บ18-3

W19-1 AA

W19-3

19-5 A

W19-9

W19-11 A

$A$

WT9-12 SA

W19-15

U19-16 SA

U18-18 A

W21-1

U22-1

W:2-2

แ22-7

W22-9

W22-10

แ22-12

W22-18

แ22-20

W22-21

422-26

H23-1

423-2

w23-4

A

H23-9

แ23-10

W23-11

SA

Anolyses

$3_{\mathrm{H}}$

129

$3_{H,} \mathrm{NO}_{3},{ }^{90} \mathrm{Sr},{ }^{99} \mathrm{TC}$

$129^{\mathrm{I}}$

$90_{\mathrm{Sr}},{ }^{99} \mathrm{TC}$

${ }^{14} \mathrm{C}$, Garma Scan, ${ }^{3}$, Alpha, Beta

1291

U, ${ }^{99} \mathrm{TC}$

$14 \mathrm{C}$

129

$14 \mathrm{C}$

$129 \mathrm{~J}$

$9_{\mathrm{TC}}$

${ }^{14} \mathrm{C}$

$129_{1}$

${ }^{14} \mathrm{c}$

129 ,

Alpha, Beta, Garma Scan, ${ }^{3} H$

${ }^{3} \mathrm{H}, \mathrm{NO}_{3}, \mathrm{U}$

${ }^{129} 9_{1}$

$\mathrm{U}$

u

${ }^{3} \mathrm{H}, \mathrm{NO}_{3},{ }^{90} \mathrm{Sr}, \mathrm{U}$

$129_{1}$

$3_{\mathrm{H},} \mathrm{NO}_{3},{ }^{90} \mathrm{Sr}, \mathrm{U}$

$129_{1}$

${ }^{3} \mathrm{H}, \mathrm{U}$

129 I

U

$9_{\text {If }}$

$\mathrm{NO}_{3} \cup,{ }^{14} \mathrm{C}$

$129_{1}$

${ }^{14} \mathrm{C}$

$129_{1}$

$\mathrm{NO}_{3}, \mathrm{U}$

$3 \mathrm{H}, \mathrm{U}$

129 I

$\mathrm{u}$

${ }^{90} \mathrm{Sr}$

129 I

${ }_{99}$

99 TC

${ }^{90} \mathrm{Sr},{ }^{99} \mathrm{Tc}$ 
IABLE B.1. (contd)

\subsection{0-Area Wells}

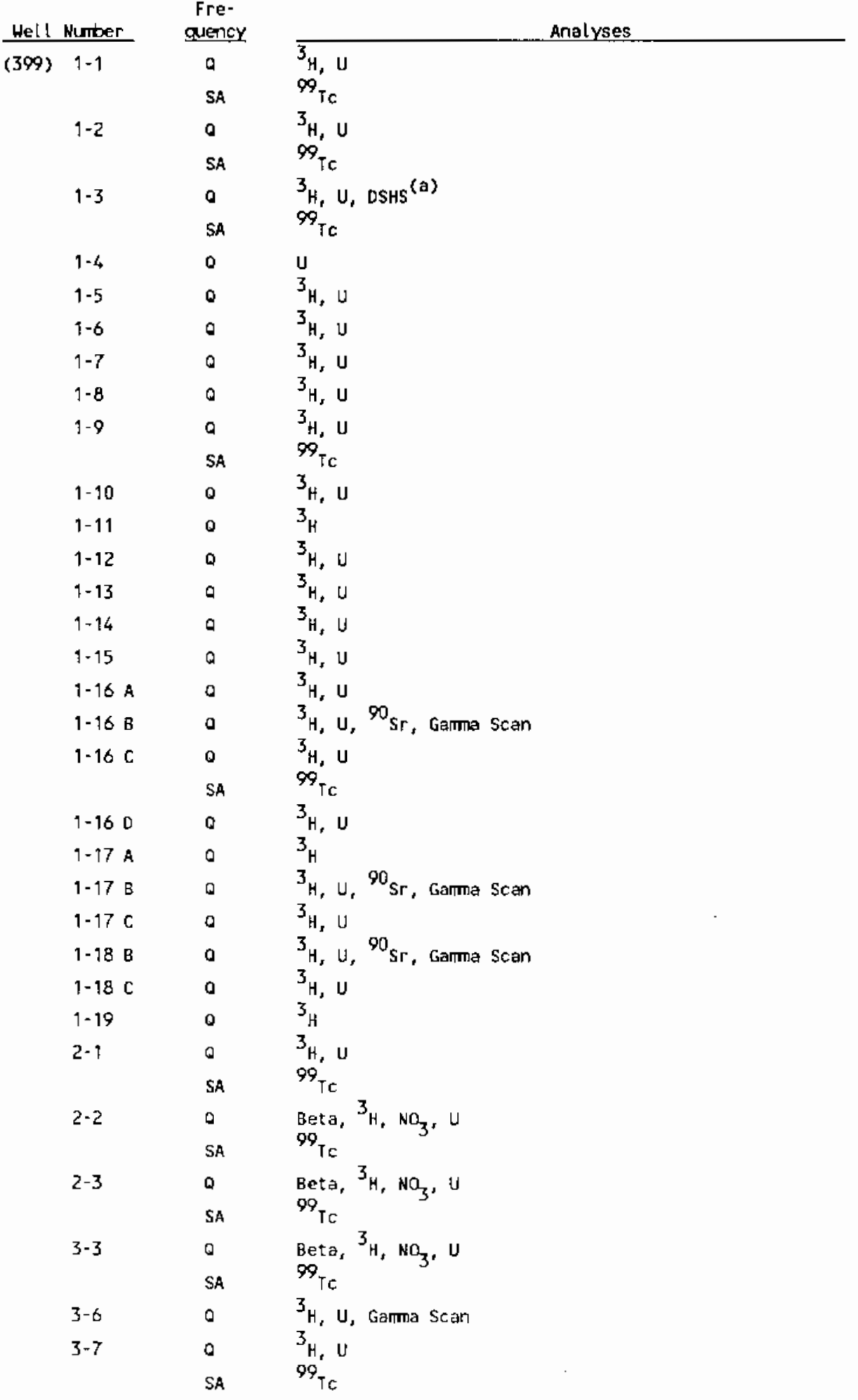

$$
\text { B. } 9
$$




\section{IABLE B.1. (contd)}

\begin{tabular}{|c|c|c|c|}
\hline Hell & Nunber. & $\begin{array}{l}\text { Fre- } \\
\text { quency }\end{array}$ & Analyses \\
\hline$(399)$ & 3-9 & Q & Beta, ${ }^{3} \mathrm{H}, \mathrm{NO}_{3}, \mathrm{U},{ }^{90} \mathrm{Sr}$ \\
\hline & & SA & 99 Tc \\
\hline & $3-10$ & 0 & ${ }^{3} \mathrm{H}, \mathrm{U},{ }^{90} \mathrm{Sr}$ \\
\hline & $3-11$ & 0 & Beta, ${ }^{3} \mathrm{H}_{2} \mathrm{NO}_{3}, \mathrm{U},{ }^{90} \mathrm{Sr}$, Gemma Scan \\
\hline & & SA & 99 Ts \\
\hline & $3-12$ & 0 & Beta, ${ }^{3} \mathrm{H}, \mathrm{NO}_{3}, \mathrm{U},{ }^{90} \mathrm{Sr}$ \\
\hline & & SA & ${ }^{49} \mathrm{Tc}$ \\
\hline & $4-1$ & a & ${ }^{3} \mathrm{H}, \mathrm{U}$, Gamma Scan \\
\hline & $4 \cdot 7$ & 0 & ${ }^{3} \mathrm{H}, \mathrm{U}$ \\
\hline & & SA & ${ }^{99}$ ic \\
\hline & $4-9$ & Q & Beta, ${ }^{3} \mathrm{H}, \mathrm{NO}_{3}, \mathrm{U},{ }^{90} \mathrm{Sr}$, Gamma Scan \\
\hline & & SA & TC \\
\hline & 4-10 & Q & Beta, ${ }^{3} \mathrm{H}, \mathrm{NO}_{3}, \mathrm{U},{ }^{90} \mathrm{Sr}$, Germa Scen \\
\hline & & SA & ${ }^{99} \mathrm{Tc}$ \\
\hline & $4-11$ & a & $3_{\mathrm{H}, \mathrm{U}}$ \\
\hline & $5-1$ & a & Gete, ${ }^{3} \mathrm{H}, \mathrm{NO}_{3}, \mathrm{U}$ \\
\hline & & SA & ${ }^{99 \mathrm{TC}}$ \\
\hline & $6-1$ & 0 & Beta, ${ }^{3} \mathrm{H}, \mathrm{NO}_{3}, \mathrm{U}$, Gamma scan \\
\hline & & SA & ${ }^{99} \mathrm{TC}$ \\
\hline & $8-1$ & 0 & Beta, ${ }^{3} \mathrm{H}, \mathrm{NO}_{3}, \mathrm{U}$, Garma Scan \\
\hline & & SA & ${ }^{99} \mathrm{Tc}$ \\
\hline & $8 \cdot 2$ & 0 & ${ }^{3} \mathrm{H}, \mathrm{U}$ \\
\hline & $8-3$ & Q & Beta, ${ }^{3} \mathrm{H}, \mathrm{NO}_{3}, \mathrm{U}$, Gamma Scan \\
\hline & & SA & 99 IC \\
\hline & $8-4$ & 0 & Beta, ${ }^{3} \mathrm{H}, \mathrm{NO}_{3}, \mathrm{U}$ \\
\hline & & SA & $99 \mathrm{TC}$ \\
\hline & & & 4.0 400-Area Wells \\
\hline Hell I & Nunber. & $\begin{array}{c}\text { Fre- } \\
\text { quency }\end{array}$ & Analyses \\
\hline (499) & so-7 & a & $3_{H}$, Garma Scan \\
\hline & so-8 & 0 & Alphe, Beta, ${ }^{3}$, Gamma Scan \\
\hline & & A & 129 \\
\hline & $\mathrm{s} 1 \cdot 7 \mathrm{~B}$ & 0 & Alpha, Beta, ${ }^{3} \mathrm{H}, \mathrm{NO}_{3}$ \\
\hline & & A & ${ }^{129} \mathrm{I}$ \\
\hline & $51-7 c$ & 0 & Alpha, Beta, ${ }^{3} \mathrm{H}, \mathrm{NO}_{3}$ \\
\hline & & A & $129{ }_{I}$ \\
\hline & $51-8 \mathrm{~A}$ & 0 & Alpha, Beta, ${ }^{3} \mathrm{H}, \mathrm{NO}_{3}$ \\
\hline & & A & ${ }^{2 q_{I}}$ \\
\hline & $51-8 \mathbf{s}$ & 0 & Alpha, Beta, ${ }^{3} \mathrm{H}, \mathrm{NO}_{3}$ \\
\hline & & A & ${ }^{129} \mathrm{I}$ \\
\hline & $\mathrm{s} 1 \cdot 8 \mathrm{C}$ & 0 & Alpha, Beta, ${ }^{3} \mathrm{H}$ \\
\hline & & A & \\
\hline
\end{tabular}

(a) Duplicate samples sent to the Washington State Department of Sociat and Health Services. 
IABLE B.1. (contd)

\subsection{0 -Area Hells}

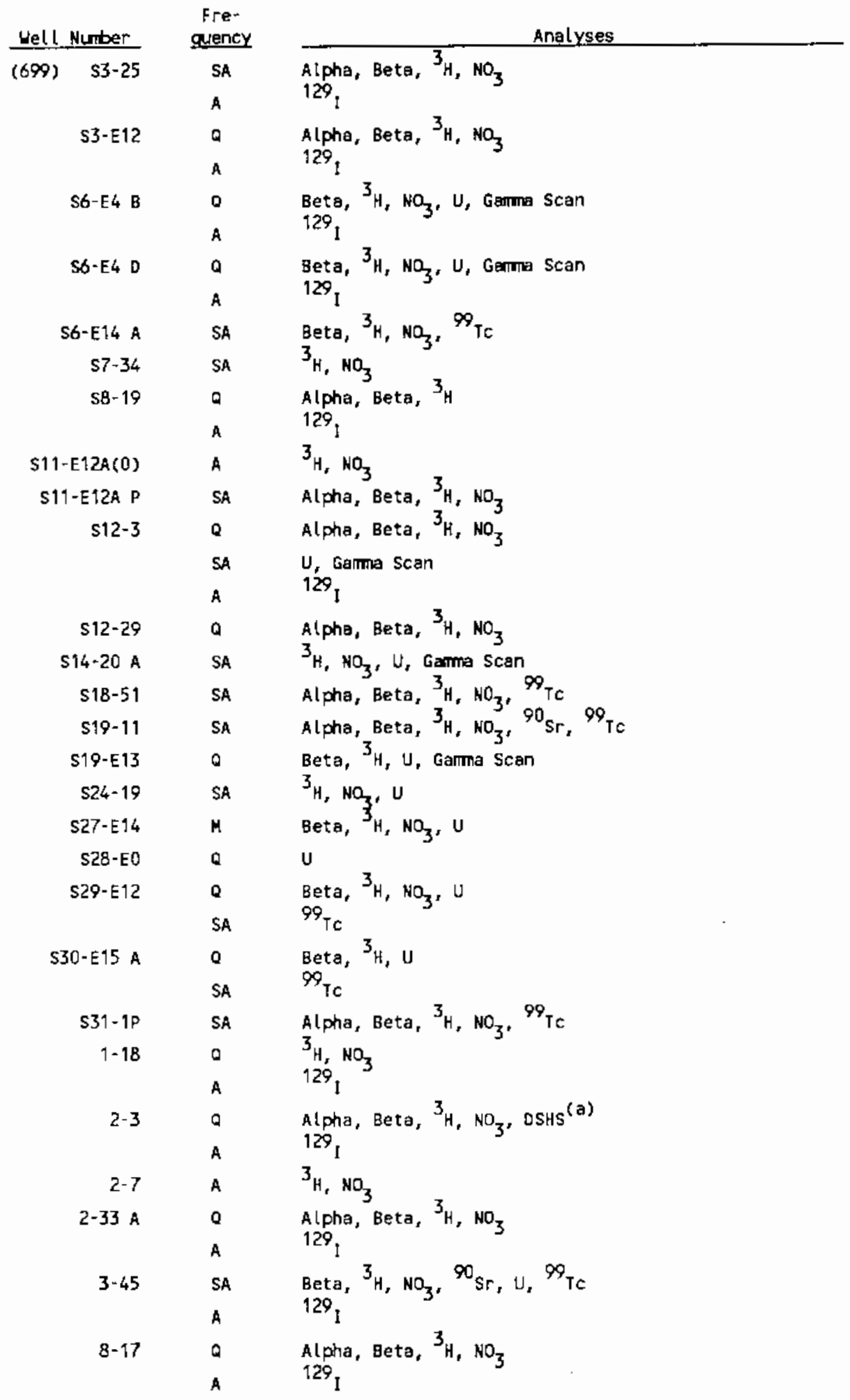


TABLE B.1. (contd)

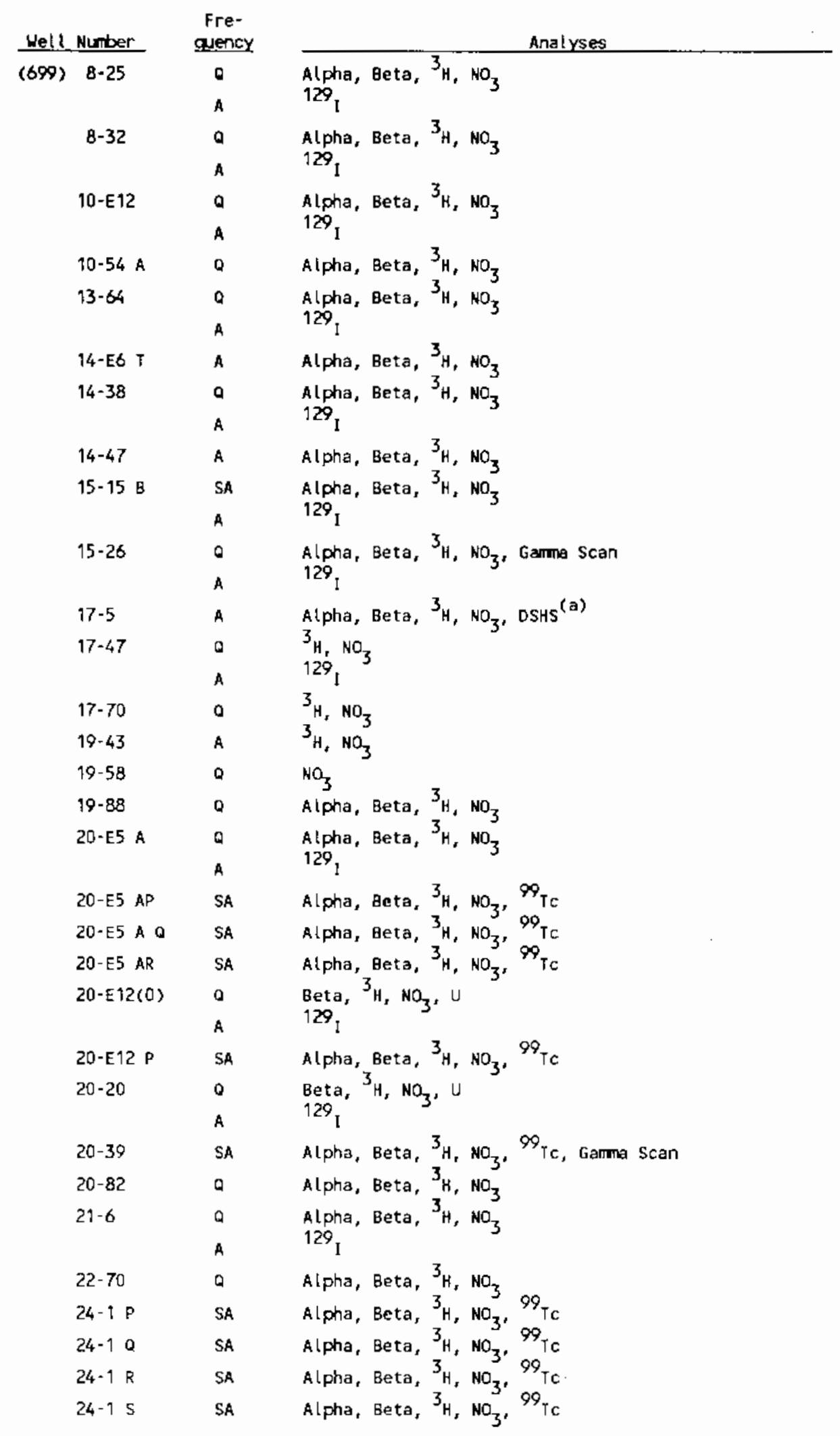


TABLE B.1. (contd)

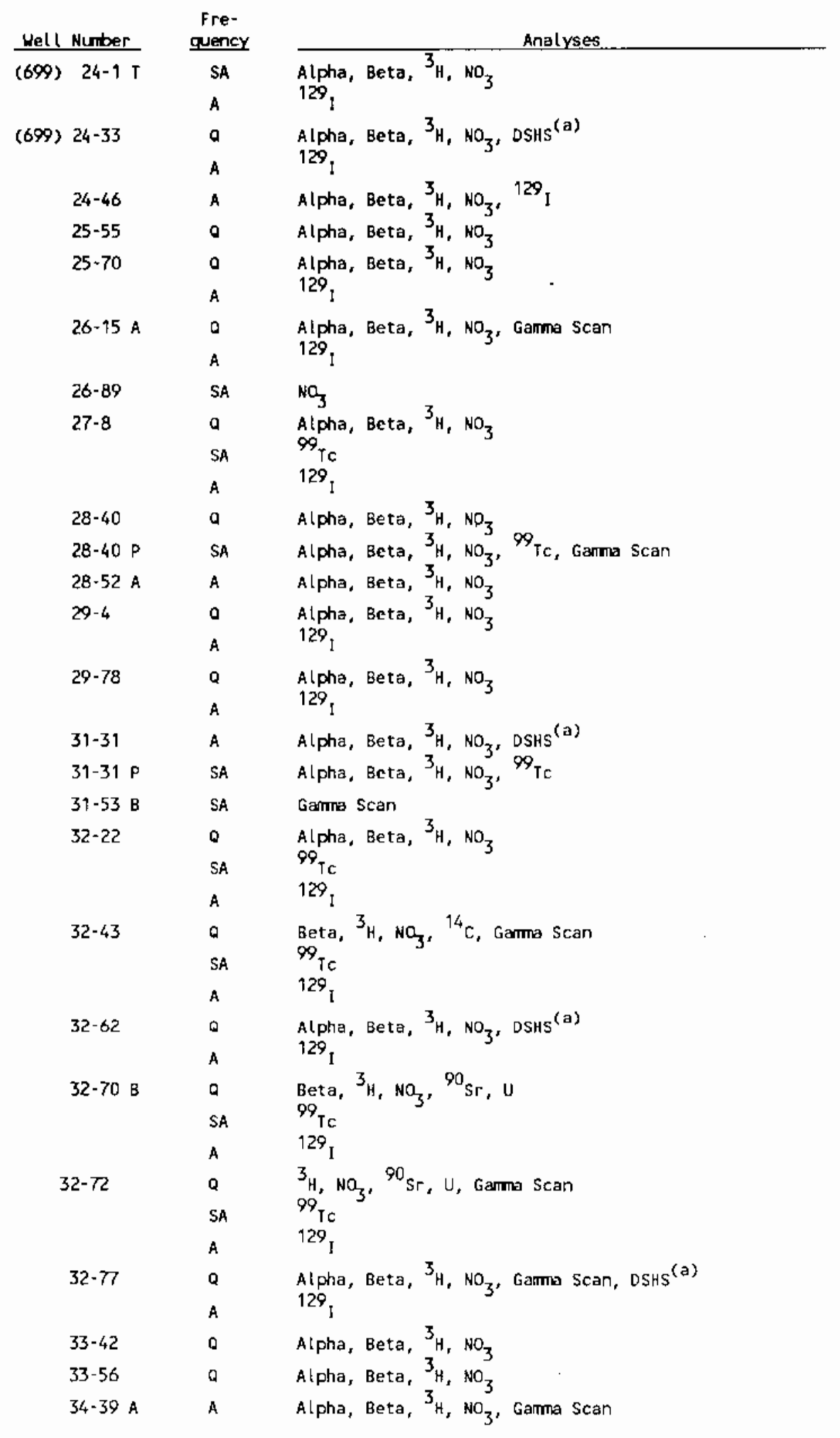


TABLE B.1. (contd)

\begin{tabular}{|c|c|c|c|}
\hline Hell & Number & $\begin{array}{c}\text { Fre- } \\
\text { guency }\end{array}$ & Analyses \\
\hline$(699)$ & $34+418$ & $Q$ & $3_{\mathrm{H}}, \mathrm{NO}_{3}$, Gamma Scan \\
\hline & $34-42$ & Q & Alpha, geta, ${ }^{3} \mathrm{H}, \mathrm{NO}_{3}$ \\
\hline & $34-51$ & Q & Alpha, Beta, ${ }^{3} \mathrm{H}, \mathrm{NO}_{3}$, Gamm Scan \\
\hline & & A & \\
\hline & $35-9$ & 0 & Alpha, Beta, ${ }^{3} \mathrm{H}, \mathrm{NO}_{3}$ \\
\hline & & A & ${ }^{129} 9^{1}$ \\
\hline & $35-66$ & 0 & geta, ${ }^{3} \mathrm{H}_{2} \mathrm{NO}_{3},{ }^{90} \mathrm{Sr}, \mathrm{U}$ \\
\hline & & SA & ${ }_{10}$ \\
\hline & & A & ${ }^{129}{ }_{1}$ \\
\hline & $35-70$ & 0 & Beta, ${ }^{3} \mathrm{H}_{2} \mathrm{NO}_{3},{ }^{90} \mathrm{Sr}, \mathrm{U},{ }^{14} \mathrm{C}, \mathrm{OSHS}^{(\mathrm{a})}$ \\
\hline & & SA & \\
\hline & & $A$ & 129 \\
\hline & $36-46 P$ & SA & Alpha, Beta, ${ }_{3}^{3}, \mathrm{NO}_{3},{ }^{99} \mathrm{TC}$ \\
\hline & $36-460$ & SA & Alpha, Beta, ${ }^{3} \mathrm{H}, \mathrm{NO}_{3},{ }^{99} \mathrm{TC}$ \\
\hline & $36-61 \mathrm{~A}$ & Q & $\mathrm{NO}_{3}$ \\
\hline & $36-61 \mathrm{~B}$ & 0 & Alpha, Beta, ${ }^{3} \mathrm{H}, \mathrm{NO}_{3}$ \\
\hline & & A & ${ }_{128}^{1}$ \\
\hline & $36-93$ & SA & Alpha, Beta, ${ }^{3} \mathrm{H}, \mathrm{NO}_{3},{ }^{90} \mathrm{Sr},{ }^{99} \mathrm{TC}$ \\
\hline & $37-E 4$ & A & Alpha, Beta, $3_{3}, \mathrm{NO}_{3}$ \\
\hline & $37-43$ & A & Alpha, Beta, ${ }^{3} \mathrm{H}, \mathrm{NO}_{3},{ }^{99} \mathrm{Tc},{ }^{14} \mathrm{C}$, Gamma Scan, ${ }^{129} \mathrm{I}$ \\
\hline & $37-82 A$ & 0 & Alpha, Beta, ${ }^{3} \mathrm{H}, \mathrm{NO}_{3}, \mathrm{DSHS}^{(\mathrm{a})}$ \\
\hline & $38-15$ & Q & Alpha, Beta, ${ }^{3} \mathrm{H}, \mathrm{NO}_{3}$, Gamma Scan \\
\hline & & A & ${ }^{129} I_{1}$ \\
\hline & $38-65$ & Q & 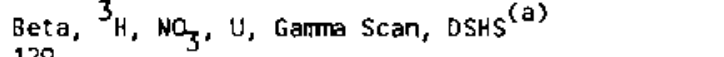 \\
\hline & & A & ${ }^{129} \mathrm{I}$ \\
\hline & $38-70$ & 0 & ${ }^{3} \mathrm{H}, \mathrm{NO}_{3},{ }^{90} \mathrm{Sr},{ }^{14} \mathrm{C},{ }^{63} \mathrm{Ni}$ \\
\hline & & A & $1299^{1}$ \\
\hline & $39-0$ & A & Alpha, Beta, ${ }_{3}^{3} \mathrm{H}_{1} \mathrm{NO}_{3}$, Gamma Scan \\
\hline & $39-39$ & 0 & Alpha, Beta, ${ }^{3} \mathrm{H}_{1} \mathrm{NO}_{3}$, Gamma Scan \\
\hline & & A & \\
\hline & $39-79$ & a & Alpha, Beta, ${ }^{3} \mathrm{H}_{1} \mathrm{NO}_{3}$, Gamma Scan \\
\hline & & A & $129^{I}$ \\
\hline & 40-1 & 0 & Alpha, Beta, ${ }^{3} \mathrm{H}, \mathrm{NO}_{3}$, Gamma Scan, DSHS ${ }^{(a)},{ }^{90} \mathrm{Sr}$ \\
\hline & & $S A$ & "TC \\
\hline & $40-33 \mathrm{~A}$ & A & Alpha, Beta, ${ }^{3} \mathrm{H}, \mathrm{NO}_{3}$, Gamma Scan, DSHS ${ }^{(a)}$ \\
\hline & $40-62$ & a & $\begin{array}{l}\text { Alpha, Beta, }{ }^{3} \mathrm{H}, \mathrm{NO}_{3} \\
129 \text {. }\end{array}$ \\
\hline & & A & ${ }_{1}{ }_{3} \quad 90$ \\
\hline & 41-1 & 0 & Beta, ${ }^{3} \mathrm{H}_{1} \mathrm{NO}_{3},{ }^{9} \mathrm{Sr}$, Gamma Scan, U, Alpha \\
\hline & & $\begin{array}{l}S A \\
A\end{array}$ & $\begin{array}{l}{ }_{1} \mathrm{TC} \\
129 \\
1\end{array}$ \\
\hline
\end{tabular}


TABLE B.1. (contd)

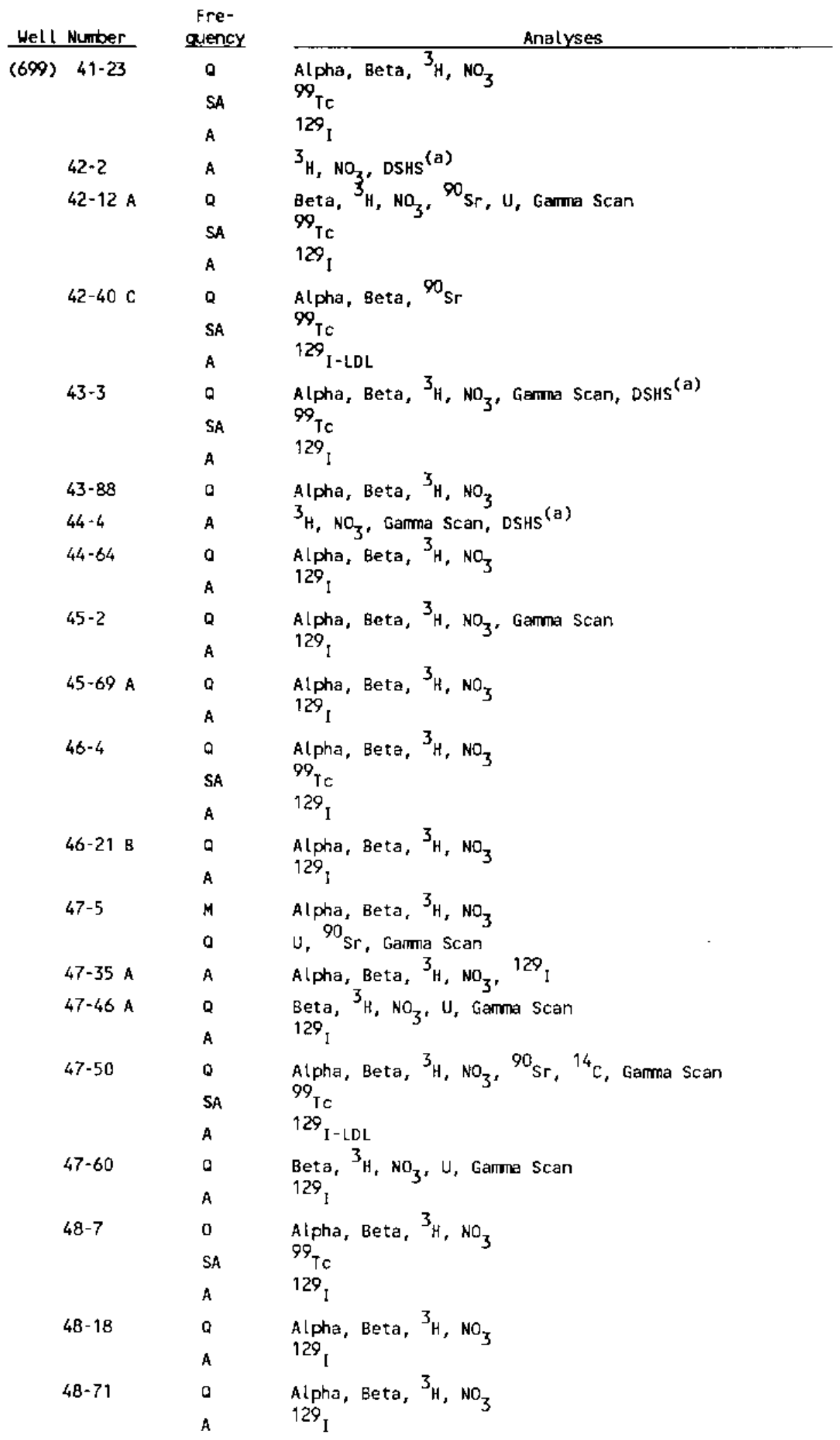

B. 15 


\section{TABLE B.1. (contd)}

\begin{tabular}{|c|c|c|}
\hline \multirow{2}{*}{$\frac{\text { Hell Ninber }}{\text { (699) } 49-13 \mathrm{E}}$} & $\begin{array}{l}\text { Fre- } \\
\text { guency }\end{array}$ & Analyses \\
\hline & $\begin{array}{l}\text { O } \\
\text { SA } \\
\text { A }\end{array}$ & $\begin{array}{l}\text { Alpha, Beta, }{ }^{3} \mathrm{H}, \mathrm{NO}_{3} \\
99_{\mathrm{Tc}} \\
129_{1}\end{array}$ \\
\hline $49-28$ & A & $\begin{array}{l}\text { Alpha, Beta, }{ }^{3} \mathrm{H}, \mathrm{NO}_{3} \\
\mathrm{PZO}_{\mathrm{I}}\end{array}$ \\
\hline $49.55 \mathrm{~A}$ & $\begin{array}{l}\text { Q } \\
\text { SA } \\
\text { A }\end{array}$ & $\begin{array}{l}\text { Beta, }{ }^{3} \mathrm{H}, \mathrm{NO}_{3},{ }^{90} \mathrm{Sr}, \mathrm{U}, \mathrm{Gamma} \text { Scan, Alpha } \\
99_{\mathrm{TC}} \\
129_{\mathrm{I}}\end{array}$ \\
\hline $49-55 \mathrm{~B}$ & $\begin{array}{l}0 \\
\text { SA } \\
\text { A }\end{array}$ & $\begin{array}{l}\text { Alpha, Beta, }{ }^{90} \mathrm{Sr} \\
99 \mathrm{TC} \\
{ }^{129} \mathrm{I}-\text { LDL }\end{array}$ \\
\hline 49.57 & $\begin{array}{l}\text { Q } \\
\text { SA } \\
A\end{array}$ & $\begin{array}{l}\text { Beta, }{ }^{3} \mathrm{H}, \mathrm{NO}_{3},{ }^{90} \mathrm{Sr}, \mathrm{U},{ }^{14} \mathrm{C} \text {, Garma scan } \\
99_{\mathrm{TC}} \\
{ }^{129} \mathrm{C}\end{array}$ \\
\hline $\begin{array}{l}49-79 \\
50-30\end{array}$ & $Q$ & $\begin{array}{l}\text { Alpha, Beta, }{ }^{3} \mathrm{H}, \mathrm{NO}_{3} \\
\text { Alpha, Beta, }{ }_{3} \mathrm{H}_{2} \mathrm{NO}_{3},{ }^{9} \mathrm{Sr}\end{array}$ \\
\hline $50-42$ & A & $\begin{array}{l}\text { Alpha, Beta, }{ }^{3} \mathrm{H}_{1} \mathrm{NO}_{3} \\
129,\end{array}$ \\
\hline $50-45$ & $\begin{array}{l}0 \\
\text { SA } \\
\text { A }\end{array}$ & $\begin{array}{l}\text { Alpha, Beta, }{ }^{90} \mathrm{Sr} \\
99_{\mathrm{TC}} \\
129_{1-L D L}\end{array}$ \\
\hline $50-48 \mathrm{~B}$ & $\begin{array}{l}Q \\
\text { SA } \\
\text { A }\end{array}$ & $\begin{array}{l}\text { Alpha, Beta, } 90_{\mathrm{Sr}} \\
999_{\mathrm{TC}} \\
{ }^{129} \mathrm{I}\end{array}$ \\
\hline $50-53$ & $\begin{array}{l}a \\
\text { SA } \\
\text { A }\end{array}$ & $\begin{array}{l}\text { Alpha, Beta, }{ }^{3} \mathrm{H}, \mathrm{NO}_{3},{ }^{90} \mathrm{Sr},{ }^{16} \mathrm{C} \text {, Garma Scan } \\
99_{\mathrm{TC}} \\
{ }^{129} \text { ! }\end{array}$ \\
\hline $50 \cdot 85$ & $Q$ & Alpha, Beta, ${ }^{3} \mathrm{H}, \mathrm{NO}_{3}$ \\
\hline $51-46$ & $\begin{array}{l}\text { Q } \\
\text { SA }\end{array}$ & $\begin{array}{l}\text { Alpha, Beta, }{ }^{90} \mathrm{Sr} \\
999_{\mathrm{Tc}} \\
129 \mathrm{I}-\mathrm{LDL}\end{array}$ \\
\hline $5 \imath-63$ & $\begin{array}{l}0 \\
A\end{array}$ & $\begin{array}{l}\text { Beta, }{ }^{3} \mathrm{H}_{2} \mathrm{NO}_{3}, \mathrm{U} \\
{ }_{129} \mathrm{I}\end{array}$ \\
\hline $51-75$ & $a$ & Alpha, Beta, ${ }^{3} \mathrm{H}, \mathrm{NO}_{3}$ \\
\hline $52-19$ & Q & $\begin{array}{l}\text { Alpha, Beta, }{ }^{3} \mathrm{H}_{1} \mathrm{NO}_{3} \\
129 \text { ! }\end{array}$ \\
\hline $52-46 \mathrm{~A}$ & $\begin{array}{l}\text { Q } \\
\text { SA } \\
\text { A }\end{array}$ & $\begin{array}{l}\text { Alpha, Beta, }{ }^{90} \mathrm{Sr} \\
99 \mathrm{TC} \\
{ }^{129} 1-\text { LOL }\end{array}$ \\
\hline $52-48$ & $\begin{array}{l}0 \\
\text { SA } \\
A\end{array}$ & $\begin{array}{l}\text { Alpha, Beta, }{ }^{90} \mathrm{Sr} \\
99 \mathrm{TC} \\
{ }^{129} \mathrm{I}-\text { LDL }\end{array}$ \\
\hline $53-50$ & SA & $\begin{array}{l}\text { Alpha, Beta, }{ }^{90} \mathrm{Sr} \\
99 \mathrm{Tc} \\
129\end{array}$ \\
\hline
\end{tabular}


TABLE B.1. (contd)

\begin{tabular}{|c|c|c|c|}
\hline Wel! & Number & $\begin{array}{l}\text { Fre- } \\
\text { quency }\end{array}$ & Analyses \\
\hline$(699)$ & $53-55 A$ & A & $1299_{1}$ \\
\hline & $53-103$ & SA & Alpha, Beta, ${ }^{3} \mathrm{H},{ }^{9} \mathrm{TC}, \mathrm{NO}_{3}$ \\
\hline & & A & $1291-L D L$ \\
\hline & $54-34$ & a & Alpha, Beta, ${ }_{3}^{3},_{3} \mathrm{NO}_{3}$ \\
\hline & $54-45 A$ & 0 & Alpha, Beta, ${ }^{3} \mathrm{H}_{3} \mathrm{NO}_{3}$ \\
\hline & $54-57$ & a & Alpha, Beta, ${ }^{00} \mathrm{Sr}$ \\
\hline & & SA & ${ }^{99}$ TC \\
\hline & & A & 129 I-LOL \\
\hline & $55-40$ & SA & ${ }_{3}^{3} \mathrm{H}_{3} \mathrm{NO}_{3}$ \\
\hline & $55-44$ & SA & ${ }^{3} \mathrm{H}_{3} \mathrm{NO}_{3}$ \\
\hline & $55-50 A(0)$ & 0 & 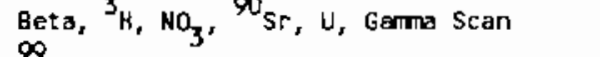 \\
\hline & & SA & ${ }_{170}^{1 \mathrm{TC}}$ \\
\hline & & A & $\int_{1}^{129}$ \\
\hline & $55-50 \mathrm{C}$ & 0 & ${ }_{129}^{3}{ }_{1} \mathrm{NO}_{3}, \mathrm{U}$, Gamma Scan \\
\hline & $55-50 \mathrm{D}$ & A & ${ }_{12 \mathrm{H}_{1}}^{3} \mathrm{NO}_{3}, \mathrm{U}$ \\
\hline & $55-70$ & A & Beta, ${ }^{3} \mathrm{H}_{2} \mathrm{NO}_{3}, \mathrm{U}$ \\
\hline & $55-76$ & a & \\
\hline & $55-89$ & SA & Beta, ${ }^{3} \mathrm{H}, \mathrm{NO}_{3},{ }^{9} \mathrm{Sr}, \mathrm{U}$, Gamma Scan \\
\hline & $56-43$ & A & $\begin{array}{l}\text { Alpha, Beta, }{ }^{3} \mathrm{H}, \mathrm{NO}_{3} \\
129\end{array}$ \\
\hline & $56-53$ & $\begin{array}{l}0 \\
\text { SA } \\
\text { A }\end{array}$ & $\begin{array}{l}\text { Alpha, Beta, }{ }^{90} \mathrm{Sr} \\
99 \mathrm{TC} \\
{ }^{129} \mathrm{~T}-\mathrm{LDL}\end{array}$ \\
\hline & $57-29 \mathrm{~A}$ & a & Alpha, Beta, ${ }^{3} \mathrm{H}, \mathrm{NO}_{3}$ \\
\hline & $59-58$ & $\begin{array}{l}0 \\
\text { SA } \\
\text { A }\end{array}$ & $\begin{array}{l}\text { Beta, }{ }^{3}, \mathrm{NO}_{3},{ }^{90} \mathrm{Sr}, \mathrm{u} \\
99_{\mathrm{TC}} \\
{ }^{129} \mathrm{I}\end{array}$ \\
\hline & $59-80 \mathrm{~B}$ & 0 & $\mathrm{NO}_{3}$ \\
\hline & $60-57$ & 0 & $\begin{array}{l}\text { Beta, }{ }^{3} \mathrm{H}, \mathrm{NO}_{3}, \mathrm{U} \\
129_{1}\end{array}$ \\
\hline & $60-60$ & $\begin{array}{l}\text { SA } \\
A\end{array}$ & $\begin{array}{l}\text { Beta, }{ }^{3} \mathrm{H}, \mathrm{NO}_{3},{ }^{90} \mathrm{Sr}, \mathrm{U},{ }^{99} \mathrm{TC}, \text { Gamma Scan } \\
{ }^{129} \mathrm{I}\end{array}$ \\
\hline & $61-37$ & 0 & Alpha, Beta, ${ }_{3}^{3} \mathrm{H}, \mathrm{NO}_{3}$ \\
\hline & $61-41$ & 0 & Al pha, Beta, ${ }_{3}^{3}{ }_{90}{ }^{N_{3}}$ \\
\hline & $61-62$ & $\begin{array}{l}0 \\
\text { SA }\end{array}$ & $\begin{array}{l}\text { Beta, }{ }^{3} \mathrm{H}, \mathrm{NO}_{3},{ }^{90} \mathrm{Sr}, \mathrm{U}, \text { Gamma Scan } \\
{ }_{\mathrm{IC}}\end{array}$ \\
\hline & & A & $\left.{ }^{129}\right]_{3}$ \\
\hline & $61-66$ & $\begin{array}{l}0 \\
S A\end{array}$ & $\begin{array}{l}\text { Beta, }{ }^{3} \mathrm{H}, \mathrm{NO}_{3},{ }^{90} \mathrm{Sr}, \mathrm{U}, \text { Gamma Scan } \\
{ }^{99} \mathrm{TC}\end{array}$ \\
\hline & & A & ${ }^{129} 1$ \\
\hline & $62-31$ & a & $\mathrm{NO}_{3}$ \\
\hline
\end{tabular}


IABLE B.1. (contd)

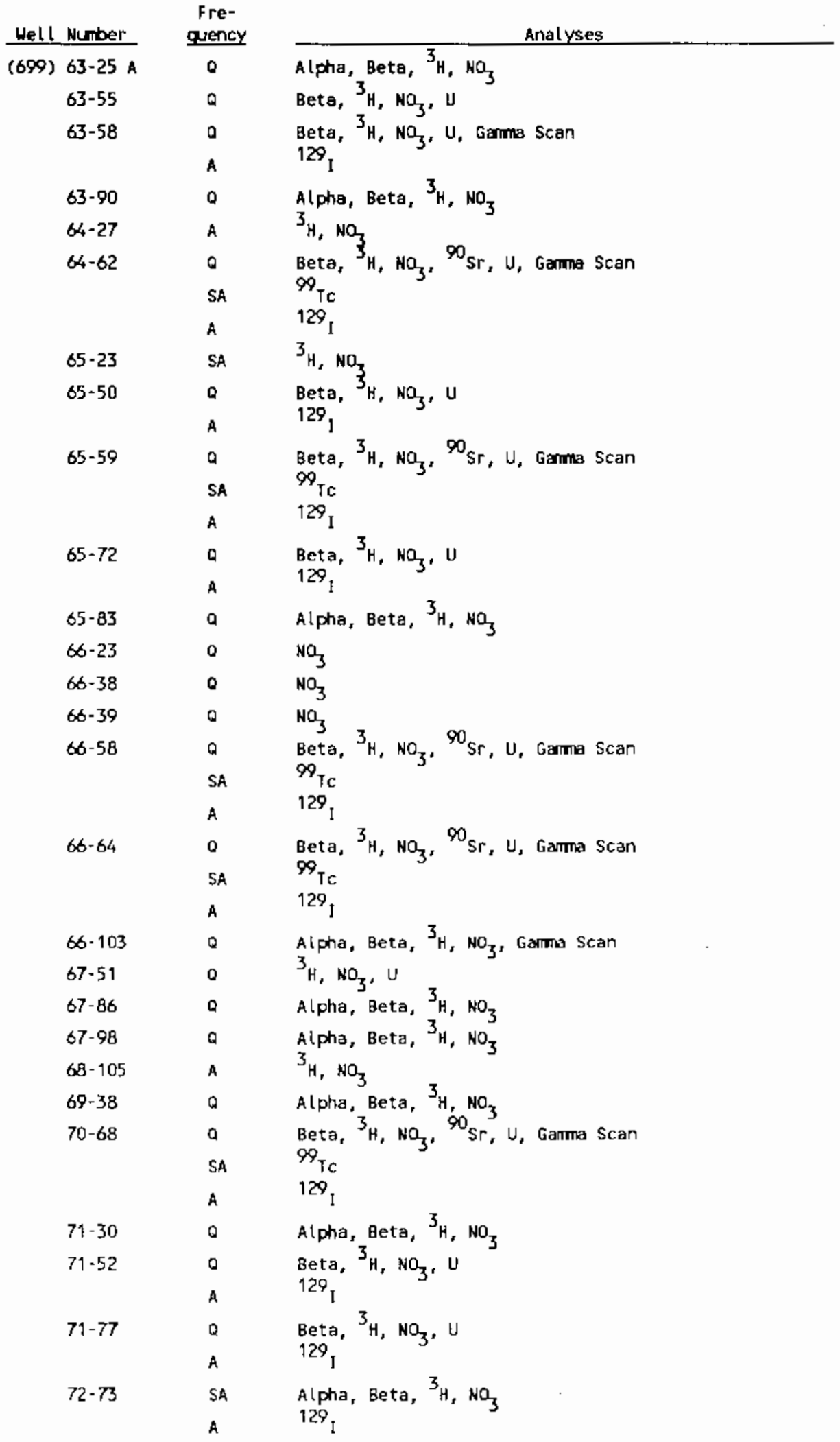


TABLE B.1. (contd)

\begin{tabular}{|c|c|c|c|}
\hline \multicolumn{2}{|c|}{ Well Number } & $\begin{array}{l}\text { Fre- } \\
\text { guency }\end{array}$ & Analyses \\
\hline \multirow[t]{22}{*}{ (699) } & $72-88$ & Q & Alpha, Beta, ${ }^{3} \mathrm{H}_{2} \mathrm{NO}_{3}$ \\
\hline & $\pi-92$ & SA & Alpha, Beta, ${ }^{3} \mathrm{H}, \mathrm{NO}_{3}$ \\
\hline & $73-61$ & 0 & Beta, ${ }^{3} \mathrm{H}, \mathrm{NO}_{3}, \mathrm{U}$ \\
\hline & & A & $129_{1}$ \\
\hline & $74-44$ & 0 & Alpha, Beta, ${ }^{3} \mathrm{H}, \mathrm{NO}_{3}$ \\
\hline & $77-36$ & A & $3_{H}, \mathrm{NO}_{3}$ \\
\hline & $77-54$ & 0 & Alpha, Beta, ${ }^{3} \mathrm{H}, \mathrm{NO}_{3}$ \\
\hline & $78-62$ & SA & $\mathrm{NO}_{3}$ \\
\hline & $80-43 P$ & SA & Alpha, Beta, ${ }^{3} \mathrm{H}, \mathrm{NO}_{3},{ }^{99} \mathrm{TC}$ \\
\hline & $80-430$ & SA & 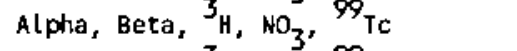 \\
\hline & $80-43 \mathrm{R}$ & SA & Alpha, Beta, ${ }^{3} \mathrm{H}, \mathrm{NO}_{3},{ }^{99} \mathrm{Tc}$ \\
\hline & $80-43 \mathrm{~s}$ & SA & 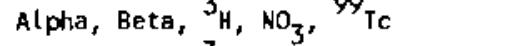 \\
\hline & $8 i-58$ & $Q$ & Alpha, Beta, ${ }^{3} \mathrm{H}, \mathrm{NO}_{3}$ \\
\hline & $83-47$ & SA & ${ }^{3} \mathrm{H},{ }^{90} \mathrm{Sr},{ }^{99} \mathrm{TC}$ \\
\hline & $84-35 A(0)$ & A & ${ }^{3} \mathrm{H}, \mathrm{NO}_{3}$ \\
\hline & $87-55$ & Q & Aloha, Beta, ${ }_{3}^{\mathrm{H}}, \mathrm{NO}_{3}$ \\
\hline & $89-35$ & $Q$ & Alpha, Beta, ${ }_{3}^{3}, \mathrm{NO}_{3}$ \\
\hline & $90-45$ & Q & Algha, Beta, ${ }_{-3}^{3}, \mathrm{NO}_{3}$ \\
\hline & $96-49$ & $Q$ & Alpha, Beta, ${ }^{3} \mathrm{H}, \mathrm{NO}_{3}, \mathrm{U},{ }^{14} \mathrm{C},{ }^{63} \mathrm{Ni}$ \\
\hline & $97-43$ & Q & Alpha, Beta, ${ }_{3}^{\mathrm{H}}, \mathrm{NO}_{3}$ \\
\hline & $97-51 \mathrm{~A}$ & a & 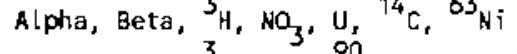 \\
\hline & $101-48 \mathrm{~B}$ & SA & $\begin{array}{l}\text { A lpha, Beta, }{ }^{3} \mathrm{H}, \mathrm{NO}_{3},{ }^{90} \mathrm{Sr} \\
99_{\mathrm{Tc}}\end{array}$ \\
\hline
\end{tabular}


TABLE B.2. Wells to Be Sampled for Hazardous Chemicals During the First Two Quarters of 1988

We11 Number
$199-\mathrm{B} 3-1$
$199-\mathrm{F} 5-4$
$199-\mathrm{F} 7-1$
$199-\mathrm{N}-28$
$199-\mathrm{N}-29(\mathrm{a})$
$299-\mathrm{E} 13-5$
$299-\mathrm{E} 13-14$
$299-\mathrm{E} 17-1$
$299-\mathrm{E} 17-6$
$299-\mathrm{E} 25-19$
$299-\mathrm{E} 25-21$
$299-\mathrm{E} 25-23$
$299-\mathrm{E} 25-24$
$299-\mathrm{E} 28-21$
$299-W 14-6$
$299-W 19-9$
$299-W 19-15$
$299-W 19-16$
$299-W 23-10$
$499-\mathrm{S} 1-7 \mathrm{C}$
$499-\mathrm{S} 1-8 \mathrm{~A}$
$499-\mathrm{S} 1-8 \mathrm{~B}$
$699-\mathrm{S} 3-\mathrm{E} 12$
$699-10-\mathrm{E} 12$
$699-12-4 D$
$699-14-38$
$699-19-43$
$699-20-39$
$699-24-33$
$699-24-46$
$699-29-78$
$699-32-70 \mathrm{~B}$
$699-32-72$
$699-32-77$
$699-35-66$
$699-36-61 \mathrm{~A}$
$699-37-\mathrm{E} 4$
$699-37-43$
$699-38-70$

We11 Number
$699-39-39$
$699-39-79$
$699-40-62$
$699-41-1$
$699-42-2$
$699-43-3$
$699-44-64$
$699-45-2$
$699-45-42(b)$
$699-46-4$
$699-47-46 A$
$699-47-5$
$699-47-50$
$699-47-60$
$699-49-55 A$
$699-49-57$
$699-49-79$
$699-50-53$
$699-50-85$
$699-55-50 C$
$699-55-76$
$699-65-72$
$699-65-83$
$699-67-86$
$699-70-68$
$699-71-30$
$699-71-52$
$699-71-77$
$699-72-73$
$699-73-61$
$699-74-44$
$699-77-36$
$699-78-62$
$699-81-58(a)$
$699-83-47$
$699-96-49$
$699-97-51 A$
$699-97-43$
$699-101-48 \mathrm{~B}$

(a) Sampled during second quarter by project other than the Site-wide Ground-Water Monitoring Project.

(b) Sampled for Westinghouse during first quarter. 


\section{APPENDIX C}

DATA LISTINGS 


\section{APPENDIX C}

\section{DATA LISTINGS}

This appendix contains tables listing the constituents for which samples taken during the period July through December 1987 had been analyzed and reported by United States Testing Company, Inc. (UST). TabTe C.1 is a key to the constituent and constituent group names used throughout the remaining tables. Some constituents appear more than once in the list in different forms (e.g., NITRATE and HNITRATE are both nitrate ion, but HNITRATE has a higher detection limit). The constituents $U$ and U-CHEM are both total uranium, but in units of $\mathrm{pC} i / \mathrm{L}$ and $\mathrm{ppb}(\mathrm{a})$, respectively. The constituent names CHRDMUM and FCHROMI represent chromium analyses done on unfiltered and filtered samples, respectively.

Each monitoring well sampled on the Hanford Site from July through December 1987 was placed into one of three groups: 1) Site-wide chemical monitoring wells (Tables C.2 and C.3), 2) compliance monitoring wells (Tables C.4 and C.5), and 3) additional Site-wide radiological and nitrate monitoring wells that were not in either of the other two networks (Tables C.6 and C.7).

Most wells were sampled once during each quarter. Some exceptions included a few wells that were sampled monthly, wells that were dropped from the chemical network after three sampling periods, and wells that were sampled semiannually for radiological constituents. Compliance monitoring in the $100-\mathrm{H}$ and 300 Areas is conducted monthly and quarterly, respectively.

Tables C.3, C.5, and $C .7$ contain all chemical results (above detection levels) and radiochemical results (for which the result is at least as large as the counting error). Blank spaces in the tables or constituents missing from the first column indicate that the results did not meet these criteria.

(a) The conversion from $\mathrm{ppb}$ uranium to $\mathrm{pC} i / \mathrm{L}$ uranium is approximately 0.699 for natural uranium. Other conversion factors may be calculated where the isotopic composition has been measured [e.g., Law, Serkowski, and Schatz (1987) report a conversion factor of 0.679 for their data]. 
The letters "NR" in the tables indicate that the analysis was not requested or the results had not yet been reported by the 1 aboratory at the time of writing.

Analyses were performed by UST following approved procedures (EPA 1982) or other standard methods. Specific conductance, $\mathrm{pH}$, and temperature were measured in the field at the time of collection, in accordance with documented procedures. The analytical procedures have been described elsewhere (Jaquish and Mitchel1 1988, Appendix D).

\section{REFERENCES}

EPA. 1982. Test Methods for Evaluating Solid Waste: Physical/Chemical Methods. 2nd ed., SW-846, Washington, D.C.

Jaquish, R. E., and P. J. Mitche11, eds. 1988. Environmental Monitoring at Hanford for 1987. PNL-6464, Pacific Northwest Laboratory, Richland, Washington.

Law, A. G., J. A. Serkowski, and A. L. Schatz. 1987. Results of the Separations Area Ground-Water Monitoring Network for 1986. RHO-RE-SR-87-24P, Rockwe11 Hanford Operations, Richland, Washington. 
TABLE C.1. Key to Constituent and Constituent Group Names Used in Tables C.2, C.3, C.4, C.5, C.6, and C.7

Individual Constituents

\begin{tabular}{|c|c|c|}
\hline Name in Tables & Units & Full Name \\
\hline ALKALIN & $\mathrm{ppb}$ & Total alkalinity in ppb $\mathrm{CaCO}_{3}$ \\
\hline ALPHA & $\mathrm{pCi} / \mathrm{L}$ & Gross alpha \\
\hline$A M-241$ & $\mathrm{pCi} / \mathrm{L}$ & Americium-241 \\
\hline AMMONIU & $\mathrm{ppb}$ & Ammonium ion \\
\hline BETA & $\mathrm{pC} i / L$ & Gross beta \\
\hline BISMUTH & $\mathrm{ppb}$ & Bismuth \\
\hline $\mathrm{Cl} 4$ & $\mathrm{pCi} / \mathrm{L}$ & Carbon-14 \\
\hline C130CCA & $\mathrm{pCi} / \mathrm{L}$ & Cis-1,3-dichloro-cyclohexane \\
\hline CITRUSR & ppb. & Citrus red \\
\hline COLIFRM & $\operatorname{mpn}(\mathrm{a})$ & Coliform bacteria \\
\hline CDNDFLD & umho & Specific conductance \\
\hline CDNDLAB & jmho & Specific conductance, laboratory \\
\hline CYANIDE & $\mathrm{ppb}$ & Cyanide \\
\hline DIOXIN & $\mathrm{ppb}$ & Dioxin \\
\hline ETHYGLY & $\mathrm{ppb}$ & Ethylene glycol \\
\hline HNITRAT & ppb & Nitrate, high detection limit \\
\hline HYMEPRA & $\mathrm{ppb}$ & 2-Hydroxy-2-methylpropanoic acid \\
\hline$I-129$ & $\mathrm{pCi} / \mathrm{L}$ & Iodine-129 \\
\hline LFLUORD & $\mathrm{ppb}$ & Fluoride, low DL \\
\hline LHYDRAZ & $\mathrm{ppb}$ & Hydrazine, low detection limit \\
\hline LPHENOL & $\mathrm{ppb}$ & Phenol, low detection limit \\
\hline NC23HC & $\mathrm{ppb}$ & Tricosane \\
\hline NC24HC & $\mathrm{ppb}$ & Tetracosane \\
\hline $\mathrm{NC} 25 \mathrm{HC}$ & ppb & Pentacosane \\
\hline $\mathrm{NC} 26 \mathrm{HC}$ & ppb & Hexacosane \\
\hline $\mathrm{NC} 27 \mathrm{HC}$ & $\mathrm{ppb}$ & Heptacosane \\
\hline $\mathrm{NC} 28 \mathrm{HC}$ & $\mathrm{ppb}$ & Octacosane \\
\hline$N I-63$ & $\mathrm{pCi} / \mathrm{L}$ & Nickel-63 \\
\hline PERCHLO & $\mathrm{ppb}$ & Perchlorate \\
\hline PHF IELD & & $\mathrm{pH}$ (measured in field) \\
\hline $\mathrm{PH}-\mathrm{LAB}$ & & ph, latoratory \\
\hline RADIUM & $\mathrm{pCi} / \mathrm{L}$ & Radium-226 \\
\hline SR 90 & $\mathrm{pCi} / \mathrm{L}$ & Strontium-90 \\
\hline STYRENE & $\mathrm{ppb}$ & Styrene \\
\hline SULFIDE & $\mathrm{ppb}$ & Sulfide \\
\hline T4CLCHL & $\mathrm{ppb}$ & Trans-4-chloro-cyclohexanol \\
\hline TC & $\mathrm{ppb}$ & Total carbon \\
\hline TC-99 & $\mathrm{pCi} / \mathrm{L}$ & Technetium-99 \\
\hline TDS & $\mathrm{ppb}$ & Total dissolved solids \\
\hline TOC & $\mathrm{ppb}$ & Total organic carbon \\
\hline TOX & $\mathrm{ppb}$ & Total organic halogen \\
\hline TOXLDL & ppb & Total organic halogen, low detection limit \\
\hline
\end{tabular}

(a) $\operatorname{mpn}=$ most probable number. 
IABLE C.1. (contd)

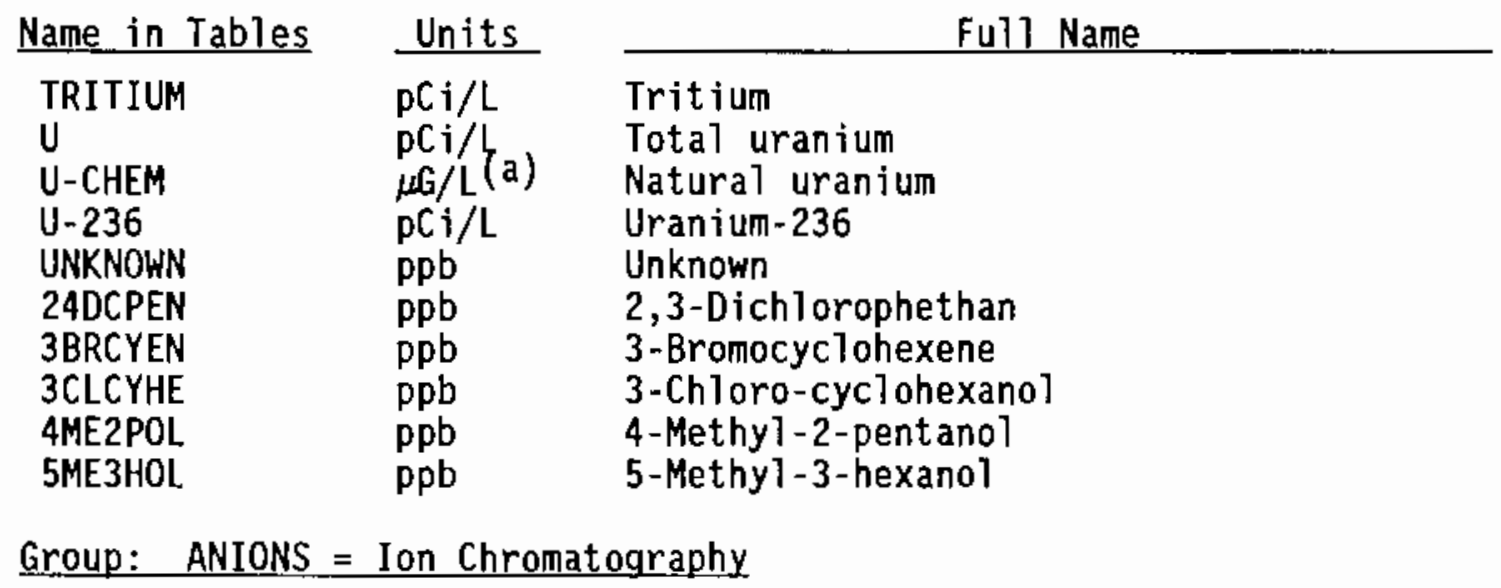

\begin{tabular}{|c|c|c|}
\hline Name in Tables & Units & Full Name \\
\hline $\begin{array}{l}\text { CHLORID } \\
\text { FLUORID } \\
\text { NITRATE } \\
\text { PHOSPHA } \\
\text { SULFATE }\end{array}$ & $\begin{array}{l}\mathrm{ppb} \\
\mathrm{ppb} \\
\mathrm{ppb} \\
\mathrm{ppb} \\
\mathrm{ppb}\end{array}$ & $\begin{array}{l}\text { Chloride } \\
\text { Fluoride } \\
\text { Nitrate } \\
\text { Phosphate } \\
\text { Sulfate }\end{array}$ \\
\hline \multicolumn{3}{|c|}{ Group: ANIONSE = Anions Enhanced (ANIONS plus the following) } \\
\hline Name in Tables & Units & Full Name \\
\hline $\begin{array}{l}\text { BROMIDE } \\
\text { NITRITE }\end{array}$ & $\begin{array}{l}\mathrm{ppb} \\
\mathrm{ppb}\end{array}$ & $\begin{array}{l}\text { Bromide } \\
\text { Nitrite }\end{array}$ \\
\hline
\end{tabular}

(a) $\mu \mathrm{g} / \mathrm{L}=\mathrm{ppb}$. 
TABLE C. . . (contd)

Group: DIRAOIN $=$ Direct Aqueous Injection

Name in Tables

Units

Fu11 Name

$\begin{array}{lll}\text { 1,1-DIM } & \mathrm{ppb} & \text { 1,1-dimethylhydrazine } \\ \text { l,2-DIM } & \mathrm{ppb} & \text { 1,2-dimethylhydrazine } \\ \text { ACETILE } & \mathrm{ppb} & \text { Acetonitrile } \\ \text { ACRYIDE } & \mathrm{ppb} & \text { Acrylamide } \\ \text { ALLYLAL } & \mathrm{ppb} & \text { Allyl alcohol } \\ \text { CHLACET } & \mathrm{ppb} & \text { Chloroacetaldehyde } \\ \text { CHLORAL } & \mathrm{ppb} & \text { Chloral } \\ \text { CHLPROP } & \mathrm{ppb} & \text { 3-chloropropionitrile } \\ \text { CYANBRO } & \mathrm{ppb} & \text { Cyanogen bromide } \\ \text { CYANCHL } & \mathrm{ppb} & \text { Cyanogen chloride } \\ \text { CYANOGN } & \mathrm{ppb} & \text { Cyanogen } \\ \text { DICPROP } & \mathrm{ppb} & \text { Dichloropropanol } \\ \text { ETHCARB } & \mathrm{ppb} & \text { Ethyl carbamate } \\ \text { ETHCYAN } & \mathrm{ppb} & \text { Ethyl cyanide } \\ \text { ETHOXID } & \mathrm{ppb} & \text { Ethylene oxide } \\ \text { FLUOROA } & \mathrm{ppb} & \text { Fluoroacetic acid } \\ \text { GLYCIDY } & \mathrm{ppb} & \text { Glycidylaldehyde } \\ \text { HYORAZI } & \mathrm{ppb} & \text { Hydrazine } \\ \text { ISOBUTY } & \mathrm{ppb} & \text { Isobutylalcohol } \\ \text { METZINE } & \mathrm{ppb} & \text { Methyl hydrazine } \\ \text { PARALDE } & \mathrm{ppb} & \text { Paraldehyde } \\ \text { PROPYLA } & \mathrm{ppb} & \text { N-propylamine } \\ \text { PROPYNO } & \mathrm{ppb} & \text { 2-propyn-1-ol }\end{array}$

Group: GAMHA = Gamma Scan

Name in Tables Units

Fu11 Name

$\begin{array}{lll}\text { C0-60 } & \text { PCi/L } & \text { Cobalt }-60 \\ \mathrm{CS}-137 & \mathrm{PC} \mathrm{i} / \mathrm{L} & \text { Cesium-137 } \\ \mathrm{RU}-103 & \mathrm{PC} \mathrm{i} / \mathrm{L} & \text { Ruthenium-103 } \\ \mathrm{RU}-106 & \mathrm{pC} \mathrm{i} / \mathrm{L} & \text { Ruthenium-106 } \\ \mathrm{SB}-125 & \mathrm{pC} \mathrm{i} / \mathrm{L} & \text { Antimony-125 }\end{array}$

Group: HERB $=$ Herbicides

Name in Tables Units

Fu11 Name

$\begin{array}{lll}2,4,5 T P & \mathrm{ppb} & 2,4,5-\mathrm{TP} \text { silvex } \\ 2,4-\mathrm{D} & \mathrm{ppb} & 2,4-\mathrm{D}\end{array}$


TABLE C.1. (contd)

Group: HERBE $=$ Herbicides, enhanced (HERB plus the following)

\begin{tabular}{|c|c|c|}
\hline Name in Tables & Units & Fu11 Name \\
\hline $2,4,5-\mathrm{T}$ & $\mathrm{ppb}$ & $2,4,5-T$ \\
\hline Group: ICPMT = & CP Metals & \\
\hline Name in Tables & Units & Full Name \\
\hline ALUMNUM & $\mathrm{ppb}$ & Aluminum, unfiltered \\
\hline BARIUM & $\mathrm{ppb}$ & Barium, unfiltered \\
\hline CADMIUM & $\mathrm{ppb}$ & Cadmium, unfi] tered \\
\hline CALCIUM & $\mathrm{ppb}$ & Calcium, unfiltered \\
\hline CHROMUM & $\mathrm{ppb}$ & Chromium, unfiltered \\
\hline COPPER & $\mathrm{ppb}$ & Copper, unfiltered \\
\hline IRON & $\mathrm{ppb}$ & Iron, unfiltered \\
\hline MAGNES & $\mathrm{ppb}$ & Magnesium, unfiltered \\
\hline MANGESE & $\mathrm{ppb}$ & Manganese, unfiltered \\
\hline NICKEL & $\mathrm{ppb}$ & Nickel, unfiltered \\
\hline POTASUM & $\mathrm{ppb}$ & Potassium, unfiltered \\
\hline SILVER & $\mathrm{ppb}$ & Silver, unfiltered \\
\hline SODIUM & $\mathrm{ppb}$ & Sodium, unfiltered \\
\hline VANADUM & $\mathrm{ppb}$ & Vanadium, unfiltered \\
\hline ZINC & $\mathrm{ppb}$ & Zinc, unfiltered \\
\hline
\end{tabular}

Group: $\quad$ ICPMTE $=$ ICP Metals, enhanced (ICPMT plus the following)

\begin{tabular}{|c|c|c|}
\hline Name in Tables & Units & Full Name \\
\hline $\begin{array}{l}\text { ANTIONY } \\
\text { BERYLUM } \\
\text { OSMIUM } \\
\text { STRONUM }\end{array}$ & $\begin{array}{l}\mathrm{ppb} \\
\mathrm{ppb} \\
\mathrm{ppb} \\
\mathrm{ppb}\end{array}$ & $\begin{array}{l}\text { Antimony, unfiltered } \\
\text { Beryllium, unfiltered } \\
\text { Osmium, unfiltered } \\
\text { Strontium, unfiltered }\end{array}$ \\
\hline \multicolumn{3}{|c|}{ Group: ICPMTF = ICP Metals, filtered } \\
\hline Name in Tables & Units & Full Name \\
\hline $\begin{array}{l}\text { FALUMIN } \\
\text { FBARIUM } \\
\text { FCADMIU } \\
\text { FCALCIU } \\
\text { FCHROMI } \\
\text { FCOPPER } \\
\text { FIRON } \\
\text { FMAGNES } \\
\text { FMANGAN } \\
\text { FNICKEL } \\
\text { FPOTASS }\end{array}$ & $\begin{array}{l}\mathrm{ppb} \\
\mathrm{ppb} \\
\mathrm{ppb} \\
\mathrm{ppb} \\
\mathrm{ppb} \\
\mathrm{ppb} \\
\mathrm{ppb} \\
\mathrm{ppb} \\
\mathrm{ppb} \\
\mathrm{ppb} \\
\mathrm{ppb}\end{array}$ & $\begin{array}{l}\text { Aluminum, filtered } \\
\text { Barium, filtered } \\
\text { Cadmium, filtered } \\
\text { Calcium, filtered } \\
\text { Chromium, filtered } \\
\text { Copper, filtered } \\
\text { Iron, filtered } \\
\text { Magnesium, filtered } \\
\text { Manganese, filtered } \\
\text { Nickel, filtered } \\
\text { Potassium, filtered }\end{array}$ \\
\hline
\end{tabular}


TABLE C.1. (contd)

Group: ICPMTF $=$ ICP Metals, filtered (continued)

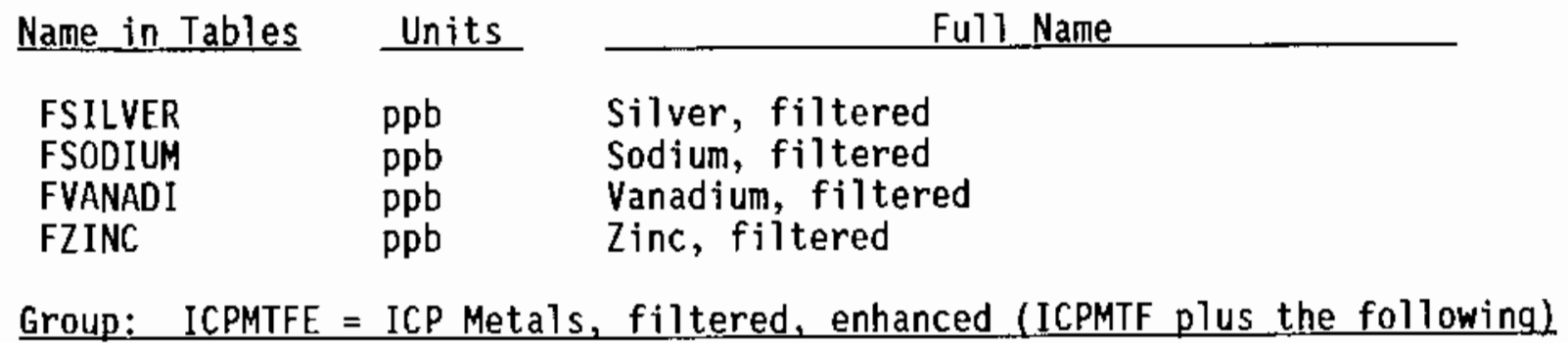

Name in Tables Units

Full Name

$\begin{array}{lll}\text { FANTIMO } & \text { ppb } & \text { Antimony, filtered } \\ \text { FBERYLL } & \text { ppb } & \text { Beryllium, filtered } \\ \text { FOSMIUM } & \text { ppb } & \text { Osmium, filtered } \\ \text { FSTRONT } & \text { ppb } & \text { Strontium, filtered }\end{array}$

Group: OMET $=0$ ther metals

Name in Tables Units

Full Name

$\begin{array}{lll}\text { ARSENIC } & p p b & \text { Arsenic } \\ \text { LEADGF } & p p b & \text { Lead (graphite furnace) } \\ \text { MERCURY } & p p b & \text { Mercury } \\ \text { SELENUM } & p p b & \text { Selenium }\end{array}$

Group: OMETE $=$ Other metals (OMET plus the following)

Name in Tables Units Ful1 Name

THALIUM $\quad p p b \quad$ Thallium

Group: OMEFE $=$ Other metals, filtered

Name in Tables Units

Fu11 Name

FARSENI $\quad \mathrm{ppb} \quad$ Arsenic, filtered

FLEAD $\quad$ ppb Lead, filtered

FMERCUR $\quad \mathrm{ppb} \quad$ Mercury, filtered

FSELENI $\quad \mathrm{ppb}$ Selenium, filtered

Group: OMETFE $=$ Other metals (OMETF plus the following)

Name in Tables Units Full Name

FTHALLI $\quad \mathrm{ppb} \quad$ ThatTium, filtered 
IABLE C.1. (contd)

Group: $\quad$ PCBS $=$ Polychlorinated Biphenyls

Name in Tables Units

Ful1 Name

$\begin{array}{lll}\text { AR1016 } & \mathrm{ppb} & \text { Arochlor } 1016 \\ \text { AR1221 } & \mathrm{ppb} & \text { Arochlor } 1221 \\ \text { AR1232 } & \mathrm{ppb} & \text { Arochlor } 1232 \\ \text { AR1242 } & \mathrm{ppb} & \text { Arochlor } 1242 \\ \text { AR1248 } & \mathrm{ppb} & \text { Arochlor } 1248 \\ \text { AR1254 } & \mathrm{ppb} & \text { Arochlor } 1254 \\ \text { AR1260 } & \mathrm{ppb} & \text { Arochlor } 1260\end{array}$

Group: PEST $=$ Pesticides

Name in Tables Units

$\begin{array}{lll}\text { ENDRIN } & \text { ppb } & \text { Endrin } \\ \text { METHLOR } & p p b & \text { Methoxychlor } \\ \text { TOXAENE } & p p b & \text { Toxaphene } \\ \text { a-BHC } & p p b & \text { Alpha-BHC } \\ \text { b-BHC } & p p b & \text { Beta-BHC } \\ \text { d-BHC } & p p b & \text { Delta-BHC } \\ \text { g-BHC } & p p b & \text { Gamma-BHC }\end{array}$

Group: $\quad$ PESTE $=$ Pesticides, enhanced (PEST plus the following)

Name in Tables Units

Fu11 Name

\begin{tabular}{|c|c|c|}
\hline $\begin{array}{l}\text { ALDRIN } \\
\text { CHLLATE } \\
\text { CHLOANE } \\
\text { DDD } \\
\text { DDE } \\
\text { DDT } \\
\text { DIELRIN } \\
\text { ENDO1 } \\
\text { ENDO2 } \\
\text { HEPTIDE } \\
\text { HEPTLOR }\end{array}$ & $\begin{array}{l}\mathrm{ppb} \\
\mathrm{ppb} \\
\mathrm{ppb} \\
\mathrm{ppb} \\
\mathrm{ppb} \\
\mathrm{ppb} \\
\mathrm{ppb} \\
\mathrm{ppb} \\
\mathrm{ppb} \\
\mathrm{ppb} \\
\mathrm{ppb}\end{array}$ & $\begin{array}{l}\text { Aldrin } \\
\text { Chlorobenzilate } \\
\text { Chlordane } \\
\text { DDD } \\
\text { DDE } \\
\text { DDT } \\
\text { Dieldrin } \\
\text { Endosulfan I } \\
\text { Endosulfan II } \\
\text { Heptchlor epoxide } \\
\text { Heptachlor }\end{array}$ \\
\hline \multicolumn{3}{|c|}{ Group: PHOSPST $=$ Phosphorus } \\
\hline Name in Tables & Units & $\ldots$ \\
\hline $\begin{array}{l}\text { CARBPHT } \\
\text { DIMETHO } \\
\text { DISULFO } \\
\text { METHPAR } \\
\text { PARATHI } \\
\text { TETEPYR }\end{array}$ & $\begin{array}{l}\mathrm{ppb} \\
\mathrm{ppb} \\
\mathrm{ppb} \\
\mathrm{ppb} \\
\mathrm{ppb} \\
\mathrm{ppb}\end{array}$ & $\begin{array}{l}\text { Carbophenothion } \\
\text { Dimethoate } \\
\text { Disulfoton } \\
\text { Methy parathion } \\
\text { Parathion } \\
\text { Tetraethylpyrophosphate }\end{array}$ \\
\hline
\end{tabular}


TABLE C.1. (contd)

Group: $\quad$ PU-ISO $=$ Plutonium Isotopes

\begin{tabular}{llll} 
Name in Tables & Units & & Full Name \\
\cline { 4 - 5 } PU-238 & & & \\
PU39-40 & PC $\mathrm{L}$ & & Plutonium-238 \\
& $\mathrm{pC} / \mathrm{L}$ & & Plutonium-239, Plutonium-240
\end{tabular}

Group: SEMVOL $=$ Semivolatile organics

Name in Tables Units Fu11. Name

12-dben

$1234 \mathrm{TE}$

$1235 T E$

123TRI

13-dben

135TRI

14-dben

HEXACHL

HEXCBEN

KEROSEN

NAPHTHA

PENTCHB

PHENOL

TETRCHB

TRIBUPH

TRICHLB

$\begin{array}{ll}\text { ppb } & 1,2-\text { dichlorobenzene } \\ \text { ppb } & 1,2,3,4 \text {-tetrachlorobenzene } \\ \text { ppb } & 1,2,3,5 \text {-tetrachlorobenzene } \\ \text { ppb } & 1,2,3 \text {-trichlorobenzene } \\ \text { ppb } & 1,3 \text {-dichlorobenzene } \\ \text { ppb } & 1,3,5 \text {-trichlorobenzene } \\ \text { ppb } & 1,4-\text { dichlorobenzene } \\ \text { ppb } & \text { Hexachlorophene } \\ \text { ppb } & \text { Hexachlorobenzene } \\ \text { ppb } & \text { Kerosene } \\ \text { ppb } & \text { Naphthalene } \\ \text { ppb } & \text { Pentachlorobenzene } \\ \text { ppb } & \text { Phenol } \\ \text { ppb } & 1,2,4,5 \text {-tetrachlorobenzene } \\ \text { ppb } & \text { Tributylphosphoric acid } \\ \text { ppb } & 1,2,4-\text { trichlorobenzene }\end{array}$

Group: SEMVOLE = Semivolatile Organics, enhanced (SEMVOL plus the following)

\begin{tabular}{|c|c|c|}
\hline Name in Tables & Units & Ful1 Name \\
\hline 1-napha & $\mathrm{ppb}$ & 1 -naphthylamine \\
\hline 2-napha & $\mathrm{ppb}$ & 2-naphthylamine \\
\hline 24-dchp & $\mathrm{ppb}$ & 2,4-dichlorophenol \\
\hline 24-dint & $\mathrm{ppb}$ & 2,4-dinitrotoluene \\
\hline $245-\operatorname{trp}$ & $\mathrm{ppb}$ & $2,4,5$-trichlorophenol \\
\hline $246-t r p$ & $\mathrm{ppb}$ & $2,4,6$-trichlorophenol \\
\hline 26-dchp & $\mathrm{ppb}$ & 2,6-dichloropheno] \\
\hline 26-dint & $\mathrm{ppb}$ & 2,6-dinitrotoluene \\
\hline ACEFENE & ppb & 2-acetylaminofluorene \\
\hline ACETOPH & ppb & Acetophenone \\
\hline AMI ISOX & $\mathrm{ppb}$ & 5-(aminomethyl)-3-isoxazolol \\
\hline AMINOYL & $\mathrm{ppb}$ & 4-aminobyphenyi \\
\hline AMITROL & ppb & Amitrole \\
\hline ANILINE & $\mathrm{ppb}$ & Aniline \\
\hline ARAMITE & $\mathrm{ppb}$ & Aramite \\
\hline AURAMIN & $p p b$ & Auramine \\
\hline
\end{tabular}


TABLE C.1. (contd)

Group: SEMVOLE = Semivolatile Organics, enhanced (SEMVOL plus the following) (continued)

\begin{tabular}{|c|c|c|}
\hline $\begin{array}{l}\text { BENDICM } \\
\text { BFNDINE }\end{array}$ & ppb & \\
\hline $\begin{array}{l}\text { BENDINE } \\
\text { BENTHOL }\end{array}$ & $\begin{array}{l}\mathrm{ppb} \\
\mathrm{ppb}\end{array}$ & $\begin{array}{l}\text { Benzidine } \\
\text { Benzenethoil }\end{array}$ \\
\hline BENZAAN & $\mathrm{ppb}$ & Benz[a] anthracene \\
\hline BENZBFL & $\mathrm{ppb}$ & Benzo $[b]$ fluoranthene \\
\hline BENZCAC & $\mathrm{ppb}$ & Benz [c] acridine \\
\hline BENZCHL & $\mathrm{ppb}$ & Benzyl chloride \\
\hline BENZJFL & $\mathrm{ppb}$ & Benzo[j]fluoranthene \\
\hline BENZOPY & $\mathrm{ppb}$ & Benzo[a]pyrene \\
\hline BIS2CHE & $\mathrm{ppb}$ & Bis(2-chloroethyl) ether \\
\hline BIS2CHM & $\mathrm{ppb}$ & Bis (2-chloroethoxy) methane \\
\hline BIS2EPH & $\mathrm{ppb}$ & Bis(2-ethylhexy1) phthalate \\
\hline BIS2ETH & $\mathrm{ppb}$ & Bis(2-chloroi sopropyl)ether \\
\hline BROPHEN & $\mathrm{ppb}$ & 4-bromopheny 1 phenyl ether \\
\hline BUTBENP & $\mathrm{ppb}$ & Butyl benzyl phthalate \\
\hline BUTDINP & $\mathrm{ppb}$ & 2-sec-buty $1-4,6$-dinitrophenol \\
\hline CHALETH & $\mathrm{ppb}$ & Chloroalkyl ethers \\
\hline CHLANIL & $\mathrm{ppb}$ & P-chloroaniline \\
\hline CHLCRES & $\mathrm{ppb}$ & P-chloro-m-cresol \\
\hline CHLEPOX & $\mathrm{ppb}$ & 1-chloro-2,3-epoxypropane \\
\hline CHLNAPH & $\mathrm{ppb}$ & 2-chloronaphthalene \\
\hline CHLNAPZ & $\mathrm{ppb}$ & Chlornaphaz ine \\
\hline CHLPHEN & $\mathrm{ppb}$ & 2-chlorophenol \\
\hline CHRYSEN & $\mathrm{ppb}$ & Chrysene \\
\hline CRESOLS & $\mathrm{ppb}$ & cresols \\
\hline CYCHDIN & $\mathrm{ppb}$ & 2-cyclohexy)-4,6-dinitrophenol \\
\hline DIBAEPY & $\mathrm{ppb}$ & Dibenzo [a, e]pyrene \\
\hline DIBAHAC & $\mathrm{ppb}$ & Dibenz $[a, h]$ acridine \\
\hline DIBAHAN & $\mathrm{ppb}$ & Dibenz $[\mathrm{a}, \mathrm{h}]$ anthracene \\
\hline DIBAHPY & $\mathrm{ppb}$ & Dibenzo $[a, h]$ pyrene \\
\hline DIBAIPY & $\mathrm{ppb}$ & Dibenzo $[\mathrm{a}, \mathrm{i}]$ pyrene \\
\hline DIBAJAC & $\mathrm{ppb}$ & Dibenz $[a, j]$ acridine \\
\hline DIBCGCA & $\mathrm{ppb}$ & $7 \mathrm{H}$-dibenzo[c,g]carbazole \\
\hline DIBPHTH & $\mathrm{ppb}$ & Di-n-butyl phthalate \\
\hline DICHBEN & $\mathrm{ppb}$ & $3,3^{\prime}$-dichlorobenzidine \\
\hline DIEPHTH & $\mathrm{ppb}$ & Diethy] phthalate \\
\hline DIHYSAF & $\mathrm{ppb}$ & Dihydrosafrole \\
\hline DIMBENZ & $\mathrm{ppb}$ & 7,12-dimethy] benz [a] anthracene \\
\hline DIMEAMB & $\mathrm{ppb}$ & P-dimethyl aminoazobenzene \\
\hline DIMETHB & $\mathrm{ppb}$ & $3,3^{\prime}$-dimethoxybenzidine \\
\hline DIMEYLB & $\mathrm{ppb}$ & $3,3^{\prime}$-dimethylbenzidine \\
\hline DIMPHAM & $\mathrm{ppb}$ & Alpha, alpha-dimethylphenethyl amine \\
\hline DIMPHEN & $\mathrm{ppb}$ & 2,4-dimethylphenol \\
\hline DIMPHTH & $\mathrm{ppb}$ & Dimethy 1 phthalate \\
\hline DINBENZ & $\mathrm{ppb}$ & Dinitrobenzene \\
\hline DINCRES & $\mathrm{ppb}$ & 4,6-dinitro-o-cresol and salts \\
\hline
\end{tabular}


IABLE C.1. (contd)

\begin{tabular}{|c|c|c|}
\hline Name in Tables & Units & Full Name \\
\hline DINPHEN & ppb & 2,4-dinjtrophenol \\
\hline DIOPHTH & $\mathrm{ppb}$ & Di-n-octyl phthalate \\
\hline DIPHAMI & $\mathrm{ppb}$ & Diphenyl amine \\
\hline DIPHHYD & $\mathrm{ppb}$ & 1,2-diphenylhydrazine \\
\hline DIPRNIT & $\mathrm{ppb}$ & Di-n-propyinitrosamine \\
\hline ETHMETS & $\mathrm{ppb}$ & Ethyl methanesulfonate \\
\hline ETHMINE & $\mathrm{ppb}$ & Ethylene imine \\
\hline FLUORAN & $\mathrm{ppb}$ & Fluoranthene \\
\hline HEXAENE & $\mathrm{ppb}$ & Hexachloropropene \\
\hline HEXCBUT & $\mathrm{ppb}$ & Hexachlorobutadiene \\
\hline HEXCCYC & $\mathrm{ppb}$ & Hexachlorocyclopent adiene \\
\hline HEXCETH & $\mathrm{ppb}$ & Hexachloroethane \\
\hline INDENOP & $\mathrm{ppb}$ & Indeno $(1,2,3$-cd) pyrene \\
\hline ISOSOLE & $\mathrm{ppb}$ & Isosafrole \\
\hline MALHYDR & $\mathrm{ppb}$ & Maleic hydrizide \\
\hline MALOILE & $\mathrm{ppb}$ & Malononitrile \\
\hline MELPHAL & $\mathrm{ppb}$ & Melphalan \\
\hline METACTO & $\mathrm{ppb}$ & 2-methyllactonitrile \\
\hline METAZIR & $\mathrm{ppb}$ & 2 -methylaziridine \\
\hline METBISC & $\mathrm{ppb}$ & $4,4^{\prime}$-methylenebis (2-chloroaniline) \\
\hline METCHAN & $\mathrm{ppb}$ & 3-methylchol anthrene \\
\hline METHAPY & $\mathrm{ppb}$ & Methapyrilene \\
\hline METHIOU & $\mathrm{ppb}$ & Methyl thiouracil \\
\hline METHNYL & $\mathrm{ppb}$ & Methol ony? \\
\hline METMSUL & $\mathrm{ppb}$ & Methyl methanesulfonate \\
\hline METPROP & $\mathrm{ppb}$ & 2-methyl-2-(methyl thio) propionaldehyde- \\
\hline NAPHQUI & $\mathrm{ppb}$ & 1,4-naphthoquinone \\
\hline NICOT IN & $\mathrm{ppb}$ & Nicotinic acid \\
\hline NITBENZ & $\mathrm{ppb}$ & Nitrobenzine \\
\hline NITPHEN & $\mathrm{ppb}$ & 4-nitrophenol \\
\hline NITRAN I & $\mathrm{ppb}$ & P-nitroaniline \\
\hline NITRPYR & $\mathrm{ppb}$ & Nitrosopyrrolidine \\
\hline NITRTOL & $\mathrm{ppb}$ & 5-nitro-o-toluidine \\
\hline NNIBUTY & $\mathrm{ppb}$ & $N$-nitrosodi-n-butylamine \\
\hline NNIDIEA & $\mathrm{ppb}$ & $\mathrm{N}$-nitrosodiethanolamine \\
\hline NNIDIEY & $\mathrm{ppb}$ & $\mathrm{N}-n$ itrosodiethylamine \\
\hline NNIDIME & $\mathrm{ppb}$ & $\mathrm{N}$-nitrosodimethylamine \\
\hline NNIMETH & $\mathrm{ppb}$ & $\mathrm{N}$-nitrosomethylethylamine \\
\hline NNIMORP & $\mathrm{ppb}$ & $\mathrm{N}$-nitrosomorphol ine \\
\hline NNINICO & $\mathrm{ppb}$ & 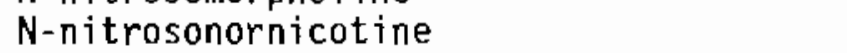 \\
\hline NNIPIPE & $\mathrm{ppb}$ & $\mathrm{N}$-n itrosopiperidine \\
\hline NNIURET & $\mathrm{ppb}$ & $\mathrm{N}-n$ itroso-N-methylurethane \\
\hline NNIVINY & $\mathrm{ppb}$ & $\mathrm{N}$-nitrosomethylvinylamine \\
\hline OTOL.HYD & $\mathrm{ppb}$ & 0 -toluidine hydrochloride \\
\hline PBENZQU & $\mathrm{ppb}$ & $\mathrm{P}$ benzoquinone \\
\hline PENTCHN & $\mathrm{ppb}$ & Pentachloronitrobenzene \\
\hline $\begin{array}{l}\text { PENTCHP } \\
-\end{array}$ & $p p b$ & Pentachlorophenol \\
\hline
\end{tabular}


IABLE C.1. (contd)

\begin{tabular}{|c|c|c|}
\hline Name in Tables & Units & Full Name \\
\hline PHENINE & $\mathrm{ppb}$ & Phenylenediamine \\
\hline PHENTIN & $\mathrm{ppb}$ & Phenacetin \\
\hline PHTHEST & $\mathrm{ppb}$ & Phthalic acid esters \\
\hline PICOLIN & $\mathrm{ppb}$ & 2-picoline \\
\hline PRONIDE & $\mathrm{ppb}$ & Pronamide \\
\hline RESERPI & $\mathrm{ppb}$ & Reserpine \\
\hline RESORCI & $\mathrm{ppb}$ & Resorcinol \\
\hline SAFROL & $\mathrm{ppb}$ & Safrol \\
\hline STRYCHN & $\mathrm{ppb}$ & Strychnine \\
\hline SYMTRIN & $\mathrm{ppb}$ & Sym-trinitrobenzene \\
\hline TETRCHP & $\mathrm{ppb}$ & $2,3,4,6$-tetrachlorophenol \\
\hline THIONOX & $\mathrm{ppb}$ & Thiofanox \\
\hline THIURAM & $\mathrm{ppb}$ & Thiuram \\
\hline TOLUDIA & $\mathrm{ppb}$ & Toluenediamine \\
\hline TRIPHOS & $\mathrm{ppb}$ & $0,0,0$-triethyl phosphorothioate \\
\hline TRISPHO & $\mathrm{ppb}$ & Tris (2,3-dibromopropyl) phosphate \\
\hline WARFRIN & $\mathrm{ppb}$ & Warfarin \\
\hline
\end{tabular}

\begin{tabular}{|c|c|c|}
\hline Name in Tables & Units & Full Name \\
\hline ACETREA & $\mathrm{ppb}$ & 1-acetyl-2-thiourea \\
\hline CHLOREA & $\mathrm{ppb}$ & $1-(0-c h l o r o p h e n y 1)$ thiourea \\
\hline DIETRO & $\mathrm{ppb}$ & Diethylstilbesterol \\
\hline ETHYREA & $\mathrm{ppb}$ & Ethylenethiourea \\
\hline NAPHREA & $\mathrm{ppb}$ & 1-naphthyl-2-thiourea \\
\hline PHENREA & $\mathrm{ppb}$ & $\mathrm{N}$-phenyl thiourea \\
\hline THIOURA & $\mathrm{ppb}$ & Thiourea \\
\hline
\end{tabular}

Name in Tables Units

Full Name

$\begin{array}{lll}U 234 & \mathrm{pC} \mathrm{C} / \mathrm{L} & \text { Uranium-234 } \\ \mathrm{U} 235 & \mathrm{pC} \mathrm{i} / \mathrm{L} & \text { Uranium-235 } \\ \mathrm{U} 238 & \mathrm{pC} i / \mathrm{L} & \text { Uranium-238 }\end{array}$


Group: $\quad$ VOLORG = Volatile organics

\begin{tabular}{|c|c|c|}
\hline Name in Tables & Unjts & Full Name \\
\hline $1,1,1-\mathrm{T}$ & $\mathrm{ppb}$ & $1,1,1$-trichloroethane \\
\hline $1,1,2-T$ & $\mathrm{ppb}$ & $1,1,2$-trichloroethane \\
\hline CHLFORM & ppb & Chloroform \\
\hline HEXONE & ppb & Hexone \\
\hline M-XYLE & ppb & Xylene-m \\
\hline METHONE & $\mathrm{ppb}$ & Methyl ethyl ketone \\
\hline METHYCH & ppb & Methylene chioride \\
\hline OPXYLE & ppb & Xylene-o,p \\
\hline PERCENE & ppb & Perchloroethylene \\
\hline TETRANE & ppb & Tetrachloromethane (carbon tetrachloride) \\
\hline TRICENE & $\mathrm{ppb}$ & Trichloroethylene (1,2-trichloroethene) \\
\hline \multicolumn{3}{|c|}{ VOLORGE $=$ Volatile Organics, enhanced (VOLORG plus the following) } \\
\hline Name in Tables & Units & Full Name \\
\hline $1,1-0 I C$ & $\mathrm{ppb}$ & 1,1-dichloroethane \\
\hline $1,2-\mathrm{DIC}$ & ppb & 1,2 -dichloroethane \\
\hline $1112-t c$ & ppb & $1,1,1,2$-tetrachlorethane \\
\hline $1122-t c$ & ppb & $1,1,2,2$-tetrachlorethane \\
\hline $123-\operatorname{trp}$ & $\mathrm{ppb}$ & $1,2,3$-trichloropropane \\
\hline ACROLIN & $\mathrm{ppb}$ & Acrolein \\
\hline ACRYILE & $\mathrm{ppb}$ & Acrylonitrile \\
\hline BENZENE & $\mathrm{ppb}$ & Benzene \\
\hline BISTHER & $\mathrm{ppb}$ & Bis(chloromethyl) ether \\
\hline BROMONE & $\mathrm{ppb}$ & Bromoacetone \\
\hline BROMORM & $\mathrm{ppb}$ & Bromoform \\
\hline CARBIDE & $\mathrm{ppb}$ & Carbon disulfide \\
\hline CHLBENZ & $\mathrm{ppb}$ & Chlorobenzene \\
\hline CHLTHER & $\mathrm{ppb}$ & 2-chloroethyl vinyl ether \\
\hline CHMTHER & ppb & Chloromethyl methyl ether \\
\hline CROTONA & $\mathrm{ppb}$ & Crotonaldehyde \\
\hline DIBRCHL & $\mathrm{ppb}$ & 1,2-dibromo-3-ch1 oropropane \\
\hline DIBRETH & $\mathrm{ppb}$ & 1,2 -dibromoethane \\
\hline DIBRMET & $\mathrm{ppb}$ & Dibromomethane \\
\hline DIBUTEN & ppb & 1,4-dichloro-2-butene \\
\hline DICDIFM & ppb & Dichlorodifluoromethane \\
\hline DICETHY & ppb & 1,1-dichloroethylene \\
\hline DICPANE & ppb & 1,2-dichloropropane \\
\hline DICPENE & $\mathrm{ppb}$ & 1,3-dichloropropene \\
\hline DIETHY & $\mathrm{ppb}$ & Diethylarsine \\
\hline DIOXANE & $\mathrm{ppb}$ & Dioxane \\
\hline ETHMETH & ppb & Ethyl methacrylate \\
\hline FORMALN & ppb & Formal in \\
\hline HYDRSUL & ppb & Hydrogen sulfide \\
\hline IODOMET & $\mathrm{ppb}$ & Iodomethane \\
\hline METACRY & ppb & Methyl methacrylate \\
\hline
\end{tabular}


IABLE C.1. (contd)

\begin{tabular}{|c|c|c|}
\hline Name in Tables & Units & Full Name \\
\hline METHACR & $\mathrm{ppb}$ & Methacrylonitrile \\
\hline METHBRO & $\mathrm{ppb}$ & Methyl bromide \\
\hline METHCHL & $\mathrm{ppb}$ & Methyl chloride \\
\hline METHTHI & $\mathrm{ppb}$ & Methanethio] \\
\hline NNDIEHY & $\mathrm{ppb}$ & $\mathrm{N}, \mathrm{N}$-diethylhydrazine \\
\hline PENTACH & $\mathrm{ppb}$ & Pentachloroethane \\
\hline PYRIDIN & $\mathrm{ppb}$ & Pyridine \\
\hline TOLUENE & $\mathrm{ppb}$ & Toluene \\
\hline TRANDCE & $\mathrm{ppb}$ & Trans-1,2-dichloroethene \\
\hline TRCMEOL & $\mathrm{ppb}$ & Trichloromethanethiol \\
\hline TRCMFLM & $\mathrm{ppb}$ & Trichloromonofluoromethane \\
\hline TRCPANE & $\mathrm{ppb}$ & Trichloropropane \\
\hline VINYIDE & $\mathrm{ppb}$ & Vinyl chloride \\
\hline
\end{tabular}


TABLE C.2. Constituents Analyzed for in Ground-Water Samples from Site-Wide Chemical Monitoring Wells

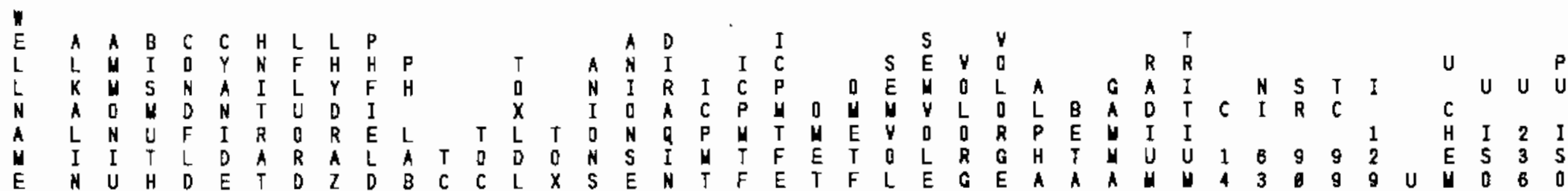

\begin{tabular}{|c|c|c|c|c|c|c|c|c|c|c|c|c|c|c|c|c|c|c|c|c|c|c|c|c|c|c|c|c|c|c|c|c|}
\hline 199-B3-1 & $x$ & $x$ & & $x$ & $x$ & $x$ & $x$ & & $x$ & $x$ & $x$ & $x$ & $x$ & $x$ & $x$ & $x$ & $x$ & $x$ & $x$ & $x$ & $x$ & $x$ & $x$ & $x$ & $x$ & & & $x$ & & & & \\
\hline $199-84-1$ & $x$ & $x$ & $x$ & $x$ & & & $x$ & & $x$ & $x$ & $x$ & $x$ & $x$ & $x$ & & $x$ & $x$ & $x$ & & $x$ & $x$ & $x$ & $x$ & $x$ & $x$ & & & $x$ & & & & \\
\hline $199-84-4$ & $x$ & $x$ & $x$ & $x$ & & & $x$ & & $x$ & $x$ & $x$ & $x$ & $x$ & $x$ & & $x$ & $x$ & $x$ & & $x$ & $x$ & $x$ & $x$ & $x$ & $x$ & & & $x$ & $x$ & & & \\
\hline $199-89-1$ & $x$ & $x$ & $x$ & $x$ & $x$ & & $x$ & $x$ & $x$ & $x$ & $x$ & $x$ & $x$ & $x$ & & $x$ & $x$ & $x$ & $x$ & $x$ & $x$ & $x$ & $x$ & $x$ & $x$ & & & & & & & \\
\hline $199-D 2-5$ & $x$ & $x$ & $x$ & $x$ & & & $x$ & $x$ & $x$ & $x$ & $x$ & $x$ & $x$ & $x$ & & $x$ & $x$ & $x$ & $x$ & $x$ & $x$ & $x$ & $x$ & $x$ & $x$ & & & $x$ & & & & \\
\hline $199-05-12$ & $x$ & $x$ & $x$ & $x$ & $x$ & & $x$ & & $x$ & $x$ & $x$ & $x$ & $x$ & $x$ & & $x$ & $x$ & $x$ & & $x$ & $x$ & $x$ & $x$ & $x$ & $x$ & & & $x$ & $x$ & & $x$ & $x$ \\
\hline $199-188-3$ & $x$ & $x$ & $x$ & $x$ & & & $x$ & & $x$ & $x$ & $x$ & $x$ & $x$ & $x$ & & $x$ & $x$ & $x$ & & $x$ & $x$ & $x$ & $x$ & $x$ & $x$ & & & & & & & \\
\hline $199-\mathrm{F} 5-1$ & $x$ & $x$ & $x$ & $x$ & $x$ & & $x$ & & $x$ & $x$ & $x$ & $x$ & $x$ & $x$ & $x$ & $x$ & $x$ & $x$ & $x$ & $x$ & $x$ & $x$ & $x$ & $x$ & $x$ & & & & & & $x$ & \\
\hline $199-\mathrm{F} 5-4$ & $x$ & $x$ & & $x$ & $x$ & $x$ & $x$ & & $x$ & $x$ & $x$ & $x$ & $x$ & $x$ & $x$ & $x$ & $x$ & $x$ & $x$ & $x$ & $x$ & $x$ & $x$ & $x$ & $x$ & & & & & & $x$ & \\
\hline $199-\mathrm{F} 5-6$ & $x$ & $x$ & $x$ & $x$ & & & $x$ & & $x$ & $x$ & $x$ & $x$ & $x$ & $x$ & & $x$ & $x$ & $x$ & & $x$ & $x$ & $x$ & $x$ & $x$ & $x$ & & & & & & $x$ & \\
\hline $199-F /-1$ & $x$ & $x$ & & $x$ & $x$ & & $x$ & $x$ & $x$ & $x$ & $x$ & $x$ & $x$ & $x$ & $x$ & $x$ & $x$ & $x$ & $x$ & $x$ & $x$ & $x$ & $x$ & $x$ & $x$ & & & & & & $x$ & \\
\hline $199-F 8-1$ & $x$ & $x$ & $x$ & $x$ & $x$ & & $x$ & & $x$ & $x$ & $x$ & $x$ & $x$ & $x$ & $x$ & $x$ & $x$ & $x$ & $x$ & $x$ & $x$ & $x$ & $x$ & $x$ & $x$ & & & $x$ & $x$ & & $x$ & \\
\hline $199-K-11$ & $x$ & $x$ & $x$ & $x$ & $x$ & & $x$ & $x$ & $x$ & $x$ & $x$ & $x$ & $x$ & $x$ & & $x$ & $x$ & $x$ & $x$ & $x$ & $x$ & $x$ & $x$ & $x$ & $x$ & & & & & & $x$ & \\
\hline $199-K-19$ & $x$ & $x$ & $x$ & $x$ & & & $x$ & $x$ & $x$ & $x$ & $x$ & $x$ & $x$ & $x$ & & $x$ & $x$ & $x$ & $x$ & $x$ & $x$ & $x$ & $x$ & $x$ & $x$ & & & & & & $x$ & \\
\hline $199-\mathrm{X}-29$ & $x$ & $x$ & $x$ & $x$ & & & $x$ & & $x$ & $x$ & $x$ & $x$ & $x$ & $x$ & & $x$ & $x$ & $x$ & & $x$ & $x$ & $x$ & $x$ & $x$ & $x$ & & & & & & $x$ & \\
\hline $199-K-22$ & $x$ & $x$ & $x$ & $x$ & $x$ & & $x$ & & $x$ & $x$ & $x$ & $x$ & $x$ & $x$ & $x$ & $x$ & $x$ & $x$ & $x$ & $x$ & $x$ & $x$ & $x$ & $x$ & $x$ & & & & & & $x$ & \\
\hline $199-\mathrm{K}-77$ & $x$ & $x$ & $x$ & $x$ & $x$ & & $x$ & & $x$ & $x$ & $x$ & $x$ & $x$ & $x$ & $x$ & $x$ & $x$ & $x$ & $x$ & $x$ & $x$ & $x$ & $x$ & $x$ & $x$ & & & & & & $x$ & \\
\hline $199-K-28$ & $x$ & $x$ & $x$ & $x$ & & & $x$ & & $x$ & $x$ & $x$ & $x$ & $x$ & $x$ & & $x$ & $x$ & $x$ & & $x$ & $x$ & $x$ & $x$ & $x$ & $x$ & & & & & & $x$ & \\
\hline $199-K-29$ & $x$ & $x$ & $x$ & $x$ & $x$ & & $x$ & & $x$ & $x$ & $x$ & $x$ & $x$ & $x$ & $x$ & $x$ & $x$ & $x$ & $x$ & $x$ & $x$ & $x$ & $x$ & $x$ & $x$ & & & & & & $x$ & \\
\hline $199-K-39$ & $x$ & $x$ & $x$ & $x$ & & & $x$ & & $x$ & $x$ & $x$ & $x$ & $x$ & $x$ & & $x$ & $x$ & $x$ & & $x$ & $x$ & $x$ & $x$ & $x$ & $x$ & & & & & & $x$ & \\
\hline $199-\mathrm{N}-6$ & $x$ & $x$ & $x$ & $x$ & & & $x$ & & $x$ & $x$ & $x$ & $x$ & $x$ & $x$ & & $x$ & $x$ & $x$ & & $x$ & $x$ & $x$ & $x$ & & & & & & & & & \\
\hline $199-N-28$ & $x$ & $x$ & $x$ & $x$ & $x$ & & $x$ & $x$ & $x$ & $x$ & $x$ & $x$ & $x$ & $x$ & $x$ & $x$ & $x$ & $x$ & $x$ & $x$ & $x$ & $x$ & $x$ & $x$ & $x$ & & & $x$ & & & & \\
\hline $199-N-29$ & $x$ & $x$ & $x$ & $x$ & $x$ & & $x$ & & $x$ & $x$ & $x$ & $x$ & $x$ & $x$ & $x$ & $x$ & $x$ & $x$ & $x$ & $x$ & $x$ & $x$ & $x$ & $x$ & $x$ & $x$ & $x$ & $x$ & $x$ & $x$ & & \\
\hline $299-E 13-5$ & $x$ & $x$ & & $x$ & $x$ & & $x$ & & $x$ & $x$ & $x$ & $x$ & $x$ & $x$ & $x$ & $x$ & $x$ & $x$ & $x$ & $x$ & $x$ & $x$ & $x$ & $x$ & $x$ & & & $x$ & & & $x$ & $x$ \\
\hline $299-\mathrm{E} 13-14$ & $x$ & $x$ & & $x$ & $x$ & & $x$ & & $x$ & $x$ & $x$ & $x$ & $x$ & $x$ & $x$ & $x$ & $x$ & $x$ & $x$ & $x$ & $x$ & $x$ & $x$ & $x$ & $x$ & & & $x$ & & & $x$ & $x$ \\
\hline $299-E 17-1$ & $x$ & $x$ & $x$ & $x$ & $x$ & $x$ & $x$ & $x$ & $x$ & $x$ & $x$ & $x$ & $x$ & $x$ & $x$ & $x$ & $x$ & $x$ & $x$ & $x$ & $x$ & $x$ & $x$ & $x$ & $x$ & & & $x$ & $x$ & $x$ & & \\
\hline 299 -Er7-5 & $x$ & $x$ & $x$ & $x$ & $x$ & $x$ & $x$ & $x$ & $x$ & $x$ & $x$ & $x$ & $x$ & $x$ & $x$ & $x$ & $x$ & $x$ & $x$ & $x$ & $x$ & $x$ & $x$ & $x$ & $x$ & $x$ & & $x$ & $x$ & $x$ & $x$ & \\
\hline
\end{tabular}


TABLE C.2. (contd)

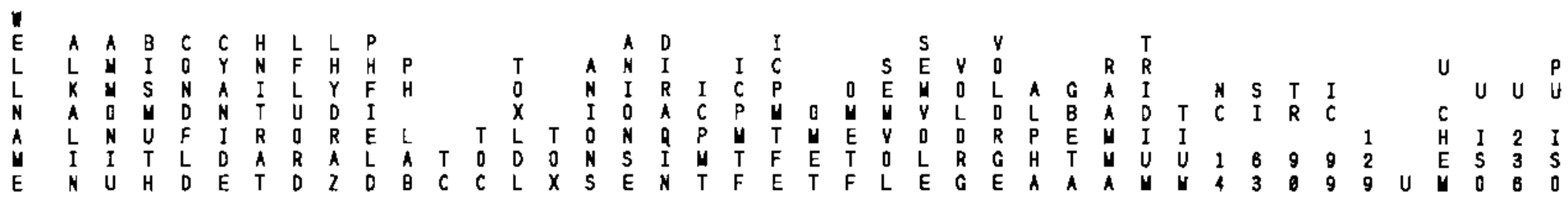

\begin{tabular}{|c|c|c|c|c|c|c|c|c|c|c|c|c|c|c|}
\hline 299-日7-6 & $x$ & $x$ & $x$ & $x$ & $x$ & $x$ & $x$ & $x$ & $x$ & $x$ & $x$ & $x$ & $x$ & \\
\hline 299-E17-9 & $x$ & $x$ & $x$ & $x$ & $x$ & $x$ & $x$ & $x$ & $x$ & $x$ & $x$ & $x$ & $x$ & \\
\hline 299-E24-2 & $x$ & $x$ & $x$ & $x$ & $x$ & $x$ & $x$ & $x$ & $x$ & $x$ & $x$ & $x$ & $x$ & \\
\hline $290-024-8$ & $x$ & $x$ & $x$ & $x$ & $x$ & $x$ & $x$ & & $x$ & $x$ & $x$ & $x$ & $x$ & \\
\hline $290-E 24-12$ & $x$ & $x$ & $x$ & $x$ & $x$ & $x$ & $x$ & $x$ & $x$ & $x$ & $x$ & $x$ & $x$ & \\
\hline 290-E25-18 & $x$ & $x$ & $x$ & $x$ & $x$ & $x$ & $x$ & & $x$ & $x$ & $x$ & $x$ & $x$ & \\
\hline $290-025-19$ & $x$ & $x$ & & $x$ & $x$ & $x$ & $x$ & & $x$ & $x$ & $x$ & $x$ & & \\
\hline $290-05-20$ & $x$ & $x$ & $x$ & $x$ & $x$ & $x$ & $x$ & & $x$ & $x$ & $x$ & $x$ & $x$ & \\
\hline $290-E 25-21$ & $x$ & $x$ & $x$ & $x$ & & $x$ & $x$ & $x$ & $x$ & $x$ & $x$ & $x$ & $x$ & \\
\hline $290-E 25-22$ & $x$ & $x$ & $x$ & $x$ & & $x$ & $x$ & $x$ & $x$ & $x$ & $x$ & $x$ & $x$ & \\
\hline $290-E 26-23$ & $x$ & $x$ & $x$ & $x$ & & $x$ & $x$ & $x$ & $x$ & $x$ & $x$ & $x$ & $x$ & \\
\hline 290-E26-24 & $x$ & $x$ & $x$ & $x$ & $x$ & $x$ & $x$ & $x$ & $x$ & $x$ & $x$ & $x$ & $x$ & \\
\hline 299-E7-5 & $x$ & $x$ & $x$ & $x$ & $x$ & & $x$ & & $x$ & $x$ & $x$ & $x$ & $x$ & \\
\hline $299-\mathrm{E} 20-21$ & $x$ & $x$ & $x$ & $x$ & $x$ & $x$ & $x$ & & $x$ & $x$ & $x$ & $x$ & $x$ & \\
\hline$x-x_{2}-1$ & $x$ & $x$ & & $x$ & $x$ & $x$ & $x$ & $x$ & $x$ & $x$ & $x$ & $x$ & $x$ & \\
\hline $299-23-1$ & $x$ & $x$ & $x$ & $x$ & $x$ & & $x$ & & $x$ & $x$ & $x$ & $x$ & $x$ & \\
\hline $292-\mathrm{CH}^{2}-3$ & $x$ & $x$ & $x$ & $x$ & $x$ & & $x$ & & $x$ & $x$ & $x$ & $x$ & $x$ & \\
\hline $299-133-5$ & $x$ & $x$ & $x$ & $x$ & $x$ & & $x$ & & $x$ & $x$ & $x$ & $x$ & $x$ & \\
\hline $299-\mathrm{EB}^{2}-8$ & $x$ & $x$ & $x$ & $x$ & $x$ & & $x$ & & $x$ & $x$ & $x$ & $x$ & $x$ & \\
\hline $299-E 33-16$ & $x$ & $x$ & & $x$ & $x$ & $x$ & $x$ & & $x$ & $x$ & $x$ & $x$ & $x$ & \\
\hline 299-E33-18 & $x$ & $x$ & $x$ & $x$ & $x$ & & $x$ & & $x$ & $x$ & $x$ & $x$ & $x$ & \\
\hline $299-\mathrm{E3}-21$ & $x$ & $x$ & $x$ & $x$ & $x$ & & $x$ & & $x$ & $x$ & $x$ & $x$ & $x$ & \\
\hline $299-\mathrm{E}^{2}-24$ & $x$ & $x$ & $x$ & $x$ & $x$ & & $x$ & & $x$ & $x$ & $x$ & $x$ & $x$ & \\
\hline $299-E 4-1$ & $x$ & $x$ & & $x$ & $x$ & & $x$ & $x$ & $x$ & $x$ & $x$ & $x$ & $x$ & \\
\hline $299-19-4$ & $x$ & $x$ & $x$ & $x$ & $x$ & & $x$ & & $x$ & $x$ & $x$ & $x$ & $x$ & \\
\hline $290-110-8$ & $x$ & $x$ & $x$ & $x$ & $x$ & $x$ & $x$ & & $x$ & $x$ & $x$ & $x$ & $x$ & \\
\hline $299-10-9$ & $x$ & $x$ & $x$ & $x$ & $x$ & $x$ & $x$ & & $x$ & $x$ & $x$ & $x$ & $\mathrm{x}$ & \\
\hline
\end{tabular}

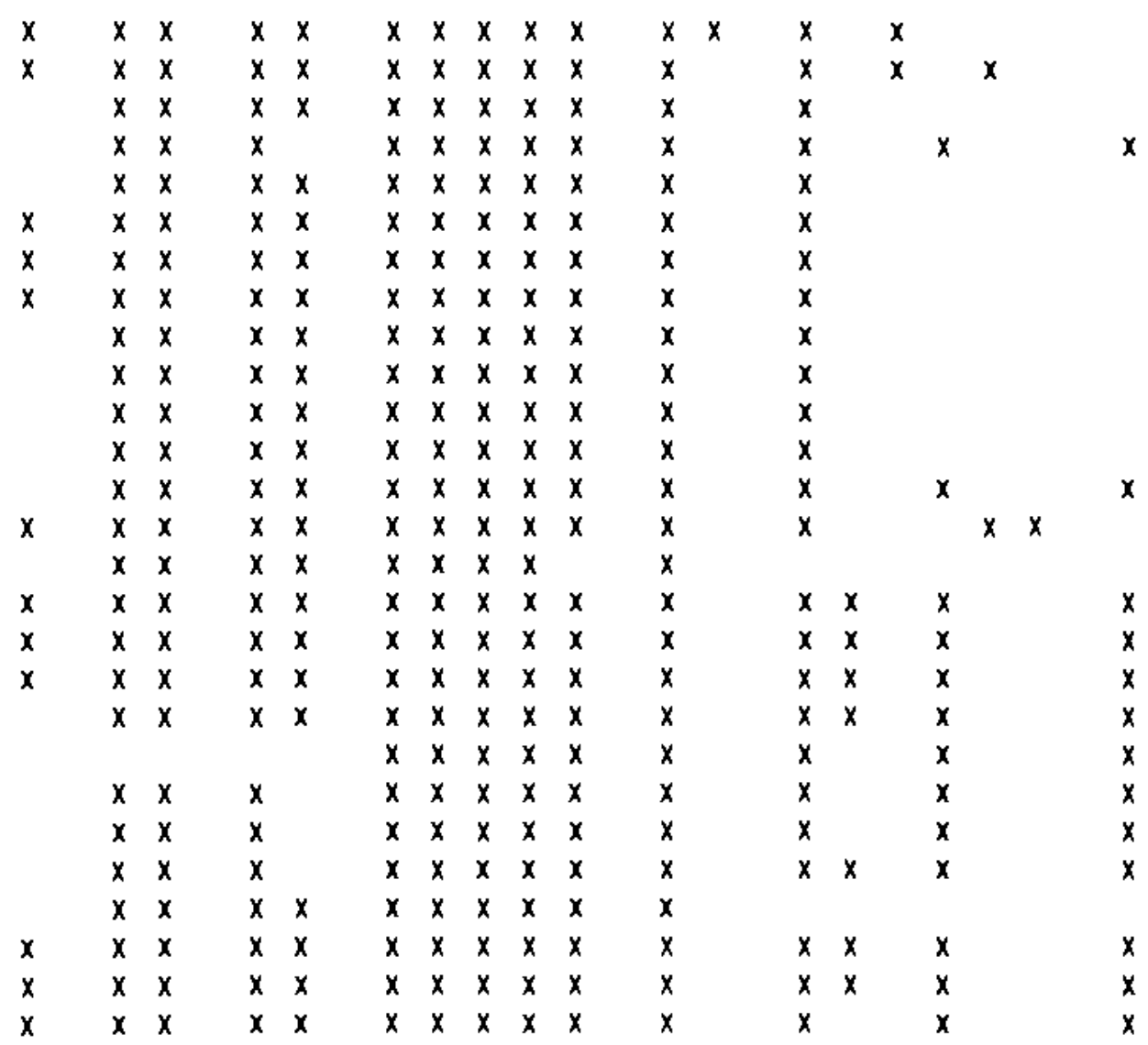




\section{TABLE C.2. (contd)}

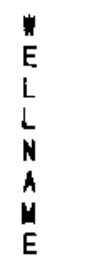

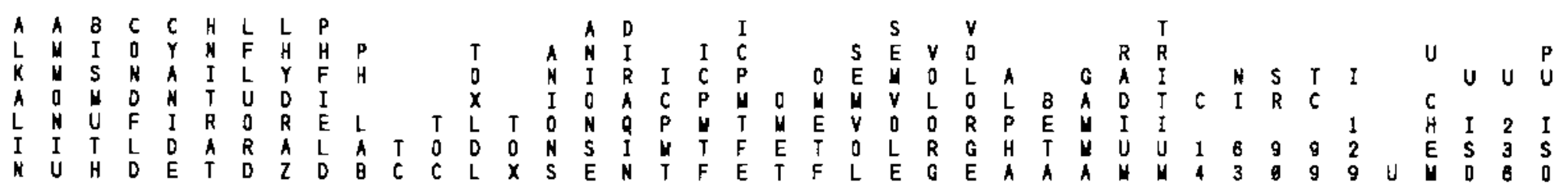

\begin{tabular}{|c|c|c|c|c|c|c|c|c|c|c|c|c|c|c|c|c|c|c|c|c|c|c|c|c|c|c|c|c|c|c|c|c|c|c|c|c|c|}
\hline $299-114-2$ & $x$ & $x$ & $x$ & $x$ & $x$ & & $x$ & & $x$ & $x$ & $x$ & $x$ & $x$ & $x$ & & $x$ & $x$ & $x$ & & $x$ & $x$ & & $x$ & $x$ & $x$ & $x$ & $x$ & & $x$ & $x$ & & & $x$ & & & & $x$ \\
\hline $299-714-5$ & $x$ & $x$ & $x$ & $x$ & $x$ & $x$ & $x$ & & $x$ & $x$ & $x$ & $x$ & $x$ & $x$ & & $x$ & $x$ & $x$ & & $x$ & $x$ & & $x$ & $x$ & $x$ & $x$ & $x$ & & $x$ & $x$ & & & $x$ & & & & $x$ \\
\hline $299-\pi 14-6$ & $x$ & $x$ & $x$ & $x$ & $x$ & $x$ & $x$ & & $x$ & $x$ & $x$ & $x$ & $x$ & $x$ & & $x$ & $x$ & $x$ & & $x$ & $x$ & & $x$ & $x$ & $x$ & $x$ & $x$ & & $x$ & $x$ & & & $x$ & & & & $x$ \\
\hline $299-\pi 15-4$ & $x$ & $x$ & $x$ & $x$ & $x$ & $x$ & $x$ & & $x$ & $x$ & $x$ & $x$ & $x$ & $x$ & & $x$ & $x$ & $x$ & & $x$ & $x$ & & $x$ & $x$ & $x$ & $x$ & $x$ & & $x$ & $x$ & & & $x$ & & & & $x$ \\
\hline $299-715-16$ & $x$ & $x$ & $x$ & $x$ & $x$ & $x$ & $x$ & $x$ & $x$ & $x$ & $x$ & $x$ & $x$ & $x$ & & $x$ & $x$ & $x$ & & $x$ & $x$ & & $x$ & $x$ & $x$ & $x$ & $x$ & & $x$ & & & & & & & & $x$ \\
\hline 292-T15-11 & $x$ & $x$ & $x$. & $x$ & $x$ & $x$ & $x$ & $x$ & $x$ & $x$ & $x$ & $x$ & $x$ & $x$ & & $x$ & $x$ & $x$ & & $x$ & $x$ & & $x$ & $x$ & $x$ & $x$ & $x$ & & $x$ & $x$ & & & & & & & $x$ \\
\hline 299-118-15 & $x$ & $x$ & & $x$ & $x$ & $x$ & $x$ & & $x$ & $x$ & $x$ & $x$ & $x$ & $x$ & & $x$ & $x$ & $x$ & & $x$ & $x$ & & $x$ & $x$ & $x$ & $x$ & $x$ & & $x$ & & & & & $x$ & $x$ & & \\
\hline $299-19-3$ & $x$ & $x$ & & $x$ & $x$ & $x$ & $x$ & & $x$ & $x$ & $x$ & $x$ & $x$ & $x$ & & $x$ & $x$ & $x$ & $x$ & $x$ & $x$ & & $x$ & $x$ & $x$ & $x$ & $x$ & & $x$ & $x$ & $x$ & $x$ & & $x$ & $x$ & $x$ & \\
\hline $299-719-5$ & $x$ & $x$ & $x$ & $x$ & $x$ & $x$ & $x$ & & $x$ & $x$ & $x$ & $x$ & $x$ & $x$ & & & $x$ & $x$ & & $x$ & $x$ & & $x$ & $x$ & $x$ & $x$ & $x$ & & $x$ & & & & $x$ & & & & \\
\hline $299-119-9$ & $x$ & $x$ & $x$ & $x$ & $x$ & $x$ & $x$ & & $x$ & $x$ & $x$ & $x$ & $x$ & $x$ & & $x$ & $x$ & $x$ & & $x$ & $x$ & & $x$ & $x$ & $x$ & $x$ & $x$ & & $x$ & $x$ & $x$ & $x$ & & $x$ & $x$ & & \\
\hline 299--119-11 & $x$ & $x$ & $x$ & $x$ & $x$ & $x$ & $x$ & & $x$ & $x$ & $x$ & $x$ & $x$ & $x$ & & $x$ & $x$ & $x$ & & $x$ & $x$ & & $x$ & $x$ & $x$ & $x$ & $x$ & & $x$ & $x$ & $x$ & $x$ & & $x$ & $x$ & $x$ & \\
\hline $299-19-13$ & $x$ & $x$ & $x$ & $x$ & $x$ & $x$ & $x$ & & $x$ & $x$ & $x$ & $x$ & $x$ & $x$ & & $x$ & $x$ & $x$ & & $x$ & $x$ & & $x$ & $x$ & $x$ & $x$ & $x$ & & $x$ & $x$ & & & & $x$ & $x$ & & \\
\hline $299-19-15$ & $x$ & $x$ & $x$ & $x$ & $x$ & $x$ & $x$ & & $x$ & $x$ & $x$ & $x$ & $x$ & $x$ & $x$ & $x$ & $x$ & $x$ & & $x$ & $x$ & & $x$ & $x$ & $x$ & $x$ & $x$ & & $x$ & $x$ & $x$ & $x$ & & $x$ & $x$ & & \\
\hline $299-119-16$ & $x$ & $x$ & $x$ & $x$ & $x$ & $x$ & $x$ & & $x$ & $x$ & $x$ & $x$ & $x$ & $x$ & $x$ & $x$ & $x$ & $x$ & & $x$ & $x$ & & $x$ & $x$ & $x$ & $x$ & $x$ & & $x$ & $x$ & $x$ & $x$ & & $x$ & $x$ & & \\
\hline $290-119-20$ & $x$ & $x$ & & $x$ & $x$ & $x$ & $x$ & & $x$ & $x$ & $x$ & $x$ & $x$ & $x$ & & $x$ & $x$ & $x$ & $x$ & $x$ & $x$ & $x$ & $x$ & $x$ & $x$ & $x$ & $x$ & $x$ & $x$ & $x$ & $x$ & & & $x$ & & & \\
\hline $299-\pi 19-24$ & $x$ & $x$ & & $x$ & $x$ & $x$ & $x$ & & $x$ & $x$ & $x$ & $x$ & $x$ & $x$ & & $x$ & $x$ & $x$ & $x$ & $x$ & $x$ & $x$ & $x$ & $x$ & $x$ & $x$ & $x$ & $x$ & $x$ & $x$ & $x$ & & & $x$ & $x$ & $x$ & $x$ \\
\hline $295-122-12$ & $x$ & $x$ & & $x$ & $x$ & $x$ & $x$ & & $x$ & $x$ & $x$ & $x$ & $x$ & $x$ & & & & & & & $x$ & & $x$ & $x$ & $x$ & $x$ & $x$ & & $x$ & $x$ & & & $x$ & & & & $x$ \\
\hline $299-122-20$ & $x$ & $x$ & & $x$ & $x$ & & $x$ & & $x$ & $x$ & $x$ & $x$ & $x$ & $x$ & & $x$ & & & & & $x$ & & $x$ & $x$ & $x$ & $x$ & $x$ & & $x$ & $x$ & $x$ & $x$ & $x$ & & & & $x$ \\
\hline $299-122-22$ & $x$ & $x$ & $x$ & $x$ & $x$ & $x$ & $x$ & & $x$ & $x$ & $x$ & $x$ & $x$ & $x$ & & & $x$ & $x$ & & $x$ & & & $x$ & $x$ & $x$ & $x$ & $x$ & & $x$ & $x$ & & & & $x$ & & & \\
\hline $299-122-26$ & $x$ & $x$ & $x$ & $x$ & $x$ & & $x$ & & $x$ & $x$ & $x$ & $x$ & $x$ & $x$ & & & $x$ & $x$ & & $x$ & $x$ & & $x$ & $x$ & $x$ & $x$ & $x$ & & $x$ & $x$ & $x$ & & $x$ & & & & $x$ \\
\hline $299-1123-10$ & $x$ & $x$ & $x$ & $x$ & $x$ & $x$ & $x$ & & $x$ & $x$ & $x$ & $x$ & $x$ & $x$ & & $x$ & $x$ & $x$ & & $x$ & $x$ & & $x$ & $x$ & $x$ & $x$ & $x$ & & $x$ & $x$ & & & & $x$ & $x$ & & \\
\hline 299-\%27-1 & $x$ & $x$ & $x$ & $x$ & $x$ & $x$ & $x$ & & $x$ & $x$ & $x$ & $x$ & $x$ & $x$ & & $x$ & $x$ & $x$ & & $x$ & $x$ & & $x$ & $x$ & $x$ & $x$ & $x$ & & $x$ & $x$ & & & & $x$ & & & \\
\hline $499-\$ 1-7 C$ & $x$ & $x$ & & $x$ & $x$ & & $x$ & $x$ & $x$ & $x$ & $x$ & $x$ & $x$ & $x$ & & & & & & & $x$ & & $x$ & $x$ & $x$ & $x$ & $x$ & & $x$ & & & & & & & & \\
\hline 499-51-84 & $x$ & $x$ & $x$ & $x$ & $x$ & & $x$ & $x$ & $x$ & $x$ & $x$ & $x$ & $x$ & $x$ & & & $x$ & $x$ & & $x$ & $x$ & & $x$ & $x$ & $x$ & $x$ & $x$ & & $x$ & & & & & & & & \\
\hline $499-S I-8 B$ & $x$ & $x$ & & & $x$ & $x$ & $x$ & & & $x$ & $x$ & $x$ & $x$ & $x$ & & $x$ & $x$ & $x$ & & $x$ & $x$ & & $x$ & $x$ & $x$ & $x$ & $x$ & & $x$ & & & & & & & & \\
\hline $699-2-3$ & $x$ & $x$ & $x$ & $x$ & $x$ & & $x$ & & $x$ & $x$ & $x$ & $x$ & $x$ & $x$ & & & $x$ & $x$ & & $x$ & & & $x$ & $x$ & $x$ & $x$ & $x$ & & $x$ & & & & & & & & \\
\hline $699-2-334$ & $x$ & $x$ & $x$ & $x$ & & & $x$ & & $x$ & $x$ & $x$ & $x$ & $x$ & $x$ & & & $x$ & $x$ & & $x$ & $x$ & & $x$ & $x$ & $x$ & $x$ & & & $x$ & & & & & & & & \\
\hline
\end{tabular}


TABLE C.2. (contd)

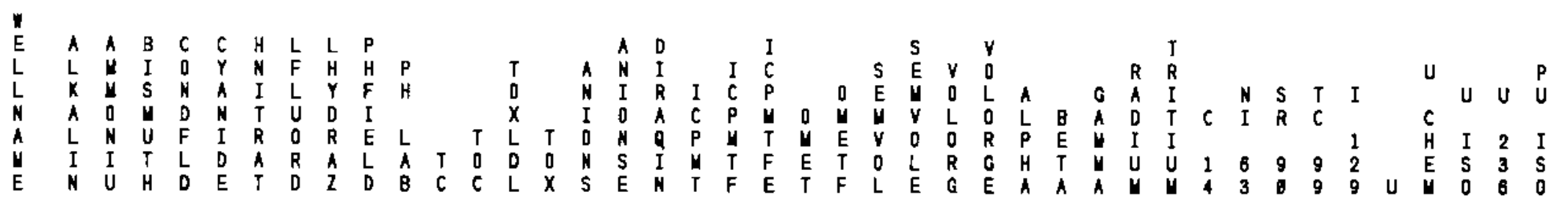

\begin{tabular}{|c|c|c|c|c|c|c|c|c|c|c|c|c|c|c|c|c|c|c|c|c|c|c|c|c|c|c|c|c|c|c|c|}
\hline \multirow[t]{2}{*}{$699-0-25$} & \multirow{2}{*}{$\begin{array}{l}x \\
x\end{array}$} & \multirow{2}{*}{$\begin{array}{l}x \\
x\end{array}$} & \multirow{2}{*}{$\begin{array}{l}x \\
x\end{array}$} & \multirow{2}{*}{$\begin{array}{l}x \\
x\end{array}$} & \multicolumn{2}{|l|}{$x$} & \multirow{2}{*}{$\begin{array}{l}x \\
x\end{array}$} & \multirow{2}{*}{$\begin{array}{l}x \\
x\end{array}$} & \multirow{2}{*}{$\begin{array}{l}x \\
x\end{array}$} & \multirow{2}{*}{$\begin{array}{l}x \\
x\end{array}$} & \multirow{2}{*}{$\begin{array}{l}x \\
x\end{array}$} & \multirow{2}{*}{$\begin{array}{l}x \\
x\end{array}$} & \multirow{2}{*}{$\begin{array}{l}x \\
x\end{array}$} & $x$ & \multicolumn{2}{|r|}{$x$} & $x$ & $x$ & \multirow{2}{*}{$\begin{array}{l}x \\
x\end{array}$} & \multirow{2}{*}{$\begin{array}{l}x \\
x\end{array}$} & \multirow{2}{*}{$\begin{array}{l}x \\
x\end{array}$} & $x$ & $x$ & $x$ & $x$ & & & & & & \\
\hline & & & & & $x$ & & & & & & & & & $x$ & $x$ & $x$ & $x$ & $x$ & & & & $x$ & $x$ & & $x$ & & & & & & \\
\hline BS-10-E12 & $x$ & $x$ & & $x$ & $x$ & $x$ & $x$ & & $x$ & $x$ & $x$ & $x$ & $x$ & $x$ & $x$ & $x$ & $x$ & $x$ & $x$ & $x$ & $x$ & $x$ & $x$ & & $x$ & & & & & & \\
\hline $690-15-28$ & $x$ & $x$ & $x$ & $x$ & $x$ & & $x$ & & $x$ & $x$ & $x$ & $x$ & $x$ & $x$ & & $x$ & $x$ & $x$ & & $x$ & $x$ & $x$ & $x$ & $x$ & $x$ & & & & & & \\
\hline $699-19-43$ & $x$ & $x$ & & $x$ & $x$ & $x$ & $x$ & & $x$ & $x$ & $x$ & $x$ & $x$ & $x$ & $x$ & $x$ & $x$ & $x$ & $x$ & $x$ & $x$ & $x$ & $x$ & & $x$ & & & & & & \\
\hline 69-20-EA & $x$ & $x$ & $x$ & $x$ & $x$ & & $x$ & & $x$ & $x$ & $x$ & $x$ & $x$ & $x$ & & $x$ & $x$ & $x$ & & $x$ & $x$ & $x$ & $x$ & $x$ & $x$ & & & & & & \\
\hline $699-20-20$ & $x$ & $x$ & $x$ & $x$ & $x$ & & & & $x$ & $x$ & $x$ & $x$ & $x$ & $x$ & & $x$ & $x$ & $x$ & $x$ & $x$ & $x$ & $x$ & $x$ & $x$ & $x$ & & & & & $x$ & \\
\hline $699-20-39$ & $x$ & $x$ & $x$ & $x$ & $x$ & & $x$ & & $x$ & $x$ & $x$ & $x$ & $x$ & $x$ & $x$ & $x$ & $x$ & $x$ & $x$ & $x$ & $x$ & $x$ & $x$ & & $x$ & & & & & & \\
\hline $699-24-33$ & $x$ & $x$ & $x$ & $x$ & $x$ & & $x$ & $x$ & $x$ & $x$ & $x$ & $x$ & $x$ & $x$ & $x$ & $x$ & $x$ & $x$ & $x$ & $x$ & $x$ & $x$ & $x$ & $x$ & $x$ & & & & & & \\
\hline $699-24-46$ & $x$ & $x$ & & $x$ & $x$ & $x$ & $x$ & & $x$ & $x$ & $x$ & $x$ & $x$ & $x$ & $x$ & $x$ & $x$ & $x$ & $x$ & $x$ & $x$ & $x$ & $x$ & & $x$ & & & & & & \\
\hline $699-20-40$ & $x$ & $x$ & $x$ & $x$ & $x$ & & $x$ & & $x$ & $x$ & $x$ & $x$ & $x$ & $x$ & & $x$ & $x$ & $x$ & $x$ & $x$ & $x$ & $x$ & $x$ & $x$ & $x$ & & & & & & \\
\hline $699-29-4$ & $x$ & $x$ & $x$ & $x$ & $x$ & & $x$ & & $x$ & $x$ & $x$ & $x$ & $x$ & $x$ & & $x$ & $x$ & $x$ & & $x$ & $x$ & $x$ & $x$ & & $x$ & & & & & & \\
\hline $699-29-78$ & $x$ & $x$ & & $x$ & $x$ & $x$ & $x$ & & $x$ & $x$ & $x$ & $x$ & $x$ & $x$ & $x$ & $x$ & $x$ & $x$ & $x$ & $x$ & $x$ & $x$ & $x$ & & $x$ & & & & & & \\
\hline $699-32-22$ & $x$ & $x$ & $x$ & $x$ & $x$ & & $x$ & & $x$ & $x$ & $x$ & $x$ & $x$ & $x$ & & $x$ & $x$ & $x$ & & $x$ & $x$ & $x$ & $x$ & $x$ & $x$ & & & $x$ & $x$ & & \\
\hline $699-32-43$ & $x$ & $x$ & & $x$ & $x$ & & $x$ & $x$ & $x$ & $x$ & $x$ & $x$ & $x$ & $x$ & & & & $x$ & & $x$ & $x$ & $x$ & $x$ & $x$ & $x$ & $\mathbf{x}$ & & $x$ & $x$ & & \\
\hline $699-32-768$ & $x$ & $x$ & & $x$ & $x$ & $x$ & $x$ & & $x$ & $x$ & $x$ & $x$ & $x$ & $x$ & $x$ & $x$ & $x$ & $x$ & $x$ & $x$ & $x$ & $x$ & $x$ & $x$ & $x$ & & & & & $x$ & \\
\hline $699-32-72$ & $x$ & $x$ & & $x$ & $x$ & $x$ & $x$ & & $x$ & $x$ & & $x$ & $x$ & $x$ & $x$ & $x$ & $x$ & $x$ & $x$ & $x$ & $x$ & $x$ & $x$ & $x$ & $x$ & & & & & $x$ & \\
\hline $699-32-\pi$ & $x$ & $x$ & & $x$ & $x$ & $x$ & $x$ & & $x$ & $x$ & $x$ & $x$ & $x$ & $x$ & $x$ & $x$ & $x$ & $x$ & $x$ & $x$ & $x$ & $x$ & $x$ & $x$ & $x$ & & & & & & \\
\hline $699-33-42$ & $x$ & $x$ & $x$ & $x$ & $x$ & & $x$ & & $x$ & $x$ & & & & $x$ & & $x$ & $x$ & $x$ & & & & $x$ & $x$ & $x$ & $x$ & & & & & & \\
\hline $699-33-86$ & $x$ & $x$ & $x$ & $x$ & $x$ & & $x$ & & $x$ & $x$ & $x$ & $x$ & $x$ & $x$ & & $x$ & $x$ & $x$ & $x$ & $x$ & $x$ & $x$ & $x$ & $x$ & $x$ & & & & & & \\
\hline $6 x-34-42$ & $x$ & $x$ & $x$ & $x$ & $x$ & & $x$ & & $x$ & $x$ & $x$ & $x$ & $x$ & $x$ & & $x$ & $x$ & $x$ & $x$ & $x$ & $x$ & $x$ & $x$ & $x$ & $x$ & & & & & & \\
\hline $699-34-51$ & $x$ & $x$ & $x$ & $x$ & $x$ & & $x$ & & $x$ & $x$ & $x$ & $x$ & $x$ & $x$ & & $x$ & $x$ & $x$ & $x$ & $x$ & $x$ & $x$ & $x$ & $x$ & $x$ & & & & & & \\
\hline $699-35-9$ & $x$ & $x$ & $x$ & $x$ & $x$ & & $x$ & $x$ & $x$ & $x$ & $x$ & $x$ & $x$ & $x$ & & $x$ & $x$ & $x$ & $x$ & $x$ & $x$ & $x$ & $x$ & $x$ & $x$ & & & & & & \\
\hline $699-35-66$ & $x$ & $x$ & & $x$ & $x$ & $x$ & $x$ & & $x$ & $x$ & $x$ & $x$ & $x$ & $x$ & $x$ & $x$ & $x$ & $x$ & $x$ & $x$ & $x$ & $x$ & $x$ & $x$ & $x$ & & & & & $x$ & \\
\hline $699-35-70$ & $x$ & $x$ & $x$ & $x$ & $x$ & & $x$ & & $x$ & $x$ & $x$ & $x$ & $x$ & $x$ & & $x$ & $x$ & $x$ & $x$ & $x$ & $x$ & $x$ & $x$ & $x$ & $x$ & $x$ & $x$ & $x$ & $x$ & $x$ & \\
\hline $699-35-784$ & $x$ & $x$ & $x$ & $x$ & $x$ & $x$ & $x$ & & $x$ & $x$ & $x$ & $x$ & $x$ & $x$ & & $x$ & $x$ & $x$ & $x$ & $x$ & $x$ & $x$ & $x$ & $x$ & $x$ & & & & & $x$ & $x$ \\
\hline $699-36-81 k$ & $x$ & $x$ & & $x$ & $x$ & $x$ & $x$ & & $x$ & $x$ & $x$ & $x$ & $x$ & $x$ & $x$ & $x$ & $x$ & $x$ & $x$ & $x$ & $x$ & $x$ & $x$ & & & & & & & & \\
\hline
\end{tabular}


TABLE C.2. (contd)

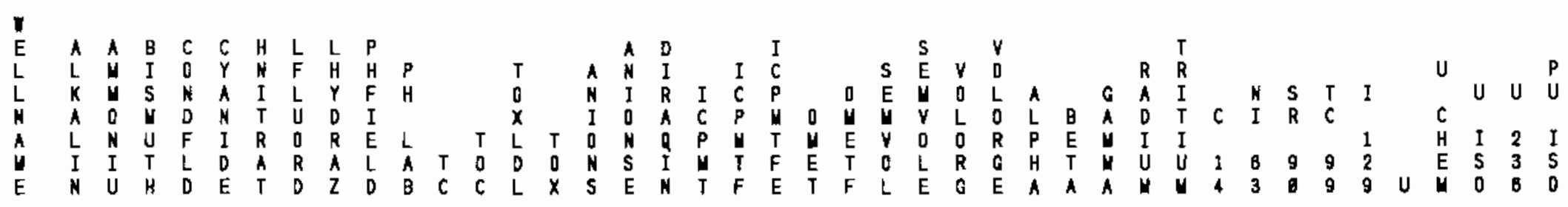

\begin{tabular}{|c|c|c|c|c|c|c|c|c|c|c|c|c|c|c|c|c|c|c|c|c|c|c|c|c|c|c|c|c|c|c|c|c|c|c|c|c|c|c|}
\hline $699-37-E 4$ & $x$ & $x$ & & $x$ & $x$ & $x$ & $x$ & & $x$ & $x$ & $x$ & $x$ & $x$ & $x$ & $x$ & & $x$ & $x$ & & $x$ & $x$ & & $x$ & $x$ & $x$ & $x$ & $x$ & & $x$ & & & $x$ & & & $x$ & & & \\
\hline $699-37-43$ & $x$ & $x$ & & $x$ & $x$ & $x$ & $x$ & & $x$ & $x$ & $x$ & $x$ & $x$ & $x$ & $x$ & & $x$ & $x$ & & $x$ & $x$ & & $x$ & $x$ & $x$ & $x$ & $x$ & & $x$ & $x$ & & & $x$ & $x$ & & & & \\
\hline $692-37-824$ & $x$ & $x$ & $x$ & $x$ & $x$ & & $x$ & & $x$ & $x$ & $x$ & $x$ & $x$ & $x$ & & & $x$ & $x$ & & $x$ & $x$ & & $x$ & $x$ & $x$ & $x$ & & & $x$ & & & & & & & & & \\
\hline $699-38-65$ & $x$ & $x$ & $x$ & $x$ & $x$ & & $x$ & & $x$ & $x$ & $x$ & $x$ & $x$ & $x$ & & & $x$ & $x$ & & $x$ & $x$ & & $x$ & $x$ & $x$ & $x$ & $x$ & & $x$ & & & & & & $x$ & & & \\
\hline $699-38-70$ & $x$ & $x$ & $x$ & $x$ & $x$ & & $x$ & $x$ & $x$ & $x$ & $x$ & $x$ & $x$ & $x$ & $x$ & & $x$ & $x$ & & $x$ & $x$ & & $x$ & $x$ & $x$ & $x$ & $x$ & & $x$ & $x$ & $x$ & $x$ & $x$ & $x$ & $x$ & $x$ & $x$ & $x$ \\
\hline $699-39-79$ & $x$ & $x$ & $x$ & $x$ & $x$ & & $x$ & $x$ & $x$ & $x$ & $x$ & $x$ & $x$ & $x$ & $x$ & & $x$ & $x$ & & $x$ & $x$ & & $x$ & $x$ & $x$ & $x$ & $x$ & & $x$ & & & & & & & & & \\
\hline $699-46-1$ & $x$ & $x$ & $x$ & $x$ & $x$ & $x$ & $x$ & & $x$ & $x$ & $x$ & $x$ & $x$ & $x$ & $x$ & & $x$ & $x$ & & $x$ & $x$ & & $x$ & $x$ & $x$ & $x$ & $x$ & & $x$ & & & $x$ & $x$ & & $x$ & & & \\
\hline $699-40-334$ & $x$ & $x$ & $x$ & $x$ & $x$ & & $x$ & & $x$ & $x$ & $x$ & $x$ & $x$ & $x$ & & & $x$ & $x$ & & $x$ & & & $x$ & $x$ & $x$ & $x$ & & & $x$ & & & & & & & & & \\
\hline $699-49-62$ & $x$ & $x$ & & $x$ & $x$ & $x$ & $x$ & & $x$ & $x$ & $x$ & $x$ & $x$ & $x$ & $x$ & & $x$ & $x$ & & $x$ & $x$ & & $x$ & $x$ & $x$ & $x$ & & & $x$ & & & & & & & & & \\
\hline $699-41-1$ & $x$ & $x$ & & $x$ & $x$ & $x$ & $x$ & & $x$ & $x$ & $x$ & $x$ & $x$ & $x$ & $x$ & & $x$ & $x$ & & $x$ & $x$ & & $x$ & $x$ & $x$ & $x$ & $x$ & & $x$ & & & $x$ & $x$ & $x$ & $x$ & & & \\
\hline $\cos -42-2$ & $x$ & $x$ & & $x$ & $x$ & $x$ & $x$ & & $x$ & $x$ & $x$ & $x$ & $x$ & $x$ & $x$ & & $x$ & $x$ & & $x$ & $x$ & & $x$ & $x$ & $x$ & $x$ & $x$ & & $x$ & & & $x$ & & & $x$ & & & \\
\hline $699-43-3$ & $x$ & $x$ & & & $x$ & $x$ & $x$ & & & $x$ & $x$ & $x$ & $x$ & $x$ & $x$ & & $x$ & $x$ & & $x$ & $x$ & & $x$ & $x$ & $x$ & $x$ & $x$ & & $x$ & & & $x$ & & & $x$ & & & \\
\hline $699-43-89$ & $x$ & $x$ & $x$ & $x$ & $x$ & & $x$ & $x$ & $x$ & $x$ & $x$ & $x$ & $x$ & $x$ & & & $x$ & $x$ & & $x$ & $x$ & & $x$ & $x$ & $x$ & $x$ & & & $x$ & & & & & & & & & \\
\hline $699-44-64$ & $x$ & $x$ & & $x$ & $x$ & $x$ & $x$ & & $x$ & $x$ & $x$ & $x$ & $x$ & $x$ & $x$ & & $x$ & $x$ & & $x$ & $x$ & & $x$ & $x$ & $x$ & $x$ & $x$ & & $x$ & & & & & & & & & \\
\hline $695-45-2$ & $x$ & $x$ & & & $x$ & $x$ & $x$ & & & $x$ & $x$ & $x$ & $x$ & $x$ & $x$ & & $x$ & $x$ & & $x$ & $x$ & & $x$ & $x$ & $x$ & $x$ & $x$ & & $x$ & & & $x$ & & & $x$ & & & \\
\hline $699-45-42$ & $x$ & $x$ & & $x$ & $x$ & $x$ & $x$ & & $x$ & $x$ & $x$ & $x$ & $x$ & $x$ & $x$ & $x$ & $x$ & $x$ & $x$ & $x$ & $x$ & $x$ & $x$ & $x$ & $x$ & $x$ & $x$ & $x$ & $x$ & & & $x$ & & & & $x$ & $x$ & \\
\hline $699-45-69 \lambda$ & $x$ & $x$ & $x$ & $x$ & $x$ & & $x$ & & $x$ & $x$ & $x$ & $x$ & $x$ & $x$ & & & $x$ & $x$ & & $x$ & $x$ & & $x$ & $x$ & $x$ & $x$ & $x$ & & $x$ & & & & & & & & & \\
\hline $699-46-4$ & $x$ & $x$ & & & $x$ & $x$ & $x$ & & & $x$ & $x$ & $x$ & $x$ & $x$ & $x$ & & $x$ & $x$ & & $x$ & $x$ & & $x$ & $x$ & $x$ & $x$ & $x$ & & $x$ & & & $x$ & $x$ & $x$ & $x$ & & & \\
\hline $699-47-5$ & $x$ & $x$ & & $x$ & $x$ & $x$ & $x$ & & $x$ & $x$ & $x$ & $x$ & $x$ & $x$ & $x$ & & $x$ & $x$ & & $x$ & $x$ & & $x$ & $x$ & $x$ & $x$ & $x$ & & $x$ & & & $x$ & & & $x$ & & & \\
\hline $699-47-464$ & $x$ & $x$ & & $x$ & $x$ & $x$ & $x$ & & $x$ & $x$ & $x$ & $x$ & $x$ & $x$ & $x$ & & $x$ & $x$ & & $x$ & $x$ & & $x$ & $x$ & $x$ & $x$ & $x$ & & $x$ & & & & & & $x$ & & & \\
\hline $699-47-50$ & $x$ & $x$ & & $x$ & $x$ & $x$ & $x$ & & $x$ & $x$ & $x$ & $x$ & $x$ & $x$ & $x$ & & & & & & $x$ & & $x$ & $x$ & $x$ & $x$ & $x$ & & $x$ & $x$ & & & $x$ & $x$ & & & & \\
\hline $699-47-66$ & $x$ & $x$ & & $x$ & $x$ & $x$ & $x$ & & $x$ & $x$ & $x$ & $x$ & $x$ & $x$ & $x$ & & $x$ & $x$ & & $x$ & $x$ & & $x$ & $x$ & $x$ & $x$ & $x$ & & $x$ & & & & & & $x$ & & & \\
\hline $699-48-7$ & $x$ & $x$ & $x$ & $x$ & $x$ & & $x$ & & $x$ & $x$ & $x$ & $x$ & $x$ & $x$ & & & $x$ & $x$ & & $x$ & & & $x$ & $x$ & $x$ & $x$ & & & $x$ & & & & & & & & & \\
\hline $699-48-18$ & $x$ & $x$ & $x$ & & $x$ & & $x$ & $x$ & & $x$ & $x$ & $x$ & $x$ & $x$ & & & $x$ & $x$ & & $x$ & $x$ & & $x$ & $x$ & $x$ & $x$ & & & $x$ & & & & & & & & & \\
\hline $699-48-71$ & $x$ & $x$ & $x$ & $x$ & $x$ & & $x$ & & $x$ & $x$ & $x$ & $x$ & $x$ & $x$ & & & $x$ & $x$ & & $x$ & & & $x$ & $x$ & $x$ & $x$ & & & $x$ & & & & & & & & & \\
\hline $699-49-55 \lambda$ & $x$ & $x$ & $x$ & $x$ & $x$ & & $x$ & $x$ & $x$ & $x$ & $x$ & $x$ & $x$ & $x$ & $x$ & & $x$ & $x$ & & $x$ & $x$ & & $x$ & $x$ & $x$ & $x$ & $x$ & & $x$ & & & & & $x$ & $x$ & & & \\
\hline $699-49-57$ & $x$ & $x$ & $x$ & $x$ & $x$ & & $x$ & & $x$ & $x$ & $x$ & $x$ & $x$ & $x$ & $x$ & & $x$ & $x$ & & $x$ & $x$ & & $x$ & $x$ & $x$ & $x$ & $x$ & & $x$ & & & $x$ & & $x$ & & & & \\
\hline
\end{tabular}




\section{TABLE C.2. (contd)}

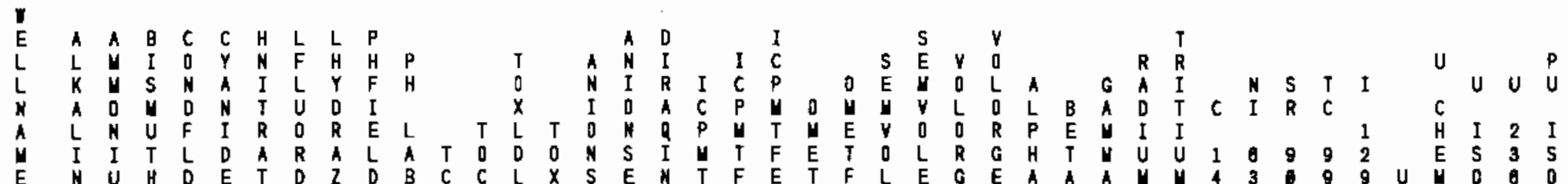

\begin{tabular}{|c|c|c|c|c|c|c|c|c|c|c|c|c|c|c|c|c|c|c|c|c|c|c|c|c|c|c|c|}
\hline $699-49-79$ & $x$ & $x$ & $x$ & $x$ & $x$ & & $x$ & $x$ & $x$ & $x$ & $x$ & $x$ & $x$ & $x$ & $x$ & $x$ & $x$ & $x$ & $x$ & $x$ & $x$ & $x$ & $x$ & & $x$ & & \\
\hline $6 x-50-63$ & $x$ & $x$ & $x$ & $x$ & $x$ & & $x$ & & $x$ & $x$ & $x$ & $x$ & $x$ & $x$ & $x$ & $x$ & $x$ & $x$ & $x$ & $x$ & $x$ & $x$ & $x$ & $x$ & $x$ & $x$ & $x$ \\
\hline $699-50-85$ & $x$ & & & $x$ & $x$ & $x$ & $x$ & & $x$ & $x$ & $x$ & $x$ & $x$ & $x$ & $x$ & $x$ & $x$ & $x$ & $x$ & $x$ & $x$ & $x$ & $x$ & & $x$ & & \\
\hline $699-51-75$ & $x$ & $x$ & $x$ & $x$ & $x$ & & $x$ & & $x$ & $x$ & $x$ & $x$ & $x$ & $x$ & & $x$ & $x$ & $x$ & & $x$ & $x$ & $x$ & $x$ & & $x$ & & \\
\hline $699-55-5 e x$ & $x$ & $x$ & & $x$ & $x$ & $x$ & $x$ & & $x$ & $x$ & $x$ & $x$ & $x$ & $x$ & $x$ & $x$ & $x$ & $x$ & $x$ & $x$ & $x$ & $x$ & $x$ & $x$ & $x$ & $x$ & \\
\hline $699-55-76$ & $x$ & $x$ & & $x$ & $x$ & $x$ & $x$ & & $x$ & $x$ & $x$ & $x$ & $x$ & $x$ & $x$ & $x$ & $x$ & $x$ & $x$ & $x$ & $x$ & $x$ & $x$ & & & & \\
\hline $699-65-72$ & $x$ & $x$ & & $x$ & $x$ & $x$ & $x$ & & $x$ & $x$ & $x$ & $x$ & $x$ & $x$ & $x$ & $x$ & $x$ & $x$ & $x$ & $x$ & $x$ & $x$ & $x$ & & $x$ & & \\
\hline $699-65-83$ & $x$ & $x$ & & $x$ & $x$ & $x$ & $x$ & & $x$ & $x$ & $x$ & $x$ & $x$ & $x$ & $x$ & $x$ & $x$ & $x$ & $x$ & $x$ & $x$ & $x$ & $x$ & & $x$ & & \\
\hline $699-67-86$ & $x$ & $x$ & & $x$ & $x$ & $x$ & $x$ & & $x$ & $x$ & $x$ & $x$ & $x$ & $x$ & $x$ & $x$ & $x$ & $x$ & $x$ & $x$ & $x$ & $x$ & $x$ & & $x$ & & \\
\hline $699-76-68$ & $x$ & $x$ & & $x$ & $x$ & $x$ & $x$ & & $x$ & $x$ & $x$ & $x$ & $x$ & $x$ & $x$ & $x$ & $x$ & $x$ & $x$ & $x$ & $x$ & $x$ & $x$ & & $x$ & & \\
\hline $699-71-30$ & $x$ & $x$ & & $x$ & $x$ & $x$ & $x$ & & $x$ & $x$ & $x$ & $x$ & $x$ & $x$ & $x$ & $x$ & $x$ & $x$ & $x$ & $x$ & $x$ & $x$ & $x$ & & $x$ & & \\
\hline $699-71-52$ & $x$ & $x$ & & $x$ & $x$ & $x$ & $x$ & & $x$ & $x$ & $x$ & $x$ & $x$ & $x$ & $x$ & $x$ & $x$ & $x$ & $x$ & $x$ & $x$ & $x$ & $x$ & & $x$ & & \\
\hline $699-71-77$ & $x$ & $x$ & & $x$ & $x$ & $x$ & $x$ & & $x$ & $x$ & $x$ & $x$ & $x$ & $x$ & $x$ & $x$ & $x$ & $x$ & $x$ & $x$ & $x$ & $x$ & $x$ & & $x$ & & \\
\hline $699-73-61$ & $x$ & $x$ & & $x$ & $x$ & $x$ & $x$ & & $x$ & $x$ & $x$ & $x$ & $x$ & $x$ & $x$ & $x$ & $x$ & $x$ & $x$ & $x$ & $x$ & $x$ & $x$ & & $x$ & & \\
\hline $699-74-44$ & $x$ & $x$ & & $x$ & $x$ & $x$ & $x$ & & $x$ & $x$ & $x$ & $x$ & $x$ & $x$ & $x$ & $x$ & $x$ & $x$ & $x$ & $x$ & $x$ & $x$ & $x$ & & $x$ & & \\
\hline $699-77-36$ & $x$ & $x$ & & & $x$ & $x$ & $x$ & & & $x$ & $x$ & $x$ & $x$ & $x$ & $x$ & $x$ & $x$ & $x$ & $x$ & $x$ & $x$ & $x$ & $x$ & & $x$ & & \\
\hline $699-77-54$ & $x$ & $x$ & $x$ & $x$ & $x$ & & $x$ & & $x$ & $x$ & $x$ & $x$ & $x$ & $x$ & & $x$ & $x$ & $x$ & & $x$ & $x$ & $x$ & $x$ & & $x$ & & \\
\hline $699-83-47$ & $x$ & $x$ & $x$ & $x$ & $x$ & & $x$ & $x$ & $x$ & $x$ & $x$ & $x$ & $x$ & $x$ & & $x$ & $x$ & $x$ & $x$ & $x$ & $x$ & $x$ & $x$ & & $x$ & & \\
\hline $699-69-35$ & $x$ & $x$ & $x$ & $x$ & $x$ & & $x$ & & $x$ & $x$ & $x$ & $x$ & $x$ & $x$ & & $x$ & $x$ & $x$ & & $x$ & $x$ & $x$ & $x$ & & $x$ & & \\
\hline $699-98-45$ & $x$ & $x$ & $x$ & $x$ & $x$ & & $x$ & & $x$ & $x$ & $x$ & $x$ & $x$ & $x$ & $x$ & $x$ & $x$ & $x$ & $x$ & $x$ & $x$ & $x$ & $x$ & & $x$ & & \\
\hline $890-96-49$ & $x$ & $x$ & & $x$ & $x$ & $x$ & $x$ & & $x$ & $x$ & $x$ & $x$ & $x$ & $x$ & $x$ & $x$ & $x$ & $x$ & $x$ & $x$ & $x$ & $x$ & $x$ & & $x$ & & \\
\hline $699-97-43$ & $x$ & $x$ & & $x$ & $x$ & $x$ & $x$ & & $x$ & $x$ & $x$ & $x$ & $x$ & $x$ & $x$ & $x$ & $x$ & $x$ & $x$ & $x$ & $x$ & $x$ & $x$ & & $x$ & & \\
\hline $699-97-514$ & $x$ & $x$ & & $x$ & $x$ & $x$ & $x$ & & $x$ & $x$ & $x$ & $x$ & $x$ & $x$ & & $x$ & $x$ & $x$ & $x$ & & & $x$ & $x$ & & $x$ & & \\
\hline $69-101-488$ & $x$ & $x$ & & $x$ & $x$ & $x$ & $x$ & & $x$ & $x$ & $x$ & $x$ & $x$ & $x$ & $x$ & $x$ & $x$ & $x$ & $x$ & $x$ & $x$ & $x$ & $x$ & & $x$ & & \\
\hline $699-53-\mathrm{E} 2$ & $x$ & $x$ & & $x$ & $x$ & $x$ & $x$ & & $x$ & $x$ & $x$ & $x$ & $x$ & $x$ & $x$ & $x$ & $x$ & $x$ & $x$ & $x$ & $x$ & $x$ & $x$ & & $x$ & & \\
\hline $699-53-25$ & $x$ & $x$ & $x$ & $x$ & $x$ & & $x$ & $x$ & $x$ & $x$ & $x$ & $x$ & $x$ & $x$ & & $x$ & $x$ & $x$ & $x$ & $x$ & $x$ & $x$ & $x$ & & $x$ & & \\
\hline $699-56 E 14 h$ & $x$ & $x$ & $x$ & $x$ & $x$ & & $x$ & & $x$ & $x$ & $x$ & $x$ & $x$ & $x$ & & $x$ & $x$ & $x$ & & $x$ & $x$ & $x$ & $x$ & & $x$ & & \\
\hline
\end{tabular}


TABLE C.2. (contd)

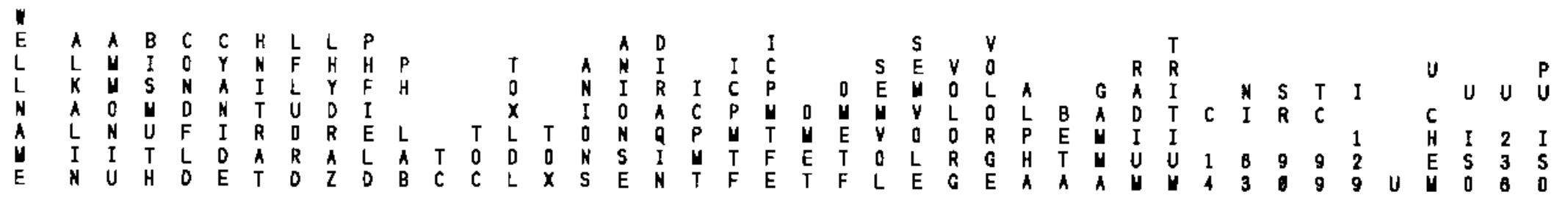

$699-58-19$ $\begin{array}{lllllllllll}x & x & x & x & x & x & x & x & x & x & x\end{array}$

EST-S11E12AP

COS-S12-3

69-S29-E12

$x \times x \quad x \quad x \quad x \quad x \quad x$

$x$

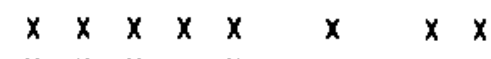

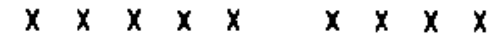
$x \times x \quad x \quad x$

$x \times x$

$\hat{x} x \quad x$

$x \times x$

$x \times x \times$

$x y x y$

699-591-1

$x \times x \times$

$x \times x$

$x \times x x$

$x \quad x \quad x$

$x \times x \times x$

$x \times x x$ 
TABLE C.3. Results for Site-Wide Chemical Monitoring Wells

\begin{tabular}{|c|c|c|c|c|c|c|c|c|c|}
\hline \multicolumn{2}{|c|}{$\begin{array}{l}\text { CONSTITUENT } \\
\text { MAME UNITS }\end{array}$} & $\begin{array}{l}\text { CDNTRACIUAL } \\
\text { DETECTION } \\
\text { LIUIT }\end{array}$ & $\begin{array}{l}\text { DRINKING } \\
\text { WATER } \\
\text { STANDARD }\end{array}$ & $\begin{array}{l}\text { SAUPLE } \\
\text { DATE }\end{array}$ & $1-83-1$ & $\begin{array}{l}\text { SAHPLE } \\
\text { DATE }\end{array}$ & $1-84-1$ & $\begin{array}{l}\text { SAHPLE } \\
\text { DATE }\end{array}$ & $1-84-4$ \\
\hline------ & ---- & $---1-1,-$ & 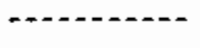 & $--+\cdots$ & $\cdots+\infty$ & $\cdots+-$ & ----- & ------ & ----- \\
\hline ALKALIN & & & & $10 / 18 / 87$ & 105000.000 & $07 / 36 / 87$ & 109086.60 & $97 / 30 / 87$ & 158084.010 \\
\hline ALPHA & $\mathrm{PCI} / \mathrm{L}$ & 4.00 & 15.60 & $16 / 19 / 87$ & $\left\{\begin{array}{l}2.24 \\
1.34\}\end{array}\right.$ & $67 / 31 / 67$ & ------ & $97 / 30 / 87$ & ------ \\
\hline BETA & PCI/L & 8.00 & 56.66 & $10 / 19 / 87$ & $\left\{\begin{array}{c}147.69 \\
11.26\}\end{array}\right.$ & $67 / 31 / 67$ & $\left\{\begin{array}{c}68.66 \\
7.88\}\end{array}\right.$ & $97 / 31 / 87$ & $\left\{\begin{array}{r}92.84 \\
9.15\}\end{array}\right.$ \\
\hline CHLORID & PPB & 500.00 & & $10 / 19 / 07$ & 9430.00 & $07 / 30 / 87$ & 8251.06 & $07 / 30 / 87$ & 8506.00 \\
\hline$C O-60$ & $\mathrm{PCI} / \mathrm{L}$ & 22.50 & 160.60 & $\begin{array}{l}07 / 02 / 87 \\
10 / 19 / 87\end{array}$ & 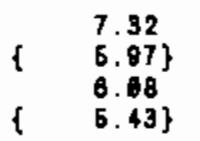 & $\begin{array}{l}17 / 30 / 87 \\
10 / 19 / 87\end{array}$ & & $\begin{array}{l}07 / 31 / 87 \\
10 / 18 / 87\end{array}$ & $\begin{array}{ll}\{\quad & 5.08 \\
\{\quad 4.54\}\end{array}$ \\
\hline CONDFLD & UNHO & 1.00 & & $10 / 19 / 07$ & 318.66 & $67 / 30 / 87$ & 307.60 & $07 / 30 / 87$ & 280.00 \\
\hline$C S-137$ & $\mathrm{PCI} / \mathrm{L}$ & 20.60 & 265.60 & $\begin{array}{l}7 / 02 / 87 \\
10 / 19 / 87\end{array}$ & $\begin{array}{l}0.07 \\
\{\quad 5.24\}\end{array}$ & $\begin{array}{l}17 / 31 / 07 \\
10 / 19 / 07\end{array}$ & ----- & $\begin{array}{l}07 / 36 / 87 \\
10 / 19 / 87\end{array}$ & $-\ldots . .$. \\
\hline FBARIUU & PPB & 6.06 & 1600.00 & $10 / 19 / 07$ & 40.00 & $67 / 30 / 07$ & 33.00 & $67 / 36 / 87$ & 22.8 \\
\hline FCALCIU & PPB & & & $11 / 10 / 87$ & 51600.00 & $67 / 36 / 67$ & 30606.00 & $67 / 30 / 87$ & 38200.60 \\
\hline FCHROMI & PPB & 10.66 & 50.00 & $10 / 10 / 87$ & 47.00 & $67 / 30 / 67$ & 13.00 & $97 / 30 / 87$ & 12.60 \\
\hline FUAGNES & PPB & 1. 0 & & $10 / 19 / 87$ & 8330.00 & $67 / 30 / 87$ & $6 \overline{19.0}$ & $67 / 30 / 87$ & 6810.01 \\
\hline FUANGAN & PPB & 5.00 & & $16 / 19 / 87$ & $\cdots+-$ & $07 / 36 / 87$ & ----- & $97 / 36 / 87$ & 5.00 \\
\hline FPOTASS & PPE & 100.00 & & $10 / 10 / 87$ & 1938.00 & $07 / 36 / 67$ & 4170.00 & $67 / 31 / 87$ & 4750.01 \\
\hline FSODIUH & PPB & 106.00 & & $10 / 19 / 87$ & 9470.00 & $97 / 36 / 87$ & 10000.06 & $07 / 30 / 87$ & 11060.00 \\
\hline FYAKADI & $\mathrm{PPB}$ & 5.66 & & $10 / 19 / 87$ & ----- & $07 / 38 / 87$ & 6.00 & $07 / 36 / 87$ & 7.00 \\
\hline FZINC & PPB & 5.06 & & $10 / 19 / 87$ & 33.00 & $07 / 30 / 87$ & +....- & $67 / 30 / 87$ & ------ \\
\hline $\begin{array}{l}*-V A \\
\mid-V A \\
-V A \\
\text { N }- \text { DE } \\
\text { NR }- \text { AN } \\
\text { VALUES } \\
\text { VATERS }\end{array}$ & $\begin{array}{l}\text { UE EXCEE } \\
\text { UE EXCEE } \\
\text { UE EXCEE } \\
\text { ECTION } \\
\text { LYSIS NC } \\
\text { ANDARD'S }\end{array}$ & $\begin{array}{l}\text { EDS PRIMARY D } \\
\text { EDS PROPOSED } \\
\text { EDS SCREENING } \\
\text { INIT WAS NDT } \\
\text { OT RERUESTED } \\
\text { ARE CDUNTIN } \\
\text { S) IN PARENTH }\end{array}$ & $\begin{array}{l}\text { RINXING WATER } \\
\text { RIUARY DRINK } \\
\text { LEVEL FDR FL } \\
\text { AYAILABLE FO } \\
\text { R NOT YET RE } \\
\text { ERRORS FOR } \\
\text { ESES ARE PRDF }\end{array}$ & $\begin{array}{l}\text { ANDARD. } \\
\text { IATER STA } \\
\text { ER INVESTI } \\
\text { OUPARISON } \\
\text { TED } \\
\text { IONUCLIDES } \\
\text { D ONLY }\end{array}$ & $\begin{array}{l}\text { ARD. } \\
\text { ITIDN. }\end{array}$ & & & & \\
\hline
\end{tabular}


TABLE C.3. (contd)

\begin{tabular}{|c|c|c|c|c|c|}
\hline $\begin{array}{c}\text { CONSTI } \\
\text { NAME }\end{array}$ & $\begin{array}{l}\text { JENT } \\
\text { UNITS }\end{array}$ & $\begin{array}{l}\text { CONTRACTUAL } \\
\text { OETECTION } \\
\text { LIUIT }\end{array}$ & $\begin{array}{l}\text { DRINKING } \\
\text { IATER } \\
\text { STANDARD }\end{array}$ & $\begin{array}{l}\text { SAUPLE } \\
\text { DATE }\end{array}$ & 1 \\
\hline HNITRAT & PPB & 2680.01 & 46080.01 & $\begin{array}{l}07 / 82 / 87 \\
10 / 19 / 87\end{array}$ & $\begin{array}{l}556 \\
363\end{array}$ \\
\hline LFLUORD & PPB & 20.06 & 4001.00 & $18 / 18 / 87$ & \\
\hline NITRATE & PPB & 504.60 & 45600.06 & $10 / 19 / 87$ & \\
\hline PH-LAB & & 1.01 & & $18 / 19 / 87$ & \\
\hline PHFIELD & & 0.10 & & $10 / 19 / 87$ & \\
\hline RU-106 & PCI $/ L$ & 172.56 & 31.10 & $17 / 62 / 87$ & \\
\hline & & & & $10 / 19 / 87$ & \\
\hline SR 00 & PCI/L & 6.11 & 8.10 & $10 / 18 / 87$ & \\
\hline SULFATE & PPB & 506.11 & & $10 / 10 / 87$ & \\
\hline TC & PPB & & & $10 / 18 / 87$ & 24 \\
\hline TC-89 & $P C I / L$ & 15.00 & 906.60 & & \\
\hline TRITIUV & PCI/L & 590.00 & 20000000 & $\begin{array}{l}17 / 02 / 87 \\
10 / 10 / 87\end{array}$ & $\begin{array}{r}25 \\
\left\{\begin{array}{r}2 \\
36\end{array}\right. \\
\left\{\begin{array}{l}2 \\
\text {. }\end{array}\right.\end{array}$ \\
\hline \multicolumn{6}{|c|}{ 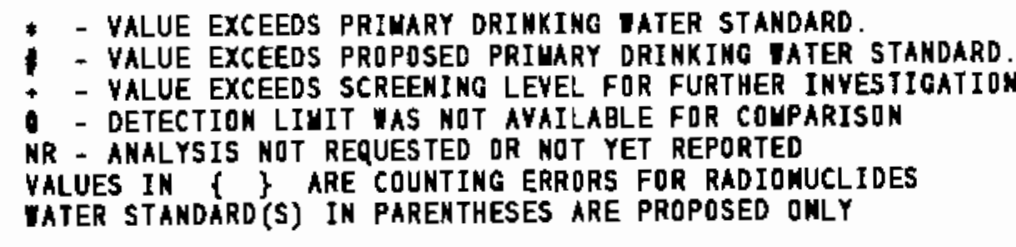 } \\
\hline
\end{tabular}

\begin{tabular}{|c|c|c|c|}
\hline $\begin{array}{c}\text { SAUPLE } \\
\text { DATE }\end{array}$ & $\begin{array}{c}1-\theta 4-1 \\
N R \\
M R\end{array}$ & $\begin{array}{c}\text { SAUPLE } \\
\text { DATE }\end{array}$ & \begin{tabular}{c}
$1-84-4$ \\
\hdashline$R R$ \\
$M R$
\end{tabular} \\
\hline $17 / 31 / 87$ & 241.00 & $07 / 30 / 87$ & 263.6 \\
\hline $07 / 36 / 07$ & 11206.00 & $67 / 31 / 07$ & 0301.00 \\
\hline $17 / 31 / 87$ & 7.68 & $07 / 30 / 87$ & 7.75 \\
\hline $97 / 31 / 87$ & 7.11 & $67 / 36 / 87$ & 7.11 \\
\hline $\begin{array}{l}67 / 31 / 87 \\
10 / 19 / 87\end{array}$ & & $\begin{array}{l}07 / 31 / 87 \\
10 / 19 / 87\end{array}$ & $\begin{array}{l}53.09 \\
69.04\end{array}$ \\
\hline $10 / 10 / 07$ & $\begin{array}{c}27.36 \\
\{\quad 2.05\} \\
\mathrm{MR}\end{array}$ & $\begin{array}{l}67 / 35 / 07 \\
10 / 19 / 87\end{array}$ & 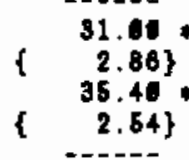 \\
\hline $07 / 36 / 67$ & 10500.06 & $67 / 30 / 87$ & 36906.01 \\
\hline $17 / 30 / 87$ & 25806.00 & $07 / 31 / 97$ & 25004.01 \\
\hline & NR & $07 / 30 / 87$ & $\left\{\begin{array}{c}88.69 \\
1.72\}\end{array}\right.$ \\
\hline $\begin{array}{l}67 / 91 / 87 \\
10 / 10 / 87\end{array}$ & 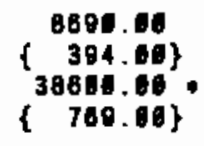 & $\begin{array}{l}67 / 30 / 87 \\
18 / 10 / 87\end{array}$ & 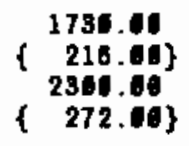 \\
\hline
\end{tabular}




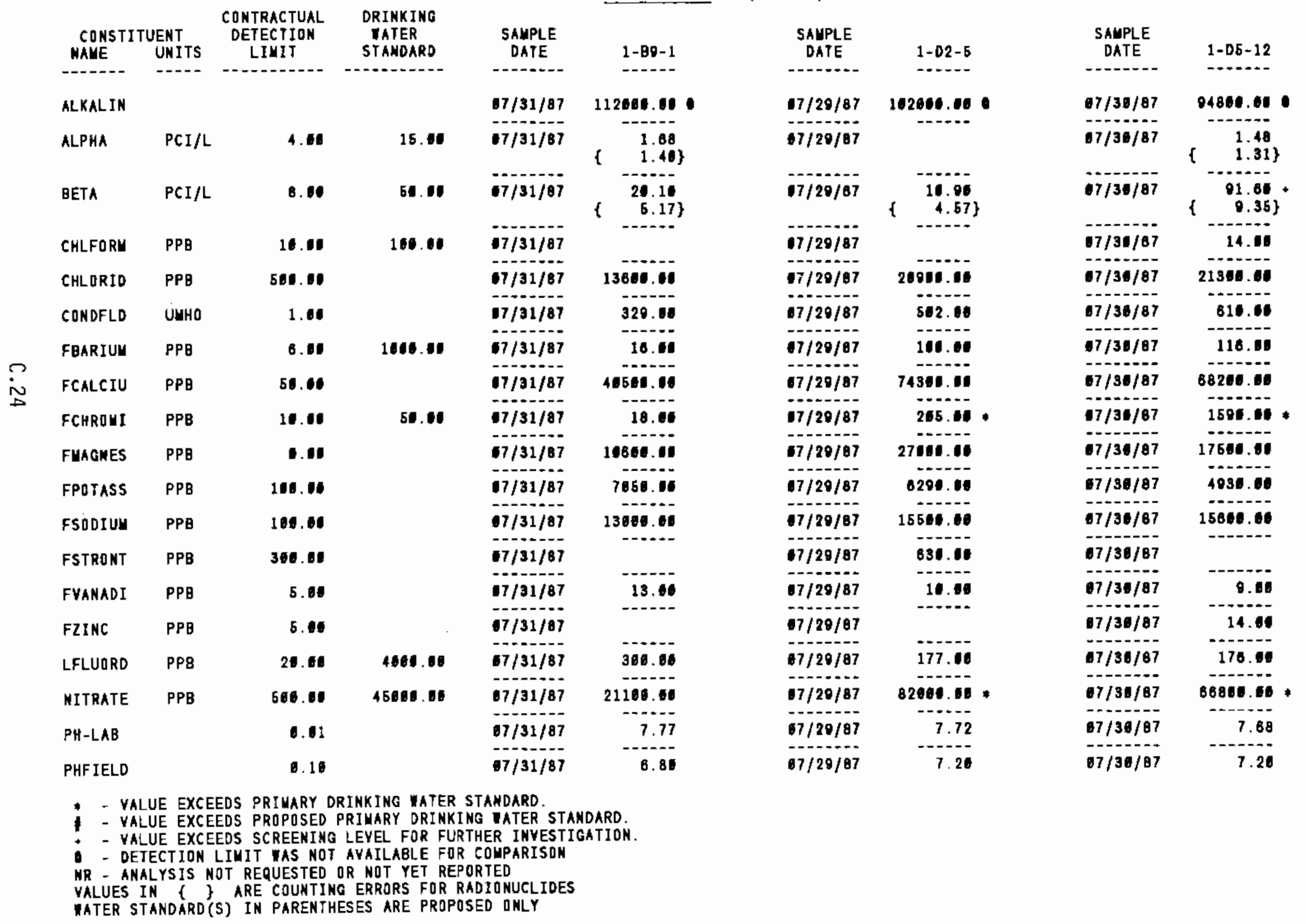




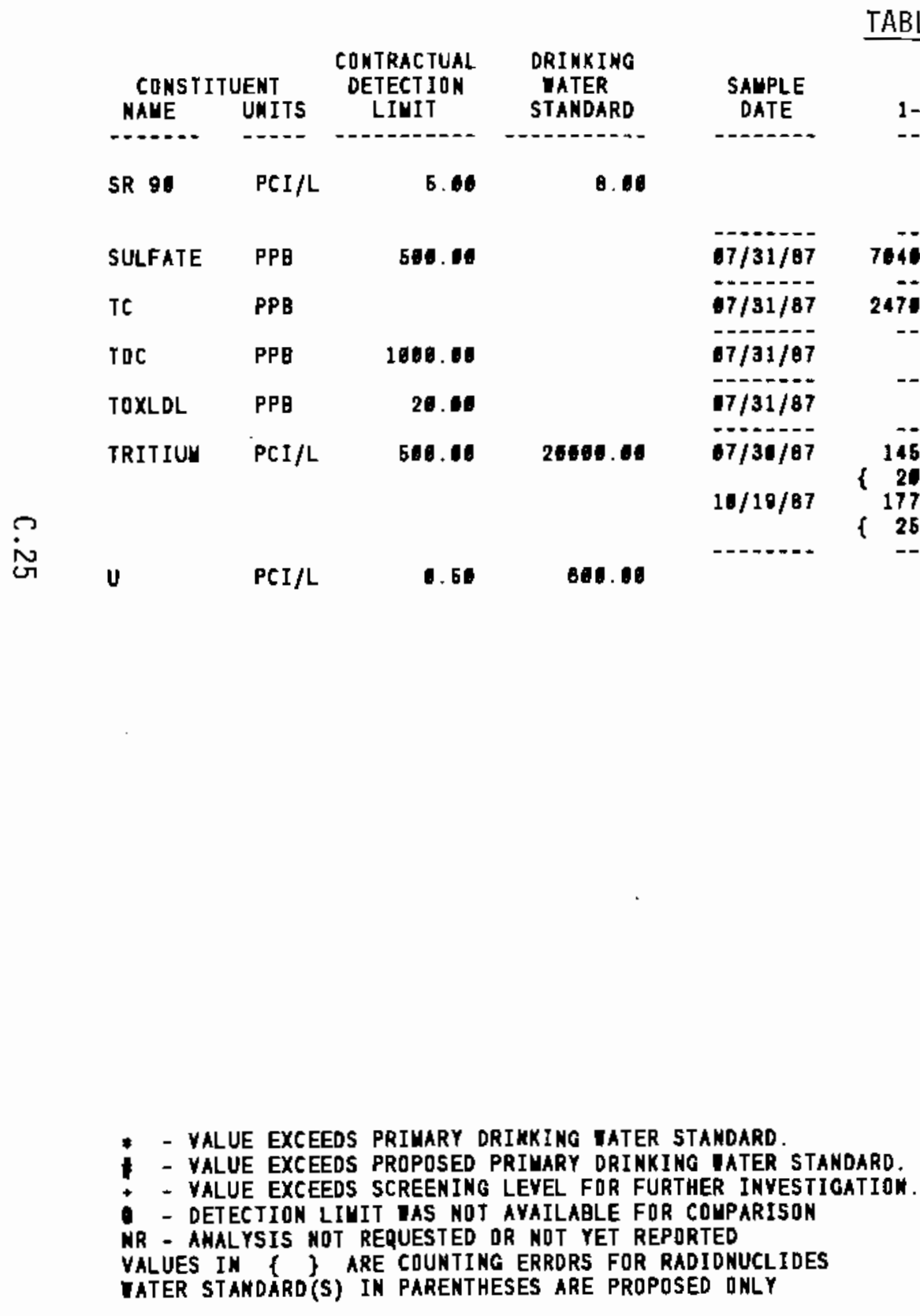

ABLE C.3. (contd)

SAYPLE

DATE

1-D5-12

KR

$10 / 14 / 87$

$16 / 14 / 87$

$07 / 29 / 87$

103080.00

-17/30/87

$\left\{\begin{array}{r}46.90 * \\ 3.37\}\end{array}\right.$

400.00

-.---..-

(2)

$67 / 29 / 87$

25100.06

$07 / 20 / 87$

1450.00

$07 / 29 / 87$

-.....

$17 / 29 / 87$

30500.00

200.09

$18 / 14 / 87$

$\{606.66\}$

250.5

$10 / 14 / 87$

$\{71.0\}$

in

NR

$07 / 30 / 87$

139801.11

24300.00

07/31/87

7ain...

$07 / 30 / 87$

$67 / 30 / 87$

$10 / 14 / 87$

NR

--no.-.

$10 / 14 / 87$

24.46

5211.0

\{ 291.01)

7760.00

\{ 388.04$\}$

NR

1.86

3. 54

UXE

MR - AHALYSIS NOT RERUESTED DR NDT YET REPDRTED

IATER STANDARO(S) IN PARENTHESES ARE PROPOSED DNLY 
TABLE C.3. (contd)

\begin{tabular}{|c|c|c|c|c|c|c|c|c|c|}
\hline $\begin{array}{c}\text { CDNSTI } \\
\text { NAME }\end{array}$ & $\begin{array}{l}\text { UENT } \\
\text { UNITS }\end{array}$ & $\begin{array}{l}\text { CONTRACTUAL } \\
\text { DETECTIOK } \\
\text { LIMIT }\end{array}$ & $\begin{array}{l}\text { ORINKING } \\
\text { VATER } \\
\text { STAKOARD }\end{array}$ & $\begin{array}{c}\text { SAMPLE } \\
\text { DATE }\end{array}$ & $1-08-3$ & $\begin{array}{c}\text { SAUPLE } \\
\text { DATE }\end{array}$ & $1-\mathrm{Fb}-1$ & $\begin{array}{c}\text { SANPLE } \\
\text { DATE }\end{array}$ & $1-F 5-4$ \\
\hline ALKALIN & & & & $67 / 36 / 97$ & $\underset{\mathrm{NR}}{54100.010}$ & $\begin{array}{l}17 / 27 / 87 \\
10 / 15 / 87\end{array}$ & $\begin{array}{l}97280.10: \\
93768.00\end{array}$ & $10 / 15 / 87$ & ${ }_{M R}^{19960 \theta^{\prime}}$ \\
\hline ALPHA & $\mathrm{PCI} / \mathrm{L}$ & 4.01 & 16.80 & $07 / 30 / 87$ & $\left.\begin{array}{ll} & 1.44 \\
& 1.11 \\
\mathrm{NR}\end{array}\right\}$ & $\begin{array}{l}07 / 27 / 87 \\
10 / 15 / 87\end{array}$ & & $18 / 15 / 87$ & $\begin{array}{ll}\left\{\begin{array}{l}7.36 \\
2.87\end{array}\right. \\
\mathrm{NR}\end{array}$ \\
\hline EETA & PCI/L & 8.06 & 50.60 & $67 / 36 / 87$ & $\left\{\begin{array}{l}0.61 \\
4.23\} \\
N R\end{array}\right.$ & $\begin{array}{l}07 / 27 / 87 \\
10 / 15 / 87\end{array}$ & 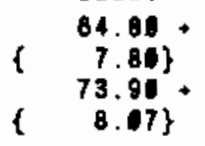 & $16 / 16 / 87$ & $\begin{array}{l}0.51 \\
4.36\} \\
\mathrm{HR}\end{array}$ \\
\hline CHLORID & PPB & 501.16 & & $17 / 38 / 87$ & 17460.06 & $\begin{array}{l}07 / 27 / 87 \\
10 / 15 / 87\end{array}$ & $\begin{array}{l}2461.01 \\
2580.06\end{array}$ & $10 / 15 / 87$ & 40000.010 \\
\hline$C 0-80$ & $\mathrm{PCI} / \mathrm{L}$ & 22.56 & 100.60 & $\begin{array}{l}17 / 36 / 87 \\
10 / 14 / 87\end{array}$ & & $\begin{array}{l}67 / 27 / 87 \\
10 / 15 / 87\end{array}$ & $\left.\begin{array}{ll} & 0.08 \\
\{.96\end{array}\right\}$ & $\begin{array}{l}67 / 12 / 87 \\
10 / 15 / 87\end{array}$ & \\
\hline CONDFLD & ЧЕНО & 1.01 & & $07 / 30 / 87$ & $\underset{\mathrm{NR}}{338.110}$ & $\begin{array}{l}07 / 27 / 87 \\
10 / 15 / 67\end{array}$ & $\begin{array}{l}230.16 \\
251.16\end{array}$ & $10 / 15 / 87$ & 704.06 \\
\hline FBARIUY & PPB & 6.00 & 1000.01 & $07 / 38 / 87$ & ${ }_{\mathrm{MR}}^{32.00}$ & $\begin{array}{l}17 / 27 / 87 \\
16 / 15 / 87\end{array}$ & $\begin{array}{l}47.10 \\
30.01\end{array}$ & $10 / 15 / 87$ & OS. \\
\hline FCALCIU & $\mathrm{PPB}$ & 50.00 & & $67 / 30 / 87$ & 38989.08 & $\begin{array}{l}97 / 27 / 67 \\
10 / 15 / 87\end{array}$ & $\begin{array}{l}38200.08 \\
30300.00\end{array}$ & $10 / 15 / 87$ & $\underset{M R}{97684.06}$ \\
\hline FCHROYI & PPB & 10.00 & 58.06 & $67 / 30 / 87$ & ${ }_{\mathrm{NR}}^{151.08}$ & $\begin{array}{l}97 / 27 / 87 \\
18 / 15 / 87\end{array}$ & & $10 / 15 / 87$ & NR \\
\hline FLUORID & PPB & 500.00 & 4080.06 & $67 / 30 / 87$ & NR & $\begin{array}{l}07 / 27 / 87 \\
10 / 15 / 87\end{array}$ & & $10 / 15 / 87$ & B11.6 \\
\hline FUAGNES & PPB & 9.65 & & $67 / 36 / 87$ & $\begin{array}{l}\text { NR } \\
\text { OA86 }\end{array}$ & $\begin{array}{l}67 / 27 / 87 \\
10 / 15 / 87\end{array}$ & $\begin{array}{l}5980.00 \\
6640.06\end{array}$ & $10 / 15 / 87$ & 20800 \\
\hline FPOTASS & $P P B$ & 100.08 & & $67 / 36 / 87$ & 4386.106 & $07 / 27 / 87$ & 2080.00 & $10 / 15 / 87$ & 8190.00 \\
\hline
\end{tabular}

- VRLUE EXCEEDS PRIUARY DRINKING TATER STANDARD.
- VRLUE EXCEEDS PROPOSED PRIUARY DRINKING WATER STAKOARO.

* - YALUE EXCEES SCREENIMG LEVEL FOR FURTHER IHYESTIGATION.

- DETECTION LIMIT "aS NOT aYrilagle FOR COMPARISON

NR - ANALYSIS NOT REQUESTED OR NOT YET REPORTED

VALUES IN $\{$, ARE COUNTING ERRORS FOR RADIONUCLIOES

WATER STANDARD'(S) IN PARENTHESES ARE PROPOSED ONLY 
TABLE C.3. (contd)

\begin{tabular}{|c|c|c|c|c|c|}
\hline $\begin{array}{l}\text { CONST I } \\
\text { MAUE }\end{array}$ & $\begin{array}{l}\text { UENT } \\
\text { UNITS }\end{array}$ & $\begin{array}{c}\text { CORTRACTUAL } \\
\text { DETECTIOAN } \\
\text { LIUIT }\end{array}$ & $\begin{array}{l}\text { DRINKING } \\
\text { IATER } \\
\text { STANDARD }\end{array}$ & $\begin{array}{l}\text { SAUPLE } \\
\text { DATE }\end{array}$ & $1-D 8-3$ \\
\hline FPDTASS & PPB & 100.01 & & & NR \\
\hline FSDDIUV & PPB & 100.00 & & $17 / 30 / 87$ & ${ }_{N R}^{5050.06}$ \\
\hline FSTRONT & PPB & 310.06 & & $67 / 31 / 87$ & NR \\
\hline FYAHADI & PPB & 5.06 & & $17 / 30 / 87$ & MR \\
\hline FZINC & PPB & 5.10 & & $07 / 35 / 97$ & WR \\
\hline HNITRAT & PPB & 2546.61 & 45000.01 & & $\begin{array}{l}\text { NR } \\
\text { NR }\end{array}$ \\
\hline LFLUBRD & PPB & 20.01 & 4010.60 & $17 / 30 / 87$ & $\underset{\mathrm{NR}}{13 \mathrm{t} . \mathrm{OI}}$ \\
\hline MI TRATE & PPB & 501.80 & 45001.00 & $07 / 31 / 87$ & 27360.01 \\
\hline PH-LAB & & 6.1 & & $07 / 35 / 87$ & $\begin{array}{l}7.69 \\
\mathrm{NR}\end{array}$ \\
\hline PHFIELO & & 0.10 & & $07 / 36 / 87$ & 7.16 \\
\hline RU-106 & PCI $/ \mathrm{L}$ & 172.50 & 30.00 & $\begin{array}{l}67 / 30 / 87 \\
10 / 14 / 87\end{array}$ & \\
\hline SULFATE & PPB & 500.00 & & $97 / 30 / 87$ & 68060.10 \\
\hline TC & PPB & & & $07 / 30 / 87$ & $\underset{\mathrm{MR}}{13900.00}$ \\
\hline TOC & PPB & 1089.00 & & $07 / 39 / 87$ & \\
\hline
\end{tabular}

\begin{tabular}{|c|c|}
\hline $\begin{array}{l}\text { SAIPLE } \\
\text { DATE }\end{array}$ & $1-F 5-1$ \\
\hline $10 / 15 / 87$ & 2580.10 \\
\hline $\begin{array}{l}17 / 27 / 87 \\
10 / 16 / 87\end{array}$ & $\begin{array}{l}3506.06 \\
4246.16\end{array}$ \\
\hline $\begin{array}{l}07 / 27 / 87 \\
10 / 16 / 87\end{array}$ & \\
\hline $\begin{array}{l}97 / 27 / 87 \\
10 / 16 / 87\end{array}$ & 7.00 \\
\hline \multirow[t]{2}{*}{$\begin{array}{l}07 / 27 / 87 \\
11 / 15 / 87\end{array}$} & \\
\hline & $\begin{array}{l}\text { MR } \\
\text { NR }\end{array}$ \\
\hline $\begin{array}{l}97 / 27 / 87 \\
10 / 16 / 87\end{array}$ & 116.6 \\
\hline $\begin{array}{l}67 / 27 / 87 \\
10 / 15 / 87\end{array}$ & $\begin{array}{r}7800.00 \\
14340.04\end{array}$ \\
\hline $\begin{array}{l}07 / 27 / 87 \\
10 / 16 / 87\end{array}$ & 7.46 \\
\hline $\begin{array}{l}07 / 27 / 87 \\
11 / 16 / 87\end{array}$ & $\begin{array}{l}8.20 \\
7.00\end{array}$ \\
\hline \multicolumn{2}{|l|}{$\begin{array}{l}17 / 27 / 87 \\
10 / 16 / 87\end{array}$} \\
\hline $\begin{array}{l}97 / 27 / 87 \\
10 / 16 / 87\end{array}$ & $\begin{array}{l}24560.01 \\
20960.10\end{array}$ \\
\hline $\begin{array}{l}07 / 27 / 87 \\
16 / 16 / 87\end{array}$ & 24301.60 \\
\hline $07 / 27 / 87$ & \\
\hline
\end{tabular}

\begin{tabular}{|c|c|}
\hline $\begin{array}{l}\text { SALPLE } \\
\text { DATE }\end{array}$ & $1-F 5-4$ \\
\hline & NR \\
\hline $16 / 15 / 87$ & 23214.00 \\
\hline $18 / 16 / 87$ & $\underset{M R}{787.06}$ \\
\hline $10 / 15 / 87$ & 16.10 \\
\hline $10 / 16 / 87$ & $\begin{array}{l}\text { 8.61 } \\
M R\end{array}$ \\
\hline $\begin{array}{l}17 / 12 / 87 \\
10 / 15 / 87\end{array}$ & $\begin{array}{l}04145.10 \\
68104.160\end{array}$ \\
\hline $10 / 16 / 87$ & $\underset{\mathrm{MR}}{165.16}$ \\
\hline $10 / 15 / 87$ & BQBSE. WR \\
\hline $19 / 18 / 87$ & $\mathrm{NR}$ \\
\hline $10 / 16 / 87$ & ${ }_{M R} \cdot 46$ \\
\hline $\begin{array}{l}17 / 102 / 87 \\
10 / 15 / 87\end{array}$ & $\left\{\begin{array}{l}56.00 \\
\{8.20\}\end{array}\right.$ \\
\hline $10 / 16 / 87$ & 125019. 10 \\
\hline $10 / 16 / 87$ & $4750 \mathrm{MR} .6 \mathrm{~s}$ \\
\hline $10 / 16 / 87$ & 1986.00 \\
\hline
\end{tabular}

* - value exceeds primary drinking tater stamdard.

- - VALUE EXCEEDS PROPOSED PRIMARY DRINKING TATER STAMDARO.

* - VALUE EXCEEDS SCREENING LEVEL FDR FURTHER INYESTIOATIDOH.

- detection linit ias not available for couparison

MR - ANALYSIS NOT RERUESTED OR MDT YET REPORTED

VALUES IN \{\} ARE COUNTING ERRORS FOR RADIDNUCLIDES

IATER STANOARD(S) IN PARENTHESES ARE PRDPOSED OKLY 
TABLE C.3. (contd)

\begin{tabular}{|c|c|c|c|c|c|c|c|c|c|}
\hline $\begin{array}{l}\text { CONSTI } \\
\text { NAME }\end{array}$ & $\begin{array}{l}\text { UENT } \\
\text { UNI TS }\end{array}$ & $\begin{array}{l}\text { CDNTRACTUAL } \\
\text { DETECTION } \\
\text { LIMIT }\end{array}$ & $\begin{array}{l}\text { ORINK ING } \\
\text { TATER } \\
\text { STANDARO }\end{array}$ & $\begin{array}{l}\text { SAUPLE } \\
\text { DATE }\end{array}$ & $1-D 8-3$ & $\begin{array}{c}\text { SAMPLE } \\
\text { DATE }\end{array}$ & $1-F 5-1$ & $\begin{array}{c}\text { SAMPLE } \\
\text { DATE }\end{array}$ & $1-F 5-4$ \\
\hline TOC & PPB & 1018.11 & & & MR & $18 / 15 / 87$ & & & NR \\
\hline TOXLDL & PPB & 20.06 & & $17 / 36 / 87$ & MR & $\begin{array}{l}17 / 27 / 87 \\
16 / 15 / 87\end{array}$ & & $10 / 15 / 87$ & 22.96 \\
\hline TRITIUN & $\mathrm{PCI} / \mathrm{L}$ & 506.66 & 20060.16 & $\begin{array}{l}17 / 36 / 87 \\
10 / 14 / 87\end{array}$ & $\begin{array}{r}4130.4 \\
271.01\} \\
4381.00 \\
\{321.06\}\end{array}$ & $\begin{array}{l}07 / 27 / 87 \\
11 / 15 / 87\end{array}$ & $\left\{\begin{array}{l}841.06 \\
177.11\end{array}\right\}$ & $\begin{array}{l}17 / 02 / 87 \\
16 / 16 / 87\end{array}$ & 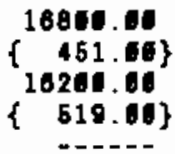 \\
\hline U & $\mathrm{PCI} / \mathrm{L}$ & 1.50 & 005.11 & & $\begin{array}{l}\text { NR } \\
\text { NR }\end{array}$ & $\begin{array}{l}67 / 27 / 87 \\
10 / 16 / 87\end{array}$ & $\begin{array}{l}0.75 \\
0.88\end{array}$ & $\begin{array}{l}07 / 12 / 87 \\
10 / 15 / 87\end{array}$ & $\begin{array}{l}6.44 \\
7.12\end{array}$ \\
\hline
\end{tabular}

$\varliminf_{\infty}^{\infty}$

* - Value exceEds PRIMary dRINKINg tater standard.

- VAlUe EXCEEDS PROPDSED PRIUARY DRIMKING Mater STANDARD.

* VALUE EXCEEDS SCREENIMG LEVEL FOR FURTHER INVESTIGATION

- DETECTIOH LIUIT IAS HDT AVAILABLE FOR COMPARISON

NR - ANALYSIS NDT RERUESTED OR NOT YET REPORTED

VALUES IN \& ARE COUNTING ERRORS FOR RADIONUCLIDES

TATER STANDARD(S) IN PARENTHESES ARE PROPOSED ONLY 
TABLE C.3. (contd)

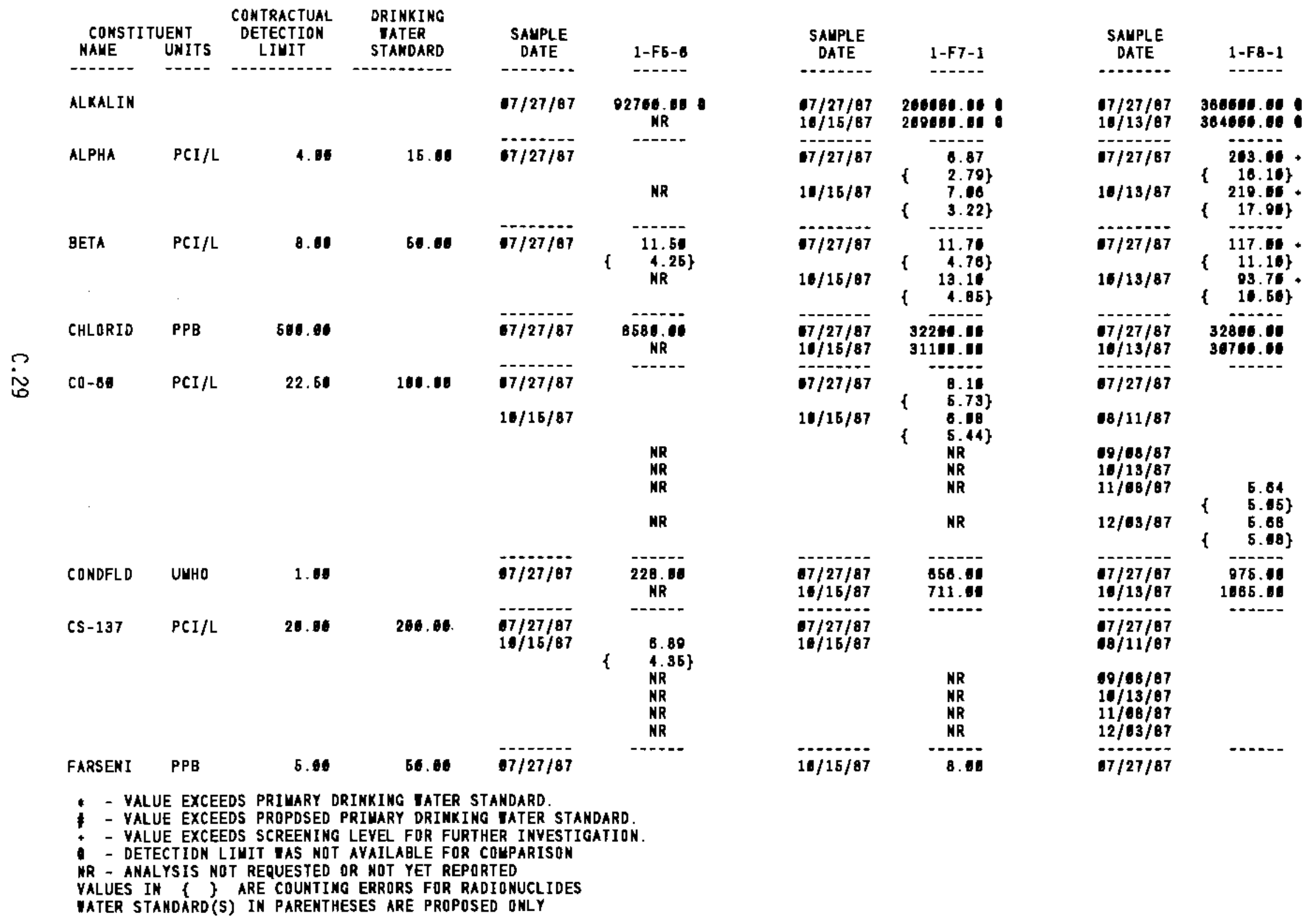


TABLE C.3. (contd)

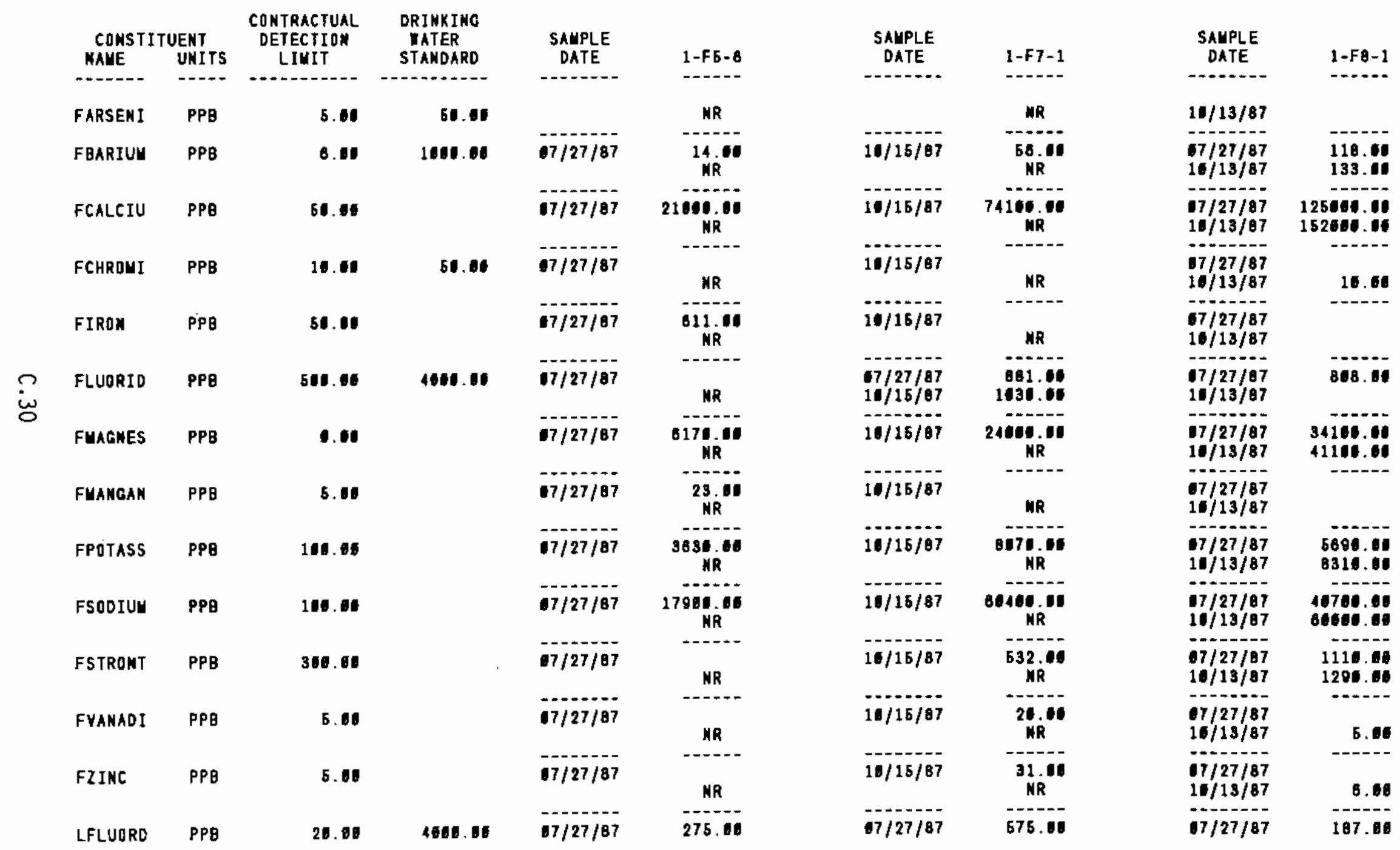

- - Value exceEds privary drinking iater standaro.

- VALUE EXCEEDS PROPOSED PRIHARY DRINKING TATER STANDARD

- YALUE EXCEEDS SCREENING LEYEL FOR FURTHER INYESTIGATIOH.

- - DETECTION LIUIT WAS NOT AVALABLE FOR COUPARISON

NR - ANALYSIS HOT REQUESTED OR NOT YET REPORTED

VALUES IN $\{$ ? ARE COUNTING ERRDRS FOR RADIONUCLIDES

IATER STAMDARD'S) IN PARENTHESES ARE PRDPOSEO DNLY 
TABLE C.3. (contd)

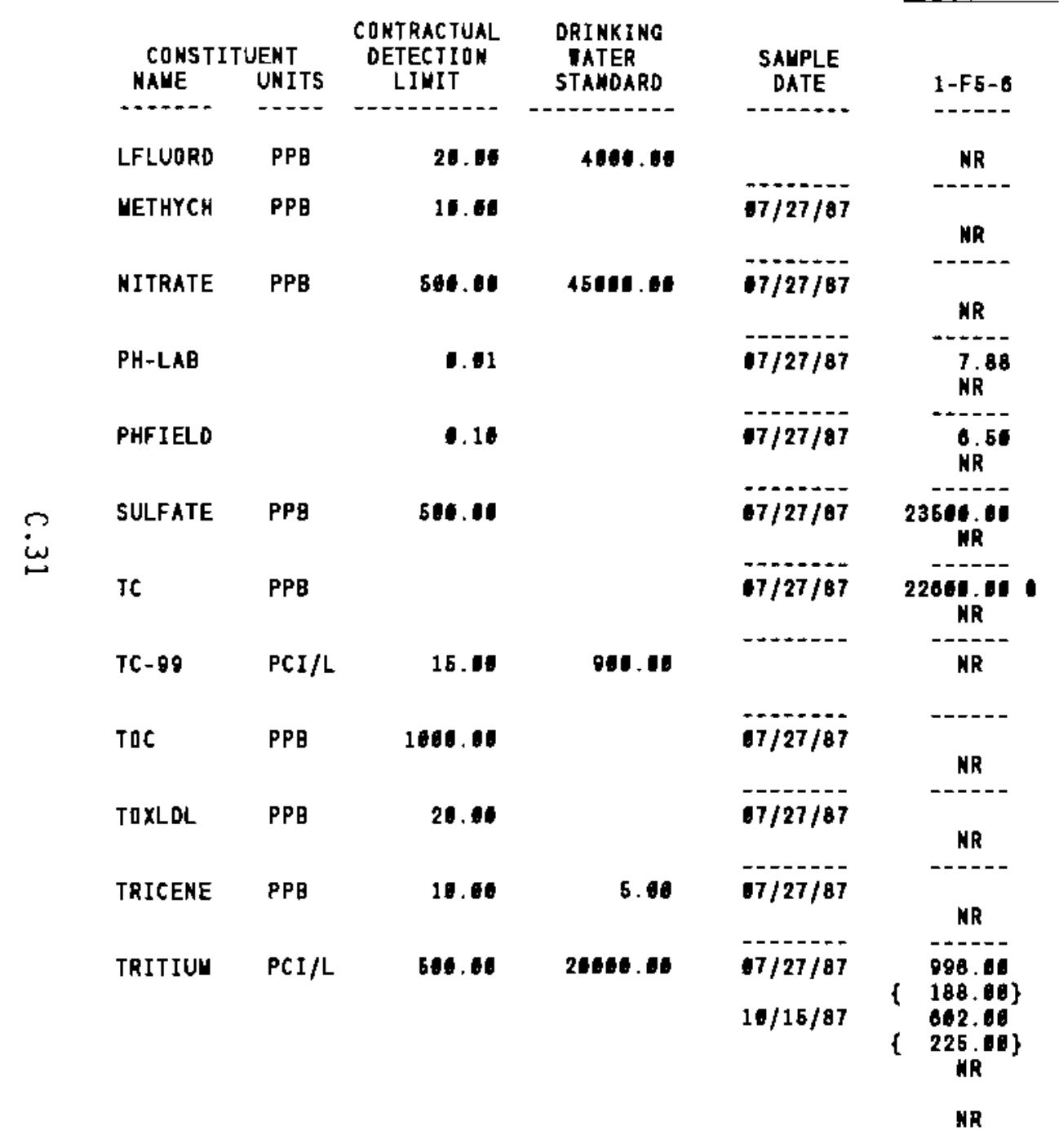

\begin{tabular}{|c|c|c|c|}
\hline $\begin{array}{c}\text { SAHPLE } \\
\text { DATE }\end{array}$ & $1-F 7-1$ & $\begin{array}{c}\text { SANPLE } \\
\text { DATE }\end{array}$ & $1-F B-1$ \\
\hline$--\cos$ & 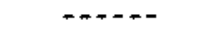 & ----n-- & 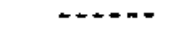 \\
\hline $10 / 15 / 87$ & 600.00 & $10 / 13 / 87$ & 197.6 \\
\hline 4797107 & ----- & $-2-1-18$ & ----- \\
\hline $\begin{array}{l}7 / 27 / 87 \\
10 / 16 / 87\end{array}$ & 34.04 & $10 / 13 / 87$ & \\
\hline 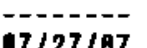 & 051"-- & 87127187 & 1849 \\
\hline $10 / 16 / 87$ & 104600.00 & $10 / 19 / 87$ & 218004.01 \\
\hline $67 / 27 / 87$ & 7.38 & $07 / 27 / 97$ & 7.51 \\
\hline $10 / 15 / 87$ & 7.57 & $16 / 13 / 87$ & 7.57 \\
\hline 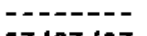 & ------ & 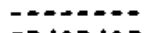 & 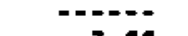 \\
\hline $\begin{array}{l}67 / 27 / 87 \\
16 / 15 / 87\end{array}$ & $\begin{array}{l}6.50 \\
7.20\end{array}$ & $\begin{array}{l}07 / 27 / 87 \\
10 / 13 / 87\end{array}$ & $\begin{array}{l}7.60 \\
7.70\end{array}$ \\
\hline 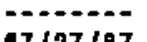 & - & 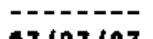 & $\overline{-1--\overline{-}}$ \\
\hline $\begin{array}{l}7 / 27 / 87 \\
10 / 15 / 87\end{array}$ & $\begin{array}{l}87401.00 \\
88640.04\end{array}$ & $\begin{array}{l}7 / 27 / 87 \\
10 / 13 / 87\end{array}$ & 89300. \\
\hline $17 / 27 / 87$ & 51000.00 & $07 / 27 / 87$ & B2800.11 \\
\hline $10 / 15 / 87$ & 524100.00 & $10 / 13 / 87$ & Bosue.16 \\
\hline \multirow{2}{*}{$\cdots$} & 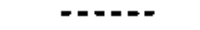 & ----n-- & \multirow{3}{*}{$\begin{array}{c}4.05 \\
1.08\}\end{array}$} \\
\hline & NR & $67 / 27 / 87$ & \\
\hline -1---1-- & $\overline{---0}$ & $-8-1-1$ & \\
\hline $\begin{array}{l}17 / 27 / 87 \\
10 / 16 / 87\end{array}$ & $\begin{array}{l}2360.00 \\
1760.00\end{array}$ & $\begin{array}{l}67 / 27 / 87 \\
10 / 13 / 87\end{array}$ & $\begin{array}{l}3030.41 \\
3660.01\end{array}$ \\
\hline $07 / 27 / 87$ & 22.30 & $07 / 27 / 87$ & 79.1 \\
\hline - & $\cdots-$ & ------ & - \\
\hline $\begin{array}{l}67 / 27 / 87 \\
16 / 15 / 87\end{array}$ & $\begin{array}{l}16.36 \% \\
12.06 \%\end{array}$ & $\begin{array}{l}07 / 27 / 87 \\
16 / 13 / 87\end{array}$ & \\
\hline $67 / 27 / 97$ & 414 & $17127 / 67$ & 13890 \\
\hline & $170.06\}$ & & $\{\quad 478.65\}$ \\
\hline $10 / 16 / 87$ & & $08 / 11 / 87$ & 12900.60 \\
\hline & NR & $09 / 08 / 87$ & 12800.00 \\
\hline & NR & $18 / 13 / 87$ & $\left\{\begin{array}{r}11801.60 \\
451.00\}\end{array}\right.$ \\
\hline
\end{tabular}

* - YaLUE EXCEEDS PRIUARY DRINKING TATER STANDARo.

* - VALUE EXCEEDS PROPOSED PRIMARY DRINKING TATER STAMDARD.

+ - VALUE EXCEEDS SCREENING LEYEL FOR FURTHER INVESTIGATION.

- DETECTION LIUIT TAS NOT AYAILABLE FOR COUPARISON

MR - ANALYSIS WOT REQUESTED OR NOT YET REPORTED

YALUES IN \& ? ARE COUNTING ERRORS FOR RADIONUCLIDES

WATER STANDARD'(S) IN PARENTHESES ARE PROPOSED ONLY 
TABLE C.3. (contd)

\begin{tabular}{|c|c|c|c|c|c|c|c|c|c|}
\hline $\begin{array}{l}\text { CONSTI } \\
\text { MAUE }\end{array}$ & $\begin{array}{l}\text { UENT } \\
\text { UNITS }\end{array}$ & $\begin{array}{c}\text { CONTRACTUAL } \\
\text { DETECTION } \\
\text { LIUIT }\end{array}$ & $\begin{array}{l}\text { ORINKING } \\
\text { TATER } \\
\text { STANDARO }\end{array}$ & $\begin{array}{l}\text { SAYPLE } \\
\text { DATE }\end{array}$ & $1-F 5-6$ & $\begin{array}{l}\text { SAUPLE } \\
\text { DATE }\end{array}$ & $1-F 7-1$ & $\begin{array}{l}\text { SAMPLE } \\
\text { DATE }\end{array}$ & $1-F \theta-1$ \\
\hline------ & --- & --------- & 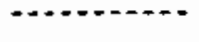 & ---n-- & ---- & - & 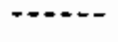 & 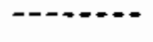 & 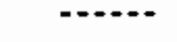 \\
\hline \multirow[t]{2}{*}{ TRITIUU } & $\mathrm{PCI} / \mathrm{L}$ & 600. 00 & 20000.10 & & NR & & NR & $11 / 08 / 87$ & $\left\{\begin{array}{l}10280.04 \\
432.46\}\end{array}\right.$ \\
\hline & & & & & MR & & MR & $12 / 03 / 87$ & $\begin{array}{r}10064.64 \\
\{\quad 428.64\}\end{array}$ \\
\hline$U$ & $\mathrm{PCI} / \mathrm{L}$ & 0.51 & 600.00 & $\begin{array}{l}07 / 27 / 87 \\
10 / 16 / 87\end{array}$ & 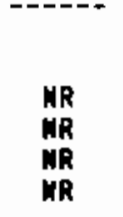 & $\begin{array}{l}07 / 27 / 87 \\
16 / 15 / 87\end{array}$ & $\begin{array}{l}4.80 \\
6.11 \\
\text { MR } \\
\text { MR } \\
\text { MR } \\
\text { MR }\end{array}$ & $\begin{array}{l}07 / 27 / 67 \\
60 / 11 / 87 \\
60 / 00 / 07 \\
10 / 13 / 87 \\
11 / 08 / 87 \\
12 / 03 / 87\end{array}$ & $\begin{array}{l}321.01 \\
343.40 \\
362.09 \\
334.01 \\
324.01 \\
336.46\end{array}$ \\
\hline UNKNOYN & PPB & 1.61 & & ------ & NR & - & NR & $10 / 13 / 87$ & 24.4 \\
\hline
\end{tabular}

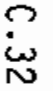

* - Value eXceEdS PRIMARY dRINKing tater stakdaro.

* - VALUE EXCEEDS PRDPOSED PRIUARY DRIMKING TATER STANDARO.

* - VALUE EXCEEDS SCREENINO LEVEL FOR FURTHER INVESTIGATION.

- - DETECTION LIUIT TAS NOT aYAILABLE FOR COUPARISON

NR - ANALYSIS NOT REQUESTED OR NDT YET REPORTED

YALUES IN \{\} ARE COUNTING ERRORS FOR RADIONUCLIDES

TATER STAMDARD(S) IN PARENTHESES ARE PROPOSED ONLY 
TABLE C.3. (contd)

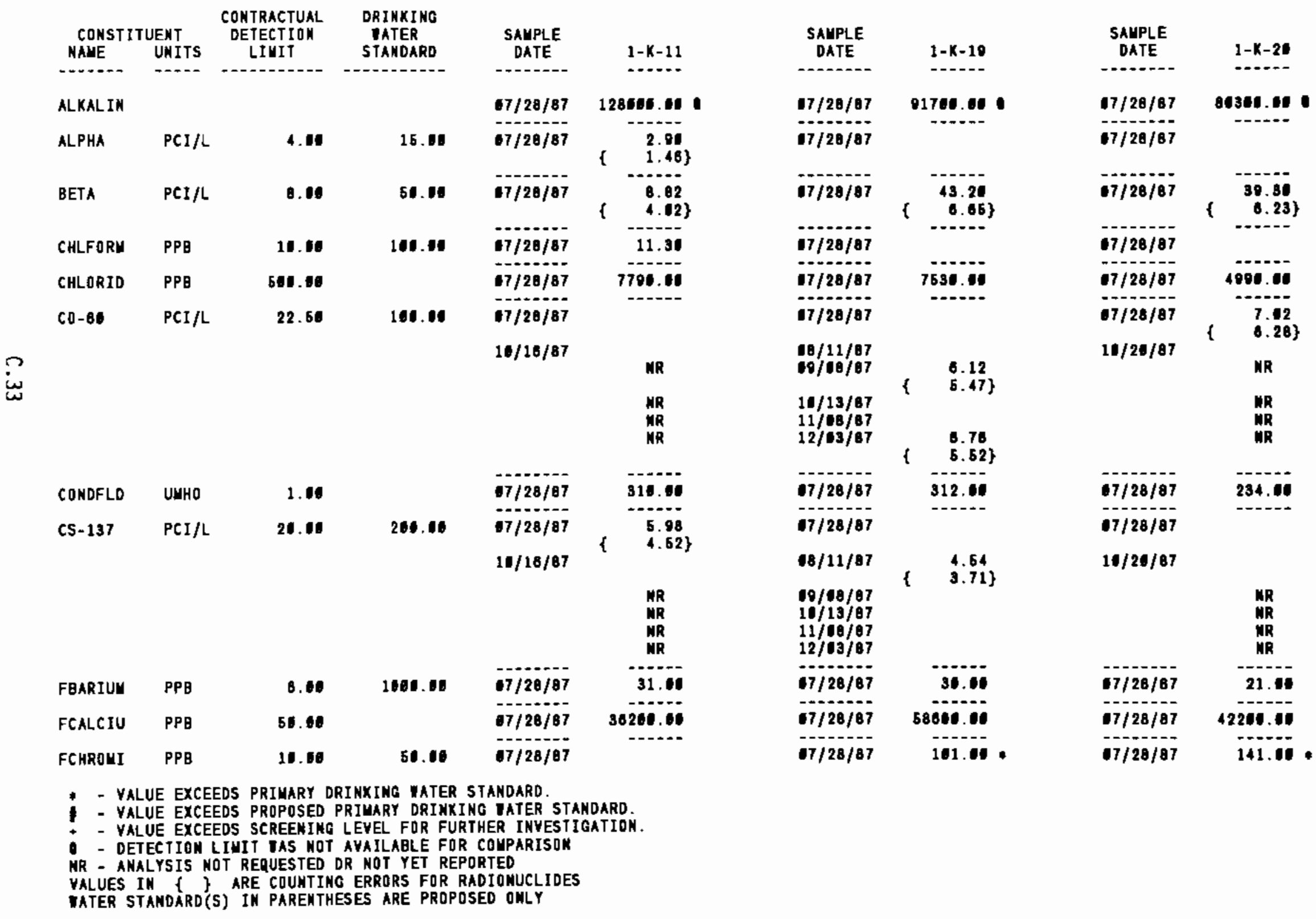


TABLE C.3. (contd)

\begin{tabular}{|c|c|c|c|c|c|}
\hline \multicolumn{2}{|c|}{ CONST ITUEAT } & $\begin{array}{c}\text { COHTRACTUAL } \\
\text { DETECTIDN } \\
\text { LIUIT }\end{array}$ & $\begin{array}{l}\text { DRINKING } \\
\text { VATER } \\
\text { STAKDARD }\end{array}$ & $\begin{array}{c}\text { SAUPLE } \\
\text { DATE }\end{array}$ & $1-x-11$ \\
\hline------- & $\cdots+$ & 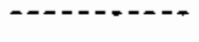 & --n--nen & $---\infty-\infty$ & ---- \\
\hline FLUORID & PPB & 500.00 & 4000.00 & $07 / 20 / 87$ & 671.00 \\
\hline FUAGNES & PPB & 00 & & $67 / 28 / 07$ & 10800.00 \\
\hline FPDTASS & PPB & 100.00 & & $67 / 28 / 07$ & 5440.00 \\
\hline FSODIUU & PPB & 100.00 & & $67 / 2 \theta / 07$ & 32166.00 \\
\hline FVANADI & PPB & 6. 10 & & $67 / 28 / 07$ & 26.00 \\
\hline FZINC & PPB & 5.110 & & $17 / 2 \theta / 07$ & \\
\hline LFLUDRO & PPB & 26.10 & 450. 00 & $67 / 28 / 07$ & 446.00 \\
\hline NITRATE & PPB & 564. 41 & 46501.00 & $07 / 28 / 07$ & 48206.00 \\
\hline PH-LAB & & 61 & & $67 / 28 / 87$ & 7.80 \\
\hline PHFIELD & & 0.10 & & $07 / 28 / 87$ & 7.10 \\
\hline SULFATE & PPB & 580.06 & & $07 / 2 \theta / \theta 7$ & 42006.00 \\
\hline TC & PPB & & & $07 / 2 \theta / 07$ & 39300.00 \\
\hline TOC & PPB & 1000.00 & & $17 / 20 / 87$ & 1700.00 \\
\hline TRITIUH & $\mathrm{PCI} / \mathrm{L}$ & 560.09 & 20080.60 & $\begin{array}{l}17 / 20 / 87 \\
10 / 10 / 87\end{array}$ & 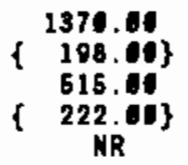 \\
\hline & & & & & NR \\
\hline & & & & & NR \\
\hline & & & & & NR \\
\hline
\end{tabular}

\begin{tabular}{|c|c|c|c|}
\hline $\begin{array}{l}\text { SAUPLE } \\
\text { DATE }\end{array}$ & $1-k-10$ & $\begin{array}{c}\text { SAMPLE } \\
\text { DATE }\end{array}$ & $1-K-20$ \\
\hline $17 / 2 \theta / 87$ & & $17 / 28 / 87$ & \\
\hline $17 / 28 / 87$ & 9161.14 & $17 / 29 / 87$ & 7430.10 \\
\hline $07 / 28 / 87$ & 2274.11 & $17 / 28 / 87$ & 2010.10 \\
\hline $07 / 28 / 87$ & 4911.11 & $07 / 28 / 87$ & 3700.10 \\
\hline $07 / 28 / 87$ & & $07 / 28 / 87$ & \\
\hline $07 / 28 / 87$ & 117.10 & $07 / 28 / 87$ & \\
\hline $07 / 28 / 87$ & 103.01 & $07 / 28 / 87$ & 112.00 \\
\hline $07 / 28 / 87$ & 68900.01. & $17 / 28 / 87$ & 20700.10 \\
\hline $07 / 28 / 87$ & 7.88 & $07 / 28 / 87$ & 0.10 \\
\hline $07 / 28 / 87$ & 7.11 & $17 / 28 / 87$ & 7.30 \\
\hline $07 / 28 / 87$ & Ge104.65 & $17 / 28 / 87$ & 50800.60 \\
\hline $07 / 2 \theta / 87$ & 18245.60 & $17 / 28 / 87$ & 18740.0 \\
\hline $07 / 2 \theta / 97$ & & $17 / 28 / 07$ & \\
\hline $\begin{array}{l}07 / 2 \theta / 87 \\
10 / 11 / 87 \\
10 / 68 / 87\end{array}$ & 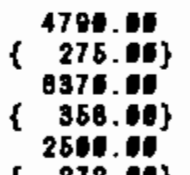 & $\begin{array}{l}17 / 28 / 87 \\
15 / 24 / 67\end{array}$ & 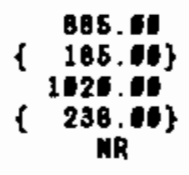 \\
\hline $16 / 13 / 87$ & $\begin{array}{r}272.00\} \\
4405.00 \\
323.00\}\end{array}$ & & MR \\
\hline $11 / 68 / 87$ & $\left\{\begin{array}{r}5106.00 \\
\{333.01\}\end{array}\right.$ & & NR \\
\hline $12 / 03 / 87$ & ( 337.06$\}$ & & NR \\
\hline
\end{tabular}

* - VALUe eXCEEdS PRIUARY dRINKING taier standard.

- VALUE EXCEEDS PRDPOSED PRIVARY DRINKIHG IATER STANDARD.

- - YALUE EXCEEDS SCREENINO LEVEL FOR FURTHER INVESTIGATION.

- DETECTION LIMIT TAS NOT AYAILABLE FOR COUPARISDN

NR - ANALYSIS NOT REQUESTED DR NOT YET REPORTED

VALUES IN I I ARE CDUNTING ERRORS FOR RADIONUCLIDES

VALUES IN $\{$ '
WATER STAKDARD'(S) IN PARENTHESES ARE PROPOSED ONLY 
TABLE C.3. (contd)

\begin{tabular}{|c|c|c|c|c|c|c|c|c|c|}
\hline $\begin{array}{l}\text { CONSTI } \\
\text { NAHE }\end{array}$ & $\begin{array}{l}\text { UENT } \\
\text { UNITS }\end{array}$ & $\begin{array}{c}\text { CONTRACTUAL } \\
\text { DETECTION } \\
\text { LILIT }\end{array}$ & $\begin{array}{l}\text { DRINKING } \\
\text { WATER } \\
\text { STANDARD }\end{array}$ & $\begin{array}{c}\text { SAYPLE } \\
\text { DATE }\end{array}$ & $1-K-11$ & $\begin{array}{l}\text { SAUPLE } \\
\text { DATE }\end{array}$ & $1-K-10$ & $\begin{array}{l}\text { SAHPLE } \\
\text { DATE }\end{array}$ & $1-k-20$ \\
\hline$-\cdots$ & ---- & 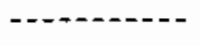 & 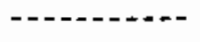 & $\cdots$ & $-\cdots-1$ & $---n$ & $-\cdot----$ & $---\infty-$ & $\cdots---$ \\
\hline U & $\mathrm{PCI} / \mathrm{L}$ & 6.56 & 601.00 & $\begin{array}{l}07 / 28 / 07 \\
10 / 16 / 87\end{array}$ & $\begin{array}{l}3.15 \\
3.74 \\
\text { NR } \\
\text { NR } \\
\text { NR } \\
\text { NR }\end{array}$ & $\begin{array}{l}17 / 28 / 87 \\
68 / 11 / 87 \\
10 / 68 / 87 \\
10 / 13 / 87 \\
11 / 60 / 87 \\
12 / 83 / 87\end{array}$ & $\begin{array}{r}1.80 \\
1.60 \\
0.77 \\
.85 \\
1.70 \\
.82\end{array}$ & $\begin{array}{l}67 / 28 / 87 \\
10 / 20 / 87\end{array}$ & $\begin{array}{l}1.98 \\
0.97 \\
\text { MR } \\
\text { MR } \\
\text { NR } \\
\text { NR }\end{array}$ \\
\hline
\end{tabular}

$\stackrel{?}{w}$

* - Value exceEds priuary orinking tater standard.

- VALUE EXCEEDS PROPOSED PRIMARY DRINKING TATER STAMDARD.

- VALUE EXCEEDS SCREENIMG LEVEL FDR FURTHER IUVESTIGATION.

- DETECTION LIUIT TAS NOT AVAILABLE FOR COUPARISON

NR - ANALYSIS NDT REQUESTED DR NOT YET REPORTED

VALUES IN \{ \} ARE CDUNTING ERRORS FOR RADIOHUCLIDES

IATER STANDARD'S) IN PARENTHESES ARE PROPOSED ONLY 
TABLE C.3. (contd)

\begin{tabular}{|c|c|c|c|c|c|}
\hline$\underbrace{\text { CONST I }}_{\text {MAUE }}$ & $\begin{array}{l}\text { UENT } \\
\text { UNITS }\end{array}$ & $\begin{array}{c}\text { CORTRACTUAL } \\
\text { DETECTION } \\
\text { LINIT }\end{array}$ & $\begin{array}{l}\text { DRINKING } \\
\text { IATER } \\
\text { STAKDARD }\end{array}$ & $\begin{array}{c}\text { SAUPLE } \\
\text { DATE }\end{array}$ & $1-K-22$ \\
\hline ALKALIN & & & & $\begin{array}{l}07 / 28 / B 7 \\
10 / 20 / B 7\end{array}$ & $\begin{array}{l}82701.01 \\
91706.10\end{array}$ \\
\hline ALPHA & $\mathrm{PCI} / \mathrm{L}$ & 4.60 & 15.00 & $\begin{array}{l}17 / 28 / 87 \\
16 / 20 / 87\end{array}$ & $\begin{array}{l}1.47 \\
\{\quad 1.08\}\end{array}$ \\
\hline AUUONIU & PPB & 50.00 & & $\begin{array}{l}07 / 28 / 87 \\
10 / 26 / 87\end{array}$ & 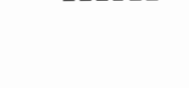 \\
\hline BETA & PCI/L & 8.10 & 50.60 & $\begin{array}{l}17 / 28 / 87 \\
16 / 21 / 87\end{array}$ & $\left\{\begin{array}{r}14.20 \\
4.41\} \\
14.10 \\
4.30\}\end{array}\right.$ \\
\hline CHLFORU & PPB & 10.00 & 101.00 & $\begin{array}{l}17 / 28 / 87 \\
10 / 26 / 87\end{array}$ & \\
\hline CHLORID & PPB & 506.01 & & $\begin{array}{l}07 / 28 / 87 \\
10 / 20 / 87\end{array}$ & $\begin{array}{l}3984.10 \\
4186.16\end{array}$ \\
\hline $60-60$ & PCI/L & 22.50 & 186.60 & $\begin{array}{l}17 / 28 / 87 \\
67 / 28 / 87 \\
10 / 21 / 87 \\
10 / 21 / 87\end{array}$ & $\begin{array}{l}\quad 6.99 \\
\{\quad 5.45\}\end{array}$ \\
\hline CONDFLD & UUHO & 1.68 & & $\begin{array}{l}17 / 28 / 87 \\
10 / 26 / 87\end{array}$ & $\begin{array}{l}2109.01 \\
277.66\end{array}$ \\
\hline$C S=137$ & PCI/L & 20.00 & 201.00 & $\begin{array}{l}17 / 28 / 87 \\
07 / 28 / 87 \\
10 / 20 / 87 \\
10 / 20 / 87\end{array}$ & 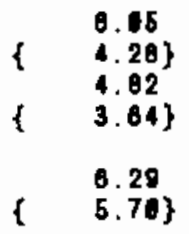 \\
\hline
\end{tabular}

\begin{tabular}{|c|c|}
\hline $\begin{array}{c}\text { SAMPLE } \\
\text { DATE }\end{array}$ & $1-K-27$ \\
\hline $\begin{array}{l}07 / 29 / 97 \\
10 / 16 / 87\end{array}$ & $\begin{array}{l}117004.01 \\
122000.00\end{array}$ \\
\hline $07 / 20 / 87$ & 4.20 \\
\hline $16 / 16 / 87$ & $\begin{array}{l}3.11 \\
1.52\}\end{array}$ \\
\hline $\begin{array}{l}97 / 29 / 87 \\
10 / 10 / 87\end{array}$ & 65.10 \\
\hline $07 / 29 / 87$ & 21.64 \\
\hline $10 / 18 / \theta 7$ & $\begin{array}{r}\quad 35.71 \\
\{\quad 6.10\}\end{array}$ \\
\hline $\begin{array}{l}07 / 29 / 87 \\
10 / 10 / 87\end{array}$ & $\begin{array}{l}13.11 \\
14.04\end{array}$ \\
\hline $\begin{array}{l}07 / 20 / 87 \\
16 / 18 / 87\end{array}$ & $\begin{array}{l}5506.00 \\
\text { 883t.06 }\end{array}$ \\
\hline \multirow[t]{2}{*}{$\begin{array}{l}17 / 29 / 87 \\
10 / 16 / 87\end{array}$} & NR \\
\hline & MR \\
\hline $\begin{array}{l}07 / 29 / 87 \\
16 / 18 / 87\end{array}$ & $\begin{array}{l}287.00 \\
350.66\end{array}$ \\
\hline \multicolumn{2}{|l|}{$67 / 29 / 87$} \\
\hline \multicolumn{2}{|l|}{$10 / 16 / 87$} \\
\hline & $\begin{array}{l}\text { NR } \\
\text { NR }\end{array}$ \\
\hline
\end{tabular}

\begin{tabular}{|c|c|}
\hline $\begin{array}{l}\text { SALPLE } \\
\text { DATE }\end{array}$ & $1-K-2 B$ \\
\hline $17 / 29 / 87$ & 102060.06 \\
\hline $07 / 29 / 87$ & $\left\{\begin{array}{l}3.35 \\
1.51\} \\
\mathrm{NR}\end{array}\right.$ \\
\hline $07 / 29 / 87$ & MR \\
\hline $7 / 20 / 87$ & $\left\{\begin{array}{c}19.58 \\
\mathrm{NR}\end{array}\right.$ \\
\hline $07 / 20 / 87$ & NR \\
\hline $17 / 28 / 87$ & 11451.10 \\
\hline $\begin{array}{l}67 / 29 / 97 \\
16 / 16 / 87\end{array}$ & NR \\
\hline & MR \\
\hline $07 / 28 / 87$ & 202.01 \\
\hline $07 / 29 / 87$ & \\
\hline $16 / 16 / 87$ & \\
\hline & $\begin{array}{l}\text { NR } \\
\text { NR }\end{array}$ \\
\hline
\end{tabular}

* - Value exceeds priuary drinking iater standard.

- VALUE EXCEEDS PROPOSED PRIUARY dRIMKIKG taTER STARDARD.

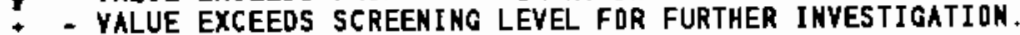

- Detection linit tas not ayallable for couparisom

NR - AKALYSIS NOT REQUESTED OR MOT YET REPORTED

VALUES IM $\{$ ARE COUNTIMG ERRORS FOR RADIONUCLIDES

IATER STANDARD'(S) IN PARENTHESES ARE PROPOSED OHLY 
TABLE C. 3. (contd)

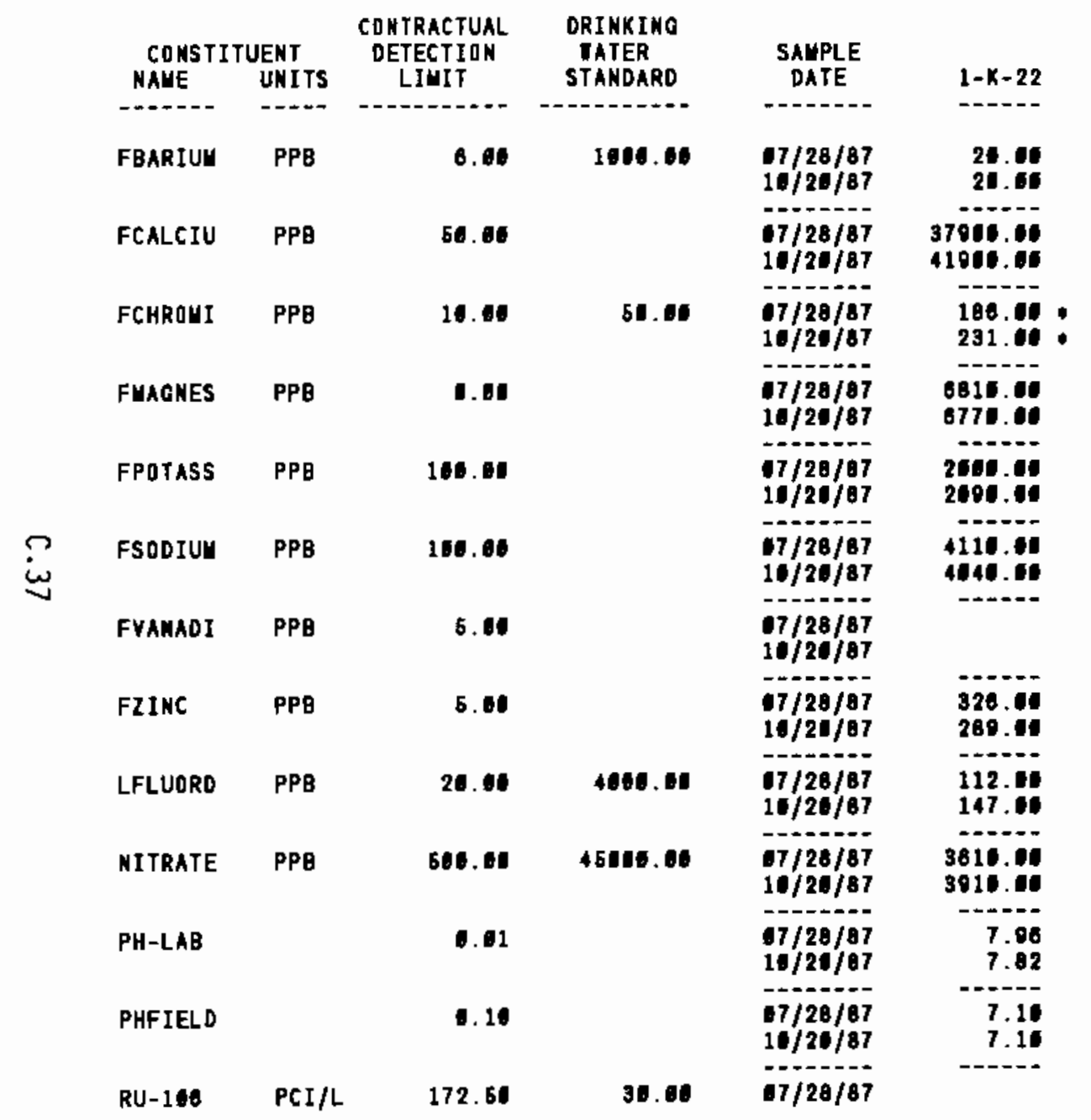

\begin{tabular}{|c|c|}
\hline $\begin{array}{c}\text { SAHPLE } \\
\text { DATE }\end{array}$ & $1-K-27$ \\
\hline 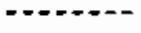 & ----- \\
\hline $\begin{array}{l}07 / 20 / 87 \\
10 / 10 / 87\end{array}$ & $\begin{array}{l}22.00 \\
24.00\end{array}$ \\
\hline $\begin{array}{l}17 / 20 / 97 \\
10 / 16 / 97\end{array}$ & $\begin{array}{l}398101.10 \\
43010.10\end{array}$ \\
\hline $\begin{array}{l}17 / 20 / 87 \\
19 / 16 / 87\end{array}$ & \\
\hline $\begin{array}{l}07 / 20 / 87 \\
10 / 16 / 87\end{array}$ & $\begin{array}{l}8174.01 \\
0665.01\end{array}$ \\
\hline $\begin{array}{l}57 / 29 / 07 \\
10 / 10 / 07\end{array}$ & 4780.06 \\
\hline $\begin{array}{l}17 / 20 / 87 \\
10 / 16 / 107\end{array}$ & $\begin{array}{l}-1-0 . \\
7050.00 \\
7080.00\end{array}$ \\
\hline $\begin{array}{l}07 / 20 / 67 \\
10 / 16 / 67\end{array}$ & $\begin{array}{r}7.11 \\
11.15\end{array}$ \\
\hline $\begin{array}{l}17 / 20 / 97 \\
16 / 18 / 07\end{array}$ & 6.00 \\
\hline $\begin{array}{l}07 / 20 / 107 \\
16 / 16 / 87\end{array}$ & $\begin{array}{l}187.01 \\
107.00\end{array}$ \\
\hline $\begin{array}{l}07 / 20 / 87 \\
11 / 16 / 87\end{array}$ & 7605.64 \\
\hline $\begin{array}{l}17 / 20 / 87 \\
16 / 16 / 87\end{array}$ & $\begin{array}{r}7.61 \\
7.79\end{array}$ \\
\hline $\begin{array}{l}07 / 20 / 87 \\
10 / 10 / 87\end{array}$ & 7.01 \\
\hline $17 / 20 / 87$ & \\
\hline
\end{tabular}

\begin{tabular}{|c|c|}
\hline $\begin{array}{l}\text { SAHPLE } \\
\text { DATE }\end{array}$ & $1-K-28$ \\
\hline --....- & $=---$ \\
\hline $07 / 20 / 87$ & $\begin{array}{l}30.01 \\
\text { NR }\end{array}$ \\
\hline $7 / 29 / 87$ & 4640106 \\
\hline $7 / 29 / 87$ & ----- \\
\hline $17 / 20 / 87$ & 12240. \\
\hline $17 / 20 / 07$ & 0210. \\
\hline $1 / 20 / 87$ & 12004.00 \\
\hline $17 / 20 / 87$ & 16.01 \\
\hline $67 / 29 / 87$ & 11.6 \\
\hline $17 / 20 / 87$ & 206.00 \\
\hline $7 / 20 / 87$ & 22000 \\
\hline $17 / 20 / 87$ & 7.50 \\
\hline $07 / 29 / 87$ & 6.01 \\
\hline $17 / 20 / 07$ & 28.31 * \\
\hline
\end{tabular}

- - VALUE EXCEEDS PRIMARY DRINKING WaTER STANDARD.

- - VALUE EXCEEDS PROPQSED PRIUARY DRINKING IATER STANDARD.

- - VALUE EXCEEDS SCREENING LEVEL FOR FURTHER INYESTIGATION.

- - DETECTION LINIT IAS MOT AYAILABLE FOR COUPARISON

NR - ANALYSIS NOT REQUESTED OR NOT YET REPORTED

VALUES IN \{ $\}$ ARE COUNTING ERRIRS FOR RADIONUCLIDES

TATER STAMDARD(S) IN PARENTHESES ARE PROPOSED ONLY 
TABLE C.3. (contd)

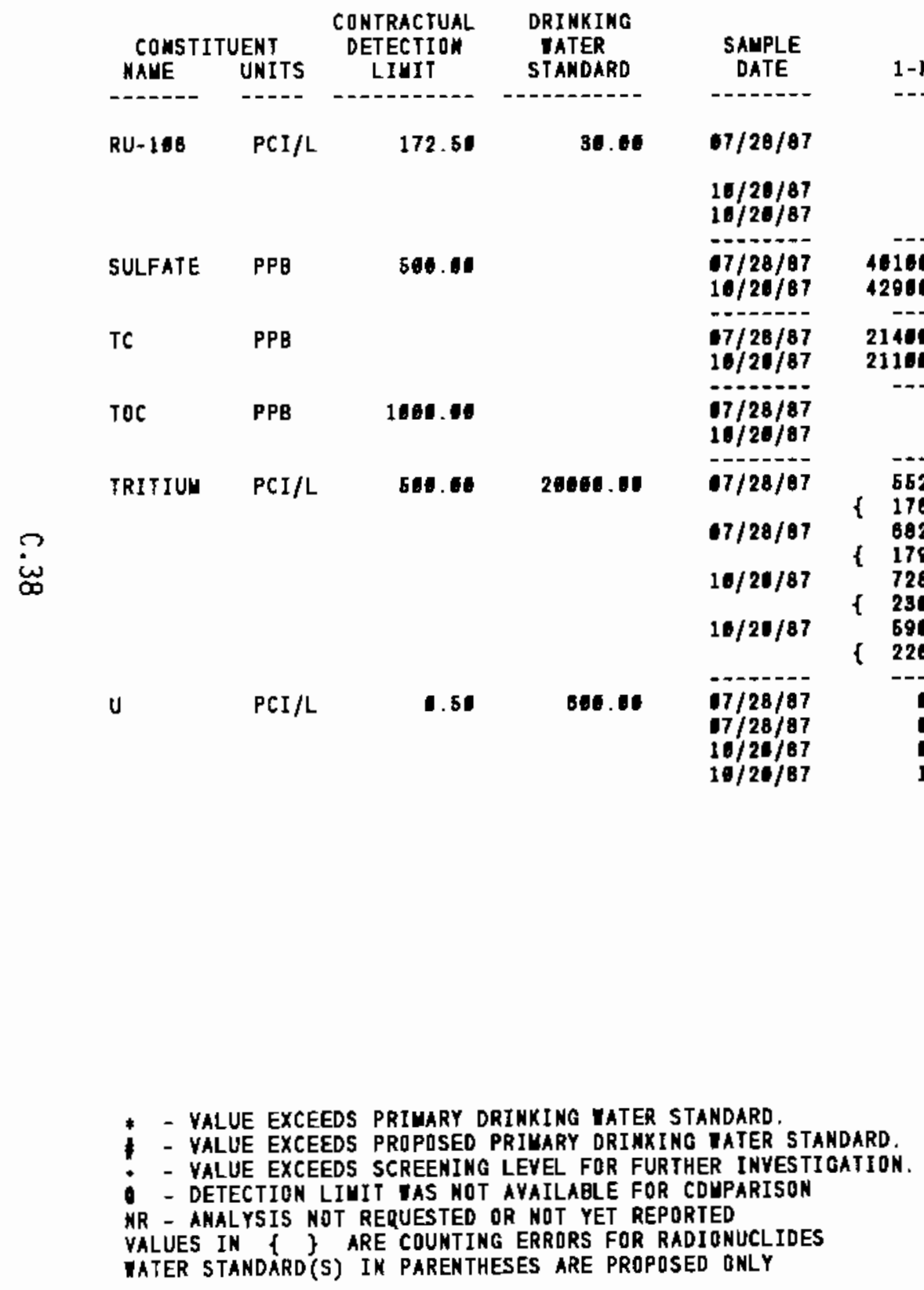

\begin{tabular}{|c|c|}
\hline $\begin{array}{l}\text { SAUPLE } \\
\text { DATE }\end{array}$ & $1-K-27$ \\
\hline \multicolumn{2}{|l|}{$10 / 16 / 87$} \\
\hline & $\underset{M R}{M R}$ \\
\hline $\begin{array}{l}17 / 29 / 87 \\
10 / 18 / 87\end{array}$ & 22409.110 \\
\hline $\begin{array}{l}17 / 29 / 87 \\
10 / 10 / 87\end{array}$ & $\begin{array}{l}39206.00 \\
28016.010\end{array}$ \\
\hline \multicolumn{2}{|l|}{$\begin{array}{l}67 / 29 / 87 \\
10 / 16 / 87\end{array}$} \\
\hline $17 / 29 / 87$ & 1350. \\
\hline $10 / 18 / 87$ & 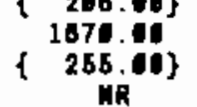 \\
\hline & MR \\
\hline $\begin{array}{l}17 / 29 / 87 \\
10 / 18 / 87\end{array}$ & $\begin{array}{l}3.0 \\
\text { M.10 } \\
\text { MR }\end{array}$ \\
\hline
\end{tabular}

SAUPLE

DATE 1-K-28

$-x-22$

160.10

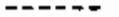

1400.00:

100.00

552.11

178.06\}

602.40

179. .9)

728.00

$230.00\}$

226.0.6

1.08
1.08
1.99
1.08

$10 / 10 / 87$

$$
\text { ( } \quad 66.70 *
$$$$
\text { NR }
$$$$
\text { NR }
$$

$07 / 28 / 87$

25000.0

NR

$07 / 29 / 87 \quad 29200.60$.

MR

$17 / 29 / 87 \quad 1766.01$

NR

$07 / 29 / 87$

$10 / 16 / 87$

4000.00

$\{280.00\}$

2980.00

\{288.06\}

NR

MP

$07 / 29 / 87$

$10 / 18 / 87$ 
TABLE C.3. (contd)

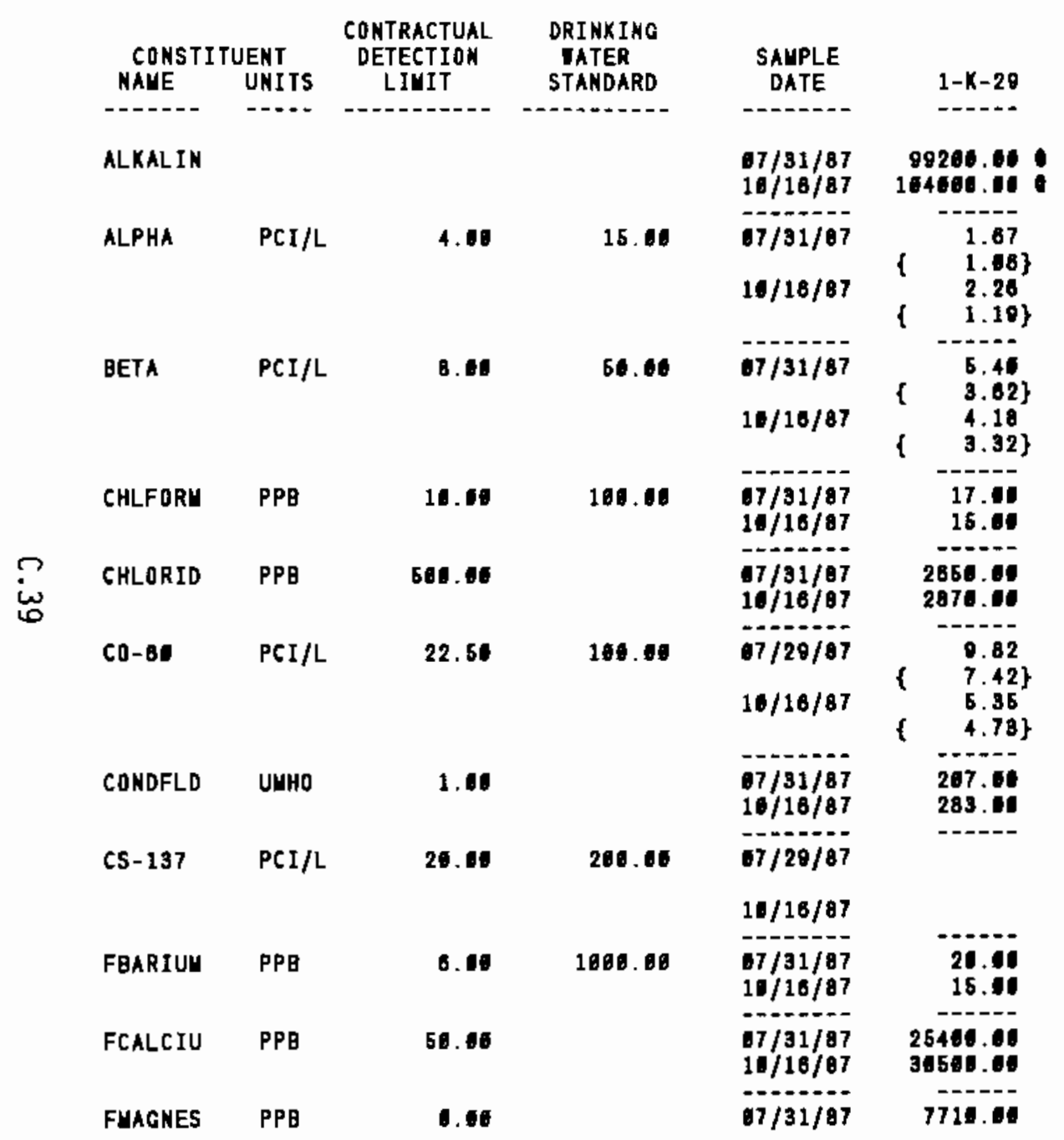

\begin{tabular}{|c|c|}
\hline $\begin{array}{l}\text { SAyPLE } \\
\text { DATE }\end{array}$ & $1-x-30$ \\
\hline $67 / 81 / 87$ & $\underset{N R}{96810.010}$ \\
\hline $17 / 31 / 87$ & $\left\{\begin{array}{l}2.33 \\
\left\{\begin{array}{l}1.36 \\
M R\end{array}\right\}\end{array}\right.$ \\
\hline $67 / 31 / 87$ & $\left\{\begin{array}{l}\text { E.75 } \\
\left.\begin{array}{l}3.61\} \\
M R\end{array}\right\}\end{array}\right.$ \\
\hline $07 / 31 / 87$ & NR \\
\hline $07 / 31 / 87$ & NR \\
\hline $\begin{array}{l}07 / 20 / 87 \\
10 / 10 / 87\end{array}$ & $\left.\begin{array}{ll} & 5.85 \\
\{\quad 5.85\end{array}\right\}$ \\
\hline $07 / 31 / 87$ & NR \\
\hline $16 / 16 / 07$ & $\left.\begin{array}{l}3.44 \\
\{\quad 3.88\end{array}\right\}$ \\
\hline $07 / 31 / 87$ & ${ }_{\mathrm{MR}}^{28.01}$ \\
\hline $17 / 31 / 87$ & 37006.01 \\
\hline $17 / 31 / 87$ & 10106.00 \\
\hline
\end{tabular}

\begin{tabular}{|c|c|}
\hline $\begin{array}{l}\text { SAMPLE } \\
\text { DATE }\end{array}$ & $1-N-8$ \\
\hline 18/103/87 & 48409.60 \\
\hline \multicolumn{2}{|l|}{ (88/09/87 } \\
\hline & MR \\
\hline $08 / 103 / 87$ & $\left\{{ }_{\mathrm{MR}}^{396.26}\right.$ \\
\hline $10 / 63 / 87$ & ...- \\
\hline $18 / 103 / 87$ & $\underset{\mathrm{NR}}{725.10}$ \\
\hline \multirow[t]{2}{*}{ - } & NR \\
\hline & NR \\
\hline $18 / 13 / 87$ & MR \\
\hline \multirow{2}{*}{-........ } & MR \\
\hline & MR \\
\hline $18 / 63 / 87$ & 14.6 \\
\hline $00 / 63 / 87$ & $177 \mathrm{MR}$ \\
\hline $08 / 03 / 87$ & 3526.64 \\
\hline
\end{tabular}

* - Value eXceEds priuary drinking tater standard

- - VALUE EXCEEDS PROPOSED PRIYARY DRINKING IATER STANDARD.

- - VALUE EXCEEDS SCREENING LEVEI FOR FURTHER INYESTIGATION.

- - DETECTIDN LIUIT TAS NOT AVAILABLE FOR COUPARISON

NR - AMALYSIS NOT REQUESTED OR NDT YET REPORTED

VALUES IN \& $\}$ ARE COUNTING ERRORS FOR RADIONUCLIDES

TATER STAMDARO(S) II PARENTHESES ARE PROPOSED ONLY 
IABLE C.3. (contd)

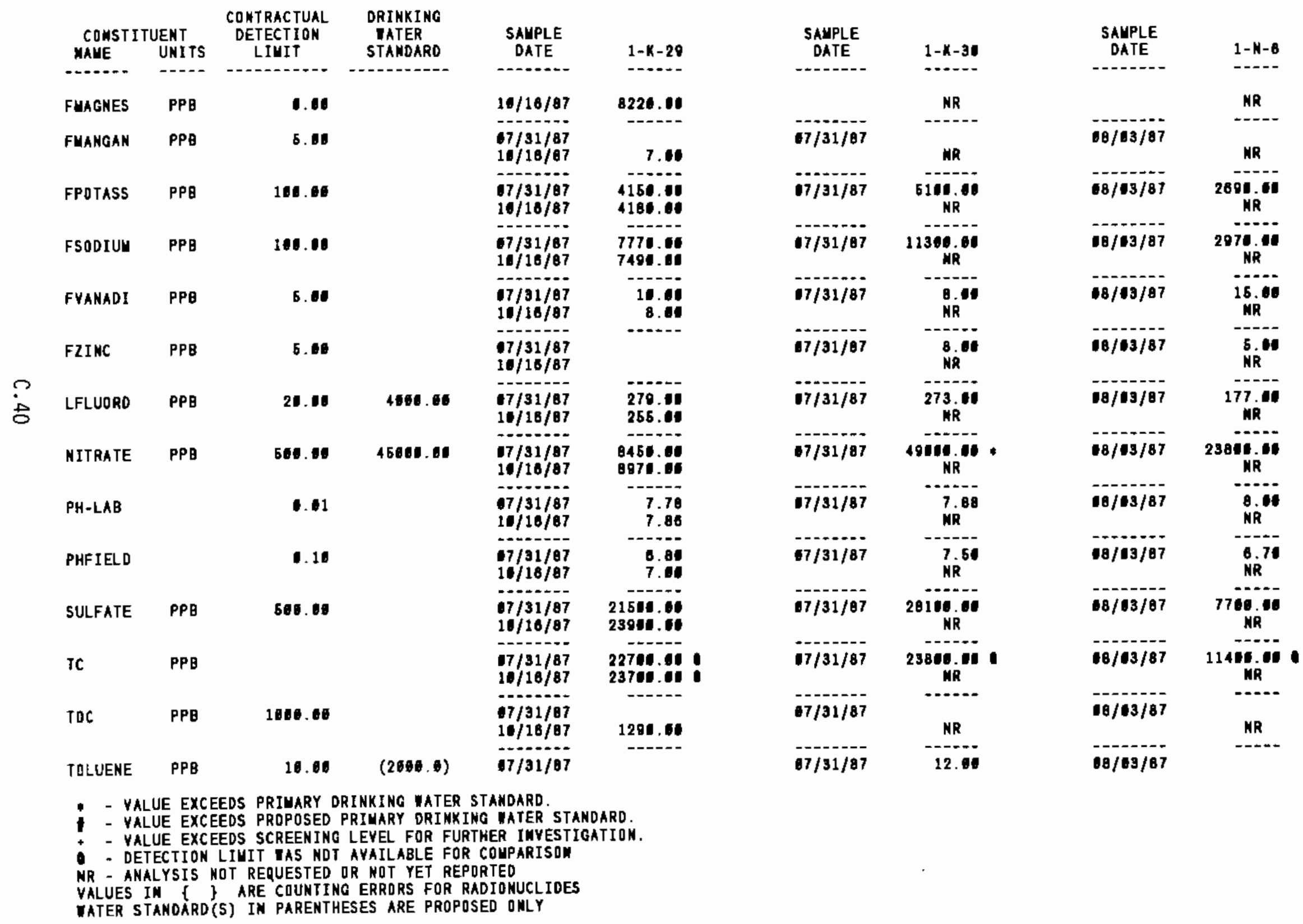


TABLE C.3. (contd)

\begin{tabular}{|c|c|c|c|c|c|c|c|c|c|}
\hline $\begin{array}{l}\text { CONSTI } \\
\text { MAHE }\end{array}$ & $\begin{array}{l}\text { UENT } \\
\text { UNITS }\end{array}$ & $\begin{array}{l}\text { CONTRACTUAL } \\
\text { DETECTION } \\
\text { LILIT }\end{array}$ & $\begin{array}{l}\text { DRINXING } \\
\text { TAIER } \\
\text { STANDARO }\end{array}$ & $\begin{array}{l}\text { SAUPLE } \\
\text { DATE }\end{array}$ & $1-k-29$ & $\begin{array}{l}\text { SALPLE } \\
\text { DATE }\end{array}$ & $1-K-3 \mid$ & $\begin{array}{l}\text { SAUPLE } \\
\text { DATE }\end{array}$ & $1-n-6$ \\
\hline$----\cdot$ & ---- & $---x+-1$ & - - - & $--\cdots--$ & ----- & 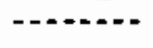 & ----- & $---1-x$ & $-\cdots$ \\
\hline TOLUENE & PPB & 10.06 & $(2800.6)$ & $10 / 16 / 87$ & & & NR & & NR \\
\hline TOXLDL & PPB & 20.16 & & $\begin{array}{l}07 / 31 / 97 \\
10 / 10 / 87\end{array}$ & 20.10 & $7 / 31 / 87$ & NR & $68 / 63 / 87$ & WR \\
\hline TRITIUU & $\mathrm{PCI} / \mathrm{L}$ & 580.06 & 26580.60 & $\begin{array}{l}10 / 29 / 07 \\
10 / 16 / 97\end{array}$ & 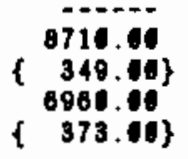 & $\begin{array}{l}17 / 29 / 87 \\
10 / 18 / 87\end{array}$ & $\begin{array}{c}730060.06 * \\
\{2046.06\} \\
1304666.66 * \\
\{4366.06\}\end{array}$ & $\cdots$ & NR \\
\hline U & $\mathrm{PCI} / \mathrm{L}$ & 6. 50 & 668.00 & $\begin{array}{l}07 / 29 / 87 \\
10 / 16 / 87\end{array}$ & $\begin{array}{r}1.34 \\
1.55\end{array}$ & $\begin{array}{l}17 / 20 / 87 \\
10 / 16 / 87\end{array}$ & $\begin{array}{r}1.65 \\
1.73\end{array}$ & & MR \\
\hline
\end{tabular}

- - yalUE EXCEEDS PRIUARY dRINKING tater STANDARD.

- VALUE EXCEEDS PROPOSED PRIUARY DRIMKING TATER STANDARD.

+ - VALUE EXCEEOS SCREENING LEVEI FoR FURTHER INVESTIGATIOK.

- DETECTION liUit ias not ayailable for colparison

NR - ANALYSIS NOT REQUESTED OR MOT YET REPORTED

YALUES IN \{\} ARE COUNTINO ERRORS FOR RADIONUCLIDES

IATER STANDARD (S) IN PARENTHESES ARE PROPOSED ONLY 
TABLE C.3. (contd)

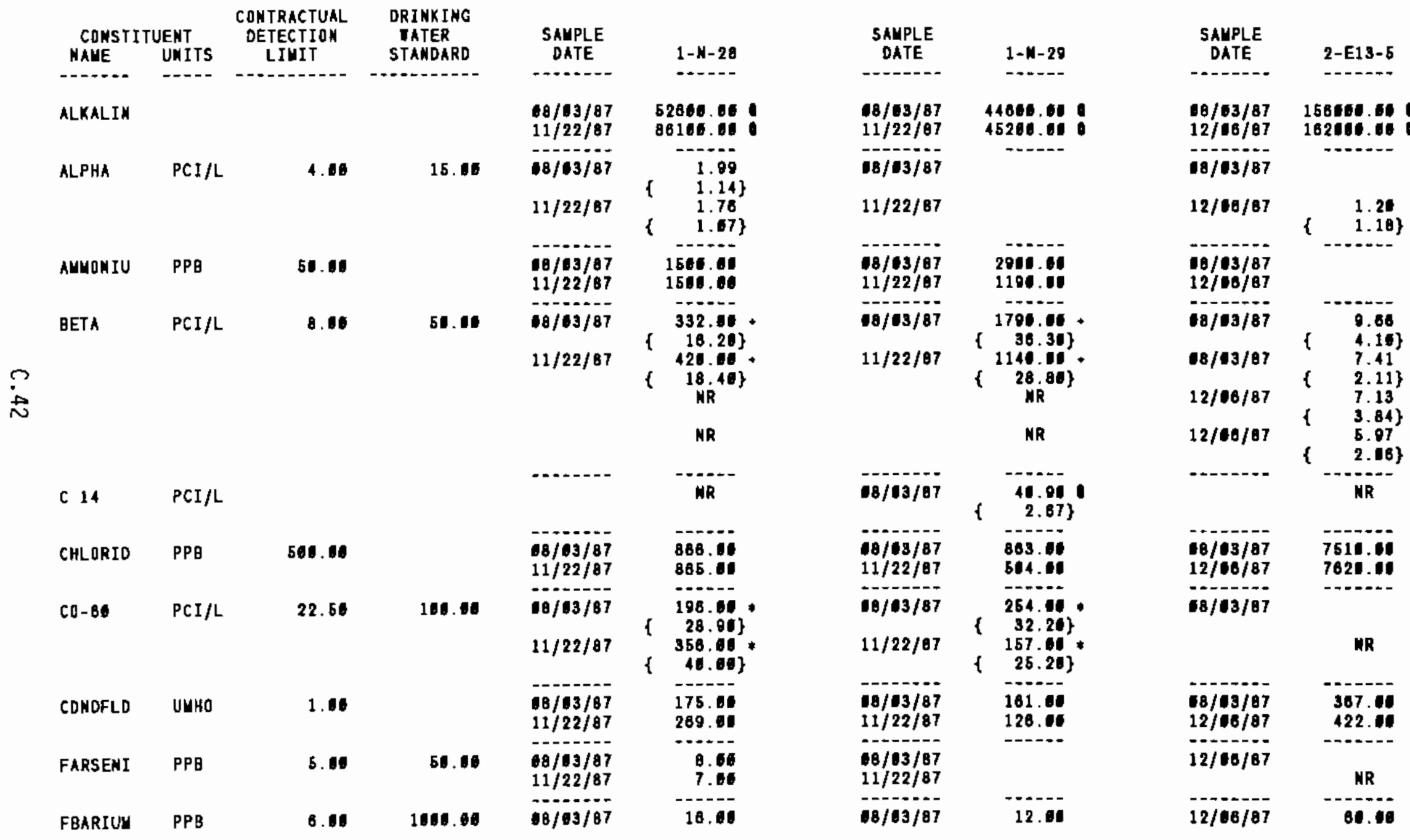

* - VALUE EXCEEDS PRIUARY DRINKING MATER STAMOARD.

- VALUE EXCEEOS PROPOSED PRIUARY DRINKING TATER STANDARD.

- YALUE EXCEEOS SCREENING LEYEL FOR FURTHER INYESTIGATION.

- DETECTIDN LIUIT TAS NOT AYAILABLE FOR COAPARISON

NR - ANALYSIS NOT REQUESTED OR NOT YET REPORTED

YALUES IN f ? ARE COUNTING ERRORS FQR RADIOHUCLIOES

VALUES IN \& ARE COUNTING ERRORS FQR RADIONUCLIOES
WATER STANDARO(S) IN PARENTHESES ARE PROPOSEO ONLY 
TABLE C.3. (contd)

\begin{tabular}{|c|c|c|c|c|c|c|c|c|c|}
\hline $\begin{array}{l}\text { CONST: } \\
\text { WAME }\end{array}$ & $\begin{array}{l}\text { TUENT } \\
\text { UMITS }\end{array}$ & $\begin{array}{c}\text { CONTRACTUAL } \\
\text { OETECTION } \\
\text { LIUIT }\end{array}$ & $\begin{array}{l}\text { DRINKING } \\
\text { WATER } \\
\text { STANDARD }\end{array}$ & $\begin{array}{c}\text { SAYPLE } \\
\text { DATE }\end{array}$ & $1-N-2 B$ & $\begin{array}{c}\text { SAUPLE } \\
\text { DATE }\end{array}$ & $1-N-2 \theta$ & $\begin{array}{c}\text { SAMPLE } \\
\text { DATE }\end{array}$ & $2-E 13-5$ \\
\hline FBARIUY & PPB & 8.60 & 1000.00 & $11 / 22 / 87$ & 32.16 & $11 / 22 / 87$ & 14.00 & & MR \\
\hline FCALCIU & PPB & 60.00 & & $\begin{array}{l}68 / 63 / 87 \\
11 / 22 / 87\end{array}$ & $\begin{array}{l}22060.100 \\
37800.00\end{array}$ & $\begin{array}{l}08 / 03 / 87 \\
11 / 22 / 87\end{array}$ & $\begin{array}{l}17000.08 \\
16405.06\end{array}$ & $12 / 08 / 07$ & 58204.101 \\
\hline FLUORID & PPB & 506.60 & 4608.06 & $\begin{array}{l}18 / 09 / 87 \\
11 / 22 / 87\end{array}$ & & $\begin{array}{l}08 / 09 / 87 \\
11 / 22 / 87\end{array}$ & & $\begin{array}{l}18 / 63 / 87 \\
12 / 06 / 87\end{array}$ & 022.00 \\
\hline FUAGNES & PPB & 0.00 & & $\begin{array}{l}08 / 03 / 87 \\
11 / 22 / 87\end{array}$ & $\begin{array}{l}4804.01 \\
7236.01\end{array}$ & $\begin{array}{l}18 / 19 / 87 \\
11 / 22 / 87\end{array}$ & $\begin{array}{l}3470.04 \\
2080.64\end{array}$ & $12 / 68 / 87$ & 18716.00 \\
\hline FPOTASS & PPB & 106.00 & & $\begin{array}{l}68 / 63 / 87 \\
11 / 22 / 87\end{array}$ & $\begin{array}{l}4181.00 \\
5311.08\end{array}$ & $\begin{array}{l}18 / 03 / 87 \\
11 / 22 / 87\end{array}$ & 3080.4 & $12 / 06 / 87$ & ${ }_{\mathrm{MR}}^{6250.06}$ \\
\hline FSODIUN & PPB & 100.00 & & $\begin{array}{l}18 / 03 / 97 \\
11 / 22 / 87\end{array}$ & $\begin{array}{l}3391.01 \\
4160.60\end{array}$ & $\begin{array}{l}18 / 108 / 87 \\
11 / 22 / 87\end{array}$ & $\begin{array}{l}2740.10 \\
2120.11\end{array}$ & $12 / 106 / 87$ & 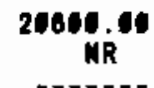 \\
\hline FVANADI & PPB & 5.61 & & $\begin{array}{l}08 / 03 / 87 \\
11 / 22 / 87\end{array}$ & $\begin{array}{l}23.11 \\
18.11\end{array}$ & $\begin{array}{l}08 / 09 / 87 \\
11 / 22 / 67\end{array}$ & $\begin{array}{l}12.00 \\
13.01\end{array}$ & $12 / 66 / 87$ & ${ }_{\mathrm{MR}}^{17.06}$ \\
\hline FZIMC & $\mathrm{PPB}$ & 5.00 & & $\begin{array}{l}88 / 103 / 87 \\
11 / 22 / 87\end{array}$ & & $\begin{array}{l}68 / 63 / 87 \\
11 / 22 / 87\end{array}$ & & $12 / 00 / 87$ & $\mathrm{MR}^{\mathrm{P.6}}$ \\
\hline$I-120$ & $\mathrm{PCI} / \mathrm{L}$ & & 500.00 & & MR & $09 / 10 / 87$ & \{ :.11 & & MR \\
\hline LFLUORD & PPB & 26.11 & 4060.00 & $\begin{array}{l}08 / 09 / 87 \\
11 / 22 / 87\end{array}$ & $\begin{array}{l}88.00 \\
74.00\end{array}$ & $\begin{array}{l}18 / 63 / 87 \\
11 / 22 / 87\end{array}$ & $\begin{array}{r}71.04 \\
104.06\end{array}$ & $\begin{array}{l}18 / 09 / 87 \\
12 / 00 / 87\end{array}$ & $\begin{array}{r}401.60 \\
464.60\end{array}$ \\
\hline UETHONE & PPB & 10.60 & & $\begin{array}{l}08 / 103 / 87 \\
11 / 22 / 87\end{array}$ & & $\begin{array}{l}18 / 03 / 87 \\
11 / 22 / 87\end{array}$ & & $\begin{array}{l}18 / 63 / 87 \\
12 / 06 / 87\end{array}$ & 30.15 \\
\hline NI-69 & $\mathrm{PCI} / \mathrm{L}$ & 10.00 & 50.00 & & NR & $08 / 09 / 87$ & $\{\quad 2.00\}$ & & MR \\
\hline NITRATE & PPB & 500.00 & 45006.60 & $\begin{array}{l}08 / 13 / 87 \\
11 / 22 / 87\end{array}$ & $\begin{array}{l}42200.60 \\
84360.60\end{array}$ & $\begin{array}{l}68 / 63 / 87 \\
11 / 22 / 87\end{array}$ & $\begin{array}{l}39400.04 \\
18106.04\end{array}$ & $\begin{array}{l}08 / 03 / 87 \\
12 / 08 / 87\end{array}$ & $\begin{array}{l}10100.00 \\
11201.00\end{array}$ \\
\hline PH-LAB & & 0.01 & & $08 / 03 / 87$ & 8.18 & $08 / 103 / 87$ & 8.28 & $08 / 03 / 87$ & 7.73 \\
\hline \multicolumn{10}{|c|}{ 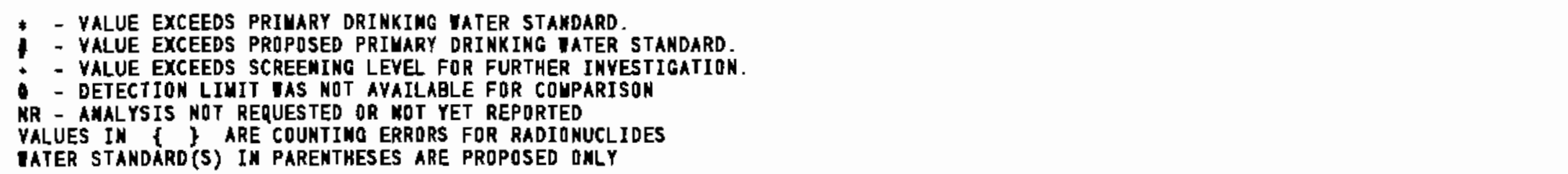 } \\
\hline
\end{tabular}


TABLE C.3. (contd)

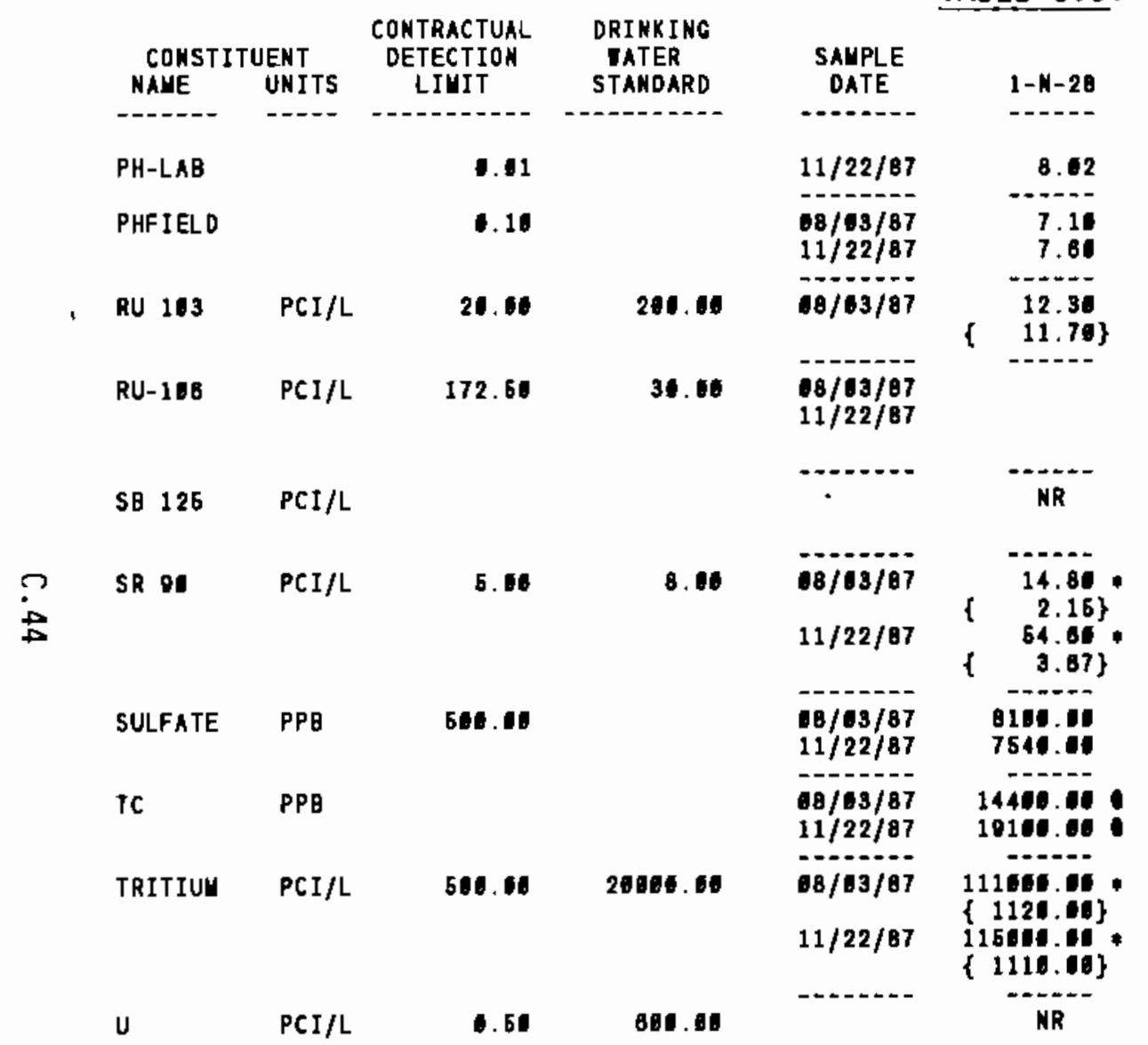

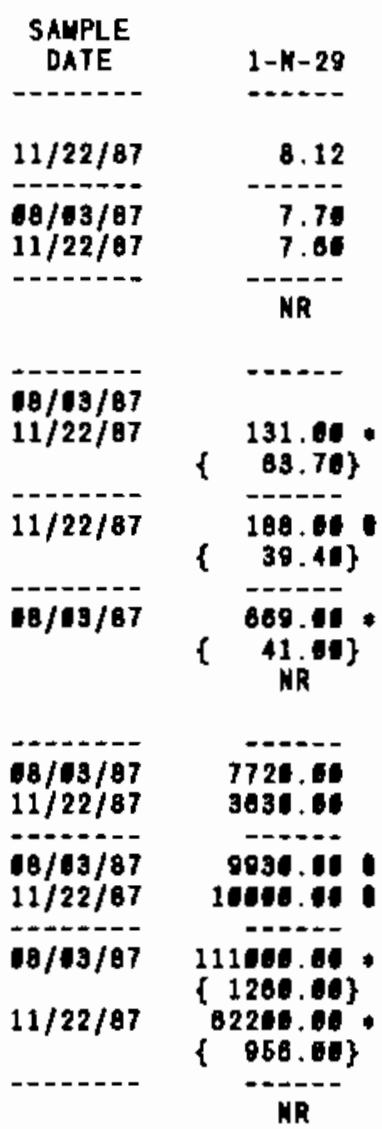

SAMPLE

$12 / 08 / 07$

(.......

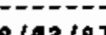

$88 / 93 / 87$

$12 / 08 / 87$

0.12

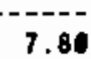

7.80

IR

$18 / 03 / 07$

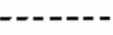

$-5$

18/03/87

NR

$8 / 03 / 87$

$12 / 06 / 87$

44890 is

46706

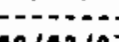

$18 / 63 / 87$

38145.48

3700.01:

$18 / 03 / 87$

12/98/B7

$68 / 03 / 87$

* - VALUE EXCEEDS PRIUARY DRIRKINg IATER STANDARD.

- VALUE EXCEEDS PRDPOSED PRIMARY DRINKING TATER STANDARD.

- VALUE EXCEEDS SCREENING LEVEL FOR FURTHER INYESTIGATION.

- DETECTION LIUIT TAS NOT AVALAGLE FOR CDUPARISON

NR - ANALYSIS NOT REQUESTED OR NOT YET REPORTED

YALUES IN $\{?$ ARE COUNTING ERRORS FOR RADIONUCLIDES

WATER STANDARD(S) IN PARENTHESES ARE PROPOSED ONLY 
TABLE C.3. (contd)

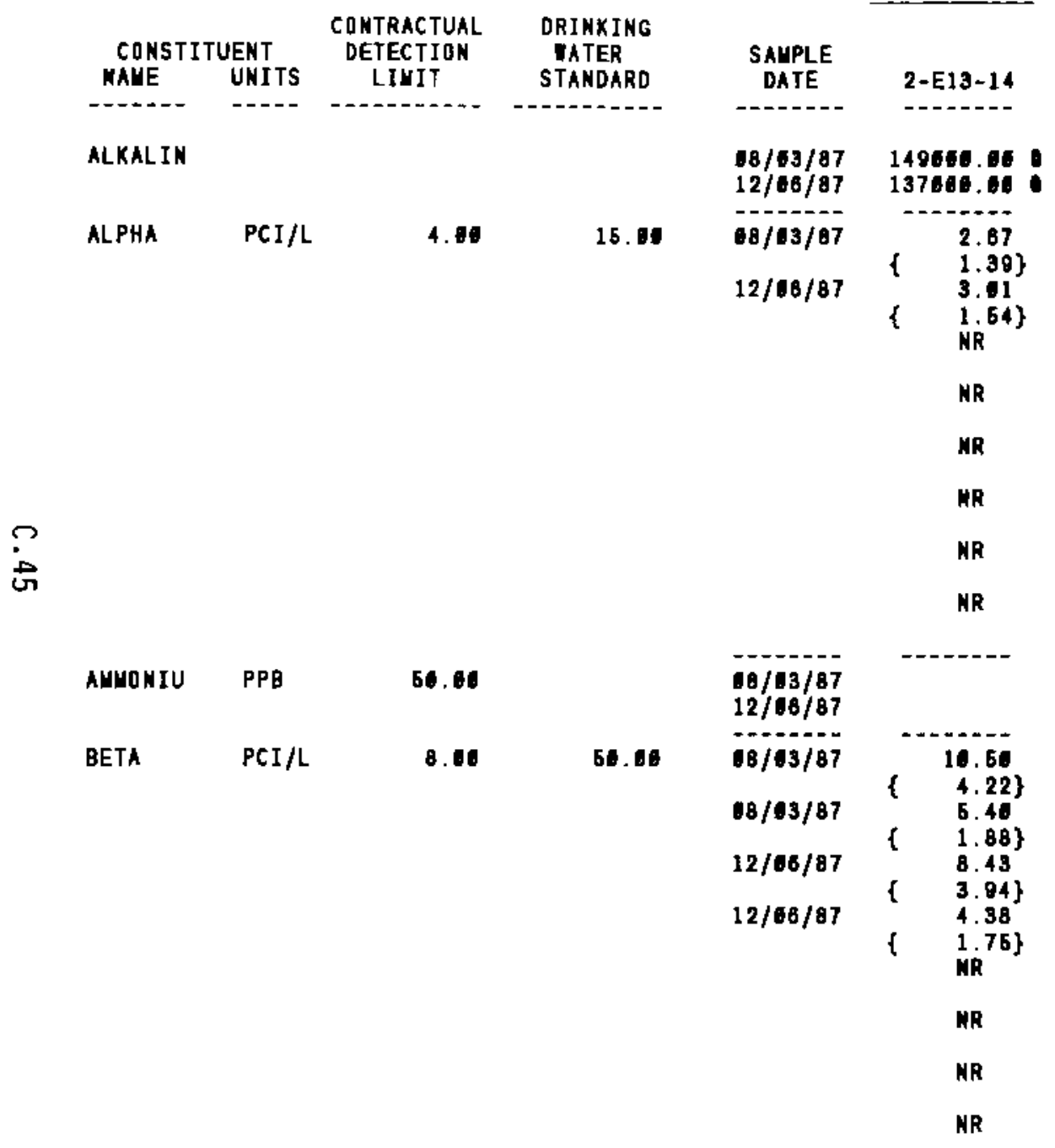

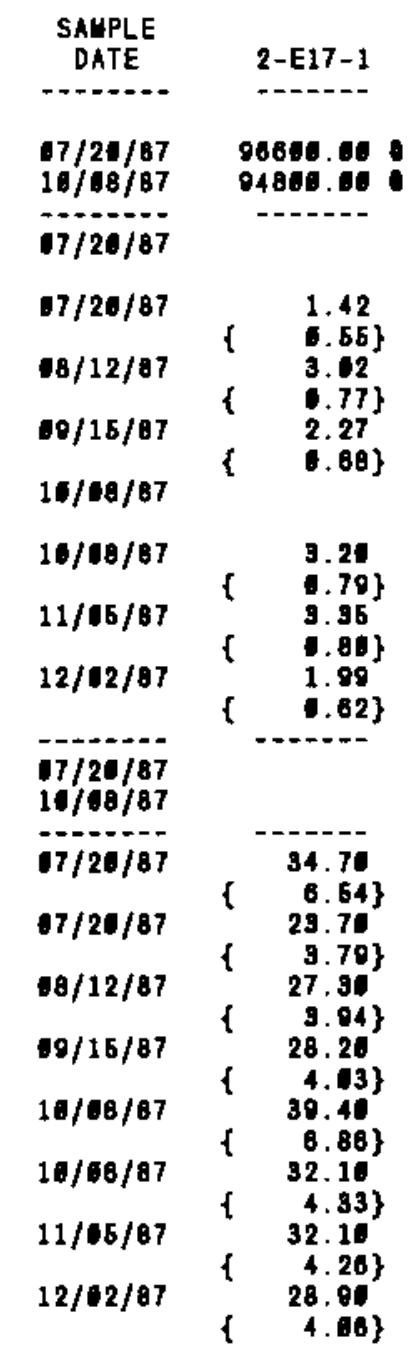

SAMPLE
DATE

$97 / 20 / 87$
$10 / 07 / 87$

$---\overline{10-}$

6.88
2.08

$67 / 20 / 07\left\{\begin{array}{l}2.08\} \\ 0.24\end{array}\right.$

68/12/87 \{ 1.07$\}$

$09 / 09 / 87$ \{ 1.11$\}$

$10 / 07 / 07\left\{\begin{array}{l}1.28\} \\ \text { f }\end{array}\right.$

\{ 2.10$\}$

$16 / 07 / 87 \quad 6.83$

11/06/07 \{ 1.16\}

12/62/87 \{ 1.12\}

\{r.2 $\begin{array}{r}1.22 \\ \ldots . .25\end{array}$

07/20/87 04.66

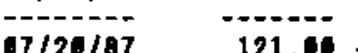

1 16.30\}.

$07 / 20 / 87$ 113.06.

18/12/87 $\left\{\begin{array}{l}7.47\} \\ 128.08\end{array}\right.$

10/09/87 $\left\{\begin{array}{l}7.03\} \\ 582.09\end{array}\right.$

$10 / 07 / 87\{18.40\}$

(22.30\}

$18 / 07 / 87 \quad 613.04+$

$11 / 65 / 87$ ( $440.84+$

$12 / 62 / 8\} \quad\{14.00\}$

$143.19+$

* - Value exceEdS PRIUARY dRINKIHg tater STAMDARD.

- - VALUE EXCEEDS PROPOSEO PRIMARY DRIMKIMG TATER STANDARD.

- - VALUE EXCEEDS SCREENING LEVEL FOR FURTHER INVESTIGATION.

- DETECTION LIUIT IAS NOT AYAILABLE FOR COUPARISON

NR - ANALYSIS NOT RERUESTED OR NOT YET REPORTED

YALUES IN \{ \} ARE COUNTING ERRORS FOR RADIONUCLIDES

IATER STAMDARD'S) IN PARENTHESES ARE PROPOSED ONLY 
TABLE C. 3. (contd)

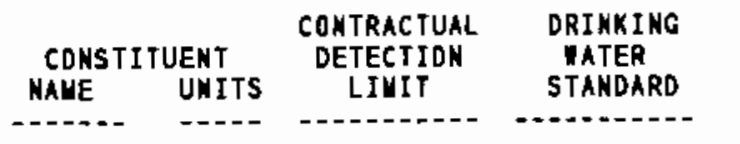

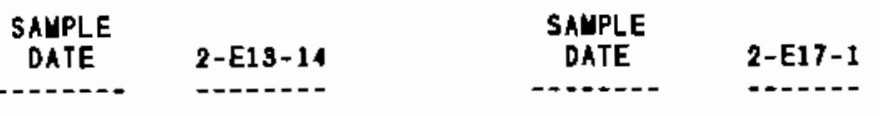

NR

C $14 \mathrm{PCI} / \mathrm{L}$

CHLORID PPB

500.06

CQ-8E

$\mathrm{PCI} / \mathrm{L}$

22. 60

100.00

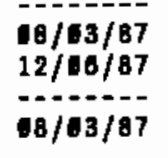

$\stackrel{8}{8}$

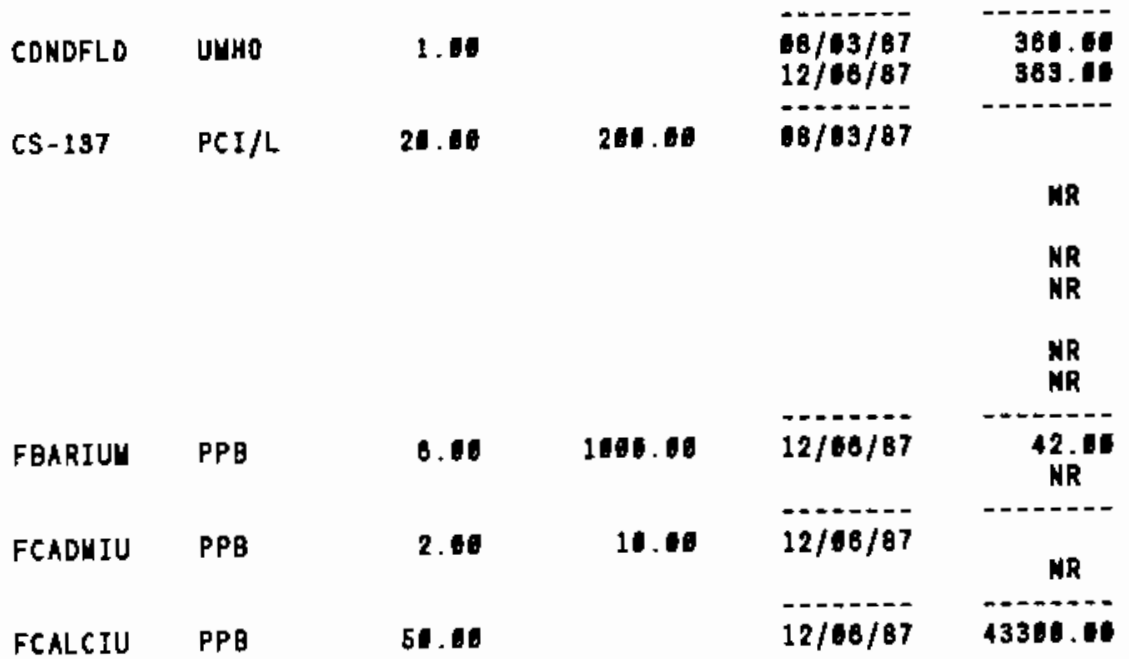

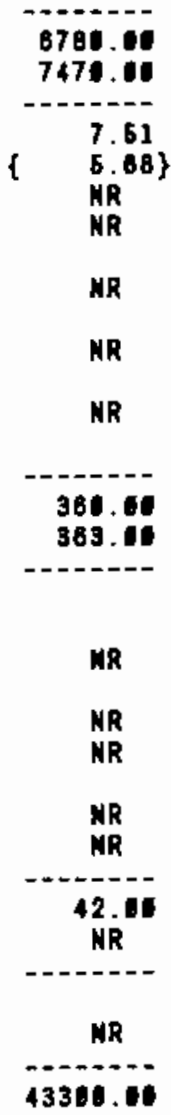

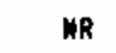

$7 / 20 / 87$

$10 / 08 / 87$

$07 / 26 / 07$

18/12/87

$09 / 15 / 87$

$10 / 08 / 87$

$11 / 05 / 87$

$12 / 02 / 67$

\section{$07 / 20 / 87$}

$10 / 08 / 87$

$07 / 20 / 87$

$68 / 12 / 87$

$10 / 15 / 87$

$10 / 08 / 87$

$11 / 65 / 97$

$17 / 20 / 87$

18/08/87

-

$07 / 26 / 87$

16/68/87

$07 / 20 / 87$

\subsection{0}

635. 10

-

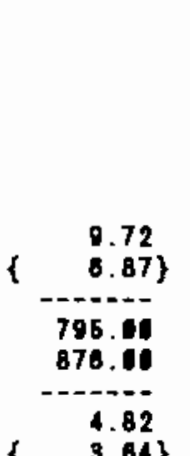

$\{\quad 3.64\}$

$\begin{array}{r}5.28 \\ 1 \quad 4.00 \\ \hline\end{array}$

-..-

95.11

-...-

2.06

93864.01
SAMPLE

DATE

2-E17-5

18/10/87

$7 / 20 / 87$

$16 / 67 / 87$

-

17/20/8?

$08 / 12 / 87$
$69 / 00 / 87$

$10 / 07 / 87$

$11 / 06 / 87$

$12 / 12 / 87$

$7 / 20 / 67$

10/07/87

$17 / 26 / 87$

08/12/87

19/09/87

16/47/87

$11 / 15 / 87$

$12 / 12 / 87$

$7 / 20 / 87$

16/07/87

10.-...--

$07 / 26 / 87$

$10 / 107 / 87$

$67 / 20 / 87$
23.50
$\{\quad 2.39\}$

8221.4

6740.64

-...-.

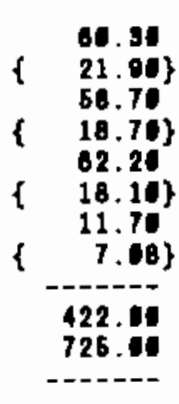

6.81

$\{4.64\}$

$\left\{\begin{array}{r}7.94 \\ 7.61\}\end{array}\right.$

39.65

85.19

85.45

47510.65

* - value exceeds priuary orinking mater standard

* - VALUE EXCEEDS PRILARY ORINKING TATER STANDARD.

- VALUE EXCEEDS PRGPOSED PRIVARY DRINKING

- - VALUE EXCEEDS SCREENING LEVEL FOR FURTHER INVESTICA

NR - ANALYSIS HOT RERUESTED OR NOT YET REPORTED

VALUES IN $\{$ ? ARE CDUNTIMG ERRDRS FOR RADIONUCLIDES

WATER STANOARD(S) IN PARENTHESES ARE PROPOSED ONLY 


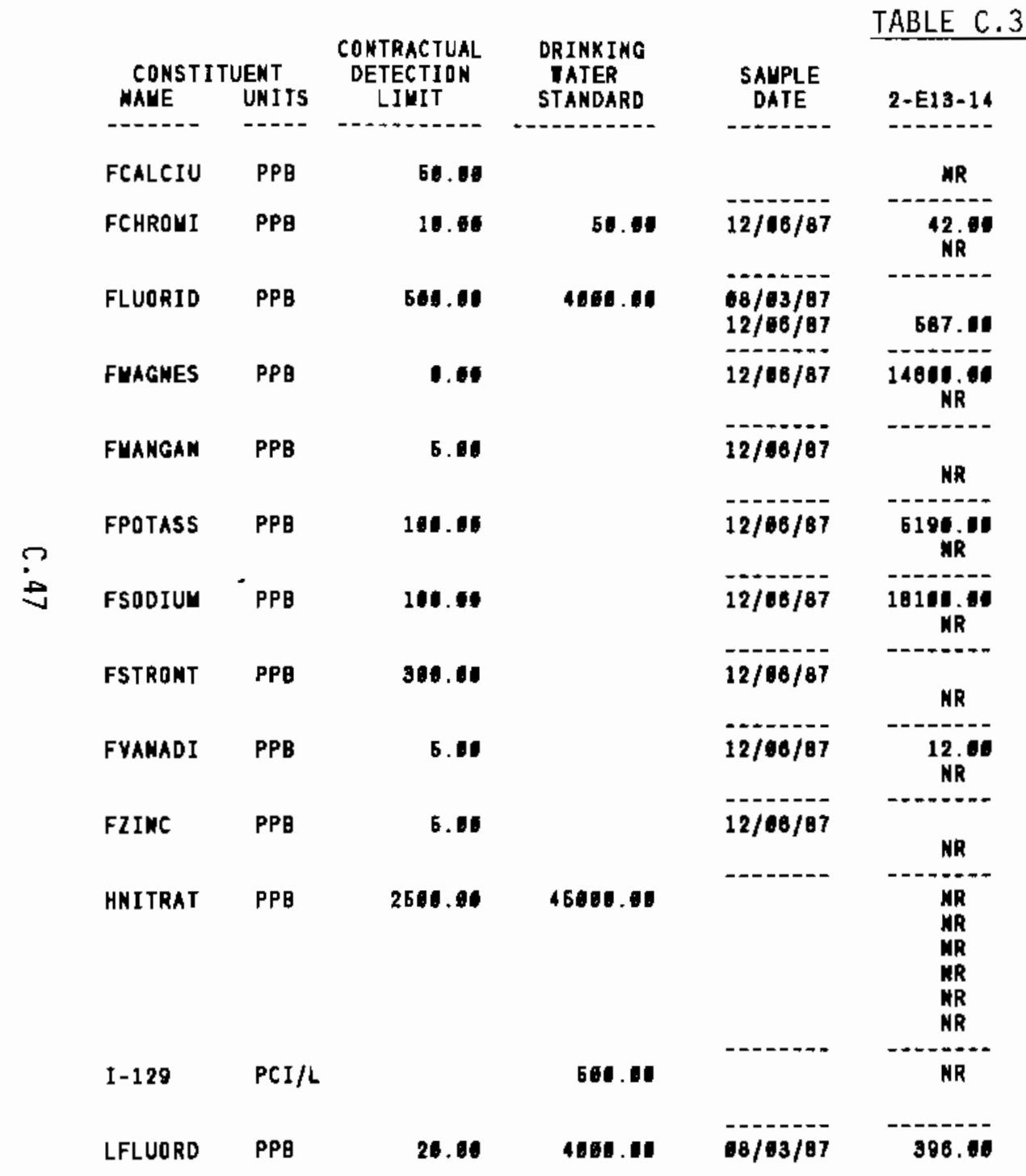

(contd)

- - yalue exceeds priyary drinking tater standard.

- - VALUE EXCEEDS PROPOSED PRIMARY DRINKING TATER STAMDARD.

+ - VALUE EXCEEDS SCREenING LEYEL fDR fURTHER INYESTIGATION.

- DEtection liUit tas Not aYAilable for couparison

MR - ANALYSIS MOT REQUESTED OR NOT YET REPDRTED

VALUES IN \{ \} ARE COUNTING ERRORS FOR RADIONUCLIDES

IATER STANDARD'S S) IN PARENTHESES ARE PROPOSED OMLY

\begin{tabular}{|c|c|}
\hline $\begin{array}{l}\text { SAUPLE } \\
\text { DATE }\end{array}$ & $2-E 17-1$ \\
\hline $11 / 108 / 87$ & 87801.00 \\
\hline $\begin{array}{l}07 / 20 / 87 \\
10 / 09 / 87\end{array}$ & \\
\hline $\begin{array}{l}67 / 20 / 87 \\
10 / 108 / 87\end{array}$ & 011.00 \\
\hline $\begin{array}{l}17 / 20 / 87 \\
10 / 108 / 87\end{array}$ & 28201.00 \\
\hline $\begin{array}{l}07 / 20 / 87 \\
10 / 08 / 87\end{array}$ & \\
\hline $\begin{array}{l}17 / 20 / 87 \\
16 / 66 / 87\end{array}$ & $\begin{array}{l}10600.06 \\
11706.06\end{array}$ \\
\hline $\begin{array}{l}1 / 20 / 87 \\
11 / 18 / 87\end{array}$ & $\begin{array}{r}32766.11 \\
38566.10\end{array}$ \\
\hline $\begin{array}{l}17 / 20 / 87 \\
10 / 108 / 87\end{array}$ & $\begin{array}{l}522.00 \\
489.00\end{array}$ \\
\hline $\begin{array}{l}97 / 25 / 87 \\
19 / 108 / 87\end{array}$ & $\begin{array}{r}13.06 \\
17.00\end{array}$ \\
\hline $\begin{array}{l}07 / 29 / 87 \\
10 / 10 / 87\end{array}$ & $\begin{array}{l}22.00 \\
17.00\end{array}$ \\
\hline $\begin{array}{l}67 / 26 / 87 \\
68 / 12 / 87 \\
69 / 16 / 87 \\
16 / 08 / 87 \\
11 / 65 / 87 \\
12 / 62 / 87\end{array}$ & 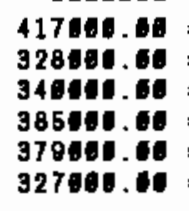 \\
\hline $18 / 10 / 87$ & $\begin{array}{r}47.30 \\
\{\quad 5.62\}\end{array}$ \\
\hline $17 / 26 / 87$ & 410.00 \\
\hline
\end{tabular}

\begin{tabular}{|c|c|}
\hline $\begin{array}{l}\text { SAAPLE } \\
\text { DATE }\end{array}$ & $2-E 17-6$ \\
\hline $16 / 07 / 87$ & 70500.06 \\
\hline $\begin{array}{l}17 / 20 / 87 \\
10 / 07 / 87\end{array}$ & \\
\hline $\begin{array}{l}07 / 20 / 87 \\
10 / 07 / 87\end{array}$ & 076.00 \\
\hline $\begin{array}{l}07 / 20 / 87 \\
10 / 07 / 87\end{array}$ & 14896.106 \\
\hline $\begin{array}{l}07 / 21 / 87 \\
10 / 67 / 87\end{array}$ & 15.06 \\
\hline $\begin{array}{l}67 / 25 / 87 \\
16 / 167 / 87\end{array}$ & 8060.10 \\
\hline $\begin{array}{l}67 / 21 / 87 \\
16 / 107 / 87\end{array}$ & 26000.00 \\
\hline $\begin{array}{l}67 / 26 / 87 \\
16 / 07 / 87\end{array}$ & 380.00 \\
\hline $\begin{array}{l}07 / 21 / 87 \\
16 / 107 / 87\end{array}$ & 15.96 \\
\hline $\begin{array}{l}07 / 21 / 87 \\
11 / 07 / 87\end{array}$ & 22.45 \\
\hline $\begin{array}{l}07 / 26 / 87 \\
18 / 12 / 87 \\
60 / 10 / 87 \\
16 / 107 / 87 \\
11 / 105 / 87 \\
12 / 112 / 87\end{array}$ & 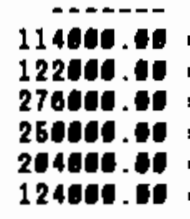 \\
\hline $08 / 16 / 87$ & $\begin{array}{r}25.00 \\
\quad 3.20\}\end{array}$ \\
\hline $77 / 20 / 87$ & 485.10 \\
\hline
\end{tabular}


TABLE C.3. (contd)

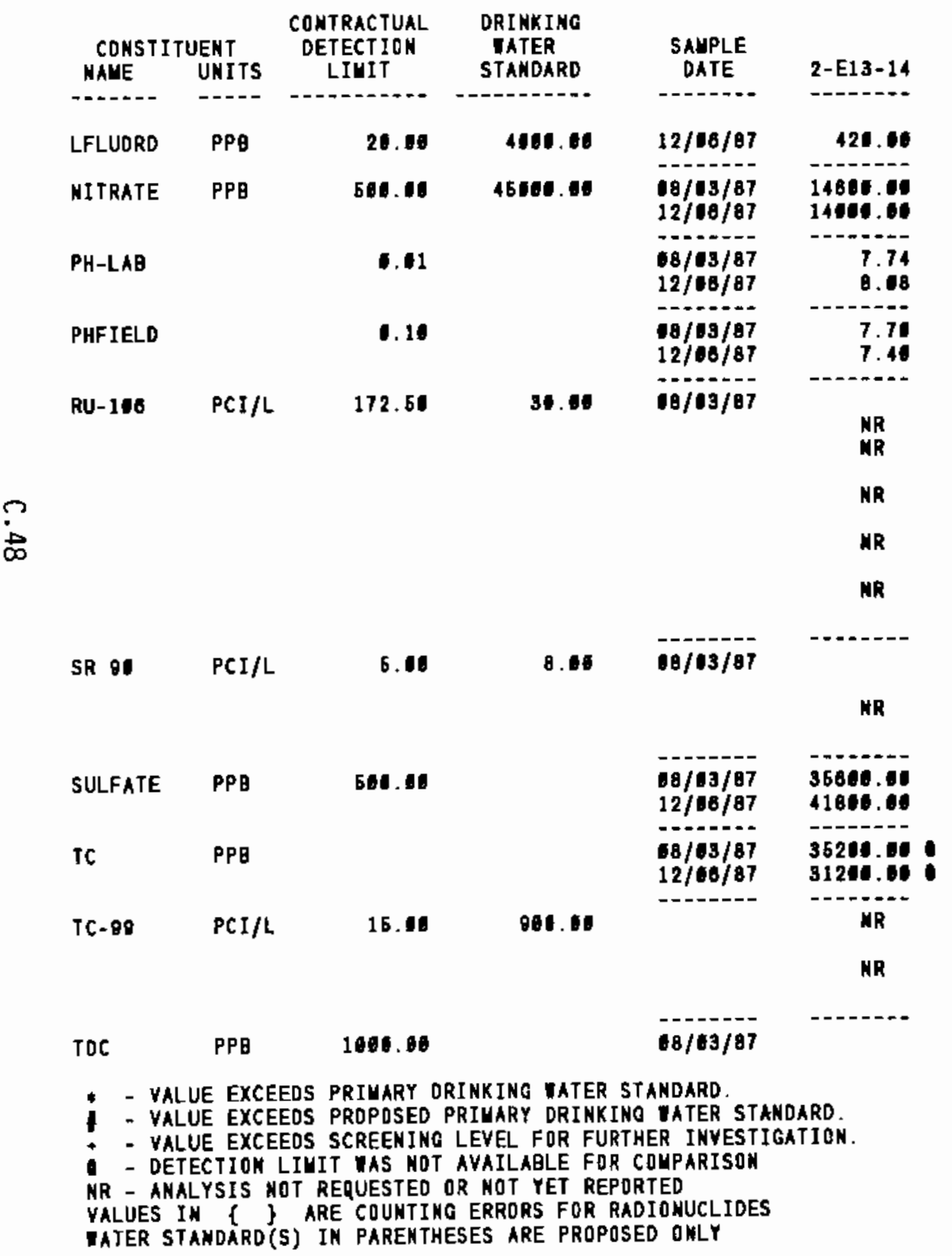

\begin{tabular}{|c|c|}
\hline $\begin{array}{c}\text { SAUPLE } \\
\text { DATE }\end{array}$ & 2-E17-1 \\
\hline - & 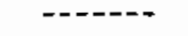 \\
\hline $10 / 08 / 07$ & 410.60 \\
\hline $\begin{array}{l}17 / 24 / 87 \\
10 / 08 / 87\end{array}$ & 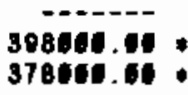 \\
\hline $\begin{array}{l}17 / 21 / 87 \\
10 / 68 / 87\end{array}$ & $\begin{array}{r}7.49 \\
7.65\end{array}$ \\
\hline $\begin{array}{l}7 / 26 / 87 \\
10 / 18 / 87\end{array}$ & $\begin{array}{l}3.08 \\
7.81\end{array}$ \\
\hline $\begin{array}{l}07 / 20 / 87 \\
68 / 12 / 87 \\
00 / 15 / 87\end{array}$ & \\
\hline $10 / 08 / 87$ & \\
\hline $11 / 65 / 67$ & \\
\hline $12 / 02 / 87$ & \\
\hline 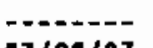 & $---2--$ \\
\hline $67 / 26 / 07$ & $\begin{array}{l}5.91 \\
1.42\}\end{array}$ \\
\hline $10 / 08 / 87$ & $\begin{array}{l}0.94 \\
1.68\}\end{array}$ \\
\hline $\begin{array}{l}7 / 26 / 87 \\
18 / 68 / 87\end{array}$ & $\begin{array}{l}41406.05 \\
41000.05\end{array}$ \\
\hline $\begin{array}{l}17 / 20 / 87 \\
18 / 108 / 87\end{array}$ & 23300.00 \\
\hline $18 / 11 / 87$ & $\left\{\begin{array}{c}32.56 \\
1.07\} \\
\text { NR }\end{array}\right\}$ \\
\hline $87 / 26 / 87$ & ---1 \\
\hline
\end{tabular}

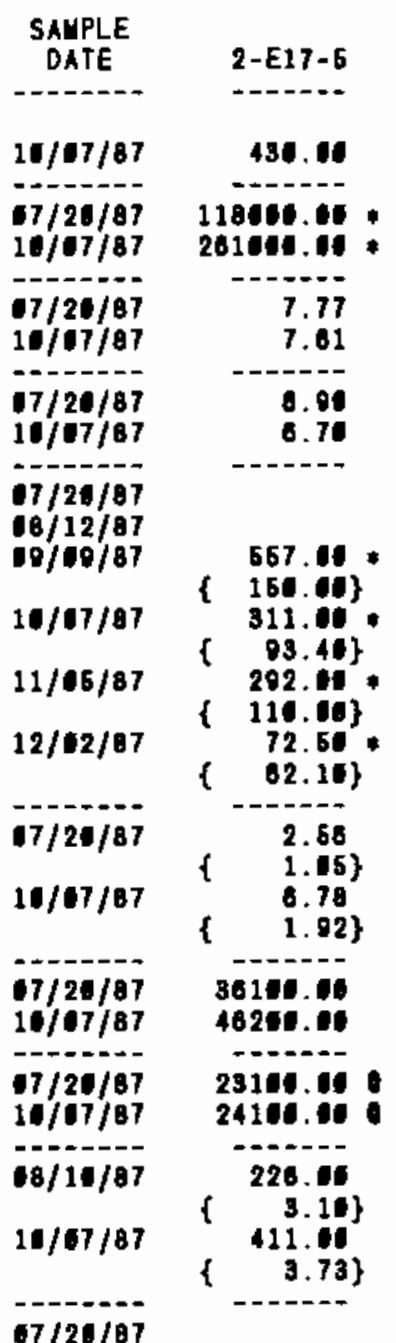

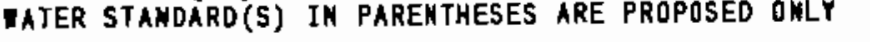


TABLE C.3. (contd)

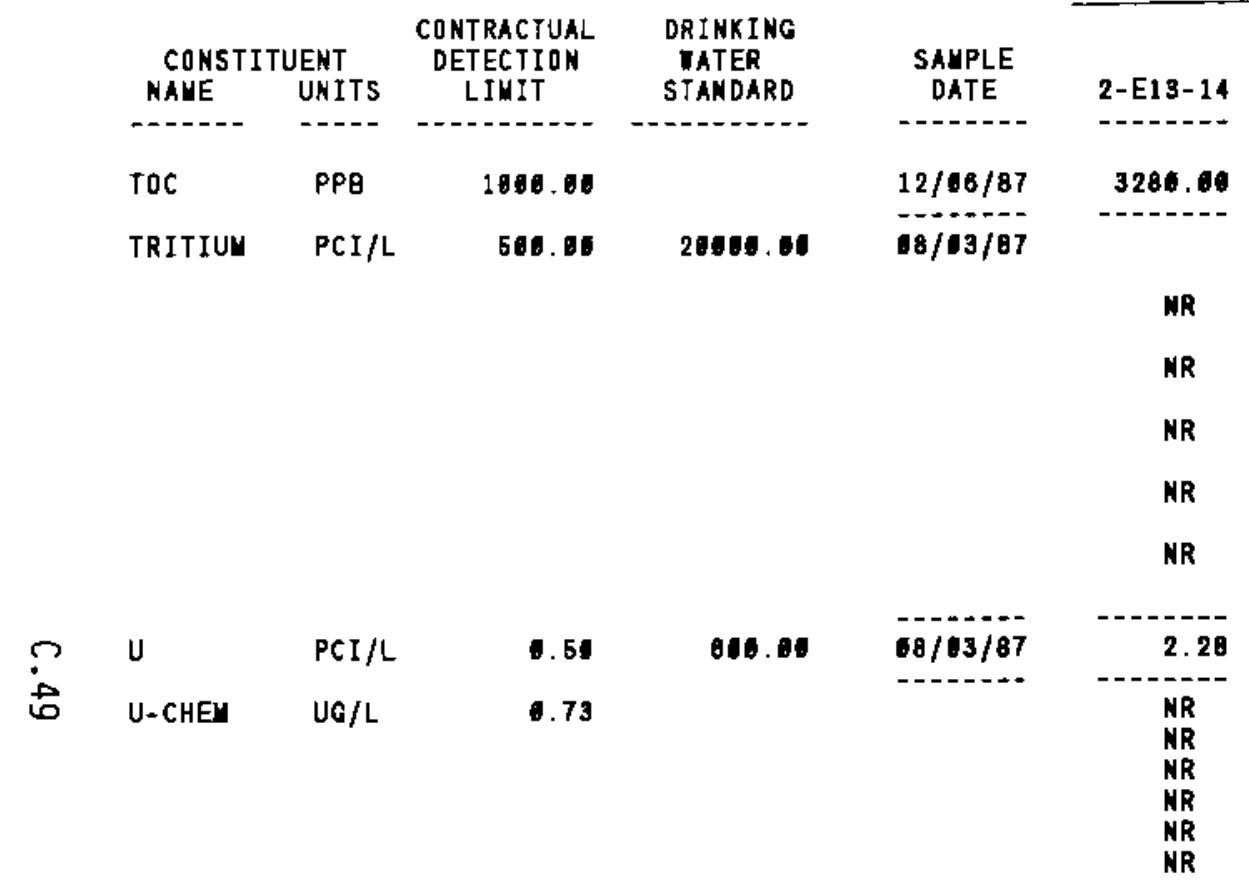

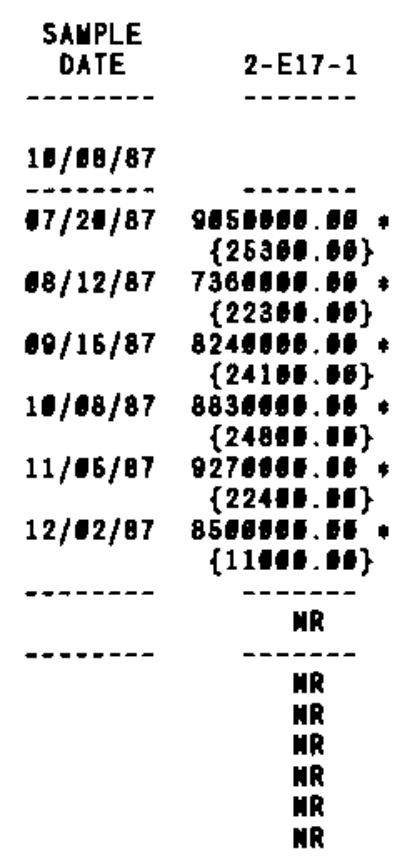

\begin{tabular}{|c|c|}
\hline $\begin{array}{l}\text { SALPLE } \\
\text { DATE }\end{array}$ & $2-E 17-6$ \\
\hline $16 / 67 / 87$ & \\
\hline $7 / 26 / 87$ & $\begin{array}{r}4528060.01 \\
\{17060.00\}\end{array}$ \\
\hline $68 / 12 / 87$ & $4276000.01 \%$ \\
\hline $69 / 69 / 87$ & 5396160 \\
\hline $16 / 67 / 87$ & 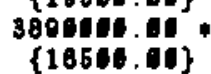 \\
\hline $11 / 65 / 87$ & 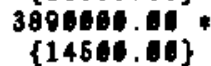 \\
\hline $12 / 02 / 87$ & $\begin{array}{c}42391060101 \\
\{7760.010\}\end{array}$ \\
\hline & WR \\
\hline $\begin{array}{l}67 / 26 / 87 \\
68 / 12 / 87 \\
60 / 00 / 87 \\
16 / 07 / 87 \\
11 / 05 / 87 \\
12 / 02 / 87\end{array}$ & $\begin{array}{r}7.42 \\
8.12 \\
16.71 \\
8.28 \\
8.88 \\
7.36\end{array}$ \\
\hline
\end{tabular}

- VALUE eXCEEDS PRIMARY dRINKING MaTER STANDARD

- YALUE EXCEEDS PROPOSED PRIMARY DRINKING TATER STAMDARD.

- - VALUE EXCEEDS SCREENING LEVEL FOR FURTHER INYESTIGATION.

- DETECTIDN LIUIT ias NOT aVALlable for couparison

NR - ANALYSIS NOT REQUESTED DR NOT YET REPORTED

YALUES IN \{ ARE COUNTIMG ERRORS FOR RADIONUCLIDES

inter STANDARo(S) in PaRENTHESES aRE PROPOSED ONLY 
TABLE C.3. (contd)

\begin{tabular}{|c|c|c|c|c|c|}
\hline $\begin{array}{c}\text { CONSTI } \\
\text { MAVE }\end{array}$ & $\begin{array}{l}\text { UENT } \\
\text { UNITS }\end{array}$ & $\begin{array}{c}\text { CONTRACTUAL } \\
\text { DETECTIOH } \\
\text { LIUIT }\end{array}$ & $\begin{array}{l}\text { DRINXING } \\
\text { IATER } \\
\text { STANDARD }\end{array}$ & $\begin{array}{l}\text { SAUPLE } \\
\text { DATE }\end{array}$ & $2-E 17-8$ \\
\hline ALKALIN & & & & $\begin{array}{l}67 / 26 / 87 \\
10 / 67 / 87\end{array}$ & $\begin{array}{l}158860.08 \\
148806.00\end{array}$ \\
\hline \multirow[t]{8}{*}{ ALPHA } & $\mathrm{PCI} / \mathrm{C}$ & 4.80 & 15.01 & $17 / 20 / 87$ & \\
\hline & & & & $10 / 07 / 97$ & \\
\hline & & & & & NR \\
\hline & & & & & NR \\
\hline & & & & & NR \\
\hline & & & & & NR \\
\hline & & & & & MR \\
\hline & & & & & MR \\
\hline BETA & $\mathrm{PCI} / \mathrm{L}$ & 8.60 & 50.00 & $07 / 26 / 87$ & 10.7 \\
\hline & & & & $67 / 20 / 87$ & 11.10 \\
\hline & & & & $68 / 12 / 87$ & 104.68. \\
\hline & & & & $69 / 09 / 87$ & $\begin{array}{r}9.87 \mathrm{~J} \\
11.80\end{array}$ \\
\hline & & & & $16 / 07 / 87$ & $\begin{array}{l}2.42\} \\
8.34\end{array}$ \\
\hline & & & & $10167 / 87$ & $\begin{array}{l}3.56\} \\
8.88\end{array}$ \\
\hline & & & & Defor & $2.0 \%$ \\
\hline & & & & $11 / 65 / 87$ & $\begin{array}{l}6.93 \\
2.027\end{array}$ \\
\hline & & & & $12 / 82 / 87$ & $\begin{array}{r}7.96 \\
\{\quad 2.18\}\end{array}$ \\
\hline CHLDRID & PPB & 506.00 & & $07 / 21 / 87$ & 3476.00 \\
\hline
\end{tabular}

\begin{tabular}{|c|c|c|c|}
\hline $\begin{array}{l}\text { SAMPLE } \\
\text { DATE }\end{array}$ & $2-E 17-8$ & $\begin{array}{l}\text { SAUPLE } \\
\text { DATE }\end{array}$ & $2-E 24-2$ \\
\hline $\begin{array}{l}17 / 26 / 87 \\
10 / 67 / 87\end{array}$ & $\begin{array}{l}106000.048 \\
124600.00\end{array}$ & $68 / 26 / 87$ & 88104.00 \\
\hline $07 / 20 / 87$ & 1.86 & $17 / 21 / 87$ & 5.68 \\
\hline $07 / 20 / 87$ & 3.43 & $08 / 12 / 87$ & 4.30 \\
\hline $18 / 12 / 87$ & $\begin{array}{l}0.81\} \\
2.10\end{array}$ & $68 / 26 / 87$ & $\begin{array}{l}0.80\} \\
1.38\end{array}$ \\
\hline $09 / 09 / 07$ & $\begin{array}{l}\{.63\} \\
3.24\end{array}$ & $10 / 10 / 87$ & $\begin{array}{l}1.32\} \\
3.88\end{array}$ \\
\hline $16 / 17 / 87$ & $\begin{array}{l}1.77\} \\
1.87\end{array}$ & $16 / 108 / 87$ & $\begin{array}{l}1.85\} \\
4.58\end{array}$ \\
\hline $10 / 0 / 68$ & (1.23\} & & $0.83\}$ \\
\hline $11 / 167 / 87$ & $\begin{array}{l}2.14 \\
.85\end{array}$ & $11 / 06 / 87$ & 4.94 \\
\hline $11 / 06 / 87$ & $2.78^{\circ}$ & $12 / 07 / 87$ & 8.62 \\
\hline $12 / 02 / 87$ & $\begin{array}{l}2.71\} \\
\{\quad 1.64\}\end{array}$ & & $\frac{1,11\}}{M R}$ \\
\hline $97 / 25 / 87$ & $\begin{array}{r}28.46 \\
5.74\}\end{array}$ & $67 / 21 / 87$ & $\begin{array}{c}18.96 \\
3.24\}\end{array}$ \\
\hline $07 / 20 / 87$ & $\begin{array}{r}24.66 \\
3.57\}\end{array}$ & $18 / 12 / 87$ & $\begin{array}{r}18.20 \\
3.71\end{array}$ \\
\hline $68 / 12 / 87$ & 34.10 & $18 / 20 / 87$ & 21.26 \\
\hline $10 / 69 / 87$ & $\begin{array}{r}4.25\} \\
14.30\end{array}$ & $90 / 99 / 87$ & $\begin{array}{r}5.48\} \\
14.18\end{array}$ \\
\hline & $2.79\}$ & & $2.72\}$ \\
\hline $10 / 07 / 87$ & $\begin{array}{r}14.40 \\
4.47 \mathrm{k}\end{array}$ & $10 / 06 / 97$ & $\begin{array}{l}21.16 \\
3.89\end{array}$ \\
\hline $10 / 07 / 87$ & $24.40^{\circ}$ & $11 / 05 / 87$ & 19.56 \\
\hline $11 / 05 / 87$ & $3.62\}$ & & $3.23\}$ \\
\hline $11 / 700 \%$ & $3.48\}$ & 1270186 & $\begin{array}{r}20.20 \\
3.37\}\end{array}$ \\
\hline $12 / 62 / 87$ & $\begin{array}{r}28.56 \\
\{\quad 3.88\}\end{array}$ & & MR \\
\hline $67 / 26 / 87$ & 6950.06 & $8 / 20 / 87$ & 5510.01 \\
\hline
\end{tabular}

- - value exceEdos primary dRinking yater standard.

- - VALUE EXCEEDS PRQPOSED PRIUARY DRINKING tater STANDARD.

- VAlUe EXCEEDS SCREenimo LeVel for further inVestigation.

- DETECTION linit tas hot available for couparison

NR - ANALYSIS NOT REQUESTED OR NOT YET REPORTED

VALUES IN IN ARE COUNTING ERRORS FOR RADIONUCLIDES

IATER STANDARO'(S) IN PARENTHESES ARE PROPOSED ONLY 


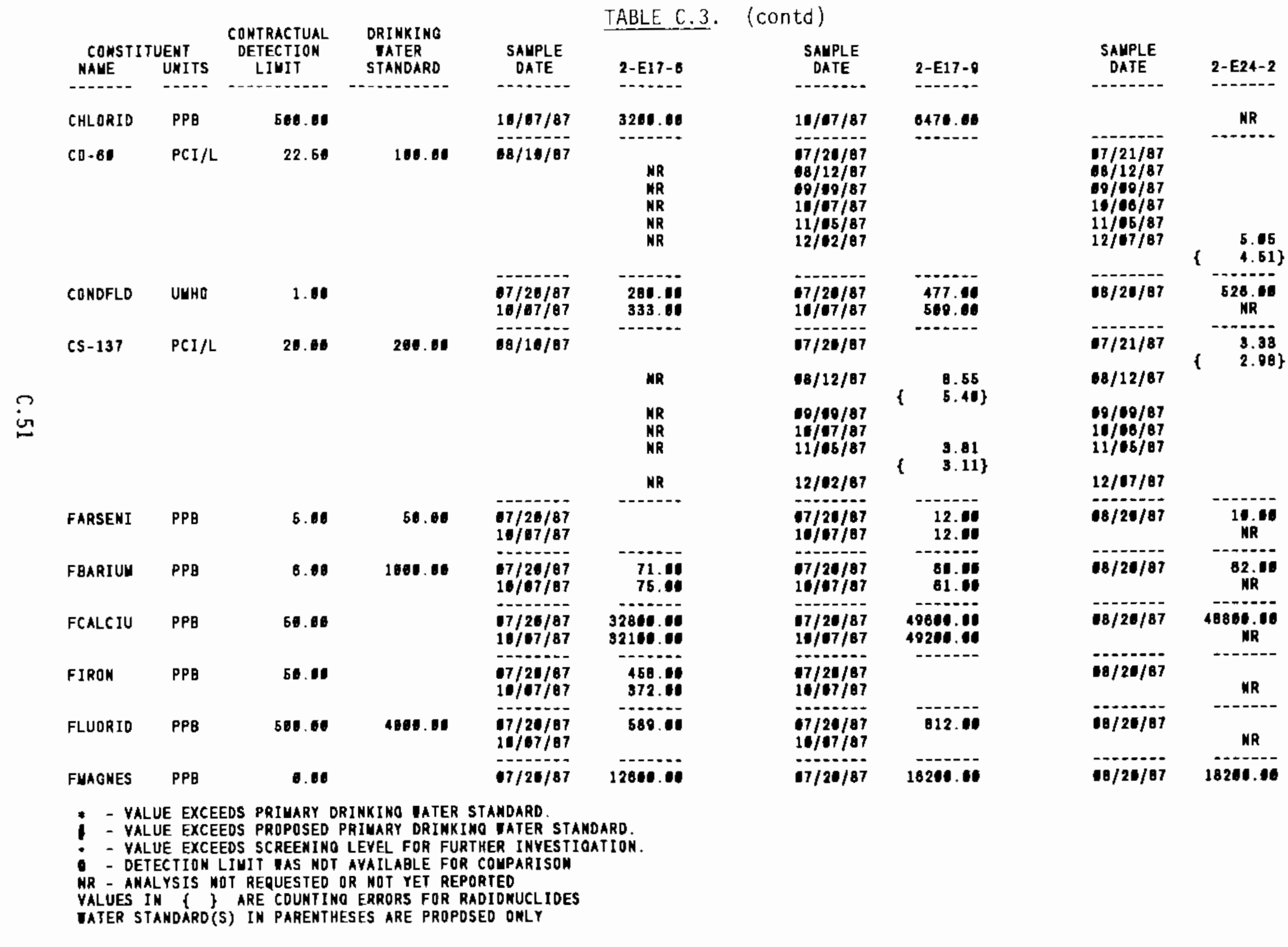


TABLE C.3. (contd)

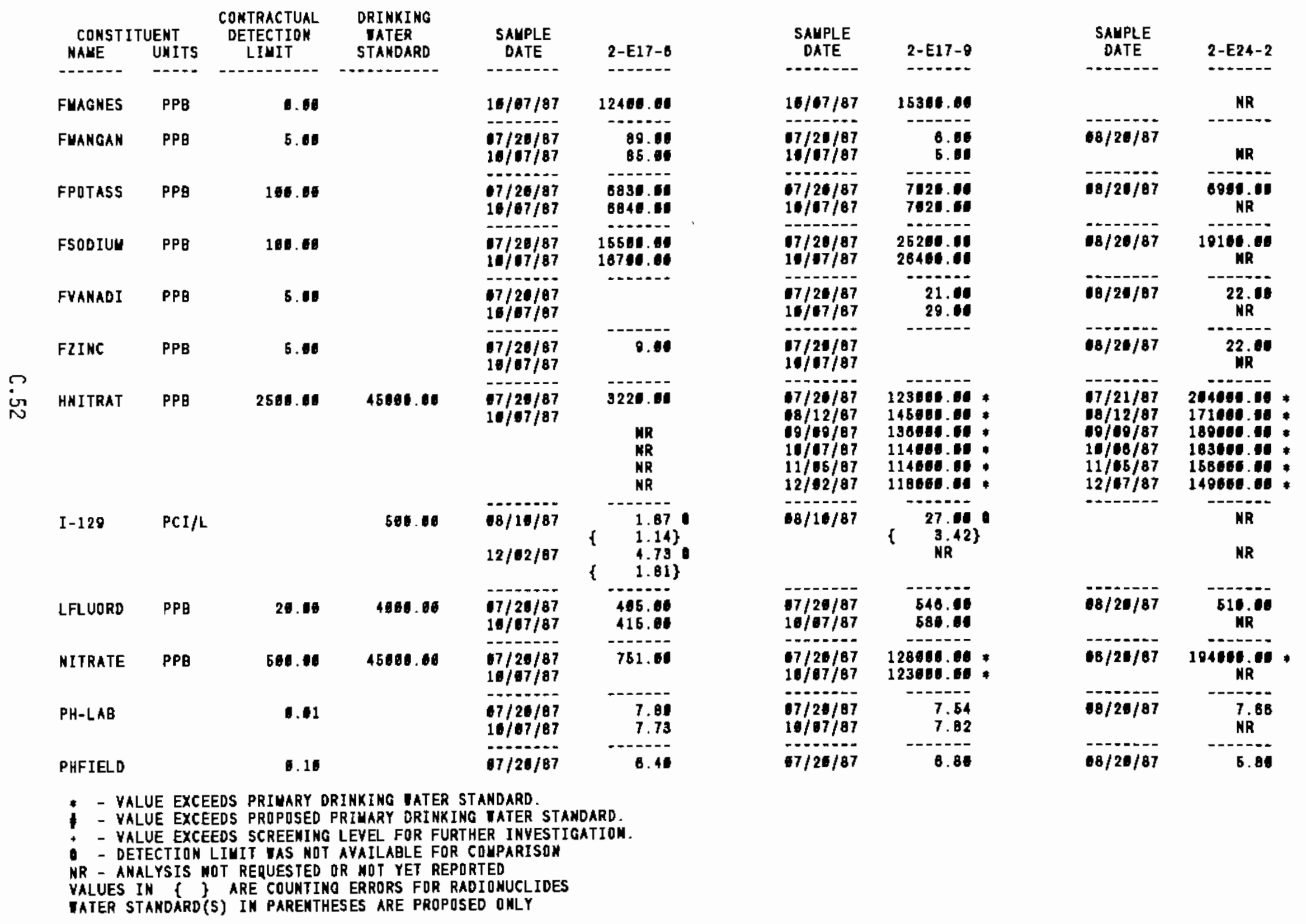


TABLE C.3. (contd)

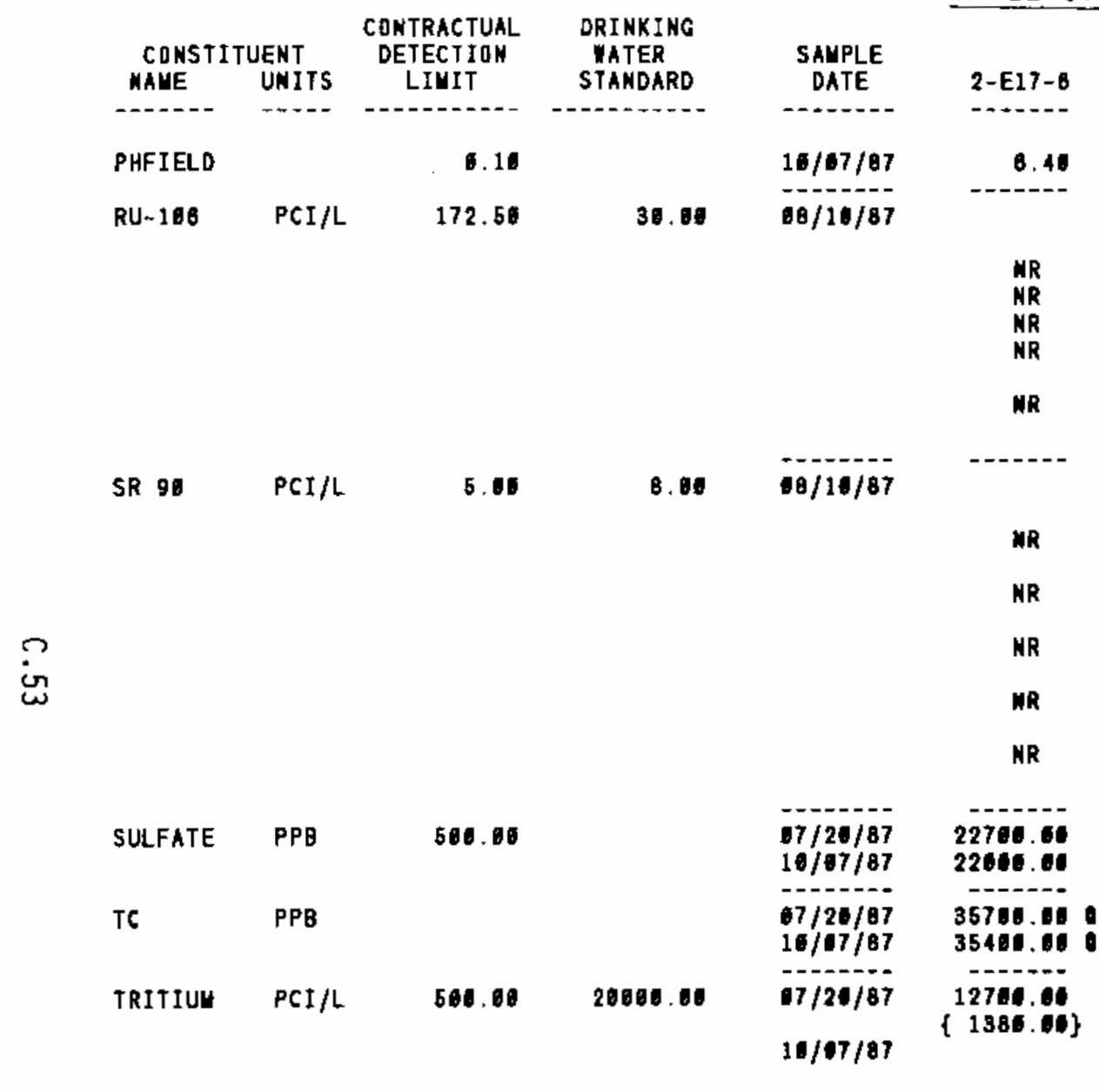

\section{NR}

NR

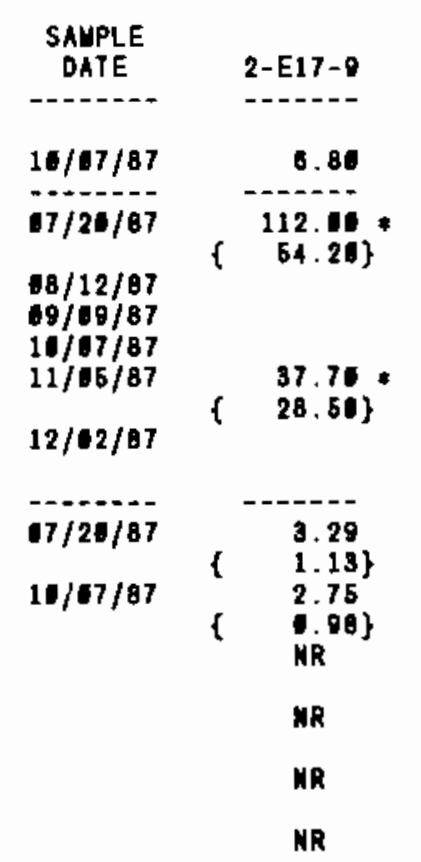

$07 / 20187 \quad 42100 .-10$

$\begin{array}{ll}67 / 26 / 97 & 42100.06 \\ 10 / 07 / 87 & 39040.15\end{array}$

$-2 .-1-2$

$17 / 20 / 87$ 27266.00

10/67/87 2564t.de

07/20/87 4030104.01.

68/12/87 680740..0\}.

19/60/87 516814.06\}

bocene. of *

$10 / 67 / 87$ 374665.08.

\{18685.04\}

\begin{tabular}{|c|c|}
\hline $\begin{array}{c}\text { SAUPLE } \\
\text { DATE }\end{array}$ & $2-E 24-2$ \\
\hline$----\cdots$ & $\cdots+-1$ \\
\hline & NR \\
\hline-----4 & --- \\
\hline $07 / 21 / 87$ & 116.0 \\
\hline & $\{50.60\}$ \\
\hline $\begin{array}{l}08 / 12 / 87 \\
10 / 60 / 87 \\
10 / 08 / 87\end{array}$ & \\
\hline $11 / 05 / 87$ & $\begin{array}{l}20.39 \\
20.20\}\end{array}$ \\
\hline $12 / 07 / 07$ & $\begin{array}{l}31.70^{\circ} \\
25.00\}\end{array}$ \\
\hline $67 / 21 / 87$ & $---1-\overline{3.34}$ \\
\hline & $1.10\}$ \\
\hline $58 / 12 / 87$ & 2.45 \\
\hline & $1.30\}$ \\
\hline $10 / 00 / 87$ & 2.44 \\
\hline $10 / 06 / 87$ & 1.77 \\
\hline & $0.71\}$ \\
\hline $11 / 06 / 87$ & 2.70 \\
\hline $12 / 07 / 87$ & $\begin{array}{l}1.06\} \\
2.68\end{array}$ \\
\hline & $1.28\}$ \\
\hline $88 / 20 / 87$ & 28054.00 \\
\hline------- & 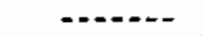 \\
\hline $68 / 20 / 87$ & 21604.01 \\
\hline$---1--\overline{-}$ & -20-10 \\
\hline $07 / 21 / 87$ & $\begin{array}{c}4856060.04 \\
\{18660.00\}\end{array}$ \\
\hline $08 / 12 / 67$ & $\begin{array}{c}4035050.00 \\
\{10540.10\}\end{array}$ \\
\hline $00 / 09 / 87$ & 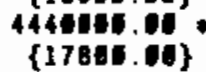 \\
\hline $16 / 08 / 87$ & $\begin{array}{l}3839190.00 \\
\{16106.60\}\end{array}$ \\
\hline
\end{tabular}

- - Value exceEds priuary dRINKINg tater standard

- VALUE EXCEEDS PROPOSED PRIMARY DRINKINO YATER STANDARD.

- VALUE EXCEEOS SCREENIHG LEVEl FOR FURTHER INVESTIGATION

- DETECTION LIUIT TAS NOT AVAILABLE FOR COUPaRISOH

NR - ANALYSIS NOT REQUESTED OR NOT YET REPORTED

VALUES IN $\{?$ ARE COUNTING ERRDRS FOR RADIONUCLIDES

TATER STANDARD(S) IN PARENTHESES ARE PRDPOSED ONLY 
TABLE C.3. (contd)

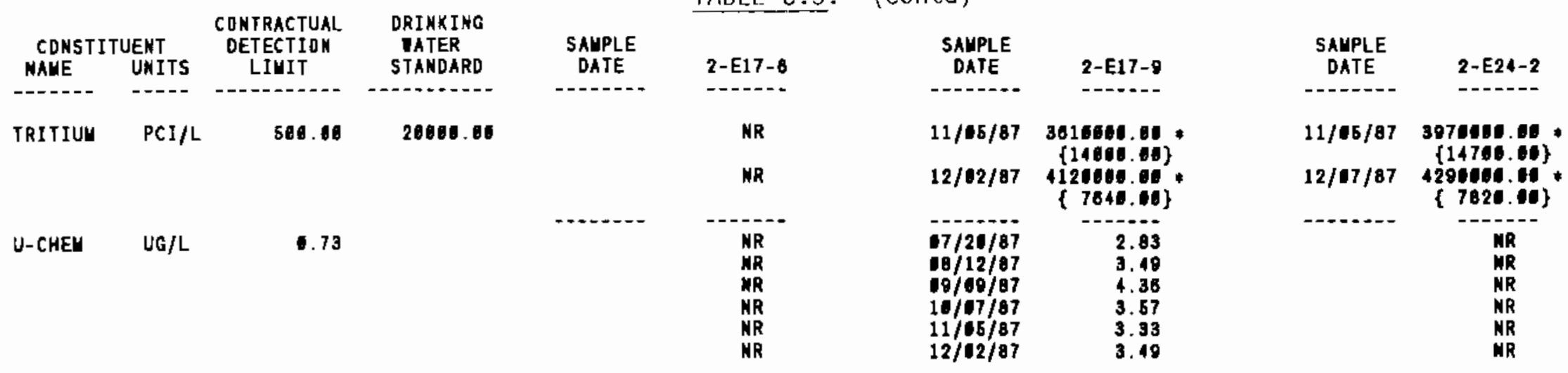

$\stackrel{?}{i}$

- - YALUE EXCEEDS PRIUARY dRINKING IATER STANDARD.

- VALUE EXCEEDS PROPOSED PRIUARY DRINKING TATER STAMDARD.

- YALUE EXCEEDS SCREENING LEYEL FOR FURTHER INYESTIGATION.

- DETECTION LIMIT IAS NDT AVAILABLE For COUPARISON

NR - AMALYSIS NDT REQUESTED OR NOT YET REPORTED

YALUES IN I $\}$ ARE COUNTING ERRORS FOR RADIONUCLIDES

IATER STANDARD'S) IN PARENTHESES ARE PROPOSED ONLY 
TABLE C.3. (contd)

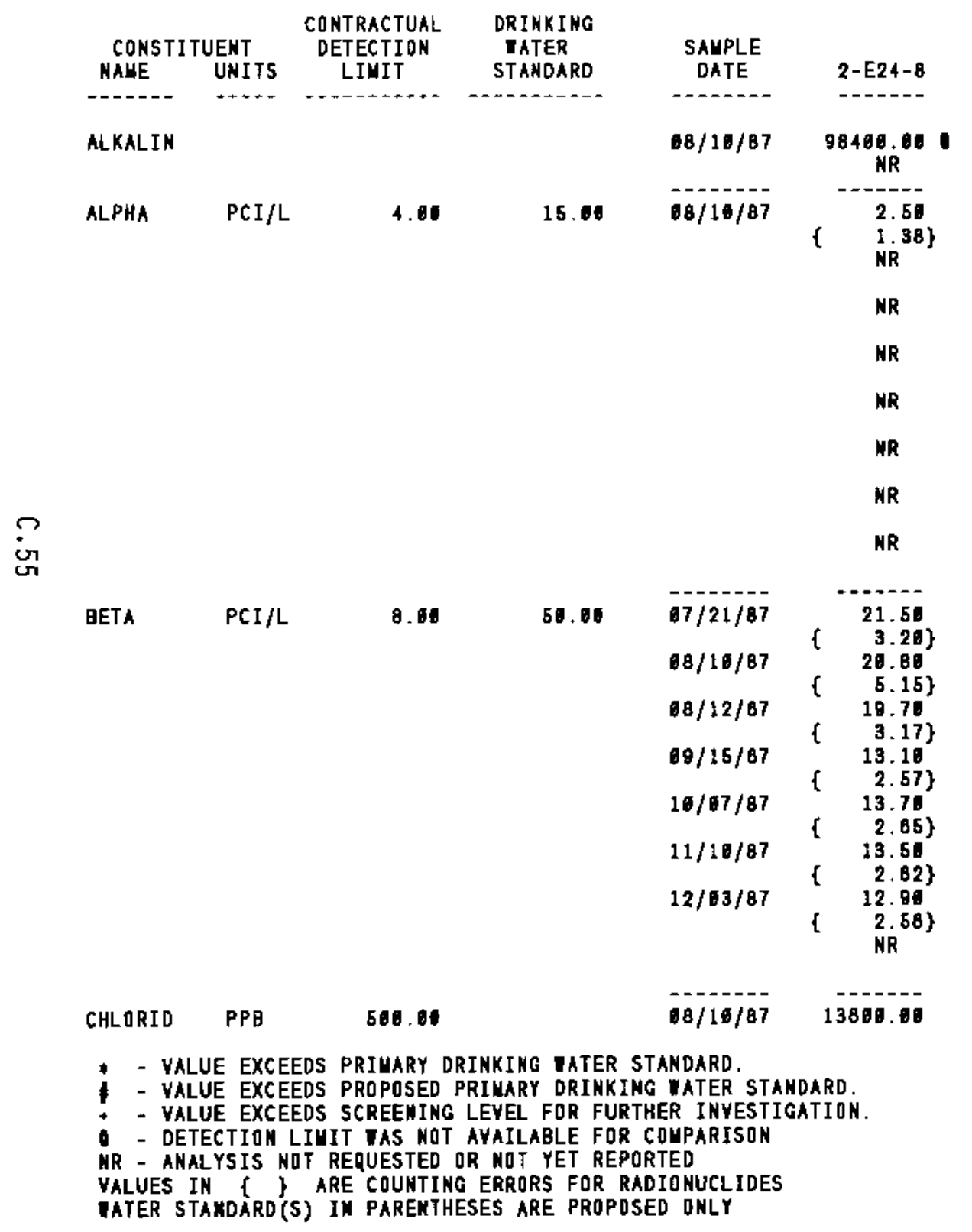

\begin{tabular}{|c|c|}
\hline $\begin{array}{l}\text { SAUPLE } \\
\text { DATE }\end{array}$ & $2-E 25-18$ \\
\hline $\begin{array}{l}67 / 16 / 87 \\
10 / 00 / 87\end{array}$ & $\begin{array}{l}86600.01 \\
88164.61\end{array}$ \\
\hline $87 / 18 / 87$ & $\begin{array}{r}1.17 \\
1.89\end{array}$ \\
\hline $87 / 18 / 87$ & 1.83 \\
\hline $88 / 13 / 87$ & 1.12 \\
\hline $89 / 13 / 87$ & $\begin{array}{l}0.52\} \\
1.29\end{array}$ \\
\hline $10 / 108 / 87$ & 10.50 \\
\hline $10 / 00 / 87$ & 1.21 \\
\hline $11 / 60 / 87$ & 1.63 \\
\hline $12 / 04 / 87$ & $\begin{array}{r}.61\} \\
1.38 \\
. .54\}\end{array}$ \\
\hline $87 / 18 / 87$ & 4 \\
\hline $87 / 16 / 87$ & \\
\hline $08 / 13 / 87$ & \\
\hline $09 / 13 / 87$ & 7.72 \\
\hline $10 / 68 / 87$ & $\begin{array}{l}2.85\} \\
5.38\end{array}$ \\
\hline $10 / 08 / 87$ & 6. \\
\hline & $1.94\}$ \\
\hline $109 / 87$ & $\begin{array}{l}5.79 \\
1.8 B\end{array}$ \\
\hline $12 / 04 / 87$ & $\begin{array}{l}8.02 \\
1.09\}\end{array}$ \\
\hline $17 / 18 / 6$ & 4716.06 \\
\hline
\end{tabular}

- VALUE EXCEEDS PRIUARY DRINKIMG IATER STANDARD.

- YALUE EXCEEDS PROPOSED PRIMARY ORINKING WATER STAMDARD.

- YALUE EXCEEDS SCREENING LEYEI FOR FURTHER INYESTIGATION.

VALUES IN I ARE COUNTING ERRORS FOR RADIONUCLIDES

IATER STAKDARD(S) IN PAREMTHESES ARE PROPOSED DNLY 


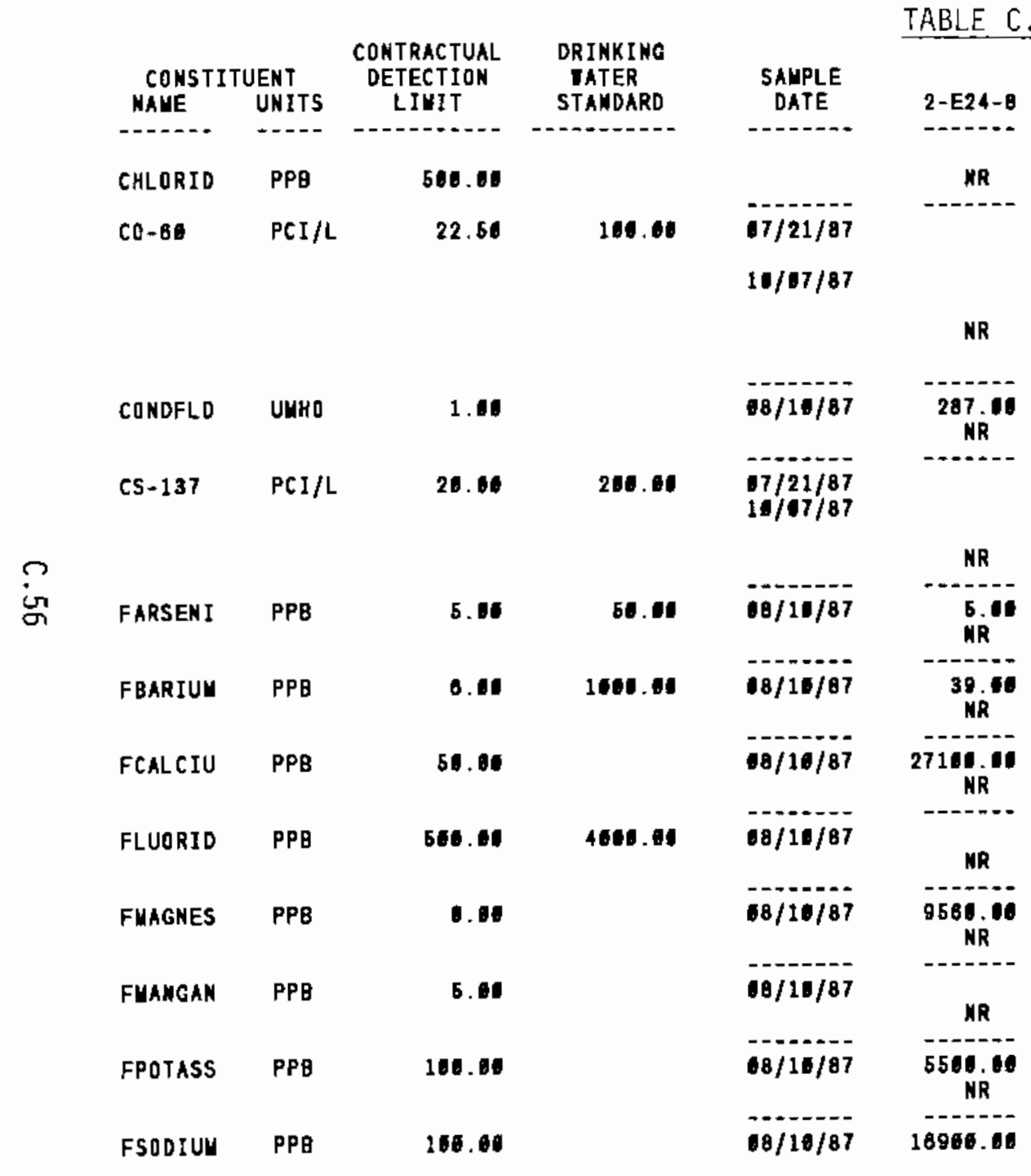

\begin{tabular}{|c|c|}
\hline $\begin{array}{l}\text { SAMPLE } \\
\text { DATE }\end{array}$ & $2-E 24-12$ \\
\hline & NR \\
\hline $17 / 13 / 87$ & 7.31 \\
\hline $09 / 16 / 87$ & 14.50 \\
\hline $10 / 13 / 87$ & $\left.\begin{array}{ll}1 & 8.83 \\
1 & 8.12 \\
\{ & 8.08\end{array}\right\}$ \\
\hline $67 / 13 / 87$ & 275.16 \\
\hline $\begin{array}{l}07 / 13 / 87 \\
60 / 16 / 87\end{array}$ & \\
\hline $10 / 13 / 87$ & \\
\hline $07 / 13 / 87$ & $\begin{array}{l}5.60 \\
\text { NR }\end{array}$ \\
\hline $07 / 13 / 87$ & WR \\
\hline $17 / 13 / 67$ & 44401.66 \\
\hline $97 / 19 / 87$ & ${ }_{\mathrm{NR}}$ \\
\hline $67 / 13 / 87$ & ${ }_{\mathrm{NR}}^{12900.01}$ \\
\hline $07 / 13 / 87$ & NR \\
\hline $67 / 13 / 87$ & SO86.01 \\
\hline $67 / 13 / 87$ & 21280.60 \\
\hline
\end{tabular}

SAMPLE

SAYTE

2-E25-18

$10 / 68 / 87$

$07 / 18 / 87$

$11 / 68 / 87$

4486.00

-

MR

$67 / 18 / 87$

$10 / 00 / 87$

254.0

241.00

$7110 / 87$

$10 / 08 / 87$

8. 39

17/16/87

$10 / 68 / 87$

i7/18/87

$10 / 08 / 87$

\{ $\mathrm{b}_{\mathrm{MR}}^{\mathrm{B}}$ \}

12.4

11.00

18.0

19.4

$07 / 16 / 87$

$10 / 08 / 87$

24590.9

24500.09

24000.01

$07 / 18 / 87$

860.10

$10 / 00 / 97$

i7/16/87

$10 / 50 / 87$

7140.60

7140.10

17/16/97

$10 / 08 / 87 \quad 7.60$

$7118 / 87$

$17 / 16 / 87$

$10 / 60 / 07$

5430.01

5106.00

$07 / 18 / 87$

1796.

- YALUE EXCEEDS PRIUARY DRIMKING YATER STAMDARD

- YALUE EXCEEDS PROPOSED PRIMARY DRIMKIMG IATER STANDARD.

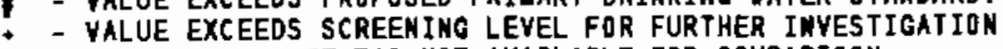

- DETECTION LIUIT IAS NOT AVAILABLE fOR COUPARISON

NR - ANALYSIS HOT REQUESTED OR NOT YET REPDRTED

YALUES IN \{ \& ARE COUNTIMG ERRORS FOR RADIONUCLIDES

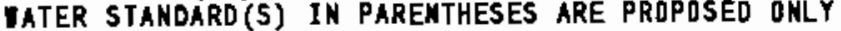


IABLE C.3. (contd)

\begin{tabular}{|c|c|c|c|c|c|}
\hline \multicolumn{2}{|c|}{ CONSTITUENT } & $\begin{array}{c}\text { CONTRACTUAL } \\
\text { DETECTION } \\
\text { LIUIT }\end{array}$ & $\begin{array}{l}\text { DRINKING } \\
\text { WATER } \\
\text { STANDARD }\end{array}$ & SAHPLE & $2-E 24-B$ \\
\hline----- & $\cdots+\infty$ & 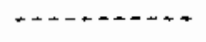 & 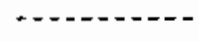 & 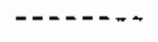 & ------ \\
\hline FSODIUU & PPB & 100.06 & & & NR \\
\hline FYANADI & PPB & 5.00 & & $18 / 10 / 87$ & $\underset{N R}{22.00}$ \\
\hline FZINC & PPB & 5.60 & & $68 / 10 / 87$ & NR \\
\hline HNITRAT & PPB & 2500.00 & 45800.06 & $\begin{array}{l}67 / 21 / 87 \\
68 / 12 / 87 \\
69 / 15 / 87 \\
16 / 17 / 87 \\
11 / 16 / 87 \\
12 / 03 / 87\end{array}$ & $\begin{array}{l}6700.01 \\
6960.04 \\
5830.00 \\
4876.00 \\
5226.04 \\
4146.01\end{array}$ \\
\hline LFLUORD & PPB & 24.00 & 4060.00 & $O B / 10 / 87$ & $\begin{array}{c}355.01 \\
\text { NR }\end{array}$ \\
\hline NITRATE & PPG & 580.00 & 45000.06 & $08 / 16 / 87$ & $\begin{array}{c}\text { NR } \\
\text { NR }\end{array}$ \\
\hline PH-LAB & & 01 & & $68 / 10 / 87$ & ${ }_{\mathrm{NR}}^{7.82}$ \\
\hline PHF IELD & & 0.10 & & $100 / 10 / 87$ & 7.10 \\
\hline$R U-166$ & $\mathrm{PCI} / \mathrm{L}$ & 172.50 & 30.00 & $07 / 21 / 87$ & . \\
\hline & & & & $10 / 67 / 87$ & $\left\{\begin{array}{l}51.40 \\
\mathbf{M R}\end{array}\right\}$ \\
\hline SR 90 & $\mathrm{PCI} / \mathrm{L}$ & 5.00 & 9.00 & $68 / 11 / 87$ & ------ \\
\hline & & & & & NR \\
\hline SULFATE & $P P B$ & 560.00 & & $08 / 10 / 87$ & 40500.00 \\
\hline $\begin{array}{l}*-V A L \\
\$-V A L \\
+-V A L \\
\text { N }- \text { DE } \\
\text { NR - AN } \\
\text { VALUES } \\
\text { WATER S }\end{array}$ & $\begin{array}{l}\text { JE EXCE } \\
\text { JE EXCE } \\
\text { JE EXCE } \\
\text { ECTION } \\
\text { YSIS N } \\
\text { S ? } \\
\text { ANDARD? }\end{array}$ & $\begin{array}{l}\text { EDS PRIUARY } \\
\text { EDS PROPOSED } \\
\text { EDS SCREENINO } \\
\text { LIUI T WAS NOT } \\
\text { OT REQUESTED } \\
\text { ARE COUNTII } \\
\text { S) IN PARENTH }\end{array}$ & $\begin{array}{l}\text { RINKING TATE } \\
\text { PRIMARY DRIN } \\
\text { LEVEL FOR FI } \\
\text { AVAILABLE F } \\
\text { OR NDT YET R } \\
\text { O ERRORS FOR } \\
\text { ESES ARE PRO }\end{array}$ & $\begin{array}{l}\text { ANDARD. } \\
\text { TATER ST } \\
\text { ER INYESTI } \\
\text { OUPARISOA } \\
\text { TED } \\
\text { IONUCLIDES } \\
\text { D DHLY }\end{array}$ & $\begin{array}{l}\text { DARD. } \\
\text { ATION. }\end{array}$ \\
\hline
\end{tabular}

\begin{tabular}{|c|c|c|c|}
\hline \multirow[t]{2}{*}{$\begin{array}{c}\text { SALPLE } \\
\text { DATE }\end{array}$} & $2-E 24-12$ & $\begin{array}{c}\text { SAMPLE } \\
\text { DATE }\end{array}$ & $2-E 25-18$ \\
\hline & NR & $10 / 08 / 87$ & 16700.00 \\
\hline $07 / 13 / 67$ & ${ }_{M R}^{21.06}$ & $\begin{array}{l}97 / 18 / 87 \\
16 / 08 / 87\end{array}$ & $\begin{array}{l}35.60 \\
38.16\end{array}$ \\
\hline $67 / 13 / 87$ & 9.11 & $\begin{array}{l}67 / 18 / 87 \\
10 / 68 / 87\end{array}$ & 8.01 \\
\hline $\begin{array}{l}07 / 13 / 87 \\
09 / 20 / 87 \\
00 / 16 / 87 \\
10 / 13 / 87 \\
11 / 10 / 87 \\
12 / 67 / 87\end{array}$ & 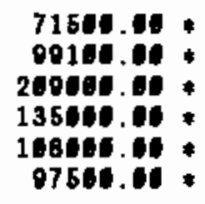 & $\begin{array}{l}07 / 16 / 87 \\
08 / 13 / 87 \\
09 / 13 / 87 \\
10 / 08 / 87 \\
11 / 09 / 87 \\
12 / 04 / 87\end{array}$ & 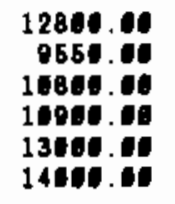 \\
\hline $17 / 13 / 87$ & $54 \mathrm{MR}_{\mathrm{MR}}$ & $\begin{array}{l}7 / 16 / 87 \\
16 / 68 / 87\end{array}$ & $\begin{array}{l}516.60 \\
476.16\end{array}$ \\
\hline $07 / 13 / 87$ & $60414 \mathrm{MR} .10$. & $\begin{array}{l}17 / 16 / 87 \\
10 / 68 / 87\end{array}$ & $\begin{array}{l}14864.06 \\
12101.06\end{array}$ \\
\hline $07 / 13 / 87$ & 7.49 & $\begin{array}{l}17 / 18 / 87 \\
10 / 68 / 67\end{array}$ & $\begin{array}{l}7.96 \\
8.05\end{array}$ \\
\hline $67 / 13 / 87$ & $\frac{8.76}{M R}$ & $\begin{array}{l}97 / 18 / 87 \\
10 / 08 / 97\end{array}$ & $\begin{array}{l}8.00 \\
7.00\end{array}$ \\
\hline $07 / 13 / 87$ & & $07 / 16 / 87$ & 40.64 \\
\hline $89 / 15 / 87$ & $\left\{\begin{array}{l}421.61 \\
102.16\end{array}\right.$ & $10 / 08 / 87$ & \\
\hline $18 / 13 / 87$ & $\left\{\begin{array}{c}183.14 \\
\{7.90\}\end{array}\right.$ & & NR \\
\hline $07 / 13 / 87$ & 4.10 & $67 / 16 / 87$ & \\
\hline $16 / 13 / 87$ & $\begin{array}{ll} & 4.31 \\
\{\quad 1.16\}\end{array}$ & $16 / 08 / 87$ & \\
\hline $67 / 13 / 87$ & 32504.010 & $67 / 16 / 87$ & 28300.06 \\
\hline
\end{tabular}


TABLE C.3. (contd)

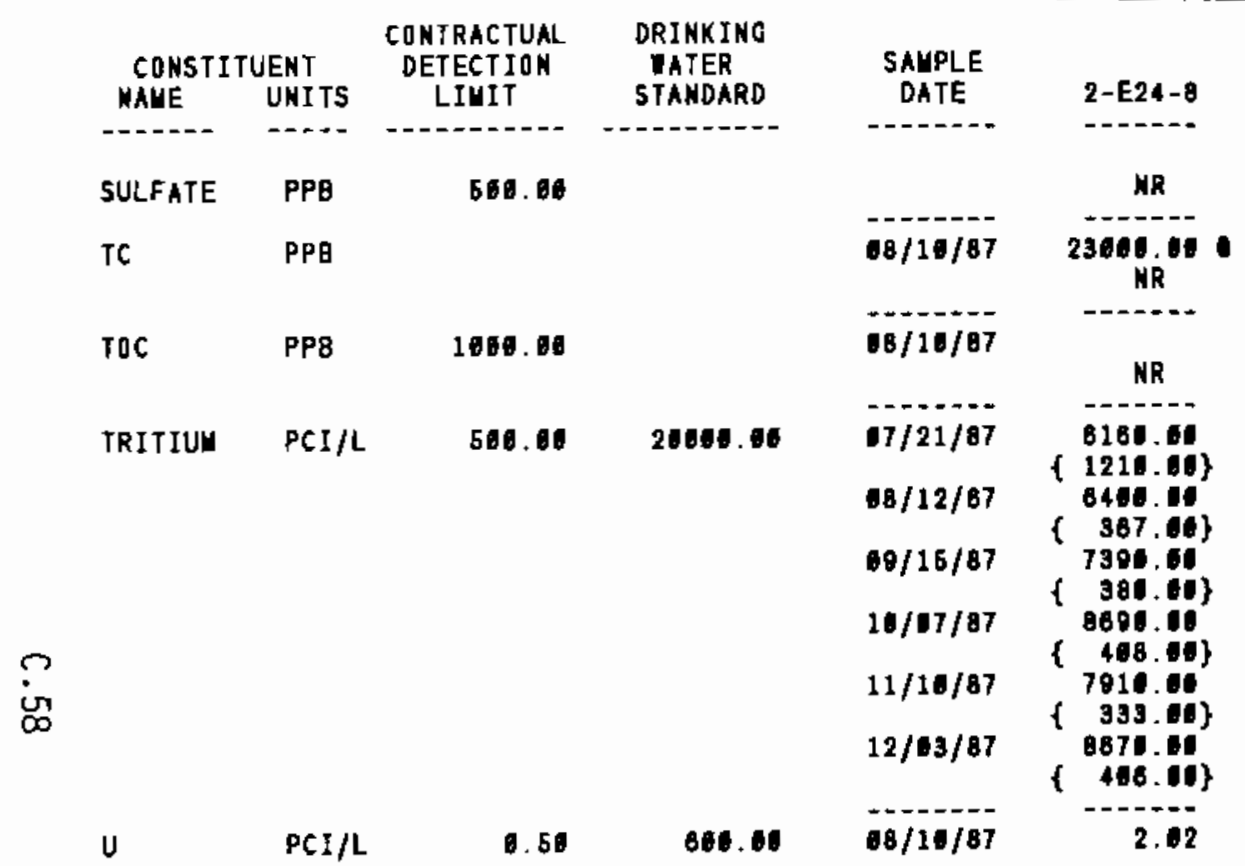

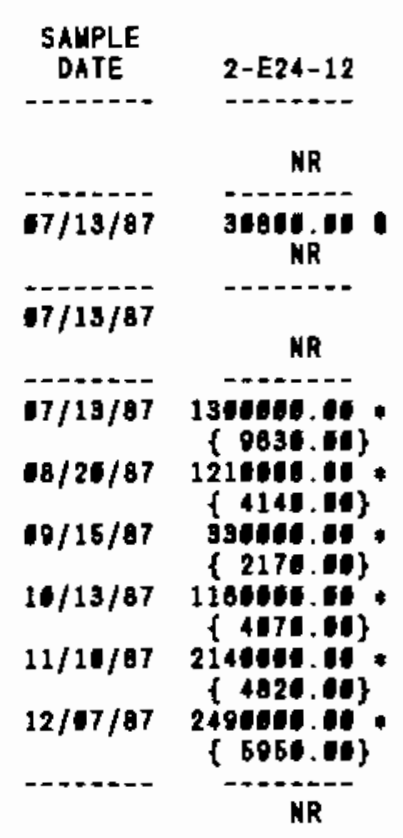

\begin{tabular}{|c|c|}
\hline $\begin{array}{l}\text { SAUPLE } \\
\text { DATE }\end{array}$ & $2-E 25-1 \theta$ \\
\hline $18 / 68 / 87$ & 35666.00 \\
\hline $\begin{array}{l}67 / 18 / 87 \\
16 / 88 / 87\end{array}$ & $\begin{array}{l}21300.00 \\
208000.00\end{array}$ \\
\hline $\begin{array}{l}07 / 18 / 87 \\
10 / 8 B / 87\end{array}$ & 1066.61 \\
\hline $07 / 10 / 87$ & 1 50000 . 00 \\
\hline $18 / 13 / 87$ & B7ofe.06 \\
\hline $09 / 13 / 87$ & 961日6.06 \\
\hline $10 / 08 / 87$ & 70010.00 \\
\hline $11 / 08 / 87$ & 82510.60 \\
\hline $12 / 04 / 87$ & $\begin{array}{l}116000.00 \\
\{1310.06\}\end{array}$ \\
\hline & NR \\
\hline
\end{tabular}

* - value exceeds priuary drinking mater standard

1 - YALUE EXCEEDS PROPOSED PRIUARY DRINKING UATER STANDARD.

- VAlUE EXCEeds SCREENING LeVEL for fURTHER INYESTIGATION.

- DETECTION LIUIT tas nOT aVAilable for couparison

NR - AMALYSIS NOT REQUESTED OR NDT YET REPORTED

VALUES IN \{\} ARE COUNTIRO ERRORS FDR RADIDNUCLIDES

TATER SIANDARD(S) IN PARENTHESES ARE PROPOSED ONLY 


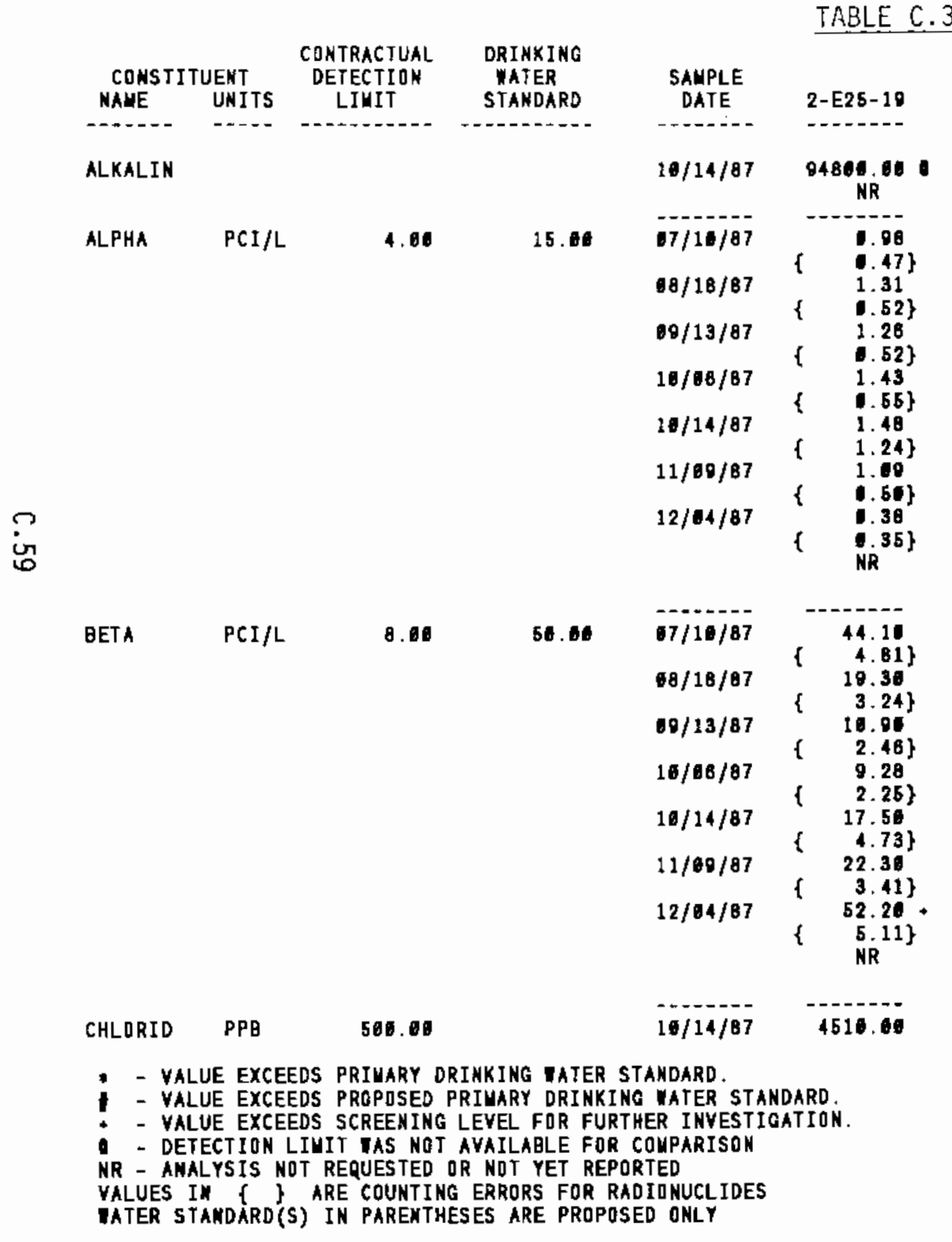

\begin{tabular}{|c|c|c|c|}
\hline $\begin{array}{c}\text { SAUPLE } \\
\text { DATE }\end{array}$ & 2-E25-20 & $\begin{array}{c}\text { SAUPLE } \\
\text { DATE }\end{array}$ & 2-E25-21 \\
\hline $\begin{array}{l}7 / 16 / 87 \\
10 / 08 / 87\end{array}$ & $\begin{array}{l}167000.010 \\
104006.00\end{array}$ & $97 / 18 / 87$ & $\underset{\mathrm{NR}}{11800 \mathrm{~s}}$ \\
\hline $17 / 16 / 87$ & $\begin{array}{l}1.91 \\
1.82\}\end{array}$ & $07 / 18 / 87$ & \\
\hline $07 / 18 / 87$ & $\begin{array}{r}1.78 \\
.58\end{array}$ & $07 / 10 / 87$ & $\begin{array}{l}0.04 \\
0.40\}\end{array}$ \\
\hline $18 / 13 / 87$ & 1.32 & $88 / 13 / 67$ & $\begin{array}{l}1.25 \\
.58\}\end{array}$ \\
\hline $69 / 13 / 87$ & 1.89 & $60 / 13 / 87$ & 1.17 \\
\hline $18 / 18 / 87$ & 1.84 & $11 / 10 / 87$ & 1.52 \\
\hline $10 / 08 / 87$ & 2.15 & $11 / 09 / 87$ & 1.74 \\
\hline $11 / 60 / 87$ & $2.10^{\circ}$ & $12 / 64 / 87$ & 1.60 \\
\hline $12 / 04 / 87$ & $\begin{array}{l}1.02\} \\
\{\quad 1.07 \\
\{\quad .63\}\end{array}$ & & $\mathbf{M R}$ \\
\hline $67 / 16 / 87$ & 24.01 & $07 / 16 / 87$ & $\begin{array}{c}17.106 \\
4.69\}\end{array}$ \\
\hline $07 / 18 / 87$ & $\begin{array}{l}9.16 \\
2.68\end{array}$ & $17 / 18 / 87$ & $\begin{array}{r}11.10^{\circ} \\
2.43\}\end{array}$ \\
\hline $08 / 13 / 87$ & 14.26 & $18 / 13 / 87$ & 12.16 \\
\hline $69 / 13 / 97$ & $\begin{array}{r}15.30 \\
2.983\end{array}$ & $00 / 13 / 87$ & 10.58 \\
\hline $10 / 08 / 87$ & $\begin{array}{r}16.20 \\
\{\quad 4.82\}\end{array}$ & $10 / 08 / 07$ & $\begin{array}{l}0.04 \\
2.21\}\end{array}$ \\
\hline $10 / 08 / 87$ & 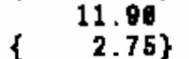 & $11 / 68 / 87$ & $\begin{array}{l}9.51 \\
1.24\}\end{array}$ \\
\hline $11 / 09 / 87$ & $\begin{array}{r}14.30 \\
\quad 2.96\end{array}$ & $12 / 04 / 87$ & 16.10 \\
\hline $12 / 04 / 87$ & $\left\{\begin{array}{r}15.50 \\
\quad 3.05\}\end{array}\right.$ & & \\
\hline $07 / 16 / 87$ & 8036.00 & $07 / 16 / 87$ & 3500.08 \\
\hline
\end{tabular}


TABLE C.3. (contd)

$\begin{array}{cccc}\text { CONSTITUENT } & \begin{array}{c}\text { CONTRACTUAL } \\ \text { DETECTION }\end{array} & \begin{array}{c}\text { DRINKING } \\ \text { WATER } \\ \text { MAME }\end{array} \text { UNITS } \\ \text { LINIT } & \text { STANDARD }\end{array}$

SAMPLE
DATE
------

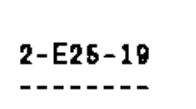

\begin{tabular}{|c|c|}
\hline $\begin{array}{c}\text { SAMPLE } \\
\text { DATE }\end{array}$ & 2-E25-20 \\
\hline $10 / 68 / 87$ & 8180.10 \\
\hline $07 / 18 / 87$ & \\
\hline $11 / 08 / 87$ & $\begin{array}{l}\text { NR } \\
\text { NR } \\
\text { NR }\end{array}$ \\
\hline & NR \\
\hline $\begin{array}{l}07 / 16 / 87 \\
10 / 10 / 87\end{array}$ & $\begin{array}{l}783.11 \\
818.11\end{array}$ \\
\hline $6 / 10 / 87$ & $\begin{array}{l}8.33 \\
5.73 k\end{array}$ \\
\hline $11 / 08 / 87$ & $\begin{array}{l}8.55 \\
5.40\} \\
\text { MR } \\
\text { MR } \\
\text { MR } \\
\text { MR }\end{array}$ \\
\hline $\begin{array}{l}7 / 18 / 87 \\
16 / 10 / 87\end{array}$ & 7.66 \\
\hline $\begin{array}{l}17 / 16 / 87 \\
16 / 88 / 87\end{array}$ & $\begin{array}{l}65.06 \\
81.11\end{array}$ \\
\hline $\begin{array}{l}07 / 16 / 87 \\
11 / 08 / 87\end{array}$ & 3.10 \\
\hline $\begin{array}{l}7 / 16 / 87 \\
10 / 08 / 87\end{array}$ & $\begin{array}{l}69101.04 \\
58200.00\end{array}$ \\
\hline $\begin{array}{l}17 / 16 / 87 \\
10 / 08 / 87\end{array}$ & \\
\hline $07 / 16 / 87$ & 1000.00 \\
\hline
\end{tabular}

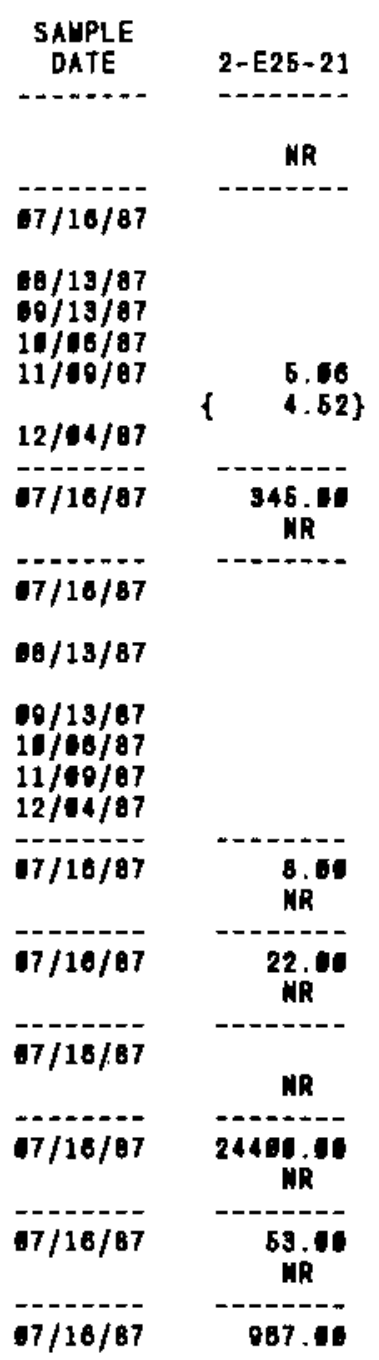

FLUORID PPB

500.00

4080.00

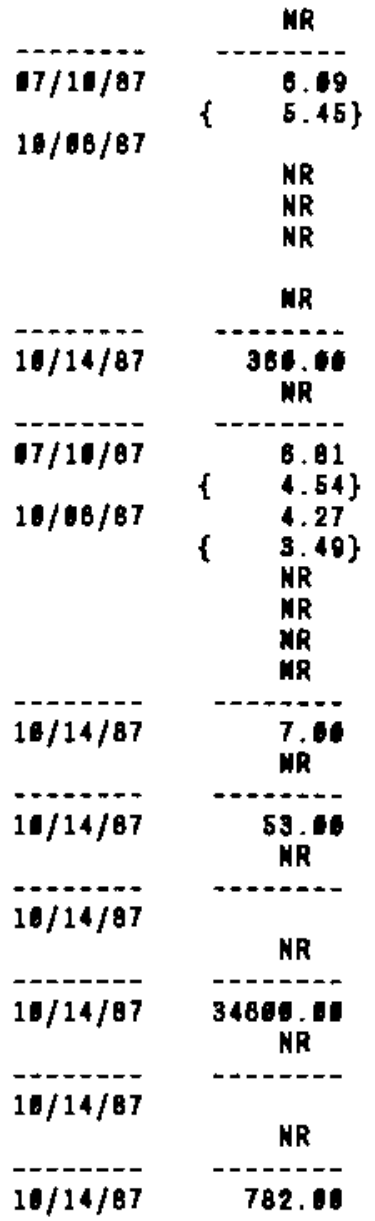

- - yalue exceeds priuary drinxing tater standard.

1 - VALUE EXCEEDS PRoposed priuary dRINKing tater standard.

- - Value eXceEds screening LeVEl for further inyestigation.

- DETECTION LIMIT IAS NOT AVAILABLE FOR COUPARISON

NR - ANALYSIS NDT REQUESTED DR NOT YET REPORTED

VALUES IN $f\}$ ARE COUNTING ERRDRS FOR RADIONUCLIDES

IATER STANDARD(S) IN PARENTHESES ARE PROPDSED ONLY 


\begin{tabular}{|c|c|c|c|c|c|}
\hline $\begin{array}{c}\text { CONSTI } \\
\text { NAUE }\end{array}$ & $\begin{array}{l}\text { FUENT } \\
\text { UNITS }\end{array}$ & $\begin{array}{l}\text { CONTRACTUAL } \\
\text { OETECTION } \\
\text { LIUIT }\end{array}$ & $\begin{array}{l}\text { DRINKING } \\
\text { WATER } \\
\text { STANDARD }\end{array}$ & $\begin{array}{c}\text { SAMPLE } \\
\text { DATE }\end{array}$ & $2-E 26-1 \theta$ \\
\hline FLUORID & PPB & 500.00 & 4800.00 & & NR \\
\hline FMAGNES & PPB & 6.60 & & $16 / 14 / 87$ & 9844.11 \\
\hline FHANGAN & PPB & 5.00 & & $16 / 14 / 87$ & NR \\
\hline FPOTASS & PPB & 100.06 & & $10 / 14 / 87$ & 8384.06 \\
\hline FSODIUU & PPB & 100.00 & & $10 / 14 / 87$ & $\begin{array}{c}30464.00 \\
\text { NR }\end{array}$ \\
\hline FSTRONT & PPB & 300.06 & & $10 / 14 / 87$ & NR \\
\hline FYAMADI & PPB & 5. 08 & & $18 / 14 / 87$ & ${ }_{M R}^{46.60}$ \\
\hline FZINC & PPB & 5.00 & & $18 / 14 / 87$ & ${ }_{M R}^{17.01}$ \\
\hline HNITRAT & PPB & 2500.06 & 45000.00 & $\begin{array}{l}67 / 16 / 87 \\
88 / 18 / 87 \\
69 / 13 / 87 \\
16 / 66 / 87 \\
11 / 69 / 87 \\
12 / 04 / 87\end{array}$ & 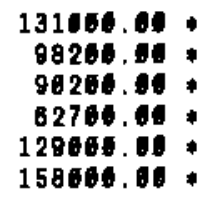 \\
\hline LFLUORD & PPB & 20.00 & 4000.80 & $18 / 14 / 87$ & NR \\
\hline WETHONE & PPB & 10.00 & & $10 / 14 / 87$ & ${ }_{\mathrm{NR}}^{28.18}$ \\
\hline NITRATE & PPB & 560.80 & 45000.60 & $10 / 14 / 87$ & $\underset{N R}{72800.01} *$ \\
\hline$P H-L A B$ & & 0.01 & & $10 / 14 / 87$ & 7.85 \\
\hline
\end{tabular}

(contd)

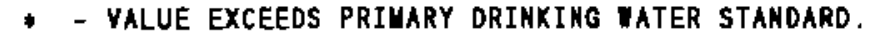

- - VALUE EXCEEDS PROPOSED PRINARY DRIMKING MATER STANDARD.

- - VALUE EXCEEDS SCREENING LEVEL FOR FURTHER INVESTIGATION.

- - DETECTION LiUIt TAS nOt aVailable for couparison

NR - ANALYSIS NOT REQUESTED OR NOT YET REPORTED

VALUES IM \{\} ARE COUNTING ERRORS FOR RADIONUCLIDES

IATER STANDARD(S) IN PARENTHESES ARE PROPOSED ONLY

\begin{tabular}{|c|c|}
\hline $\begin{array}{l}\text { SAMPLE } \\
\text { DATE }\end{array}$ & 2-E25-29 \\
\hline $10 / 68 / 87$ & \\
\hline $\begin{array}{l}7 / 16 / 87 \\
16 / 66 / 87\end{array}$ & $\begin{array}{l}18541.11 \\
18308.61\end{array}$ \\
\hline $\begin{array}{l}67 / 18 / 87 \\
10 / 08 / 87\end{array}$ & \\
\hline $\begin{array}{l}07 / 18 / 87 \\
10 / 00 / 87\end{array}$ & $\begin{array}{l}13100.00 \\
12790.06\end{array}$ \\
\hline $\begin{array}{l}17 / 18 / 87 \\
10 / 1 / 8 / 87\end{array}$ & $\begin{array}{l}18000.18 \\
45800.08\end{array}$ \\
\hline $\begin{array}{l}67 / 18 / 87 \\
10 / 00 / 87\end{array}$ & 345.11 \\
\hline $\begin{array}{l}17 / 18 / 87 \\
10 / 100 / 87\end{array}$ & $\begin{array}{l}12.11 \\
48.11\end{array}$ \\
\hline $\begin{array}{l}67 / 18 / 87 \\
10 / 08 / 67\end{array}$ & $\begin{array}{r}23.11 \\
8.11\end{array}$ \\
\hline $\begin{array}{l}67 / 16 / 87 \\
68 / 13 / 97 \\
09 / 13 / 87 \\
16 / 68 / 87 \\
11 / 69 / 87 \\
12 / 64 / 87\end{array}$ & 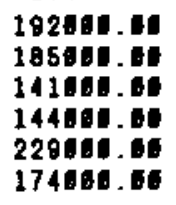 \\
\hline $\begin{array}{l}07 / 10 / 87 \\
10 / 68 / 87\end{array}$ & $\begin{array}{l}786.60 \\
868.66\end{array}$ \\
\hline $\begin{array}{l}07 / 16 / 87 \\
10 / 08 / 87\end{array}$ & \\
\hline $\begin{array}{l}17 / 16 / 87 \\
10 / 08 / 87\end{array}$ & $\begin{array}{l}178000.00 \\
146006.00\end{array}$ \\
\hline $07 / 18 / 87$ & 7.88 \\
\hline
\end{tabular}

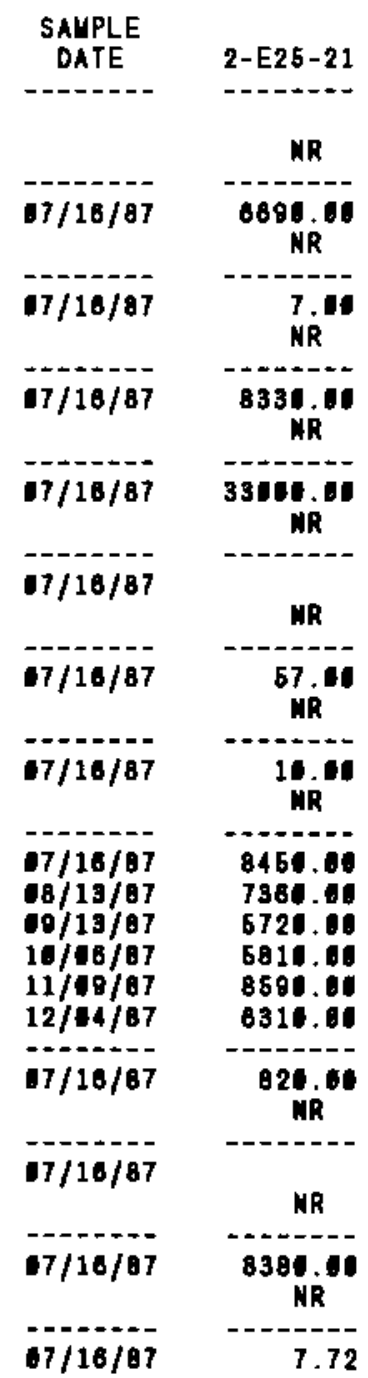


TABLE C.3. (contd)

\begin{tabular}{|c|c|c|}
\hline CONSTITUENT & $\begin{array}{c}\text { CONTRACTUAL } \\
\text { DETECTIOA }\end{array}$ & $\begin{array}{l}\text { DRINXING } \\
\text { WATER }\end{array}$ \\
\hline MAUE UNITS & & STANOARD \\
\hline
\end{tabular}

$\begin{array}{cc}\text { SAYPLE } \\ \text { DATE }\end{array} 2-\mathrm{E25-10}$

\begin{tabular}{|c|c|}
\hline $\begin{array}{l}\text { SANPLE } \\
\text { OATE }\end{array}$ & $2-E 25-20$ \\
\hline 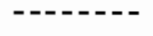 & $\cdots-$ \\
\hline $10 / 68 / 87$ & 7.74 \\
\hline $\begin{array}{l}07 / 16 / 87 \\
10 / 88 / 87\end{array}$ & 8.01 \\
\hline $67 / 18 / 87$ & - \\
\hline $10 / 08 / 87$ & 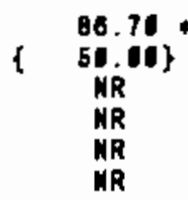 \\
\hline $\begin{array}{l}67 / 16 / 87 \\
16 / 18 / 87\end{array}$ & 90304.06 \\
\hline $\begin{array}{l}17 / 16 / 87 \\
10 / 68 / 87\end{array}$ & 28846.00 \\
\hline $\begin{array}{l}07 / 16 / 87 \\
10 / 60 / 87\end{array}$ & 2070.00 \\
\hline $\begin{array}{l}97 / 10 / 87 \\
10 / 68 / 87\end{array}$ & 1800.10 \\
\hline $7 / 16 / 87$ & 631584.66 \\
\hline $68 / 13 / 87$ & 567640.64 \\
\hline $00 / 13 / 87$ & $\begin{array}{l}286004 \\
\{2026.00\}\end{array}$ \\
\hline $10 / 08 / 87$ & $\begin{array}{l}311060.00 \\
\{2110.00\}\end{array}$ \\
\hline $11 / 00 / 07$ & $\begin{array}{l}614000.00 \\
\{3370.01\}\end{array}$ \\
\hline $12 / 04 / 87$ & $\begin{array}{l}050000.14 \\
\text { (3504.06) }\end{array}$ \\
\hline
\end{tabular}

\begin{tabular}{|c|c|}
\hline \multirow{2}{*}{$\begin{array}{l}\text { SAUPLE } \\
\text { DATE }\end{array}$} & \multirow{2}{*}{$2-E 25-21$} \\
\hline & \\
\hline & NR \\
\hline $07 / 16 / 87$ & 7.60 \\
\hline - - - - & $\begin{array}{c}\text { MR } \\
-\end{array}$ \\
\hline $07 / 16 / 87$ & $56.71 *$ \\
\hline $18 / 13 / 87$ & .315 \\
\hline $\begin{array}{l}09 / 18 / 87 \\
10 / 08 / 87 \\
11 / 00 / 87 \\
12 / 04 / 87\end{array}$ & \\
\hline$\because-1$ & - \\
\hline $17 / 16 / 87$ & N6400.10 \\
\hline $67 / 16 / 87$ & 36100.00 \\
\hline - - & ------ \\
\hline $67 / 16 / 87$ & MR \\
\hline $07 / 19 / 87$ & \\
\hline & MR \\
\hline $17 / 16 / 87$ & $\left\{\begin{array}{l}5070.06 \\
1180.10\}\end{array}\right.$ \\
\hline $88 / 13 / 87$ & $\begin{array}{r}4344.10 \\
324.06\}\end{array}$ \\
\hline $60 / 13 / 87$ & $\begin{array}{r}4316.11 \\
+310.04\}\end{array}$ \\
\hline $10 / 96 / 07$ & 5260.06 \\
\hline $11 / 09 / 87$ & $\left\{\begin{array}{r}5010.00 \\
339.06\}\end{array}\right.$ \\
\hline $12 / 64 / 87$ & $\begin{array}{r}4836.00 \\
\{\quad 932.01\}\end{array}$ \\
\hline
\end{tabular}

- - VALUE eXCEeds PRIMARY dRINKING vaTER STandaro.

* - VALUE EXCEEDS PROPOSED PRIUARY DRINKING IATER STANDARO

- - VALUE EXCEEDS SCREENINO LEVEl For FURTHER INVESTIGATION.

- DETECTION LIUIT UAS NOT AYAILABLE FOR COMPARISON

MR - AMALYSIS NOT REQUESTED OR NOT YET REPORTED

VALUES IN $\{$ A ARE CDUNTINO ERRORS FOR RADIONUCLIDES

DATER STAMDARD(S) IN PARENTHESES ARE PROPOSED ONLY 
TABLE C.3. (contd)

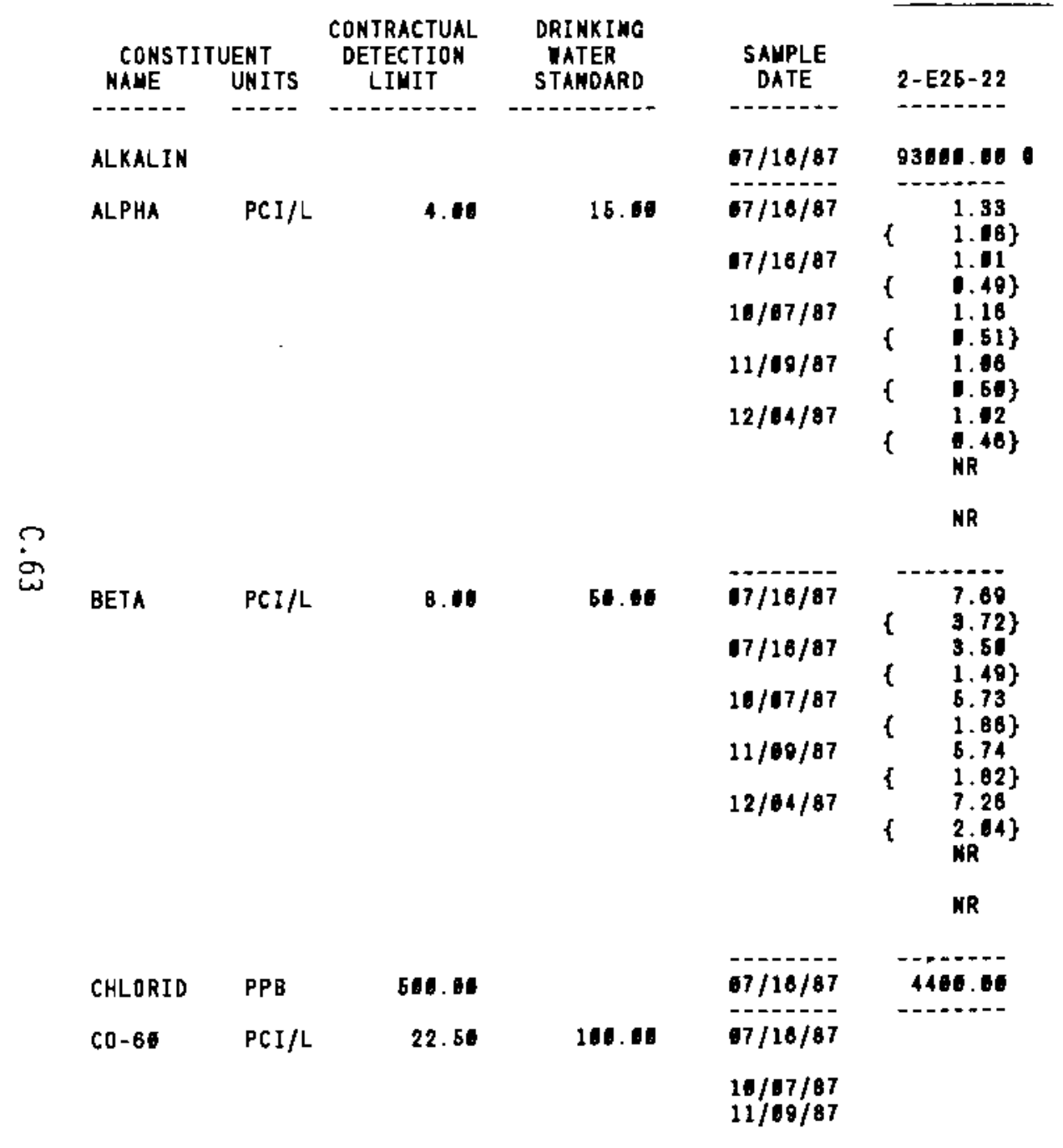

\begin{tabular}{|c|c|}
\hline $\begin{array}{l}\text { SALPLE } \\
\text { DATE }\end{array}$ & $2-E 25-23$ \\
\hline 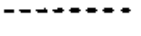 & 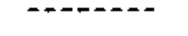 \\
\hline $07 / 17 / 87$ & 105000.00 \\
\hline $07 / 17 / 87$ & 1.63 \\
\hline $07 / 17 / 87$ & 1.63 \\
\hline $18 / 13 / 87$ & 1.12 \\
\hline $19 / 13 / 87$ & 0.78 \\
\hline $10 / 00 / 87$ & $\begin{array}{l}.72\} \\
.75 \\
.92\}\end{array}$ \\
\hline $11 / 00 / 87$ & C. 87 \\
\hline $12 / 04 / 87$ & $\{\quad 014\}$ \\
\hline $17 / 17 / 87$ & 21.20 \\
\hline $07 / 17 / 87$ & $\left\{\begin{array}{r}5.12 \\
14.60\end{array}\right.$ \\
\hline $18 / 13 / 87$ & $\left\{\begin{array}{r}2.75\} \\
14.65\end{array}\right.$ \\
\hline $19 / 19 / 87$ & $\begin{array}{r}13.04 \\
\{\quad 2.63\}\end{array}$ \\
\hline $16 / 69 / 87$ & $\begin{array}{r}14.60^{\circ} \\
\quad 2.67\}\end{array}$ \\
\hline $11 / 00 / 87$ & 13.25 \\
\hline $12 / 64 / 87$ & $\begin{array}{r}14.70 \\
\{\quad 2.79\}\end{array}$ \\
\hline $17 / 17 / 87$ & 1680.60 \\
\hline $17 / 17 / 87$ & ----- \\
\hline $\begin{array}{l}00 / 13 / 87 \\
00 / 13 / 87\end{array}$ & \\
\hline
\end{tabular}

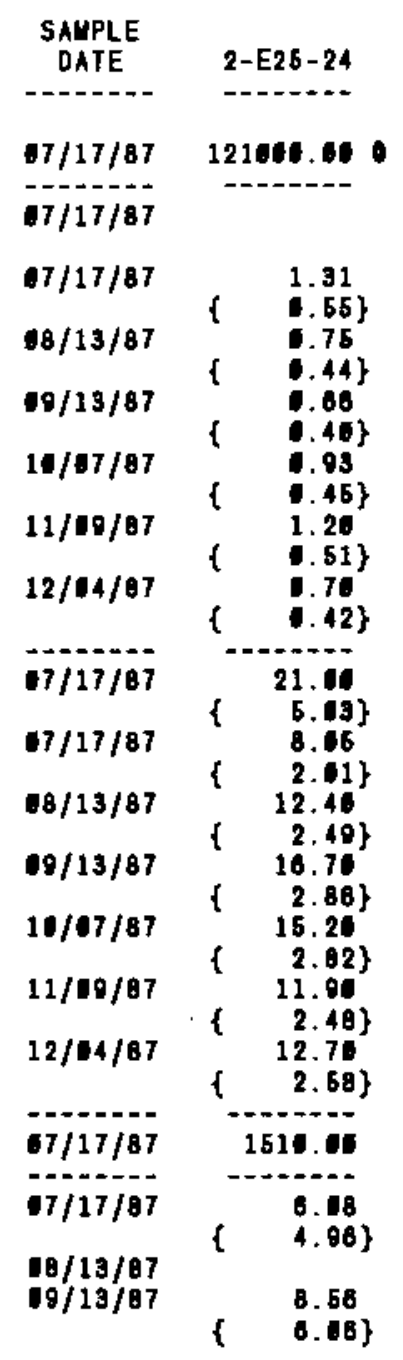

- - value exceeds priuary drinkino tater standard.

1 - YALUE EXCEEDS PROPOSED PRIUARY DRINKING IATER STANDARD.

- YALUE EXCEEDS SCREEKING LEVEL FDR FURTHER INYESTIGATION.

- - DETECTION LIHIT IAS NOT AVAILABLE FOR COUPARISON

NR - AMALYSIS NOT REQUESTED OR MOT YET REPORTED

VALUES IN $T$ ARE COUNTING ERRORS FOR RADIONUCLIDES

IATER STANDARD'(S) IN PARENTHESES ARE PROPOSED ONLY 
TABLE C.3. (contd)

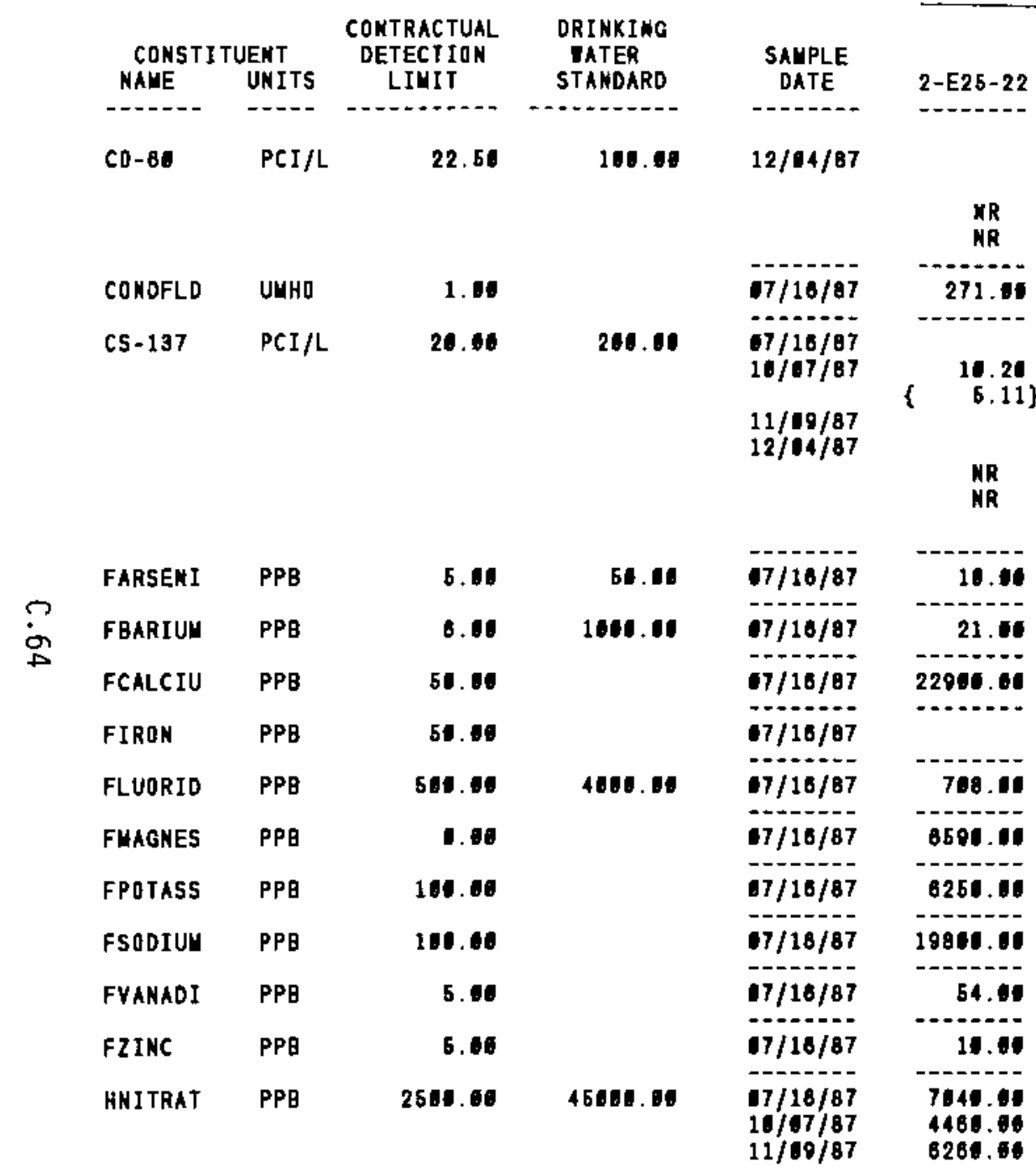

\begin{tabular}{|c|c|}
\hline $\begin{array}{l}\text { SAUPLE } \\
\text { DATE }\end{array}$ & 2-E26-23 \\
\hline $10 / 60 / 87$ & \\
\hline $\begin{array}{l}11 / 09 / 87 \\
12 / 04 / 87\end{array}$ & \\
\hline $67 / 17 / 87$ & 321.00 \\
\hline $\begin{array}{l}17 / 17 / 87 \\
18 / 13 / 87\end{array}$ & \\
\hline $\begin{array}{l}09 / 13 / 97 \\
10 / 00 / 87 \\
11 / 40 / 87 \\
12 / 04 / 87\end{array}$ & $\left\{\begin{array}{l}8.48 \\
4.91\end{array}\right]$ \\
\hline $07 / 17 / 87$ & 17.41 \\
\hline $07 / 17 / 87$ & 37.01 \\
\hline $07 / 17 / 87$ & 26300.00 \\
\hline $17 / 17 / 87$ & 05.00 \\
\hline $07 / 17 / 87$ & 001.00 \\
\hline $07 / 17 / 87$ & 4000.00 \\
\hline $07 / 17 / 87$ & 7820.66 \\
\hline $07 / 17 / 87$ & 18460.06 \\
\hline $07 / 17 / 87$ & 130.66 \\
\hline $07 / 17 / 87$ & 17.06 \\
\hline $\begin{array}{l}07 / 17 / 878 \\
8 / 13 / 87 \\
60 / 13 / 87\end{array}$ & 3971.6E \\
\hline
\end{tabular}

SAMPLE

DATE

------

2-E25-24

$10 / 107 / 87 \quad 11.21$

$11 / 00 / 87$

$12 / 04 / 87$

$17 / 17 / 87$

-7i17/87

$08 / 13 / 87$

$09 / 13 / 87$

$19 / 107 / 87$

$11 / 00 / 87$
$12 / 04 / 87$

R

10.10

17...

iา117/87

$07 / 17 / 07$

$67 / 17 / 87$

7/17/87

17/17/87

17/17/87

$07 / 17 / 87$

17/17/87

$17 / 17 / 87$

97/17/87

:7/17/87

$17 / 17 / 87$

-------

$07 / 17 / 87$

$18 / 13 / 87$

$10 / 13 / 87$ $\left\{\begin{array}{r}11.21 \\ 7.94\}\end{array}\right.$

250.010

…….

17.10

(17....

32.11

26060.06

-------

948.06

5016.06

o796.06

24655

146.00

0.06

3280.01

* - Value exceEds primary drinking tater stamdard.

1 - VALUE EXCEEDS PROPOSED PRIUARY DRINKING IATER STAMDARD.

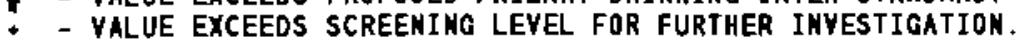

- DETECTION LIUIT UAS nOt AVAILABLE fOR COUPARISON

NR - ANALYSIS NOT RERUESTED OR NOT YET REPORTED

YALUES IN \& I ARE COUNTING ERRORS FOR RADIONUCLIDES

IATER STANDARD(S) IN PARENTHESES ARE PROPOSED ONLY 
TABLE C.3. (contd)

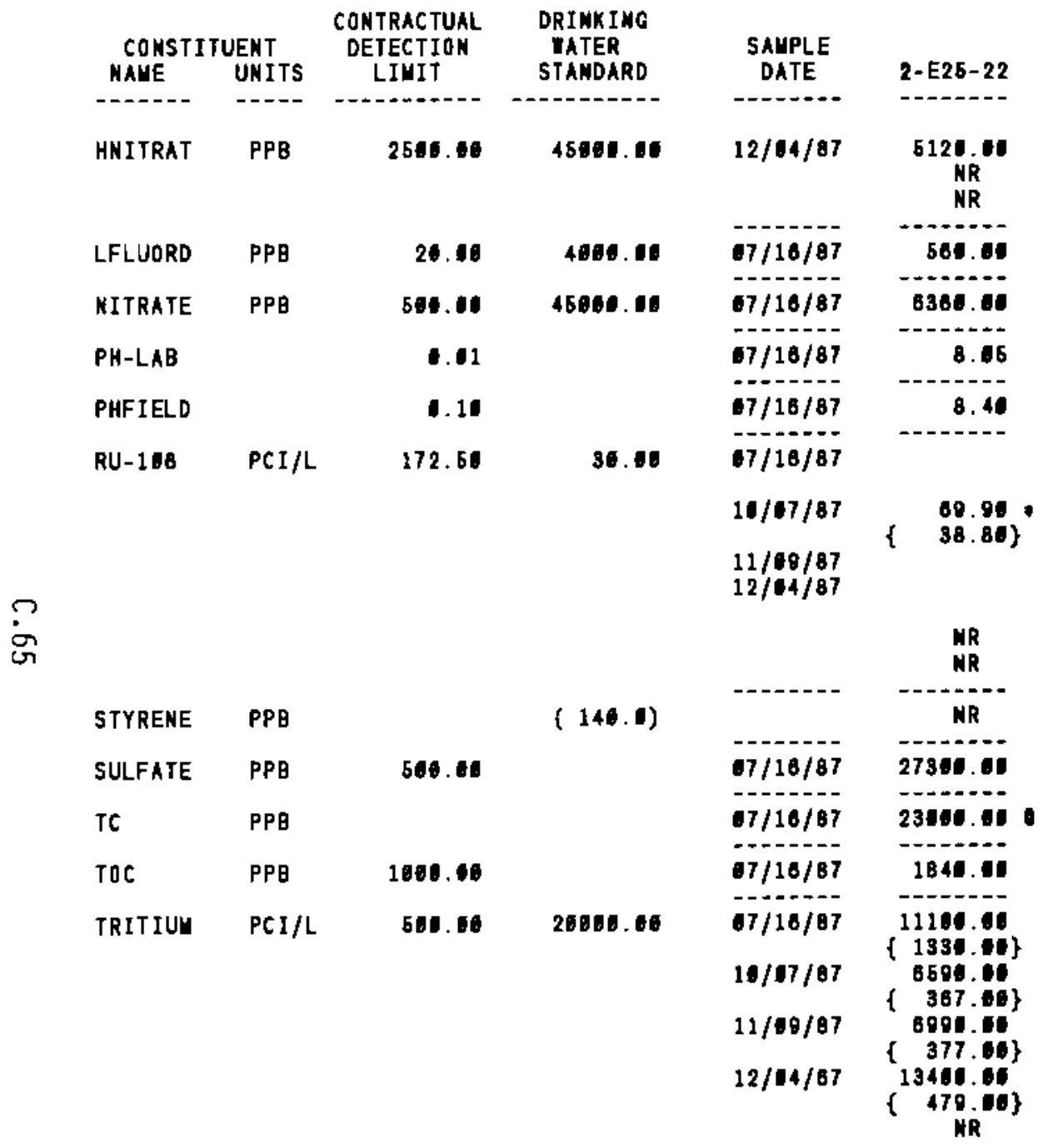

\begin{tabular}{|c|c|}
\hline $\begin{array}{c}\text { SAUPLE } \\
\text { DATE }\end{array}$ & 2-E25-23 \\
\hline $\begin{array}{l}11 / 49 / 87 \\
11 / 09 / 87 \\
12 / 04 / 87\end{array}$ & 2556.00 \\
\hline $07 / 17 / 87$ & 525.10 \\
\hline $17 / 17 / 87$ & 2540.01 \\
\hline $97 / 17 / 87$ & 7.09 \\
\hline $7 / 17 / 87$ & 7.30 \\
\hline $\begin{array}{l}17 / 17 / 87 \\
09 / 13 / 87\end{array}$ & $\begin{array}{l}50.11 \\
\{\quad 40.71\}\end{array}$ \\
\hline $\begin{array}{l}10 / 13 / 87 \\
10 / 109 / 87\end{array}$ & \\
\hline $\begin{array}{l}11 / 49 / 87 \\
12 / 04 / 87\end{array}$ & \\
\hline $11 / 17 / 87$ & 11.00 \\
\hline $67 / 17 / 87$ & 28910.00 \\
\hline $61 / 17 / 87$ & 270100.00 \\
\hline $67 / 17 / 87$ & \\
\hline $97 / 17 / 87$ & $\begin{array}{r}1886.4 \\
222.64\}\end{array}$ \\
\hline $18 / 13 / 87$ & $\left\{\begin{array}{l}320.60 \\
177.06\}\end{array}\right.$ \\
\hline $09 / 13 / 87$ & \\
\hline $10 / 09 / 87$ & \\
\hline $11 / 09 / 87$ & $\begin{array}{l}478.80 \\
\{\quad 212.00\}\end{array}$ \\
\hline
\end{tabular}

SAMPLE

DATE

2-E25-24

-.-....

$10 / 47 / 87$

$11 / 00 / 87$

$12 / 04 / 87$

67/17/87

$07 / 17 / 87$

07/17/87

07/17/87

07/17/87

$19 / 13 / 87$

$69 / 13 / 87$
$10 / 67 / 87$

$11 / 00 / 87$

$12 / 04 / 87$

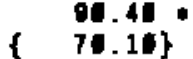

$07 / 17 / 8$

$07 / 17 / 87$

67/17/87

$67 / 17 / 87$

$68 / 13 / 87$

$19 / 13 / 97$

$10 / 07 / 87$

$11 / 60 / 87$

890.06

1071.00

7.88

-1.80
-7.40

$-\cdot--\cdot-$

......

NR

278st.06

31200.10 .

1171.

$\{225.06\}$

710.00

$187.00\}$

216.10

491.10

$\{220.16\}$

218.60

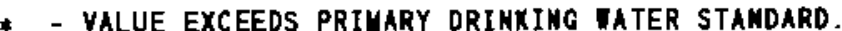

* - VALUE EXCEEDS PRIUARY ORINKING EXCEEDS PROPOSED PRIMARY DRIMKIKG WATER STANDARD.

- VALUE EXCEEDS PROPOSED PRIMARY DRIMKIRG TATER STANDARD.

- DETECTION LIUIT VAS NOT AVAILABLE FOR COUPARISON

MR - ANALYSIS NOT REQUESTED OR NOT YET REPORTED

VALUES IN \{ ARE COUNIIHG ERRORS FOR RADIONUCLIDES

IATER STANDARD'(S) IN PARENTHESES ARE PROPOSED ONLY 
TABLE C.3. (contd)

\begin{tabular}{ccc}
\multicolumn{2}{c}{ CONSTITUENT } & $\begin{array}{c}\text { CONTRACTUAL } \\
\text { DETECTION } \\
\text { HAHE }\end{array}$ UNITS \\
LIUIT
\end{tabular}

TRITIUU PCI/L

500.00
DRINKING

TATER

STANDARD

-

20000. 09
SAUPLE

DATE

- DATE-

2-E25-22

MR
SAMPLE

DATE

- - - - -

$12 / 64 / 87$
2-E25-23

469.106

$\{213.08\}$
SAUPLE

DATE

2-E25-24

- - -

$12 / 04 / 87$

748.01

รั

- - VALUE eXceEds priuary dRINKINg tater stakdard

- - VALUE EXCEEDS PROPOSED PRIUARY DRINXING UATER STANDARO.

- - VALUE EXCEEDS SCREENINg LEVEL For FURTHER INVESTIGATION.

- DETECTION LIUIT IAS MOT AVALABLE FOR COMPARISON

NR - ANALYSIS NOT REQUESTED OR NDT YET REPORTED

VALUES IN \{ $\}$ ARE COUNTING ERRORS FOR RADIONUCLIDES

TATER STANDARD(S) IN PARENTHESES ARE PROPOSED ORLY 


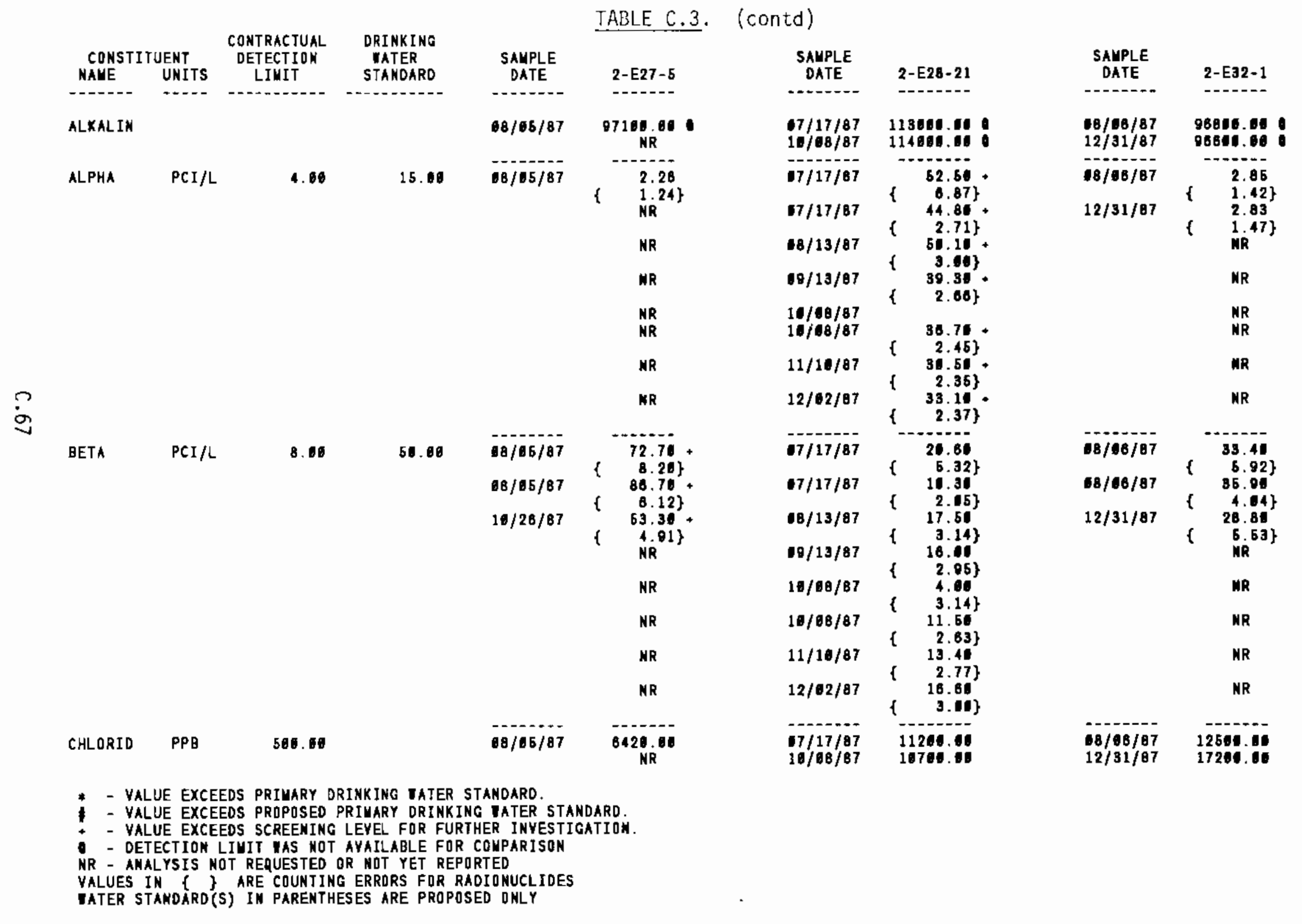




\begin{tabular}{|c|c|c|c|c|c|c|c|c|c|}
\hline $\begin{array}{c}\text { CONSTIT } \\
\text { MANE }\end{array}$ & $\begin{array}{l}\text { UENT } \\
\text { UNITS }\end{array}$ & $\begin{array}{c}\text { CONTRACTUAL } \\
\text { DETECTIOX } \\
\text { LIUIT T }\end{array}$ & $\begin{array}{l}\text { ORINKING } \\
\text { TATER } \\
\text { STANDARD }\end{array}$ & $\begin{array}{c}\text { SAMPLE } \\
\text { DATE }\end{array}$ & TABLE C & $\begin{array}{l}\text { itd) } \\
\text { SAMPLE } \\
\text { DATE }\end{array}$ & $2-E 28-21$ & $\begin{array}{c}\text { SAUPLE } \\
\text { DATE }\end{array}$ & 2-E32-1 \\
\hline$C O-86$ & PCI/L & 22.60 & 200.60 & $\begin{array}{l}18 / 05 / 87 \\
10 / 28 / 87\end{array}$ & $\begin{array}{l}\text { NR } \\
\text { MR } \\
\text { NR } \\
\text { NR }\end{array}$ & $\begin{array}{l}07 / 17 / 87 \\
68 / 13 / 87 \\
99 / 13 / 87 \\
16 / 1 / 8 / 87 \\
11 / 11 / 87 \\
12 / 02 / 87\end{array}$ & 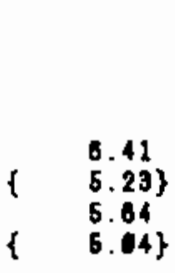 & & $\begin{array}{l}\text { NR } \\
\text { NR } \\
\text { NR } \\
\text { NR } \\
\text { NR } \\
\text { NR }\end{array}$ \\
\hline CONDFLD & UМHO & 1.80 & & $08 / 05 / 87$ & ${ }_{\mathrm{MR}}^{276.64}$ & $\begin{array}{l}17 / 17 / 87 \\
16 / 108 / 87\end{array}$ & $\begin{array}{l}551.11 \\
528.11\end{array}$ & $\begin{array}{l}08 / 68 / 87 \\
12 / 31 / 87\end{array}$ & $\begin{array}{l}275.10 \\
270.00\end{array}$ \\
\hline CS-137 & $\mathrm{PCI} / \mathrm{L}$ & 20.90 & 200.00 & $\begin{array}{l}18 / 05 / 87 \\
10 / 26 / 87\end{array}$ & $\begin{array}{c}12.01 \\
\{\quad 8.38\} \\
M R \\
M R \\
N R \\
N R\end{array}$ & $\begin{array}{l}17 / 17 / 87 \\
08 / 13 / 87 \\
68 / 13 / 87 \\
14 / 88 / 87 \\
11 / 16 / 87 \\
12 / 02 / 87\end{array}$ & $\begin{array}{ll} & 3.78 \\
\{\quad 3.38\} \\
\{\quad 3.82 \\
\{\quad 3.84\}\end{array}$ & & $\begin{array}{l}\text { MR } \\
\text { MR } \\
\text { NR } \\
\text { MR } \\
\text { MR } \\
\text { MR }\end{array}$ \\
\hline FARSENI & $\mathrm{PPB}$ & 5.06 & 50.66 & $08 / 05 / 87$ & MR & $\begin{array}{l}07 / 17 / 87 \\
16 / 108 / 87\end{array}$ & - - - & $\begin{array}{l}68 / 48 / 87 \\
12 / 31 / 87\end{array}$ & $\begin{array}{l}6.10 \\
0.110\end{array}$ \\
\hline FBARIUU & $P P B$ & 6.08 & 1080.08 & $08 / 06 / 87$ & ${ }_{\mathrm{NR}}^{17.60}$ & $\begin{array}{l}17 / 17 / 87 \\
11 / 08 / 87\end{array}$ & $\begin{array}{l}43.11 \\
48.11\end{array}$ & $\begin{array}{l}18 / 06 / 87 \\
12 / 31 / 87\end{array}$ & $\begin{array}{l}37.16 \\
31.06\end{array}$ \\
\hline FCALCIU & PPB & 60.00 & & $68 / 65 / 87$ & $\begin{array}{c}8324.110 \\
\text { NR }\end{array}$ & $\begin{array}{l}07 / 17 / 87 \\
10 / 60 / 87\end{array}$ & $\begin{array}{l}87200.01 \\
\text { B3100.00 }\end{array}$ & $\begin{array}{l}98 / 58 / 87 \\
12 / 31 / 87\end{array}$ & $\begin{array}{l}24200.60 \\
30000.06\end{array}$ \\
\hline FLUORID & $\mathrm{PPB}$ & 600.00 & 4680.00 & $18 / 05 / 87$ & NR & $\begin{array}{l}17 / 17 / 87 \\
16 / 108 / 87\end{array}$ & 674.6 & $\begin{array}{l}08 / 06 / 87 \\
12 / 91 / 87\end{array}$ & $\begin{array}{l}684.11 \\
805.11\end{array}$ \\
\hline FUAGMES & PPB & 0.60 & & $68 / 06 / 87$ & $\underset{\text { NR }}{8381.06}$ & $\begin{array}{l}17 / 17 / 87 \\
10 / 08 / 87\end{array}$ & $\begin{array}{l}15760.11 \\
157106.01\end{array}$ & $\begin{array}{l}18 / 100 / 87 \\
12 / 31 / 87\end{array}$ & 9810.18 \\
\hline FPOTASS & $\mathrm{PPB}$ & 108.60 & & $08 / 05 / 87$ & Bo10.01 & $07 / 17 / 87$ & 5320.01 & $10 / 06 / 87$ & 548E. 14 \\
\hline 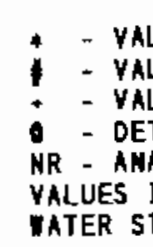 & 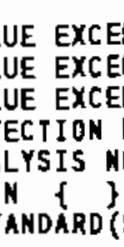 & $\begin{array}{l}\text { EDS PRTNARY D } \\
\text { EDS PRDPQSED } \\
\text { EDS SCREENING } \\
\text { LIUIT FAS NOT } \\
\text { OT RERUESTED } \\
\text { ARE COUNTIN } \\
\text { (S) IN PARENTH }\end{array}$ & $\begin{array}{l}\text { RINKING IATEF } \\
\text { RIWARY DRIN } \\
\text { LEVEL FOR F } \\
\text { AVAILAOLE FC } \\
\text { R NOT YET RE } \\
\text { EERRORS FOR } \\
\text { ESES ARE PROF }\end{array}$ & $\begin{array}{l}\text { ANOARO } \\
\text { WATER STA } \\
\text { ER INYESTI } \\
\text { OUPARISOH } \\
\text { TED } \\
\text { IONUCLIDES } \\
\text { D DHLY }\end{array}$ & ATON. & & & & \\
\hline
\end{tabular}




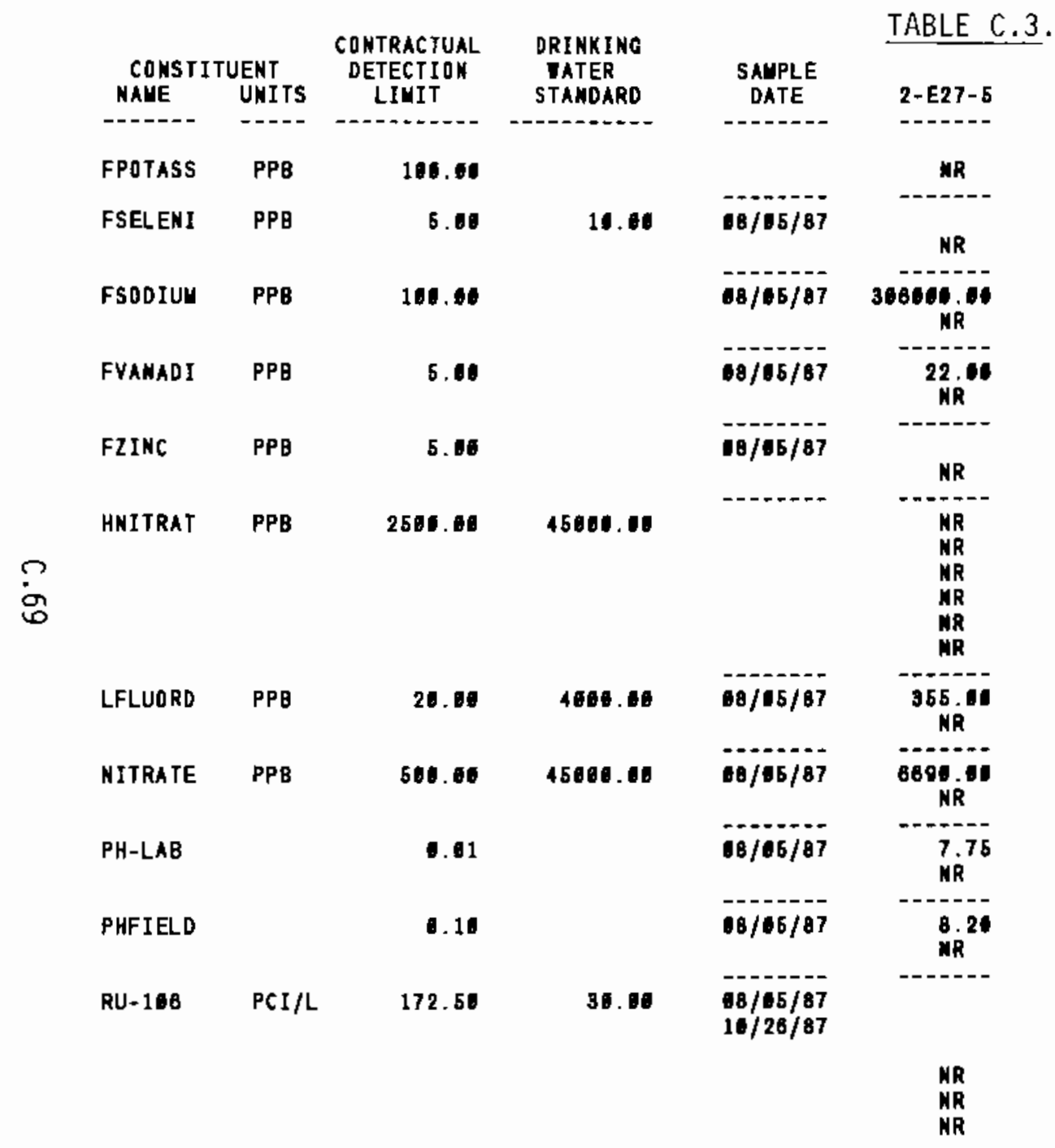

(contd)

\begin{tabular}{|c|c|c|c|}
\hline $\begin{array}{l}\text { SALPLE } \\
\text { DATE }\end{array}$ & $2-E 28-21$ & $\begin{array}{c}\text { SAUPLE } \\
\text { DATE }\end{array}$ & 2-E92-1 \\
\hline $10 / 08 / 07$ & 5680.00 & $12 / 31 / 87$ & 5288.10 \\
\hline $\begin{array}{l}17 / 17 / 87 \\
10 / 68 / 87\end{array}$ & 5.66 & $\begin{array}{l}\text { 88/08/87 } \\
12 / 31 / 97\end{array}$ & \\
\hline $\begin{array}{l}07 / 17 / 87 \\
10 / 60 / 87\end{array}$ & $\begin{array}{l}22801.10 \\
227101.110\end{array}$ & $\begin{array}{l}08 / 06 / 87 \\
12 / 31 / 87\end{array}$ & 20204.100 \\
\hline $\begin{array}{l}07 / 17 / 87 \\
10 / 08 / 87\end{array}$ & $\begin{array}{l}13.0 \\
17.01\end{array}$ & $\begin{array}{l}68 / 66 / 87 \\
12 / 31 / 87\end{array}$ & 24.06 \\
\hline $\begin{array}{l}67 / 17 / 87 \\
16 / 08 / 87\end{array}$ & 11.00 & $\begin{array}{l}18 / 68 / 87 \\
12 / 31 / 87\end{array}$ & $\begin{array}{r}8.06 \\
13.65\end{array}$ \\
\hline $\begin{array}{l}17 / 17 / 87 \\
98 / 13 / 87 \\
19 / 13 / 87 \\
11 / 68 / 87 \\
11 / 1 / 87 \\
12 / 16 / 87\end{array}$ & 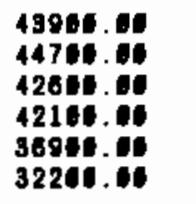 & $10 / 08 / 87$ & 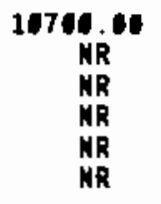 \\
\hline $\begin{array}{l}07 / 17 / 87 \\
10 / 00 / 87\end{array}$ & $\begin{array}{l}475.11 \\
489.11\end{array}$ & $\begin{array}{l}08 / 08 / 87 \\
12 / 31 / 87\end{array}$ & $\begin{array}{l}595.11 \\
680.14\end{array}$ \\
\hline $\begin{array}{l}17 / 17 / 87 \\
10 / 08 / 87\end{array}$ & $\begin{array}{l}44304.01 \\
42501.11\end{array}$ & $\begin{array}{l}08 / 08 / 87 \\
12 / 31 / 87\end{array}$ & $\begin{array}{l}\text { 105es. II } \\
10804.10\end{array}$ \\
\hline $\begin{array}{l}67 / 17 / 87 \\
10 / 08 / 87\end{array}$ & $\begin{array}{l}7.63 \\
7.65\end{array}$ & $\begin{array}{l}80 / 18 / 87 \\
12 / 31 / 87\end{array}$ & $\begin{array}{l}7.82 \\
7.83\end{array}$ \\
\hline $\begin{array}{l}67 / 17 / 87 \\
16 / 08 / 87\end{array}$ & $\begin{array}{l}7.30 \\
6.80\end{array}$ & $\begin{array}{l}00 / 18 / 87 \\
12 / 31 / 87\end{array}$ & $\begin{array}{l}7.20 \\
7.60\end{array}$ \\
\hline $\begin{array}{l}07 / 17 / 87 \\
0 \theta / 13 / 87\end{array}$ & $\begin{array}{l}60.80 \\
42.10\end{array}$ & & $\begin{array}{l}\text { NR } \\
\text { MR }\end{array}$ \\
\hline $\begin{array}{l}19 / 13 / 87 \\
10 / 68 / 87 \\
11 / 16 / 87\end{array}$ & & & $\begin{array}{l}\text { NR } \\
\text { NR } \\
\text { NR }\end{array}$ \\
\hline
\end{tabular}

- - value exceeds primary dRINking tater standard.

- YALUE EXCEEDS PROPOSED PRIMARY DRINKIMG TATER STANDARD.

- VALUE EXCEEDS SCREEMING LEVEL FOR FURTHER INVESTIOATION.

- - DETECTION LIUIT TAS MOT AVAILABLE FOR COUPARISON

- DETECTION LIMIT IAS MOT AVAILABLE FOR COYPAT
NR - AHALYSIS NDT REQUESTED OR NOT YET REPORTED

VALUES IN \{\} ARE CDUNTING ERRORS FOR RADIONUCLIDES

IATER STANDARD(S) IN PAREATHESES ARE PROPOSED ONLY 


\begin{tabular}{|c|c|c|c|c|c|}
\hline \multicolumn{2}{|c|}{ CONSTITUENT } & $\begin{array}{c}\text { CONTRACTUAL } \\
\text { DETECTIDN } \\
\text { LIUIT }\end{array}$ & $\begin{array}{l}\text { DRINKING } \\
\text { WATER } \\
\text { STANDARD }\end{array}$ & $\begin{array}{l}\text { SALPLE } \\
\text { DATE }\end{array}$ & $2-E 27-5$ \\
\hline------ & $\ldots$. & - & 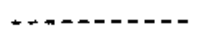 & ------ & ------ \\
\hline$R U=180$ & $\mathrm{PCI} / \mathrm{L}$ & 172.50 & 30.00 & & NR \\
\hline SULFATE & PPB & 500.00 & & $18 / 65 / 87$ & 33500.00 \\
\hline TC & PPB & & & $18 / 05 / 67$ & 22900.01 \\
\hline \multirow[t]{6}{*}{ TRITIUH } & $P C I / L$ & 580.00 & 20861.00 & $0 B / 06 / 87$ & $\left\{\begin{array}{c}3450.00 \\
293.09\} \\
N R\end{array}\right\}$ \\
\hline & & & & & NR \\
\hline & & & & & NR \\
\hline & & & & & NR \\
\hline & & & & & NR \\
\hline & DC II & $5 a$ & ont & $--1---$ & $---1--$ \\
\hline U 234 & $\mathrm{PCI} / \mathrm{L}$ & 6.10 & 32.10 & & NR \\
\hline U 235 & $\mathrm{PCI} / \mathrm{L}$ & 0.10 & 32.00 & $-\cdots$ & NR \\
\hline U 238 & $\mathrm{PCI} / \mathrm{L}$ & 0.10 & 4.80 & & NR \\
\hline U-CHEM & UO/L & 0.73 & & & $\begin{array}{l}\text { NR } \\
\text { NR } \\
\text { NR } \\
\text { NR } \\
\text { NR } \\
\text { NR }\end{array}$ \\
\hline 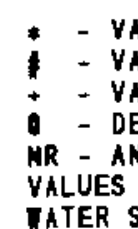 & $\begin{array}{l}\text { UE EXCE } \\
\text { UE EXCE } \\
\text { UE EXCE } \\
\text { ECTION } \\
\text { LYSIS N } \\
\text { N \& \} } \\
\text { AMDARD? }\end{array}$ & $\begin{array}{l}\text { EOS PRIMARY } \\
\text { EDS PROPOSED } \\
\text { EOS SCREENINO } \\
\text { LIUI TAS NOT } \\
\text { OT REQUESTED } \\
\text { ARE COUNTI } \\
\text { S) IN PARENTH }\end{array}$ & $\begin{array}{l}\text { RINKING IATEF } \\
\text { PRIVARY DRINI } \\
\text { LEVEL FOR FI } \\
\text { AYAILAELE F } \\
\text { OR NOT YET RE } \\
\text { O ERRORS FOR } \\
\text { ESES ARE PROF }\end{array}$ & $\begin{array}{l}\text { ANDARO. } \\
\text { TATER ST } \\
\text { ER INYEST } \\
\text { OUPARISON } \\
\text { TED } \\
\text { IONUCLIDES } \\
\text { D ONLY }\end{array}$ & $\begin{array}{l}\text { OARD. } \\
\text { ATION. }\end{array}$ \\
\hline
\end{tabular}

\section{contd)}

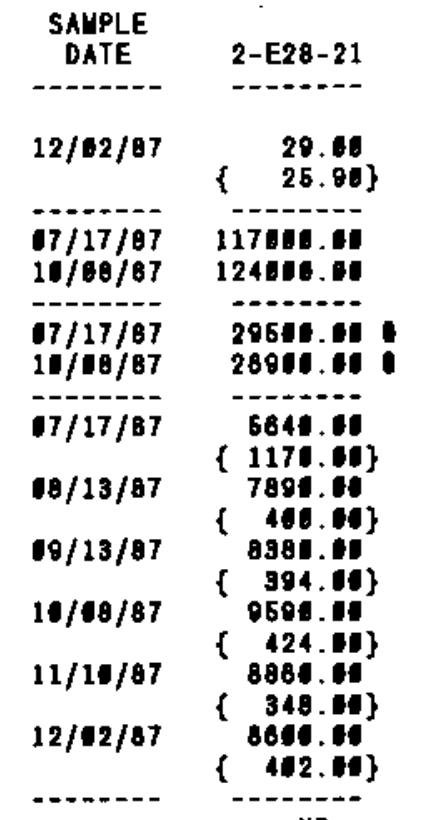

SALPLE

2-E32-1

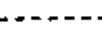

MR

NR

$08 / 06 / 87$

337.0. 10

-.-.-- -....

10/06/87 22814.te

12/31/87 21900.06

$00 / 06 / 87$

7564.10

$\{3306\}$

MR

NR

NR

NR

NR

NR

OT/17/87 23.34

$07 / 17 / 87$

$\left\{\begin{array}{r}23.34 \\ \quad 0.43\}\end{array}\right.$

$7 / 17 / 87$

$\left\{\begin{array}{l}1.1 \\ 0\end{array}\right.$

$\left\{\begin{array}{r}23.24 \\ \quad .49\}\end{array}\right.$

97/17/87

$97 / 17$

$08 / 13 / 87$

$60 / 13 / 87$

$14 / 98 / 87$

$11 / 10 / 87$

$12 / 02 / 87$

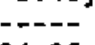

84.81

58.41

61.81

62.61

44.20

48.60

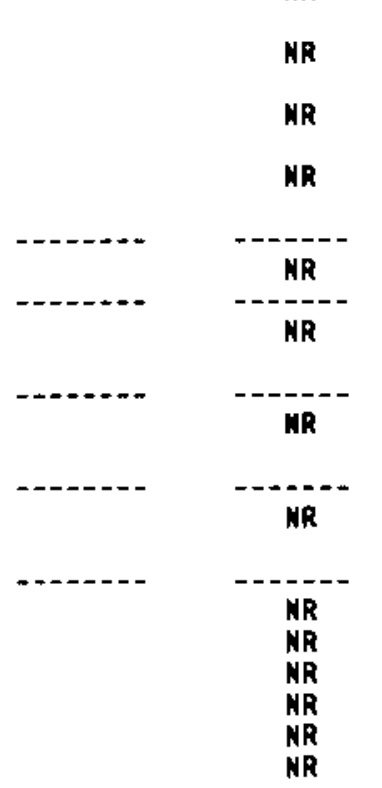


TABLE C.3. (contd)

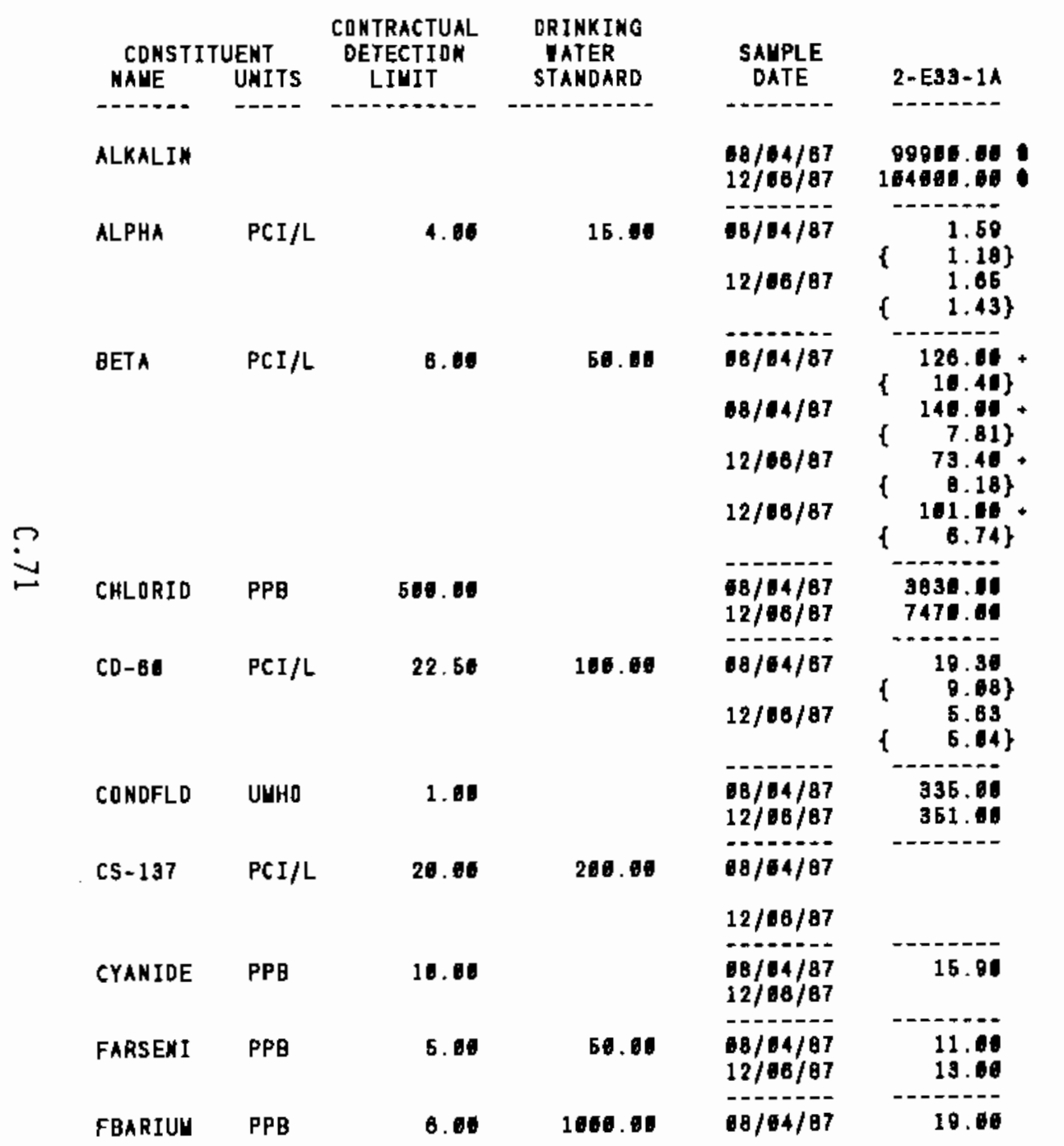

\begin{tabular}{|c|c|}
\hline $\begin{array}{l}\text { SAUPLE } \\
\text { DATE }\end{array}$ & $2-E 33-3$ \\
\hline $\begin{array}{l}8 / 08 / 07 \\
12 / 06 / 87\end{array}$ & $\begin{array}{l}06300.00 \\
09466.06\end{array}$ \\
\hline $18 / 46 / 87$ & 1.24 \\
\hline $12 / 06 / 87$ & $\left.\begin{array}{ll} & 1.98 \\
\{\quad 1.32\end{array}\right\}$ \\
\hline $68 / 106 / 87$ & $\begin{array}{r}253.66 \\
\{14.76\}\end{array}$ \\
\hline $10 / 06 / 87$ & $\begin{array}{r}438.10 \\
28.01\}\end{array}$ \\
\hline $12 / 00 / 87$ & $\begin{array}{r}27.71 \\
5.56\end{array}$ \\
\hline $12 / 06 / 87$ & $\begin{array}{r}29.90 \\
\{\quad 3.78\}\end{array}$ \\
\hline $\begin{array}{l}68 / 06 / 87 \\
12 / 08 / 87\end{array}$ & $\begin{array}{l}3531.06 \\
3746.06\end{array}$ \\
\hline $68 / 06 / 87$ & $\begin{array}{r}24.10 \\
\end{array}$ \\
\hline $12 / 06 / 87$ & $\begin{array}{r}7.46 \\
\{\quad 5.84\}\end{array}$ \\
\hline $\begin{array}{l}88 / 06 / 87 \\
12 / 06 / 07\end{array}$ & $\begin{array}{r}360.00 \\
363.00\end{array}$ \\
\hline $08 / 08 / 87$ & $\begin{array}{r}5.11 \\
\quad 362\}\end{array}$ \\
\hline $12 / 00 / 87$ & \\
\hline $\begin{array}{l}08 / 68 / 87 \\
12 / 68 / 07\end{array}$ & 38.71 \\
\hline $\begin{array}{l}68 / 08 / 87 \\
12 / 08 / 87\end{array}$ & $\begin{array}{l}12.00 \\
13.00\end{array}$ \\
\hline $08 / 08 / 87$ & 18.66 \\
\hline
\end{tabular}

\begin{tabular}{|c|c|}
\hline $\begin{array}{l}\text { SAMPLE } \\
\text { DATE }\end{array}$ & 2-E93-5 \\
\hline $\begin{array}{l}88 / 16 / 87 \\
12 / 68 / 87\end{array}$ & $\begin{array}{r}97800.008 \\
101060.048\end{array}$ \\
\hline $08 / 10 / 97$ & \\
\hline $12 / 08 / 87$ & \\
\hline $88 / 10 / 87$ & 245.00 \\
\hline $18 / 10 / 87$ & $\begin{array}{l}13.606 \\
266.60\end{array}$ \\
\hline $12 / 06 / 87$ & $\begin{array}{l}18.78\} \\
169.08\end{array}$ \\
\hline & $11.80\}$ \\
\hline $12 / 18 / 07$ & $\left\{\begin{array}{r}244.08 \\
\{11.56\}\end{array}\right.$ \\
\hline $\begin{array}{l}68 / 10 / 87 \\
12 / 106 / 97\end{array}$ & 5240.60 \\
\hline $08 / 10 / 87$ & 30.80 \\
\hline $12 / 08 / 87$ & $\left\{\begin{array}{l}13.08 \\
\{\quad 11.58\}\end{array}\right.$ \\
\hline $\begin{array}{l}08 / 18 / 87 \\
12 / 166 / 87\end{array}$ & $\begin{array}{l}311.60 \\
345.80\end{array}$ \\
\hline $16 / 10 / 87$ & \\
\hline $12 / 08 / 87$ & \\
\hline $\begin{array}{l}18 / 10 / 87 \\
12 / 06 / 87\end{array}$ & $\begin{array}{l}28.40 \\
15.80\end{array}$ \\
\hline $\begin{array}{l}08 / 16 / 87 \\
12 / 68 / 87\end{array}$ & $\begin{array}{l}8.00 \\
8.00\end{array}$ \\
\hline $88 / 10 / 87$ & 37.00 \\
\hline
\end{tabular}

* - VAlUE exceEds priuary dRINXING iater standard.

* - VALUE EXCEEDS PROPOSEO PRIUARY ORINKIMG IATER STANDARD.

- YALUE EXCEEDS SCREENING LEVEL FOR FURTHER INYESTIGATION.

- detectidn liuit tas rot ayailable for couparison

NR - ANALYSIS NOT REQUESTED OR NOT YET REPORTED

YALUES IN $\{$ \& ARE COUNTING ERRORS FOR RADIONUCLIDES

WATER STANDARD(S) IN PARENTHESES ARE PRDPDSED ONLY 
TABLE C.3. (contd)

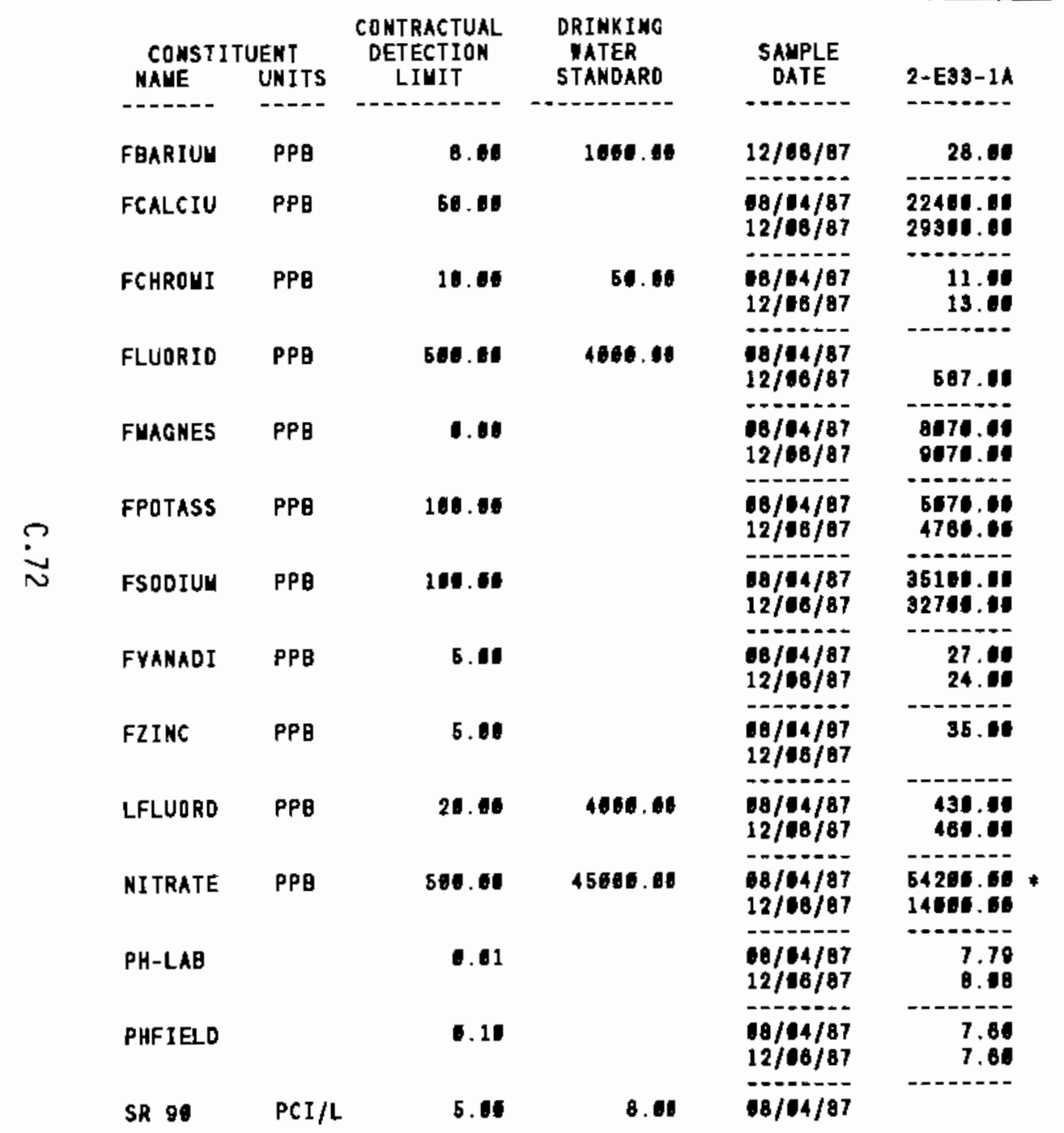

\begin{tabular}{|c|c|}
\hline $\begin{array}{l}\text { SAUPLE } \\
\text { DATE }\end{array}$ & $2-E 33-3$ \\
\hline $12 / 08 / 87$ & 20.01 \\
\hline $\begin{array}{l}98 / 108 / 87 \\
12 / 06 / 87\end{array}$ & $\begin{array}{l}25501.01 \\
29206.01\end{array}$ \\
\hline $\begin{array}{l}68 / 18 / 87 \\
12 / 66 / 87\end{array}$ & 16.0 \\
\hline $\begin{array}{l}98 / 188 / 87 \\
12 / 16 / 87\end{array}$ & $\begin{array}{l}781.11 \\
700.01\end{array}$ \\
\hline $\begin{array}{l}68 / 08 / 87 \\
12 / 00 / 87\end{array}$ & $\begin{array}{l}8244.11 \\
8190.11\end{array}$ \\
\hline $\begin{array}{l}18 / 68 / \theta 7 \\
12 / 06 / \theta 7\end{array}$ & $\begin{array}{l}5060.60 \\
1870.00\end{array}$ \\
\hline $\begin{array}{l}08 / 08 / 87 \\
12 / 06 / 87\end{array}$ & 32000.00 \\
\hline $\begin{array}{l}68 / 006 / 87 \\
12 / 00 / 87\end{array}$ & 29.10 \\
\hline $\begin{array}{l}08 / 06 / 87 \\
12 / 00 / 87\end{array}$ & \\
\hline $\begin{array}{l}88 / 06 / 87 \\
12 / 06 / 87\end{array}$ & $\begin{array}{l}805.01 \\
806.01\end{array}$ \\
\hline $\begin{array}{l}86 / 00 / 87 \\
12 / 08 / 87\end{array}$ & 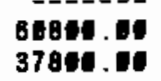 \\
\hline $\begin{array}{l}88 / 18 / 87 \\
12 / 08 / 87\end{array}$ & $\begin{array}{l}7.72 \\
8.16\end{array}$ \\
\hline $\begin{array}{l}08 / 106 / 87 \\
12 / 06 / 87\end{array}$ & $\begin{array}{l}7.51 \\
7.51\end{array}$ \\
\hline $8 / 168 / 87$ & \\
\hline
\end{tabular}

\begin{tabular}{|c|c|}
\hline $\begin{array}{l}\text { SAMPLE } \\
\text { OATE }\end{array}$ & $2-E 33-5$ \\
\hline $12 / 96 / 87$ & 37.10 \\
\hline $\begin{array}{l}18 / 10 / 87 \\
12 / 06 / 87\end{array}$ & $\begin{array}{l}27364.60 \\
36466.66\end{array}$ \\
\hline $\begin{array}{l}08 / 16 / 87 \\
12 / 18 / 87\end{array}$ & 11.01 \\
\hline $\begin{array}{l}18 / 11 / 87 \\
12 / 06 / 87\end{array}$ & 532.11 \\
\hline $\begin{array}{l}18 / 10 / 87 \\
12 / 06 / 87\end{array}$ & $\begin{array}{r}9460.00 \\
19800.06\end{array}$ \\
\hline $\begin{array}{l}08 / 10 / 87 \\
12 / 06 / 87\end{array}$ & $\begin{array}{l}5670.06 \\
6266.66\end{array}$ \\
\hline $\begin{array}{l}18 / 16 / 87 \\
12 / 00 / 87\end{array}$ & $\begin{array}{l}23004.11 \\
21506.00\end{array}$ \\
\hline $\begin{array}{l}18 / 11 / 87 \\
12 / 08 / 87\end{array}$ & $\begin{array}{l}24.10 \\
23.10\end{array}$ \\
\hline $\begin{array}{l}18 / 16 / 87 \\
12 / 06 / 87\end{array}$ & $\begin{array}{l}7.00 \\
8.00\end{array}$ \\
\hline $\begin{array}{l}18 / 10 / 87 \\
12 / 06 / 87\end{array}$ & $\begin{array}{l}375.10 \\
430.61\end{array}$ \\
\hline $\begin{array}{l}68 / 16 / 87 \\
12 / 68 / 87\end{array}$ & $\begin{array}{l}37915.110 \\
34615.110\end{array}$ \\
\hline $\begin{array}{l}18 / 11 / 87 \\
12 / 08 / 87\end{array}$ & $\begin{array}{l}7.88 \\
8.11\end{array}$ \\
\hline $\begin{array}{l}18 / 10 / 87 \\
12 / 86 / 87\end{array}$ & 7.10 \\
\hline $18 / 10 / 07$ & \\
\hline
\end{tabular}

- - Value exceeds privary dRinking iater stamdaro.

* - YALUE EXCEEDS PROPOSED PRIUARY DRINKIMG IATER STANDARD.

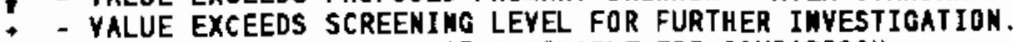

- - detection livit ias not ayailable for couparison

NR - ANALYSIS NOT REQUESTED OR NOT YET REPORTED

VALUES IM \{ $\}$ ARE COUNTING ERRORS FOR RADIOHUCLIDES

IATER STANDARD(S) IN PARENTHESES ARE PRLPOSED OMLY 
TABLE C.3. (contd)

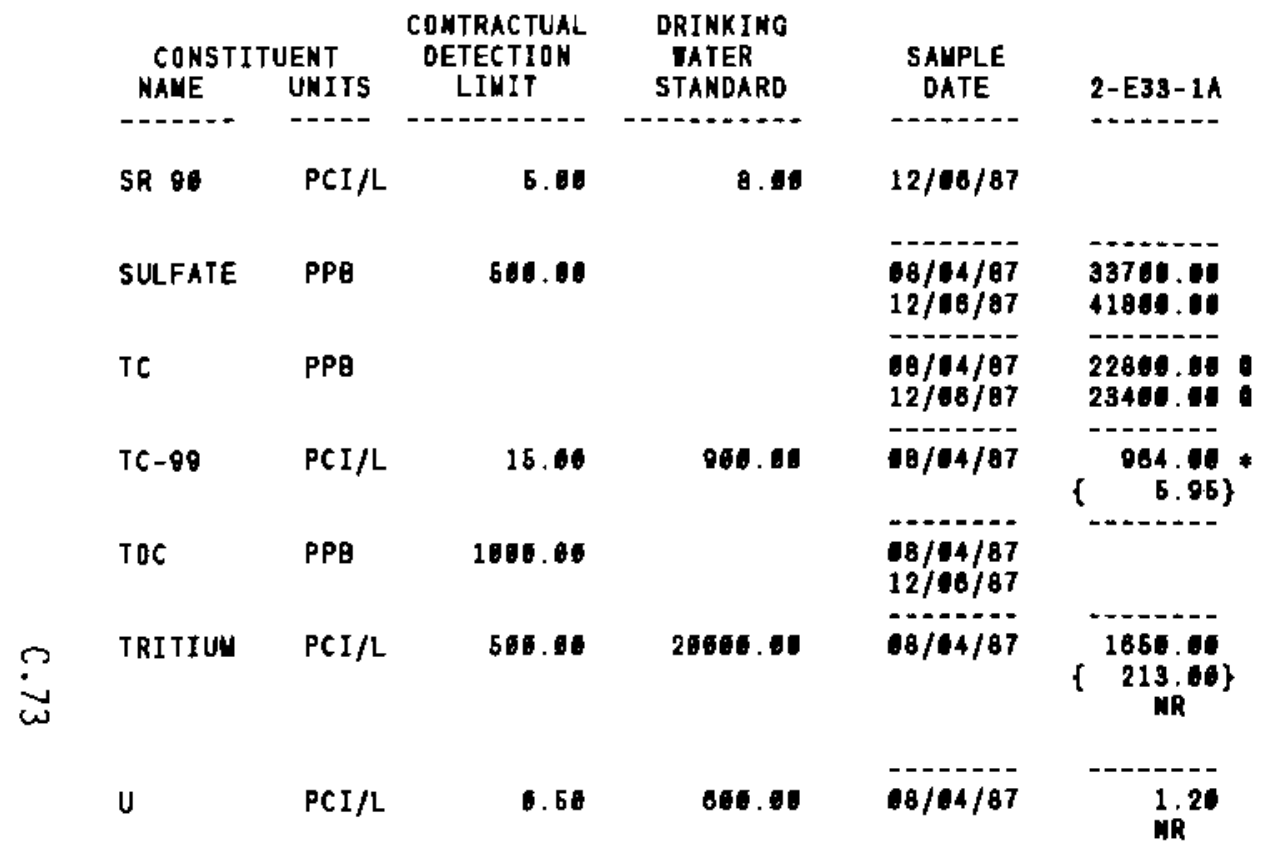

\begin{tabular}{|c|c|}
\hline $\begin{array}{l}\text { SALPLE } \\
\text { DATE }\end{array}$ & $2-E 33-3$ \\
\hline & NR \\
\hline -ー-ー-ー- & 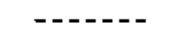 \\
\hline $\begin{array}{l}18 / 108 / 87 \\
12 / 06 / 87\end{array}$ & $\begin{array}{l}34400.00 \\
33606.00\end{array}$ \\
\hline $\begin{array}{l}10 / 16 / 87 \\
12 / 46 / 87\end{array}$ & 22501.00 \\
\hline $08 / 06 / 07$ & $\left\{\begin{array}{r}1466.66 \\
6.35\}\end{array}\right.$ \\
\hline $\begin{array}{l}-1-1-- \\
08 / 96 / 97 \\
12 / 66 / 87\end{array}$ & - \\
\hline $98 / 60 / 07$ & $\left\{\begin{array}{l}058.06 \\
181.06\}\end{array}\right.$ \\
\hline $12 / 06 / 87$ & $\left\{\begin{array}{r}1890.00 \\
256.06\}\end{array}\right.$ \\
\hline $\begin{array}{l}18 / 68 / 97 \\
68 / 68 / 07\end{array}$ & $\begin{array}{l}1.17 \\
1.73\end{array}$ \\
\hline
\end{tabular}

\begin{tabular}{|c|c|}
\hline $\begin{array}{c}\text { SAMPLE } \\
\text { DATE }\end{array}$ & $2-E 33-5$ \\
\hline$\ldots . . .$. & 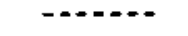 \\
\hline $12 / 108 / 87$ & $\begin{array}{l}.83 \\
.76\}\end{array}$ \\
\hline $\begin{array}{l}08 / 10 / 87 \\
12 / 16 / 87\end{array}$ & 41840.06 \\
\hline $\begin{array}{l}08 / 16 / 87 \\
12 / 06 / 87\end{array}$ & 22800.06 \\
\hline $18 / 18 / 87$ & $\left\{\begin{array}{c}108.15 \\
12.16\}\end{array}\right.$ \\
\hline $\begin{array}{l}10 / 16 / 67 \\
12 / 68 / 67\end{array}$ & 1430.10 \\
\hline $19 / 16 / 67$ & $\left\{\begin{array}{c}2480.00 \\
274.10\}\end{array}\right.$ \\
\hline $18 / 10 / 87$ & 1.17 \\
\hline
\end{tabular}

* - Value exceeds priuary dRInking tater standard.

- VALUE EXCEEDS PROPOSED PRIMARY DRINKING TATER STAMDARD.

- - VALUE EXCEEDS SCREENING LEVEL FOR FURTHER INYESTIGATION.

- DETECTION LIUIT UAS hOT AVALABLE FOR COUPARISON

NR - ANALYSIS NOT REQUESTED OR NOT YET REPORTED

YALUES IN I ARE COUNTING ERRORS FOR RADIONUCLIDES

IATER STANDARD(S) IN PARENTHESES ARE PROPOSED OHLY 
TABLE C.3. (contd)

\begin{tabular}{|c|c|c|c|c|c|}
\hline $\begin{array}{l}\text { CONSTIT } \\
\text { NAME }\end{array}$ & $\begin{array}{l}\text { JENT } \\
\text { UNITS }\end{array}$ & $\begin{array}{l}\text { CONTRACTUAL } \\
\text { OETECTION } \\
\text { LIUIT }\end{array}$ & $\begin{array}{l}\text { ORINKING } \\
\text { DATER } \\
\text { STANOARD }\end{array}$ & $\begin{array}{c}\text { SAUPLE } \\
\text { DATE }\end{array}$ & 2-E33-8 \\
\hline AL KALIN & & & & $68 / 04 / 87$ & 104000.00 \\
\hline ALPHA & $\mathrm{PCI} / \mathrm{L}$ & 4.08 & 15.06 & $08 / 04 / 87$ & $\begin{array}{ll}\{\quad 2.38 \\
\quad 1.25\}\end{array}$ \\
\hline BETA & $\mathrm{PCI} / \mathrm{L}$ & 0.10 & 60.00 & $\begin{array}{l}68 / 84 / 87 \\
68 / 64 / 87 \\
10 / 28 / 87\end{array}$ & 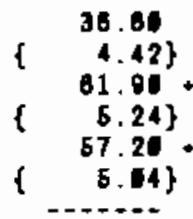 \\
\hline CHLORID & $\mathrm{PPB}$ & 500.06 & & $00 / 64 / 87$ & 4710.16 \\
\hline COMDFLO & UUHO & 1.00 & & $68 / 84 / 87$ & 234.00 \\
\hline CS-137 & PCI/L & 24.00 & 200.00 & $\begin{array}{l}08 / 04 / 87 \\
11 / 28 / 87\end{array}$ & $\left\{\begin{array}{l}5.51 \\
3 \quad 3.91\end{array}\right\}$ \\
\hline FARSENI & PPB & 6.60 & 6I.W & $18 / 04 / 87$ & 7.6 \\
\hline FBARIUU & PPB & 6.10 & 1000.00 & $08 / 04 / 07$ & 27.11 \\
\hline FCALCIU & $\mathrm{PPB}$ & 50.66 & & $08 / 04 / 87$ & 25911.010 \\
\hline FUAGMES & PPB & 6.00 & & $68 / 04 / 87$ & 8534.11 \\
\hline FPOTASS & PPB & 106.60 & & $108 / 64 / 87$ & 5386.01 \\
\hline FSODIUY & $\mathrm{PPB}$ & 180.00 & & $68 / 04 / 87$ & 12849.66 \\
\hline FVANADI & $\mathrm{PPB}$ & 5.06 & & $106 / 04 / 07$ & 22.10 \\
\hline FZIMC & PPB & 5.00 & & $00 / 64 / 07$ & 6.10 \\
\hline HNITRAT & $\mathrm{PPB}$ & 2506.00 & 46800.66 & & $\begin{array}{l}\text { NR } \\
\text { NR }\end{array}$ \\
\hline
\end{tabular}

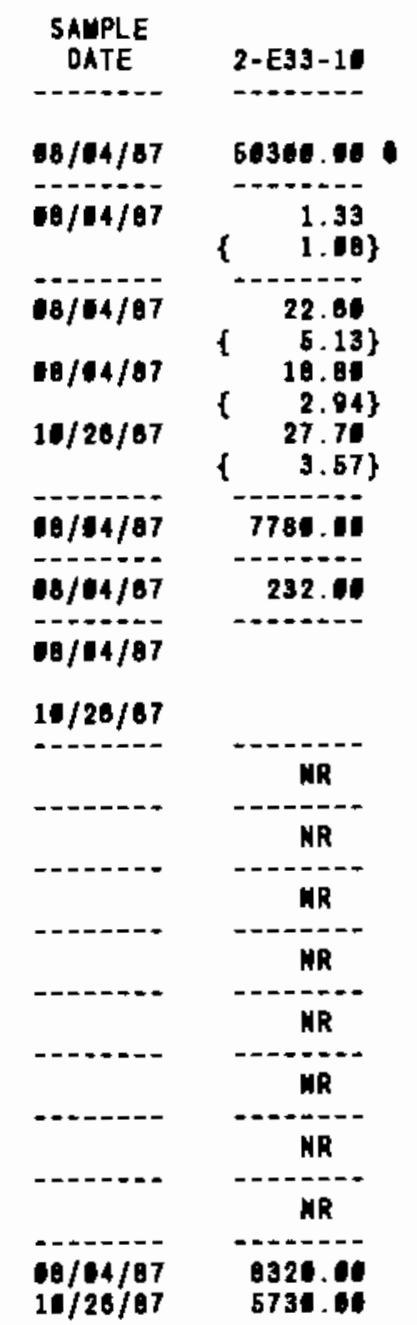

\begin{tabular}{|c|c|}
\hline $\begin{array}{l}\text { SAMPLE } \\
\text { DATE }\end{array}$ & 2-E33-18 \\
\hline $08 / 04 / 87$ & PBBES. II \\
\hline $08 / 04 / 87$ & $\left.\begin{array}{ll}1.50 \\
\{\quad 1.04\end{array}\right\}$ \\
\hline $18 / 04 / 87$ & $\left\{\begin{array}{r}14.50 \\
\quad 4.32\}\end{array}\right.$ \\
\hline $18 / 04 / 07$ & 16.06 \\
\hline $10 / 28 / 87$ & $\begin{array}{r}10.81 \\
\{\quad 2.33\}\end{array}$ \\
\hline $16 / 04 / 07$ & 3568.06 \\
\hline $00 / 04 / 87$ & 277.60 \\
\hline $68 / 04 / 87$ & \\
\hline $15 / 25 / 87$ & \\
\hline $18 / 44 / 87$ & 6.11 \\
\hline $08 / 04 / 87$ & 12.40 \\
\hline $16 / 04 / 87$ & 0680.00 \\
\hline $08 / 14 / 87$ & 7690.16 \\
\hline $10 / 04 / 87$ & 4784.64 \\
\hline $10 / 04 / 87$ & 2siven.00 \\
\hline $08 / 14 / 87$ & 21.06 \\
\hline $66 / 144 / 87$ & 6.01 \\
\hline
\end{tabular}

- - yalue exceeds prigary drinking tater standard.

- YALUE EXCEES PRDPOSED PRIYARY DRINKINO TATER STANDARO.

* - Value eXceEds screenimo leyel for further investioation.

- - oETECTION Livit vas not avaILable for comparison

NR - ANALYSIS NOT REQUESTED OR MOT YET REPORTED

VALUES IN if ARE COUMTINO ERRORS FOR RADIONUCLIDES

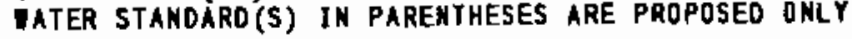


TABLE C. 3. (contd)

\begin{tabular}{|c|c|c|c|c|c|}
\hline $\begin{array}{c}\text { CONSTI } \\
\text { MAUE }\end{array}$ & $\begin{array}{l}\text { UENT } \\
\text { UNITS }\end{array}$ & $\begin{array}{c}\text { CONTRACTUAL } \\
\text { OETECTIOAN } \\
\text { LIUIT }\end{array}$ & $\begin{array}{l}\text { DRINKING } \\
\text { VATER } \\
\text { STAKOARD }\end{array}$ & $\begin{array}{l}\text { SALPLE } \\
\text { DATE }\end{array}$ & $2-E 33-8$ \\
\hline LFLUORD & PPB & 20.00 & 40000.00 & $08 / 64 / 87$ & 328.60 \\
\hline NITRATE & PPB & 500.66 & 45000.00 & $18 / 14 / 87$ & 8886.06 \\
\hline PH-LAB & & 0.01 & & $10 / 04 / 87$ & 7.71 \\
\hline PHFIELD & & 0.10 & & $08 / 04 / 87$ & 7.20 \\
\hline RU-160 & $\mathrm{PCI} / \mathrm{L}$ & $172.5 \mathrm{I}$ & 36.16 & $\begin{array}{l}18 / 64 / 87 \\
16 / 28 / 87\end{array}$ & $\left\{\begin{array}{l}59.91 \\
\{\quad 54.30\end{array}\right.$ \\
\hline \multirow[t]{2}{*}{ SR 91} & $P C I / L$ & 5.06 & B.10 & $18 / 84 / 87$ & \\
\hline & & & & $10 / 28 / 87$ & \\
\hline SULFATE & PPB & 500.00 & & $10 / 04 / 87$ & 28800.01 \\
\hline TC & PPB & & & $18 / 04 / 87$ & 240000.00 \\
\hline TC -98 & $\mathrm{PCI} / \mathrm{L}$ & 15.00 & 900.00 & $88 / 04 / 87$ & $\begin{array}{c}102.09 \\
\{\quad 2.01\}\end{array}$ \\
\hline TRITIUU & $\mathrm{PCI} / \mathrm{L}$ & 586.68 & 20986.06 & $08 / 04 / 87$ & $\begin{array}{c}\begin{array}{c}4580.06 \\
278.06 \\
N R\end{array} \\
\text { NR }\end{array}$ \\
\hline u & $\mathrm{PCI} / \mathrm{L}$ & 1.50 & 600.08 & $68 / 04 / 87$ & 1.40 \\
\hline
\end{tabular}

\begin{tabular}{|c|c|}
\hline $\begin{array}{c}\text { SANPLE } \\
\text { DATE }\end{array}$ & $2-E 33-10$ \\
\hline 88/104/87 & 466.16 \\
\hline $18 / 04 / 87$ & 5821.01 \\
\hline $10 / 04 / 87$ & 7.78 \\
\hline $08 / 04 / 87$ & 7.39 \\
\hline \multicolumn{2}{|l|}{$\begin{array}{l}08 / 04 / 87 \\
10 / 26 / 87\end{array}$} \\
\hline \multicolumn{2}{|l|}{$18 / 64 / 87$} \\
\hline & NR \\
\hline $08 / 64 / 87$ & 27004.00 \\
\hline \multirow[t]{2}{*}{$68 / 64 / 87$} & 22300.00 \\
\hline & NR \\
\hline & $\begin{array}{r}3040.00 \\
246.06\}\end{array}$ \\
\hline $16 / 28 / 87$ & $\left\{\begin{array}{r}4380.00 \\
289.60\}\end{array}\right.$ \\
\hline $18 / 104 / 87$ & 1.42 \\
\hline
\end{tabular}

\begin{tabular}{|c|c|}
\hline $\begin{array}{l}\text { SAMPLE } \\
\text { DATE }\end{array}$ & 2-E.AS-18 \\
\hline $18 / 04 / 87$ & 335. \\
\hline $18 / 04 / 87$ & 15704.60 \\
\hline $10 / 04 / 87$ & 7.84 \\
\hline $08 / 04 / 87$ & 7.65 \\
\hline $\begin{array}{l}08 / 104 / 87 \\
10 / 26 / 87\end{array}$ & \\
\hline $18 / 94 / 87$ & $\left\{\quad \begin{array}{l}2.91 \\
M R\end{array}\right.$ \\
\hline $08 / 04 / 87$ & 30300.00 \\
\hline $18 / 04 / 87$ & 22100.60 \\
\hline & MR \\
\hline $00 / 04 / 87$ & $\left\{\begin{array}{c}1010.00 \\
220.4\end{array}\right\}$ \\
\hline $18 / 04 / 87$ & \\
\hline
\end{tabular}

* - Value exceeds priuary drinkimg tater standaro.

- VALUE EXCEEDS PROPOSED PRIUARY DRINKING IATER STANDARo.

- VALUE EXCEEDS SCREENING LEYEL FOR FURTHER INYESTIGATION.

- DETECTION LIUIT IAS NOT AYALABLE FOR COUPARISON

MR - ANALYSIS NOT REQUESTED OR NOT YET REPDRTED

VALUES IN \{ $\}$ ARE COUNTING ERRORS FOR RADIONUCLIDES

WATER STAMDARD(S) IN PARENTHESES ARE PROPOSED ONLY 


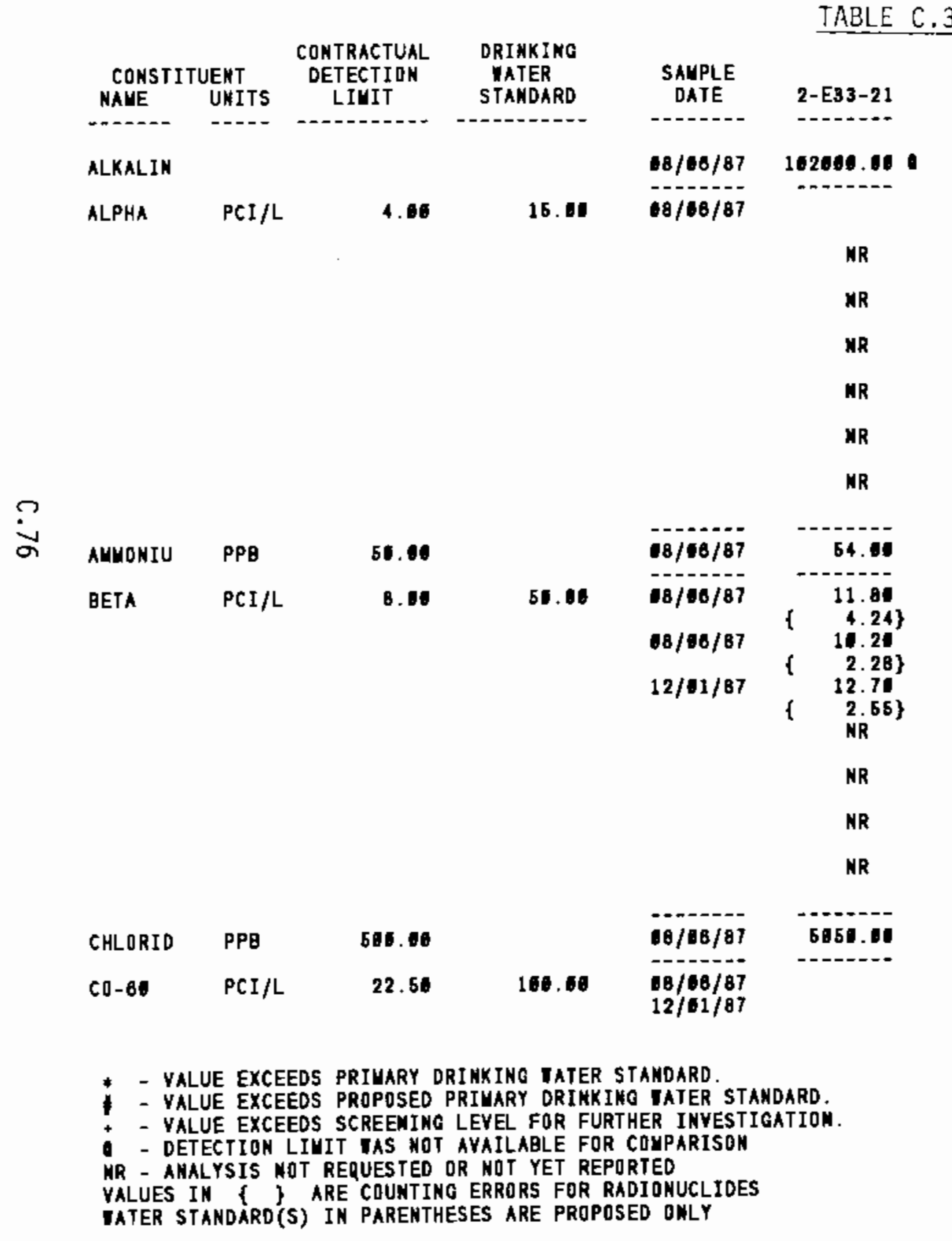

(contd) 


\begin{tabular}{|c|c|c|c|c|c|}
\hline $\begin{array}{c}\text { CONSTI } \\
\text { NAME }\end{array}$ & $\begin{array}{l}\text { UENT } \\
\text { UNITS }\end{array}$ & $\begin{array}{c}\text { CONTRACTUAL } \\
\text { DETECTIOH } \\
\text { LIUIT }\end{array}$ & $\begin{array}{l}\text { DRINKIMG } \\
\text { IATER } \\
\text { STANDARD }\end{array}$ & $\begin{array}{l}\text { SAMPLE } \\
\text { DATE }\end{array}$ & $2-E 33-21$ \\
\hline CO-6E & $\mathrm{PCI} / \mathrm{L}$ & 22.66 & 160.60 & & $\begin{array}{l}\text { NR } \\
\text { NR } \\
\text { MR } \\
\text { NR }\end{array}$ \\
\hline COMOFLD & UUHO & 1.60 & & $18 / 06 / 87$ & 243.06 \\
\hline CS-137 & $\mathrm{PCI} / \mathrm{L}$ & 26.61 & 200.00 & $\begin{array}{l}18 / 66 / 97 \\
12 / 61 / 87\end{array}$ & $\begin{array}{l}\text { MR } \\
\text { NR } \\
\text { NR } \\
\text { NR }\end{array}$ \\
\hline FARSENI & PPB & 6.6 & 51.00 & $08 / 06 / 87$ & 5.06 \\
\hline FBARIUU & PPB & 6.11 & 1080.00 & $18 / 60 / 87$ & 37.66 \\
\hline FCADHIU & PPB & 2.11 & 10.00 & $10 / 00 / 07$ & 2.61 \\
\hline FCALCIU & PPB & 50.10 & & $10 / 06 / 87$ & 23500.01 \\
\hline FIRON & PPB & 50.80 & & $10 / 06 / 97$ & \\
\hline FLUARID & PPB & 500.60 & 4006.00 & $18 / 08 / 87$ & \\
\hline FUAGMES & $\mathrm{PPB}$ & 0.06 & & $08 / 06 / 87$ & 8510.00 \\
\hline FHAMGAM & PPB & 6.10 & & $18 / 06 / 87$ & \\
\hline FPOTASS & PPB & 100.00 & & $68 / 08 / 87$ & 5170.06 \\
\hline FSODIUY & PPB & 108.00 & & $108 / 06 / 87$ & 12100.06 \\
\hline FSTRONT & PPB & 300.08 & & $68 / 06 / 87$ & \\
\hline FYANADI & PPB & 5.10 & & $10 / 08 / 87$ & 22.60 \\
\hline
\end{tabular}

(contd)

* - VALUE EXCEedS PRIUAaY dRINKIMG tater stamoard.

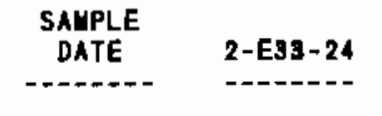

\begin{tabular}{|c|c|}
\hline & $\begin{array}{l}\text { NR } \\
\text { NR } \\
\text { NR } \\
\text { NR }\end{array}$ \\
\hline $18 / 06 / 87$ & 286.60 \\
\hline \multirow[t]{3}{*}{$\begin{array}{l}18 / 96 / 87 \\
10 / 28 / 87\end{array}$} & \\
\hline & NR \\
\hline & $\begin{array}{l}\text { NR } \\
\text { NR }\end{array}$ \\
\hline $68 / 08 / 87$ & 6.10 \\
\hline $68 / 06 / 97$ & 32.14 \\
\hline $08 / 06 / 87$ & 2.0 \\
\hline $10 / 06 / 87$ & 25865.04 \\
\hline \multicolumn{2}{|l|}{$00 / 06 / 87$} \\
\hline $08 / 06 / 87$ & \\
\hline $08 / 00 / 87$ & 8948.00 \\
\hline \multicolumn{2}{|l|}{$18 / 00 / 87$} \\
\hline $68 / 08 / 87$ & 5060.60 \\
\hline $68 / 80 / 87$ & 16400.01 \\
\hline $68 / 68 / 87$ & \\
\hline $68 / 06 / 87$ & 24.80 \\
\hline
\end{tabular}

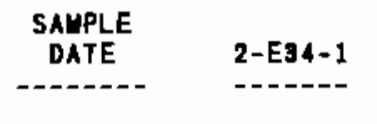

\begin{tabular}{|c|c|}
\hline $\begin{array}{l}90 / 15 / 97 \\
10 / 07 / 87\end{array}$ & 6.83 \\
\hline $\begin{array}{l}11 / 10 / 87 \\
12 / 93 / 87\end{array}$ & \\
\hline $67 / 21 / 87$ & 546.60 \\
\hline $\begin{array}{l}67 / 21 / 87 \\
68 / 13 / 87 \\
60 / 16 / 87\end{array}$ & 4.82 \\
\hline $15 / 07 / 87$ & $\begin{array}{l}3.64] \\
9.98\end{array}$ \\
\hline & 8.32] \\
\hline $\begin{array}{l}11 / 16 / 87 \\
12 / 43 / 87\end{array}$ & \\
\hline $07 / 21 / 07$ & \\
\hline $07 / 21 / 87$ & 36.06 \\
\hline $67 / 21 / 87$ & \\
\hline $07 / 21 / 87$ & 68106.40 \\
\hline $07 / 21 / 87$ & 118.00 \\
\hline $07 / 21 / 87$ & 682.00 \\
\hline $07 / 21 / 97$ & 17205.6 \\
\hline $07 / 21 / 87$ & 12.11 \\
\hline $07 / 21 / 07$ & ad16.01 \\
\hline $07 / 21 / 07$ & 24065.00 \\
\hline $07 / 21 / 87$ & 306.00 \\
\hline $07 / 21 / 87$ & \\
\hline
\end{tabular}

* - VALUE EXCEEDS PRDPOSED PRIMARY DRINKING IATER STANDARD.

* - value eXceEds screening LeVel for further investigation.

- - Detection liyit yas not available for couparison

HR - ANALYSIS NOT REQUESTED OR NOT YET REPORTED

YALUES IN \{ \} ARE COUNTING ERRORS FOR RADIONUCLIDES

IATER STAKDARD(S) IN PARENTHESES ARE PROPOSED ONLY 
TABLE C.3. (contd)

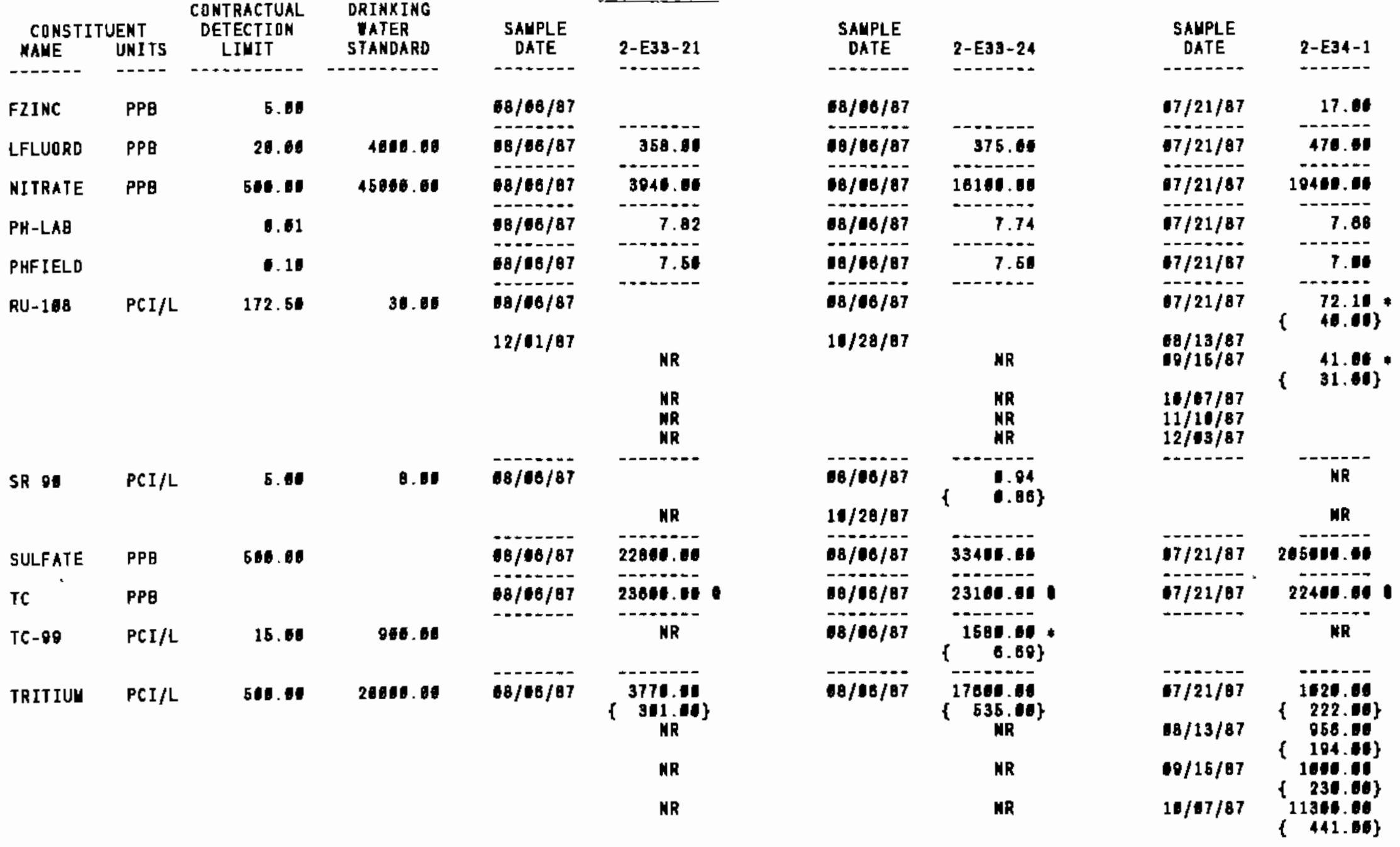

- - yalue exceEdos PRIMARY dRINKINg Tater STANDARD.

- VALUE EXCEEDS PROPOSED PRIMARY DRINKING MATER STANDARD.

- YALE EXCEEDS SCREENING LEVEL FOR FURTHER INYESTIGATION.

- DETECTION LIUIT TAS NDT AVAILABLE FOR COUPARISON

NR - DETECTIUN LIMT REQUESTED OR NOT YET REPORTED

VALUES IN I ARE COUNTING ERRORS FOR RADIDNUCLIDES

YALUES IN I \& ARE COUNTING ERRORS FOR RADIDNUCLIDES
IATER STANDARD(S) IN PARENTHESES ARE PROPOSED ONLY 
TABLE C. 3. (contd)

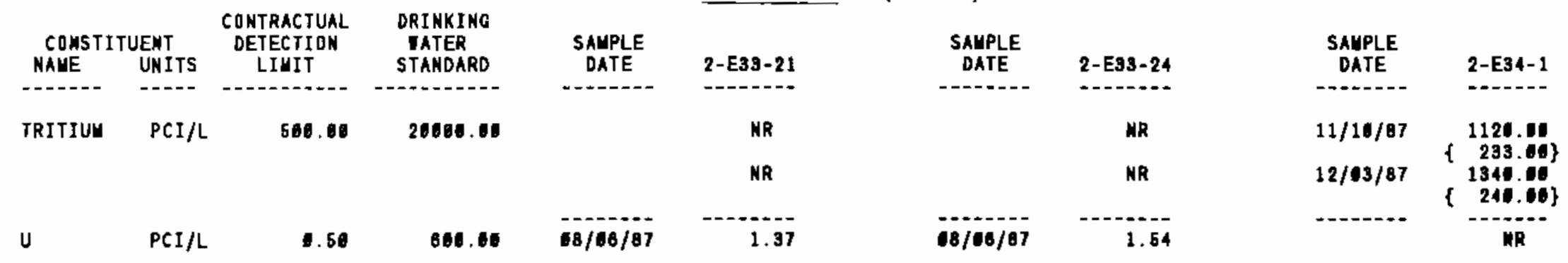

$\stackrel{?}{0}$

\footnotetext{
* VALUe exceEdS primary drinking mater standard.

- VALUE EXCEEDS PRDPDSED PRIUARY DRINKING TATER STANDARD.

- YALUE EXCEEDS SCREENING LEVEL FUR FURTHER INVESTIGATION.

- DETECTION LIUIT WAS NOT AYAILABLE FOR COUPARISON

NR - ANALYSIS NOT REQUESTED OR MOT YET REPORTED

VALUES IN \{\} ARE COUNTIMG ERRORS FOR RADIONUCLIDES

TATER STANDARD(S) IN PARENTHESES ARE PRDPOSED ONLY
} 
IABLE C.3. (contd)

\begin{tabular}{|c|c|c|c|c|c|c|c|c|c|}
\hline$\underset{\text { MAME }}{\text { CONSTI }}$ & $\begin{array}{l}\text { UENT } \\
\text { UNITS }\end{array}$ & $\begin{array}{c}\text { CONTRACTUAL } \\
\text { DETECTION } \\
\text { LIUIT }\end{array}$ & $\begin{array}{l}\text { DRINKING } \\
\text { TATER } \\
\text { STANDARD }\end{array}$ & $\begin{array}{l}\text { SAMPLE } \\
\text { DATE }\end{array}$ & $2-10-4$ & $\begin{array}{c}\text { SAUPLE } \\
\text { DATE }\end{array}$ & $2-110-8$ & $\begin{array}{c}\text { SAUPLE } \\
\text { DATE }\end{array}$ & $2-110-0$ \\
\hline ALKALIN & & & & $\begin{array}{l}67 / 22 / 87 \\
12 / 86 / 87\end{array}$ & $\begin{array}{l}157000.008: \\
184089.08\end{array}$ & $\begin{array}{l}68 / 18 / 87 \\
12 / 08 / 87\end{array}$ & $\begin{array}{l}136000.18: \\
136600.06:\end{array}$ & $\begin{array}{l}08 / 19 / 87 \\
12 / 66 / 87\end{array}$ & $\begin{array}{l}170040.010 \\
170000.08\end{array}$ \\
\hline ALPHA & $\mathrm{PCI} / \mathrm{L}$ & 4.00 & 15.00 & & $\begin{array}{ll} & 2.38 \\
\{\quad 1.03\}\end{array}$ & $10 / 10 / 87$ & & $18 / 18 / 87$ & \\
\hline & & & & $12 / 06 / 87$ & $\begin{array}{l}\text { NR } \\
\text { MR }\end{array}$ & $\begin{array}{l}18 / 10 / 87 \\
12 / 06 / 87 \\
12 / 06 / \theta 7\end{array}$ & \begin{tabular}{ll}
\{ & 1.52 \\
\{ & $0.58\}$ \\
\{ & 1.19 \\
\{ & 1.73 \\
\{ & 1.58 \\
\hdashline & 0.50
\end{tabular} & $\begin{array}{l}08 / 18 / 87 \\
12 / 06 / 87 \\
12 / 06 / 87\end{array}$ & $\begin{array}{l}1.58 \\
: .59\} \\
5.21 \\
3.40\} \\
2.57 \\
1.71\}\end{array}$ \\
\hline BETA & $\mathrm{PCI} / \mathrm{L}$ & 8.80 & 59.01 & $\begin{array}{l}07 / 22 / 87 \\
07 / 22 / 87 \\
12 / 88 / 87 \\
12 / 08 / 87\end{array}$ & 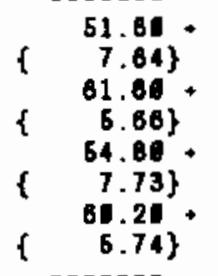 & $\begin{array}{l}68 / 19 / 87 \\
18 / 19 / 87 \\
12 / 06 / 87 \\
12 / 06 / 87\end{array}$ & 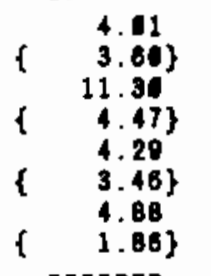 & $\begin{array}{l}18 / 10 / 87 \\
18 / 19 / 87 \\
12 / 108 / 87 \\
12 / 08 / 87\end{array}$ & 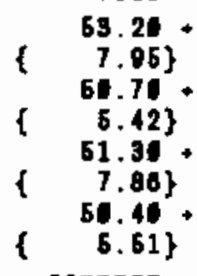 \\
\hline CHLFORY & PPB & 10.00 & 100.00 & $\begin{array}{l}67 / 22 / 87 \\
12 / 18 / 87\end{array}$ & $\begin{array}{l}10.00 \\
18.00\end{array}$ & $\begin{array}{l}10 / 19 / 87 \\
12 / 100 / 87\end{array}$ & & $\begin{array}{l}18 / 19 / 87 \\
12 / 06 / 87\end{array}$ & $\begin{array}{l}24.01 \\
14.01\end{array}$ \\
\hline CHLORIO & PPB & 500.00 & & $\begin{array}{l}17 / 22 / 87 \\
12 / 06 / 87\end{array}$ & $\begin{array}{l}17780.06 \\
16980.00\end{array}$ & $\begin{array}{l}08 / 19 / 87 \\
12 / 06 / 87\end{array}$ & $\begin{array}{l}4390.60 \\
3970.60\end{array}$ & $\begin{array}{l}18 / 10 / 87 \\
12 / 06 / 87\end{array}$ & $\begin{array}{l}19010.01 \\
10516.11\end{array}$ \\
\hline $\mathrm{CO}-68$ & PCI/L & 22.50 & 100.00 & $67 / 22 / 87$ & 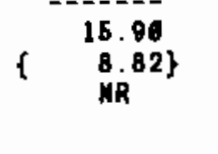 & $\begin{array}{l}18 / 19 / 87 \\
12 / 86 / 87\end{array}$ & & $\begin{array}{l}18 / 10 / 87 \\
12 / 18 / 87\end{array}$ & $\left(\begin{array}{r}13.60 \\
7.85\end{array}\right)$ \\
\hline CONDFLD & UUHO & 1.00 & & $\begin{array}{l}67 / 22 / 87 \\
12 / 08 / 87\end{array}$ & $\begin{array}{l}753.60 \\
752.16\end{array}$ & $\begin{array}{l}18 / 19 / 07 \\
12 / 06 / 87\end{array}$ & $\begin{array}{l}342.00 \\
314.00\end{array}$ & $\begin{array}{l}08 / 10 / 87 \\
12 / 06 / 87\end{array}$ & 1056.06 \\
\hline FARSEFI & PPE & 5.06 & 58.66 & $\begin{array}{l}07 / 22 / 87 \\
12 / 06 / 87\end{array}$ & $\begin{array}{l}7.00 \\
8.00\end{array}$ & $\begin{array}{l}68 / 10 / 97 \\
12 / 66 / 87\end{array}$ & 5.60 & $\begin{array}{l}18 / 19 / 87 \\
12 / 06 / 67\end{array}$ & $\begin{array}{l}16.01 \\
17.60\end{array}$ \\
\hline FBARIUU & PPB & 8.60 & 1800.00 & $07 / 22 / 87$ & 22.60 & $18 / 18 / 87$ & 34.10 & $18 / 19 / 87$ & 69.11 \\
\hline
\end{tabular}

* - value exceeds priuary orinking tater standaro.

- YALUE EXCEEDS PROPOSED PRIYARY DRINKIMG TATER STANDARO.

- yALUE EXCEEDS SCREEkING LEVEI FOR FURTHER INYESTIGATION.

- DETECTION LINIT MS NOT AVAILABLE FOR COUPARISOM

NR - ANALYSIS NOT REQUESTED OR NOT YET REPORTED

YALUES IN I $f$ ARE COUHTING ERRDRS FOR RADIONUCLIDES

IATER STANDARD(S) IN PARENTHESES ARE PROPOSED ONLY 


\begin{tabular}{|c|c|c|c|c|}
\hline$\underbrace{\text { CONSTI }}_{\text {MANE }}$ & $\begin{array}{l}\text { UENT } \\
\text { UNITS }\end{array}$ & $\begin{array}{l}\text { CONTRACTUAL } \\
\text { DETECTION } \\
\text { LINIT }\end{array}$ & $\begin{array}{l}\text { DRINKIWG } \\
\text { WATER } \\
\text { STANDARD }\end{array}$ & $\begin{array}{l}\text { SAUPLE } \\
\text { DATE }\end{array}$ \\
\hline FBARIUUY & PPB & B.8 & 1000.01 & $12 / 68 / 87$ \\
\hline FCALCIU & PPB & 56.60 & & $\begin{array}{l}07 / 22 / 87 \\
12 / 06 / 87\end{array}$ \\
\hline FCHROUI & PPB & 10.80 & 59.00 & $\begin{array}{l}17 / 22 / 87 \\
12 / 06 / 87\end{array}$ \\
\hline FIRDN & PPB & 66.11 & & $\begin{array}{l}67 / 22 / 87 \\
12 / 66 / 87\end{array}$ \\
\hline FLUORID & PPB & 500.06 & 4140.00 & $\begin{array}{l}17 / 22 / 87 \\
12 / 06 / 87\end{array}$ \\
\hline FUAGNES & PPB & 0.00 & & $\begin{array}{l}97 / 22 / 87 \\
12 / 06 / 87\end{array}$ \\
\hline FPOTASS & PPB & 180.60 & & $\begin{array}{l}07 / 22 / 87 \\
12 / 06 / 87\end{array}$ \\
\hline F5ODIUU & PPB & 100.00 & & $\begin{array}{l}07 / 22 / 87 \\
12 / 66 / 87\end{array}$ \\
\hline FYANADI & PPB & 5.00 & & $\begin{array}{l}07 / 22 / 87 \\
12 / 06 / 87\end{array}$ \\
\hline FZINC & PPB & 6.0 & & $\begin{array}{l}07 / 22 / 87 \\
12 / 66 / 87\end{array}$ \\
\hline HNITRAT & PPB & 2568.86 & 45800.00 & \\
\hline LFLUORD & PPB & 26.00 & 4040.00 & $\begin{array}{l}07 / 22 / 87 \\
12 / 06 / 87\end{array}$ \\
\hline NITRATE & PPB & 580.00 & 45064.60 & $\begin{array}{l}67 / 22 / 87 \\
12 / 68 / 87\end{array}$ \\
\hline PH-LAB & & 0.01 & & $87 / 22 / 87$ \\
\hline
\end{tabular}

TABLE C.3. (contd)

\begin{tabular}{|c|c|c|c|c|}
\hline $2-10-4$ & $\begin{array}{c}\text { SAMPLE } \\
\text { DATE }\end{array}$ & $2-516-8$ & $\begin{array}{l}\text { SAMPLE } \\
\text { DATE }\end{array}$ & $2-110-0$ \\
\hline 34.00 & $12 / 106 / 87$ & 28.66 & $12 / 08 / 87$ & 60.06 \\
\hline $\begin{array}{l}10401.11 \\
24806.10\end{array}$ & $\begin{array}{l}08 / 10 / 87 \\
12 / 08 / 87\end{array}$ & $\begin{array}{l}20206.60 \\
33400.00\end{array}$ & $\begin{array}{l}18 / 18 / 87 \\
12 / 06 / 87\end{array}$ & 390365.116 \\
\hline \multirow[t]{2}{*}{$60.01:$} & $\begin{array}{l}18 / 10 / 87 \\
12 / 06 / 87\end{array}$ & & $\begin{array}{l}06 / 19 / 87 \\
12 / 08 / 87\end{array}$ & $\begin{array}{l}136.00 \\
152.00\end{array}$ \\
\hline & $\begin{array}{l}18 / 18 / 87 \\
12 / 08 / 97\end{array}$ & 69.61 & $\begin{array}{l}18 / 18 / 87 \\
12 / 68 / 87\end{array}$ & \\
\hline 3511.06 & $\begin{array}{l}18 / 19 / 87 \\
12 / 06 / 87\end{array}$ & 1120.00 & $\begin{array}{l}18 / 19 / 87 \\
12 / 06 / 87\end{array}$ & 5380.60 \\
\hline $\begin{array}{l}7040.00 \\
7506.00\end{array}$ & $\begin{array}{l}68 / 10 / 87 \\
12 / 10 / 87\end{array}$ & $\begin{array}{l}11600.00 \\
11406.01\end{array}$ & $\begin{array}{l}68 / 10 / 87 \\
12 / 06 / 87\end{array}$ & $\begin{array}{l}13900.10 \\
15901.01\end{array}$ \\
\hline 2480.06 & $\begin{array}{l}10 / 19 / 87 \\
12 / 08 / 87\end{array}$ & 3826.010 & $\begin{array}{l}08 / 19 / 87 \\
12 / 86 / 67\end{array}$ & $\begin{array}{l}4580.06 \\
5000.10\end{array}$ \\
\hline 48006.06 & $\begin{array}{l}08 / 19 / 87 \\
12 / 66 / 87\end{array}$ & $\begin{array}{l}18500.00 \\
24300.00\end{array}$ & $\begin{array}{l}18 / 19 / 87 \\
12 / 06 / 87\end{array}$ & $\begin{array}{l}167000.10 \\
185000.01\end{array}$ \\
\hline \multirow[t]{2}{*}{51.6} & $\begin{array}{l}19 / 19 / 87 \\
12 / 08 / 87\end{array}$ & 29.60 & $\begin{array}{l}18 / 19 / 97 \\
12 / 06 / 97\end{array}$ & $\begin{array}{l}56.01 \\
06.01\end{array}$ \\
\hline & $\begin{array}{l}08 / 19 / 87 \\
12 / 66 / 87\end{array}$ & 27.11 & $\begin{array}{l}18 / 10 / 87 \\
12 / 06 / 87\end{array}$ & 0.06 \\
\hline NR & $\begin{array}{l}08 / 19 / 87 \\
12 / 06 / 87\end{array}$ & & $\begin{array}{l}18 / 19 / 87 \\
12 / 06 / 87\end{array}$ & 381600.6 \\
\hline $\begin{array}{l}2984.00 \\
3150.00\end{array}$ & $\begin{array}{l}18 / 19 / 87 \\
12 / 06 / 87\end{array}$ & $\begin{array}{r}508.00 \\
1260.06\end{array}$ & $\begin{array}{l}18 / 19 / 87 \\
12 / 06 / 87\end{array}$ & 4701.6 \\
\hline 17000.00 & $\begin{array}{l}18 / 19 / 87 \\
12 / 06 / 87\end{array}$ & $\begin{array}{l}2606.01 \\
1821.61\end{array}$ & $\begin{array}{l}18 / 18 / 87 \\
12 / 68 / 87\end{array}$ & $\begin{array}{l}378106.06 \\
361006.00\end{array}$ \\
\hline 7.95 & $68 / 19 / 87$ & 7.93 & $68 / 19 / 07$ & 7.81 \\
\hline
\end{tabular}

- - yalue EXCEEDS PRIMARY DRINKING Tater STANDARD.

- YALUE EXCEEDS PROPOSED PRIMARY DRINKING PATER STANDARD.

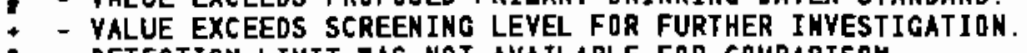

- DETECTION LIMIT TAS NOT aVAILABLE FOR COUPARISON

NR - AMALYSIS NOT REQUESTED OR NOT YET REPARTED

YALUES IN $f$ I ARE CDUNTING ERRORS FOR RADIONUCLIDES

inTER STANDARD(S) IN PARENTHESES ARE PROPOSED ONLY 


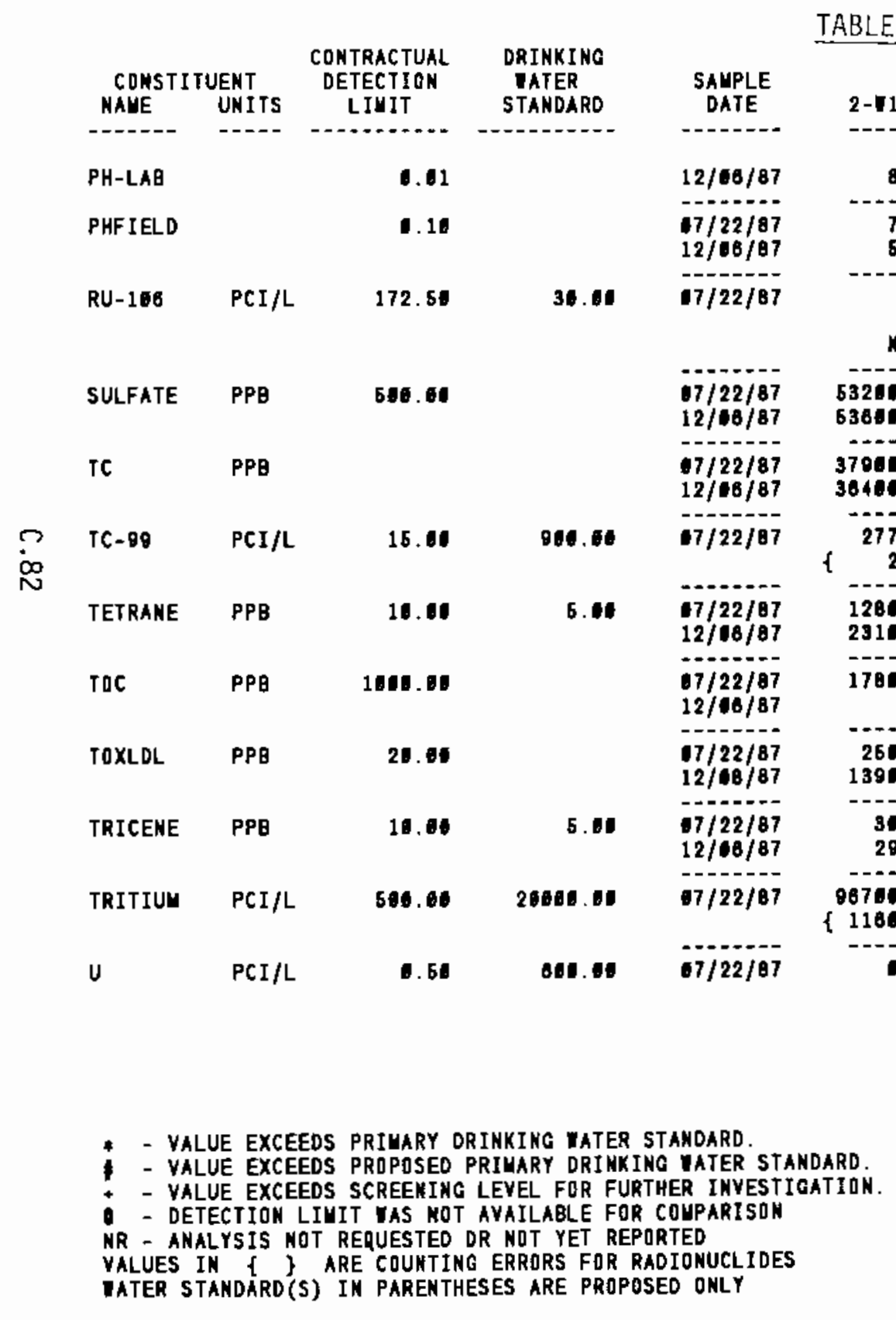

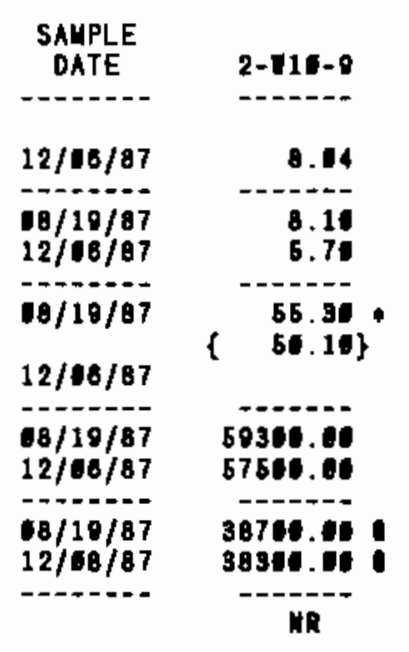

$10 / 10 / 87$

$12 / 08 / 87$

1450.04

1220.00

18/19/87

$12 / 06 / 97$

18/10/87

$12 / 08 / 87$

19/19/07

$12 / 08 / 07$

$08 / 19 / 07$

3760.01

$261.09\}$

1204.00

926.05

18.0

$10.00 *$

70900.01

\{ 885.00\}

$\begin{array}{lllr}10 / 19 / 87 & 1.28 & 68 / 19 / 87 & 2.10\end{array}$

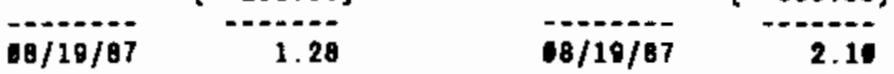

- VALUE EXCEEDS PRIUARY ORINKIMG WATER STAMDARD.

IONUCLIDES

IATER STANDARD(S) IN PARENTHESES ARE PROPOSED ONLY 
TABLE C.3. (contd)

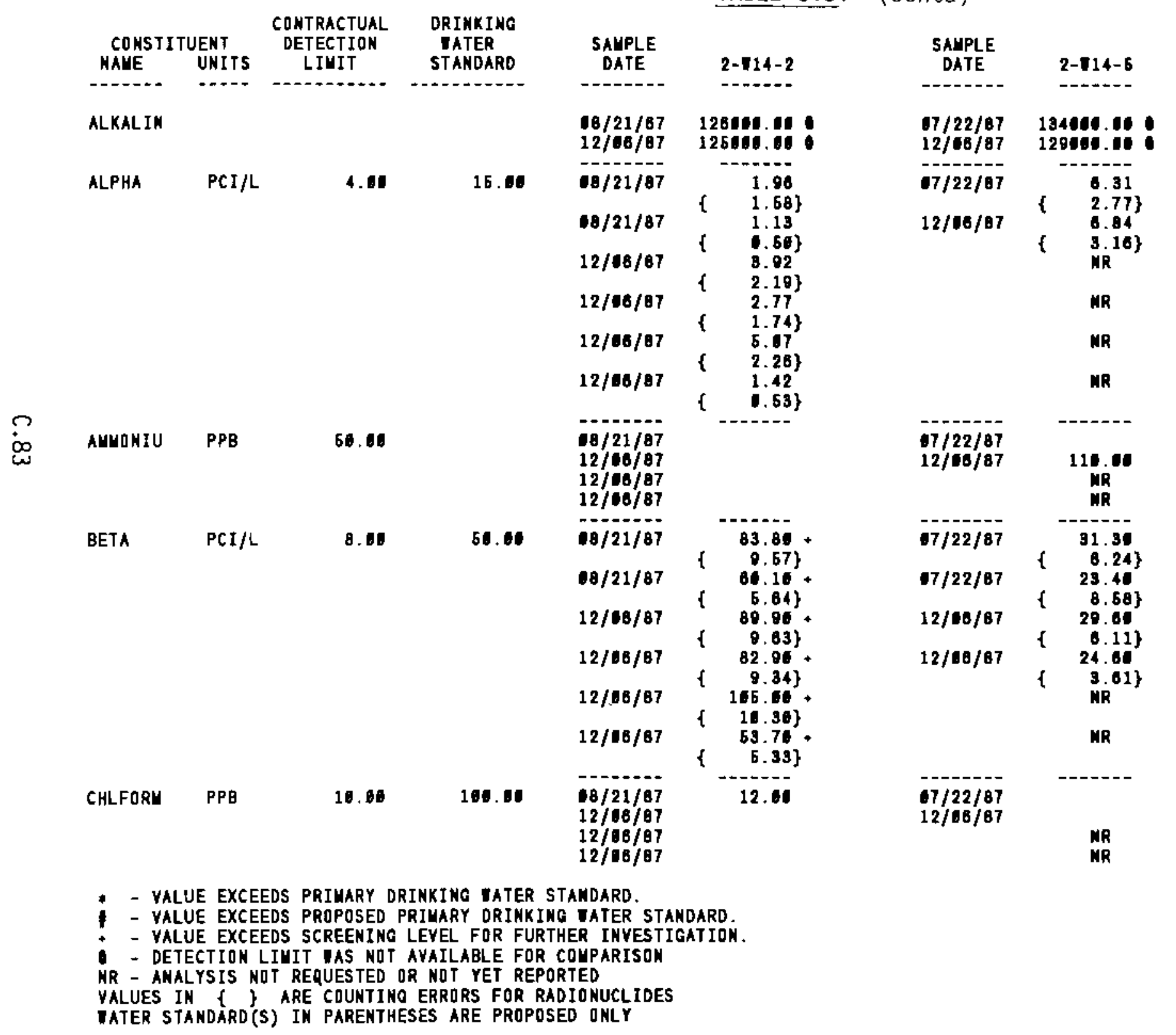

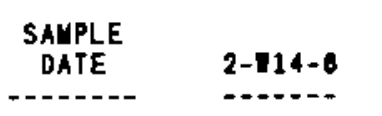

$18 / 21 / 97 \quad 125010.10$ $12 / 16 / 87 \quad 120000.01$. -....... $12 / 16 / 87$ $\left\{\begin{array}{l}2.74 \\ 1.73\} \\ \mathrm{NR}\end{array}\right.$

NR

MR

NR

$08 / 21 / 87$ $12 / 10 / 87$

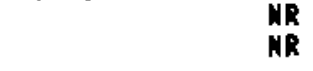

$68 / 21 / 87$

$18 / 21 / 87$

$12 / 16 / 87$

$12 / 18 / 87$

(1)

.........

$98 / 21 / 87$
$12 / 18 / 87$

- YALUE EXCEEDS PRIMARY DRINKIM

TATER STANDARD'S S IM PARENTHESES ARE PROPOSED ONLY 
TABLE C.3. (contd)

\begin{tabular}{|c|c|c|c|c|c|}
\hline \multicolumn{2}{|c|}{ CONSTITUENT } & $\begin{array}{c}\text { CONTRACTUAL } \\
\text { DETECTION } \\
\text { LIUIT }\end{array}$ & $\begin{array}{l}\text { DRINKING } \\
\text { TATER } \\
\text { STANDARD }\end{array}$ & $\begin{array}{c}\text { SAMPLE } \\
\text { DATE }\end{array}$ & $2-\pi 14-2$ \\
\hline----- & $m . .$. & - - & 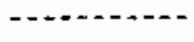 & ------ & $\cdots+\cdots$ \\
\hline CHLORID & PPE & 500.00 & & $\begin{array}{l}08 / 21 / 87 \\
12 / 06 / 87 \\
12 / 68 / 87 \\
12 / 86 / 87\end{array}$ & $\begin{array}{l}52800.06 \\
47800.06 \\
48800.00 \\
46500.06\end{array}$ \\
\hline $\mathrm{CO}-6 \mathrm{O}$ & $\mathrm{PCI} / \mathrm{L}$ & 22. 50 & 100.01 & $68 / 21 / 07$ & . \\
\hline & & & & $12 / 96 / 87$ & \\
\hline CONDFLD & UМHO & 1.69 & & $\begin{array}{l}18 / 21 / 87 \\
12 / 06 / 87\end{array}$ & $\begin{array}{l}507.06 \\
577.06\end{array}$ \\
\hline CS -137 & $\mathrm{PCI} / \mathrm{L}$ & 20.65 & 280.60 & $88 / 21 / 87$ & $4--2-2$ \\
\hline & & & & $12 / 108 / 87$ & $\begin{array}{l}6.35 \\
4.02\}\end{array}$ \\
\hline CYANIDE & PPB & 16.00 & & $\begin{array}{l}18 / 21 / 87 \\
12 / 08 / 87\end{array}$ & $\begin{array}{r}62.00 \\
115.00\end{array}$ \\
\hline FALUMIN & PPB & 150.00 & & $\begin{array}{l}18 / 21 / 87 \\
12 / 06 / 87 \\
12 / 66 / 87 \\
12 / 96 / 87\end{array}$ & 516.01 \\
\hline FBARIUY & PPB & B. 60 & 1008.00 & $\begin{array}{l}18 / 21 / 87 \\
12 / 06 / 87 \\
12 / 06 / 87 \\
12 / 06 / 87\end{array}$ & $\begin{array}{r}84.60 \\
96.00 \\
102.00 \\
88.00\end{array}$ \\
\hline FCADHIU & PPB & 2.00 & 10.06 & $\begin{array}{l}08 / 21 / 87 \\
12 / 66 / 87 \\
12 / 68 / 87 \\
12 / 88 / 87\end{array}$ & -....-- \\
\hline FCALCIU & PPB & 50.00 & & $\begin{array}{l}18 / 21 / 67 \\
12 / 68 / 87 \\
12 / 06 / 67\end{array}$ & $\begin{array}{l}63800.00 \\
68700.00 \\
64900.00\end{array}$ \\
\hline
\end{tabular}

\begin{tabular}{|c|c|}
\hline $\begin{array}{l}\text { SAUPLE } \\
\text { DATE }\end{array}$ & $2-114-5$ \\
\hline$---1-\ldots$ & $---1--$ \\
\hline $\begin{array}{l}07 / 22 / 87 \\
12 / 06 / 87\end{array}$ & $\begin{array}{c}54501.08 \\
46200.00 \\
\text { WR } \\
\text { WR }\end{array}$ \\
\hline \multirow[t]{2}{*}{$67 / 22 / 87$} & ----- \\
\hline & MR \\
\hline $\begin{array}{l}07 / 22 / 87 \\
12 / 06 / 87\end{array}$ & 611.06 \\
\hline - & $---\cdots$ \\
\hline $67 / 22 / 87$ & $\begin{array}{l}8.65 \\
6.40\} \\
N R\end{array}$ \\
\hline $\begin{array}{l}17 / 22 / 87 \\
12 / 16 / 87\end{array}$ & ----- \\
\hline 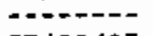 & $\cdots$ \\
\hline $\begin{array}{l}17 / 22 / 87 \\
12 / 16 / 87\end{array}$ & $\begin{array}{c}153.00 \\
108.01 \\
\text { NR } \\
\text { NR }\end{array}$ \\
\hline $\begin{array}{l}17 / 22 / 87 \\
12 / 16 / 87\end{array}$ & $\begin{array}{l}43.65 \\
36.85 \\
\text { KR } \\
\text { WR }\end{array}$ \\
\hline \multirow[t]{2}{*}{$\begin{array}{l}67 / 22 / 87 \\
12 / 08 / 87\end{array}$} & 6.00 \\
\hline & $\begin{array}{l}\text { MR } \\
\text { MR }\end{array}$ \\
\hline $\begin{array}{l}07 / 22 / 87 \\
12 / 06 / 87\end{array}$ & $\begin{array}{c}25460.00 \\
22309.01 \\
\text { NR }\end{array}$ \\
\hline
\end{tabular}

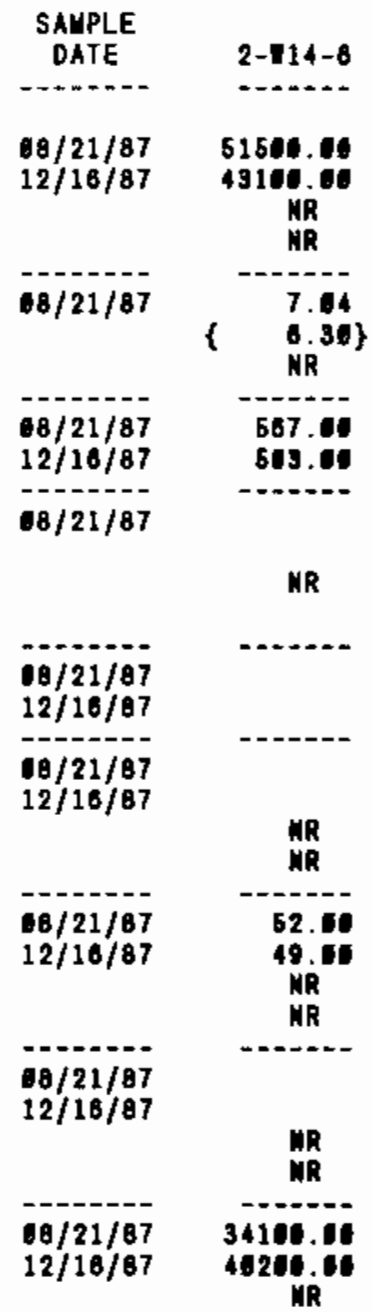

- Y YLUE EXCEEDS PRIUARY DRINIKIMO TATER STANDARD.

- - VALUE EXCEEDS PROPOSED PRIUARY DRINKIMG TATER STAMDARD

+ - VALUE EXCEEDS SCREENING LEVEL FOR FURTHER INYESTIOATION.

- DETECTIDN LIHIT US NOT AVAILABLE FOR COMPARISON

NR - AKALYSIS NOT REQUESTED OR NOT YET REPDRTED

VALUES IN $\{$ \& ARE COUNTING ERRDRS FOR RADIONUCLIDES

TATER STANDARO(S) IN PARENTHESES ARE PROPOSED ONLY 
TABLE C. 3. (contd)

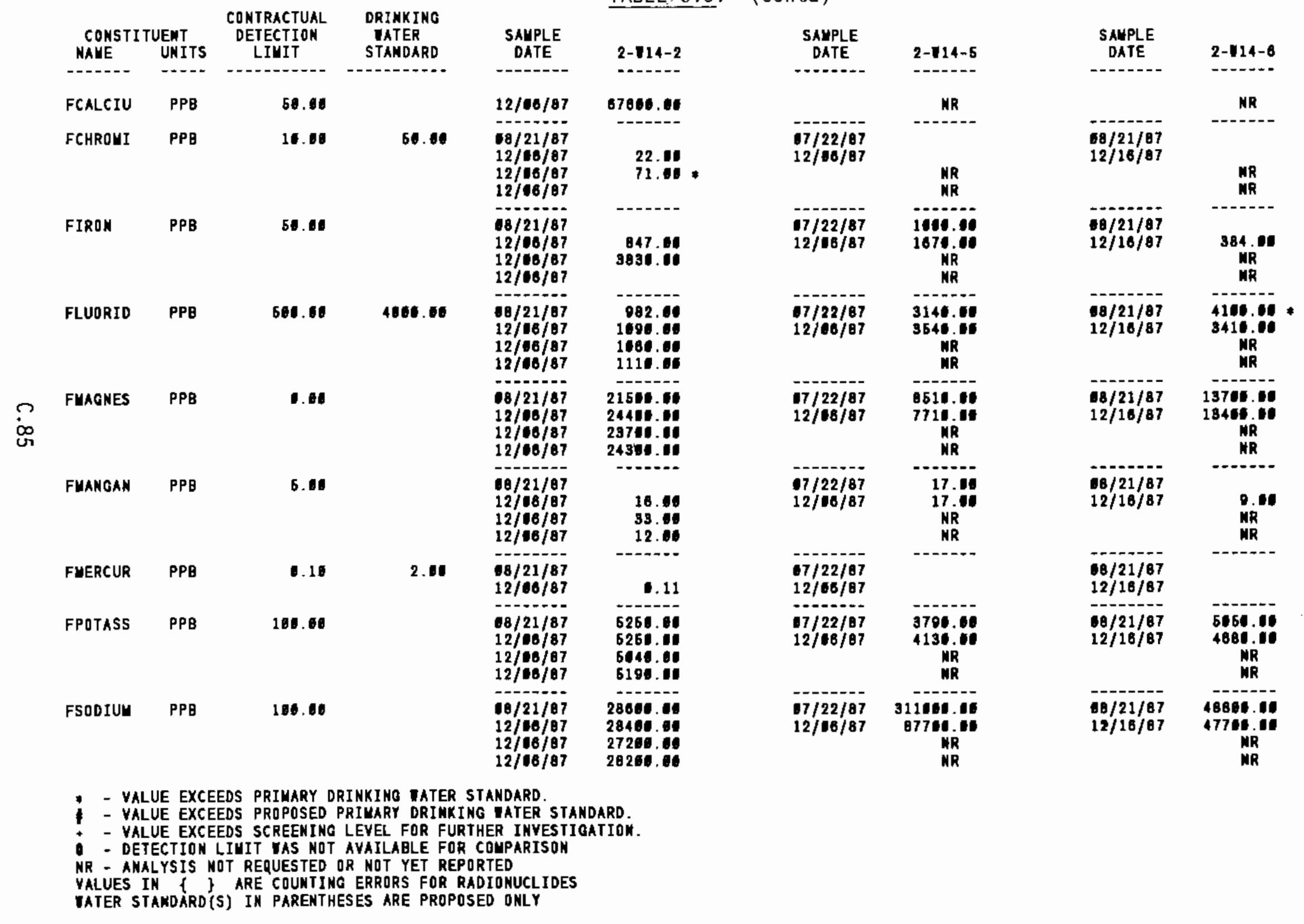


TABLE C.3. (contd)

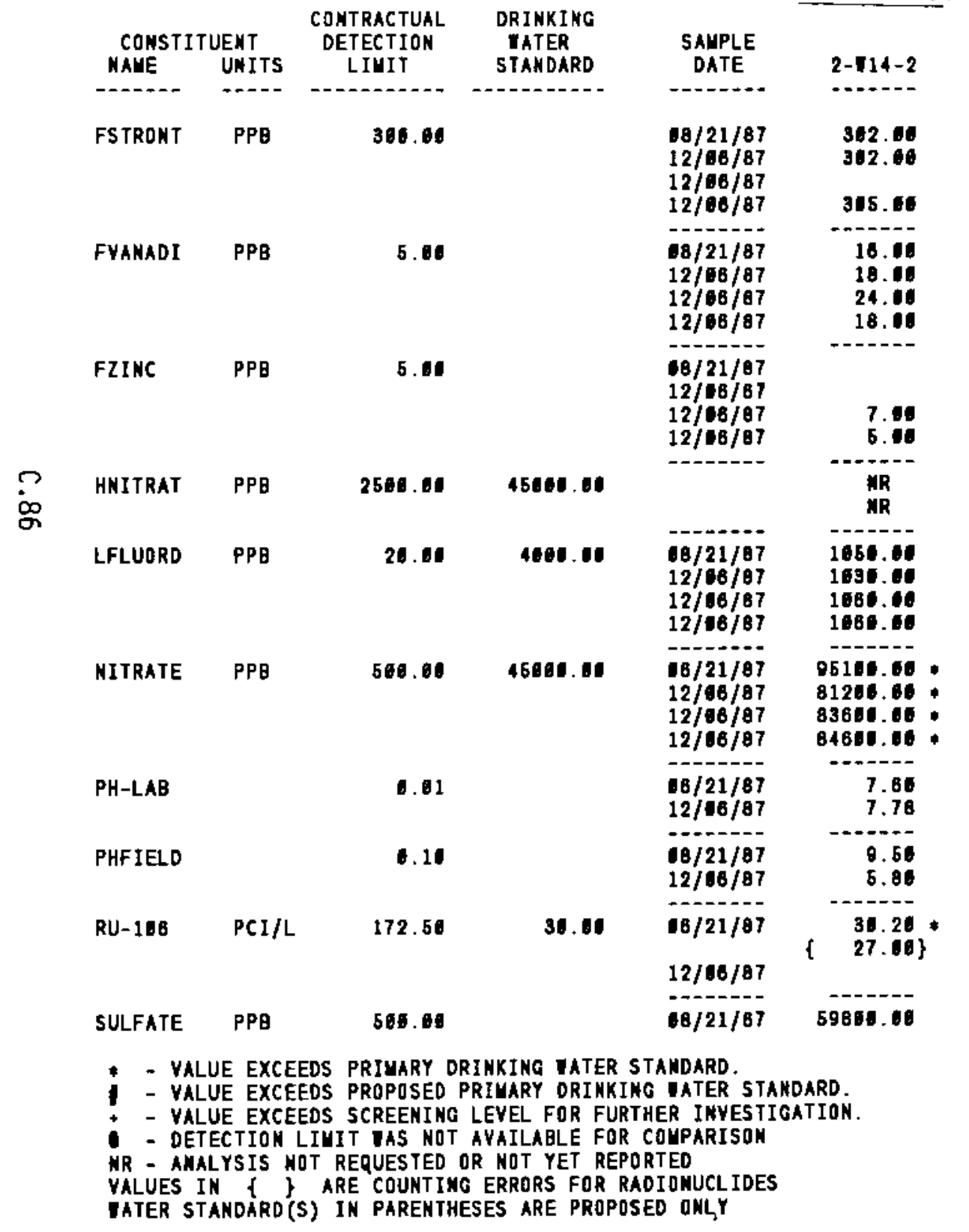

SAUPLE

2-714-6

DATE 2-T14-5

$17 / 22 / 87$

$12 / 66 / 87$

NR

NR

c7/22/87

$12 / 06 / 87$

104.09

87.00

NR

NR

17/22/87 30.00

$12 / 10 / 87$

NR

MR

$17 / 22 / 87$ os5

$17 / 22 / 87$

$12106 / 81$

$17 / 22 / 87$

28066.66

$-2-1$

2964. 6

MR

$-7 / 2-2 / 87$

$12 / 06 / 67$

63911.41*

29804.10

HP

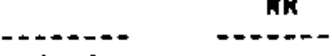

$07 / 22 / 87$
$12 / 66 / 87$

7.98
8.02

a.

5. 89

$12 / 18 / 87$

- $7 / 22 / 87$

$------$

NR

$67 / 22 / 87$
$68 / 21 / 87$
$12 / 18 / 87$

\begin{tabular}{|c|c|}
\hline & $\begin{array}{l}\text { WR } \\
\text { KR }\end{array}$ \\
\hline $\begin{array}{l}68 / 21 / 97 \\
12 / 16 / 07\end{array}$ & $\begin{array}{l}28.01 \\
\text { NR } \\
\text { NR }\end{array}$ \\
\hline
\end{tabular}

$18 / 21 / 87$

$12 / 18 / 87$

\section{NR}

$68 / 21 / 07$

$12 / 16 / 87$

18/21/87

$18 / 21 / 87$
$12 / 16 / 87$

20100. 00

4400.00

3800.00

NR
NR

19/21/87

$12 / 16 / 87$

9480

34806. 01

MR

MR

09/21/87 $\quad 7.76$

12/16/87

08/21/a7

$12 / 16 / 87$

$08 / 21 / 87$

7.77

0.0

7.2

NR

$68 / 21 / 87$ 81500.00

- VALUE EXCEEDS PRI

- YALUE EXCEEDS PROPOSED PRIMARY DRINKIMG TATER STAHDARD.

- VALUE EXCEEOS SCREENING LEVEL FOR FURTHER IBYESTIGATION. 


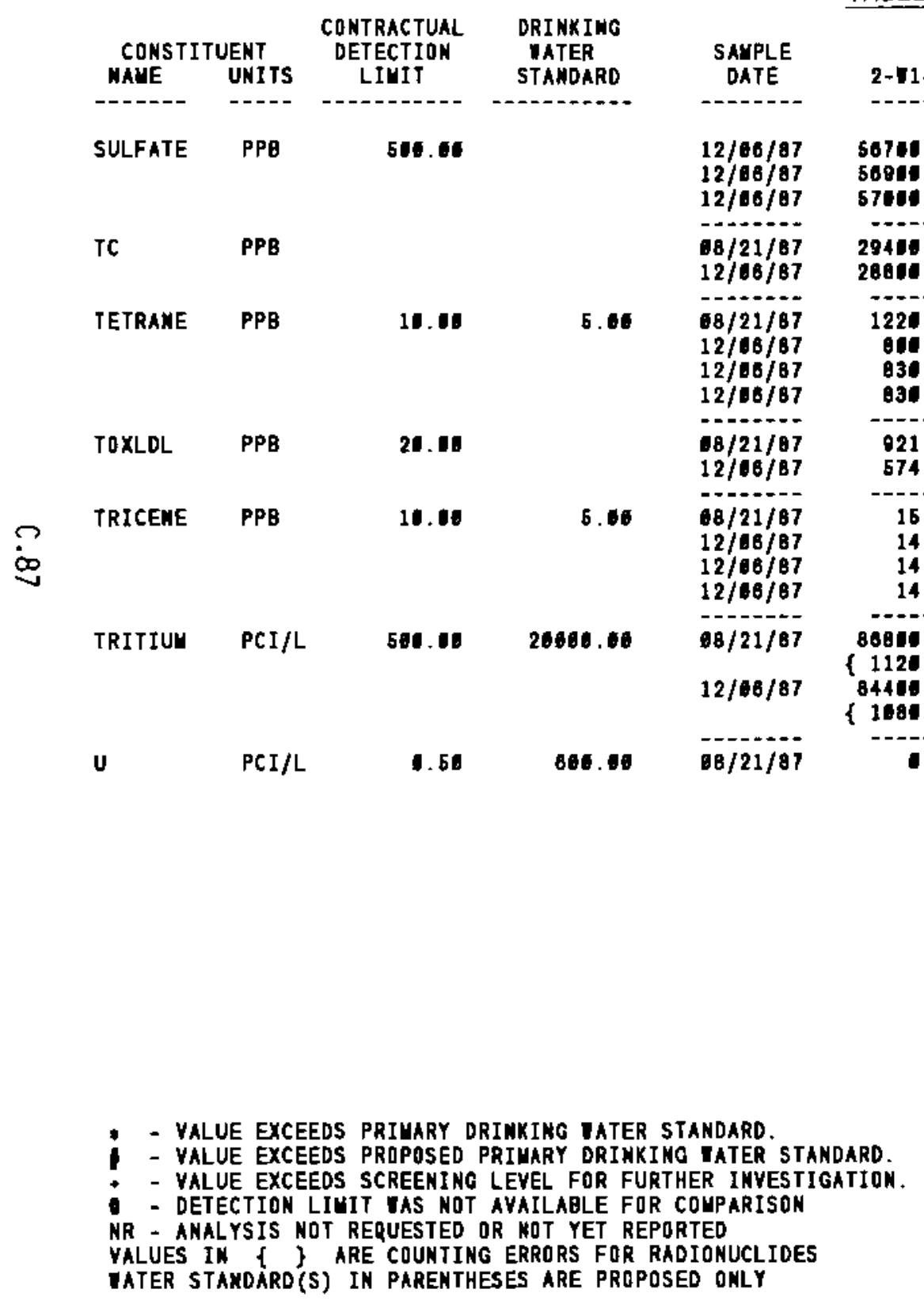

TABLE C.3. (contd)

SAMPLE

\begin{tabular}{|c|c|c|c|}
\hline DATE & $2-714-5$ & DATE & $2-714-8$ \\
\hline$---1, \ldots$ & 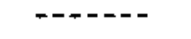 & $\ldots$ & ------ \\
\hline $12 / 06 / 97$ & $\underset{\substack{M R \\
M R}}{55460.00}$ & $12 / 16 / 87$ & $\begin{array}{c}49400.08 \\
\text { NR } \\
\text { MR }\end{array}$ \\
\hline - & $-\cdots-$ & ------- & 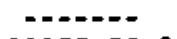 \\
\hline $\begin{array}{l}07 / 22 / 87 \\
12 / 66 / 87\end{array}$ & $\begin{array}{l}33256.00 \\
29664.00\end{array}$ & $\begin{array}{l}18 / 21 / 87 \\
12 / 16 / 87\end{array}$ & $\begin{array}{l}20200.45 \\
28040.01\end{array}$ \\
\hline $97 / 22 / 87$ & 402.00 & $8 / 21 / 87$ & 850.00 \\
\hline $12 / 00 / 07$ & $\begin{array}{c}\text { MR } \\
\text { MR }\end{array}$ & $12 / 16 / 87$ & $\underset{\text { MR }}{\text { NRR }}$ \\
\hline$---1--1$ & -4 & 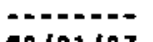 & 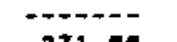 \\
\hline $\begin{array}{l}17 / 22 / 87 \\
12 / 88 / 87\end{array}$ & $\begin{array}{l}340.01 \\
224.11\end{array}$ & $\begin{array}{l}18 / 21 / 87 \\
12 / 16 / 87\end{array}$ & $\begin{array}{l}371.00 \\
280.00\end{array}$ \\
\hline 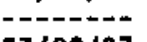 & $-\infty--$ & - & $-0-0--0$ \\
\hline \multirow{2}{*}{$\begin{array}{l}17 / 22 / 87 \\
12 / 08 / 87\end{array}$} & & $\begin{array}{l}08 / 21 / 87 \\
12 / 10 / 87\end{array}$ & \\
\hline & $\begin{array}{l}\text { NR } \\
\text { MR }\end{array}$ & & $\begin{array}{l}\text { NR } \\
\text { NR }\end{array}$ \\
\hline$=-1$ & חי- & ----1--- & $--10-\overline{10}$ \\
\hline $17 / 22 / 87$ & $\begin{array}{l}6021.06 \\
1234.06\}\end{array}$ & $18 / 21 / 87$ & {$\left[\begin{array}{r}29804.60 \\
675.60\}\end{array}\right.$} \\
\hline $12 / 06 / 87$ & $\begin{array}{r}6218.00 \\
\{360.00\}\end{array}$ & $12 / 16 / 87$ & $\begin{array}{r}16561.00 \\
\quad 518.06\}\end{array}$ \\
\hline $7 / 22 / 87$ & 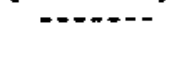 & $18 / 21 / 87$ & 2.00 \\
\hline
\end{tabular}

YALUES IH

ATER STANOARD(S) IN PARENTHESES ARE PROPOSED ONLY 
TABLE C.3. (contd)

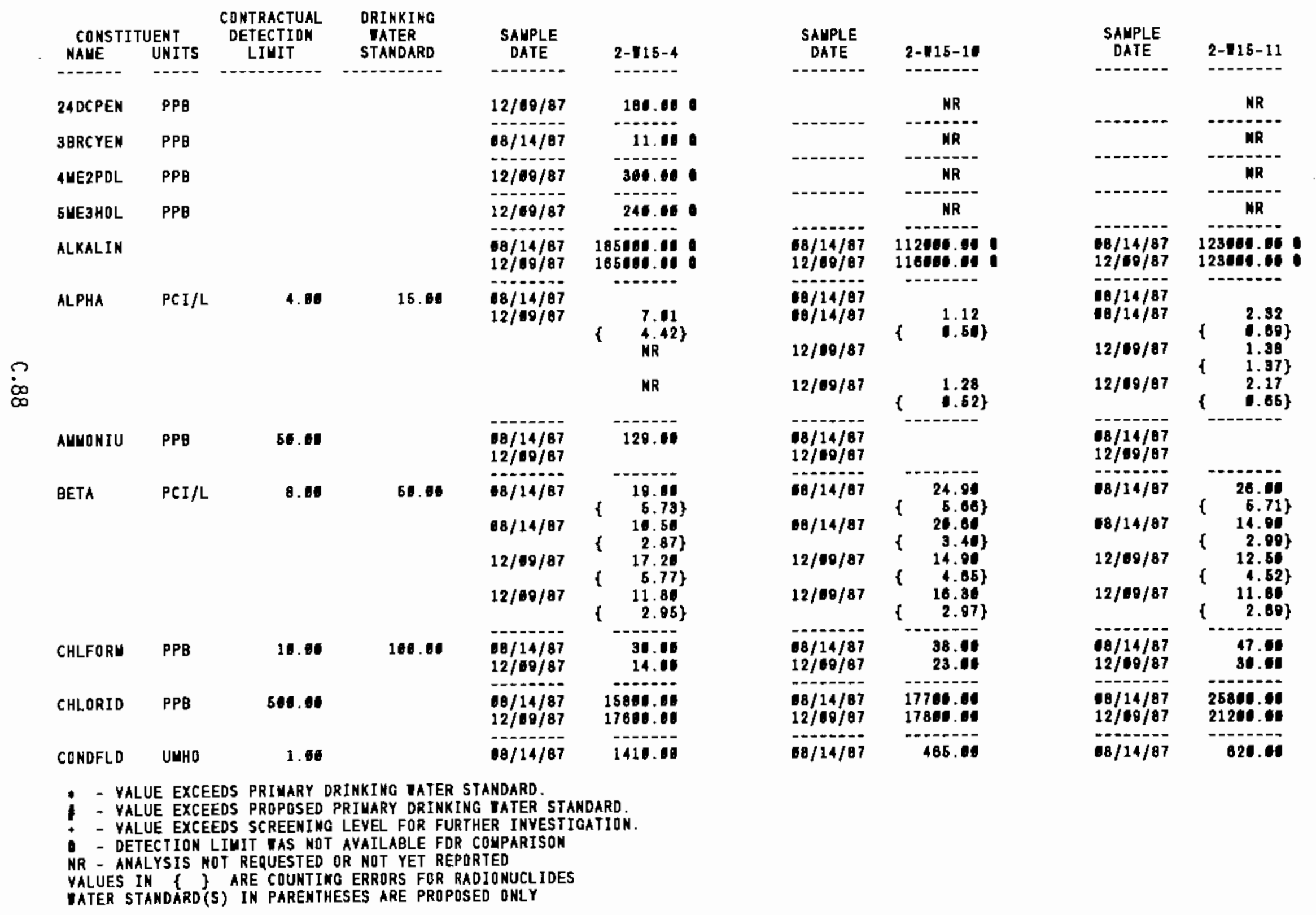


TABLE C. .3. (contd)

\begin{tabular}{|c|c|c|c|c|c|}
\hline $\begin{array}{c}\text { CONSTI } \\
\text { NAYE }\end{array}$ & $\begin{array}{l}\text { UENT } \\
\text { UNITS }\end{array}$ & $\begin{array}{c}\text { CONTRACTUAL } \\
\text { DETECTION } \\
\text { LIUIT }\end{array}$ & $\begin{array}{l}\text { ORINKING } \\
\text { WATER } \\
\text { STAMDARD }\end{array}$ & $\begin{array}{c}\text { SAMPLE } \\
\text { DATE }\end{array}$ & $2-115-4$ \\
\hline CONDFLD & UNHO & 1.68 & & $12 / 09 / 87$ & 1354.00 \\
\hline CS-137 & PCI/L & 26.00 & 208.60 & $08 / 14 / 87$ & NR \\
\hline FARSENI & PPB & 6. 10 & 56.0 & $60 / 14 / 87$ & MR \\
\hline FBARIUU & $\mathrm{PPB}$ & 0.10 & 1806.60 & $08 / 14 / 87$ & ${ }_{M R}^{56.01}$ \\
\hline FCALCIU & PPB & 66.00 & & $00 / 14 / 87$ & $\begin{array}{c}28106.00 \\
M R\end{array}$ \\
\hline FCHROMI & PPB & 10.6 & 56.60 & $08 / 14 / 87$ & NR \\
\hline FIRON & PPB & 50.00 & & $18 / 14 / 87$ & $\underset{\mathrm{NR}}{289.91}$ \\
\hline FLEAD & PPB & 5.60 & 50.90 & $68 / 14 / 87$ & MR \\
\hline FLUORID & PPB & 510.01 & 4008.01 & $\begin{array}{l}08 / 14 / 87 \\
12 / 09 / 87\end{array}$ & $\begin{array}{l}1964.64 \\
12764.04\end{array}$ \\
\hline FUAGNES & PPB & 6.00 & & $08 / 14 / 87$ & $\begin{array}{l}8836.00 \\
M R\end{array}$ \\
\hline FuANGAN & PPB & 5.80 & & $68 / 14 / 87$ & MR \\
\hline FPOTASS & PPB & 108.60 & & $08 / 14 / 87$ & W34. \\
\hline FSODIUM & PPB & 108.00 & & $68 / 14 / 87$ & $26906 \mathrm{NR}_{\mathrm{NR}}^{\mathrm{ON}}$ \\
\hline FSTRONT & PPB & 364.66 & & $18 / 14 / 87$ & \\
\hline
\end{tabular}

\begin{tabular}{|c|c|}
\hline $\begin{array}{c}\text { SAMPLE } \\
\text { DATE }\end{array}$ & $2-716-10$ \\
\hline $12 / 00 / \theta 7$ & 485.00 \\
\hline $188 / 14 / 87$ & MR \\
\hline $\begin{array}{l}08 / 14 / 87 \\
12 / 00 / 87\end{array}$ & \\
\hline $\begin{array}{l}08 / 14 / 07 \\
12 / 00 / 87\end{array}$ & $\begin{array}{l}58.11 \\
62.01\end{array}$ \\
\hline $\begin{array}{l}68 / 14 / 87 \\
12 / 09 / 87\end{array}$ & $\begin{array}{l}47600.11 \\
\text { 58610. }\end{array}$ \\
\hline $\begin{array}{l}18 / 14 / 87 \\
12 / 09 / 87\end{array}$ & 13.06 \\
\hline $\begin{array}{l}68 / 14 / 67 \\
12 / 10 / 87\end{array}$ & \\
\hline $\begin{array}{l}08 / 14 / 87 \\
12 / 100 / 87\end{array}$ & \\
\hline $\begin{array}{l}08 / 14 / 87 \\
12 / 00 / 87\end{array}$ & 566.16 \\
\hline $\begin{array}{l}18 / 14 / 87 \\
12 / 99 / 87\end{array}$ & $\begin{array}{l}17549.04 \\
18394.00\end{array}$ \\
\hline $\begin{array}{l}68 / 14 / 87 \\
12 / 00 / 87\end{array}$ & \\
\hline $\begin{array}{l}68 / 14 / 87 \\
12 / 10 / 87\end{array}$ & $\begin{array}{l}4760.00 \\
44600.100\end{array}$ \\
\hline $\begin{array}{l}18 / 14 / 87 \\
12 / 00 / 87\end{array}$ & $\begin{array}{l}17500.00 \\
10010.60\end{array}$ \\
\hline $18 / 14 / 87$ & \\
\hline
\end{tabular}

\begin{tabular}{|c|c|}
\hline $\begin{array}{l}\text { SAUPLE } \\
\text { DATE }\end{array}$ & 2-T16-11 \\
\hline $12 / 00 / 87$ & 587.00 \\
\hline $\begin{array}{l}08 / 14 / 87 \\
12 / 09 / 87\end{array}$ & $\begin{array}{r}8.78 \\
+\quad 72\end{array}$ \\
\hline $\begin{array}{l}18 / 14 / 87 \\
12 / 100 / 87\end{array}$ & \\
\hline $\begin{array}{l}8 / 14 / \theta 7 \\
12 / 60 / \theta 7\end{array}$ & $\begin{array}{l}76.14 \\
70.11\end{array}$ \\
\hline $\begin{array}{l}08 / 14 / 87 \\
12 / 00 / 87\end{array}$ & 61196.06 \\
\hline $\begin{array}{l}18 / 14 / 87 \\
12 / 09 / 87\end{array}$ & $\begin{array}{l}36.00 \\
80.01\end{array}$ \\
\hline $\begin{array}{l}08 / 14 / 87 \\
12 / 60 / 87\end{array}$ & \\
\hline $\begin{array}{l}68 / 14 / 87 \\
12 / 09 / 87\end{array}$ & \\
\hline $\begin{array}{l}68 / 14 / 87 \\
12 / 00 / 87\end{array}$ & 891.10 \\
\hline $\begin{array}{l}68 / 14 / 87 \\
12 / 60 / 87\end{array}$ & 21200.41 \\
\hline $\begin{array}{l}18 / 14 / 87 \\
12 / 00 / 87\end{array}$ & \\
\hline $\begin{array}{l}68 / 14 / 87 \\
12 / 00 / 87\end{array}$ & $\begin{array}{l}5346.00 \\
4926.01\end{array}$ \\
\hline $\begin{array}{l}88 / 14 / 87 \\
12 / 69 / 97\end{array}$ & $\begin{array}{l}25000.01 \\
22000.01\end{array}$ \\
\hline $88 / 14 / 87$ & 915.4 \\
\hline
\end{tabular}

- - value exceEds primary dRinking tater standard.

- VALUE EXCEEDS PROPOSED PRIMARY DRINKKING IATER STANDARD.

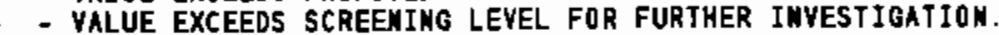

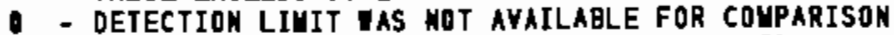

NR - ANALYSIS NOT REQUESTED OR MOT YET REPORTED

YALUES IN if ARE COUNTINO ERRORS FOR RADIONUCLIDES

WATER STANDARD(S) IN PARENTHESES ARE PROPOSED ONLY 


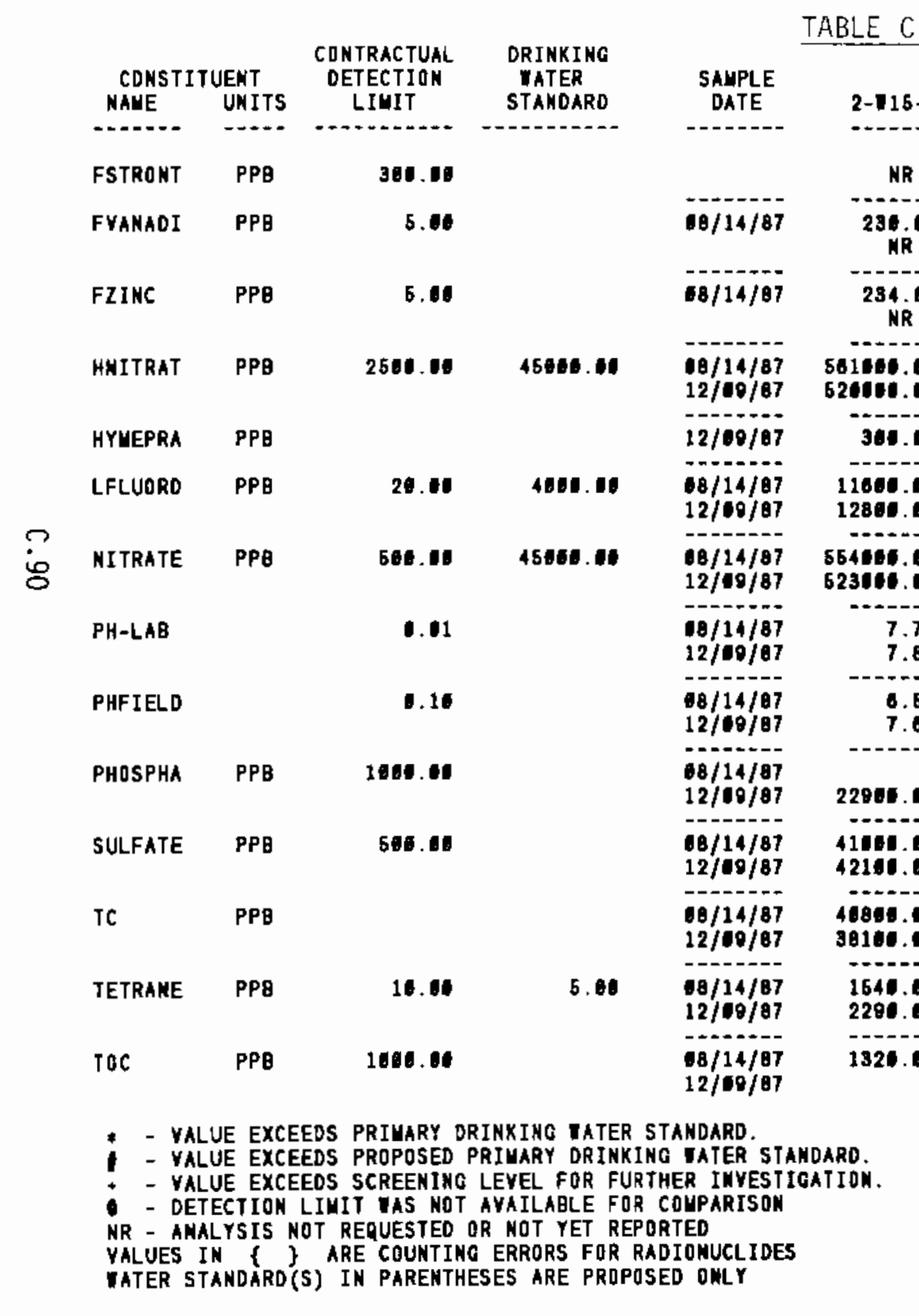

\begin{tabular}{|c|c|c|c|}
\hline $\begin{array}{l}\text { SALPLE } \\
\text { DATE }\end{array}$ & $2-715-10$ & $\begin{array}{l}\text { SALPLE } \\
\text { DATE }\end{array}$ & $2-\pi 15-11$ \\
\hline -..-- & 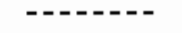 & $-\ldots-c$ & - \\
\hline $12 / 09 / 87$ & & $12 / 80 / 87$ & 318.01 \\
\hline $\begin{array}{l}18 / 14 / 87 \\
12 / 10 / 67\end{array}$ & 27.11 & $\begin{array}{l}10 / 14 / 87 \\
12 / 69 / 87\end{array}$ & 24.19 \\
\hline $\begin{array}{l}88 / 14 / 87 \\
12 / 09 / 87\end{array}$ & ----- & $\begin{array}{l}-18 / 14 / 67 \\
12 / 69 / 87\end{array}$ & $\begin{array}{l}6.00 \\
0.06\end{array}$ \\
\hline $\begin{array}{l}68 / 14 / 87 \\
12 / 60 / 87\end{array}$ & 101010.00 & $\begin{array}{l}68 / 14 / 97 \\
12 / 90 / 87\end{array}$ & $\begin{array}{l}142010 \\
133040.05\end{array}$ \\
\hline - & NR & +---- & NR \\
\hline $\begin{array}{l}18 / 14 / 87 \\
12 / 00 / 87\end{array}$ & $\begin{array}{l}360.00 \\
345.00\end{array}$ & $\begin{array}{l}18 / 14 / 87 \\
12 / 09 / 87\end{array}$ & 440.10 \\
\hline $\begin{array}{l}08 / 14 / 87 \\
12 / 00 / 87\end{array}$ & 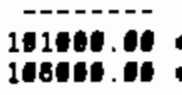 & $\begin{array}{l}00 / 14 / 87 \\
12 / 00 / 87\end{array}$ & $\begin{array}{l}142806.00 \\
126001.06\end{array}$ \\
\hline $\begin{array}{l}10 / 14 / 67 \\
12 / 60 / 67\end{array}$ & $\begin{array}{r}7.62 \\
7.82\end{array}$ & $\begin{array}{l}18 / 14 / 87 \\
12 / 09 / 87\end{array}$ & $\begin{array}{r}7.77 \\
7.89\end{array}$ \\
\hline $18 / 14 / 87$ & 7.10 & $18 / 14 / 87$ & 7.11 \\
\hline $\begin{array}{l}12 / 10 / 87 \\
08 / 14 / 87 \\
12 / 00 / 87\end{array}$ & ינים & $\begin{array}{l}12 / 69 / 87 \\
18 / 14 / 87 \\
12 / 00 / 87\end{array}$ & 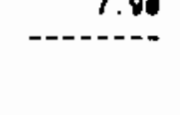 \\
\hline $\begin{array}{l}98 / 14 / 87 \\
12 / 80 / 87\end{array}$ & $\begin{array}{l}42310.06 \\
63916.06\end{array}$ & $\begin{array}{l}08 / 14 / 87 \\
12 / 69 / 87\end{array}$ & 65460.00 \\
\hline $\begin{array}{l}00 / 14 / 87 \\
12 / 00 / 87\end{array}$ & $\begin{array}{l}\overline{26314.04} \\
27194.61\end{array}$ & $\begin{array}{l}68 / 14 / 87 \\
12 / 69 / 87\end{array}$ & 20345.46 \\
\hline $\begin{array}{l}18 / 14 / 87 \\
12 / 09 / 87\end{array}$ & 2220.10 & $\begin{array}{l}18 / 14 / 07 \\
12 / 00 / 07\end{array}$ & 2734.06 \\
\hline $\begin{array}{l}08 / 14 / 87 \\
12 / 09 / 87\end{array}$ & ------- & $\begin{array}{l}18 / 14 / 87 \\
12 / 69 / 87\end{array}$ & \\
\hline
\end{tabular}




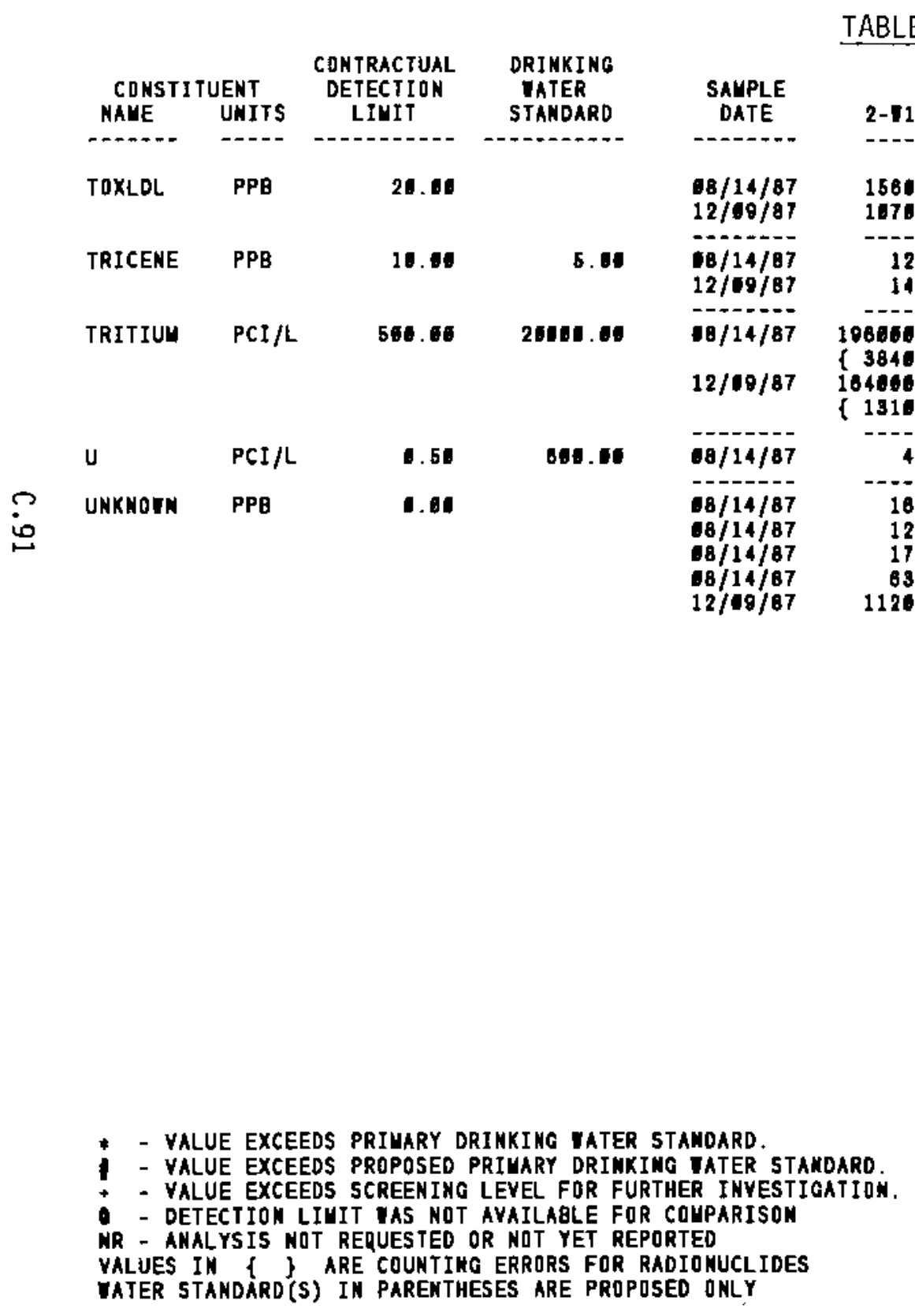


TABLE C.3. (contd)

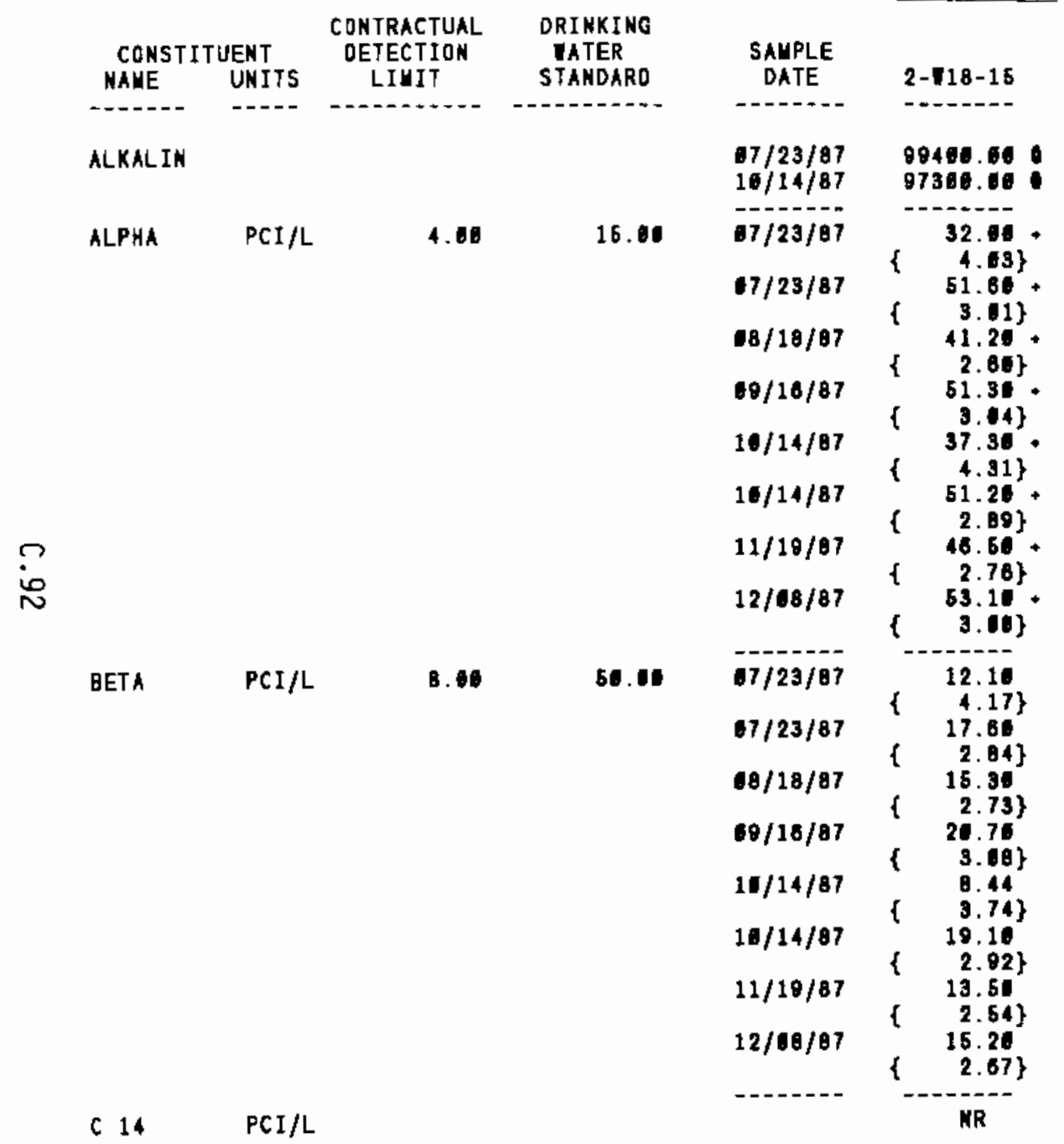

\begin{tabular}{|c|c|c|c|}
\hline $\begin{array}{l}\text { SAUPLE } \\
\text { DATE }\end{array}$ & $2-119-3$ & $\begin{array}{c}\text { SALPLE } \\
\text { DATE }\end{array}$ & $2-119-5$ \\
\hline $11 / 16 / 87$ & 198001.010 & $08 / 20 / 87$ & 98411.00 \\
\hline $\begin{array}{l}07 / 22 / 87 \\
68 / 13 / 87\end{array}$ & $\left\{\begin{array}{c}7180.11 \\
385.89 \\
0880.06\end{array}\right\}$ & $18 / 21 / 87$ & $\begin{array}{l}7.93 \\
2.23\} \\
\mathrm{NR}\end{array}$ \\
\hline $10 / 15 / 87$ & $\begin{array}{c}368.06\} \\
5871.06 \\
\{321.06\}\end{array}$ & & MR \\
\hline $16 / 15 / 87$ & 4140.00 & & NR \\
\hline $10 / 15 / 87$ & $48 B 1.40$ & & NR \\
\hline $11 / 11 / 87$ & 4740.06 & & NR \\
\hline $12 / 09 / 87$ & $\left(\begin{array}{c}287 . .04 \\
\mathrm{HR}\end{array}\right)$ & & $\begin{array}{l}\text { MR } \\
\text { MR }\end{array}$ \\
\hline $17 / 22 / 87$ & $7131.40+$ & $68 / 20 / 87$ & 26.96 \\
\hline $68 / 13 / 67$ & \{006.60\}. & $08 / 20 / 87$ & $\begin{array}{r}25.10 \\
3.35\}\end{array}$ \\
\hline $08 / 15 / 87$ & $\left\{\begin{array}{r}8051.08 \\
497.00\end{array}\right\}$ & $12 / 61 / 87$ & $\begin{array}{r}30.30 \\
3.69\}\end{array}$ \\
\hline $10 / 15 / 87$ & 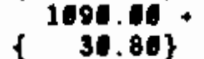 & & WR \\
\hline $10 / 15 / 87$ & 4850.60 & & NR \\
\hline $11 / 11 / 87$ & 4854.08 & & MR \\
\hline $12 / 08 / 87$ & $\left(\begin{array}{c}4050.60 \\
416\end{array}\right\}$ & & $\begin{array}{l}\text { WR } \\
\text { MR }\end{array}$ \\
\hline $18 / 28 / 87$ & $\left\{\begin{array}{l}9.08 \\
\{\quad 2.13\}\end{array}\right.$ & & NR \\
\hline
\end{tabular}

* - Value exceeds primary drinkimg tater stahdard.

* - YALUE EXCEEDS PROPOSED PRIUARY DRINKING IATER STANDARD.

- YLLUE EXCEEDS SCREENING LEVEL FOR FURTHER INYESTIGATION.

- - DETECTIOH LIUIT IAS NOT aVAILABLE for COMPARISOK

NR - ANALYSIS NOT REQUESTED OR NOT YET REPORTED

VALUES IN \{ ? ARE COUNTING ERRORS FOR RADIONUCLIDES

WATER STAMOARD'S) IN PARENTHESES ARE PROPOSED ONLY 
TABLE C.3. (contd)

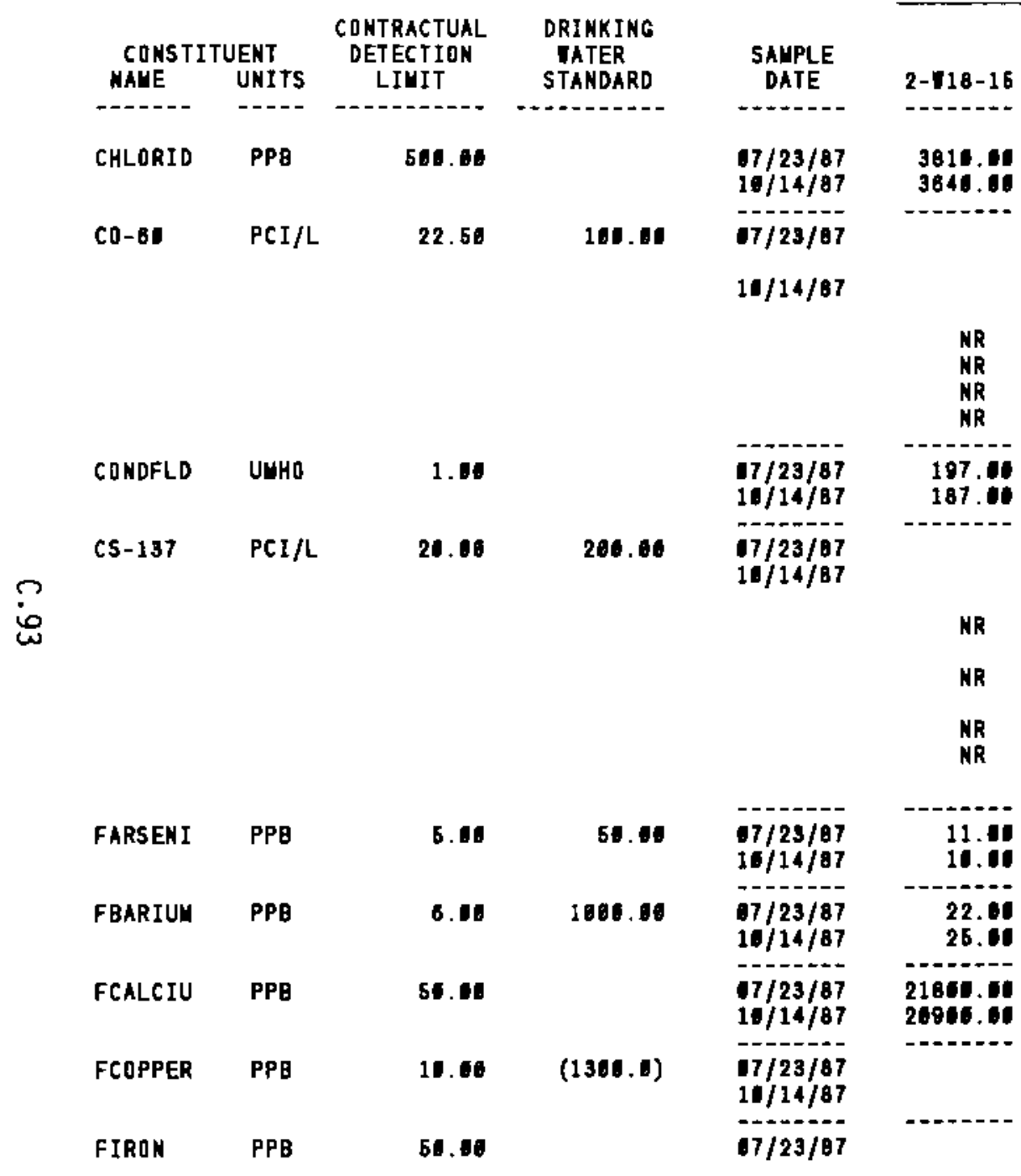

\begin{tabular}{|c|c|}
\hline $\begin{array}{c}\text { SAUPLE } \\
\text { DATE }\end{array}$ & $2-119-3$ \\
\hline $10 / 16 / 87$ & $10166_{N R} 16$ \\
\hline $07 / 22 / 87$ & \\
\hline $18 / 13 / 87$ & $\begin{array}{r}28.86 \\
B .42\end{array}$ \\
\hline $\begin{array}{l}08 / 15 / 87 \\
16 / 15 / 87 \\
11 / 11 / 87 \\
12 / 68 / 87\end{array}$ & \\
\hline $16 / 16 / 87$ & ${ }_{\mathrm{MR}}^{\mathrm{BNO}}$ \\
\hline $\begin{array}{l}17 / 22 / 87 \\
18 / 13 / 87\end{array}$ & 3.11 \\
\hline $10 / 16 / 87$ & $\begin{array}{l}8.12 \\
7.96\}\end{array}$ \\
\hline $10 / 16 / 87$ & 4.82 \\
\hline $\begin{array}{l}11 / 11 / 87 \\
12 / 40 / 87\end{array}$ & $\left.\begin{array}{ll} & 5.10 \\
\{\quad 9.59\end{array}\right\}$ \\
\hline $10 / 16 / 87$ & NR \\
\hline $10 / 15 / 87$ & 49.00 \\
\hline $10 / 16 / 87$ & 42000.00 \\
\hline $16 / 15 / 87$ & 12.11 \\
\hline $10 / 15 / 87$ & 245.60 \\
\hline
\end{tabular}

\begin{tabular}{|c|c|}
\hline $\begin{array}{l}\text { SAYPLE } \\
\text { DATE }\end{array}$ & $2-119-5$ \\
\hline $10 / 20 / 07$ & $\underset{N R}{4230.06}$ \\
\hline \multirow[t]{2}{*}{$68 / 21 / 87$} & $\left\{\begin{array}{l}5.67 \\
\left.\quad \begin{array}{l}5.17 \\
N R\end{array}\right\}\end{array}\right.$ \\
\hline & $\begin{array}{l}\text { NR } \\
\text { NR } \\
\text { NR } \\
\text { NR }\end{array}$ \\
\hline $18 / 26 / 87$ & 234.06 \\
\hline \multirow[t]{4}{*}{$08 / 20 / 87$} & MR \\
\hline & MR \\
\hline & MR \\
\hline & $\begin{array}{l}\text { NR } \\
\text { NR }\end{array}$ \\
\hline $18 / 20 / 87$ & $\stackrel{\theta .01}{M R}$ \\
\hline $68 / 25 / 87$ & ${ }_{M R}^{22.16}$ \\
\hline $68 / 26 / 87$ & $\underset{\mathrm{MR}}{18600}$ \\
\hline $08 / 20 / 87$ & MR \\
\hline $18 / 20 / 87$ & Bo.so \\
\hline
\end{tabular}

- - Value exceeds priuary drinxing tater stamdaro.

- VALUE EXCEEDS PROPOSED PRIUARY DRINKIMG TATER STANDARD.

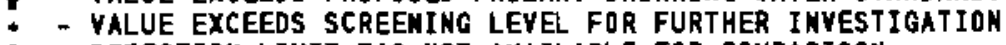

- Detection liuit ias mot available for couparison

NR - ANALYSIS NOT REQUESTED OR NOT YET REPORTED

VALUES IN \{ $\}$ ARE COUNTING ERRORS FOR RADIONUCLIDES

IATER STANDARO(S) IN PARENTHESES ARE PROPOSED OHLY 
TABLE C.3. (contd)

\begin{tabular}{|c|c|c|c|c|c|c|c|c|c|}
\hline $\begin{array}{c}\text { CDNSTI } \\
\text { NAUE }\end{array}$ & $\begin{array}{l}\text { TUENT } \\
\text { UNITS }\end{array}$ & $\begin{array}{c}\text { CONTRACTUAL } \\
\text { OETECTION } \\
\text { LIMIT }\end{array}$ & $\begin{array}{l}\text { ORINKING } \\
\text { IATER } \\
\text { STANDARD }\end{array}$ & $\begin{array}{c}\text { SAMPLE } \\
\text { DATE }\end{array}$ & $2-118-15$ & $\begin{array}{l}\text { SAMPLE } \\
\text { DATE }\end{array}$ & $2-518-3$ & $\begin{array}{c}\text { SAMPLE } \\
\text { DAIE }\end{array}$ & $2-710-5$ \\
\hline FIRON & PPB & 50.60 & & $10 / 14 / 87$ & & & NR & & NR \\
\hline FLEAD & PPB & 5.86 & 50.00 & $\begin{array}{l}67 / 23 / 87 \\
16 / 14 / 87\end{array}$ & 5.01 & $18 / 15 / 67$ & NR & $88 / 20 / 87$ & MR \\
\hline FLUDRID & $\mathrm{PPB}$ & 580.80 & 4006.06 & $\begin{array}{l}17 / 23 / 87 \\
16 / 14 / 87\end{array}$ & & $16 / 15 / 87$ & $\underset{\mathrm{NR}}{985.00}$ & $08 / 20 / 87$ & MR \\
\hline FUAGNES & PPB & 0.60 & & $\begin{array}{l}17 / 23 / 87 \\
10 / 14 / 87\end{array}$ & $\begin{array}{l}8100.06 \\
8390.00\end{array}$ & $10 / 16 / 87$ & 14106.06 & $68 / 26 / 87$ & $\underset{N R}{B 220.00}$ \\
\hline FUAMGAN & PPQ & 5.80 & & $\begin{array}{l}17 / 23 / 87 \\
10 / 14 / 87\end{array}$ & 5.11 & $10 / 15 / 87$ & MR & $68 / 26 / 87$ & NR \\
\hline FPOTASS & PPB & 181.80 & & $\begin{array}{l}17 / 23 / 87 \\
10 / 14 / 87\end{array}$ & 3384.110 & $10 / 16 / 87$ & ${ }_{\mathrm{NR}}^{4636}$ & $68 / 20 / 87$ & $\begin{array}{c}3204.01 \\
\text { NA }\end{array}$ \\
\hline FSOOIUM & PPB & 100.80 & & $\begin{array}{l}07 / 23 / 97 \\
10 / 14 / 87\end{array}$ & $\begin{array}{r}8000.10 \\
10300.01\end{array}$ & $10 / 16 / 07$ & $\underset{\mathrm{WR}}{77100.60}$ & $68 / 26 / 87$ & ${ }_{M R}^{24360.00}$ \\
\hline FYAMAOI & PPB & 5.80 & & $\begin{array}{l}17 / 23 / 87 \\
10 / 14 / 87\end{array}$ & $\begin{array}{l}19.10 \\
21.00\end{array}$ & $10 / 16 / 87$ & MR & $68 / 26 / 87$ & 27.60 \\
\hline FZIMC & PPB & 5.80 & & $\begin{array}{l}07 / 23 / 87 \\
10 / 14 / 87\end{array}$ & 8.00 & $10 / 16 / 07$ & $\begin{array}{l}16.61 \\
\text { MR }\end{array}$ & $68 / 26 / 87$ & NR \\
\hline HWITRAT & PPB & 2500.00 & 46006.60 & $\begin{array}{l}07 / 23 / 87 \\
80 / 18 / 87 \\
09 / 16 / 87 \\
10 / 14 / 87 \\
11 / 19 / 87 \\
12 / 08 / 87\end{array}$ & 2740.10 & $\begin{array}{l}07 / 22 / 87 \\
18 / 13 / 87 \\
09 / 16 / 87 \\
10 / 16 / 87 \\
11 / 11 / 87 \\
12 / 09 / 87\end{array}$ & 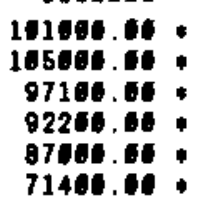 & $\begin{array}{l}68 / 20 / 97 \\
12 / 01 / 87\end{array}$ & $\begin{array}{c}4740.60 \\
301.10 \\
\text { KR } \\
\text { MR } \\
\text { NR } \\
\text { MR }\end{array}$ \\
\hline $1-128$ & $\mathrm{PCI} / \mathrm{L}$ & & 506.60 & & NR & $10 / 20 / 87$ & $\left\{\begin{array}{r}32.01 \\
3.02\}\end{array}\right.$ & & MR \\
\hline LFLUDRD & PPB & 20.00 & 4008.06 & $\begin{array}{l}67 / 23 / 87 \\
16 / 14 / 87\end{array}$ & $\begin{array}{l}230.01 \\
250.06\end{array}$ & $10 / 16 / 87$ & NR & $00 / 20 / 87$ & ${ }_{\mathrm{NR}}^{460.01}$ \\
\hline NITRATE & PPB & 500.60 & 45000.00 & $07 / 23 / 87$ & 1280.00 & $10 / 16 / 87$ & 93000.06 & $10 / 26 / 87$ & 928.68 \\
\hline \multicolumn{10}{|c|}{ 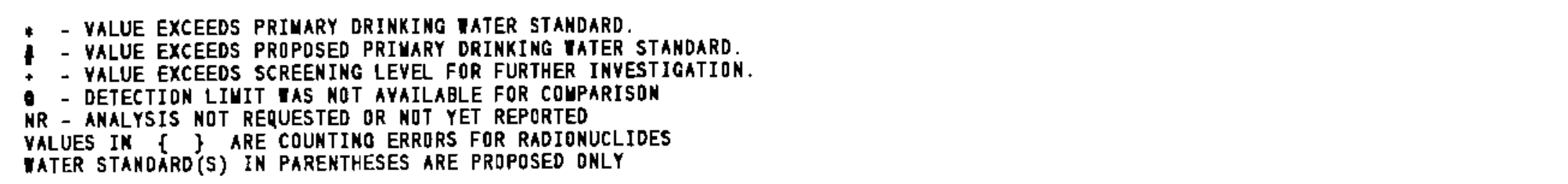 } \\
\hline
\end{tabular}


TABLE C.3. (contd)

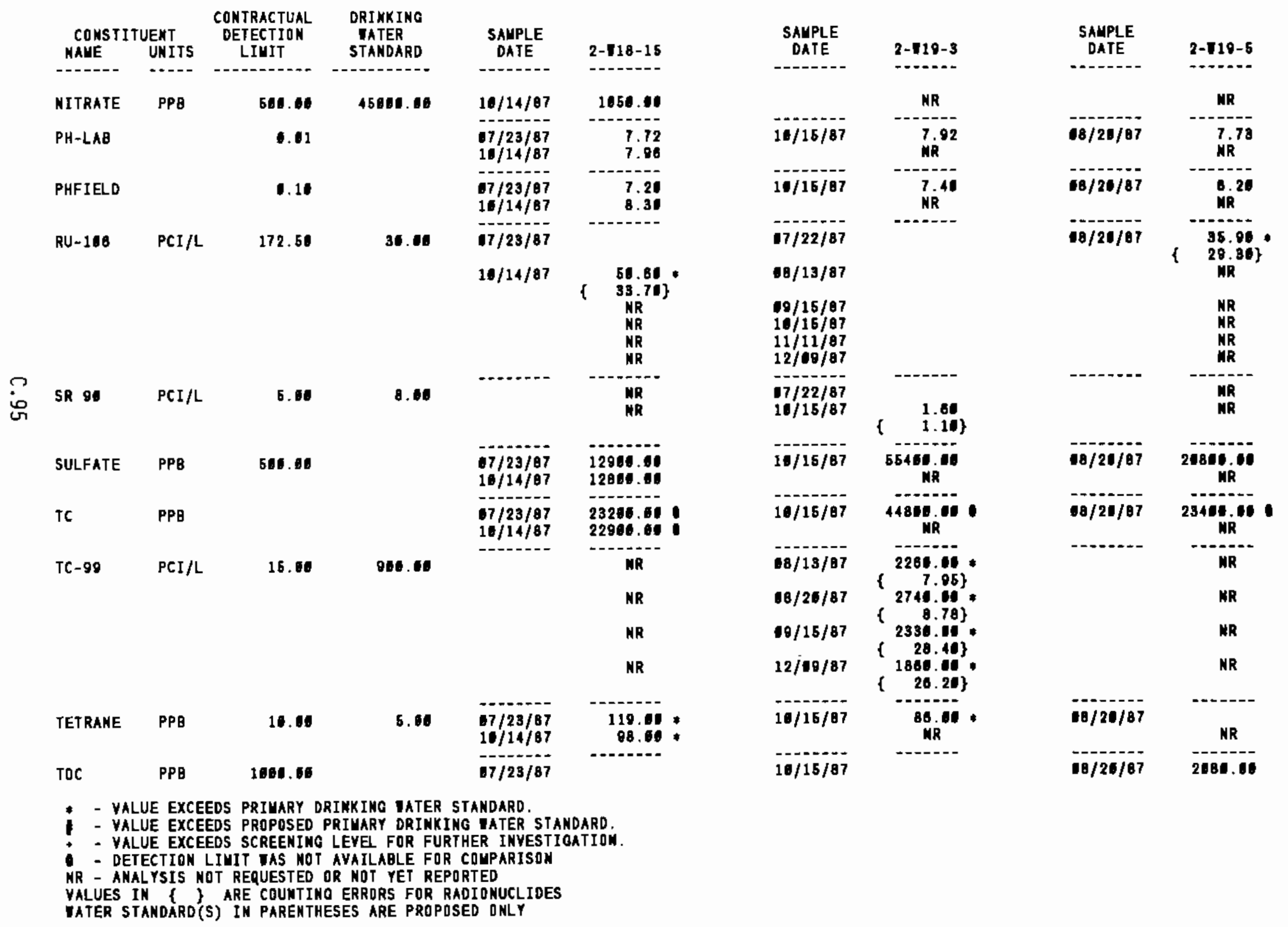


TABLE C.3. (contd)

$\begin{array}{cccc}\text { CONSTITUENT } & \begin{array}{c}\text { CONTRACTUAL } \\ \text { DETECTIDN }\end{array} & \begin{array}{c}\text { ORINKING } \\ \text { IATER } \\ \text { NAME }\end{array} \text { UMITS } \\ \text { LIMIT } & \text { STANDARD }\end{array}$

\begin{tabular}{cr}
$\begin{array}{c}\text { SAUPLE } \\
\text { DATE }\end{array}$ & $2-118-16$ \\
$-10 / 14 / 87$ & \\
\hdashline $1 / 23 / 87$ & 85.40 \\
$10 / 14 / 87$ & 81.20 \\
\hdashline $7 / 23 / 87$ & - \\
$08 / 18 / 87$ & 335.16 \\
$69 / 16 / 87$ & $210.66\}$ \\
$10 / 14 / 87$ & \\
$11 / 10 / 87$ & \\
$12 / 68 / 87$ &
\end{tabular}

号

$\begin{array}{llr}\text { TOC } & \text { PPB } & \mathbf{1 0 0 8 . 0 8} \\ \text { TOXLDL } & \text { PPB } & 20.06\end{array}$

TRITIUY PCI/L

690.60

20000.00

\begin{tabular}{|c|c|}
\hline \multirow{2}{*}{$\begin{array}{c}\text { SAMPLE } \\
\text { DATE }\end{array}$} & $2-110-3$ \\
\hline & NR \\
\hline $10 / 16 / 87$ & 89.00 \\
\hline $17 / 22 / 87$ & $\left\{\begin{array}{r}1410.06 \\
248.06\}\end{array}\right\}$ \\
\hline $18 / 13 / 87$ & 1290.10 \\
\hline $89 / 15 / 87$ & $\begin{array}{r}995.10 \\
\{\quad 233.10\end{array}$ \\
\hline $10 / 15 / 87$ & $\begin{array}{l}1420.10 \\
244.03\end{array}$ \\
\hline $11 / 11 / 87$ & 1110.01 \\
\hline $12 / 09 / 07$ & $\left\{\begin{array}{l}021.0 \\
187.4\}\end{array}\right.$ \\
\hline - non & MR \\
\hline $07 / 22 / 87$ & $\left\{\begin{array}{c}3430.01 \\
09.40\}\end{array}\right.$ \\
\hline $60 / 16 / 07$ & $\left\{\begin{array}{l}3190.08 \\
08.71\end{array}\right\}$ \\
\hline $67 / 22 / 87$ & 140.01 * \\
\hline $69 / 16 / 87$ & $\begin{array}{r}133.11 \\
14.01\end{array}$ \\
\hline $67 / 22 / 87$ & $\left\{\begin{array}{c}3470.01 \\
00.71\}\end{array}\right.$ \\
\hline $00 / 16 / 07$ & $\left\{\begin{array}{r}3160.01 \\
68.40\end{array}\right\}$ \\
\hline $60 / 16 / 87$ & $\left\{\begin{array}{r}55.41 \\
0.95\end{array}\right\}$ \\
\hline $67 / 22 / 87$ & 0870.01 \\
\hline
\end{tabular}

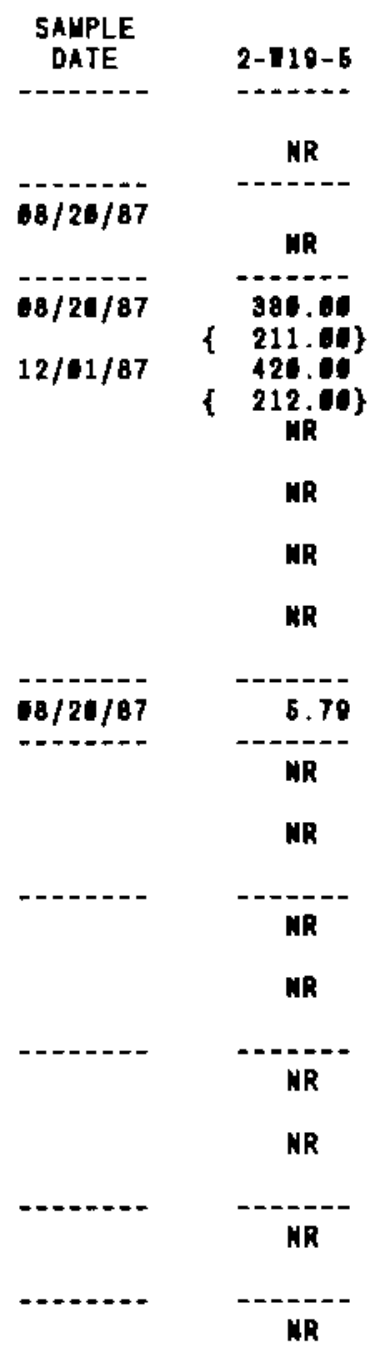

$\begin{array}{lll}U-C H E V ~ U G / L & 1.73 & 07 / 23 / 87\end{array}$

* - YALUE EXCEEDS PRIUARY DRINKING TATER STANDARD.
- YALUE EXCEEDS PRDPOSED PRIMARY DRIMKING TATER STANDARO

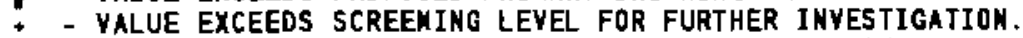

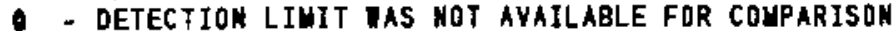

NR - AMALYSIS MOT REQUESTED OR NDT YET REPORTED

YALUES IN $\{$ ARE COUNTING ERRORS FDR RADIDNUCLIDES

VATER STANDARD(S) IN PARENTHESES ARE PROPOSED DNLY 
IABLE C.3. (contd)

\begin{tabular}{|c|c|c|c|c|c|c|c|c|c|}
\hline $\begin{array}{l}\text { CONSTI } \\
\text { MALE }\end{array}$ & $\begin{array}{l}\text { UENT } \\
\text { UNITS }\end{array}$ & $\begin{array}{c}\text { CONTRACTUAL } \\
\text { DETECTION } \\
\text { LIHIT }\end{array}$ & $\begin{array}{l}\text { DRINKING } \\
\text { WATER } \\
\text { STANDARD }\end{array}$ & $\begin{array}{c}\text { SAUPLE } \\
\text { DATE }\end{array}$ & $2-118-15$ & $\begin{array}{l}\text { SAUPLE } \\
\text { DATE }\end{array}$ & $2-110-3$ & $\begin{array}{c}\text { SAHPLE } \\
\text { DATE }\end{array}$ & $2-\$ 18-5$ \\
\hline$--\infty \ldots$ & --- & - & 1 & $\ldots$ & $--\cdots$ & - - & ----- & -.--- & $---\ldots$ \\
\hline U-CHEM & UG/L & 0.73 & & $\begin{array}{l}68 / 18 / 87 \\
69 / 16 / 87 \\
10 / 14 / 87 \\
11 / 18 / 87 \\
12 / 08 / 87\end{array}$ & $\begin{array}{l}87.70 \\
88.08 \\
70.20 \\
76.40 \\
71.30\end{array}$ & $\begin{array}{l}08 / 13 / 87 \\
09 / 15 / 87 \\
10 / 16 / 87 \\
11 / 11 / 87 \\
12 / 09 / 87\end{array}$ & $\begin{array}{l}9530.06 \\
8280.06 \\
8590.06 \\
7251.00 \\
5870.00\end{array}$ & & $\begin{array}{l}\text { MR } \\
\text { MR } \\
\text { MR } \\
\text { MR } \\
\text { MR }\end{array}$ \\
\hline
\end{tabular}

$\stackrel{?}{i}$

- VAlUe exceeds prinary dRINKing mater standaro.

- VALUE EXCEEDS PROPOSED PRIUARY DRINKING WATER STANDARD.

- VALUE EXCEEDS SCREENING LEYEL FOR FURTHER INYESTIGATION.

- - DETECTION LIUIT TAS NOT AVAILABLE FOR COMPARISON

NR - ANALYSIS NOT REQUESIED OR NOT YET REPORTED

VALUES IN 1 ? ARE COUNTING ERRDRS FOR RADIOHUCLIDES

IATER STAMDARD(S) IN PARENTHESES ARE PROPOSED ONLY 
TABLE C.3. (contd)

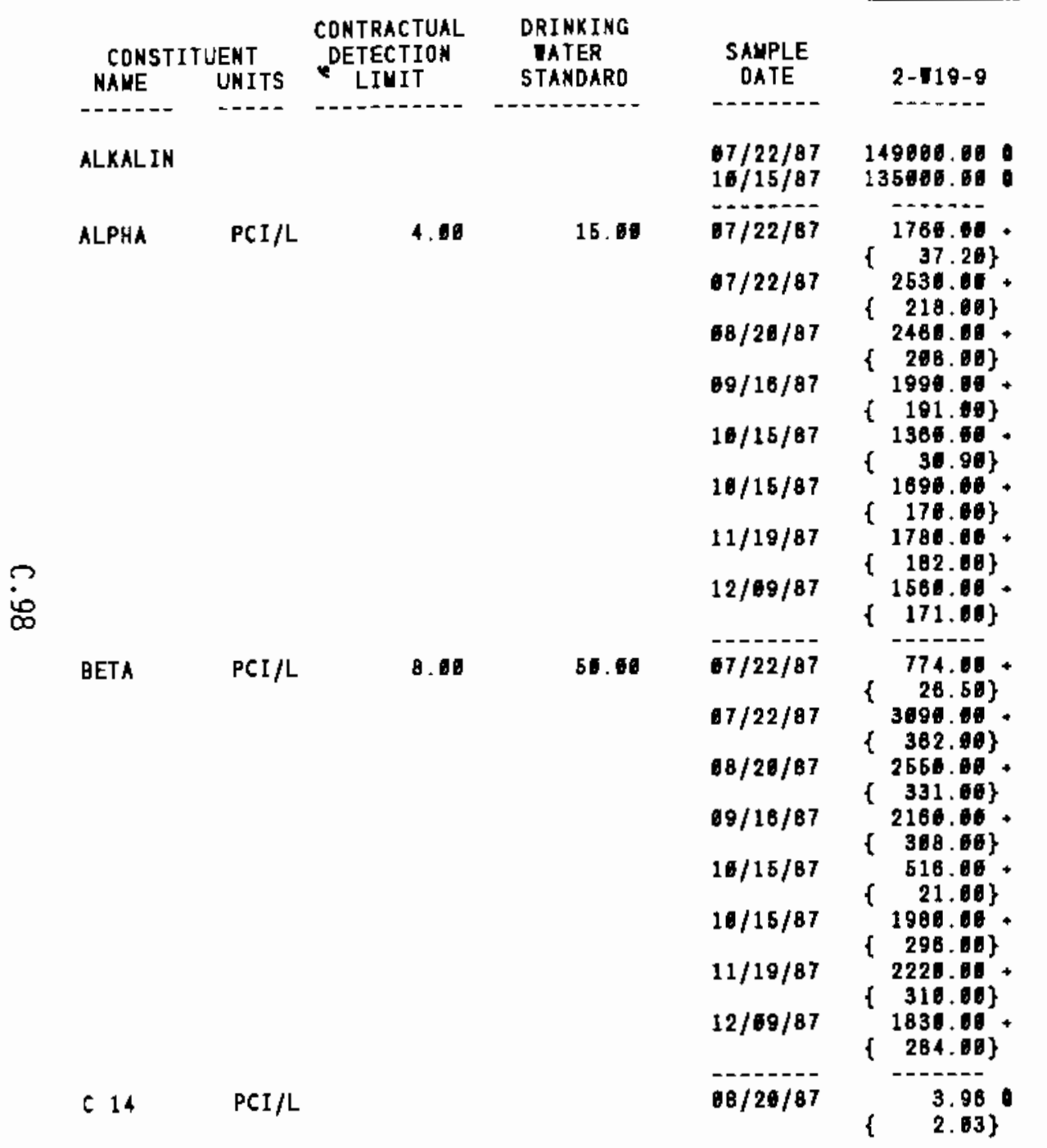

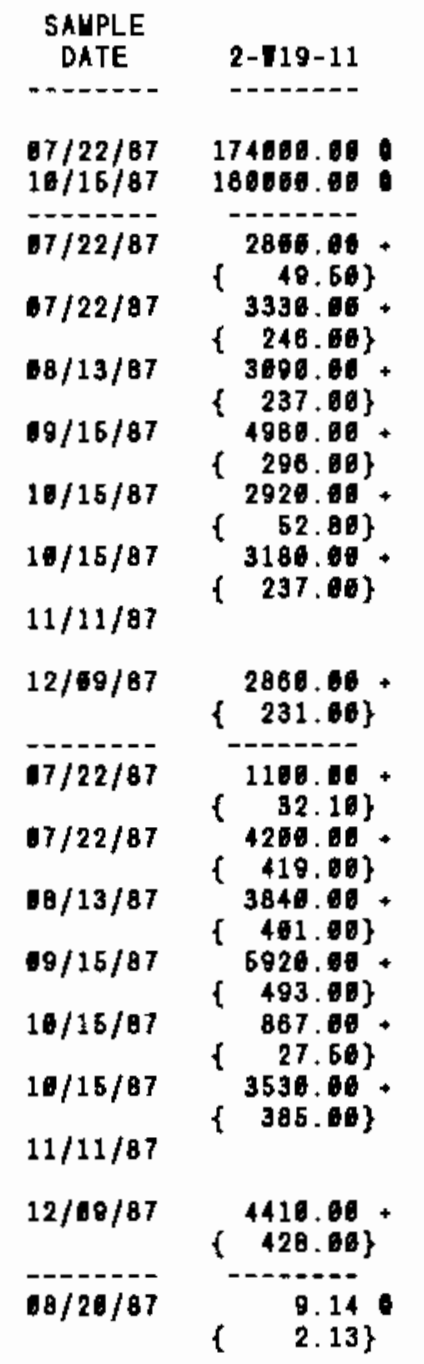

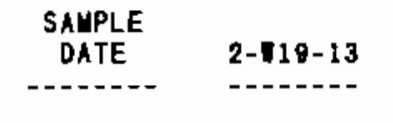

$10 / 14 / 87$ 145080.60:

$07 / 21 / 87-5,11$

$07 / 21 / 87\left\{\begin{array}{l}1.97 \\ \text { 1 }\end{array}\right.$

68/13/87 \{ 1.31$\}$

$68 / 13 / 87,8.85$

$08 / 18 / 87$ \& 8.68

$18 / 14 / 87\{1.25\}$

10/14/87 \{ 2.18\}

$11 / 11 / 87 \quad\{\quad 1.15\}$

$11 / 11 / 87-7.47$

$12 / 08 / 87\left\{\begin{array}{l}1.16\} \\ \text { (1) }\end{array}\right.$

…... $\quad$........

26.70
$\left\{\begin{array}{r}5.84 \\ 5\end{array}\right]$

$07 / 21 / 87$ 21.28

$08 / 13 / 87\left\{\begin{array}{l}3.27\} \\ \text { a }\end{array}\right.$

$\left\{\begin{array}{l}3.41\} \\ 3.98\end{array}\right.$

69/16/87 15.58

$16 / 14 / 87 \quad\left\{\begin{array}{r}2.78\} \\ 14.88\end{array}\right.$

$10 / 14 / 87\left\{\begin{array}{l}4.59 \\ \text { f }\end{array}\right.$

$1111 / 87\left\{\begin{array}{l}\text { 3.17 } \\ \text { 2. }\end{array}\right.$

$11 / 11 / 87 \quad\left\{\begin{array}{r}22.28 \\ 3.36\}\end{array}\right.$

$12 / 08 / 87$

$-\cdot-\cdot-\cdot$

$\{\quad 3.11\}$

NR

* - yalue exceeds privary dRINKirg tater standaro.

- VALUE EXCEEDS PROPOSED PRIUARY DRINKING TATER STAMDARD.

- VALUE EXCEEDS SCREENING LEVEL FOR FURTHER INYESTIGATION.

- DETECTION LIUIT IAS NOT AVAILABLE FOR COUPARISON

NR - AHALYSIS NOT REQUESTED OR NOT YET REPORTED

VALUES IN \{ $\}$ ARE COUNTING ERRORS FOR RADIONUCLIDES

IATER STANDARD(S) IN PARENTHESES ARE PROPOSEO OHLY 
TABLE C.3. (contd)

\begin{tabular}{cccc}
$\begin{array}{c}\text { CONSTITUENT } \\
\text { MALE }\end{array}$ UNITS & $\begin{array}{c}\text { CONTRACTUAL } \\
\text { OETECTION } \\
\text { LIUIT }\end{array}$ & $\begin{array}{c}\text { DRINXING } \\
\text { NATER } \\
\text { STANDARD }\end{array}$ \\
\hline & - & & \\
CHLORID & PPB & &
\end{tabular}

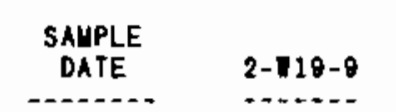

\begin{tabular}{|c|c|}
\hline $\begin{array}{c}\text { SAMPLE } \\
\text { DATE }\end{array}$ & $2-18-11$ \\
\hline & $--1--10$ \\
\hline $\begin{array}{l}7 / 22 / 87 \\
10 / 16 / 87\end{array}$ & $\begin{array}{l}21880.66 \\
19780.60\end{array}$ \\
\hline $67 / 22 / 87$ & 2.73 \\
\hline $\begin{array}{l}08 / 13 / 87 \\
00 / 15 / 87\end{array}$ & $\begin{array}{r}6.40 \\
\{\quad 5.23\}\end{array}$ \\
\hline $\begin{array}{l}10 / 15 / 87 \\
11 / 11 / 87\end{array}$ & \\
\hline $12 / 108 / 87$ & \\
\hline $\begin{array}{l}67 / 22 / 87 \\
10 / 15 / 87\end{array}$ & $\begin{array}{l}621.00 \\
570.00\end{array}$ \\
\hline $07 / 22 / 87$ & \\
\hline
\end{tabular}

SAUPLE
DATE

2-719-13

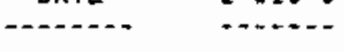

22400.00

$07 / 22 / 87$
$16 / 15 / 87$

CO-80 PCI/L

22.59

100.60

$68 / 24 / 87$

CONDFLD UUHO

1.00

$10 / 16 / 87$

20.06

200.00

$88 / 26 / 87$

CS-137 PCI/L

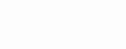

FBARIUY PPB

8.00

1000.00

7

$07 / 22 / 87$
$10 / 15 / 87$

B7/22/87

$10 / 16 / 87$

97/22/87

$07 / 22 / 87$
$10 / 16 / 87$

7/22/87

$10 / 16 / 87$

ब7/22/87

$10 / 15 / 87$

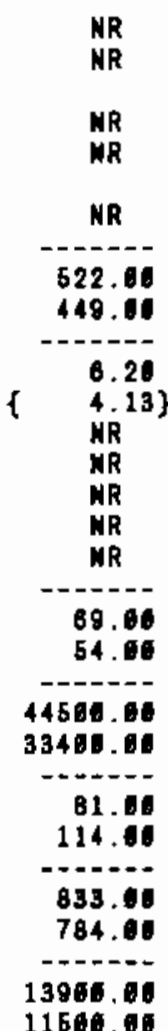

FMAGNES PPB

๑. 08

11500.00

* - VALUE EXCEEDS PRIUARY DRIHKING IATER STANDARD.

+ - VALUE EXCEEDS SCREENING LEVEL FOR FURTHER INVESTIGATION.

+ - VALUE EXCEEDS SCREENING LEVEL FDR FURTHER INYESTIGA

MR - ANALYSIS NOT REQUESTED OR NOT YET REPORTED

VALUES IN \{ \} ARE COUNTING ERRORS FOR RADIONUCLIDES

TATER STANDARD(S) IN PARENTHESES ARE PROPOSED ONLY 
TABLE_. 3. (contd)

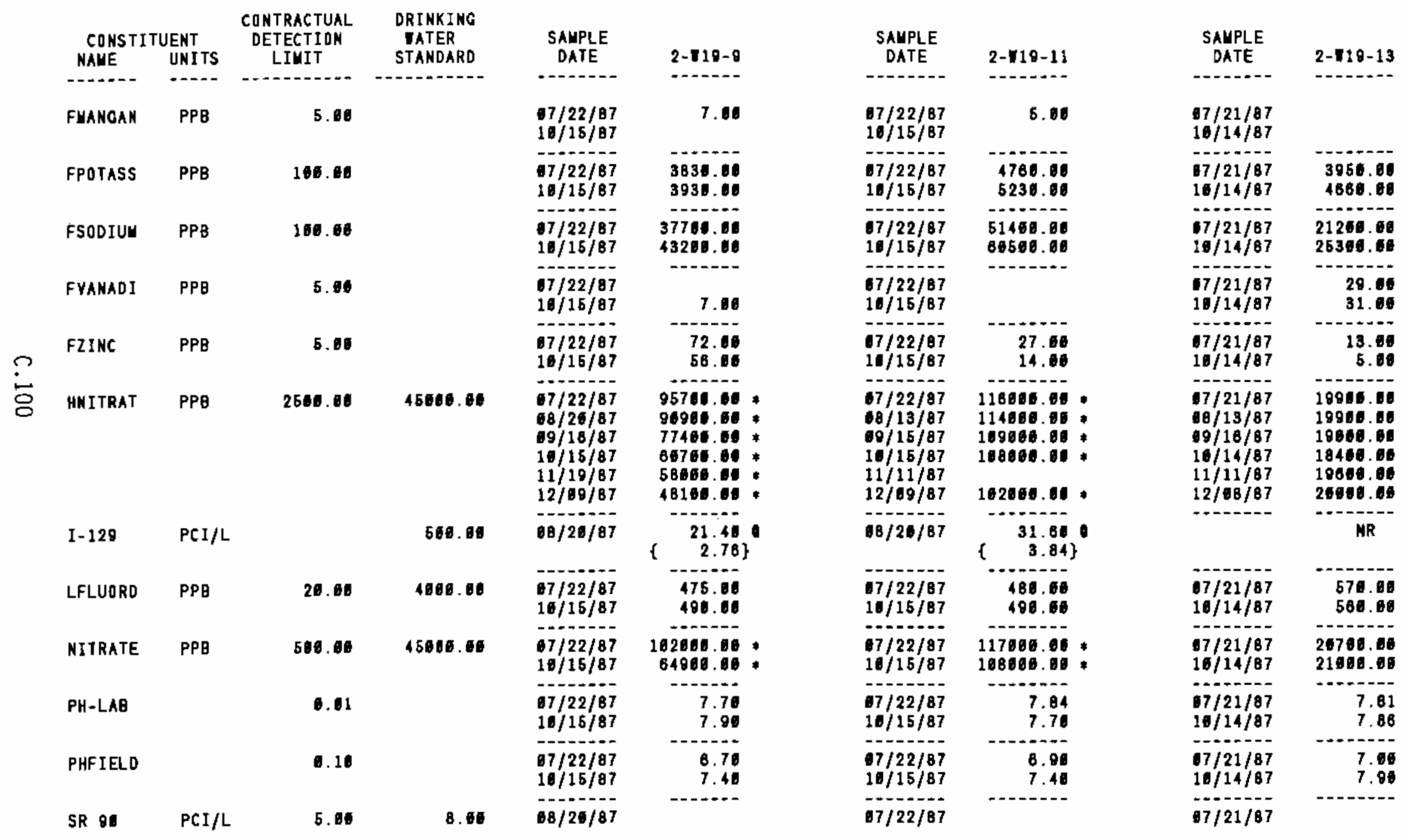

* - yalue eXCeEds PRIMary dRinking tater standard.

- VALUE EXCEEDS PROPOSED PRIMARY DRINKING VATER STANDARD.

- - YALUE EXCEEOS SCREEMING LEVEL FOR FURTHER INVESTIGATION.

- DETECTION LIUIT WAS NOT AVAILABLE FOR COUPARISON

NR - ANALYSIS KOT REQUESTED OR NOT YET REPORTED

VALUES IN \{ \} ARE COUNTING ERRORS FOR RADIONUCLIDES

WATER STANDARO'(S) IN PARENTHESES ARE PROPOSED DNLY 
IABLE C.3. (contd)

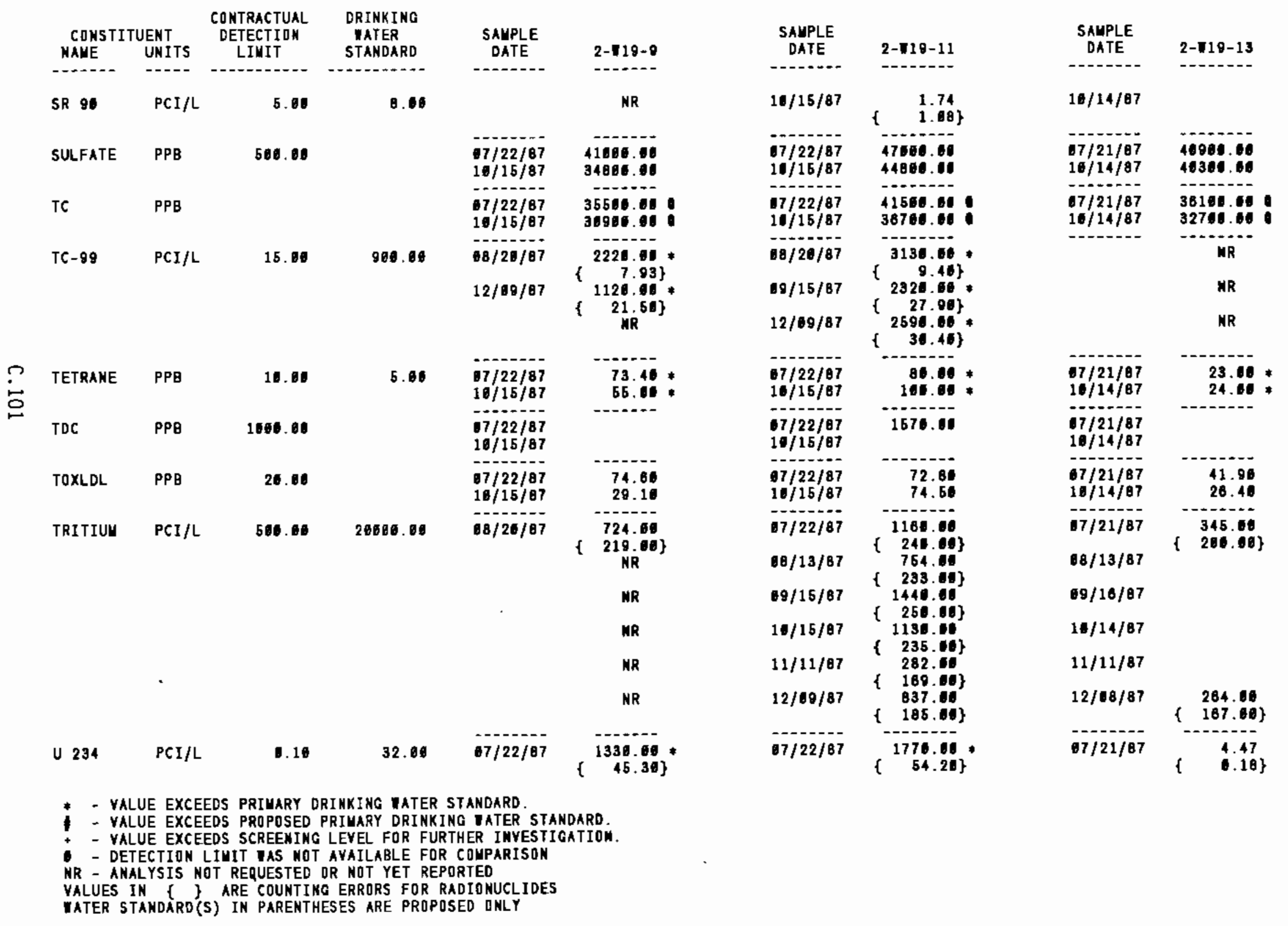


TABLE C.3. (contd)

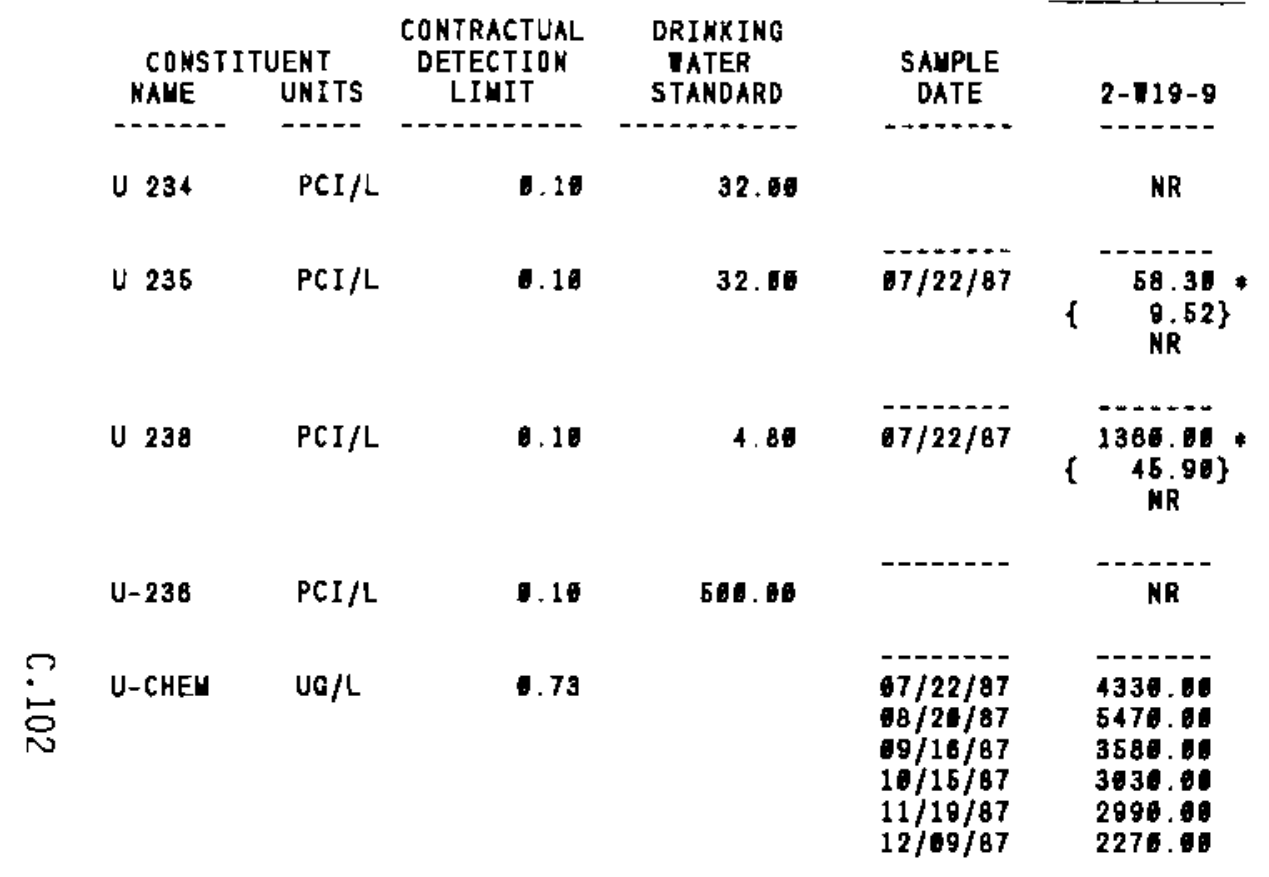

\begin{tabular}{|c|c|}
\hline $\begin{array}{l}\text { SAUPLE } \\
\text { DATE }\end{array}$ & $2-110-11$ \\
\hline ים & 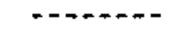 \\
\hline $19 / 15 / 87$ & $\left\{\begin{array}{r}2620.65 \\
66.16\}\end{array}\right.$ \\
\hline $07 / 22 / 87$ & 102.00 \\
\hline $19 / 15 / 87$ & $\begin{array}{l}\left\{\begin{array}{c}13.10\} \\
113.00\end{array}\right. \\
\{\quad 13.80\}\end{array}$ \\
\hline $7 / 22 / 87$ & 2610.00 \\
\hline $00 / 15 / 87$ & 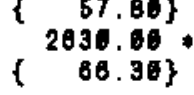 \\
\hline $19 / 15 / 87$ & $\begin{array}{r}54.21 \\
\{\quad 9.73\}\end{array}$ \\
\hline $\begin{array}{l}77 / 22 / 87 \\
6 / 13 / 87 \\
60 / 15 / 67 \\
16 / 15 / 87 \\
11 / 11 / 87 \\
12 / 69 / 67\end{array}$ & $\begin{array}{r}5430.00 \\
4910.00 \\
7900.00 \\
6170.00 \\
2.00 \\
4480.00\end{array}$ \\
\hline
\end{tabular}

\begin{tabular}{|c|c|}
\hline $\begin{array}{c}\text { SAUPLE } \\
\text { DATE }\end{array}$ & $2-119-13$ \\
\hline 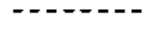 & $-\cdots+-1$ \\
\hline & NR \\
\hline - - - - - & - \\
\hline $67 / 21 / 07$ & ๑. 17 \\
\hline & $\begin{array}{l}0.04\} \\
N R\end{array}$ \\
\hline --- & 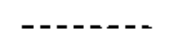 \\
\hline $67 / 21 / 87$ & $\begin{array}{ll}\left\{\quad \begin{array}{l}4.64 \\
\text { NR }\end{array}\right. & .17\}\end{array}$ \\
\hline$--1-1$ & NR \\
\hline - - - - - & - - - \\
\hline $\begin{array}{l}67 / 21 / 87 \\
68 / 13 / 87 \\
69 / 16 / 87 \\
10 / 14 / 87 \\
11 / 11 / 87 \\
12 / 68 / 87\end{array}$ & $\begin{array}{r}12.90 \\
8.88 \\
12.08 \\
10.80 \\
10.40 \\
7.83\end{array}$ \\
\hline
\end{tabular}

* - Value exceEds primary dRINXing mater standard.

- - VALUE EXCEEDS PRDPOSED PRIMARY DRINKING WATER STANDARD.

* - VALUE EXCEEDS SCREENING LEVEL FOR FURTHER INYESTIGATION.

- DETECTION LIUIT IAS NDT AVAILABLE FDR COMPARISON

NR - AMALYSIS NOT REQUESTED OR NOT YEI REPORTED

VALUES IN \{\} ARE COUKTING ERRORS FOR RADIONUCLIDES

WATER STANDARD(S) IN PARENTHESES ARE PROPOSED ONLY 
TABLE C.3. (contd)

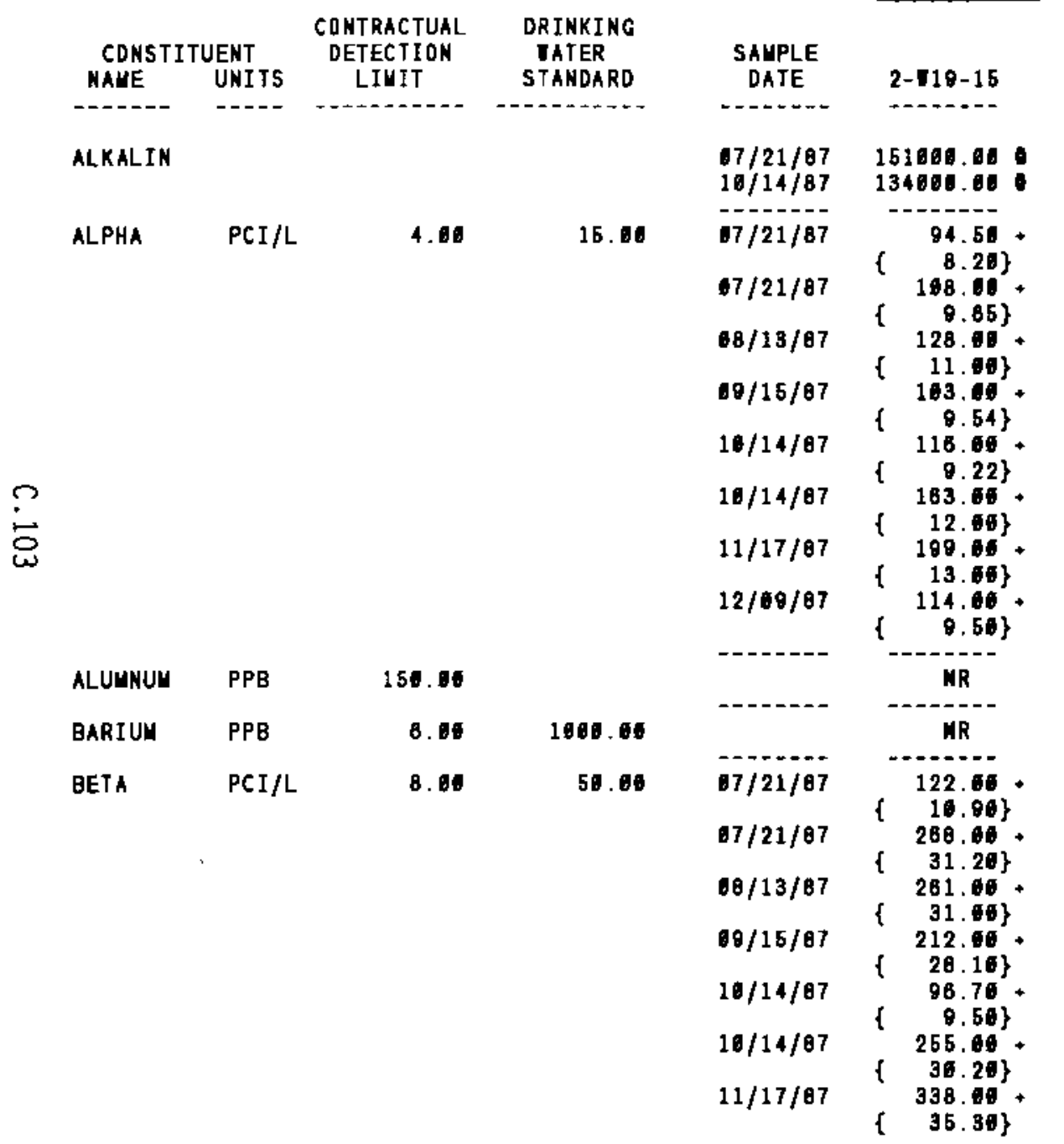

\begin{tabular}{|c|c|c|c|}
\hline $\begin{array}{l}\text { SAMPLE } \\
\text { DATE }\end{array}$ & $2-118-16$ & $\begin{array}{l}\text { SAHPLE } \\
\text { DATE }\end{array}$ & $2-11 \theta-20$ \\
\hline 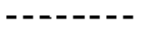 & - - & -- - - & - \\
\hline $\begin{array}{l}67 / 23 / 87 \\
16 / 14 / 87\end{array}$ & $\begin{array}{l}135000.06 \\
127000.00\end{array}$ & $\begin{array}{l}07 / 23 / 87 \\
12 / 66 / 87\end{array}$ & $\begin{array}{l}124000.00 \\
124000.00\end{array}$ \\
\hline $87 / 23 / 87$ & $\begin{array}{r}1070.00 \\
27.00\}\end{array}$ & $97 / 23 / 87$ & $\begin{array}{r}248.00 \\
23.48\}\end{array}$ \\
\hline $07 / 23 / 87$ & $1380.00+$ & $97 / 23 / 87$ & $282.09+$ \\
\hline $00 / 13 / 87$ & $1270.09+$ & $68 / 13 / 87$ & 309.04 . \\
\hline $19 / 16 / 87$ & $1190.00+$ & $99 / 16 / 87$ & $\begin{array}{l}11.09 \mathrm{k} \\
104.09\end{array}+$ \\
\hline $10 / 14 / 87$ & $\left\{\begin{array}{c}883.08+ \\
\{\quad 24.78\}\end{array}\right.$ & $11 / 19 / 87$ & $\begin{array}{c}245.00+ \\
10.70\}\end{array}$ \\
\hline $10 / 14 / 67$ & 1246.00 & $12 / 06 / 87$ & $\begin{array}{c}172.69+ \\
18.60 \%\end{array}$ \\
\hline $11 / 17 / 87$ & $\left\{\begin{array}{r}1620.00 \\
\{\quad 137.00\}\end{array}\right.$ & $12 / 86 / 87$ & $\begin{array}{c}319.00 \\
12.10\}\end{array}$ \\
\hline $12 / 00 / 07$ & $\left\{\begin{array}{r}1170.00 \\
\{144.00\end{array}\right\}$ & $12 / 86 / 87$ & $\begin{array}{r}344.00 \\
\{\quad 12.70\}\end{array}$ \\
\hline-- & NR & $12 / 60 / 87$ & 869.65 \\
\hline 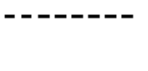 & NR & $12 / 68 / 67$ & 210.06 \\
\hline $97 / 23 / 87$ & 432.06 & $67 / 23 / 87$ & 945.06 \\
\hline $07 / 23 / 87$ & $\begin{array}{r}2008.06 \\
\left\{\begin{array}{c}18.06\} \\
298.06\end{array}\right\}\end{array}$ & $67 / 23 / 67$ & $\begin{array}{c}597.60 \\
27.36\}\end{array}$ \\
\hline $08 / 13 / 07$ & $\begin{array}{r}1820.06 \\
292.00\}\end{array}$ & $68 / 13 / 67$ & $\begin{array}{r}724.00 \\
\{39.09\}\end{array}$ \\
\hline $09 / 16 / 07$ & $\begin{array}{r}1650.06 \\
272.68\}\end{array}$ & $69 / 16 / 87$ & $\begin{array}{r}846.09 \\
\{\quad 32.60\}\end{array}$ \\
\hline $10 / 14 / 87$ & $\left\{\begin{array}{c}350.68 \\
\quad 17.16\end{array}\right\}$ & $11 / 19 / 87$ & $\left\{\begin{array}{r}1130.00 \\
37.80\end{array}\right\}$ \\
\hline $10 / 14 / 87$ & $\begin{array}{r}1446.06 \\
\{\quad 256.06\}\end{array}$ & $12 / 06 / 87$ & $\begin{array}{r}778.08+ \\
\{\quad 30.30\}\end{array}$ \\
\hline $11 / 17 / 87$ & $\left\{\begin{array}{r}1768.00 \\
281.60\}\end{array}\right.$ & $12 / 06 / 87$ & $\left\{\begin{array}{c}1076.08 \\
36.86\}\end{array}\right.$ \\
\hline
\end{tabular}

- - Value eXceeds priuhry dRINKINg tater stahoard.

- VALUE EXCEEDS PROPOSED PRIUARY DRIHKING MATER STANDARD.

- VALUE EXCEEDS SCREEMIMG LEVEL FOR FURTHER INYESTIGATION.

- - DETECTION LIUIT WAS NOT AVAILABLE FOR COUPARISON

NR - ANALYSIS NOT REQUESTED OR MOT YET REPORTED

VALUES IN \{ $\}$ ARE COUNTING ERRORS FOR RADIDNUCLIDES

IATER STANDARD(S) IN PARENTHESES ARE PROPOSED DNLY 
TABLE C.3. (contd)

\begin{tabular}{|c|c|c|c|c|c|c|c|c|c|}
\hline$\underset{\text { NANE }}{\text { CONSTI }}$ & $\begin{array}{l}\text { UENT } \\
\text { UNITS }\end{array}$ & $\begin{array}{c}\text { CONTRACTUAL } \\
\text { DETECTION } \\
\text { LIMIT }\end{array}$ & $\begin{array}{l}\text { DRINKING } \\
\text { TATER } \\
\text { STANDARD }\end{array}$ & $\begin{array}{c}\text { SAMPLE } \\
\text { DATE }\end{array}$ & $2-518-15$ & $\begin{array}{c}\text { SAUPLE } \\
\text { DATE }\end{array}$ & $2-119-16$ & $\begin{array}{l}\text { SALPLE } \\
\text { DATE }\end{array}$ & $2-118-26$ \\
\hline BETA & $\mathrm{PCI} / \mathrm{L}$ & 8.80 & 50.00 & $12 / 69 / 87$ & $\left\{\begin{array}{l}81.90 \\
\{\quad 11.80\end{array}\right\}^{+}$ & $12 / 09 / 87$ & $\left\{\begin{array}{r}1426.00 \\
254.09\}\end{array}\right.$ & $12 / 68 / 87$ & $\left\{\begin{array}{l}115 \mathrm{~s} .06 \\
38.40\end{array}\right\}$ \\
\hline C 14 & $\mathrm{PCI} / \mathrm{L}$ & & & $18 / 18 / 87$ & $\begin{array}{r}7.60 \\
\{\quad 2.10\}\end{array}$ & $68 / 18 / 07$ & $\begin{array}{l}4.12 \\
\{\quad 2.63\}\end{array}$ & & NR \\
\hline CALCIUU & $\mathrm{PPB}$ & 50.00 & & & NR & & MR & $12 / 86 / 87$ & 247000.06 \\
\hline CHLORID & PPB & 500.00 & & $\begin{array}{l}07 / 21 / 87 \\
16 / 14 / 87 \\
11 / 17 / 87 \\
-\end{array}$ & $\begin{array}{l}25460.60 \\
21160.06 \\
24960.06\end{array}$ & $\begin{array}{l}07 / 23 / 87 \\
16 / 14 / 97 \\
11 / 17 / 87 \\
-\end{array}$ & $\begin{array}{l}15986.00 \\
15286.00 \\
14666.06\end{array}$ & $\begin{array}{l}07 / 23 / 87 \\
12 / 68 / 87\end{array}$ & $\begin{array}{c}33211.60 \\
20510.68 \\
\text { NR }\end{array}$ \\
\hline CHROMUY & PPB & 10.00 & 50.60 & & NR & & MR & $12 / 08 / 87$ & 10.00 \\
\hline CO-BO & $\mathrm{PCI} / \mathrm{L}$ & 22.50 & 160.66 & $68 / 18 / 87$ & & $68 / 18 / 87$ & & $07 / 23 / 87$ & $\begin{array}{l}4.72 \\
\{\quad 3.15\}\end{array}$ \\
\hline & & & & & 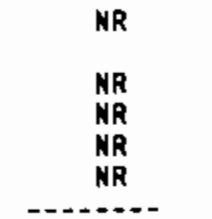 & & $\begin{array}{l}\text { MR } \\
\text { MR } \\
\text { MR } \\
\text { NR } \\
----\end{array}$ & $\begin{array}{l}18 / 13 / 87 \\
10 / 18 / 87 \\
11 / 19 / 87 \\
12 / 96 / 87 \\
12 / 08 / 87 \\
-\end{array}$ & $\begin{array}{r}16.76 \\
\{\quad 6.25\}\end{array}$ \\
\hline CONDFLD & UNHO & 1.00 & & $\begin{array}{l}07 / 21 / 87 \\
10 / 14 / 87\end{array}$ & $\begin{array}{r}488.60 \\
428.60 \\
\end{array}$ & $\begin{array}{l}67 / 23 / 87 \\
16 / 14 / 87\end{array}$ & $\begin{array}{r}418.10 \\
370.06\end{array}$ & $\begin{array}{l}67 / 23 / 87 \\
12 / 08 / 87 \\
-\end{array}$ & $\begin{array}{r}1888.60 \\
1751.06\end{array}$ \\
\hline CS-137 & $\mathrm{PCI} / \mathrm{L}$ & 20.00 & 200.00 & $08 / 18 / 87$ & $\begin{array}{l}\text { NR } \\
\text { NR } \\
\text { NR } \\
\text { NR } \\
\text { NR }\end{array}$ & $68 / 18 / 87$ & $\begin{array}{l}\text { NR } \\
\text { NR } \\
\text { NR } \\
\text { NR } \\
\text { NR }\end{array}$ & $\begin{array}{l}07 / 23 / 87 \\
68 / 13 / 67 \\
69 / 16 / 87 \\
11 / 18 / 87 \\
12 / 68 / 87 \\
12 / 68 / 87\end{array}$ & $\begin{array}{r}88.80 \\
\{\quad 8.19\}\end{array}$ \\
\hline FALUUIN & PPB & 156.68 & & $\begin{array}{l}67 / 21 / 87 \\
10 / 14 / 87\end{array}$ & & $\begin{array}{l}07 / 23 / 87 \\
10 / 14 / 87\end{array}$ & & $\begin{array}{l}17 / 23 / 87 \\
12 / 98 / 87\end{array}$ & 350.00 \\
\hline FBARIUU & PPB & 6.00 & 1800.00 & $\begin{array}{l}07 / 21 / 87 \\
10 / 14 / 87\end{array}$ & $\begin{array}{l}51.06 \\
44.66\end{array}$ & $\begin{array}{l}87 / 23 / 87 \\
10 / 14 / 87\end{array}$ & $\begin{array}{l}43.00 \\
41.00\end{array}$ & $\begin{array}{l}67 / 23 / 87 \\
12 / 06 / 87\end{array}$ & $\begin{array}{l}284.60 \\
189.06\end{array}$ \\
\hline \multicolumn{10}{|c|}{ 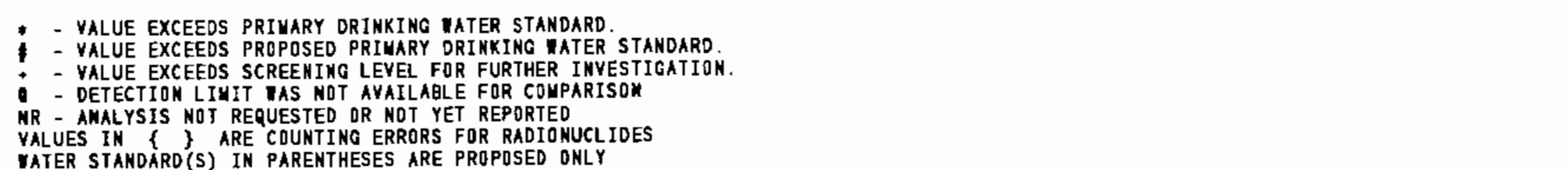 } \\
\hline
\end{tabular}


TABLE C.3. (contd)

\begin{tabular}{|c|c|c|c|c|c|}
\hline $\begin{array}{l}\text { CONSTI } \\
\text { HAUE }\end{array}$ & $\begin{array}{l}\text { UENT } \\
\text { UNITS }\end{array}$ & $\begin{array}{l}\text { CONFRACTUAL } \\
\text { DETECTION } \\
\text { LIMIT }\end{array}$ & $\begin{array}{l}\text { DRINKING } \\
\text { IATER } \\
\text { STANOARD }\end{array}$ & $\begin{array}{c}\text { SAMPLE } \\
\text { DATE }\end{array}$ & $2-119-15$ \\
\hline$-\cdots--$ & $-\cdots-$ & - - - & - & $-\ldots-1$ & $-+\cdots$ \\
\hline FCALCIU & PPB & 50.00 & & $\begin{array}{l}67 / 21 / 87 \\
16 / 14 / 87\end{array}$ & $\begin{array}{l}58900.00 \\
48480.09\end{array}$ \\
\hline FIRQN & PPQ & 56.06 & & $\begin{array}{l}07 / 21 / 87 \\
16 / 14 / 87\end{array}$ & \\
\hline FLUORID & $\mathrm{PPB}$ & 560.06 & 4046.08 & $\begin{array}{l}07 / 21 / 87 \\
10 / 14 / 87 \\
11 / 17 / 87\end{array}$ & $\begin{array}{l}702.60 \\
653.60 \\
861.00\end{array}$ \\
\hline FUAGNES & PPB & 1.86 & & $\begin{array}{l}97 / 21 / 87 \\
10 / 14 / 87\end{array}$ & $\begin{array}{l}19980.04 \\
16766.00\end{array}$ \\
\hline FYANGAN & PPB & 5.06 & & $\begin{array}{l}67 / 21 / 87 \\
18 / 14 / 87\end{array}$ & $\begin{array}{l}12.60 \\
14.60\end{array}$ \\
\hline FNICKEL & PPB & 10.00 & & $\begin{array}{l}67 / 21 / 87 \\
10 / 14 / 87\end{array}$ & 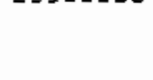 \\
\hline FPDTASS & PPB & 108.80 & & $\begin{array}{l}87 / 21 / 87 \\
10 / 14 / 87\end{array}$ & $\begin{array}{l}5120.00 \\
4840.00\end{array}$ \\
\hline FSODIUH & PPB & 180.00 & & $\begin{array}{l}67 / 21 / 87 \\
10 / 14 / 87\end{array}$ & $\begin{array}{l}28506.00 \\
28700.00\end{array}$ \\
\hline FSTRDNT & PPB & 300.00 & & $\begin{array}{l}07 / 21 / 87 \\
19 / 14 / 87\end{array}$ & -..--n \\
\hline FVANADI & PPB & 5.80 & & $\begin{array}{l}07 / 21 / 87 \\
10 / 14 / 87\end{array}$ & $\begin{array}{l}21.00 \\
25.00\end{array}$ \\
\hline FZINC & PPB & 6.00 & & $\begin{array}{l}07 / 21 / 87 \\
10 / 14 / 87\end{array}$ & $\begin{array}{l}23.00 \\
21.06\end{array}$ \\
\hline HMITRAT & PPB & 2500.00 & 45000.00 & $\begin{array}{l}67 / 21 / 87 \\
68 / 13 / 87 \\
99 / 16 / 87 \\
10 / 14 / 87\end{array}$ & $\begin{array}{l}83560.00 \\
82600.00 \\
65500.00 \\
58800.00\end{array}$ \\
\hline
\end{tabular}

\begin{tabular}{|c|c|}
\hline $\begin{array}{c}\text { SAHPLE } \\
\text { OATE }\end{array}$ & $2-19-16$ \\
\hline - - - & - \\
\hline $\begin{array}{l}07 / 23 / 87 \\
10 / 14 / 87\end{array}$ & $\begin{array}{l}36100.00 \\
30200.00\end{array}$ \\
\hline $\begin{array}{l}87 / 23 / 87 \\
10 / 14 / 87\end{array}$ & $-+-1 .-4$ \\
\hline $\begin{array}{l}67 / 29 / 87 \\
16 / 14 / 87 \\
11 / 17 / 87\end{array}$ & $\begin{array}{l}824.00 \\
747.00 \\
761.00\end{array}$ \\
\hline$--1-1$ & - \\
\hline $\begin{array}{l}67 / 23 / 87 \\
16 / 14 / 87\end{array}$ & $\begin{array}{l}11300.00 \\
10400.60\end{array}$ \\
\hline $\begin{array}{l}07 / 23 / 87 \\
16 / 14 / 87\end{array}$ & 5.69 \\
\hline $\begin{array}{l}07 / 23 / 87 \\
10 / 14 / 87\end{array}$ & \\
\hline $\begin{array}{l}07 / 23 / 87 \\
16 / 14 / 87\end{array}$ & 3770.00 \\
\hline $\begin{array}{l}07 / 23 / 87 \\
16 / 14 / 87\end{array}$ & 38700.00 \\
\hline $\begin{array}{l}07 / 23 / 87 \\
10 / 14 / 87\end{array}$ & $\cdots$ \\
\hline $\begin{array}{l}07 / 23 / 87 \\
10 / 14 / 87\end{array}$ & $\begin{array}{r}13.06 \\
17.06\end{array}$ \\
\hline $\begin{array}{l}17 / 23 / 87 \\
16 / 14 / 87\end{array}$ & $\begin{array}{r}15.00 \\
0.00\end{array}$ \\
\hline $\begin{array}{l}97 / 23 / 87 \\
68 / 13 / 87 \\
09 / 16 / 87 \\
18 / 14 / 87\end{array}$ & $\begin{array}{l}55900.00 \\
48900.00 \\
47100.00 \\
41980.00\end{array}$ \\
\hline
\end{tabular}

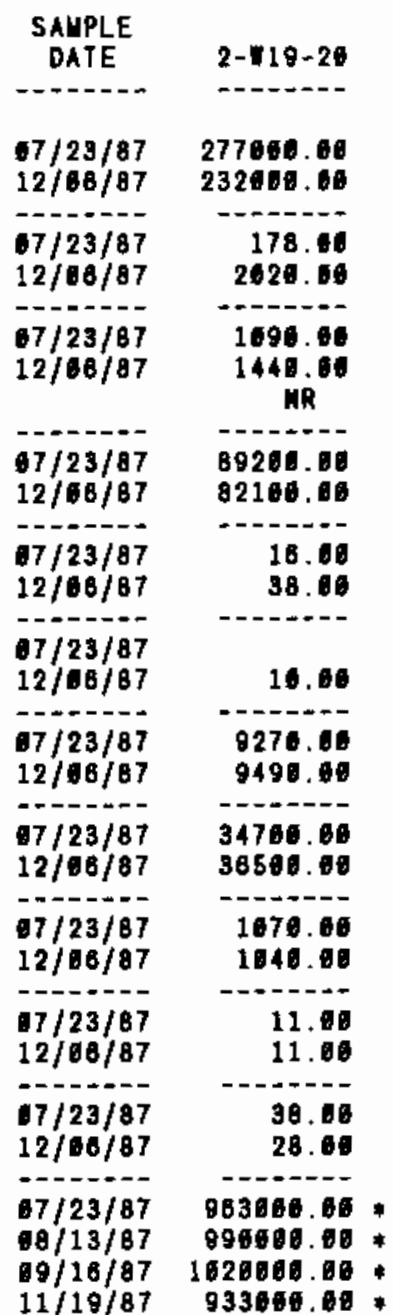

* - VALUE EXCEEDS PRIMARY dRINKING MaTER STANDARD.

* - VALUE EXCEEDS PROPOSED PRIMARY ORINKING TATER STANDARO

- - VALUE EXCEEDS SCREEMING LEVEL FDR FURTHER INVESTIGATION.

- DETECTIOH LIUIT IAS NOT AVAILABLE FOR COUPARISON

- DETECTION LIHIT TAS NOT AVAILABLE FOR COUPARI
NR - ANALYSIS NOT REQUESTED OR NOT YET REPORTED

VALUES IN \& $\}$ ARE COUNTING ERRORS FOR RADIONUCLIDES

WATER STANDARO(S) IN PARENTHESES ARE PROPOSED ONLY 
TABLE C.3. (contd)

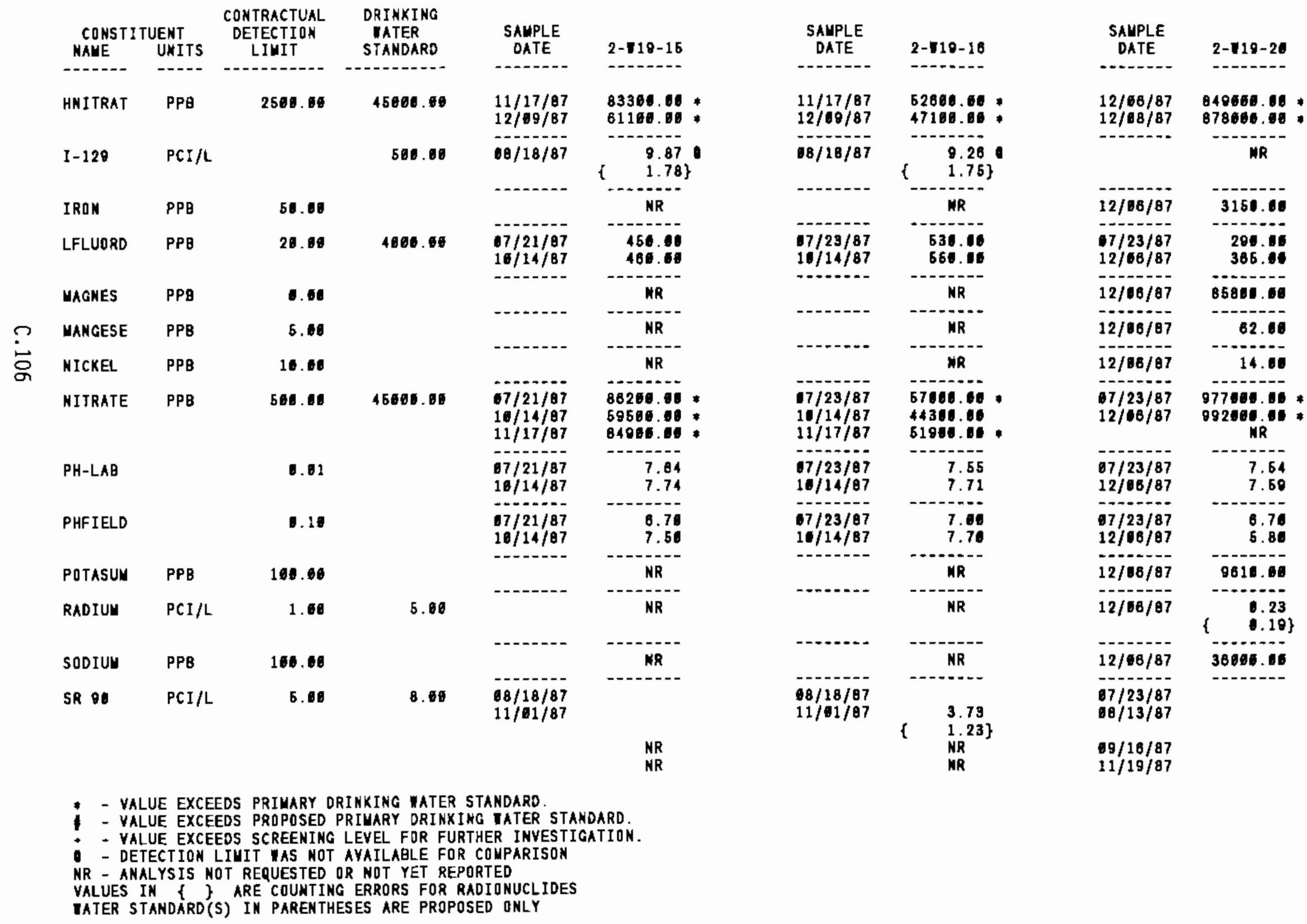


TABLE C.3. (contd)

\begin{tabular}{|c|c|c|c|c|c|}
\hline $\begin{array}{l}\text { CONSTI } \\
\text { NAHE }\end{array}$ & $\begin{array}{l}\text { UENT } \\
\text { UNI ITS }\end{array}$ & $\begin{array}{l}\text { CONTRACTUAL } \\
\text { DETECTION } \\
\text { LIMIT }\end{array}$ & $\begin{array}{l}\text { DRINKING } \\
\text { IATER } \\
\text { STANDARD }\end{array}$ & $\begin{array}{l}\text { SAUPLE } \\
\text { DATE }\end{array}$ & $2-18-15$ \\
\hline SR 96 & $\mathrm{PCI} / \mathrm{L}$ & 5.00 & 8.00 & & $\begin{array}{l}\text { NR } \\
\text { NR }\end{array}$ \\
\hline SULFATE & PPB & 680.08 & & $\begin{array}{l}67 / 21 / 87 \\
10 / 14 / 87 \\
11 / 17 / 87\end{array}$ & $\begin{array}{l}51800.08 \\
43208.08 \\
58600.00\end{array}$ \\
\hline TC & PPB & & & $\begin{array}{l}97 / 21 / 87 \\
18 / 14 / 87\end{array}$ & $\begin{array}{l}38884.01 \\
32980.06\end{array}$ \\
\hline TC -90 & $\mathrm{PCI} / \mathrm{L}$ & 16.00 & 960.08 & $\begin{array}{l}68 / 18 / 87 \\
12 / 69 / 87\end{array}$ & $\begin{array}{c}586.86 \\
4.16\} \\
784.86 \\
\{\quad 7.33\}\end{array}$ \\
\hline TETRANE & PPB & 10.06 & 5.60 & $\begin{array}{l}07 / 21 / 87 \\
10 / 14 / 87\end{array}$ & $\begin{array}{l}69.00 \\
78.09\end{array}$ \\
\hline TOC & PPB & 1000.00 & & $\begin{array}{l}67 / 21 / 87 \\
1 \% / 14 / 87\end{array}$ & \\
\hline TOXLDL & PPB & 20.00 & & $\begin{array}{l}07 / 21 / 87 \\
10 / 14 / 87\end{array}$ & $\begin{array}{l}52.96 \\
82.80\end{array}$ \\
\hline TRITIUU & $\mathrm{PCI} / \mathrm{L}$ & 500.00 & 20600.00 & 88/18/87 & 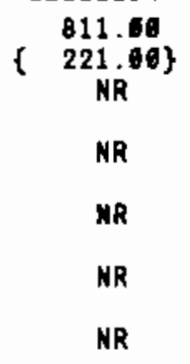 \\
\hline U 234 & $\mathrm{PCI} / \mathrm{L}$ & $\theta .10$ & 32.60 & $67 / 21 / 87$ & $\begin{array}{r}85.10 \\
\{\quad 6.86\}\end{array}$ \\
\hline
\end{tabular}

\begin{tabular}{|c|c|}
\hline $\begin{array}{c}\text { SAUPLE } \\
\text { DATE }\end{array}$ & \begin{tabular}{c}
$2-\$ 19-18$ \\
\hdashline$R$ \\
MR
\end{tabular} \\
\hline $\begin{array}{l}07 / 23 / 87 \\
19 / 14 / 87 \\
11 / 17 / 87\end{array}$ & $\begin{array}{l}34468.08 \\
32380.60 \\
31566.06\end{array}$ \\
\hline $\begin{array}{l}97 / 23 / 87 \\
10 / 14 / 87\end{array}$ & $\begin{array}{l}32360.08 \\
28408.01\end{array}$ \\
\hline $86 / 18 / 87$ & 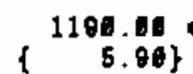 \\
\hline $12 / 09 / 87$ & $\begin{array}{r}1838.60 \\
\{24.80\}\end{array}$ \\
\hline $\begin{array}{r}67 / 23 / 87 \\
10 / 14 / 87\end{array}$ & $\begin{array}{r}113.01 \\
115.8\end{array}$ \\
\hline $\begin{array}{l}07 / 23 / 87 \\
10 / 14 / 87\end{array}$ & \\
\hline $\begin{array}{l}07 / 23 / 87 \\
10 / 14 / 87\end{array}$ & $\begin{array}{l}01.30 \\
83.20\end{array}$ \\
\hline $68 / 18 / 87$ & $\begin{array}{c}323.66 \\
\left\{\begin{array}{c}204.06 \\
\text { NR }\end{array}\right\}\end{array}$ \\
\hline & NR \\
\hline & MR \\
\hline & NR \\
\hline & MR \\
\hline $87 / 23 / 87$ & $\begin{array}{c}862.00 \\
\{\quad 22.10\}\end{array}$ \\
\hline
\end{tabular}

SAUPLE

OATE

2-719-20

$12 / 06 / 87$

$12 / 08 / 87$

67/23/87

$12 / 96 / 87$

58000 NR

$07 / 23 / 87$ 12/66/87

304010.05 28765.

$12 / 68 / 87$

1169s

$27.26\}$

NR

$07 / 23 / 87$

$77 / 23 / 87$
$12 / 06 / 87$

67/23/87

12/96/B

? $123 / 87$

$12 / 08 / 87$

$7 / 23 / 87$

67/23/87

09/13/87

-9/18/87

$11 / 19 / 87$

12/08/87

$12 / 68 / 87$

$-2$

$39.71 *$

36. 06 *

1870.6

-...--

30.0

$------$

1240.00
$241.00\}$

1846.06

\{ 250.06

$\{258.09)$

1578.09

$\{205.06\}$

1410.86

\{249.60\}

1570.09

( 204.00

NR

- Value exceeds privary dRinking tater standaro.

- VALUE EXCEEDS PROPOSED PRIUARY DRIMKING VATER STAMDARD

- - VALUE EXCEEDS SCREENING LEYEL FOR FURTHER INYESTIG

- DETECTIDN LIUIT TAS NOT AVAILABLE FOR COUPARISON

NR - ANALYSIS NOT REQUESTED OR HOT YET REPORTED

VALUES IN \& \& ARE COUNTING ERRIRS FOR RADIONUCLIDES

TATER STANDARD(S) IN PARENTHESES ARE PROPOSED ONLY 


\begin{tabular}{|c|c|c|}
\hline $\begin{array}{l}\text { CONSTI I } \\
\text { HANE }\end{array}$ & $\begin{array}{l}\text { UENT } \\
\text { UNITS }\end{array}$ & $\begin{array}{l}\text { CONTRACTUAL } \\
\text { DEIECTION } \\
\text { LIUIT }\end{array}$ \\
\hline U 235 & $\mathrm{PCI} / \mathrm{L}$ & 0.10 \\
\hline U $23 B$ & $\mathrm{PCI} / \mathrm{L}$ & 0.10 \\
\hline U-CHEU & UG/L & 0.73 \\
\hline VAKADUU & PPB & 5.00 \\
\hline ZINC & PPB & 5.00 \\
\hline
\end{tabular}

TABLE C.3. (contd)

\begin{tabular}{|c|}
\hline $2-118-15$ \\
\hline $\begin{array}{l}2.72 \\
1.42\}\end{array}$ \\
\hline $\begin{array}{c}87.70 \\
B . B B\}\end{array}$ \\
\hline $\begin{array}{l}264.66 \\
222.64 \\
204.06 \\
192.60 \\
358.60 \\
280.64\end{array}$ \\
\hline NR \\
\hline NR \\
\hline
\end{tabular}

\begin{tabular}{|c|c|}
\hline $\begin{array}{l}\text { SAUPLE } \\
\text { DATE }\end{array}$ & $2-19-16$ \\
\hline $97 / 23 / 87$ & $\left\{\begin{array}{l}28.50 \\
4.80\end{array}\right.$ \\
\hline $97 / 23 / 97$ & $\begin{array}{c}685.60 \\
\{\quad 22.50\}\end{array}$ \\
\hline $\begin{array}{l}07 / 23 / 87 \\
08 / 13 / 87 \\
90 / 18 / 87 \\
10 / 14 / 87 \\
11 / 17 / 87 \\
12 / 80 / 87\end{array}$ & $\begin{array}{l}1938.06 \\
1588.08 \\
2910.06 \\
1858.08 \\
2140.08 \\
1586.00\end{array}$ \\
\hline & NR \\
\hline
\end{tabular}

32.08

67/21/87

4.80

$67 / 21 / 87$

- $7 / 21 / 87$

$08 / 13 / 87$

$09 / 15 / 87$

$10 / 14 / 87$

$11 / 17 / 87$

$12 / 00 / 87$

....-...

NR

NR

NR

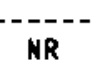

NR

$67 / 23 / 87$

98/13/67

09/16/87

$11 / 18 / \mathrm{B} 7$

12/66/87

$12 / 68 / B 7$

…구

$12 / 66 / 87$

$12 / 60 / 87$

467.6

441.00

435.10

458.60

544.0

498. 05

11.06

33. 06

* - yalue exceEds priuary drinking water stanoard.

- YALUE EXCEEDS PROPOSED PRIMARY DRINKING WATER STAMDARD.

+ - VALUE EXCEEDS SCREENING LEVEL FOR FURTHER INYESTIGATION.

- DETECTION LIUIT WAS NOT AVAILABLE fOR COMPARISON

NR - ANALYSIS NDT REQUESTED OR NOT YET REPORTED

YALUES IN \{ $\}$ ARE COUNTING ERRORS FOR RADIDNUCLIDES

WATER STANDARD(S) IN PARENTHESES ARE PROPOSED ONLY 
TABLE C.3. (contd)

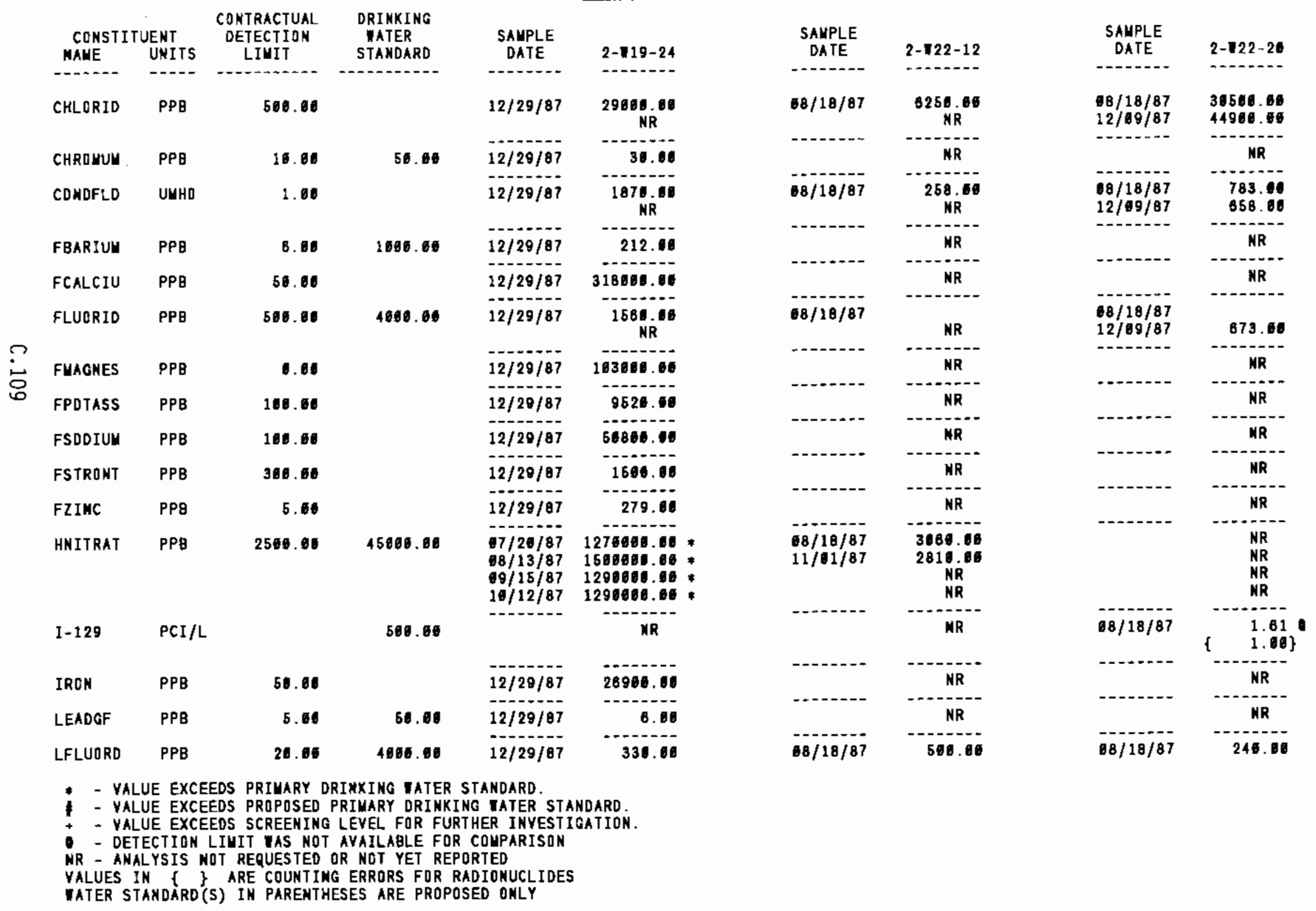


TABLE C.3. (contd)

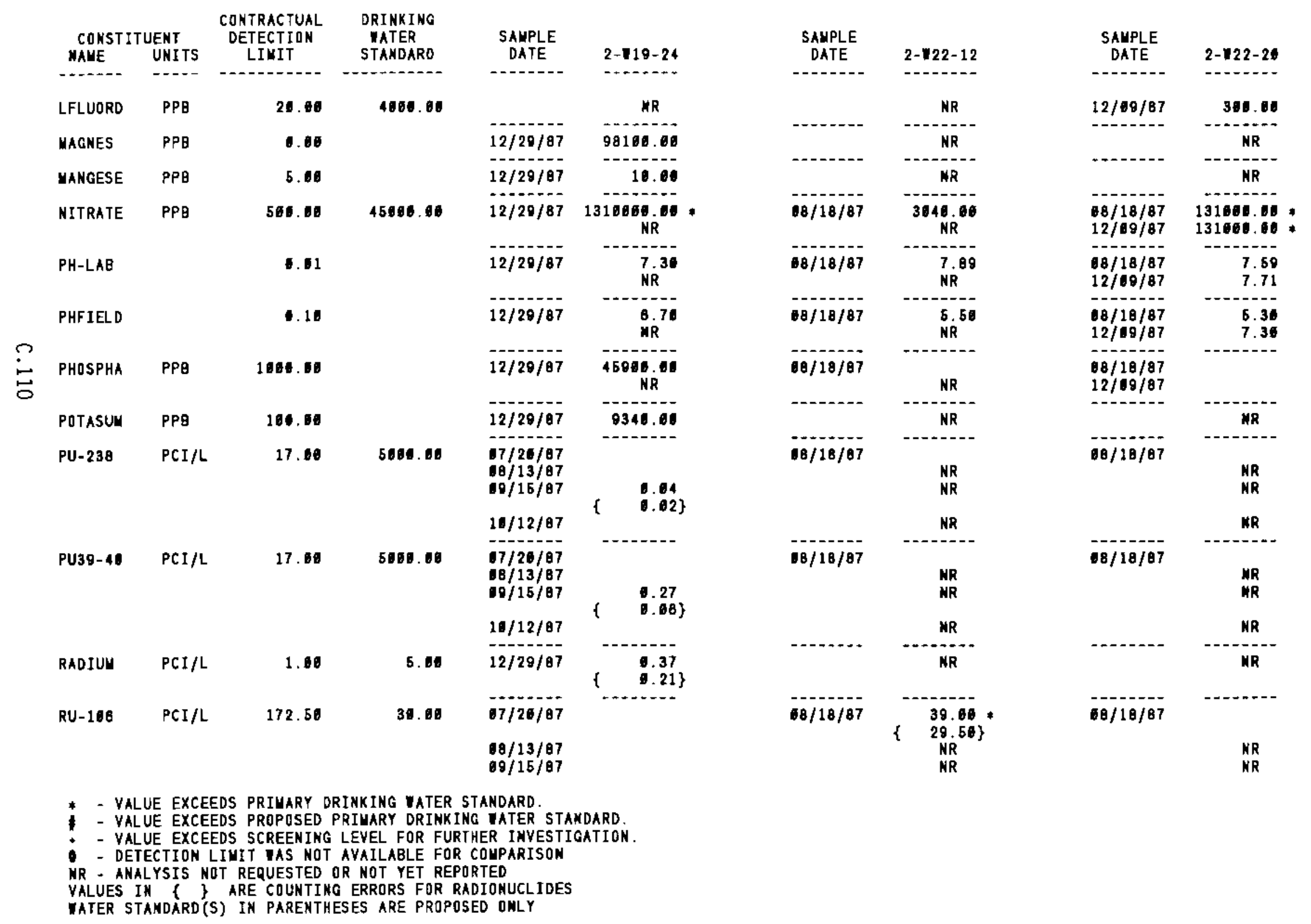


TABLE C.3. (contd)

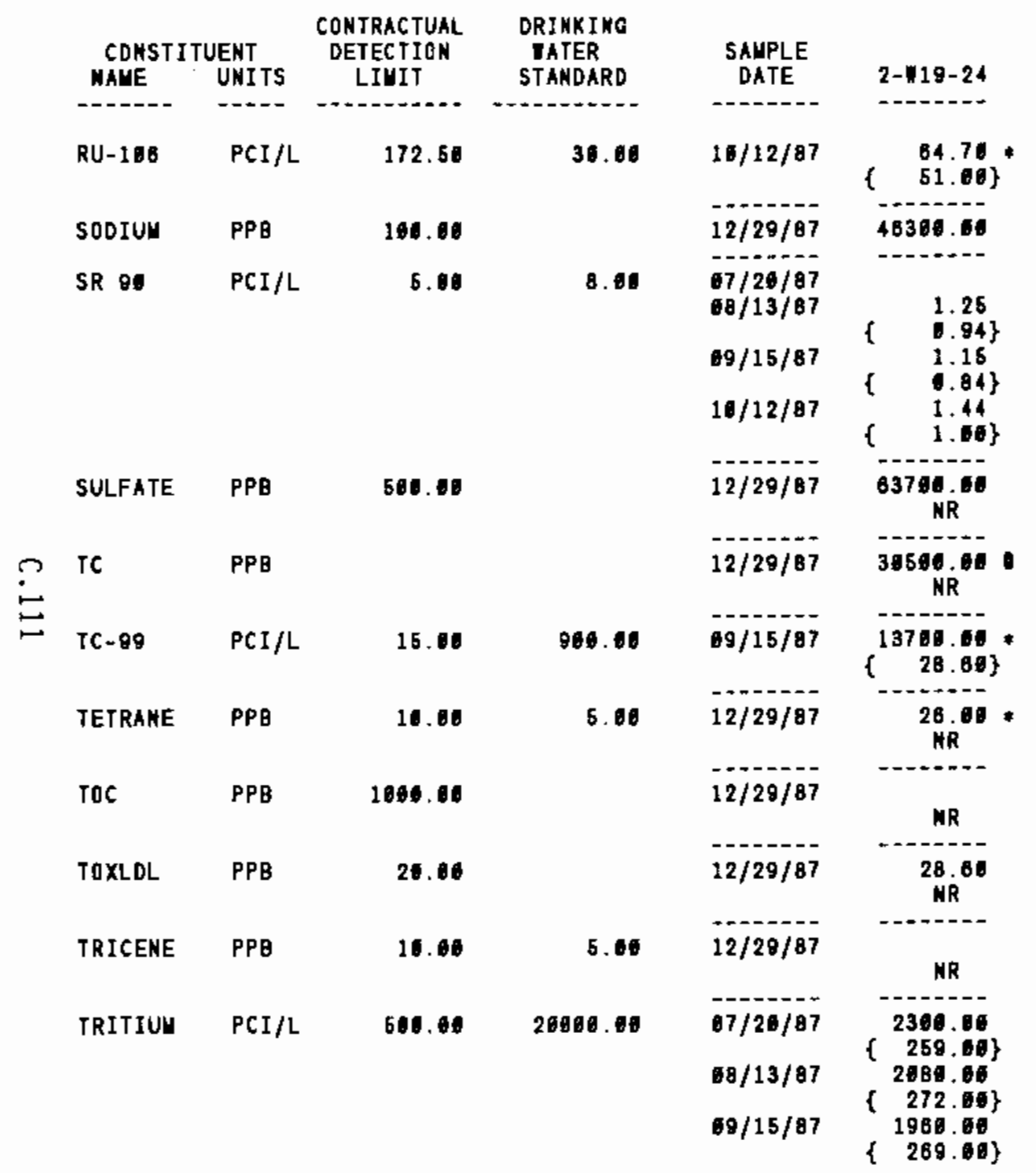
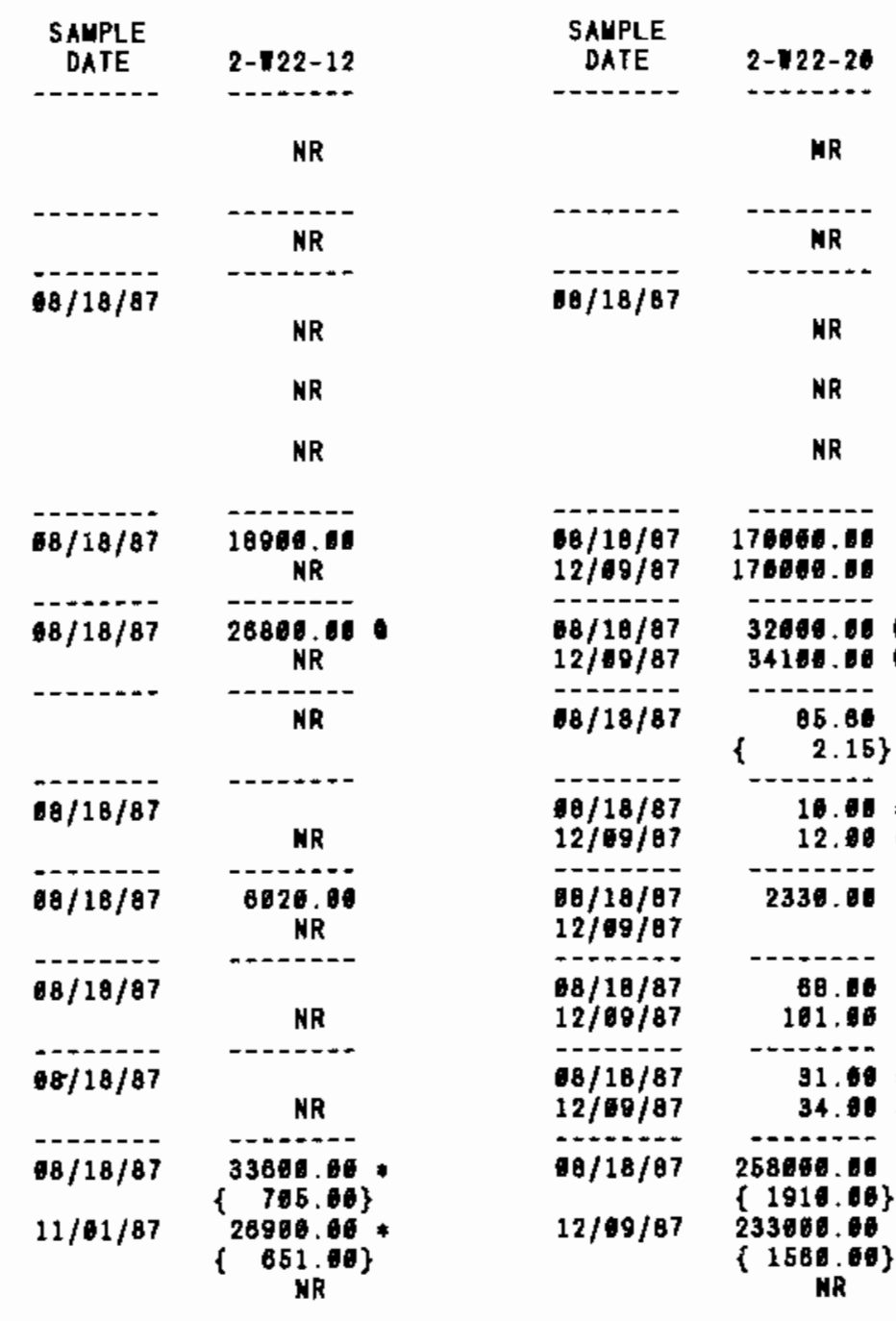

MR

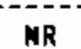

$08 / 18 / 87$

NR

NR

NR

$68 / 18 / 87$

12/09/87 176009.06

170964

$68 / 18 / 97$

$12 / 49 / 87$

32969

$08 / 18 / 87$

34106.06

* - YALUE EXCEEDS PRIUARY DRINKING WATER STANDARD.

- VALUE EXCEEDS PROPISED PRIUARY DRINKING VATER STANDARO.

+ - VALUE EXCEEDS SCREENING LEVEL FOR FURTHER INVESTIGATION.

- DETECTION LINIT IAS NOT AVAILABLE FOR COUPARISON

NR - AKALYSIS NOT RERUESTED OR NOT YET REPORTED

VALUES IN $\{$ \} ARE COUNTING ERRORS FOR RADIONUCLIDES

WATER STANOARD(S) IN PARENTHESES ARE PROPOSED ONLY

$18 / 18 / 87$

$12 / 69 / 87$

B5.68

$\left\{\begin{array}{r}2.15\} \\ -2.05\end{array}\right.$

$00 / 10 / 87 \quad 2330.06$

$12 / 99 / 87$

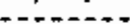

$68 / 18 / 87$

$12 / 68 / 87$

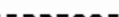

68/18/87

$12 / 60 / 87$

68.06

101.06

91.00 .

98/18/87

34.00

$12 / 09 / 87$

258000.

\{1910.66\}

233000.00.

$1580.00\}$ 
TABLE C.3. (contd)

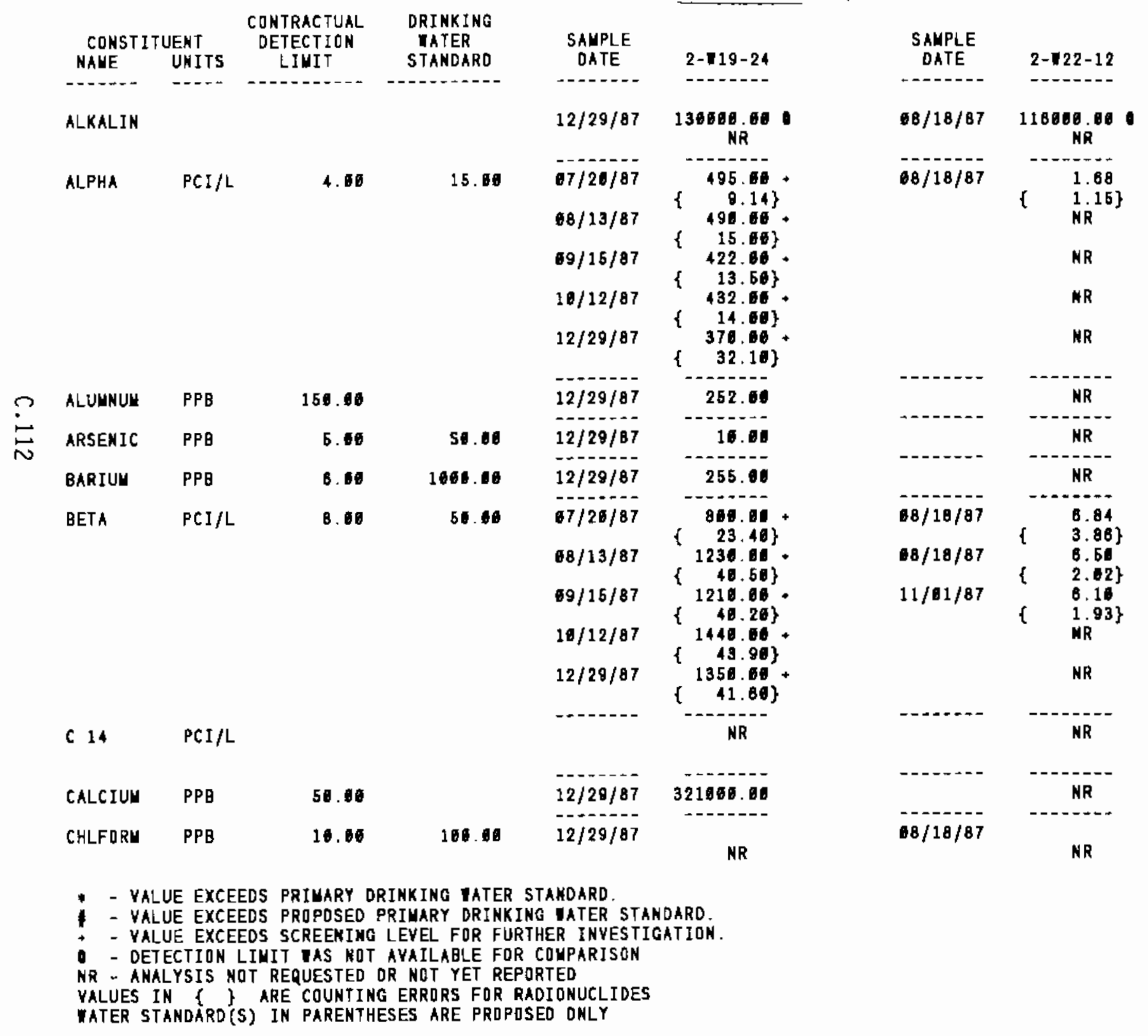


TABLE C.3. (contd)

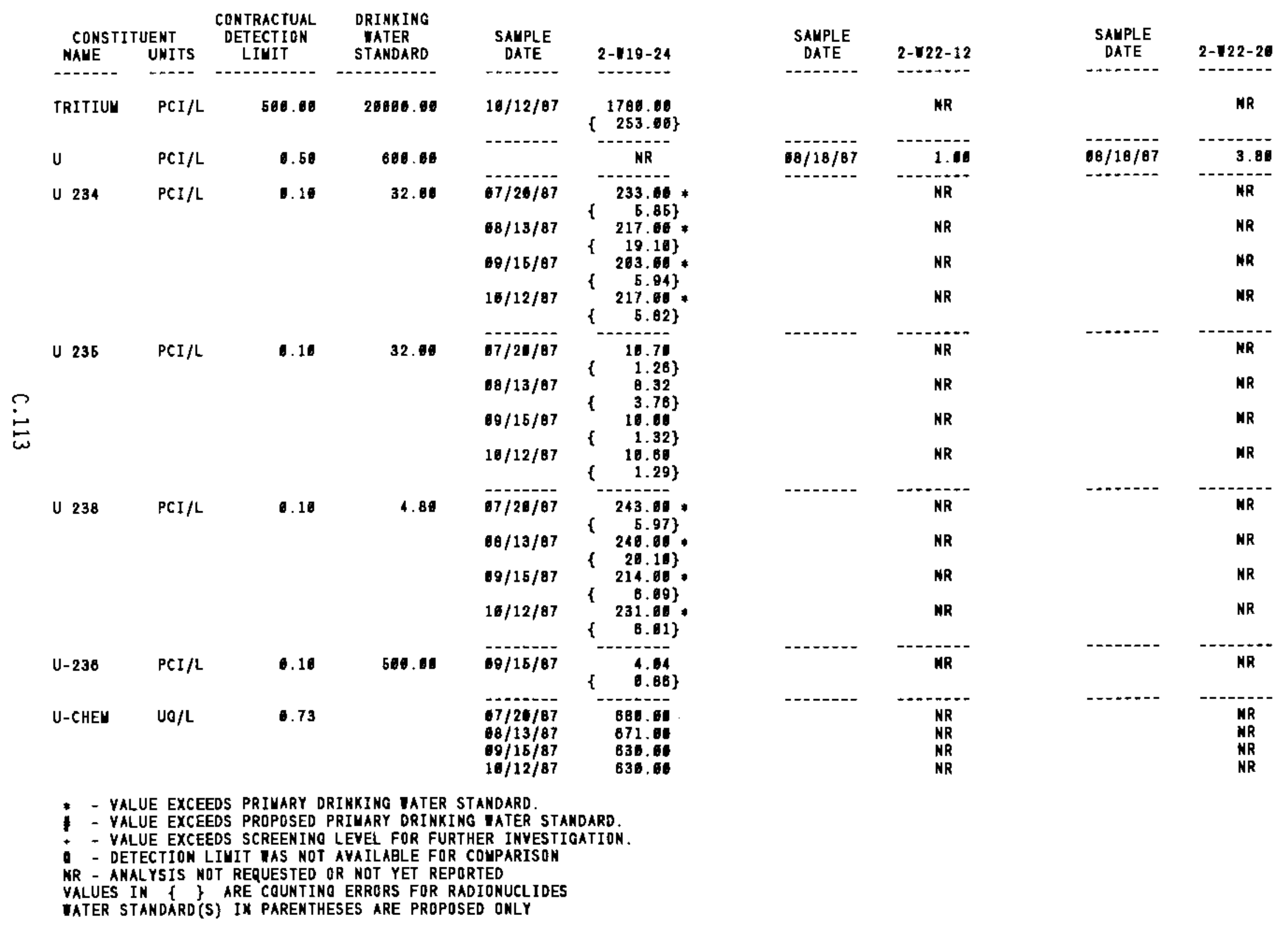


TABLE C.3. (contd)

\begin{tabular}{|c|c|c|c|c|c|c|c|c|c|}
\hline $\begin{array}{l}\text { CONSTI } \\
\text { NAUE }\end{array}$ & $\begin{array}{l}\text { UENT } \\
\text { UNITS }\end{array}$ & $\begin{array}{l}\text { CONIRACTUAL } \\
\text { DETECTION } \\
\text { LINIT }\end{array}$ & $\begin{array}{l}\text { DRINKING } \\
\text { WAFER } \\
\text { STANDARD }\end{array}$ & $\begin{array}{c}\text { SAUPLE } \\
\text { DATE }\end{array}$ & $2-19-24$ & $\begin{array}{l}\text { SAUPLE } \\
\text { DATE }\end{array}$ & $2-122-12$ & $\begin{array}{c}\text { SAYPLE } \\
\text { DATE }\end{array}$ & $2-722-20$ \\
\hline------ & $\cdots+$ & $---1---1$ & - - - & $-\cdots-1$ & $\cdots---$ & $-\cdots++-0$ & $---n-$ & $-\cdots---$ & ------ \\
\hline YANADUU & $\mathrm{PPB}$ & 5.60 & & $12 / 29 / 87$ & 58.06 & & NR & & NR \\
\hline ZINC & PPB & 5.00 & & $12 / 29 / 87$ & 787.60 & & $N R$ & & NR \\
\hline
\end{tabular}

* - VALUE EXCEEDS PRIMARY DRINKING Tater STANDARD.

- - YALUE EXCEEDS PROPDSED PRIHARY DRIMKIHG WATER STANDARD

* - VALUE EXCEEDS SCREENING LEVEL FOR FURTHER INVESTIGATION.

- DETECTIDN LIUIT WAS NDT AYAILABLE FOR CONPARISON

HR - ANALYSIS NDT REQUESTED OR NOT YET REPORTED

YALUES IN \{\} ARE COUNTING ERRORS FOR RADIONUCLIDES

WATER STANDARD(S) IN PARENTHESES ARE PROPDSED QNLY 
TABLE C.3. (contd)

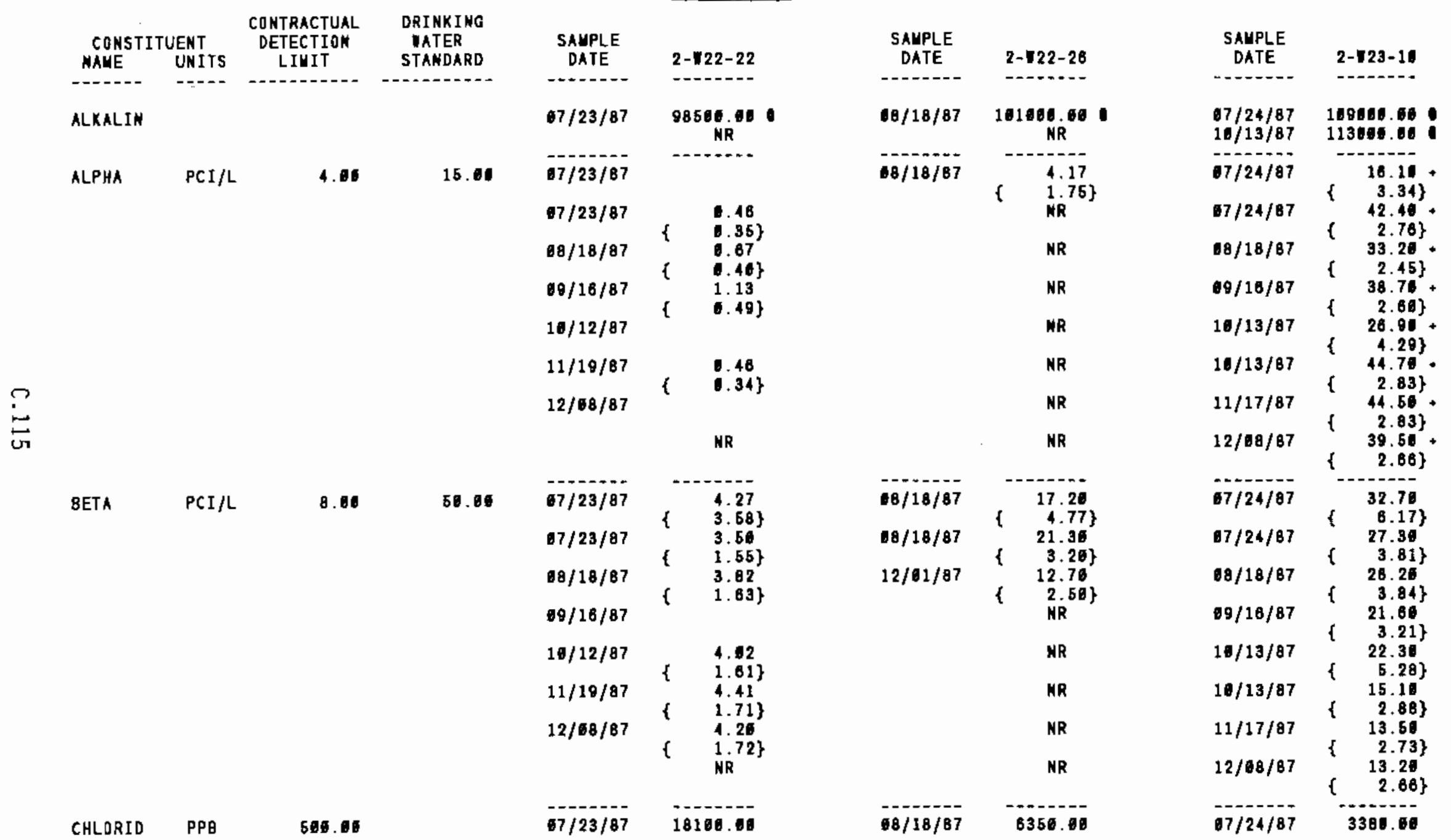

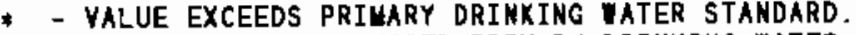

- YALUE EXCEDS PROPOSED PRIUARY DRINKING IATER STANDARD

- - VALUE EXCEEDS SCREENIRG LEVEL FDR FURTHER INYESTIGATION.

- DETECTION LIUIT UAS NOT AYAILABLE FOR COMPARISON

NR - AMALYSIS NOT REQUESTED OR NOT YET REPORTED

YALUES IN I ARE COUKTING ERRORS FOR RADIONUCLIDES

IATER STANDARD'S) IN PARENTHESES AFE F:OOPOSED OKLY 
IABLE C.3. (contd)

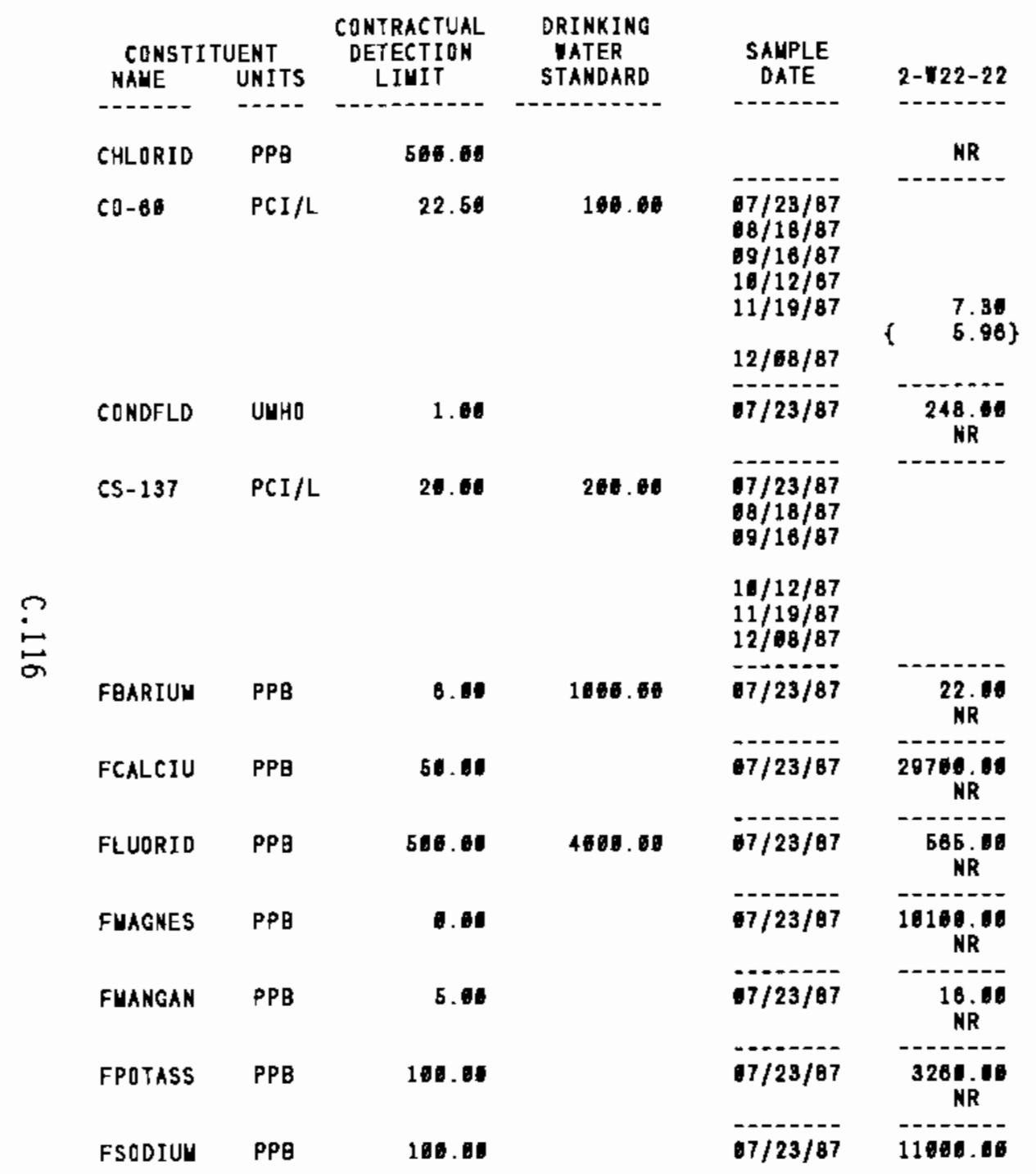

\begin{tabular}{|c|c|c|c|}
\hline \multirow[t]{2}{*}{$\begin{array}{c}\text { SAHPLE } \\
\text { DATE }\end{array}$} & $2-22-28$ & $\begin{array}{c}\text { SAYPLE } \\
\text { DATE }\end{array}$ & $2-123-10$ \\
\hline & NR & $10 / 13 / 87$ & 3528.60 \\
\hline \multirow[t]{2}{*}{$80 / 1 \theta / 87$} & $\begin{array}{l}\text { NR } \\
\text { KR } \\
\text { NR } \\
\text { NR }\end{array}$ & $\begin{array}{l}17 / 24 / 87 \\
60 / 18 / 87 \\
69 / 16 / 87 \\
16 / 13 / 87 \\
11 / 17 / 87\end{array}$ & \\
\hline & NR & $12 / 08 / 07$ & \\
\hline$O B / 1 \theta / 87$ & $\begin{array}{c}234.06 \\
M R\end{array}$ & $\begin{array}{l}97 / 24 / 87 \\
10 / 13 / 87\end{array}$ & 533.00 \\
\hline \multirow[t]{2}{*}{$88 / 18 / \theta 7$} & $\begin{array}{l}\text { NR } \\
\text { MR }\end{array}$ & $\begin{array}{l}67 / 24 / 87 \\
68 / 10 / 07 \\
69 / 16 / 87\end{array}$ & 5.88 \\
\hline & $\begin{array}{l}\text { NR } \\
\text { NR } \\
\text { NR }\end{array}$ & $\begin{array}{l}19 / 13 / 87 \\
11 / 17 / 87 \\
12 / 60 / 87\end{array}$ & \\
\hline $86 / 18 / 87$ & NR & $\begin{array}{l}67 / 24 / 87 \\
10 / 13 / 67\end{array}$ & $\begin{array}{l}88.06 \\
74.06\end{array}$ \\
\hline $88 / 18 / 87$ & 19500.00 & $\begin{array}{l}97 / 24 / 87 \\
16 / 13 / 87\end{array}$ & $\begin{array}{l}56800.00 \\
55500.06\end{array}$ \\
\hline $88 / 18 / 87$ & NR & $\begin{array}{l}97 / 24 / 87 \\
10 / 13 / 87\end{array}$ & \\
\hline $88 / 18 / 87$ & $\underset{\mathrm{NR}}{7188}$ & $\begin{array}{l}07 / 24 / 87 \\
16 / 13 / 87\end{array}$ & $\begin{array}{l}19586.00 \\
21066.08\end{array}$ \\
\hline $68 / 18 / 87$ & NR & $\begin{array}{l}07 / 24 / 87 \\
16 / 13 / 67\end{array}$ & \\
\hline $68 / 18 / 87$ & $\begin{array}{c}3280.08 \\
\text { NR }\end{array}$ & $\begin{array}{l}67 / 24 / 87 \\
16 / 13 / 87\end{array}$ & $\begin{array}{l}4210.66 \\
5890.66\end{array}$ \\
\hline $68 / 10 / 07$ & 20000.00 & $07 / 24 / 67$ & 20400.00 \\
\hline
\end{tabular}

- - Value exceeds PRIUARy dRINking mater stanoard.

* - YALUE EXCEEDS PROPOSED PRIUARY DRINKING TATER STANDARD.

+ - yalue exceEdS SCREening leyel far fuRTHer inyestigation.

- DETECTIDN LiUit us not ayailagle for couparison

NR - ANALYSIS MOT RERUESTED OR NOT YET REPORTED

YALUES IN \{\} ARE COUNIING ERRORS FOR RADIONUCLIDES

vaTER STANDARD(S) IN PARENTHESES ARE PROPDSED DNLY 
TABLE C.3. (contd)

\begin{tabular}{|c|c|c|c|c|c|}
\hline $\begin{array}{l}\text { CONSTI } \\
\text { NAHE }\end{array}$ & $\begin{array}{l}\text { TUEKT } \\
\text { UNITS }\end{array}$ & $\begin{array}{c}\text { CONTRACTUAL } \\
\text { DETECTIDN } \\
\text { LIUIT }\end{array}$ & $\begin{array}{l}\text { DRINKING } \\
\text { IATER } \\
\text { STANDARO }\end{array}$ & $\begin{array}{l}\text { SAMPLE } \\
\text { DATE }\end{array}$ & 2-T22-22 \\
\hline FSDDIUM & PPB & 100.08 & & & NR \\
\hline FYANADI & PPB & 5.80 & & $07 / 23 / 87$ & 9.00 \\
\hline FZINC & PPB & 5.98 & & $67 / 23 / 87$ & MR \\
\hline HNITRAT & PPB & 2560.66 & 45060.00 & $\begin{array}{l}17 / 23 / 87 \\
08 / 18 / 87 \\
09 / 16 / 87 \\
18 / 12 / 87 \\
11 / 18 / 87 \\
12 / 68 / 87\end{array}$ & $\begin{array}{l}3886.00 \\
4881.00\end{array}$ \\
\hline LFLUORD & PPB & 20.00 & 4000.08 & $67 / 23 / 87$ & ${ }_{\mathrm{MR}}^{460.00}$ \\
\hline MITRATE & PPB & 500.010 & 45000.00 & $1 / 23 / 87$ & 1324.10 \\
\hline$P H-L A B$ & & 6.1 & & $67 / 23 / 87$ & $\mathrm{MR}^{\mathrm{B}}$ \\
\hline PHF IELD & & 1.10 & & $07 / 23 / 87$ & ${ }_{M R}^{8.80}$ \\
\hline SULFATE & PPB & 508.08 & & $07 / 23 / 87$ & 19700.6 \\
\hline TC & PPB & & & $67 / 23 / 87$ & 23806.68 \\
\hline TC-99 & $\mathrm{PCI} / \mathrm{L}$ & 15.00 & 800.80 & & NR \\
\hline TRITIUU & $\mathrm{PCI} / \mathrm{L}$ & 506.00 & 20006.00 & $07 / 23 / 87$ & $\begin{array}{r}1970.06 \\
240.06\} \\
1806.06 \\
217.01\}\end{array}$ \\
\hline
\end{tabular}

\begin{tabular}{|c|c|c|c|}
\hline \multirow[t]{2}{*}{$\begin{array}{l}\text { SAYPLE } \\
\text { DATE }\end{array}$} & $2-522-26$ & $\begin{array}{l}\text { SAUPLE } \\
\text { DATE }\end{array}$ & $2-123-10$ \\
\hline & NR & $10 / 13 / 87$ & 26286.06 \\
\hline $08 / 18 / 87$ & 31.60 & $\begin{array}{l}17 / 24 / 87 \\
18 / 13 / 87\end{array}$ & $\begin{array}{l}15.60 \\
20.06\end{array}$ \\
\hline $88 / 18 / 87$ & MR & $\begin{array}{l}67 / 24 / 87 \\
16 / 13 / 87\end{array}$ & 22.00 \\
\hline & $\begin{array}{l}\text { NR } \\
\text { NR } \\
\text { NR } \\
\text { NR } \\
\text { NR } \\
\text { NR }\end{array}$ & $\begin{array}{l}07 / 24 / 87 \\
88 / 18 / 87 \\
69 / 18 / 87 \\
10 / 13 / 87 \\
11 / 17 / 87 \\
12 / 88 / 87\end{array}$ & 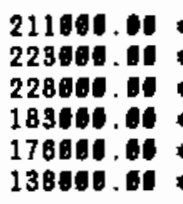 \\
\hline $08 / 18 / 87$ & $\underset{N R}{455.60}$ & $\begin{array}{l}07 / 24 / 87 \\
18 / 13 / 87\end{array}$ & $\begin{array}{l}218.10 \\
228.10\end{array}$ \\
\hline $08 / 18 / 87$ & 0060.00 & $\begin{array}{l}67 / 24 / 87 \\
10 / 13 / 87\end{array}$ & $\begin{array}{l}216604.00 \\
180660.00\end{array}$ \\
\hline $68 / 18 / 87$ & $\begin{array}{l}7.75 \\
\mathrm{NR}\end{array}$ & $\begin{array}{l}87 / 24 / 87 \\
10 / 13 / 87\end{array}$ & $\begin{array}{r}7.65 \\
7.65\end{array}$ \\
\hline $08 / 18 / 87$ & $\begin{array}{l}5.10 \\
\text { NR }\end{array}$ & $\begin{array}{l}07 / 24 / 87 \\
10 / 13 / 87\end{array}$ & 7.11 \\
\hline $68 / 18 / 87$ & 10506.01 & $\begin{array}{l}07 / 24 / 87 \\
16 / 13 / 87\end{array}$ & $\begin{array}{l}12286.06 \\
12706.06 .\end{array}$ \\
\hline $08 / 18 / 87$ & 23450.01 & $07 / 24 / 87$ & 28485.06 \\
\hline $08 / 18 / 87$ & $\begin{array}{r}102.06 \\
2.27\end{array}$ & & NR \\
\hline $68 / 18 / 87$ & 25068.60 & $07 / 24 / 87$ & $\begin{array}{l}821600.06 \\
(8460.00\end{array}$ \\
\hline $12 / 61 / 87$ & $\begin{array}{c}31460.60 \\
\{\quad 090.00\}\end{array}$ & $68 / 18 / 87$ & $\begin{array}{l}780086.90 \\
\{3336.00\}\end{array}$ \\
\hline
\end{tabular}

* - value exceEds priuary dRinking hater standard

- - VALUE EXCEEDS PROPOSED PRIMARY DRINXING IATER STANDARD.

- - YaLUE EXCEEDS SCREENING LEYEL FBR FURTHER INYESTIOATION.

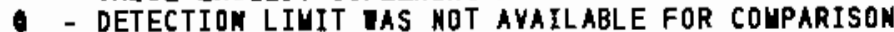

NR - ANALYSIS NOT REQUESTED OR NOT YET REPORTED

VALUES IN \{ $\}$ ARE COUNTING ERRORS FOR RADIONUCLIDES

WATER STANDARD(S) IN PARENTHESES ARE PROPOSED ONLY 
TABLE C.3. (contd)

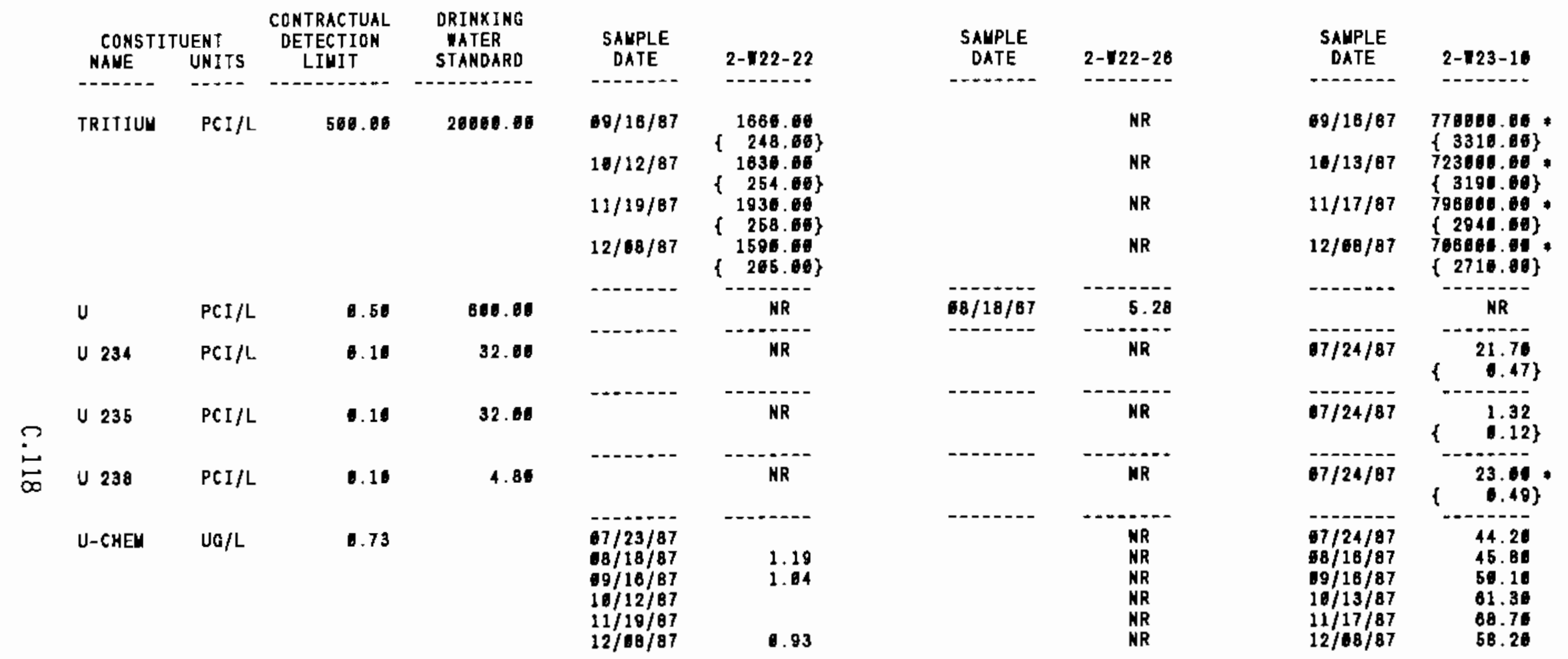

- YALUE EXCEEDS PRIMARY DRINKIKG taTER STANDARD.

- VALUE EXCEEDS PROPOSED PRIUARY DRINKIHG WATER STANDARD.

- YALUE EXCEEDS SCREENIKG LEYEL FOR FURTHER INYESTIGATIDN

- DETECTIDN LIHIT UAS NDT AYAILABLE FOR COMPARISON

NR - ANALYSIS NOT RERUESTED OR MDT YET REPORTED

YALUES IM \{ $\}$ ARE COUNTING ERRORS FOR RADIONUCLIDES

TATER STANDARD(S) IN PARENTHESES ARE PROPOSED ONLY 
TABLE C.3. (contd)

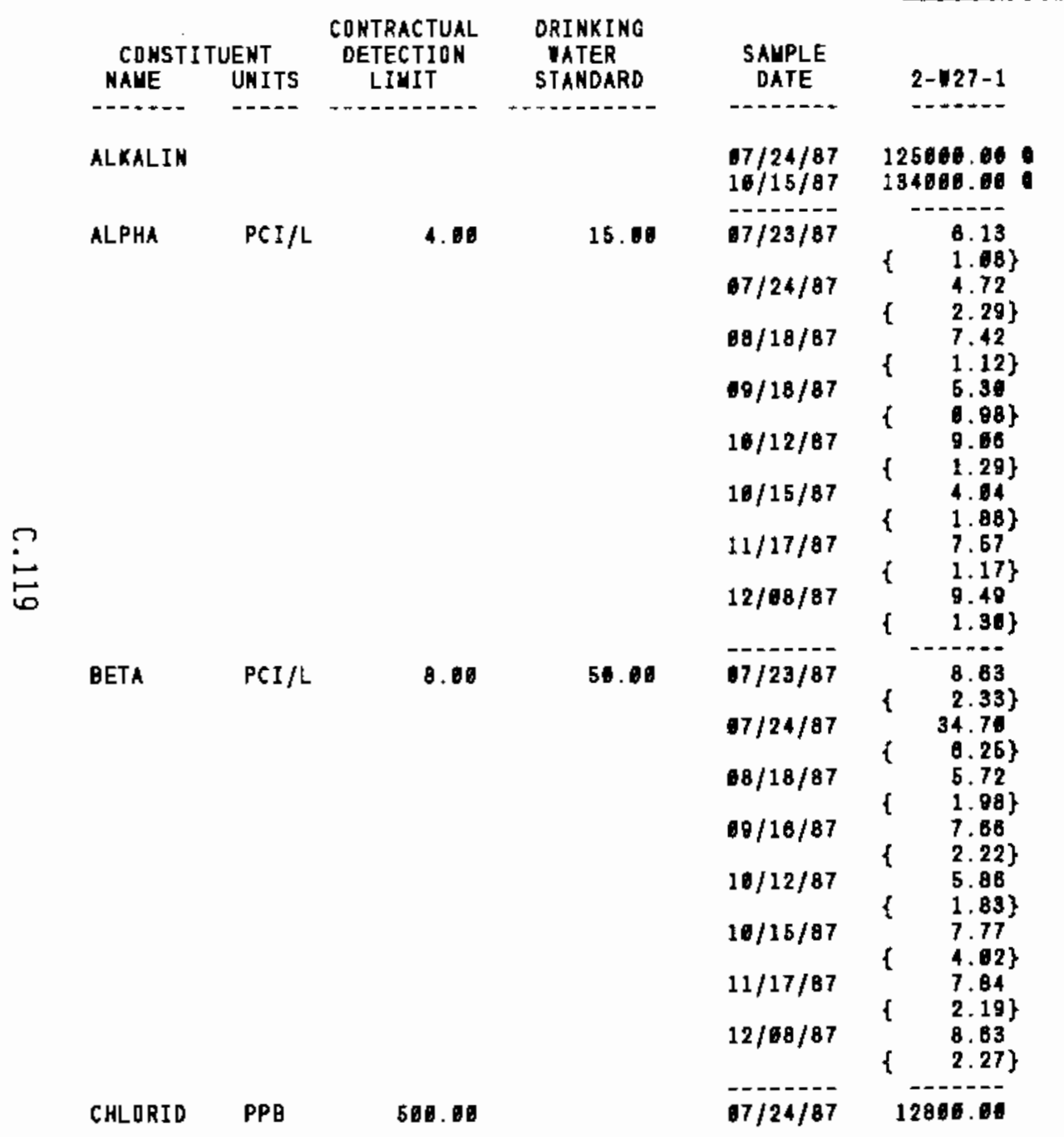

\begin{tabular}{|c|c|}
\hline $\begin{array}{l}\text { SAYPLE } \\
\text { DATE }\end{array}$ & $4-51-76$ \\
\hline $07 / 28 / 87$ & $259000_{N R}=0$ \\
\hline \multirow[t]{7}{*}{$07 / 28 / 87$} & $\left.\begin{array}{ll} & 3.76 \\
2.01\} & \\
\text { NR }\end{array}\right\}$ \\
\hline & NR \\
\hline & NR \\
\hline & NR \\
\hline & NR \\
\hline & NR \\
\hline & MR \\
\hline \multirow[t]{8}{*}{$7 / 28 / 87$} & 28.46 \\
\hline & $\mathrm{NR}^{8.16\}}$ \\
\hline & NR \\
\hline & NR \\
\hline & NR \\
\hline & NR \\
\hline & MR \\
\hline & NR \\
\hline $67 / 28 / 87$ & 13000.06 \\
\hline
\end{tabular}

\begin{tabular}{|c|c|}
\hline $\begin{array}{c}\text { SAMPLE } \\
\text { DATEE }\end{array}$ & $4-S 1-8 A$ \\
\hline $87 / 28 / 87$ & 131080.00 \\
\hline \multirow[t]{7}{*}{$07 / 28 / 87$} & $\begin{array}{ll} & \begin{array}{l}3.47 \\
1.89 \\
\mathrm{NR}\end{array}\end{array}$ \\
\hline & NR \\
\hline & $M R$ \\
\hline & NR \\
\hline & NR \\
\hline & NR \\
\hline & MR \\
\hline \multirow[t]{8}{*}{$97 / 28 / 87$} & 23.50 \\
\hline & 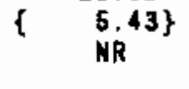 \\
\hline & MR \\
\hline & MR \\
\hline & NR \\
\hline & NR \\
\hline & NR \\
\hline & NR \\
\hline & 16161.00 \\
\hline
\end{tabular}

* - VALUE eXCEEDS PRIMARY dRIMKING "ater STANDARD

- - YALUE EXCEEDS PROPOSED PRIMARY DRINKING VATER STANDARD.

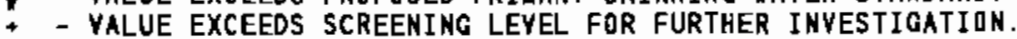

- DETECTIDN LIUIT tas hot AVAilable for couparison

NR - ANALYSIS NOT REQUESTED OR NOT YET REPORTED

VRLUES IY I I ARE COUNTING ERRORS FOR RADIONUCLIDES

MATER STANDARD'S) IN PARENTHESES ARE PROPDSED ONLY 
TABLE C. 3. (contd)

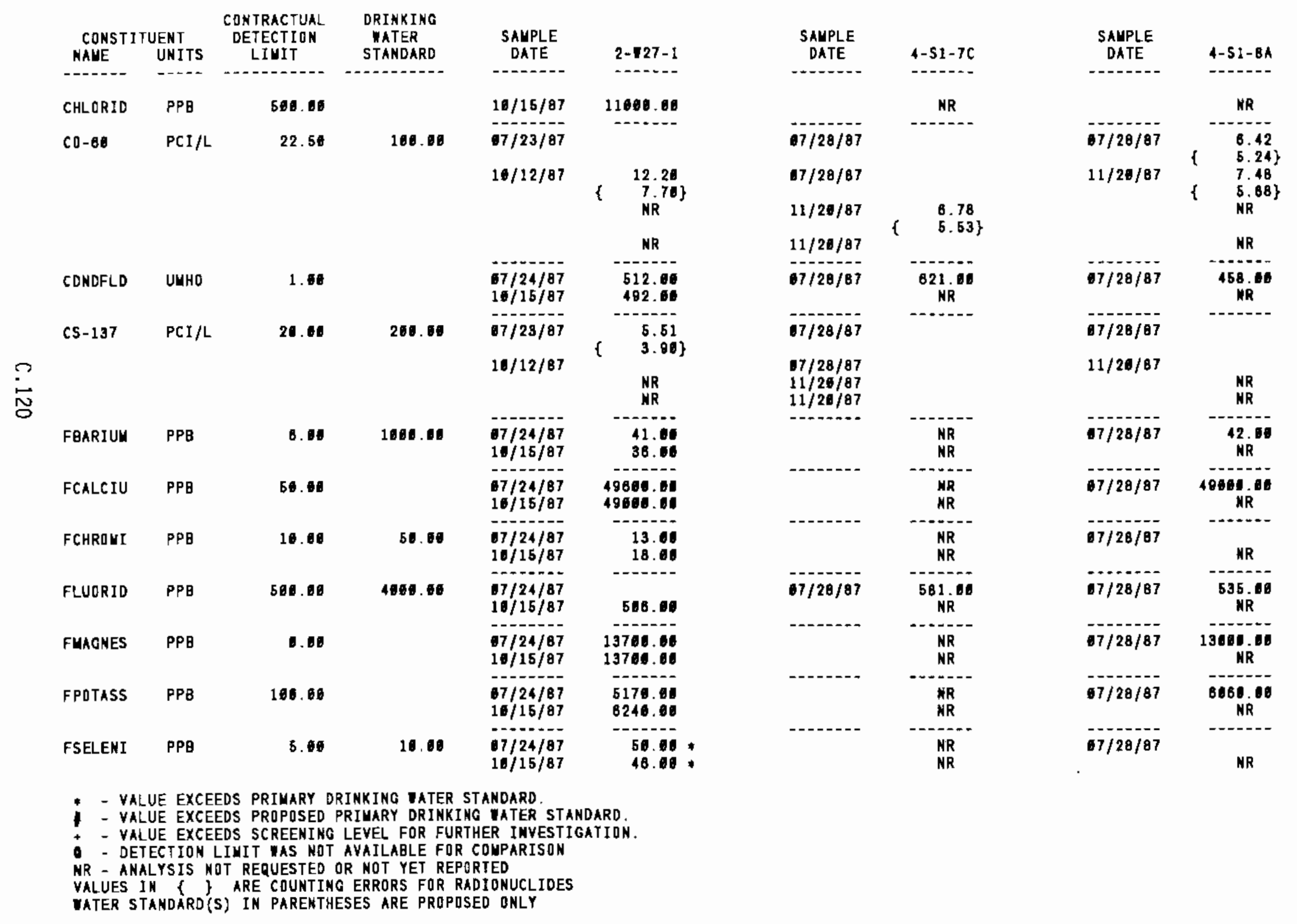


TABLE C.3. (contd)

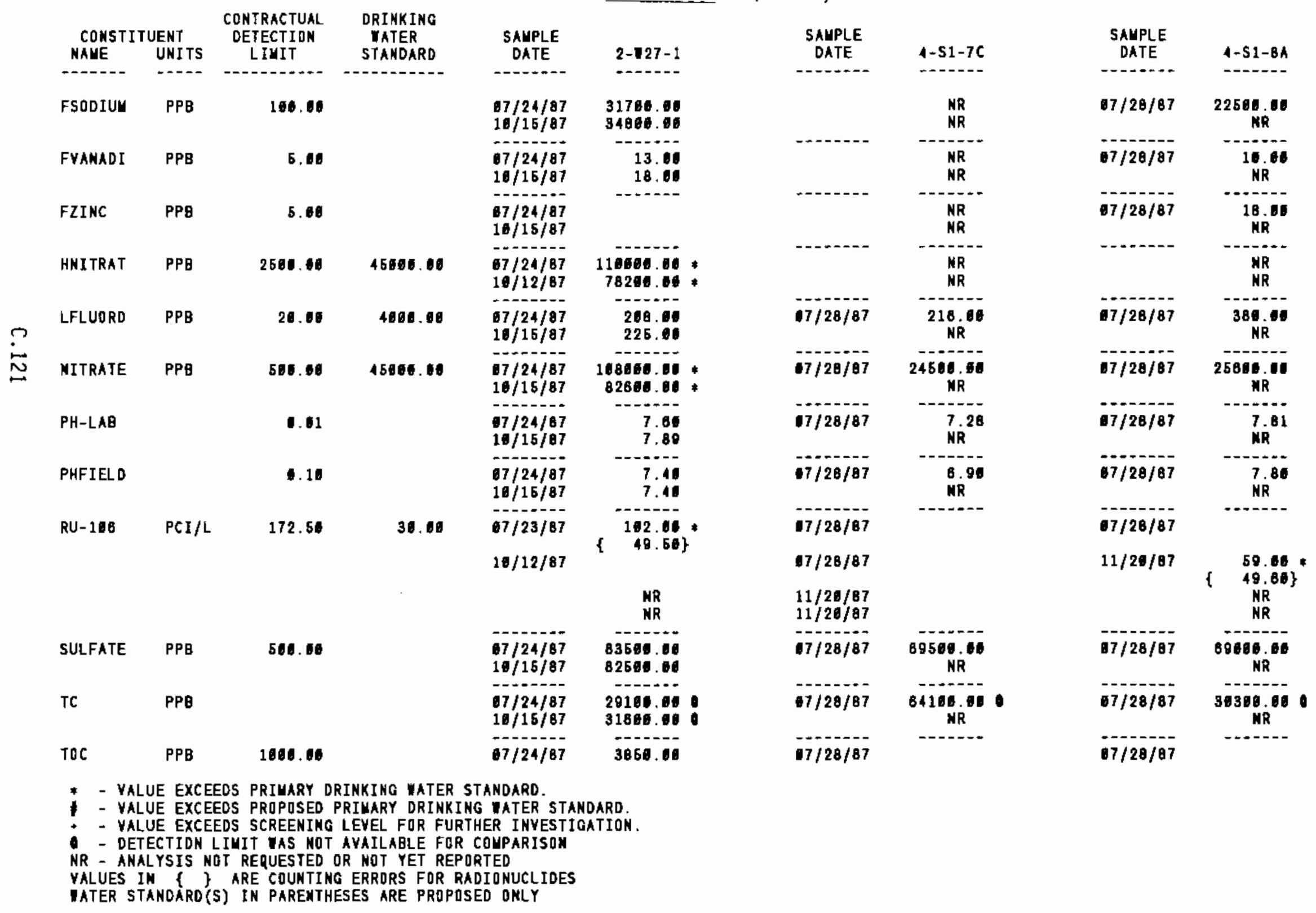


TABLE C.3. (contd)

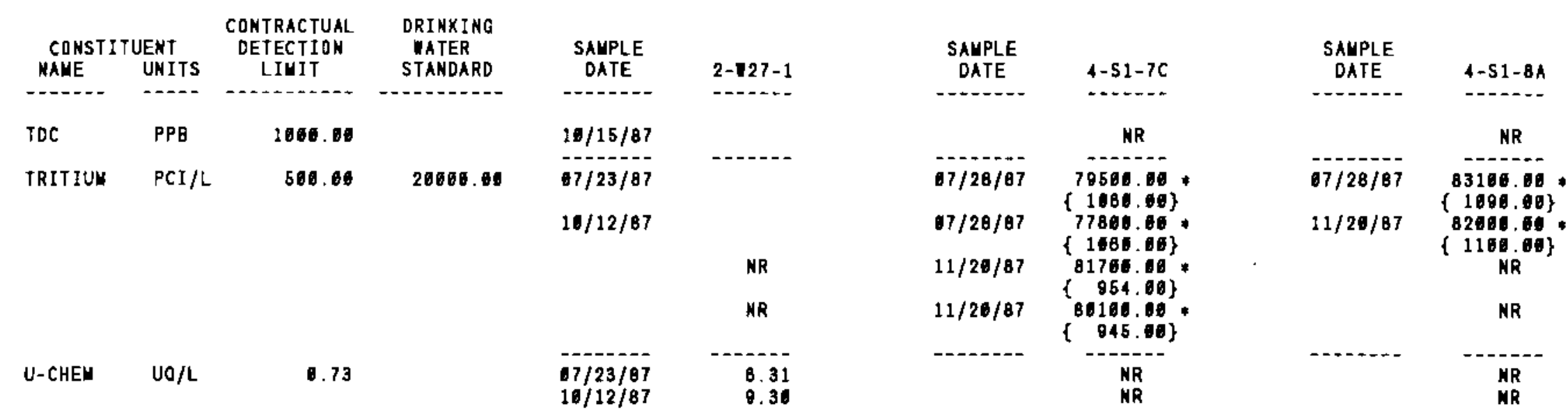

$\stackrel{n}{N}$

- YALUE EXCEEDS PRIUARY DRINKING TATER STANDARD.

- VALUE EXCEEDS PRDPDSED PRIUARY DRINKING WATER STANDARD.

+ - VALUE EXCEEDS SCREENING LEVEl FDR FURTHER INYESTIGATION

- DETECTION LIUIT TAS NOT AVAILABLE FOR COUPARISUN

NR - ANALYSIS NDT REQUESTED OR KOT YET REPDRTED

VALUES IN \{\} ARE COUNTING ERRORS FOR RAOIONUCLIDES

WATER STAMDARD'(S) IN PARENTHESES ARE PROPOSED ONLY 
TABLE C.3. (contd)

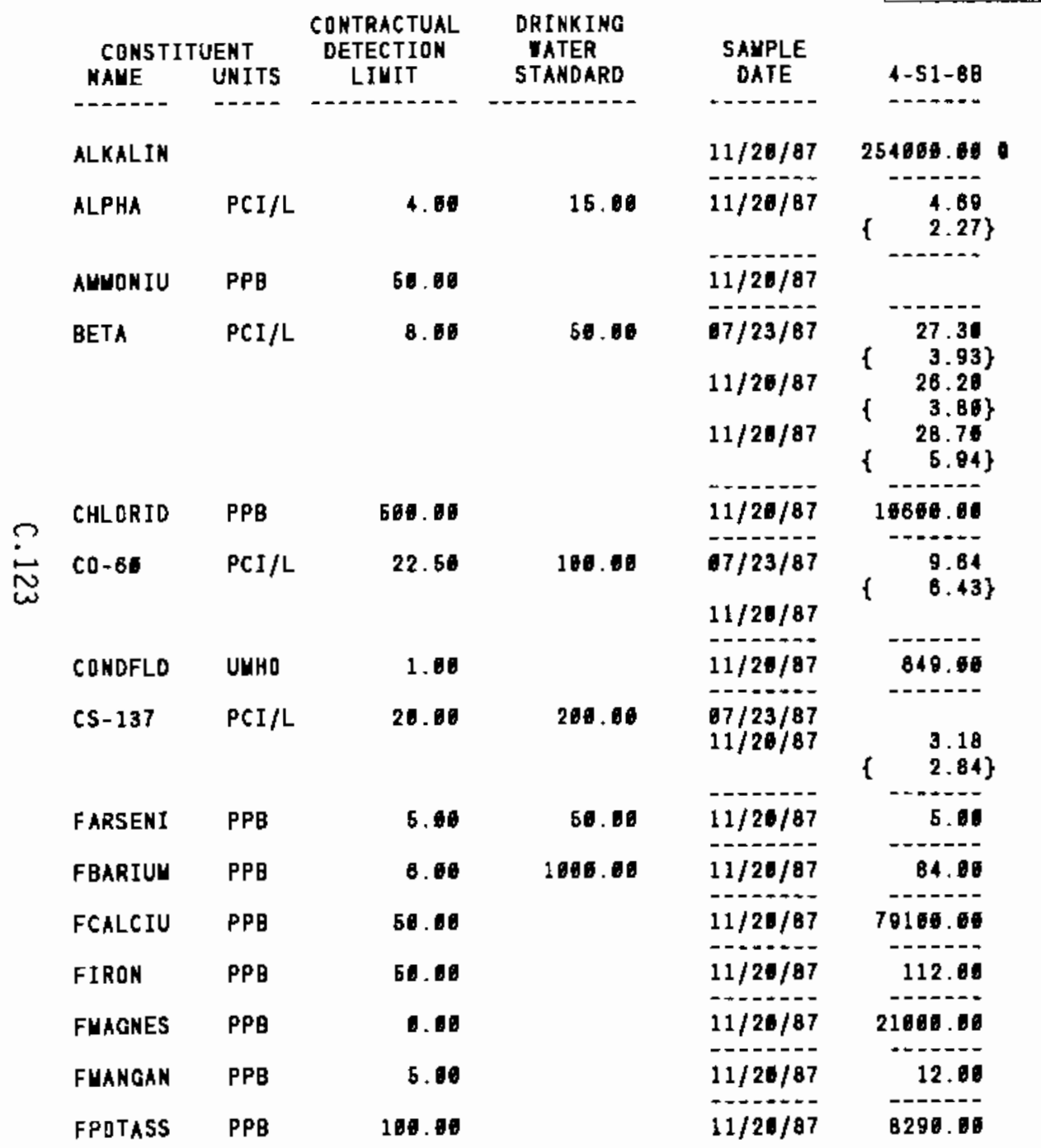

\begin{tabular}{|c|c|}
\hline $\begin{array}{l}\text { SALPLE } \\
\text { OATE }\end{array}$ & $0-2-3$ \\
\hline------- & ---- \\
\hline $09 / 09 / 87$ & 121000.60 \\
\hline $69 / 69 / 87$ & $\begin{array}{l}2.25 \\
1.39\}\end{array}$ \\
\hline $10 / 90 / 87$ & ---- \\
\hline $69 / 09 / 87$ & $\begin{array}{c}27.96 \\
5.78\} \\
N R\end{array}$ \\
\hline & NR \\
\hline ----U & ---- \\
\hline $68 / 60 / 87$ & 8030.00 \\
\hline $69 / 09 / 87$ & $\begin{array}{l}5.68 \\
5.68\}\end{array}$ \\
\hline $16 / 25 / 87$ & \\
\hline $99 / 60 / 97$ & 321.06 \\
\hline $\begin{array}{l}09 / 09 / 87 \\
10 / 25 / 87\end{array}$ & \\
\hline $09 / 69 / 87$ & $m$ \\
\hline $68 / 60 / 87$ & 46.00 \\
\hline $69 / 60 / 87$ & 38000.00 \\
\hline $00 / 00 / 07$ & $\ldots$ \\
\hline -...-_ & ----- \\
\hline $89 / 09 / 87$ & 11100.00 \\
\hline $60 / 09 / 87$ & \\
\hline $69 / 00 / 87$ & $58 \overline{70.00}$ \\
\hline
\end{tabular}

\begin{tabular}{|c|c|}
\hline $\begin{array}{c}\text { SANPLE } \\
\text { DATE }\end{array}$ & $8-2-33 A$ \\
\hline 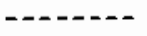 & - \\
\hline$B 8 / 24 / 87$ & 135000.00 \\
\hline $68 / 24 / 87$ & $\left\{\begin{array}{l}2.69 \\
\quad 1.33\end{array}\right\}$ \\
\hline $98 / 24 / 87$ & 77.00 \\
\hline \multirow{3}{*}{$08 / 24 / 87$} & ----- \\
\hline & NR \\
\hline & MR \\
\hline - - - - & $-1-1$ \\
\hline $68 / 24 / 87$ & 5830.06 \\
\hline ים & NR \\
\hline & NR \\
\hline $88 / 24 / 87$ & 294.18 \\
\hline------ & NR \\
\hline $8 / 24 / 87$ & ------ \\
\hline $68 / 24 / 87$ & 31.00 \\
\hline$-\cdots-10$ & 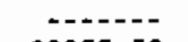 \\
\hline $08 / 24 / 87$ & 33864.00 \\
\hline $68 / 24 / 87$ & 01.00 \\
\hline $68 / 24 / 87$ & 11000.06 \\
\hline $68 / 24 / 87$ & $--1 .-1$ \\
\hline $08 / 24 / 87$ & 4250.06 \\
\hline
\end{tabular}

* - VAlUE eXceEdS prigary dRINKING tater standard.

- - VALUE EXCEEDS PROPOSED PRIMARY ORINKIMg TATER STANDARD.

- - VALUE EXCEEDS SCREENING LEVEL FOR FURTHER INVESTIGATION.

- DETECTION LIUIT IAS NOT AVALABLE FDR COUPARISON

NR - ANALYSIS NOT REQUESTED OR NOT YET REPORTED

YALUES IN \& \& ARE COUNTING ERRORS FOR RADIONUCLIDES

TATER STANDARD(S) IN PARENTHESES ARE PROPOSED ONLY 
TABLE C.3. (contd)

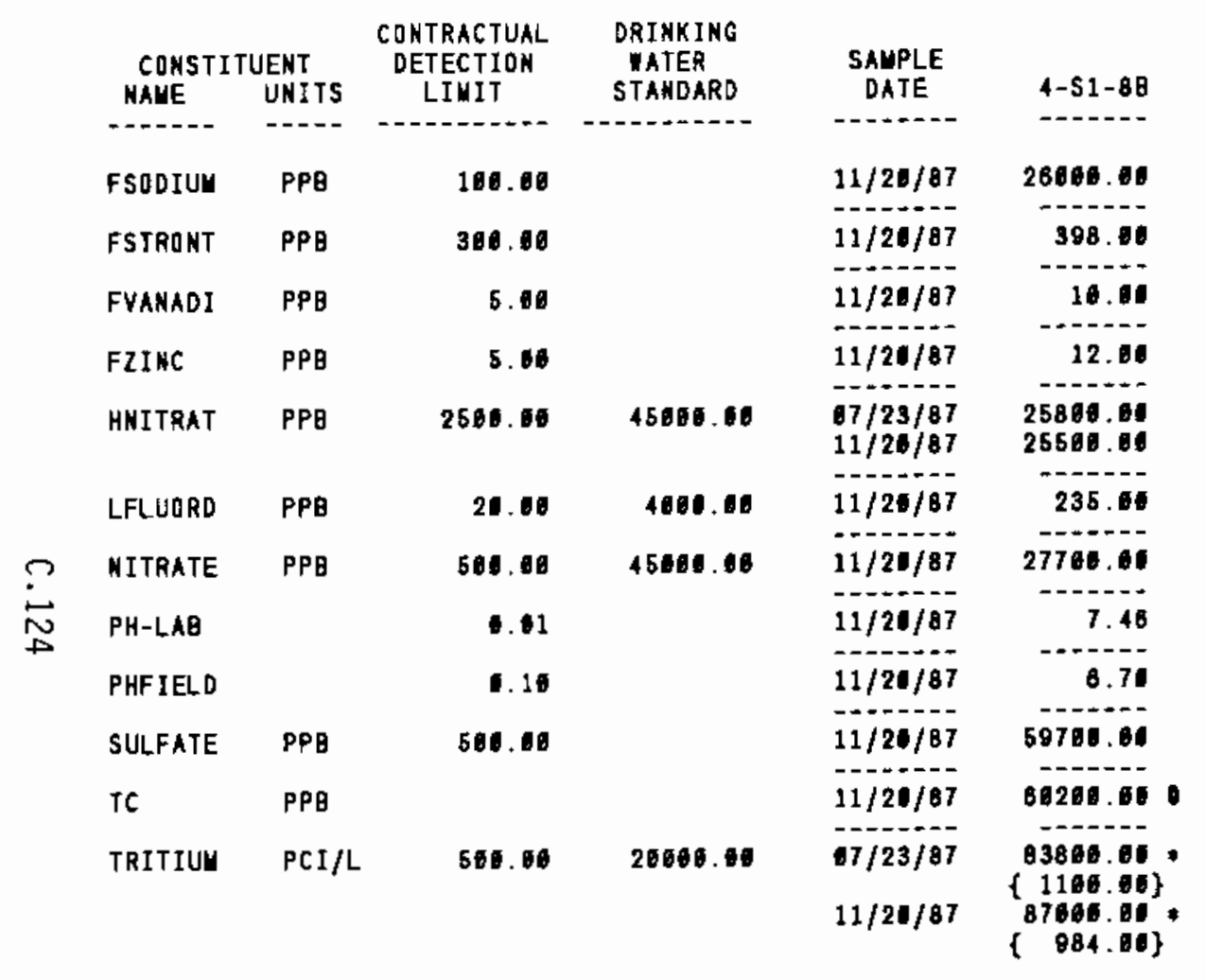

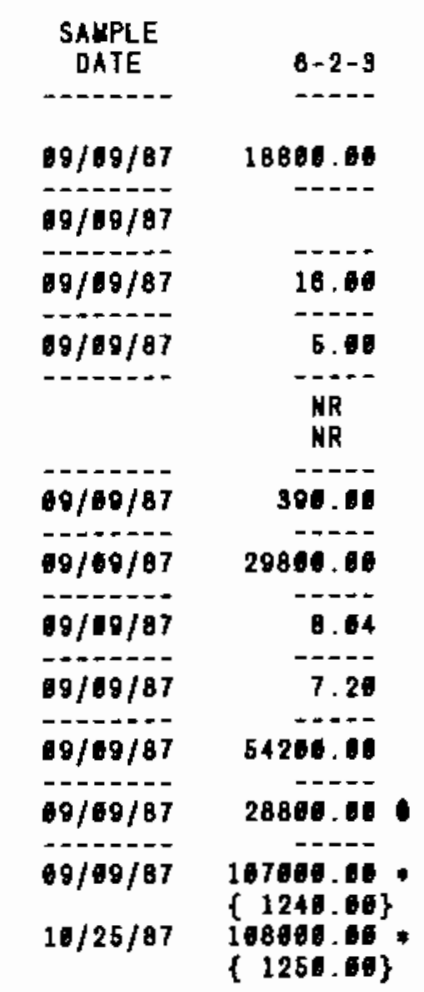

\begin{tabular}{|c|c|}
\hline $\begin{array}{l}\text { SAMPLE } \\
\text { DATE }\end{array}$ & $6-2-33 A$ \\
\hline - & 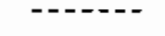 \\
\hline $08 / 24 / 87$ & 11800.00 \\
\hline $88 / 24 / 87$ & \\
\hline $68 / 24 / 87$ & 11.00 \\
\hline $08 / 24 / 87$ & $\cdots$ \\
\hline 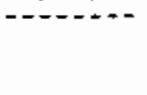 & $\begin{array}{l}\text { NR } \\
\text { NR }\end{array}$ \\
\hline $98 / 24 / 87$ & 394.08 \\
\hline $88 / 24 / 87$ & 2810.06 \\
\hline $88 / 24 / 87$ & $--1--\overline{7}$ \\
\hline$--.-1--$ & - \\
\hline $98 / 24 / 87$ & 6.70 \\
\hline 881248 & 3959 \\
\hline $18 / 24 / 87$ & 33580.06 \\
\hline $08 / 24 / 87$ & 31900.00 \\
\hline $88 / 24 / 87$ & \\
\hline $10 / 25 / 87$ & \\
\hline
\end{tabular}

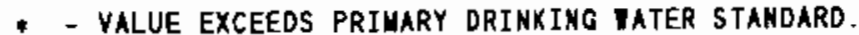

- - YALUE EXCEEDS PROPDSFD PRIUARY DRINKING TATER STANDARD.

- - YALUE EXCEEDS SCREENING LEYEL FOR FURTHER IMYESTIGATION.

- - DETECTIDN LIUIT IAS NOT AVAILABLE FOR COUPARISOH

NR - ANALYSIS NOT RERUESTED OR NOT YET REPORTED

VALUES IN ( ) ARE COUNTIMG ERRDRS FOR RADIONUCLIDES

WATER STANDARD(S) IN PARENTHESES ARE PROPOSED ONLY 


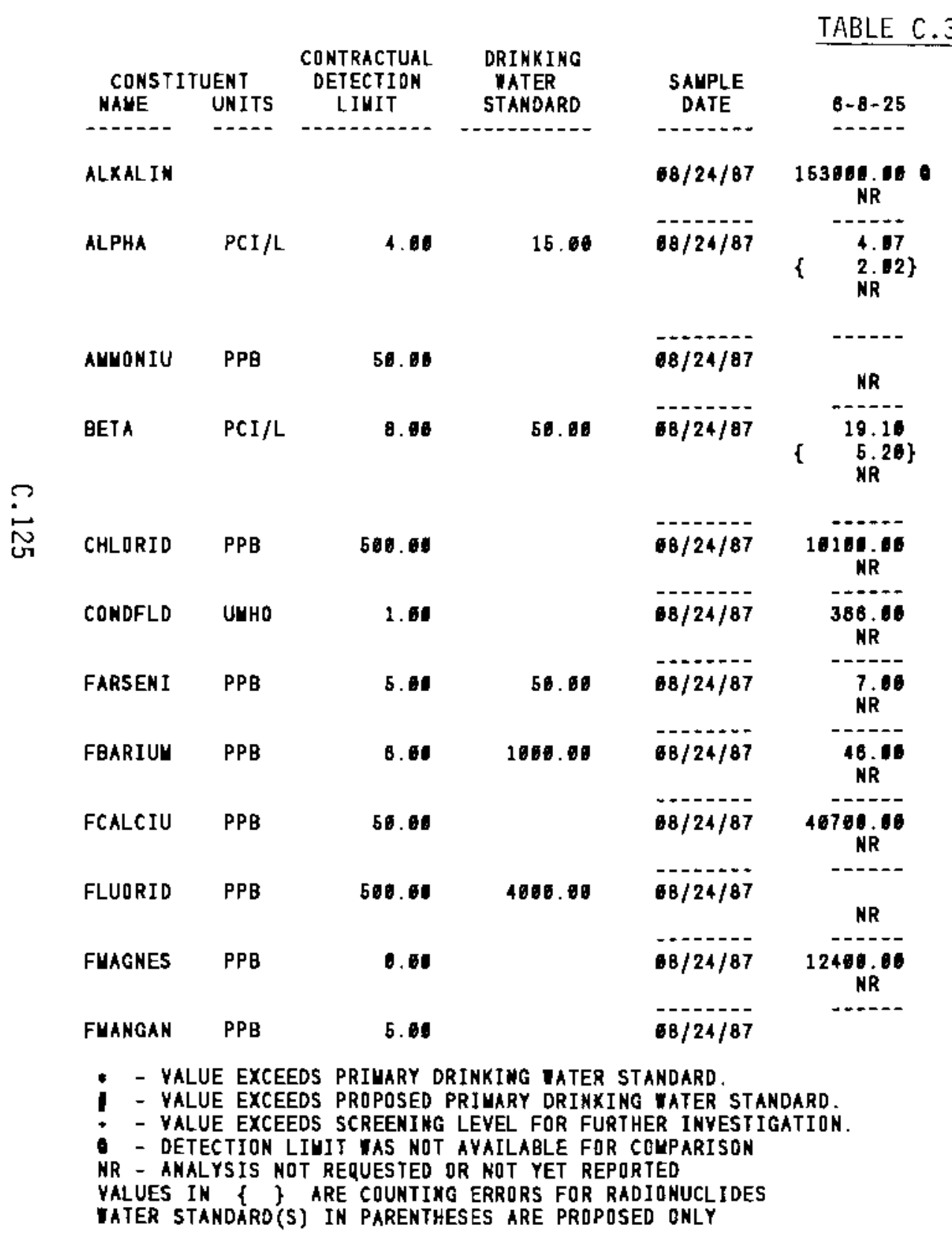

\begin{tabular}{|c|c|c|}
\hline $\begin{array}{c}\text { SAMPLE } \\
\text { DATE }\end{array}$ & \multicolumn{2}{|c|}{$6-9-E 2$} \\
\hline $\begin{array}{l}68 / 24 / 87 \\
16 / 27 / 87\end{array}$ & $\begin{array}{l}129000 \\
134600\end{array}$ & 1.068 \\
\hline $18 / 24 / 87$ & & .53 \\
\hline $10 / 27 / 87$ & $\begin{array}{ll} & 1 \\
1 & 1\end{array}$ & $\begin{array}{l}.71 \\
1.18\}\end{array}$ \\
\hline $\begin{array}{l}08 / 24 / 87 \\
10 / 27 / 87\end{array}$ & $\begin{array}{r}240 \\
88\end{array}$ & 3.00 \\
\hline $68 / 24 / 87$ & & .85 \\
\hline $16 / 27 / 87$ & $\left\{\begin{array}{l}4 \\
1\end{array}\right.$ & $\begin{array}{l}26 \\
.59\}\end{array}$ \\
\hline $\begin{array}{l}68 / 24 / 87 \\
10 / 27 / 87\end{array}$ & $\begin{array}{l}4580 \\
4648\end{array}$ & 1.00 \\
\hline $\begin{array}{l}08 / 24 / 87 \\
10 / 27 / 87\end{array}$ & $\begin{array}{l}258 \\
291\end{array}$ & 1.08 \\
\hline $\begin{array}{l}08 / 24 / 87 \\
16 / 27 / 87\end{array}$ & & 3.00 \\
\hline $\begin{array}{l}08 / 24 / 87 \\
16 / 27 / 87\end{array}$ & $\begin{array}{l}44 . \\
38 .\end{array}$ & 1.00 \\
\hline $\begin{array}{l}08 / 24 / 87 \\
10 / 27 / 87\end{array}$ & $\begin{array}{l}21780 \\
23000\end{array}$ & .00 \\
\hline $\begin{array}{l}08 / 24 / 87 \\
10 / 27 / 87\end{array}$ & 588 & 8.08 \\
\hline $\begin{array}{l}88 / 24 / 87 \\
10 / 27 / 87\end{array}$ & $\begin{array}{r}16100 \\
9880\end{array}$ & 8.08 \\
\hline $68 / 24 / 87$ & 20 & 8.00 \\
\hline
\end{tabular}

\begin{tabular}{|c|c|}
\hline $\begin{array}{l}\text { SAMPLE } \\
\text { DATE }\end{array}$ & $B-10-E 12$ \\
\hline $18 / 27 / 97$ & 258806.00 \\
\hline $10 / 27 / 87$ & $\left.\begin{array}{ll}\{ & \left.\begin{array}{l}1.69 \\
1.33\end{array}\right\} \\
\mathrm{NR}\end{array}\right\}$ \\
\hline $18 / 27 / 87$ & NR \\
\hline $18 / 27 / 87$ & $\left\{\begin{array}{l}9.60 \\
\mathrm{NR}\end{array}\right.$ \\
\hline $10 / 27 / 87$ & NR \\
\hline $10 / 27 / 87$ & 404.01 \\
\hline $18 / 27 / 67$ & KR \\
\hline $18 / 27 / 87$ & ${ }_{\mathrm{NR}}^{67.61}$ \\
\hline $10 / 27 / 87$ & NR \\
\hline $16 / 27 / 87$ & $\begin{array}{c}520.00 \\
\mathrm{NR}\end{array}$ \\
\hline $16 / 27 / 87$ & 24500.010 \\
\hline
\end{tabular}


TABLE C.3. (contd)

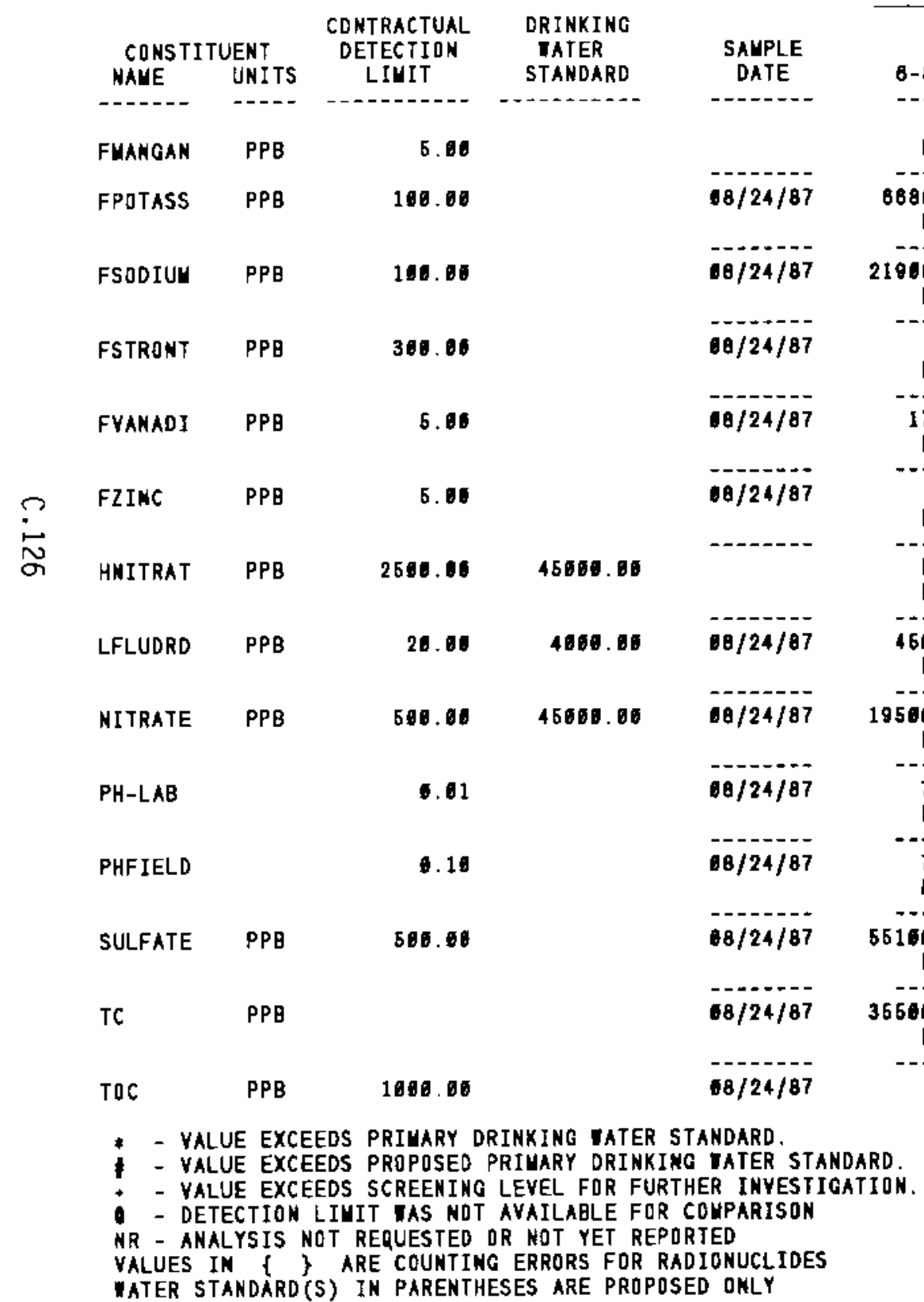

\begin{tabular}{|c|c|}
\hline $\begin{array}{l}\text { SALPLEE } \\
\text { DATE }\end{array}$ & $8-8-E 2$ \\
\hline $11 / 27 / 87$ & 24.66 \\
\hline $\begin{array}{l}68 / 24 / 87 \\
16 / 27 / 87\end{array}$ & $\begin{array}{l}8370.06 \\
8278.06\end{array}$ \\
\hline $\begin{array}{l}88 / 24 / 87 \\
16 / 27 / 87\end{array}$ & $\begin{array}{l}23500.00 \\
24600.00\end{array}$ \\
\hline $\begin{array}{l}08 / 24 / 87 \\
16 / 27 / 87\end{array}$ & \\
\hline $\begin{array}{l}60 / 24 / 87 \\
10 / 27 / 87\end{array}$ & \\
\hline $\begin{array}{l}06 / 24 / 87 \\
16 / 27 / 87\end{array}$ & 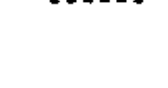 \\
\hline & $\begin{array}{l}\text { NR } \\
\text { NR }\end{array}$ \\
\hline $\begin{array}{l}68 / 24 / 87 \\
18 / 27 / 87\end{array}$ & $\begin{array}{l}460.60 \\
426.06\end{array}$ \\
\hline $\begin{array}{l}08 / 24 / 87 \\
18 / 27 / 87\end{array}$ & $\begin{array}{l}2070.00 \\
1476.00\end{array}$ \\
\hline $\begin{array}{l}88 / 24 / 87 \\
10 / 27 / 87\end{array}$ & $\begin{array}{l}7.93 \\
8.18\end{array}$ \\
\hline $\begin{array}{l}09 / 24 / 87 \\
10 / 27 / 87\end{array}$ & $\begin{array}{l}7.06 \\
7.90\end{array}$ \\
\hline $\begin{array}{l}08 / 24 / 87 \\
18 / 27 / 87\end{array}$ & $\begin{array}{l}29800.00 \\
28866.00\end{array}$ \\
\hline $\begin{array}{l}88 / 24 / 87 \\
18 / 27 / 87\end{array}$ & 32100.00 \\
\hline $08 / 24 / 87$ & 1370.00 \\
\hline
\end{tabular}

\begin{tabular}{|c|c|}
\hline \multirow[t]{2}{*}{$\begin{array}{c}\text { SAMPLE } \\
\text { DATE }\end{array}$} & $0-10-E 12$ \\
\hline & NR \\
\hline $16 / 27 / 87$ & $\underset{N R}{8800.06}$ \\
\hline $10 / 27 / 87$ & 19100.010 \\
\hline $10 / 27 / 87$ & 522.61 \\
\hline $10 / 27 / 87$ & ${ }_{\mathrm{NR}}^{14.00}$ \\
\hline $10 / 27 / 87$ & $\begin{array}{l}9.11 \\
\text { MR }\end{array}$ \\
\hline $\begin{array}{l}07 / 28 / 87 \\
10 / 27 / 87\end{array}$ & $\begin{array}{l}18800.00 \\
20300.00\end{array}$ \\
\hline $10 / 27 / 87$ & $\begin{array}{l}248.06 \\
\text { NR }\end{array}$ \\
\hline $10 / 27 / 87$ & 22900.00 \\
\hline $10 / 27 / 87$ & $\begin{array}{l}7.84 \\
\text { NR }\end{array}$ \\
\hline $10 / 27 / 87$ & $\begin{array}{l}7.68 \\
\mathrm{NR}\end{array}$ \\
\hline $18 / 27 / 87$ & 29104.68 \\
\hline $10 / 27 / 87$ & $\frac{69000.00}{\mathrm{KR}}$ \\
\hline $10 / 27 / 87$ & \\
\hline
\end{tabular}


TABLE C.3. (contd)

\begin{tabular}{|c|c|c|c|c|c|c|c|c|c|}
\hline $\begin{array}{l}\text { CONST I } \\
\text { WANE }\end{array}$ & $\begin{array}{l}\text { UENT } \\
\text { UNITS }\end{array}$ & $\begin{array}{l}\text { CONTRACTUAL } \\
\text { DETEIION } \\
\text { LIMIT }\end{array}$ & $\begin{array}{l}\text { DRIAK ING } \\
\text { WATER } \\
\text { STANDARD }\end{array}$ & $\begin{array}{c}\text { SAHPLE } \\
\text { DATE }\end{array}$ & $6-8-25$ & $\begin{array}{c}\text { SAHPLE } \\
\text { DATE }\end{array}$ & $6-8-E 2$ & $\begin{array}{c}\text { SAUPLE } \\
\text { DATE }\end{array}$ & $6-10-E 12$ \\
\hline 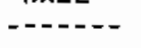 & $\ldots$ & 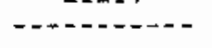 & - - & $\ldots . .$. & $\ldots-$. & $\ldots . . .-$ & -... & $-\ldots+-$ & -......- \\
\hline TOC & $P P B$ & 1080.00 & & & NR & $10 / 27 / 87$ & & & NR \\
\hline TRITIUU & $\mathrm{PCI} / \mathrm{L}$ & 500.00 & 20600.00 & $\begin{array}{l}08 / 24 / 87 \\
10 / 25 / 87\end{array}$ & $\begin{array}{c}38200.00 \\
\left\{\begin{array}{c}852.00\} \\
34808.09\end{array}\right. \\
\{724.00\}\end{array}$ & $\begin{array}{l}68 / 24 / 87 \\
1 / 27 / 87\end{array}$ & $\begin{array}{l}710.00 \\
183.00\}\end{array}$ & $\begin{array}{l}07 / 29 / 87 \\
16 / 27 / 87\end{array}$ & 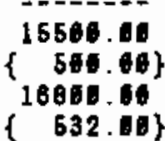 \\
\hline
\end{tabular}

- - VALUE EXCEEDS PRIUARY DRINKINg vater standaro.

- VALUE EXCEEDS PROPOSED PRIMARY DRINKING UATER STANOARD.

- - yalue exceEds sCREening level for further inyestigation.

- DETECTION LIHIT TAS NOT AVAILABLE FDR COUPARISON

NR - ANALYSIS NDT REQUESTED OR NOT YET REPORTED

VALUES IN \& ? ARE COUNTING ERRORS FOR RADIONUCLIDES

WATER STAMDARD'(S) IN PARENTHESES ARE PROPOSED ONLY 
TABLE C.3. (contd)

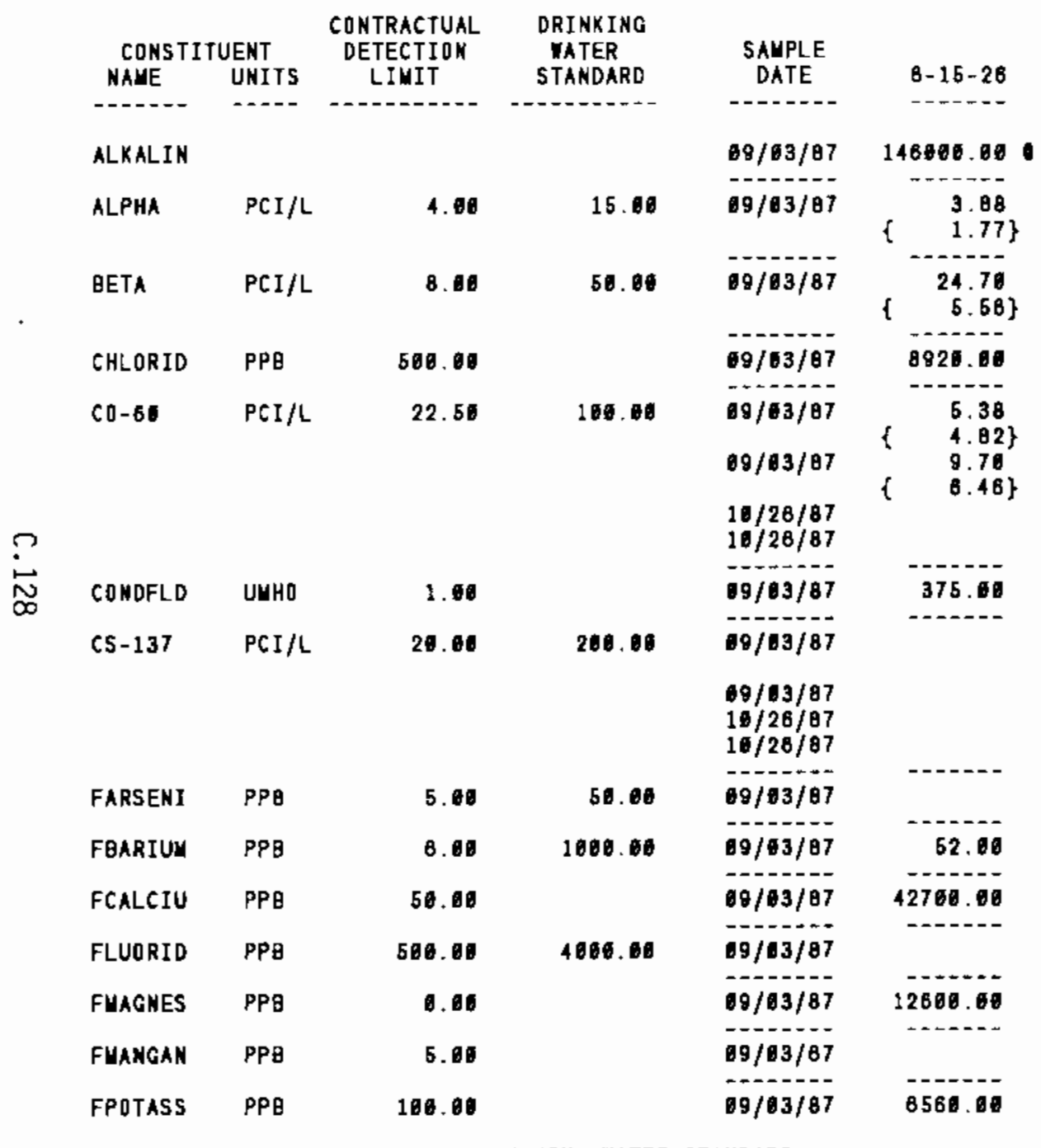

\begin{tabular}{|c|c|}
\hline $\begin{array}{l}\text { SAMPLE } \\
\text { DATE }\end{array}$ & $6-18-43$ \\
\hline$+\ldots+\infty$ & - \\
\hline $12 / 28 / 87$ & 116000.00 \\
\hline $12 / 2 \theta / 87$ & $\begin{array}{ll} & 3.61 \\
\{\quad 1.62\}\end{array}$ \\
\hline $12 / 28 / 87$ & $\begin{array}{l}\quad 0.55 \\
\{\quad 3.61\}\end{array}$ \\
\hline $12 / 28 / 87$ & 7880.00 \\
\hline - ה- & NR \\
\hline & NR \\
\hline & $\begin{array}{l}\text { NR } \\
\text { NR }\end{array}$ \\
\hline $12 / 28 / 87$ & 312.06 \\
\hline------ & NR \\
\hline & $\begin{array}{l}\text { NR } \\
\text { NR } \\
\text { NR }\end{array}$ \\
\hline $12 / 20 / 87$ & ------ \\
\hline $12 / 20 / 87$ & 56.60 \\
\hline $12 / 28 / 87$ & 42300.00 \\
\hline$---1---$ & ---- \\
\hline $12 / 28 / 87$ & 574.09 \\
\hline $12 / 28 / 67$ & 16700.00 \\
\hline $12 / 28 / 87$ & 8.00 \\
\hline $12 / 28 / 87$ & 5710.60 \\
\hline
\end{tabular}

\begin{tabular}{|c|c|}
\hline $\begin{array}{l}\text { SAUPLE } \\
\text { DATE }\end{array}$ & $8-20-E 5 A$ \\
\hline 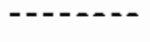 & ט-n \\
\hline $68 / 28 / 87$ & 118000.00 \\
\hline $68 / 28 / 87$ & $\left\{\begin{array}{l}2.93 \\
1.32\}\end{array}\right.$ \\
\hline $08 / 28 / 87$ & $\left\{\begin{array}{c}17.26 \\
\{\quad 4.83\}\end{array}\right.$ \\
\hline $68 / 28 / 87$ & 8400.00 \\
\hline $68 / 25 / 87$ & \\
\hline \multicolumn{2}{|l|}{$10 / 25 / 87$} \\
\hline & $\begin{array}{l}\text { NR } \\
\text { NR }\end{array}$ \\
\hline $68 / 28 / 87$ & 304.00 \\
\hline $8 / 25 / 67$ & 5.34 \\
\hline $10 / 25 / 87$ & $\begin{array}{l}3.77\} \\
\text { NR } \\
\text { NR }\end{array}$ \\
\hline $88 / 28 / 87$ & 8.00 \\
\hline $08 / 28 / 87$ & 64.06 \\
\hline $08 / 28 / 87$ & 34900.00 \\
\hline $8 / 28 / 87$ & 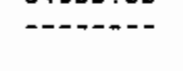 \\
\hline $6 B / 28 / 87$ & 10300.60 \\
\hline $60 / 28 / 87$ & $\cdots+\cdots$ \\
\hline $68 / 28 / 87$ & 5376.69 \\
\hline
\end{tabular}

* - VAlUE EXCEEDS PRIUARY DRINKING vater standaro.

- VALUE EXCEEDS PROPOSED PRIUARY DRINKING WATER STANDARO.

- - VALUE EXCEEDS SCREENINg LEVEL FQR FURTHER INVESTIGATION.

- DETECTION LIMIT IAS XOT AVAILABLE FOR COUPARISIN

NR - ANALYSIS NOT REQUESTED OR NOT YET REPORTED

VALUES IN \& ) ARE CDUNTING ERRORS FOR RADIONUCLIDES

TATER STAMDARD(S) IN PARERTHESES ARE PRDPOSED ONLY 
TABLE C.3. (contd)

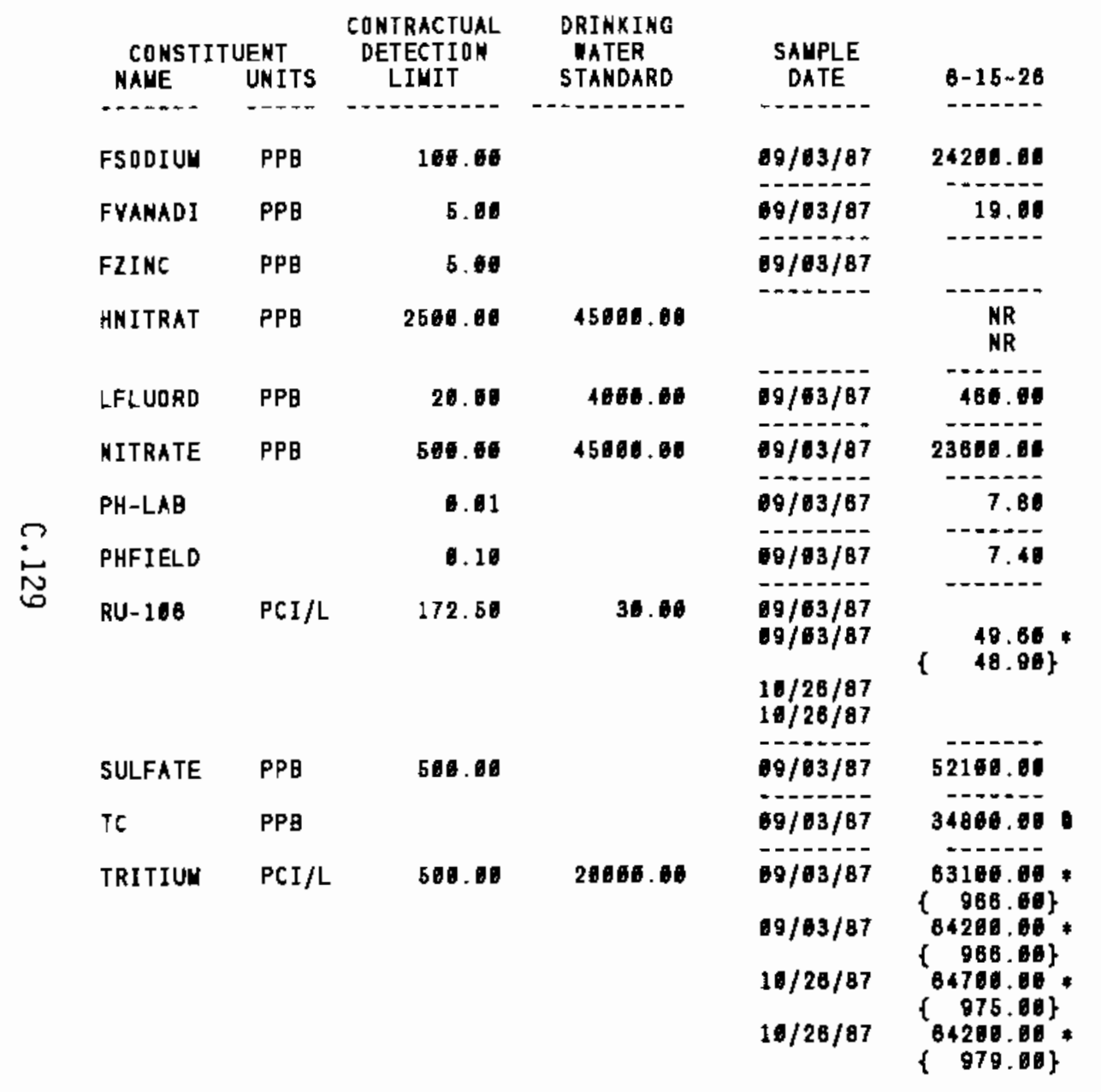

\begin{tabular}{|c|c|c|c|}
\hline $\begin{array}{l}\text { SAUPLE } \\
\text { DATE }\end{array}$ & $8-19-43$ & $\begin{array}{c}\text { SALPLE } \\
\text { DATE }\end{array}$ & $B \sim 26-E 5 A$ \\
\hline $12 / 28 / 87$ & 20100.00 & $08 / 28 / 87$ & 12900.06 \\
\hline $12 / 28 / 87$ & 7.00 & $08 / 28 / 87$ & 19.00 \\
\hline $12 / 28 / 87$ & 218.00 & $08 / 28 / 87$ & \\
\hline $\begin{array}{l}89 / 27 / 87 \\
12 / 28 / 87\end{array}$ & $\begin{array}{r}12866.07 \\
9326.06\end{array}$ & & $\begin{array}{l}\text { NR } \\
\text { NR }\end{array}$ \\
\hline $12 / 28 / 87$ & 410.00 & $68 / 28 / 87$ & 278.6 \\
\hline $12 / 28 / 87$ & 16160.80 & $68 / 28 / 87$ & 24400.66 \\
\hline $12 / 2 B / 87$ & 7.92 & $88 / 28 / 87$ & 8.19 \\
\hline \multirow[t]{2}{*}{$12 / 28 / 87$} & 7.76 & $08 / 28 / 87$ & 7.48 \\
\hline & $\begin{array}{l}\text { NR } \\
\text { NR } \\
\text { NR } \\
\text { NR }\end{array}$ & $\begin{array}{l}08 / 25 / 87 \\
18 / 25 / 87\end{array}$ & $\left\{\begin{array}{c}73.50 \\
68.88\} \\
M R \\
M R\end{array}\right\}$ \\
\hline $12 / 28 / 87$ & 80400.00 & $08 / 28 / 87$ & 32660.00 \\
\hline $12 / 2 B / 87$ & 25300.000 & $68 / 28 / 07$ & 28980.090 \\
\hline $88 / 27 / 87$ & & $68 / 25 / 87$ & 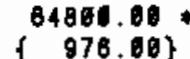 \\
\hline $12 / 28 / 87$ & MR & $10 / 25 / 87$ & $\left\{\begin{array}{c}67468.68 \\
064.80 \\
M R\end{array}\right\}$ \\
\hline & NR & & MR \\
\hline
\end{tabular}

* - Value exceeds priuary drinking vater standard.

- VALUE EXCEEDS PROPOSED PRIMARY DRINKING IATER STANDARD.

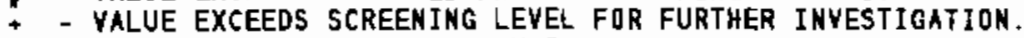

- DETECTIOK LIHIT Yas nOt aVaILable for couparison

NR - ANALYSIS NOT REQUESTED OR NOT YET REPORTED

VALUES IN \{ $\}$ ARE CDUNTING ERRORS FDR RADIONUCLIDES

WATER STANDARD'(S) IN PARENTHESES ARE PROPOSED OHLY 
TABLE C.3. (contd)

\begin{tabular}{|c|c|c|c|c|c|}
\hline \multicolumn{2}{|c|}{ CONSTITUENT } & $\begin{array}{c}\text { CONTRACTUAL } \\
\text { OETECTIOK } \\
\text { LIUIT }\end{array}$ & $\begin{array}{l}\text { DRINKIHG } \\
\text { IATER } \\
\text { STANDARD }\end{array}$ & $\begin{array}{l}\text { SANPLE } \\
\text { DATE }\end{array}$ & $8-20-20$ \\
\hline$\cdots-\cdots$ & ---- & - - - & - - & $-----n$ & ----- \\
\hline $1,1,1-T$ & PPB & 10.00 & 260.66 & $68 / 26 / 87$ & NR \\
\hline ALKALIN & & & & $08 / 26 / 87$ & 108006.00 \\
\hline ALPHA & $\mathrm{PCI} / \mathrm{L}$ & 4.00 & 15.60 & $08 / 26 / 87$ & $\begin{array}{ll}\left\{\quad \begin{array}{l}2.75 \\
1.53\end{array}\right\} & \mathrm{NR}\end{array}$ \\
\hline AHMONIU & PPB & 50.60 & & $68 / 26 / 87$ & S3. \\
\hline BETA & $\mathrm{PCI} / \mathrm{L}$ & 8.60 & 56.06 & $68 / 26 / 87$ & $\left\{\begin{array}{c}29.70 \\
\text { B. } \\
\text { NR }\end{array}\right\}$ \\
\hline CHLORID & $\mathrm{PPB}$ & 500.00 & & $88 / 26 / 87$ & $\begin{array}{c}14000.00 \\
\text { NR }\end{array}$ \\
\hline$C O-60$ & $\mathrm{PCI} / \mathrm{L}$ & 22.50 & 100.00 & $\begin{array}{l}68 / 28 / 87 \\
18 / 28 / 87\end{array}$ & $\begin{array}{r}17.36 \\
\{\quad 8.64\}\end{array}$ \\
\hline CDNDFLD & UUH口 & 1.60 & & $68 / 26 / 87$ & 339.68 \\
\hline FARSENI & PPB & 6.06 & 50.00 & $18 / 26 / 87$ & 10.00 \\
\hline FBARIUU & PPB & B. 10 & $1600: 00$ & $O B / 26 / 87$ & ${ }_{\mathrm{NR}}^{64.60}$ \\
\hline FCALCIU & PPB & 68.66 & & $68 / 28 / 87$ & $\begin{array}{c}38100.06 \\
N 8\end{array}$ \\
\hline FLUORID & PPB & 580.06 & 4060.06 & $68 / 26 / 87$ & \\
\hline $\begin{array}{l}:-V_{A} \\
t-V_{A} \\
+-V_{A} \\
0-D E \\
\text { NR }-A N \\
\text { VALUES } \\
\text { WATERS }\end{array}$ & $\begin{array}{l}\text { UE EXCE } \\
\text { UE EXCE } \\
\text { UE EXCE } \\
\text { ECTIQN } \\
\text { LYSIS H } \\
\text { N \{ \} } \\
\text { ANDARD? }\end{array}$ & $\begin{array}{l}\text { EDS PRIHARY } \\
\text { EDS PROPOSED } \\
\text { EDS SCREENIK } \\
\text { LINIT NAS NO } \\
\text { OT REQUESTED } \\
\text { ARE COUNTI } \\
\text { S) IN PAREMT }\end{array}$ & $\begin{array}{l}\text { RINKING VATE } \\
\text { PRIUARY DRIN } \\
\text { LEVEL FOR F } \\
\text { AVAILABLE F } \\
\text { OR NOT YET R } \\
\text { G ERRBRS FOR } \\
\text { ESES ARE PRO }\end{array}$ & $\begin{array}{l}\text { ANDARD. } \\
\text { IATER ST, } \\
\text { ER INVEST } \\
\text { OMPARISON } \\
\text { TED } \\
\text { IONUCLIOES } \\
\text { O ONLY }\end{array}$ & $\begin{array}{l}\text { DARD. } \\
\text { ATIOX. }\end{array}$ \\
\hline
\end{tabular}

\begin{tabular}{|c|c|}
\hline $\begin{array}{l}\text { SAUPLE } \\
\text { DATE }\end{array}$ & $6-28-39$ \\
\hline - - & ------ \\
\hline $\begin{array}{l}08 / 25 / 87 \\
11 / 19 / 87\end{array}$ & \\
\hline $\begin{array}{l}8 / 25 / 87 \\
11 / 19 / 87\end{array}$ & $\begin{array}{l}123000.00 \\
129000.06\end{array}$ \\
\hline $18 / 25 / 87$ & 1.81 \\
\hline $11 / 19 / 87$ & 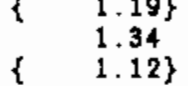 \\
\hline $\begin{array}{l}18 / 25 / 87 \\
11 / 19 / 87\end{array}$ & -..... \\
\hline $\begin{array}{l}68 / 25 / 87 \\
11 / 18 / 87\end{array}$ & $\left\{\begin{array}{l}9.36 \\
4.86\}\end{array}\right.$ \\
\hline $\begin{array}{l}68 / 25 / 87 \\
11 / 19 / 87\end{array}$ & $\begin{array}{l}-1--1-- \\
4580.60 \\
4540.00\end{array}$ \\
\hline$\cdots-1-1$ & MR \\
\hline & NR \\
\hline $11 / 19 / 87$ & 270.00 \\
\hline $\begin{array}{l}08 / 25 / 87 \\
11 / 19 / 87\end{array}$ & 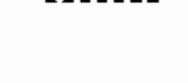 \\
\hline $\begin{array}{l}88 / 25 / 87 \\
11 / 10 / 87\end{array}$ & $\begin{array}{l}29.60 \\
30.60\end{array}$ \\
\hline $\begin{array}{l}0 / 25 / 87 \\
11 / 18 / 87\end{array}$ & 27000.00 \\
\hline $88 / 25 / 87$ & ----- \\
\hline
\end{tabular}

\begin{tabular}{|c|c|}
\hline $\begin{array}{l}\text { SAHPLE } \\
\text { DATE }\end{array}$ & $6-24-33$ \\
\hline$-\ldots+\ldots$ & ----- \\
\hline $\begin{array}{l}98 / 25 / 87 \\
11 / 16 / 87\end{array}$ & $\begin{array}{l}18.00 \\
23.00\end{array}$ \\
\hline $\begin{array}{l}08 / 25 / 87 \\
11 / 16 / 87\end{array}$ & $\begin{array}{l}192000.00 \\
184000.00\end{array}$ \\
\hline $8 / 25 / 87$ & 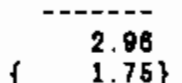 \\
\hline $11 / 16 / 87$ & $\begin{array}{r}3.88 \\
\{\quad 1.82\}\end{array}$ \\
\hline $\begin{array}{l}68 / 25 / 87 \\
11 / 10 / 87\end{array}$ & \\
\hline $08 / 25 / 87$ & 14.86 \\
\hline $11 / 10 / 87$ & $\begin{array}{c}4.83\} \\
24.96 \\
\{\quad 5.48\}\end{array}$ \\
\hline $\begin{array}{l}08 / 25 / 87 \\
11 / 10 / 87\end{array}$ & $\begin{array}{l}-780.0 \\
8280.00 \\
8790.00\end{array}$ \\
\hline $68 / 25 / 97$ & $-\ldots, n$ \\
\hline $11 / 16 / 87$ & \\
\hline $11 / 16 / 87$ & 494.00 \\
\hline $\begin{array}{l}98 / 25 / 87 \\
11 / 10 / 87\end{array}$ & 5.06 \\
\hline $\begin{array}{l}68 / 25 / 07 \\
11 / 10 / 07\end{array}$ & $\begin{array}{l}57.06 \\
81.00\end{array}$ \\
\hline $\begin{array}{l}08 / 25 / 87 \\
11 / 16 / 87\end{array}$ & $\begin{array}{r}49300.00 \\
59700.00\end{array}$ \\
\hline $68 / 25 / 87$ & \\
\hline
\end{tabular}




\section{TABLE C.3. (contd)}

\begin{tabular}{|c|c|c|c|c|c|}
\hline$\underset{\text { NAME }}{\text { CONSTI }}$ & $\begin{array}{l}\text { UENT } \\
\text { UNITS }\end{array}$ & $\begin{array}{c}\text { CONTRACTUAL } \\
\text { DETECTION } \\
\text { LIUIT }\end{array}$ & $\begin{array}{l}\text { DRINKIHG } \\
\text { IAIER } \\
\text { STANDARD }\end{array}$ & $\begin{array}{l}\text { SAUPLE } \\
\text { DATE }\end{array}$ & $8-20-20$ \\
\hline FLUURID & PPB & 500.00 & 4800.00 & & NR \\
\hline FUAGMES & $P P B$ & 0.06 & & $88 / 20 / 87$ & ${ }_{\text {MR }}^{80401}$ \\
\hline Fyangan & PPB & 6.00 & & $19 / 26 / 87$ & NR \\
\hline FPOTASS & PPB & 100.06 & & $68 / 20 / 87$ & Boge.eI \\
\hline FSDDIUY & PPB & 104.01 & & $18 / 28 / 87$ & $1006 \mathrm{NR}$ \\
\hline FVANADI & PPB & 5.06 & & $68 / 26 / 87$ & 13.00 \\
\hline LFLUQRD & PPB & 20.00 & 40180.66 & & MR \\
\hline УETHYCH & PPB & 10.00 & & $08 / 28 / 67$ & $\begin{array}{l}13.14 \\
\text { NR }\end{array}$ \\
\hline NItRATE & PPB & 600.06 & 45000.00 & $08 / 26 / 87$ & $\underset{\mathrm{MR}}{36064.60}$ \\
\hline PH-LAB & & 6.01 & & $10 / 28 / \theta 7$ & $\mathrm{MR}^{7.68}$ \\
\hline PHFIELD & & 1. 10 & & $8 / 26 / 87$ & 7.80 \\
\hline SULFATE & PPB & 584.06 & & $6 / 26 / 87$ & $\$ 2100.01$ \\
\hline TC & PPB & & & $88 / 28 / 87$ & $\underset{\mathrm{HR}}{25380 \mathrm{~L}}$ \\
\hline TRITIUY & $\mathrm{PCI} / \mathrm{L}$ & 506.80 & 20000.00 & $18 / 26 / 87$ & $\left\{\begin{array}{l}180100.94 \\
\{1429.06\}\end{array}\right.$ \\
\hline
\end{tabular}

\begin{tabular}{|c|c|c|c|}
\hline $\begin{array}{c}\text { SAUPLE } \\
\text { DATE }\end{array}$ & $6-20-39$ & $\begin{array}{c}\text { SAUPLE } \\
\text { DATE }\end{array}$ & $6-24-38$ \\
\hline $11 / 10 / 87$ & 537.00 & $11 / 1 / / 87$ & 678.19 \\
\hline $\begin{array}{l}08 / 25 / 87 \\
11 / 19 / 87\end{array}$ & $\begin{array}{l}9574.14 \\
0840.11\end{array}$ & $\begin{array}{l}68 / 25 / 87 \\
11 / 11 / 87\end{array}$ & $\begin{array}{l}14400.10 \\
10201.01\end{array}$ \\
\hline $\begin{array}{l}18 / 25 / 87 \\
11 / 10 / 87\end{array}$ & $\begin{array}{r}8.10 \\
14.10\end{array}$ & $\begin{array}{l}18 / 25 / 87 \\
11 / 10 / 87\end{array}$ & \\
\hline $\begin{array}{l}08 / 25 / 87 \\
11 / 19 / 87\end{array}$ & 4709.6 & $\begin{array}{l}08 / 26 / 87 \\
11 / 16 / 87\end{array}$ & $\begin{array}{l}6850.01 \\
8330.10\end{array}$ \\
\hline $\begin{array}{l}68 / 25 / 87 \\
11 / 10 / 87\end{array}$ & $\begin{array}{l}14800.40 \\
15760.4\end{array}$ & $\begin{array}{l}60 / 25 / 87 \\
11 / 10 / 87\end{array}$ & 24101010 \\
\hline $\begin{array}{l}10 / 25 / 87 \\
11 / 10 / 87\end{array}$ & $\begin{array}{l}5.00 \\
8.04\end{array}$ & $\begin{array}{l}18 / 25 / 87 \\
11 / 10 / 07\end{array}$ & $\begin{array}{l}17.00 \\
18.00\end{array}$ \\
\hline $\begin{array}{l}08 / 25 / 87 \\
11 / 19 / 87\end{array}$ & 428.61 & $\begin{array}{l}98 / 25 / 87 \\
11 / 10 / 87\end{array}$ & $\begin{array}{l}496.00 \\
406.01\end{array}$ \\
\hline $\begin{array}{l}98 / 25 / 87 \\
11 / 19 / 87\end{array}$ & & $\begin{array}{l}08 / 25 / 87 \\
11 / 10 / 87\end{array}$ & \\
\hline $\begin{array}{l}68 / 26 / 87 \\
11 / 10 / 87\end{array}$ & 4310.01 & $\begin{array}{l}98 / 25 / 87 \\
11 / 16 / 87\end{array}$ & 23200.06 \\
\hline $\begin{array}{l}08 / 25 / 87 \\
11 / 19 / 87\end{array}$ & $\begin{array}{l}7.68 \\
7.83\end{array}$ & $\begin{array}{l}60 / 25 / 87 \\
11 / 10 / 87\end{array}$ & $\begin{array}{r}7.46 \\
7.42 \\
\end{array}$ \\
\hline $11 / 10 / 87$ & 8.96 & $11 / 10 / 87$ & 7.10 \\
\hline $\begin{array}{l}88 / 25 / 87 \\
11 / 19 / 87\end{array}$ & $\begin{array}{l}25000.11 \\
26980.40\end{array}$ & $\begin{array}{l}18 / 25 / 87 \\
11 / 16 / 87\end{array}$ & $\begin{array}{l}45160.06 \\
46506.06\end{array}$ \\
\hline $\begin{array}{l}08 / 25 / 87 \\
11 / 19 / 87\end{array}$ & 28065.01 & $\begin{array}{l}88 / 25 / 87 \\
11 / 10 / 87\end{array}$ & $\begin{array}{l}46100.09 \\
47760.00\end{array}$ \\
\hline $08 / 25 / 87$ & & $08 / 25 / 87$ & $\begin{array}{r}85586.00 \\
\{\quad 973.00\}\end{array}$ \\
\hline
\end{tabular}

- VALUE eXCEeds PRIUARY dRINKing vater standard.

- VALUE EXCEEDS PROPOSED PRIMARY DRINKING IATER STANDARD.

- - YALUE EXCEEDS SCREENING LEVEL For fURTHER INYESTIGATION.

- DETECTION LiMIt tas Not ayatLable for cDUPARISOM

NR - ANALYSIS NOT RERUESTED OR NOT YET REPORTED

VALUES IN $\{$ ? ARE COUNTING ERRORS FOR RADIONUCLIDES

IATER STANDARD(S) IN PAREMTHESES ARE PROPOSED OKLY 
TABLE C.3. (contd)

\begin{tabular}{|c|c|c|c|c|c|c|c|c|c|}
\hline $\begin{array}{l}\text { COHSTI } \\
\text { NAME }\end{array}$ & $\begin{array}{l}\text { UENT } \\
\text { UNITS }\end{array}$ & $\begin{array}{l}\text { CONIRACTUAL } \\
\text { DETECTION } \\
\text { LIUIT }\end{array}$ & $\begin{array}{l}\text { DRIMKING } \\
\text { TATER } \\
\text { STAMDARD }\end{array}$ & $\begin{array}{l}\text { SALPLE } \\
\text { DATE }\end{array}$ & $0-20-20$ & $\begin{array}{l}\text { SAMPLE } \\
\text { DATE }\end{array}$ & $8-28-39$ & $\begin{array}{c}\text { SAMPLE } \\
\text { DATE }\end{array}$ & $6-24-3 z$ \\
\hline$--n$ & $\cdots+-$ & 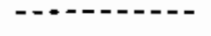 & $+-1-1-30$ & +----- & - n-en & 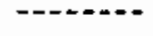 & ------ & --no- & ----- \\
\hline TRITIUY & $\mathrm{PCI} / \mathrm{L}$ & 566.00 & 20606.00 & $10 / 20 / 07$ & $\left\{\begin{array}{l}186000.15 \\
\{154.4\end{array} *\right.$ & $11 / 19 / 87$ & & $11 / 10 / 87$ & $\begin{array}{l}196004.06 \\
\{1240.06\}\end{array}$ \\
\hline U & $\mathrm{PCI} / \mathrm{L}$ & 0.50 & 664.65 & $\begin{array}{l}18 / 26 / 87 \\
18 / 28 / 87\end{array}$ & $\begin{array}{l}3.17 \\
2.01\end{array}$ & & MR & & MR \\
\hline
\end{tabular}

$?$

* - VALUE EXCEEDS PRIHARY dRIMKIMg TATER STANDARD.

- - VALUE EXCEEDS PROPOSED PRIUARY DRINKINO TATER STANDARD.

- - VALUE EXCEEDS SCREENING LEYEL FOR FuRTHER InVESTIGATION.

- - DETECTION LIUIT IAS NDT AYAILABLE FOR COUPARISON

NR - ANALYSIS NOT REQUESTED OR HOT YET REPORTED

YALUES IN $\{$ ? ARE COUNTING ERRORS FOR RADIONUCLIDES

WATER STANDARO(S) IN PARENTHESES ARE PROPOSED ONLY 
TABLE C.3. (contd)

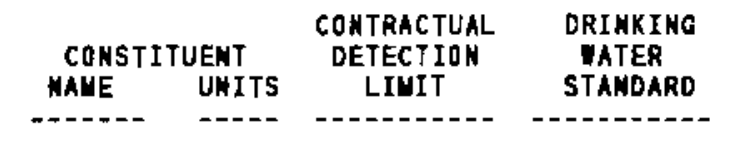

\begin{tabular}{|c|c|}
\hline $\begin{array}{l}\text { SAYPLE } \\
\text { DATE }\end{array}$ & $8-24-48$ \\
\hline$-+-1--$ & ------- \\
\hline $11 / 16 / 87$ & 137000.00 \\
\hline $11 / 10 / 87$ & $\begin{array}{ll} & 1.92 \\
\{\quad 1.32\}\end{array}$ \\
\hline $11 / 10 / 87$ & $\left\{\begin{array}{r}10.00 \\
4.00\}\end{array}\right.$ \\
\hline $11 / 10 / 87$ & 4684.04 \\
\hline $11 / 11 / 07$ & 331.0 \\
\hline 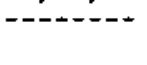 & $\mathbf{M R}$ \\
\hline
\end{tabular}

\begin{tabular}{|c|c|}
\hline $\begin{array}{l}\text { SAMPLE } \\
\text { DATE }\end{array}$ & $8-28-40$ \\
\hline $09 / 51 / 87$ & 134000.06 \\
\hline $00 / 01 / 87$ & $\left.\begin{array}{ll}\{ & 1.05 \\
1.05\end{array}\right\}$ \\
\hline $60 / 01 / 87$ & $\begin{array}{c}25.41 \\
\{\quad 5.32\}\end{array}$ \\
\hline $09 / 01 / 87$ & 0460.10 \\
\hline $00 / 01 / 87$ & 333.6 \\
\hline $08 / 64 / 87$ & 4.14 \\
\hline $10 / 26 / 87$ & $\begin{array}{l}\{\quad 6.26\} \\
\{\quad 4.58\}\end{array}$ \\
\hline $00 / 61 / 87$ & 30.06 \\
\hline $60 / 01 / 87$ & gosen.00 \\
\hline $60 / 01 / 87$ & 12006.00 \\
\hline $10 / 01 / 87$ & 5970.06 \\
\hline $10 / 01 / 87$ & 10704.00 \\
\hline $00 / 01 / 87$ & 21.01 \\
\hline & MR \\
\hline $00 / 01 / 87$ & 480.00 \\
\hline $00 / 01 / 87$ & 13201.06 \\
\hline $09 / 101 / 07$ & 7.87 \\
\hline $09 / 01 / 87$ & 6.80 \\
\hline
\end{tabular}

\begin{tabular}{|c|c|}
\hline $\begin{array}{l}\text { SAMPLE } \\
\text { DATE }\end{array}$ & $6-29-4$ \\
\hline $10 / 10 / 87$ & 123000.60 \\
\hline $10 / 16 / 87$ & $\left.\begin{array}{l}4.45 \\
1.81\}\end{array}\right\}$ \\
\hline $89 / 11 / 87$ & $\begin{array}{r}26.20 \\
\{\quad 5.52\}\end{array}$ \\
\hline $19 / 11 / 87$ & 8520.01 \\
\hline \multirow[t]{3}{*}{$00 / 10 / 87$} & 201.00 \\
\hline & NR \\
\hline & NR \\
\hline $00 / 10 / 07$ & 65.60 \\
\hline $00 / 10 / 07$ & 41000.60 \\
\hline $09 / 11 / 87$ & sest.es \\
\hline $10 / 11 / 87$ & 5426.00 \\
\hline $10 / 11 / 87$ & 13200.00 \\
\hline $00 / 11 / 87$ & 17.60 \\
\hline & NR \\
\hline $09 / 10 / 87$ & 267.00 \\
\hline $10 / 10 / 87$ & soave.00 \\
\hline $99 / 16 / 87$ & 7.81 \\
\hline $09 / 16 / 87$ & 7.11 \\
\hline
\end{tabular}

* - value exceeds primary drinximg mater stamdard.

\begin{tabular}{|c|c|}
\hline $11 / 10 / 87$ & 76.10 \\
\hline $11 / 10 / 87$ & 92101.04 \\
\hline $11 / 16 / 87$ & 12856.00 \\
\hline $11 / 16 / 87$ & 6376.60 \\
\hline $11 / 16 / 87$ & 17280.16 \\
\hline $11 / 11 / 87$ & 5.11 \\
\hline $\begin{array}{l}08 / 27 / 87 \\
11 / 11 / 87\end{array}$ & $\begin{array}{l}6501.64 \\
7790.01\end{array}$ \\
\hline $11 / 10 / 87$ & 465.06 \\
\hline $11 / 10 / 87$ & 8400.00 \\
\hline $11 / 11 / 07$ & 7.88 \\
\hline $11 / 11 / 87$ & 7.80 \\
\hline
\end{tabular}

NR

1800.06

FCALCIU PPB

FHAGNES PPB

FPOTASS PPB

FSODIUY PPB

FYANAOI PPB

HNITRAT PPB

LFLUORD PPB

NItRATE PPB

509. 60

๑. 10

\begin{tabular}{|c|c|c|}
\hline 45060.00 & $\begin{array}{l}68 / 27 / 87 \\
11 / 11 / 87\end{array}$ & $\begin{array}{l}6500.05 \\
7790.01\end{array}$ \\
\hline 4000.00 & $11 / 10 / 87$ & 465.05 \\
\hline & $11 / 10 / 87$ & 8400.00 \\
\hline & $11 / 11 / 97$ & 7.88 \\
\hline & $11 / 11 / 87$ & 7.81 \\
\hline
\end{tabular}

- YALUE EXCEEDS PROPOSED PRIUARY DRINKING TATER STANDARD.

- - YALUE EXCEEDS SCREENING LEVEL FDR FURTHER INYESTIGATION.

- DETECTIDN LIUIT TAS NOT AVAILABLE FOR CDUPARISON

NR - ANALYSIS NOT REQUESTED OR NOT YET REPORTED

YALUES IN \& \} ARE COUNTING ERRORS FOR RADIONUCLIDES

WATER STANDARD(S) IN PARENTHESES ARE PROPOSEO DNLY 
IABLE C.3. (contd)

\begin{tabular}{|c|c|c|c|c|c|c|c|c|c|}
\hline $\begin{array}{c}\text { CONSTI } \\
\text { MANE }\end{array}$ & $\begin{array}{l}\text { UENT } \\
\text { UHITS }\end{array}$ & $\begin{array}{l}\text { CONTRACTUAL } \\
\text { DETECTION } \\
\text { LIUIT }\end{array}$ & $\begin{array}{c}\text { DRINKING } \\
\text { WATER } \\
\text { STANDARO }\end{array}$ & $\begin{array}{c}\text { SAUPLE } \\
\text { DATE }\end{array}$ & $6-24-46$ & $\begin{array}{c}\text { SALPLE } \\
\text { DATE }\end{array}$ & $6-28-46$ & $\begin{array}{c}\text { SAMPLE } \\
\text { DATE }\end{array}$ & $8-20-4$ \\
\hline SULFATE & PPB & 506.06 & & $11 / 11 / 87$ & 28860.00 & $99 / 01 / 87$ & 41304.01 & $10 / 10 / 87$ & 32710.16 \\
\hline TC & PPB & & & $11 / 10 / 87$ & 31380.00 & $00 / 01 / 87$ & 96000.010 & $10 / 16 / 87$ & 20500.01 \\
\hline TRITIUN & $\mathrm{PCI} / \mathrm{L}$ & 500.00 & 20080.01 & $\begin{array}{l}08 / 27 / 87 \\
11 / 10 / 87\end{array}$ & & $\begin{array}{l}18 / 44 / 87 \\
10 / 20 / 87\end{array}$ & $\left\{\begin{array}{l}12301.18 \\
457.06\end{array}\right.$ & $\begin{array}{l}18 / 25 / 87 \\
10 / 25 / 87\end{array}$ & 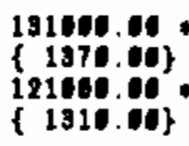 \\
\hline
\end{tabular}

* - value exceeds primary drinking tater standaro.

- YALUE EXCEEDS PROPOSED PRIMARY DRINKINO TATER STAMDARD.

- VALUE EXCEEDS SCREENING LEYEL FOR FURTHER INVESTIGATION.

- DEJECTION LIUIT IAS NOT AVAILABLE FOR COUPARISON

NR - ANALYSIS NOT REQUESTED OR NOT YET REPORTED

VALUES IN ( ) ARE COUNTING ERRORS FOR RADIONUCLIDES

IATER STANDARD(S) IN PARENTHESES ARE PROPOSED ONLY 
TABLE C.3. (contd)

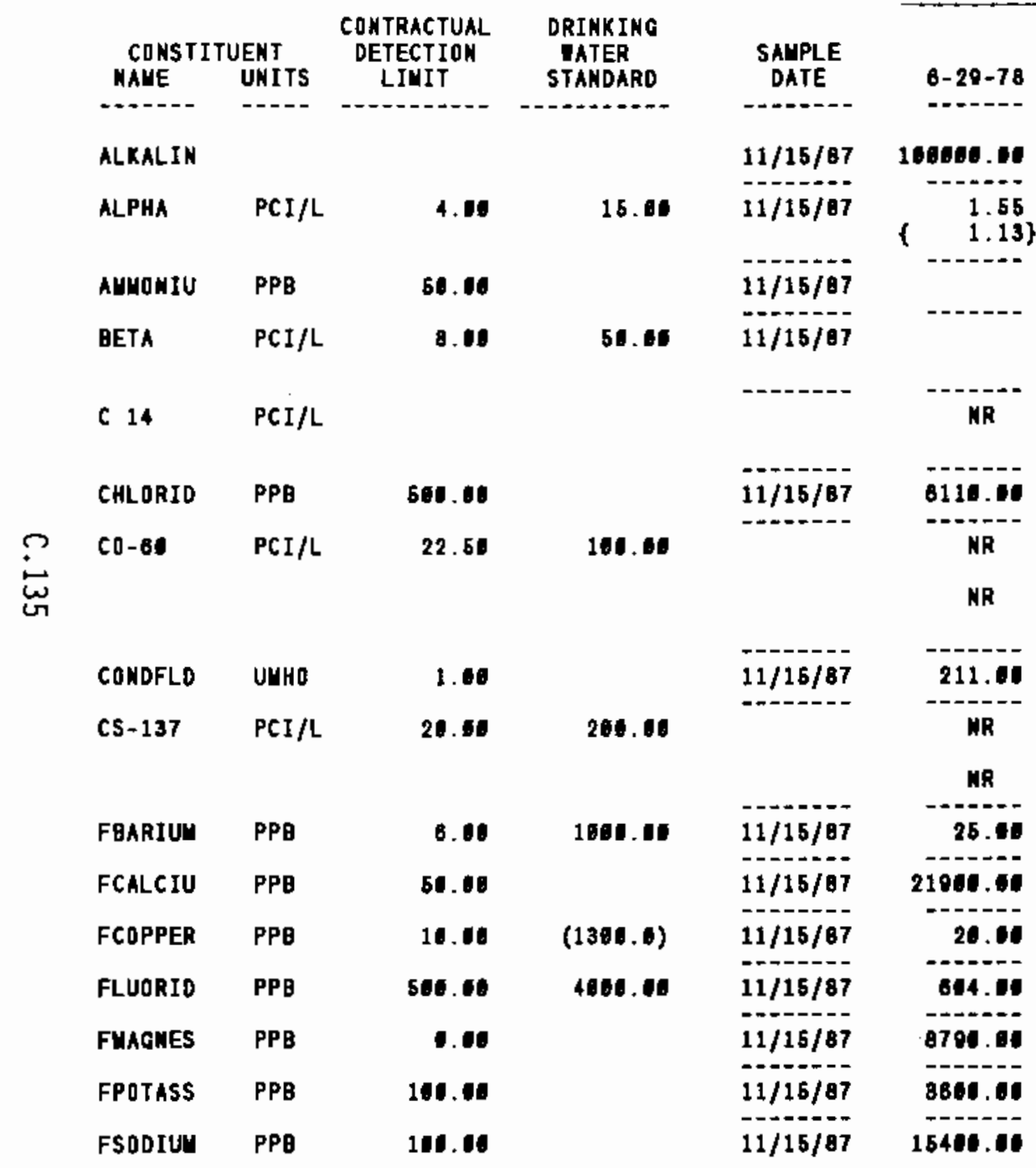

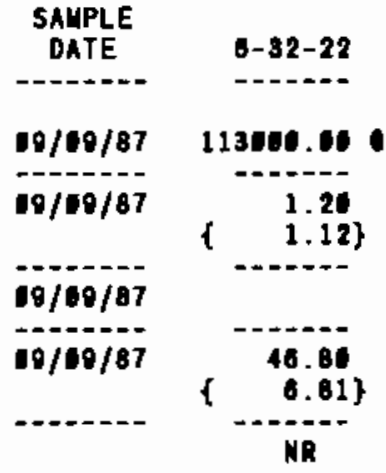

$10 / 00 / 8$

$00 / 09 / 87$

$11 / 19 / 87$

$10 / 69 / 87$

19/69/87

$11 / 13 / 87$

$10 / 09 / 87$

$60 / 09 / 87$

$60 / 09 / 87$

$00 / 09 / 87$

$10 / 60 / 97$

10/00/87

$00 / 09 / 87$

1060.00

$-----$

(11.45

$\{\quad 7.18$

$\left\{\begin{array}{r}8.78 \\ \quad 5.62\} \\ \hline\end{array}\right.$

310.0

6.60

$\{\quad 4.38\}$

37.19

-.......

31360.14

-..--

………

9ost.un

6e8s.us

24260.10
SAYPLE

DATE

$00 / 27 / 87$ 103060.01

$08 / 27 / 87 \quad 4.96$

-........

$00 / 27 / 07$

$\{\quad 1.02\}$

18/27/87 54.38 .

$10 / 00 / 87$
$-18 / 27 / 87$

$60 / 60 / 07$

$10 / 28 / 87$

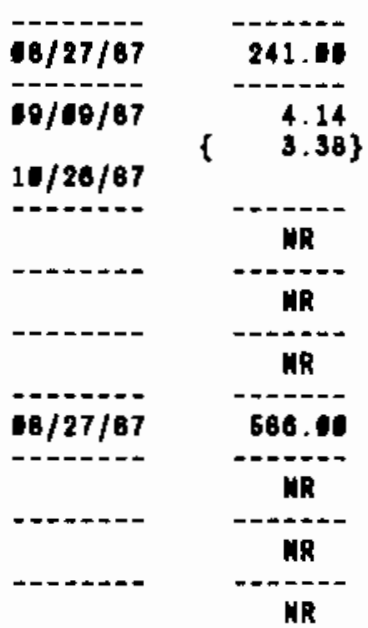

* - yalue exceeds primary orinkino tater standoaro.

- VALUE EXCEEDS PROPOSED PRIMARY ORINKIMG iATER STAMDARD.

* - VALUE EXCEEDS SCREENing LEYel for fURTHER INYESTIOATIOn.

- DETECTION LIUIT ias hot AVATLABLE fOR COUPARISOX

MR - ANALYSIS NOT REQUESTED OR NOT YET REPORTED

VALUES IM I ARE COUMIIMG ERRORS FOR RADIONUCLIDES

DATER STANDARD(S) IN PARENTHESES ARE PROPOSED OMLY 
TABLE C.3. (contd)

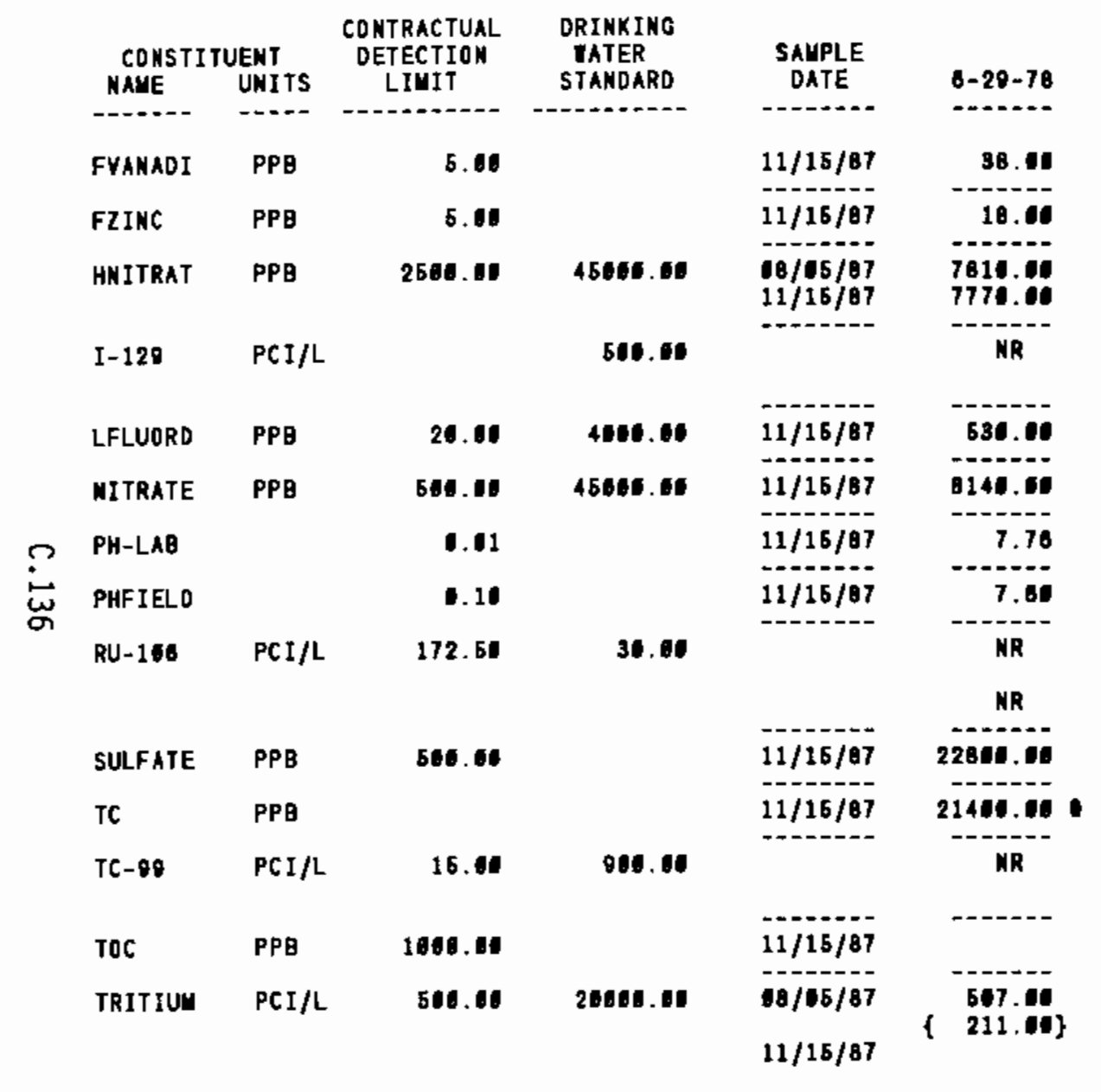

\begin{tabular}{|c|c|}
\hline $\begin{array}{l}\text { SAUPLE } \\
\text { DATE }\end{array}$ & $6-32-22$ \\
\hline - - - & -ancen \\
\hline $00 / 00 / 87$ & 19.16 \\
\hline $69 / 19 / 87$ & 5.16 \\
\hline 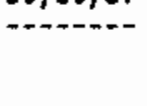 & $\begin{array}{l}\text { NR } \\
\text { NR }\end{array}$ \\
\hline $0 / 14 / 87$ & $\left\{\begin{array}{r}3.87 \\
\quad \quad .33\}\end{array}\right.$ \\
\hline $09 / 00 / 87$ & 400.01 \\
\hline $19 / 10 / 87$ & sobes. \\
\hline $00 / 10 / 87$ & B. 12 \\
\hline $\begin{array}{l}0 / 19 / a 7 \\
60 / 09 / 87\end{array}$ & 7.30 \\
\hline $11 / 13 / 87$ & \\
\hline $10 / 60 / 87$ & 48404.46 \\
\hline $10 / 00 / 87$ & 27140.06 \\
\hline $60 / 60 / 07$ & $\left\{\begin{array}{r}218.06 \\
2.00\}\end{array}\right.$ \\
\hline $10 / 60 / 87$ & \\
\hline $9 / 60 / 87$ & 282600 10 * \\
\hline $11 / 13 / 87$ & 25851.40* \\
\hline
\end{tabular}

\begin{tabular}{|c|c|}
\hline $\begin{array}{l}\text { SAHPLE } \\
\text { OATE }\end{array}$ & $6-32-43$ \\
\hline -ー-ー--ー & ----- \\
\hline & NR \\
\hline 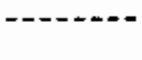 & ----- \\
\hline - & NR \\
\hline $00 / 04 / 87$ & 5.51 \\
\hline & $0.44\}$ \\
\hline $08 / 27 / 87$ & 825.00 \\
\hline $18 / 27 / 87$ & 40000. \\
\hline - & 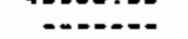 \\
\hline $18 / 27 / 87$ & 7.87 \\
\hline $98 / 27 / 87$ & 0.40 \\
\hline$-0-1-1$ & - \\
\hline $09 / 00 / 87$ & $\begin{array}{r}48.00 \\
14.01\end{array}$ \\
\hline $16 / 28 / 87$ & \\
\hline $18 / 27 / 87$ & 80896.0 \\
\hline$---c-1$ & $\ldots$ \\
\hline $18 / 27 / 67$ & 20506.00 \\
\hline $0 / 109 / 87$ & 85.08 \\
\hline & $\{\quad 1.67\}$ \\
\hline $6 / 27 / 87$ & 1070.6 \\
\hline ג-- & \\
\hline $60 / 60 / 87$ & 464601.01 \\
\hline $10 / 26 / 87$ & $\{40500.00$ \\
\hline
\end{tabular}

- - yalUe eXceEds PRIUARY oRINKINg vater staMdard.

- - YALUE EXCEEDS PROPOSEO PRIUARY DRINKING TATER STANDARD.

- YALUE EXCEEDS SCREENING LEVEL FOR FURTHER INYESTIGATION.

- DETECTION LIUIT IAS nOt aYaILABLE For couparison

NR - ANALYSIS NOT REQUESTED OR NOT YET REPORTED

YALUES IN I A ARE COUNTING ERRORS FOR RAOIONUCLIDES

IATER STANDARD'(S) IN PARENTHESES ARE PROPOSED ONLY 
TABLE C.3. (contd)

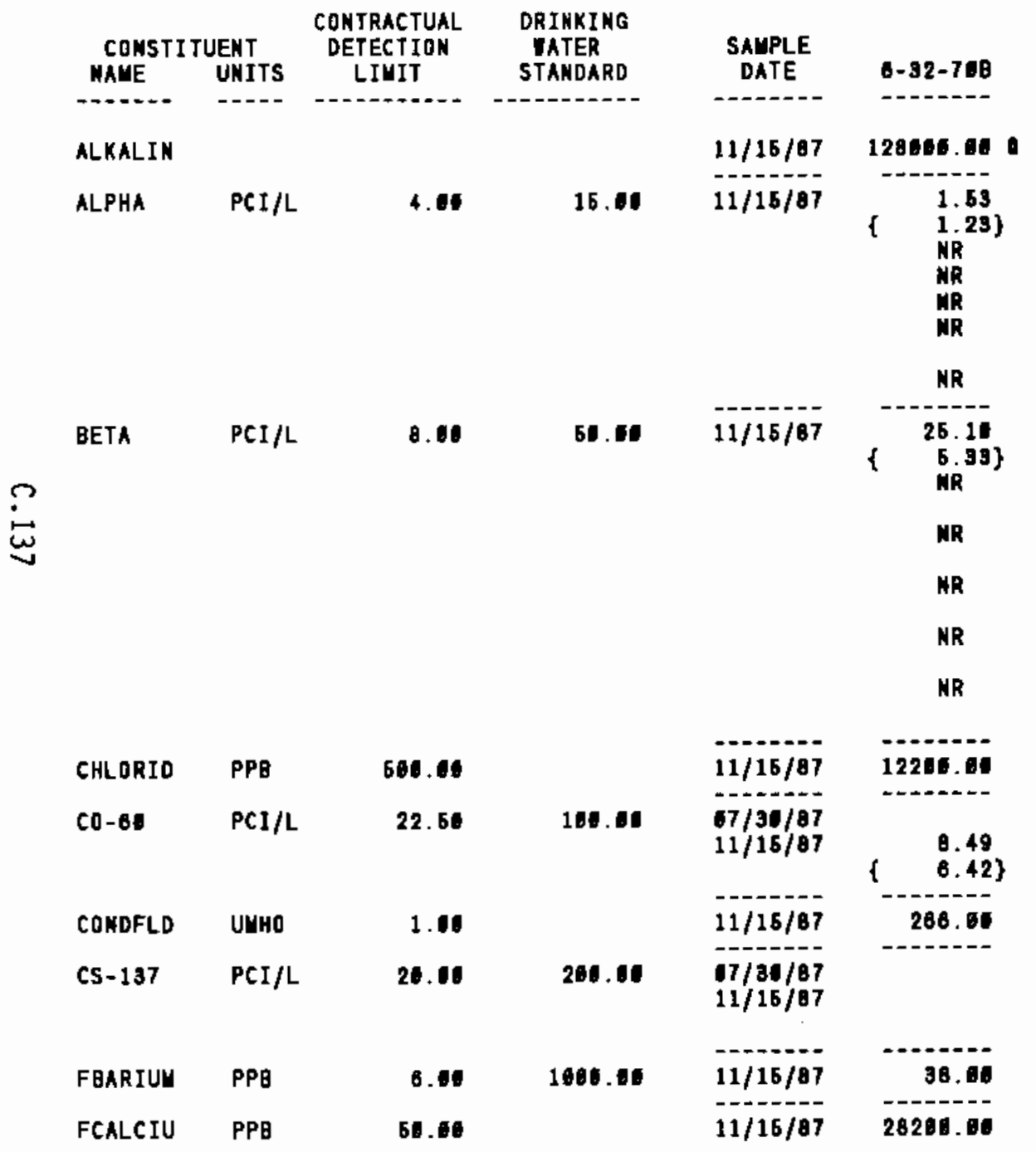

\begin{tabular}{|c|c|c|c|}
\hline $\begin{array}{c}\text { SAUPLE } \\
\text { DATE }\end{array}$ & $8-32-72$ & $\underset{\text { DATE }}{\text { SAMPLE }}$ & $6-32-77$ \\
\hline $10 / 14 / 87$ & 97364.01 & $11 / 16 / 07$ & 103950.000 \\
\hline $17 / 08 / 87$ & $\begin{array}{l}0.47 \\
\{.35\}\end{array}$ & $11 / 15 / 87$ & \\
\hline $\begin{array}{l}0 / 198 / 87 \\
10 / 14 / 87 \\
10 / 14 / 87 \\
12 / 11 / 87\end{array}$ & 6.84 & & $\begin{array}{l}\text { NR } \\
\text { NR } \\
\text { NR } \\
\text { NR }\end{array}$ \\
\hline $12 / 07 / 87$ & $0.30\}$ & & MR \\
\hline $67 / 08 / 87$ & $\left\{\begin{array}{r}13.01 \\
2.02\}\end{array}\right.$ & $11 / 15 / 87$ & \\
\hline $10 / 09 / 87$ & 5.15 & & MR \\
\hline $15 / 14 / 87$ & 5.68 & & WR \\
\hline $11 / 14 / 87$ & $\begin{array}{r}1.48\} \\
\quad \quad 3.18 \\
\{\quad 1.45\}\end{array}$ & & MR \\
\hline $12 / 01 / 87$ & 13.80 & & NR \\
\hline $12 / 07 / 87$ & $\begin{array}{l}4.75 \\
\{\quad 1.78\}\end{array}$ & & NR \\
\hline $16 / 14 / 87$ & 18700.010 & $11 / 15 / 07$ & 7530.44 \\
\hline $\begin{array}{l}17 / 06 / 87 \\
12 / 01 / 87\end{array}$ & & $\begin{array}{l}18 / 05 / 87 \\
11 / 15 / 07\end{array}$ & \\
\hline $10 / 14 / 87$ & 231.06 & $11 / 15 / 87$ & 213.06 \\
\hline $\begin{array}{l}07 / 08 / 87 \\
12 / 01 / 87\end{array}$ & $\begin{array}{ll} & 5.96 \\
\{\quad 3.97\}\end{array}$ & $\begin{array}{l}98 / 65 / 87 \\
11 / 15 / 87\end{array}$ & \\
\hline $10 / 14 / 87$ & 17.00 & $11 / 15 / 87$ & 27.01 \\
\hline $11 / 14 / 87$ & 22500.00 & $11 / 15 / 87$ & 22145.01 \\
\hline
\end{tabular}

* - YALUE EXCEedS PRILARY DRIMXING tater STAMDARD.

- VALUE EXCEEDS PROPOSED PRIUARY DRIHKING IATER STAMDARD.

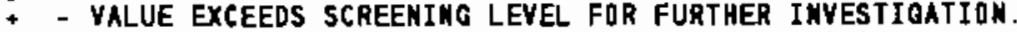

- DETECTION LIUIT IAS mOT AVAILABLE for COUPARISON

HR - ANALYSIS NOT REQUESTED OR MOT YET REPORTED

VALUES IN \{\} ARE COUNTIMO ERRORS FOR RAOIONUCLIDES

IATER STAMDARD(S) IN PARENTHESES ARE PROPOSED OMLY 


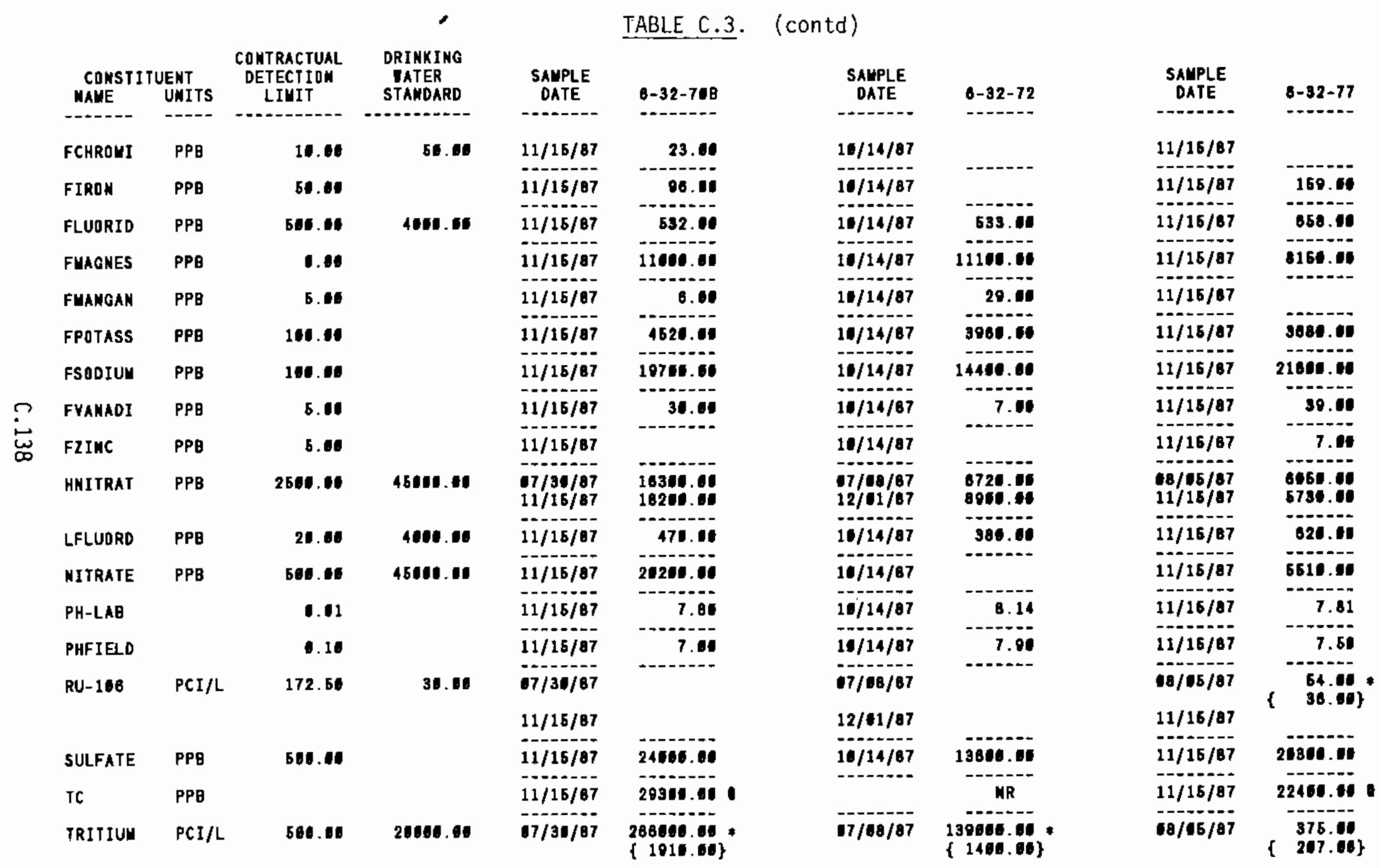

* - yalue eXceEdS privary dRINKIMg tater standard.

- - YALUE EXCEEDS PROPOSED PRIUARY DRINKIMG TATER STANDARD.

- - YALUE EXCEEDS SCREENING LEVEL FDR FURTHER INYESTIGATION.

- DETECTION LIUIT IAS MOT AVAILABLE FOR COYPARISON

NR - ANALYSIS NOT REQUESTED OR NDT YET REPORTED

YALUES IN \{ \& ARE COUNTING ERRORS FDR RADIONUCLIOES

IATER STAMDARD'(S) IN PARENTHESES ARE PROPOSED ONLY 
TABLE C.3. (contd)

\begin{tabular}{|c|c|c|c|c|c|c|c|c|c|}
\hline $\begin{array}{c}\text { CONSTI } \\
\text { MANE }\end{array}$ & $\begin{array}{l}\text { UENT } \\
\text { UNITS }\end{array}$ & $\begin{array}{c}\text { CONTRACTUAL } \\
\text { DETECIION } \\
\text { LIUIT }\end{array}$ & $\begin{array}{l}\text { DRINKING } \\
\text { VATER } \\
\text { STANDARD }\end{array}$ & $\begin{array}{c}\text { SAYPLE } \\
\text { DATE }\end{array}$ & $6-32-768$ & $\begin{array}{l}\text { SALPLE } \\
\text { DATE }\end{array}$ & $0-32-72$ & $\begin{array}{l}\text { SAUPLE } \\
\text { DATE }\end{array}$ & $6-32-77$ \\
\hline TRITIUU & $\mathrm{PCI} / \mathrm{L}$ & 600.00 & 20000.01 & $11 / 15 / 87$ & $\begin{array}{l}273506.60 \\
(1726.66)\end{array}$ & $12 / 101 / 87$ & \{148680.186) & $11 / 15 / 87$ & \\
\hline u & $\mathrm{PCI} / \mathrm{L}$ & 8.50 & 660.11 & $\begin{array}{l}17 / 35 / 87 \\
11 / 15 / 87\end{array}$ & $\begin{array}{l}1.26 \\
1.18\end{array}$ & $\begin{array}{l}1 / 08 / 87 \\
12 / 01 / 87\end{array}$ & & & $\underset{M R}{M R}$ \\
\hline
\end{tabular}

$\stackrel{?}{\stackrel{\omega}{\omega}}$

* - value exceEds primary dRIMKino tater standard.

- VALUE EXCEEDS PRDPOSED PRIMARY DRINKIMO TATER STANDARD.

- - VALUE EXCEEDS SCREENING LEVEL FOR FURTHER INYESTIGATIOH.

- - DETECTION LIUIT TAS MOT AVAILABLE FOR COUPARISON

NR - ANALYSIS NOT REQUESTED OR NOT YET REPORTED

VALUES IM \& $]$ ARE COUNTINO ERRORS FOR RADIONUCLIDES

IATER STAMDARD(S) IN PARENTHESES ARE PROPOSED ONLY 
TABLE C.3. (contd)

\begin{tabular}{|c|c|c|c|c|c|c|c|c|c|}
\hline $\begin{array}{l}\text { CONSTI } \\
\text { MANE }\end{array}$ & $\begin{array}{l}\text { JEFT } \\
\text { UNITS }\end{array}$ & $\begin{array}{c}\text { CONTRACTUAL } \\
\text { DEIECTION } \\
\text { LIMIT }\end{array}$ & $\begin{array}{l}\text { DRINKINO } \\
\text { IATER } \\
\text { STANDARD }\end{array}$ & $\begin{array}{c}\text { SALPLE } \\
\text { DATE }\end{array}$ & $6-33-42$ & $\begin{array}{l}\text { SAUPLE } \\
\text { DATE }\end{array}$ & $\theta-33-5 \theta$ & $\begin{array}{l}\text { SALPLE } \\
\text { DATE }\end{array}$ & 8-34-42 \\
\hline ALKALIN & & & & $18 / 27 / 87$ & 98400.001 & $108 / 27 / 87$ & 178666.010 & $00 / 01 / 87$ & 101060.01 \\
\hline ALPHA & $\mathrm{PCI} / \mathrm{L}$ & 4.08 & 15.00 & $18 / 27 / 87$ & $\left.\begin{array}{ll}\{\quad & 2.09 \\
1.53\end{array}\right\}$ & $68 / 27 / 87$ & $\left.\begin{array}{ll} & 2.11 \\
\{\quad 1.36\end{array}\right\}$ & $10 / 01 / 87$ & $\left.\begin{array}{ll} & 2.41 \\
\{\quad 1.50\end{array}\right\}$ \\
\hline AHYONIU & PPB & 50.00 & & $08 / 27 / 87$ & 78.6 & $18 / 27 / 87$ & & $10 / 01 / 87$ & \\
\hline BETA & PCI $/ \mathrm{L}$ & 8.18 & 50.00 & $08 / 27 / 07$ & $\left\{\begin{array}{l}89.10 \\
\quad 7.70\}\end{array}\right.$ & $18 / 27 / 87$ & $\left.\begin{array}{ll} & 7.22 \\
\{\quad 4.43\end{array}\right\}$ & $10 / 61 / 07$ & $\left\{\begin{array}{c}14.50 \\
4.79\end{array}\right.$ \\
\hline CHLORID & PPB & 580.08 & & $08 / 27 / 07$ & 5876.01 & $10 / 27 / 87$ & 7000.10 & $00 / 01 / 87$ & 4946.65 \\
\hline $00-80$ & $\mathrm{PCI} / \mathrm{L}$ & 22.51 & 100.00 & $\begin{array}{l}07 / 90 / 07 \\
10 / 25 / 87\end{array}$ & $\left\{\begin{array}{r}16.20 \\
8.89\end{array}\right\}$ & $\begin{array}{l}17 / 30 / 87 \\
10 / 26 / 87\end{array}$ & & $\begin{array}{l}17 / 30 / 87 \\
10 / 25 / 87\end{array}$ & $\begin{array}{l}5.07 \\
4.63\}\end{array}$ \\
\hline CONDFLD & UМHO & 1.11 & & $18 / 27 / 87$ & 224.60 & $00 / 27 / 87$ & 370.06 & $09 / 01 / 87$ & 272.16 \\
\hline CS-137 & PCI $/ \mathrm{L}$ & 21.10 & 200.00 & $\begin{array}{l}07 / 30 / 87 \\
10 / 25 / 87\end{array}$ & & $\begin{array}{l}07 / 30 / 87 \\
10 / 26 / 87\end{array}$ & 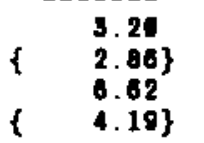 & $\begin{array}{l}07 / 80 / 87 \\
11 / 25 / 87\end{array}$ & \\
\hline FARSEMI & $\mathrm{PPB}$ & Б. 68 & 58.00 & $08 / 27 / 07$ & 8.01 & $18 / 27 / 87$ & & $09 / 61 / 07$ & \\
\hline FBARIUU & PPQ & 6.60 & 1000.00 & $18 / 27 / 97$ & 95.01 & $18 / 27 / 87$ & 40.00 & $00 / 61 / 87$ & 26.50 \\
\hline FCALCIU & PPB & Б0. 00 & & $10 / 27 / 07$ & 24300.00 & $18 / 27 / 87$ & 38565.91 & $00 / 01 / 87$ & 20200.60 \\
\hline FIRON & PPB & 60.00 & & $18 / 27 / 87$ & 54.00 & $08 / 27 / 87$ & & $10 / 01 / 87$ & \\
\hline FLUDRID & $\mathrm{PPB}$ & 500. 00 & 4880.60 & $08 / 27 / 87$ & 678.00 & $10 / 27 / 87$ & & $19 / 01 / 87$ & 563.01 \\
\hline FHAGNES & PPB & 0.01 & & $18 / 27 / 87$ & 8836.61 & $18 / 27 / 87$ & 14100.10 & $69 / 01 / 87$ & 7236.06 \\
\hline FMANGAN & PPB & 5.00 & & $68 / 27 / 87$ & 15.01 & $18 / 27 / 87$ & & $69 / 01 / 87$ & \\
\hline FPOTASS & $\mathrm{PPB}$ & 160.18 & & $08 / 27 / 87$ & 5336.01 & $80 / 27 / 87$ & 5289.11 & $60 / 01 / 87$ & 5076.16 \\
\hline
\end{tabular}

* - value exceeds priuary drinking tater standard.

* - YALUE EXCEES PROPOSED PRIMARY DRINKING TATER STAMDARD.

- - YALE EXCEEDS SCREENING LEVEI FOR FURTHER INYESTIGATION.

- DETECTION LIUIT IAS NOT AVAILABLE FOR COYPARISON

NR - ANALYSIS NDT REQUESTED OR NOT YET REPORTED

VALUES IN $f$; ARE COUNTING ERRORS FOR RADIDNUCLIDES

IATER STANDARD(S) IN PARENTHESES ARE PROPOSED ONLY 
IABLE C.3. (contd)

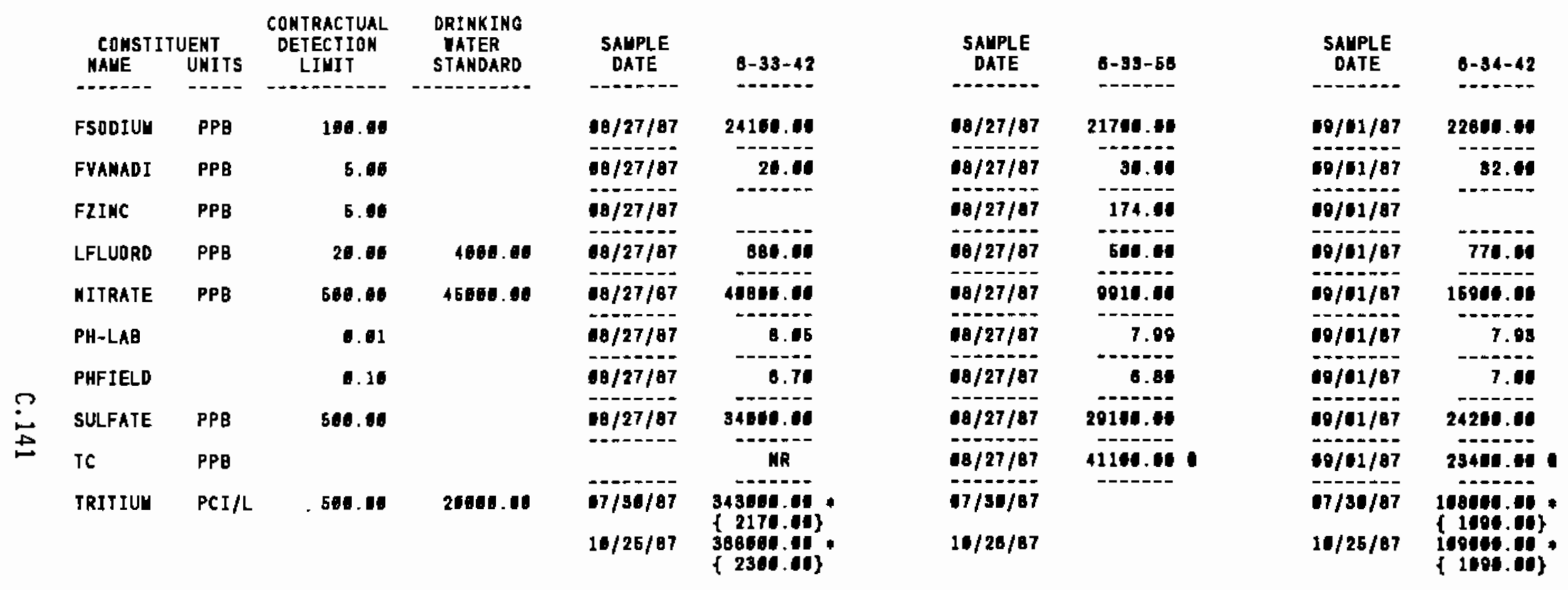

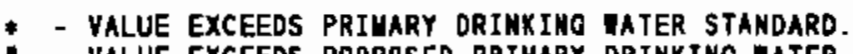

- VAlUE eXCEEDS PRoposed priuary dRINKIng tater staMdard.

* - VALUE EXCEEDS SCREENINO LEVEL FOR FURTHER IMVESTIGATION.

- DETECTIOK LIMIT IAS NDT AVAILABLE FOR COMPARISON

NR - ANALYSIS NOT REQUESTED OR HOT YET REPDRTED

VALUES IN I A ARE COUNTIMG ERRORS FOR RADIOMUCLIDES

TaTER STANDARd(S) IN PAREMTHESES ARE PROPOSED ONLY 
TABLE C.3. (contd)

\begin{tabular}{|c|c|c|c|c|c|}
\hline $\begin{array}{c}\text { CONSTI } \\
\text { MAME }\end{array}$ & $\begin{array}{l}\text { JENT } \\
\text { UNITS }\end{array}$ & $\begin{array}{l}\text { CONTRACTUAL } \\
\text { DETECTION } \\
\text { LIUIT }\end{array}$ & $\begin{array}{l}\text { DRINKING } \\
\text { TATER } \\
\text { STANDARD }\end{array}$ & $\begin{array}{c}\text { SAMPLE } \\
\text { DATE }\end{array}$ & $6-34-61$ \\
\hline ALKALIM & & & & $69 / 23 / 87$ & 157000.00 \\
\hline ALPHA & PCI/L & 4.10 & 15.81 & $19 / 23 / 07$ & $\begin{array}{ll}\{\quad & 2.05 \\
\quad & 1.26\}\end{array}$ \\
\hline AMMONIU & PPB & 56.06 & & $19 / 23 / 87$ & \\
\hline BETA & PCI/L & 8.10 & 56.06 & $00 / 23 / 87$ & $\begin{array}{l}7.58 \\
\{\quad 3.88\} \\
\end{array}$ \\
\hline CHLORID & PPB & 596.11 & & $19 / 28 / 87$ & 6740.10 \\
\hline CONDFLD & UWHO & 1.01 & & $19 / 23 / 87$ & 338.66 \\
\hline CS-137 & $\mathrm{PCI} / \mathrm{L}$ & 24.10 & 201.10 & $\begin{array}{l}17 / 30 / 87 \\
10 / 26 / 87\end{array}$ & $\left\{\begin{array}{l}7.89 \\
\{\quad 4.01\}\end{array}\right.$ \\
\hline FARSEMI & PPB & 5.00 & 60.01 & $09 / 23 / 87$ & \\
\hline FBARIUY & PPB & 8.6 & 1069.64 & $09 / 23 / 87$ & 47.11 \\
\hline FCALCIU & PPB & 50.64 & & $10 / 23 / 87$ & 45600.00 \\
\hline FCHROYI & PPB & 10.00 & 56.01 & $19 / 23 / 87$ & \\
\hline FUAGNES & PPB & 1.60 & & $10 / 23 / 87$ & 13346.01 \\
\hline FHANGAM & PPB & 5.60 & & $18 / 23 / 87$ & 5.60 \\
\hline FPQTASS & PPB & 100.00 & & $19 / 23 / 87$ & 5731.6 \\
\hline FSODIUY & PPB & 180.00 & & $19 / 23 / 67$ & 22201.60 \\
\hline FVANADI & PPB & 6.06 & & $19 / 23 / 87$ & 28.80 \\
\hline FZINC & PPB & 5.60 & & $10 / 23 / 07$ & 6.11 \\
\hline HMITRAT & PPB & 2580.60 & 46096.00 & & NR \\
\hline
\end{tabular}

\begin{tabular}{|c|c|c|c|}
\hline $\begin{array}{l}\text { SAMPLE } \\
\text { DATE }\end{array}$ & $6-35-9$ & $\begin{array}{c}\text { SAMPLE } \\
\text { DATE }\end{array}$ & $6-36-80$ \\
\hline $18 / 25 / 87$ & 122000.010 & $12 / 61 / 87$ & 166064.00 \\
\hline $18 / 25 / 87$ & $-2-0$ & $12 / 01 / 87$ & $\left\{\begin{array}{l}2.64 \\
\{\quad 1.40\end{array}\right\}$ \\
\hline $08 / 25 / 87$ & & $12 / 01 / 87$ & 69.10 \\
\hline $66 / 25 / 67$ & $\begin{array}{r}48.24 \\
\quad 7.24\} \\
\end{array}$ & $12 / 01 / 87$ & $\begin{array}{r}14.06 \\
4.03\}\end{array}$ \\
\hline $18 / 26 / 87$ & 19800.00 & $12 / 11 / 87$ & 1695e.11 \\
\hline $08 / 25 / 87$ & 388.01 & $12 / 101 / 07$ & 400.66 \\
\hline $18 / 25 / 87$ & & $08 / 10 / 07$ & \\
\hline $10 / 25 / 87$ & & $12 / 01 / 07$ & \\
\hline $18 / 25 / 87$ & 5.00 & $12 / 01 / 87$ & \\
\hline $10 / 25 / 87$ & 65.11 & $12 / 01 / 87$ & 44.01 \\
\hline $68 / 25 / 87$ & 97000.00 & $12 / 01 / 87$ & 45000.01 \\
\hline $18 / 26 / 87$ & 11.00 & $12 / 01 / 87$ & 24.01 \\
\hline $18 / 25 / 87$ & 11300.00 & $12 / 01 / 87$ & 13500.00 \\
\hline $18 / 25 / 87$ & & $12 / 61 / 87$ & \\
\hline $88 / 25 / 87$ & 6376.00 & $12 / 1 / 87$ & 4871.61 \\
\hline $08 / 25 / 87$ & 10851.00 & $12 / 01 / 87$ & 108040 \\
\hline $18 / 25 / 87$ & 16.10 & $12 / 11 / 87$ & 28.10 \\
\hline \multirow[t]{2}{*}{$08 / 25 / 87$} & & $12 / 11 / 87$ & 11.6 \\
\hline & NR & $18 / 19 / 87$ & 29965 to \\
\hline
\end{tabular}

- - yalue exceeds primary drimkimg tater standard.

1 - VALUE EXCEEDS PROPOSED PRIUAARY DRINKing IATER STAMDARD.

- - VALUE EXCEEDS SCREENIRG LEVEL FoR FURTHER INYESTIGATIOH.

- DETECTION LIUIT IAS nOt AVAILABLE for couparison

NR - ANALYSIS NOT REQUESTED DR NOT YET REPORTED

VALUES IN \& $?$ ARE COUNTING ERRORS FOR RADIONUCLIDES

iater Standard(S) In parentheses are proposed only 
TABLE C.3. (contd)

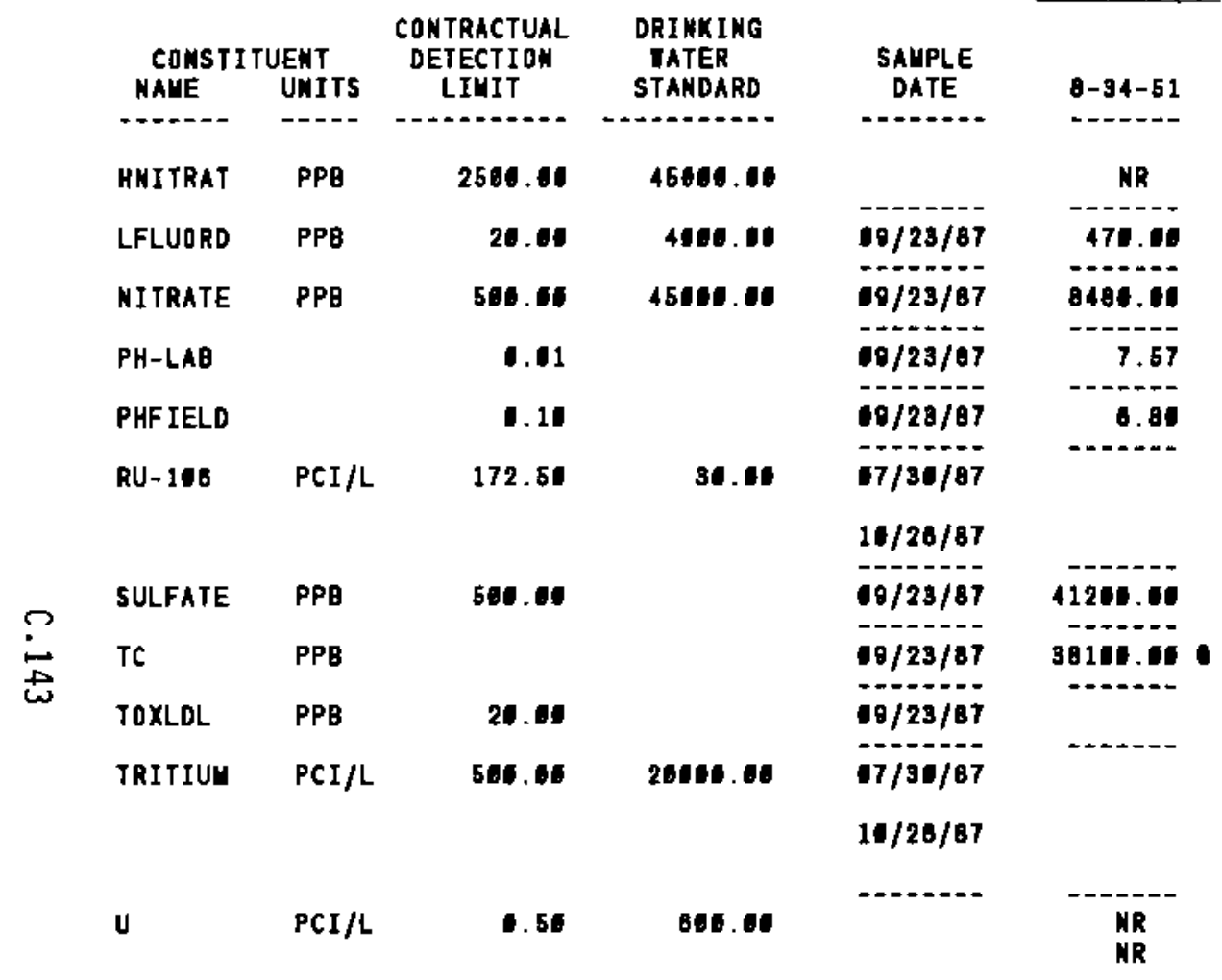

\begin{tabular}{|c|c|}
\hline \multirow{3}{*}{$\begin{array}{c}\text { SAMPLE } \\
\text { DATE }\end{array}$} & $a-35-9$ \\
\hline & - \\
\hline & HR \\
\hline$-1-1-19$ & \\
\hline 68/25/87 & 370.00 \\
\hline $68 / 25 / 97$ & 37314.16 \\
\hline ") & 78 \\
\hline 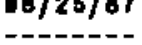 & $\begin{array}{r}1.18 \\
------\end{array}$ \\
\hline $18 / 25 / 87$ & 7.20 \\
\hline $18 / 26 / 87$ & - \\
\hline $10 / 25 / 87$ & \\
\hline $08 / 25 / 87$ & 44100.00 \\
\hline & - \\
\hline $08 / 25 / 87$ & 2760s. \\
\hline $18 / 25 / 87$ & -+-0 \\
\hline 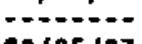 & - \\
\hline $08 / 25 / 87$ & $\frac{105010.00}{\{1600.00\}}$ \\
\hline $10 / 25 / 87$ & $\{1689.60\}$ \\
\hline & NR \\
\hline
\end{tabular}

\begin{tabular}{|c|c|}
\hline $\begin{array}{l}\text { SAMPLE } \\
\text { DATE }\end{array}$ & $8-36-60$ \\
\hline 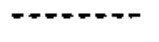 & - \\
\hline $12 / 01 / 87$ & 21806.01 \\
\hline $12 / 01 / 87$ & 470.00 \\
\hline $12 / 01 / 07$ & 24201.01 \\
\hline 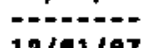 & \\
\hline $12 / 01 / 07$ & 7.70 \\
\hline $12 / 01 / 87$ & 10 \\
\hline $18 / 10 / 07$ & $\begin{array}{r}27.60 \\
\quad 24.80\}\end{array}$ \\
\hline $12 / 01 / 67$ & \\
\hline & 20464.6 \\
\hline $12 / 01 / 87$ & sones. is \\
\hline $12 / 01 / 87$ & $40 . \overline{c 0}$ \\
\hline $08 / 10 / 07$ & $\begin{array}{c}120401.04 \\
\text { (4126.00) }\end{array}$ \\
\hline $12 / 01 / 67$ & (4106.00) \\
\hline $\begin{array}{l}08 / 10 / 97 \\
12 / 01 / 07\end{array}$ & $\begin{array}{l}1.84 \\
1.81\end{array}$ \\
\hline
\end{tabular}

* - Value exceeds primary drinking tater stamdard.

- VALUE EXCEEDS PROPOSED PRIUARY DRIMKING TATER STANDARD.

- - VALUE EXCEEDS SCREENIMO LEVEL FOR FURTHER INYESTIGATION.

- DETECTION LIUIT TAS NOT AVAILABLE FOR COUPARISON

NR - AMALYSIS NOT REQUESTED OR NOT YET REPORTED

VALUES IN ( ) ARE COUNTINO ERRORS FOR RADIONUCLIDES

JATER STANDARD'(S) IN PAREMTHESES ARE PROPOSED ONLY 
IABLE C.3. (contd)

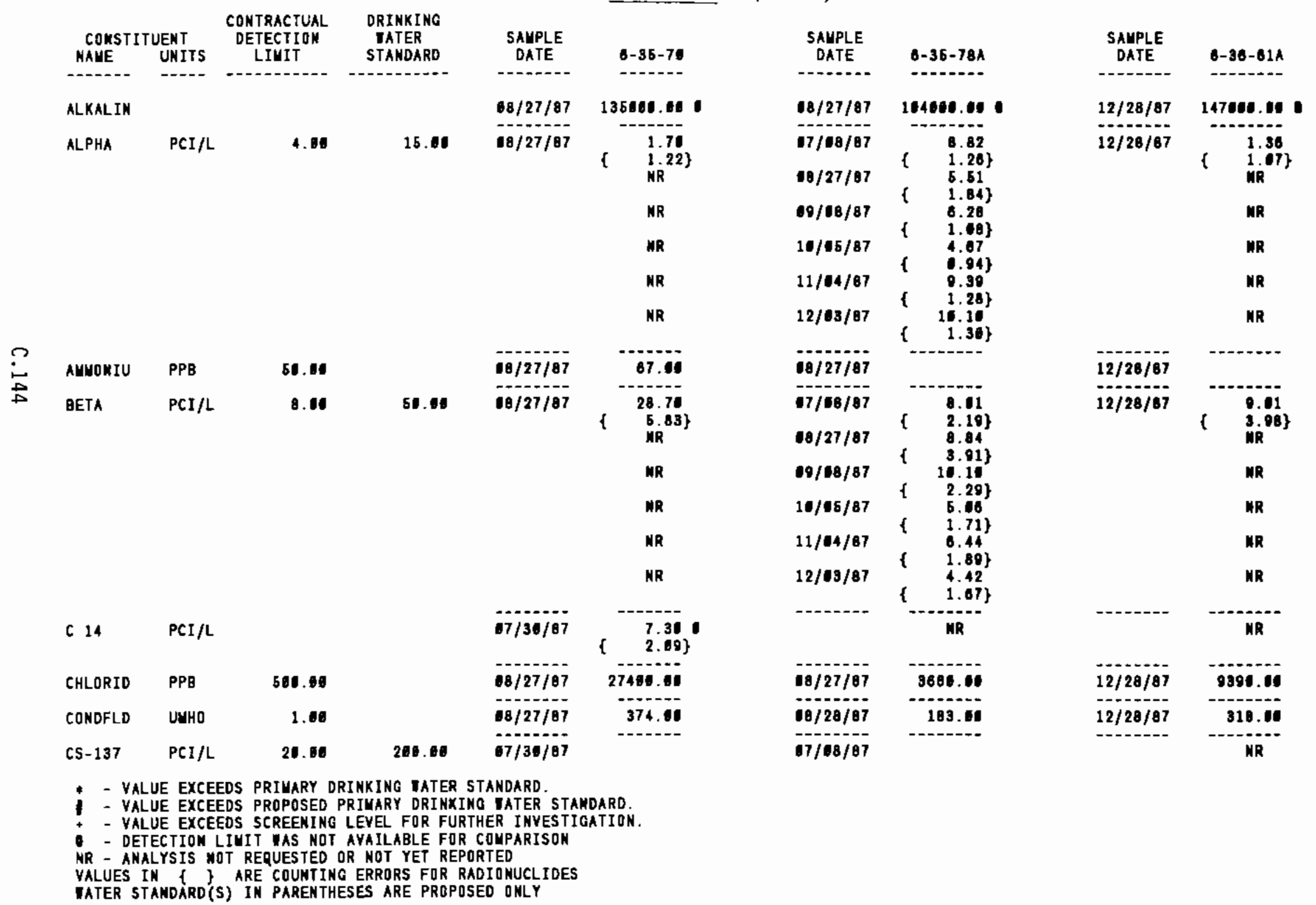


TABLE C.3. (contd)

\begin{tabular}{|c|c|c|c|c|c|c|c|c|c|}
\hline $\begin{array}{c}\text { CONSIIT } \\
\text { NAVE }\end{array}$ & $\begin{array}{l}\text { UENT } \\
\text { UNI TS }\end{array}$ & $\begin{array}{c}\text { CONTRACTUAL } \\
\text { DETECTION } \\
\text { LIUIT }\end{array}$ & $\begin{array}{l}\text { DRIWKING } \\
\text { TATER } \\
\text { STANDARD }\end{array}$ & $\begin{array}{c}\text { SAMPLE } \\
\text { DATE }\end{array}$ & $8-36-76$ & $\begin{array}{c}\text { SAMPLE } \\
\text { DATE }\end{array}$ & 6-35-78A & $\begin{array}{c}\text { SAMPLE } \\
\text { DATE }\end{array}$ & $6-38-81 A$ \\
\hline$C 5-137$ & $\mathrm{PCI} / \mathrm{L}$ & 20.00 & 206.00 & $10 / 20 / 87$ & & $10 / 05 / 87$ & $\left\{\begin{array}{l}9.86 \\
\quad 8.46\}\end{array}\right.$ & & NR \\
\hline FBARIUU & $\mathrm{PPB}$ & 0.00 & 1601.06 & $68 / 27 / 87$ & 42.01 & $68 / 27 / 87$ & 20.00 & $12 / 28 / 87$ & 58.60 \\
\hline FCALCIU & PPB & 50.00 & & $88 / 27 / 87$ & 37700.6 & $18 / 27 / 87$ & 16100.00 & $12 / 28 / 87$ & 42640.6 \\
\hline FCHROYI & PPB & 10.00 & 50.00 & $88 / 27 / 87$ & & $18 / 27 / 87$ & & $12 / 20 / 87$ & 16.65 \\
\hline FIRON & $\mathrm{PPB}$ & 50.10 & & $18 / 27 / 87$ & 94.11 & $80 / 27 / 87$ & 162.06 & $12 / 28 / 87$ & 139.60 \\
\hline FLUORID & PPB & 580.10 & 4006.00 & $18 / 27 / 87$ & & $80 / 27 / 87$ & 679.00 & $12 / 28 / \theta 7$ & \\
\hline FWAGNES & PPB & 0.00 & & $88 / 27 / 87$ & 13406.01 & $08 / 27 / 87$ & 8186.06 & $12 / 28 / 07$ & 13601.65 \\
\hline FUANGAN & PPB & 5.10 & & $18 / 27 / 87$ & 43.08 & $18 / 27 / 07$ & 40.106 & $12 / 28 / 87$ & \\
\hline FPOTASS & PPB & 180.80 & & $68 / 27 / 87$ & 5834.01 & $18 / 27 / 87$ & 2886.06 & $12 / 28 / 87$ & 4771.00 \\
\hline FSODIUU & PPB & 189.00 & & $88 / 27 / 87$ & 21800.00 & $18 / 27 / 87$ & 20000.00 & $12 / 28 / 87$ & 16705.00 \\
\hline FVAMADI & PPB & 5.00 & & $08 / 27 / 87$ & 23.64 & $80 / 27 / 87$ & 0.00 & $12 / 28 / 87$ & 30.00 \\
\hline FZINC & PPB & 6.00 & & $88 / 27 / 87$ & 16.00 & $80 / 27 / 87$ & 18.60 & $12 / 28 / 87$ & \\
\hline HNI TRAT & PPB & 2500.00 & 45000.00 & & MR & $\begin{array}{l}07 / 08 / 87 \\
16 / 65 / 87\end{array}$ & & $\begin{array}{l}07 / 90 / 87 \\
11 / 10 / 87\end{array}$ & $\begin{array}{l}18701.06 \\
21060.04\end{array}$ \\
\hline I-129 & $\mathrm{PCI} / \mathrm{L}$ & & 506.00 & $07 / 09 / 87$ & $\left\{\begin{array}{r}47.20^{\circ} \\
5.46\}\end{array}\right.$ & & NR & & MR \\
\hline LFLUORD & PPB & 20. 10 & 4868.60 & $88 / 27 / 87$ & 395.61 & $18 / 27 / 87$ & 535.18 & $12 / 28 / 87$ & 406.00 \\
\hline METHYCH & $\mathrm{PPB}$ & 10.00 & & $08 / 27 / 87$ & & $68 / 27 / 87$ & 12.00 & $12 / 28 / 87$ & \\
\hline NITRATE & PPB & 500.01 & 45000.00 & $90 / 27 / 87$ & 29400.00 & $68 / 27 / 87$ & 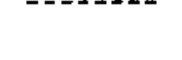 & $12 / 28 / 87$ & 22304.01 \\
\hline PH-LAB & & 1.11 & & $10 / 27 / 87$ & 7.82 & $68 / 27 / 87$ & 7.84 & $12 / 28 / 87$ & 7.70 \\
\hline PHFIELD & & 0.10 & & $88 / 27 / 87$ & 6.90 & $88 / 28 / 87$ & 8.81 & $12 / 28 / 87$ & 7.40 \\
\hline \multicolumn{10}{|c|}{ 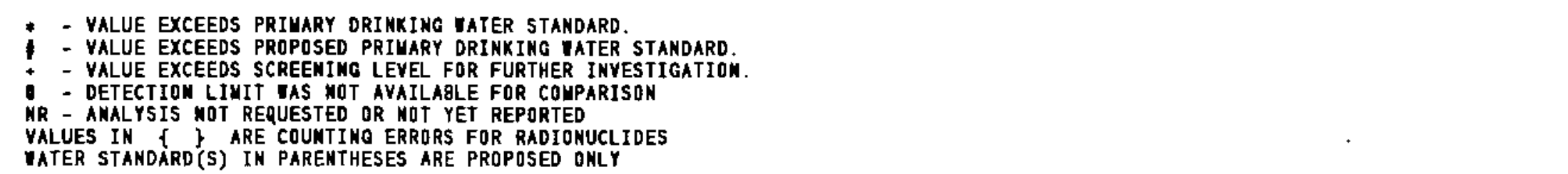 } \\
\hline
\end{tabular}


TABLE C.3. (contd)

\begin{tabular}{|c|c|c|c|c|c|c|c|c|c|}
\hline $\begin{array}{l}\text { CONSTI } \\
\text { WAME }\end{array}$ & $\begin{array}{l}\text { UENT } \\
\text { UNITS }\end{array}$ & $\begin{array}{c}\text { CONTRACTUAL } \\
\text { DETECTION } \\
\text { LIUIT }\end{array}$ & $\begin{array}{l}\text { DRINKING } \\
\text { DATER } \\
\text { STAMDARD }\end{array}$ & $\begin{array}{c}\text { SAUPLE } \\
\text { DATE }\end{array}$ & $8-35-70$ & $\begin{array}{l}\text { SAUPLE } \\
\text { DATE }\end{array}$ & $8-36-7 B A$ & $\begin{array}{l}\text { SAHPLE } \\
\text { DATE }\end{array}$ & $6-38-61 A$ \\
\hline 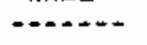 & $\ldots .$. & 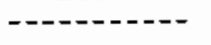 & 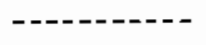 & $--1-1$ & ------ & $+-\infty-\infty$ & - & 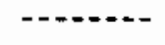 & $---\infty+-$ \\
\hline SULFATE & PPB & 501.90 & & $08 / 27 / 87$ & 36100.60 & $16 / 27 / 87$ & 14195. 01 & $12 / 28 / 87$ & 31600.00 \\
\hline TC & PPB & & & $18 / 27 / 87$ & 31446.01 & $08 / 27 / 87$ & 16000.00 & $12 / 28 / 87$ & \\
\hline$T C-80$ & $\mathrm{PCI} / \mathrm{L}$ & 15.00 & 900.60 & $17 / 36 / 87$ & $\left\{\begin{array}{r}112.00 \\
2.00\}\end{array}\right.$ & & WR & & NR \\
\hline TRITIUU & $\mathrm{PCI} / \mathrm{L}$ & 506.60 & 26000.08 & $17 / 30 / 87$ & $1346100.00 \%$ & $07 / 08 / 87$ & - & $---1-0$ & NR \\
\hline & & & & $10 / 28 / 87$ & $\begin{array}{c}1240000.10 \\
\{4161.06\} \\
\text { NR } \\
\text { NR } \\
\text { NR }\end{array}$ & $\begin{array}{l}19 / 48 / 87 \\
10 / 05 / 87 \\
11 / 04 / 87 \\
12 / 08 / 87\end{array}$ & & & $\begin{array}{l}\text { NR } \\
\text { MR } \\
\text { MR } \\
\text { NR }\end{array}$ \\
\hline $\mathbf{u}$ & $\mathrm{PCI} / \mathrm{L}$ & 6.60 & 860.66 & $\begin{array}{l}6 / 30 / 97 \\
10 / 26 / 87\end{array}$ & $\begin{array}{r}2.18 \\
2.34\end{array}$ & -ルー-ー- & $\begin{array}{l}\text { MR } \\
\text { MR }\end{array}$ & & MR \\
\hline U 234 & $\mathrm{PCI} / \mathrm{L}$ & 0.10 & 32.00 & & NR & $67 / 08 / 07$ & $\left\{\begin{array}{l}5.05 \\
\quad .26\}\end{array}\right.$ & & NR \\
\hline U 235 & PCI/L & 6.10 & 32.66 & & MR & $07 / 08 / 87$ & $\{\quad 0.24$ & & MR \\
\hline U 238 & $\mathrm{PCI} / \mathrm{L}$ & 0.10 & 4.00 & ---.-.-. & NR & $17 / 08 / 87$ & $\begin{array}{c}4.78 \\
\quad 1.26\}\end{array}$ & & MR \\
\hline U-CHEU & UG/L & e. 73 & & & $\begin{array}{c}\text { MR } \\
\text { MR } \\
\text { NR } \\
\text { NR } \\
\text { NR }\end{array}$ & $\begin{array}{l}07 / 08 / 87 \\
09 / 08 / 87 \\
10 / 65 / 87 \\
11 / 64 / 87 \\
12 / 109 / 87\end{array}$ & $\begin{array}{r}13.20 \\
7.90 \\
7.85 \\
11.70 \\
11.56\end{array}$ & & $\begin{array}{l}\text { MR } \\
\text { NR } \\
\text { MR } \\
\text { MR } \\
\text { NR }\end{array}$ \\
\hline
\end{tabular}

- - YaLUE eXCEEDS PRIUARY dRINKING tater STANDARD.

- YALUE EXCEEDS PROPOSED PRIUARY DRINKING IATER STANDARD

- VALUE EXCEEDS SCREEMING LEVEL FOR FURTHER INYESTIGATION.

- DETECTION LIUIT TAS nOt aVAILABLE for couparison

NR - ANALYSIS NOT REQUESTED OR MOT YET REPORTED

VALUES IN \{\} ARE COUNTING ERRORS FOR RADIOHUCLIDES

TATER STAMDARD(S) IN PARENTHESES ARE PROPOSED OALY 
TABLE C.3. (contd)

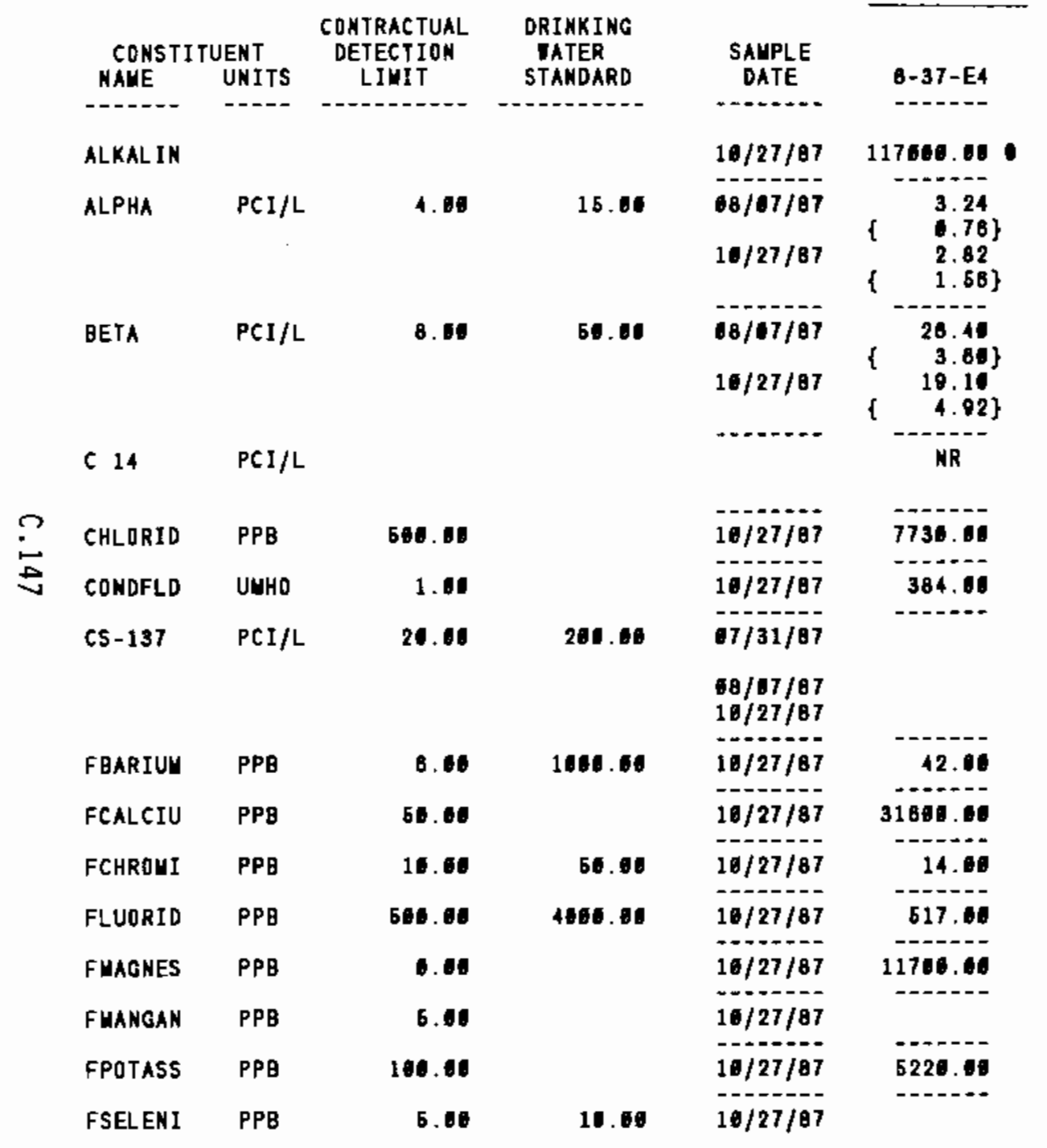

\begin{tabular}{|c|c|}
\hline $\begin{array}{l}\text { SAMPLEE } \\
\text { DATE }\end{array}$ & $6-37-49$ \\
\hline $11 / 16 / 87$ & 104000000 \\
\hline $11 / 15 / 87$ & $\left\{\begin{array}{l}3.28 \\
\left\{\begin{array}{l}2.26 \\
M R\end{array}\right\}\end{array}\right.$ \\
\hline $11 / 15 / 87$ & $\left\{\begin{array}{l}9.10 \\
4.00\} \\
M R\end{array}\right\}$ \\
\hline $00 / 10 / 87$ & $\begin{array}{r}8.38 \\
\{\quad 2.77\}\end{array}$ \\
\hline $11 / 16 / 87$ & 11706.00 \\
\hline $11 / 15 / 87$ & 518.06 \\
\hline $60 / 10 / 87$ & 12.96 \\
\hline $11 / 16 / 87$ & \\
\hline $11 / 16 / 87$ & 78.00 \\
\hline $11 / 16 / 87$ & 60460.00 \\
\hline $11 / 15 / 87$ & \\
\hline $11 / 16 / 87$ & 899.16 \\
\hline $11 / 15 / 87$ & 16304.00 \\
\hline $11 / 16 / 87$ & 5.10 \\
\hline $11 / 16 / 87$ & 7891.06 \\
\hline $11 / 16 / 87$ & \\
\hline
\end{tabular}

* - value exceeds priuary drinxing tater standard

1 - VALUE EXCEEDS PROPOSED PRIMARY DRINKING YATER STANDARD.

- VALUE EXCEEDS SCREENINO LEVEI FOR FURTHER INVESTIGATION.

- DETECTION liUit tas hot aVAILABle for coyparison

NR - ANALYSIS NOT RERUESTED OR NOT YET REPORTED

VALUES IN \& $\}$ ARE COUNTINO ERRORS FOR RADIONUCLIDES

IATER STANDARD(S) IN PARENTHESES ARE PROPOSED ONLY 
TABLE C.3. (contd)

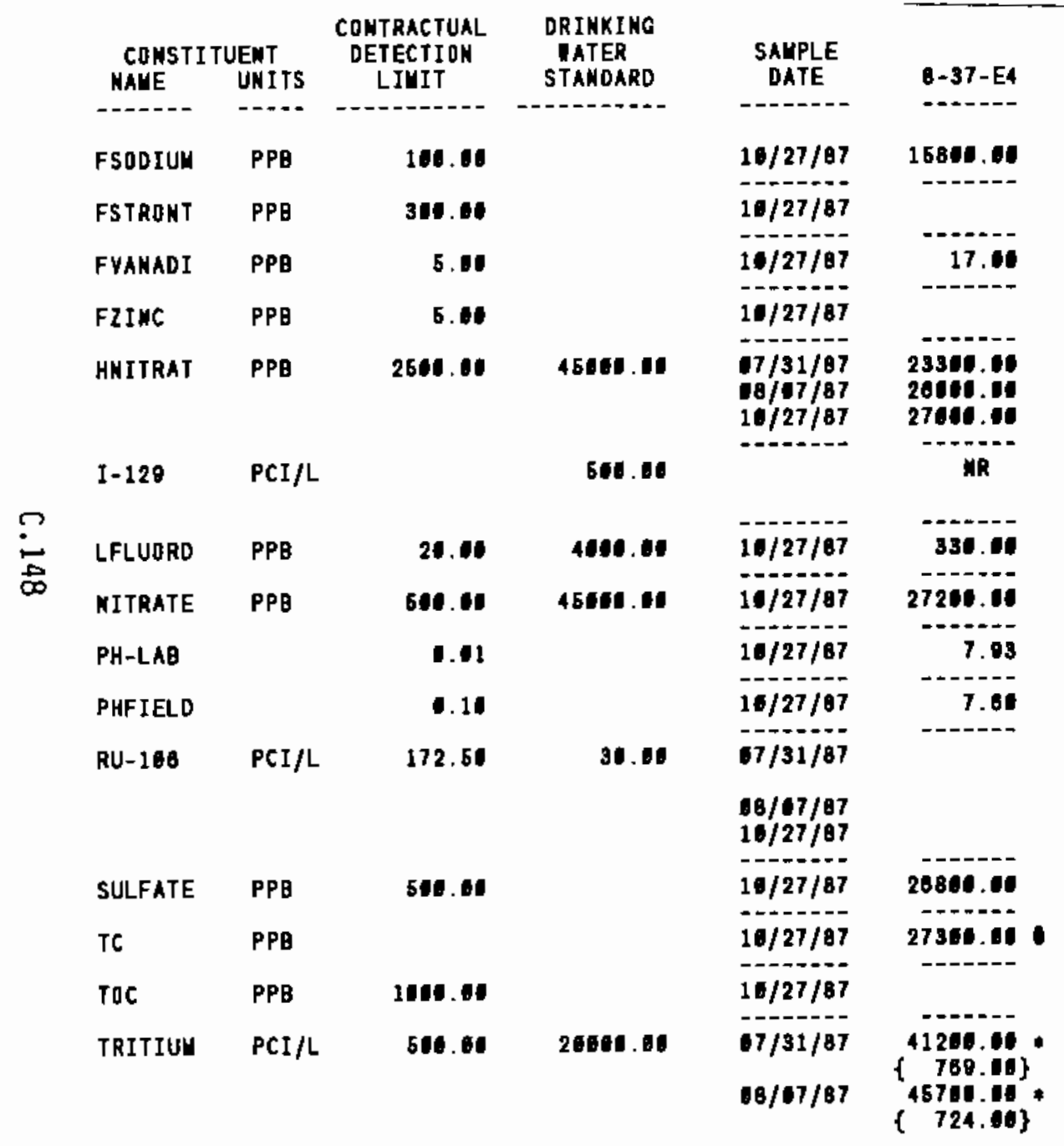

\begin{tabular}{|c|c|c|c|}
\hline $\begin{array}{c}\text { SAUPLE } \\
\text { DATE }\end{array}$ & $8-37-43$ & $\begin{array}{c}\text { SAMPLE } \\
\text { DATE }\end{array}$ & $6-37-82 A$ \\
\hline $11 / 15 / 87$ & 40548 . & $99 / 23 / 87$ & 21504 st \\
\hline $11 / 15 / 87$ & 358.00 & $00 / 23 / 87$ & \\
\hline $11 / 16 / 87$ & 21.10 & $09 / 23 / 87$ & 30.01 \\
\hline $11 / 15 / 87$ & 8.60 & $09 / 23 / 87$ & \\
\hline $\begin{array}{l}09 / 10 / 87 \\
11 / 15 / 87\end{array}$ & 10204.00 & & $\begin{array}{l}\text { NR } \\
\text { NR } \\
\text { MR }\end{array}$ \\
\hline $60 / 10 / 07$ & 16.71 & & NR \\
\hline $11 / 16 / 87$ & 511.61 & $09 / 28 / 87$ & 224.6 \\
\hline $11 / 15 / 87$ & 1070e.01 & $08 / 23 / 87$ & 47104.00 \\
\hline $11 / 16 / 87$ & 7.80 & $00 / 29 / 87$ & 0.77 \\
\hline $11 / 16 / 87$ & 7.81 & $19 / 23 / 87$ & 0.11 \\
\hline $09 / 10 / 87$ & 71.80 & & MR \\
\hline $11 / 15 / 87$ & $\begin{array}{l}t \quad 43.30) \\
\text { MR }\end{array}$ & & $\underset{M R}{M R}$ \\
\hline $11 / 15 / 87$ & 218000 .60 & $00 / 23 / 87$ & 54300.00 \\
\hline $11 / 16 / 87$ & 22766.01 & $88 / 23 / 87$ & 2060.01 \\
\hline $11 / 15 / 87$ & & $00 / 23 / 87$ & 1930.06 \\
\hline $60 / 10 / 87$ & 11000.06 & $18 / 21 / 87$ & \\
\hline $11 / 15 / 87$ & $\left\{\begin{array}{l}58004.06 \\
\{028.06\end{array}\right.$ & $11 / 20 / 87$ & $\left\{\begin{array}{l}498.00 \\
175.04\end{array}\right.$ \\
\hline
\end{tabular}

* - VAlue exceeds primary drinking tater STANDARD.

- VALUE EXCEEDS PROPDSED PRTUARY DRINKING YATER STANDARD.

- Y YAUE EXCEEDS SCREENINg LEVEL FOR FURTHER IMVESTIGATIDN.

- DETECTION LIUIT IAS MOT AVAILABLE fOR COMPARISON

HR - ANALYSIS NOT REQUESTED OR NOT YET REPORTED

VALUES IN I I ARE COUNTING ERRDRS FOR RADIONUCLIDES

IATER STANDARD'S) IN PARENTHESES ARE PROPOSED ONLY 
TABLE C.3. (contd)

\begin{tabular}{|c|c|c|c|c|c|c|c|c|c|}
\hline$\underset{\text { MAYE }}{\text { CONSTI }}$ & $\begin{array}{l}\text { UENT } \\
\text { UNITS }\end{array}$ & $\begin{array}{c}\text { CONTRACTUAL } \\
\text { DETECTION } \\
\text { LIUIT }\end{array}$ & $\begin{array}{l}\text { DRINKING } \\
\text { IATER } \\
\text { STANDARD }\end{array}$ & $\begin{array}{c}\text { SAMPLE } \\
\text { DATE }\end{array}$ & $\theta-37-E_{4}$ & $\begin{array}{c}\text { SAMPLE } \\
\text { DATE }\end{array}$ & $6-37-43$ & $\begin{array}{c}\text { SAMPLE } \\
\text { DATE }\end{array}$ & $6-37-82 A$ \\
\hline IRITIUY & $\mathrm{PCI} / \mathrm{L}$ & 600.00 & 20061.00 & $10 / 27 / 87$ & $\left\{\begin{array}{c}49460.06 \\
\{870.86\}\end{array}\right.$ & & MR & & MR \\
\hline u & $\mathrm{PCI} / \mathrm{L}$ & 0.60 & 600.60 & $68 / 07 / 87$ & 2.24 & & MR & & MR \\
\hline
\end{tabular}

$\overbrace{i}^{0}$

* - VALUE EXCEEDS PRIUARY DRIMKING IATER STANDARD.

- VALUE EXCEEDS PROPOSED PRIUARY DRINKING IATER STANDARD.

- - VALUE EXCEEDS SCREEMING LEVEI FOR FURTHER INYESTIGATION.

- DETECTION LIUIT TAS HOT AVAILABLE FOR COUPARISON

NR - ANALYSIS NOT REQUESTED OR NOT YET REPORTED

YALUES IN \{\} ARE COUNTING ERRDRS FOR RADIONUCLIDES

IATER STAMDARD(S) IN PARENTHESES ARE PRDPOSED ONLY 
TABLE C.3. (contd)

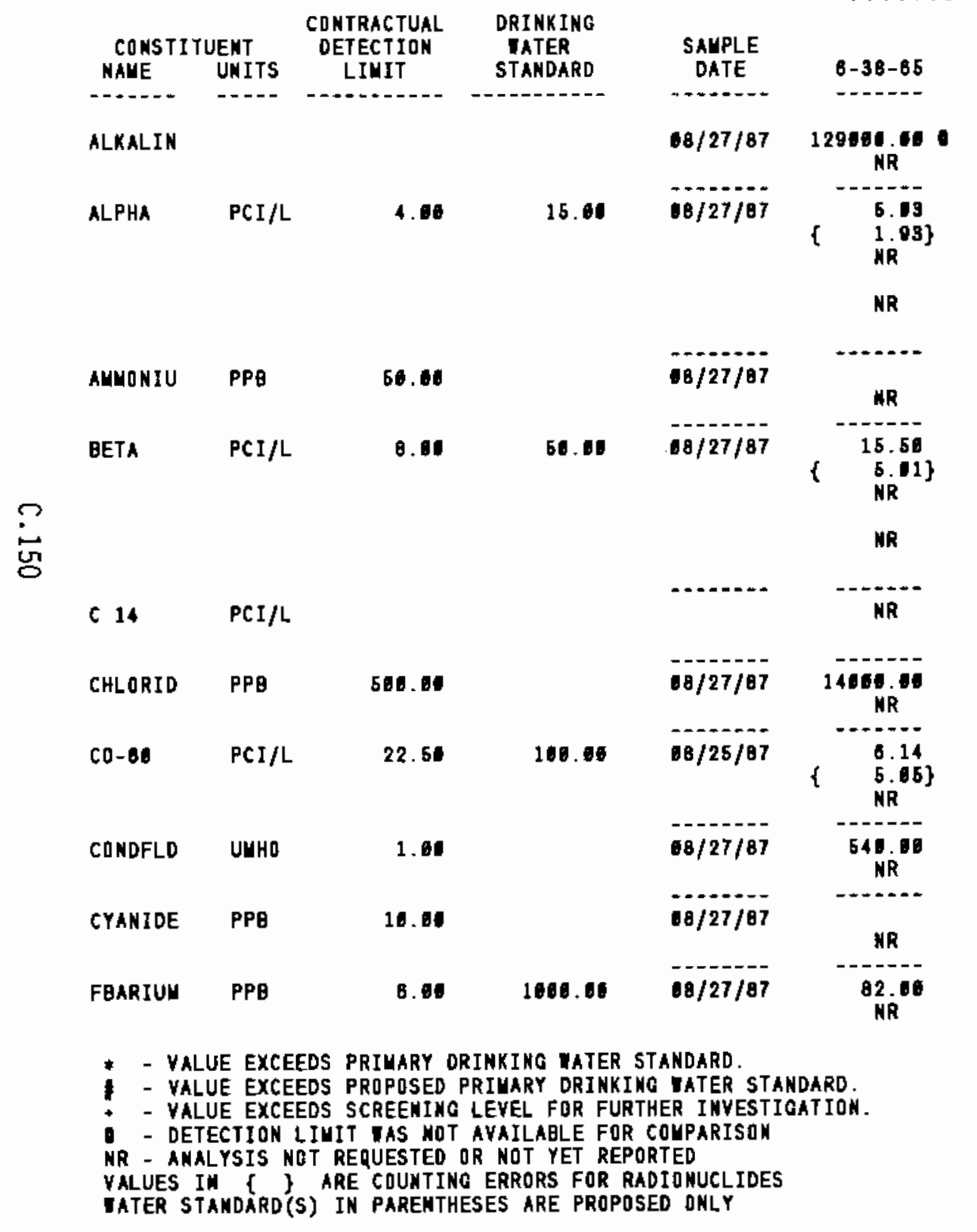

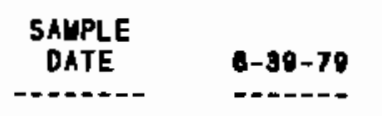

18/27/87 96041.64:

12/02/87 99440.06

18/27/a7 4.05

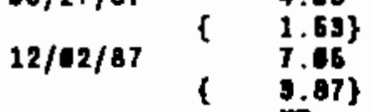

in

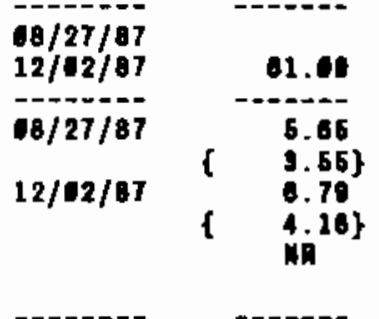

$\operatorname{mo}$

18/27/87

$12 / 02 / 87 \quad 3430.00$

$18 / 25 / 87 \quad 0.43$

( 6.25$\}$

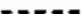

$18 / 27 / 87$

$12 / 02 / 07$

$68 / 27 / 67$

12/02/87

$6 / 27 / 87$

$12 / 02 / 87$
170.60

194.00

----.

21.9

20.11

$08 / 27187$

$12 / 01 / 87 \quad B B .6 B$ 
IABLE C.3. (contd)

\begin{tabular}{|c|c|c|c|c|c|}
\hline $\begin{array}{c}\text { CONSTI I } \\
\text { NAWE }\end{array}$ & $\begin{array}{l}\text { UENT } \\
\text { UNITS }\end{array}$ & $\begin{array}{l}\text { CONTRACTUAL } \\
\text { DETECTION } \\
\text { LIMIT }\end{array}$ & $\begin{array}{l}\text { DRINXING } \\
\text { IATER } \\
\text { STANDARD }\end{array}$ & $\begin{array}{l}\text { SAUPLE } \\
\text { DATE }\end{array}$ & $8-38-85$ \\
\hline FCALCIU & PPB & 50.10 & & $08 / 27 / 87$ & 53916.00 \\
\hline FIRON & PPB & 51.06 & & $68 / 27 / 87$ & NR \\
\hline FLUORID & PPB & 600.00 & 4600.00 & $60 / 27 / 87$ & NR \\
\hline FUAGNES & PPB & 1.09 & & $18 / 27 / 87$ & 21800.106 \\
\hline FUANGAN & PPB & 5.60 & & $68 / 27 / 87$ & $\begin{array}{l}5.16 \\
\text { NR }\end{array}$ \\
\hline FPOTASS & PPB & 186.04 & & $18 / 27 / 87$ & NR \\
\hline FSODIUU & PPB & 166.60 & & $68 / 27 / 87$ & $\underset{N R}{24200.00}$ \\
\hline FSTRONT & PPB & 360.60 & & $68 / 27 / 87$ & ${ }_{\mathrm{HR}}^{346}$ \\
\hline FYAMADI & PPB & 5.06 & & $18 / 27 / 87$ & $\underset{M R}{28.010}$ \\
\hline FZINC & PPB & 5.80 & & $68 / 27 / 87$ & MR \\
\hline LFLUORD & PPB & 20.00 & 4000.00 & $08 / 27 / 87$ & $\begin{array}{c}348.01 \\
\text { NR }\end{array}$ \\
\hline UETHYCH & PPB & 10.00 & & $08 / 27 / 87$ & $\underset{\text { NR }}{336104}$ \\
\hline HI- 83 & $\mathrm{PCI} / \mathrm{L}$ & 18.00 & 58.60 & & NR \\
\hline
\end{tabular}

\begin{tabular}{|c|c|}
\hline $\begin{array}{l}\text { SAUPLE } \\
\text { DATE }\end{array}$ & $8-38-71$ \\
\hline $\begin{array}{l}68 / 27 / 87 \\
12 / 61 / 87\end{array}$ & $\begin{array}{l}78000.110 \\
88104.110\end{array}$ \\
\hline $\begin{array}{l}18 / 27 / 87 \\
12 / 01 / 87\end{array}$ & \\
\hline $\begin{array}{l}10 / 27 / 87 \\
12 / 01 / 87\end{array}$ & 888.01 \\
\hline $\begin{array}{l}18 / 27 / 87 \\
12 / 61 / 87\end{array}$ & $\begin{array}{l}36060.01 \\
31800.01\end{array}$ \\
\hline $\begin{array}{l}18 / 27 / 87 \\
12 / 01 / 87\end{array}$ & 22.10 \\
\hline $\begin{array}{l}18 / 27 / 87 \\
12 / 11 / 87\end{array}$ & $\begin{array}{l}8481.06 \\
5841.04\end{array}$ \\
\hline $\begin{array}{l}18 / 27 / 87 \\
12 / 01 / 87\end{array}$ & $\begin{array}{l}21204.11 \\
21406.16\end{array}$ \\
\hline $\begin{array}{l}18 / 27 / 87 \\
12 / 01 / 87\end{array}$ & $\begin{array}{r}496.66 \\
485.66\end{array}$ \\
\hline $\begin{array}{l}18 / 27 / 87 \\
12 / 01 / 87\end{array}$ & $\begin{array}{l}10.60 \\
26.06\end{array}$ \\
\hline $\begin{array}{l}68 / 27 / 87 \\
12 / 1 / / 87\end{array}$ & $\begin{array}{l}16.00 \\
21.00\end{array}$ \\
\hline $\begin{array}{l}09 / 27 / 87 \\
12 / 01 / 87\end{array}$ & 428.10 \\
\hline $\begin{array}{l}18 / 27 / 87 \\
12 / 11 / 87\end{array}$ & 17.01 \\
\hline $10 / 25 / 07$ & $\left\{\begin{array}{l}4.60 \\
2.06\end{array}\right.$ \\
\hline
\end{tabular}

SAUPLE

DATE

0-39-70

$08 / 27 / 87$

22301. IS

$08 / 27 / 87$

$12 / 02 / 87$

$-7-127 / 87$

$00 / 27 / 87$
$12 / 102 / 87$

$10 / 27 / 87$

$12 / 02 / 87$

$8 / 27 / 87$

$12 / 102 / 87$

0107107

$10 / 27 / 07$
$12 / 02 / 07$

$18 / 27 / 87$

$12 / 02 / 87$

$18 / 27 / 87$

$12 / 12 / 87$

10.0.-.

$09 / 27 / 87$
$12 / 12 / 07$

$8 / 27 / 87$

12/02/87

$68 / 27 / 87$

$12 / 02 / 87$

-......-

$18 / 27 / 87$

$12 / 102 / 87$

67. 15

-

5226.01

5846. 10

-..--

2920.01

2680.01

206es.06

18164.

617.

19.00

17.00

415.00

MR

4.68

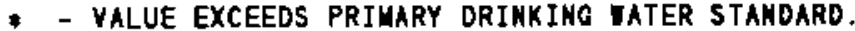

- VALUE EXCEEDS PROPOSED PRIUARY DRINKING TATER STANDARD.

* VALUE EXCEEDS SCREENING LEVEL FOR FURTHER INVESTIGATION.

- DETECTION LIUIT IAS NOT AVAILABLE FOR COUPARISON

NR - ANALYSIS NOT REQUESTED OR NOT YET REPORTED

VALUES IN \{\} ARE COUNTING ERRORS FOR RADIONUCLIDES

TATER STANDARO'(S) IN PARENTHESES ARE PROPOSED ONLY 
TABLE C.3. (contd)

\begin{tabular}{|c|c|c|c|c|c|}
\hline $\begin{array}{c}\text { CONSTI } \\
\text { NAME }\end{array}$ & $\begin{array}{l}\text { UEYT } \\
\text { UNI TS }\end{array}$ & $\begin{array}{l}\text { CONTRACTUAL } \\
\text { DETECTION } \\
\text { LIUIT }\end{array}$ & $\begin{array}{l}\text { DRINKING } \\
\text { DATER } \\
\text { STANDARD }\end{array}$ & $\begin{array}{c}\text { SAUPLE } \\
\text { DATE }\end{array}$ & $8-38-65$ \\
\hline MITRATE & PPB & 580.00 & 46680.00 & $08 / 27 / 87$ & $\underset{\mathrm{NR}}{180009.16} *$ \\
\hline PH-LAB & & 0.01 & & $18 / 27 / 87$ & ${ }_{M R}^{7.91}$ \\
\hline PHFIELD & & 0.10 & & $08 / 27 / 87$ & $\begin{array}{l}\text { B.80 } \\
\mathrm{KR}\end{array}$ \\
\hline$R U-106$ & $\mathrm{PCI} / \mathrm{L}$ & 172.50 & 36.06 & $18 / 25 / 87$ & $\{\underbrace{65.10}_{\mathrm{MR}}\}^{54.10}$ \\
\hline SULFATE & PPB & 501.6 & & $18 / 27 / 87$ & $3918 \mathrm{MR}$ \\
\hline TC & PPB & & & $08 / 27 / 87$ & 38004. If \\
\hline TC-90 & $\mathrm{PCI} / \mathrm{L}$ & 15.60 & 904.64 & & $\begin{array}{l}\text { MR } \\
\text { MR } \\
\text { MR }\end{array}$ \\
\hline TETRANE & PPB & 10.10 & 6.60 & $88 / 27 / 87$ & MR \\
\hline TOC & PPB & 1000.01 & & $68 / 27 / 87$ & MR \\
\hline TOXLDL & PPB & 20.80 & & $68 / 27 / 87$ & 27604.16 \\
\hline TRITIUE & $\mathrm{PCI} / \mathrm{L}$ & 581.00 & 20000.00 & $\begin{array}{l}68 / 25 / 87 \\
16 / 26 / 87\end{array}$ & $\begin{array}{l}389000.06 \\
\{2320.06\} \\
359006.84 \\
\{2236.08\}\end{array}$ \\
\hline
\end{tabular}

\begin{tabular}{|c|c|c|c|}
\hline $\begin{array}{c}\text { SAYPLE } \\
\text { DATE }\end{array}$ & $8-36-76$ & $\begin{array}{c}\text { SAMPLE } \\
\text { DATE }\end{array}$ & $8-38-70$ \\
\hline $\begin{array}{l}18 / 27 / 87 \\
12 / 11 / 87\end{array}$ & $202960.00:$ & $\begin{array}{l}18 / 27 / 87 \\
12 / 02 / 87\end{array}$ & $\begin{array}{l}2390.10 \\
8230.11\end{array}$ \\
\hline $\begin{array}{l}08 / 27 / 87 \\
12 / 01 / 87\end{array}$ & $\begin{array}{l}7.81 \\
7.68\end{array}$ & $\begin{array}{l}18 / 27 / 87 \\
12 / 62 / 87\end{array}$ & $\begin{array}{l}\text { 6. } 12 \\
\text { B. } 27\end{array}$ \\
\hline $\begin{array}{l}68 / 27 / 87 \\
12 / 01 / 87\end{array}$ & $\begin{array}{l}0.80 \\
7.76\end{array}$ & $\begin{array}{l}18 / 27 / 87 \\
12 / 02 / 87\end{array}$ & $\begin{array}{l}7.31 \\
8.10\end{array}$ \\
\hline $08 / 26 / 07$ & & $18 / 25 / 87$ & \\
\hline $12 / 01 / 87$ & & $12 / 02 / 87$ & \\
\hline $\begin{array}{l}68 / 27 / 87 \\
12 / 01 / 87\end{array}$ & $\begin{array}{l}48806.04 \\
46865.40\end{array}$ & $\begin{array}{l}18 / 27 / 87 \\
12 / 62 / 87\end{array}$ & $\begin{array}{l}14001.06 \\
14660.06\end{array}$ \\
\hline $\begin{array}{l}18 / 27 / 87 \\
12 / 01 / 87\end{array}$ & 29201.00 & $\begin{array}{l}10 / 27 / 87 \\
12 / 02 / 87\end{array}$ & $\begin{array}{l}21406.01 \\
23901.01\end{array}$ \\
\hline $18 / 25 / 87$ & $\left\{\begin{array}{c}1389.61 \\
6.24\}\end{array}\right.$ & & FR \\
\hline $90 / 15 / 87$ & 2860.60 & & MR \\
\hline $12 / 19 / 07$ & $\left\{\begin{array}{r}2576.98 \\
8.99\}\end{array}\right.$ & & NR \\
\hline $\begin{array}{l}08 / 27 / 87 \\
12 / 11 / 87\end{array}$ & $\begin{array}{r}35.04 \\
45.01\end{array}$ & $\begin{array}{l}08 / 27 / 87 \\
12 / 102 / 07\end{array}$ & $\begin{array}{r}264.44 \\
380.14\end{array}$ \\
\hline $\begin{array}{l}68 / 27 / 87 \\
12 / 1 / 87\end{array}$ & 1124.00 & $\begin{array}{l}08 / 27 / 87 \\
12 / 02 / 87\end{array}$ & 1170.01 \\
\hline $\begin{array}{l}68 / 27 / 87 \\
12 / 1 / 87\end{array}$ & $\begin{array}{r}53.20 \\
52.10 \\
-\end{array}$ & $\begin{array}{l}08 / 27 / 87 \\
12 / 62 / 87\end{array}$ & $\begin{array}{r}102.4 \\
123.16\end{array}$ \\
\hline $18 / 25 / 97$ & $\left\{\begin{array}{r}1271.0 \\
242.10\end{array}\right\}$ & $60 / 26 / 87$ & \\
\hline $12 / 11 / 87$ & $\left\{\begin{array}{r}1080.10\} \\
231.10\}\end{array}\right.$ & $12 / 02 / 87$ & \\
\hline
\end{tabular}

- - VALUE eXCEeds priuary dRIMKING tater stakdard.

- VALUE EXCEEDS PROPOSED PRIMARY DRINKIMG IATER STANDARO.

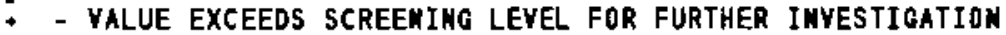

- DETECTION limit Ias not ayailable for couparison

NR - ANALYSIS NOT REQUESTED OR NOT YET REPORTED

VALUES IN \{\} ARE COUNTING ERRORS FOR RADIOHUCLIDES

IATER STANDARD(S) IN PARENTHESES ARE PROPOSED ONLY 


\begin{tabular}{|c|c|c|c|c|c|}
\hline $\begin{array}{l}\text { CONST } \\
\text { NAHE }\end{array}$ & $\begin{array}{l}\text { UENT } \\
\text { UNITS }\end{array}$ & $\begin{array}{c}\text { CONTRACTUAL } \\
\text { DETECTION } \\
\text { LIUIT }\end{array}$ & $\begin{array}{l}\text { DRINK ING } \\
\text { TATER } \\
\text { STANDARD }\end{array}$ & $\begin{array}{c}\text { SALPLE } \\
\text { DATE }\end{array}$ & $6-38-65$ \\
\hline----- & $-\infty \ldots$ & $x-2+\infty+\infty$ & $--1-+-1+-$ & $+\infty-200-0$ & $+\infty+\infty-\infty$ \\
\hline U & $\mathrm{PCI} / \mathrm{L}$ & 0.50 & 600.06 & $\begin{array}{l}18 / 25 / 87 \\
10 / 26 / 87\end{array}$ & $\begin{array}{l}1.57 \\
1.54\end{array}$ \\
\hline U 234 & $\mathrm{PCI} / \mathrm{L}$ & 0.10 & 32.60 & & NR \\
\hline U 235 & $\mathrm{PCI} / \mathrm{L}$ & 6.16 & 32.00 & & NR \\
\hline U 238 & PCI /L & 5.10 & 4.80 & & MR \\
\hline$U-236$ & $\mathrm{PCI} / \mathrm{L}$ & t. 10 & 500.00 & & MR \\
\hline U-CHEU & UG/L & 0.73 & & ---1 & NR \\
\hline
\end{tabular}

SAMPLE DATE - - $8-38-76$

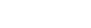 $\begin{array}{ll}08 / 25 / 87 & 36.40 \\ 12 / 01 / 87 & 39.20\end{array}$ $19 / 15 / 87$ 23.20

1.80

$-2-10$<smiles>[13CH][13CH][13CH]</smiles>

$60 / 16 / 87$

$\quad .79$
$\quad \quad .35\}$

$10 / 16 / 87$

$\because-1-1-1$

$19 / 15 / 87$

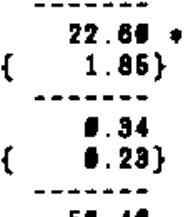

$12 / 03 / 87$
SAMPLE

DATE

8-30-70

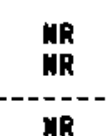

MR
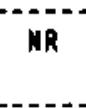

NR

NR

NR

* - YaLUE EXCEEDS PRIUARY DRINKING tater STANDARD.

- - YALUE EXCEEDS PROPOSED PRIUARY DRIMKING WATER STANDARD.

- - YALUE EXCEEDS SCREENING LEYEL FOR FURTHER INYESTIGATION.

- DETECTION LIUIT IAS NOT AYAILABLE FOR COUPARISON

MR - AMALYSIS NOT REQUESTED OR NOT YET REPORTED

VALUES IN \{ $\}$ ARE COUNTING ERRORS FOR RADIONUCLIDES

TATER STANDARD(S) IN PARENTHESES ARE PROPOSED ONLY 
TABLE C.3. (contd)

\begin{tabular}{|c|c|c|c|c|c|}
\hline $\begin{array}{l}\text { CONSTIT } \\
\text { MANE }\end{array}$ & $\begin{array}{l}\text { TUENT } \\
\text { UNITS }\end{array}$ & $\begin{array}{c}\text { CONTRACTUAL } \\
\text { DETECTION } \\
\text { LIMIT }\end{array}$ & $\begin{array}{l}\text { DRINKING } \\
\text { IATER } \\
\text { STAMDARD }\end{array}$ & $\begin{array}{c}\text { SAMPLE } \\
\text { DATE }\end{array}$ & $8-46-1$ \\
\hline ALKALIN & & & & $\begin{array}{l}19 / 01 / 87 \\
10 / 27 / 87\end{array}$ & $\begin{array}{l}1280100.00 \\
125000.04\end{array}$ \\
\hline ALPHA & PCI/L & 4.00 & 16.00 & $\begin{array}{l}08 / 07 / 87 \\
09 / 61 / 87 \\
11 / 27 / 87\end{array}$ & $\begin{array}{l}3.78 \\
0.85\} \\
1.79 \\
1.25\} \\
3.28 \\
1.49\}\end{array}$ \\
\hline BETA & $\mathrm{PCI} / \mathrm{L}$ & 8.00 & 50.00 & $\begin{array}{l}68 / 07 / 87 \\
10 / 61 / 87 \\
10 / 27 / 87\end{array}$ & 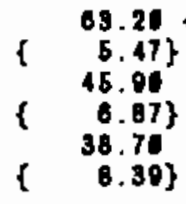 \\
\hline CHLORIO & PPB & 600.09 & & $\begin{array}{l}69 / 01 / 67 \\
10 / 27 / 97\end{array}$ & $\begin{array}{r}9360.010 \\
11160.00\end{array}$ \\
\hline CONDFLD & UHHO & 1.01 & & $\begin{array}{l}09 / 01 / 87 \\
16 / 27 / 87\end{array}$ & $\begin{array}{l}301.11 \\
464.08\end{array}$ \\
\hline CYANIDE & PPB & 10.6 & & $\begin{array}{l}09 / 61 / 87 \\
16 / 27 / 87\end{array}$ & 11.50 \\
\hline FARSENI & PPB & 5.66 & 51.06 & $\begin{array}{l}10 / 61 / 87 \\
10 / 27 / 87\end{array}$ & \\
\hline FBARIUU & $\mathrm{PPB}$ & 6.00 & 1806.00 & $\begin{array}{l}10 / 01 / 87 \\
10 / 27 / 87\end{array}$ & $\begin{array}{l}58.00 \\
64.01\end{array}$ \\
\hline FCALCIU & PPB & 50.00 & & $\begin{array}{l}00 / 01 / 87 \\
16 / 27 / 87\end{array}$ & $\begin{array}{l}38200.80 \\
41160.06\end{array}$ \\
\hline FCHRONI & PPB & 16.00 & 50.66 & $\begin{array}{l}10 / / 1 / 87 \\
10 / 27 / 87\end{array}$ & 10.10 \\
\hline FIRON & PPB & & & $69 / 01 / 87$ & \\
\hline \multicolumn{6}{|c|}{ 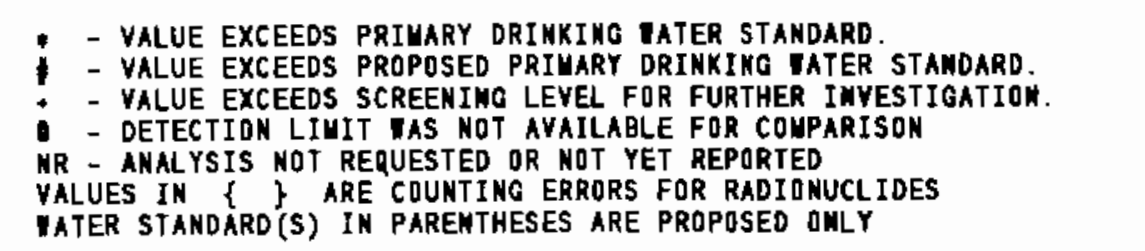 } \\
\hline
\end{tabular}

\begin{tabular}{|c|c|}
\hline $\begin{array}{l}\text { SAUPLE } \\
\text { DATE }\end{array}$ & $6-40-33 A$ \\
\hline $60 / 03 / 67$ & 188est.106 \\
\hline \multirow[t]{3}{*}{$10 / 63 / 87$} & \\
\hline & NR \\
\hline & NR \\
\hline \multirow{3}{*}{$19 / 03 / 87$} & \\
\hline & $\left.\begin{array}{l}\begin{array}{l}0.18 \\
3.08\end{array} \\
M R\end{array}\right\}$ \\
\hline & NR \\
\hline $10 / 03 / 87$ & 3289.60 \\
\hline $10 / 63 / 87$ & 261 WR \\
\hline $00 / 03 / 87$ & MR \\
\hline $10 / 09 / 87$ & B.6. \\
\hline $69 / 09 / 07$ & MR. \\
\hline $60 / 63 / 67$ & 1104. \\
\hline $00 / 03 / 87$ & \\
\hline & MR \\
\hline $09 / 01 / 87$ & 128.16 \\
\hline
\end{tabular}

\begin{tabular}{|c|c|}
\hline $\begin{array}{l}\text { 5ALPLE } \\
\text { DATE }\end{array}$ & $0-40-62$ \\
\hline $12 / 15 / 87$ & 141006. NO \\
\hline \multirow[t]{2}{*}{$12 / 15 / 87$} & $\left.\begin{array}{ll} & 1.30 \\
1.24\end{array}\right\}$ \\
\hline & MR \\
\hline 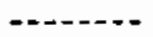 & \\
\hline \multirow[t]{2}{*}{$12 / 15 / 87$} & $\begin{array}{l}7.80 \\
\text { MR }\end{array}$ \\
\hline & MR \\
\hline $12 / 15 / 87$ & 9946.10 \\
\hline $12 / 16 / 87$ & 452.06 \\
\hline $12 / 15 / 07$ & MR \\
\hline $12 / 15 / 87$ & MR \\
\hline $12 / 15 / 87$ & 97.0 \\
\hline $12 / 16 / 87$ & 49141.00 \\
\hline $12 / 15 / 97$ & MR \\
\hline $12 / 16 / 87$ & \\
\hline
\end{tabular}

- YaLUE EXCEEDS Primary dRIMKIMG IATER STANDARD.

- YALUE EXCEEDS PROPOSED PRIMARY DRINKIMG GATER STAMDARD.

MR - ANALYSIS NOT RERUESTED OR NOT YET REPORTED

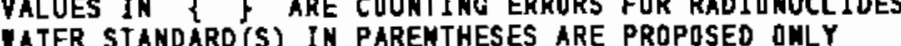


TABLE C.3. (contd)

\begin{tabular}{|c|c|c|c|c|c|c|c|c|c|}
\hline \multicolumn{2}{|c|}{$\begin{array}{l}\text { CONSTITUENT } \\
\text { MAUE UNITS }\end{array}$} & $\begin{array}{l}\text { CONTRACTUAL } \\
\text { DETECTION } \\
\text { LIMIT }\end{array}$ & $\begin{array}{l}\text { DRINKINO } \\
\text { IATER } \\
\text { STANDARD }\end{array}$ & $\begin{array}{c}\text { SAMPLE } \\
\text { DATE }\end{array}$ & $8-46-1$ & $\begin{array}{l}\text { SAMPLE } \\
\text { DATE }\end{array}$ & B-41-33A & $\begin{array}{c}\text { SAMPLE } \\
\text { OATE }\end{array}$ & $0-41-82$ \\
\hline FIROM & PPB & 58.68 & & $10 / 27 / 87$ & & & NR & & MR \\
\hline FLUORID & PPB & 580.00 & 4008.06 & $\begin{array}{l}89 / 91 / 87 \\
10 / 27 / 87\end{array}$ & 580.80 & $09 / 03 / 87$ & ${ }_{\text {NR }}^{856.10}$ & $12 / 15 / 87$ & ${ }_{\mathrm{NR}}^{561.01}$ \\
\hline FUAGMES & $\mathrm{PPB}$ & 0.96 & & $\begin{array}{l}69 / 61 / 87 \\
10 / 27 / 87\end{array}$ & $\begin{array}{l}12401.06 \\
12801.18\end{array}$ & $00 / 03 / 87$ & $\begin{array}{c}3798.110 \\
M R\end{array}$ & $12 / 15 / 87$ & NR \\
\hline FuANGAN & PPB & 5.60 & & $\begin{array}{l}89 / 91 / 87 \\
10 / 27 / 87\end{array}$ & & $60 / 03 / 87$ & 44.06 & $12 / 15 / 87$ & MR \\
\hline FPOTASS & PPB & 100.80 & & $\begin{array}{l}80 / 01 / 87 \\
10 / 27 / 87\end{array}$ & $\begin{array}{l}5386.80 \\
5746.00\end{array}$ & $60 / 09 / 87$ & ${ }_{\mathrm{MR}}^{036 \mathrm{AOA}}$ & $12 / 16 / 87$ & E226.110 \\
\hline FSOdIUY & PPB & 108.06 & & $\begin{array}{l}60 / 01 / 87 \\
10 / 27 / 87\end{array}$ & $\begin{array}{l}18300.60 \\
19106.60\end{array}$ & $60 / 03 / 87$ & ${ }_{M R}^{51800} \cdot 09$ & $12 / 15 / 87$ & 1840 MR $^{0.00}$ \\
\hline FSTRONT & PPB & 360.64 & & $\begin{array}{l}89 / 61 / 87 \\
18 / 27 / 87\end{array}$ & 3e8.6 & $10 / 03 / 87$ & MR & $12 / 15 / 87$ & MR \\
\hline FVANADI & PPB & 6.81 & & $\begin{array}{l}69 / 61 / 87 \\
10 / 27 / 87\end{array}$ & $\begin{array}{l}19.01 \\
18.80\end{array}$ & $19 / 03 / 87$ & MR & $12 / 16 / 87$ & 26.10 \\
\hline HNITRAT & PPB & 2580.08 & 45060.81 & $\begin{array}{l}88 / 117 / 87 \\
98 / 25 / 87 \\
10 / 27 / 87\end{array}$ & $\begin{array}{l}38960.80 \\
39696080 \\
39560.80\end{array}$ & & $\begin{array}{l}\text { MR } \\
\text { MR } \\
\text { WR } \\
.\end{array}$ & $\begin{array}{l}67 / 30 / 87 \\
12 / 15 / 87\end{array}$ & $\begin{array}{l}49600.00 \\
4720.10\end{array}$ \\
\hline LFLUORD & PPB & 28.80 & 4860.06 & $\begin{array}{l}90 / 91 / 87 \\
10 / 27 / 87\end{array}$ & 385.00 & $69 / 103 / 87$ & ${ }_{\mathrm{MR}}^{045.10}$ & $12 / 16 / 87$ & ${ }_{M R}^{415.01}$ \\
\hline MITRATE & PPB & 581.86 & 45868.66 & $\begin{array}{l}89 / 01 / 87 \\
19 / 27 / 87\end{array}$ & $\begin{array}{l}35186.68 \\
48896.60\end{array}$ & $00 / 13 / 87$ & NR & $12 / 15 / 87$ & $4720 \mathrm{NR} .01$. \\
\hline PH-LAB & & 0.01 & & $\begin{array}{l}89 / 01 / 87 \\
19 / 27 / 87\end{array}$ & $\begin{array}{l}7.73 \\
7.98\end{array}$ & $00 / 109 / 87$ & $\begin{array}{l}7.94 \\
M R\end{array}$ & $12 / 15 / 87$ & 7.77 \\
\hline PHFIELD & & 0.18 & & $\begin{array}{l}80 / 01 / 87 \\
10 / 27 / 87\end{array}$ & $\begin{array}{l}8.78 \\
7.80\end{array}$ & $69 / 03 / 87$ & 7.50 & $12 / 15 / 87$ & 7.81 \\
\hline SULFATE & PPB & 680.08 & & $09 / 01 / 87$ & 33900.00 & $69 / 09 / 87$ & & $12 / 15 / 87$ & 48461.06 \\
\hline 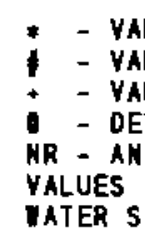 & $\begin{array}{l}\text { UE EXCE } \\
\text { UE EXCE } \\
\text { UEE EXCE } \\
\text { ECTION } \\
\text { YSIS } \\
\text { YNIS } \\
\text { ENDARD? }\end{array}$ & $\begin{array}{l}\text { SS PRIYARY D } \\
\text { S PROPOSED } \\
\text { S SCRENING } \\
\text { SIT YNS NOT } \\
\text { REQUESTED } \\
\text { ARE CDUNTIM } \\
\text { IN PARENTH }\end{array}$ & $\begin{array}{l}\text { NOT YET RI } \\
\text { RROR FOR } \\
\text { S ARE PROA }\end{array}$ & 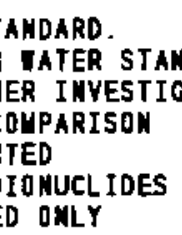 & $\begin{array}{l}\text { ARD. } \\
\text { IION. }\end{array}$ & & & & \\
\hline
\end{tabular}


TABLE C.3. (contd)

\begin{tabular}{|c|c|c|c|c|c|c|c|}
\hline$\underset{\text { NAME }}{\text { CONSTI }}$ & $\begin{array}{l}\text { UENT } \\
\text { UNITS }\end{array}$ & $\begin{array}{c}\text { CORTRACTUAL } \\
\text { DETECTIDN } \\
\text { LIUIT }\end{array}$ & $\begin{array}{l}\text { DRINKING } \\
\text { VATER } \\
\text { STANDARD }\end{array}$ & $\begin{array}{l}\text { SAMPLE } \\
\text { DATE }\end{array}$ & $6-40-1$ & $\begin{array}{c}\text { SAYPLE } \\
\text { DATE }\end{array}$ & $0-40-33 A$ \\
\hline SULFATE & PPB & 561.80 & & $10 / 27 / 87$ & 38910.16 & & $M R$ \\
\hline TC & PPB & & & $\begin{array}{l}68 / 61 / 87 \\
16 / 27 / 87\end{array}$ & $\begin{array}{l}28500.00: \\
28816\end{array}$ & $10 / 03 / 87$ & $3940 \mathrm{HR}_{\mathrm{KR}}$ \\
\hline IC -80 & PCI/L & 15.00 & 901.10 & $10 / 27 / 87$ & $\left\{\begin{array}{r}278.61 \\
2.94\end{array}\right\}$ & & WR \\
\hline TRITIUU & $\mathrm{PCI} / \mathrm{L}$ & 501.00 & 20060.00 & $\begin{array}{l}08 / 67 / 87 \\
10 / 25 / 87 \\
10 / 27 / 87\end{array}$ & 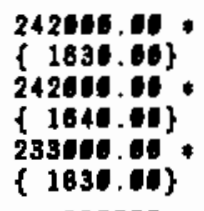 & $\begin{array}{l}10 / 03 / 87 \\
12 / 08 / 87\end{array}$ & NR \\
\hline u & $\mathrm{PCI} / \mathrm{L}$ & 0.50 & 064.60 & $68 / 67 / 87$ & 2.81 & & MR \\
\hline
\end{tabular}

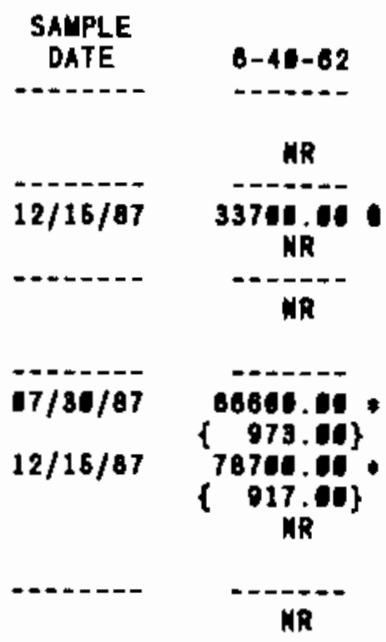

* - Value exceeds priuary drinking tater standard.

- VALUE EXCEEdS PROPOSED PRIUARY dRINKING tater Standaro.

- VALUE EXCEEDS SCREENING LEVEI For FURTHER INVESTIGATIOM.

- DETECTION LIUIT tas NOT AVAILABLE fOR COMPARISON

NR - AMALYSIS NDT REQUESTED OR NOT YET REPORTED

VALUES IN $\{$ ARE COUNIING ERRORS FOR RADIONUCLIDES

VATER STANDARD(S) IN PARENTHESES ARE PROPQSED DNLY 


\section{TABLE C.3. (contd)}

\begin{tabular}{|c|c|c|c|c|c|}
\hline $\begin{array}{c}\text { CONSTI I } \\
\text { NANE }\end{array}$ & $\begin{array}{l}\text { UENT } \\
\text { UNITS }\end{array}$ & $\begin{array}{l}\text { CONTRACTUAL } \\
\text { DETECTION } \\
\text { LIUIT }\end{array}$ & $\begin{array}{l}\text { DRINKING } \\
\text { TATER } \\
\text { STANDARD }\end{array}$ & $\begin{array}{l}\text { SAMPLEE } \\
\text { DATEE }\end{array}$ & $6-41-1$ \\
\hline ALKALIN & & & & $18 / 27 / 87$ & 124000.00 \\
\hline ALPHA & $\mathrm{PCI} / \mathrm{L}$ & 4.00 & 15.86 & $\begin{array}{l}68 / 67 / 87 \\
16 / 27 / 87\end{array}$ & $\begin{array}{ll} & 3.63 \\
\{ & 1.80\} \\
\{ & 4.62 \\
\{ & 1.89\}\end{array}$ \\
\hline BETA & $\mathrm{PCI} / \mathrm{L}$ & 8.66 & 56.6 & $\begin{array}{l}68 / 107 / 87 \\
10 / 27 / 87\end{array}$ & $\begin{array}{cc} & 64.46 \\
\{\quad 5.61\} \\
40.16 \\
\{\quad 8.44\}\end{array}$ \\
\hline CHLORID & PPB & 508.00 & & $18 / 27 / 87$ & 11100.00 \\
\hline$C 0.61$ & $\mathrm{PCI} / \mathrm{L}$ & 22 . 64 & 100.00 & $08 / 17 / 87$ & \\
\hline CONDFLD & UWHO & 1.00 & & $10 / 27 / 87$ & 408.106 \\
\hline CYAMIDE & PPB & 10.00 & & $10 / 27 / 87$ & 10.10 \\
\hline FBARIUU & PPB & 6.60 & 1000.06 & $10 / 27 / 87$ & 60.100 \\
\hline FCALCIU & PPB & 50.60 & & $18 / 27 / 87$ & 46800.00 \\
\hline FCHROUI & PPB & 11.01 & 50.66 & $18 / 27 / 87$ & 10.00 \\
\hline FLUORID & PPB & 500.64 & 4800.00 & $10 / 27 / 87$ & 585.10 \\
\hline FHAGKES & PPB & 0.00 & & $10 / 27 / 87$ & 12700.08 \\
\hline FPOTASS & PPB & 108.6 & & $18 / 27 / 87$ & 5688.00 \\
\hline FSODIUU & PPB & 180.01 & & $11 / 27 / 87$ & 18804.10 \\
\hline FSTRONT & PPB & 386.66 & & $10 / 27 / 87$ & 321.00 \\
\hline FVAMADI & PPB & 5.01 & & $10 / 27 / 87$ & 18.00 \\
\hline
\end{tabular}

\begin{tabular}{|c|c|}
\hline $\begin{array}{l}\text { SALPLE } \\
\text { DATE }\end{array}$ & $6-42-2$ \\
\hline $11 / 27 / 87$ & 125000.00 \\
\hline $10 / 197 / 87$ & 2.88 \\
\hline $19 / 27 / 87$ & $\begin{array}{l}9.17 \\
1.69\}\end{array}$ \\
\hline $18 / 07 / 87$ & 61.50 \\
\hline $10 / 27 / 87$ & $\left\{\begin{array}{r}41.20 \\
\quad 0.40\}\end{array}\right.$ \\
\hline $10 / 27 / 87$ & 10906.01 \\
\hline $08 / 07 / 87$ & $\left\{\begin{array}{r}15.11 \\
\quad B .07\}\end{array}\right.$ \\
\hline $10 / 27 / 87$ & 406.01 \\
\hline $10 / 27 / 87$ & \\
\hline $10 / 27 / 87$ & 46.16 \\
\hline $10 / 27 / 87$ & 40100.60 \\
\hline $10 / 27 / 87$ & \\
\hline $10 / 27 / 87$ & 587.16 \\
\hline $10 / 27 / 87$ & 12804.00 \\
\hline $10 / 27 / 87$ & 5868.10 \\
\hline $18 / 27 / 87$ & 19464.60 \\
\hline $11 / 27 / 87$ & 318.10 \\
\hline $10 / 27 / 87$ & 18.00 \\
\hline
\end{tabular}

\begin{tabular}{|c|c|}
\hline $\begin{array}{l}\text { SAMPLEE } \\
\text { DATE }\end{array}$ & $8-43-3$ \\
\hline $10 / 28 / 87$ & 125000.00 \\
\hline $08 / 07 / 07$ & 3. 53 \\
\hline $10 / 28 / 87$ & $\begin{array}{l}2.77 \\
1.47\}\end{array}$ \\
\hline $18 / 07 / 87$ & 46.50 \\
\hline $10 / 28 / 87$ & $\left.\begin{array}{rr}\{ & 8.76 \\
\{\quad 7.10\end{array}\right\}$ \\
\hline $10 / 28 / 87$ & 11800.00 \\
\hline $08 / 67 / 87$ & $\begin{array}{l}8.50 \\
0.40\}\end{array}$ \\
\hline & $\mathrm{MR}$ \\
\hline $11 / 28 / 87$ & \\
\hline $11 / 28 / 07$ & 53.01 \\
\hline $10 / 28 / 87$ & 42890.04 \\
\hline $16 / 28 / 87$ & ii.uo \\
\hline $10 / 28 / 87$ & 1480.00 \\
\hline $10 / 28 / 87$ & 13740.14 \\
\hline $10 / 28 / 87$ & 5280.06 \\
\hline $10 / 28 / 87$ & 20014.66 \\
\hline $10 / 28 / 87$ & 342.16 \\
\hline $10 / 28 / 87$ & 22.10 \\
\hline
\end{tabular}

* - value exceeds priuary drinking tater standard.

- YALUE EXCEEDS PROPOSED PRIMARY DRINKING IATER STANDARD.

- YALUE EXCEEDS SCREENINO LEYEL FOR FURTHER INYESTIgATION.

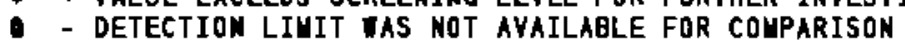

HR - AFALYSIS MOT REQUESTED OR NOT YET REPORTED

YALUES IN \{\} ARE COUNTING ERRORS FOR RADIONUCLIDES

IATER STANDARD'(S) IN PARENTHESES ARE PROPOSED ONLY 
TABLE C.3. (contd)

\begin{tabular}{|c|c|c|c|c|c|}
\hline $\begin{array}{c}\text { CONSTI } \\
\text { MANE }\end{array}$ & $\begin{array}{l}\text { UENT } \\
\text { UNITS }\end{array}$ & $\begin{array}{c}\text { CONTRACTUAL } \\
\text { DETECTION } \\
\text { LIUIT }\end{array}$ & $\begin{array}{l}\text { DRINKIMG } \\
\text { IATER } \\
\text { STANDARD }\end{array}$ & $\begin{array}{l}\text { SAUPLE } \\
\text { DATE }\end{array}$ & $6-41-1$ \\
\hline HNI TRAT & PPB & 2500.06 & 45060.00 & $68 / 07 / 87$ & $\begin{array}{c}30804.01 \\
\text { MR } \\
\text { MR }\end{array}$ \\
\hline I-129 & $\mathrm{PCI} / \mathrm{L}$ & & 600.11 & $08 / 67 / 87$ & $\begin{array}{l}: 17 \\
\{\quad: .1\}\end{array}$ \\
\hline LFLUORD & PPB & 21.00 & 4010 .te & $18 / 27 / 87$ & 370.6 \\
\hline NI TRATE & PPB & 506.01 & 45860.68 & $10 / 27 / 87$ & 41400.66 \\
\hline PH-LAB & & 0.61 & & $10 / 27 / 67$ & 7.00 \\
\hline PHFIELD & & 0.10 & & $10 / 27 / 87$ & 7.50 \\
\hline$R U-106$ & $\mathrm{PCI} / \mathrm{L}$ & 172.54 & 31.16 & $08 / 07 / 87$ & $\left\{\begin{array}{l}87.68 \\
\{\quad 53.39\end{array}\right.$ \\
\hline SULFATE & $\mathrm{PPB}$ & 500.01 & & $10 / 27 / 87$ & 39600.65 \\
\hline IC & PPB & & & $10 / 27 / 87$ & 31000.00 \\
\hline TC-89 & PCI/L & 15.01 & 900.01 & $08 / 07 / 07$ & $\left\{\begin{array}{c}308.00 \\
9.13\end{array}\right\}$ \\
\hline TRITIUU & $\mathrm{PCI} / \mathrm{L}$ & 506.06 & 20006.60 & $\begin{array}{l}68 / 67 / 87 \\
68 / 07 / 87 \\
68 / 07 / 87 \\
10 / 27 / 87 \\
18 / 27 / 87\end{array}$ & 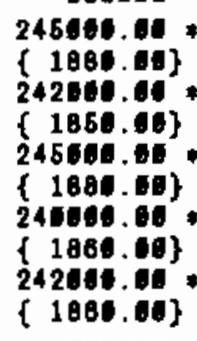 \\
\hline $\mathrm{U}$ & $\mathrm{PCI} / \mathrm{L}$ & 6.51 & 600.00 & $68 / 07 / 87$ & 2.53 \\
\hline
\end{tabular}

\begin{tabular}{|c|c|c|c|}
\hline $\begin{array}{c}\text { SAUPLE } \\
\text { DATE }\end{array}$ & $8-42-2$ & $\begin{array}{c}\text { SAUPLE } \\
\text { DATE }\end{array}$ & $0-43-3$ \\
\hline \multirow[t]{2}{*}{$\begin{array}{l}07 / 31 / 87 \\
08 / 67 / 87 \\
10 / 27 / 87 \\
-1\end{array}$} & $\begin{array}{l}34460.00 \\
38866.00 \\
38866.08\end{array}$ & $\begin{array}{l}07 / 31 / 87 \\
18 / 67 / 87 \\
10 / 27 / 87\end{array}$ & $\begin{array}{l}34501.41 \\
37400.14 \\
38700.41\end{array}$ \\
\hline & MR & & WR \\
\hline $10 / 27 / 87$ & 375.10 & $10 / 28 / 87$ & 386.01 \\
\hline $10 / 27 / 87$ & 30701.16 & $10 / 28 / 87$ & s8560.01 \\
\hline $11 / 27 / 87$ & 7.03 & $10 / 28 / 87$ & 7.80 \\
\hline $1 / / 27 / 87$ & 7.60 & & NR \\
\hline $68 / 67 / 87$ & & $18 / 07 / 87$ & \\
\hline $10 / 27 / 67$ & 30200.40 & $10 / 20 / 87$ & 41200.00 \\
\hline \multirow[t]{2}{*}{$10 / 27 / 87$} & s1610.65 & $10 / 28 / 87$ & 32500.00 \\
\hline & MR & & MR \\
\hline \multirow{4}{*}{$\begin{array}{l}07 / 31 / 87 \\
08 / 07 / 87 \\
11 / 27 / 87\end{array}$} & 214600 & $67 / 31 / 87$ & 2285 \\
\hline & $222060.00^{*}$ & $18 / 07 / 87$ & 236000 \\
\hline & (1814..S\}) & $10 / 27 / 87$ & \{1834.66\} \\
\hline & MR & & MR \\
\hline $88 / 07 / 87$ & 3.04 & $08 / 67 / 87$ & 2.61 \\
\hline
\end{tabular}

- - yalue exceeds priyary dRinking tater standard.

- - YALUE EXCEEDS PROPOSED PRIHARY DRINKING TATER STANOARD.

+ - VALUE EXCEEDS SCREENING LEYEL FOR FURTHER INYESTIGATION.

- DETECtion liUit tas nOt ayailable for cDuparison

NR - AMALYSIS NOT REQUESTED OR NDT YET REPORTED

YALUES IN \& A ARE COUNTINO ERRORS FOR RADIDNUCLIDES

IATER STAKDARD (5) IN PARENTHESES ARE PROPDSED ONLY 


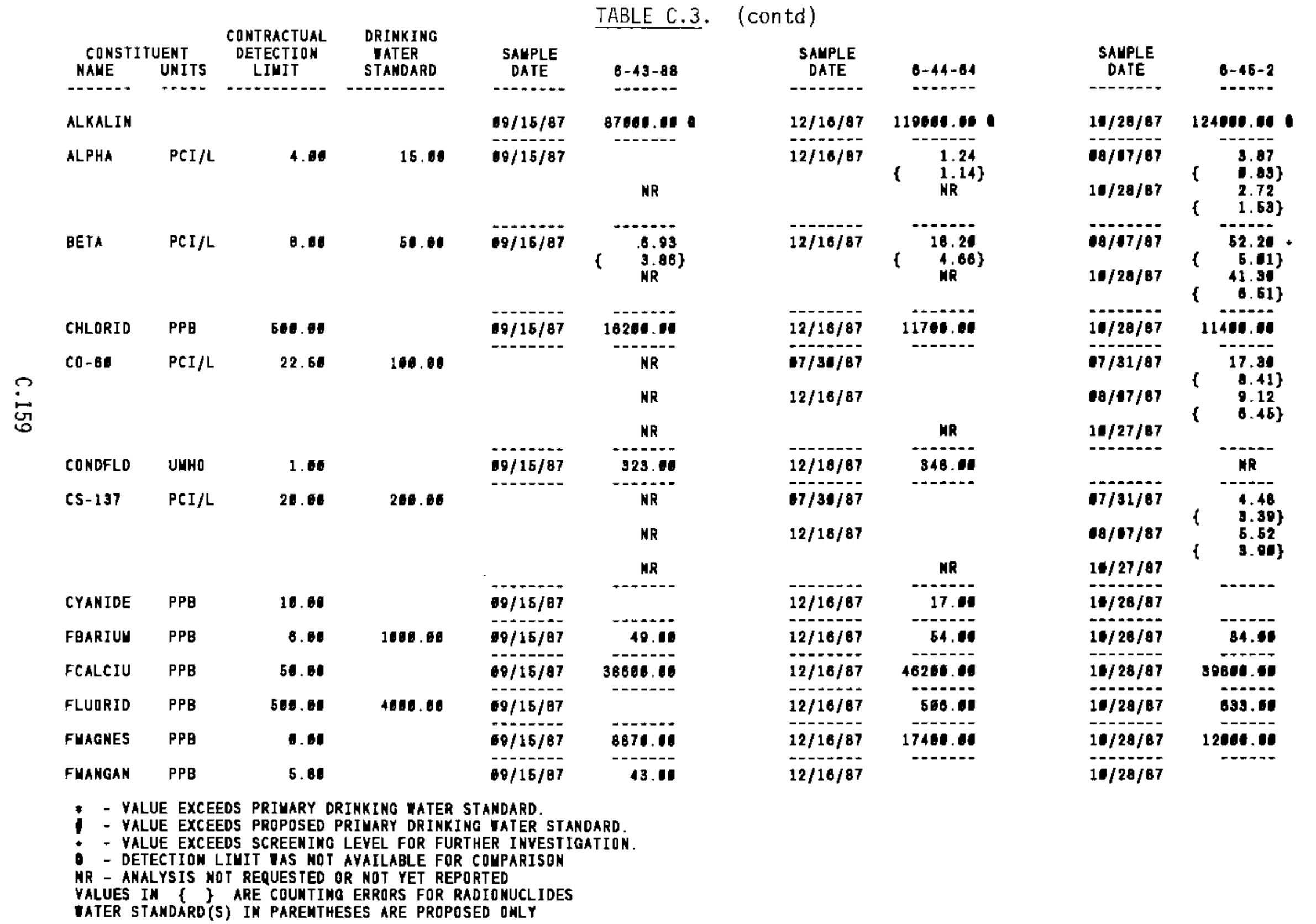


TABLE C.3. (contd)

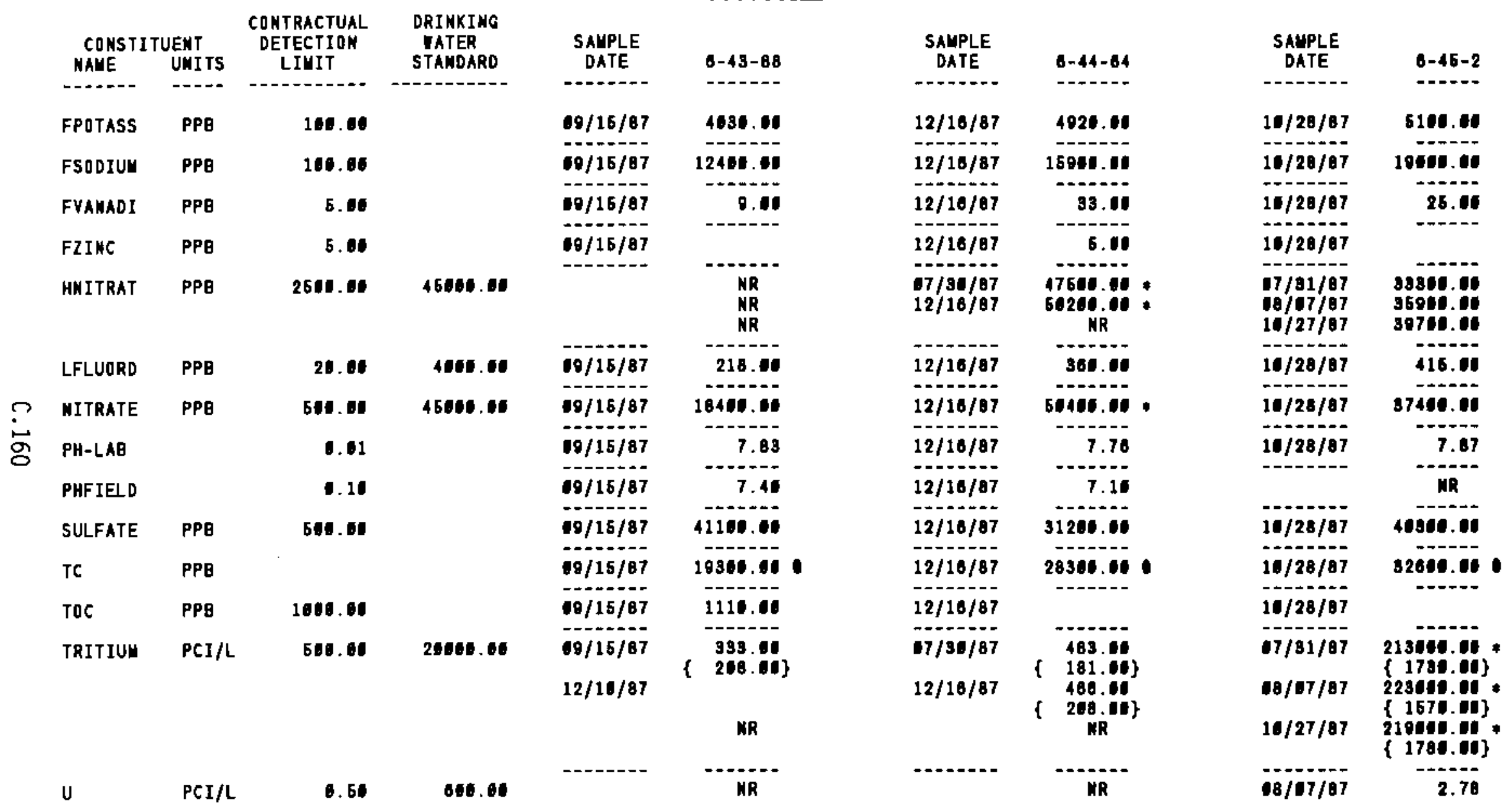

- - Value exceEds priuary dRinkino mater standard.

- YALUE EXCEEDS PROPOSED PRIUARY DRINKINO IATER STANDARD.

+ - YALUE EXCEEDS SCREENING LEVEI FOR FURTHER INYESTIGATION.

- DETECTION liuit tas nOt ayailable for couparison

MR - ANALYSIS HOT RERUESTED OR NOT YET REPORTED

VALUES IN i $f$ ARE COUNTING ERRORS FOR RADIONUCLIDES

IATER STANDARD'S) IN PARENTHESES ARE PROPOSED ONLY 
TABLE C.3. (contd)

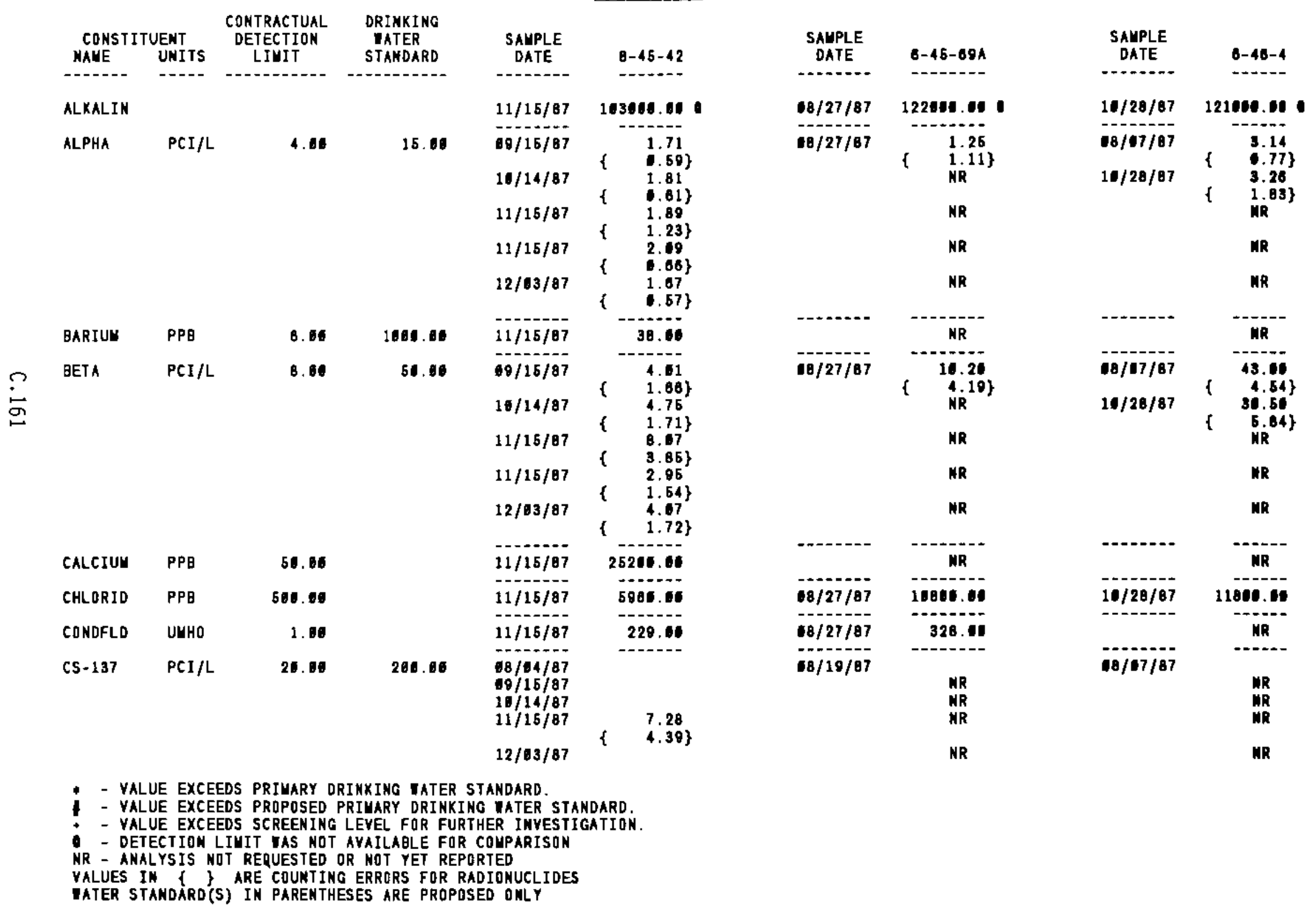


TABLE C. 3. (contd)

\begin{tabular}{|c|c|c|c|c|c|}
\hline \multicolumn{2}{|c|}{ CONSTITUENT } & $\begin{array}{c}\text { CONTRACTUAL } \\
\text { DETECTION } \\
\text { LIUIT }\end{array}$ & $\begin{array}{l}\text { DRINKING } \\
\text { TATER } \\
\text { STAMDARD }\end{array}$ & $\begin{array}{c}\text { SALPLE } \\
\text { DATE }\end{array}$ & $8-4$ \\
\hline FBARIUYY & PPB & 0.110 & 1800.06 & $11 / 15 / 87$ & \\
\hline FCALCIU & $\mathrm{PPB}$ & 51.11 & & $11 / 15 / 87$ & \\
\hline FCHROMI & PPB & 10.01 & 50.61 & $11 / 15 / 87$ & \\
\hline FLUORID & PPB & 500.11 & 4060.01 & $11 / 15 / 87$ & \\
\hline FUAGMES & PPB & 0.10 & & $11 / 15 / 87$ & 10 \\
\hline FuAMGAN & $\mathrm{PPB}$ & 5.11 & & $11 / 15 / 87$ & \\
\hline FPOTASS & PPB & 106.10 & & $11 / 15 / 87$ & \\
\hline FSODIUN & $\mathrm{PPB}$ & 101.01 & & $11 / 18 / 87$ & \\
\hline FSTRONT & PPB & 306.10 & & $11 / 16 / 87$ & \\
\hline FVAMADI & PPB & 5. 10 & & $11 / 15 / 87$ & \\
\hline FZINC & $\mathrm{PPB}$ & 5.11 & & $11 / 16 / 87$ & \\
\hline HNITRAT & PPB & 2506.01 & 45060.100 & $88 / 04 / 87$ & \\
\hline$I-128$ & $\mathrm{PCI} / \mathrm{L}$ & & 500.00 & n........... & \\
\hline IRON & PPB & 50.60 & & $11 / 15 / 87$ & \\
\hline LFLUORD & PPB & 20.10 & 4060.60 & $11 / 15 / 87$ & \\
\hline WAGMES & $\mathrm{PPB}$ & 0.00 & & $11 / 15 / 87$ & 104 \\
\hline MANGESE & $\mathrm{PPB}$ & 5.00 & & $11 / 15 / 87$ & \\
\hline NITRATE & $\mathrm{PPB}$ & 500.10 & 46000.00 & $11 / 15 / 87$ & 747 \\
\hline $\begin{array}{l}-V A \\
!=V A \\
-V A \\
:-D E \\
\text { NR - AN } \\
\text { VALUES } \\
\text { MATER S }\end{array}$ & $\begin{array}{l}\text { UE EXCE } \\
\text { UE EXCE } \\
\text { UE EXCE } \\
\text { ECTIDN } \\
\text { LYSIS } \\
\text { A }\{ \\
\text { AMOARD? }\end{array}$ & $\begin{array}{l}\text { EDS PRINARY D } \\
\text { EDS PROPOSED } \\
\text { EOS SCREENING } \\
\text { IIIT IAS NDT } \\
\text { T REQUESTED } \\
\text { ARE COUNTIN } \\
\text { S) IN PARENTH }\end{array}$ & $\begin{array}{l}\text { NKING NATE } \\
\text { INARY ORIN } \\
\text { EVEI FOR F } \\
\text { VAILABLE F } \\
\text { NOT YET RE } \\
\text { ERRORS FOR } \\
\text { ES ARE PRO }\end{array}$ & $\begin{array}{l}\text { ANDARO. } \\
\text { WATER STA } \\
\text { ER INVESTI } \\
\text { OMPARISOH } \\
\text { TED } \\
\text { IONUCLIDES } \\
\text { D ONLY }\end{array}$ & TION \\
\hline
\end{tabular}

\begin{tabular}{|c|c|}
\hline $\begin{array}{l}\text { SAUPLE } \\
\text { DATE }\end{array}$ & $6-46-4$ \\
\hline $10 / 28 / 87$ & 36.10 \\
\hline $16 / 28 / 87$ & 9016.010 \\
\hline $16 / 20 / 87$ & 11.0 \\
\hline $10 / 28 / 87$ & 848.10 \\
\hline $10 / 28 / 87$ & 12850.00 \\
\hline $10 / 28 / 87$ & \\
\hline $11 / 28 / 87$ & 6046.06 \\
\hline $10 / 28 / 87$ & 22201.00 \\
\hline $10 / 28 / 87$ & 316.01 \\
\hline $10 / 28 / 87$ & 26.01 \\
\hline $10 / 28 / 87$ & \\
\hline $\begin{array}{l}07 / 31 / 87 \\
08 / 07 / 87 \\
10 / 27 / 87\end{array}$ & $\begin{array}{l}25145.010 \\
264160.10 \\
27040.10\end{array}$ \\
\hline $17 / 31 / 87$ & $\begin{array}{l}0.16 \\
\quad 0.11\}\end{array}$ \\
\hline & $\mathrm{MR}$ \\
\hline $18 / 28 / 87$ & 485.11 \\
\hline & NR \\
\hline & NR \\
\hline $6 / 28 / 87$ & 28400.10 \\
\hline
\end{tabular}


TABLE C.3. (contd)

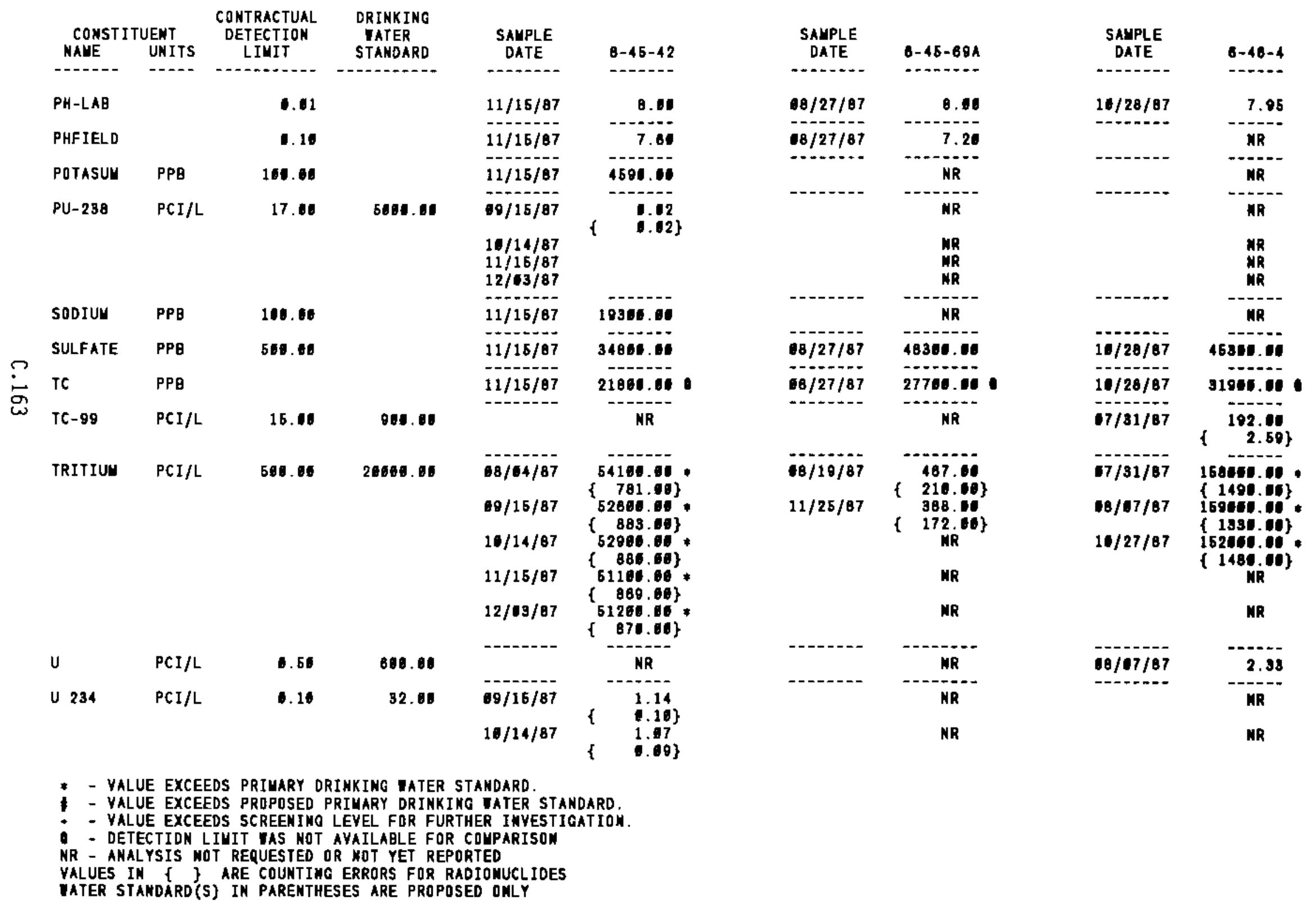


TABLE C.3. (contd)

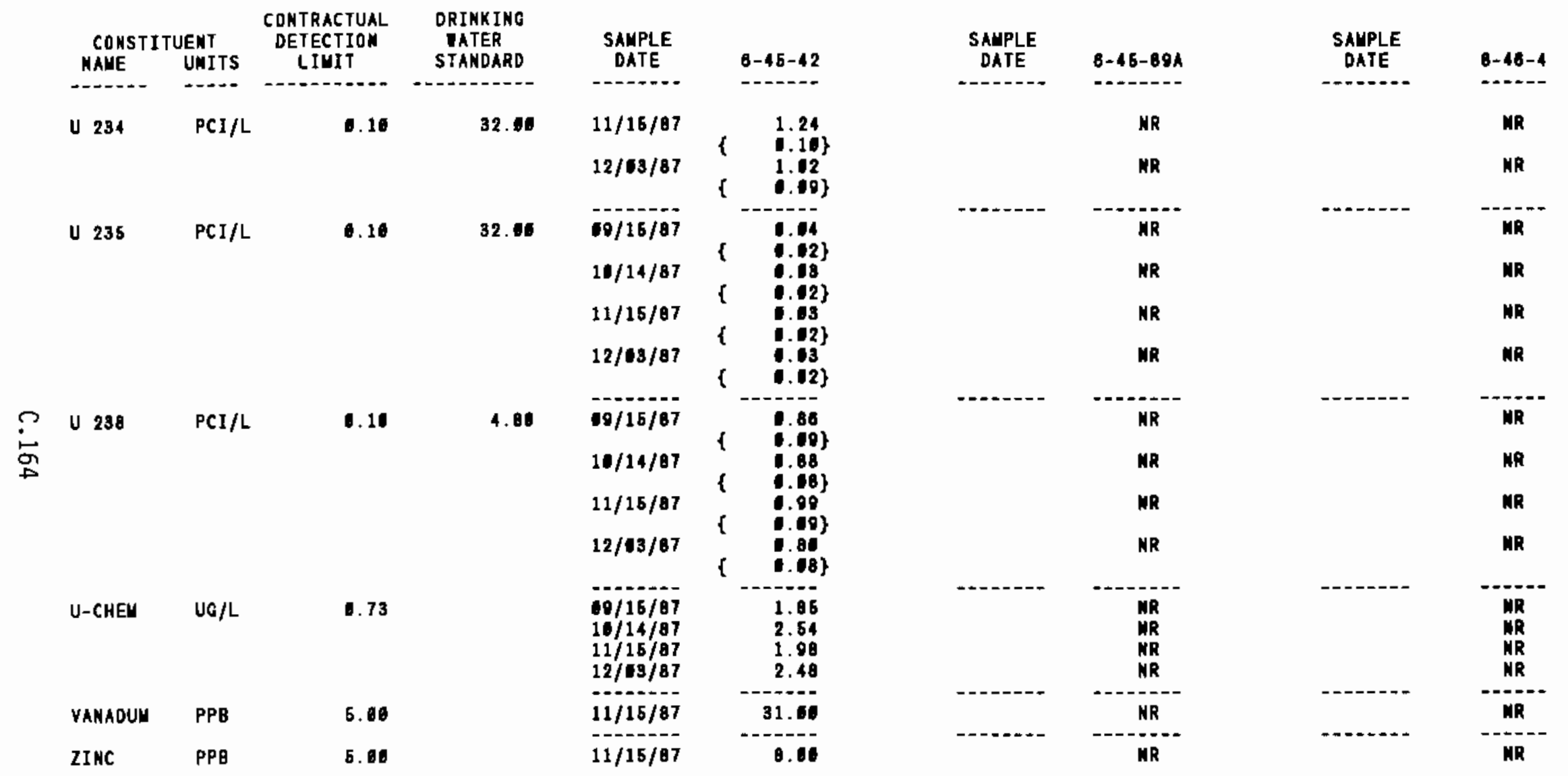

* - value exceeds primary drinking yater standard.

* - VALUE EXCEEDS PROPOSED PRIMARY ORINKING IATER STANDARD.

- VALUE EXCEEDS SCREENING LEVEL FOR FURTHER IMVESTIGATIDN.

- DETECTION LINIT UAS NOT AVAILABLE FoR COUPARISON

HR - AMALYSIS MOT REQUESTED DR NOT YET REPDRTED

VALUES IN \& $\}$ ARE COUNTING ERRORS FOR RADIONUCLIDES

WATER STANDARD(S) IN PARENTHESES ARE PROPOSED ONLY 
TABLE C.3. (contd)

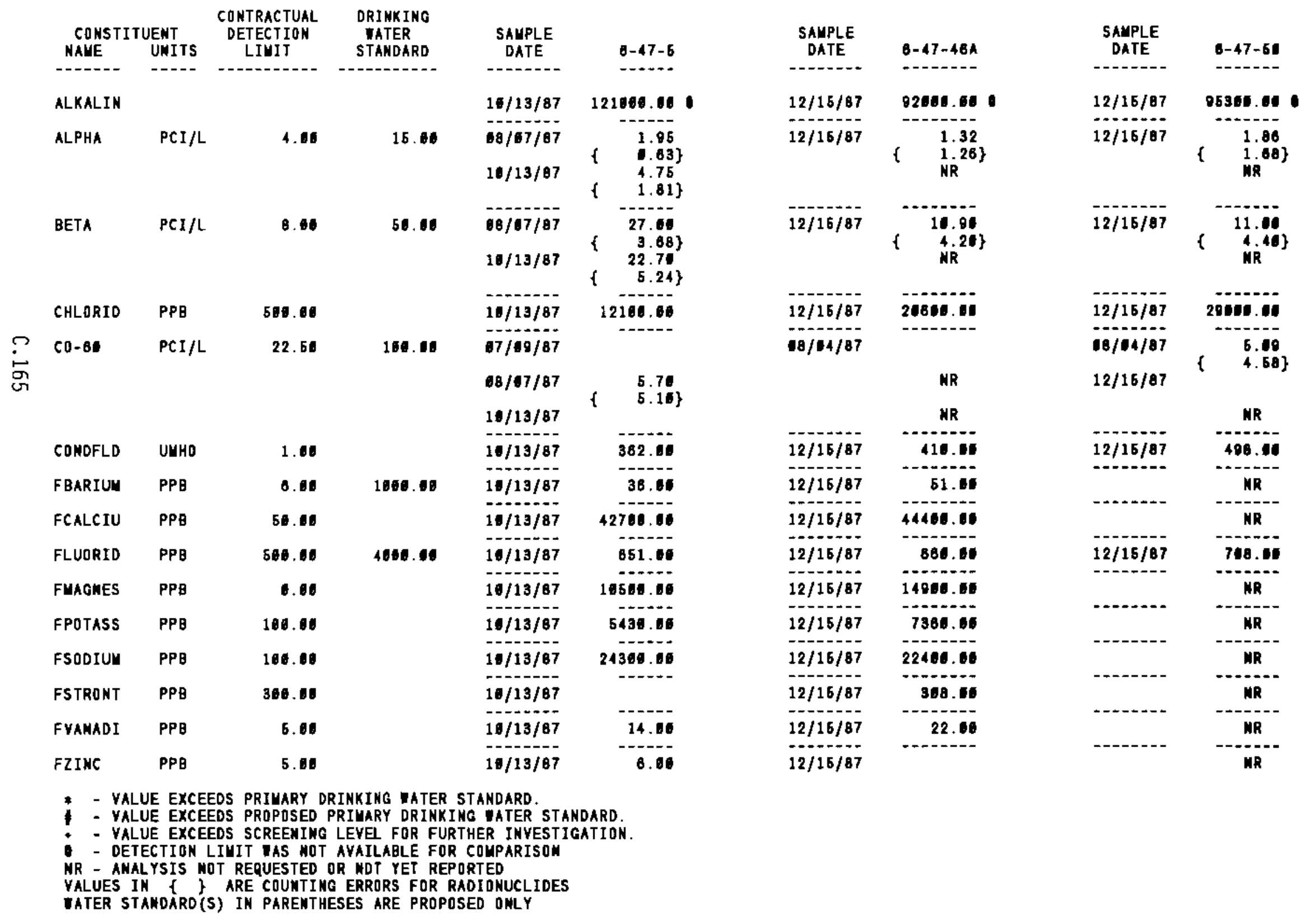


TABLE C.3. (contd)

\begin{tabular}{|c|c|c|c|c|c|}
\hline $\begin{array}{l}\text { CONSTI } \\
\text { NAHE }\end{array}$ & $\begin{array}{l}\text { UEWT } \\
\text { UNITS }\end{array}$ & $\begin{array}{l}\text { COKTRACTUAL } \\
\text { DETECTIDN } \\
\text { LINIT }\end{array}$ & $\begin{array}{l}\text { DRINKING } \\
\text { IATER } \\
\text { STANDARD }\end{array}$ & $\begin{array}{l}\text { SAMPLE } \\
\text { DATE }\end{array}$ & $0-47-5$ \\
\hline HNI TRAT & PPB & 2500.01 & 45000.66 & $\begin{array}{l}17 / 09 / 87 \\
10 / 07 / 87 \\
08 / 11 / 87 \\
19 / 19 / 87 \\
16 / 13 / 87 \\
11 / 08 / 87 \\
12 / 93 / 87\end{array}$ & 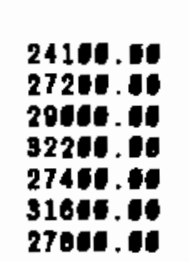 \\
\hline$I-12 \theta$ & $\mathrm{PCI} / \mathrm{L}$ & & 500.00 & & MR \\
\hline LFLUORD & PPB & 26.06 & 4061.00 & $1 / / 13 / 87$ & 300.10 \\
\hline NITRATE & PPB & 500.100 & 45010.00 & $10 / 13 / 87$ & 28060.06 \\
\hline PH-LAB & & 1.1 & & $11 / 13 / 87$ & 7.70 \\
\hline PHF IELD & & 1.10 & & $16 / 13 / 97$ & 7.44 \\
\hline RU-106 & PCI /L & 172.50 & 30. 6 & $\begin{array}{l}07 / 60 / 87 \\
18 / 07 / 87 \\
10 / 13 / 87\end{array}$ & $\{\quad 87.80\}$ \\
\hline SULFATE & PPB & 500.0 & & $10 / 13 / 87$ & 50700.00 \\
\hline TC & PPB & & & $10 / 13 / 87$ & 28100.51 \\
\hline TRITIUY & $\mathrm{PCI} / \mathrm{L}$ & 508.01 & 26060.00 & $\begin{array}{l}17 / 69 / 87 \\
68 / 107 / 87 \\
08 / 11 / 87 \\
09 / 08 / 87 \\
10 / 13 / 67\end{array}$ & 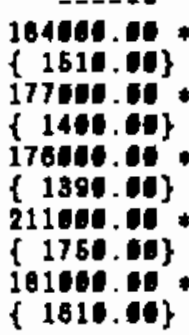 \\
\hline
\end{tabular}

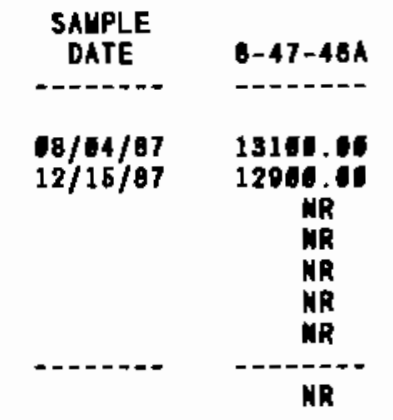

19/15/87

$12 / 15 / 87$

$12 / 15 / 87$

$12 / 15 / 87$

$00 / 04 / 87$

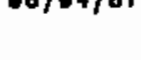

$12 / 15 / 87$

$12 / 15 / 87$

$12 / 16 / 87$

$00 / 04 / 87$

$12 / 15 / 87$

5os.us

14400.06

7.80

7.81

88.20.

$\{40.10\}$

98000.00

20860.00

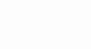

SAMPLE

DATE

6-47-50

10/16/87 0764.06

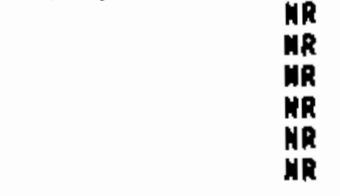

$10 / 04 / 87$
NR

$12 / 15 / 87$

$12 / 15 / 87$

$12 / 15 / 87$

$12 / 15 / 07$

$08 / 04 / 87$

$12 / 15 / 87$

$12 / 15 / 97$

12/16/97

$12 / 15 / 87$

$00 / 16 / 87$

$\{\quad: 01$.

580.10

a1se.

9100.0.

7.80

0.86

(....-.

WR

101900.00

23460.01.

23460.10

HR

MR

IR

MR

NR

- - value exceeds priuary dRinking tater stamdard.

- VALUE EXCEEDS PROPOSED PRIUARY DRINKING YATER STAMDARD.

- - YALUE EXCEEDS SCREENING LEYEL FOR FURTHER INYESTIGATION.

- - DETECTIDN LIUIT UAS NOT AYAILAQLE FOR COUPARISON

NR - ANALYSIS NOT RERUESTED OR MOT YET REPORTEO

YALUES IN I ARE COUNTING ERRORS FOR RADIONUCLIDES

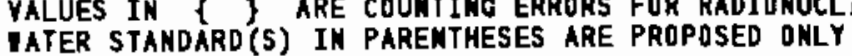


TABLE C.3. (contd)

\begin{tabular}{|c|c|c|c|c|c|c|c|c|c|}
\hline $\begin{array}{l}\text { CONSTI } \\
\text { NAUE }\end{array}$ & $\begin{array}{l}\text { UENT } \\
\text { UNITS }\end{array}$ & $\begin{array}{c}\text { CONTRACTUAL } \\
\text { DETECTION } \\
\text { LIUIT }\end{array}$ & $\begin{array}{l}\text { DRINKING } \\
\text { TATER } \\
\text { STANDARD }\end{array}$ & $\begin{array}{l}\text { SAHPLE } \\
\text { DATE }\end{array}$ & $0-47-5$ & $\begin{array}{l}\text { SAMPLE } \\
\text { DATE }\end{array}$ & $8-47-464$ & $\begin{array}{l}\text { SAUPLE } \\
\text { DATE }\end{array}$ & $6-47-60$ \\
\hline------ & ---- & 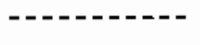 & - - & $m-\infty-\infty$ & ----- & $\cdots$ & $--+\infty$ & 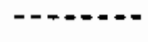 & -...... \\
\hline \multirow[t]{2}{*}{ TRITIUU } & PCI /L & 560.00 & 20005.00 & $11 / 08 / 87$ & $\begin{array}{l}206060.00 \\
\{1726.00\}\end{array}$ & & NR & & NR \\
\hline & & & & $12 / 03 / 87$ & $\{1814.00\}$ & & MR & & NR \\
\hline u & $\mathrm{PCI} / \mathrm{L}$ & 0.60 & 605.60 & $\begin{array}{l}17 / 00 / 87 \\
88 / 67 / 87 \\
10 / 13 / 87\end{array}$ & $\begin{array}{l}1.71 \\
2.10 \\
2.20\end{array}$ & $08 / 04 / 07$ & $\begin{array}{l}1.43 \\
M R \\
M R\end{array}$ & & $\begin{array}{l}\text { MR } \\
\text { NR } \\
\text { MR }\end{array}$ \\
\hline
\end{tabular}

- - value exceeds primary drinking tater stamdard.

- - VALUE EXCEEDS PROPOSED PRIMARY DRINKING IATER STANDARD.

- VALUE EXCEEDS SCREENINO LEVEL FOR FURTHER INYESTIGATION

- DETECTION LIUIT IAS NOT AVAILABLE FOR COUPARISON

NR - ANALYSIS NOT RERUESTED OR NOT YET REPORTED

VALUES IN I ? ARE COUNTIMG ERRORS FOR RADIONUCLIDES

WATER STANDARD(S) IN PARENTHESES ARE PROPLSED ONLY 
TABLE C. .3. (contd)

\begin{tabular}{|c|c|c|c|c|c|}
\hline \multicolumn{2}{|c|}{$\begin{array}{l}\text { COMSTITUENT } \\
\text { NAYE UNITS }\end{array}$} & $\begin{array}{c}\text { CONTRACTUAL } \\
\text { DETECTION } \\
\text { LIUIT }\end{array}$ & $\begin{array}{l}\text { DRINKING } \\
\text { IATER } \\
\text { STAMDARD }\end{array}$ & $\begin{array}{l}\text { SAUPLE } \\
\text { DATE }\end{array}$ & $0-47-60$ \\
\hline ALKAL IN & & & & $11 / 30 / 87$ & 128000.01 \\
\hline ALPHA & $\mathrm{PCI} / \mathrm{L}$ & 4.00 & 16.60 & $11 / 36 / 87$ & \\
\hline AUHOMIU & PPB & 58.06 & & $11 / 38 / 87$ & 83.66 \\
\hline BETA & PCI/L & 8.60 & 58.00 & $11 / 30 / 87$ & $\begin{array}{r}\quad \text { B.23 } \\
\{\quad 3.87\}\end{array}$ \\
\hline CHLORID & PPB & 506.60 & & $11 / 30 / 87$ & 9996.01 \\
\hline COMDFLD & UMHO & 1.60 & & $11 / 30 / 87$ & 345.06 \\
\hline FARSEMI & PPB & 5.60 & 60.60 & $11 / 36 / 87$ & 9.6 \\
\hline FBARIUU & $\mathrm{PPB}$ & 0.00 & 1066.06 & $11 / 35 / 87$ & 39.60 \\
\hline FCALCIU & PPB & 58.69 & & $11 / 36 / 87$ & 37000.10 \\
\hline FLUORID & PPB & 508.00 & 4018.61 & $11 / 30 / 87$ & 720.06 \\
\hline FUAGNES & PPB & 0.00 & & $11 / 30 / 87$ & 12360.06 \\
\hline FPOTASS & PPE & 108.66 & & $11 / 30 / 87$ & 6716.01 \\
\hline FSODIUU & PPB & 100.06 & & $11 / 38 / 87$ & 25501.00 \\
\hline FYANADI & PPB & 5.00 & & $11 / 36 / 87$ & 17.00 \\
\hline FZINC & PPB & 5.06 & & $11 / 31 / 87$ & \\
\hline HNITRAT & PPg & 2560.06 & 45600.00 & $\begin{array}{l}08 / 94 / 87 \\
11 / 39 / 87\end{array}$ & $\begin{array}{l}21700.00 \\
22700.00\end{array}$ \\
\hline LFLUORD & PPB & 28.06 & 4600.61 & $11 / 30 / 87$ & 655.06 \\
\hline METHYCH & PPB & 10.06 & & $11 / 30 / 87$ & \\
\hline 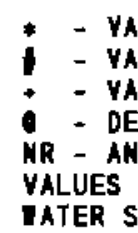 & $\begin{array}{l}\text { JE EXCEE } \\
\text { JE EXCEE } \\
\text { JE EXCEE } \\
\text { ECTIDN } \\
\text { YSIS NC } \\
\text { \{ \} } \\
\text { NOARD's }\end{array}$ & $\begin{array}{l}\text { DS PRIUARY D D } \\
\text { DS PROPOSED } \\
\text { DS SCREENING } \\
\text { IVIT NAS NOT } \\
\text { T REQUESTED } \\
\text { ARE COUNTIN } \\
\text { ) IN PARENTH }\end{array}$ & $\begin{array}{l}\text { INKING WATER } \\
\text { RIMARY DRIN } \\
\text { LEYEL FOR FL } \\
\text { AVAILABLE F } \\
\text { R NOT YET RE } \\
\text { ERRORS FOR } \\
\text { SES ARE PROF }\end{array}$ & $\begin{array}{l}\text { ANDARD. } \\
\text { TATER STA } \\
\text { ER INYESTI } \\
\text { OUPARISON } \\
\text { TED } \\
\text { IONUCLIDES } \\
\text { D ONLY }\end{array}$ & $\begin{array}{l}\text { DARO. } \\
\text { ATIOK. }\end{array}$ \\
\hline
\end{tabular}

\begin{tabular}{|c|c|}
\hline $\begin{array}{c}\text { SAMPLE } \\
\text { DATE }\end{array}$ & $0-46-7$ \\
\hline $10 / 61 / 87$ & 104000.06 \\
\hline $09 / 01 / 87$ & $\begin{array}{r}1.95 \\
\{\quad 0.95\}\end{array}$ \\
\hline $60 / 01 / 07$ & \\
\hline $10 / 01 / 87$ & $\begin{array}{r}8.80 \\
\{\quad 9.35\} \\
\end{array}$ \\
\hline $10 / 01 / 87$ & 4400.1. \\
\hline $09 / 11 / 87$ & 217.00 \\
\hline $10 / 11 / 87$ & \\
\hline $60 / 01 / 87$ & 33.01 \\
\hline $10 / 01 / 07$ & 201060.00 \\
\hline $60 / 01 / 87$ & \\
\hline $00 / 01 / 87$ & B180.160 \\
\hline $19 / 61 / 87$ & 2091.66 \\
\hline $19 / 61 / 87$ & 8771.60 \\
\hline $10 / 101 / 87$ & 6. 19 \\
\hline \multirow[t]{2}{*}{$99 / 11 / 87$} & \\
\hline & $\begin{array}{l}\text { MR } \\
\text { MR }\end{array}$ \\
\hline $00 / 01 / 87$ & 178.40 \\
\hline $60 / 01 / 87$ & \\
\hline
\end{tabular}

\begin{tabular}{|c|c|}
\hline $\begin{array}{l}\text { SAUPLE } \\
\text { DATE }\end{array}$ & $8-48-18$ \\
\hline $18 / 25 / 87$ & 110000.6 \\
\hline $18 / 25 / 87$ & $\begin{array}{r}2.40 \\
\{\quad 1.99\}\end{array}$ \\
\hline $18 / 25 / 87$ & \\
\hline $18 / 25 / 87$ & $\left\{\begin{array}{r}11.16 \\
4.35\}\end{array}\right.$ \\
\hline $68 / 25 / 87$ & 0436.06 \\
\hline & WR \\
\hline $18 / 25 / 87$ & \\
\hline $18 / 25 / 87$ & 34.64 \\
\hline $18 / 25 / 87$ & s3000.40 \\
\hline $18 / 26 / 87$ & \\
\hline $08 / 25 / 87$ & 12260.60 \\
\hline $08 / 25 / 87$ & 5810.60 \\
\hline $10 / 26 / 87$ & 13360.01 \\
\hline $08 / 25 / 87$ & 15.11 \\
\hline $08 / 25 / 87$ & 5.61 \\
\hline & $\begin{array}{c}\text { MR } \\
\text { MR } \\
-1\end{array}$ \\
\hline $18 / 25 / 87$ & 250.01 \\
\hline $18 / 25 / 87$ & 80.01 \\
\hline
\end{tabular}


TABLE C.3. (contd)

\begin{tabular}{|c|c|c|c|c|c|c|c|c|c|}
\hline $\begin{array}{l}\text { CONSTI } \\
\text { NAHE }\end{array}$ & $\begin{array}{l}\text { UENT } \\
\text { UNITS }\end{array}$ & $\begin{array}{l}\text { CONTRACTUAL } \\
\text { DETECTION } \\
\text { LIUIT }\end{array}$ & $\begin{array}{l}\text { ORINKINO } \\
\text { WATER } \\
\text { STANDARD }\end{array}$ & $\begin{array}{l}\text { SAUPLE } \\
\text { DATE }\end{array}$ & $8-47-88$ & $\begin{array}{l}\text { SAUPLE } \\
\text { DATE }\end{array}$ & $6-48-7$ & $\begin{array}{l}\text { SAMPLE } \\
\text { DATE }\end{array}$ & $8-48-18$ \\
\hline----- & ---- & 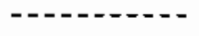 & - - & $---c--$ & ----- & $-\cdots---$ & ---- & ------ & $---\infty--$ \\
\hline NITRATE & PPB & 500.00 & 45006.00 & $11 / 36 / 87$ & 24300.10 & $18 / 01 / 87$ & 6350.00 & $18 / 25 / 87$ & 6681.01 \\
\hline PH-LAB & & 0.01 & & $11 / 30 / 87$ & 8.44 & $10 / 61 / 87$ & 7.39 & $10 / 25 / 67$ & 7.70 \\
\hline PHF IELD & & 0.10 & & $11 / 30 / 87$ & 7.66 & $10 / 81 / 87$ & 6.30 & & MR \\
\hline SULFATE & PPB & 500.00 & & $11 / 30 / 87$ & 49560.00 & $10 / 81 / 87$ & 20604.6 & $18 / 25 / 87$ & 52206.5 \\
\hline TC & PPB & & & $11 / 30 / 87$ & 28446.60 & $10 / 61 / 87$ & $28 B \overline{c o s}$ & $68 / 25 / 87$ & 20564.65 \\
\hline TRITIUM & $\mathrm{PCI} / \mathrm{L}$ & 500.09 & 20690.60 & $\begin{array}{l}68 / 04 / 87 \\
11 / 3 \% / 07\end{array}$ & & $\begin{array}{l}18 / 25 / 87 \\
11 / 21 / 87\end{array}$ & $\left\{\begin{array}{l}277.60 \\
188.00\}\end{array}\right.$ & $\begin{array}{l}68 / 25 / 07 \\
11 / 02 / 87\end{array}$ & $\begin{array}{l}302.00 \\
\{\quad 167.00\}\end{array}$ \\
\hline $\mathbf{U}$ & $\mathrm{PCI} / \mathrm{L}$ & 6.50 & 600.06 & $18 / 04 / 87$ & 1.55 & $--+\infty-0$ & NR & $\cdots$ & $\mathrm{NR}$ \\
\hline
\end{tabular}

- - VALUE EXCEEDS PRIMARY DRINKINg IATER STAMDARD.

- - VALUE EXCEEDS PROPOSED PRIMARY DRINKINO VATER STAMDARD.

- - VALUE EXCEEDS SCREENING LEVEL FOR fURTHER INVESTIGATION.

- DETECTION linit tas nat available for couparison

NR - ANALYSIS NOT REQUESTED OR NOT YET REPORTED

VALUES IN \{ $\}$ ARE COUNTING ERRDRS FOR RADIONUCLIOES

IATER STANDARD(S) IN PARENTHESES ARE PROPOSED ONLY 
TABLE C. 3. (contd)

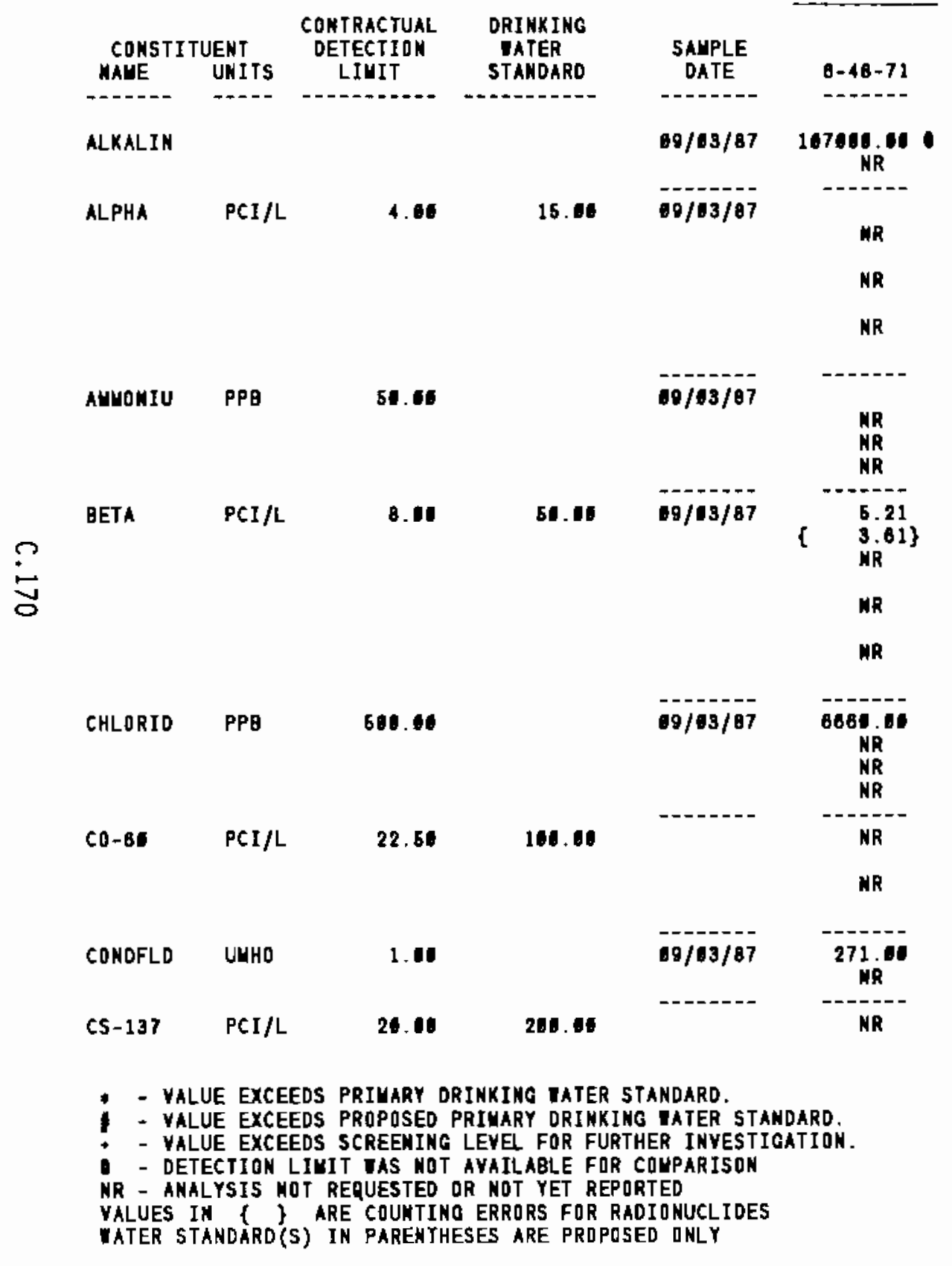

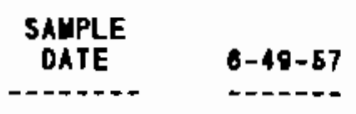

$09 / 16 / 87$ 165000.04 $11 / 34 / 87$ 10246e.65

\section{9/10/07}

$18 / 26 / 87$

$11 / 31 / 87 \quad 4.11$

$11 / 80 / 07$ \{ 2.48$\}$

$11 / 31 / 87$ ( 1.05$)$

\{ $\quad 0.14\}$

19/28/87

$11 / 31 / 87 \quad 640.08$

$11 / 35 / 67 / 67 \quad 640.01$

$11 / 96 / 87 \quad 626.00$

$18 / 26 / 87$ 1180.01.

$11 / 30 / 97$ \{ 33.30$\}$.

$11 / 36 / 87\{36.76\}$

\{ 33.64$\}$

1100.00 .

(33.60)

08/28/87 16246.0

$11 / 90 / 97$ 1504.

$11 / 90 / 97$ 15196.01

$11 / 30 / 87 \quad 15106.00$

/8/25/87

$11 / 36 / 87$

$10 / 20 / 87$

$11 / 36 / 87$

$\left\{\begin{array}{c}182.04 \\ 30.06\}\end{array}\right.$

108.66 .

( 29.80)

733.

abs.

08/25/67
10/10/87

$11 / 30 / 07 \quad\left\{\begin{array}{l}1.58 \\ 1.15\end{array}\right.$

$11 / 30 / 87$

$11 / 96 / 87$

$10 / 10 / 87$

$11 / 36 / 87$

$11 / 31 / 87$

$11 / 36 / 87$

og/1s/ar

$11 / 30 / 87$

$11 / 30 / 87$

$11 / 30 / 87$

$60 / 10 / 87$

11/30/87

$11 / 80 / 87$

$11 / 80 / 87$

$08 / 25 / 97$

$11 / 86 / 87$

$00 / 16 / 87$

$11 / 34 / 87$

$68 / 25 / 87$
68.4

180.00

$\{12.60\}$

209.01.

15.01)

( 16.70$)$

281.00 .

( 16.00$)$

365s.06

4215. 0

4100.06

4100.16

-...--

24.7

10.34

11.50)

294.

337.41

9.33
$4.09\}$ 
TABLE C. .3. (contd)

\begin{tabular}{|c|c|c|c|c|c|}
\hline \multicolumn{2}{|c|}{ CONST ITUENT } & $\begin{array}{l}\text { CONTRACTUAL } \\
\text { DETECTION } \\
\text { LIMIT }\end{array}$ & $\begin{array}{c}\text { DRINKING } \\
\text { ATER } \\
\text { STANDARD }\end{array}$ & $\begin{array}{c}\text { SAUPLE } \\
\text { DATE }\end{array}$ & $6-48-71$ \\
\hline------- & ---- & 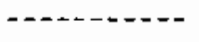 & - & $---n-\infty$ & ------- \\
\hline C5-137 & $\mathrm{PCI} / \mathrm{L}$ & 26.66 & 206.04 & & NR \\
\hline CYANIDE & PPB & 10.00 & & $00 / 63 / 67$ & $\begin{array}{l}N R \\
N R \\
N R\end{array}$ \\
\hline FARSENI & PPB & 5.66 & 56.01 & $09 / 63 / 07$ & $\begin{array}{l}\text { NR } \\
\text { NRR } \\
\text { MR }\end{array}$ \\
\hline FBARIUU & PPB & 6.66 & 1000.00 & $09 / 13 / 87$ & $\begin{array}{l}28.11 \\
\text { NR } \\
\text { NR } \\
\text { NR }\end{array}$ \\
\hline FBERYLL & PPB & 6.66 & & $00 / 03 / 87$ & $\begin{array}{l}\text { NR } \\
\text { MR } \\
\text { NR }\end{array}$ \\
\hline FCADUIL & $\mathrm{PPB}$ & 2.06 & 18.00 & $09 / 63 / 87$ & $\begin{array}{l}\text { NR } \\
\text { NR } \\
\text { MR }\end{array}$ \\
\hline FCALCIU & $\mathrm{PPB}$ & 60.00 & & $69 / 63 / 87$ & $\begin{array}{c}\text { NR } \\
\text { NR } \\
\text { NR }\end{array}$ \\
\hline FCHROHI & PPB & 10.00 & 50.00 & $69 / 03 / 87$ & $\begin{array}{l}\text { NR } \\
\text { NR } \\
\text { NR }\end{array}$ \\
\hline FIRON & PPB & 50.06 & & $60 / 03 / 87$ & \\
\hline
\end{tabular}

\begin{tabular}{|c|c|}
\hline $\begin{array}{l}\text { SAUPLE } \\
\text { DATE }\end{array}$ & $8-40-55 A$ \\
\hline $11 / 30 / 07$ & \\
\hline$---1-n$ & \\
\hline $\begin{array}{l}10 / 26 / 87 \\
11 / 31 / 87\end{array}$ & $\begin{array}{c}271.00 \\
342.60 \\
\text { NR } \\
\text { NR }\end{array}$ \\
\hline $18 / 28 / 87$ & $=-$ \\
\hline $\begin{array}{l}11 / 91 / 97 \\
11 / 30 / 87 \\
11 / 36 / 97\end{array}$ & $\begin{array}{l}0.14 \\
0.01\end{array}$ \\
\hline $8198 / 87$ & $\cdots$ \\
\hline $\begin{array}{l}8 / 26 / 67 \\
11 / 36 / 97 \\
11 / 36 / 97 \\
11 / 36 / 87\end{array}$ & $\begin{array}{l}48.05 \\
60.05 \\
52.00 \\
51.00\end{array}$ \\
\hline $\begin{array}{l}18 / 26 / 87 \\
11 / 36 / 87 \\
11 / 36 / 87 \\
11 / 30 / 87\end{array}$ & \\
\hline $\begin{array}{l}16 / 26 / 87 \\
11 / 30 / 87 \\
11 / 30 / 87 \\
11 / 36 / 87\end{array}$ & \\
\hline $\begin{array}{l}18 / 26 / 97 \\
11 / 36 / 87 \\
11 / 30 / 87 \\
11 / 36 / 87\end{array}$ & 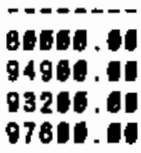 \\
\hline $18 / 28 / 87$ & $-\infty---$ \\
\hline $\begin{array}{l}8 / 26 / 87 \\
11 / 30 / 87 \\
11 / 36 / 87 \\
11 / 36 / 87\end{array}$ & $\begin{array}{l}10.00 \\
10.01\end{array}$ \\
\hline $8 / 26 / 87$ & 180.00 \\
\hline
\end{tabular}

\begin{tabular}{|c|c|}
\hline $\begin{array}{l}\text { SAMPLE } \\
\text { DATE }\end{array}$ & $0-40-57$ \\
\hline & \\
\hline $1 / 36 / 87$ & $\begin{array}{r}5.47 \\
1 \quad 3.87\end{array}$ \\
\hline $\begin{array}{l}60 / 10 / 87 \\
11 / 30 / 87 \\
11 / 36 / 87 \\
11 / 30 / 87\end{array}$ & $\begin{array}{l}19.00 \\
81.90 \\
48.90 \\
61.56\end{array}$ \\
\hline $\begin{array}{l}00 / 10 / 87 \\
11 / 36 / 87 \\
11 / 36 / 87 \\
11 / 86 / 87\end{array}$ & $\begin{array}{l}18.04 \\
11.04 \\
11.00 \\
11.00\end{array}$ \\
\hline $\begin{array}{l}-10 / 10 / 87 \\
11 / 30 / 87 \\
11 / 80 / 87 \\
11 / 36 / 87\end{array}$ & $\begin{array}{l}21.01 \\
27.11 \\
29.10 \\
28.10\end{array}$ \\
\hline $\begin{array}{l}09 / 14 / 87 \\
11 / 90 / 87 \\
11 / 30 / 87 \\
11 / 30 / 87\end{array}$ & 6.11 \\
\hline $\begin{array}{l}-9 / 10 / 87 \\
11 / 36 / 87 \\
11 / 36 / 87 \\
11 / 36 / 87\end{array}$ & 2.06 \\
\hline $\begin{array}{l}19 / 10 / 87 \\
11 / 90 / 87 \\
11 / 30 / 97 \\
11 / 30 / 87\end{array}$ & $\begin{array}{l}10714.00 \\
24104.00 \\
24804.00\end{array}$ \\
\hline $\begin{array}{l}10 / 10 / 87 \\
11 / 30 / 87 \\
11 / 30 / 87 \\
11 / 30 / 87\end{array}$ & $\begin{array}{l}11.00 \\
11.01 \\
16.00\end{array}$ \\
\hline & \\
\hline
\end{tabular}

* - VALUE EXCEEDS PRIMARY DRIMKIMG taTER STAMDARD.

* VALUE EXCEEDS PROPOSED PRIMARY DRINKIMG TATER STAMDARD.

* - VALUE EXCEEDS SCREENING LEVEL FOR FURTHER INVESTIGATION.

- - DETECTION LIUIT TAS NOT AVAILABLE FOR COUPARISON

NR - AMALYSIS NDT REQUESTED DR NOT YET REPORTED

VALUES IN \{ $\}$ ARE COUNTINO ERRORS FOR RADIONUCLIDES

WATER STANDARO(S) IN PARENTHESES ARE PROPOSED ONLY 
TABLE C.3. (contd)

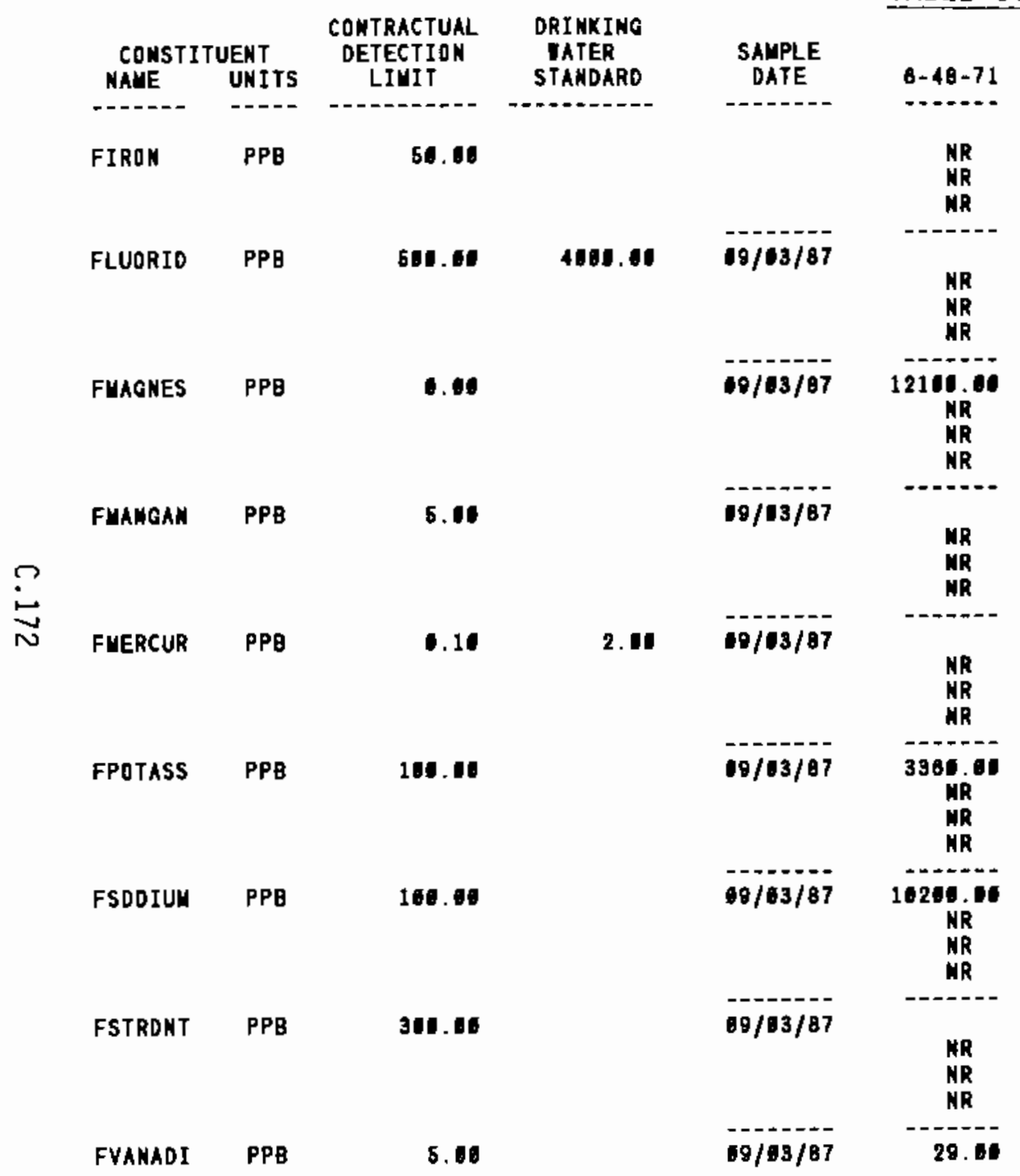

* - value exceeds primary drinking tater stamdard.

- VALUE EXCEEDS PROPOSED PRIUARY DRINKIMG IATER STAMDARD.

* - VALUE EXCEEDS SCREENINo LEVEL FOR FURTHER INYESTIOATION.

- DETECTION LIUIT TAS NOT AVAILABLE FOR COUPARISON

NR - ANALYSIS NOT RERUESTED OR NOT YET REPORTED

VALUES IN \{\} ARE COUNTING ERRORS FOR RADIONUCLIDES

WATER STANDARD(S) IN PARENTHESES ARE PROPOSED ONLY

\begin{tabular}{|c|c|}
\hline $\begin{array}{l}\text { SALPLE } \\
\text { DATE }\end{array}$ & $8-49-65 A$ \\
\hline & \\
\hline $\begin{array}{l}11 / 30 / 87 \\
11 / 30 / 87 \\
11 / 30 / 87\end{array}$ & $\begin{array}{l}118.00 \\
136.06 \\
145.06\end{array}$ \\
\hline $\begin{array}{l}18 / 28 / 87 \\
11 / 30 / 87 \\
11 / 30 / 97 \\
11 / 30 / 87\end{array}$ & $\begin{array}{l}777.00 \\
746.00 \\
727.01\end{array}$ \\
\hline $\begin{array}{l}18 / 28 / 87 \\
11 / 30 / 87 \\
11 / 36 / 87 \\
11 / 36 / 87\end{array}$ & $\begin{array}{l}24800.10 \\
20140.10 \\
28200.04 \\
28856.01\end{array}$ \\
\hline $\begin{array}{l}08 / 26 / 87 \\
11 / 30 / 87 \\
11 / 36 / 87 \\
11 / 36 / 87\end{array}$ & $\begin{array}{l}24.04 \\
23.06 \\
23.10 \\
23.6\end{array}$ \\
\hline \multirow[t]{2}{*}{$\begin{array}{l}18 / 28 / 87 \\
11 / 36 / 87\end{array}$} & \\
\hline & $\begin{array}{l}\text { NR } \\
\text { NR }\end{array}$ \\
\hline $\begin{array}{l}8 / 26 / 87 \\
11 / 30 / 87 \\
11 / 36 / 87 \\
11 / 36 / 87\end{array}$ & $\begin{array}{l}11004.05 \\
11704.5 \\
11000.04 \\
11700.00\end{array}$ \\
\hline $\begin{array}{l}18 / 28 / 87 \\
11 / 96 / 87 \\
11 / 30 / 87 \\
11 / 30 / 87\end{array}$ & $\begin{array}{l}4450.00 \\
45680.00 \\
48656.00 \\
45460.06\end{array}$ \\
\hline $\begin{array}{l}-1 / 28 / 87 \\
11 / 36 / 87 \\
11 / 36 / 87 \\
11 / 36 / 87\end{array}$ & $\begin{array}{l}439.14 \\
444.11 \\
486.11 \\
463.01\end{array}$ \\
\hline $8 / 26 / 87$ & 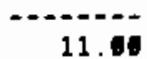 \\
\hline
\end{tabular}

SAUPLE

DATE

- - -...-

$11 / 30 / 87$

$11 / 30 / 87$

$11 / 36 / 87$

$0 / 16 / 87$

$11 / 30 / 87$

$11 / 31 / 87$

$11 / 30 / 07$

0

09/10/87

$11 / 30 / 87$

$11 / 30 / 87$

$11 / 30 / 67$

00/10/87

$11 / 36 / 87$

1/8.

11/31/87

11/30/87

10/19/87

$11 / 3 / 87$

$11 / 36 / 87$

$11 / 31 / 67$

09/10/87

$11 / 30 / 87$

$11 / 36 / 87$

$11 / 31 / 97$

$00 / 16 / 87$

$11 / 36 / 87$

$11 / 35 / 67$

09/10/87

$11 / 30 / 87$

$11 / 30 / 87$

$11 / 30 / 87$

60/10/87
8-40-57

-.---.-

81.

1040. if

1040.65

6020.01

7630.40

$707 . .46$

7635.01

-...-.

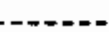

6.12
6.12

5204

5591

501.

6481.

5480.15

462 10.

46961.

48964. U1

46016. If

-.-.-..

38.68 
TABLE C.3. (contd)

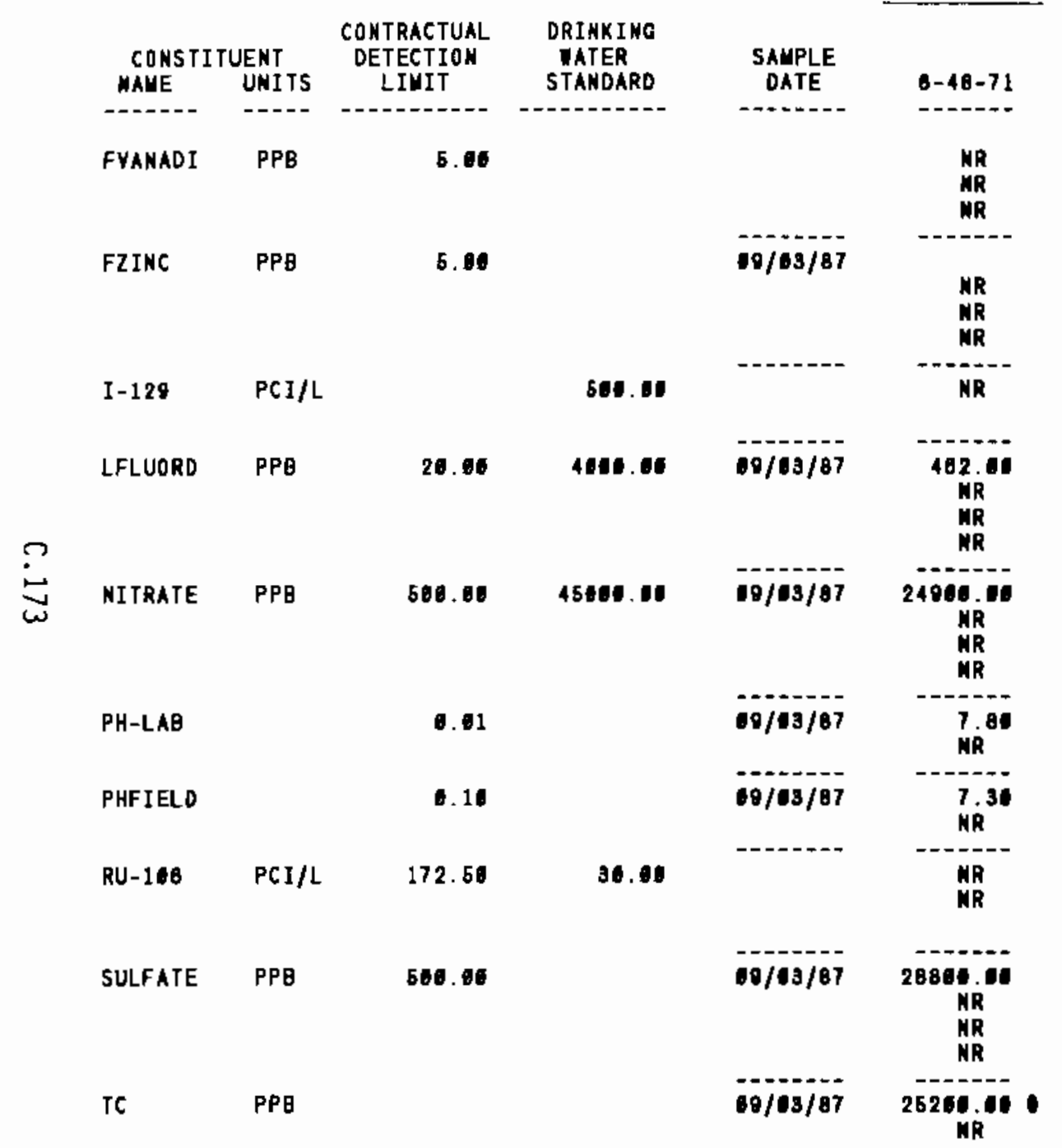

\begin{tabular}{|c|c|}
\hline $\begin{array}{l}\text { SAUPLE } \\
\text { DATE }\end{array}$ & $6-49-55 A$ \\
\hline $\begin{array}{l}11 / 30 / 87 \\
11 / 30 / 87 \\
11 / 96 / 87\end{array}$ & $\begin{array}{l}10.60 \\
10.06 \\
10.06\end{array}$ \\
\hline $\begin{array}{l}68 / 26 / 87 \\
11 / 30 / 87 \\
11 / 30 / 87 \\
11 / 30 / 87\end{array}$ & \\
\hline $10 / 64 / 87$ & $\left\{\begin{array}{l}06 \\
\text { (1.01\} }\end{array}\right.$ \\
\hline $\begin{array}{l}10 / 26 / 87 \\
11 / 30 / 87 \\
11 / 30 / 87 \\
11 / 30 / 87\end{array}$ & $\begin{array}{l}428.60 \\
455.60 \\
461.60 \\
469.60\end{array}$ \\
\hline $\begin{array}{l}13 / 26 / 87 \\
11 / 85 / 87 \\
11 / 31 / 87 \\
11 / 34 / 87\end{array}$ & 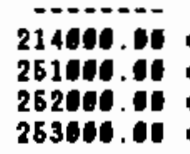 \\
\hline $\begin{array}{l}18 / 20 / 87 \\
11 / 30 / 97\end{array}$ & 7.98 \\
\hline $\begin{array}{l}08 / 28 / 87 \\
11 / 36 / 87\end{array}$ & $\begin{array}{l}7.56 \\
7.76\end{array}$ \\
\hline $\begin{array}{l}68 / 25 / 87 \\
11 / 31 / 87\end{array}$ & \\
\hline $\begin{array}{l}10 / 28 / 87 \\
11 / 30 / 87 \\
11 / 30 / 87 \\
11 / 30 / 87\end{array}$ & 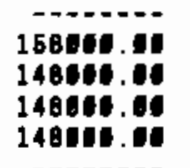 \\
\hline $\begin{array}{l}08 / 28 / 87 \\
11 / 36 / 87\end{array}$ & $\begin{array}{l}17611000 \\
23164.00\end{array}$ \\
\hline
\end{tabular}

\begin{tabular}{|c|c|}
\hline $\begin{array}{l}\text { SAUPLE } \\
\text { DATE }\end{array}$ & $0-40-57$ \\
\hline $\begin{array}{l}11 / 36 / 87 \\
11 / 90 / 87 \\
11 / 31 / 97\end{array}$ & $\begin{array}{l}38.40 \\
36.40 \\
25.40\end{array}$ \\
\hline $\begin{array}{l}09 / 10 / 87 \\
11 / 30 / 87 \\
11 / 90 / 87 \\
11 / 30 / 87\end{array}$ & 7.00 \\
\hline $09 / 04 / 87$ & $\begin{array}{l}0.26 \\
\{\quad 02\}\end{array}$ \\
\hline $\begin{array}{l}19 / 11 / 87 \\
11 / 90 / 87 \\
11 / 90 / 97 \\
11 / 30 / 87\end{array}$ & $\begin{array}{l}1090.41 \\
1004.40 \\
1030.40\end{array}$ \\
\hline $\begin{array}{l}60 / 10 / 87 \\
11 / 30 / 87 \\
11 / 80 / 87 \\
11 / 30 / 87\end{array}$ & 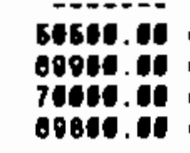 \\
\hline $\begin{array}{l}19 / 16 / 87 \\
11 / 36 / 87\end{array}$ & $\begin{array}{l}7.92 \\
8.07\end{array}$ \\
\hline $\begin{array}{l}09 / 16 / 87 \\
11 / 35 / 87\end{array}$ & $\begin{array}{l}7.20 \\
7.76\end{array}$ \\
\hline $\begin{array}{l}09 / 25 / 87 \\
11 / 39 / 87\end{array}$ & $\left\{\begin{array}{l}51.56 \\
\{\quad 34.46\}\end{array}\right.$ \\
\hline $\begin{array}{l}10 / 10 / 87 \\
11 / 90 / 87 \\
11 / 90 / 87 \\
11 / 30 / 87\end{array}$ & 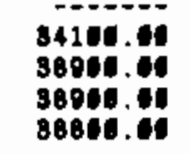 \\
\hline $\begin{array}{l}19 / 110 / 97 \\
11 / 30 / 87\end{array}$ & $\begin{array}{l}24210.01 \\
24100.01\end{array}$ \\
\hline
\end{tabular}

* - VALUE EXCEEDS PRTMARY DRIMKING TaTER STAMDARD

- VALUE EXCEEDS PRoPDSED PRIMARY DRINKING TATER STANDARD.

* - VAlUe eXCEEdS SCREening LeVel for further inyestioation.

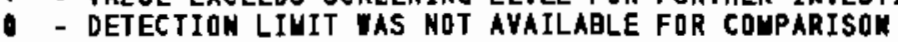

NR - ANALYSIS NDT REQUESTED OR NOT YET REPORTED

VALUES IT \{\} ARE CDUNTING ERRORS FOR RADIONUCLIDES

itTER STANDARO'(S) IM PARENTHESES ARE PROPOSED DNLY 
TABLE C.3. (contd)

\begin{tabular}{|c|c|c|c|c|c|c|c|c|c|}
\hline $\begin{array}{c}\text { CONSTI } \\
\text { NAME }\end{array}$ & $\begin{array}{l}\text { UENT } \\
\text { UNITS }\end{array}$ & $\begin{array}{c}\text { CONTRACTUAL } \\
\text { DETECTIOA } \\
\text { LIUIT }\end{array}$ & $\begin{array}{l}\text { DRINKING } \\
\text { TATER } \\
\text { STANDARD }\end{array}$ & $\begin{array}{c}\text { SAHPLE } \\
\text { DATE }\end{array}$ & $6-48-71$ & $\begin{array}{c}\text { SAMPLE } \\
\text { DATE }\end{array}$ & $0-49-55 A$ & $\begin{array}{c}\text { SAUPLE } \\
\text { DATE }\end{array}$ & $0-40-67$ \\
\hline$-\cdots---$ & ---- & 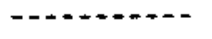 & $--1-1,-$ & 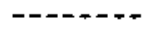 & $\ldots . .$. & ------- & - - & - & $\cdots--$ \\
\hline TRITIUM & PCI/L & 500.90 & 20000.00 & $\begin{array}{l}68 / 25 / 87 \\
12 / 16 / 87\end{array}$ & & $\begin{array}{l}08 / 25 / 87 \\
11 / 30 / 87\end{array}$ & $\begin{array}{c}16900.00 \\
536.00\} \\
\{516.50\}\end{array}$ & $\begin{array}{l}08 / 26 / 87 \\
11 / 30 / 87\end{array}$ & $\begin{array}{r}1720.04 \\
254.04\} \\
3840.00 \\
\{208.01\}\end{array}$ \\
\hline$U$ & $\mathrm{PCI} / \mathrm{L}$ & 0.50 & 860.65 & ------ & MR & $18 / 25 / 87$ & 3.29 & & MR \\
\hline
\end{tabular}

$\sum_{D}$

* - yalue exceeds primary dRinking iater standaro.

- VALUE EXCEEDS PROPOSEO PRIMARY DRINKING YaTER STANDARD.

- VALUE EXCEEDS SCREENING LEVEL FOR FURTHER INVESTIGATIOH.

- DETECTION livit ias not available for couparison

HR - ANALYSIS RDT REQUESTED OR NOT YET REPORTED

VALUES IN f ARE COUNTINO ERRORS FOR RADIONUCLIDES

IATER STANDARD(S) IN PARENTHESES ARE PROPOSED ONLY 
TABLE C.3. (contd)

\begin{tabular}{|c|c|c|}
\hline CONSTITUENT & $\begin{array}{c}\text { CONTRACTUAL } \\
\text { DETECT IDN }\end{array}$ & $\begin{array}{l}\text { DRINK ING } \\
\text { ATER }\end{array}$ \\
\hline NAUE UNITS & LIMIT & STANDARD \\
\hline
\end{tabular}
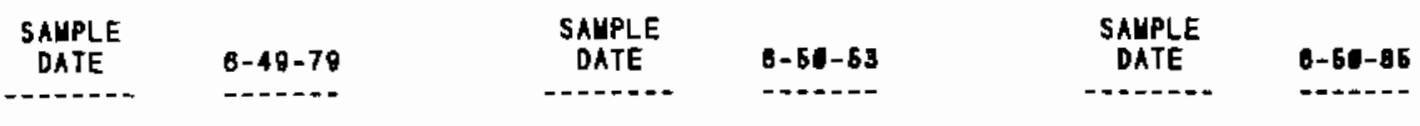

ALKALIN

$08 / 26 / 87$ $11 / 36 / 87$

98400.04
103060.00

ALPHA PCI/L

4.60

15.90

$68 / 28 / 87$

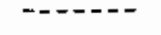

$11 / 90 / 87$

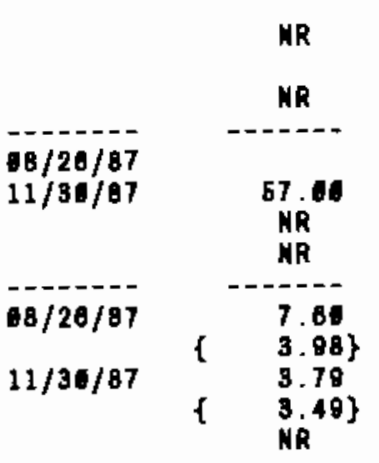

\section{MR}

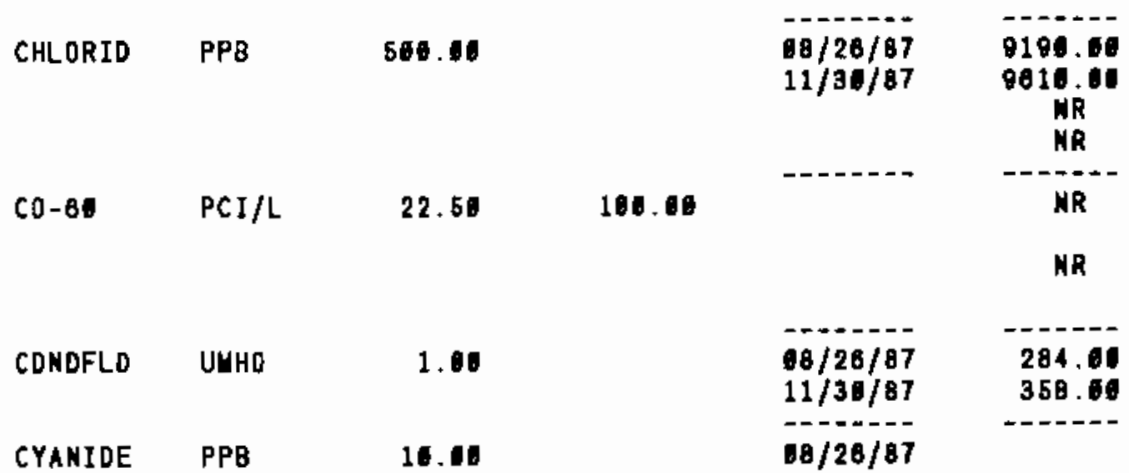

- - Value eXCEeds PRIMARY dRINXING TaTER STAMDaRD.

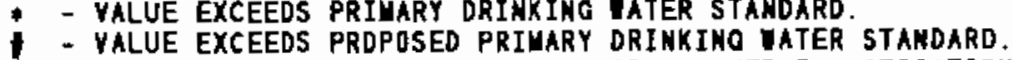

- VALUE EXCEEDS SCREENINg LEVEL FOR FURTHER IMVESTIGATIDN.

- DETECTION LIUIT WAS NOT AVAILABLE FOR CDUPARISON

NR - ANALYSIS NOT REQUESTED OR NOT YET REPORTED

VALUES IN $\{$ \& ARE COUNTING ERRORS FQR RADIONUCLIOES

IATER STANDARD(S) IN PARENTHESES ARE PROPOSED OHLY

\begin{tabular}{|c|c|c|c|}
\hline $\begin{array}{l}68 / 20 / 87 \\
12 / 67 / 87\end{array}$ & $\begin{array}{l}\text { 68500. } \\
69045\end{array}$ & $12 / 11 / 87$ & 110001.09 \\
\hline $18 / 20 / 87$ & 2.76 & $12 / 11 / 87$ & 1.64 \\
\hline & $\begin{array}{l}2.12\} \\
0.10\end{array}$ & & $\frac{1.20\}}{M R}$ \\
\hline & $\begin{array}{l}4.89\} \\
4.78\end{array}$ & & \\
\hline $12 / 10780$ & $\{\quad 4.58\}$ & & $\mathbf{N K}$ \\
\hline $12 / 17 / 67$ & & & $\mathbf{M R}$ \\
\hline $88 / 28 / 87$ & 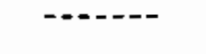 & --+-- & -ne \\
\hline $\begin{array}{l}12 / 67 / 87 \\
12 / 07 / 67 \\
12 / 67 / 67\end{array}$ & & & $\begin{array}{l}\text { NR } \\
\text { NR } \\
\text { MR }\end{array}$ \\
\hline G) & $\overline{1350.04}$ & 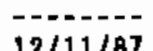 & 9.67 \\
\hline $08 / 20 / 01$ & $\{35.46\}$ & $12 / 11 / 87$ & $\begin{array}{l}9.67 \\
3.28\}\end{array}$ \\
\hline $12 / 07 / 87$ & $\left(\begin{array}{c}2850.10 \\
68.16\end{array}\right)$ & & NR \\
\hline $12 / 57 / 87$ & $\begin{array}{r}2570.00 \\
51.64\}\end{array}$ & & WR \\
\hline $12 / 07 / 87$ & $\left\{\begin{array}{l}2770.14 \\
(55.26)\end{array}\right.$ & & WR \\
\hline - & $\cdots--\infty$ & 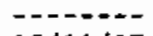 & 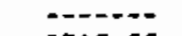 \\
\hline $\begin{array}{l}18 / 26 / 67 \\
12 / 07 / 87 \\
12 / 07 / 87 \\
12 / 67 / 87\end{array}$ & $\begin{array}{l}40800.60 \\
112010.60 \\
119640.96 \\
113640.06\end{array}$ & $12 / 11 / 87$ & $\begin{array}{c}\text { NR } \\
\text { NR } \\
\text { NR }\end{array}$ \\
\hline $00 / 25 / 87$ & 411.01 & 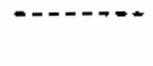 & MR \\
\hline $12 / 67 / 87$ & $\begin{array}{c}487.85\} \\
\{\quad 46.40\}\end{array}$ & & NR \\
\hline 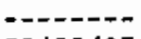 & $-\ldots$ & - & $-\infty+\infty-\infty$ \\
\hline $\begin{array}{l}08 / 26 / 87 \\
12 / 67 / 87\end{array}$ & $\begin{array}{l}1268.05 \\
1152.05\end{array}$ & $12 / 11 / 87$ & $\begin{array}{c}283 . \mathrm{HI} \\
\mathrm{MR}\end{array}$ \\
\hline /8/26/87 & 971.60 & $12 / 11 / 87$ & \\
\hline
\end{tabular}


TABLE C.3. (contd)

\begin{tabular}{|c|c|c|c|c|c|}
\hline $\begin{array}{c}\text { CONSTI } \\
\text { NAUE }\end{array}$ & $\begin{array}{l}\text { UENT } \\
\text { UNITS }\end{array}$ & $\begin{array}{c}\text { CONTRACTUAL } \\
\text { DETECTIOH } \\
\text { LIHIT }\end{array}$ & $\begin{array}{l}\text { DRINKING } \\
\text { IATER } \\
\text { STAMOARD }\end{array}$ & $\begin{array}{l}\text { SALPLE } \\
\text { DATE }\end{array}$ & $0-40-7 \theta$ \\
\hline CYANIDE & PPB & 18.00 & & $11 / 91 / 87$ & $\begin{array}{l}\text { MR } \\
\text { NR }\end{array}$ \\
\hline FBARIUU & PPB & 6.16 & 1060.00 & $\begin{array}{l}08 / 26 / 87 \\
11 / 36 / 87\end{array}$ & $\begin{array}{l}31.09 \\
M R \\
M R\end{array}$ \\
\hline FCALCIU & PPB & 58.01 & & $\begin{array}{l}68 / 28 / 87 \\
11 / 30 / 87\end{array}$ & $\begin{array}{c}37301.00 \\
44500.00 \\
\text { NR } \\
\text { HR }\end{array}$ \\
\hline FIRON & PPB & 60.00 & & $\begin{array}{l}08 / 26 / 87 \\
11 / 30 / 87\end{array}$ & $\underset{M R}{\mathrm{MR}}$ \\
\hline FLUORID & PPB & 500.06 & 4166.06 & $\begin{array}{l}18 / 28 / 87 \\
11 / 30 / 67\end{array}$ & $\begin{array}{l}\text { NR } \\
\text { MR }\end{array}$ \\
\hline FUAGNES & PPB & 0.01 & & $\begin{array}{l}18 / 28 / 97 \\
11 / 36 / 87\end{array}$ & $\begin{array}{c}13760.60 \\
14400.06 \\
M R \\
M R\end{array}$ \\
\hline FPDTASS & PPB & 100.08 & & $\begin{array}{l}18 / 26 / 87 \\
11 / 30 / 87\end{array}$ & $\begin{array}{c}385 t .01 \\
337 \\
\text { NR } \\
\text { NR }\end{array}$ \\
\hline FSELENI & PPB & 5.10 & 10.00 & $\begin{array}{l}98 / 28 / 87 \\
11 / 36 / 87\end{array}$ & $\begin{array}{l}\text { MR } \\
\text { NR }\end{array}$ \\
\hline FSODIUU & PPB & 100.00 & & $08 / 28 / 87$ & 8680.60 \\
\hline
\end{tabular}

\begin{tabular}{|c|c|c|c|}
\hline $\begin{array}{l}\text { SAMPLE } \\
\text { DATE }\end{array}$ & $6-60-53$ & $\begin{array}{c}\text { SAMPLE } \\
\text { DATE }\end{array}$ & $6-60-86$ \\
\hline $\begin{array}{l}12 / 07 / 87 \\
12 / 07 / 87 \\
12 / 07 / 87\end{array}$ & $\begin{array}{r}822.00 \\
1120.00 \\
1125.00\end{array}$ & & $\underset{M R}{M R}$ \\
\hline $\begin{array}{l}18 / 26 / 87 \\
12 / 07 / 87 \\
12 / 67 / 87 \\
12 / 07 / 87\end{array}$ & $\begin{array}{l}71.06 \\
70.4 \\
71.04 \\
71.06\end{array}$ & $12 / 11 / 87$ & $\underset{M R}{M_{M R}}$ \\
\hline $\begin{array}{l}18 / 28 / 87 \\
12 / 07 / 87 \\
12 / 07 / 87 \\
12 / 87 / 87\end{array}$ & 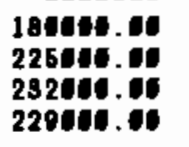 & $12 / 11 / 07$ & ${ }_{\mathrm{MR}}^{\mathrm{MPR}}$ \\
\hline $\begin{array}{l}18 / 28 / 87 \\
12 / 67 / 87 \\
12 / 67 / 87 \\
12 / 07 / 87\end{array}$ & $\begin{array}{l}246.16 \\
283.11 \\
292.11 \\
200.11\end{array}$ & $12 / 11 / 87$ & $\underset{M R}{M R}$ \\
\hline $\begin{array}{l}18 / 26 / 87 \\
12 / 97 / 87 \\
12 / 97 / 87 \\
12 / 07 / 87\end{array}$ & $\begin{array}{l}1130.00 \\
1130.00 \\
1196.00\end{array}$ & $12 / 11 / 87$ & $\underset{M R}{M R}$ \\
\hline $\begin{array}{l}68 / 28 / 87 \\
12 / 07 / 87 \\
12 / 97 / 87 \\
12 / 67 / 87\end{array}$ & 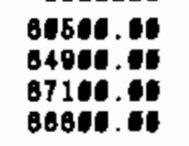 & $12 / 11 / 87$ & 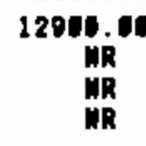 \\
\hline $\begin{array}{l}18 / 28 / 87 \\
12 / 67 / 87 \\
12 / 67 / 87 \\
12 / 67 / 87\end{array}$ & $\begin{array}{l}14500.06 \\
14406.06 \\
14004.01 \\
14904.04\end{array}$ & $12 / 11 / 87$ & $\underset{\substack{M R \\
\text { MR }}}{360.06}$ \\
\hline $\begin{array}{l}88 / 28 / 87 \\
12 / 07 / 87 \\
12 / 07 / 87 \\
12 / 87 / 87\end{array}$ & $\begin{array}{l}11.00 \\
13.00 \\
15.60 \\
14.00\end{array}$ & $12 / 11 / 87$ & $\begin{array}{l}\text { MR } \\
\text { MR } \\
\text { MR }\end{array}$ \\
\hline $68 / 26 / 87$ & 55610.00 & $12 / 11 / 87$ & 8874.1 \\
\hline
\end{tabular}

* - value exceeds prigary dRinking tater standard.

- YALUE EXCEEDS PROPOSED PRILARY DRINKING IATER STAMDARD.

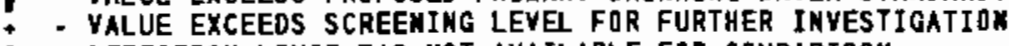

- DETECTION LIUIT ias MOT aYaILable fOR COMParisom

MR - ANALYSIS NOT REQUESTED OR NOT YET REPORTED

YALUES IN $(?$ ARE COUMTING ERRORS FOR RADIONUCLIDES

TATER STANDARD(S) IN PARENTHESES ARE PROPOSED ONLY 
TABLE C.3. (contd)

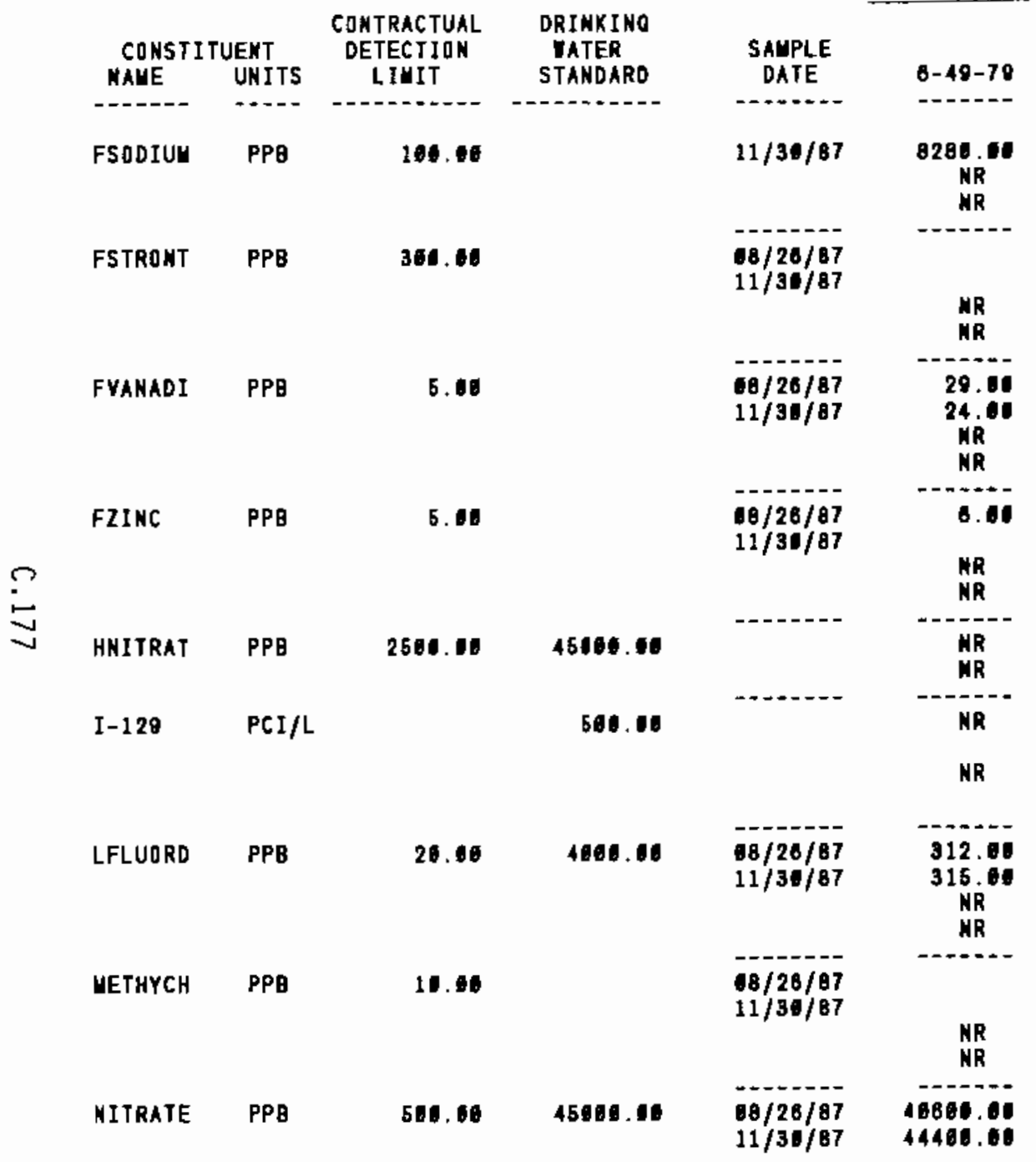

\begin{tabular}{|c|c|}
\hline $\begin{array}{c}\text { SAUPLE } \\
\text { DATE }\end{array}$ & $8-50-63$ \\
\hline $\begin{array}{l}12 / 07 / 87 \\
12 / 07 / 87 \\
12 / 67 / 87\end{array}$ & $\begin{array}{l}69200.01 \\
61101.01 \\
61684.60\end{array}$ \\
\hline $\begin{array}{l}18 / 26 / 87 \\
12 / 17 / 87 \\
12 / 67 / 87 \\
12 / 07 / 87\end{array}$ & $\begin{array}{l}867.19 \\
910.11 \\
930.11 \\
059.60\end{array}$ \\
\hline $\begin{array}{l}08 / 28 / 87 \\
12 / 07 / 97 \\
12 / 17 / 87 \\
12 / 107 / 87\end{array}$ & $\begin{array}{r}11.04 \\
7.06\end{array}$ \\
\hline \multirow[t]{2}{*}{$\begin{array}{l}10 / 26 / 87 \\
12 / 67 / 87 \\
12 / 67 / 87 \\
12 / 07 / 67\end{array}$} & $\begin{array}{r}8.100 \\
8.00 \\
0.00 \\
18.01\end{array}$ \\
\hline & $\begin{array}{l}\text { NR } \\
\text { NR }\end{array}$ \\
\hline $19 / 104 / 87$ & 0.14 \\
\hline $60 / 04 / 87$ & 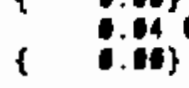 \\
\hline $\begin{array}{l}18 / 26 / 87 \\
12 / 67 / 87 \\
12 / 17 / 87 \\
12 / 07 / 67\end{array}$ & $\begin{array}{l}310.14 \\
360.01 \\
295.06 \\
306.01\end{array}$ \\
\hline $\begin{array}{l}18 / 26 / 87 \\
12 / 67 / 87 \\
12 / 67 / 87 \\
12 / 67 / 87\end{array}$ & 22360.11 \\
\hline $\begin{array}{l}88 / 28 / 87 \\
12 / 07 / 87\end{array}$ & 568000. If \\
\hline
\end{tabular}

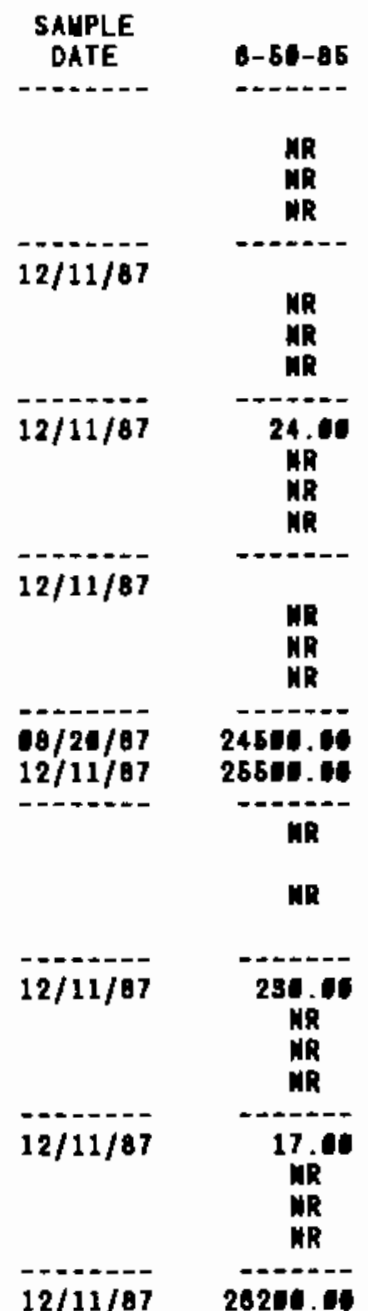

* - Value exceeds priuary dRINKIng tater standard

- VALUE EXCEEDS PROPOSED PRIMARY DRINKING iATER STANDARD.

- VALUE EXCEEDS SCREENING LEVEL FoR FURTHER INYESTIGATION.

- - OETECTION LIUIT NAS NOT AVAJLABLE FOR COMPARISON

MR - ANALYSIS HOT REQUESTED OR WDT YET REPORTED

VALUES IN 5 ARE COUNTING ERRORS FOR RADIOMUCLIDES

IATER STANDARD(S) IN PARENTHESES ARE PROPDSED ONLY 


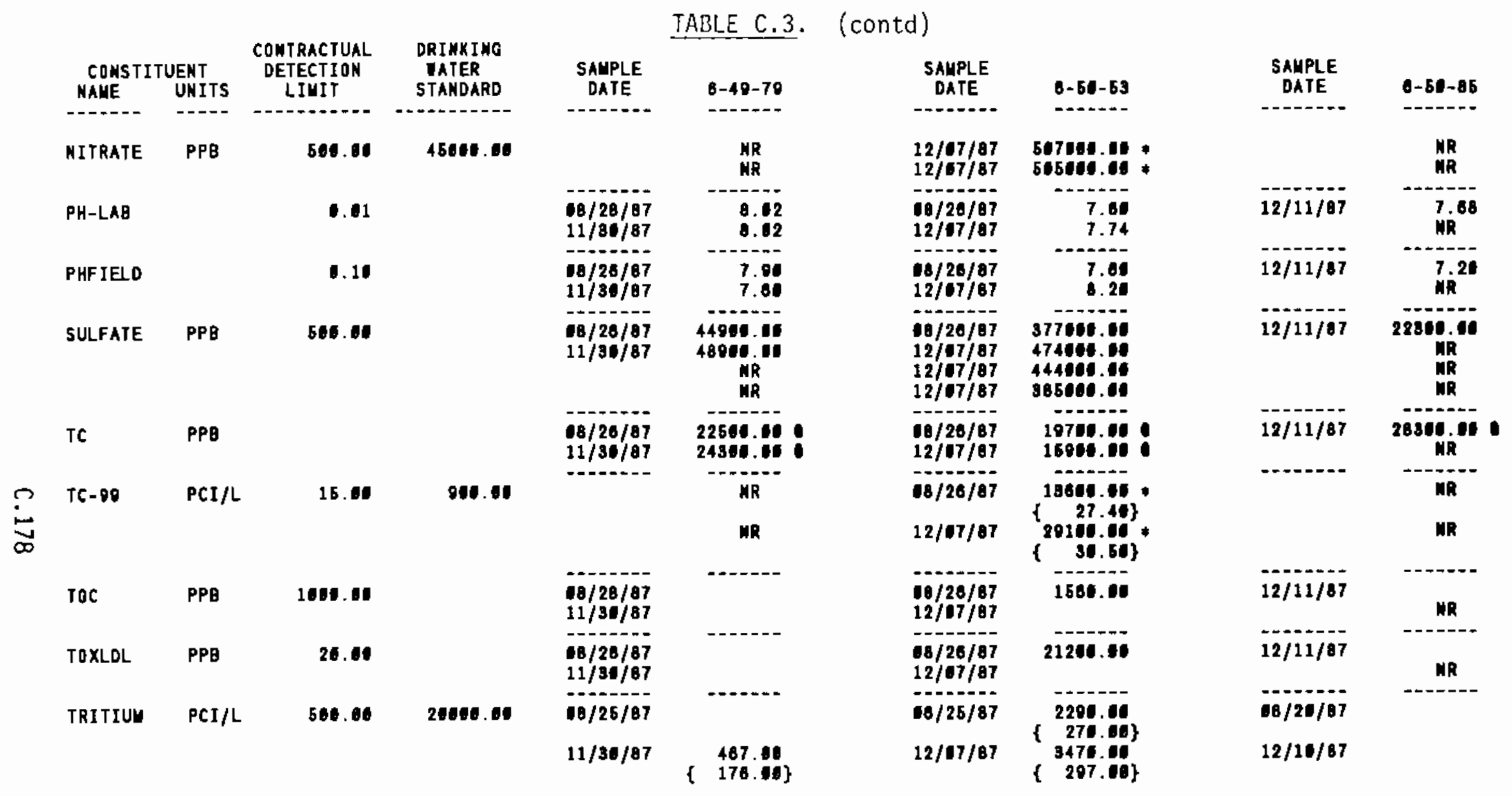

- - yalue exCEeds primary dRINKING iater standaro

- VALUE EXCEEDS PROPOSED PRIMARY DRINKING IATER STANDARRD.

- VALUE EXCEEDS SCREENING LEYEL FOR FURTHER INVESTIgation.

- deTECTiOn linit ias not ayailable for comparison

MR - ANALYSIS NOT REQUESTED OR NOT YET REPORTED

YALUES IN $\&$ ARE COUNTING ERRORS FOR RADIDNUCLIDES

IATER STANDARD(S) IN PAREMTHESES ARE PROPDSED ONLY 
TABLE C.3. (contd)

\begin{tabular}{|c|c|c|c|c|c|}
\hline \multicolumn{2}{|c|}{$\begin{array}{l}\text { CONSTITUENT } \\
\text { NALE UNITS }\end{array}$} & $\begin{array}{l}\text { CONTRACTUAL } \\
\text { DETECTIDH } \\
\text { LIUIT }\end{array}$ & $\begin{array}{l}\text { DRINKING } \\
\text { IATER } \\
\text { STANDARD }\end{array}$ & $\begin{array}{l}\text { SAMPLE } \\
\text { DATE }\end{array}$ & $8-51-75$ \\
\hline ALKALIN & & & & $69 / 48 / 87$ & 123040.06 \\
\hline \multirow[t]{2}{*}{ ALPHA } & $\mathrm{PCI} / \mathrm{L}$ & 4.60 & 15.76 & $19 / 108 / 87$ & \\
\hline & & & & & $\underset{N R}{M R}$ \\
\hline AMYONIU & PPB & 50.00 & & $19 / 188 / 87$ & \\
\hline \multirow[t]{2}{*}{ BETA } & $\mathrm{PCI} / \mathrm{L}$ & 8.06 & 50.60 & $09 / 08 / 87$ & 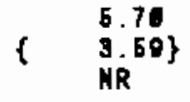 \\
\hline & & & & & NR \\
\hline CHLORID & PPB & 600.66 & & $19 / 08 / 87$ & 3321.60 \\
\hline CONDFLD & UUHO & 1.00 & & $19 / 108 / 87$ & 279.10 \\
\hline$C S-137$ & PCI/L & 28.01 & 200.00 & & NR \\
\hline FBARIUU & PPB & 8.01 & 1000.01 & $09 / 08 / 87$ & 32.60 \\
\hline FCALCIU & PPQ & 60.00 & & $19 / 108 / 87$ & 28600.00 \\
\hline FIRON & PPB & 58.60 & & $69 / 08 / 87$ & \\
\hline FUAGNES & PPB & 0.60 & & $00 / 08 / 87$ & 12600.10 \\
\hline FUANGAM & PPB & 5.01 & & $10 / 06 / 87$ & 43.01 \\
\hline FPOTASS & PPB & 100.00 & & $10 / 08 / 87$ & 3716.00 \\
\hline FSODIUY & PPB & 100.06 & & $09 / 108 / 87$ & 0931.06 \\
\hline FVANADI & PPB & 6.08 & & $60 / 08 / 07$ & 10.00 \\
\hline \multicolumn{6}{|c|}{ 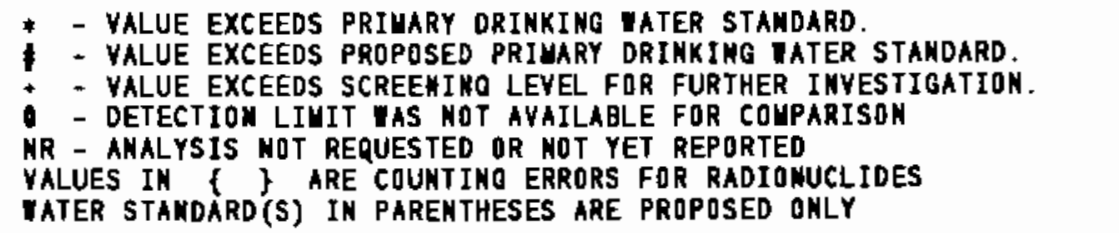 } \\
\hline
\end{tabular}

\begin{tabular}{|c|c|}
\hline $\begin{array}{l}\text { SAMPLE } \\
\text { DATE }\end{array}$ & $8-65-600$ \\
\hline $11 / 26 / 87$ & 116040.00 \\
\hline $10 / 28 / 87$ & 0.76 \\
\hline $\begin{array}{l}11 / 25 / 87 \\
12 / 67 / 07\end{array}$ & $\begin{array}{l}1.75 \\
\{.41\}\end{array}$ \\
\hline $11 / 25 / 87$ & \\
\hline $08 / 28 / 87$ & 5.69 \\
\hline $11 / 25 / 87$ & 0.05 \\
\hline $12 / \bullet 7 / 87$ & $\begin{array}{r}6.26 \\
\{\quad 1.81\}\end{array}$ \\
\hline $11 / 26 / 87$ & 5701.110 \\
\hline $11 / 26 / 87$ & 212.00 \\
\hline $68 / 26 / 87$ & $\begin{array}{r}0.40 \\
\{.06\}\end{array}$ \\
\hline $11 / 25 / 87$ & 13.60 \\
\hline $11 / 25 / 97$ & 36600.06 \\
\hline $11 / 26 / 87$ & \\
\hline $11 / 28 / 87$ & 0376.06 \\
\hline $11 / 25 / 87$ & \\
\hline $11 / 26 / 87$ & 4881.61 \\
\hline $11 / 25 / 87$ & 5639.10 \\
\hline $11 / 25 / 87$ & 12.0 \\
\hline
\end{tabular}

\begin{tabular}{|c|c|}
\hline $\begin{array}{l}\text { SAMPLE } \\
\text { DATE }\end{array}$ & $0-65-76$ \\
\hline $12 / 62 / 87$ & 01704.01 \\
\hline \multicolumn{2}{|l|}{$12 / 12 / 87$} \\
\hline & $\underset{N R}{M R}$ \\
\hline $12 / 12 / 87$ & 94.06 \\
\hline \multirow[t]{2}{*}{$12 / 12 / 87$} & 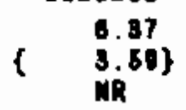 \\
\hline & MR \\
\hline $12 / 62 / 87$ & 22000.06 \\
\hline \multirow[t]{2}{*}{$12 / \$ 2 / 87$} & 270.06 \\
\hline & $\mathrm{MR}$ \\
\hline $12 / 102 / 87$ & 28.06 \\
\hline $12 / 12 / 87$ & 34700.06 \\
\hline $12 / 02 / 87$ & 08.01 \\
\hline $12 / 02 / 87$ & 14506.01 \\
\hline $12 / 02 / 87$ & 47.11 \\
\hline $12 / 62 / 87$ & 4170.00 \\
\hline $12 / 12 / 87$ & 9180.41 \\
\hline $12 / 02 / 87$ & \\
\hline
\end{tabular}

VATER STAMDARD'(S) IN PARENTHESES ARE PROPOSED ONLY 
TABLE C.3. (contd)

\begin{tabular}{|c|c|c|c|c|c|c|c|c|c|}
\hline$\underbrace{\text { CONSTI }}_{\text {NAVE }}$ & $\begin{array}{l}\text { UENT } \\
\text { UNITS }\end{array}$ & $\begin{array}{c}\text { CONTRACTUAL } \\
\text { DETECTION } \\
\text { LIMIT }\end{array}$ & $\begin{array}{l}\text { DRINKING } \\
\text { IATER } \\
\text { STANDARO }\end{array}$ & $\begin{array}{c}\text { SAUPLE } \\
\text { DATE }\end{array}$ & $8-51-75$ & $\begin{array}{c}\text { SAUPLE } \\
\text { DATE }\end{array}$ & $6-55-5 D C$ & $\underset{\text { DAIE }}{\text { SAMPLE }}$ & $8-65-76$ \\
\hline FZIMC & PPB & 5.6 & & $69 / 68 / 87$ & 31.60 & $11 / 25 / 87$ & & $12 / 62 / 87$ & \\
\hline LFLUDRD & PPB & 26.06 & 4806.08 & $00 / 08 / 87$ & 346.16 & $11 / 25 / 87$ & 231.11 & $12 / 02 / 07$ & 271.6 \\
\hline PHFIELD & & 0.10 & & $00 / 06 / 87$ & 7.00 & $11 / 25 / 87$ & 6.91 & $12 / 02 / 87$ & 7.70 \\
\hline SULFATE & PPB & 506.06 & & $68 / 68 / 87$ & 27551.16 & $11 / 25 / 87$ & 16264.06 & $12 / 62 / 87$ & 42016.06 \\
\hline TC & PPB & & & $09 / 08 / 87$ & 27001.010 & $11 / 26 / 07$ & $26444.00 \circ$ & $12 / 02 / 07$ & 22101.10 \\
\hline
\end{tabular}

* - VALUE EXCEEDS PRIYARY DRIMKING IATER STAMDARD.

1 - YALUE EXCEEDS PROPDSED PRIMARY DRIMKING TATER STAMDARD.

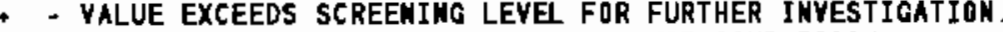

- DETECTION LIYIT IAS ROT AVAILABLE fOR COYPARISON

MR - ANALYSIS MOT RERUESTED OR NOT YET REPORTED

YALUES IN A ARE COUMTING ERRORS FOR RADIONUCLIOES

WATER STANDARO'(S) IN PARENTHESES ARE PROPOSED DNLY 


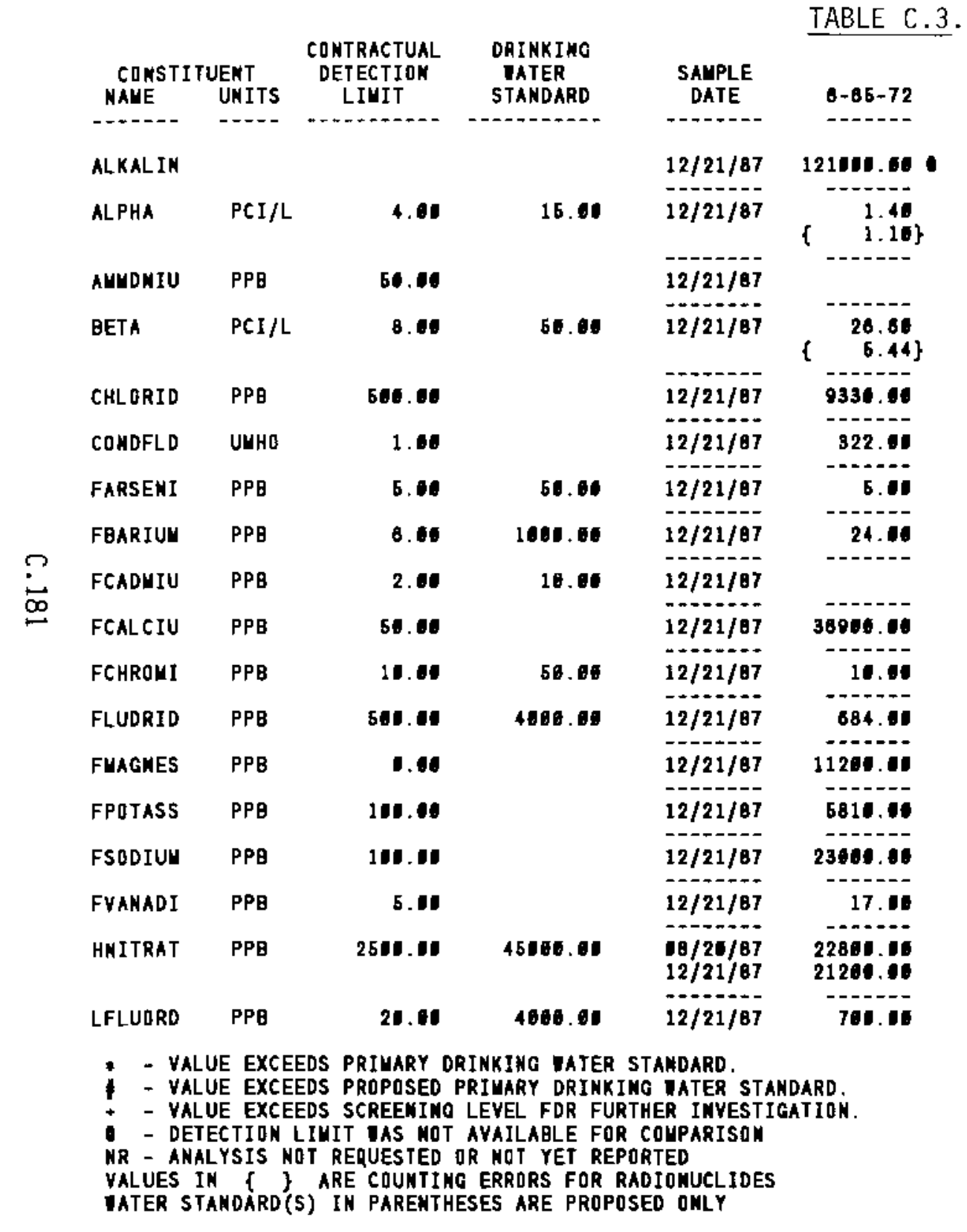

contd)

\begin{tabular}{|c|c|c|c|}
\hline $\begin{array}{l}\text { SALPLE } \\
\text { OATE }\end{array}$ & $8-65-83$ & $\begin{array}{c}\text { SAUPLE } \\
\text { DATE }\end{array}$ & $6-67-86$ \\
\hline $12 / 62 / 87$ & 118108.008 & $12 / 22 / 07$ & 118000.00 \\
\hline $12 / 102 / 87$ & & $12 / 22 / 87$ & \\
\hline $12 / 02 / 107$ & 73.60 & $12 / 22 / 07$ & \\
\hline $12 / 02 / 87$ & $\left\{\begin{array}{l}9.32 \\
\{\quad 4.47\}\end{array}\right.$ & $12 / 22 / 87$ & $\begin{array}{ll} & 4.76 \\
& 3.52\}\end{array}$ \\
\hline $12 / 02 / 107$ & 6320.00 & $12 / 22 / 07$ & 4961.01 \\
\hline $12 / 102 / 87$ & 290.61 & $12 / 22 / 87$ & 286.11 \\
\hline $12 / 02 / 07$ & & $12 / 22 / 87$ & \\
\hline $12 / 02 / 87$ & 9.10 & $12 / 22 / 87$ & 17.11 \\
\hline $12 / 12 / 07$ & & $12 / 22 / 87$ & 2. 14 \\
\hline $12 / 102 / 87$ & 36364.01 & $12 / 22 / 87$ & 38406.00 \\
\hline $12 / 102 / 87$ & 28.114 & $12 / 22 / 87$ & 18.00 \\
\hline $12 / 102 / 87$ & 518.01 & $12 / 22 / 87$ & 516.01 \\
\hline $12 / 02 / 87$ & 18964.01 & $12 / 22 / 07$ & 9900.45 \\
\hline $12 / 62 / 87$ & 8711.16 & $12 / 22 / 87$ & 8496.06 \\
\hline $12 / 62 / 87$ & 13000.64 & $12 / 22 / 87$ & 10006.06 \\
\hline $12 / 62 / 87$ & 30.66 & $12 / 22 / 87$ & 17.00 \\
\hline $\begin{array}{l}08 / 10 / 97 \\
12 / 02 / 87\end{array}$ & 4890.16 & $\begin{array}{l}08 / 19 / 87 \\
12 / 22 / 87\end{array}$ & $\begin{array}{l}2984.44 \\
2736.40\end{array}$ \\
\hline $12 / 02 / 87$ & 420.10 & $12 / 22 / 07$ & 416.0 \\
\hline
\end{tabular}

- VALUE EXCEEDS PRIUARY DRINKING taTER STAkDARD.

- VALUE EXCEEDS PROPASED PRIMARY DRINKIMG TATER STAMDARD.

.

VALUES IN i I ARE COUNTING ERRORS FOR RADIOHUCLIDES

IATER STAMDARD(S) IN PARENTHESES ARE PROPOSED ONLY 
TABLE C.3. (contd)

\begin{tabular}{|c|c|c|c|c|c|c|c|c|c|}
\hline $\begin{array}{l}\text { CDNSTI } \\
\text { NAUE }\end{array}$ & $\begin{array}{l}\text { UENT } \\
\text { UNITS }\end{array}$ & $\begin{array}{l}\text { CQNTRACTUAL } \\
\text { DETECTION } \\
\text { LIUIT }\end{array}$ & $\begin{array}{l}\text { DRINKING } \\
\text { WATER } \\
\text { STANDARD }\end{array}$ & $\begin{array}{c}\text { SAWPLE } \\
\text { DATE }\end{array}$ & $6-65-72$ & $\begin{array}{c}\text { SAHPLE } \\
\text { DATE }\end{array}$ & $6-65-83$ & $\begin{array}{c}\text { SANPLE } \\
\text { DATE }\end{array}$ & $8-67-86$ \\
\hline------ & --- & 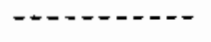 & $---1----$ & ------ & $--n+\infty$ & 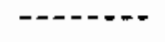 & 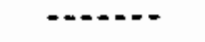 & $-\ldots$ & $-----\infty$ \\
\hline NITRATE & PPB & 580.00 & 45000.00 & $12 / 21 / 87$ & 22260.00 & $12 / 02 / 87$ & 4700.66 & $12 / 22 / 87$ & 2810.01 \\
\hline PH-LAB & & 0.01 & & $12 / 21 / 87$ & 7.91 & $12 / 02 / 87$ & 7.94 & $12 / 22 / 87$ & 7.00 \\
\hline PHFIELD & & $\$ .10$ & & $12 / 21 / 87$ & 7.38 & $12 / 12 / 87$ & 7.60 & $12 / 22 / 87$ & 6.81 \\
\hline SULFATE & PPB & 560.00 & & $12 / 21 / 87$ & 46100.66 & $12 / 02 / 07$ & 40904.0 & $12 / 22 / 87$ & soces \\
\hline TC & PPB & & & $12 / 21 / 87$ & 26966.010 & $12 / 62 / 87$ & 28704.00 & $12 / 22 / 87$ & 26501.01 \\
\hline TRITIUU & $\mathrm{PCI} / \mathrm{L}$ & 6อง. & 20006.65 & $\begin{array}{l}68 / 26 / 87 \\
12 / 21 / 87\end{array}$ & $\begin{array}{c}3354.00 \\
201.06\} \\
3046.06 \\
276.04\}\end{array}$ & $\begin{array}{l}18 / 10 / 87 \\
12 / 12 / 67\end{array}$ & $\begin{array}{r}132.04 \\
237.06\} \\
886.04 \\
228.06\}\end{array}$ & $\begin{array}{l}08 / 18 / 87 \\
12 / 22 / 87\end{array}$ & $\begin{array}{l}\quad 082.00 \\
226.06\} \\
051.04 \\
\{\quad 218.06\}\end{array}$ \\
\hline
\end{tabular}

$\stackrel{?}{\stackrel{\mathscr{D}}{\mathrm{d}}}$

* - value exceeds prinary drinking vater standard.

- VALUE EXCEEDS PROPOSED PRIUARY DRINKIHG TATER STAMDARD.

- - YALUE EXCEEDS SCREENING LEVEl FOR FURTHER INYESTIGATIOH.

- DETECTION LIUIT IAS NOT AVAILABLE FOR COUPARISON

NR - AMALYSIS NOT REQUESTED OR NOT YET REPORTED

VALUES IN \{\} ARE COUKTING ERRORS FOR RADIONUCLIDES

WATER STANDARD(S) IN PARENTHESES ARE PROPOSED ONLY 
TABLE C.3. (contd)

\begin{tabular}{|c|c|c|c|c|c|}
\hline $\begin{array}{c}\text { CONSTI } \\
\text { WANE }\end{array}$ & $\begin{array}{l}\text { UENT } \\
\text { UNI IS }\end{array}$ & $\begin{array}{c}\text { CONTRACTUAL } \\
\text { DETECTIINN } \\
\text { LIUIT }\end{array}$ & $\begin{array}{l}\text { DRINKING } \\
\text { TATER } \\
\text { STANDARD }\end{array}$ & $\begin{array}{c}\text { SAUPLE } \\
\text { DATE }\end{array}$ & $8-71-68$ \\
\hline ALKALIN & & & & $12 / 21 / 87$ & 118056.180 \\
\hline ALPHA & $P C I / L$ & 4.10 & 15.86 & $12 / 21 / 87$ & \\
\hline ALUONIU & PPB & 50.60 & & $12 / 21 / 87$ & 57.16 \\
\hline BETA & $\mathrm{PCI} / \mathrm{L}$ & 8.66 & 50.60 & $12 / 21 / 07$ & $\left\{\begin{array}{c}13.30 \\
4.18\}\end{array}\right.$ \\
\hline CHLORIO & PPB & 56e.18 & & $12 / 21 / \theta 7$ & 5820.40 \\
\hline CONDFLD & UWHO & 1.10 & & $12 / 21 / \theta 7$ & 238.10 \\
\hline FARSENI & PPB & 6.00 & 50.01 & $12 / 21 / 87$ & \\
\hline FEARIUU & PPB & 8.00 & 1000.08 & $12 / 21 / 87$ & 17.10 \\
\hline FCALCIU & PPB & 60.00 & & $12 / 21 / 87$ & 31200.65 \\
\hline FCHROUI & PPB & 16.00 & 51.00 & $12 / 21 / 87$ & \\
\hline FLUDRID & PPB & 586.06 & 4000.00 & $12 / 21 / 87$ & \\
\hline FUAGNES & PPB & 0.60 & & $12 / 21 / 87$ & 8810.10 \\
\hline FPOTASS & PPB & 188.68 & & $12 / 21 / 87$ & 4491.10 \\
\hline FSODIUU & PPB & 108.66 & & $12 / 21 / 07$ & 12765.60 \\
\hline FSTRONT & PPB & 980.06 & & $12 / 21 / 87$ & \\
\hline FVANADI & PPB & 5.01 & & $12 / 21 / 87$ & 15.00 \\
\hline HNITRAT & PPB & 2508.80 & 45060.06 & $\begin{array}{l}18 / 28 / 97 \\
12 / 21 / 87\end{array}$ & $\begin{array}{l}3454.06 \\
3216.00\end{array}$ \\
\hline LFLUDRO & PPB & 20.60 & 4660.06 & $12 / 21 / 87$ & 384.64 \\
\hline \multicolumn{6}{|c|}{ 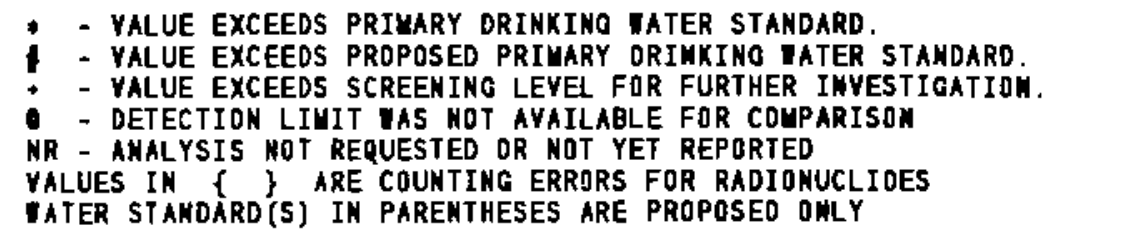 } \\
\hline
\end{tabular}

\begin{tabular}{|c|c|c|c|}
\hline $\begin{array}{l}\text { SAMPLE } \\
\text { DATE }\end{array}$ & $6-71-36$ & $\begin{array}{l}\text { SAUPLE } \\
\text { DATE }\end{array}$ & $8-71-52$ \\
\hline $12 / 13 / 87$ & 260000.010 & $12 / 13 / 87$ & 116406.00 \\
\hline $12 / 13 / 87$ & $\begin{array}{l}7.03 \\
\{\quad 2.81\}\end{array}$ & $12 / 13 / 07$ & $\left\{\begin{array}{l}1.86 \\
\{\quad 1.23\end{array}\right\}$ \\
\hline $12 / 13 / 87$ & & $12 / 18 / 07$ & \\
\hline $12 / 13 / 87$ & $\left.\begin{array}{l}7.13 \\
\{\quad 3.80\end{array}\right\}$ & $12 / 13 / 07$ & $\begin{array}{r}\quad 5.01 \\
\{\quad 3.52\}\end{array}$ \\
\hline $12 / 13 / 87$ & 21600.10 & $12 / 13 / 07$ & $\$ 114.10$ \\
\hline $12 / 13 / 87$ & 534.110 & $12 / 13 / 07$ & 276.16 \\
\hline $12 / 13 / 87$ & 6.11 & $12 / 18 / 07$ & \\
\hline $12 / 19 / 87$ & 47.01 & $12 / 13 / 97$ & 30.61 \\
\hline $12 / 13 / 87$ & sesse.0s & $12 / 13 / 07$ & 37716.10 \\
\hline $12 / 13 / 87$ & & $12 / 13 / 07$ & 18.60 \\
\hline $12 / 13 / 87$ & 810.00 & $12 / 13 / 07$ & \\
\hline $12 / 13 / 07$ & 18100.00 & $12 / 13 / 87$ & 11610.00 \\
\hline $12 / 13 / 67$ & 6190.00 & $12 / 13 / 87$ & 6310.0 \\
\hline $12 / 13 / 87$ & 43266.00 & $12 / 13 / 87$ & 18900.01 \\
\hline $12 / 13 / 87$ & 646.00 & $12 / 13 / 07$ & \\
\hline $12 / 13 / 87$ & 6.16 & $12 / 13 / 87$ & 17.01 \\
\hline $\begin{array}{l}68 / 25 / 87 \\
12 / 19 / 87\end{array}$ & $\begin{array}{l}25606.06 \\
28615.010\end{array}$ & $\begin{array}{l}18 / 23 / 87 \\
12 / 19 / 87\end{array}$ & $\begin{array}{l}8084.14 \\
8034.15\end{array}$ \\
\hline $12 / 13 / 87$ & 476.06 & $12 / 13 / 87$ & 376.06 \\
\hline
\end{tabular}


TABLE C.3. (contd)

\begin{tabular}{|c|c|c|c|c|c|c|c|c|c|}
\hline $\begin{array}{l}\text { CONSTI } \\
\text { NAME }\end{array}$ & $\begin{array}{l}\text { UENT } \\
\text { UNITS }\end{array}$ & $\begin{array}{l}\text { CONTRACTUAL } \\
\text { DETECTION } \\
\text { LINIT }\end{array}$ & $\begin{array}{l}\text { DR INKING } \\
\text { TATER } \\
\text { STANDARD }\end{array}$ & $\begin{array}{l}\text { SAHPLE } \\
\text { OATE }\end{array}$ & $8-70-68$ & $\begin{array}{l}\text { SAUPLE } \\
\text { DATE }\end{array}$ & $8-71-34$ & $\begin{array}{l}\text { SAHPLE } \\
\text { DATE }\end{array}$ & $6-71-62$ \\
\hline----- & ---- & - - - & $-1-\log$ & ------- & $----n$ & 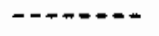 & $--1-2$ & -...-- & - - \\
\hline NITRATE & PPB & 580.00 & 46000.00 & $12 / 21 / 87$ & 3271.66 & $12 / 13 / 87$ & 292106.00 & $12 / 13 / 87$ & 7141.01 \\
\hline PH-LAB & & 0.01 & & $12 / 21 / 87$ & 8.17 & $12 / 13 / 07$ & 7.86 & $12 / 13 / 07$ & 6.11 \\
\hline PHFIELD & & 0.1 & & $12 / 21 / 87$ & 7.64 & $12 / 13 / 87$ & 7.31 & $12 / 13 / 87$ & 7.10 \\
\hline SULFATE & PPG & & & $12 / 21 / 87$ & 16000.10 & $12 / 13 / 67$ & se600.50 & $12 / 13 / 87$ & 60800.40 \\
\hline TC & PPQ & & & $12 / 21 / 87$ & 26365.01 & $12 / 13 / 87$ & 46360.00 & $12 / 13 / 87$ & 27500.00 \\
\hline TRITIUN & $\mathrm{PCI} / \mathrm{L}$ & 586.60 & 20466.00 & $\begin{array}{l}08 / 26 / 87 \\
12 / 21 / 87\end{array}$ & $\begin{array}{r}1784.06 \\
258.04\} \\
1704.04 \\
246.06\}\end{array}$ & $\begin{array}{l}08 / 26 / 87 \\
12 / 13 / 87\end{array}$ & & $\begin{array}{l}10 / 23 / 87 \\
12 / 18 / 87\end{array}$ & $\left\{\begin{array}{r}1120.06 \\
281.00\} \\
1095.00 \\
226.04\}\end{array}\right.$ \\
\hline
\end{tabular}

$\stackrel{?}{\stackrel{2}{p}}$

- - value exceEds priuary drinking inter standaro.

- VALUE EXCEEDS PROPOSED PRIUARY DRINKING IATER STANDARD

- YALUE EXCEEDS SCREENING LEVEL FOR FURTHER INYESTIGATION.

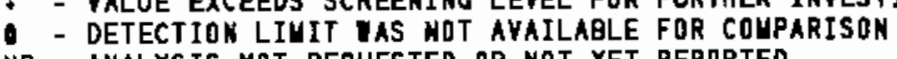

NR - ANALYSIS MOT REQUESTED OR NOT YET REPORTEO

VALUES IN $\{$ \} ARE COUKTING ERRORS FOR RADIONUCLIDES

IATER STANDARD(S) IN PARENTHESES ARE PROPOSED ONLY 
TABLE C. 3. (contd)

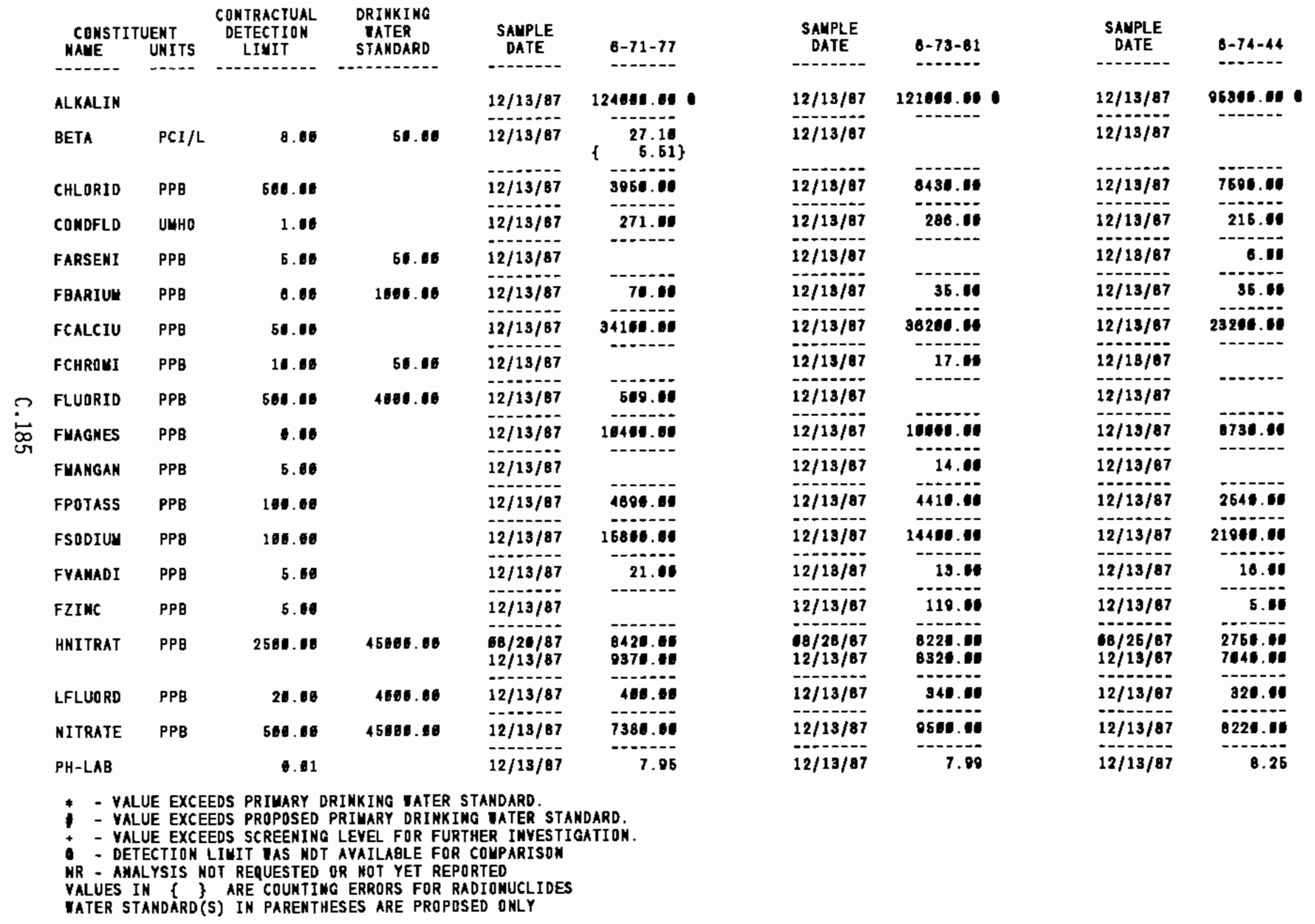


TABLE C.3. (contd)

\begin{tabular}{|c|c|c|c|c|c|c|c|c|c|}
\hline $\begin{array}{c}\text { CONSTI } \\
\text { NANE }\end{array}$ & $\begin{array}{l}\text { UENT } \\
\text { UNITS }\end{array}$ & $\begin{array}{c}\text { CONTRACTUAL } \\
\text { DETECTIOAN } \\
\text { LIUIT }\end{array}$ & $\begin{array}{l}\text { DRINKING } \\
\text { IATER } \\
\text { STAHDARD }\end{array}$ & $\begin{array}{c}\text { SAUPLE } \\
\text { DATE }\end{array}$ & $8-71-77$ & $\begin{array}{c}\text { SAMPLE } \\
\text { DATE }\end{array}$ & $0-73-61$ & $\begin{array}{l}\text { SAMPLEE } \\
\text { DATEE }\end{array}$ & $0-74-44$ \\
\hline PHF IELD & & 6.16 & & $12 / 13 / 87$ & 6.95 & $12 / 18 / 87$ & 7.20 & $12 / 13 / 87$ & 7.41 \\
\hline SULFATE & $\mathrm{PPB}$ & 500.00 & & $12 / 13 / 87$ & 24300.100 & $12 / 13 / 87$ & 40506.06 & $12 / 13 / 67$ & 38554. 10 \\
\hline TC & PPB & & & $12 / 19 / 87$ & 20466.01 & $12 / 13 / 87$ & 27100.00 & $12 / 19 / 87$ & 21800.00 \\
\hline TRITIUM & $\mathrm{PCI} / \mathrm{L}$ & 580.60 & 25000.06 & $\begin{array}{l}68 / 20 / 87 \\
12 / 13 / 67\end{array}$ & $\left\{\begin{array}{r}3181.00 \\
288.01 \\
3840.16 \\
285.16\end{array}\right\}$ & $\begin{array}{l}18 / 26 / 87 \\
12 / 13 / 87\end{array}$ & & $\begin{array}{l}08 / 26 / 87 \\
12 / 19 / 87\end{array}$ & \\
\hline
\end{tabular}

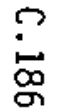

- - value exceeds priuary drinking tater standard.

1 - VALUE EXCEEDS PROPOSED PRIUARY DRINKINO TATER STANDARO

- VALUE EXCEEDS SCREEKING LEVEL FOR FURTHER INVESTIGATION.

- DETECTION LIUIT tas nOT AVAILABLE For COMPARISON

NR - ANALYSIS NOT REQUESTED OR NOT YET REPORTED

YALUES IN \{ $\}$ ARE COUNTING ERRORS FOR RADIONUCLIDES

IATER STAMDARO(S) IN PARENTHESES ARE PROPOSED ONLY 
TABLE C.3. (contd)

\begin{tabular}{|c|c|c|c|c|c|}
\hline$\underset{\text { NAHE }}{\text { CONSTI }}$ & $\begin{array}{l}\text { TUENT } \\
\text { UNI TS }\end{array}$ & $\begin{array}{c}\text { CONTRACIUAL } \\
\text { DETECTION } \\
\text { LIMIT }\end{array}$ & $\begin{array}{l}\text { DRINKING } \\
\text { VATER } \\
\text { STANDARD }\end{array}$ & $\begin{array}{c}\text { SAMPLE } \\
\text { DATE }\end{array}$ & $6-77-38$ \\
\hline ALKALIN & & & & $12 / 13 / 07$ & 228000.00 \\
\hline ALPHA & $\mathrm{PCI} / \mathrm{L}$ & 4.00 & 15.00 & $12 / 13 / 87$ & 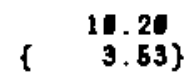 \\
\hline AHUONIU & PPB & 50.01 & & $12 / 13 / 87$ & \\
\hline BETA & $\mathrm{PCI} / \mathrm{L}$ & B. 10 & 50.60 & $12 / 13 / 87$ & $\begin{array}{l}\quad 8.58 \\
\{\quad 4.13\}\end{array}$ \\
\hline CHLORIO & PPB & 580.01 & & $12 / 13 / 87$ & 224040 \\
\hline CONDFLO & UNHO & 1.00 & & & NR \\
\hline FARSENI & PPB & 6.00 & 50.06 & $12 / 13 / 87$ & 6.6 \\
\hline FBARIUU & PPB & 8.98 & 1800.06 & $12 / 13 / 67$ & 55.65 \\
\hline FCALCIU & PPB & 50.01 & & $12 / 13 / 87$ & 65000.06 \\
\hline FCHROHI & PPB & 10.60 & 56.06 & $12 / 13 / 87$ & \\
\hline FIRON & PPB & 50.01 & & $12 / 13 / 87$ & \\
\hline FLUORIO & PPB & 500.00 & 4060.80 & $12 / 13 / 87$ & 933.66 \\
\hline FHAGNES & PPB & 0.00 & & $12 / 13 / 87$ & 18000.00 \\
\hline FWANOAM & PPB & 5.68 & & $12 / 13 / 87$ & \\
\hline FPOTASS & PPB & 100.00 & & $12 / 13 / 87$ & 5130.06 \\
\hline FSODIUV & PPB & 100.06 & & $12 / 13 / 87$ & 04106.00 \\
\hline FSTRONT & $\mathrm{PPB}$ & 306.60 & & $12 / 13 / 87$ & 633.00 \\
\hline FYAMADI & PPB & 5.60 & & $12 / 13 / 87$ & 18.00 \\
\hline HNITRAT & PPB & 2580.60 & 45000.00 & $08 / 25 / 87$ & 61106.08 \\
\hline
\end{tabular}

\begin{tabular}{|c|c|}
\hline $\begin{array}{l}\text { SAMPLE } \\
\text { DATE }\end{array}$ & $8-77-54$ \\
\hline $09 / 15 / 87$ & 117600.66 \\
\hline $00 / 15 / 87$ & $\begin{array}{ll} & 1.28 \\
\{\quad 1.02\}\end{array}$ \\
\hline $60 / 15 / 87$ & \\
\hline $69 / 15 / 87$ & $\left.\begin{array}{ll} & 7.18 \\
\{\quad 3.86\end{array}\right\}$ \\
\hline $69 / 15 / 87$ & 5030.110 \\
\hline $00 / 16 / 87$ & 918.10 \\
\hline $10 / 16 / 87$ & \\
\hline $60 / 16 / 87$ & 25.6 \\
\hline $00 / 15 / 07$ & 32500.66 \\
\hline $09 / 15 / 87$ & \\
\hline $09 / 15 / 07$ & \\
\hline $09 / 15 / 87$ & \\
\hline $09 / 15 / 87$ & 10400.00 \\
\hline $09 / 15 / 87$ & \\
\hline $08 / 15 / 87$ & 4520.00 \\
\hline $19 / 16 / 87$ & 15106.14 \\
\hline $10 / 15 / 87$ & \\
\hline $10 / 15 / 87$ & 21.16 \\
\hline & NR \\
\hline
\end{tabular}

\begin{tabular}{|c|c|}
\hline $\begin{array}{l}\text { SAYPLE } \\
\text { DATE }\end{array}$ & $8-03-47$ \\
\hline $10 / 00 / 07$ & 11094s.00 \\
\hline $10 / 00 / 07$ & $\begin{array}{r}8.94 \\
(\quad 1.87\}\end{array}$ \\
\hline $09 / 00 / 87$ & 66.06 \\
\hline $10 / 00 / 07$ & $\left\{\begin{array}{r}11.10 \\
4.34\end{array}\right.$ \\
\hline $10 / 08 / 87$ & 6534.01 \\
\hline $10 / 08 / 87$ & 280.01 \\
\hline $10 / 108 / 87$ & \\
\hline $10 / 68 / 87$ & 39.10 \\
\hline $10 / 68 / 87$ & sosed. 06 \\
\hline $00 / 08 / 87$ & 44.60 \\
\hline $00 / 00 / 87$ & 398.01 \\
\hline $00 / 00 / 07$ & \\
\hline $09 / 08 / 87$ & 11300.06 \\
\hline $09 / 08 / 87$ & 8.16 \\
\hline $10 / 08 / 87$ & 3020.10 \\
\hline $10 / 68 / 87$ & 20aces wo \\
\hline $00 / 18 / 97$ & \\
\hline $00 / 08 / 87$ & 10.06 \\
\hline & NR \\
\hline
\end{tabular}

* - value exceeds primary drinkino tater standard.

1 - VALUE EXCEEDS PROPOSED PRIUARY DRIMKING TATER STANDARD.

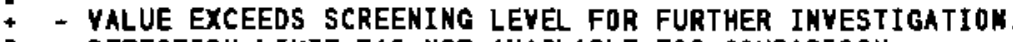

- - detection linit ias not available for couparison

MR - ANALYSIS NDT REQUESTED OR NOT YET REPORTED

VALUES IN \& \& ARE COUNTING ERRORS FOR RADIOHUCLIDES

IATER STAMDARD(S) IN PARENTHESES ARE PROPOSED OHLY 
TABLE C.3. (contd)

\begin{tabular}{|c|c|}
\hline $\begin{array}{c}\text { CONST } \\
\text { NAUE }\end{array}$ & $\begin{array}{l}\text { UENT } \\
\text { UNITS }\end{array}$ \\
\hline HNITRAT & PPB \\
\hline LFLUORD & $P P B$ \\
\hline NI JRATE & PPB \\
\hline PH-LAB & \\
\hline PHFIELD & \\
\hline SULFATE & PPB \\
\hline TC & PPB \\
\hline TOC & PPB \\
\hline JRICENE & PPB \\
\hline TRITIUY & $\mathrm{PCI} / \mathrm{L}$ \\
\hline
\end{tabular}

\begin{tabular}{|c|c|c|c|}
\hline $\begin{array}{c}\text { CDMTRACTUAL } \\
\text { DETECTION } \\
\text { LIUIT }\end{array}$ & $\begin{array}{l}\text { DRINKING } \\
\text { TATER } \\
\text { STANDARD }\end{array}$ & $\begin{array}{l}\text { SAUPLE } \\
\text { DATEE }\end{array}$ & $6-77-36$ \\
\hline 2586.00 & 45600.00 & $12 / 13 / 87$ & 48981.64 \\
\hline 28.60 & 4006.66 & $12 / 13 / 87$ & 690.11 \\
\hline 506.00 & 45000.00 & $12 / 13 / 87$ & 70100.60 \\
\hline 0.1 & & $12 / 13 / 87$ & 7.84 \\
\hline$\$ .10$ & & & NR \\
\hline \multirow[t]{2}{*}{586.60} & & $12 / 13 / 87$ & 58200.110 \\
\hline & & $12 / 19 / 87$ & 56100.90 \\
\hline 1000.00 & & $12 / 13 / 87$ & 1280.10 \\
\hline 10.40 & 5.10 & $12 / 13 / 87$ & 34.01 \\
\hline \multirow[t]{2}{*}{568.10} & 26064.06 & $08 / 25 / 87$ & \\
\hline & & $12 / 13 / 87$ & \\
\hline
\end{tabular}

\begin{tabular}{|c|c|}
\hline \multirow[t]{2}{*}{$\begin{array}{c}\text { SAUPLE } \\
\text { DATE }\end{array}$} & $0-77-54$ \\
\hline & NR \\
\hline $00 / 15 / 87$ & 365.86 \\
\hline $19 / 15 / 87$ & 7040.06 \\
\hline $00 / 16 / 87$ & 7.77 \\
\hline $10 / 15 / 87$ & 7.69 \\
\hline $60 / 16 / 87$ & 41740.16 \\
\hline $10 / 15 / 87$ & 28710.00 \\
\hline $19 / 15 / 87$ & \\
\hline $60 / 16 / 87$ & \\
\hline $10 / 69 / 87$ & \\
\hline $12 / 13 / 87$ & \\
\hline
\end{tabular}

\begin{tabular}{|c|c|}
\hline $\begin{array}{l}\text { SAMPLE } \\
\text { DATE }\end{array}$ & $8-83-47$ \\
\hline & WR \\
\hline $19 / 00 / 87$ & 480.00 \\
\hline $19 / 09 / 07$ & 5826.16 \\
\hline $18 / 68 / 87$ & 7.00 \\
\hline $10 / 108 / 87$ & 6.11 \\
\hline $60 / 108 / 87$ & 60305.06 \\
\hline $10 / 08 / 87$ & 27850.01 \\
\hline $00 / 08 / 07$ & \\
\hline $69 / 08 / 97$ & \\
\hline $10 / 108 / 87$ & $\left\{\begin{array}{l}710.01 \\
222.00 \\
\text { MR }\end{array}\right\}$ \\
\hline
\end{tabular}

* - value exceEos primary drinkimg iater standard.

1 - VALUE EXCEEDS PROPOSED PRIUARY DRINKING VATER STANDARD.

* - YALUE EXCEEDS SCREENING LEVEL For fuRTHER INYESTIGATION.

- DETECTION LiUIT IAS hOT AVAILABLE fOR COUPARISOM

NR - ANALYSIS NOT REQUESTED OR NDT YET REPBRTED

VALUES IN \{ $\}$ ARE COUNTING ERRORS FDR RADIDNUCLIDES

IATER STANDARD(S) IN PARENTHESES ARE PROPOSED DMLY 
IABLE C.3. (contd)

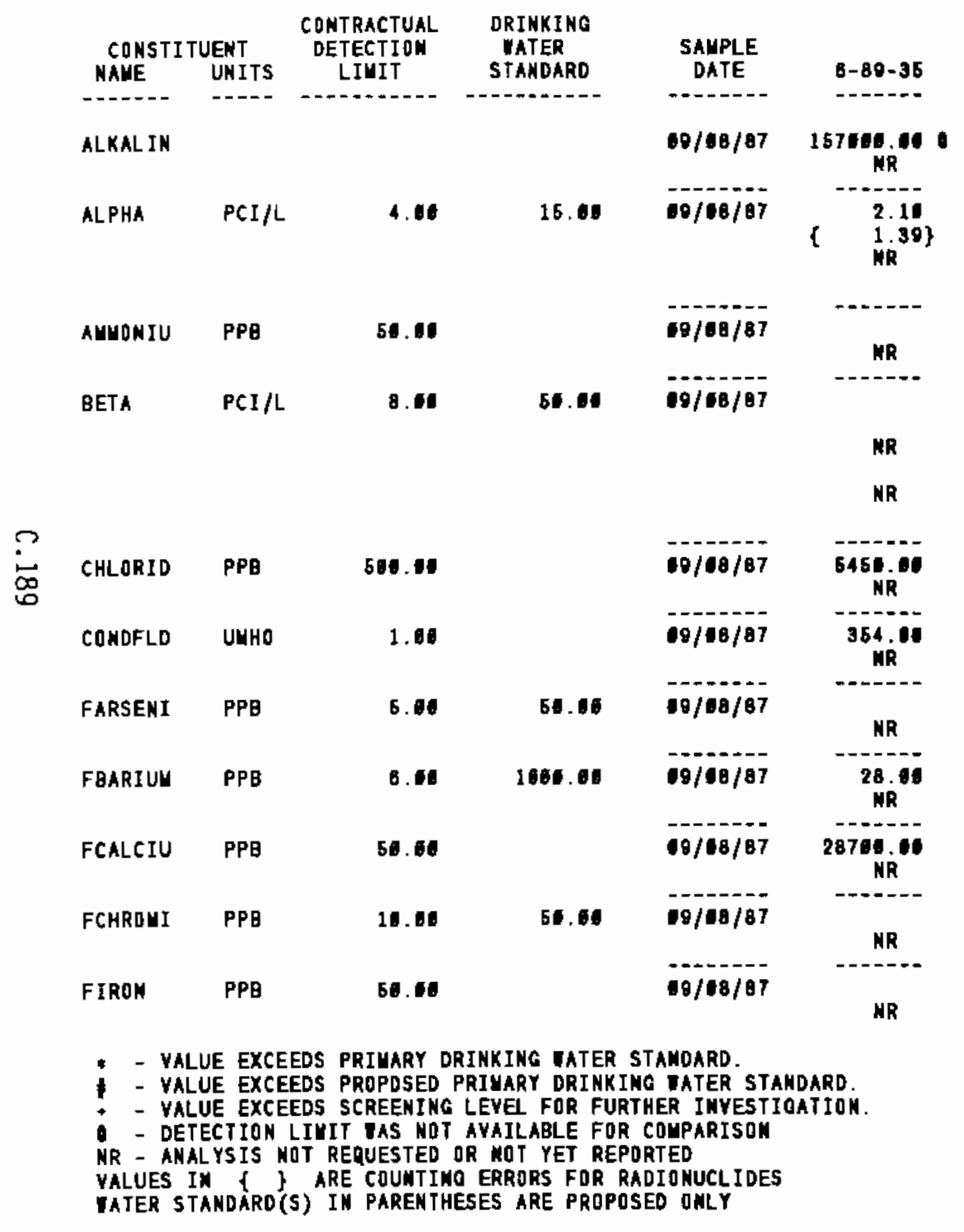

\begin{tabular}{|c|c|}
\hline $\begin{array}{l}\text { SALPLE } \\
\text { DATE }\end{array}$ & $6-90-45$ \\
\hline ---n-- & ------ \\
\hline $\begin{array}{l}19 / 08 / 87 \\
12 / 13 / 87\end{array}$ & $\begin{array}{l}122000.00 \\
122006\end{array}$ \\
\hline $99 / 108 / 97$ & 1.23 \\
\hline $12 / 13 / 87$ & 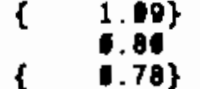 \\
\hline $\begin{array}{l}00 / 08 / 87 \\
12 / 13 / 87\end{array}$ & 103.40 \\
\hline $18 / 40 / 07$ & 6.67 \\
\hline $12 / 13 / 87$ & $\begin{array}{l}3.68\} \\
4.07 \\
3.29\} \\
\mathrm{HR}\end{array}$ \\
\hline - & - \\
\hline $\begin{array}{l}0 / 18 / 87 \\
12 / 13 / 87\end{array}$ & $\begin{array}{l}4890.06 \\
4806.06\end{array}$ \\
\hline $\begin{array}{l}10 / 18 / 87 \\
12 / 13 / 87\end{array}$ & 235.00 \\
\hline $\begin{array}{l}19 / 08 / 87 \\
12 / 13 / 87\end{array}$ & 5.6 \\
\hline $\begin{array}{l}10 / 10 / 87 \\
12 / 19 / 87\end{array}$ & 20.00 \\
\hline $\begin{array}{l}10 / 08 / 87 \\
12 / 13 / 87\end{array}$ & $\begin{array}{l}26800.40 \\
32501.10\end{array}$ \\
\hline $\begin{array}{l}10 / 08 / 87 \\
12 / 13 / 87\end{array}$ & 10.00 \\
\hline $\begin{array}{l}90 / 68 / 87 \\
12 / 13 / 87\end{array}$ & $\begin{array}{r}706.09 \\
1154.00\end{array}$ \\
\hline
\end{tabular}

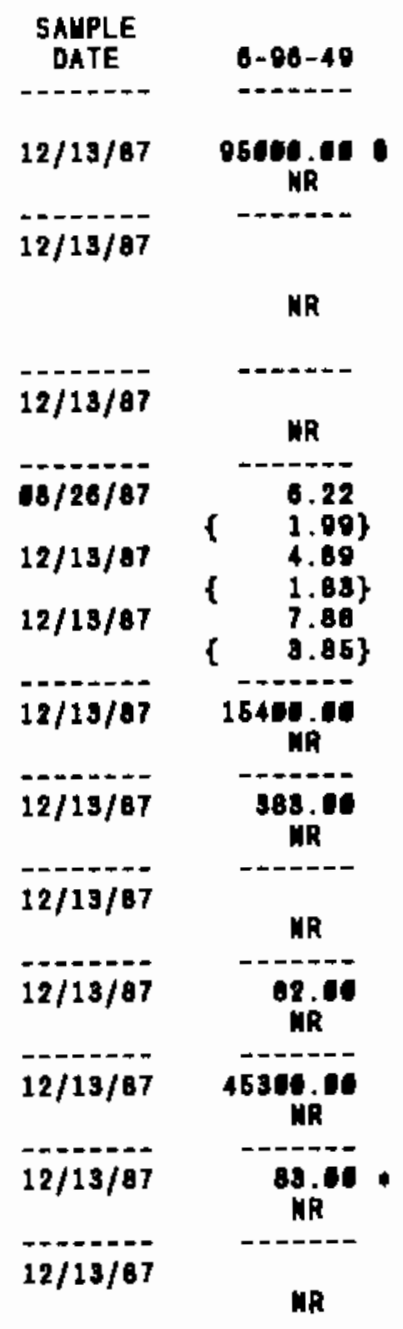


TABLE C.3. (contd)

\begin{tabular}{|c|c|c|c|c|c|}
\hline \multicolumn{2}{|c|}{$\begin{array}{l}\text { CONSTI TUENT } \\
\text { NAUE UNITS }\end{array}$} & $\begin{array}{c}\text { CONTRACTUAL } \\
\text { DETECTION } \\
\text { LIVIT }\end{array}$ & $\begin{array}{l}\text { DRINK ING } \\
\text { TATER } \\
\text { STANDARD }\end{array}$ & $\begin{array}{l}\text { SALPLE } \\
\text { DATE }\end{array}$ & $0-89-35$ \\
\hline$-\cdots-$ & $-\ldots$ & $---1-1-1-1$ & 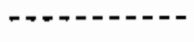 & 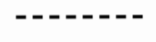 & $\ldots$ \\
\hline FLUORID & PPB & 500.06 & 4960.10 & $19 / 68 / 87$ & NR \\
\hline FWAONES & PPB & 0.00 & & $19 / 08 / 87$ & 11600.06 \\
\hline FWANGAK & PPB & 5.00 & & $10 / 08 / 87$ & NR \\
\hline FPOTASS & PPB & 180.06 & & $10 / 00 / 07$ & $\begin{array}{c}4356.00 \\
\mathrm{NR}\end{array}$ \\
\hline FSODIUU & PPB & 100.5 & & $99 / 18 / 87$ & $\begin{array}{c}\text { 30004 .06 } \\
\text { NR }\end{array}$ \\
\hline FYANADI & PPB & Б.110 & & $10 / 08 / 87$ & 19.6 \\
\hline FZINC & PPB & 6.10 & & $50 / 08 / 07$ & 5. 10 \\
\hline HNI TRAT & PPB & 2506.100 & 45006.65 & & $\begin{array}{l}\text { NR } \\
\text { NR }\end{array}$ \\
\hline LFLUORD & PPB & 20.00 & 4606.10 & $19 / 50 / 87$ & $\underset{\mathrm{NR}}{660.96}$ \\
\hline NITRATE & PPB & 560.66 & 45000.00 & $19 / 06 / 87$ & 941日..t. \\
\hline PH-LAB & & 1.01 & & $19 / 08 / 87$ & $\begin{array}{l}7.83 \\
\text { NR }\end{array}$ \\
\hline PHF IELD & & 0.16 & & $00 / 08 / 87$ & 7.20 \\
\hline SULFATE & PPB & 580.06 & & $00 / 08 / 87$ & 30500.01 \\
\hline
\end{tabular}

\begin{tabular}{|c|c|}
\hline $\begin{array}{l}\text { SAYPLE } \\
\text { DATE }\end{array}$ & $8-90-46$ \\
\hline $\begin{array}{l}09 / 18 / 87 \\
12 / 13 / 87\end{array}$ & 589.10 \\
\hline $\begin{array}{l}10 / 08 / 87 \\
12 / 13 / 87\end{array}$ & $\begin{array}{l}11460.06 \\
11000.06\end{array}$ \\
\hline $\begin{array}{l}19 / 188 / 87 \\
12 / 13 / 87\end{array}$ & $\begin{array}{l}61.11 \\
84.10\end{array}$ \\
\hline $\begin{array}{l}60 / 08 / 87 \\
12 / 19 / 87\end{array}$ & $\begin{array}{l}3900.10 \\
3770.0\end{array}$ \\
\hline $\begin{array}{l}19 / 18 / 87 \\
12 / 13 / 87\end{array}$ & $\begin{array}{l}17006.04 \\
16005.00\end{array}$ \\
\hline $\begin{array}{l}18 / 08 / 87 \\
12 / 13 / 87\end{array}$ & $\begin{array}{l}25.64 \\
13.60\end{array}$ \\
\hline $\begin{array}{l}19 / 18 / 87 \\
12 / 13 / 87\end{array}$ & $\begin{array}{l}57.10 \\
98.11\end{array}$ \\
\hline & $\begin{array}{l}\text { XR } \\
\text { NR }\end{array}$ \\
\hline $\begin{array}{l}19 / 08 / 87 \\
12 / 13 / 87\end{array}$ & $\begin{array}{l}535.16 \\
596.16\end{array}$ \\
\hline $\begin{array}{l}09 / 08 / 87 \\
12 / 13 / 87\end{array}$ & $\begin{array}{l}6011.00 \\
7098.40\end{array}$ \\
\hline $\begin{array}{l}10 / 08 / 87 \\
12 / 13 / 87\end{array}$ & $\begin{array}{l}7.79 \\
7.84\end{array}$ \\
\hline $\begin{array}{l}08 / 68 / 87 \\
12 / 13 / 87\end{array}$ & $\begin{array}{l}5.54 \\
0.44\end{array}$ \\
\hline $\begin{array}{l}09 / 18 / 88 \\
12 / 13 / 87\end{array}$ & $\begin{array}{l}33369.10 \\
32766.10\end{array}$ \\
\hline
\end{tabular}

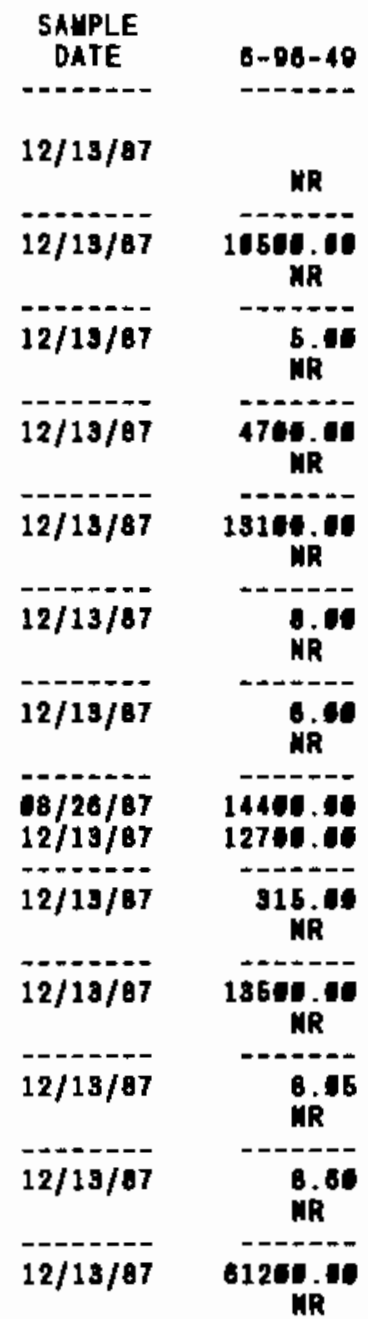

- - YALUE EXCEEDS PRIMARY DRINKINO MATER STANDARD.

- - VALUE EXCEEDS PROPOSED PRIMARY DRINKING TATER STANDARD.

- - VALUE EXCEEDS SCREENING LEYEL FOR FURTHER INYESTIGATION.

- DETECTION LIVIT IAS NOT AYAILABLE FoR COYPARISON

NR - AHALYSIS MDT RERUESTED OR NOT YET REPORTED

VALUES IN $f$ ARE COUNTING ERRORS FOR RADIONUCLIDES

IATER STANDARD(S) in PARENTHESES ARE PROPBSED ONLY 


\begin{tabular}{|c|c|c|c|c|c|c|c|c|c|}
\hline $\begin{array}{l}\text { CONSII } \\
\text { MANE }\end{array}$ & $\begin{array}{l}\text { TUENT } \\
\text { UN I IS }\end{array}$ & $\begin{array}{l}\text { CQNTRACTUAL } \\
\text { DETECT ION } \\
\text { LINTT }\end{array}$ & $\begin{array}{l}\text { DRINKIMG } \\
\text { WATER } \\
\text { STAMDARD }\end{array}$ & $\begin{array}{l}\text { SAUPLE } \\
\text { DATE }\end{array}$ & $8-89-36$ & $\begin{array}{c}\text { SAHPLE } \\
\text { OATE }\end{array}$ & $0-90-45$ & $\begin{array}{l}\text { SAMPLE } \\
\text { DATE }\end{array}$ & $8-96-40$ \\
\hline$-\ldots . . .-$ & $-\cdots$ & 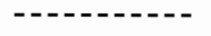 & 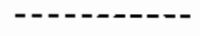 & $---1-4$ & $--\infty-\infty$ & - & $-\ldots-0$ & $---1--0$ & - - \\
\hline TC & PPE & & & $00 / 08 / 87$ & 37400 NR & $\begin{array}{l}10 / 08 / 87 \\
12 / 13 / 87\end{array}$ & 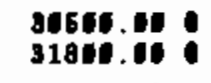 & $12 / 13 / 07$ & $22400_{\mathrm{NR}}$ \\
\hline TOC & PPB & 1646.60 & & $10 / 08 / 87$ & MR & $\begin{array}{l}00 / 08 / 87 \\
12 / 13 / 87\end{array}$ & 1185. & $12 / 13 / 87$ & MR \\
\hline TRITIUM & $\mathrm{PCI} / \mathrm{L}$ & 585.00 & 20001.06 & $\begin{array}{l}10 / 68 / 87 \\
11 / 68 / 87\end{array}$ & $\begin{array}{l}534.10 \\
217.00 \\
(\quad 687.00 \\
222.01\end{array}$ & $\begin{array}{l}10 / 08 / 87 \\
12 / 18 / 07\end{array}$ & $\begin{array}{c}8525.10 \\
207.16\} \\
8206.50 \\
207.56\}\end{array}$ & $\begin{array}{l}00 / 20 / 87 \\
12 / 13 / 87\end{array}$ & $\left\{\begin{array}{r}14806.00 \\
428.06\} \\
13785.46 \\
412.06\}\end{array}\right.$ \\
\hline
\end{tabular}

$\overbrace{\infty}^{\infty}$

* - yalue exceeds primary dRinking tater standard.

- - YALUE EXCEEDS PROPOSED PRIUARY DRINKING TATER STANOARO.

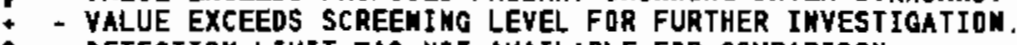

- DETECTIOM LINIT tas NDT AVAILABLE for COMPARISOH

MR - AMALYSIS MOT REQUESTED OR NOT YET REPORTED

VALUES IN \& \& ARE COUNIING ERRORS FOR RADIONUCLIDES

PATER STANDARD'S) IN PARENTHESES ARE PROPOSED ONLY 
TABLE C.3. (contd)

\begin{tabular}{|c|c|c|c|c|c|}
\hline $\begin{array}{c}\text { CONSTI } \\
\text { NAUE }\end{array}$ & $\begin{array}{l}\text { UENT } \\
\text { UNITS }\end{array}$ & $\begin{array}{c}\text { CONTRACTUAL } \\
\text { DETECTION } \\
\text { LINIT }\end{array}$ & $\begin{array}{l}\text { DRINKING } \\
\text { VATER } \\
\text { SIANDARO }\end{array}$ & $\underset{\text { DAMTE }}{\text { SAMPLE }}$ & $9-97-43$ \\
\hline ALKAL IN & & & & $12 / 13 / 87$ & 1200100.00 \\
\hline ALPHA & $P C I / L$ & 4.00 & 16.00 & $12 / 13 / 87$ & $\left(\begin{array}{l}1.20 \\
1.12\}\end{array}\right.$ \\
\hline BETA & $\mathrm{PCI} / \mathrm{L}$ & 8.60 & 60.16 & $\begin{array}{l}68 / 26 / 87 \\
12 / 13 / 87 \\
12 / 13 / 87\end{array}$ & $\begin{array}{ll} & 5.13 \\
\{ & 1.88\} \\
\{ & 8.76 \\
\{ & 2.14\} \\
\{ & 3.72 \\
\{ & 3.43\}\end{array}$ \\
\hline CHLORID & PPB & 500.00 & & $12 / 13 / 87$ & 10106. It \\
\hline CONDFLD & UМHD & 1.6 & & $12 / 13 / 87$ & 378.10 \\
\hline FBARIUU & PPB & 6.01 & 1001.80 & $12 / 13 / 87$ & 49.11 \\
\hline FCADUIU & $\mathrm{PPB}$ & 2.00 & 10.01 & $12 / 13 / 87$ & \\
\hline FCALCIU & PPB & 56.06 & & $12 / 13 / 87$ & 46100.66 \\
\hline FCHROUI & PPB & 10.01 & 60.01 & $12 / 13 / 87$ & 101.00 \\
\hline FUAGNES & PPB & 0.00 & & $12 / 13 / 87$ & 11060.66 \\
\hline FPOTASS & PPB & 100.60 & & $12 / 13 / 87$ & 3790.06 \\
\hline FSODIUV & $\mathrm{PPB}$ & 100.00 & & $12 / 13 / 87$ & 21800.00 \\
\hline FVAMADI & $\mathrm{PPB}$ & 6. 10 & & $12 / 13 / 87$ & \\
\hline FZIMC & PPB & 6.16 & & $12 / 13 / 87$ & 6.61 \\
\hline HHITRAT & PPB & 2501.01 & 46600.00 & $\begin{array}{l}18 / 20 / 87 \\
12 / 19 / 87\end{array}$ & $\begin{array}{l}20400.60 \\
19600.08\end{array}$ \\
\hline LFLUORD & $\mathrm{PPB}$ & 20.00 & 4000.00 & $12 / 13 / 67$ & 350.10 \\
\hline
\end{tabular}

\begin{tabular}{|c|c|}
\hline $\begin{array}{l}\text { SALPLE } \\
\text { DATE }\end{array}$ & $8-07-511$ \\
\hline $12 / 13 / 87$ & 88100.06 \\
\hline $12 / 13 / 87$ & \\
\hline $12 / 13 / 87$ & \\
\hline & MR \\
\hline & WR \\
\hline $12 / 13 / 87$ & $250 \mathrm{se}$ \\
\hline $12 / 13 / 87$ & 360.00 \\
\hline $12 / 13 / 87$ & 91.01 \\
\hline $12 / 19 / 87$ & \\
\hline $12 / 13 / 87$ & 66501.14 \\
\hline $12 / 18 / 87$ & 116.00 \\
\hline $12 / 13 / 87$ & 12500.01 \\
\hline $12 / 13 / 87$ & 3880.00 \\
\hline $12 / 13 / 87$ & 11604.60 \\
\hline $12 / 13 / 87$ & \\
\hline $12 / 13 / 97$ & B.u \\
\hline $\begin{array}{l}68 / 26 / 87 \\
12 / 13 / 87\end{array}$ & 20200.00 \\
\hline $12 / 13 / 87$ & 346.00 \\
\hline
\end{tabular}

\begin{tabular}{|c|c|}
\hline $\begin{array}{l}\text { SAUPLE } \\
\text { DATE }\end{array}$ & $8-101-488$ \\
\hline $12 / 21 / 87$ & 78806.05 \\
\hline $12 / 21 / 87$ & \\
\hline $12 / 21 / 87$ & $\left\{\quad \begin{array}{l}4.37 \\
\text { MR }\end{array}\right\}$ \\
\hline & MR \\
\hline $12 / 21 / 87$ & 016.00 \\
\hline $12 / 21 / 67$ & 149.66 \\
\hline $12 / 21 / 87$ & 19.61 \\
\hline $12 / 21 / 87$ & 2.6 \\
\hline $12 / 21 / 87$ & 20050.00 \\
\hline $12 / 21 / 87$ & \\
\hline $12 / 21 / 87$ & 6700.11 \\
\hline $12 / 21 / 87$ & 2414.110 \\
\hline $12 / 21 / 87$ & 4740.60 \\
\hline $12 / 21 / 87$ & 10.10 \\
\hline $12 / 21 / \theta 7$ & \\
\hline $\begin{array}{l}08 / 28 / 87 \\
12 / 21 / 87\end{array}$ & \\
\hline $12 / 21 / 87$ & 198.01 \\
\hline
\end{tabular}

- - VALUE eXceEds primary dRINKING tater standard

- - YALUE EXCEEDS PROPOSED PRIMARY DRINKING IATER STAMDARO.

- - YALUE EXCEEDS SCREEMINO LEVEL FOR FURTHER INVESTIGATIOH.

- detection linit tas not available for couparison

KR - AYALYSIS MOT REQUESTED OR NOT YET REPDRTED

VALUES IM \{ $\}$ ARE COUNTING ERRORS FOR RADIONUCLIDES

PATER STAKDARD(S) IN PARENTHESES ARE PROPDSED ONLY 
TABLE C.3. (contd)

\begin{tabular}{|c|c|c|c|c|c|c|c|c|c|}
\hline $\begin{array}{l}\text { CONSTI } \\
\text { MAHE }\end{array}$ & $\begin{array}{l}\text { UENT } \\
\text { UNITS }\end{array}$ & $\begin{array}{c}\text { CORTRACTUAL } \\
\text { DETECTIDAN } \\
\text { LIUIT }\end{array}$ & $\begin{array}{l}\text { DRINKING } \\
\text { IATER } \\
\text { STANDARD }\end{array}$ & $\begin{array}{l}\text { SAUPLE } \\
\text { DATE }\end{array}$ & $8-97-43$ & $\begin{array}{c}\text { SAMPLE } \\
\text { DATE }\end{array}$ & $8-97-51 A$ & $\underset{\text { DATE }}{\text { SAPLE }}$ & $8-101-408$ \\
\hline NITRATE & PPB & 500.08 & 45060.66 & $12 / 13 / 87$ & 21300.01 & $12 / 18 / 67$ & $217 \mathrm{des}$ & $12 / 21 / 87$ & 9as.es \\
\hline$P H-L A B$ & & 0.01 & & $12 / 13 / 87$ & 7.03 & $12 / 13 / 87$ & 7.61 & $12 / 21 / 87$ & 7.01 \\
\hline PHF IELD & & t.1. & & $12 / 13 / 87$ & 8.10 & $12 / 13 / 87$ & 7.71 & $12 / 21 / 97$ & 7.26 \\
\hline SULFATE & PPB & 565.60 & & $12 / 13 / 87$ & 62000.01 & $12 / 13 / 87$ & 88504. & $12 / 21 / 87$ & 1176.es \\
\hline TC & PPB & & & $12 / 13 / 87$ & 28400.010 & $12 / 13 / 87$ & 20064.01 & $12 / 21 / 87$ & 18106.64 \\
\hline TRITIUV & PCI $/ \mathrm{L}$ & 600.10 & 26000.00 & $\begin{array}{l}18 / 26 / 87 \\
12 / 13 / 87\end{array}$ & $\begin{array}{r}9634.16 \\
363.00\} \\
9746.08 \\
358.08\}\end{array}$ & $\begin{array}{l}68 / 26 / 87 \\
12 / 13 / 87\end{array}$ & 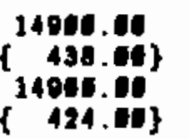 & $\begin{array}{l}18 / 20 / 07 \\
12 / 21 / 87\end{array}$ & \\
\hline
\end{tabular}

$\overbrace{\omega}^{\infty}$

* - Value exceEds primary dRINKIng vater standard.

- VALUE EXCEEDS PROPOSED PRIMARY DRINKING IATER STAHDARD.

- VALUE EXCEEDS SCREENIMO LEVEI FOR FURTHER INVESTIGATION.

- DETECTION LINIT IAS NOT AYAILABLE FOR COUPARISON

MR - AMALYSIS NOT REQUESTED OR HOT YET REPDRTED

VALUES IN I I ARE COUMTING ERRORS FOR RADIONUCLIDES

IATER STANDARD(S) IN PARENTHESES ARE PROPOSED ONLY 
TABLE C. $3 . \quad$ (contd)

\begin{tabular}{|c|c|c|c|c|c|}
\hline $\begin{array}{l}\text { CONSTI } \\
\text { NAUE }\end{array}$ & $\begin{array}{l}\text { JENT } \\
\text { UNITS }\end{array}$ & $\begin{array}{l}\text { CDNTRACTUAL } \\
\text { DETECTION } \\
\text { LIUIT }\end{array}$ & $\begin{array}{l}\text { DRINKING } \\
\text { TATER } \\
\text { STANDARD }\end{array}$ & $\begin{array}{c}\text { SAUPLE } \\
\text { DATE }\end{array}$ & B-S3-E12 \\
\hline ALKAL IN & & & & $10 / 27 / 87$ & 116000.00 \\
\hline ALPHA & $\mathrm{PCI} / \mathrm{L}$ & 4.10 & 15.6 & $10 / 27 / 97$ & $\begin{array}{ll} & 1.10 \\
\{\quad 1.83\end{array}$ \\
\hline AUYONIU & PPB & 56.66 & & $10 / 27 / 87$ & \\
\hline BETA & $\mathrm{PCI} / \mathrm{L}$ & 8.11 & 56.66 & $14 / 27 / 87$ & $\begin{array}{ll} & 4.84 \\
\{\quad 3.52\}\end{array}$ \\
\hline CHLORID & PPA & 580.10 & & $11 / 27 / 87$ & B251.10 \\
\hline CONDFLD & UNHO & 1.66 & & $10 / 27 / 87$ & 278.00 \\
\hline FARSENI & PPB & 5.10 & 50.10 & $10 / 27 / 87$ & 6.10 \\
\hline FBARIUY & PPB & 6.10 & 1600.08 & $16 / 27 / 87$ & 37.10 \\
\hline FCALCIU & PPB & 58.60 & & $11 / 27 / 87$ & 94200.06 \\
\hline FIRUN & PPB & 60.16 & & $11 / 27 / 87$ & \\
\hline FLUORID & PPB & 500.11 & 4064.00 & $10 / 27 / 87$ & 688.60 \\
\hline FUAGMES & PPB & 0.10 & & $18 / 27 / 87$ & 16700.06 \\
\hline FUANGAM & PPB & 5.10 & & $10 / 27 / 87$ & \\
\hline FPOTASS & PPB & 100.06 & & $10 / 27 / 87$ & 5021.68 \\
\hline FSELENI & PPB & 5.90 & 10.00 & $10 / 27 / 87$ & \\
\hline FSODIUY & PPB & 106.00 & & $18 / 27 / 87$ & 14701.01 \\
\hline FVANADI & PPB & 6.00 & & $10 / 27 / 87$ & 20.00 \\
\hline HNITRAT & PPB & 2581.06 & 45000.00 & $\begin{array}{l}67 / 24 / 87 \\
10 / 27 / 87\end{array}$ & $\begin{array}{l}21904.66 \\
22000.65\end{array}$ \\
\hline
\end{tabular}

\begin{tabular}{|c|c|}
\hline $\begin{array}{l}\text { SAUPLE } \\
\text { DATE }\end{array}$ & $0-53-25$ \\
\hline $10 / 15 / 87$ & 148000.10 \\
\hline $00 / 15 / 87$ & $\left\{\begin{array}{l}4.20 \\
1.91\}\end{array}\right.$ \\
\hline $10 / 15 / 87$ & 88.16 \\
\hline $09 / 15 / 87$ & $\left\{\begin{array}{r}11.71 \\
\{\quad 4.43\}\end{array}\right.$ \\
\hline $00 / 16 / 87$ & 22746.06 \\
\hline $08 / 15 / 87$ & 465.11 \\
\hline $09 / 15 / 87$ & \\
\hline $10 / 16 / 07$ & 04.00 \\
\hline $10 / 15 / 07$ & 40201.01 \\
\hline $00 / 15 / 87$ & 179.10 \\
\hline $00 / 16 / 87$ & \\
\hline $10 / 15 / 87$ & 12244.00 \\
\hline $19 / 15 / 87$ & 145.61 \\
\hline $00 / 16 / 87$ & 6838.106 \\
\hline $00 / 15 / 87$ & \\
\hline $19 / 15 / 87$ & 2220106 \\
\hline $09 / 15 / 87$ & \\
\hline & $\begin{array}{c}\text { MR } \\
\text { MR }\end{array}$ \\
\hline
\end{tabular}

\begin{tabular}{|c|c|}
\hline $\begin{array}{l}\text { SAYPLE } \\
\text { DATE }\end{array}$ & B-SB-E14A \\
\hline $1 / 28 / 87$ & 106000.00 \\
\hline $68 / 28 / 87$ & 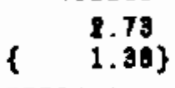 \\
\hline $08 / 28 / 87$ & \\
\hline $18 / 28 / 87$ & $\left\{\begin{array}{l}7.10 \\
8.06\end{array}\right\}$ \\
\hline $18 / 28 / 07$ & 18106.0 \\
\hline $68 / 28 / 87$ & 280.00 \\
\hline $10 / 28 / 87$ & 1.10 \\
\hline $18 / 28 / 87$ & 18.00 \\
\hline $68 / 28 / 87$ & 23500.00 \\
\hline $18 / 28 / 87$ & \\
\hline $08 / 28 / 07$ & \\
\hline $08 / 28 / 87$ & 10000.01 \\
\hline $18 / 26 / 87$ & 1.00 \\
\hline $88 / 28 / 87$ & 5880.06 \\
\hline $08 / 28 / 87$ & 6.010 \\
\hline $18 / 28 / 87$ & 13650.06 \\
\hline $18 / 28 / 87$ & 17.01 \\
\hline & $\underset{M R}{M R}$ \\
\hline
\end{tabular}

- Value exceeds priuary drinkihg Mater standard.

- YALUE EXCEEDS PRDPOSED PRIMARY DRINKINO IATER STANDARD.

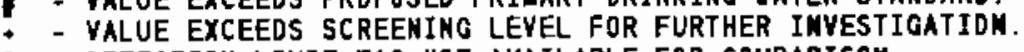

- DETECTION LIUIT TAS NOT AVRILABLE FOR COMPARISON

NR - AKALYSIS NGT REQUESTED OR NOT YET REPORTED

VALUES IN \& t ARE COUNTING ERRGRS FOR RADIDNUCLIDES

TATER STAMDARD(S) IN PARENTHESES ARE PROPOSED ONLY 
TABLE C.3. (contd)

\begin{tabular}{|c|c|c|c|c|c|}
\hline $\begin{array}{c}\text { CONSTI I } \\
\text { NAHE }\end{array}$ & $\begin{array}{l}\text { UENT } \\
\text { UNI TS }\end{array}$ & $\begin{array}{l}\text { CONTRACTUAL } \\
\text { DETECTION } \\
\text { LIMIT }\end{array}$ & $\begin{array}{l}\text { DRIMKING } \\
\text { FATER } \\
\text { STANDARD }\end{array}$ & $\begin{array}{c}\text { SAMPLE } \\
\text { DATE }\end{array}$ & $8-53-E 12$ \\
\hline LFLUORD & $\mathrm{PPB}$ & 20.60 & 4600.80 & $10 / 27 / 87$ & 374.04 \\
\hline NC23HC & $\mathrm{PPB}$ & & & & NR \\
\hline NC24HC & PPB & & & & NR \\
\hline MC25HC & PPB & & & & NR \\
\hline $\mathrm{MC} 2 \mathrm{BHC}$ & PPB & & & & NR \\
\hline MC27HC & PPB & & & & NR \\
\hline NC28HC & PPB & & & & NR \\
\hline NI TRATE & PPB & 600.00 & 46080.06 & $10 / 27 / 87$ & 25004.66 \\
\hline PH-LAB & & 1.11 & & $16 / 27 / 87$ & 8.20 \\
\hline PHFIEID & & 0.16 & & $16 / 27 / 87$ & 7.80 \\
\hline SULFATE & PPB & 560.66 & & $10 / 27 / 07$ & 24680.11 \\
\hline TC & PPB & & & $10 / 27 / 87$ & 205010 \\
\hline TDXLDL & PPB & 20.00 & & $10 / 27 / 87$ & \\
\hline TRITIUN & PCI/L & 5ES. 10 & 200000.00 & $\begin{array}{l}17 / 24 / 87 \\
10 / 27 / 87\end{array}$ & $\begin{array}{c}4965.01 \\
277.00\} \\
6940.01 \\
299.06\}\end{array}$ \\
\hline
\end{tabular}

\begin{tabular}{|c|c|}
\hline DATE & 8-53-25 \\
\hline $18 / 15 / 07$ & 601.00 \\
\hline $19 / 15 / 87$ & 11.00 \\
\hline $10 / 15 / 07$ & 13.00 \\
\hline $00 / 15 / 87$ & 17.10 \\
\hline $00 / 16 / 87$ & 16.01 \\
\hline $60 / 16 / 67$ & 10.01 \\
\hline $09 / 15 / 87$ & 13.6 \\
\hline $00 / 15 / 87$ & \\
\hline $10 / 15 / 87$ & 7.00 \\
\hline $10 / 15 / 87$ & 7.11 \\
\hline $60 / 15 / 87$ & 71000.6 \\
\hline $00 / 16 / 87$ & 34761.00 \\
\hline $10 / 15 / 87$ & 37.60 \\
\hline $10 / 00 / 87$ & \\
\hline
\end{tabular}

MR

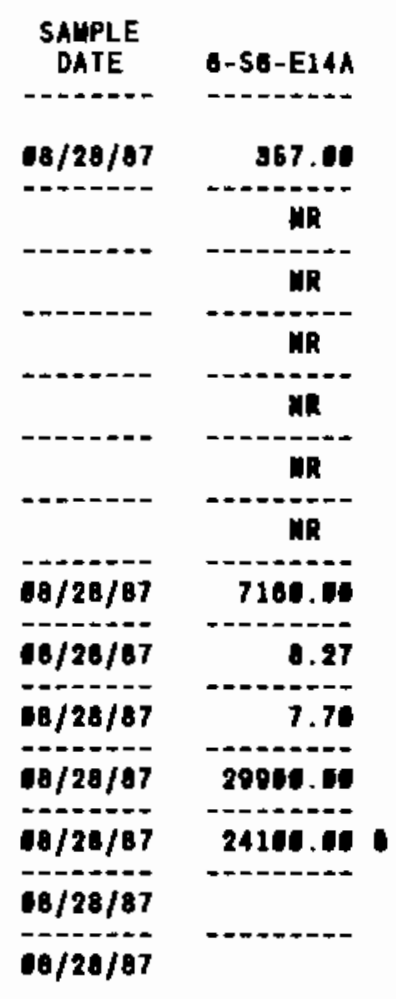

MR

* - Value exceeds priuary drinking tater stamdard.

- - VALUE EXCEEOS PROPOSED PRIUARY DRINKING IATER STANDARD.

- VALUE EXCEEDS SCREENING LEVEL FOR FURTHER INVESTIGATION.

- DETECTION LIUIT UAS NOT AVAILABLE FOR COUPARISON

NR - AMALYSIS NOT REQUESTED QR NOT YET REPORTED

VALUES IN \{ $\}$ ARE COUNTING ERRORS FOR RADIONUCLIDES

IATER STAMDARD(S) IN PARENTHESES ARE PROPDSED DNLY 


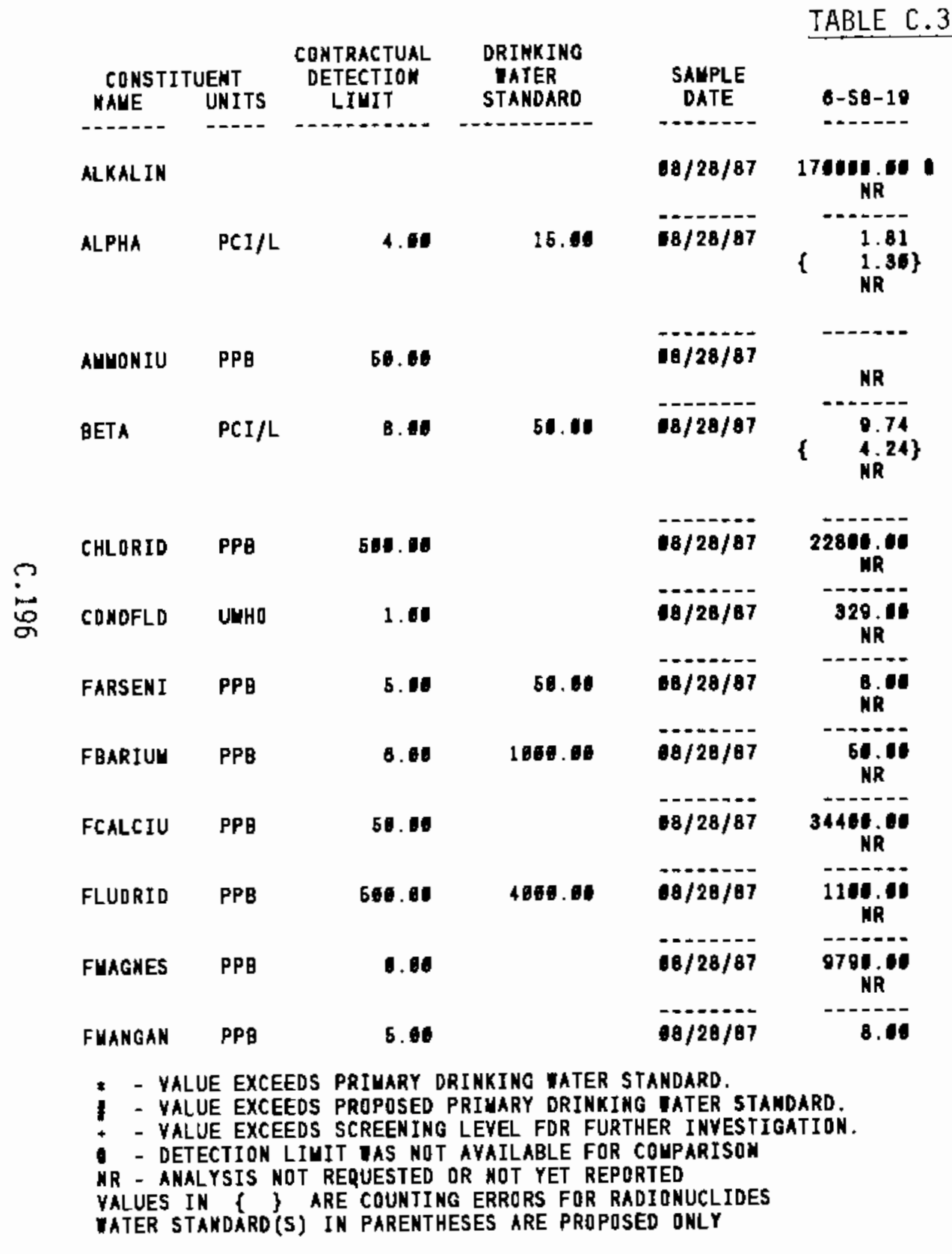

\begin{tabular}{|c|c|}
\hline $\begin{array}{l}\text { SALPLE } \\
\text { DATE }\end{array}$ & $6-S 11-E 12 A P$ \\
\hline$+=-0-0-$ & $--1+-2-1-1$ \\
\hline $12 / 29 / 87$ & 188500.10 \\
\hline \multicolumn{2}{|c|}{$12 / 20 / 87$} \\
\hline & WR \\
\hline $12 / 20 / B 7$ & $\underset{\mathrm{KR}}{118.00}$ \\
\hline $12 / 20 / 97$ & $\begin{array}{ll}(1.38 \\
\operatorname{MR}^{2}\end{array}$ \\
\hline $12 / 20 / 87$ & 16300.60 \\
\hline $12 / 20 / 87$ & $\begin{array}{c}288.01 \\
\text { NR }\end{array}$ \\
\hline & $\begin{array}{l}\text { MR } \\
\text { MR }\end{array}$ \\
\hline & NR \\
\hline & $\begin{array}{l}\text { MR } \\
\text { MR }\end{array}$ \\
\hline $12 / 29 / 87$ & $\frac{906.11}{\mathrm{HR}}$ \\
\hline & $\begin{array}{l}\text { MR } \\
\text { MR }\end{array}$ \\
\hline & MR \\
\hline
\end{tabular}

\begin{tabular}{|c|c|}
\hline $\begin{array}{c}\text { SAMPLE } \\
\text { DATE }\end{array}$ & $6-512-3$ \\
\hline - & \\
\hline $\begin{array}{l}68 / 28 / 87 \\
10 / 27 / 87\end{array}$ & $\begin{array}{l}129004.04 \\
120516.04\end{array}$ \\
\hline $18 / 28 / 07$ & 9.14 \\
\hline $18 / 27 / 87$ & 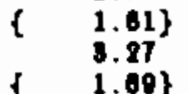 \\
\hline $\begin{array}{l}68 / 28 / 87 \\
16 / 27 / 87\end{array}$ & \\
\hline $18 / 28 / 87$ & \\
\hline $10 / 27 / 87$ & $\begin{array}{c}\{\quad 0.09\} \\
\{\quad 11.04 \\
\{\quad 4.26\}\end{array}$ \\
\hline $\begin{array}{l}10 / 28 / 87 \\
10 / 27 / 87\end{array}$ & 27001.00 \\
\hline $\begin{array}{l}18 / 26 / 87 \\
16 / 27 / 67\end{array}$ & 386.00 \\
\hline $\begin{array}{l}18 / 28 / 87 \\
16 / 27 / 87\end{array}$ & \\
\hline $\begin{array}{l}18 / 26 / 87 \\
18 / 27 / 87\end{array}$ & 60.01 \\
\hline $\begin{array}{l}68 / 28 / 97 \\
16 / 27 / 97\end{array}$ & 42904.06 \\
\hline $\begin{array}{l}10 / 28 / 87 \\
10 / 27 / 87\end{array}$ & 887.00 \\
\hline $\begin{array}{l}08 / 28 / 87 \\
10 / 27 / 87\end{array}$ & 10900.06 \\
\hline $18 / 28 / 87$ & \\
\hline
\end{tabular}


TABLE C.3. (contd)

\begin{tabular}{|c|c|c|c|c|c|}
\hline$\underbrace{\text { CONSTI }}_{\text {MANE }}$ & $\begin{array}{l}\text { TUENT } \\
\text { UNITS }\end{array}$ & $\begin{array}{c}\text { CONTRACTUAL } \\
\text { DETECIIOA } \\
\text { LIVIT }\end{array}$ & $\begin{array}{l}\text { ORIMKING } \\
\text { IATER } \\
\text { STAMDARD }\end{array}$ & $\begin{array}{c}\text { SAUPLE } \\
\text { DATE }\end{array}$ & $8-58-10$ \\
\hline FUANGAN & PPE & 5.00 & & & NR \\
\hline FPOTASS & PPB & 181.00 & & $18 / 28 / 87$ & 7810.10 \\
\hline FSODIUY & PPB & 100.00 & & $68 / 2 \theta / 07$ & ${ }_{\mathrm{KR}}^{33501 . \mathrm{i}}$ \\
\hline FYAKADI & PPB & 5.66 & & $08 / 28 / 87$ & 14. 16 \\
\hline FZINC & PPB & 5. 60 & & $10 / 28 / 87$ & MR \\
\hline LFLUORO & PPB & 24.00 & 4601.60 & $18 / 28 / 87$ & 1344.16 \\
\hline MI TRATE & PPB & 506.01 & 45000.00 & $68 / 28 / 87$ & MR \\
\hline$P H-L A B$ & & 0.1 & & $18 / 28 / 87$ & ${ }_{\text {MR }}^{8.05}$ \\
\hline PHFIELD & & 0.10 & & $8 B / 28 / 87$ & $7,4.4$ \\
\hline SULFATE & PPB & 566.61 & & $08 / 28 / 87$ & $\underset{\text { NR }}{18806}$ \\
\hline TC & PPB & & & $18 / 28 / 87$ & $\begin{array}{c}38460.00 \\
\text { NR }\end{array}$ \\
\hline TOXLDL & PPB & 20.00 & & $18 / 28 / 87$ & MR \\
\hline$v$ & PCI $/ \mathrm{L}$ & 6.50 & 806.00 & & MR \\
\hline
\end{tabular}

\begin{tabular}{|c|c|}
\hline $\begin{array}{l}\text { SAYPLE } \\
\text { DATE }\end{array}$ & B-511-E12AP \\
\hline & NR \\
\hline & $\begin{array}{l}\text { NR } \\
\text { NR }\end{array}$ \\
\hline & NR \\
\hline & $\begin{array}{l}\text { MR } \\
\text { MR }\end{array}$ \\
\hline & $\begin{array}{l}\text { MR } \\
\text { MR }\end{array}$ \\
\hline $12 / 20 / 87$ & 82: 10 \\
\hline $12 / 20 / 87$ & MR \\
\hline $12 / 29 / 87$ & $7_{M R}^{.90}$ \\
\hline $12 / 29 / 87$ & ${ }_{\mathrm{MR}}^{7.86}$ \\
\hline $12 / 29 / 87$ & MR \\
\hline $12 / 29 / 87$ & 37509.100 \\
\hline $12 / 28 / 87$ & MR \\
\hline
\end{tabular}

SALPLE

DATE 6-512-3

$10 / 27 / 07$

68/28/97 0158.08

10/27/87 B985.16

10/28/87 20100.00

$11 / 27 / 07 \quad 25996.06$

$00 / 2 B / 87 \quad 11$

$11 / 27 / 97 \quad 0.6$

-1828/87

$\begin{array}{ll}88 / 28 / 87 & 60.06 \\ 10 / 27 / 87 & 60.01\end{array}$

$00 / 28 / 97 \quad 669.06$

$11 / 27 / 97 \quad 525.11$

$18 / 28 / 87 \quad 12100.00$

$10 / 27 / 87 \quad 12201.06$

-18/20/07

$\begin{array}{ll}08 / 28 / 87 & 8.67 \\ 10 / 27 / 87 & 0.01\end{array}$

$08 / 28 / 87 \quad 7.30$

16/27/67 $\quad 7.71$

$03 / 28 / 87$ 53894.

$11 / 27 / 87 \quad 53706.06$

$08 / 28 / 87$ 28201.48

$10 / 27 / 87$ 33161.6:

$08 / 28 / 87 \quad 36.30$

$10 / 27 / 87$

$08 / 28 / 87$

2.94

* - value exceeds privary drimkino tater stamdard.

- VALUE EXCEEDS PROPOSED PRIUARY DRIMKING IATER STAMDARD.

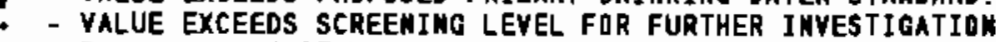

- DETECTION LIUIT UAS nOt AVAILABLE fOR COMPARISON

HR - ANALYSIS MOT RERUESTEO OR HOT YET REPORTED

VALUES IN $\{$ \& ARE COUNTING ERRORS FOR RADIONUCLIDES

WATER STANDARO(S) IN PARENTHESES ARE PROPOSED OMLY 
TABLE C.3. (contd)

\begin{tabular}{|c|c|c|c|c|c|c|c|}
\hline$\underset{\text { MAHE }}{\text { CONSII }}$ & $\begin{array}{l}\text { TUENT } \\
\text { UNITS }\end{array}$ & $\begin{array}{c}\text { CONTRACTUAL } \\
\text { DETECTION } \\
\text { LINIT }\end{array}$ & $\begin{array}{l}\text { DRINKING } \\
\text { TATER } \\
\text { STANDARD }\end{array}$ & $\begin{array}{c}\text { SAUPLE } \\
\text { DATE }\end{array}$ & B-528-E12 & $\begin{array}{c}\text { SAUPLE } \\
\text { DATE }\end{array}$ & $0-531-1$ \\
\hline ALKALIN & & & & $00 / 10 / 07$ & 148000.00 & $18 / 24 / 87$ & 91110.60 \\
\hline BETA & $\mathrm{PCI} / \mathrm{L}$ & 8.00 & 56.06 & $69 / 16 / 87$ & $\begin{array}{r}3.80 \\
\{\quad 3.48\}\end{array}$ & $68 / 24 / 87$ & $\begin{array}{r}5.74 \\
\{\quad 3.81\}\end{array}$ \\
\hline CHLORID & PPB & 5os.6 & & $18 / 10 / 87$ & 12266.00 & $18 / 24 / 87$ & 6334. 16 \\
\hline CONOFLD & UNRO & 1.00 & & $09 / 10 / 87$ & 310.11 & $18 / 24 / 87$ & 107.06 \\
\hline FARSENI & PPB & 5.60 & 50.10 & $10 / 10 / 87$ & & $18 / 24 / 87$ & 6.06 \\
\hline FBARIUU & PPB & 0.10 & 1060.10 & $10 / 14 / 87$ & 43.6 & $18 / 24 / 87$ & 10.10 \\
\hline FCALCIU & PPB & 50.11 & & $00 / 10 / 87$ & 46400.10 & $68 / 24 / 87$ & 227es. W \\
\hline FUAGMES & PPB & 0.60 & & $00 / 10 / 87$ & 0000.00 & $18 / 24 / 87$ & 5834.66 \\
\hline FPOTASS & PPB & 160.0 & & $00 / 14 / 87$ & 6561.11 & $18 / 24 / 87$ & 4110.14 \\
\hline FSODIUY & PPB & 101.10 & & $00 / 10 / 07$ & 21100.00 & $68 / 24 / 87$ & B616.01 \\
\hline FYAKADI & PPB & 5.00 & & $00 / 10 / 87$ & 12.00 & $18 / 24 / 07$ & 14.6 \\
\hline FZINC & PPB & 5.10 & & $00 / 10 / 87$ & 10.11 & $18 / 24 / 87$ & \\
\hline LFLUORD & PPB & 20.10 & 4080.00 & $10 / 10 / 87$ & 350.00 & $18 / 24 / 87$ & 250.00 \\
\hline NITRATE & PPB & 584.60 & 46000.05 & $90 / 14 / 87$ & 22316.00 & $18 / 24 / 87$ & 3480.06 \\
\hline$P H-L A B$ & & 6.61 & & $09 / 11 / 87$ & 7.68 & $18 / 24 / 87$ & 7.91 \\
\hline PHF IELD & & 6.10 & & $10 / 10 / 87$ & 0.80 & $18 / 24 / 87$ & 7.30 \\
\hline SULFATE & PPB & 500.100 & & $09 / 14 / 87$ & 32314.66 & $18 / 24 / 87$ & 18095.06 \\
\hline TC & PPB & & & $00 / 10 / 87$ & 36600.010 & $18 / 24 / 87$ & 37166.15 \\
\hline TOC & PPB & 1686.10 & & $10 / 10 / 87$ & & $68 / 24 / 87$ & 1190.06 \\
\hline $\begin{array}{l}*-V A \\
\vdots=V A \\
:-V A \\
:-D E \\
\text { KR }=A N \\
\text { VALUES } \\
\text { VATER S }\end{array}$ & $\begin{array}{l}\text { LUE EXCEEI } \\
\text { LUE EXCEEI } \\
\text { LUE EXCEE } \\
\text { TECTION L } \\
\text { ALYSIS ND } \\
\text { IN \& \& } \\
\text { TANDARD'S }\end{array}$ & $\begin{array}{l}\text { EDS PRIUARY DF } \\
\text { EDS PROPOSED } \\
\text { EDS SCREENING } \\
\text { IUIT WAS NDT } \\
\text { DT REQUESTED } \\
\text { ARE COUNTIMC } \\
\text { S) IN PARENTHE }\end{array}$ & $\begin{array}{l}\text { INKING WATER } \\
\text { RIMARY DRINK } \\
\text { LEVEL FOR FU } \\
\text { AYAILABLE FO } \\
\text { R NOT YET RE } \\
\text { IERRORS FOR } \\
\text { SES ARE PROP }\end{array}$ & $\begin{array}{l}\text { ANDARD. } \\
\text { IATER STA } \\
\text { ER INYESTI } \\
\text { OUPARISON } \\
\text { IED } \\
\text { IONUCLIDES } \\
\text { D ONLY }\end{array}$ & $\begin{array}{l}\text { DARO. } \\
\text { ATIOH. }\end{array}$ & & \\
\hline
\end{tabular}


TABLE C.3. (contd)

\begin{tabular}{|c|c|c|c|c|c|c|c|}
\hline $\begin{array}{l}\text { CONST } \\
\text { NAME }\end{array}$ & $\begin{array}{l}\text { UENT } \\
\text { UNITS }\end{array}$ & $\begin{array}{c}\text { CONTRACTUAL } \\
\text { DETECTIDN } \\
\text { LIMIT }\end{array}$ & $\begin{array}{l}\text { DRINXING } \\
\text { WATER } \\
\text { STAMDARD }\end{array}$ & $\begin{array}{l}\text { SAMPLE } \\
\text { DATE }\end{array}$ & B-529-E12 & $\begin{array}{l}\text { SANPLE } \\
\text { DATE }\end{array}$ & $8-531-1$ \\
\hline u & $\mathrm{PCI} / \mathrm{L}$ & 0.50 & 060.00 & $\begin{array}{l}00 / 11 / 87 \\
10 / 21 / 87\end{array}$ & $\begin{array}{l}2.28 \\
2.41\end{array}$ & & $\begin{array}{l}\text { UR } \\
\text { NR }\end{array}$ \\
\hline
\end{tabular}

- - value exceeds priuary drimking tater stamdard.

1 - VALUE EXCEEDS PROPOSED PRIMARY DRINKIMG TATER STANDARD.

- VALUE EXCEEDS SCREENINO LEYEL FOR FURTHER INYESTIOATION.

- DETECTION LIUIT AS NOT AYAILABLE FOR COMPARISON

TU REQUESTED OR NOT YET REPORTED

YALUES IN \{ ARE COU:TING ERRORS FOR RADIONUCLIDES

WATER STANDARD(S) IN PARENTHESES ARE PROPOSED ONLY 
TABLE C.4. Constituents Analyzed for in Ground-Water Samples from Compliance Monitoring Wells

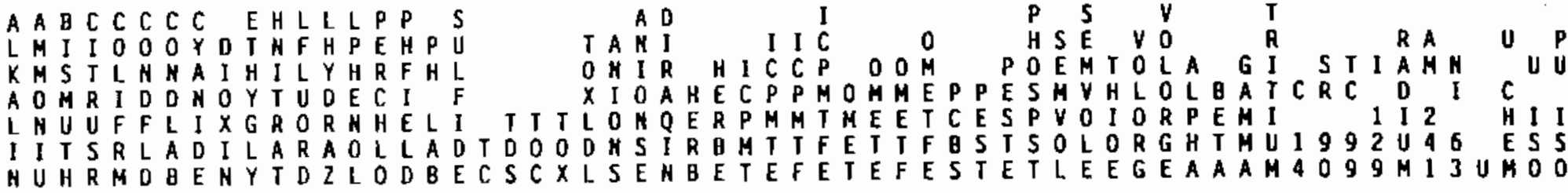

$\begin{array}{ll} & \\ 1-H 3-1 \\ 1-H 3-2 A \\ 1-H 3-2 B \\ 1-H 3-2 C \\ 1-H 4 \cdot-3 \\ 1-H 4-4 \\ 1-H 4-5 \\ 1-H 4-6 \\ 1-H 4-7 \\ 1-H 4-9 \\ 1-H 4-9 \\ 1-H 4-10 \\ 1-H 4-11 \\ 1-H 4-12 A \\ 1-H 4-12 B \\ 1-H 4-12 C \\ 1-H 4-13 \\ 1-H 4-14 \\ 1-H 4-15 A \\ 1-H 4-15 B \\ 1-H 4-16 \\ 1-H 4-17 \\ 1-H 4-18 \\ 1-H-2 \\ 1-H-3 \\ 1-N-4\end{array}$

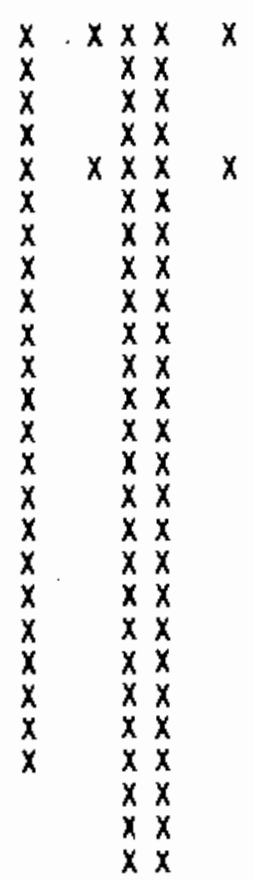

\begin{tabular}{|c|c|c|}
\hline \multicolumn{2}{|c|}{$x \times x$} & $x \times \times \times$ \\
\hline$x$ & $x \times$ & $x \times \times \times$ \\
\hline$x$ & $x \times$ & $x \times x \times$ \\
\hline$x$ & $x \ddot{x}$ & $x \times \times x$ \\
\hline$x$ & $x x$ & \\
\hline$x$ & $x x$ & $x \times x x$ \\
\hline$x$ & $x \times \cdot$ & $x \times x \times$ \\
\hline$x$ & $x \times$ & $x \times x \times$ \\
\hline$x$ & $x x$ & $x \times \times x$ \\
\hline$x$ & $x x$ & $x \times x \times$ \\
\hline$x$ & $x x$ & $x \times x \times$ \\
\hline$x$ & $x x$ & $x \times \times \times$ \\
\hline$x$ & $x \times$ & $x \times x \times$ \\
\hline$x$ & $x \times$ & $x \times \times \times$ \\
\hline$x$ & $x \times$ & $x \times x \times$ \\
\hline$x$ & $x \times$ & $x \times x \times$ \\
\hline$x$ & & $x \times x \times$ \\
\hline$x$ & $x x$ & $x \times \times \times$ \\
\hline$x$ & $x \times$ & $x \times x \times$ \\
\hline$x$ & $x \times$ & $x \times x \times$ \\
\hline$x$ & $x \times$ & $x \times \times x$ \\
\hline$x$ & $x x$ & $x \times x \times$ \\
\hline$x$ & $8 y$ & $x \times x$ \\
\hline & $\hat{x}$ & \\
\hline & & \\
\hline
\end{tabular}

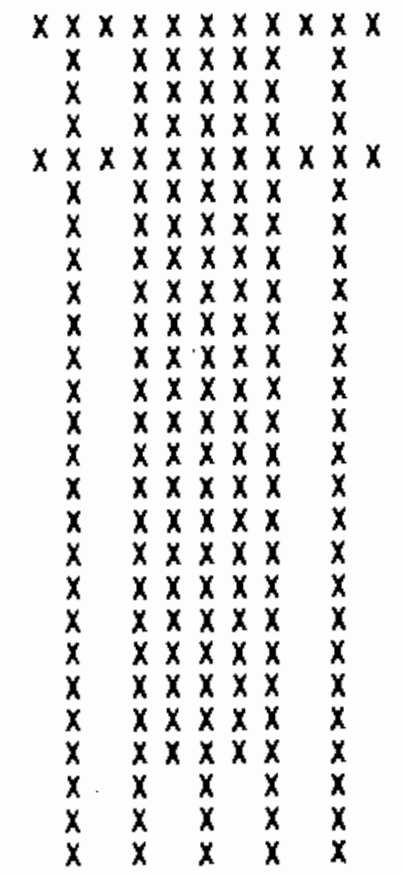

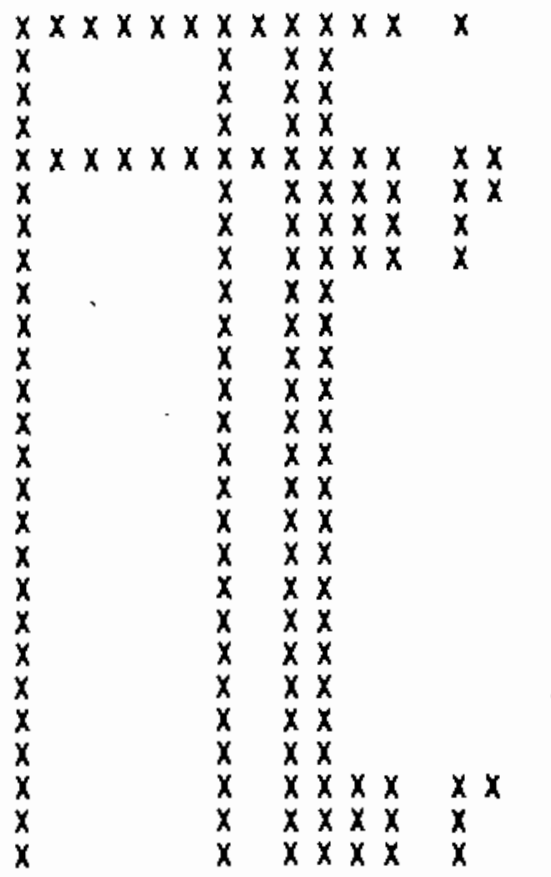

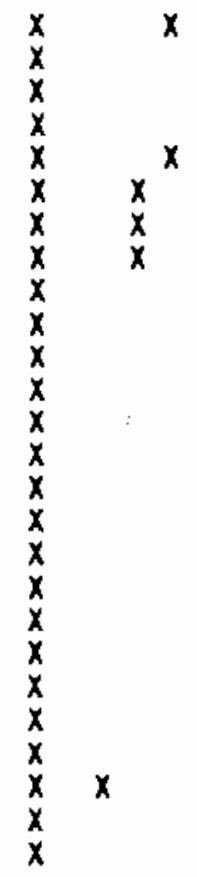




\title{
TABLE C.4. (contd)
}

\begin{abstract}
A A B C C C C C E HLLLPP S LMI I O OOOYYOTNFH PEHPU XMSTL H H A 1 H I L Y HRF H L A O R R D N OYTUDECI F

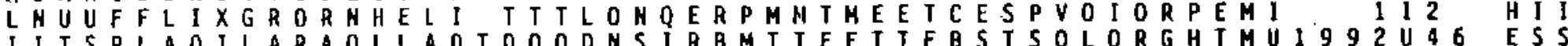

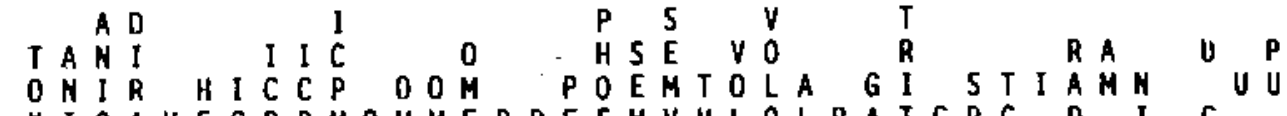
$X$ I OA HE C P P M OMHEPPESMVHLOLBATCRC D I C

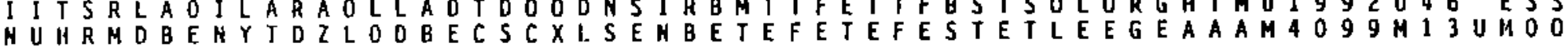

$\begin{array}{cc} & \\ & 1-N-14 \\ & 1-N-27 \\ & 1-N-31 \\ & 1-N-32 \\ & 1-H-36 \\ & 1-N-39 \\ 0 \quad & 1-N-41 \\ 0 & 1-H-42 \\ 1-N-49 \\ 1-N-52 \\ 1-N-58 \\ 1-N-59 \\ 1-N-60 \\ 1-N-61 \\ 2-E 25-25 \\ 2-E 25-26 \\ 2-E 25-27 \\ 2-E 25-28 \\ 3-1-1 \\ 3-1-2 \\ 3-1-3 \\ 3-1-4 \\ 3-1-5 \\ 3-1-6 \\ 3-1-7 \\ 3-1-8\end{array}$

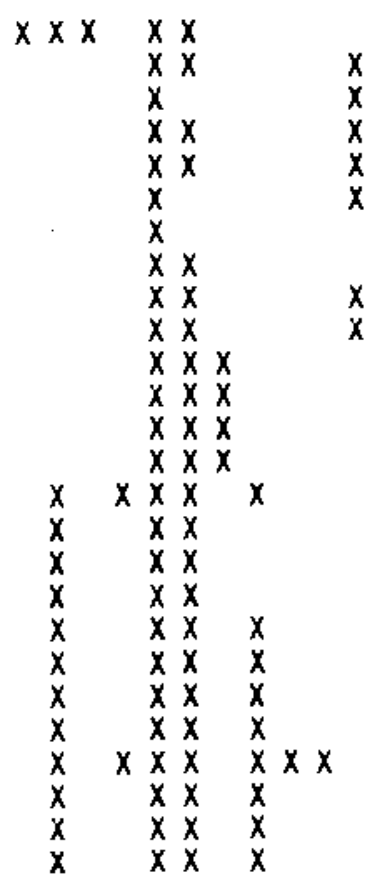

\begin{tabular}{|c|c|c|c|c|c|}
\hline & & $x \times$ & $x$ & $X$ & $x$ \\
\hline$x$ & & $x$ & & & \\
\hline$x$ & & & & & \\
\hline$x$ & & $x$ & & & \\
\hline$x$ & & $x$ & & & \\
\hline$x$ & & . & & & \\
\hline & & $x$ & & & \\
\hline$x$ & & $x$ & & & \\
\hline$x$ & & $x$ & & & \\
\hline & $x$ & $x x$ & & $x$ & \\
\hline & $x$ & $x \times$ & & $x$ & \\
\hline & $x$ & $x \times$ & & $x$ & \\
\hline & $x$ & $x \times$ & & $x$ & \\
\hline
\end{tabular}

$\begin{array}{lllll}x & x & x & x & x \\ x & x & x & x & x \\ x & x & x & x & x \\ x & x & x & x & x \\ x & x & x & x & x \\ x & x & x & x & x \\ x & x & x & x & x \\ x & x & x & x & x \\ x & x & x & x & x \\ x & x & x & x & x \\ x & x & x & x & x \\ x & x & x & x & x \\ x & x & x & x & x \\ x & x & x & x & x\end{array}$

$x$
$x$
$x$
$x$
$x$
$x$
$x$
$x$
$x$
$x$
$x$
$x$
$x$
$x$

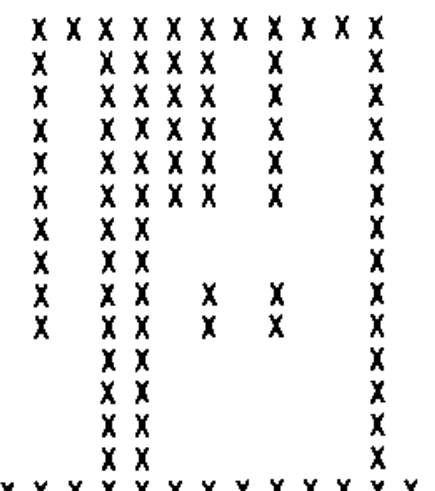

$\begin{array}{llllllllll}x & x & x & x & x & x & x & x & x & x\end{array}$

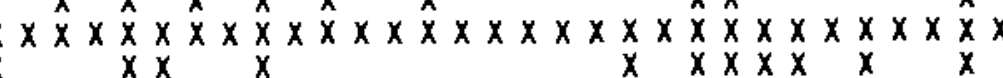

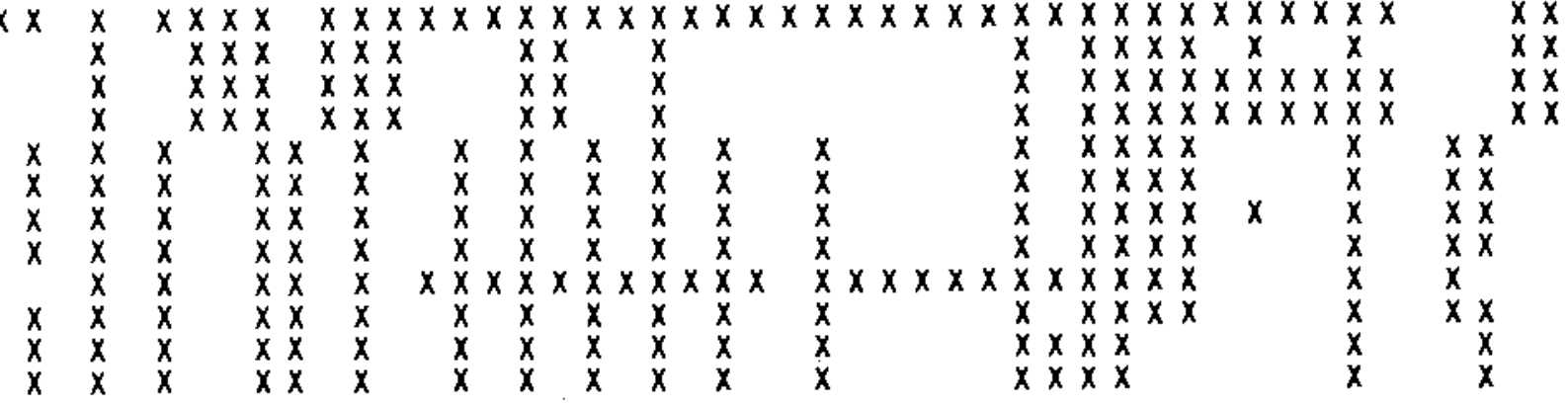


TABLE C.4. (contd)

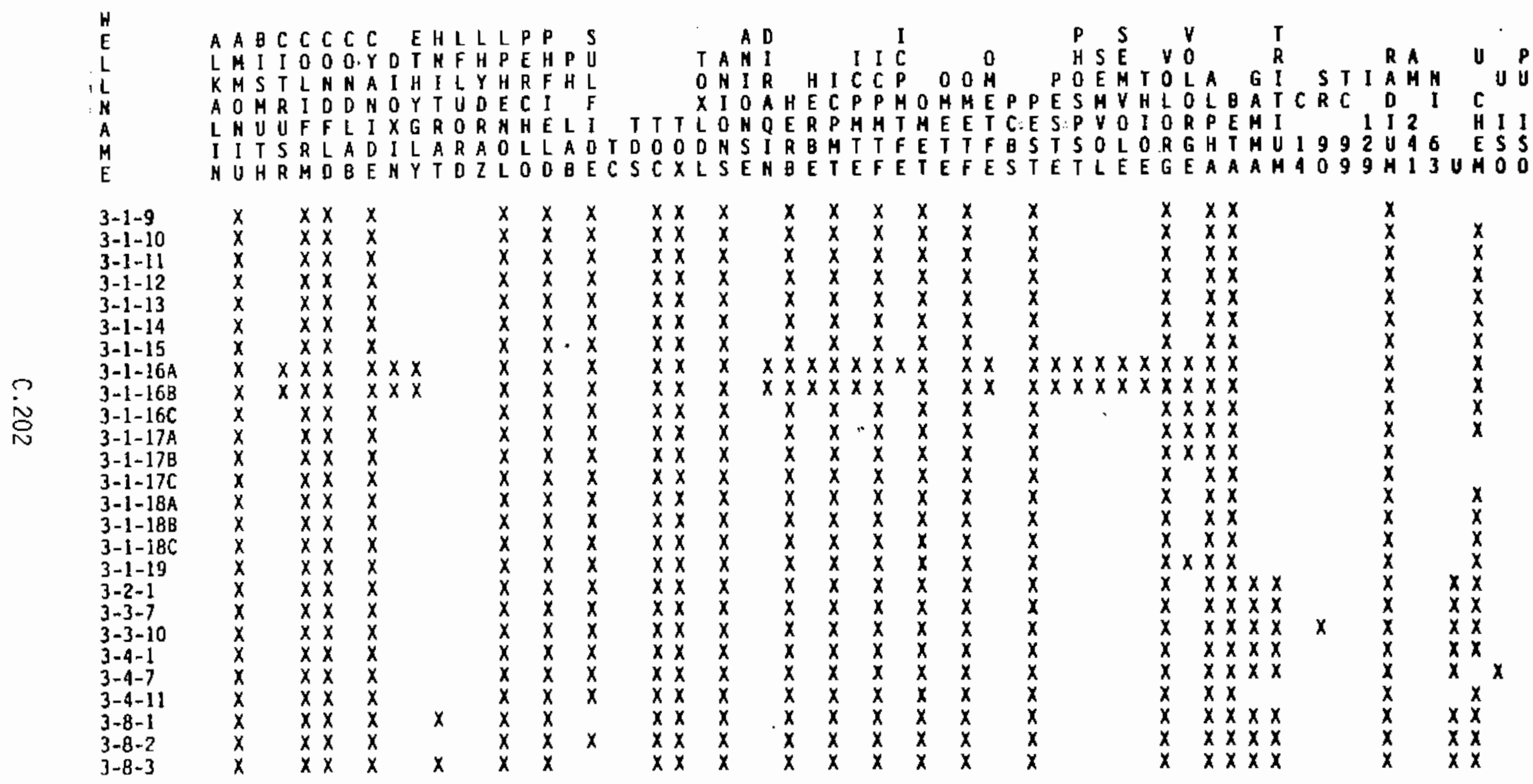




\section{IABLE C.4. (contd)}

$$
\begin{aligned}
& W \\
& E \\
& L \\
& L \\
& H \\
& A \\
& M \\
& E
\end{aligned}
$$

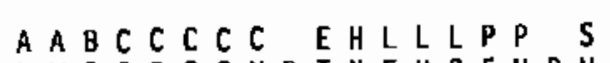

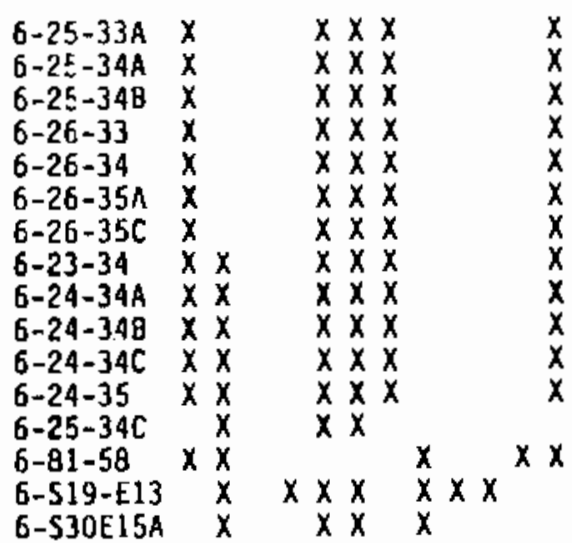

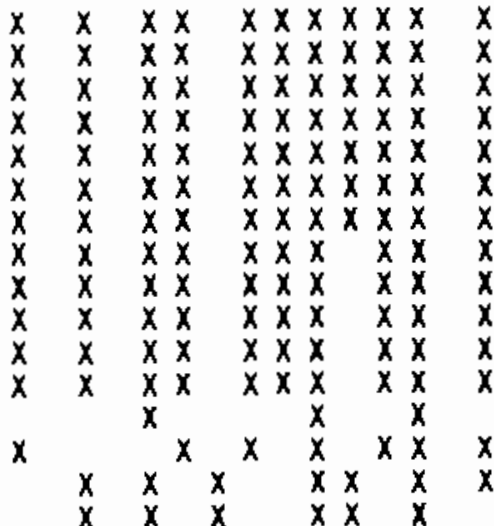

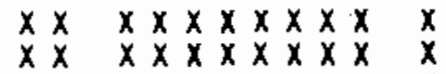

$\begin{array}{llllllllllll}x & x & x & x & x & x & x & x & x & x & x \\ x & x & x & x & x & x & x & x & x & x & x \\ x & x & x & x & x & x & x & x & x & x & x\end{array}$

$\begin{array}{llllllllll}x & x & x & x & x & x & x & x & x & x \\ x & x & x & x & x & x & x & x & x & x \\ x & x & x & x & x & x & x & x & x\end{array}$

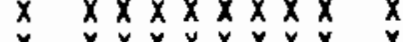

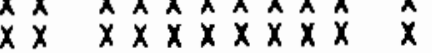

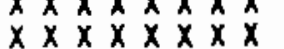

$\times \times \times \times \times \times \times \times \times \times$

$x \times x \times x \times x \times x$

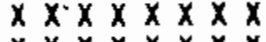

$x \times \times \times \times \times \times x$ $x$

6 -S30E15A $X$

$x \times x \times x \times$ 
TABLE C.5. Results for Compliance Monitoring Wells

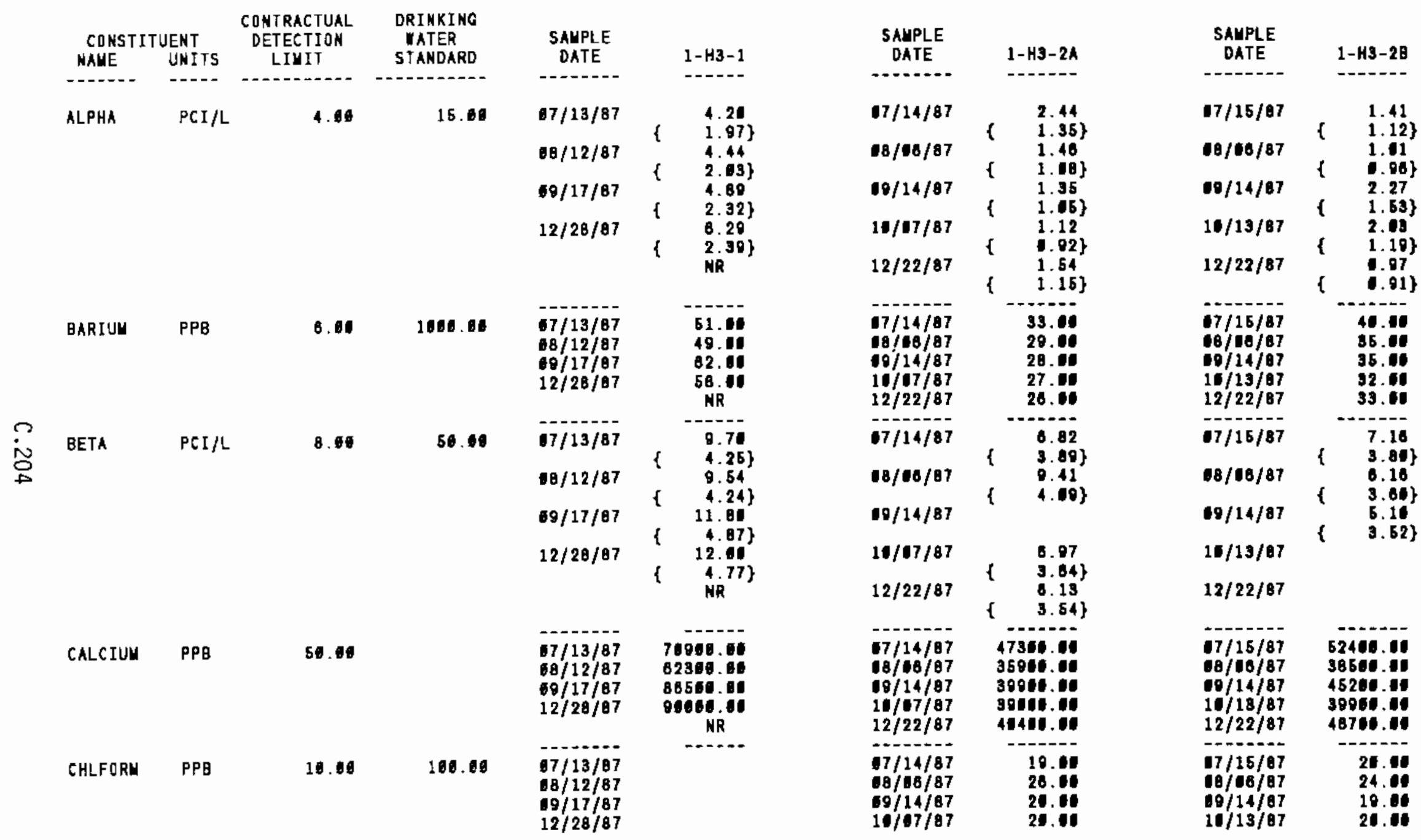

- - Value exceeds priUary dRINKINg vater standard.

- VALUE EXCEEDS PROPOSED PRIMARY DRINKING TATER STANDARD

- YALUE EXCEEDS SCREENING LEVEL FOR FURTHER INVESTIGATION.

- DETECTION liUit tas nOt aVailable for COMParison

NR - ANALYSIS NOT REQUESTED OR NOT YET REPORTED

YALUES IN \& ARE COUNTING ERRORS FOR RADIONUCLIDES

WATER STANDARO(S) IN PARENTHESES ARE PROPOSED ONLY 
TABLE C.5. (contd)

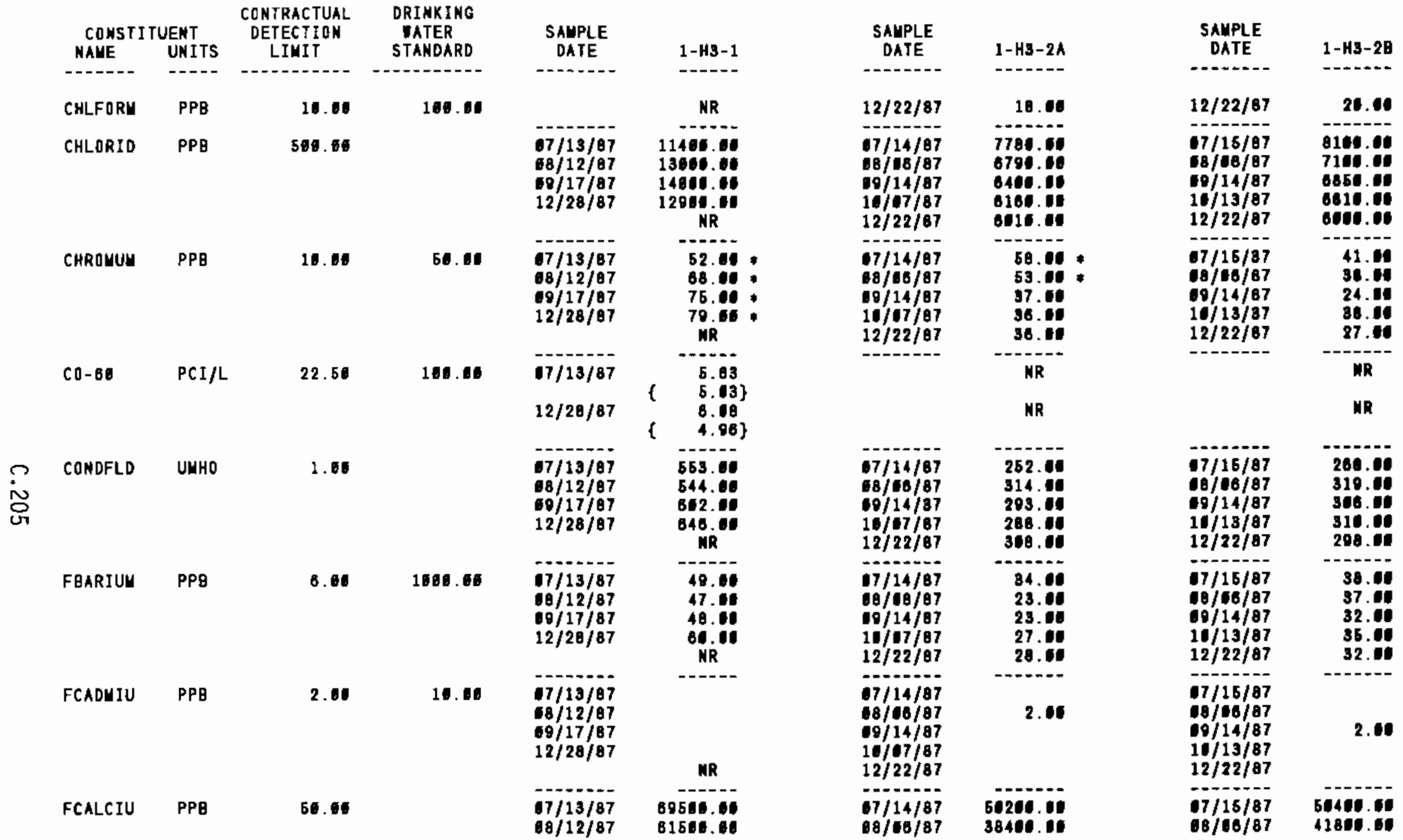

- VALUE EXCEEDS PRIUARY DRINKING TATER STANDARD.

- - VALUE EXCEEOS PROPOSED PRIUARY DRINKING TATER STANDARD

- - VALUE EXCEEDS SCREENIMG LEVEL FOR FURTHER INVESTIGATION.

- - DETECTION LIUIT UAS NOT AVAILABLE FOR CDUPARISOM

NR - ANALYSIS NOT REQUESTEO OR NOT YET REPORTED

VALUES IN \{\} ARE COUNTING ERRORS FOR RADIONUCLIDES

TATER STAMDARO(S) IN PARENTHESES ARE PROPOSED ONLY 
TABLE C.5. (contd)

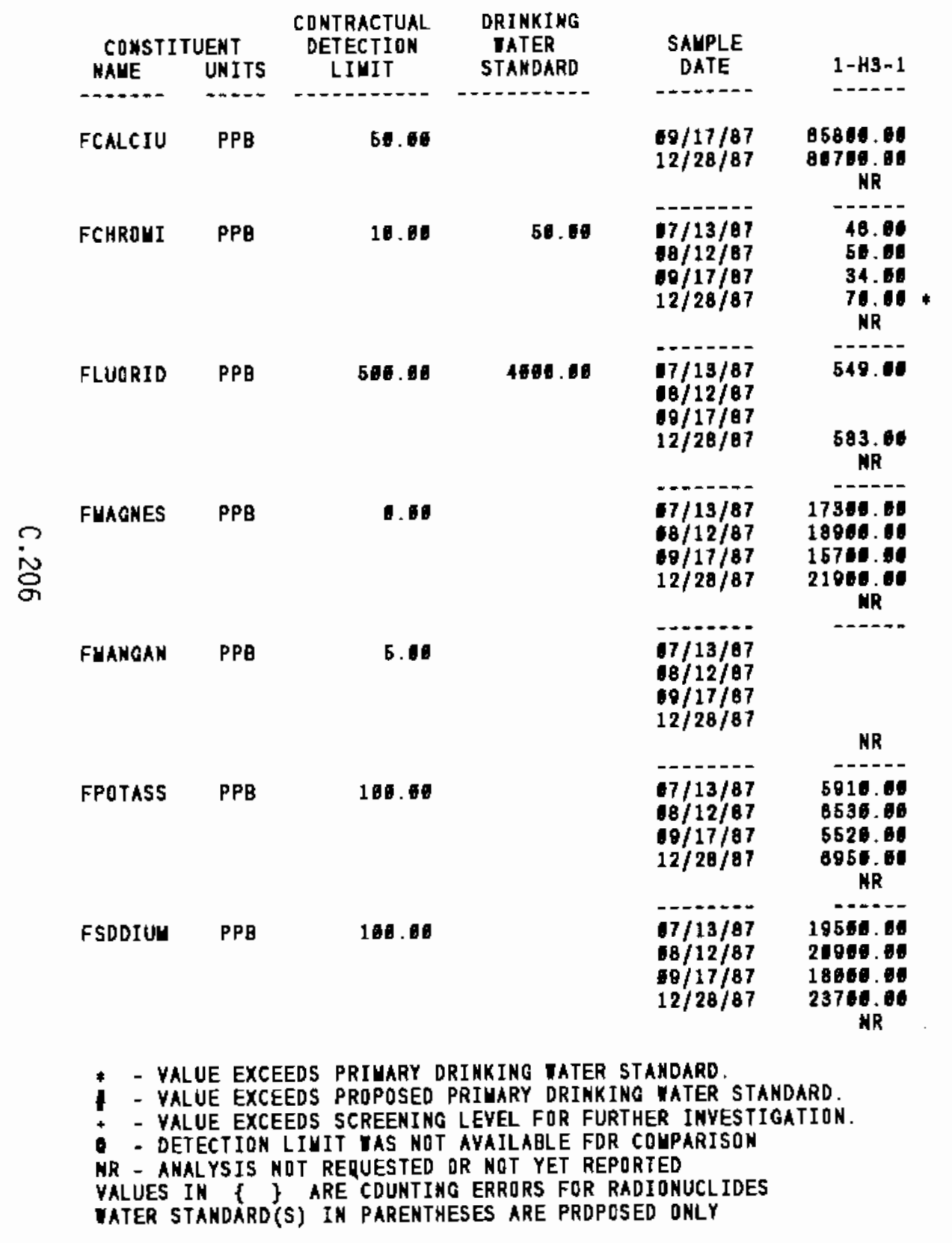

\begin{tabular}{|c|c|}
\hline $\begin{array}{l}\text { SALPLE } \\
\text { DATE }\end{array}$ & $1-\mathrm{Hg}-2 \mathrm{~A}$ \\
\hline $\begin{array}{l}00 / 14 / 87 \\
10 / 07 / 87 \\
12 / 22 / 87\end{array}$ & $\begin{array}{l}46100.04 \\
40756.06 \\
39106.06\end{array}$ \\
\hline $\begin{array}{l}1 / 14 / 87 \\
60 / 68 / 87 \\
19 / 14 / 87 \\
10 / 67 / 87 \\
12 / 22 / 87\end{array}$ & $\begin{array}{l}51.00 \\
46.00 \\
32.01 \\
20.01 \\
36.01\end{array}$ \\
\hline $\begin{array}{l}07 / 14 / 87 \\
68 / 66 / 87 \\
69 / 14 / 87 \\
18 / 67 / 87 \\
12 / 22 / 87\end{array}$ & \\
\hline $\begin{array}{l}7 / 14 / 87 \\
00 / 08 / 87 \\
09 / 14 / 87 \\
10 / / 97 / 87 \\
12 / 22 / 87\end{array}$ & 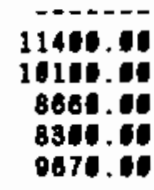 \\
\hline $\begin{array}{l}07 / 14 / 87 \\
08 / 66 / 87 \\
19 / 11 / 87 \\
10 / 107 / 87 \\
12 / 22 / 87\end{array}$ & \\
\hline $\begin{array}{l}67 / 14 / 87 \\
68 / 68 / 97 \\
00 / 14 / 87 \\
16 / 07 / 87 \\
12 / 22 / 87\end{array}$ & $\begin{array}{l}4789.60 \\
4496.10 \\
4210.10 \\
3836.10 \\
4846.00\end{array}$ \\
\hline $\begin{array}{l}07 / 14 / 87 \\
98 / 08 / 87 \\
09 / 14 / 87 \\
10 / 07 / 87 \\
12 / 22 / 87\end{array}$ & $\begin{array}{l}12804.00 \\
12904.00 \\
11800.00 \\
14104.00 \\
14200.00\end{array}$ \\
\hline
\end{tabular}

SAMPLE DATE $1-H 3-28$

09/14/87 45895.4

$10 / 13 / 87$ 4159/.

$12 / 22 / 87$ 41001.01

$0715 / 87 \quad 87.40$

$\begin{array}{ll}07 / 15 / 87 & 87.11 \\ 68 / 06 / 87 & 40.16\end{array}$

$19 / 14 / 87 \quad 32.16$

$11 / 13 / 87$ 31.H

$12 / 22 / 87$ 23.11

-115197

$60 / 00 / 87$

$10 / 14 / 87$

$10 / 13 / 87$

12/22/87

67/15/87

$18 / 18 / 87$

$6 / 14 / 87$

16/13/87

$12 / 22 / 87$

$07 / 15 / 87$

97/15/87

$09 / 14 / 87$

$10 / 13 / 87$

$12 / 22 / 87$

$7 / 15 / 87$

$18 / 60 / 87$

$10 / 14 / 87$

$10 / 13 / 87$

$12 / 22 / 87$

$07 / 15 / 67$

$18 / 06 / 87$

$69 / 14 / 87$

$10 / 13 / 87$

$12 / 22 / 87$

11201

11165.

2944.01

10105.65

1016.0

85.00

63.11

68.11

48.11

28. 01

sues.

658s.0

4970.06

512.

5131.06

10200.

1000.18

9871.1S

16106.00

11200.10 
TABLE C. .. (contd)

\begin{tabular}{|c|c|c|c|c|c|}
\hline \multicolumn{2}{|c|}{ CONSTITUENT } & $\begin{array}{c}\text { CENTRACTUAL } \\
\text { DETECTION } \\
\text { LIUIT }\end{array}$ & $\begin{array}{l}\text { DRINEING } \\
\text { WATER } \\
\text { STANOARO }\end{array}$ & $\begin{array}{c}\text { SANPLE } \\
\text { DATE }\end{array}$ & 1-H3-1 \\
\hline$-\cdots-+-$ & $-\cdots$ & 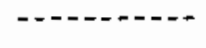 & - & $\cdots--1$ & ---- \\
\hline FSTRONT & PPB & 364.06 & & $\begin{array}{l}67 / 13 / 87 \\
18 / 12 / 87 \\
60 / 17 / 87 \\
12 / 26 / 87\end{array}$ & $\begin{array}{c}420.06 \\
456.09 \\
417.06 \\
518.04 \\
\text { MR }\end{array}$ \\
\hline FVANADI & PPB & 5.00 & & $\begin{array}{l}87 / 13 / 87 \\
68 / 12 / 87 \\
69 / 17 / 87 \\
12 / 28 / 87\end{array}$ & $\begin{array}{l}10.00 \\
10.01 \\
\text { B. } \\
\text { NR }\end{array}$ \\
\hline \multirow[t]{2}{*}{ FZINC } & PPB & 6. 10 & & $\begin{array}{l}17 / 13 / 87 \\
8 / 12 / 87 \\
10 / 17 / 87 \\
12 / 28 / 87\end{array}$ & $\begin{array}{l}6.00 \\
7.68\end{array}$ \\
\hline & & & & & MR \\
\hline IRON & PPB & 50.08 & & $\begin{array}{l}67 / 18 / 87 \\
08 / 12 / 87 \\
60 / 17 / 87 \\
12 / 28 / 87\end{array}$ & $\begin{array}{l}53.64 \\
240.68 \\
\text { MR }\end{array}$ \\
\hline WAGNES & PPB & 0.60 & & $\begin{array}{l}67 / 13 / 87 \\
18 / 12 / 87 \\
69 / 17 / 87 \\
12 / 28 / 87\end{array}$ & $\begin{array}{l}17300.06 \\
18468.04 \\
10900.00 \\
22401.06\end{array}$ \\
\hline UANGESE & PPB & 5.00 & & $\begin{array}{l}97 / 13 / 87 \\
66 / 12 / 87 \\
09 / 17 / 87 \\
12 / 28 / 87\end{array}$ & $\stackrel{9.60}{\text { MR }}$ \\
\hline METHYCH & PPB & 10.00 & & $\begin{array}{l}17 / 13 / 87 \\
68 / 12 / 67\end{array}$ & $\cdots-$ \\
\hline \multicolumn{6}{|c|}{ 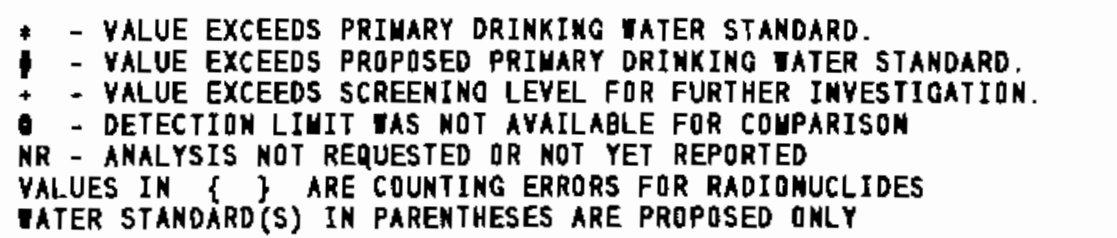 } \\
\hline
\end{tabular}

\begin{tabular}{|c|c|}
\hline $\begin{array}{l}\text { SAMPLE } \\
\text { DATE }\end{array}$ & $1-H 3-2 A$ \\
\hline -..-non & $---\infty+\infty$ \\
\hline $\begin{array}{l}07 / 14 / 87 \\
10 / 66 / 87 \\
00 / 14 / 87 \\
10 / 87 / 87 \\
12 / 22 / 87\end{array}$ & \\
\hline $\begin{array}{l}7 / 14 / 87 \\
88 / 08 / 87 \\
19 / 14 / 87 \\
16 / 67 / 87 \\
12 / 22 / 87\end{array}$ & $\begin{array}{r}10.00 \\
18.00 \\
10.00 \\
9.00\end{array}$ \\
\hline $\begin{array}{l}17 / 14 / 87 \\
18 / 68 / 87 \\
0 / 14 / 87 \\
10 / 67 / 87 \\
12 / 22 / 87\end{array}$ & \\
\hline $\begin{array}{l}97 / 14 / 87 \\
68 / 108 / 87 \\
99 / 14 / 87 \\
16 / 87 / 87 \\
12 / 22 / 87\end{array}$ & $\begin{array}{l}98.00 \\
80.40 \\
02.01 \\
72.40 \\
50.51\end{array}$ \\
\hline $\begin{array}{l}07 / 14 / 87 \\
08 / 66 / 87 \\
08 / 14 / 87 \\
10 / 07 / 87 \\
12 / 22 / 87\end{array}$ & 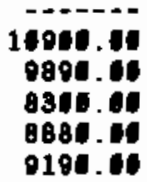 \\
\hline $\begin{array}{l}07 / 14 / 87 \\
08 / 66 / 87 \\
0 / 14 / 67 \\
16 / 67 / 87 \\
12 / 22 / 87\end{array}$ & -..-- \\
\hline $\begin{array}{l}97 / 14 / 87 \\
06 / 98 / 87\end{array}$ & \\
\hline
\end{tabular}

SALPLE DATE -

$1-43-2 B$

07/16/87

16/68/87

60/14/87

$10 / 13 / 67$

-בר-...

6/16/a7

09/10/87

$6 / 14 / 87$
$00 / 1 / 87$

$6 / 14 / 87$
$1 / 13 / 87$

$1 / 13 / 67$
$12 / 22 / 67$

$1 / 15 / 67$

68/68/87

09/14/87

16/13/87

$12 / 22 / 87$

67/15/87

$67 / 15 / 87$
$68 / 18 / 87$

60/14/87

1//13/67

12/22/07

-1/15/87

1810187



19/14/87

$10 / 13 / 87$

12/22/87

1/15/a7

$08 / 06 / 87$

18/14/87

$10 / 13 / 87$

$12 / 22 / 87$

$12 / 22 / 87$

$7 / 16 / 87$

$57 / 16 / 87$
$68 / 16 / 87$ 
TABLE C.5. (contd)

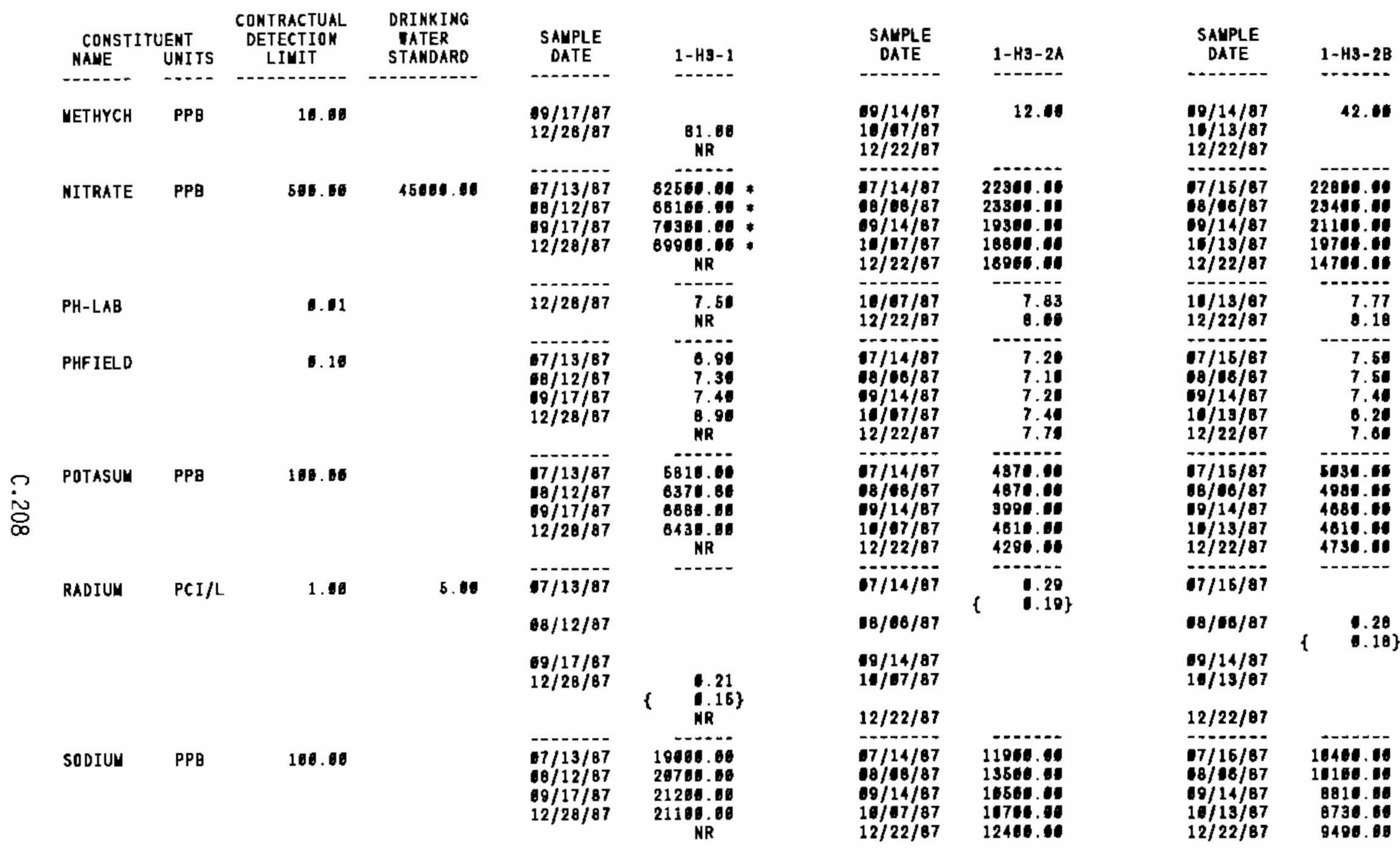

* - VALUE EXCEEOS PRIUARY dRINKING iater STANDARD

* - VALUE EXCEEOS PROPOSED PRIUARY DRINKING IATER STANDARD.

- VALUE EXCEEDS SCREENING LEVEL FOR FURTHER INYESTIGATION.

- DETECTION LIUI TAS NOT AVAILABLE FOR COUPARISON

NR - ANALYSIS NOT REQUESTED OR NOT YET REPORTED

VALUES IN \& $\}$ ARE COUNTING ERRORS FOR RADIONUCLIDES

WATER STANDARD'S) IN PARENTHESES ARE PROPOSED ONLY 
TABLE C.5. (coritd)

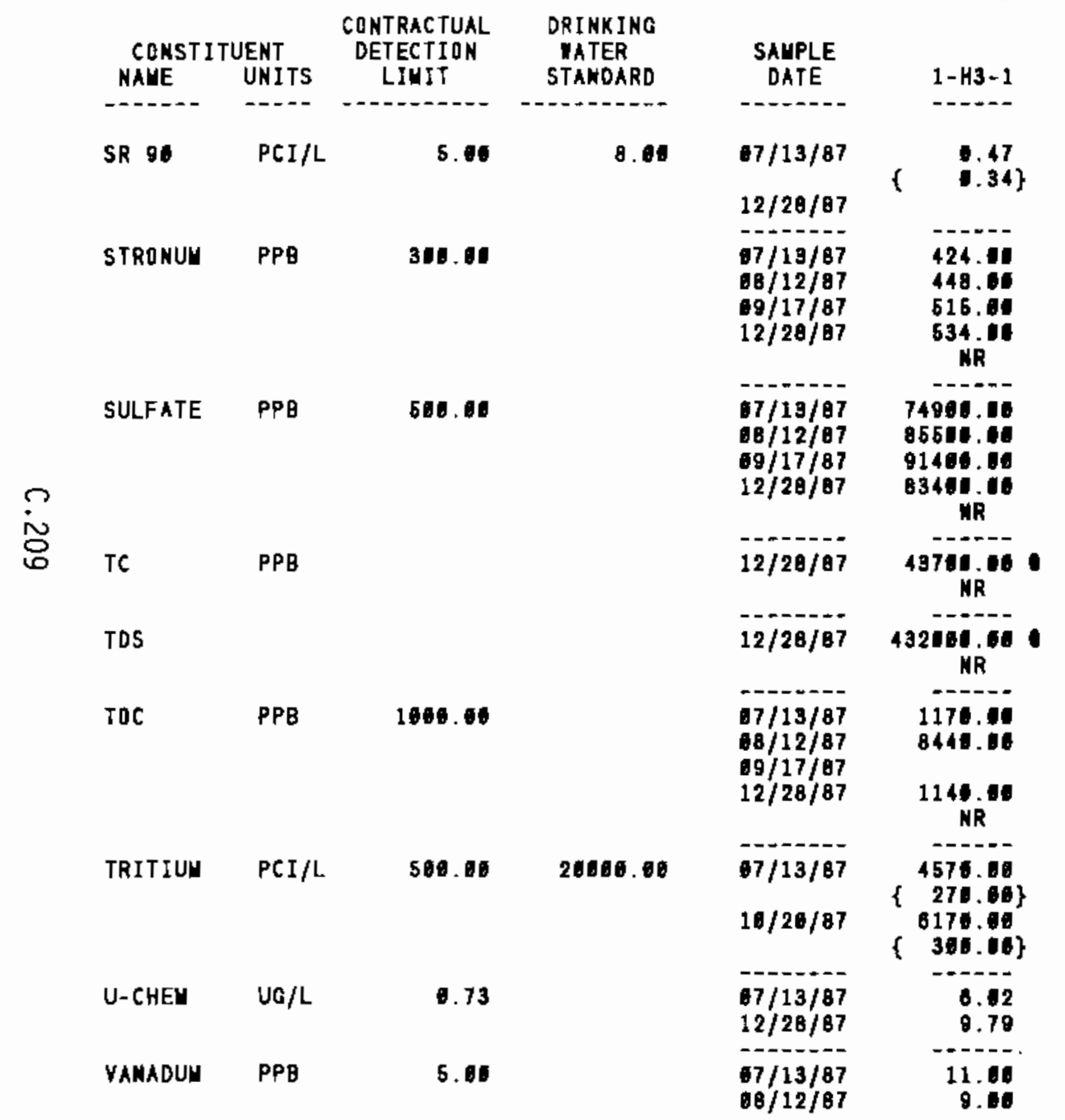

\begin{tabular}{|c|c|c|c|}
\hline $\begin{array}{c}\text { SAUPLE } \\
\text { DATE }\end{array}$ & $1-H_{3}-2 A$ & $\begin{array}{c}\text { SAMPLE } \\
\text { DATE }\end{array}$ & $1-\mathbf{H 3 - 2 B}$ \\
\hline & MR & & NR \\
\hline & NR & & NR \\
\hline $\begin{array}{l}17 / 14 / 87 \\
08 / 68 / 87 \\
19 / 14 / 87 \\
10 / 67 / 87 \\
12 / 22 / 87\end{array}$ & & $\begin{array}{l}07 / 15 / 87 \\
18 / 66 / 87 \\
00 / 14 / 87 \\
10 / 18 / 87 \\
12 / 22 / 87\end{array}$ & \\
\hline $\begin{array}{l}17 / 14 / 87 \\
68 / 06 / 87 \\
09 / 14 / 87 \\
16 / 67 / 87 \\
12 / 22 / 67\end{array}$ & 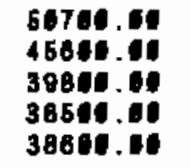 & $\begin{array}{l}17 / 16 / 87 \\
68 / 16 / 87 \\
19 / 14 / 87 \\
16 / 13 / 87 \\
12 / 22 / 87\end{array}$ & 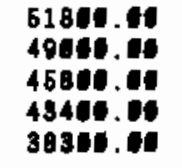 \\
\hline $\begin{array}{l}10 / 07 / 87 \\
12 / 22 / 87\end{array}$ & $\begin{array}{l}250 \mathrm{~A} \\
24 \mathrm{Bat}\end{array}$ & $\begin{array}{l}10 / 13 / 87 \\
12 / 22 / 87\end{array}$ & $\begin{array}{l}26104.00 \\
23000.16\end{array}$ \\
\hline $\begin{array}{l}18 / 97 / 07 \\
12 / 22 / 87\end{array}$ & $\begin{array}{l}208464.06 \\
217640.01\end{array}$ & $\begin{array}{l}11 / 13 / 87 \\
12 / 22 / 87\end{array}$ & $\begin{array}{l}224000.18 \\
100400.18\end{array}$ \\
\hline $\begin{array}{l}1 / 14 / 87 \\
68 / 06 / 87 \\
68 / 14 / 87 \\
16 / 67 / 87 \\
12 / 22 / 87\end{array}$ & & $\begin{array}{l}07 / 15 / 87 \\
08 / 96 / 87 \\
00 / 14 / 87 \\
10 / 19 / 87 \\
12 / 22 / 87\end{array}$ & 1020.00 \\
\hline & MR & & NR \\
\hline & $N R$ & & NR \\
\hline . & $\begin{array}{l}\text { NR } \\
\text { NR }\end{array}$ & - & $\begin{array}{l}\text { MR } \\
M R\end{array}$ \\
\hline $\begin{array}{l}07 / 14 / 87 \\
188 / 08 / 87\end{array}$ & 10.00 & $\begin{array}{l}87 / 16 / 87 \\
6 / 108 / 87\end{array}$ & 9.16 \\
\hline
\end{tabular}

* - VALUE EXCEEDS PRIHARY DRINKING waTER STANDARD.

- VALUE EXCEEDS PROPOSED PRIHARY DRINKING TATER STANOARD.

- - YALUE EXCEEDS SCREENING LEYEL FUR FURTHER INYESTIGATION.

- DETECTION LIVIT WAS NOT AVAILABLE FDR COMPARISON

NR - ANALYSIS NOT REQUESTED DR NOT YET REPDRTED

YALUES IN I A ARE COUNTINO ERRORS FOR RADIONUCLIDES

IATER STANDARD'S) IN PARENTHESES ARE PRDPDSED ONLY 


\begin{tabular}{|c|c|c|c|c|c|c|c|c|c|}
\hline $\begin{array}{c}\text { CONSTI } \\
\text { NAME }\end{array}$ & $\begin{array}{l}\text { TUERT } \\
\text { UNITS }\end{array}$ & $\begin{array}{l}\text { CONTRACTUAL } \\
\text { DETECTION } \\
\text { LIMIT }\end{array}$ & $\begin{array}{l}\text { DRINKING } \\
\text { IATER } \\
\text { STANDARD }\end{array}$ & $\begin{array}{c}\text { SAMPLE } \\
\text { DATE }\end{array}$ & $1-\mathrm{H}_{3}-1$ & $\begin{array}{c}\text { SAMPLE } \\
\text { DATE }\end{array}$ & $1-\mathrm{H}_{3}-2 \mathrm{~A}$ & $\begin{array}{l}\text { SAMPLE } \\
\text { DATE }\end{array}$ & 1-H3-2B \\
\hline VANADUU & PPB & 5.00 & & $\begin{array}{l}19 / 17 / 87 \\
12 / 28 / 87\end{array}$ & $\begin{array}{c}12.11 \\
7.81 \\
N R\end{array}$ & $\begin{array}{l}69 / 14 / 87 \\
10 / 678787 \\
12 / 22 / 87\end{array}$ & 10.80 & $\begin{array}{l}09 / 14 / 87 \\
11 / 19887 \\
12 / 22 / 87\end{array}$ & 0.01 \\
\hline ZINC & PPB & 5.06 & & $\begin{array}{l}87 / 13 / 87 \\
88 / 12 / 87 \\
89 / 17 / 87 \\
12 / 28 / 87\end{array}$ & $\begin{array}{l}7.10 \\
5.10 \\
N R\end{array}$ & $\begin{array}{l}97 / 14 / 87 \\
08 / 0187 \\
89 / 14 / 87 \\
10 / 107 / 87 \\
12 / 22 / 87\end{array}$ & $\begin{array}{l}7.10 \\
6.10 \\
6.10\end{array}$ & $\begin{array}{l}07 / 15 / 87 \\
08 / 168 / 87 \\
09 / 14 / 87 \\
16 / 13 / 87 \\
12 / 22 / 87\end{array}$ & 8.40 \\
\hline
\end{tabular}

* - Value exceeds primary dRINking tater standard

1 - VALUE EXCEEDS PROPOSED PRIUARY DRINKIng tater STAMDARD.

* - YALUE EXCEEDS SCREENING LEYEL FOR FURTHER INVESTIGATION.

- detection livit yas not available for couparisok

NR - AMALYSIS NOT REQUESTED OR NOT YET REPORTED

VALUES IN \& ARE COUNTIKG ERRORS FOR RADIONUCLIDES

WATER STANDARD'S( IN PARENTHESES ARE PROPOSED ONLY 
IABLE C.5. (contd)

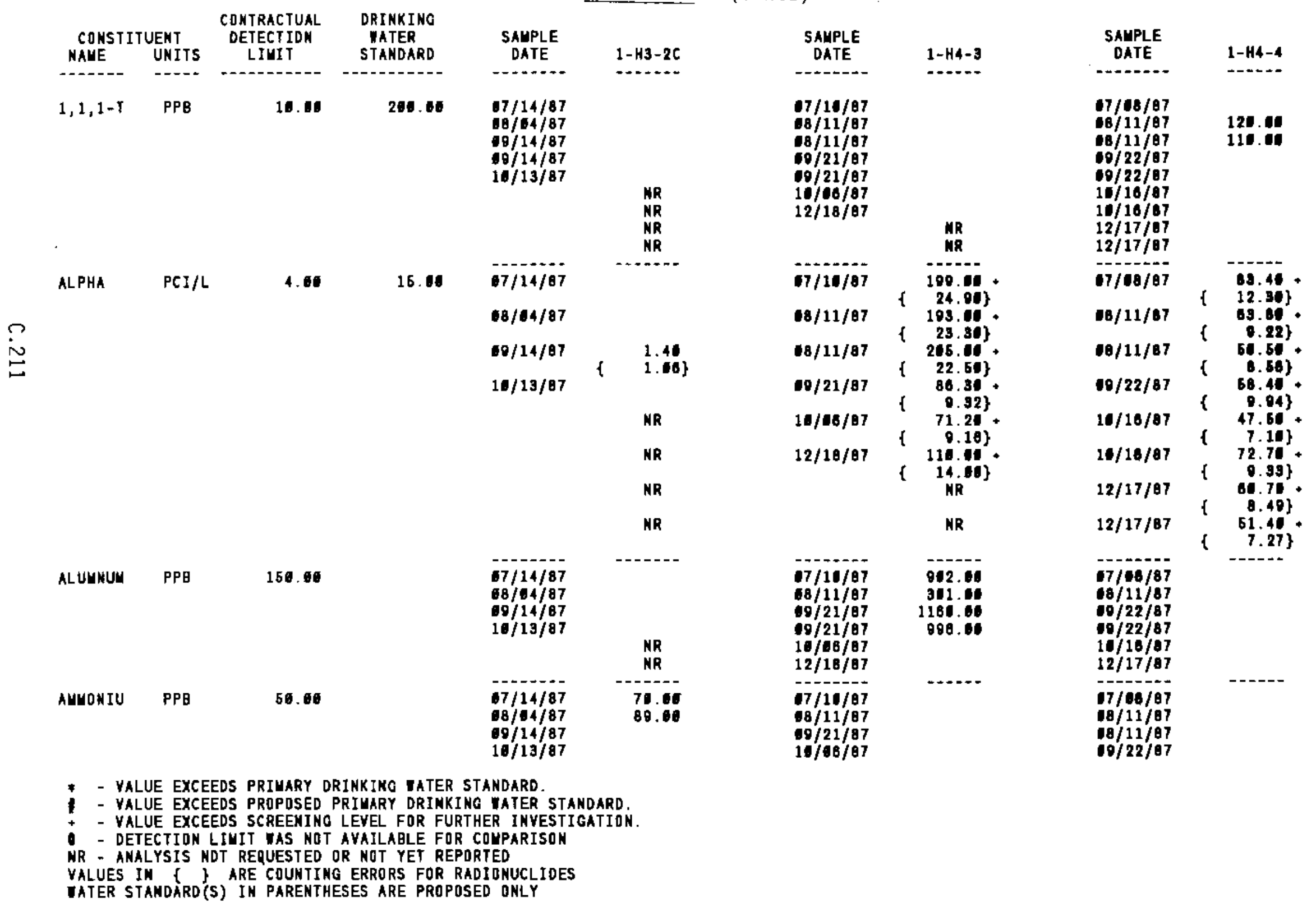




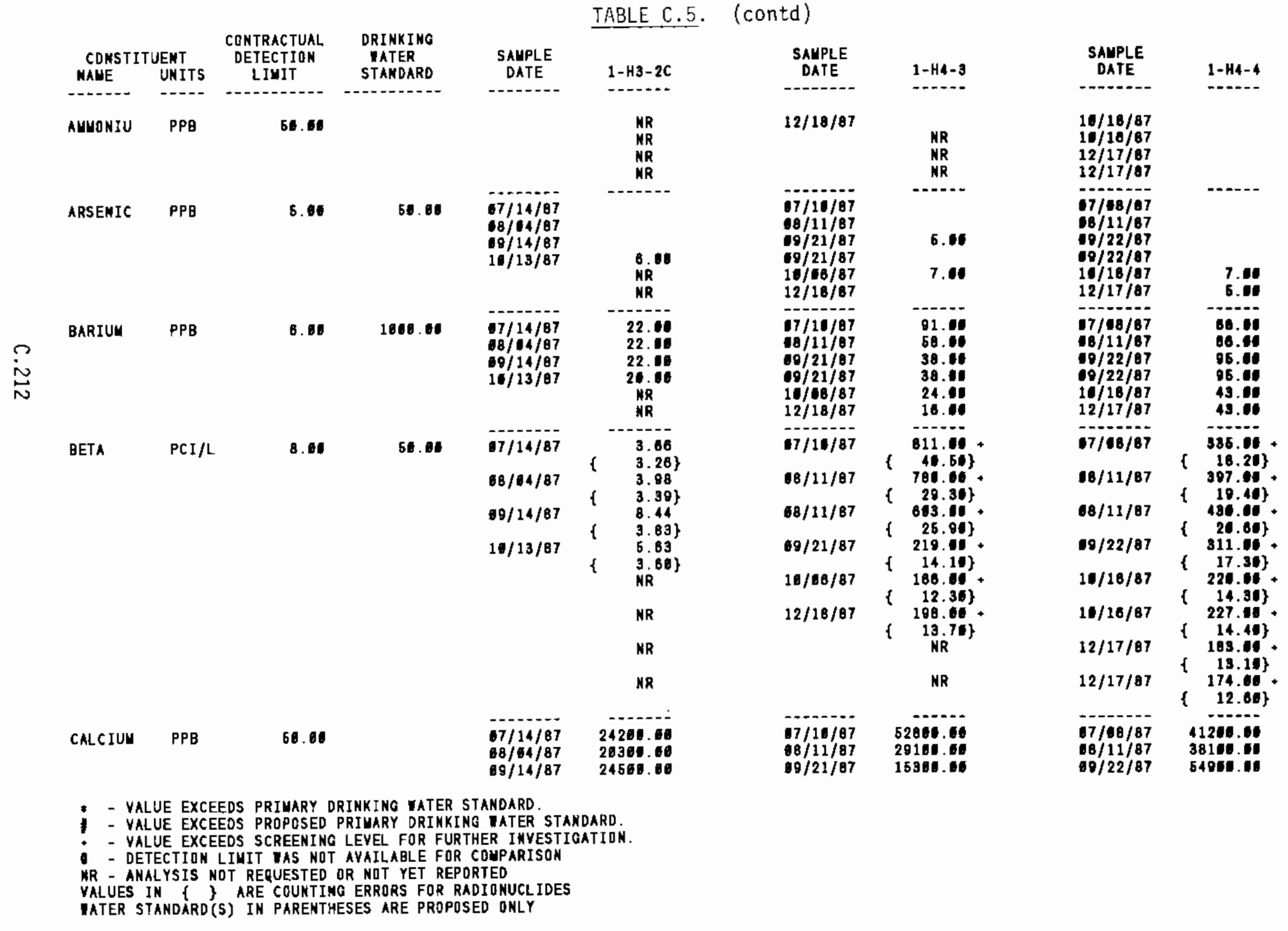




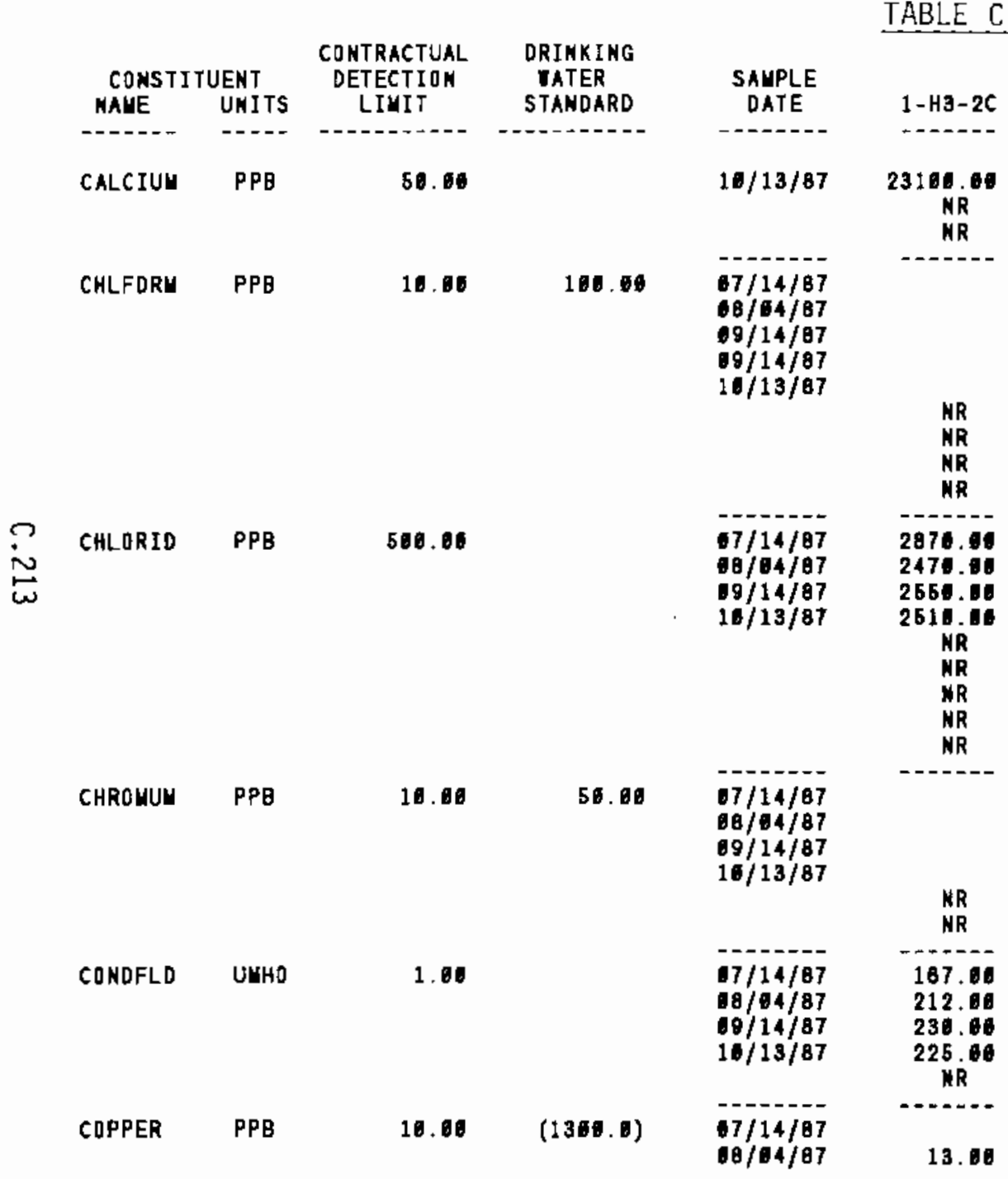

SALAPLE

DATE $1-H 4-3$

SAUPLE

DATE 1-H4-4

69/22/87 55900.

$\begin{array}{llll}10 / 66 / 87 & 13466.06 & 16 / 16 / 87 & 27180.60 \\ 12 / 18 / 87 & 12460.00 & 12 / 17 / 87 & 4334.60\end{array}$

$\begin{array}{llll}10 / 66 / 87 & 13466.06 & 16 / 16 / 87 & 27180.60 \\ 12 / 18 / 87 & 12460.00 & 12 / 17 / 87 & 4334.60\end{array}$

77/18/87

9 $111 / 87$

a)/11/87

12.01

9 $121 / 87-18.00$

$69 / 21 / 87 \quad 18.00$

$\begin{array}{llll}10 / 06 / 87 & 15.00 & 10 / 16 / 87 & 11.68 \\ 12 / 18 / 87 & 17.06 & 10 / 18 / 87 & 11.6\end{array}$

$10 / 16 / 87$

$12 / 17 / 87$

- $7108 / 87$

$67 / 10 / 87 \quad 10609.60$

$8 / 11 / 87 \quad 10500.06$

$8 / 11 / 87 \quad 10386.04$

$0 / 21 / 87 \quad 8156.00$

8120.00

8541.60

$12 / 18 / 87$

9110.00

NR

$07 / 08 / 8$

$68 / 11 / 67$

$68 / 11 / 97$

$19 / 22 / 87$

$19 / 22 / 87$

$10 / 18 / 87$

$10 / 18 / 87$

$12 / 17 / 07$

$12 / 17 / 87$

7110187

$07 / 10 / 87$

$68 / 11 / 87$
$09 / 21 / 87$

397.10

0708187

$69 / 21 / 87$
$69 / 21 / 87$

308.60

$07 / 68 / 87$

242.00

$68 / 11 / 87$

$60 / 22 / 87$

$16 / 66 / 87$

245.00

$10 / 16 / 87$

$12 / 18 / 87$

07/10/87

242.00

$12 / 17 / 8$

2020.

17/68/87

$88 / 11 / 87$

$09 / 21 / 87$

$10 / 66 / 87$

1621.09

0102187

1128.00

937.06

$12 / 18 / 87$

926.0

19/10/87

$12 / 17 / 87$

$07 / 10 / 07$

48.00

$07 / 68 / 87$

$68 / 11 / 87 \quad 25.60$

$98 / 11 / 87$

6134.

8750.10

8710.0

6896.00

6736.01

6260.01

8340.00

5950.10

6958.00

281.01

289.01

289.0

$337.00:$

337.00 .

217.00

234.08 *

752.60

021.69

1224.01

709.05

- VALUE EXCEEDS PRIUARY DRINKING TATER STAMDARD

- - VALUE EXCEEDS PROPBSED PRIUARY DRINKING TATER STANDARD.

- - yalue exCeEds screening leyel for fuRTHER inVestigation.

- DETECTION LIUIT TAS NOT AYAILABLE FOR COUPARISON

NR - ANALYSIS NOT RERUESTED OR NOT YET REPDRTED

YALUES IN \{ $\}$ ARE COUNTIHG ERRORS FOR RADIONUCLIDES

waTER STANDARD(S) IN PARENTHESES ARE PROPOSED ONLY 
TABLE C.5. (contd)

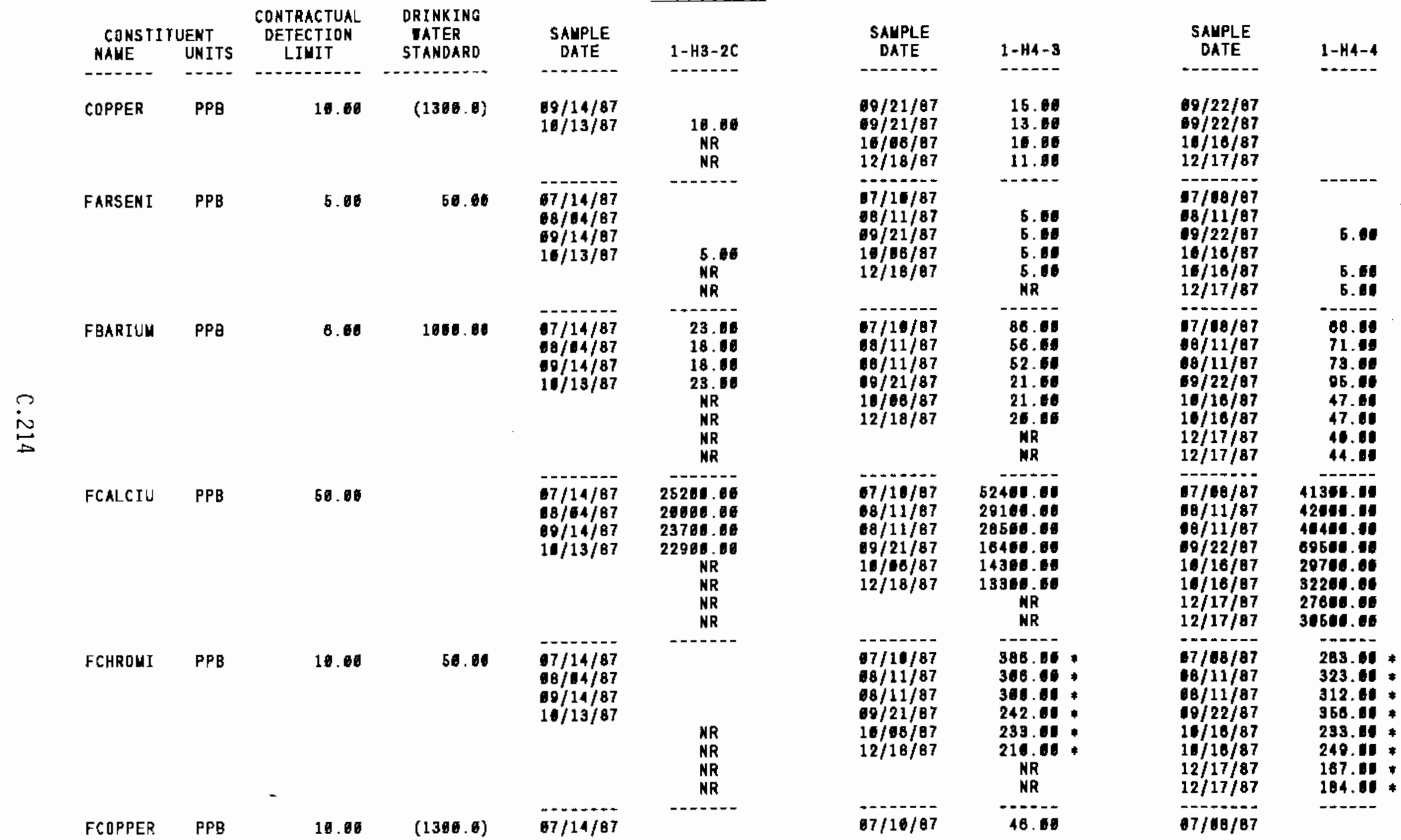

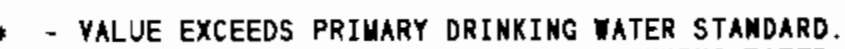

- VALUE EXCEEDS PROPOSED PRIMARY DRINKING TATER STANDARD.

- VALUE EXCEEDS SCREENING LEVEL FOR FURTHER INYESTIGATION

- DETECtion linit ias nOt available for couparison

NR - ANALYSIS NOT REQUESTED DR MOT YET REPORTED

YALUES IN \{\} ARE COUNTING ERRORS FOR RADIDNUCLIDES

WATER STANDARD(S) IN PARENTHESES ARE PROPOSED ONLY 
TABLE C.5. (contd)

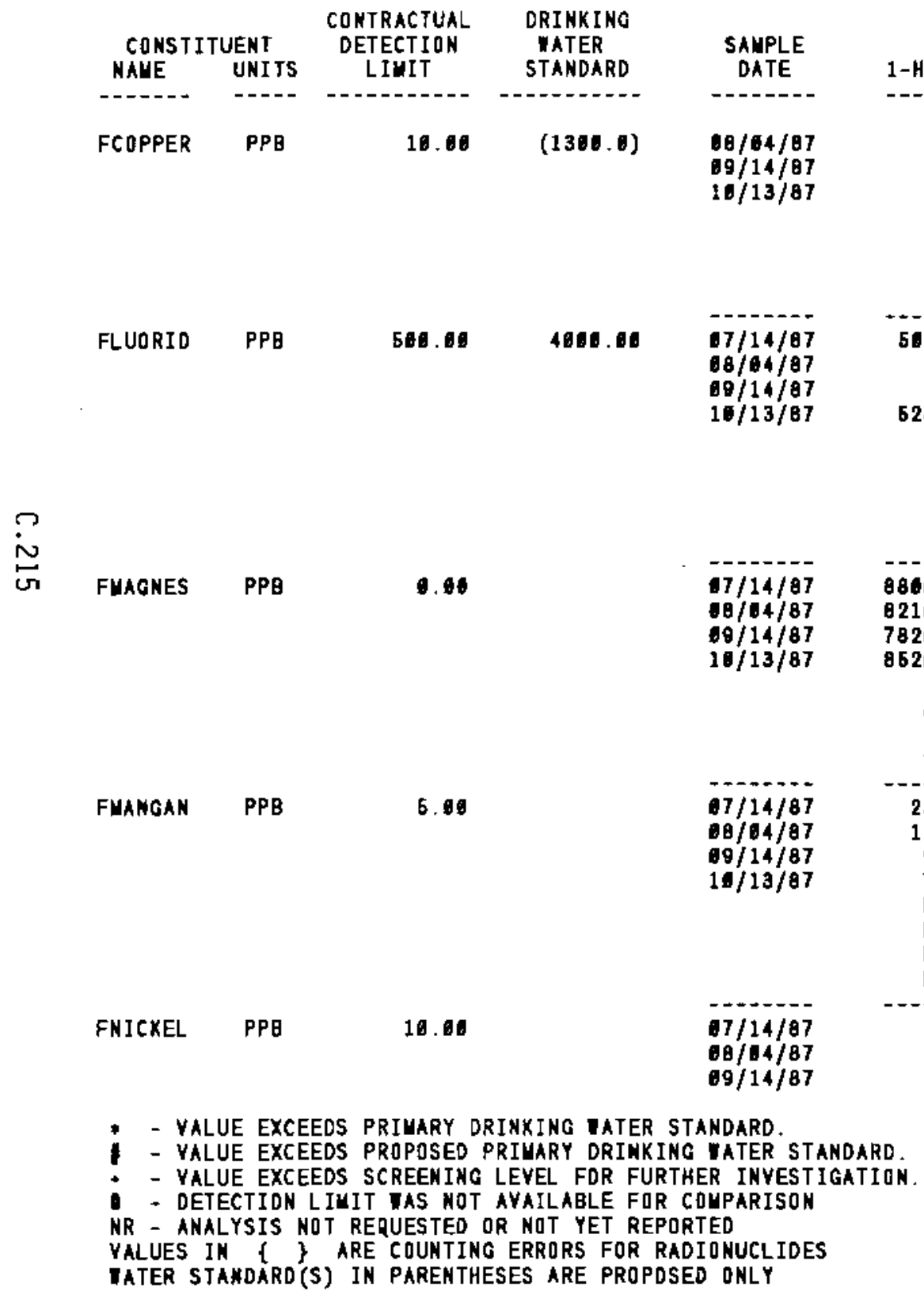

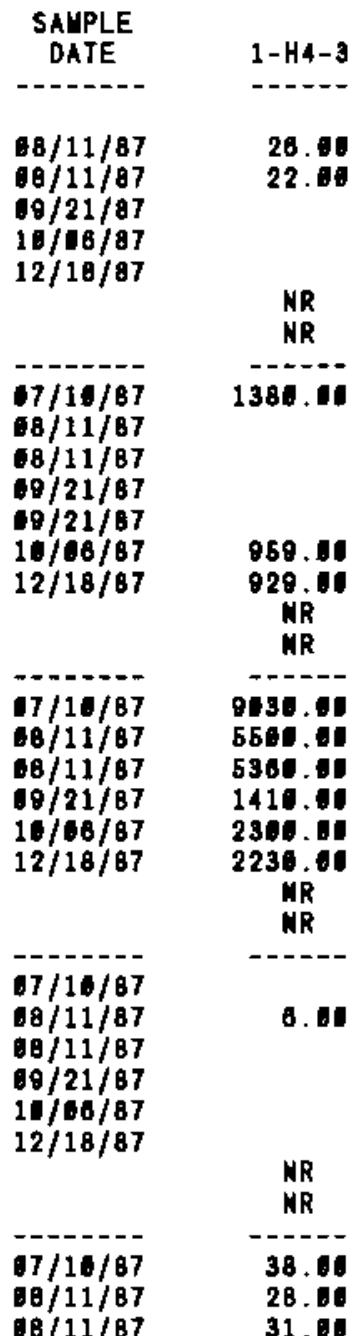
DATE

1-H4-4

$----$

NR

MR
MR
MR

NR

500.00

628.00

NR
NR
NR

NR
NR
NR

NR
NR

9860.00

0210.0

7820.00

.0

HR

MR

WR

23.60

11.60

9.60

NR

NR

NR

NR

38.10

31.00
-------

68/11/87

68/11/87

09/22/87

$16 / 16 / 87$

$18 / 18 / 87$

$12 / 17 / 87$

$12 / 17 / 87$

$12 / 17 / 87$

$17 / 68 / 87$

$00 / 11 / 87$

01/11/87

10/22/87

00/22/87

$14 / 18 / 87$

$10 / 16 / 87$

$12 / 17 / 67$

$12 / 17 / 87$

17/68/87

10/11/87

18/11/87

10/22/87

$16 / 16 / 87$

$16 / 16 / 8$

12/17/9

12/17/9

$12 / 17 / 87$

67/08/87

$68 / 11 / 87$

8/11/87

$50 / 22 / 87$

10/18/87

$10 / 18 / 87$

$12 / 17 / 87$

$12 / 17 / 87$

$12 / 17 / 67$

17/68/87

98/11/87

0/11/87
755

546.01

640.0

515.09

B85.

653.0

685.00

6800.00

7201.41

71430.

8740.01

4746.

4831.

435.0

4376.0

469. 04

--.-.

10.60

17.6

15. 
TABLE C.5. (contd)

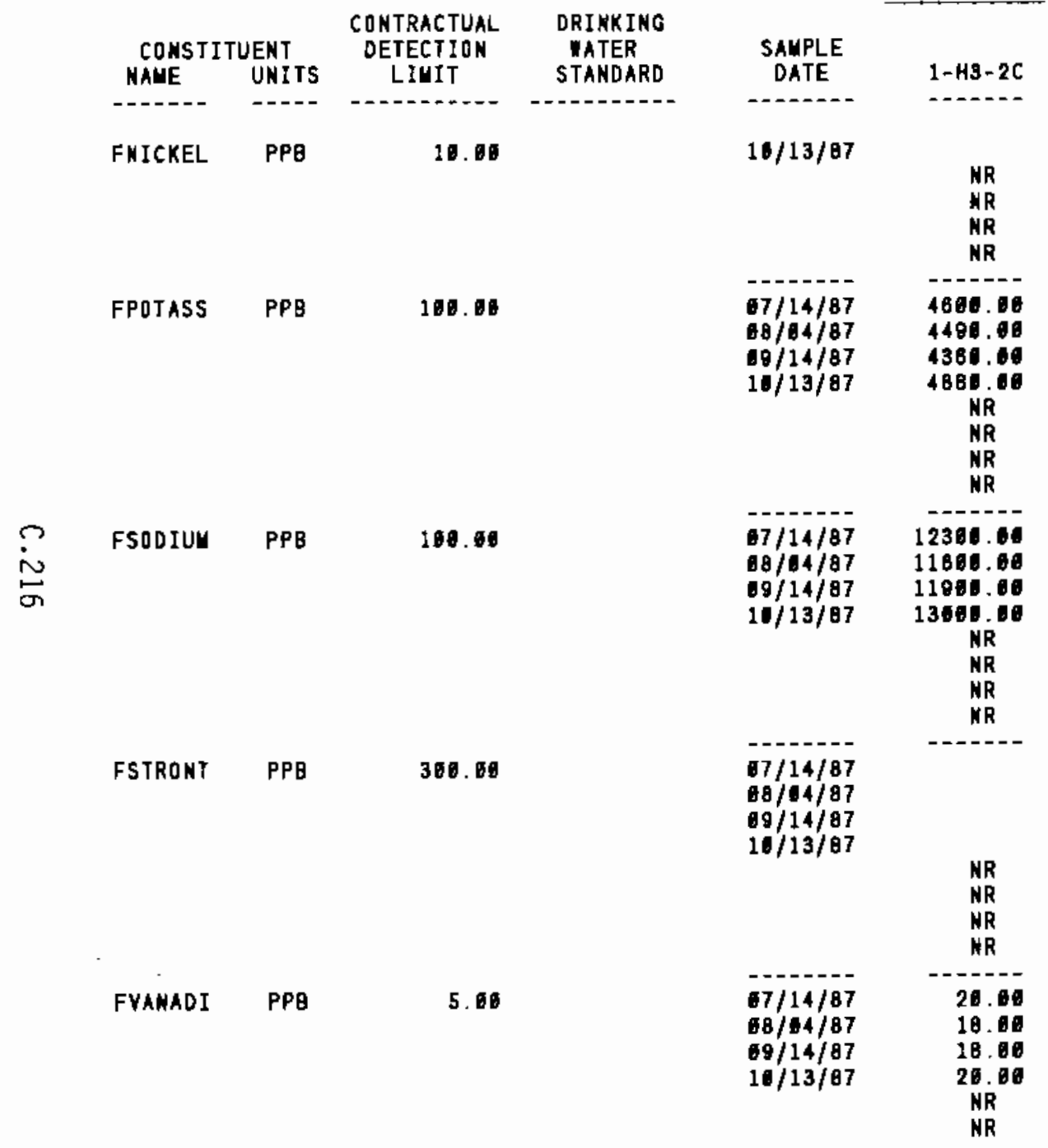

\begin{tabular}{|c|c|}
\hline $\begin{array}{c}\text { SALPLE } \\
\text { DATE }\end{array}$ & 1-H4-3 \\
\hline ------- & ……- \\
\hline $\begin{array}{l}00 / 21 / 87 \\
10 / 06 / 87 \\
12 / 18 / 87\end{array}$ & $\begin{array}{c}11.00 \\
11.00 \\
\text { NR } \\
\text { NR }\end{array}$ \\
\hline $\begin{array}{l}67 / 10 / 87 \\
68 / 11 / 87 \\
68 / 11 / 87 \\
60 / 21 / 87 \\
10 / 06 / 87 \\
12 / 18 / 87\end{array}$ & $\begin{array}{c}7840.08 \\
6176.00 \\
6040.00 \\
2200.66 \\
3609.16 \\
3480.10 \\
\text { NR } \\
\text { MR }\end{array}$ \\
\hline $\begin{array}{l}67 / 10 / 87 \\
68 / 11 / 87 \\
18 / 11 / 87 \\
69 / 21 / 87 \\
10 / 16 / 87 \\
12 / 18 / 87\end{array}$ & $\begin{array}{c}442000.00 \\
310040.06 \\
314600.06 \\
222000.00 \\
178000.01 \\
191000.00 \\
\text { KR } \\
\text { KR }\end{array}$ \\
\hline \multirow[t]{2}{*}{$\begin{array}{l}07 / 10 / 87 \\
68 / 11 / 87 \\
68 / 11 / 87 \\
09 / 21 / 87 \\
10 / 06 / 87 \\
12 / 18 / 87\end{array}$} & 312.6 \\
\hline & $\begin{array}{l}\text { MR } \\
\text { MR }\end{array}$ \\
\hline $\begin{array}{l}67 / 10 / 87 \\
68 / 11 / 87 \\
68 / 11 / 87 \\
68 / 21 / 87 \\
10 / 86 / 87 \\
12 / 18 / 87\end{array}$ & $\begin{array}{r}11.00 \\
8.00 \\
6.00 \\
11.00 \\
0.00\end{array}$ \\
\hline
\end{tabular}

\begin{tabular}{l} 
SAHPLE \\
DATE \\
\hline
\end{tabular}

10/22/87 14.16

$10 / 16 / 87$
$10 / 16 / 87$

$10 / 18 / 87$

$12 / 17 / 87$

$07 / 08 / 97$

$68 / 11 / 87$

$68 / 11 / 87$

$60 / 22 / 87$

$10 / 16 / 87$

10/16/87

$12 / 17 / 87$

$12 / 17 / 87$

17/08/07

$68 / 11 / 87$

$08 / 11 / 87$

$08 / 22 / 87$

$10 / 18 / 87$

$10 / 10 / 87$

$12 / 17 / 67$

$12 / 17 / 87$

17/08/87

$08 / 11 / 87$

$08 / 11 / 87$

-9/22/87

10/16/87

$16 / 16 / 87$

$12 / 17 / 87$

$12 / 17 / 87$

$17 / 08 / 87$

$69 / 11 / 87$

$00 / 11 / 87$

99/22/07

10/16/87

$10 / 16 / 67$
5109.00

5000.06

5834.

7224 .

7226.10

5440.00

4874.0

4150.00

4164.64

137656

145040.00

148090.96

198410

19940.0

11706. 06

115000.06

00709.64

-----

332.00

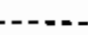

5. 00

(1)

6.06

- - value exceeds prinary dRINking mater standard.

- - VALUE EXCEEDS PROPOSED PRIUARY DRINKING TATER STANDARD.

- - VALUE EXCEEDS SCREENING LEVEL FOR FURTHER INYESTIGATION.

- DETECTION LIUIT TAS NOT AVAILABLE FOR COUPARISON

NR - ANALYSIS NOT REQUESTED OR NOT YET REPORTED

VALUES IN $f$ A ARE COUHTING ERRORS FOR RADIONUCLIDES

IATER STANDARD(S) IN PARENTHESES ARE PROPOSED ONLY 
TABLE C.5. (contd)

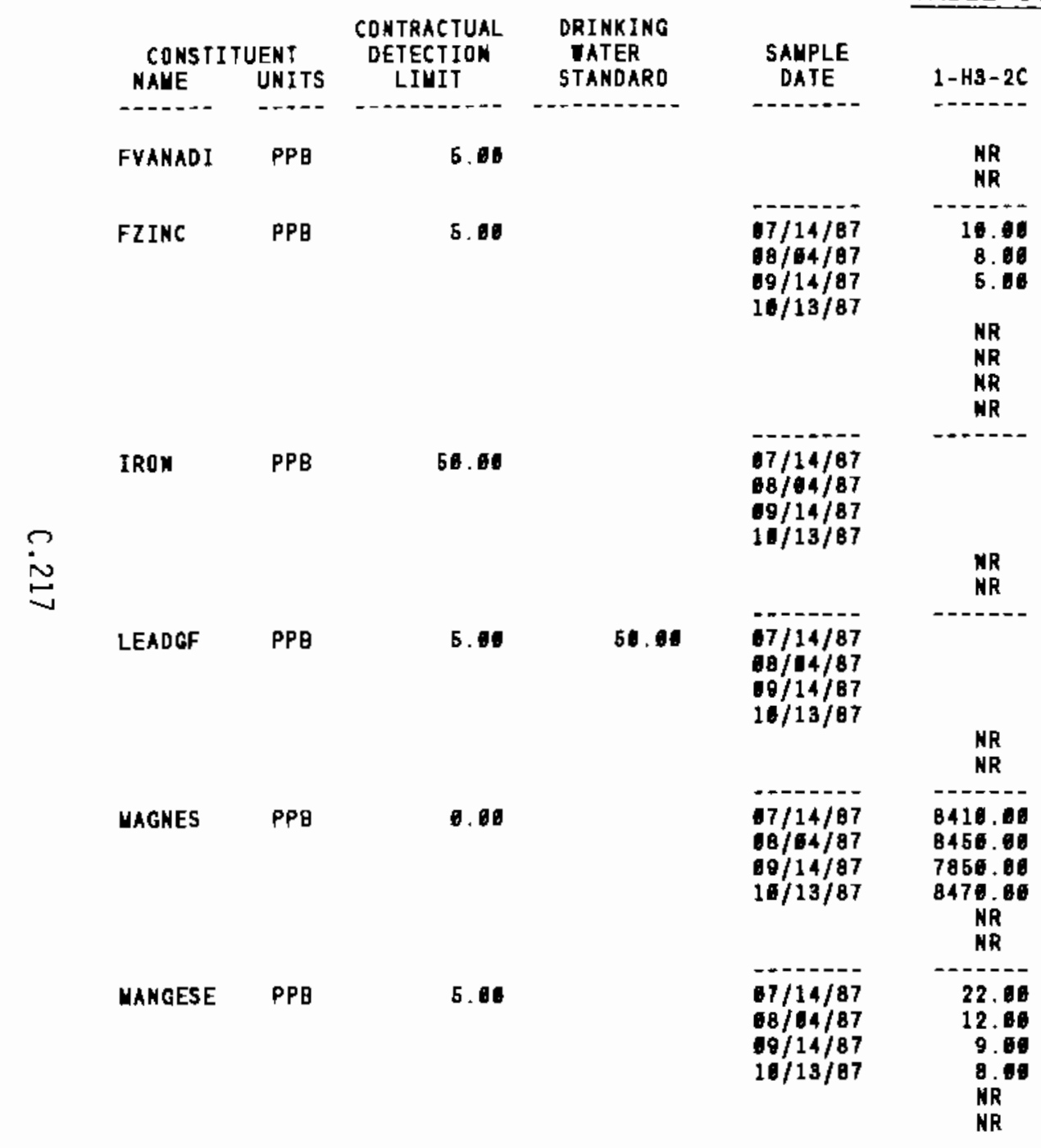

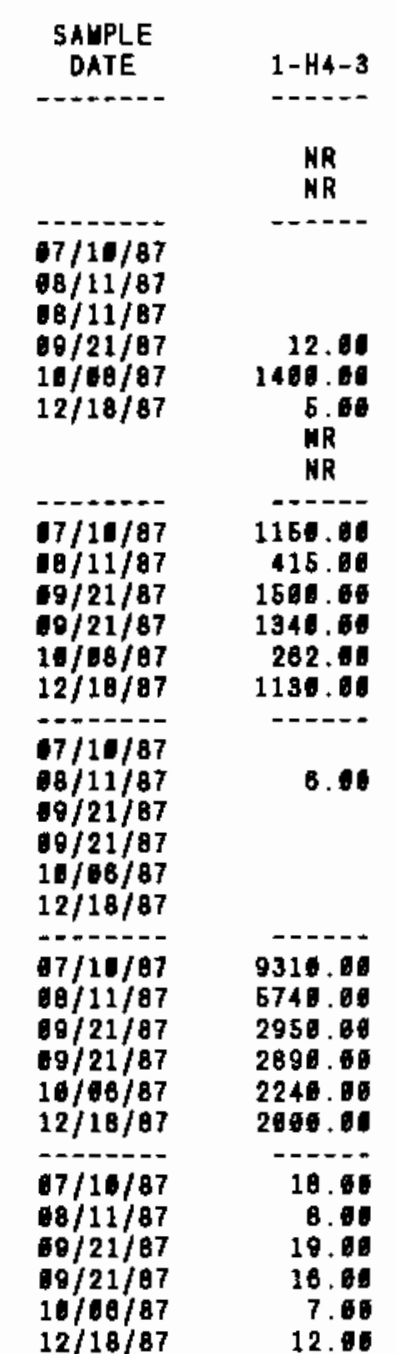

\begin{tabular}{|c|c|}
\hline $\begin{array}{l}\text { SALPLE } \\
\text { DATE }\end{array}$ & $1-\mathrm{H}_{4}-4$ \\
\hline - n-n-o- & \\
\hline $\begin{array}{l}12 / 17 / 87 \\
12 / 17 / 87\end{array}$ & 6. 06 \\
\hline 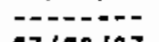 & 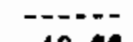 \\
\hline $\begin{array}{l}17 / 08 / 87 \\
68 / 11 / 87 \\
88 / 11 / 87 \\
10 / 22 / 87 \\
16 / 16 / 87 \\
18 / 18 / 87 \\
12 / 17 / 87 \\
12 / 17 / 87\end{array}$ & $\begin{array}{r}49.00 \\
60.00 \\
61.04 \\
125.06 \\
53.06 \\
58.40 \\
90.00 \\
111.06\end{array}$ \\
\hline $\begin{array}{l}07 / 68 / 87 \\
60 / 11 / 87 \\
09 / 22 / 87 \\
69 / 22 / 87 \\
10 / 18 / 87 \\
12 / 17 / 87\end{array}$ & $\begin{array}{r}53.00 \\
128.40 \\
238.4 \\
114.80 \\
863.60\end{array}$ \\
\hline $\begin{array}{l}67 / 98 / 87 \\
68 / 11 / 87 \\
69 / 22 / 87 \\
19 / 22 / 87 \\
16 / 18 / 87 \\
12 / 17 / 87\end{array}$ & \\
\hline $\begin{array}{l}67 / 08 / 87 \\
68 / 11 / 87 \\
69 / 22 / 87 \\
69 / 22 / 87 \\
10 / 18 / 87 \\
12 / 17 / 87\end{array}$ & $\begin{array}{l}6780.00 \\
8800.00 \\
8060.60 \\
8210.06 \\
4360.06 \\
4788.06\end{array}$ \\
\hline $\begin{array}{l}67 / 68 / 87 \\
60 / 11 / 87 \\
00 / 22 / 87 \\
60 / 22 / 87 \\
19 / 16 / 87 \\
12 / 17 / 07\end{array}$ & 15.00 \\
\hline
\end{tabular}

* - VALUE EXCEEDS PRIYARY DRINKING MATER STANDARD.

- VALUE EXCEEDS SCREENIMG LEVEL FOR FURTHER INYESTIGATION.

- DETECTION LIMIT US NOT AYALABLE FOR COMPARISON

NR - ANALYSIS NDT RERUESTED DR NDT YET REPORTED

VALUES IN \{\} ARE CDUNTING ERRDRS FOR RADIONUCLIDES

TATER STANDARD(S) IN PARENTHESES ARE PRDPDSED OHLY 
TABLE C.5. (contd)

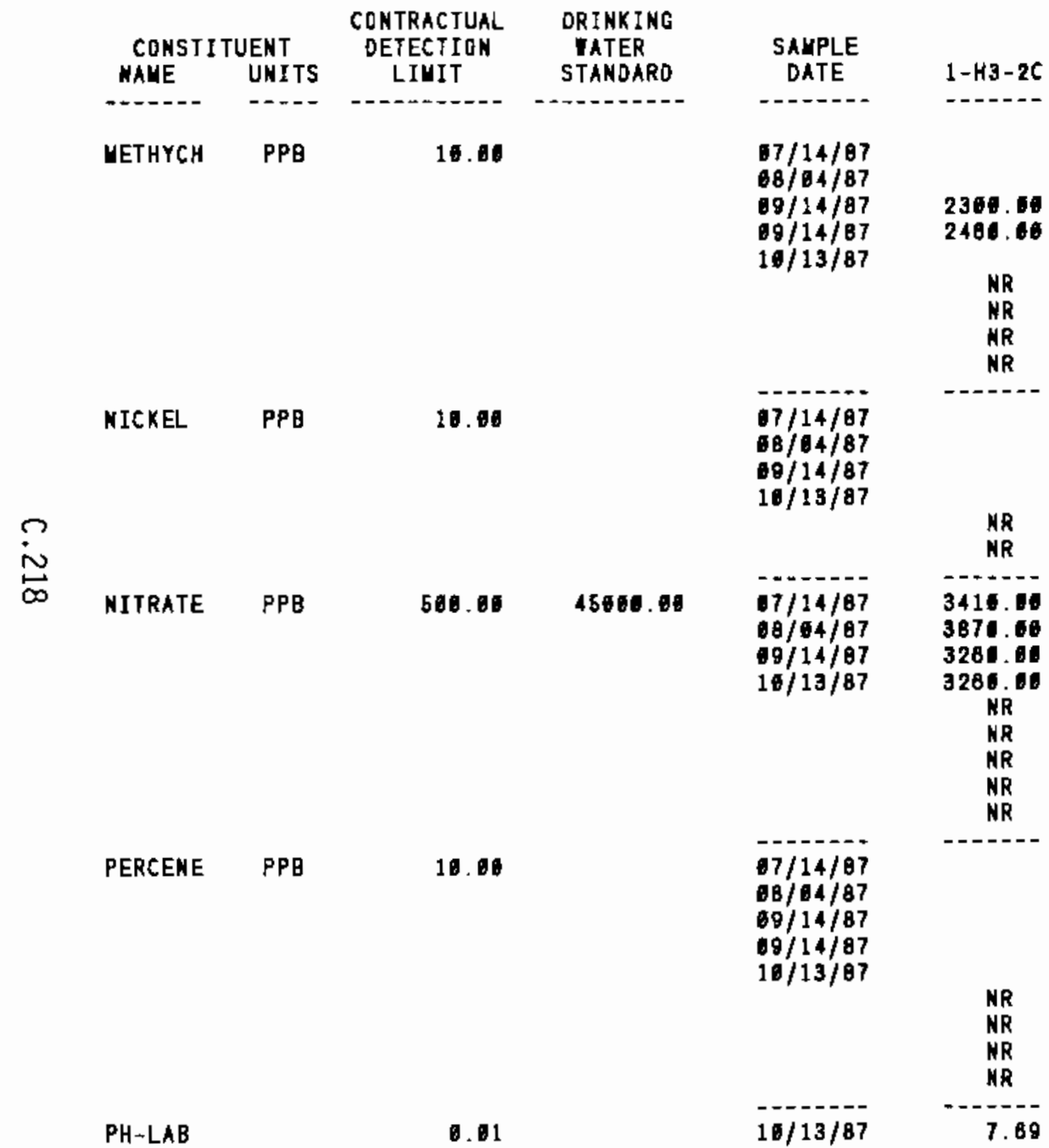

\begin{tabular}{|c|c|}
\hline $\begin{array}{c}\text { SAYPLEE } \\
\text { DATE }\end{array}$ & $1-\mathrm{H} 4-3$ \\
\hline \multirow[t]{2}{*}{$\begin{array}{l}67 / 18 / 87 \\
08 / 11 / 87 \\
06 / 11 / 87 \\
69 / 21 / 87 \\
60 / 21 / 87 \\
10 / 96 / 97 \\
12 / 18 / 87\end{array}$} & \\
\hline & $\begin{array}{l}\text { NR } \\
\text { NR }\end{array}$ \\
\hline $\begin{array}{l}07 / 18 / 87 \\
68 / 11 / 87 \\
69 / 21 / 87 \\
09 / 21 / 87 \\
1010897 \\
12 / 18 / 87\end{array}$ & $\begin{array}{l}43.06 \\
29.01 \\
10.00 \\
13.80 \\
13.80 \\
31.00\end{array}$ \\
\hline $\begin{array}{l}67 / 10 / 87 \\
68 / 11 / 87 \\
68 / 11 / 87 \\
69 / 21 / 87 \\
69 / 21 / 87 \\
11 / 66 / 87 \\
12 / 18 / 87\end{array}$ & 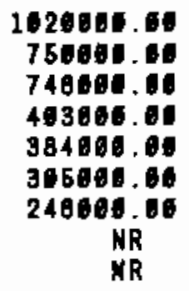 \\
\hline \multirow[t]{2}{*}{$\begin{array}{l}97 / 16 / 87 \\
68 / 11 / 87 \\
08 / 11 / 87 \\
89 / 21 / 87 \\
99 / 21 / 87 \\
19 / 81 / 87 \\
12 / 18 / 87\end{array}$} & \\
\hline & $\begin{array}{l}\text { NR } \\
\text { NR }\end{array}$ \\
\hline 8 & 7.97 \\
\hline
\end{tabular}

SAMPLE

DATE 1-H4-4

- DAT--. -

$97 / 68 / 87$

$08 / 11 / 87$

88/11/87

99/22/87

09/22/87

6801. 16

$10 / 16 / 87$

8460.60

$18 / 18 / 87$

$12 / 17 / 87$

$12 / 17 / 87$

$07 / 08 / 87$

10/11/87

$60 / 22 / 87$

$09 / 22 / 87$

$10118 / 87$

$12 / 17 / 87$

17/0187

$09 / 11 / 87$

98/11/87

$69 / 22 / 87$

89/22/87

$10 / 18 / 97$

$12 / 17 / 87$

$12 / 17 / 87$

12/17/87

$07 / 08 / 87$

$60 / 11 / 87$

$68 / 11 / 87$

$10 / 22 / 87$

$08 / 22 / 87$

10/16/87

10/16/87

$12 / 17 / 87$

$12 / 17 / 87$

$10 / 18 / 87$

10.00

11.0

25.60

10.

10.6

2081100.

980.4. .

374600.04

$612660.01 \%$

231060.06 *

231000.00 ;

139000.00

$139900.00 *$

-.-.-

62.66

52.00

- - Value exceEds pRIMary dRinking mater standard.

- VALUE EXCEEDS PROPOSED PRIMARY DRINKING waTER STANOARD

- VALUE EXCEEDS SCREEMING LEVEL FOR FURTHER IAVESTIGATIDN.

- DETECTION LIUIT TAS NOT AVAILABLE FOR COMPARISON

NR - ANALYYSIS NOT REQUESTED OR NOT YET REPORTED

YALUES IN I \& ARE COUNTING ERRORS FOR RADIONUCLIDES

IATER STANDARD(S) IN PAREXTHESES ARE PROPISED ONLY 
TABLE C.5. (contd)

\begin{tabular}{|c|c|c|c|}
\hline $\begin{array}{l}\text { CDNSTI } \\
\text { NAUE }\end{array}$ & $\begin{array}{l}\text { TUENT } \\
\text { UNITS }\end{array}$ & $\begin{array}{c}\text { CONTRACTUAL } \\
\text { OETECTION } \\
\text { LIUIT }\end{array}$ & $\begin{array}{l}\text { DRINKING } \\
\text { IATER } \\
\text { STAKDARD }\end{array}$ \\
\hline$--\ldots+-$ & $---\infty$ & 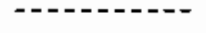 & $\ldots+\ldots+\cdots$ \\
\hline PH-LAB & & 0.1 & \\
\hline PHFIELD & & 1.10 & \\
\hline
\end{tabular}

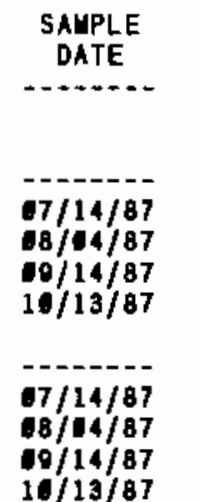

$1-\mathrm{H3}-2 \mathrm{C}$

SAUPLE

DATE

1- $\mathrm{H} 4-3$

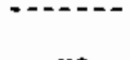

-

-...-

\begin{tabular}{rr}
$12 / 18 / 87$ & 8.31 \\
\hline $7 / 10 / 87$ & 7.91 \\
$68 / 11 / 87$ & 7.10 \\
$10 / 21 / 87$ & 8.10 \\
$10 / 06 / 87$ & 7.81 \\
$12 / 18 / 87$ & 7.50
\end{tabular}

PHOSPHA PPB 1000.00
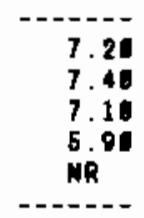

1/10/87

$68 / 11 / 87$

68/11/87

$60 / 21 / 87$

$09 / 21 / 87$

$10 / 06 / 87$

$12 / 18 / 07$

D

11706

MR

1-H4-4

---..-

\begin{tabular}{lr}
$12 / 17 / 87$ & 7.01 \\
$017 / 08 / 87$ & 7.46 \\
$18 / 11 / 87$ & 6.96 \\
$60 / 22 / 87$ & 7.10 \\
$11 / 16 / 87$ & \\
$12 / 17 / 87$ & 7.68 \\
\hline
\end{tabular}

- $7 / 8 / 87$

68/11/87

68/11/87

$00 / 22 / 87$

19/22/87

10/16/a7

10/16/a7

12/17/97

$12 / 17 / 67$

-

$7 / 00 / 87$

$08 / 11 / 87$

$00 / 22 / 87$

19/22/07

10/16/97

12/17/87

$67 / 00 / 87$

18/11/87

18/22/07

$10 / 16 / 87$

$12 / 17 / 87$

17/11/8?

$10 / 21 / 87$

7/ $-18 / 87$

$17 / 08 / 87$
$18 / 11 / 87$

\subsection{0}

0520.14

5340

5340.01

5684.10

B385. 04

6805.04

4201.01

4150.11

- 10

.10
$\quad .17\}$

$\{.18$

○.15\}
POTASUH PPB 100.60

-

NR

$07 / 14 / 87 \quad 4420.60$

$10 / 04 / 87$

$60 / 14 / 87$

4519.60

4370.50

4521.80

WR

RADIUW PCI/L

5.60

$67 / 14 / 87$

21....

$18 / 14 / 87$

\{ $\quad .18\}$

$00 / 14 / 87$

$10 / 13 / 87$

\begin{tabular}{|c|c|c|c|c|c|}
\hline$R U-196$ & $\mathrm{PCI} / \mathrm{L}$ & 172.66 & 30.00 & 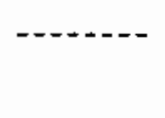 & NR \\
\hline SILVER & PPB & 10.01 & 50.09 & $\begin{array}{l}67 / 14 / 87 \\
68 / 04 / 87\end{array}$ & \\
\hline
\end{tabular}

\begin{tabular}{|c|c|}
\hline $\begin{array}{l}07 / 16 / 87 \\
90 / 11 / 87 \\
00 / 21 / 87 \\
10 / 21 / 87 \\
10 / 06 / 87 \\
12 / 18 / 87\end{array}$ & $\begin{array}{l}7910.04 \\
6240.44 \\
4281.41 \\
4219.14 \\
3914.54 \\
3498.64\end{array}$ \\
\hline $17 / 11 / 87$ & \\
\hline $08 / 11 / 87$ & $\begin{array}{r}0.21\} \\
0.28 \\
.21\end{array}$ \\
\hline
\end{tabular}

MR

10.0

240.04

210.15

3010.15

$09 / 21 / 87$

$10 / 16 / 87$

$12 / 18 / 87$

$17 / 10 / 87$

$12 / 18 / 87$

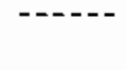

-.2.--

$67 / 18 / 87$
$68 / 11 / 87$

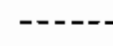

11.0

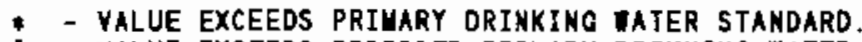

- - VALUE EXCEEDS PRDPOSED PRIUARY DRINKING VATER STANDARD.

- - VAlUE EXCEEDS SCREENIMO LEVEL FOR FURTHER INYESTIOATION.

- - DETECTIDN LIUIT IAS NUT AVAILABLE FOR COMPARISDN

NR - ANALYSIS NOT REQUESTED OR NOT YET REPORTED

YALUES IN \& 3 ARE COUNTING ERRORS FOR RADIOHUCLIDES

IATER STANDARD(S) IN PARENTHESES ARE PROPOSED OMLY 
TABLE C.5. (contd)

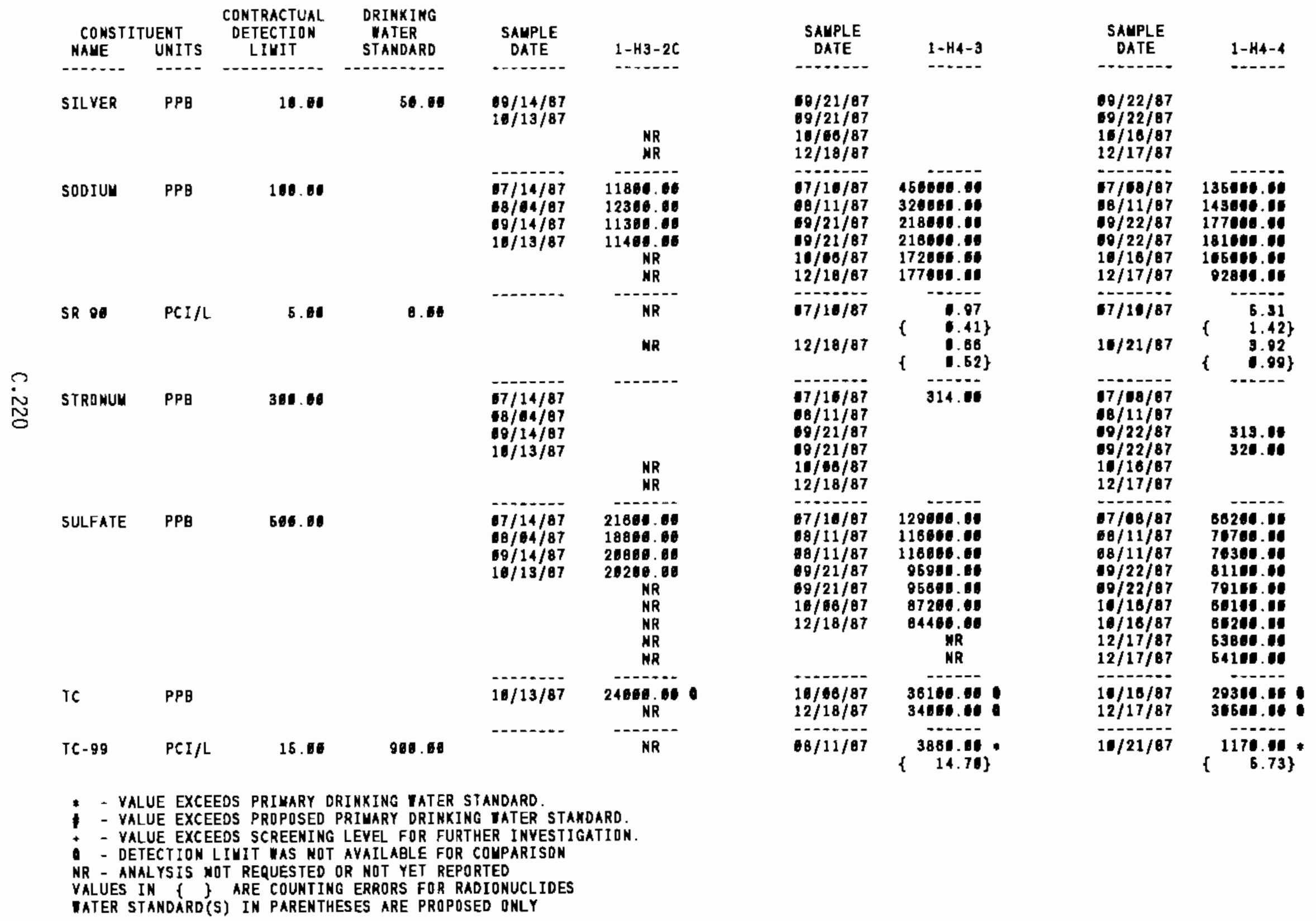


TABLE C.5. (contd)

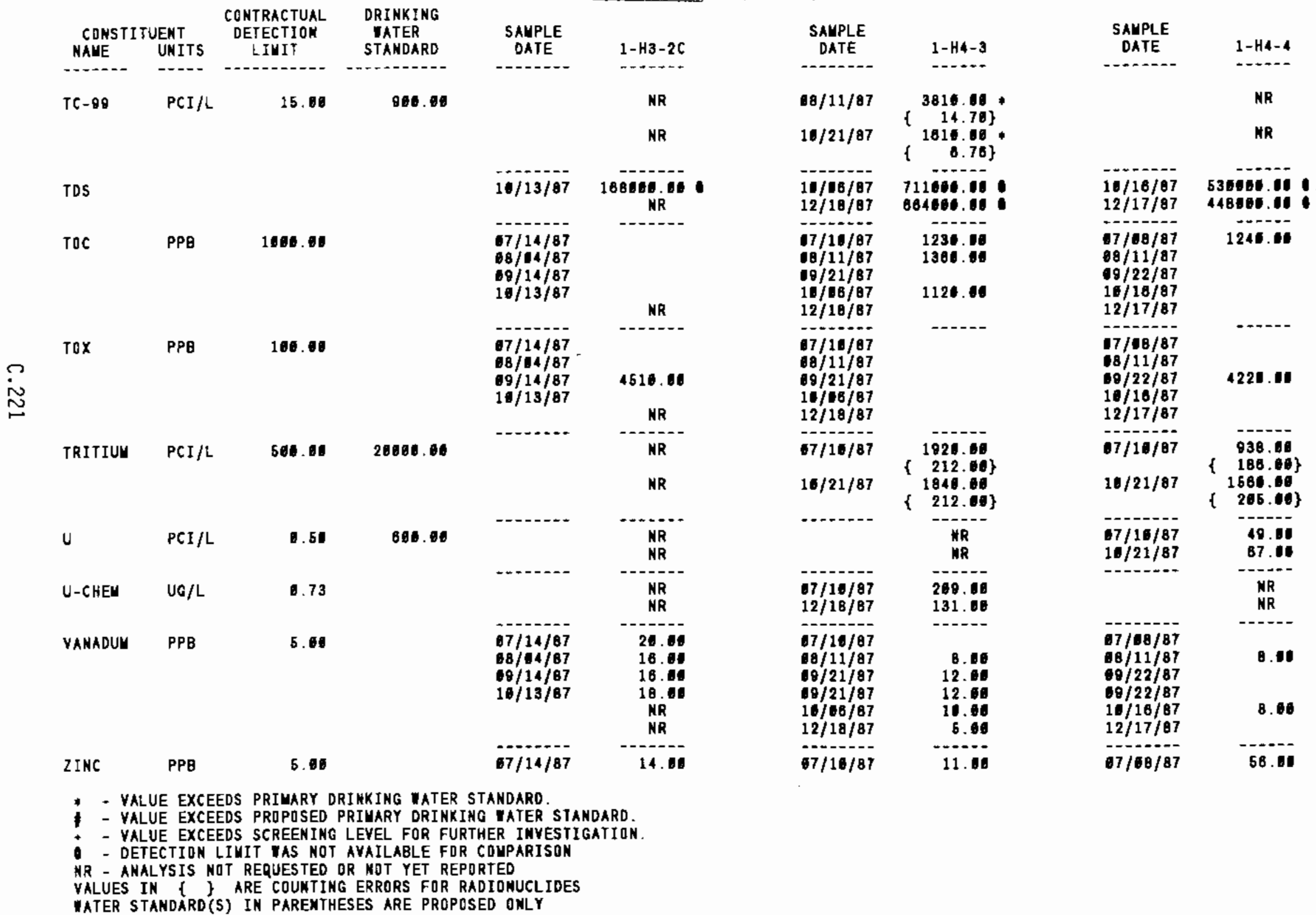


TÁBLE C.5. (contd)

\begin{tabular}{|c|c|c|c|c|c|c|c|c|c|}
\hline \multicolumn{2}{|c|}{ CONSTITUENT } & $\begin{array}{l}\text { CONTRACTUAL } \\
\text { DETECTION } \\
\text { LINIT }\end{array}$ & $\begin{array}{l}\text { DRINKING } \\
\text { SATER } \\
\text { STANDARO }\end{array}$ & $\begin{array}{l}\text { SAUPLE } \\
\text { DATE }\end{array}$ & $1-\mathrm{H3}-2 \mathrm{C}$ & $\begin{array}{c}\text { SAMPLE } \\
\text { DATE }\end{array}$ & $1-\mathrm{H}_{4}-3$ & $\begin{array}{l}\text { SALPLE } \\
\text { DATE }\end{array}$ & $1-\mathrm{H}_{4}-4$ \\
\hline 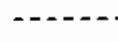 & $\cdots--$ & 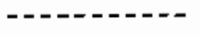 & 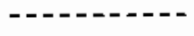 &.----- & $\cdots--$ & 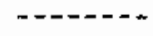 & "-- - & - & ---- \\
\hline ZINC & PPB & 5.60 & & $\begin{array}{l}68 / 64 / 87 \\
69 / 14 / 87 \\
10 / 13 / 87\end{array}$ & $\begin{array}{c}13.00 \\
12.00 \\
10.00 \\
N R \\
\text { NR }\end{array}$ & $\begin{array}{l}08 / 11 / 87 \\
09 / 21 / 87 \\
09 / 21 / 87 \\
16 / 06 / 87 \\
12 / 18 / 87\end{array}$ & $\begin{array}{l}5.00 \\
6.00\end{array}$ & $\begin{array}{l}68 / 11 / 87 \\
69 / 22 / 87 \\
69 / 22 / 87 \\
10 / 18 / 87 \\
12 / 17 / 87\end{array}$ & $\begin{array}{r}79.01 \\
98.60 \\
98.01 \\
53.00 \\
328.00\end{array}$ \\
\hline
\end{tabular}

* - value exceeds primary drinking tater standard.

* - VALUE EXCEEDS PRDPOSED PRIUARY DRIMKING WaTER STANDARD.

- - VALUE EXCEEDS SCREEMING LEVEL FOR FURTHER INVESTIGATION.

- - DETECTION LIUIT IAS NOT AVALABLE FOR COMPARISOM

NR - ANALYSIS NOT REQUESTED DR NOT YET REPORTED

VALUES IN $\{$ \& ARE COUNTING ERRDRS FOR RADIONUCLIDES

WATER STANOARO(S) IN PARENTHESES ARE PROPOSED ONLY 
TABLE C.5. (contd)

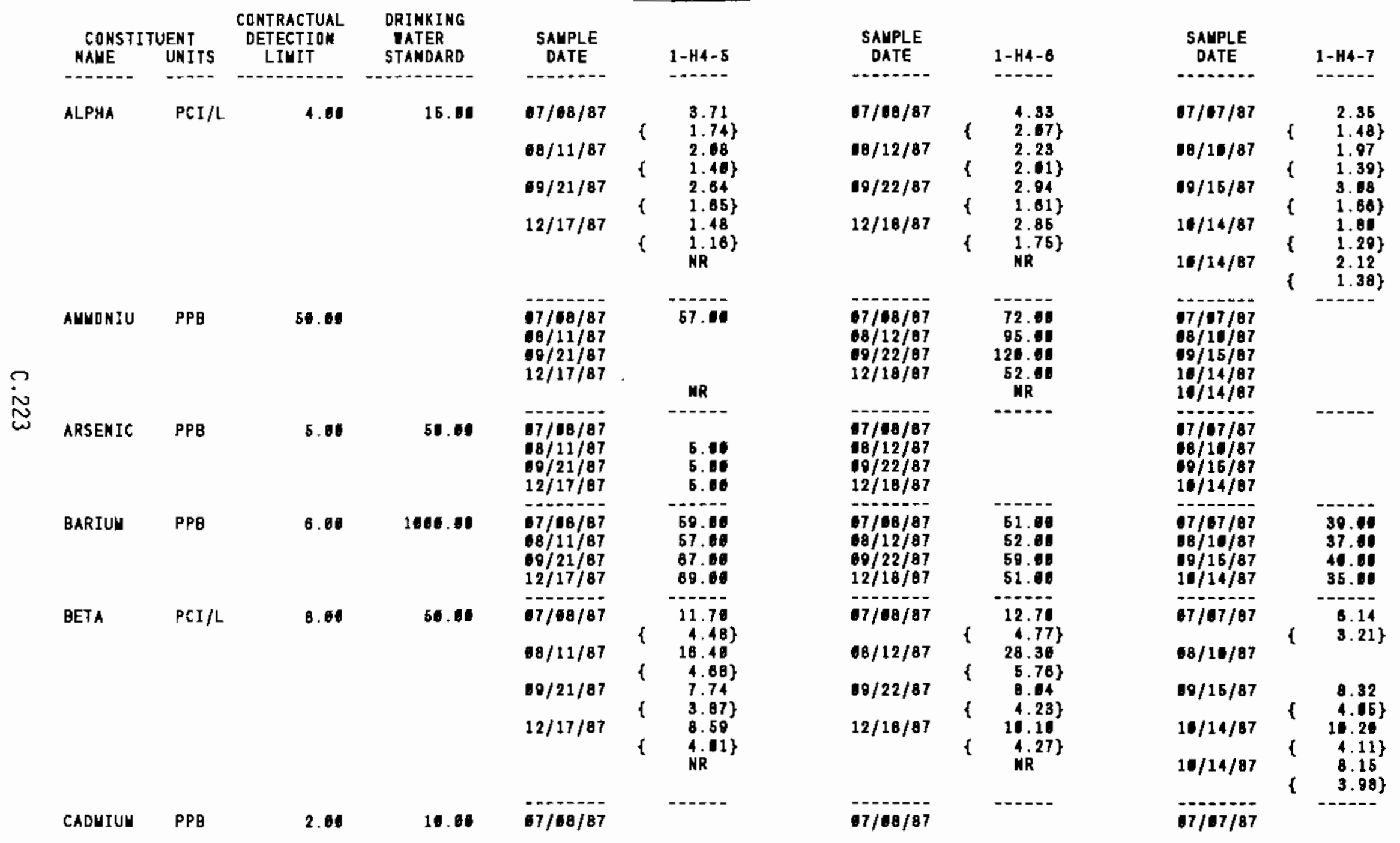

* - yalue exceeds priuary dRinxing tater standard

* VALUE EXCEEDS PROPOSED PRIMARY DRINKING PATER STANDARD.

- - VALUE EXCEEDS SCREENING LEYEL FOR FURTHER INYESTIGATION.

- DETECTION LIMIt IAS NOT AYAILABLE for COMPARISON

NR - AMALYSIS NOT RERUESTED OR NOT YET REPORTED

VALUES IN \{ \} ARE COUMTIMG ERRORS FDR RADIONUCLIDES

nater STandard(S) in PaRentheses are proposed onLy 
TABLE C.5. (contd)

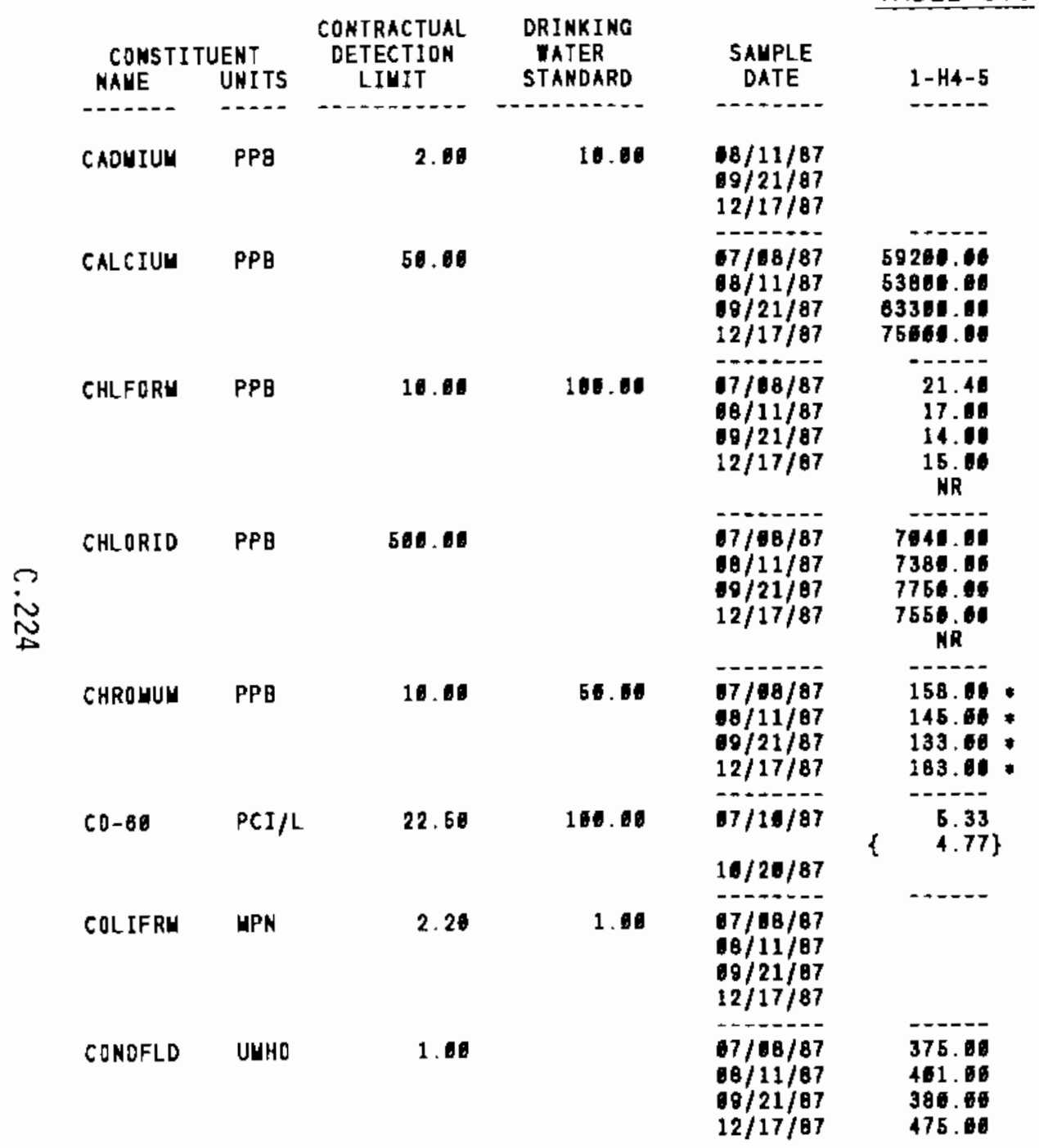

\begin{tabular}{|c|c|}
\hline $\begin{array}{l}\text { SAMPLE } \\
\text { DATE }\end{array}$ & $1-\mathrm{H} 4-6$ \\
\hline ---n-- & $\cdots$ \\
\hline $\begin{array}{l}08 / 12 / 87 \\
08 / 22 / 87 \\
12 / 18 / 87\end{array}$ & 2.00 \\
\hline $\begin{array}{l}07 / 86 / 87 \\
08 / 12 / 87 \\
00 / 22 / 87 \\
12 / 18 / 87\end{array}$ & $\begin{array}{l}89200.00 \\
53801.06 \\
68000.06 \\
71066.00\end{array}$ \\
\hline \multirow[t]{2}{*}{$\begin{array}{l}07 / 88 / 87 \\
08 / 12 / 87 \\
0 / 22 / 87 \\
12 / 18 / 87\end{array}$} & \\
\hline & NR \\
\hline $\begin{array}{l}97 / 18 / 87 \\
6 / 12 / 87 \\
69 / 22 / 87 \\
12 / 18 / 87\end{array}$ & $\begin{array}{c}15200 \\
15860 \\
15800 \\
14604 \\
\text { NR }\end{array}$ \\
\hline $\begin{array}{l}67 / 68 / 87 \\
68 / 12 / 87 \\
69 / 22 / 87 \\
12 / 16 / 87\end{array}$ & $\begin{array}{l}82.00 \\
85.00 \\
78.00\end{array}$ \\
\hline $07 / 16 / 87$ & 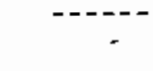 \\
\hline $1 / / 20 / 87$ & \\
\hline $\begin{array}{l}07 / 08 / 87 \\
08 / 12 / 87 \\
09 / 22 / 87 \\
12 / 18 / 87\end{array}$ & $-\cdots$ \\
\hline $\begin{array}{l}97 / 98 / 87 \\
68 / 12 / 87 \\
69 / 22 / 87 \\
12 / 18 / 87\end{array}$ & $\begin{array}{l}--.-1 \\
472.00 \\
544.00 \\
541.00 \\
511.00\end{array}$ \\
\hline
\end{tabular}

SAUPLE

DATE

1-H4-7

$68 / 10 / 87$

$08 / 15 / 87$

$10 / 14 / 87$

67/07/87

$06 / 16 / 87$

$08 / 16 / 87$

$10 / 14 / 87$

$10 / 14 / 87$

$07 / 07 / 87$

$08 / 16 / 87$

$09 / 15 / 87$

$10 / 14 / 87$

$97 / 67 / 87$

68/10/87

$69 / 15 / 87$

$10 / 14 / 87$

18/14/87

67/67/87

68/11/87

$00 / 15 / 87$

$10 / 14 / 87$

$10 / 14 / 67$

- $-1 . . .$.

85104.11

47260.00

55864.60

64301. 10

19.00

21.

18. 4

17.50

$-1 .-2$

9060.

2260.60

8811. 14

0170.0

9274.01

124.

118.00 .

112.00 *

117.09 .

NR

NR

$07 / 07 / 87$

$08 / 14 / 87$

$08 / 15 / 87$

$10 / 14 / 87$

67/07/87

$00 / 10 / 87$

$08 / 10 / 87$

$10 / 15 / 87$

* - VALUE EXCEEDS PRIHARY DRINKING TATER STANDARD.

- - VALUE EXCEEDS PROPOSED PRIMARY DRINKING VATER STANDARD.

+ - YALUE EXCEEOS SCREENING LEYEL FOR FURTHER INYESTIOATION.

- DETECTION LIUIT WAS NOT AYAILABLE FDR COUPARISOH

NR - ANALYSIS NOT REQUESTED OR NOT YET REPORTED

VALUES IN \{\} ARE COUNTING ERRORS FOR RADIONUCLIDES

IATER STANDARDIS) IN PARENTHESES ARE PROPOSED ONLY 
TABLE C.5. (contd)

\begin{tabular}{|c|c|c|c|c|c|c|c|c|c|}
\hline $\begin{array}{c}\text { CONSTI } \\
\text { NAUE }\end{array}$ & $\begin{array}{l}\text { UENT } \\
\text { U*ITS }\end{array}$ & $\begin{array}{c}\text { CONTRACTUAL } \\
\text { DETECTION } \\
\text { LIUIT }\end{array}$ & $\begin{array}{l}\text { DRINKING } \\
\text { IATER } \\
\text { STAMDARD }\end{array}$ & $\begin{array}{c}\text { SAMPLE } \\
\text { DATE }\end{array}$ & $1-\mathrm{H} 4-5$ & $\begin{array}{c}\text { SAMPLE } \\
\text { DATE }\end{array}$ & $1-\mathrm{H} 4-6$ & $\begin{array}{c}\text { SAYPLE } \\
\text { DATE }\end{array}$ & $1-\mathrm{H} 4-7$ \\
\hline CQPPER & PPB & 10.06 & $(1906.0)$ & $\begin{array}{l}67 / 68 / 87 \\
68 / 11 / 87 \\
69 / 21 / 87 \\
12 / 17 / 87\end{array}$ & 20.00 & $\begin{array}{l}07 / 08 / 87 \\
06 / 12 / 87 \\
09 / 22 / 87 \\
12 / 18 / 87\end{array}$ & 11.00 & $\begin{array}{l}07 / 07 / 87 \\
0 / 10 / 87 \\
9 / 15 / 87 \\
10 / 14 / 87\end{array}$ & 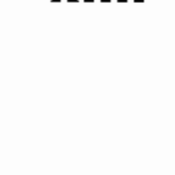 \\
\hline FARSENI & PPB & 6.10 & 60.01 & $\begin{array}{l}07 / 68 / 87 \\
6 / 11 / 87 \\
00 / 21 / 87 \\
12 / 17 / 87\end{array}$ & $\begin{array}{l}7.00 \\
5.01 \\
5.00 \\
\text { NR }\end{array}$ & $\begin{array}{l}07 / 08 / 87 \\
08 / 12 / 87 \\
10 / 22 / 87 \\
12 / 18 / 87\end{array}$ & NR & $\begin{array}{l}17 / 107 / 87 \\
18 / 16 / 87 \\
10 / 16 / 87 \\
10 / 14 / 87 \\
10 / 14 / 87\end{array}$ & $\cdots$ \\
\hline FBARIUU & PPB & 8.69 & 1080.01 & $\begin{array}{l}07 / 68 / 87 \\
19 / 11 / 87 \\
09 / 21 / 87 \\
12 / 17 / 87\end{array}$ & $\begin{array}{l}63.06 \\
81.66 \\
85.60 \\
88.66 \\
\text { NR }\end{array}$ & $\begin{array}{l}17 / 98 / 87 \\
68 / 12 / 87 \\
69 / 22 / 87 \\
12 / 18 / 87\end{array}$ & $\begin{array}{l}47.16 \\
48.16 \\
47.16 \\
60.66\end{array}$ & $\begin{array}{l}67 / 07 / 87 \\
08 / 10 / 87 \\
00 / 15 / 87 \\
10 / 14 / 87 \\
10 / 14 / 87\end{array}$ & $\begin{array}{l}39.16 \\
46.06 \\
31.10 \\
38.10 \\
41.0\end{array}$ \\
\hline FCALCIU & PPB & 50.06 & & $\begin{array}{l}67 / 68 / 87 \\
68 / 11 / 87 \\
68 / 21 / 87 \\
12 / 17 / 87\end{array}$ & 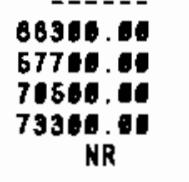 & $\begin{array}{l}17 / 18 / 87 \\
18 / 12 / 87 \\
18 / 22 / 87 \\
12 / 18 / 87\end{array}$ & $\begin{array}{l}64104.01 \\
57744.01 \\
67596.01 \\
72114.41 \\
\text { NR }\end{array}$ & $\begin{array}{l}17 / 17 / 87 \\
68 / 10 / 87 \\
60 / 15 / 87 \\
10 / 14 / 87 \\
10 / 14 / 87\end{array}$ & $\begin{array}{l}80700.01 \\
40860.66 \\
57168.60 \\
57406.00 \\
83500.09\end{array}$ \\
\hline FCHRQHI & PPB & 16.01 & 50.01 & $\begin{array}{l}07 / 68 / 87 \\
0 / 11 / 87 \\
09 / 21 / 87 \\
12 / 17 / 87\end{array}$ & $\begin{array}{l}164.16 \\
168.68 \\
148.06 \\
142.06 \\
\text { NR }\end{array}$ & $\begin{array}{l}7 / 08 / 87 \\
68 / 12 / 87 \\
69 / 22 / 87 \\
12 / 18 / 87\end{array}$ & $\begin{array}{l}41.06 \\
56.0 \% \\
52.00 \\
89.00 \\
\text { NR }\end{array}$ & $\begin{array}{l}07 / 97 / 87 \\
08 / 16 / 87 \\
00 / 15 / 87 \\
16 / 14 / 87 \\
16 / 14 / 87\end{array}$ & $\begin{array}{l}118.11 \\
125.18 \\
118.06 \\
123.6 \\
128.06\end{array}$ \\
\hline FIRON & PPB & 58.80 & & $\begin{array}{l}07 / 98 / 97 \\
68 / 11 / 87 \\
6 / 21 / 87 \\
12 / 17 / 87\end{array}$ & $\begin{array}{c}169.00 \\
\text { NR }\end{array}$ & $\begin{array}{l}07 / 66 / 87 \\
19 / 12 / 87 \\
19 / 22 / 87 \\
12 / 18 / 87\end{array}$ & $\begin{array}{l}55.00 \\
58.06 \\
\text { MR }\end{array}$ & $\begin{array}{l}67 / 07 / 87 \\
68 / 10 / 87 \\
69 / 15 / 87 \\
10 / 14 / 87 \\
16 / 14 / 87\end{array}$ & \\
\hline FLUURID & PPB & 500.00 & 4080.00 & $\begin{array}{l}07 / 68 / 87 \\
08 / 11 / 87 \\
09 / 21 / 87\end{array}$ & & $\begin{array}{l}67 / 08 / 87 \\
68 / 12 / 87 \\
69 / 22 / 87\end{array}$ & & $\begin{array}{l}67 / 67 / 87 \\
08 / 16 / 87 \\
00 / 15 / 87\end{array}$ & \\
\hline \multicolumn{10}{|c|}{ 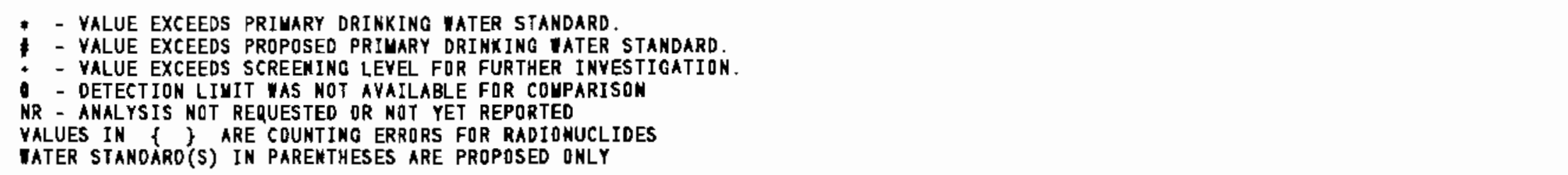 } \\
\hline
\end{tabular}




\begin{tabular}{|c|c|c|c|c|}
\hline $\begin{array}{c}\text { CONSTI } \\
\text { NAME }\end{array}$ & $\begin{array}{l}\text { UENT } \\
\text { UHITS }\end{array}$ & $\begin{array}{c}\text { CONTRACTUAL } \\
\text { DETECTION } \\
\text { LIUIT }\end{array}$ & $\begin{array}{l}\text { DRINKING } \\
\text { IAIER } \\
\text { STANDARD }\end{array}$ & $\begin{array}{l}\text { SAMPLE } \\
\text { DATE }\end{array}$ \\
\hline FLUORID & PPQ & 500.00 & 4068.06 & $12 / 17 / 07$ \\
\hline FUAGNES & PPB & 0.00 & & $\begin{array}{l}07 / 08 / 87 \\
98 / 11 / 87 \\
99 / 21 / 87 \\
12 / 17 / 87\end{array}$ \\
\hline FWANGAN & PPB & 5.10 & & $\begin{array}{l}17 / 98 / 87 \\
08 / 11 / 87 \\
09 / 21 / 87 \\
12 / 17 / 87\end{array}$ \\
\hline FPOTASS & PPB & 100.66 & & $\begin{array}{l}87 / 68 / 87 \\
88 / 11 / 87 \\
69 / 21 / 87 \\
12 / 17 / 87\end{array}$ \\
\hline FSODIUM & PPB & 100.60 & & $\begin{array}{l}07 / 18 / 87 \\
08 / 11 / 87 \\
69 / 21 / 87 \\
12 / 17 / 87\end{array}$ \\
\hline FSTRONT & PPB & 300.00 & & $\begin{array}{l}17 / 108 / 87 \\
88 / 11 / 87 \\
69 / 21 / 87 \\
12 / 17 / 87\end{array}$ \\
\hline FVAMADI & PPB & 5.06 & & $\begin{array}{l}17 / 08 / 87 \\
18 / 11 / 87 \\
69 / 21 / 87 \\
12 / 17 / 87\end{array}$ \\
\hline FZIHC & PPB & 5.60 & & $07 / 6 B / 87$ \\
\hline
\end{tabular}

TABLE C.5. (contd)

$1-H 4-5$
MR
11601.00
11501.06
12609.16

\begin{tabular}{|c|}
\hline $\begin{array}{l}\text { SAHPLE } \\
\text { DATE }\end{array}$ \\
\hline $12 / 18 / 87$ \\
\hline $\begin{array}{l}07 / 68 / 87 \\
08 / 12 / 87 \\
00 / 22 / 87 \\
12 / 18 / 87\end{array}$ \\
\hline $\begin{array}{l}07 / 10 / 87 \\
68 / 12 / 87 \\
60 / 22 / 87 \\
12 / 10 / 87\end{array}$ \\
\hline
\end{tabular}

1-H4-6

SAUPLE

DATE 1-H4-7

MR

5820.06

4890.6

5891. 64

4874.6

NR

12900.04

11300.01

12660.0

NR

594.65

NR

$10 / 14 / 87$

$10 / 14 / 87$

$1 / 107 / 87$

1 5094. 10

14208.0

15700.00

NR

$08 / 10 / 87$

99/15/87

$11 / 14 / 87$
$10 / 14 / 87$

13100.5

11709.

11104.

…--

------

13200.01

17/07/87

54.60

72.05
78.55

53.6

NR

$08 / 16 / 87$

$09 / 16 / 87$

$19 / 14 / 87$

$14 / 14 / 87$

$67 / 07 / 87$

$17 / 68 / 87$

osso.io

0450.10

.8/12/87

19/22/87

6591.11

6140.11

NR

$68 / 10 / 87$

es71. 15

$69 / 16 / 87$

$10 / 14 / 87$

$10 / 14 / 87$

17/08/87

26200.00

8/12/87

24100. 01

2430s. II

25200 .

12/18/87

369 .

NR

$07108 / 87$

343...

$369.00 \quad 07 / 108 / 87$

361.08

326.08

326.

NR

$8 / 12 / 87$
$09 / 22 / 87$

343.08

351.08

355.00

$12 / 10 / 87$

NR

8.0

67/08/67

G.

$6.05 \quad 08 / 12 / 87$

$12 / 18 / 87$

7.06

6.00

NR

52.00

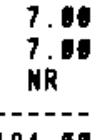

$67 / 68 / 87$

$67 / 67 / 87$

68/10/87

$60 / 16 / 87$

$10 / 14 / 87$

$10 / 14 / 87$

-----

$7 / 8787$

$67 / 87 / 87$

88/16/87

$69 / 16 / 87$

$10 / 14 / 87$

$10 / 14 / 87$

$67 / 67 / 87$

$68 / 10 / 87$

69/16/87

$10 / 14 / 87$

$10 / 14 / 87$

$67 / 07 / 87$

5305.1

c181.

B160.

617. 5

13841.6

11901.5

12100.60

13114.6

12800.6

325.6

341.16

323. 14

0

0.06

5..6

124.8

* - VALUE EXCEEDS PRIMARY DRINKING waTER STANDARo.

- V VLUE EXCEEDS PROPOSED PRIUARY DRINKING IATER STANDARD.

+ - VALUE EXCEEDS SCREENING LEVEL FOR FURTHER INYESTIGATION.

- - detection liUit tas nOt ayailable for couparison

HR - ANALYSIS NOT REQUESTED OR NOT YET REPORTED

VALUES IN \{\} ARE COUNTING ERRORS FOR RAOIONUCLIDES

WATER STANDARD(S) IN PARENTHESES ARE PRDPDSED ONLY 
TABLE C.5. (contd)

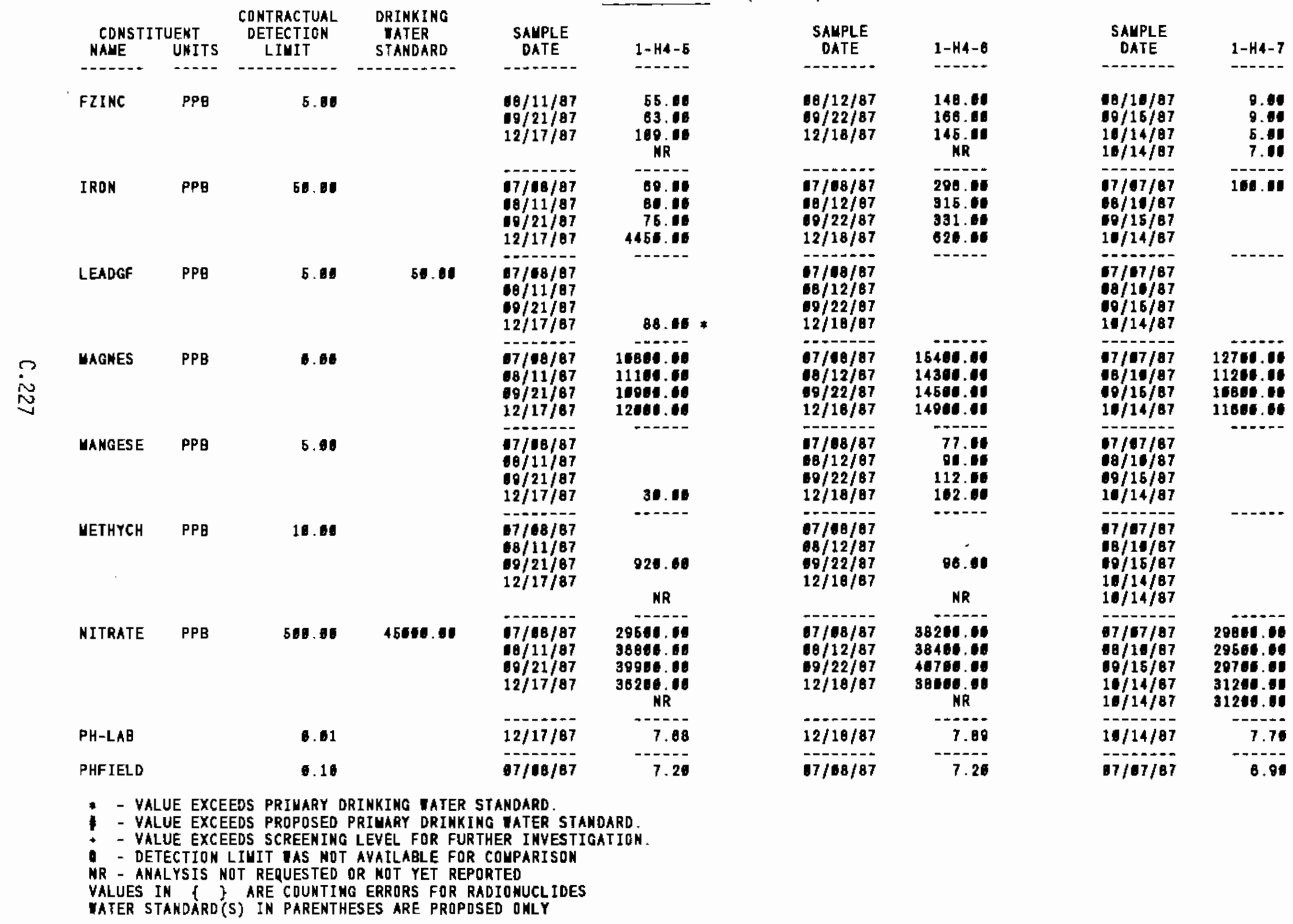


TABLE C.5. (contd)

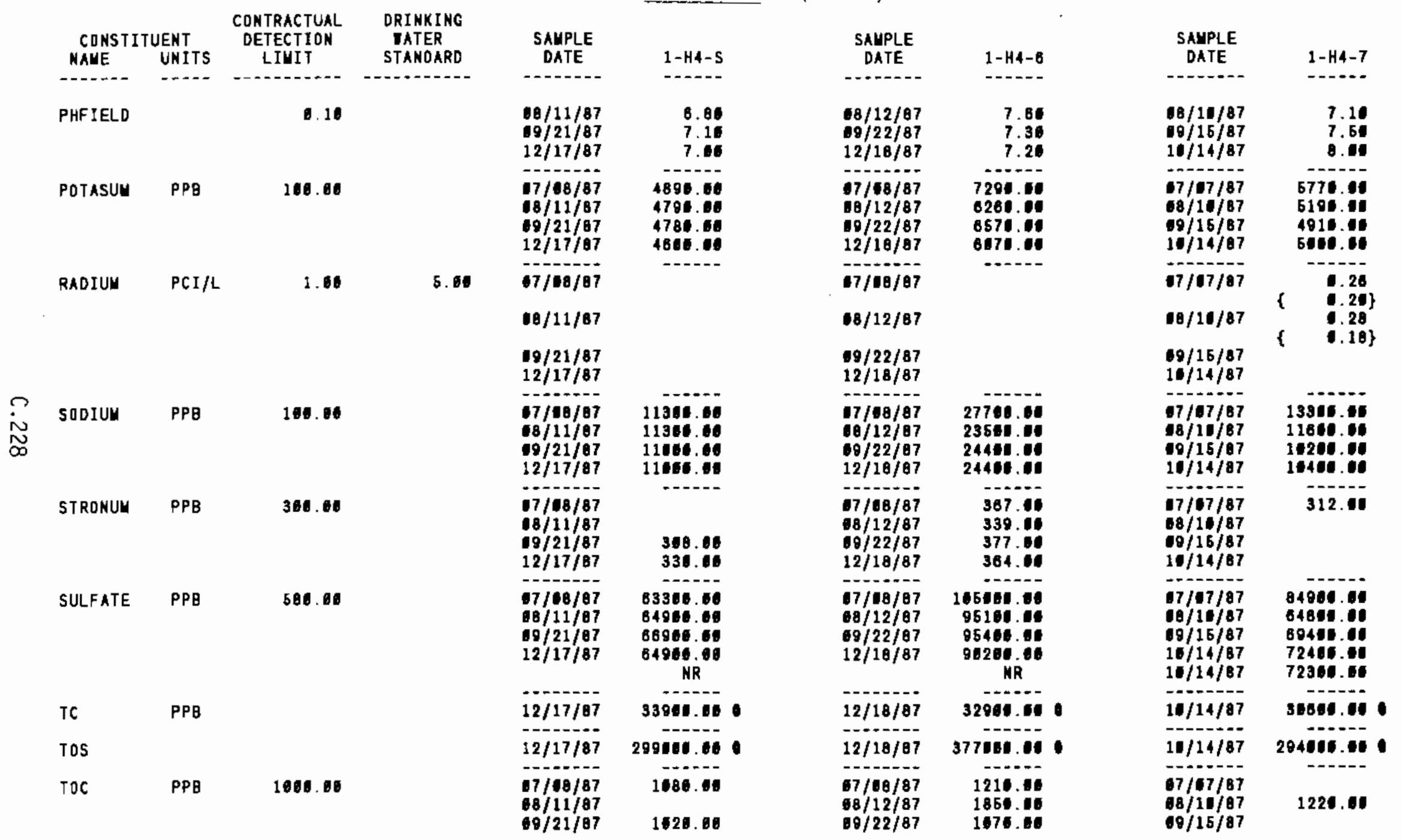

- - VaLUe eXCEEDS priuary dRINKING mater standaro.

- - VALUE EXCEEOS PROPOSED PRIMARY DRIMKIHG IATER STANDARD.

- - YALUE EXCEEOS SCREENING LEVEL FOR FURTHER INVESTIGATION.

- DETECTION linit was Not aVailable for couparison

NR - ANALYSIS NOT REQUESTED OR NOT YET REPQRTED

YALUES IN I I ARE COUNTING ERRORS FOR RADIONUCLIOES

WATER STAMDARD(S) IN PARENTHESES ARE PRDPDSED OMLY 


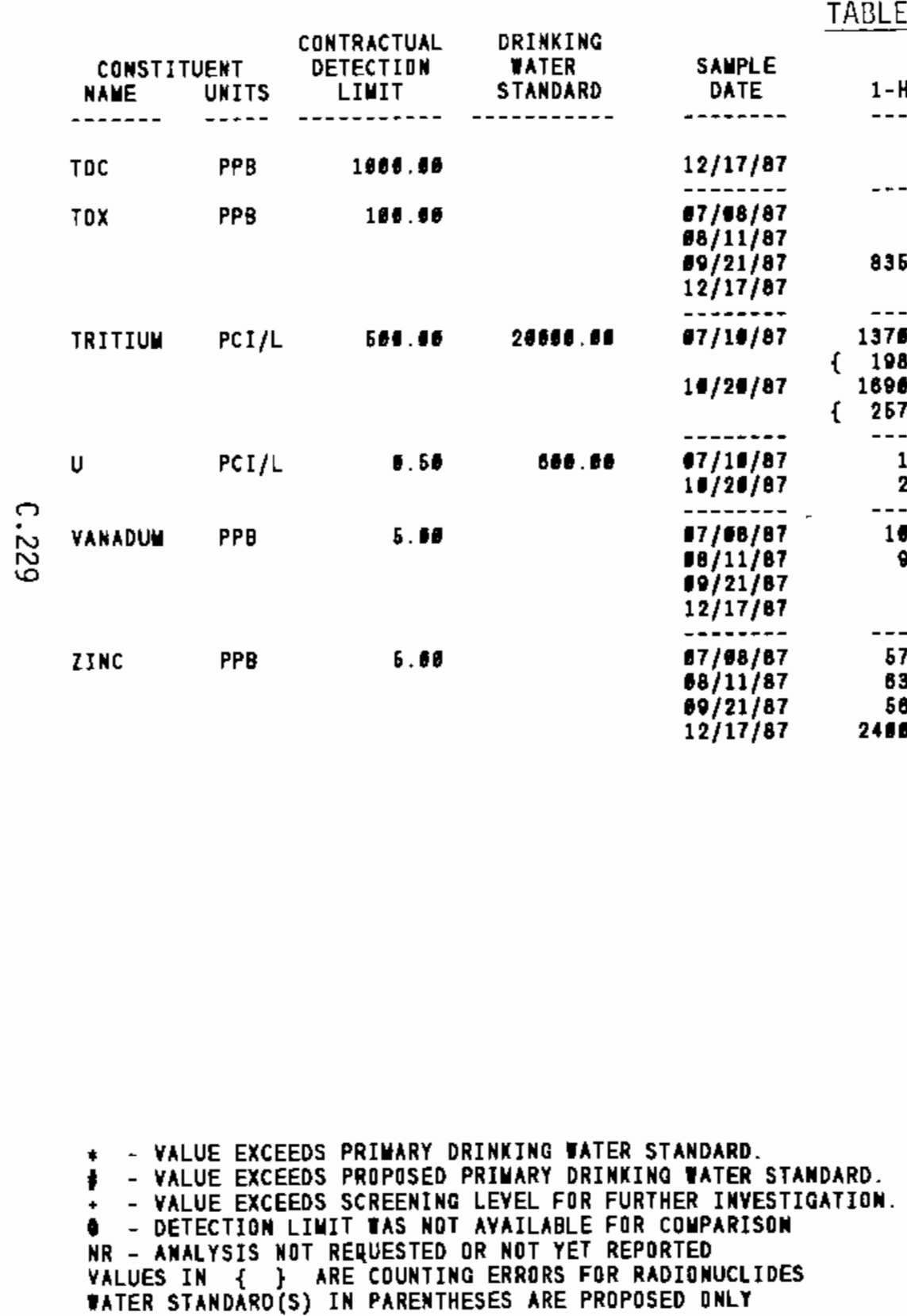

DATE 1-H4-6

SAMPLE

1-H4-7

$10 / 14 / 87$

1-H4-7

17/07/67

$18 / 16 / 87$

$00 / 16 / 67$

$16 / 14 / 87$

$10 / 22 / 87$

12/18/87

17/10/07

$10 / 20 / 87$

440

10/....-

NR

$188.00\}$
1686.00

4040.60

269.60
5471.08

267.0.

17/10/97

348.00)

3. 39

1.85

$10 / 21 / 87$

3.58

67/58/87

(1)

8.16

$18 / 12 / 87$

8.

12/18/87

-......

07/08/87

189.0

$68 / 12 / 87$

18/22/87

157.6

12/18/87

269 . 10

1090.10

....

63.60

16.

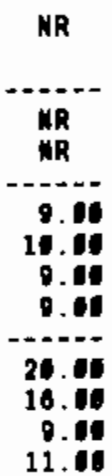

\footnotetext{
YALUES IN \{\} ARE COUNTING ERRORS FQR RADIONUCLIDES
WATER STANDARO(S) IN PARENTHESES ARE PROPOSED DNLY
} 


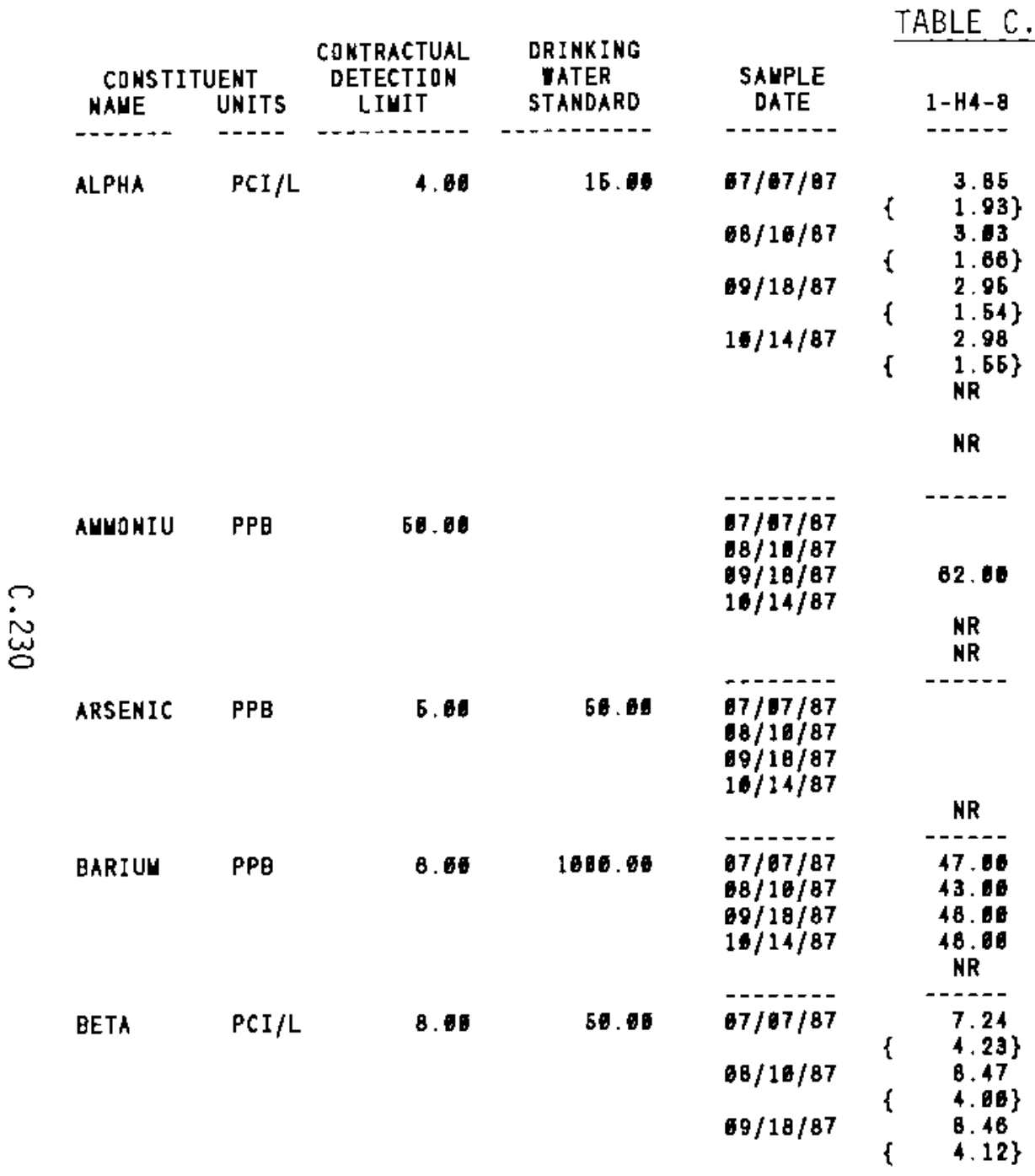

(contd)

* - VALUE eXCEEDS PRIMARY dRINXING water STANDARD.

* - Value EXCEEDS PRDPOSED PRIUARY DRINKING IATER STANDARD.

- YALUE EXCEEDS SCREENING LEYEL FOR FUR THER INYESTIGATION.

- DETECTIDN LIUIT UAS NOT AVAILABLE FOR COUPARISON

NR - ANALCYISIS NOT REQUESTED OR NOT YET REPORTED

YALUES IN \{ $\}$ ARE COUNTING ERRORS FDR RADIDNUCLIDES

IATER STANDARD (S) IM PARENTHESES ARE PROPOSED OKLY

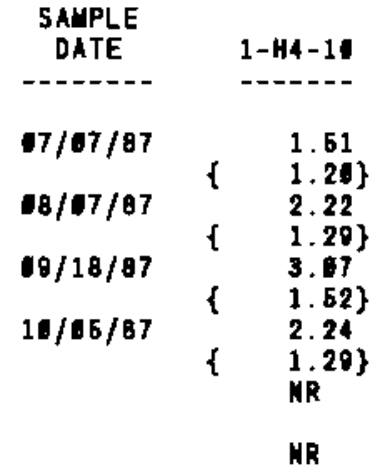

$07 / 07 / 87$

$18 / 107 / 87$

$10 / 10 / 87$

$10 / 05 / 87$

54.06

$09 / 15 / 87$

$10 / 108 / 87$

$12 / 28 / 07$

$07 / 107 / 87$

$08 / 10 / 87$

$09 / 15 / 87$

$10 / 08 / 97$

$12 / 2 B / 07$

$07 / 07 / 67$

08/10/87

$09 / 15 / 87$

16/06/87

$12 / 28 / 87$

$67 / 07 / 87$

08/10/87

$09 / 15 / 87$

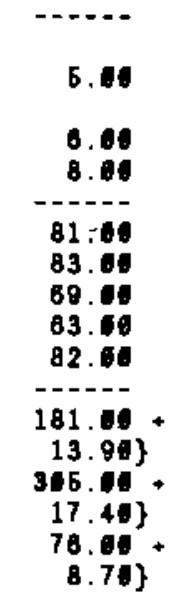

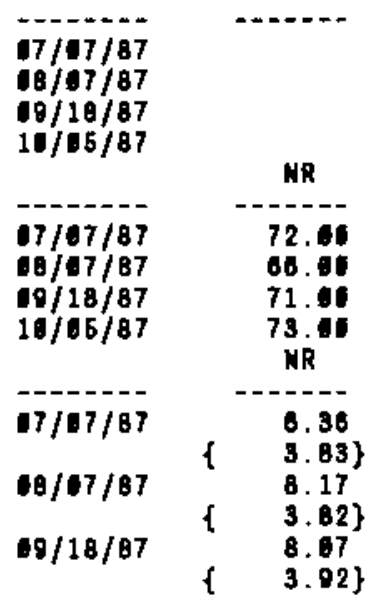


TABLE C.5. (contd)

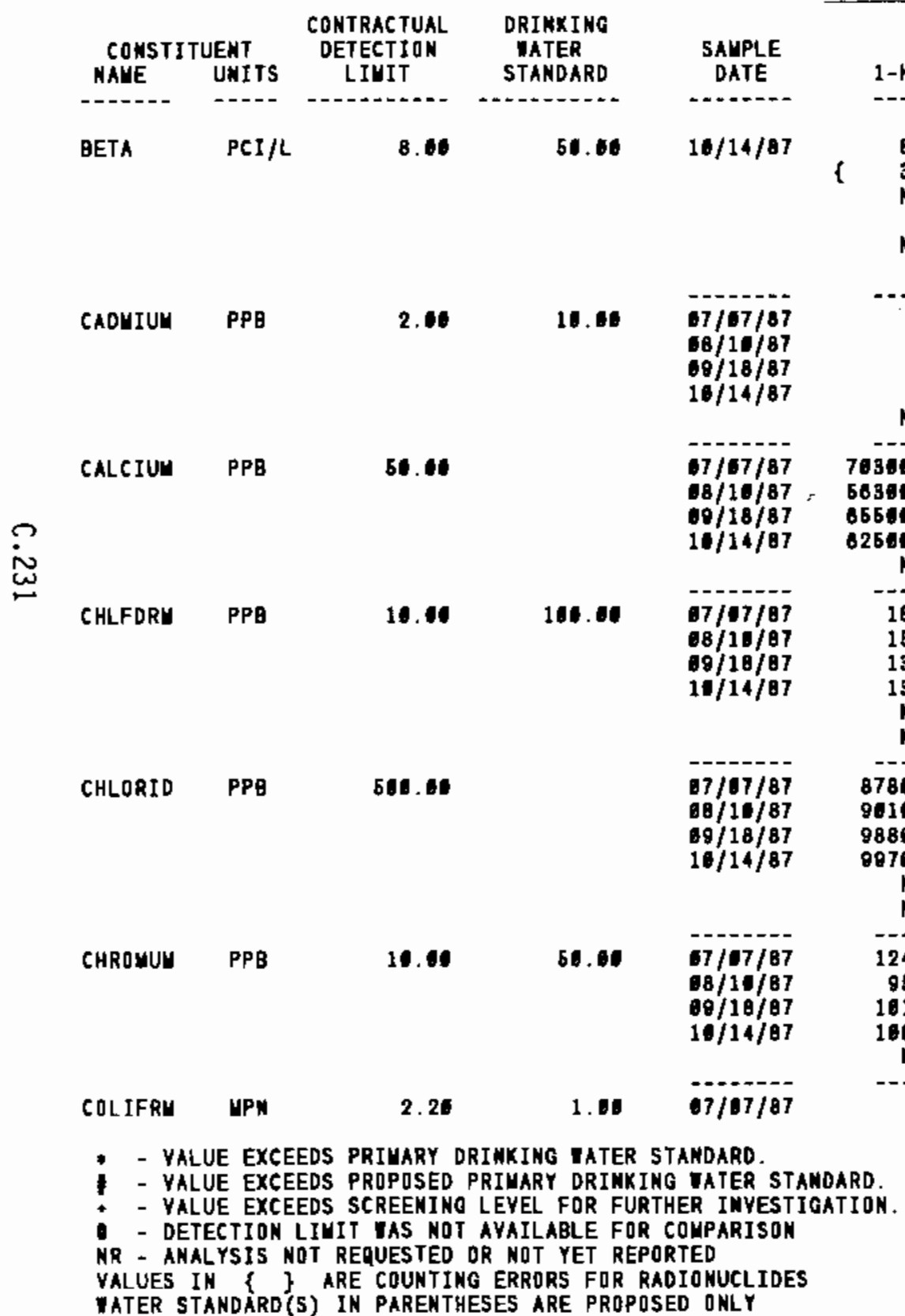

\begin{tabular}{|c|c|}
\hline $\begin{array}{l}\text { SANPLE } \\
\text { DATE }\end{array}$ & $1-H 4-9$ \\
\hline --1-- & 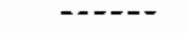 \\
\hline $16 / 68 / 87$ & 84.904 \\
\hline $12 / 28 / 87$ & $184.94+$ \\
\hline $12 / 28 / 87$ & $\begin{array}{r}12.90\} \\
128.00 \\
\{\quad 10.30\}\end{array}$ \\
\hline $\begin{array}{l}17 / 67 / 87 \\
0 / 16 / 87 \\
19 / 16 / 87 \\
10 / 86 / 87 \\
12 / 28 / 87\end{array}$ & 2.00 \\
\hline 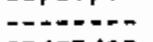 & -- \\
\hline $\begin{array}{l}67 / 07 / 87 \\
68 / 16 / 87 \\
00 / 16 / 87 \\
10 / 08 / 87 \\
12 / 28 / 87\end{array}$ & 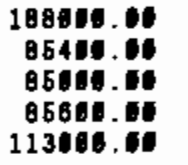 \\
\hline - & $\cdots$ \\
\hline $\begin{array}{l}07 / 07 / 87 \\
08 / 10 / 87 \\
09 / 15 / 87 \\
16 / 86 / 87 \\
12 / 28 / 87 \\
12 / 28 / 87\end{array}$ & $\begin{array}{l}18.00 \\
16.00 \\
12.00 \\
13.00 \\
12.04 \\
14.06\end{array}$ \\
\hline דים & 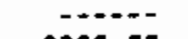 \\
\hline $\begin{array}{l}67 / 67 / 87 \\
08 / 16 / 87 \\
69 / 15 / 87 \\
10 / 68 / 87 \\
12 / 28 / 87 \\
12 / 28 / 87\end{array}$ & $\begin{array}{r}9320.00 \\
16866.00 \\
9340.04 \\
8970.00 \\
16700.00 \\
16806.00\end{array}$ \\
\hline $\begin{array}{l}07 / 07 / 87 \\
88 / 16 / 87 \\
69 / 15 / 87 \\
16 / 66 / 87 \\
12 / 28 / 87\end{array}$ & $\begin{array}{l}130.00 \\
118.00 \\
128.00 \\
141.00 \\
119.00\end{array}$ \\
\hline $97 / 67 / 87$ & \\
\hline
\end{tabular}

SAMPLE

$$
\text { DATE }
$$

1-H4-10

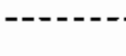

$18 / 06 / 87$

$\quad 6.23$
$\quad 3.02\}$

Q.67
3.99 )
NR

NR

R

NR

70300.01

58301.11

5604. It

MR

....

15.60

13.6

19.60

MP

-T-

9010.00

9880.06

870.00

NR

NR

24.00

101.00 :

160.60

MR

$07 / 67 / 87$
NR

1)/07/87

- 1 7/ 7

$0 / 10 / 87$

$10 / 05 / 87$

MR

$07 / 97 / 87$

08/47/87

$00 / 28 / 87$

43800.

35706.t.

44100. 06

43350.116

NR

-7/47/87

$68 / 07 / 87$

$00 / 18 / 87$

$10 / 65 / 87$

-2...

$17 / 07 / 87$
$6 / 67 / 87$

$18 / 1 / 87$
$60 / 18 / 87$

$10 / 85 / 87$

710719

1/07/97

$10 / 05 / 87$

07/97/87
MR

5049.

5641.14

6810.0

6320.01

750.0

NR

77.0

$78.06 *$

67.

67.11

MR 


\section{TABLE C.5. (contd)}

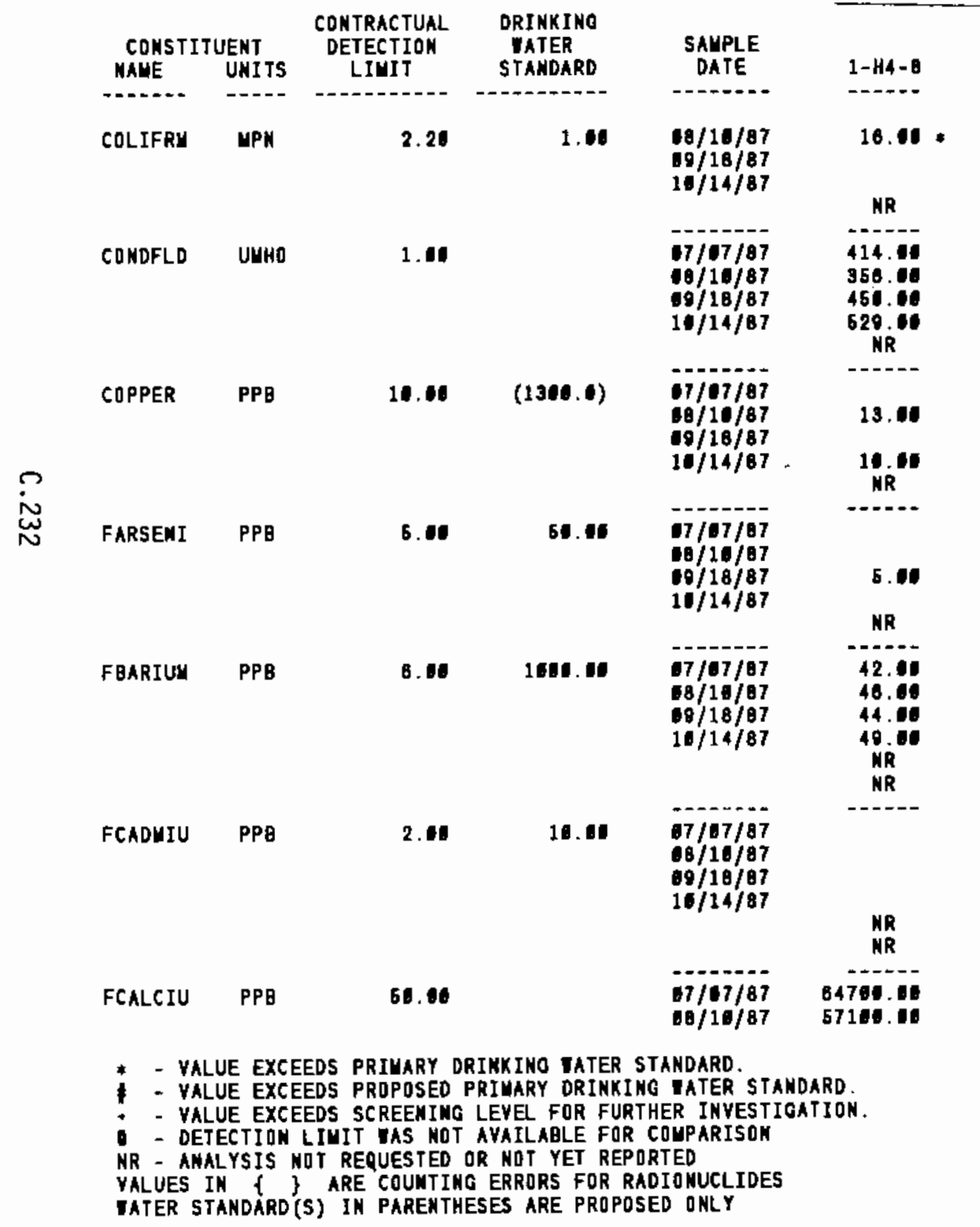

SALPLE

DATE 1-H4-10

$00 / 15 / 87$

$10 / 06 / 87$

$12 / 28 / 87$

$07 / 07 / 87$

$68 / 10 / 87$

$00 / 16 / 87$

$12 / 28 / 87$

740.11

020.10

606.10

780.06

-7/07/87

$68 / 10 / 87$

$69 / 16 / 87$

10/08/87

12/26/87

$17 / 97 / 87$

$68 / 10 / 87$

$0 / 15 / 07$

$16 / 06 / 87$

$12 / 28 / 87$

17/67/87

$68 / 10 / 87$

$60 / 16 / 87$

$10 / 06 / 87$

$12 / 28 / 07$

$12 / 26 / 87$

$7 / 67 / 87$

$18 / 11 / 87$

$69 / 15 / 87$

$10 / 08 / 87$

$12 / 28 / 87$

$12 / 28 / 07$

$07 / 07 / 87$

10/16/87 $+-\cdot-$

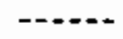

7.01

(日)

59. 15

60.01

87.11

84.10

-....

2.01

113006.00

01406.06
18/07/97

$60 / 18 / 87$

11/05/87

MR

17/07/B7

-

$08 / 07 / 8$

$10 / 55 / 87$

1/07/07

$68 / 67 / 87$

118/87

$10 / 65 / 87$

294.01

235.10

387.6

310.00

MR

16.06

11. 1

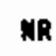

$07 / 07 / 97$

19/07/a7

00/18/07

$16 / 06 / 87$

ต7/47/87

08/17/67

$00 / 19 / 67$

$00 / 19 / 67$
$10 / 06 / 07$

10

17/97/87

$16 / 67 / 87$

$19 / 18 / 87$

$14 / 55 / 87$

$07 / 07 / 87$

68/07/87 


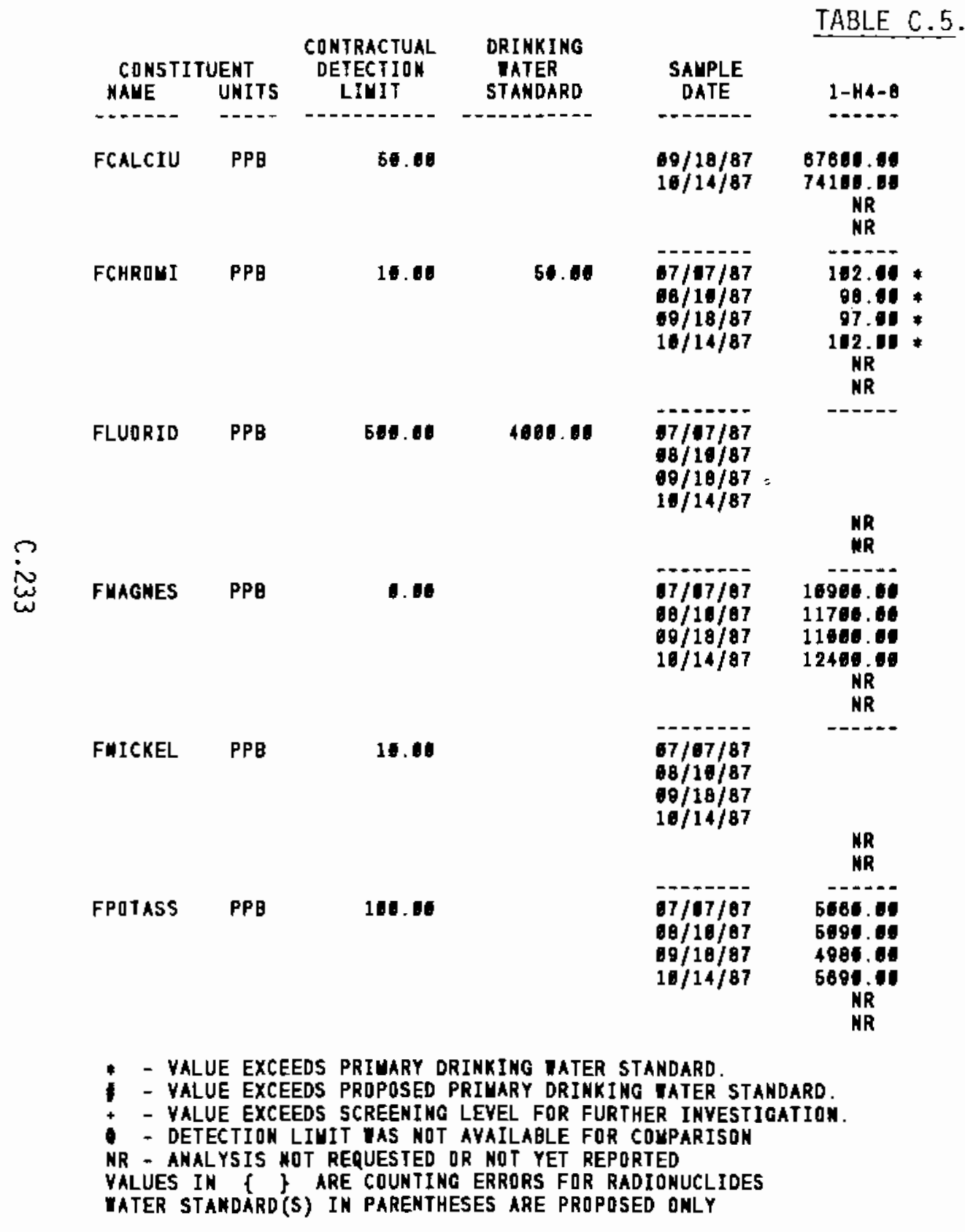

\begin{tabular}{|c|c|}
\hline $\begin{array}{c}\text { SALPLE } \\
\text { DATE }\end{array}$ & $1-H_{4}-8$ \\
\hline$\cdots-1-1$ & ----- \\
\hline $\begin{array}{l}69 / 15 / 87 \\
10 / 06 / 87 \\
12 / 28 / 87 \\
12 / 28 / 87\end{array}$ & $\begin{array}{r}83408.00 \\
80300.06 \\
198908.00 \\
108000.00\end{array}$ \\
\hline $\begin{array}{l}17 / 07 / 87 \\
18 / 14 / 87 \\
19 / 16 / 87 \\
10 / 68 / 87 \\
12 / 28 / 87 \\
12 / 28 / 87\end{array}$ & $\begin{array}{l}119.00 \\
110.00 \\
120.08 \\
133.00 \\
186.04 \\
106.00\end{array}$ \\
\hline $\begin{array}{l}07 / 67 / 87 \\
68 / 10 / 87 \\
00 / 16 / 87 \\
16 / 96 / 87 \\
12 / 28 / 87 \\
12 / 28 / 87\end{array}$ & $\begin{array}{l}650.65 \\
697.65\end{array}$ \\
\hline $\begin{array}{l}17 / 07 / 87 \\
18 / 16 / 87 \\
10 / 16 / 87 \\
10 / 60 / 87 \\
12 / 28 / 87 \\
12 / 28 / 87\end{array}$ & $\begin{array}{l}21500.01 \\
21460.04 \\
14406.01 \\
13406.01 \\
23100.01 \\
22100.04\end{array}$ \\
\hline $\begin{array}{l}07 / 97 / 87 \\
08 / 10 / 87 \\
99 / 16 / 87 \\
10 / 86 / 87 \\
12 / 28 / 87 \\
12 / 28 / 87\end{array}$ & 10.00 \\
\hline $\begin{array}{l}07 / 07 / 87 \\
08 / 10 / 87 \\
00 / 15 / 87 \\
10 / 96 / 87 \\
12 / 28 / 87 \\
12 / 28 / 87\end{array}$ & $\begin{array}{l}7290.06 \\
6680.06 \\
5834.04 \\
6038.04 \\
7176.00 \\
6766.01\end{array}$ \\
\hline
\end{tabular}

\begin{tabular}{|c|c|}
\hline $\begin{array}{c}\text { SAHPLE } \\
\text { DATE }\end{array}$ & 1-H4-18 \\
\hline$--\cdot---$ & ------- \\
\hline $\begin{array}{l}98 / 18 / 87 \\
10 / 66 / 87\end{array}$ & $\begin{array}{c}43300.14 \\
43404.01 \\
\text { KR } \\
\text { MR }\end{array}$ \\
\hline $\begin{array}{l}17 / 07 / 87 \\
18 / 97 / 87 \\
19 / 18 / 87 \\
10 / 15 / 87\end{array}$ & $\begin{array}{c}68.01 \\
92.01 \\
O 2.01 \\
\text { MR } \\
\text { MR }\end{array}$ \\
\hline \multirow[t]{2}{*}{$\begin{array}{l}17 / 67 / 87 \\
18 / 67 / 87 \\
60 / 18 / 87 \\
10 / 65 / 87\end{array}$} & 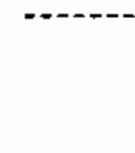 \\
\hline & $\begin{array}{l}\text { NA } \\
\text { MR }\end{array}$ \\
\hline $\begin{array}{l}07 / 107 / 87 \\
10 / 67 / 87 \\
19 / 18 / 87 \\
10 / 05 / 87\end{array}$ & 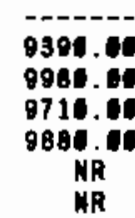 \\
\hline
\end{tabular}

\section{$07 / 07 / 87$}

68/67/87 $60 / 18 / 87$
$10 / 05 / 87$

$87 / 167 / 87$

$88 / 67 / 87$

$69 / 18 / 87$
$10 / 06 / B 7$ 


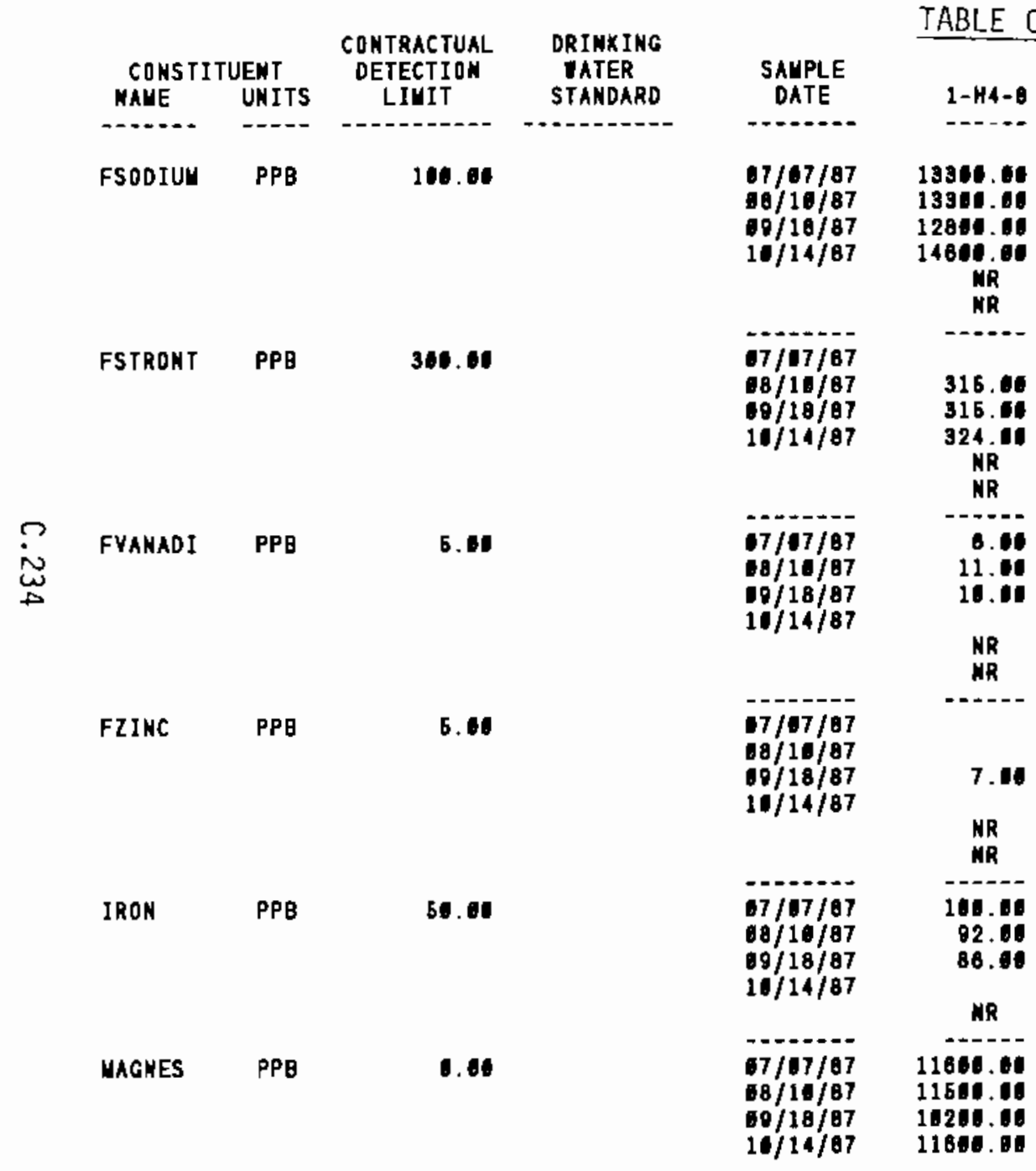

* - VALUE EXCEEDS PRIMARY DRINKING TATER STANDARO.

- - VALUE EXCEEdS PROPDSED PRIMARY DRINKING taTER STANDARD.

- VALUE EXCEEDS SCREENING LEYEL FOR FURTHER INVESTIGATIOK.

- DETECTION LIUIT TAS nOt aYAILABLE for COUPARISON

MR - ANALYSIS NOT REQUESTED OR NOT YET REPORTED

YALUES IN \{\} ARE COUNTING ERRDRS FOR RADIONUCLIOES

VATER STANDARO'(S) IN PARENTHESES ARE PROPOSEO DNLY 
TABLE C.5. (contd)

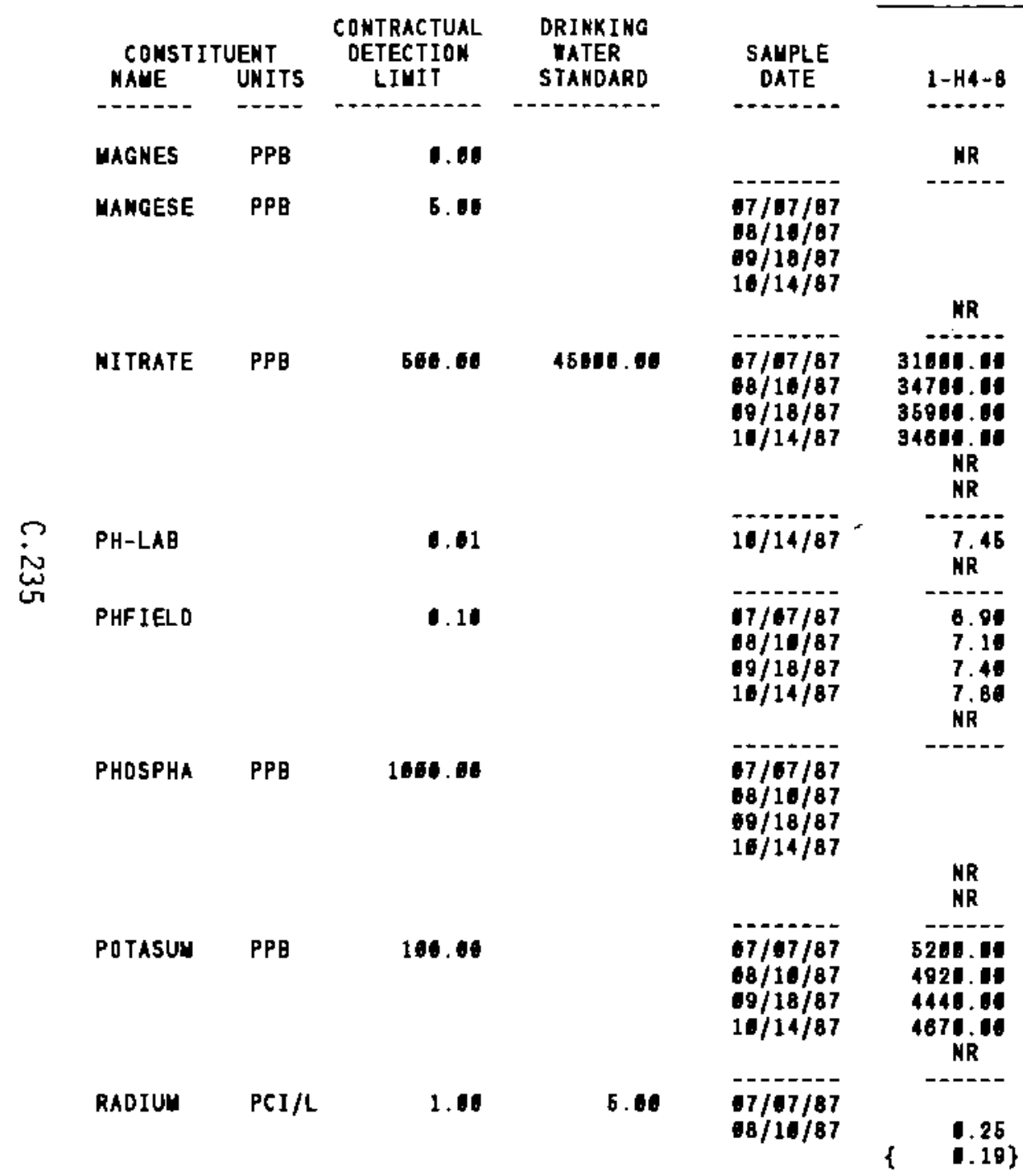

\begin{tabular}{|c|c|}
\hline $\begin{array}{l}\text { SAMPLE } \\
\text { DATE }\end{array}$ & $1-\mathrm{H}_{4}-\mathrm{O}$ \\
\hline $12 / 28 / 87$ & 21300.00 \\
\hline $\begin{array}{l}07 / 07 / 87 \\
08 / 10 / 87 \\
09 / 15 / 87 \\
10 / 06 / 87 \\
12 / 28 / 87\end{array}$ & 5.60 \\
\hline $\begin{array}{l}67 / 97 / 87 \\
68 / 10 / 87 \\
09 / 15 / 87 \\
16 / 08 / 87 \\
12 / 28 / 87 \\
12 / 28 / 87\end{array}$ & 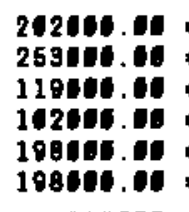 \\
\hline $\begin{array}{l}10 / 06 / 87 \\
12 / 28 / 87\end{array}$ & $\begin{array}{r}7.37 \\
7.58 \\
\end{array}$ \\
\hline $\begin{array}{l}67 / 97 / 87 \\
68 / 16 / 87 \\
69 / 16 / 87 \\
10 / 06 / 87 \\
12 / 28 / 87\end{array}$ & $\begin{array}{l}8.70 \\
7.40 \\
7.60 \\
7.80 \\
8.06\end{array}$ \\
\hline $\begin{array}{l}07 / 07 / 87 \\
08 / 10 / 87 \\
00 / 15 / 87 \\
10 / 08 / 87 \\
12 / 28 / 87 \\
12 / 28 / 87\end{array}$ & $\begin{array}{l}6580.40 \\
8580.80\end{array}$ \\
\hline $\begin{array}{l}07 / 07 / 87 \\
08 / 16 / 87 \\
19 / 16 / 87 \\
11 / 18 / 87 \\
12 / 28 / 67\end{array}$ & $\begin{array}{l}8506.10 \\
0146.80 \\
5860.01 \\
5286.80 \\
8256.00\end{array}$ \\
\hline $\begin{array}{r}17 / 67 / 87 \\
8 / 10 / 87\end{array}$ & \\
\hline
\end{tabular}

\begin{tabular}{|c|c|}
\hline $\begin{array}{l}\text { SA'PLE } \\
\text { DATE }\end{array}$ & $1-\mathrm{H}_{4-10}$ \\
\hline & NR \\
\hline \multirow[t]{2}{*}{$\begin{array}{l}07 / 07 / 87 \\
08 / 07 / 87 \\
09 / 18 / 87 \\
16 / 105 / 87\end{array}$} & \\
\hline & NR \\
\hline $\begin{array}{l}97 / 07 / 87 / 87 \\
08 / 97 / 87 \\
69 / 18 / 87 / 187 \\
10 / 06 / 87\end{array}$ & 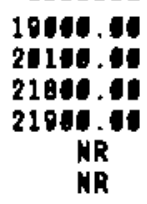 \\
\hline $11 / 65 / 87$ & ${ }_{\mathrm{NR}}^{7.11}$ \\
\hline $\begin{array}{l}7 / 67 / 87 \\
0 / 67 / 87 \\
69 / 18 / 87 \\
10 / 05 / 87\end{array}$ & $\begin{array}{l}8.50 \\
8.90 \\
7.31 \\
7.60 \\
\text { MR }\end{array}$ \\
\hline
\end{tabular}

$07 / 07 / 67$

68/107/87

$9 / 18 / 87$

$10 / 105 / 87$

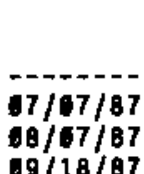

$11 / 05 / 87$

4605.10

4340.06

4110.00

4170

$07 / 07 / 87$

$08 / 87 / 87$

* - VALUE eXCEeds PRIUARY dRINKING Iater standard.

- VALUE EXCEEDS PROPOSED PRIUARY oRINKING TATER STANDARD.

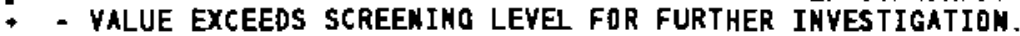

- DEtection livit ias not available for coMparison

HR - AMALYSIS NOT REQUESTED OR NOT YET REPORTED

VALUES IN \& $\}$ ARE COUNTING ERRORS FOR RADIONUCLIDES

VATER STAMDARD (S) IN PARENTHESES ARE PRGPOSED ONLY 
TABLE C.5. (contd)

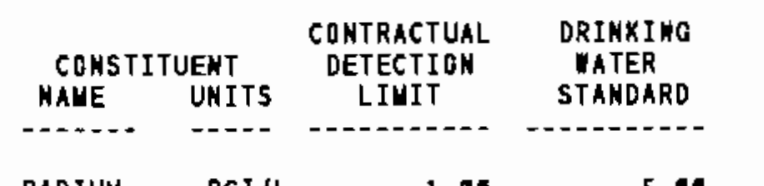
DATE

$1-\mathrm{H4}-8$

SAMPLE

1-H4-9

SALPLE

DATE

1-H4-10

RADIUN PCI/L

1.06

$80 / 18 / 87$
$10 / 14 / 87$

19/15/87

$10 / 108 / 87$

$12 / 28 / 87$

NR

$07 / 07 / 87$

SODIUN PPB

160.64

$67 / 07 / 87$

13800.60

12906.

$\begin{array}{ll}09 / 18 / 87 & 11160.00 \\ 10 / 14 / 97 & 11600.00\end{array}$

$10 / 14 / 87$

NR

STRONUM PPB

380.49

17/19/87

321.06

18/10/87

$10 / 10 / 87$

10/14/87 — 307.69

NR

$67 / 07 / 87$

$67 / 07 / 87$
$6 / 10 / 87$
$10 / 10 / 87$

-

71501.0

74000.08

76900.00

NR

11/14/87

$68 / 16 / 87$

$60 / 15 / 87$

$10 / 06 / 87$

$12 / 28 / 87$

-

17/07/87

$68 / 10 / 87$

$18 / 15 / 87$

$12 / 28 / 87$

$07 / 07 / 87$

$08 / 16 / 87$

$0 / 15 / 97$

10/08/87

$12 / 28 / 87$

$12 / 28 / 87$

$\overline{16 / 14 / 87}$

323\%日.

$16 / 06 / 87$

NR

$12 / 28 / 87$

$19 / 14 / 87 \quad 307860.60$

-

$10 / 08 / 87$

TDS

NR

17/07/87 1120.00

TOC

PPB

1000.09

$08 / 16 / 87$

08/18/87

$10 / 14 / 87$

-

97/67/87

$6 / 16 / 87$

19/15/87

$10 / 06 / 07$

$12 / 28 / 87$

NR

VAMAOUM

PPB

5. 10

$17 / 07 / 87$

$68 / 10 / 87$

……-

$67 / 07 / 87$

$08 / 16 / 87$

00/18/87

5.00
11.00

$00 / 15 / 87$

$\left\{\begin{array}{c}0.16 \\ .15\}\end{array}\right.$

3100.

27866.66

2414.

22411.60

31800.00

408.60

481.11

385.

367.4

514.00

7089

70806

07261.

7921.

74801.04

B5a44.010

86364.06

$360 \mathrm{coc}$

38460.01:

401006.00

584000.018

1686.

142.

1940. 0

7

7.6

0.6
$09 / 18 / 87$

$10 / 05 / 87$

NR

17/47/87

08/107/87

$19 / 18 / 87$

$10 / 06 / 87$

14710.11

13290.01

13201.00

NR

17/07/87

$18 / 07 / 87$

09/18/07

$10 / 05 / 87$

07/07/87

$08 / 07 / 87$

19/10/87

$10 / 06 / 87$

$10 / 06 / 67$

$10 / 0187$

$10 / 05 / 97$

-.-.-.-

$07 / 67 / 87$

00/17/87

$09 / 18 / 87$

$10 / 16 / 87$

$67 / 07 / 87$

$68 / 97 / 87$

$68 / 07 / 87$
$69 / 10 / 87$

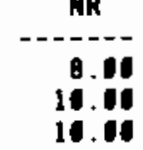

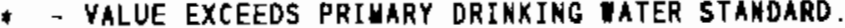

- - VALUE EXCEEDS PROPOSED PRIUARY DRINKIMG VATER STANDARD

- - VALUE EXCEEDS SCREENING LEVEL FQR FURTHER INVESTIGATION.

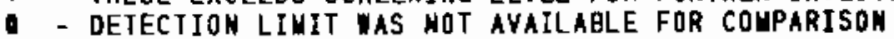

HR - ANALYSIS NOT REQUESTED OR NOT YET REPORTED

VALUES IN \{ $\}$ ARE COUNTING ERRORS FOR RADIONUCL IDES

IATER STAMDARO'(S) IN PARENTHESES ARE PROPOSED ONLY 
TABLE C.5. (contd)

\begin{tabular}{|c|c|c|c|c|c|c|c|c|c|}
\hline $\begin{array}{l}\text { CDNSTI } \\
\text { NAUE }\end{array}$ & $\begin{array}{l}\text { UENT } \\
\text { UNITS }\end{array}$ & $\begin{array}{c}\text { CONTRACTUAL } \\
\text { DETECTIDN } \\
\text { LIMIT }\end{array}$ & $\begin{array}{c}\text { DRINKIHG } \\
\text { NATER } \\
\text { STANDARD }\end{array}$ & $\begin{array}{c}\text { SAMPLE } \\
\text { DATE }\end{array}$ & $1-\mathrm{H}_{4}-8$ & $\begin{array}{c}\text { SAWPLE } \\
\text { DATE }\end{array}$ & $1-\mathrm{H} 4-9$ & $\begin{array}{c}\text { SALPLE } \\
\text { DATE }\end{array}$ & $1-14-16$ \\
\hline$\cdots+\cdots$ & --- & 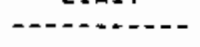 & - - - & - - & ----- & $-\cdots-\infty$ & ---- & $-\ldots$ & ---2 \\
\hline YAMADUN & PPB & 5.00 & & $10 / 14 / 87$ & $\begin{array}{l}5.60 \\
\text { NR }\end{array}$ & $\begin{array}{l}10 / 06 / 87 \\
12 / 28 / 87\end{array}$ & 7.00 & $10 / 65 / 87$ & 10.10 \\
\hline ZINC & PPB & 6.00 & & $\begin{array}{l}07 / 67 / 87 \\
08 / 16 / 87 \\
09 / 16 / 87 \\
10 / 14 / 87\end{array}$ & $\begin{array}{l}16.06 \\
22.64 \\
8.64 \\
\text { NR }\end{array}$ & $\begin{array}{l}-1 / 57 / 87 \\
10 / 16 / 87 \\
10 / 15 / 87 \\
10 / 86 / 87 \\
12 / 28 / 87\end{array}$ & $\begin{array}{r}6.06 \\
24.00 \\
6.00 \\
6.00\end{array}$ & $\begin{array}{l}07 / 07 / 87 \\
08 / 07 / 87 \\
69 / 18 / 67 \\
16 / 65 / 87\end{array}$ & $\begin{array}{l}0.00 \\
12.00 \\
\text { NR }\end{array}$ \\
\hline
\end{tabular}

$\stackrel{\Gamma}{\underset{\omega}{\omega}}$

* - value EXCEEDS PRIMARY dRINKIng Mater stanoard.

- VALUE EXCEEDS PROPDSED PRIUARY DRINKINO WATER STANDARD.

- - VALUE EXCEEDS SCREENING LEYEL FDR FURTHER INYESTIGATION.

- DETECTION LiHIt TAS nOT AVAILABLE FOR COMPARISON

NR - ANALYSIS NOT REQUESTED OR NOT YET REPORTED

VALUES IN \& \} ARE COUNTING ERRORS FOR RADIONUCLIDES

WATER STANOARD(S) IN PARENTHESES ARE PRDPOSED ONLY 


\section{TABLE C.5. (contd)}

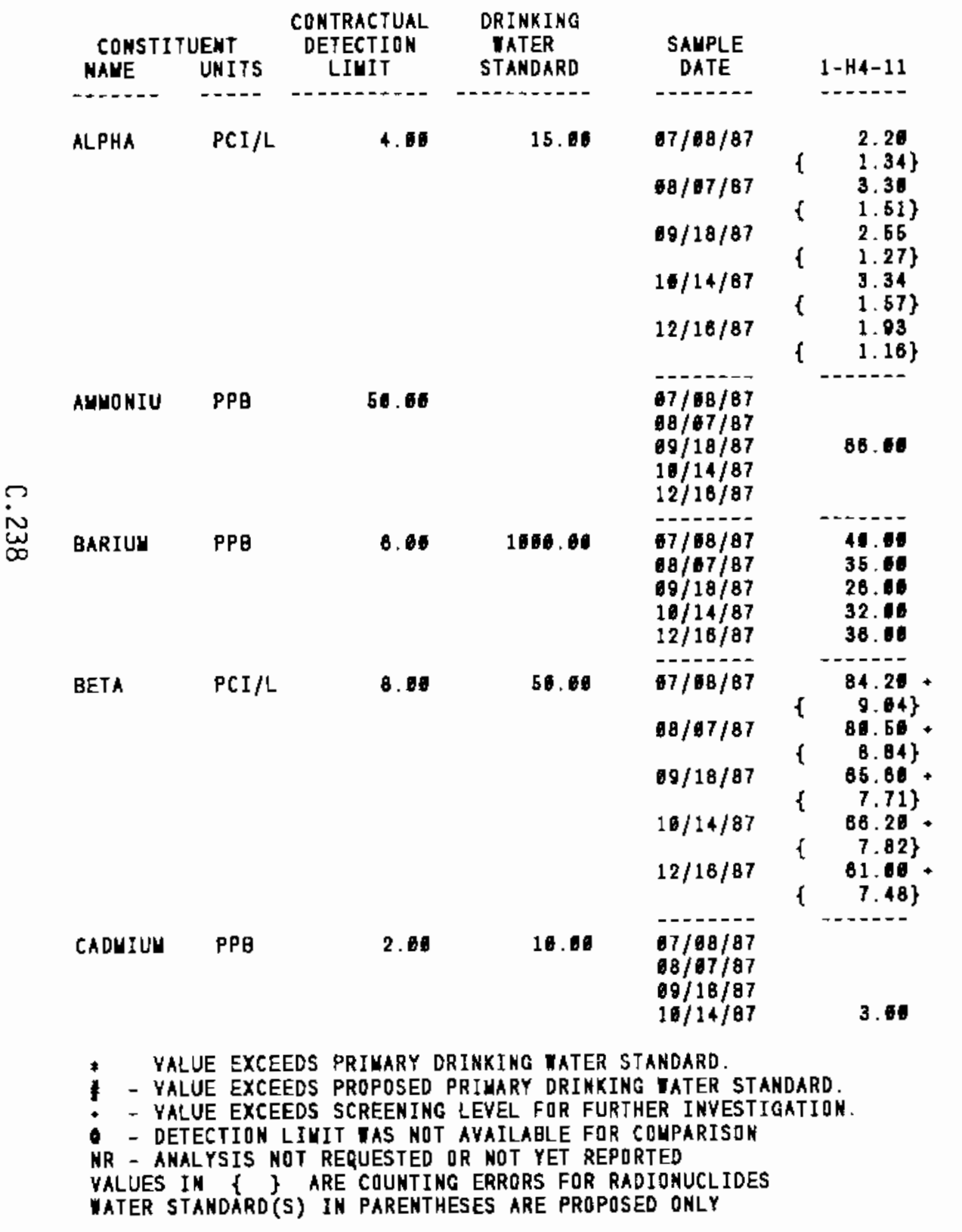

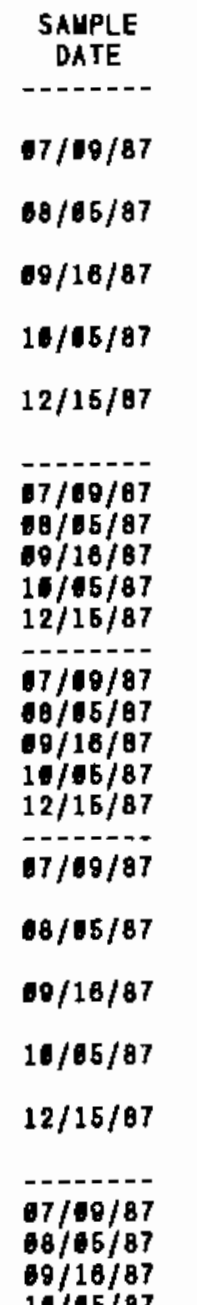

$1-H 4-12 A$

-

$15.50+$

20.28.

(3.89\}

14.70

$3.28\}$

$3.19\}^{\circ}$

8.85

$\left\{\begin{array}{l}8.46\} \\ \text {. }\end{array}\right.$

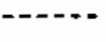

88. 10

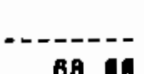

71.01

64.80

73.66

B日.t.

59.80.

( 7.64$\}$

$60.36+$

$7: 79\}$

58.60 .

$\{7.59\}$

45.10

8.55

38.89
$0.47\}$
SAMPLE

DATE

1-H4-12日

$17 / 09 / 87$

\{ 2.10$\}$

8.4
$+\quad 2.36$

$09 / 16 / 87\left\{\begin{array}{l}2.36\} \\ 0.64\end{array}\right.$

\{ 2.50$\}$

5.18
$1.96\}$

17/00/87

18

-

$09 / 16 / 87$

$10 / 16 / 87$

$12 / 16 / 87$

$07 / 60 / 87$

$08 / 05 / 87$

69/18/87

16/66/87

$12 / 16 / 67$

$07 / 00 / 87$

08/05/87

$19 / 18 / 87$

$16 / 68 / 87$

$12 / 15 / 87$

$-1-10$

$68 / 05 / 87$

69/18/87

16/06/87 
TABLE C.5. (contd)

\begin{tabular}{|c|c|c|c|c|}
\hline \multicolumn{2}{|c|}{$\begin{array}{l}\text { CONSTITUENT } \\
\text { MAME UNITS }\end{array}$} & $\begin{array}{c}\text { CONTRACTUAL } \\
\text { DEIECTION } \\
\text { LIWIT }\end{array}$ & $\begin{array}{l}\text { DRINKING } \\
\text { IATER } \\
\text { STANDARD }\end{array}$ & $\begin{array}{c}\text { SANPLE } \\
\text { DATE }\end{array}$ \\
\hline.----- & $\cdots--$ & 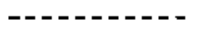 & - & ------ \\
\hline CADUIUM & PPB & 2.65 & 10.00 & $12 / 16 / 87$ \\
\hline CALCIUU & PPB & 60.61 & & $\begin{array}{l}97 / 68 / 87 \\
98 / 67 / 87 \\
69 / 18 / 87 \\
18 / 14 / 87 \\
12 / 16 / 87\end{array}$ \\
\hline CHLFORU & PPB & 10.01 & 15. & $\begin{array}{l}67 / 08 / 87 \\
68 / 107 / 87 \\
6 / 18 / 87 \\
16 / 14 / 87 \\
12 / 16 / 87\end{array}$ \\
\hline CHLORID & PPB & 500.00 & & $\begin{array}{l}--1 / 6 / 87 \\
07 / 96 / 87 \\
18 / 97 / 87 \\
19 / 18 / 87 \\
16 / 14 / 87 \\
12 / 18 / 87\end{array}$ \\
\hline CHRDUUN & PPB & 10.00 & 50.66 & $\begin{array}{l}67 / 18 / 87 \\
68 / 07 / 87 \\
69 / 18 / 87 \\
16 / 14 / 87 \\
12 / 16 / 87\end{array}$ \\
\hline COLIFRU & MPN & 2.20 & 1.66 & $\begin{array}{l}-97 / 68 / 87 \\
68 / 67 / 87 \\
69 / 18 / 87 \\
16 / 14 / 87 \\
12 / 18 / 87\end{array}$ \\
\hline CONDFLD & UעHO & 1.00 & & $\begin{array}{l}----- \\
67 / 68 / 87 \\
68 / 07 / 87 \\
69 / 18 / 87 \\
19 / 14 / 87 \\
12 / 18 / 87\end{array}$ \\
\hline COPPER & PPB & 10.00 & (1310.0) & $97 / 66 / 87$ \\
\hline
\end{tabular}

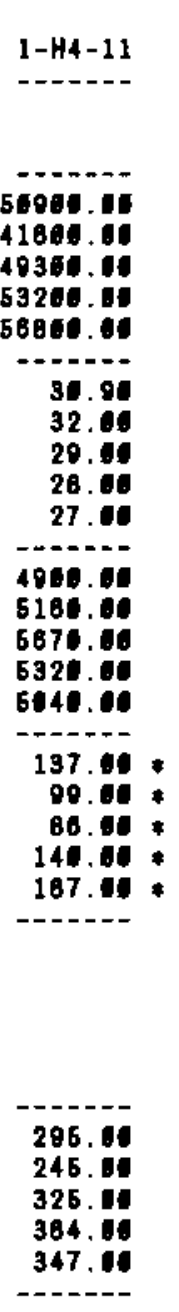

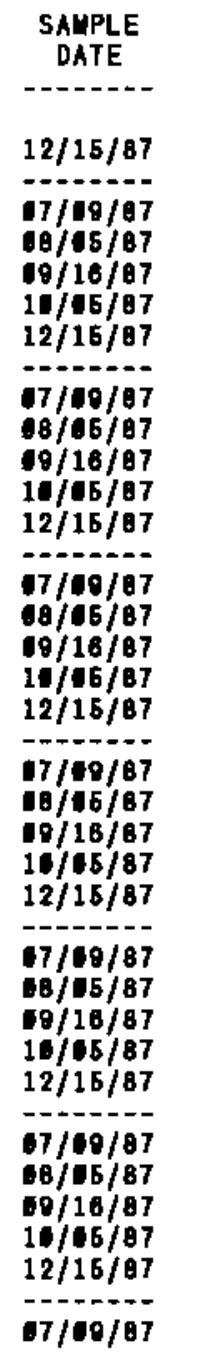

1-H4-12A

SAMPLE

DATE

1-H4-12B

$12 / 15 / 87$

2.00

B7801. 11

56564.00

73106.09

79400.01

73004.01

........

21.01

19.00

17.01

17.00

6706.0

8896.00

7676.0

B17.. 1

7170.0

215.01

215.01

181.4 .

197.1

$177.10 *$

67/09/87

$68 / 45 / 87$

60/18/87

$16 / 06 / 87$

$12 / 16 / 07$

$7 / 19187$

68/06/87

$6 / 18 / 87$

$00 / 18 / 87$
$10 / 08 / 87$

$12 / 15 / 97$

$67 / 09 / 87$

68/05/87

60/16/87

19/48/87

$12 / 15 / 87$

$-7 / 197$

$07 / 60 / 87$

18/55/87

$19 / 16 / 87$

$10 / 66 / 87$

12/15/87

$67 / 00 / 07$

16.16*

8/95/87

$118 / 07$

18/18/87

$1 / 48 / 87$

$12 / 16 / 87$

$07 / 00 / 87$

$08 / 55 / 87$

$09 / 16 / 87$

$10 / 06 / 67$

$12 / 15 / 87$

$67 / 00 / 67$

57194

57104.00

55104.01

71810.01

67919.01

70414. 11

10.4

18.41

18.0

17.11

17.00

6761.01

6051.61

7390.66

7015.06

7170.06

-

104.01.

102.09

171.04

178.01

184.11 :

434.0

618.0

616.60

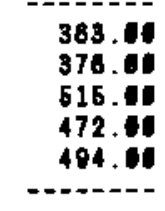

* - ValUE eXCEEDS PRIUARY dRINKING tater STANDARD.

- VALUE EXCEEDS PROPOSED PRIMARY DRINKIMG IATER STANDARD.

* - YALUE EXCEEDS SCREENIMg LEVEL FOR FURTHER IMYESTIGATIOA.

- - DETECTION LiUIT IAS NOT aYailable for coyparison

NR - AKALYSIS NOT REQUESTED OR NOT YET REPORTED

YALUES IN 5 ARE COUNTINO ERRORS FDR RADIONUCLIDES

WATER STANDARD(S) IN PARENTHESES ARE PROPDSED ONLY 
TABLE C.5. (contd)

\begin{tabular}{ccrc} 
CONSTITUENT & $\begin{array}{c}\text { CONTRACTUAL } \\
\text { DETECTION } \\
\text { NAME }\end{array}$ UNITS & $\begin{array}{c}\text { DRINKING } \\
\text { WATER } \\
\text { STANDARD }\end{array}$ \\
\hline COPPER & PPB & 10.06 & $(1366.6)$
\end{tabular}

$\begin{array}{cc}\begin{array}{c}\text { SAUPLE } \\ \text { DATE }\end{array} & 1-H 4-11 \\ & \\ 0 / 07 / 87 & \\ 09 / 18 / 87 & \\ 10 / 14 / 87 & 274.00 \\ 12 / 16 / 87 & \end{array}$

SALPLE
DATE
-

$08 / 05 / 87$

$09 / 16 / 87$

$10 / 05 / 87$

$12 / 15 / 87$

FBARIUU PPB

6. 601006.01

- $7 / 18 / 87$

FCALCIU PPB

50.00

in

FCHROUI PPB

10.61

50. 81

FWAGNES PPB

60

FPOTASS PPE

100.00

FSODIUU PPB

160.06
68/07/67

$09 / 18 / 87$

$1 / 14 / 87$

$12 / 16 / 87$

39.11

41.00

33.0

35.10

41.6

49600.08

47564.10

5eget. 40

48196

63800.60

$16 / 14 / 67$

$12 / 18 / 87$

-.......

$97 / 08 / 87$
$08 / 07 / 87$

$08 / 07 / 87$
$09 / 18 / 87$

$99 / 18 / 87$

12/16/87

17/88/87

19/07/87

19/18/87

$18 / 14 / 87$

$18 / 14 / 87$
$12 / 18 / 87$

ต7/0日/07

$68 / 67 / 87$

69/18/67

$10 / 14 / 87$

$12 / 16 / 87$

$07 / 08 / 87$

$66 / 07 / 87$

$09 / 10 / 07$
$10 / 14 / 87$
134.10

114.10

87.00

127.00 :

7470.46

7981.40

6940.10

7690.08

7040.00

2620.60

2800.00

2860.60

2871. 10

2820.00

7940.06

7940.00

7770.00
B910.00

8806.00
$07 / 09 / 87$

68/65/87

$19 / 18 / 87$

$10 / 15 / 67$

$12 / 15 / 87$

67/09/87

$08 / 56 / 87$

19/16/87

$10 / 16 / 87$

12/15/87

17/0/87

$97 / 109 / 87$
$18 / 06 / 87$

$69 / 16 / 87$

$10 / 06 / 87$

$12 / 15 / 87$

$17 / 09 / 87$

08/05/87

09/16/87

$16 / 65 / 87$

12/15/87

$4 / 09 / 87$

$10 / 06 / 87$

$09 / 18 / 67$

$10 / 05 / 87$

12/15/87

- $7100 / 87$

$08 / 05 / 67$

$08 / 66 / 87$

$10 / 65 / 87$
SAMPLE

DATE

1-H4-12B

10/05/87

69/16/87

$10 / 08 / 87$

12/16/87

$17 / 00 / 87$

$08 / 05 / 87$

69/16/87

$16 / 08 / 87$

$12 / 15 / 87$

0/10/87

8/05/87

$60 / 18 / 87$

$10 / 06 / 87$

12/16/87

17/00/a7

18/56/87

$0 / 65 / 87$
$00 / 18 / 87$

$00 / 18 / 87$
$10 / 68 / 87$

$10 / 98 / 87$
$12 / 15 / 87$

17/09/87

18/06/87

00/16/8?

10/06/87

12/15/87

$97 / 60 / 87$

$08 / 55 / 87$

$60 / 16 / 87$

$10 / 68 / 87$

$12 / 15 / 87$

$7 / 98 / 87$

$07 / 99 / 87$

$18 / 05 / 87$

$09 / 16 / 87$

$10 / 98 / 87$

09.01

98.0

116.01

จ8. 06

167.6

82801 11

55804.01

68241.00

68304.6

71680.06

1089.

108.06

187.6 :

176.14

180.19 *

150.00 .

1070e.

10000.00

10019 of

10600.06

11560.66

5630.60

5186.

4806.10

4821.11

$509 \% .11$

1.0

18860.60

28400.60

12914.

12001.

12400.06

10800.00

* - VALUE EXCEEDS PRIUARY DRINKING TATER STANDARD.

- - VALUE EXCEEDS PROPOSED PRIMARY DRINKING TATER STANDARD.

+ - VALUE EXCEEDS SCREENING LEVEL FOR FURTHER INYESTIGATION.

- DETECTION LIUIT WAS NOT AVAILABLE FOR COUPARISDN

HR - ANALYSIS NOT REQUESTED OR NDT YET REPORTED

VALUES IN \{\} ARE COUNTING ERRORS FOR RADIONUCLIDES

WATER STANDARD'(S) IN PARENTHESES ARE PROPOSED ONLY 
TABLE C.5. (contd)

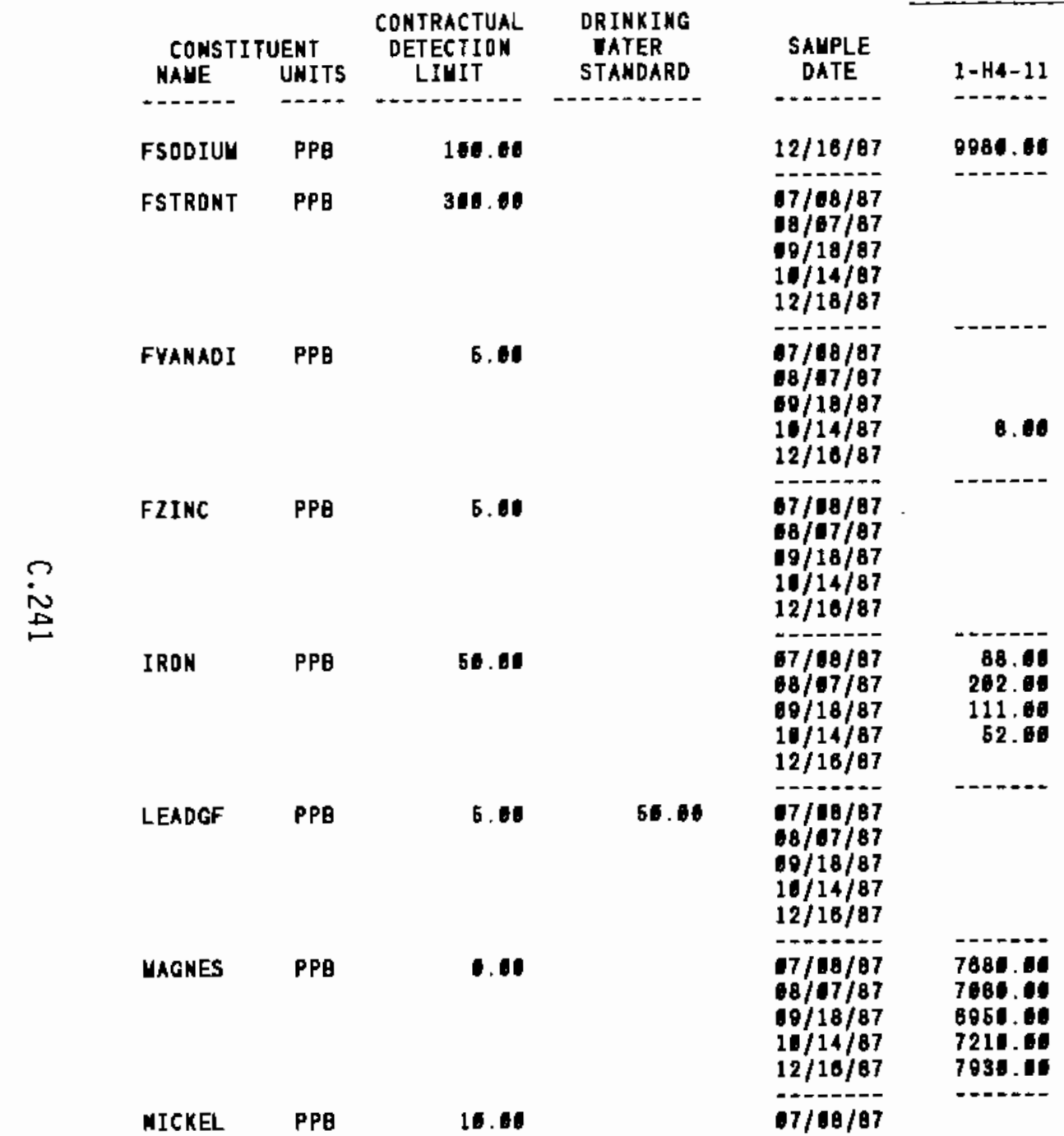

\begin{tabular}{|c|c|}
\hline $\begin{array}{l}\text { SAMPLE } \\
\text { DATE }\end{array}$ & $1-H 4-12 A$ \\
\hline - & \\
\hline $12 / 16 / 87$ & 13401.00 \\
\hline $\begin{array}{l}07 / 09 / 87 \\
68 / 05 / 87 \\
19 / 18 / 87 \\
18 / 95 / 87 \\
12 / 15 / 87\end{array}$ & $\begin{array}{l}303.00 \\
316.00 \\
398.11 \\
347.00 \\
318.00\end{array}$ \\
\hline $\begin{array}{l}17 / 00 / 87 \\
18 / 55 / 87 \\
0 / 18 / 87 \\
10 / 66 / 87 \\
12 / 16 / 87\end{array}$ & $\begin{array}{l}7.01 \\
8.01\end{array}$ \\
\hline $\begin{array}{l}-17 / 60 / 87 \\
0 / 65 / 87 \\
0 / 16 / 87 \\
10 / 66 / 87 \\
12 / 16 / 87\end{array}$ & 0.10 \\
\hline $\begin{array}{l}07 / 00 / 87 \\
08 / 65 / 87 \\
09 / 16 / 87 \\
11 / 65 / 87 \\
12 / 16 / 87\end{array}$ & \\
\hline $\begin{array}{l}7 / 10 / 87 \\
18 / 85 / 87 \\
09 / 16 / 87 \\
10 / 85 / 87 \\
12 / 15 / 87\end{array}$ & 5.10 \\
\hline $\begin{array}{l}07 / 10 / 87 \\
88 / 85 / 87 \\
99 / 18 / 87 \\
10 / 85 / 87 \\
12 / 15 / 87\end{array}$ & $\begin{array}{l}11100.00 \\
11000 \\
14200.01 \\
16400.00 \\
10700 .\end{array}$ \\
\hline $67 / 60 / 87$ & \\
\hline
\end{tabular}

SAUPLE DATE

1-H4-12B DATE

$12 / 16 / 87 \quad 12206.00$

$07 / 09 / 87$

68/05/87 315.01

69/16/87 341.01

$10 / 10 / 87$

$12 / 15 / 97$

------

$07 / 09 / 87$

68/06/87

$6 / 16 / 87$

$10 / 00 / 87$

$12 / 15 / 07$

$07 / 09 / 87$

$68 / 65 / 87$

$0 / 16 / 87$

19/16/8/87

$12 / 15 / 87$

7.

7.16

7.05

5.61

-

1.10

1..1

10.4

12.04

$07 / 09 / 87$

$08 / 05 / 87$

$69 / 10 / 87$

$16 / 06 / 87$

$12 / 16 / 87$

$07 / 197$

$07 / 09 / 87$
$08 / 05 / 87$

$08 / 05 / 87$
$00 / 16 / 87$

$16 / 08 / 87$

$12 / 15 / 87$

-7/ 9187

$18 / 65 / 87$

$00 / 16 / 87$

10/06/87

$12 / 15 / 87$

07/09/87

10194

11100.0

1170.

10700.04

12200.06

* - VAlUE eXCEEDS PRIUARY dRINKING tater STARDARD.

- - VALUE EXCEEDS PROPOSED PRIMARY DRIHKING TATER STANDARD.

- YALUE EXCEEDS SCREENING LEYEL FOR FURTHER INYESTIGATIDN,

- DETECTION LIUIT IAS NOT AVAILABLE FOR CDYPARISON

NR - ANALYSIS NOT RERUESTED OR NOT YET REPORTED

NR - ANALYSIS NOT REQUESTED OR NOT Y

VALUES IN \{ $\}$ ARE COUNTING ERRORS FOR RADIONUCLIDES
WATER STAMARD(S) IN PARENTHESES ARE PRDPOSED DNLY 
TABIE C.5. (contd)

\begin{tabular}{|c|c|c|c|c|c|c|c|c|c|}
\hline $\begin{array}{l}\text { CONSTI } \\
\text { NANE }\end{array}$ & $\begin{array}{l}\text { UENT } \\
\text { UNITS }\end{array}$ & $\begin{array}{l}\text { CONTRACTUAL } \\
\text { DETECTIDN } \\
\text { LIUIT }\end{array}$ & $\begin{array}{l}\text { DRINKING } \\
\text { TATER } \\
\text { STANDARD }\end{array}$ & $\begin{array}{l}\text { SAUPLE } \\
\text { DATE }\end{array}$ & $1-\mathrm{H} 4-11$ & $\begin{array}{c}\text { SAUPLE } \\
\text { DATE }\end{array}$ & $1-H 4-12 A$ & $\begin{array}{l}\text { SAMPLE } \\
\text { DATE }\end{array}$ & $1-H 4-12 B$ \\
\hline MICKEL & PPQ & 10.00 & & $\begin{array}{l}08 / 07 / 87 \\
69 / 18 / 87 \\
16 / 14 / 87 \\
12 / 16 / 87\end{array}$ & 01.00 & $\begin{array}{l}18 / 15 / 87 \\
60 / 18 / 87 \\
10 / 05 / 87 \\
12 / 15 / 87\end{array}$ & & $\begin{array}{l}18 / 05 / 87 \\
60 / 16 / 87 \\
10 / 68 / 87 \\
12 / 15 / 87\end{array}$ & \\
\hline NITRATE & PPB & 500.00 & 45060.78 & $\begin{array}{l}17 / 68 / 87 \\
08 / 67 / 87 \\
69 / 18 / 87 \\
18 / 14 / 87 \\
12 / 18 / 87\end{array}$ & $\begin{array}{l}36700.00 \\
32609.06 \\
28200.00 \\
28800.00 \\
28606\end{array}$ & $\begin{array}{l}07 / 69 / 87 \\
60 / 65 / 87 \\
69 / 18 / 87 \\
18 / 05 / 87 \\
12 / 16 / 87\end{array}$ & 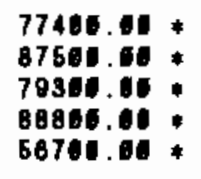 & $\begin{array}{l}67 / 00 / 87 \\
08 / 56 / 87 \\
10 / 18 / 87 \\
16 / 06 / 87 \\
12 / 15 / 87\end{array}$ & $\begin{array}{l}49300.00 \\
58408.60 \\
80816.01 \\
55006.08 \\
52601.04\end{array}$ \\
\hline PH-LAB & & e. 1 & & $\begin{array}{l}10 / 11 / 87 \\
12 / 16 / 87\end{array}$ & $\begin{array}{l}7.78 \\
7.78\end{array}$ & $\begin{array}{l}10 / 05 / 87 \\
12 / 15 / 87\end{array}$ & $\begin{array}{l}7.86 \\
7.77\end{array}$ & $\begin{array}{l}11 / 68 / 87 \\
12 / 15 / 87\end{array}$ & $\begin{array}{l}7.03 \\
7.74\end{array}$ \\
\hline PHF IELD & & 0.10 & & $\begin{array}{l}07 / 98 / 87 \\
68 / 97 / 87 \\
69 / 18 / 87 \\
16 / 14 / 87 \\
12 / 16 / 87\end{array}$ & $\begin{array}{l}7.30 \\
7.50 \\
7.40 \\
8.80 \\
7.54\end{array}$ & $\begin{array}{l}67 / 09 / 87 \\
68 / 05 / 87 \\
09 / 16 / 87 \\
11 / 05 / 87 \\
12 / 15 / 87\end{array}$ & $\begin{array}{l}7.40 \\
7.80 \\
7.58 \\
7.31 \\
9.40\end{array}$ & $\begin{array}{l}17 / 10 / 87 \\
10 / 65 / 87 \\
19 / 18 / 87 \\
10 / 86 / 87 \\
12 / 15 / 87\end{array}$ & $\begin{array}{l}7.40 \\
6.80 \\
7.50 \\
8.41 \\
8.21\end{array}$ \\
\hline \multirow[t]{3}{*}{ RADIUN } & \multirow[t]{3}{*}{ PCI/L } & \multirow[t]{3}{*}{1.60} & \multirow[t]{3}{*}{5.80} & $07 / 08 / 87$ & & $67 / 69 / 87$ & \multirow{3}{*}{ 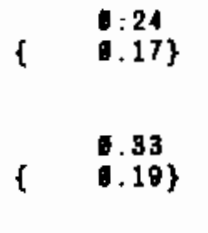 } & $07 / 09 / 87$ & \multirow[t]{3}{*}{$\quad \begin{array}{l}0.48 \\
\{.23\}\end{array}$} \\
\hline & & & & $\begin{array}{l}68 / 07 / 87 \\
69 / 18 / 87 \\
10 / 24 / 87\end{array}$ & & $\begin{array}{l}08 / 05 / 87 \\
60 / 18 / 87 \\
10 / 05 / 87\end{array}$ & & $\begin{array}{l}16 / 65 / 87 \\
99 / 16 / 87 \\
10 / 06 / 87\end{array}$ & \\
\hline & & & & $12 / 16 / 87$ & & $12 / 15 / 87$ & & $12 / 15 / \theta 7$ & \\
\hline SODIUY & PPB & 100.00 & & $\begin{array}{l}07 / 08 / 87 \\
08 / 07 / 87 \\
59 / 18 / 87 \\
10 / 14 / 87 \\
12 / 16 / 87\end{array}$ & $\begin{array}{l}8020.06 \\
7220.06 \\
8886.00 \\
7320.00 \\
8550.00\end{array}$ & $\begin{array}{l}07 / 69 / 87 \\
68 / 66 / 87 \\
68 / 16 / 87 \\
18 / 65 / 87 \\
12 / 15 / 87\end{array}$ & $\begin{array}{l}17580.00 \\
19500.00 \\
15700.00 \\
18700.00 \\
13800.00\end{array}$ & $\begin{array}{l}17 / 60 / 87 \\
08 / 65 / 87 \\
00 / 16 / 87 \\
10 / 06 / 87 \\
12 / 15 / 87\end{array}$ & $\begin{array}{l}12360.00 \\
13945.04 \\
12046.00 \\
11040.65 \\
12000.06\end{array}$ \\
\hline
\end{tabular}

* - yalue exceeds primary drinkikg vater standard.

- - VALUE EXCEEDS PROPDSED PRIUARY DRINKING TATER STAKDARD.

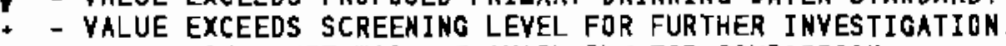

- DETECTION liuit as not available for couparison

NR - ANALYSIS NOT REQUESTED OR NOT YET REPORTED

VALUES IN \{\} ARE COUNTING ERRORS FDR RADIONUCLIDES

IATER STANDARD(S) IN PARENTHESES ARE PROPOSED OMLY 
TABLE C.5. (contd)

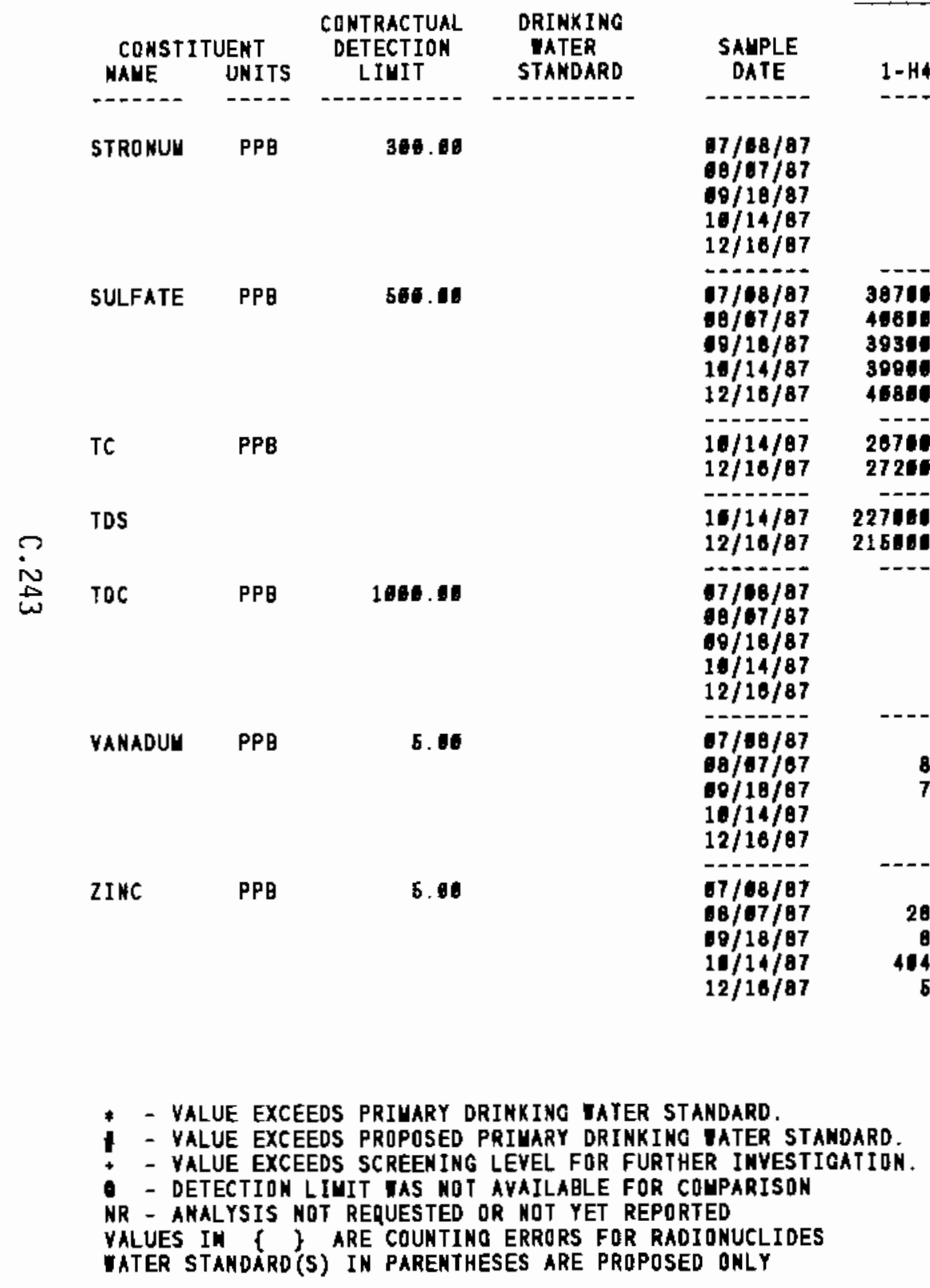

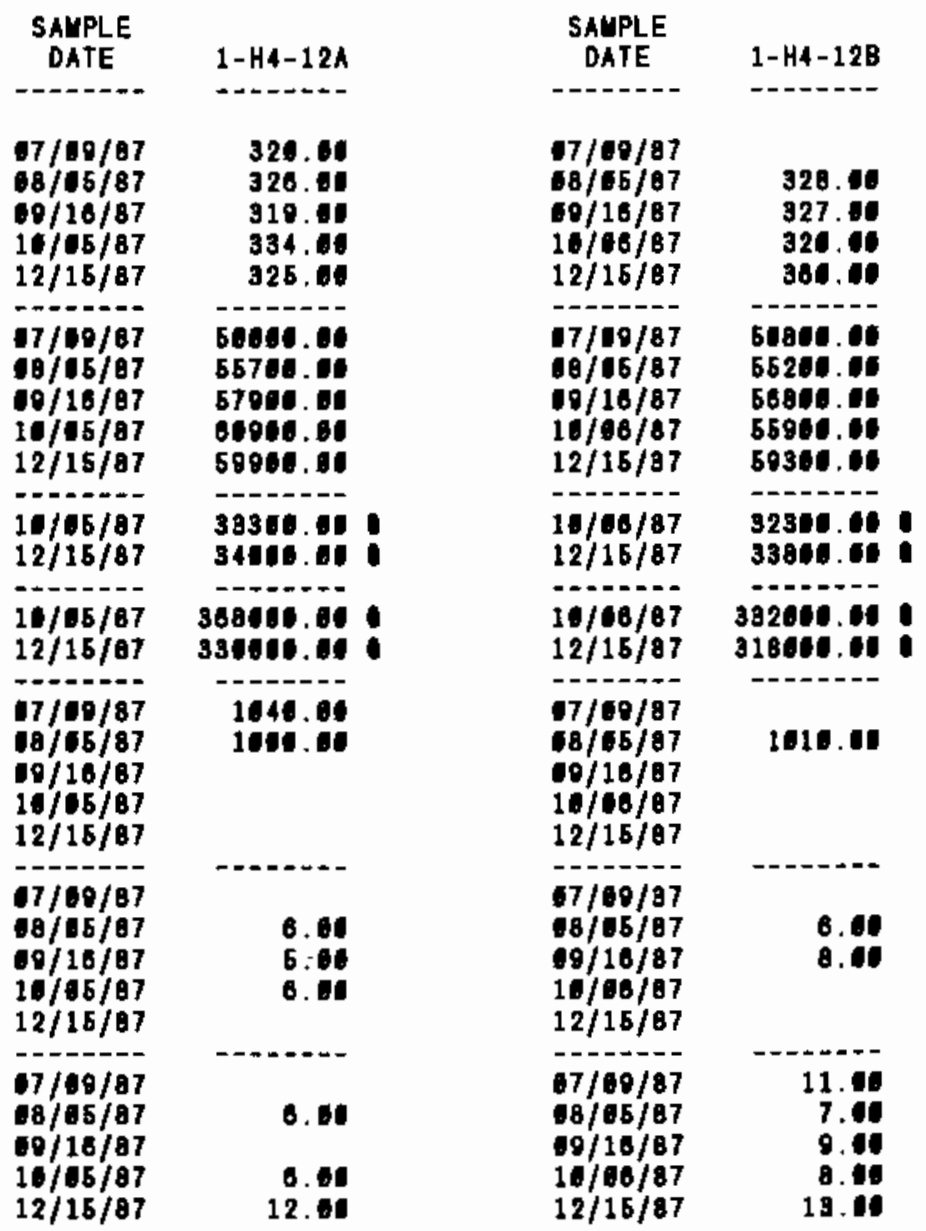

* - Value exceEds pRiuary dRINKINg tater standard.

- - VALUE EXCEEDS PROPOSED PRIUARY DRINKING TATER STAMDARD.

- DETECTION LIUIT TAS NOT AYAILABLE FOR COUPARISON

VALUES IH $(?$ ARE COUNTINO ERRORS FOR RADIONUCLIDES

IATER STANDARD(S) IN PARENTHESES ARE PROPOSED ONLY 


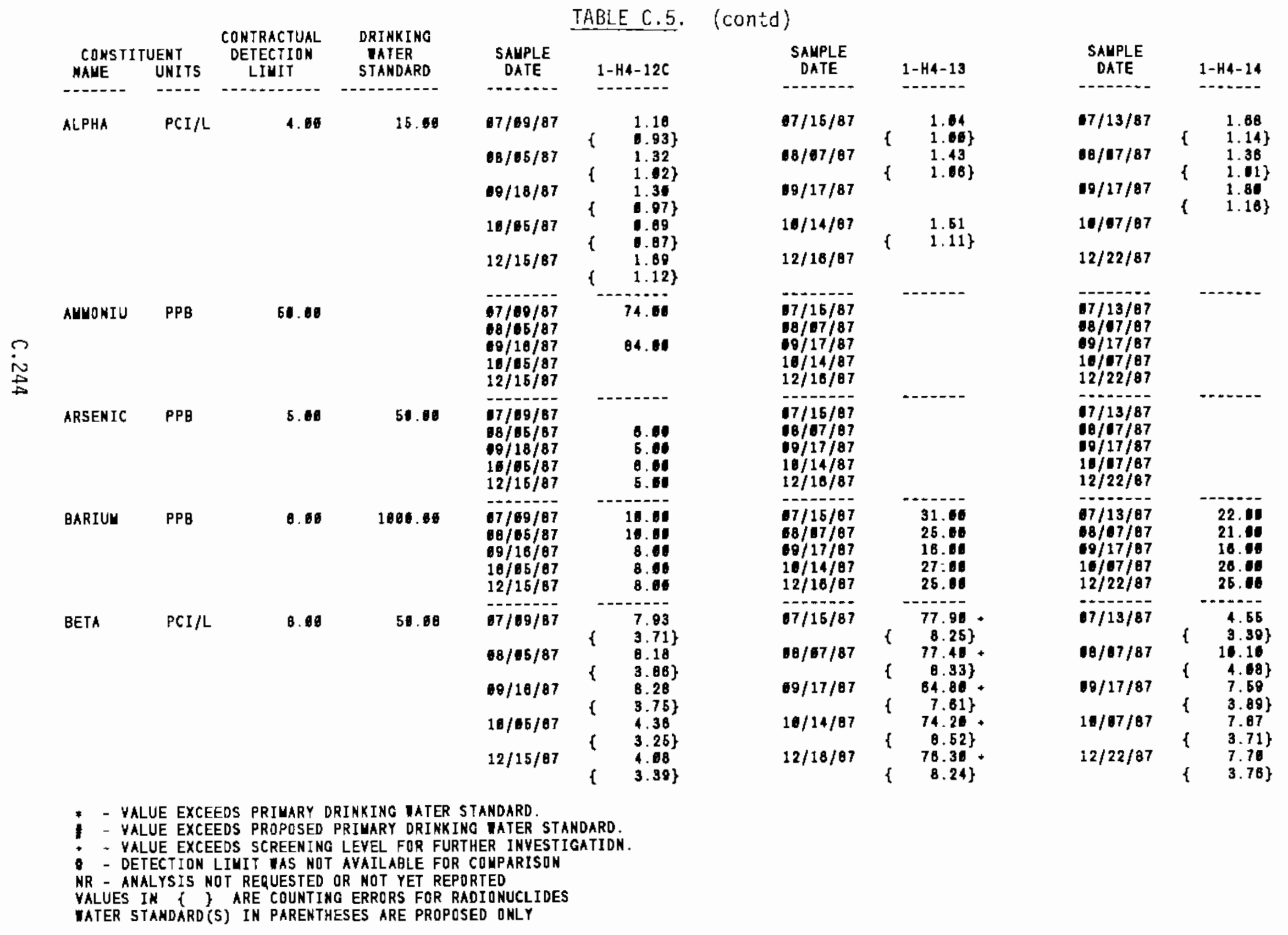


TABLE C.5. (contd)

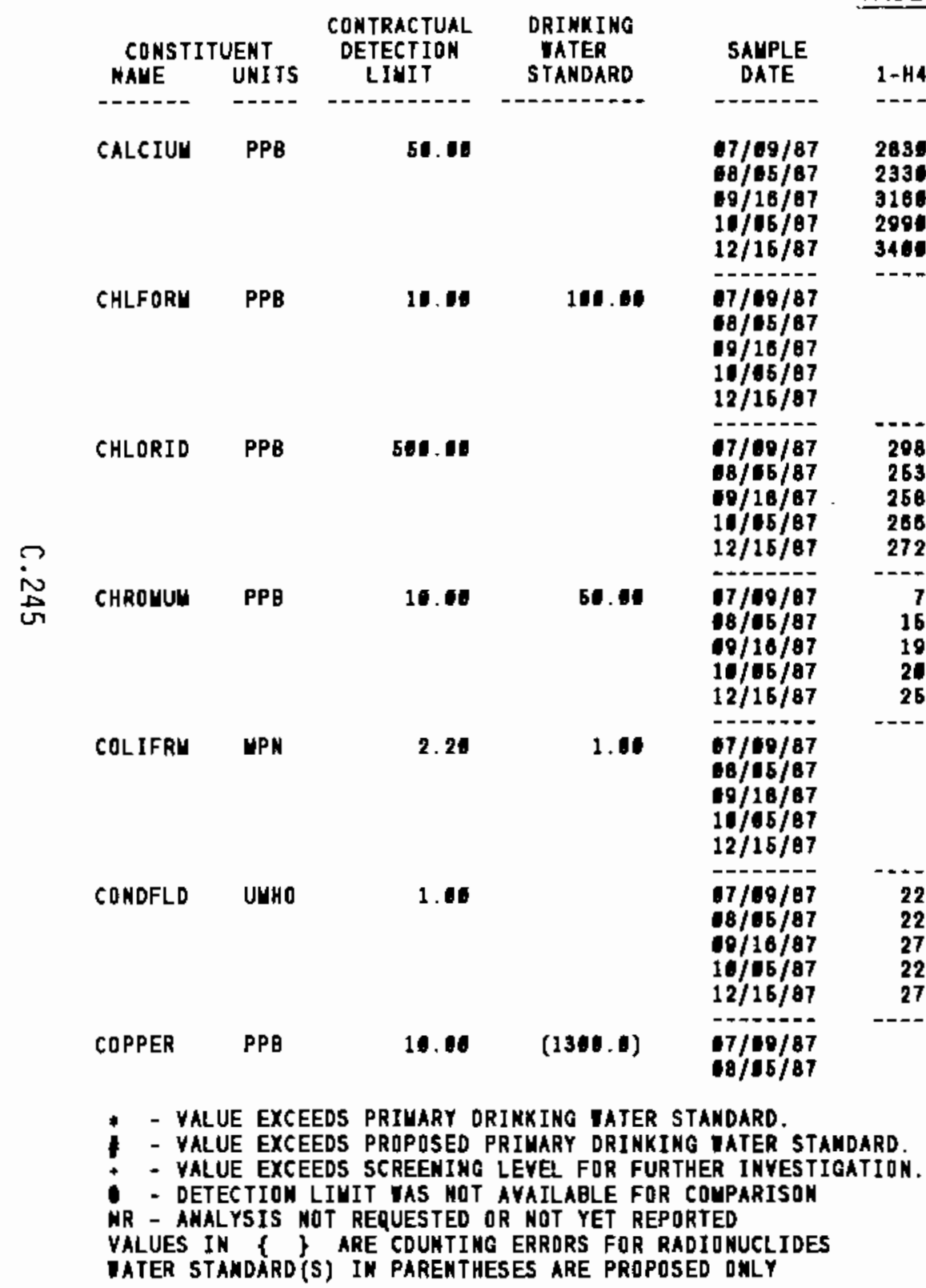

SAUPLE

SAMPLE

1-H4-14

$1-\mathrm{H} 4-12 \mathrm{C}$

DATE 1-H4-13

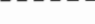

40500.00

$\begin{array}{ll}07 / 15 / 87 & 42211.61 \\ 68 / 67 / 87 & 31400.61 \\ 09 / 17 / 87 & 37496.01 \\ 11 / 14 / 87 & 36900.10\end{array}$

$07 / 13 / 87$

68/97/87

10/17/97

3414.10

42511.46

36900.10

$12 / 22 / 87$

4271.16

$12 / 18 / 87$ 4461. 16

07/19/87

4800.01

67/16/87 29.61

68/67/87 93.60

99/17/87 29.06

$\begin{array}{ll}11 / 14 / 97 & 29.64 \\ 12 / 18 / 97 & 24.06\end{array}$

$00 / 07 / 87$

36.04

00/17/87

37.61

4550

$07 / 16 / 87$

08/07/87

$09 / 17 / 87$

$10 / 14 / 87$

4531.04

12/22/87

-7/13/97

18/97/87

(9/17/87

4820.06

2580.0

2720.00

$12 / 18 / 87$

4626.

77.00

17/15/87

4246.11

$10 / 67 / 07$

12/22/07

28.11

$10 / 67 / 87$

94.10 :

$11 / 14 / 87$

$268.16 * 12 / 16 / 97$

21.10

23.41

35.10

48.09

$07 / 15 / 87$

$08 / 107 / 87$

19/17/87

$16 / 14 / 87$

$12 / 16 / 87$

2.20

$97 / 13 / 87$

$90 / 67 / 87$

$10 / 17 / 87$

$10 / 107 / 67$

$12 / 22 / 87$

33.01

28.00

26.40

4839.00

4884.10

5524.14

5960.01

B030.01

270.10

287.00

203.01 *

306.00 :

331.0

-....

-.-...

17/15/B7

10/47/87

19/17/87

1. 114/87

$12 / 18 / 87$

07/15/97

$68 / 07 / 87$

$07 / 13 / 87$

$00 / 17 / 87$

$0 / 17 / 87$
$10 / 07 / 87$

$12 / 22 / 87$

216.00

${ }_{186} 10$

17/13/87

$68 / 07 / 87$

$60 / 17 / 87$

252 .

298. 1

285.11

10/07/87

$12 / 22 / 87$

17/13/87

68/07/87

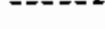

WATER STAMDARD(S) IN PARENTHESES ARE PROPOSED OMLY 
TABLE C.5. (contd)

\begin{tabular}{|c|c|c|c|c|c|}
\hline $\begin{array}{l}\text { CONSTI } \\
\text { NAME }\end{array}$ & $\begin{array}{l}\text { UENT } \\
\text { UN ITS }\end{array}$ & $\begin{array}{l}\text { CONTRACTUAL } \\
\text { DETECTION } \\
\text { LIUIT }\end{array}$ & $\begin{array}{l}\text { DRINKING } \\
\text { WATER } \\
\text { SIANDARD }\end{array}$ & $\begin{array}{c}\text { SAMPLE } \\
\text { DATE }\end{array}$ & $1-\mathrm{H} 4-12 \mathrm{C}$ \\
\hline---1 & $-\ldots$ & - & 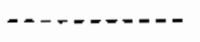 & +----- & - - \\
\hline COPPER & PPB & 18.60 & $(1300.0)$ & $\begin{array}{l}69 / 16 / 87 \\
16 / 66 / 87 \\
12 / 15 / 87\end{array}$ & 26.00 \\
\hline FARSENI & PPB & 5.66 & 50.00 & $\begin{array}{l}67 / 09 / 87 \\
08 / 65 / 87 \\
69 / 18 / 87 \\
18 / 65 / 87 \\
12 / 15 / 87\end{array}$ & $\begin{array}{l}7.01 \\
7.00 \\
0.00 \\
7.06 \\
8.00\end{array}$ \\
\hline FBARIUU & PPB & 8.00 & 1000.00 & $\begin{array}{l}07 / 109 / 87 \\
10 / 15 / 87 \\
89 / 18 / 87 \\
10 / 06 / 87 \\
12 / 16 / 87\end{array}$ & $\begin{array}{r}11.00 \\
0.00 \\
12.00 \\
10.00 \\
9.00\end{array}$ \\
\hline FCADUIU & PPB & 2.00 & 10.68 & $\begin{array}{l}07 / 10 / 87 \\
08 / 65 / 87 \\
00 / 16 / 87 \\
16 / 65 / 87 \\
12 / 16 / 87\end{array}$ & 2. 10 \\
\hline FCALCIU & PPB & 50.60 & & $\begin{array}{l}97 / 09 / 87 \\
88 / 65 / 87 \\
60 / 16 / 87 \\
16 / 65 / 87 \\
12 / 15 / 87\end{array}$ & $\begin{array}{l}29700.00 \\
23700.00 \\
29200.00 \\
33960.00 \\
31400.00\end{array}$ \\
\hline FCHROUI & PPB & 10.60 & 50.80 & $\begin{array}{l}97 / 69 / 87 \\
88 / 05 / 87 \\
60 / 16 / 87 \\
10 / 65 / 87 \\
12 / 15 / 87\end{array}$ & $\begin{array}{r}74.08 \\
147.60 \\
189.06 \\
263.06 \\
234.06\end{array}$ \\
\hline FIRON & PPB & 50.00 & & $\begin{array}{l}07 / 69 / 87 \\
08 / 85 / 87 \\
60 / 16 / 87 \\
10 / 05 / 87 \\
12 / 15 / 87\end{array}$ & 93.60 \\
\hline
\end{tabular}

\begin{tabular}{|c|}
\hline $\begin{array}{l}\text { SAUPLE } \\
\text { DATE }\end{array}$ \\
\hline $\begin{array}{l}69 / 17 / 87 \\
10 / 14 / 87 \\
12 / 16 / 87\end{array}$ \\
\hline $\begin{array}{l}07 / 16 / 87 \\
0 / 67 / 87 \\
0 / 17 / 87 \\
10 / 14 / 87 \\
12 / 16 / 87\end{array}$ \\
\hline $\begin{array}{l}17 / 15 / 87 \\
88 / 07 / 87 \\
69 / 17 / 87 \\
16 / 14 / 87 \\
12 / 16 / 87\end{array}$ \\
\hline $\begin{array}{l}67 / 15 / 87 \\
68 / 07 / 87 \\
09 / 17 / 87 \\
10 / 14 / 87 \\
12 / 16 / 87\end{array}$ \\
\hline $\begin{array}{l}07 / 16 / 87 \\
88 / 67 / 87 \\
09 / 17 / 87 \\
10 / 14 / 87 \\
12 / 18 / 87\end{array}$ \\
\hline $\begin{array}{l}07 / 15 / 87 \\
68 / 07 / 87 \\
09 / 17 / 87 \\
10 / 14 / 67 \\
12 / 16 / 87\end{array}$ \\
\hline $\begin{array}{l}7 / 15 / 87 \\
08 / 87 / 87 \\
69 / 17 / 87 \\
16 / 14 / 87 \\
12 / 18 / 87\end{array}$ \\
\hline
\end{tabular}

1-H4-13

SAMPLE

DATE I-H4-14

$00 / 17 / 87$ $16 / 67 / 87$ 12/22/87

G/13/87

68/1/ 187

$68 / 07 / 87$

$60 / 17 / 87$
$10 / 67 / 87$

$10 / 67 / 87$

$12 / 22 / 87$

$67 / 13 / 87$

10/07/87

$89 / 17 / 87$

$10 / 07 / 67$

$12 / 22 / 07$

$17 / 13 / 87$

$08 / 67 / 87$

09/17/87

$10 / 07 / 87$

$12 / 22 / 87$

41909

$07 / 13 / 87$

41900.0

37464.00

37406

44560.60

$07 / 13 / 8$

$60 / 17 / 07$

10/07/67

12/22/07

$---7 .-$

25.60
26.09

22.60

48.01

- $40 .$.

67/13/87

$08 / 07 / 87$

69/17/07

$16 / 07 / 87$

$12 / 22 / 87$

-

$07 / 13 / 87$

$00 / 47 / 87$

$09 / 17 / 87$
$18 / 8 / 87$

$12 / 22 / 87$

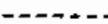

6. 14

27

24.00

23.01

27.11

28.00

-.-.-.

410301.00

36301.06

46351.06

43866.11

14646.64

288.10

261.06

287.06 *

$314.01 *$

284. $11 *$

90. 0

- - value exceeds privary drinking hater standard.

- VALUE EXCEEDS PROPOSED PRIMARY DRINKING TATER STANDARD

+ - VALUE EXCEEDS SCREENIKG LEVEL FOR FURTHER INVESTIGATION.

- DETECTION LINIT UAS NDT AYAILABLE FOR COUPARISON

NR - ANALYSIS NOT REQUESTEO OR NOT YET REPORTED

VALUES IN \{ \} ARE CDUNTING ERRORS FOR RADIQNUCLIDES

WATER STANDARD(S) IN PARENTHESES ARE PROPOSED DNLY 
TABLE C.5. (contd)

\begin{tabular}{|c|c|c|}
\hline $\begin{array}{l}\text { CONSTITUENT } \\
\text { WAME UNITS }\end{array}$ & $\begin{array}{c}\text { CONTRACTUAL } \\
\text { OETECTION } \\
\text { LIHIT }\end{array}$ & $\begin{array}{l}\text { DRINKING } \\
\text { WATER } \\
\text { STANDARD }\end{array}$ \\
\hline---- & - . & 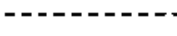 \\
\hline
\end{tabular}

\begin{tabular}{|c|c|}
\hline $\begin{array}{l}\text { SALPLEE } \\
\text { DATE }\end{array}$ & $1-\mathrm{H} 4-12 \mathrm{C}$ \\
\hline & \\
\hline $\begin{array}{l}17 / 09 / 87 \\
18 / 05 / 87 \\
19 / 18 / 87 \\
/ / 05 / 87 \\
2 / 15 / 87\end{array}$ & $\begin{array}{l}13060.01 \\
11200.00 \\
16700.00 \\
18016.00 \\
11706.00\end{array}$ \\
\hline $\begin{array}{l}17 / 09 / 97 \\
18 / 05 / 97 \\
10 / 16 / 87 \\
1 / 65 / 97 \\
12 / 16 / 87\end{array}$ & $\begin{array}{r}23.00 \\
12.00 \\
7.00 \\
0.66\end{array}$ \\
\hline $\begin{array}{l}17 / 60 / 87 \\
10 / 65 / 87 \\
19 / 16 / 87 \\
1 / 65 / 87 \\
12 / 15 / 87\end{array}$ & $\begin{array}{l}23.04 \\
19.60 \\
33.08 \\
27.04 \\
29.04\end{array}$ \\
\hline $\begin{array}{l}17 / 60 / 87 \\
18 / 65 / 87 \\
9 / 16 / 87 \\
1 / 65 / 87 \\
2 / 16 / 87\end{array}$ & $\begin{array}{l}5380.04 \\
4774.64 \\
4584.04 \\
4580.64 \\
4871.60\end{array}$ \\
\hline $\begin{array}{l}17 / 69 / 87 \\
18 / 65 / 87 \\
19 / 16 / 87 \\
16 / 65 / 87 \\
12 / 15 / 87\end{array}$ & $\begin{array}{l}5831.06 \\
4731.06 \\
4121.00 \\
4698.66 \\
5256.06\end{array}$ \\
\hline $\begin{array}{l}7 / 09 / 87 \\
8 / 65 / 87 \\
9 / 16 / 87 \\
6 / 65 / 87 \\
12 / 15 / 67\end{array}$ & $\begin{array}{l}11.00 \\
22.00 \\
25.00 \\
27.60 \\
25.00\end{array}$ \\
\hline $\begin{array}{l}7 / 60 / 87 \\
38 / 65 / 87\end{array}$ & 9.01 \\
\hline
\end{tabular}

SAUPLE DATE

$1-\mathrm{H} 4-13$

\section{$07 / 15 / 87$}

68/107/87

09/17/87

$10 / 14 / 07$

12/16/87

-..2.

$67 / 15 / 87$

$08 / 07 / 87$
$60 / 17 / 87$

10/17/87

$10 / 14 / 87$

$--115 / 87$

7)

60/17/87

10/14/97

$12 / 16 / 87$

67/15/a7

$68 / 07 / 87$

$60 / 17 / 87$

$16 / 14 / 87$

$12 / 16 / 87$

- $7 / 15 / 87$

98/16/87

68/17/87

1./14/87

$10 / 14 / 87$

-7/15/87

6/107/87

90/17/87

$10 / 14 / 87$

$12 / 16 / 87$

17/16/87

68/67/87

\subsection{0}

6690.11

6091.11

Bo10. II

7500.01

-.-...

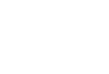

-. -

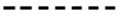

2384.

2170.

2630.00

2800.01

2200.01

7379.11

6810.01

6321.11

7420.61

7280.66

8.5

6. 10

-.-.-.

12.01
SAUPLE DATE

1-H4-14

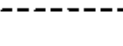

17/13/87

60/07/B7

$09 / 17 / B 7$

\%/N7/87

$12 / 22 / 87$

- $7 / 13 / 87$

17/13/87

$0 / 07 / 87$
$00 / 17 / 87$

$0 / 17 / 87$
$10 / 107 / 87$

$12 / 22 / 87$

$17 / 13 / 87$

18/67/87

19/17/97

$11 / 67 / 87$

12/22/87

17/13/07

18/67/67

$00 / 17 / 87$

$16 / 67 / 87$

$12 / 22 / 87$

-

$07 / 13 / 87$

B/ $/ 97 / 87$

$60 / 17 / 87$

$10 / 07 / 87$

12/22/87

$7 / 13 / 67$

18/67/87

19/17/87

\begin{tabular}{l}
$12 / 22 / 87$ \\
\hline
\end{tabular}

-7/13/87

$68 / 07 / 87$
7966.00

8301. II

707. 19

7986.10

8720.11

--.---.

4 ก

4140.06

9701.00

3055. 4

7920.01

7964.61

7430. .t

7610.06

8896.00

10.0

7.14

7.

7.

7.01.

....--

7.61

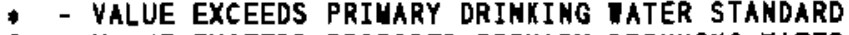

- VALUE EXCEEDS PROPOSED PRIMARY DRINKING TATER STANDARD

- VALUE EXCEEDS SCREENING LEYEL FOR FURTHER INYESTIGATION.

- DETECTION LIMIT IAS NDT AVAILABLE FOR COLPARISON

NR - AMALYSIS NDT REQUESTED OR NDT YET REPORTED

VALUES IM i $;$ ARE COUNTING ERRORS FOR RADIDNUCLIDES

WATER STAMDARD(S) IN PARENTHESES ARE PROPOSED ONLY 
TABLE C.5. (contd)

\begin{tabular}{|c|c|c|c|c|c|}
\hline $\begin{array}{l}\text { CONSTI } \\
\text { NAUE }\end{array}$ & $\begin{array}{l}\text { UENT } \\
\text { UNITS }\end{array}$ & $\begin{array}{l}\text { CONTRACTUAL } \\
\text { DETECTION } \\
\text { LIUIT }\end{array}$ & $\begin{array}{l}\text { DRINKING } \\
\text { TAYER } \\
\text { STANDARD }\end{array}$ & $\begin{array}{l}\text { SAUPLE } \\
\text { DATE }\end{array}$ & $1-\mathrm{H} 4-12 \mathrm{C}$ \\
\hline - . - & ---- & 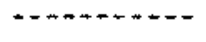 & 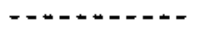 & $\cdots+-1$ & $--.-1--$ \\
\hline FZINC & PPB & 5.00 & & $\begin{array}{l}69 / 16 / 87 \\
11 / 05 / 87 \\
12 / 15 / 87\end{array}$ & $\begin{array}{r}7.60 \\
184.06 \\
6.06\end{array}$ \\
\hline IRON & PPB & 56.66 & & $\begin{array}{l}17 / 60 / 87 \\
10 / 65 / 87 \\
10 / 16 / 87 \\
10 / 65 / 87 \\
12 / 15 / 87\end{array}$ & $\begin{array}{r}141.01 \\
79.01 \\
123.00 \\
98.4 \\
84.0\end{array}$ \\
\hline MAGNES & PPB & 0.0 & & $\begin{array}{l}-17 / 10 / 87 \\
18 / 15 / 87 \\
10 / 18 / 87 \\
10 / 195 / 87 \\
12 / 15 / 87\end{array}$ & $\begin{array}{l}11400.00 \\
11900.00 \\
10910.00 \\
10500.00 \\
11000.00\end{array}$ \\
\hline 点 & PPB & 5.60 & & $\begin{array}{l}17 / 10 / 87 \\
18 / 15 / 87 \\
99 / 18 / 87 \\
19 / 16 / 87 \\
12 / 15 / 87\end{array}$ & $\begin{array}{r}22.00 \\
12.04 \\
6.00\end{array}$ \\
\hline NICXEL & PPB & 10.60 & & $\begin{array}{l}97 / 69 / 87 \\
08 / 05 / 87 \\
19 / 18 / 87 \\
19 / 05 / 87 \\
12 / 15 / 87\end{array}$ & $\begin{array}{l}22.00 \\
21.06 \\
38.60 \\
27.06 \\
34.06\end{array}$ \\
\hline NITRATE & PPB & 500.00 & 45006.60 & $\begin{array}{l}97 / 99 / 87 \\
98 / 05 / 87 \\
99 / 18 / 87 \\
10 / 16 / 87 \\
12 / 15 / 87\end{array}$ & $\begin{array}{l}3720.00 \\
5320 \\
6140 \\
5781.06 \\
5884.05\end{array}$ \\
\hline PH-LAB & & 0.01 & & $\begin{array}{l}10 / 05 / 87 \\
12 / 15 / 87\end{array}$ & $\begin{array}{l}7.84 \\
7.78\end{array}$ \\
\hline PHFIELD & & 0.10 & & $\begin{array}{l}07 / 09 / 87 \\
08 / 05 / 87\end{array}$ & $\begin{array}{l}7.30 \\
6.90\end{array}$ \\
\hline
\end{tabular}

\begin{tabular}{|c|c|}
\hline $\begin{array}{l}\text { SAUPLE } \\
\text { DATE }\end{array}$ & $1-14-13$ \\
\hline - & \\
\hline $\begin{array}{l}09 / 17 / 87 \\
10 / 14 / 87 \\
12 / 16 / 87\end{array}$ & $\begin{array}{l}7.60 \\
8.06 \\
8.06\end{array}$ \\
\hline $\begin{array}{l}97 / 15 / 87 \\
08 / 87 / 87 \\
09 / 17 / 87 \\
16 / 14 / 87 \\
12 / 16 / 87\end{array}$ & 84.06 \\
\hline $\begin{array}{l}-7 / 15 / 87 \\
18 / 17 / 87 \\
9 / 17 / 87 \\
10 / 14 / 87 \\
12 / 10 / 87\end{array}$ & $\begin{array}{l}7180.04 \\
6320.00 \\
6980.00 \\
6418.04 \\
7145.00\end{array}$ \\
\hline $\begin{array}{l}67 / 15 / 87 \\
19 / 17 / 87 \\
10 / 17 / 87 \\
11 / 14 / 87 \\
12 / 18 / 87\end{array}$ & ---1 \\
\hline $\begin{array}{l}67 / 15 / 87 \\
88 / 17 / 87 \\
19 / 17 / 87 \\
18 / 14 / 87 \\
12 / 16 / 87\end{array}$ & \\
\hline $\begin{array}{l}07 / 15 / 87 \\
08 / 07 / 87 \\
00 / 17 / 87 \\
10 / 14 / 67 \\
12 / 16 / 67\end{array}$ & $\begin{array}{l}23008.00 \\
21604.00 \\
19084.00 \\
19804.00 \\
19700.00\end{array}$ \\
\hline $\begin{array}{l}11 / 14 / 87 \\
12 / 16 / 87\end{array}$ & $\begin{array}{l}7.30 \\
7.47\end{array}$ \\
\hline $\begin{array}{l}07 / 15 / 87 \\
60 / 07 / 87\end{array}$ & $\begin{array}{l}8.80 \\
7.08\end{array}$ \\
\hline
\end{tabular}

SAUPLE

DATE

1-H4-14

00/17/87

$16 / 07 / 67$

12/22/87

07/13/87

e 187187

$87 / 87$

$19 / 17 / 87$

$10 / 67 / 87$

$12 / 22 / 87$

$7 / 13 / 87$

$08 / 07 / 87$

$09 / 17 / 87$

$10 / 17 / 87$

$12 / 22 / 87$

-1.-107

$07 / 13 / 87$

$08 / 57 / 87$

19/17/87

$10 / 07 / 87$

$12 / 22 / 87$

$67 / 13 / 87$

08187

$09 / 17 / 87$

$69 / 17 / 87$

(10/87

$12 / 22 / 87$

67/13/87

$08 / 197 / 87$

$09 / 17 / 87$

$10 / 107 / 87$

$12 / 22 / 87$

17964.01

10254.11

$12 / 22 / 87$ 19801.

$10 / 07 / 87$

10800.60

$12 / 22 / 87$

7.75

$97 / 13 / 87$

7896.10

7040.10

------

- VAlUe exceeds priuary dRinking tater standaro

- VALUE EXCEEDS PROPOSED PRIUARY DRINKING IATER STANDARD.

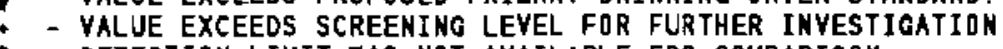

- DETECTION LIUIT IAS NOT AVAILABLE FDR COMPARISON

KR - ANALYSIS NOT REQUESTED OR NDT YET REPORTED

VALUES IN \{\} ARE CDUNTING ERRORS FOR RADIONUCLIDES

water StANDARD(S) IN PARENTHESES aRE PROPJSED ONLY 
TABLE C.5. (contd)

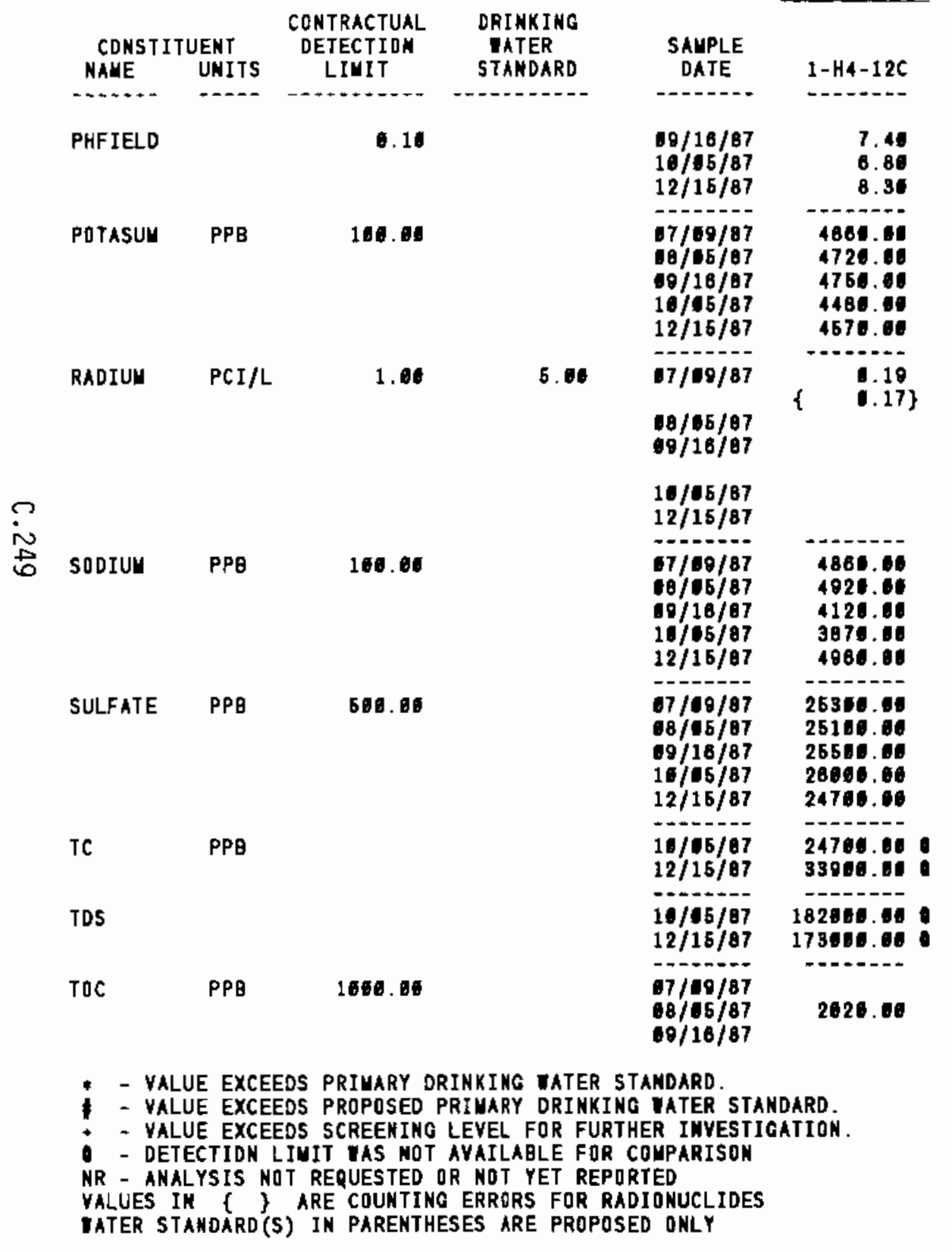

\begin{tabular}{|c|c|}
\hline $\begin{array}{l}\text { SAHPLE } \\
\text { DATE }\end{array}$ & $1-H 4-13$ \\
\hline ------- & 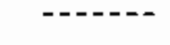 \\
\hline $\begin{array}{l}09 / 17 / 87 \\
16 / 14 / 87 \\
12 / 16 / 87\end{array}$ & $\begin{array}{l}6.90 \\
7.30 \\
7.60\end{array}$ \\
\hline $\begin{array}{l}07 / 15 / 87 \\
08 / 07 / 87 \\
09 / 17 / 87 \\
10 / 14 / 87 \\
12 / 16 / 87\end{array}$ & $\begin{array}{l}2100.00 \\
2830.00 \\
1090.00 \\
1804.00 \\
2010.00\end{array}$ \\
\hline $7 / 15 / 87$ & $\begin{array}{r}0.18 \\
.18\end{array}$ \\
\hline $\begin{array}{l}08 / 107 / 87 \\
99 / 17 / 87\end{array}$ & $\begin{array}{l}0.18 \\
\{\quad 0.15\}\end{array}$ \\
\hline $\begin{array}{l}10 / 14 / 87 \\
12 / 16 / 87\end{array}$ & \\
\hline $\begin{array}{l}67 / 15 / 87 \\
68 / 07 / 87 \\
9 / 17 / 87 \\
10 / 14 / 87 \\
12 / 16 / 87\end{array}$ & $\begin{array}{l}8641.64 \\
6370.06 \\
6480.60 \\
5930.06 \\
6350.06\end{array}$ \\
\hline $\begin{array}{l}17 / 16 / 87 \\
0 / 07 / 87 \\
99 / 17 / 87 \\
10 / 14 / 87 \\
12 / 16 / 87\end{array}$ & $\begin{array}{l}32300.00 \\
33900.00 \\
33606.00 \\
34606.00 \\
33968.00\end{array}$ \\
\hline$--1-20$ & ----- \\
\hline $\begin{array}{l}16 / 14 / 87 \\
12 / 16 / 87\end{array}$ & $\begin{array}{l}22360.00 \\
23960.60\end{array}$ \\
\hline 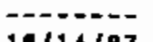 & a \\
\hline $\begin{array}{l}10 / 14 / 87 \\
12 / 16 / 87\end{array}$ & $\begin{array}{l}165060.06 \\
187080.06\end{array}$ \\
\hline $\begin{array}{l}07 / 16 / 87 \\
08 / 67 / 87 \\
09 / 17 / 87\end{array}$ & \\
\hline
\end{tabular}

\begin{tabular}{|c|c|}
\hline $\begin{array}{l}\text { SALPLE } \\
\text { DATE }\end{array}$ & $1-H 4-14$ \\
\hline - - - - & ---- \\
\hline $\begin{array}{l}69 / 17 / 87 \\
10 / 07 / 87 \\
12 / 22 / 87\end{array}$ & $\begin{array}{l}7.50 \\
7.41 \\
7.50\end{array}$ \\
\hline $\begin{array}{l}07 / 13 / 87 \\
68 / 67 / 87 \\
09 / 17 / 87 \\
10 / 07 / 87 \\
12 / 22 / 87\end{array}$ & $\begin{array}{l}-1010.06 \\
3950.04 \\
4110.06 \\
4420.04\end{array}$ \\
\hline $67 / 13 / 87$ & $\begin{array}{r}-0.20 \\
\quad 0.18\}\end{array}$ \\
\hline $\begin{array}{l}68 / 07 / 87 \\
68 / 17 / 87 \\
10 / 67 / 87 \\
12 / 22 / 87\end{array}$ & $\left\{\begin{array}{l}.15 \\
.14\}\end{array}\right.$ \\
\hline $\begin{array}{l}07 / 13 / 87 \\
0 / 17 / 87 \\
09 / 17 / 87 \\
10 / 07 / 67 \\
12 / 22 / 87\end{array}$ & $\begin{array}{l}-1-0 .- \\
7504.04 \\
7534.04 \\
7464.00 \\
7324.04 \\
7764.10\end{array}$ \\
\hline $\begin{array}{l}67 / 13 / 87 \\
68 / 67 / 97 \\
69 / 17 / 87 \\
16 / 67 / 07 \\
12 / 22 / 97\end{array}$ & 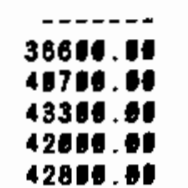 \\
\hline $\begin{array}{l}16 / 07 / 87 \\
12 / 22 / 87\end{array}$ & 23700.01 \\
\hline $\begin{array}{l}10 / 07 / 87 \\
12 / 22 / 87\end{array}$ & $\begin{array}{l}211040.00 \\
228000.01\end{array}$ \\
\hline $\begin{array}{l}07 / 13 / 87 \\
08 / 07 / 87 \\
09 / 17 / 87\end{array}$ & \\
\hline
\end{tabular}


TABLE C.5. (contd)

\begin{tabular}{|c|c|c|c|}
\hline$\underset{\text { CONSTI }}{\text { CONE }}$ & $\begin{array}{l}\text { UENT } \\
\text { UNITS }\end{array}$ & $\begin{array}{l}\text { CONTRACTUAL } \\
\text { DETECTIDN } \\
\text { LIVIT }\end{array}$ & $\begin{array}{l}\text { DRINKING } \\
\text { IATER } \\
\text { STANDARD }\end{array}$ \\
\hline$T D C$ & PPB & 1000.00 & \\
\hline VAMADUU & PPB & 5.00 & \\
\hline ZINC & PPB & 5.00 & \\
\hline
\end{tabular}

\begin{tabular}{|c|c|}
\hline $\begin{array}{l}\text { SAMPLE } \\
\text { DATE }\end{array}$ & $1-H 4-12 C$ \\
\hline $\begin{array}{l}10 / 85 / 87 \\
12 / 15 / 87\end{array}$ & \\
\hline $\begin{array}{l}17 / 00 / 87 \\
18 / 65 / 87 \\
09 / 16 / 87 \\
16 / 65 / 87 \\
12 / 15 / 87\end{array}$ & $\begin{array}{l}10.06 \\
10.00 \\
29.00 \\
25.00 \\
22.00\end{array}$ \\
\hline $\begin{array}{l}6 / 109 / 87 \\
6 / 06 / 87 \\
10 / 16 / 87 \\
16 / 85 / 87 \\
12 / 15 / 87\end{array}$ & $\begin{array}{r}16.00 \\
7.06 \\
14.00 \\
11.00 \\
25.00\end{array}$ \\
\hline
\end{tabular}

SAMPLE

DATE

-.....

1-H4-13

$12 / 10 / 87$

iา/15/97

$10 / 07 / 87$

$00 / 17 / 87$

10/14/87

$12 / 10 / 87$

--...--

$07 / 16 / 87$

68/107/87

69/17/87

$10 / 14 / 87$

$12 / 10 / 87$
SALPLE

DATE

-........

$10 / 07 / 87$

$12 / 22 / 87$

67/13/97

$19 / 107 / 87$

19/17/87

$18 / 07 / 87$

$12 / 22 / 87$

$12 / 22 / 87$

$07 / 13 / 97$

08/67/87

$18 / 17 / 87$

19/107/87

$12 / 22 / 87$
1-H4-14

-.-.-.-

7. 00

14.010

6. 69

12.00

5.00

* - YALUE EXCEEDS PRIUARY dRINKING IATER STANDARD.

- YALUE EXCEFDS PROPOSED PRIMARY DRINKING TATER STANDARD.

- VALUE EXCEEDS SCREENING LEVEL FOR FURTHER INVESTIGATION.

- DETECTION LIUIT TAS NOT AYAILABLE FOR COUPARISON

NR - ANALYSIS NOT REQUESTED OR NOT YET REPORTED

VALUES IN \& ARE COUMTING ERRORS FOR RADIONUCLIDES

TATER STAMDARO'S) IN PARENTHESES ARE PROPOSED DNLY 


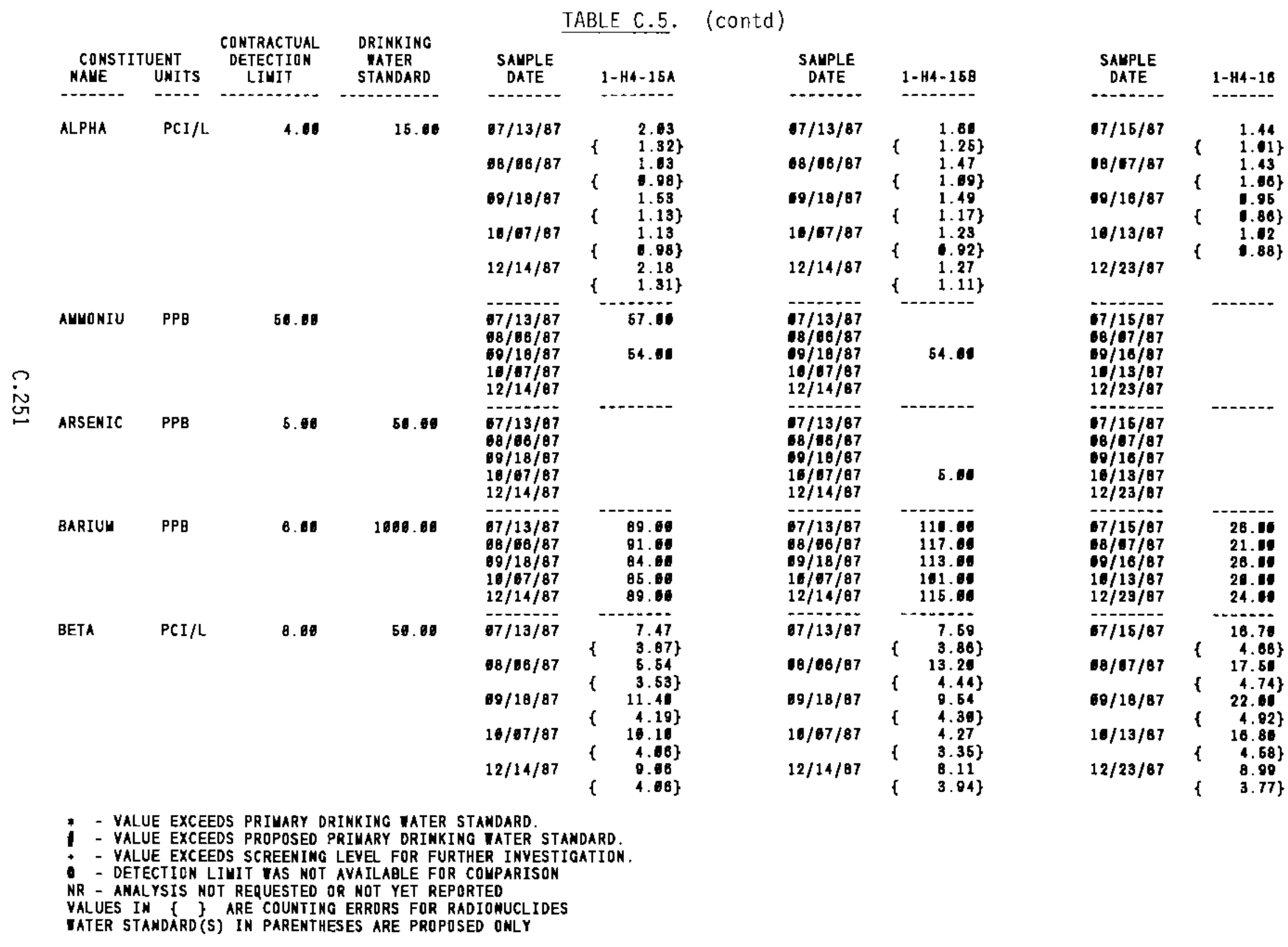


TABLE C.5. (contd)

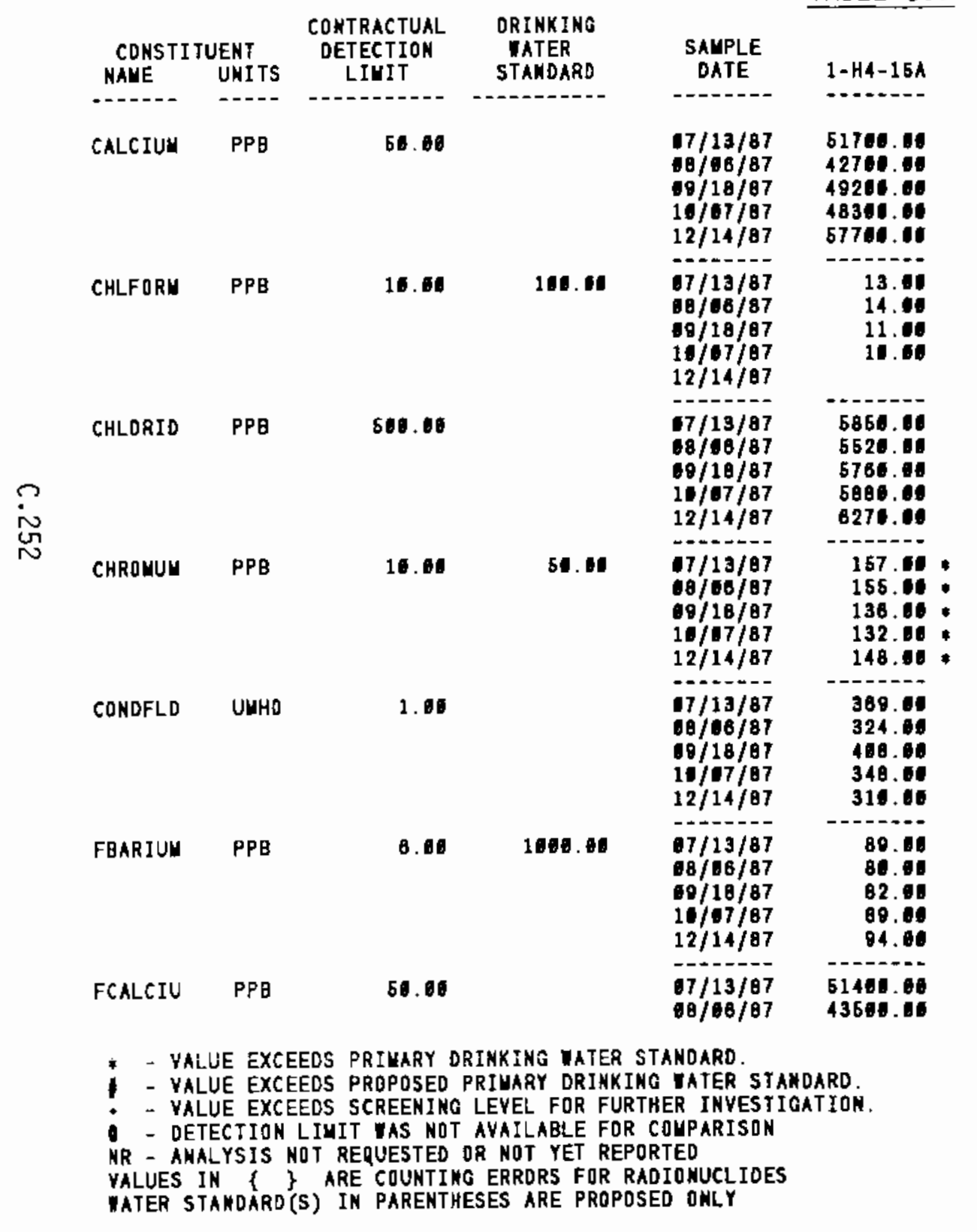

SAMPLE
OATE
-

$07 / 15 / 87 \quad 42201.06$

$68 / 67 / 87 \quad 34761.06$

$09 / 16 / 87$ 41811.06

90/19/87 37810.61

$12 / 23 / 87$ 44501.61

$07115 / 87$

16/107/87 37.06

$99 / 16 / 87 \quad 26.06$

$10 / 13 / 87 \quad 22.06$

$12 / 23 / 87$

77..... - -

$67 / 15 / 87 \quad 5800.60$

$68 / 07 / 87 \quad 8236.10$

$19 / 10 / 87 \quad 4726.10$

$11 / 13 / 87$ 6310.06

$12 / 23 / 87 \quad 5581.00$

67/15/87

$0197 / 87$

$87 / 15 / 87$

$69 / 18 / 87$

$12 / 23 / 87$

$07 / 15 / 87$

$08 / 67 / 87$

69/16/87

$16 / 13 / 87$

$12 / 23 / 87$

-------

$97 / 15 / 87$

$68 / 07 / 87$

$08 / 18 / 87$

$18 / 13 / 87$

$12 / 23 / 87$

17/15/87

$00 / 67 / 87$ 
TABLE C.5. (contd)

\begin{tabular}{ccc} 
CONSTITUERT & $\begin{array}{c}\text { CONTRACTUAL } \\
\text { DETECTION }\end{array}$ & $\begin{array}{c}\text { DRINKING } \\
\text { RATER } \\
\text { STANDERO }\end{array}$ \\
\hline
\end{tabular}

FCALCIU

FCHROUI PPB

16.00

Eอ. 19

FIRON

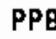

50. 00

in

FLEAD

PPB

5. 01

50.06

FUAGNES PPB

c.

FMANGAN PPB

5. 00

FPQTASS PPB

100.00

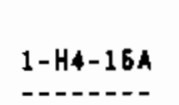

50101.06

48100.00

58700.06

155.69

147.6

141.06

141.00

147.0 *

$16 / 07 / 67$

$12 / 14 / 87$

$07 / 13 / 97$

98/06/87

$69 / 10 / 87$

$10 / 67 / 87$

$12 / 14 / 87$

17/13/87

18/66/87

$9 / 18 / 87$

10/07/87

$12 / 14 / 87$

-7/13/87

98/46/87

99/18/87

$12 / 14 / B 7$

67/13/87

$68 / 08 / 87$

$69 / 18 / 87$

$16 / 97 / 87$

$12 / 14 / 87$

07/13/67

$68 / 66 / 87$

$09 / 18 / 87$

$10 / 07 / 87$

$12 / 14 / 87$
76.06

10800.00

10500.00

10100.00

16506.00

11700.60

---.--

1

5140.10
5370.00
5470.00
5360.00
5090.00

\begin{tabular}{|c|c|}
\hline $\begin{array}{c}\text { SAUPLE } \\
\text { DATE }\end{array}$ & $1-H 4-15 B$ \\
\hline 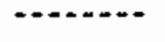 & 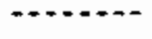 \\
\hline $\begin{array}{l}9 / 18 / 87 \\
10 / 67 / 87 \\
12 / 14 / 87\end{array}$ & $\begin{array}{l}47300.00 \\
48800.08 \\
65000.00\end{array}$ \\
\hline $\begin{array}{l}67 / 13 / 97 \\
60 / 66 / 87 \\
99 / 18 / 87 \\
10 / 07 / 87 \\
12 / 14 / 87\end{array}$ & $\begin{array}{l}149.00 \\
163.00 \\
145.00 \\
156.00 \\
151.00\end{array}$ \\
\hline $\begin{array}{l}07 / 13 / 67 \\
68 / 66 / 87 \\
69 / 18 / 87 \\
10 / 07 / 87 \\
12 / 14 / 87\end{array}$ & $\cdots$ \\
\hline $\begin{array}{l}7 / 13 / 87 \\
66 / 66 / 87 \\
68 / 18 / 87 \\
16 / 67 / 97 \\
12 / 14 / 87\end{array}$ & 6.16 \\
\hline $\begin{array}{l}07 / 13 / 87 \\
68 / 86 / 87 \\
09 / 18 / 87 \\
16 / 87 / 87 \\
12 / 14 / 87\end{array}$ & 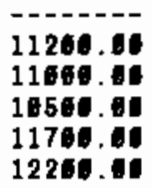 \\
\hline $\begin{array}{l}67 / 13 / 87 \\
68 / 08 / 87 \\
69 / 18 / 87 \\
16 / 97 / 87 \\
12 / 14 / 87\end{array}$ & 6. 00 \\
\hline $\begin{array}{l}67 / 13 / 87 \\
68 / 66 / 97 \\
69 / 18 / 87 \\
16 / 67 / 87 \\
12 / 14 / 87\end{array}$ & $\begin{array}{l}5176.04 \\
5216.00 \\
5336.00 \\
8116.00 \\
4828.00\end{array}$ \\
\hline
\end{tabular}

SAHPLE

DATE

1-H4-16

19/16/87

$11 / 13 / 87$

12/23/87

39800.06

37200.6

42100.6

$07 / 15 / 87$

88/07/87

$60 / 16 / 87$

$16 / 13 / 87$

$12 / 23 / 87$

$7 / 15 / 87$

$07 / 16 / 87$

$18 / 67 / 67$
$9 / 16 / 87$

$09 / 16 / 67$
$10 / 13 / 87$

$12 / 23 / 87$

$07 / 15 / 87$

08/16/87

19/18/87

19/19/87

$10 / 19 / 87$

12/29/87

$07 / 15 / 87$

08/07/87

$09 / 16 / 07$

10/13/87

$12 / 23 / 87$

07/15/87

$07 / 15 / 27$

-

$09 / 16 / 87$

$16 / 13 / 87$

$12 / 23 / 87$

$07 / 15 / 67$

$08 / 67 / 87$

$09 / 16 / 87$

$10 / 13 / 87$

$12 / 23 / 87$
4090

4990.00

5558.06

476.00

5820.09

2780.00

281. 01

2880. 01

3836.96

3080.00

* - ValUe exceEds primary dRINking tater standard

| - VALUE EXCEEDS PRDPOSED PRIMARY DRINKING VATER STAMDARD.

- - VALUE EXCEEDS SCREENING LEVEL FOR FURTHER INVESTIGATION.

- DETECTION LIHIT WAS NOT AVALLABLE FOR COUPARISON

NR - ANALYSIS NOT REQUESTED OR NOT YET REPDRTED

YALUES IN $\{$ ARE CDUNTING ERRORS FOR RADIONUCLIDES

IATER STANDARD'S) IN PARENTHESES ARE PROPOSED ONLY 
TABLE C.5. (contd)

\begin{tabular}{|c|c|c|c|c|c|}
\hline $\begin{array}{c}\text { CONSTI } \\
\text { NAME }\end{array}$ & ENT & $\begin{array}{l}\text { CONTRACTUAL } \\
\text { DETETTION } \\
\text { LIMIT }\end{array}$ & $\begin{array}{l}\text { DRINKENG } \\
\text { MATER } \\
\text { STANDARD }\end{array}$ & $\begin{array}{c}\text { SAMPLE } \\
\text { DATE }\end{array}$ & $1-H 4-15 A$ \\
\hline Fsodiuy & PPB & 180.86 & & $\begin{array}{l}87 / 13 / 87 \\
888988787 \\
88 / 18 / 87 \\
10 / 07 / 87 \\
12 / 14 / 87\end{array}$ & 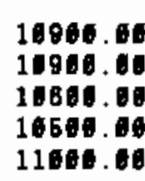 \\
\hline FYANADI & PPB & 5. 69 & & $\begin{array}{l}87 / 13 / 87 \\
88 / 68 / 87 \\
89 / 18 / 87 \\
16 / 07 / 87 \\
12 / 14 / 87\end{array}$ & $\begin{array}{r}18.08 \\
11.80 \\
0.80 \\
7.80\end{array}$ \\
\hline FZINC & PPB & 5.00 & & $\begin{array}{l}07 / 13 / 87 \\
88 / 86 / 87 \\
89 / 18 / 87 \\
16 / 67 / 87 \\
12 / 14 / 87\end{array}$ & \\
\hline IRON & PPB & 58.80 & & $\begin{array}{l}97 / 13 / 87 \\
98 / 88 / 87 \\
69 / 18 / 87 \\
16 / 67 / 87 \\
12 / 14 / 87\end{array}$ & $\begin{array}{r}549.11 \\
56.00\end{array}$ \\
\hline WAGNES & PPB & 0.80 & & $\begin{array}{l}97 / 13 / 87 \\
88 / 68 / 87 \\
89 / 18 / 87 \\
16 / 67 / 87 \\
12 / 14 / 87\end{array}$ & 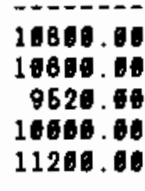 \\
\hline WANGESE & PPB & 5.80 & & $\begin{array}{l}97 / 13 / 87 \\
98 / 8887 \\
89 / 18 / 87 \\
11 / 878787 \\
12 / 14 / 87\end{array}$ & \\
\hline NITRATE & PPB & 568.68 & 45000.20 & $\begin{array}{l}87 / 13 / 87 \\
80 / 66 / 87\end{array}$ & $\begin{array}{l}28480.06 \\
29806.06\end{array}$ \\
\hline
\end{tabular}

\begin{tabular}{|c|c|}
\hline $\begin{array}{l}\text { SAMPLE } \\
\text { DATE }\end{array}$ & $1-\mathrm{HA}-15 \mathrm{~B}$ \\
\hline 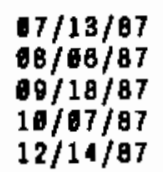 & 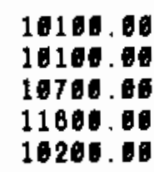 \\
\hline $\begin{array}{l}07 / 13 / 87 \\
88 / 96 / 87 \\
89 / 18 / 87 \\
10 / 67 / 87 \\
12 / 14 / 87 \\
9 / 87\end{array}$ & $\begin{array}{r}14.66 \\
8.68 \\
10.68 \\
16.80\end{array}$ \\
\hline $\begin{array}{l}8 / 13 / 87 \\
86 / 68 / 87 \\
09 / 18 / 87 \\
19 / 87 / 87 \\
12 / 14 / 87\end{array}$ & $\begin{array}{l}35.68 \\
22.88 \\
21.68 \\
27.68 \\
24.08\end{array}$ \\
\hline $\begin{array}{l}07 / 13 / 87 \\
08 / 86 / 87 \\
09 / 18 / 87 \\
16 / 87 / 87 \\
12 / 14 / 87 \\
9 / 4 / 87\end{array}$ & 98.06 \\
\hline $\begin{array}{l}97 / 13 / 87 \\
8 B / 68 / 87 \\
69 / 18 / 87 \\
18 / 97 / 87 \\
12 / 14 / 87\end{array}$ & $\begin{array}{l}10980.68 \\
11306.08 \\
10580.08 \\
9798.08 \\
11460.06\end{array}$ \\
\hline $\begin{array}{l}8 / 19 / 87 \\
68 / 96 / 87 \\
69 / 18 / 87 \\
19 / 97 / 87 \\
12 / 14 / 87\end{array}$ & 6.68 \\
\hline $\begin{array}{l}07 / 13 / 87 \\
88 / 08 / 87\end{array}$ & $\begin{array}{l}23208.06 \\
24480.00\end{array}$ \\
\hline
\end{tabular}

\begin{tabular}{|c|c|}
\hline $\begin{array}{l}\text { SAMPLE } \\
\text { DATE }\end{array}$ & $1-H_{4}-1 B$ \\
\hline $\begin{array}{l}87 / 15 / 87 \\
88 / 6787 \\
89 / 16 / 87 \\
10 / 13 / 87 \\
12 / 23 / 87\end{array}$ & 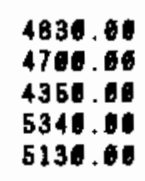 \\
\hline $\begin{array}{l}07 / 15 / 87 \\
08 / 67 / 87 \\
69 / 16 / 87 \\
16 / 13 / 87 \\
12 / 23 / 87\end{array}$ & 5.80 \\
\hline $\begin{array}{l}67 / 15 / 87 \\
68 / 07 / 87 \\
99 / 16 / 87 \\
16 / 13 / 87 \\
12 / 23 / 87\end{array}$ & \\
\hline $\begin{array}{l}97 / 15587 \\
08 / 97 / 87 \\
98 / 18 / 87 \\
16 / 13 / 87 \\
12 / 23 / 87\end{array}$ & 64.86 \\
\hline $\begin{array}{l}87 / 15 / 87 \\
68 / 97 / 87 \\
69 / 18 / 87 \\
16 / 13 / 87 \\
12 / 23 / 87\end{array}$ & $\begin{array}{l}5170.80 \\
5886.00 \\
4886.06 \\
4979.86 \\
5390.86\end{array}$ \\
\hline $\begin{array}{l}07 / 15 / 87 \\
08 / 97 / 87 \\
99 / 18 / 87 \\
16 / 13 / 87 \\
12 / 23 / 87\end{array}$ & \\
\hline $\begin{array}{l}07 / 15 / 87 \\
88 / 97 / 87\end{array}$ & $\begin{array}{l}15968.06 \\
18 B 8 \theta \text {. }\end{array}$ \\
\hline
\end{tabular}

* - value exceeds primary drinxing tater stanoard.

1 - VALUE EXCEEDS PROPOSED PRIUARY DRINKING IATER STANDARD

* - VALUE EXCEeds SCREening LeVel for fURTHER inVestigation.

- detection linit was not ayailable for comparisdn

NR - ANALYSIS NOT REQUESTED DR NOT YET REPDRTED

VALUES IN \{\} ARE COUNTING ERRORS FOR RADIOKUCLIDES

IATER STANDARD(S) IN PARENTHESES ARE PROPOSED ONLY 
TABLE C.5. (contd)

\begin{tabular}{|c|c|c|c|c|}
\hline$\underset{\text { WAUE }}{\text { CONSTI }}$ & $\begin{array}{l}\text { UENT } \\
\text { UHITS }\end{array}$ & $\begin{array}{l}\text { COKTRACTUAL } \\
\text { DETECTION } \\
\text { LIUIT }\end{array}$ & $\begin{array}{l}\text { DRINKING } \\
\text { IATER } \\
\text { STANDARD }\end{array}$ & $\begin{array}{c}\text { SAYPLE } \\
\text { DATE }\end{array}$ \\
\hline NITRATE & PPB & 500.06 & 46000.00 & $\begin{array}{l}00 / 18 / 87 \\
10 / 67 / 87 \\
12 / 14 / 87\end{array}$ \\
\hline PH-LAB & & 0.1 & & $\begin{array}{l}10 / 07 / 87 \\
12 / 14 / 87\end{array}$ \\
\hline PHFIELD & & 1.10 & & $\begin{array}{l}17 / 13 / 87 \\
09 / 96 / 87 \\
09 / 18 / 87 \\
10 / 07 / 87 \\
12 / 14 / 87\end{array}$ \\
\hline POTASUH & PPB & 100.00 & & $\begin{array}{l}67 / 13 / 87 \\
68 / 06 / 87 \\
09 / 18 / 87 \\
10 / 07 / 87 \\
12 / 14 / 87\end{array}$ \\
\hline \multirow[t]{5}{*}{ RADIUY } & $\mathrm{PCI} / \mathrm{L}$ & 1.60 & 5.68 & $67 / 13 / 87$ \\
\hline & & & & $08 / 08 / 07$ \\
\hline & & & & $08 / 18 / 87$ \\
\hline & & & & $10 / 97 / 87$ \\
\hline & & & & $12 / 14 / 87$ \\
\hline SODIUU & PPB & 100.00 & & $\begin{array}{l}67 / 13 / 87 \\
68 / 68 / 87 \\
60 / 18 / 87 \\
16 / 97 / 87 \\
12 / 14 / 87\end{array}$ \\
\hline SULFATE & PPB & 500.06 & & $\begin{array}{l}07 / 13 / 87 \\
08 / 00 / 87 \\
99 / 10 / 87\end{array}$ \\
\hline
\end{tabular}

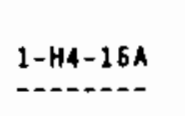

28160.60

2890100

30500.06

7.45

$\begin{array}{r}7.45 \\ 7.69 \\ \hline\end{array}$

0.56

6.56

6.70

7.08

7.40
-.2.

5481.40

6330.00

5104. 06

652.. 1

6984. 14

-.-.....

.16
$\{\quad 15\}$

.

$-----\overline{5}$

11400.0

10901.06

10504.

10700. 14

46400.06

46360.00

49600. 00

\begin{tabular}{|c|c|}
\hline $\begin{array}{l}\text { SAHPLE } \\
\text { DATE }\end{array}$ & $1-H 4-15 \theta$ \\
\hline ------- & 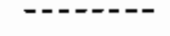 \\
\hline $\begin{array}{l}0 / 18 / 87 \\
10 / 07 / 87 \\
12 / 14 / 87\end{array}$ & $\begin{array}{l}24860.00 \\
25540.01 \\
28501.05\end{array}$ \\
\hline $\begin{array}{l}14 / 67 / 87 \\
12 / 14 / 87\end{array}$ & $\begin{array}{r}7.74 \\
7.69\end{array}$ \\
\hline $\begin{array}{l}07 / 13 / 87 \\
68 / 18 / 87 \\
68 / 18 / 87 \\
10 / 07 / 87 \\
12 / 14 / 87\end{array}$ & $\begin{array}{r}8.20 \\
6.75 \\
7.51 \\
7.01 \\
7.51\end{array}$ \\
\hline $\begin{array}{l}-\overline{-}--- \\
07 / 13 / 87 \\
00 / 06 / 87 \\
69 / 18 / 87 \\
10 / 67 / 87 \\
12 / 14 / 67\end{array}$ & $\begin{array}{l}5096.00 \\
5426.64 \\
5116.54 \\
5124.64 \\
4944.56\end{array}$ \\
\hline $7 / 13 / 87$ & a. \\
\hline & \\
\hline $99 / 18 / 87$ & $\begin{array}{l}.16\} \\
.21 \\
.15\}\end{array}$ \\
\hline $10 / 07 / 87$ & $\begin{array}{r}63 \\
6.25\end{array}$ \\
\hline $12 / 14 / 87$ & $\begin{array}{l}8.26 \\
1.17\}\end{array}$ \\
\hline $\begin{array}{l}7 / 13 / 87 \\
0 / 66 / 87 \\
09 / 18 / 87 \\
10 / 67 / 87 \\
12 / 14 / 87\end{array}$ & $\begin{array}{r}9810.01 \\
11300.4 \\
16180.40 \\
9504.40 \\
10400.46\end{array}$ \\
\hline $\begin{array}{l}97 / 13 / 87 \\
68 / 16 / 87 \\
69 / 18 / 87\end{array}$ & $\begin{array}{l}-1-0 .-1- \\
43860.00 \\
45166.00 \\
47366.00\end{array}$ \\
\hline
\end{tabular}

SAMPLE DATE

1-H4-16

-

$60 / 16 / 8$

$12 / 23 / 87$

5320.04

6585. Ut

13016.64

11/13/87 -...-

$\begin{array}{ll}11 / 13 / 87 & 7.73 \\ 12 / 23 / 87 & 7.62\end{array}$

e7/15/87

$67 / 15 / 87 \quad 7.30$

18/07/87 7.60

$10 / 13 / 97$

12/29/87

$77 / 15 / 87$

$07 / 16 / 87$

2671.6

2874.6

2550.00

2810.10

$69 / 16 / 8$

$10 / 13 / 87$

2680. 14

$1 / 16 / 87$

18/67/87

10/16/87

$10 / 13 / 87$

$12 / 23 / 07$

$67 / 15 / 87$

1890

6/47/87

46010.10

$68 / 67 / 87$

18/18/87

$10 / 13 / 8$

4440.0

4160.00

4481.08

27356.0

$17 / 15 / 87$

$18 / 07 / 87$

29840. 10

* - VALUE EXCEEDS PRIUARY DRINKING IATER STANDARD.
- VALUE EXCEEDS PROPOSED PRIMARY DRINKING WATER STANDARD.

- VALUE EXCEEDS SCREENING LEVEL FOR FURTHER INYESTIGATIDK.

- VALUE EXCEEDS SCREENING LEVEL FOR FURTHER INYESTIGA
- DETECTION LIUIT IAS NOT AVAILAGLE FOR COMPARISON

NR - ANALYSIS NOT RERUESTED OR NOT YET REPORTEO

YALUES IN \{ $\}$ ARE COUNTING ERRDRS FOR RADIONUCLIDES

WATER STANDARD(S) IN PARENTHESES ARE PROPOSED ONLY 
TABLE C.5. (contd)

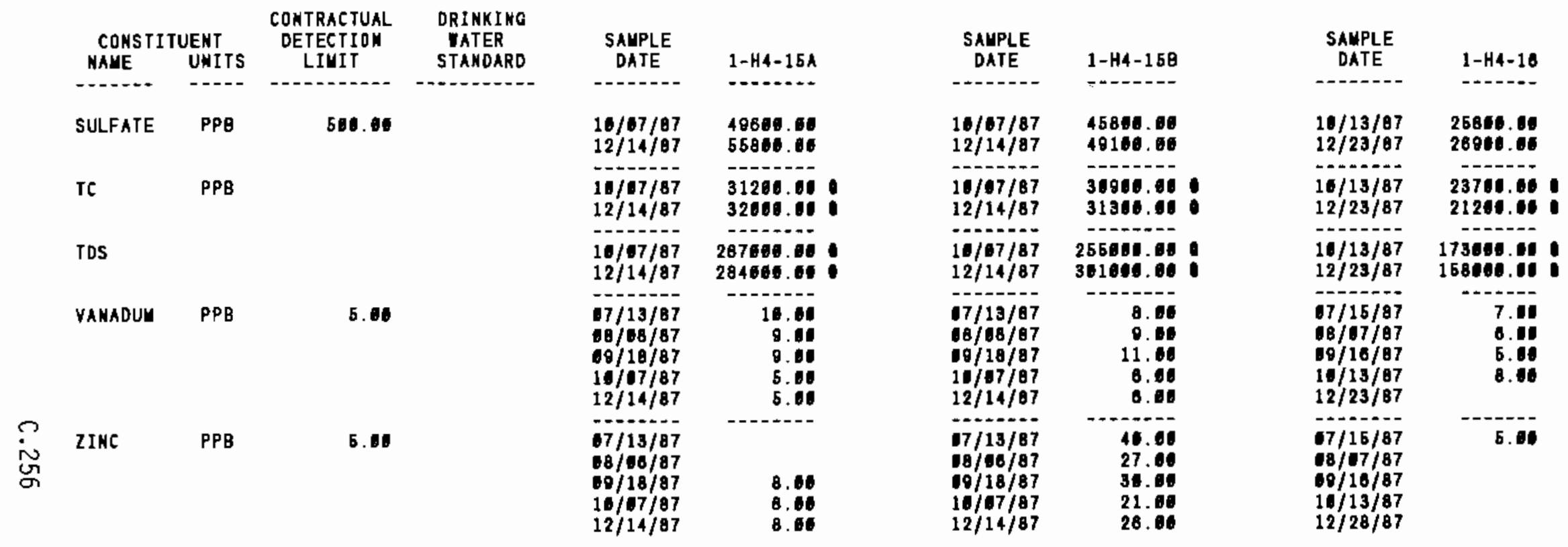

* - VALUE EXCEEDS PRIMARY DRINKING MATER STANDARD.

* - VALUE EXCEEDS PROPOSED PRIMARY DRINKING IATER STAANARD.

- VALUE EXCEEDS SCREEMING LEYEL FOR FURTHER INYESTIGAJION.

- DETECTION LIMIT IAS HOT AVAILABLE FOR COUPARISON

NR - AMALYSIS NOT RERUESTED DR NOT YET REPORTED

YALUES IN \{ $\}$ ARE COUNTINO ERRORS FOR RADIOMUCLIDES

YATER STANDARD(S) IN PARENTHESES ARE PROPOSED ONLY 


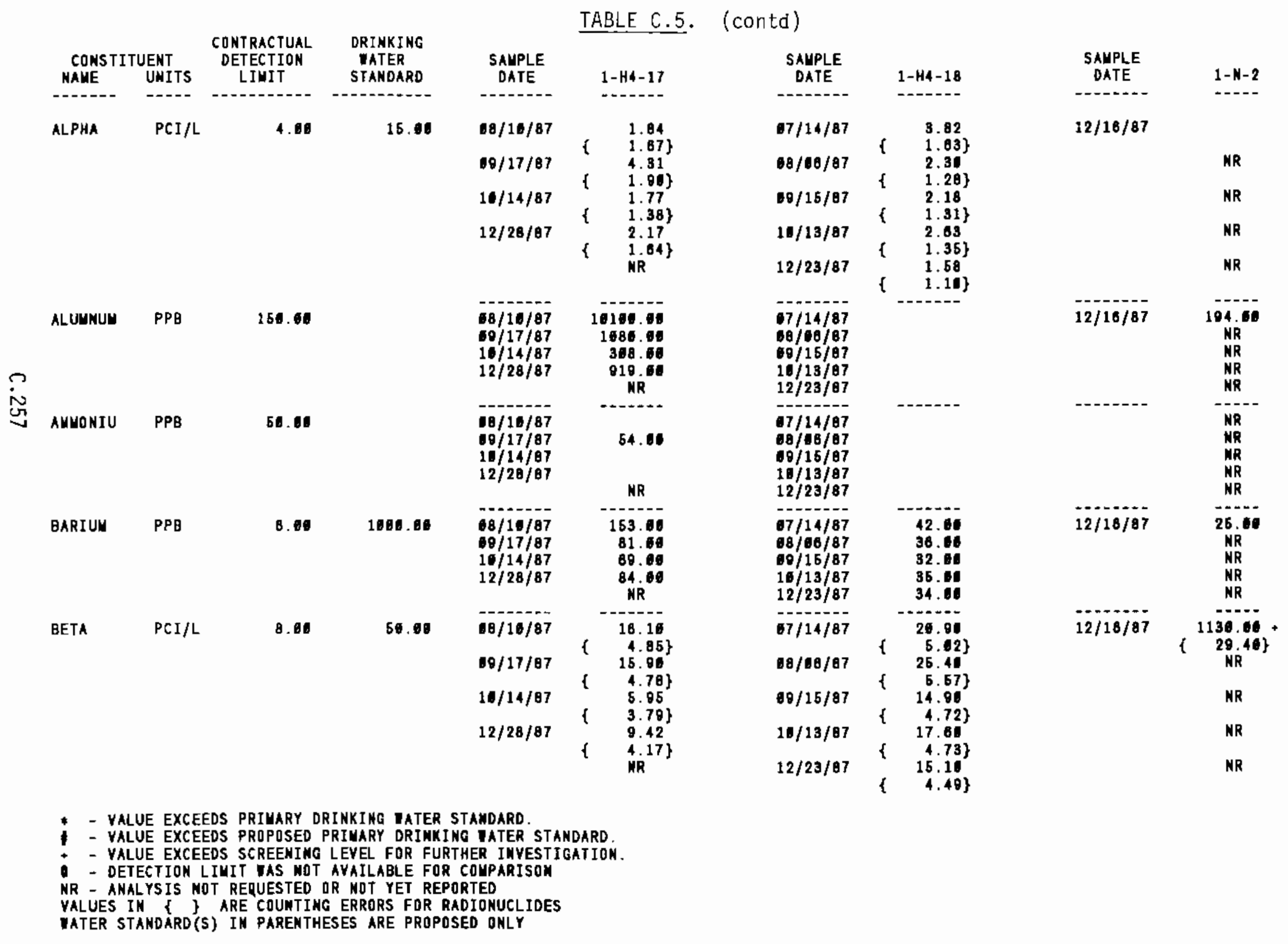


TABLE C. .5 . (contd)

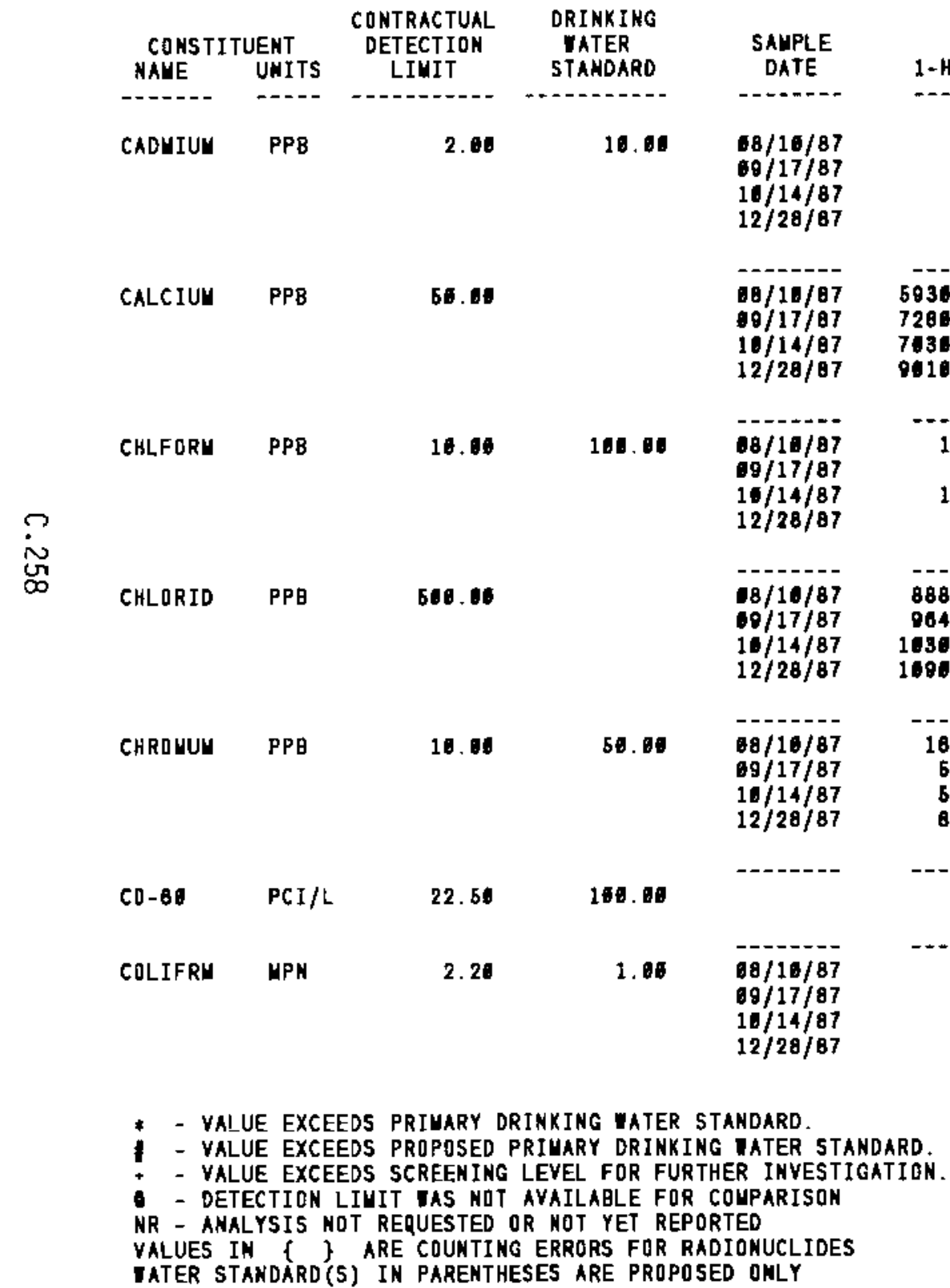

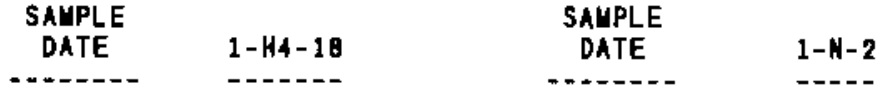

$67 / 14 / 87 \quad 12 / 16 / 97$

18/108/87

$69 / 15 / 87$

$10 / 13 / 87$

$12 / 23 / 87$

$17 / 14 / 87$

$18 / 08 / 87$

$0 / 15 / 87$

10/19/87

$12 / 29 / 87$

$07 / 14 / 87$

$08 / 166 / 87$

60/15/87

$10 / 13 / 87$

$12 / 23 / 87$

$67 / 14 / 87$

$68 / 00 / 87$

$10 / 15 / 87$

$16 / 13 / 87$

$12 / 23 / 87$

……-

$17 / 14 / 87$

$0 / 68 / 87$
$0 / 15 / 87$

$9 / 16 / 87$
$10 / 13 / 87$

$12 / 23 / 87$

56.00

$80.60 *$

NR

6006e.

9712187

$07 / 14 / 97$

$\begin{array}{ll}2.20 * & 09 / 15 / 87 \\ \text { NR } & 10 / 13 / 87 \\ 12 / 29 / 87\end{array}$

$\begin{array}{ll}2.20 * & 09 / 15 / 87 \\ & 10 / 13 / 87 \\ \text { NR } & 12 / 29 / 87\end{array}$
40000.

40000. 01

47096.10

43506.0

29.09

33.0

28.0

25.6

24.66

6041. 60

4760.10

5231. It

5164.6

4854.01

$---10$

262.80

260.

237.60

253.00

MR

\section{NR \\ $\underset{N R}{N R}$}

12/16/87 287

$12 / 16 / 87 \quad 28700$

MR

NR

NR

$----\overline{10}$

$12 / 16 / 87$ -

MR

$\underset{N R}{N R}$

MR

$12 / 16 / 87 \quad 1200.00$

NR

NR

NR

NR

$12 / 16 / 87$

NR

$M R$

NR

$99 / 11 / 87$

30.30

-...-..-

$12 / 16 / 87$
15. 4.)

NR

NR

NR

- DETECTION liUIT TAS NIT AVAILABLE FOR COUPARISON

VALUES IN \{ ? ARE COUNTING ERRORS FQR RADIONUCLIDES

TATER STANDARD(5) IN PARENTHESES ARE PROPOSED ONLY 
TABLE C.5. (contd)

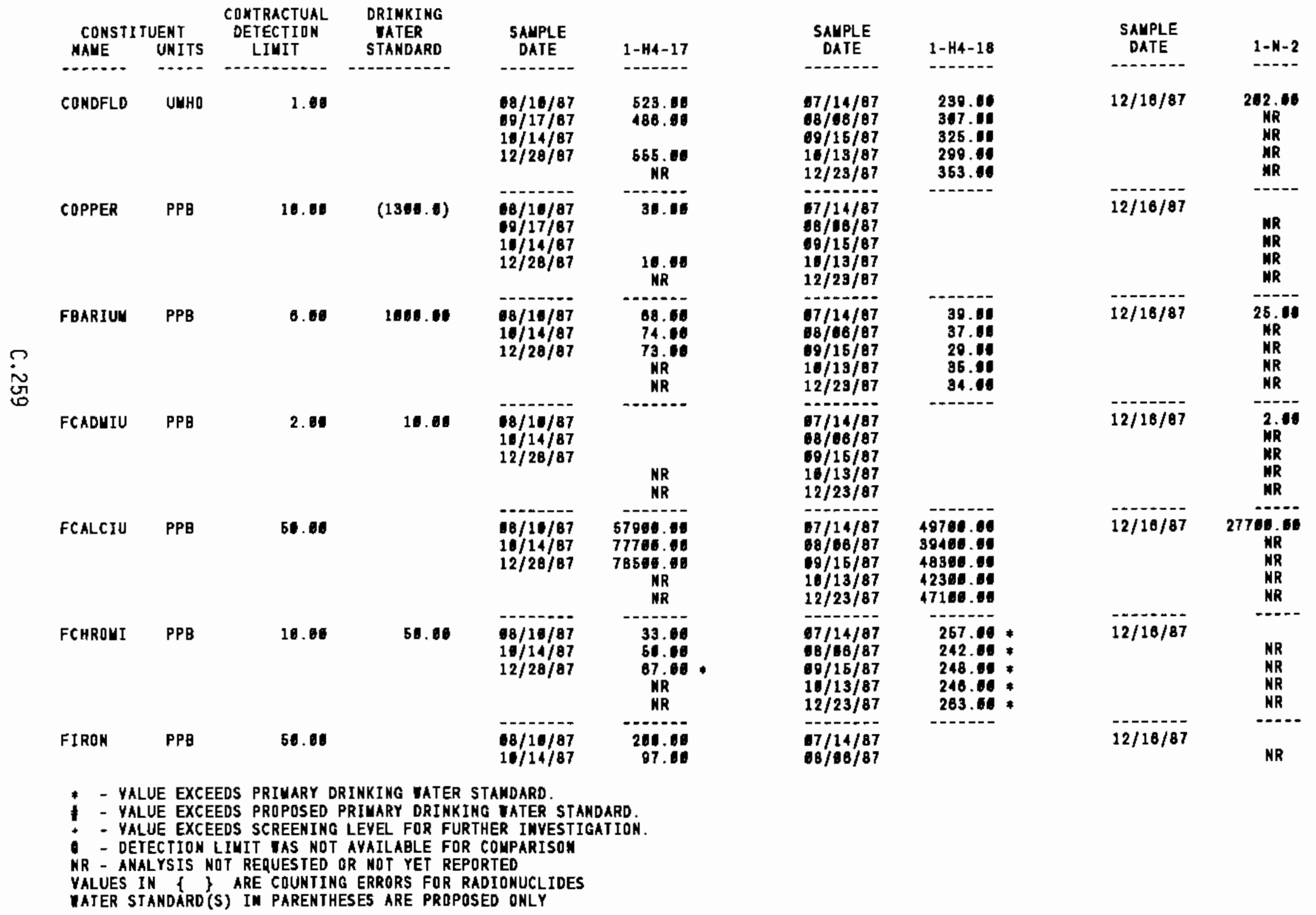




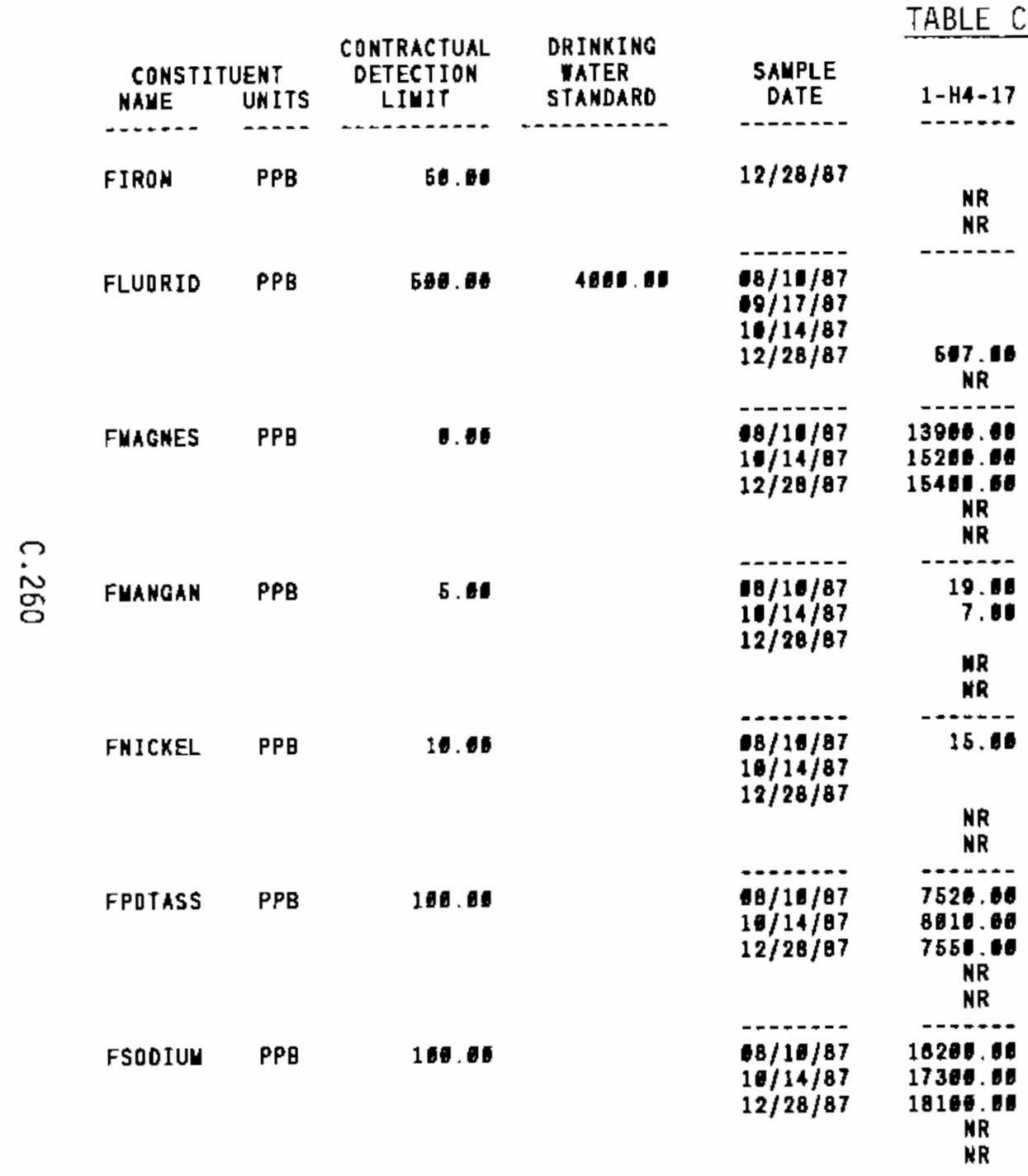

(contd) 
TABLE C.5. (contd)

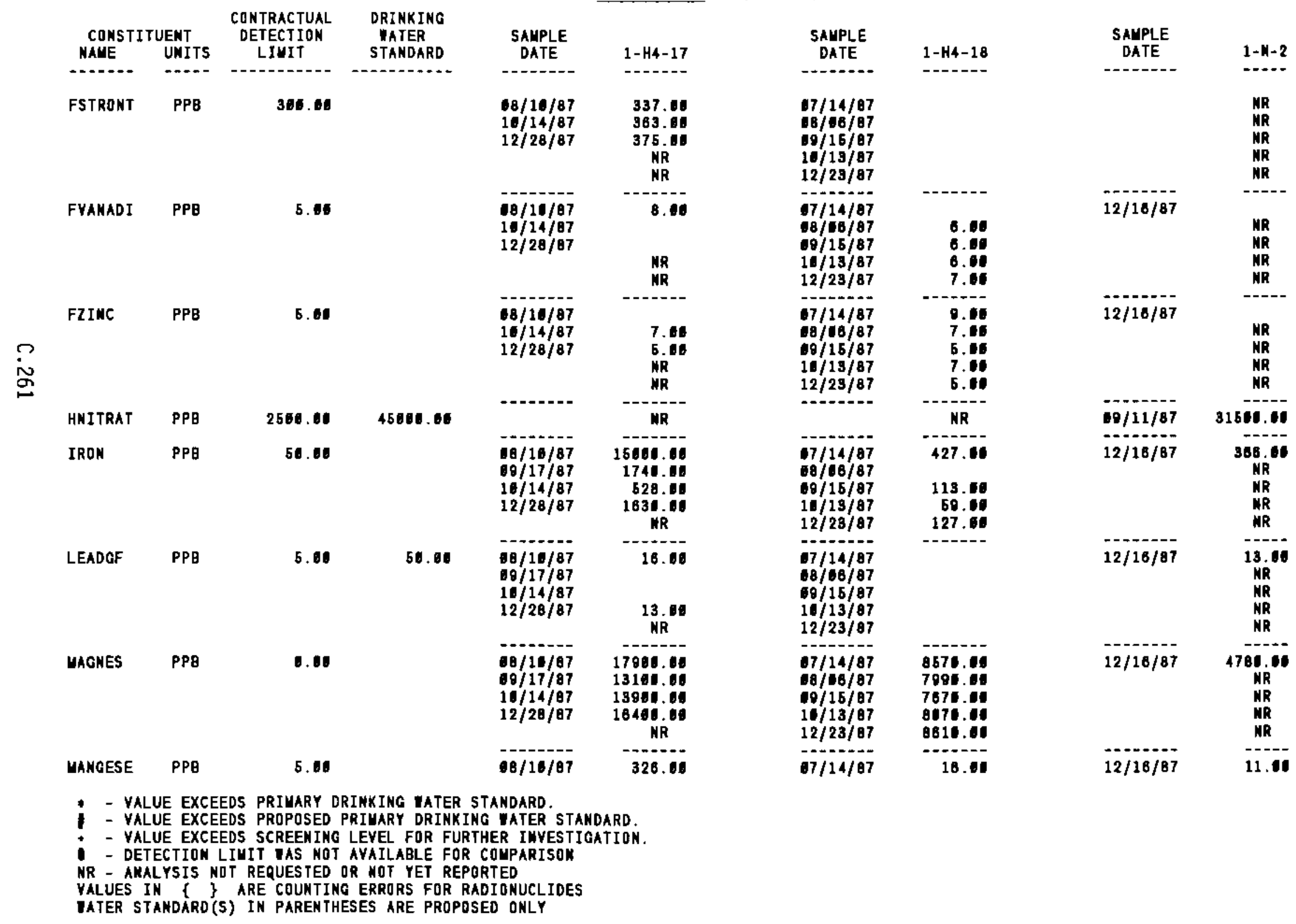


TABLE C.5. (contd)

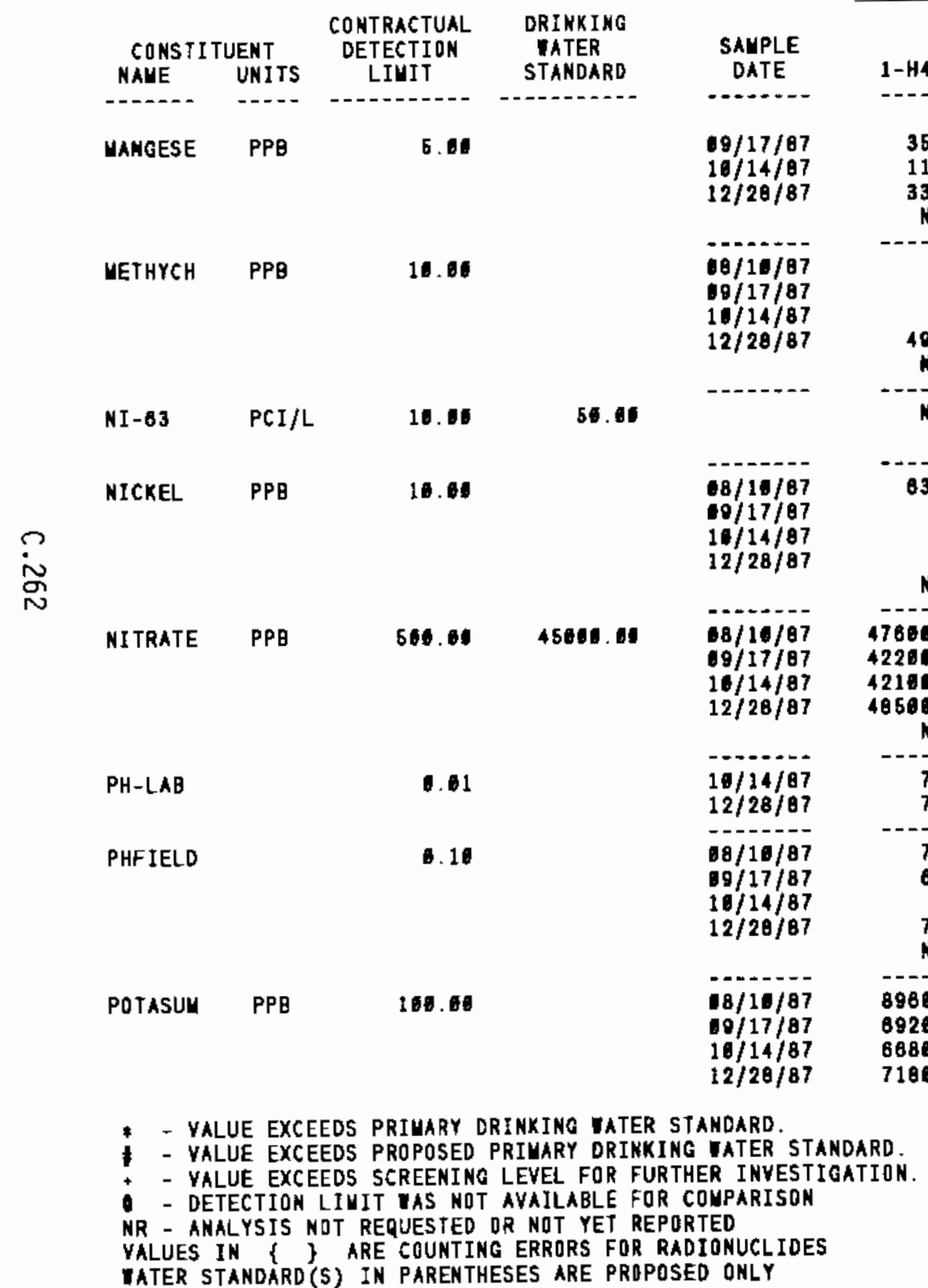

\begin{tabular}{|c|c|c|c|}
\hline $\begin{array}{c}\text { SAUPLE } \\
\text { DATE }\end{array}$ & $1-H 4-18$ & $\begin{array}{c}\text { SAMPLE } \\
\text { DATE }\end{array}$ & $1-N-2$ \\
\hline $\begin{array}{l}08 / 86 / 87 \\
60 / 15 / 87 \\
10 / 13 / 87 \\
12 / 23 / 87\end{array}$ & & & $\begin{array}{l}\text { MR } \\
\text { NR } \\
\text { NR } \\
\text { NR }\end{array}$ \\
\hline $\begin{array}{l}67 / 14 / 87 \\
68 / 08 / 87 \\
19 / 16 / 97 \\
1 \% / 13 / 87 \\
12 / 29 / 87\end{array}$ & - & $12 / 16 / 87$ & $\begin{array}{l}\text { KR } \\
\text { NR } \\
\text { NR } \\
\text { NR }\end{array}$ \\
\hline & MR & $19 / 11 / 87$ & $\begin{array}{l}4.10 \\
2.00\}\end{array}$ \\
\hline $\begin{array}{l}07 / 14 / 87 \\
98 / 48 / 87 \\
60 / 15 / 87 \\
16 / 13 / 87 \\
12 / 23 / 87\end{array}$ & - & $12 / 16 / 87$ & $\begin{array}{l}\text { NR } \\
\text { NR } \\
\text { NR } \\
\text { MR }\end{array}$ \\
\hline $\begin{array}{l}6 / 14 / 87 \\
08 / 68 / 87 \\
62 / 15 / 87 \\
16 / 13 / 87 \\
12 / 23 / 87\end{array}$ & $\begin{array}{l}25005.04 \\
26080.00 \\
23601.01 \\
23800.00 \\
28196.00\end{array}$ & $12 / 16 / 87$ & $\begin{array}{c}3401 \mathrm{~N} .00 \\
\text { NR } \\
\text { NR } \\
\text { NR } \\
\text { NR }\end{array}$ \\
\hline $\begin{array}{l}10 / 13 / 87 \\
12 / 23 / 87\end{array}$ & $\begin{array}{l}7.67 \\
7.57\end{array}$ & & $\begin{array}{l}\text { NR } \\
\text { NR }\end{array}$ \\
\hline $\begin{array}{l}67 / 14 / 87 \\
60 / 08 / 87 \\
19 / 16 / 87 \\
11 / 13 / 87 \\
12 / 23 / 87\end{array}$ & $\begin{array}{l}7.60 \\
7.10 \\
7.40 \\
5.70 \\
8.48\end{array}$ & $12 / 16 / 87$ & $\begin{array}{l}7.40 \\
M R \\
M R \\
M R \\
N R\end{array}$ \\
\hline $\begin{array}{l}67 / 14 / 87 \\
68 / 06 / 67 \\
69 / 16 / 87 \\
10 / 13 / 67\end{array}$ & $\begin{array}{l}4144.08 \\
4094.00 \\
3826.00 \\
3850.08\end{array}$ & $12 / 16 / 87$ & $\begin{array}{c}1650.66 \\
\text { MR } \\
\text { NR } \\
\text { NR }\end{array}$ \\
\hline
\end{tabular}

- YALUE EXCEEDS PROPOSED PRIMARY DRIMKING aATER STAMDaRD.

- VALUE EXCEEDS SCREENING LEVEL FOR FURTHER INYEST

YALUES IN

VATER STANDARD'S) IN PARENTHESES ARE PROPOSED ONLY

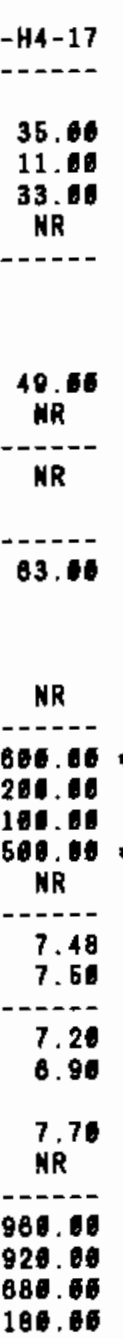


TABLE C.5. (contd)

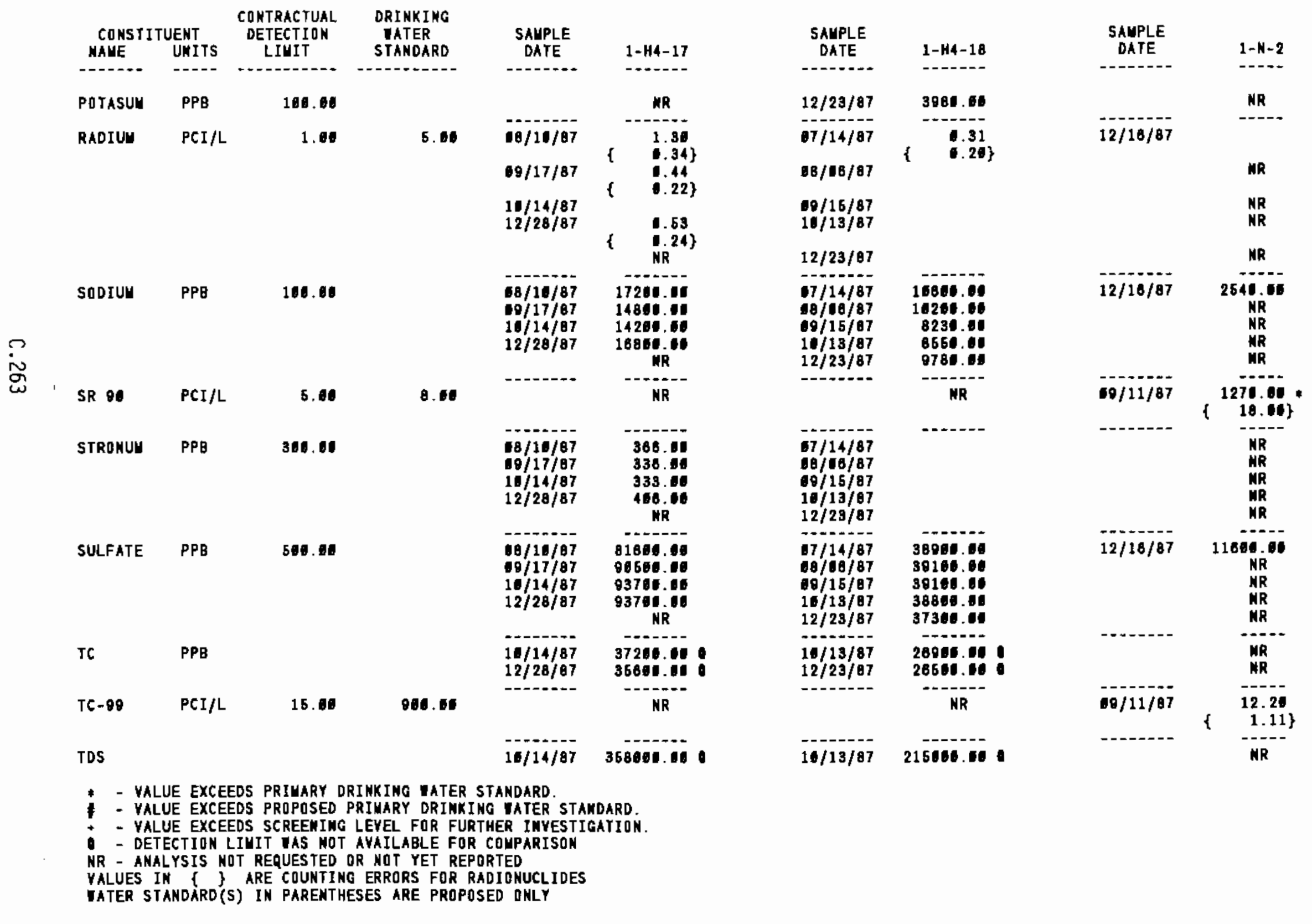


TAELE C.5. (contd)

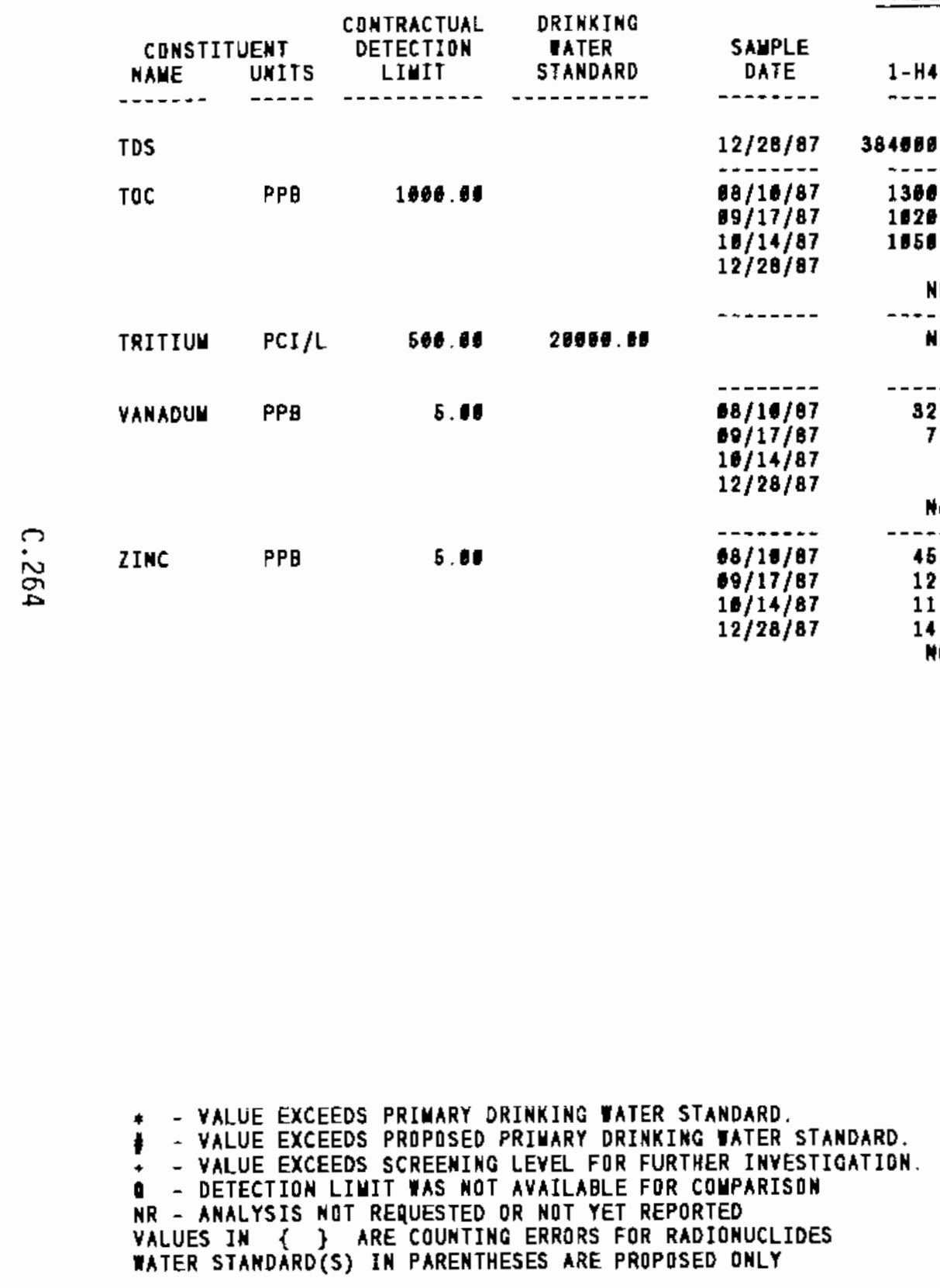

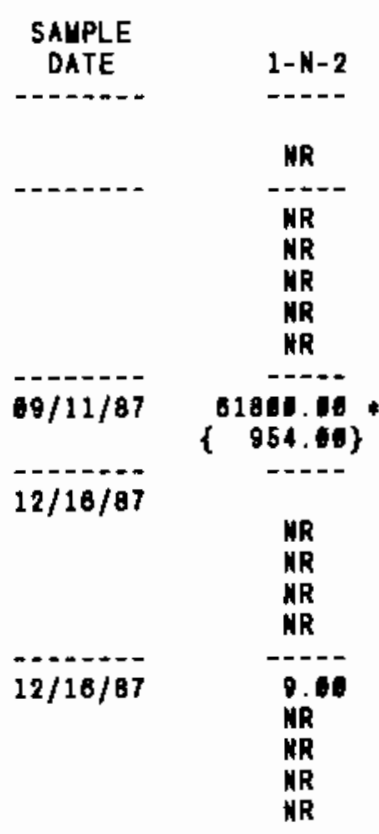

NR - ANALYSIS MBT REQUESTED OR NOT YET REPORTED

WATER STAMDARD(S) IN PARENTHESES ARE PROPQSED ONLY 
TABLE C.5. (contd)

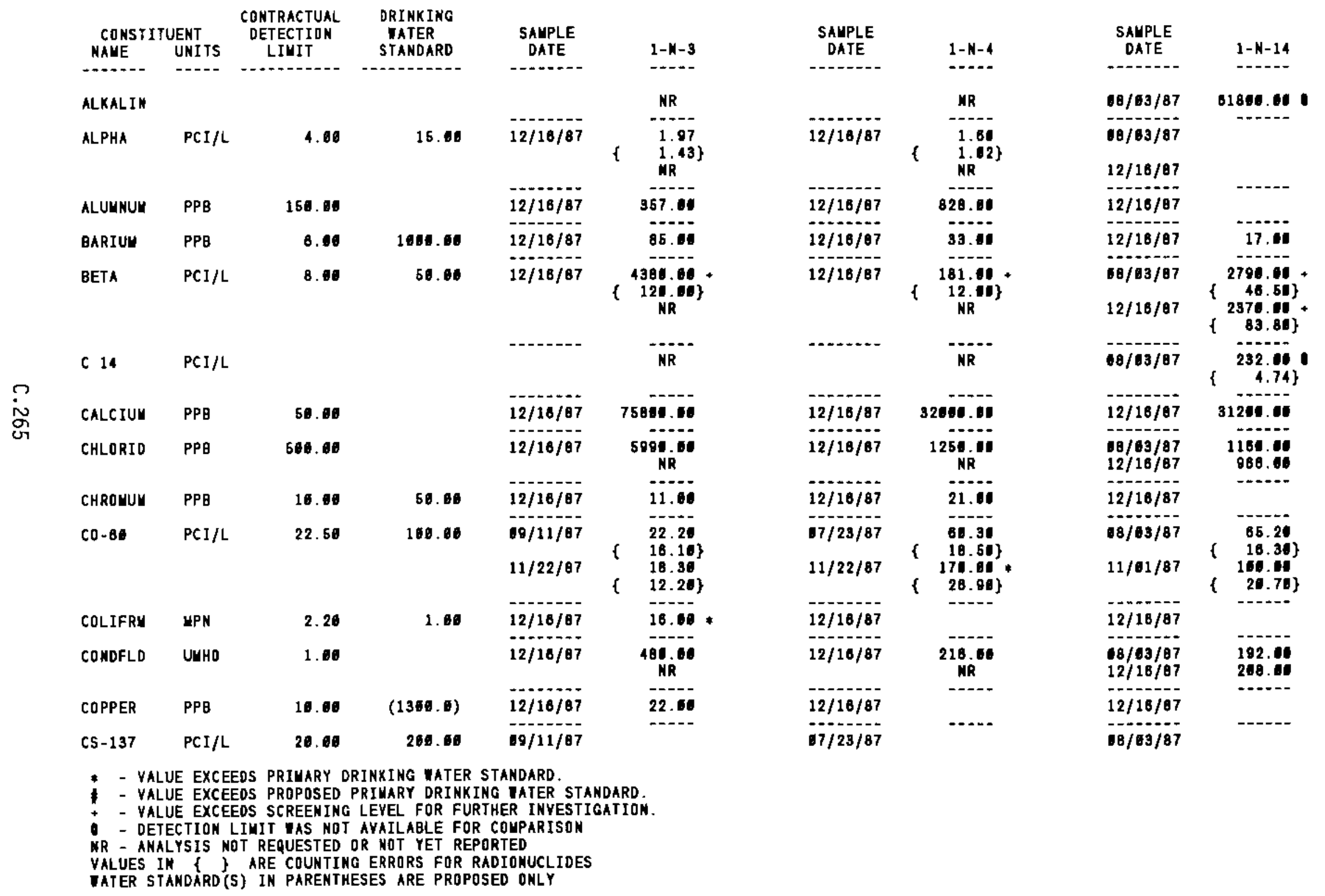




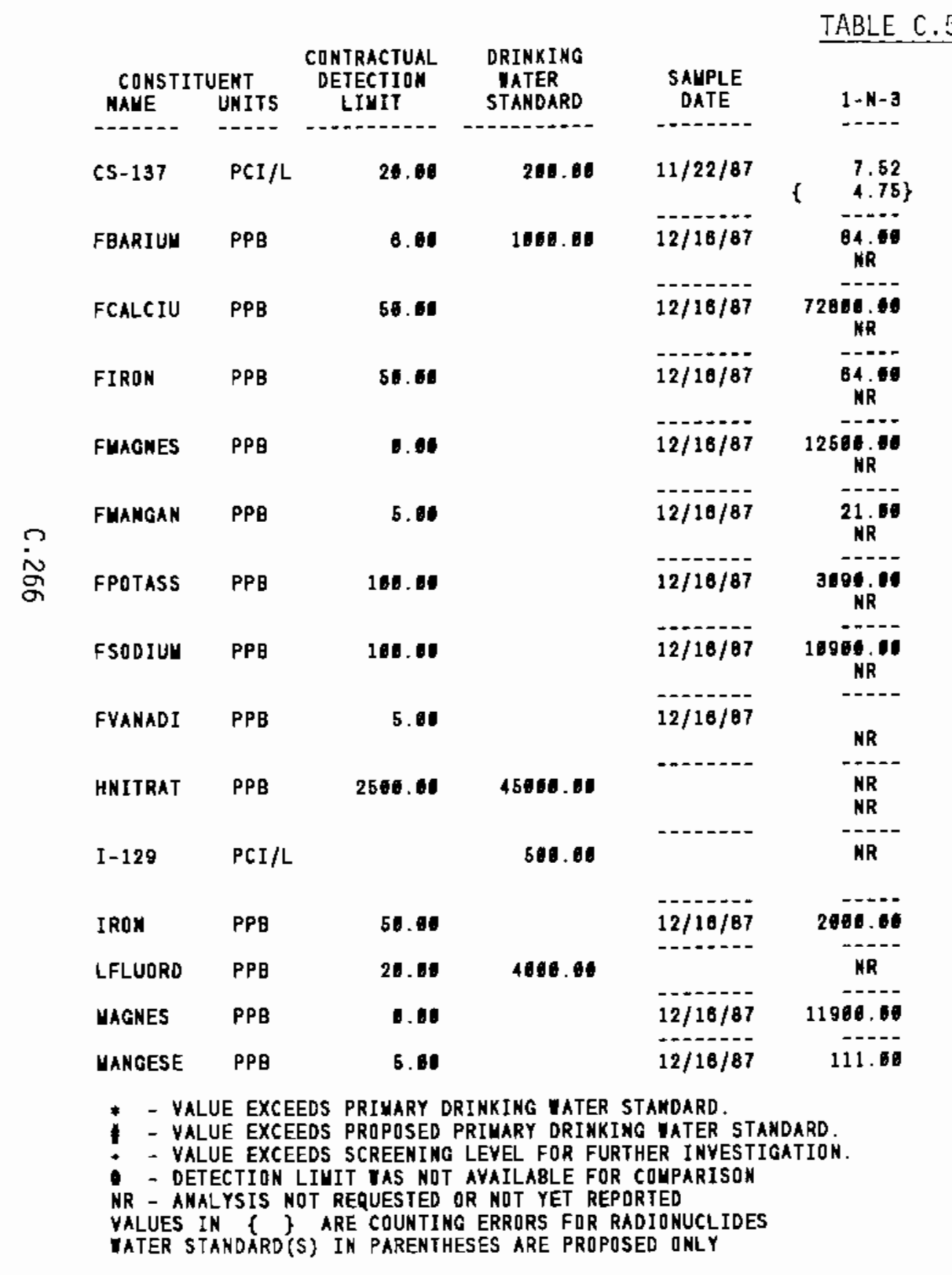

contd)

\begin{tabular}{|c|c|c|c|}
\hline $\begin{array}{c}\text { SAHPLE } \\
\text { DATE }\end{array}$ & $1-N-4$ & $\begin{array}{c}\text { SAMPLE } \\
\text { DATE }\end{array}$ & $1-N-14$ \\
\hline $11 / 22 / 87$ & $\begin{array}{l}8.95 \\
\text { B.14\} }\end{array}$ & $11 / 01 / 87$ & \\
\hline $12 / 16 / 87$ & ${ }_{\text {NR }}^{27.60}$ & $\begin{array}{l}68 / 09 / 87 \\
12 / 18 / 87\end{array}$ & $\begin{array}{l}17.00 \\
20.00\end{array}$ \\
\hline $12 / 18 / 87$ & 30600.60 & $\begin{array}{l}08 / 193 / 87 \\
12 / 18 / 87\end{array}$ & $\begin{array}{l}25200.00 \\
28804.00\end{array}$ \\
\hline $12 / 16 / 87$ & NR & $\begin{array}{l}18 / 83 / 87 \\
12 / 16 / 87\end{array}$ & \\
\hline $12 / 16 / 87$ & 5510.60 & $\begin{array}{l}08 / 63 / 87 \\
12 / 16 / 87\end{array}$ & $\begin{array}{l}4904.00 \\
\text { so16.01 }\end{array}$ \\
\hline $12 / 16 / 87$ & NR & $\begin{array}{l}18 / 63 / 87 \\
12 / 16 / 87\end{array}$ & \\
\hline $12 / 18 / 87$ & ${ }_{M R}^{308 B .60}$ & $\begin{array}{l}18 / 103 / 87 \\
12 / 16 / 87\end{array}$ & $\begin{array}{l}1806.80 \\
1806.06\end{array}$ \\
\hline $12 / 16 / 07$ & 4426.60 & $\begin{array}{l}10 / 103 / 87 \\
12 / 16 / 87\end{array}$ & $\begin{array}{l}3876.60 \\
3910.66\end{array}$ \\
\hline $12 / 18 / 87$ & 8.60 & $\begin{array}{l}16 / 03 / 87 \\
12 / 16 / 87\end{array}$ & $\begin{array}{l}8.60 \\
7.06\end{array}$ \\
\hline \multirow[t]{2}{*}{$\begin{array}{l}97 / 23 / 87 \\
11 / 22 / 87\end{array}$} & $\begin{array}{l}18460.00 \\
33400.00\end{array}$ & & $\begin{array}{l}\text { NR } \\
\text { NR }\end{array}$ \\
\hline & NR & $69 / 10 / 87$ & $\begin{array}{l}0.010 \\
0.00\}\end{array}$ \\
\hline \multirow[t]{2}{*}{$12 / 16 / 87$} & 3080.06 & $12 / 16 / 87$ & 168.11 \\
\hline & NR & $08 / 03 / 87$ & 161.06 \\
\hline $12 / 16 / 87$ & 5440.60 & $12 / 18 / 87$ & 4870.00 \\
\hline $12 / 18 / 87$ & 73.60 & $12 / 16 / 87$ & \\
\hline
\end{tabular}


TABLE C.5. (contd)

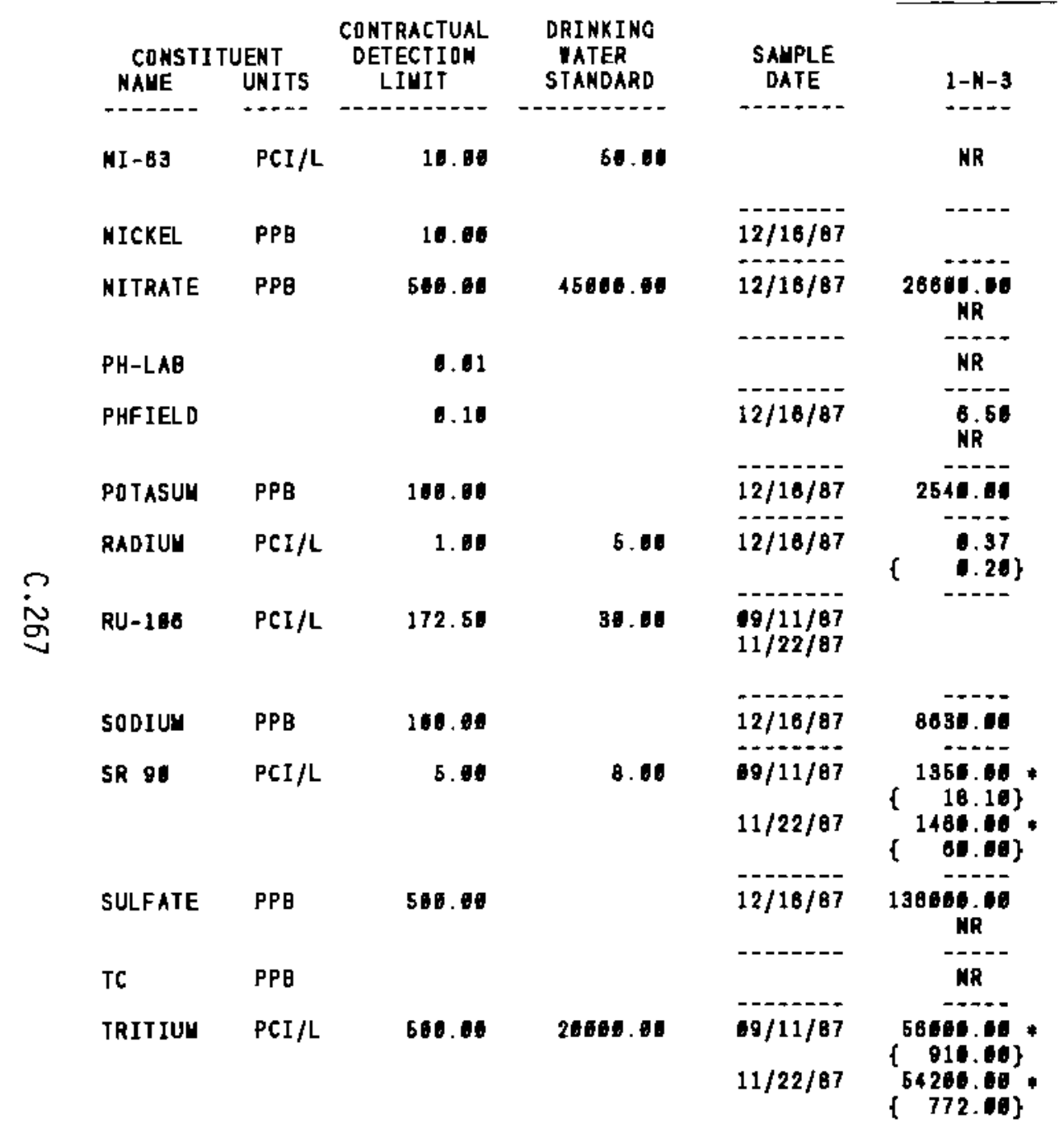

\begin{tabular}{|c|c|}
\hline \multirow{2}{*}{$\begin{array}{c}\text { SAUPLE } \\
\text { DATE }\end{array}$} & $1-N-4$ \\
\hline & NR \\
\hline $12 / 18 / 87$ & 33.6 \\
\hline $12 / 16 / 87$ & 33800.60 \\
\hline . & NR \\
\hline $12 / 18 / 87$ & 7.21 \\
\hline $12 / 18 / 87$ & 2680.01 \\
\hline $12 / 18 / 87$ & \\
\hline $\begin{array}{l}07 / 23 / 87 \\
11 / 22 / 87\end{array}$ & \\
\hline & $\{\quad 82.71\}$ \\
\hline $12 / 16 / 87$ & 3700.01 \\
\hline $07 / 23 / 87$ & 11.14 \\
\hline $11 / 22 / 87$ & $\begin{array}{r}14.31 \\
\{\quad 1.91\}\end{array}$ \\
\hline $12 / 18 / 87$ & 13200.01 \\
\hline - & NR \\
\hline $07 / 23 / 87$ & 162984.06 \\
\hline $11 / 22 / 87$ & $\begin{array}{l}138060.66 \\
\{1376.66)\end{array}$ \\
\hline
\end{tabular}

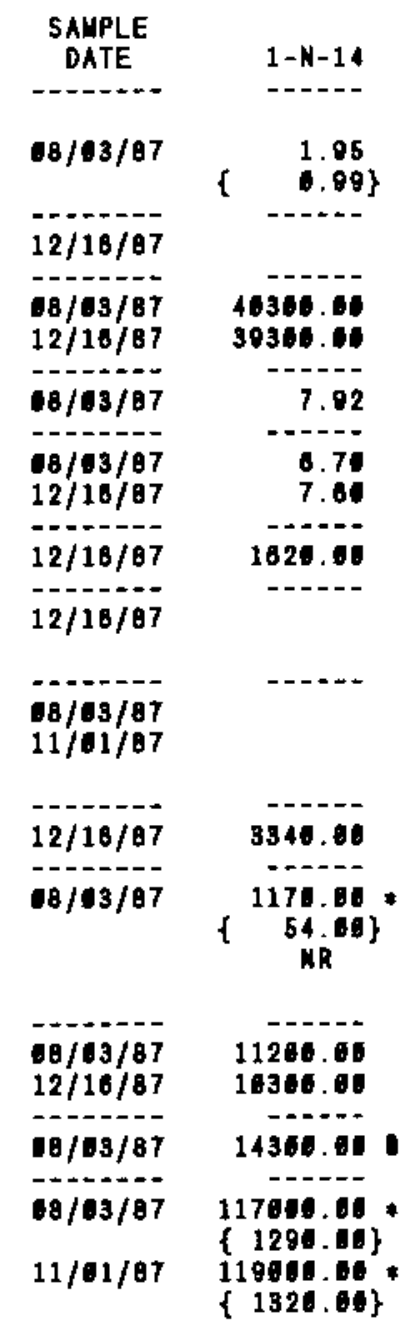

* - value eXCEeds privary dRinking vater standaro.

1 - VALUE EXCEEDS PROPOSED PRIMARY DRINXIKG IATER STAMDARD.

- - VAlUE EXCEEDS SCREENING LeVEL For FURTHER INVESTIGATION.

- DETECTion limit is not available for couparisok

MR - ANALYSIS NOT REQUESTED OR MOT YET REPORTED

VALUES IN \{ \} ARE COUNTING ERRORS FOR RADIONUCLIDES

IATER STANDRRD'(S) IN PARENTHESES ARE PROPOSED DNLY 


\begin{tabular}{|c|c|c|c|c|c|c|c|c|c|}
\hline \multirow{2}{*}{\multicolumn{2}{|c|}{ CDNSTITUENT }} & \multirow{2}{*}{$\begin{array}{l}\text { CONTRACTUAL } \\
\text { DETECTIOM } \\
\text { LIUIT }\end{array}$} & \multirow{2}{*}{$\begin{array}{l}\text { DRINKING } \\
\text { WATER } \\
\text { STAMOARD }\end{array}$} & \multirow[b]{2}{*}{$\begin{array}{l}\text { SALPLE } \\
\text { DATE }\end{array}$} & \multicolumn{4}{|c|}{ 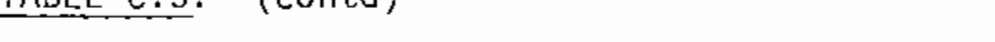 } & \multirow[b]{2}{*}{$1-N-14$} \\
\hline & & & & & $1-N-3$ & $\begin{array}{l}\text { SAUPLE } \\
\text { DATE }\end{array}$ & $1-N-4$ & $\begin{array}{c}\text { SAUPLE } \\
\text { DATE }\end{array}$ & \\
\hline------ & --- & - & $--\infty-1--1$ & $-\cdots-\cdots$ & ----- & ------ & $\cdots$ & $\cdots+\cdots$ & ----- \\
\hline VAHADUM & PPB & 5.00 & & $12 / 16 / 87$ & & $12 / 10 / 87$ & 9.00 & $12 / 16 / 87$ & \\
\hline ZINC & PPB & 5.00 & & $12 / 10 / 87$ & B. & $12 / 16 / 97$ & 62.00 & $12 / 16 / 97$ & 9.00 \\
\hline
\end{tabular}

* - VALUE EXCEEDS PRIUARY ORINKING VATER STANDARD.

- VALUE EXCEEDS PRDPOSEO PRIYARY DRINKING IaTER STAMDARO.

- VALUE EXCEEDS SCREENING LEVEL FOR FURTHER INVESTIGATIOR.

- DETECTION LIUIT TAS NOT AVAILABLE FOR COMPARISON

NR - ANALYSIS NDT REQUESTED DR NOT YET REPORTED

VALUES IN $\{$ ARE CDUNTIMG ERRORS FOR RADIONUCLIDES

MATER STANDARO(S) IN PARENTHESES ARE PROPOSED DNLY 
TABLE C.5. (contd)

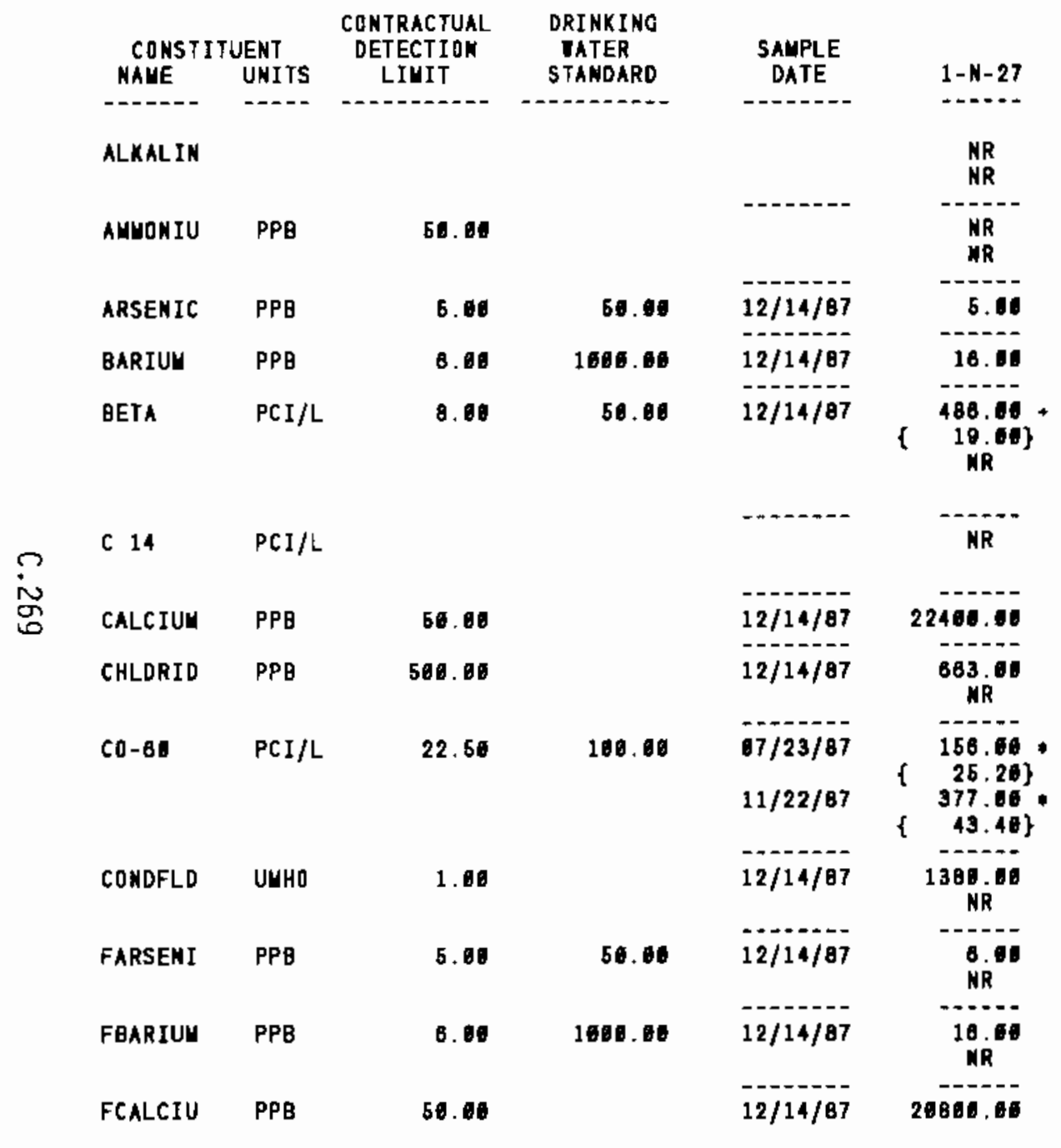

\begin{tabular}{|c|c|}
\hline $\begin{array}{l}\text { SALPLE } \\
\text { DATE }\end{array}$ & $1-N-29$ \\
\hline -ー-ー--- & 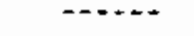 \\
\hline $\begin{array}{l}08 / 63 / 87 \\
11 / 22 / 87\end{array}$ & $\begin{array}{l}44600.000 \\
45200.650\end{array}$ \\
\hline $\begin{array}{l}8 / 03 / 87 \\
11 / 22 / 87\end{array}$ & $\begin{array}{l}-\overline{-10-} \\
2800.01 \\
1100.01\end{array}$ \\
\hline ---no-s & NR \\
\hline------- & NR \\
\hline $68 / 03 / 87$ & $\left\{\begin{array}{r}1701.10 \\
36.30\}\end{array}\right.$ \\
\hline $11 / 22 / 87$ & $\left\{\begin{array}{r}1146.00 \\
28.08\}\end{array}\right.$ \\
\hline $10 / 103 / 87$ & $\begin{array}{r}4.09 \\
\{\quad 2.87\}\end{array}$ \\
\hline$-2-1+2$. & MR \\
\hline $\begin{array}{l}06 / 63 / 87 \\
11 / 22 / 87\end{array}$ & 863.65 \\
\hline $18 / 63 / 87$ & 254.11 \\
\hline $11 / 22 / 67$ & $\begin{array}{c}\left\{\begin{array}{c}32.26\} \\
157.00\end{array}\right. \\
\{\quad 25.20\}\end{array}$ \\
\hline $\begin{array}{l}6 / 93 / 87 \\
11 / 22 / 87\end{array}$ & $\begin{array}{l}161.00 \\
126.08\end{array}$ \\
\hline $\begin{array}{l}08 / 03 / 87 \\
11 / 22 / 87\end{array}$ & ----- \\
\hline $\begin{array}{l}08 / 63 / 87 \\
11 / 22 / 87\end{array}$ & 12.00 \\
\hline $08 / 63 / 87$ & 17800.00 \\
\hline
\end{tabular}

SAUPLE
DATE
-

MR

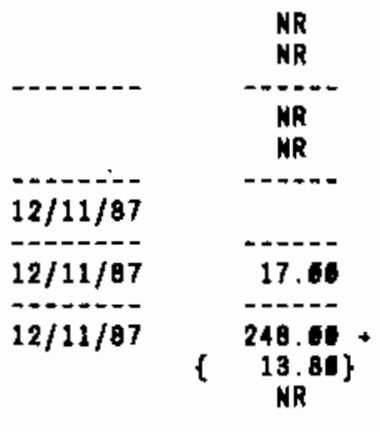

NR

$12 / 11 / 87 \quad 22900.10$

-

$12 / 11 / 87$

80/11/87 195.10,

$11 / 01 / 87$ \{ 36.70$\}$

\{ 33.70$\}$

NR

NR

12/11/87

$12 / 11 / 07$ NR

$12 / 11 / 87 \quad 13.61$

$12 / 11 / 87 \quad 22300.00$

* - value exceeds privary drinking tater standard.

- - VALUE EXCEEDS PROPOSED PRIUARY DRINKING VATER STANDARD.

- - YALUE EXCEEDS SCREENINO LEYEL FOR FURTHER INYESTIGATION.

- DETECTIDN LIYIT YAS NOT AVAILABLE FOR COUPARISON

NR - ANALYSIS NOT REQUESTED OR NOT YET REPORTED

VALUES IN \{ \} ARE CDUNTIMG ERRORS FOR RADIONUCLIDES

WATER STANDARD(5) IN PARENTHESES ARE PROPOSED ONLY 
TABLE C. 5. (contd)

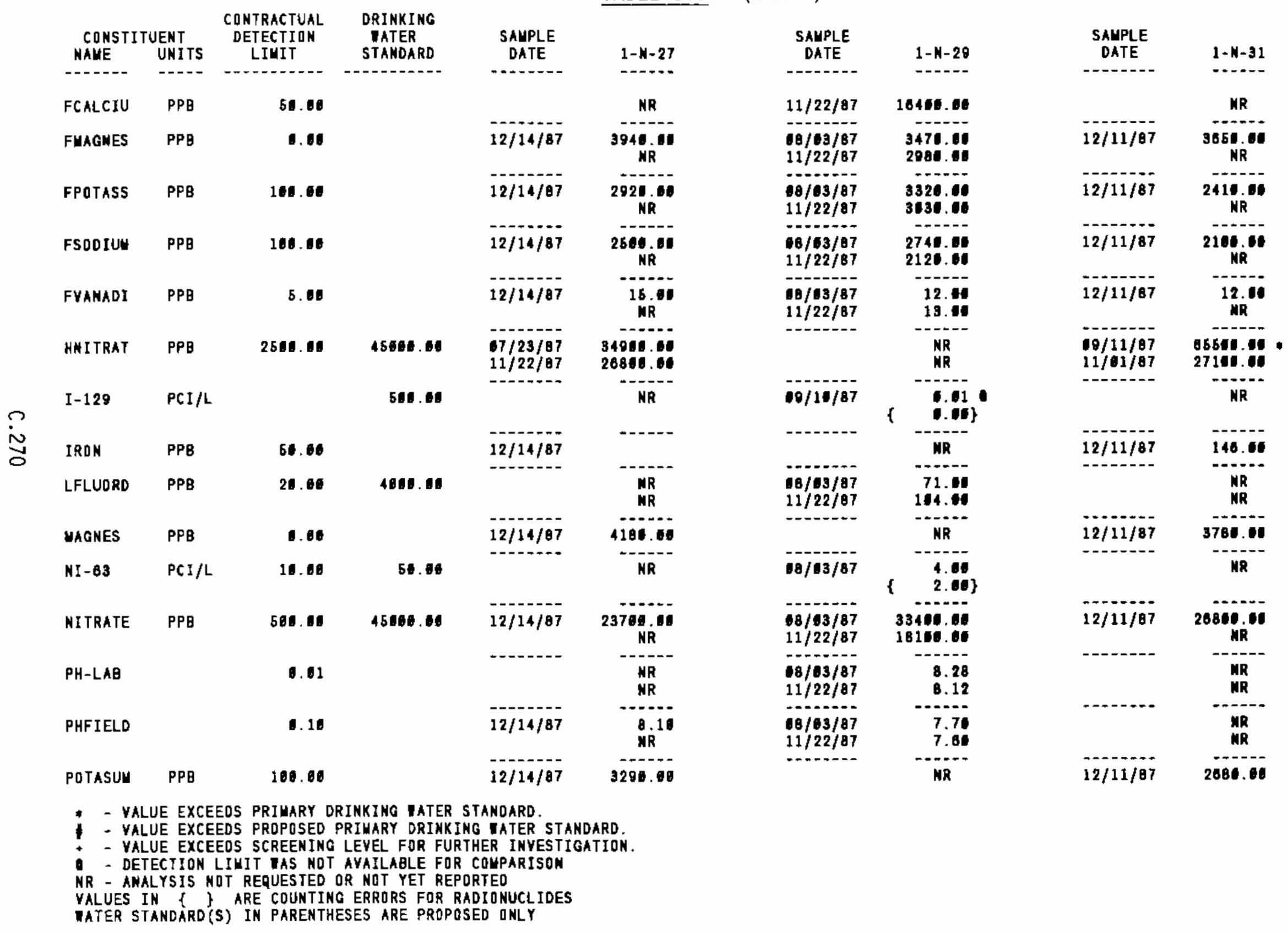


TABLE C.5. (contd)

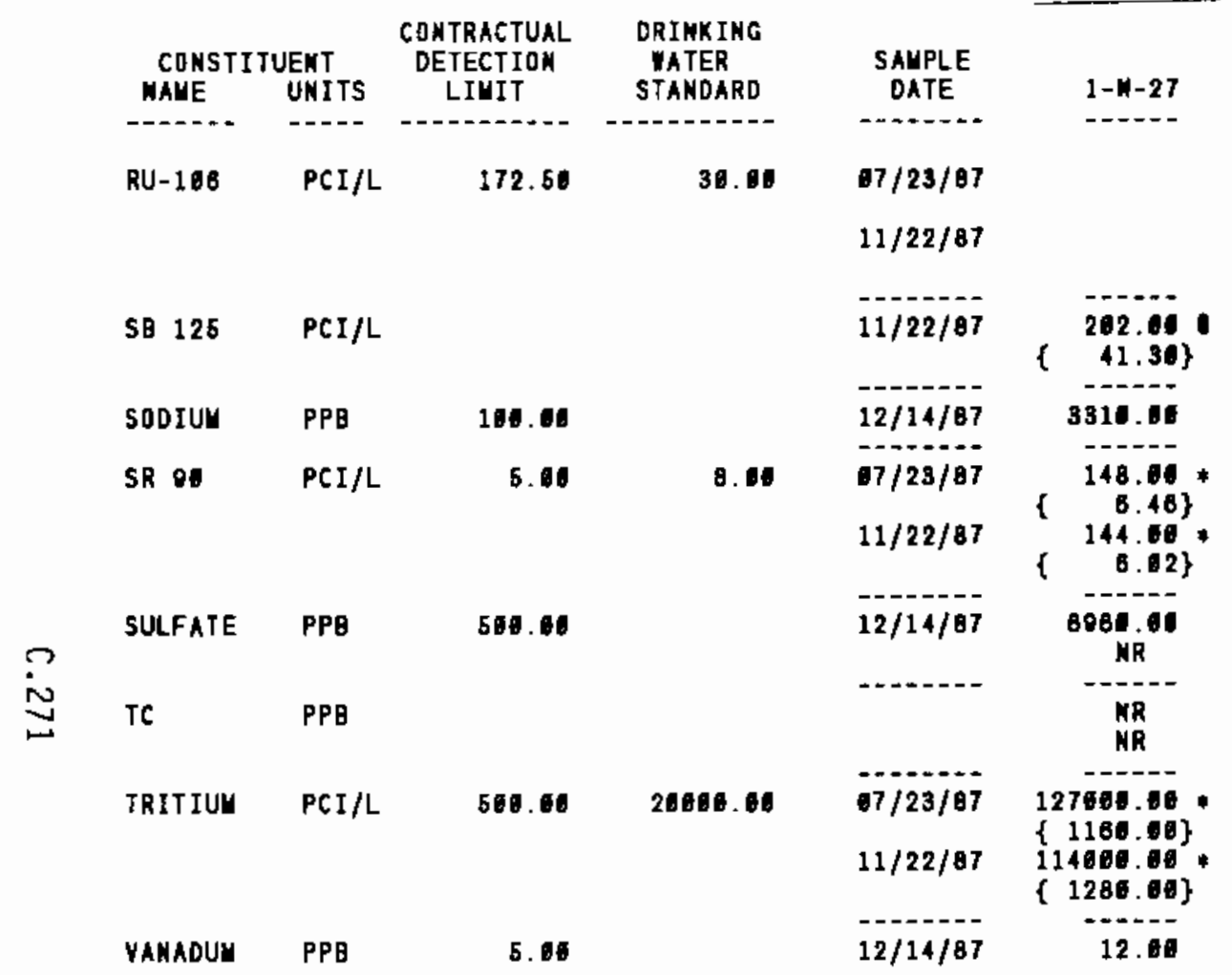

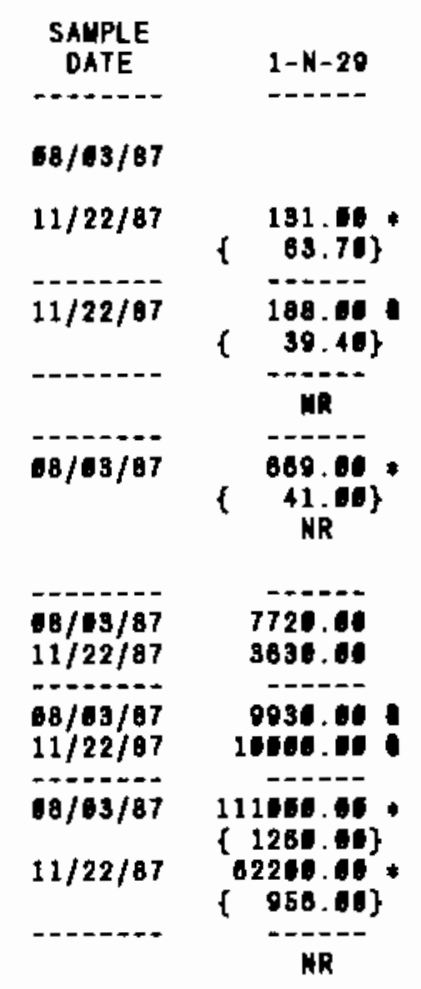

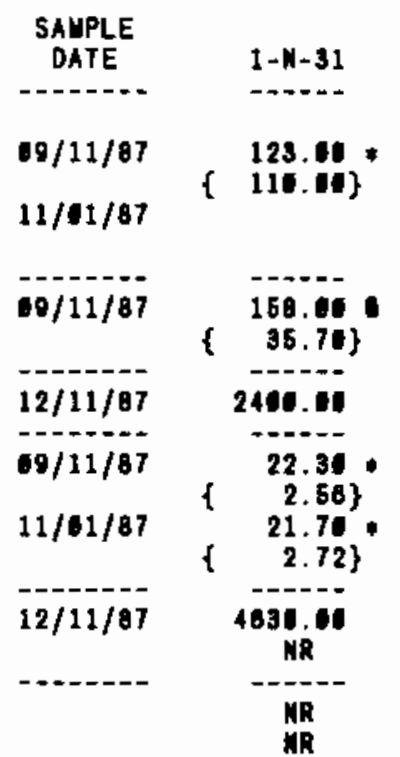

$69 / 11 / 87$

$00 / 11 / 87$

$11 / 61 / 87$

$12 / 11 / 87$

141000.00

\{1426.00\}

\{1125.00\}

6.0

* - VALUE EXCEEDS PRIUARY ORINKING WATER STANDARD.

- VALUE EXCEEDS SCREENINO LEVEL FOR FURTHER IMVESTIGATION.

- DETECTION LIVIT TAS NOT AVAILABLE FOR COLPARISON

NR - ANALYSIS NOT REQUESTED OR NOT YET REPORTED

YALUES IN \& ? ARE COUNTING ERRORS FOR RADIOHUCLIDES

WATER STANDARO(S) IN PARENTHESES ARE PROPDSED ONLY 
TABLE C.5. (contd)

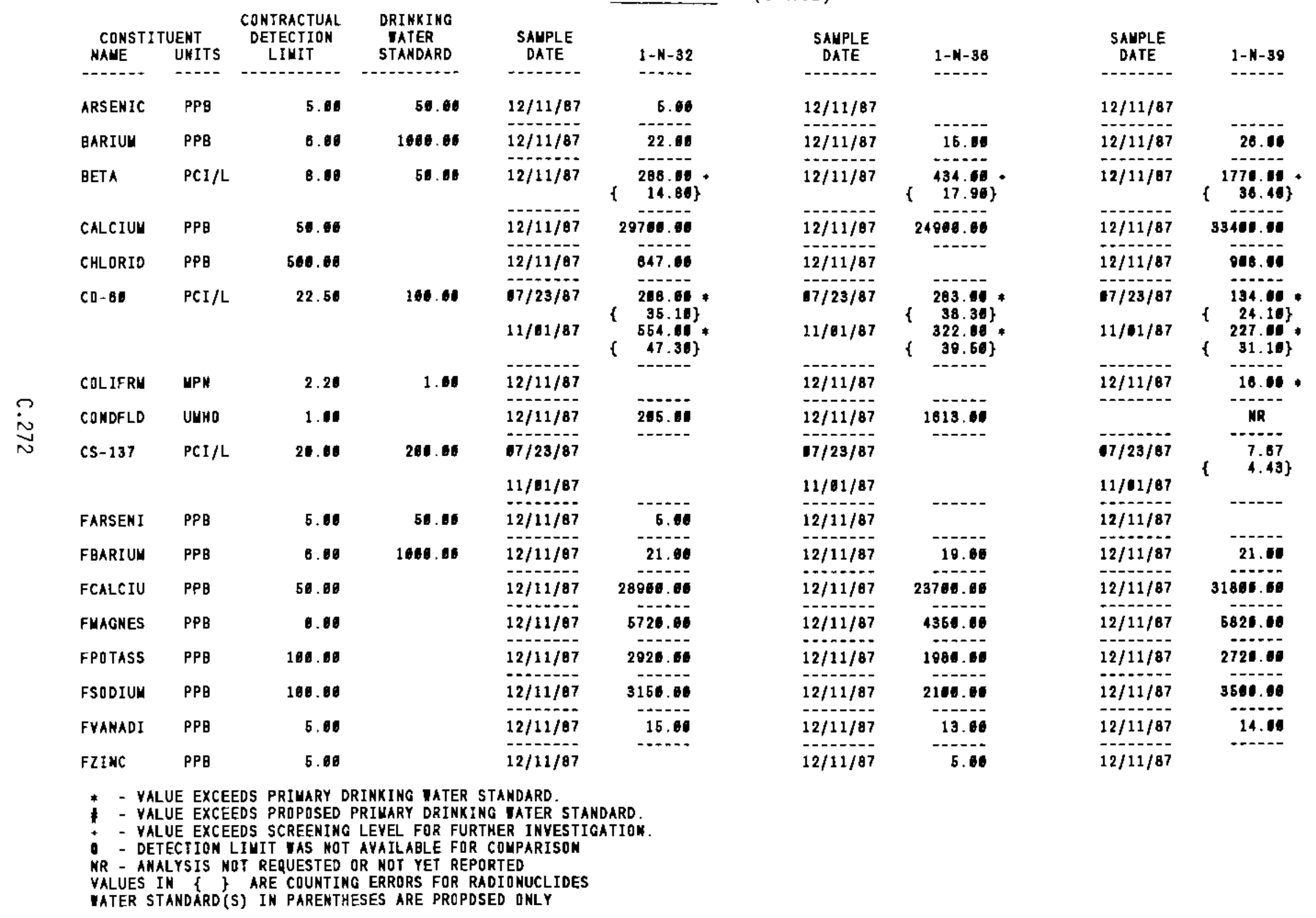


TABLE C.5. (contd)

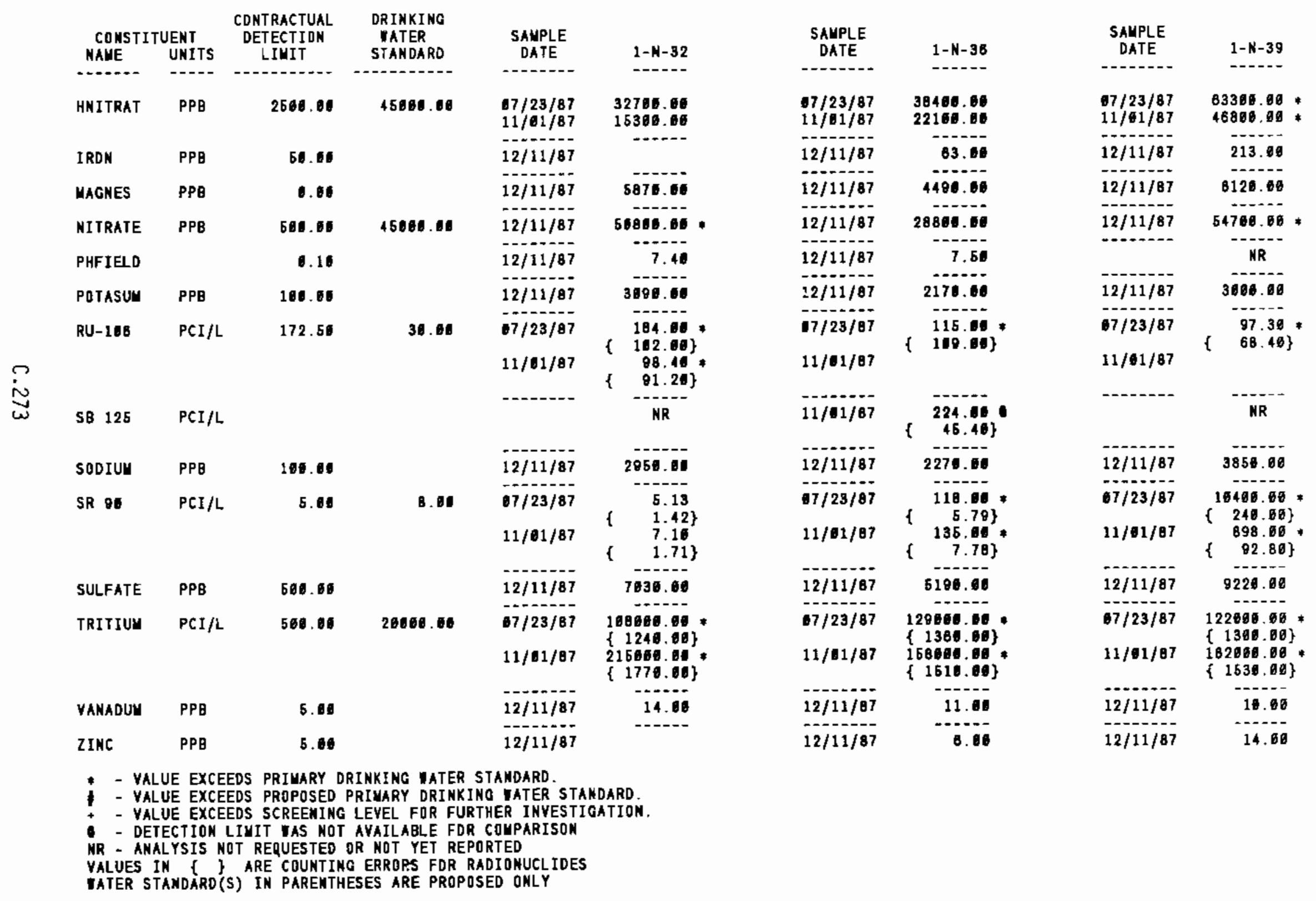




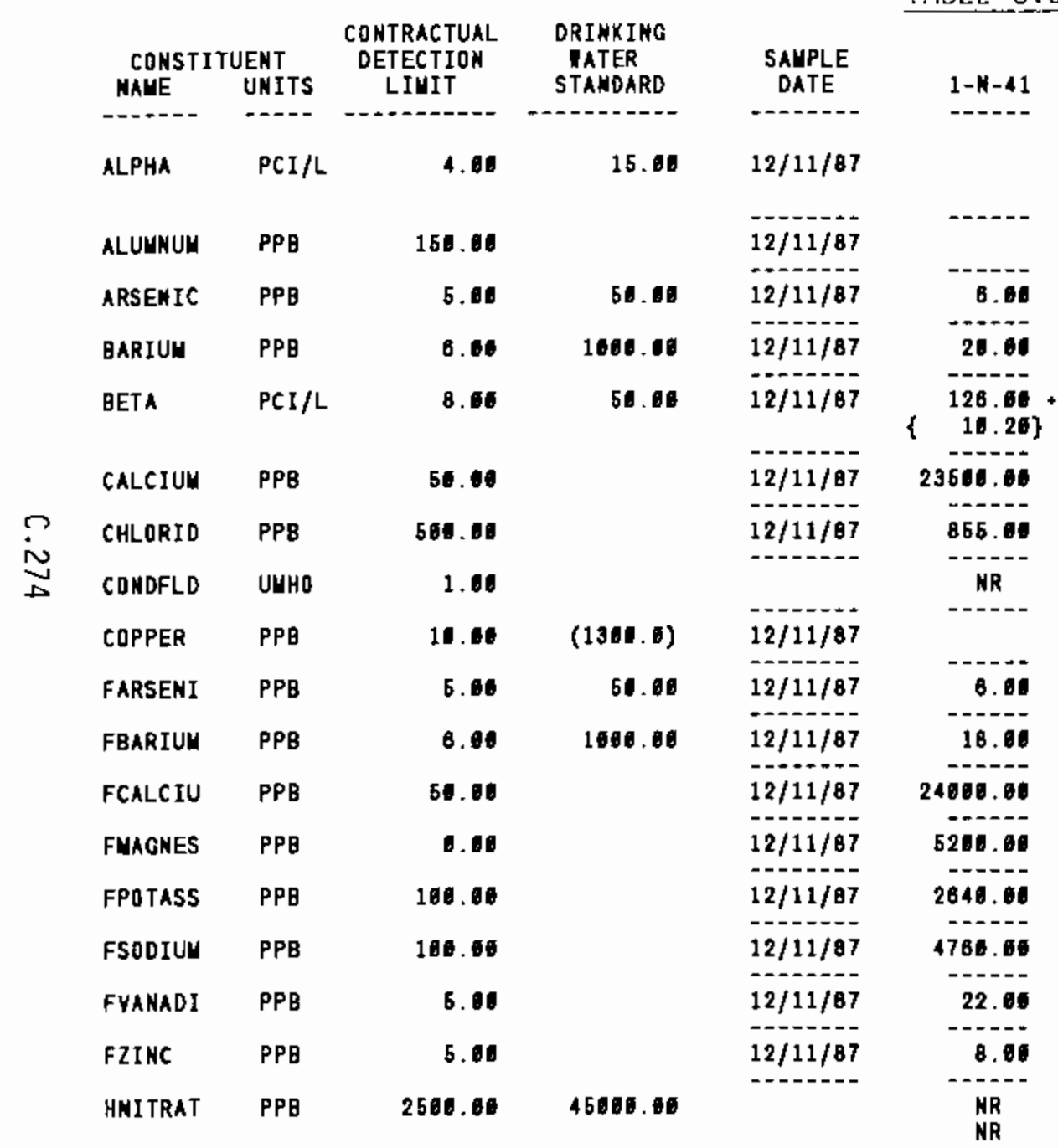

\begin{tabular}{|c|c|}
\hline $\begin{array}{l}\text { SALPLE } \\
\text { DATE }\end{array}$ & $1-N-42$ \\
\hline $12 / 14 / 87$ & \\
\hline $12 / 14 / 87$ & 276.60 \\
\hline $12 / 14 / 87$ & 8.00 \\
\hline $12 / 14 / 87$ & 23.00 \\
\hline $12 / 14 / 07$ & $\left(\begin{array}{r}127.00 \\
10.20\end{array}\right)$ \\
\hline $12 / 14 / 87$ & 25700.01 \\
\hline $12 / 14 / 87$ & 1006.10 \\
\hline $12 / 14 / 87$ & 1519.00 \\
\hline $12 / 14 / 87$ & 38.61 \\
\hline $12 / 14 / 87$ & 7.65 \\
\hline $12 / 14 / 87$ & 18.00 \\
\hline $12 / 14 / 07$ & 23460.00 \\
\hline $12 / 14 / 87$ & 6420.10 \\
\hline $12 / 14 / 87$ & 2700.01 \\
\hline $12 / 14 / 87$ & 5530.80 \\
\hline $12 / 14 / 87$ & 24.60 \\
\hline $12 / 14 / 87$ & \\
\hline & $\begin{array}{l}\text { NR } \\
\text { NR }\end{array}$ \\
\hline
\end{tabular}

\begin{tabular}{|c|c|}
\hline $\begin{array}{l}\text { SAMPLE } \\
\text { DATE }\end{array}$ & $1-11-49$ \\
\hline $12 / 14 / 87$ & $\begin{array}{l}: .85 \\
.83\}\end{array}$ \\
\hline $12 / 14 / 87$ & \\
\hline $12 / 14 / 87$ & 5.10 \\
\hline $12 / 14 / 87$ & 22.16 \\
\hline $12 / 14 / 87$ & $\left\{\begin{array}{r}134.66 \\
16.56\end{array}\right.$ \\
\hline $12 / 14 / 87$ & 32890.00 \\
\hline $12 / 14 / 87$ & 848.06 \\
\hline $12 / 14 / 87$ & 1721.00 \\
\hline $12 / 14 / 87$ & 116.11 \\
\hline $12 / 14 / 87$ & \\
\hline $12 / 14 / 87$ & 21.00 \\
\hline $12 / 14 / 87$ & 32100.06 \\
\hline $12 / 14 / 87$ & 8381.00 \\
\hline $12 / 14 / 87$ & 2880.00 \\
\hline $12 / 14 / 87$ & 3994.11 \\
\hline $12 / 14 / 87$ & 16.66 \\
\hline $12 / 14 / 07$ & 12.00 \\
\hline $\begin{array}{l}07 / 23 / 87 \\
11 / 01 / 87\end{array}$ & $\begin{array}{l}82180.08 \\
47586.08\end{array}$ \\
\hline
\end{tabular}

* - yalue exceeds priuary drinking nater standard.

- VALUE EXCEEDS PROPOSED PRIUARY DRINKING TATER STANDARD.

- VALUE EXCEEDS SCREENINg LEYEL For FURTHER INYESTIGATION.

- - detection linit uas not ayailable for couparison

NR - ANALYSIS NOT REQUESTED OR NOT YET REPORTED

VALUES IN I A ARE COUNTINO ERRORS FOR RADIONUCLIDES

IATER STANDARD(S) IN PARENTHESES ARE PROPDSED ONLY 
TABLE C.5. (contd)

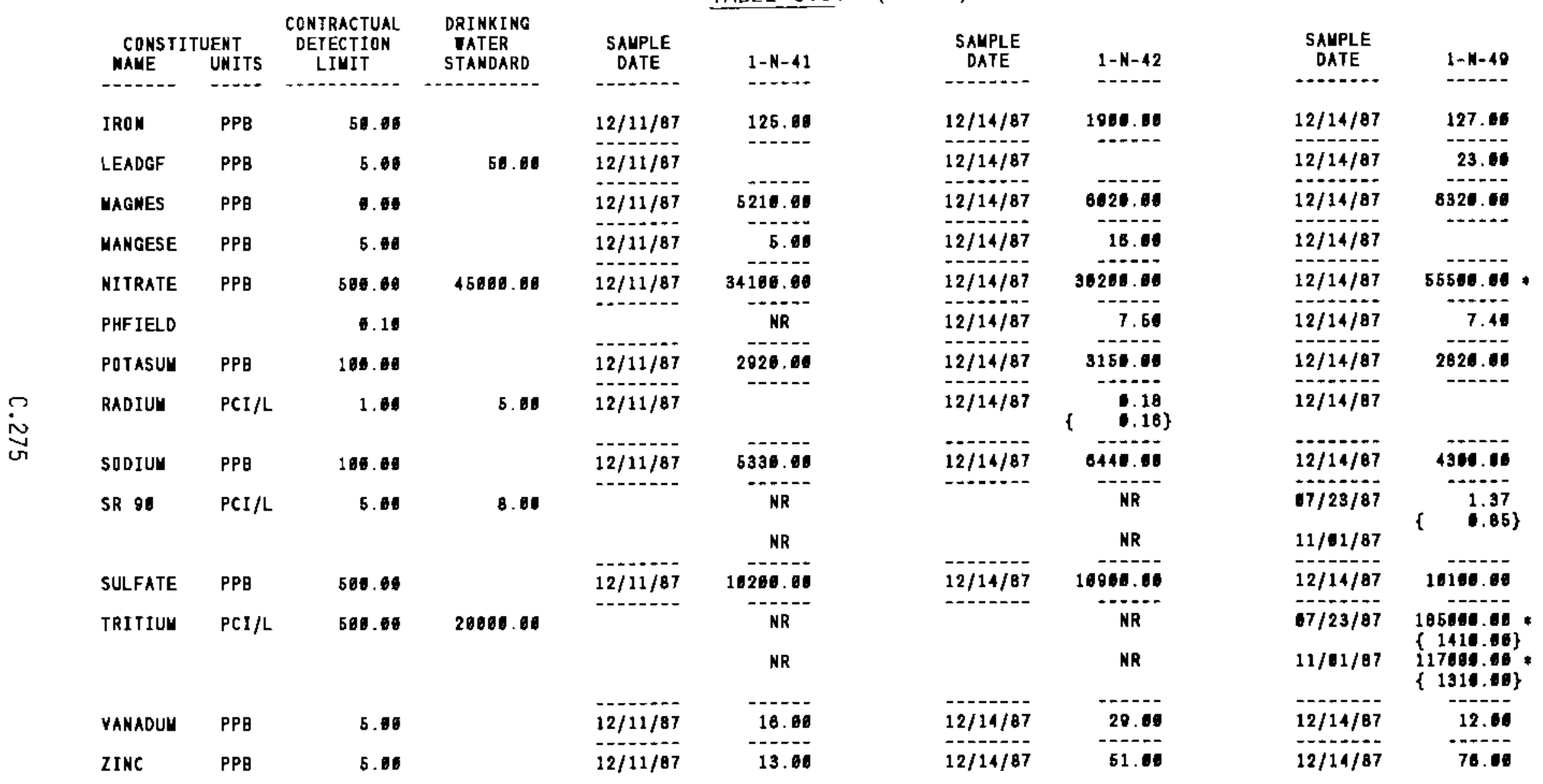

* - Value exceEds primary drinking tater standard.

- - VALUE EXCEEDS PROPOSED PRIUARY DRINKING MATER STAMDARD.

- VALUE EXCEEDS SCREENIMg LEVEL FDR FURTHER INYESTIGATION.

- DETECTION LIUIT IAS NOT AVAILABLE fOR CDUPARISON

MR - ANALYSIS NOT REQUESTED OR KOT YET REPORTED

VALUES IN \& \& ARE COUNTING ERRLRS FOR RADIONUCLIDES

IATER STANDARD(S) IN PARENTHESES ARE PRDPDSED DNLY 


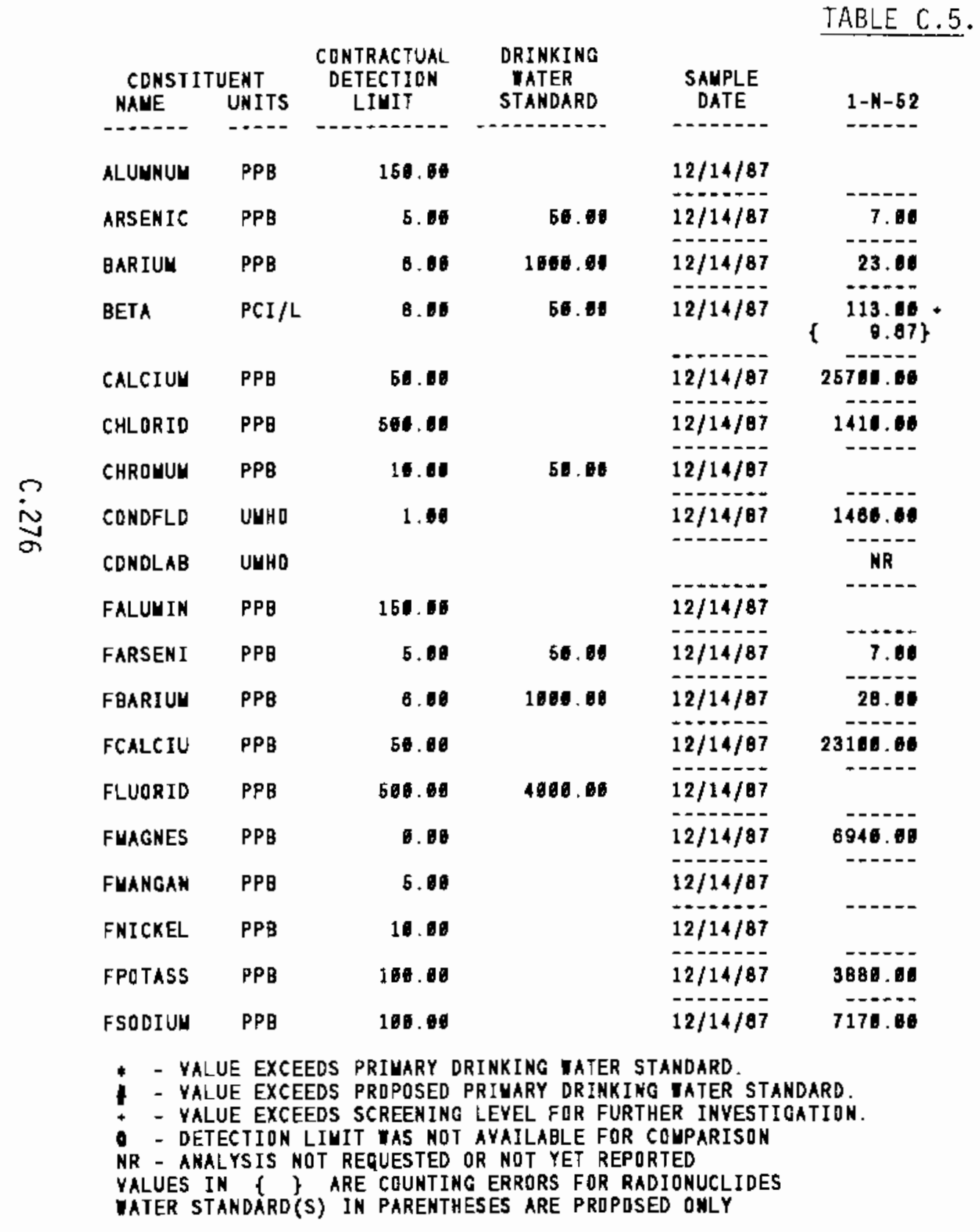

contd)

\begin{tabular}{|c|c|c|c|}
\hline $\begin{array}{c}\text { SALPLE } \\
\text { DATE }\end{array}$ & $1-N-58$ & $\begin{array}{l}\text { SAMPLE } \\
\text { DATE }\end{array}$ & $1-N-59$ \\
\hline $12 / 64 / 87$ & & $12 / 04 / 87$ & 341.60 \\
\hline $12 / 04 / 87$ & 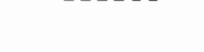 & $12 / 04 / 87$ & \\
\hline $12 / 04 / 87$ & 33.16 & $12 / 04 / 87$ & 24.00 \\
\hline $12 / 04 / 87$ & $\begin{array}{r}7.84 \\
4.58\end{array}$ & $12 / 04 / 87$ & \\
\hline $12 / 64 / 87$ & 41460.00 & $12 / 04 / 87$ & 47900.60 \\
\hline $12 / 04 / 87$ & Б780.10 & $12 / 14 / 87$ & 5656.06 \\
\hline $12 / 104 / 87$ & 14.66 & $12 / 04 / 87$ & 12.00 \\
\hline $12 / 04 / 87$ & 1476.66 & $12 / 04 / 87$ & 1623.16 \\
\hline $12 / 64 / 87$ & $148.00 \circ$ & $12 / 44 / 87$ & 151.16 \\
\hline $12 / 64 / 87$ & & $12 / 04 / 87$ & 215.10 \\
\hline $12 / 04 / 87$ & & $12 / 04 / 87$ & \\
\hline $12 / 04 / 87$ & 28.10 & $12 / 04 / 87$ & 23.00 \\
\hline $12 / 04 / 87$ & 43580.00 & $12 / 04 / 87$ & 48160.18 \\
\hline $12 / 04 / 87$ & 1398.60 & $12 / 04 / 87$ & 1520.06 \\
\hline $12 / 04 / 87$ & 8058.06 & $12 / 64 / 87$ & 10904.86 \\
\hline $12 / 64 / 87$ & & $12 / 64 / 87$ & 189.10 \\
\hline $12 / 64 / 87$ & & $12 / 64 / 87$ & 10.00 \\
\hline $12 / 04 / 87$ & 3210.00 & $12 / 84 / 87$ & 3586.60 \\
\hline $12 / 04 / 87$ & 301000.00 & $12 / 84 / 87$ & 298006.08 \\
\hline
\end{tabular}


TABLE C.5. (contd)

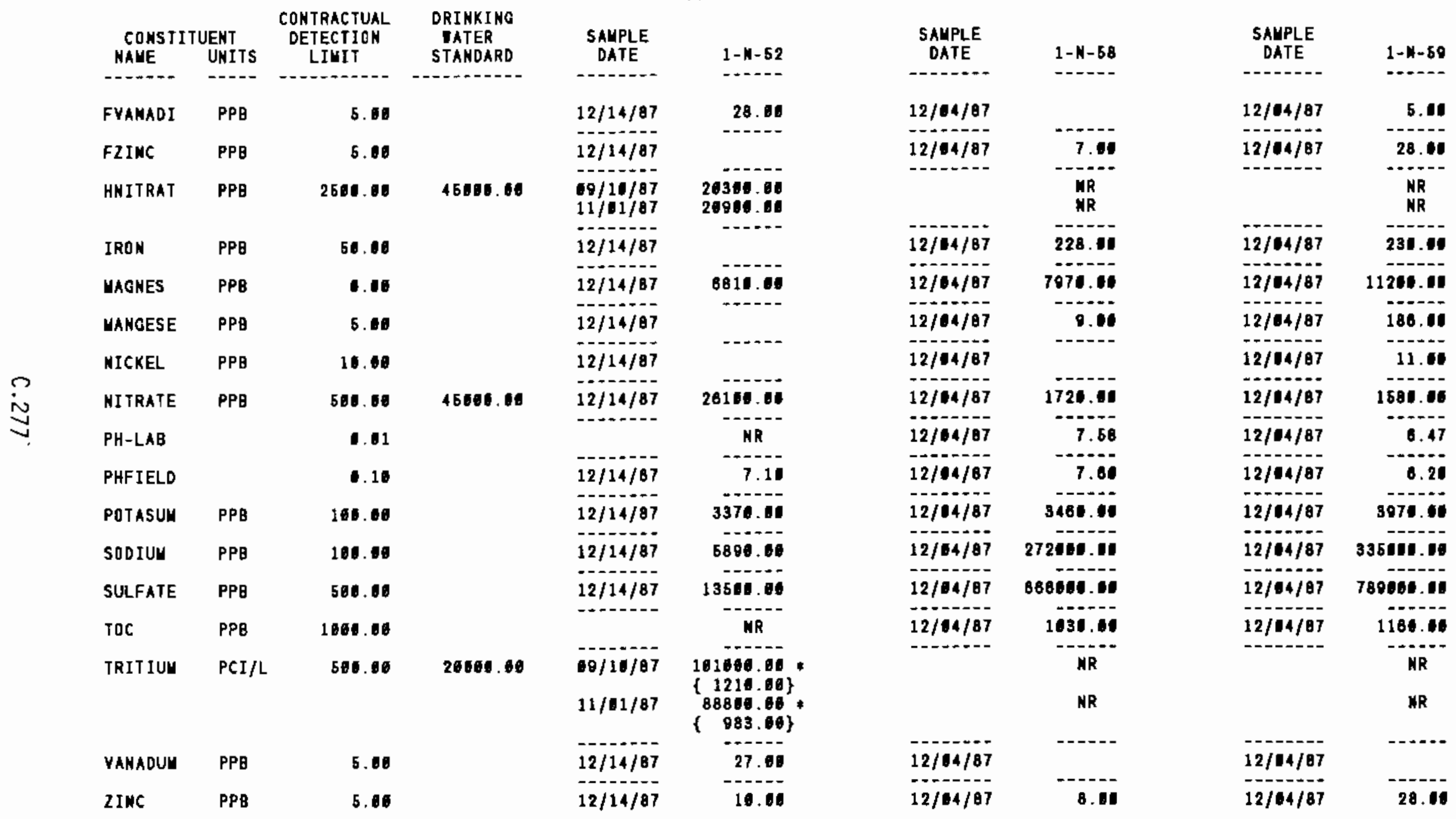

- - yalue exceeds primary drinking tajer standard.

- VALUE EXCEEDS PROPOSED PRIUARY ORINKIMG IATER STAMDARD.

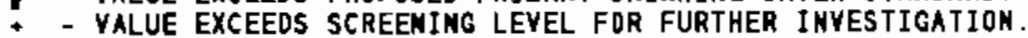

- DETECTION LIUIT TAS NOT AYAILABLE for COMPARISON

NR - ANALYSIS NOT REQUESTED OR NOT YET REPORTED

VALUES IN \{ $\}$ ARE COUNTING ERRORS FOR RADIONUCLIDES

WATER STANDARO(S) IN PARENTHESES ARE PROPOSED ONLY 
TABLE C.5. (contd)

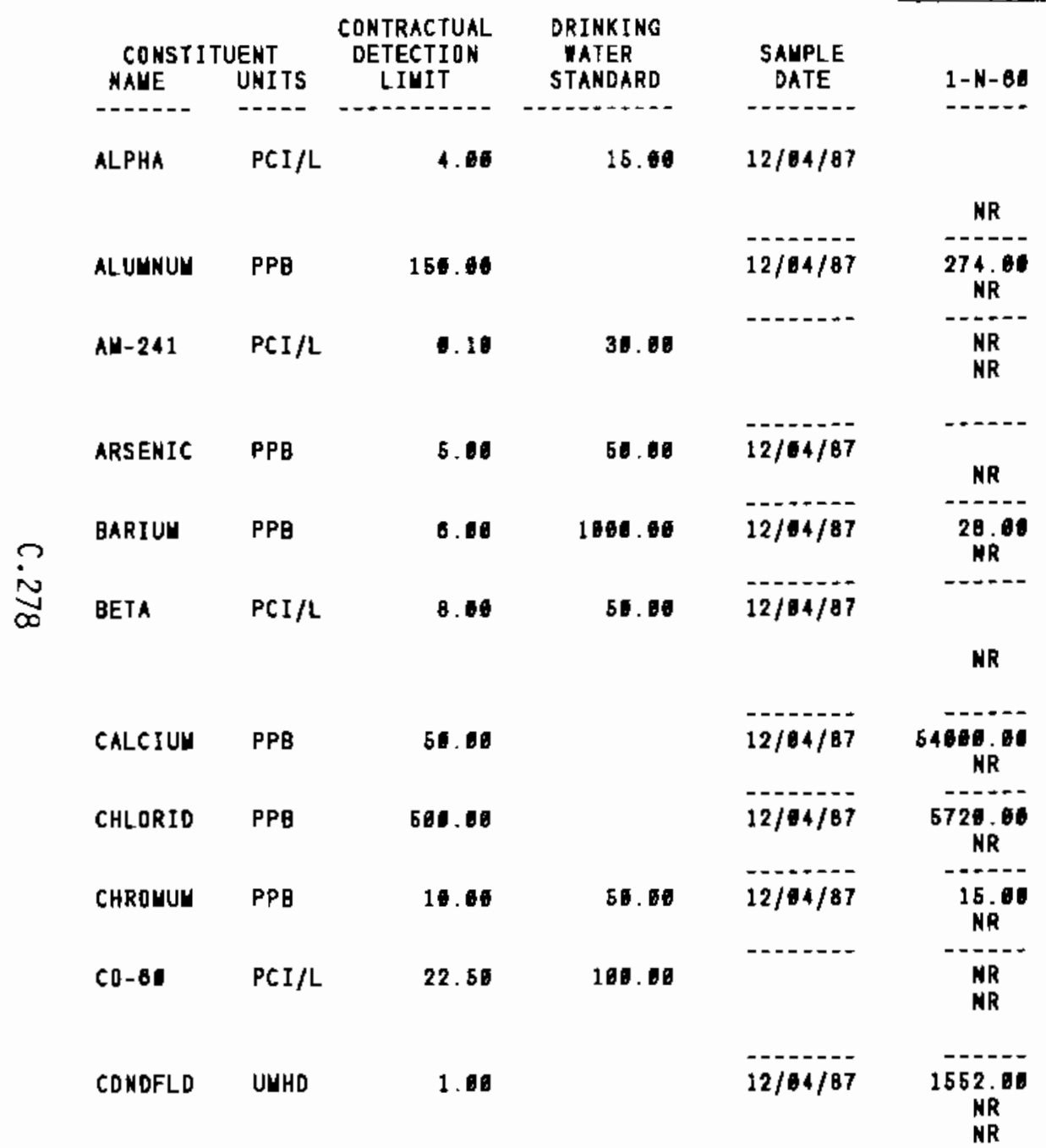

\begin{tabular}{|c|c|}
\hline $\begin{array}{c}\text { SAMPLE } \\
\text { DATE }\end{array}$ & $1-N-61$ \\
\hline 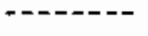 & 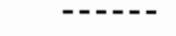 \\
\hline \multicolumn{2}{|l|}{$12 / 04 / 87$} \\
\hline & NR \\
\hline \multirow{2}{*}{$12 / 14 / 87$} & \\
\hline & NR \\
\hline \multirow[t]{2}{*}{ 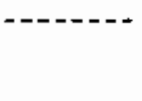 } & 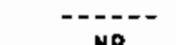 \\
\hline & $\begin{array}{l}\text { NR } \\
\text { NR }\end{array}$ \\
\hline 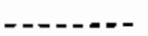 & ---- \\
\hline $12 / 64 / 87$ & \\
\hline - - - - & \\
\hline $12 / 04 / 87$ & WR \\
\hline \multirow{2}{*}{$12 / 44 / 87$} & 8.62 \\
\hline & $\begin{array}{l}\text { E.02 } \\
\text { MR }\end{array}$ \\
\hline 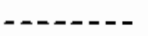 & ----- \\
\hline $12 / 64 / 87$ & ${ }_{\mathrm{NR}}^{01800.01}$ \\
\hline 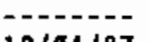 & $-\overline{c-1}$ \\
\hline $12 / 64 / 87$ & $\begin{array}{c}3684.11 \\
\text { NR }\end{array}$ \\
\hline inialor & $\overline{0}$ \\
\hline $12 / 64 / 87$ & ${ }_{N R}^{28.80}$ \\
\hline --n--- & ---1-- \\
\hline & $\begin{array}{l}\text { NR } \\
\text { NR }\end{array}$ \\
\hline …-1- & ----- \\
\hline $12 / 64 / 67$ & $\begin{array}{c}1502.00 \\
\text { NR } \\
\text { NR }\end{array}$ \\
\hline
\end{tabular}

\begin{tabular}{|c|c|}
\hline $\begin{array}{c}\text { SAHPLE } \\
\text { DATE }\end{array}$ & 2-E25-25 \\
\hline -ー---ー- & \\
\hline $07 / 01 / 87$ & $\begin{array}{l}1.04 \\
1.08\end{array}$ \\
\hline $69 / 36 / 87$ & \\
\hline $\begin{array}{l}07 / 01 / 87 \\
69 / 36 / 87\end{array}$ & --- \\
\hline$---1-2$ & n \\
\hline $\begin{array}{l}67 / 61 / 87 \\
69 / 30 / 87\end{array}$ & $\begin{array}{r}6.62 \\
.62\}\end{array}$ \\
\hline $\begin{array}{l}07 / 61 / 87 \\
09 / 30 / 87\end{array}$ & 5.00 \\
\hline $\begin{array}{l}07 / 81 / 87 \\
09 / 36 / 87\end{array}$ & $\begin{array}{l}19.10 \\
19.11\end{array}$ \\
\hline $1 / 1 / 87$ & 5.54 \\
\hline $69 / 34 / 07$ & $\begin{array}{l}\left\{\begin{array}{l}3.41 \\
\{\end{array}\right\} \\
\{\quad 3.67 \\
\{.63\}\end{array}$ \\
\hline $\begin{array}{l}07 / 61 / 67 \\
69 / 30 / 87\end{array}$ & 17590.00 \\
\hline------ & - \\
\hline $\begin{array}{l}07 / 1 / 87 \\
60 / 36 / 87\end{array}$ & $\begin{array}{l}3840.10 \\
2586.10\end{array}$ \\
\hline $\begin{array}{l}67 / 01 / 87 \\
69 / 36 / 87\end{array}$ & \\
\hline $67 / 01 / 07$ & $--1 . . .$. \\
\hline $69 / 30 / 67$ & $\begin{array}{l}8.07 \\
5.43\}\end{array}$ \\
\hline $\begin{array}{l}07 / 01 / 87 \\
07 / 01 / 87 \\
07 / 01 / 87\end{array}$ & $\begin{array}{l}136.40 \\
196.46 \\
135.46\end{array}$ \\
\hline
\end{tabular}

* VALUE EXCEeds PRIMARY dRINKING vater STANDARD.

- VALUE EXCEEDS PROPOSED PRIUARY DRINXIHG MaTER STAMDaRd.

- VALUE EXCEEDS SCREENING LEVEL FOR FURTHER INVESTIGATIBN.

- - DETECTION LIUIT IAS NOT AVAILABLE FOR COMPARISON

NR - ANALYSIS MOT REQUESTED OR NOT YET REPORTED

VALUES IN \{ $\}$ ARE COUNTING ERRORS FOR RADIONUCLIOES

TATER STANDARD(S) IN PARENTHESES ARE PRDPOSED ONLY 
TABLE C.5. (contd

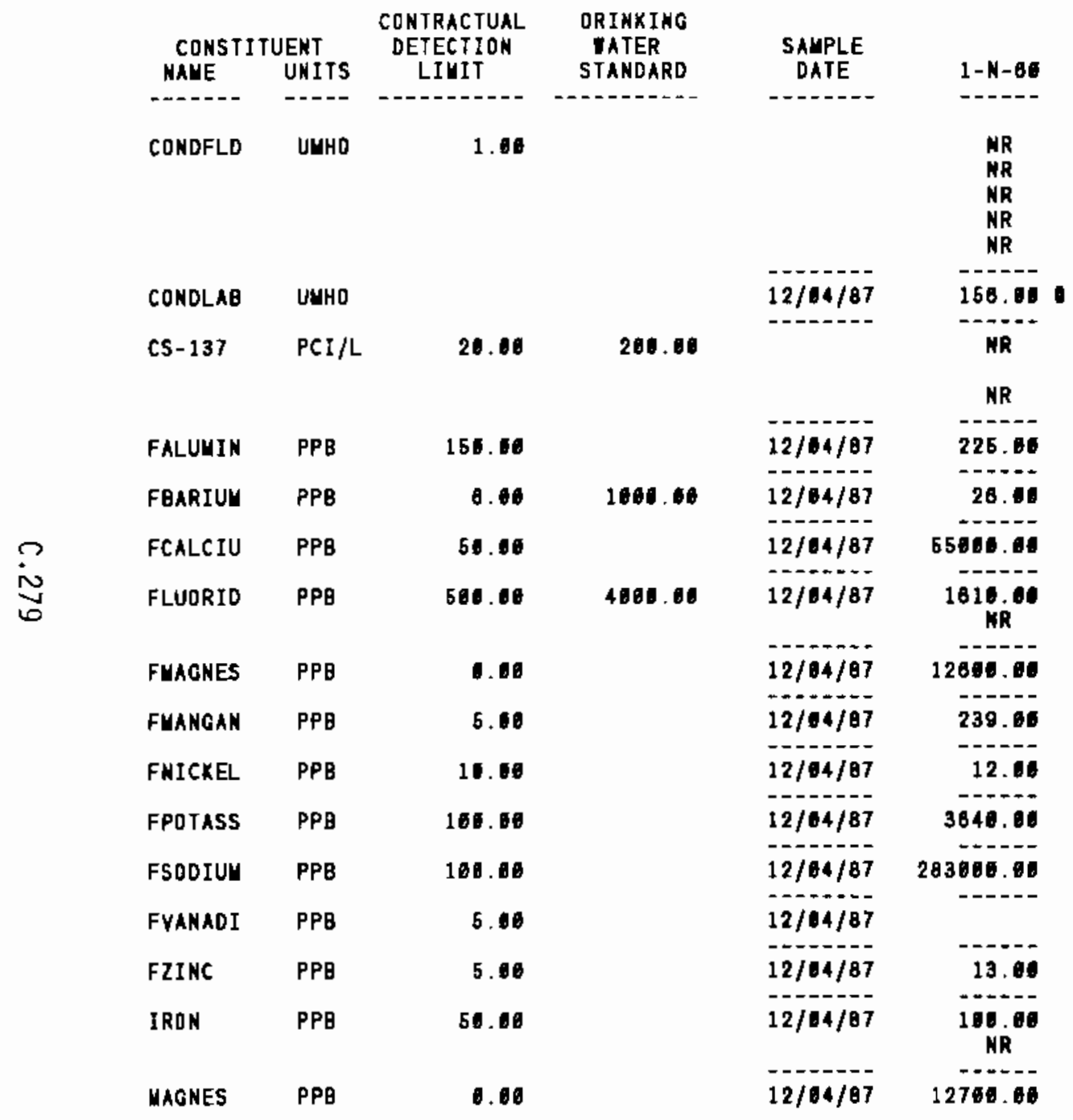

\begin{tabular}{|c|c|}
\hline \multirow[t]{2}{*}{$\begin{array}{c}\text { SAUPLE } \\
\text { OATE }\end{array}$} & $1-N-B 1$ \\
\hline & $\begin{array}{l}\text { MR } \\
\text { NR } \\
\text { NR } \\
\text { NR } \\
\text { NR }\end{array}$ \\
\hline \multirow[t]{3}{*}{$12 / 04 / 87$} & 141.00 \\
\hline & RR \\
\hline & NR \\
\hline $12 / 04 / 87$ & \\
\hline $12 / 14 / 87$ & 58.10 \\
\hline $12 / 04 / 87$ & 95300.00 \\
\hline $12 / 04 / 87$ & 1476.00 \\
\hline $12 / 04 / 87$ & 18200.60 \\
\hline $12 / 04 / 87$ & 127.60 \\
\hline $12 / 104 / 87$ & 10.00 \\
\hline $12 / 04 / 87$ & 2970.16 \\
\hline $12 / 04 / 07$ & 292000.00 \\
\hline $12 / 04 / 87$ & \\
\hline $12 / 04 / 87$ & 18.01 \\
\hline $12 / 04 / 87$ & ${ }_{\mathrm{NR}}^{162.00}$ \\
\hline $12 / 04 / 87$ & 18600.08 \\
\hline
\end{tabular}

\begin{tabular}{|c|c|}
\hline $\begin{array}{l}\text { SAMPLE } \\
\text { DATEE }\end{array}$ & $2-E 25-25$ \\
\hline \multirow[t]{2}{*}{$\begin{array}{l}67 / 91 / 87 \\
69 / 30 / 87 \\
69 / 30 / 87 \\
69 / 30 / 87 \\
69 / 30 / 87 \\
\ldots . . . .\end{array}$} & $\begin{array}{l}136.00 \\
151.00 \\
151.00 \\
151.00 \\
151.10\end{array}$ \\
\hline & NR \\
\hline $07 / 01 / 87$ & $\begin{array}{r}7.86 \\
5 \quad 8.93\end{array}$ \\
\hline $69 / 30 / 87$ & \\
\hline $67 / 01 / 87$ & \\
\hline $67 / 01 / 87$ & 18.00 \\
\hline $7 / 61 / 87$ & 18200.00 \\
\hline $07 / 91 / 67$ & \\
\hline $07 / 1 / 87$ & 6490.06 \\
\hline $07 / 01 / 87$ & \\
\hline $07 / 11 / 87$ & \\
\hline $67 / 1 / 1 / 87$ & 3010.6 \\
\hline $67 / 61 / 87$ & 7660.08 \\
\hline $67 / 01 / 87$ & 10.00 \\
\hline $107 / 1 / 87$ & 8.11 \\
\hline $\begin{array}{l}67 / 91 / 97 \\
69 / 36 / 87\end{array}$ & $\begin{array}{l}293.18 \\
249.10\end{array}$ \\
\hline $07 / 81 / 87$ & 5316.06 \\
\hline
\end{tabular}

- - value exceeds priyary drinking vater standard

1 - YALUE EXCEEDS PROPOSED PRIMARY DRTNKING IaTER STANDARD.

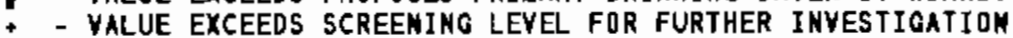

- - detection linit ias not ayailagle for couparison

NR - ANALYSIS NOT RERUESTED OR NOT YET REPURTED

VALUES IN \{\} ARE COUNTING ERRORS FOR RADIONUCLIDES

IATER STANDARD(S) IN PARENTHESES ARE PROPOSED ONLY 
TABLE C.5. (contd)

\begin{tabular}{|c|c|c|c|c|c|}
\hline $\begin{array}{l}\text { CONSTI } \\
\text { NAUE }\end{array}$ & $\begin{array}{l}\text { TUENT } \\
\text { UNITS }\end{array}$ & $\begin{array}{c}\text { CONTRACTUAL } \\
\text { DETECTION } \\
\text { LIHIT }\end{array}$ & $\begin{array}{l}\text { DRINKING } \\
\text { IATER } \\
\text { STANDARD }\end{array}$ & $\begin{array}{l}\text { SAUPLE } \\
\text { DATE }\end{array}$ & $1-N-60$ \\
\hline----- & ---- & $-\cdots+-1$ & $---1-1-\cdots$ & $\ldots-n$ & ------ \\
\hline YAGNES & PPB & 0.00 & & & NR \\
\hline WAMGESE & PPB & 6.00 & & $12 / 04 / 87$ & $\begin{array}{c}246.06 \\
\mathrm{NR}\end{array}$ \\
\hline NICKEL & PPB & 10.00 & & $12 / 64 / 87$ & 15.60 \\
\hline NITRATE & PPB & 500.68 & 45809.00 & $12 / 04 / 87$ & 1590.00 \\
\hline$P H=L A B$ & & 6. 1 & & $12 / 04 / B 7$ & 6.27 \\
\hline PHFIELD & & 1.10 & & $12 / 04 / 87$ & $\begin{array}{l}6.10 \\
\text { MR } \\
\text { MR } \\
\text { WR } \\
\text { MR } \\
\text { WR } \\
\text { NR } \\
\text { NR }\end{array}$ \\
\hline POTASUL & PPB & 168.60 & & $12 / 64 / 87$ & 4050.00 \\
\hline RADIUU & $\mathrm{PCl} / \mathrm{L}$ & 1.00 & 5.00 & $12 / 64 / 87$ & $\left\{\begin{array}{l}0.18 \\
\text { NR }\end{array}\right.$ \\
\hline SODIUV & PPB & 160.00 & & $12 / 64 / 87$ & $\begin{array}{c}320000.00 \\
N R\end{array}$ \\
\hline SULFATE & PPB & 500.00 & & $12 / 64 / 87$ & $\begin{array}{c}\text { 819000.00 } \\
\text { NR }\end{array}$ \\
\hline TC & PPB & & & & NR \\
\hline TDS & & & & & NR \\
\hline
\end{tabular}

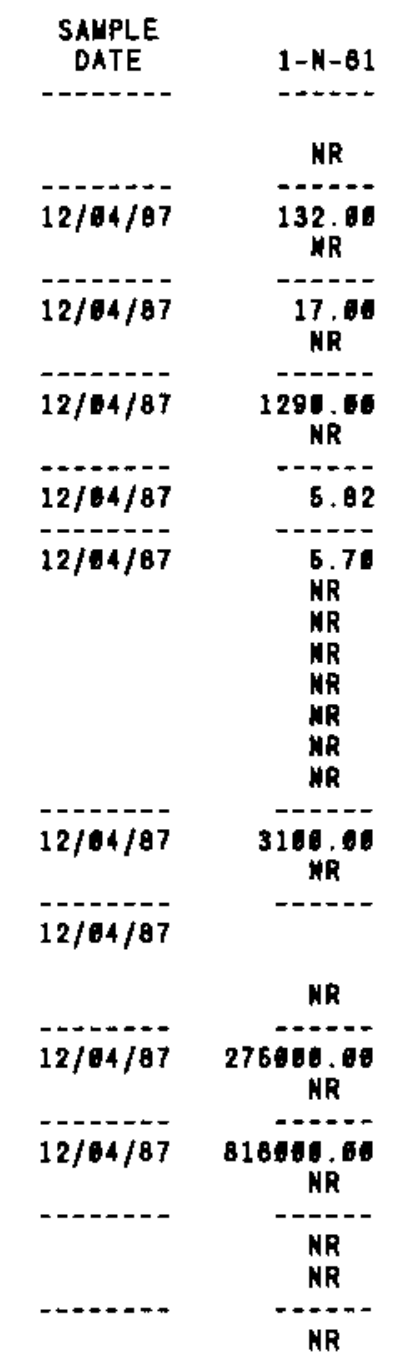

\begin{tabular}{|c|c|}
\hline $\begin{array}{l}\text { SALPLE } \\
\text { DATE }\end{array}$ & 2-E25-25 \\
\hline - & -ーー-ー- \\
\hline $09 / 30 / 87$ & 4780.00 \\
\hline $\begin{array}{l}67 / 61 / 87 \\
69 / 30 / 87\end{array}$ & \\
\hline $\begin{array}{l}07 / 61 / 87 \\
00 / 30 / 87\end{array}$ & ------ \\
\hline $\begin{array}{l}1 / 1 / 87 \\
19 / 31 / 87\end{array}$ & $\begin{array}{r}1085.65 \\
785.64\end{array}$ \\
\hline 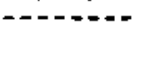 & MR \\
\hline $\begin{array}{l}07 / 01 / 87 \\
07 / 01 / 87 \\
07 / 61 / 87 \\
07 / 01 / 87 \\
9 / 36 / 87 \\
90 / 30 / 87 \\
09 / 36 / 87 \\
90 / 30 / 87\end{array}$ & $\begin{array}{r}7.26 \\
7.36 \\
7.46 \\
7.56 \\
7.06 \\
7.96 \\
7.86 \\
7.86\end{array}$ \\
\hline $\begin{array}{l}97 / 01 / 87 \\
0 / 30 / 87\end{array}$ & $\begin{array}{l}3856.06 \\
4396.60\end{array}$ \\
\hline $07 / 01 / 87$ & \\
\hline $19 / 30 / 87$ & \\
\hline $\begin{array}{l}07 / 01 / 87 \\
98 / 30 / 87\end{array}$ & $\begin{array}{l}7005.00 \\
8090.00\end{array}$ \\
\hline $\begin{array}{l}07 / 01 / 87 \\
69 / 36 / 87\end{array}$ & $\begin{array}{l}14700.04 \\
12204.04\end{array}$ \\
\hline $\begin{array}{l}07 / 61 / 87 \\
09 / 36 / 87\end{array}$ & $\begin{array}{l}\text { 16849.40 } \\
16204.10\end{array}$ \\
\hline $07 / 01 / 87$ & 17500.06 \\
\hline
\end{tabular}

* - Value exceeds priuary dRINKINg tater stahdard.

- - VALUE EXCEEDS PROPOSED PRIUARY DRINKING VATER STANDARD

- - YALUE EXCEEDS SCREENING LEVEL FOR FURTHER INYESTIGATION.

- DETECTIDN LIUIT UAS NOT AVALABLE FOR COUPARISDN

NR - ANALYSIS NOT REQUESTED OR NOT YET REPORTED

VALUES IN \{ $\}$ ARE COUNTING ERRORS FOR RADIONUCLIDES

WATER STANDARD(S) IN PARENTHESES ARE PROPOSED ONLY 
TABLE C.5. (contd)

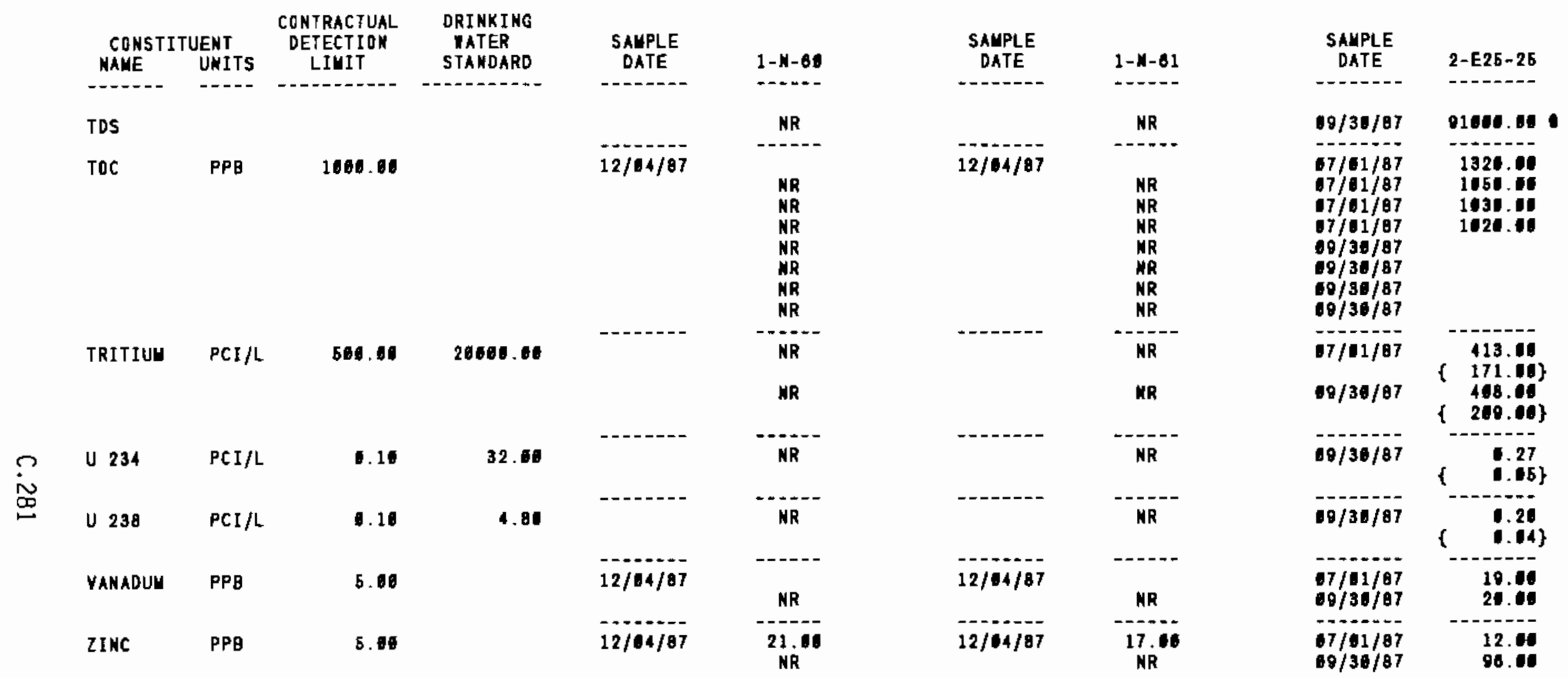

* - Value exceeds priuary drinking tater standaro.

\# - VALUE EXCEEDS PROPOSED PRIUARY DRINKING TATER STANDARD

* - YALUE EXCEEDS SCREENING LEVEL FOR FURTHER INYESTIGATION.

- DETECTION LIUIT AAS NOT AVAILABLE FOR COUPARISON

NR - ANALYSIS NDT REQUESTED OR NOT YET REPORTED

YALUES IN \{ $\}$ ARE COUNTING ERRDRS FOR RADIONUCLIDES

IATER STANDARD(S) IN PARENTHESES ARE PRDPOSED ONLY 
TABLE C.5. (contd)

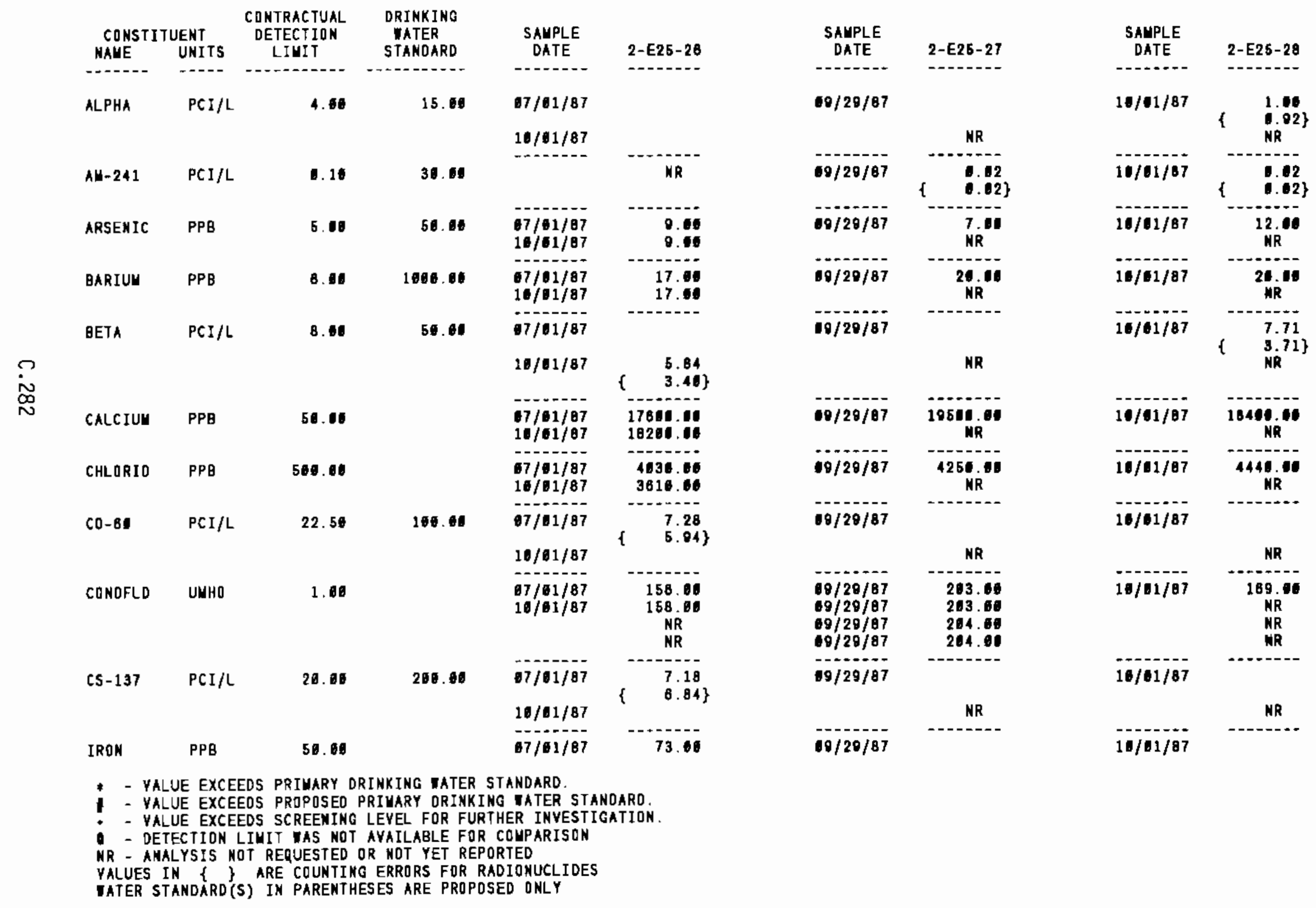


TABLE C.5. (contd)

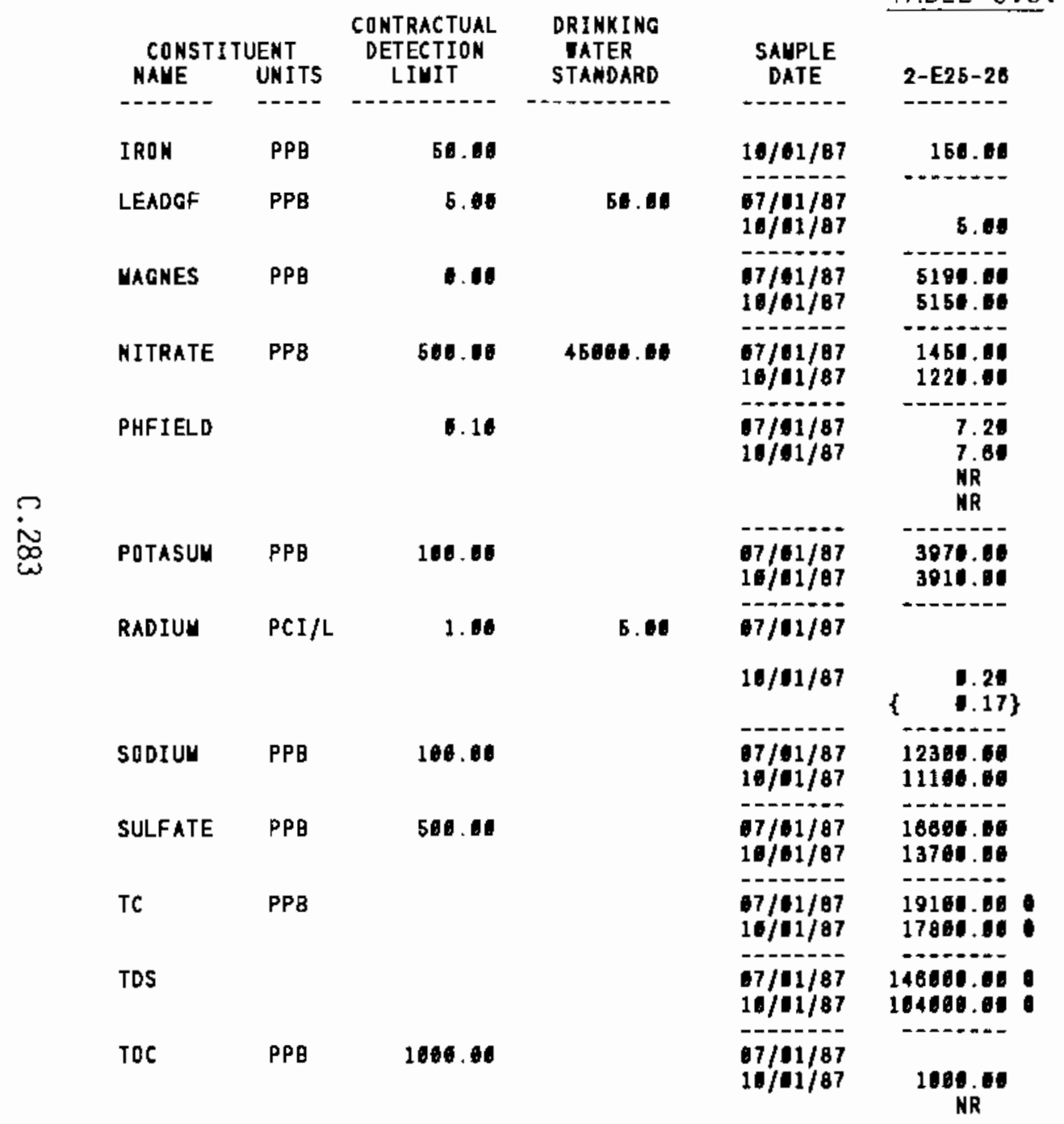

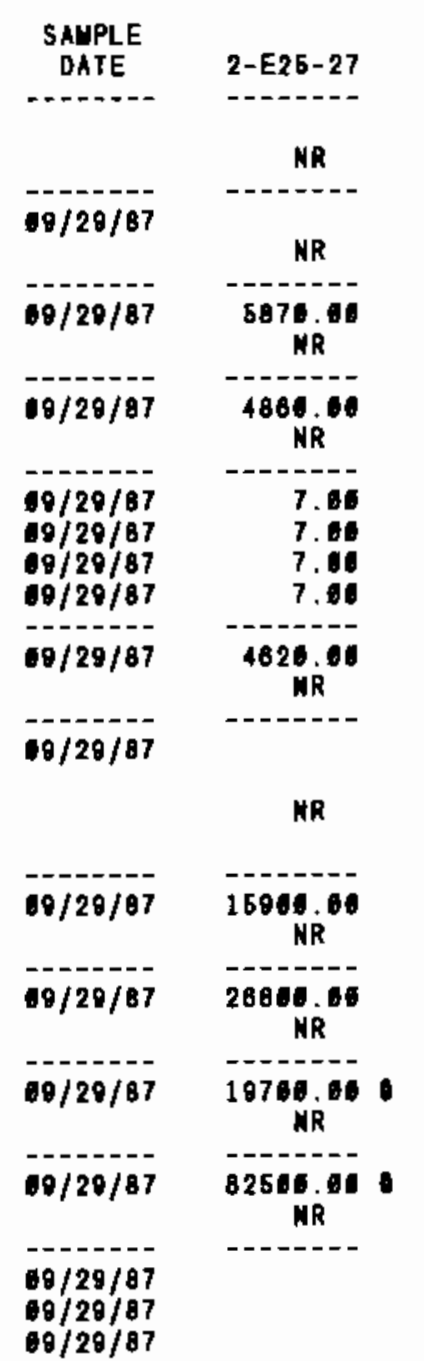

\begin{tabular}{|c|c|}
\hline $\begin{array}{l}\text { SANPLE } \\
\text { DATE }\end{array}$ & $2-E 25-28$ \\
\hline & $-\cdots-c-+\infty$ \\
\hline & NR \\
\hline \multirow{2}{*}{$10 / 01 / 87$} & \\
\hline & HR \\
\hline 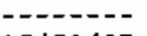 & 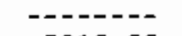 \\
\hline $10 / 01 / 87$ & NA \\
\hline 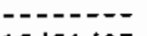 & ------- \\
\hline $16 / 61 / 87$ & 1120.00 \\
\hline 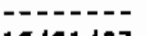 & $---\overline{7}$ \\
\hline $10 / 81 / 87$ & $\begin{array}{l}7.60 \\
\text { MR } \\
\text { NR } \\
\text { MR }\end{array}$ \\
\hline $10 / 61 / 87$ & 3940.61 \\
\hline & NR \\
\hline $10 / 01 / 87$ & 0.18 \\
\hline & $\left\{\mathrm{NR}^{15\}}\right.$ \\
\hline --ー---- & -----U- \\
\hline $10 / 01 / 87$ & $\frac{\mathrm{NR}}{12308.60}$ \\
\hline - & 13719 \\
\hline $10 / 01 / 81$ & NR \\
\hline $16 / 61 / 87$ & 10000.00 \\
\hline 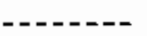 & $--\cdots$ \\
\hline $10 / 61 / B 7$ & 114000.00 \\
\hline $16 / 61 / 87$ & \\
\hline & $\begin{array}{l}\text { NR } \\
\text { NR }\end{array}$ \\
\hline
\end{tabular}

- - value exceEds primary dRinking tater standard

- - VALUE EXCEEDS PROPOSED PRIMARY DRINKING TATER STAMDARD.

* - YALUE EXCEEDS SCREENING LEVEL FOR FURTHER INYESTIGATION.

- - detection liUit tas not aVAilable for colnparison

NR - ANALYSIS NDT REQUESTED OR NDT YET REPDRTED

VALUES IN \{ $\}$ ARE COUNTING ERRORS FOR RADIONUCLIDES

IATER STANDARD(S) IN PARENTHESES ARE PROPDSED ONLY 
TABLE C.5. (contd)

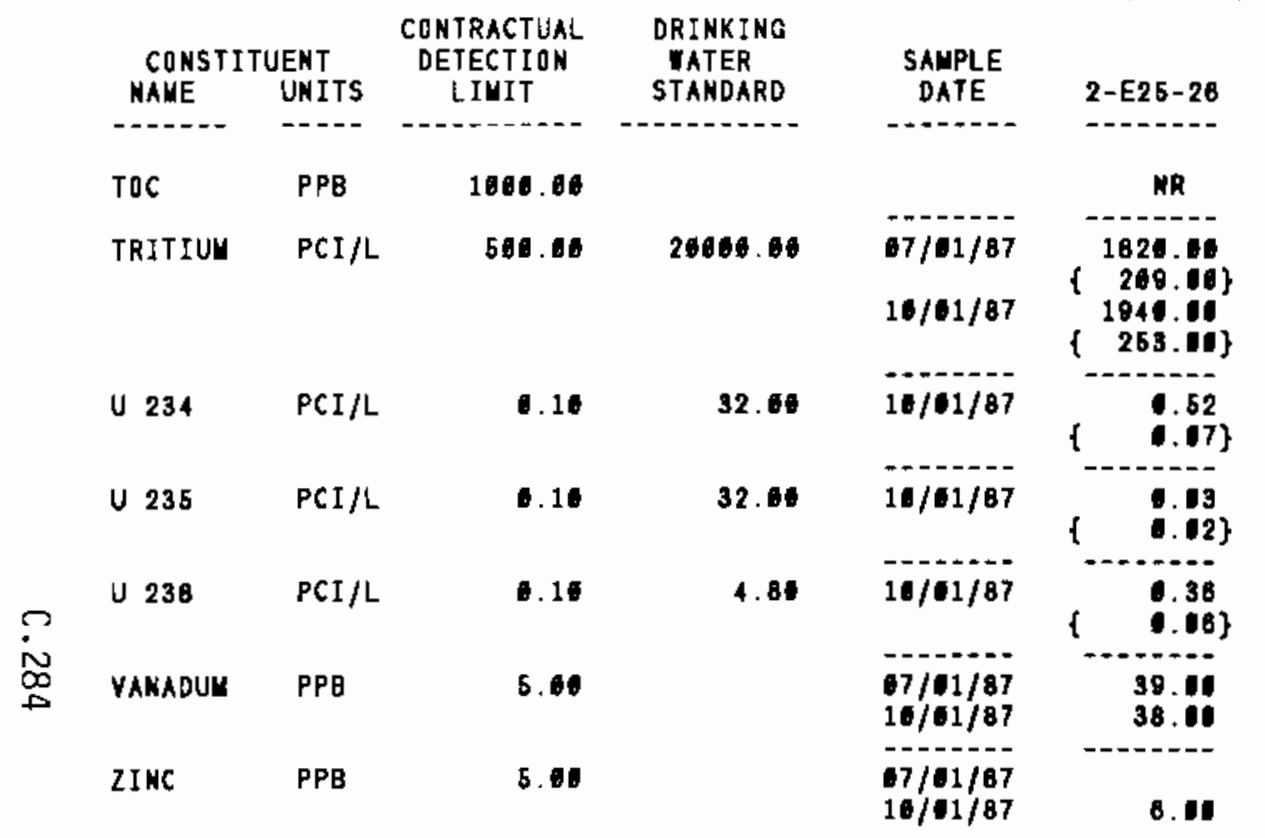

\begin{tabular}{|c|c|}
\hline $\begin{array}{c}\text { SAUPLE } \\
\text { DATE }\end{array}$ & 2-E25-27 \\
\hline $09 / 29 / 87$ & \\
\hline $19 / 29 / 87$ & $\left\{\begin{array}{c}98715.18 \\
415.08 \\
N R\end{array}\right\}$ \\
\hline $90 / 20 / 87$ & $(\quad: .58)$ \\
\hline $09 / 29 / 87$ & \\
\hline $08 / 29 / 87$ & $\begin{array}{l}: .51 \\
0.07\}\end{array}$ \\
\hline $80 / 29 / 87$ & ${ }_{\mathrm{NR}}^{36.11}$ \\
\hline $08 / 28 / 87$ & NR \\
\hline
\end{tabular}

\begin{tabular}{|c|c|}
\hline \multirow[t]{2}{*}{$\begin{array}{l}\text { SALPLE } \\
\text { DATE }\end{array}$} & $2-E 25-28$ \\
\hline & NR \\
\hline $10 / 61 / 87$ & $\left\{\begin{array}{c}1770.61 \\
250.60 \\
N R\end{array}\right\}$ \\
\hline $10 / 61 / 87$ & $\begin{array}{r}1.52 \\
1.07\}\end{array}$ \\
\hline $10 / 61 / 87$ & $\begin{array}{l}1.03 \\
\{\quad 1.2\}\end{array}$ \\
\hline $10 / 01 / 87$ & $\begin{array}{l}\{.43 \\
\{\quad .06\}\end{array}$ \\
\hline $10 / 61 / 87$ & $\begin{array}{c}46.06 \\
\text { NR }\end{array}$ \\
\hline $16 / 1 / 87$ & NR. 10 \\
\hline
\end{tabular}

- - value exceeds priuary drinking mater standard.

- - VALUE EXCEEDS PROPOSED PRIHARY DRINKING WATER STANDARD.

+ - YALUE EXCEEDS SCREENIHG LEVEL FOR FURTHER INYESTIGATION.

- DETECTION LIMIT IAS NDT AYAILABLE FOR COUPARISOM

NR - AMALYSIS NOT REQUESTED OR NOT YET REPORTED

VALUES IN $\{$ \& ARE COUNTIHG ERRORS FOR RADIONUCLIDES

TATER STANDARD(S) IN PARENTHESES ARE PROPOSED DNLY 
TABLE C.5. (contd)

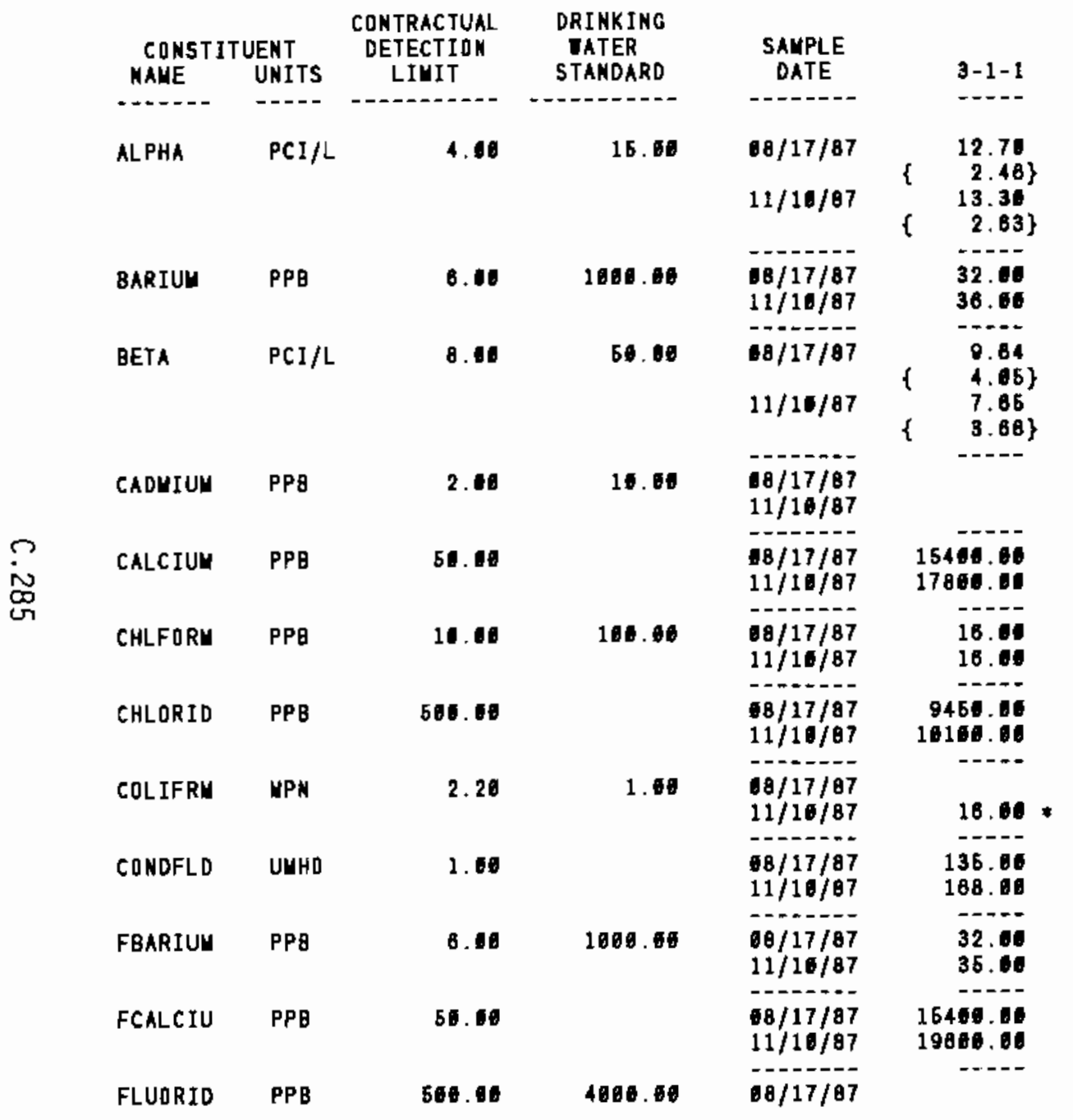

\begin{tabular}{|c|c|}
\hline $\begin{array}{l}\text { SAUPLE } \\
\text { DATE }\end{array}$ & $3-1-2$ \\
\hline מ- & $-\cdots$ \\
\hline $18 / 14 / 87$ & $\begin{array}{l}0.92 \\
2.263\end{array}$ \\
\hline $11 / 09 / 87$ & $\begin{array}{r}12.68 \\
2.58\}\end{array}$ \\
\hline 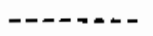 & $-\cdots$ \\
\hline $\begin{array}{l}08 / 14 / 87 \\
11 / 18 / 87\end{array}$ & $\begin{array}{l}22.00 \\
27.16\end{array}$ \\
\hline 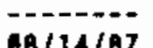 & 1238 \\
\hline $68 / 14 / 87$ & $\begin{array}{r}12.30 \\
4.05\}\end{array}$ \\
\hline $11 / 68 / 87$ & $\begin{array}{r}8.37 \\
\{\quad 3.45\}\end{array}$ \\
\hline 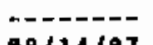 & --- \\
\hline $\begin{array}{l}88 / 14 / 87 \\
11 / 09 / 87\end{array}$ & 2.00 \\
\hline $\begin{array}{l}98 / 14 / 87 \\
11 / 68 / 87\end{array}$ & $\begin{array}{l}19700 \\
21000\end{array}$ \\
\hline $\begin{array}{l}68 / 14 / 07 \\
11 / 00 / 07\end{array}$ & $\begin{array}{l}15.00 \\
11.00\end{array}$ \\
\hline $\begin{array}{l}18 / 14 / 87 \\
11 / 89 / 87\end{array}$ & $\begin{array}{l}8650.05 \\
8540.06\end{array}$ \\
\hline $88 / 14 / 07$ & -... \\
\hline $11 / 00 / 87$ & 0.20 \\
\hline $\begin{array}{l}08 / 14 / 87 \\
11 / 60 / 87\end{array}$ & $\begin{array}{l}177.08 \\
198.00\end{array}$ \\
\hline$-1-1$ & $-\cdots$ \\
\hline $\begin{array}{l}68 / 14 / 87 \\
11 / 09 / 87\end{array}$ & $\begin{array}{l}22.80 \\
24.80\end{array}$ \\
\hline $\begin{array}{l}98 / 14 / 87 \\
11 / 69 / 87\end{array}$ & $\begin{array}{l}17806.00 \\
23100.06\end{array}$ \\
\hline $68 / 14 / 87$ & \\
\hline
\end{tabular}

\begin{tabular}{|c|c|}
\hline $\begin{array}{c}\text { SALPLE } \\
\text { DATE }\end{array}$ & $3-1-3$ \\
\hline 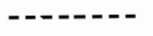 & --- \\
\hline $68 / 18 / 87$ & $\begin{array}{l}24.30 \\
3.62\} \\
\text { MR }\end{array}$ \\
\hline $08 / 18 / 87$ & 21.6 \\
\hline $8 / 18 / B 7$ & $\begin{array}{l}46.08 \\
8.56\} \\
\text { MR }\end{array}$ \\
\hline $68 / 18 / 87$ & MR \\
\hline $08 / 18 / 87$ & $1570 \mathrm{NR}^{10}$ \\
\hline $18 / 18 / 67$ & MR \\
\hline $68 / 18 / 87$ & $1114 . \mathrm{MR}$ \\
\hline $88 / 18 / 87$ & NR \\
\hline $08 / 18 / 87$ & $\begin{array}{l}\text { MR } \\
\text { MR }\end{array}$ \\
\hline $68 / 18 / 87$ & 21.00 \\
\hline $68 / 18 / 87$ & 10064 MR \\
\hline 68/28/87 & 523.08 \\
\hline
\end{tabular}

* - yalue eXceEdS priUary dRINKing vater standard.

- - YALUE EXCEEDS PROPOSED PRIUARY DRINKING IATER STANDARD.

- - YALUE EXCEEDS SCREENING LEYEI FOR FURTHER INYESTIGATION.

- - DETECTIQN LIUIT IAS NOT AYAILABLE FOR COUPARISON

NR - ANALYSIS HOT REQUESTED DR NOT YET REPORTED

YALUES IN \{ ? ARE COUNTIMG ERRORS FOR RADIONUCLIDES

WATER STANDARD(S) IN PARERTHESES ARE PROPOSED ONLY 


\begin{tabular}{|c|c|c|c|c|c|}
\hline$\underset{\text { NAME }}{\text { CDNSTI }}$ & $\begin{array}{l}\text { FUENT } \\
\text { UNITS }\end{array}$ & $\begin{array}{l}\text { CDNTRACTUAL } \\
\text { DETECTION } \\
\text { LIUIT }\end{array}$ & $\begin{array}{l}\text { DRINKING } \\
\text { WATER } \\
\text { STANDARD }\end{array}$ & $\begin{array}{l}\text { SAMPLE } \\
\text { DATE }\end{array}$ & $3-1-1$ \\
\hline FLUORID & PPB & 500.00 & 4088.80 & $11 / 10 / 87$ & 558.18 \\
\hline FUAGNES & PPB & 0.00 & & $\begin{array}{l}10 / 17 / 87 \\
11 / 10 / 87\end{array}$ & $\begin{array}{l}3470.06 \\
3756.06\end{array}$ \\
\hline FPOTASS & PPB & 166.06 & & $\begin{array}{l}18 / 17 / 87 \\
11 / 10 / 87\end{array}$ & $\begin{array}{l}2830.00 \\
2380.06\end{array}$ \\
\hline FSODIUU & PPB & 100.00 & & $\begin{array}{l}18 / 17 / 87 \\
11 / 10 / 87\end{array}$ & $\begin{array}{l}9378.60 \\
9926.08\end{array}$ \\
\hline FYAMADI & PPB & 5.66 & & $\begin{array}{l}68 / 17 / 87 \\
11 / 16 / 87\end{array}$ & 8.06 \\
\hline FZINC & $\mathrm{PPB}$ & 5.06 & & $\begin{array}{l}08 / 17 / 87 \\
11 / 16 / 87\end{array}$ & 5.81 \\
\hline IRON & PPB & 50.00 & & $\begin{array}{l}08 / 17 / 87 \\
11 / 11 / 87\end{array}$ & $\begin{array}{l}91.10 \\
51.16\end{array}$ \\
\hline HAGKES & $\mathrm{PPB}$ & 0.60 & & $\begin{array}{l}68 / 17 / 87 \\
11 / 10 / 87\end{array}$ & $\begin{array}{l}3526.60 \\
3868.06\end{array}$ \\
\hline MAMGESE & PPB & 5.60 & & $\begin{array}{l}68 / 17 / 87 \\
11 / 16 / 87\end{array}$ & 0.00 \\
\hline MITRATE & PPB & 600.00 & 45000.00 & $\begin{array}{l}08 / 17 / 87 \\
11 / 10 / 87\end{array}$ & $\begin{array}{l}1670.01 \\
1950.06\end{array}$ \\
\hline PHFIELD & & 6.10 & & $\begin{array}{l}08 / 17 / 87 \\
11 / 11 / 87\end{array}$ & $\begin{array}{l}8.80 \\
7.30\end{array}$ \\
\hline PDTASUI & $\mathrm{PPB}$ & 100.00 & & $\begin{array}{l}68 / 17 / 87 \\
11 / 1 / 87\end{array}$ & $\begin{array}{l}2180.60 \\
2598.60\end{array}$ \\
\hline$R U-168$ & $\mathrm{PCI} / \mathrm{L}$ & 172.50 & 38.08 & $\begin{array}{l}07 / 21 / 87 \\
10 / 15 / 87\end{array}$ & \\
\hline
\end{tabular}

NR (contd)

\begin{tabular}{|c|c|}
\hline $\begin{array}{c}\text { SAMPLE } \\
\text { DATE }\end{array}$ & $3-1-2$ \\
\hline 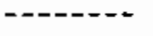 & $-\ldots$ \\
\hline $11 / 09 / 87$ & 519.00 \\
\hline $\begin{array}{l}0 / 14 / 87 \\
11 / 09 / 87\end{array}$ & $\begin{array}{l}420.00 \\
4910.00\end{array}$ \\
\hline $\begin{array}{l}18 / 14 / 87 \\
11 / 00 / 87\end{array}$ & $\begin{array}{l}2760.01 \\
3260.00\end{array}$ \\
\hline $\begin{array}{l}68 / 14 / 87 \\
11 / 60 / 87\end{array}$ & $\begin{array}{r}9800.06 \\
10900.00\end{array}$ \\
\hline $\begin{array}{l}08 / 14 / 87 \\
11 / 09 / 87\end{array}$ & 7.66 \\
\hline $\begin{array}{l}00 / 14 / 87 \\
11 / 08 / 87\end{array}$ & --- \\
\hline $\begin{array}{l}10 / 14 / 87 \\
11 / 60 / 87\end{array}$ & 58.90 \\
\hline $\begin{array}{l}8 / 14 / 87 \\
11 / 09 / 87\end{array}$ & $\begin{array}{l}4746.00 \\
4940.00\end{array}$ \\
\hline $\begin{array}{l}68 / 14 / 87 \\
11 / 09 / 87\end{array}$ & $--\infty$ \\
\hline $\begin{array}{l}08 / 14 / 87 \\
11 / 09 / 87\end{array}$ & $\begin{array}{l}3410.00 \\
3368.00\end{array}$ \\
\hline $\begin{array}{l}08 / 14 / 87 \\
11 / 00 / 87\end{array}$ & $\begin{array}{l}7.20 \\
7.10\end{array}$ \\
\hline $\begin{array}{l}8 / 14 / 87 \\
11 / 60 / 87\end{array}$ & $\begin{array}{l}2840.06 \\
3610.06\end{array}$ \\
\hline $\begin{array}{l}07 / 22 / 87 \\
16 / 19 / 87\end{array}$ & $\left\{\begin{array}{l}35.80 \\
29.36\} \\
M R\end{array}\right.$ \\
\hline
\end{tabular}

\begin{tabular}{|c|c|}
\hline $\begin{array}{l}\text { SAMPLE } \\
\text { OATE }\end{array}$ & $3-1-3$ \\
\hline - - - & \\
\hline & NR \\
\hline $88 / 18 / 97$ & 3534.10 \\
\hline & (n) \\
\hline - & $-\overline{---0}$ \\
\hline $68 / 18 / 87$ & 2145.46 \\
\hline$-\cdots$ & ---- \\
\hline $08 / 18 / 87$ & ${ }_{\mathrm{NR}}^{13860.00}$ \\
\hline $8 \mathrm{~s} / \mathrm{s} / \theta 7$ & $\cdots$ \\
\hline $08 / 18 / 01$ & NR \\
\hline$------\overline{-}$ & --- \\
\hline $18 / 18 / 87$ & $\begin{array}{l}5.16 \\
\text { HR }\end{array}$ \\
\hline$\because--1--$ & $\cdots$ \\
\hline $68 / 18 / 87$ & NR \\
\hline "---- & ---- \\
\hline $68 / 18 / 87$ & $\underset{N R}{3530.00}$ \\
\hline $88 / 18 / 87$ & $\cdots$ \\
\hline 0071070 & NR \\
\hline ------- & ---- \\
\hline $68 / 18 / 87$ & ${ }^{2020.00}$ \\
\hline $08 / 18 / 87$ & 6.86 \\
\hline - & MR \\
\hline $68 / 18 / 87$ & 2180.00 \\
\hline
\end{tabular}

$67 / 22 / 87$

$68 / 18 / 87$

$10 / 20 / 87$

* - VALUE EXCEEDS PRIMARY DRIMKING MaTER STAMDARD.

* - VALUE EXCEEDS PROPOSED PRIMARY DRINKIMG TATER STAMDARD.

- - VALUE EXCEEDS SCREENING LEVEL FOR FURTHER INYESTIGATION

- - DETECTION LIMIT IAS NOT AVAILABLE FOR COUPARISOH

- DETECTION LIUIT IAS NOT AVAILABLE FOR CONPA
NR - AMALYSIS NOT RERUESTED OR NDT YET REPORTED

YALUES IN I I ARE COUNTING ERRORS FOR RADIONUCLIDES

VATER STANDARO'(S) IN PARERTHESES ARE PRDPDSED ONLY
IATE 


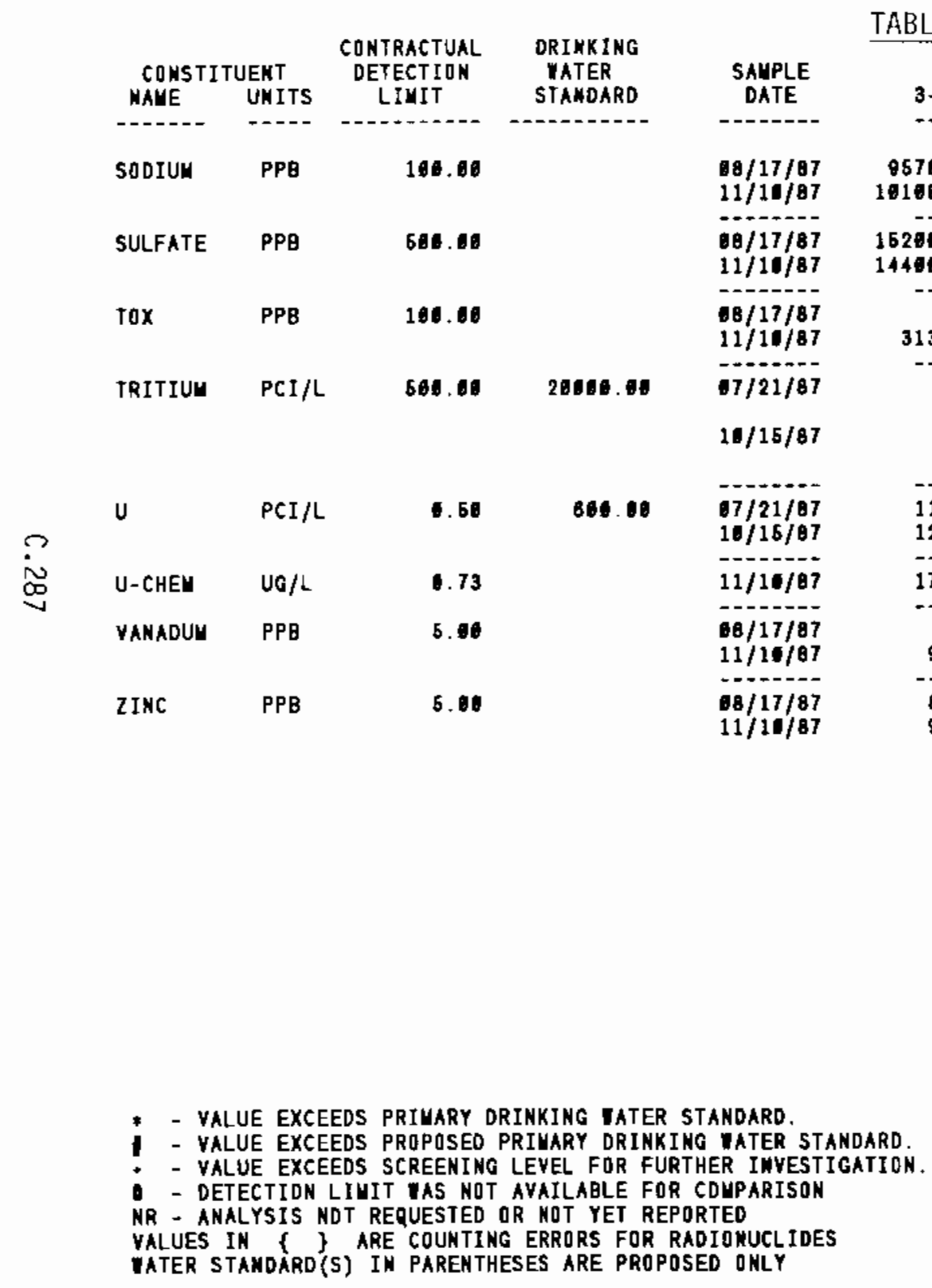

ABLE C.5. (contd)

$\begin{array}{lll} & \text { SAMPLE } & \\ 3-1-1 & \text { DATE } & \mathbf{3 - 1 - 2}\end{array}$

$\begin{array}{ll}98 / 14 / 87 & 10160.68 \\ 11 / 00 / 87 & 11160.66\end{array}$

$18 / 14 / 87 \quad 18740.86$

$\begin{array}{ll}9 / 14 / 87 & 18700.60 \\ 11 / 09 / 87 & 18460.66\end{array}$

(11/...-

$98 / 14 / 87$

-11.-

11.86

17.41

9.61

8.69

9.66
-1/22/87

$1 / 19 / 87$

24506. 66

1980.06

(214.06)

477.06

\{ 209.00\}

$07122 / 87 \quad 18.68$

$\begin{array}{rr}07 / 22 / 87 & 18.68 \\ 10 / 19 / 87 & 8.21\end{array}$

$11 / 00 / 87 \quad 15.80$

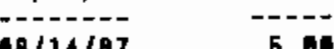

$11 / 09 / 87$

$08 / 14 / 87$

$11 / 09 / 87$

9.00

5.00
SALPLE

DATE

3-1-3

$68 / 18 / 87$

14106.06

$08 / 18 / 87 \quad 14686.06$

$\mathrm{NR}$

$8 / 18 / 87$

67/22/87

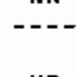

NR

$10 / 20 / 87$

374.6

174.6)

$-7122 / 87$

$07 / 22 / 87$
$16 / 26 / 87$

$-17-$

17.90
27.39

$08 / 18 / 87$

o8/18/97

$80 / 18 / 87$

- YALUE EXCEEDS PRIUARY DRINKING TATER STANDARD.

VALUE EXCEEDS PROPOSEO PRIMARY DRINKING WATER STANDARD.

- DETECTIDN LIUIT IAS NOT AVAILABLE FOR CDUPARISON

YALUES IN $f$ S ARE COUNTING ERRORS FOR RADIONUCLIDES

MTER STANDARD(S) IN PAREMTHESES ARE PROPOSED ONLY 
TABLEC.5. (contd)

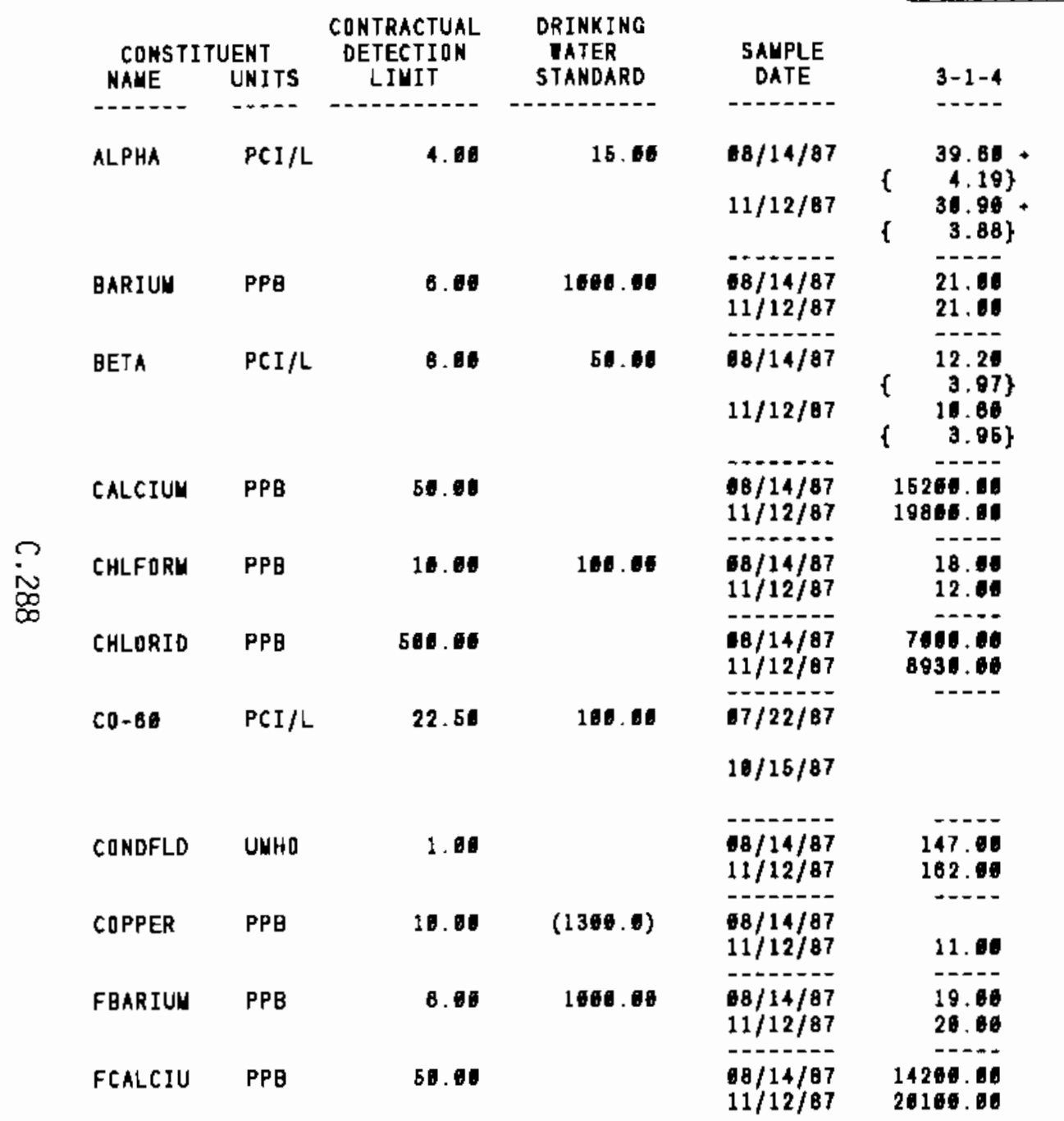

\begin{tabular}{|c|c|c|c|}
\hline $\begin{array}{l}\text { SAUPLE } \\
\text { DATE }\end{array}$ & $3-1-5$ & $\begin{array}{c}\text { SAUPLE } \\
\text { DATE }\end{array}$ & $3-1-6$ \\
\hline $08 / 28 / 87$ & $26.20+$ & $8 B / 17 / 87$ & 12.70 \\
\hline $68 / 26 / 87$ & $\begin{array}{l}\left\{\begin{array}{c}3.78\} \\
31.50 \\
\{\quad 4.06\}\end{array} .\right.\end{array}$ & $11 / 20 / 87$ & $\begin{array}{l}2.68\} \\
8.88 \\
2.32\}\end{array}$ \\
\hline $60 / 26 / 87$ & $\begin{array}{l}52.11 \\
\text { NR }\end{array}$ & $\begin{array}{l}6 / 17 / 87 \\
11 / 21 / 87\end{array}$ & $\begin{array}{l}22.19 \\
18.10\end{array}$ \\
\hline $08 / 28 / 87$ & 23.06 & $08 / 17 / 87$ & $\begin{array}{l}3.37 \\
3.86\}\end{array}$ \\
\hline $8 / 26 / 87$ & $\begin{array}{r}\quad 27.70 \\
\{\quad 5.77\}\end{array}$ & $11 / 20 / 87$ & $\begin{array}{l}4.30 \\
3.37\}\end{array}$ \\
\hline $0 B / 2 B / 87$ & $4806 \mathrm{NR}$ & $\begin{array}{l}68 / 17 / 87 \\
11 / 21 / 87\end{array}$ & 18090.01 \\
\hline $\begin{array}{l}08 / 28 / 87 \\
08 / 26 / 87\end{array}$ & $\begin{array}{l}17.00 \\
10.00\end{array}$ & $\begin{array}{l}00 / 17 / 87 \\
11 / 20 / 87\end{array}$ & $\begin{array}{l}16.00 \\
16.01\end{array}$ \\
\hline $\begin{array}{l}68 / 28 / 87 \\
68 / 28 / 87\end{array}$ & $\begin{array}{l}115060.60 \\
114000.60\end{array}$ & $\begin{array}{l}18 / 17 / 87 \\
11 / 26 / 87\end{array}$ & 6816.66 \\
\hline $07 / 23 / 87$ & & $67 / 22 / 87$ & $\begin{array}{l}8.43 \\
5.25\end{array}$ \\
\hline $10 / 13 / 07$ & & $16 / 16 / 87$ & $\begin{array}{l}\quad 8.10 \\
\{\quad 5.73\}\end{array}$ \\
\hline$B / 26 / B 7$ & $\underset{N R}{458.08}$ & $\begin{array}{l}68 / 17 / 87 \\
11 / 26 / 87\end{array}$ & $\begin{array}{l}137.01 \\
183.00\end{array}$ \\
\hline $68 / 26 / 87$ & MR & $\begin{array}{l}08 / 17 / 87 \\
11 / 20 / 87\end{array}$ & 13.86 \\
\hline $\begin{array}{l}88 / 26 / 87 \\
8 / 26 / 87\end{array}$ & $\begin{array}{l}83.06 \\
86.06\end{array}$ & $\begin{array}{l}18 / 17 / 87 \\
11 / 26 / 87\end{array}$ & $\begin{array}{l}22.100 \\
24.10 \\
-. . .\end{array}$ \\
\hline $\begin{array}{l}08 / 26 / 87 \\
80 / 26 / 87\end{array}$ & $\begin{array}{l}58700.06 \\
57300.06\end{array}$ & $\begin{array}{l}98 / 17 / 87 \\
11 / 20 / 87\end{array}$ & $\begin{array}{l}1680600 \\
21806\end{array}$ \\
\hline
\end{tabular}

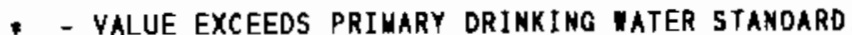

- VALUE EXCEEDS PROPOSED PRIMARY ORINKING TATER STANDARD.

- - VALUE EXCEEDS SCREENING LEYEL FOR FURTHER INYESTIGATION.

- DETECTION LIUIT WAS NOT AYAILABLE FOR COUPARISON

NR - ANALYSIS NOT REQUESTED OR NOT YET REPORTED

YALUES IN \{ \& ARE COUNTING ERRORS FOR RADIONUCLIDES

IATER STANDARD(S) IK PARENTHESES ARE PRDPOSED ONLY 


\begin{tabular}{|c|c|c|c|c|c|}
\hline $\begin{array}{c}\text { CONSTI } \\
\text { RAUE }\end{array}$ & $\begin{array}{l}\text { UENT } \\
\text { UMISS }\end{array}$ & $\begin{array}{c}\text { CONTRACTUAL } \\
\text { DERECTIION } \\
\text { LIUIT }\end{array}$ & $\begin{array}{l}\text { DRINKING } \\
\text { VATER } \\
\text { STANDARD }\end{array}$ & $\begin{array}{c}\text { SAUPLE } \\
\text { DATE }\end{array}$ & $3-1-4$ \\
\hline FCOPPER & PPB & 10.00 & $(1386.0)$ & $\begin{array}{l}08 / 14 / 87 \\
11 / 12 / 87\end{array}$ & 12.60 \\
\hline FIRON & PPB & 50.00 & & $\begin{array}{l}08 / 14 / 87 \\
11 / 12 / 87\end{array}$ & 154.60 \\
\hline FUAGNES & PPB & 0.00 & & $\begin{array}{l}10 / 14 / 87 \\
11 / 12 / 87\end{array}$ & $\begin{array}{l}3526.80 \\
4376.00\end{array}$ \\
\hline FPOTASS & PPB & 106.60 & & $\begin{array}{l}08 / 14 / 87 \\
11 / 12 / 87\end{array}$ & $\begin{array}{l}2150.00 \\
2108.00\end{array}$ \\
\hline FSODIUU & PPB & 168.68 & & $\begin{array}{l}88 / 14 / 87 \\
11 / 12 / 87\end{array}$ & $\begin{array}{l}8628.06 \\
7680.06\end{array}$ \\
\hline FSTRONT & PPB & 300.00 & & & $\begin{array}{l}\text { NR } \\
\text { NR }\end{array}$ \\
\hline FVAMADI & PPB & 5.00 & & $\begin{array}{l}68 / 14 / 87 \\
11 / 12 / 87\end{array}$ & 5.60 \\
\hline FZINC & PPB & 5.80 & & $\begin{array}{l}8 / 14 / 87 \\
11 / 12 / 87\end{array}$ & $\begin{array}{r}5.60 \\
13.00\end{array}$ \\
\hline IRON & PPB & 58.00 & & $\begin{array}{l}08 / 14 / 87 \\
11 / 12 / 87\end{array}$ & $\begin{array}{l}\text { 82. 68 } \\
89.80\end{array}$ \\
\hline WAGMES & PPg & 0.00 & & $\begin{array}{l}08 / 14 / 87 \\
11 / 12 / 87\end{array}$ & $\begin{array}{l}3570.86 \\
4140.86\end{array}$ \\
\hline WETHYCH & PPB & 10.00 & & $\begin{array}{l}18 / 14 / 87 \\
11 / 12 / 87\end{array}$ & 3040.06 \\
\hline NITRATE & PPB & 506.60 & 45606.00 & $\begin{array}{l}08 / 14 / 87 \\
11 / 12 / 87\end{array}$ & $\begin{array}{r}898.60 \\
1686.06\end{array}$ \\
\hline PHF IELD & & 0.16 & & $\begin{array}{l}68 / 14 / 87 \\
11 / 12 / 87\end{array}$ & $\begin{array}{l}8.60 \\
8.98\end{array}$ \\
\hline
\end{tabular}

ABLE C.5. (contd)

- - value exceeds primary drinkimg iater standard.

1 - VALUE EXCEEDS PRDPDSED PRIMARY DRINKING TATER STANDARD.

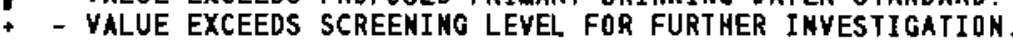

- - DETECTION LIUIt IAS not aVAilagle for couparison

NR - ANALYSIS NOT RERUESTED DR NOT YET REPORTED

YALUES IN \{\} ARE COUNTING ERRORS FDR RADIONUCLIDES

IATER STANDARD(S) IN PARENTHESES ARE PROPOSED DNLY

\begin{tabular}{|c|c|}
\hline $\begin{array}{l}\text { SAYPLE } \\
\text { DATE }\end{array}$ & $3-1-5$ \\
\hline $\begin{array}{l}08 / 26 / 87 \\
68 / 26 / 87\end{array}$ & 11.00 \\
\hline $\begin{array}{l}68 / 26 / 87 \\
6 / 26 / 87\end{array}$ & 76.10 \\
\hline $\begin{array}{l}80 / 26 / 87 \\
80 / 26 / 97\end{array}$ & $\begin{array}{l}13281.60 \\
13100.06\end{array}$ \\
\hline $\begin{array}{l}18 / 28 / 87 \\
18 / 28 / 87\end{array}$ & $\begin{array}{l}3980.16 \\
3980.06\end{array}$ \\
\hline $\begin{array}{l}8 / 28 / 87 \\
08 / 28 / 87\end{array}$ & $\begin{array}{l}17000.00 \\
17696.06\end{array}$ \\
\hline $\begin{array}{l}08 / 28 / 87 \\
09 / 26 / 87\end{array}$ & 314.00 \\
\hline $\begin{array}{l}09 / 26 / 87 \\
09 / 28 / 87\end{array}$ & 7.11 \\
\hline $\begin{array}{l}68 / 26 / 87 \\
68 / 28 / 87\end{array}$ & 8.18 \\
\hline $88 / 28 / 87$ & NR \\
\hline $08 / 28 / 87$ & 11000 NR \\
\hline $\begin{array}{l}68 / 26 / 87 \\
68 / 26 / 87\end{array}$ & 21.06 \\
\hline $\begin{array}{l}68 / 26 / 87 \\
68 / 26 / 87\end{array}$ & $\begin{array}{l}1290.88 \\
1410.86\end{array}$ \\
\hline $08 / 26 / 87$ & $\stackrel{6}{N R}^{6.56}$ \\
\hline
\end{tabular}

\begin{tabular}{|c|c|}
\hline $\begin{array}{l}\text { SAUPLE } \\
\text { DATE }\end{array}$ & $3-1-0$ \\
\hline $\begin{array}{l}68 / 17 / 87 \\
11 / 28 / 87\end{array}$ & 12.06 \\
\hline $\begin{array}{l}68 / 17 / 87 \\
11 / 20 / 87\end{array}$ & \\
\hline $\begin{array}{l}18 / 17 / 87 \\
11 / 20 / 87\end{array}$ & $\begin{array}{l}4686.110 \\
4636.41\end{array}$ \\
\hline $\begin{array}{l}18 / 17 / 87 \\
11 / 28 / 87\end{array}$ & $\begin{array}{l}2226.66 \\
2450.66\end{array}$ \\
\hline $\begin{array}{l}98 / 17 / 87 \\
11 / 26 / 87\end{array}$ & $\begin{array}{l}6758.96 \\
7185.86\end{array}$ \\
\hline & $\begin{array}{l}\text { MR } \\
\text { IRR }\end{array}$ \\
\hline $\begin{array}{l}8 / 17 / 87 \\
11 / 20 / 87\end{array}$ & 10.60 \\
\hline $\begin{array}{l}68 / 17 / 87 \\
11 / 26 / 87\end{array}$ & $\begin{array}{r}13.00 \\
6.00\end{array}$ \\
\hline $\begin{array}{l}68 / 17 / 87 \\
11 / 26 / 87\end{array}$ & 71.00 \\
\hline $\begin{array}{l}88 / 17 / 87 \\
11 / 20 / 87\end{array}$ & $\begin{array}{l}3910.06 \\
4365.06\end{array}$ \\
\hline $\begin{array}{l}68 / 17 / 87 \\
11 / 20 / 87\end{array}$ & \\
\hline $\begin{array}{l}68 / 17 / 87 \\
11 / 20 / 87\end{array}$ & $\begin{array}{l}1136.06 \\
1976.65\end{array}$ \\
\hline $\begin{array}{l}08 / 17 / 87 \\
11 / 26 / 87\end{array}$ & $\begin{array}{l}8.80 \\
8.70\end{array}$ \\
\hline
\end{tabular}


TABLE C.5. (contd)

\begin{tabular}{|c|c|c|c|c|c|}
\hline $\begin{array}{c}\text { CONSTI } \\
\text { MAUE }\end{array}$ & $\begin{array}{l}\text { TUENT } \\
\text { UNI I S }\end{array}$ & $\begin{array}{l}\text { CONTRACIUAL } \\
\text { DETECTION } \\
\text { LIUIT }\end{array}$ & $\begin{array}{l}\text { DRINKING } \\
\text { IATER } \\
\text { STANDARD }\end{array}$ & $\underset{\text { DATE }}{\text { SAMPLE }}$ & $3-1-4$ \\
\hline POTASUI & PPB & 106.61 & & $\begin{array}{l}88 / 14 / 87 \\
11 / 12 / 87\end{array}$ & $\begin{array}{l}2090.40 \\
2000.00\end{array}$ \\
\hline \multirow[t]{2}{*}{ RADIUM } & PCI/L & 1.00 & 5.60 & $08 / 14 / 87$ & \\
\hline & & & & $11 / 12 / 87$ & \\
\hline SODIUV & PPB & 160.00 & & $\begin{array}{l}18 / 14 / 87 \\
11 / 12 / 87\end{array}$ & $\begin{array}{l}7360.06 \\
7150.10\end{array}$ \\
\hline SULFATE & PPB & 500.01 & & $\begin{array}{l}68 / 14 / 87 \\
11 / 12 / 87\end{array}$ & $\begin{array}{l}13600.00 \\
13800.06\end{array}$ \\
\hline TOX & PPB & 100.60 & & $\begin{array}{l}18 / 14 / 87 \\
11 / 12 / 87\end{array}$ & 4770.06 \\
\hline TRITIUU & $\mathrm{PCI} / \mathrm{L}$ & 580.86 & 20000.00 & $\begin{array}{l}67 / 22 / 87 \\
10 / 15 / 87\end{array}$ & \\
\hline$u$ & PCI/L & 0.50 & 806.06 & $\begin{array}{l}07 / 22 / 87 \\
10 / 15 / 87\end{array}$ & $\begin{array}{l}20.46 \\
10.18\end{array}$ \\
\hline U-CHEM & UG $/ \mathrm{L}$ & 0.73 & & $11 / 12 / 87$ & 38.10 \\
\hline YANADUY & PPB & 5.90 & & $\begin{array}{l}68 / 14 / 87 \\
11 / 12 / 87\end{array}$ & 6.010 \\
\hline ZINC & PPB & 5.00 & & $\begin{array}{l}68 / 14 / 87 \\
11 / 12 / 87\end{array}$ & $\begin{array}{r}9.00 \\
39.00\end{array}$ \\
\hline
\end{tabular}

\begin{tabular}{|c|c|c|c|}
\hline $\begin{array}{c}\text { SALPLE } \\
\text { DATE }\end{array}$ & $3-1-5$ & $\begin{array}{c}\text { SAMPLE } \\
\text { DATE }\end{array}$ & $3-1-6$ \\
\hline $68 / 28 / 87$ & $357 \mathrm{NR}_{\mathrm{R}} .60$ & $\begin{array}{l}58 / 17 / 87 \\
11 / 20 / 87\end{array}$ & $\begin{array}{l}2206.00 \\
2418.00\end{array}$ \\
\hline $00 / 28 / 87$ & $\left\{\begin{array}{l}.19 \\
M R\end{array}\right.$ & $\begin{array}{l}68 / 17 / 07 \\
11 / 26 / 87\end{array}$ & \\
\hline $08 / 28 / 87$ & 14191.10 & $\begin{array}{l}08 / 17 / 87 \\
11 / 21 / 87\end{array}$ & $\begin{array}{l}\text { BB46. } \\
\text { B87U. }\end{array}$ \\
\hline $\begin{array}{l}08 / 26 / 87 \\
88 / 28 / 87\end{array}$ & $\begin{array}{l}12300.00 \\
12364.06\end{array}$ & $\begin{array}{l}08 / 17 / 87 \\
11 / 20 / 87\end{array}$ & $\begin{array}{l}13000.01 \\
12800.00\end{array}$ \\
\hline 08/28/87 & NR & $\begin{array}{l}08 / 17 / 87 \\
11 / 26 / 87\end{array}$ & \\
\hline $\begin{array}{l}67 / 23 / 87 \\
10 / 13 / 87\end{array}$ & $\left\{\begin{array}{l}417.66 \\
200.06\end{array}\right\}$ & $\begin{array}{l}87 / 22 / 87 \\
10 / 15 / 87\end{array}$ & \\
\hline $\begin{array}{l}07 / 23 / 87 \\
11 / 13 / 87\end{array}$ & $\begin{array}{l}18.70 \\
22.98\end{array}$ & $\begin{array}{l}97 / 22 / 87 \\
10 / 15 / 87\end{array}$ & $\begin{array}{l}8.97 \\
0.47\end{array}$ \\
\hline & MR & $11 / 21 / 87$ & 13.80 \\
\hline $8 / 28 / 87$ & NR & $\begin{array}{l}00 / 17 / 87 \\
11 / 29 / 87\end{array}$ & 6.00 \\
\hline $88 / 28 / 87$ & MR & $\begin{array}{l}68 / 17 / 87 \\
11 / 20 / 87\end{array}$ & $\begin{array}{l}0.00 \\
6.00\end{array}$ \\
\hline
\end{tabular}

* - yalue exceeds primary drinking iater standard.

* - VALUE EXCEEDS PRIMARY DRINATA PROPOSED PRIMARY DRINKING MATER STANDARD.

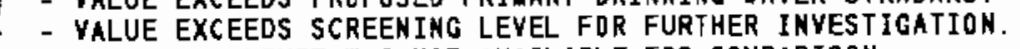

- DETECTION LIUIT MAS NOT AVAILABLE FDR COMPARISON

RR - ANALYSIS NOT RERUESTED OR NOT YET REPORTED

VALUES IN \{\} ARE COUNTING ERRIRS FOR RADIONUCLIDES

nATER STANDARD(S) IN PARENTHESES ARE PROPDSED OKLY 
TABLE C.5. (contd)

\begin{tabular}{|c|c|c|c|c|c|c|c|c|c|}
\hline $\begin{array}{c}\text { CONSTI } \\
\text { NAYE }\end{array}$ & $\begin{array}{l}\text { UENT } \\
\text { UNITS }\end{array}$ & $\begin{array}{l}\text { CONTRACTUAL } \\
\text { DETECTION } \\
\text { LIMIT }\end{array}$ & $\begin{array}{l}\text { DRINKING } \\
\text { IATER } \\
\text { STANDARD }\end{array}$ & $\underset{\text { DATE }}{\text { SAMPLE }}$ & $3-1-7$ & $\begin{array}{c}\text { SAMPLE } \\
\text { DATE }\end{array}$ & $3-1-\theta$ & $\begin{array}{c}\text { SAYPLE } \\
\text { DATE }\end{array}$ & $3-1-8$ \\
\hline ALPHA & $\mathrm{PCI} / \mathrm{L}$ & 4.00 & 15.00 & $\begin{array}{l}18 / 17 / 87 \\
11 / 13 / 87\end{array}$ & $\begin{array}{c}30.20 \\
\left\{\quad \begin{array}{c}3.95\} \\
27.98 \\
\{\quad 3.83\}\end{array}\right. \\
\end{array}$ & $\begin{array}{l}18 / 20 / 87 \\
11 / 13 / 87\end{array}$ & $\begin{array}{c}24.79 \\
3.36\} \\
24.68 \\
3.47\}\end{array}$ & $68 / 18 / 87$ & NR \\
\hline AVIDNIU & PPB & 56.06 & & $\begin{array}{l}0 / 17 / 87 \\
11 / 19 / 87\end{array}$ & & $\begin{array}{l}68 / 21 / 87 \\
11 / 13 / 87\end{array}$ & & $68 / 18 / 87$ & $\underset{\mathrm{HR}}{125.00}$ \\
\hline BARIUM & PPB & B. 80 & 1080.00 & $\begin{array}{l}68 / 17 / 97 \\
11 / 13 / 07\end{array}$ & 22.60 & $\begin{array}{l}68 / 20 / 87 \\
11 / 13 / 97 \\
-\end{array}$ & $\begin{array}{l}31.66 \\
38.61\end{array}$ & $08 / 18 / 87$ & $\begin{array}{l}110.60 \\
\mathrm{NR}\end{array}$ \\
\hline BETA & $\mathrm{PCI} / \mathrm{L}$ & 8.08 & 66.00 & $\begin{array}{l}08 / 17 / 87 \\
11 / 13 / 87\end{array}$ & $\begin{array}{c}49.86 \\
\{\quad 8.86\} \\
29.76 \\
\{\quad 8.53\}\end{array}$ & $\begin{array}{l}68 / 20 / 87 \\
11 / 13 / 87\end{array}$ & $\begin{array}{c}29.84 \\
\{\quad 5.68\} \\
28.20 \\
\{\quad 5.22\} \\
-\end{array}$ & $68 / 10 / 87$ & $\begin{array}{c}14.10 \\
\text { NR }\end{array}$ \\
\hline CAOHIUU & PPB & 2.00 & 10.60 & $\begin{array}{l}08 / 17 / 87 \\
11 / 13 / 87\end{array}$ & 2.66 & $\begin{array}{l}68 / 20 / 87 \\
11 / 13 / 87\end{array}$ & & $08 / 18 / 87$ & MR \\
\hline CALCIUM & $\mathrm{PPB}$ & 50.00 & & $\begin{array}{l}68 / 17 / 87 \\
11 / 13 / 87\end{array}$ & $\begin{array}{l}18289.66 \\
27806.11\end{array}$ & $\begin{array}{l}18 / 20 / 87 \\
11 / 13 / 87 \\
0\end{array}$ & $\begin{array}{r}14401.00 \\
21400.00\end{array}$ & $18 / 18 / 87$ & $\begin{array}{c}1530 \mathrm{MR} \\
\mathrm{MR}\end{array}$ \\
\hline CHLFORM & $\mathrm{PPB}$ & 10.00 & 165.00 & $\begin{array}{l}98 / 17 / 87 \\
11 / 13 / 87\end{array}$ & $\begin{array}{l}18.00 \\
13.00\end{array}$ & $\begin{array}{l}68 / 20 / 07 \\
11 / 13 / 07\end{array}$ & & $18 / 18 / 87$ & NR \\
\hline CHLORID & PPB & 500.80 & & $\begin{array}{l}18 / 17 / 87 \\
11 / 13 / 87\end{array}$ & $\begin{array}{r}11200.00 \\
39408.06 \\
0\end{array}$ & $\begin{array}{l}68 / 26 / 87 \\
11 / 13 / 87 \\
-\end{array}$ & $\begin{array}{r}10200.00 \\
24301000 \\
-\end{array}$ & $68 / 18 / 87$ & NR \\
\hline CHROMUY & $\mathrm{PPB}$ & 10.0 & 68.00 & $\begin{array}{l}98 / 17 / 87 \\
11 / 13 / 87\end{array}$ & & $\begin{array}{l}08 / 21 / 87 \\
11 / 13 / 87 \\
\end{array}$ & & $66 / 18 / 87$ & 18.60 \\
\hline CONDFLD & Чино & 1.60 & & $\begin{array}{l}08 / 17 / 87 \\
11 / 13 / 87\end{array}$ & $\begin{array}{l}152.10 \\
245.61\end{array}$ & $\begin{array}{l}88 / 20 / 87 \\
11 / 13 / 87\end{array}$ & $\begin{array}{l}200.80 \\
244.06\end{array}$ & $08 / 18 / 87$ & $\begin{array}{c}256.60 \\
M R\end{array}$ \\
\hline COPPER & $\mathrm{PPB}$ & 10.00 & $(1300.0)$ & $\begin{array}{l}08 / 17 / 87 \\
11 / 13 / 87\end{array}$ & 25.00 & $\begin{array}{l}88 / 20 / 87 \\
11 / 13 / 87\end{array}$ & & $68 / 18 / 87$ & NR \\
\hline FEARIUY & PPB & 6.60 & 1000.00 & $68 / 17 / 87$ & 22.68 & $68 / 20 / 87$ & 28.60 & $68 / 18 / 87$ & 120.00 \\
\hline \multicolumn{10}{|c|}{ 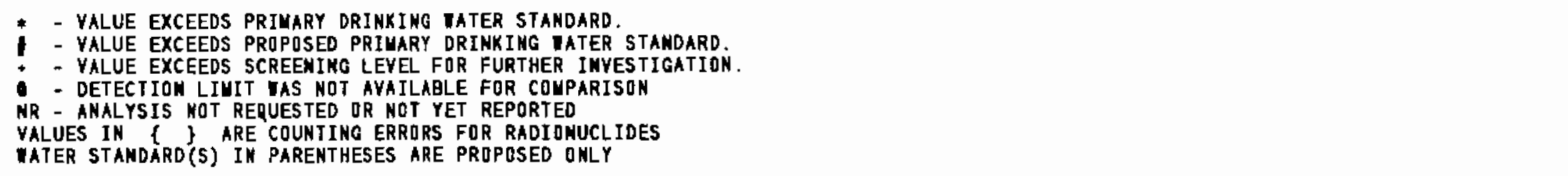 } \\
\hline
\end{tabular}


TABLE C.5. (contd)

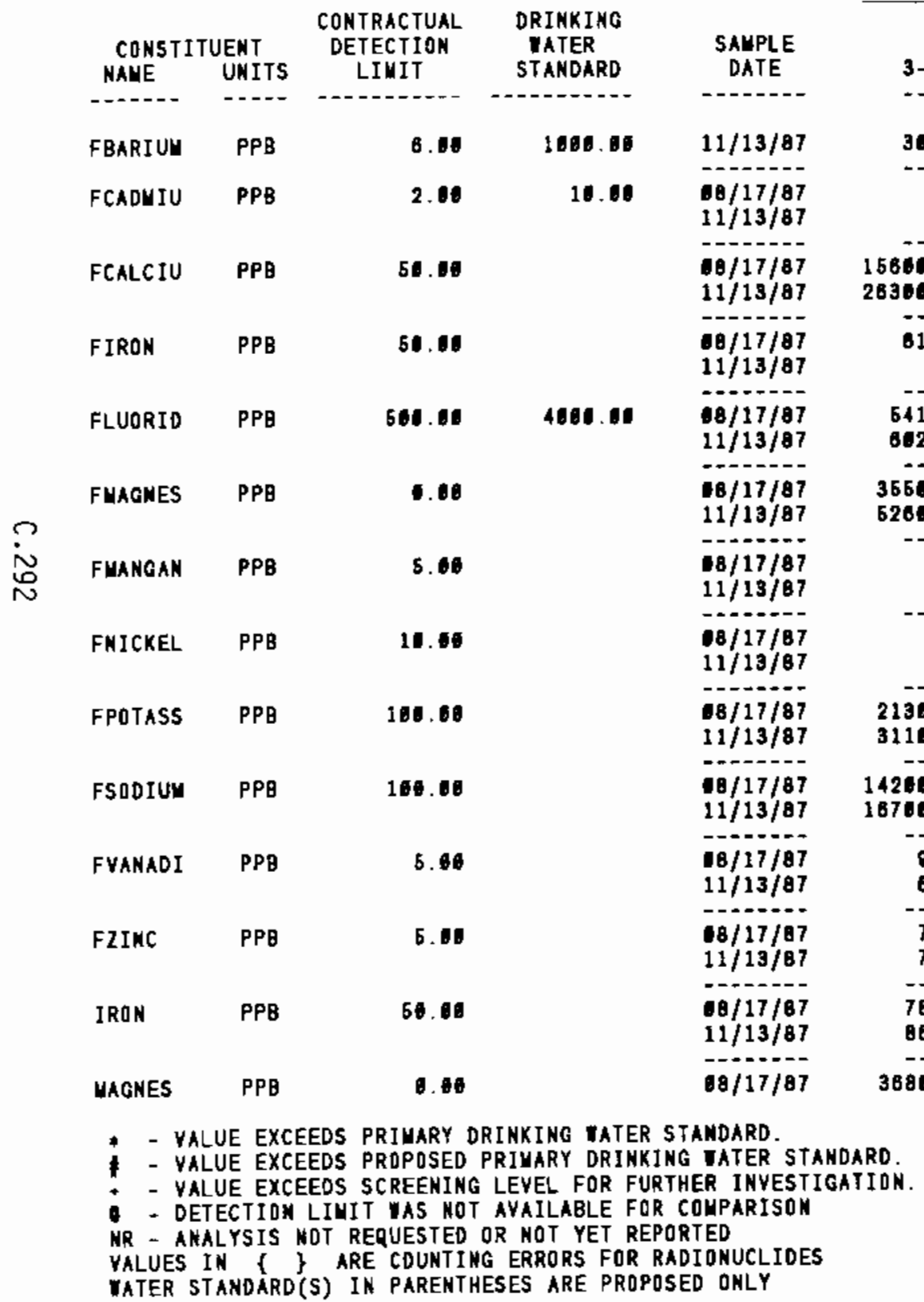

\begin{tabular}{|c|c|}
\hline $\begin{array}{l}\text { SALPLE } \\
\text { DATE }\end{array}$ & $3-1-8$ \\
\hline$--n \ldots$ & ---- \\
\hline $11 / 13 / 87$ & 38.00 \\
\hline $\begin{array}{l}10 / 26 / 87 \\
11 / 13 / 87\end{array}$ & \\
\hline$---1-$ & $\cdots$ \\
\hline $\begin{array}{l}18 / 26 / 87 \\
11 / 13 / 87\end{array}$ & $\begin{array}{l}15700.00 \\
21700.00\end{array}$ \\
\hline $\begin{array}{l}10 / 26 / 87 \\
11 / 13 / 87\end{array}$ & \\
\hline$\because+--$ & --- \\
\hline $\begin{array}{l}68 / 26 / 87 \\
11 / 13 / 87\end{array}$ & $\begin{array}{l}680.00 \\
722.00\end{array}$ \\
\hline $\begin{array}{l}68 / 20 / 87 \\
11 / 13 / 87\end{array}$ & $\begin{array}{l}5186.0 \\
8285.04\end{array}$ \\
\hline $\begin{array}{l}68 / 20 / 87 \\
11 / 13 / 87\end{array}$ & $\begin{array}{l}-1.0 \\
17.01\end{array}$ \\
\hline $\begin{array}{l}08 / 20 / 87 \\
11 / 13 / 87\end{array}$ & ---- \\
\hline $\begin{array}{l}58 / 20 / 87 \\
11 / 13 / 87\end{array}$ & $\begin{array}{r}4110.00 \\
4760.00\end{array}$ \\
\hline $\begin{array}{l}10 / 20 / 87 \\
11 / 13 / 87\end{array}$ & $\begin{array}{l}21600 \\
25100\end{array}$ \\
\hline $\begin{array}{l}09 / 26 / 87 \\
11 / 13 / 87\end{array}$ & \\
\hline $\begin{array}{l}68 / 20 / 87 \\
11 / 13 / 87\end{array}$ & 18.0 \\
\hline $\begin{array}{l}68 / 25 / 87 \\
11 / 13 / 87\end{array}$ & $\ldots$ \\
\hline $88 / 21 / 87$ & 5230.06 \\
\hline
\end{tabular}

\begin{tabular}{|c|c|}
\hline $\begin{array}{l}\text { SAUPLE } \\
\text { DATE }\end{array}$ & $3-1-8$ \\
\hline 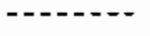 & \\
\hline & NR \\
\hline$-1-10$ & 2. \\
\hline $08 / 18 / 07$ & HR \\
\hline$\cdots-1-1$ & $2=$ \\
\hline $18 / 18 / 87$ & $\underset{\mathrm{NR}}{16760}$ \\
\hline$\cdots-1-1$ & \\
\hline $68 / 18 / 87$ & 281.1. \\
\hline - - & $-\ldots$ \\
\hline $18 / 18 / 87$ & $\underset{\mathrm{MR}}{881.6}$ \\
\hline -...... & -1 \\
\hline $18 / 18 / 67$ & $\underset{\mathrm{MR}}{7241.01}$ \\
\hline$-1-1-4$ & $\overline{-1}$ \\
\hline $18 / 18 / 87$ & MR \\
\hline 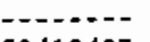 & - \\
\hline $18 / 18 / 87$ & MR \\
\hline 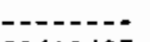 & 55 \\
\hline $08 / 18 / 87$ & ${ }_{N R}^{5540.00}$ \\
\hline$\because-1-1-$ & 619 \\
\hline $88 / 18 / 87$ & 51906.60 \\
\hline $8 / 18 / 87$ & \\
\hline & NR \\
\hline $68 / 18 / 87$ & \\
\hline & NR \\
\hline $08 / 18 / 87$ & 427.16 \\
\hline & NK \\
\hline $68 / 18 / 87$ & 7280.00 \\
\hline
\end{tabular}




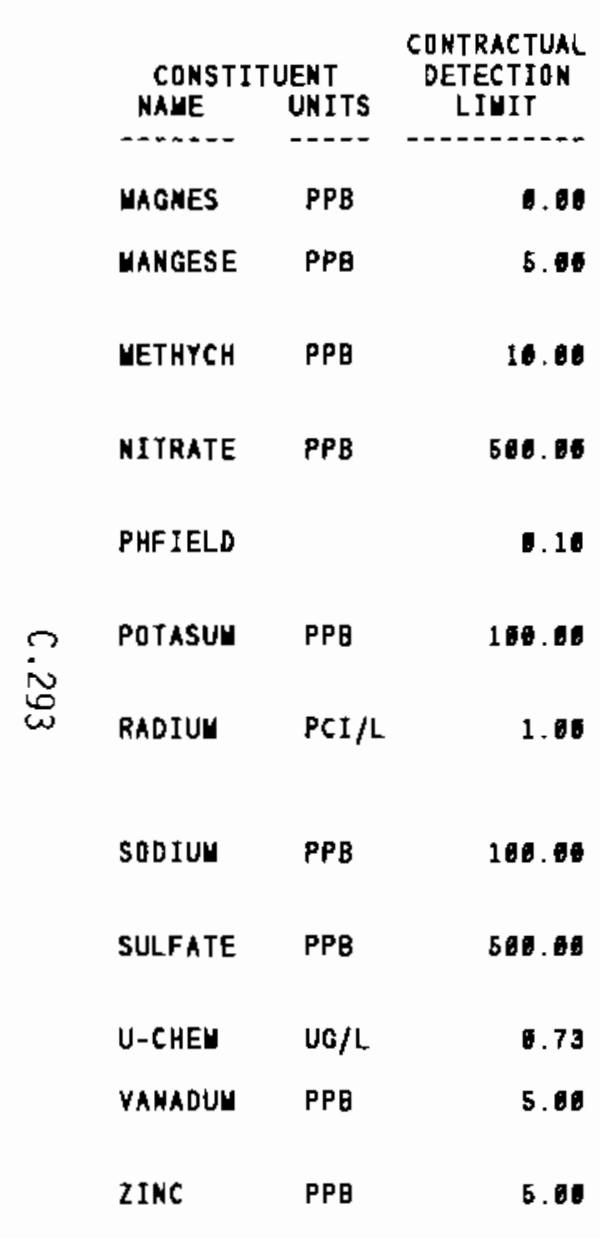

TABLE C.5. (contd)

DRINXING
WATER
STANDARD

\begin{tabular}{|c|c|}
\hline $\begin{array}{l}\text { SAMPLE } \\
\text { DATE }\end{array}$ & $3-1-7$ \\
\hline $11 / 13 / 87$ & 5336.10 \\
\hline $\begin{array}{l}18 / 17 / 87 \\
11 / 13 / 87\end{array}$ & \\
\hline $\begin{array}{l}88 / 17 / 87 \\
11 / 13 / 87\end{array}$ & \\
\hline $\begin{array}{l}68 / 17 / 87 \\
11 / 13 / 87\end{array}$ & $\begin{array}{l}2261.60 \\
1021.00\end{array}$ \\
\hline $\begin{array}{l}88 / 17 / 87 \\
11 / 13 / 87\end{array}$ & $\begin{array}{l}6.80 \\
8.48\end{array}$ \\
\hline $\begin{array}{l}08 / 17 / 87 \\
11 / 13 / 87\end{array}$ & $\begin{array}{r}2280.08 \\
3180.00\end{array}$ \\
\hline $\begin{array}{l}68 / 17 / 87 \\
11 / 13 / 87\end{array}$ & \\
\hline
\end{tabular}

.........

0.17107

$68 / 17 / 87$
$11 / 13 / 87$

14006.09

$08 / 17 / 67$

$11 / 13 / 87$

18060.00

16200.00

14580.00

$11 / 13 / 87$

37.70

$88 / 17 / 87$

$11 / 13 / 87$

$00 / 17 / 87$

$11 / 13 / 87$

\begin{tabular}{|c|c|}
\hline $\begin{array}{l}\text { SAUPLE } \\
\text { DATE }\end{array}$ & $3-1-8$ \\
\hline $11 / 13 / 87$ & 6010.00 \\
\hline $\begin{array}{l}08 / 26 / 87 \\
11 / 23 / 87\end{array}$ & $\begin{array}{l}11.08 \\
13.00\end{array}$ \\
\hline $\begin{array}{l}08 / 20 / 87 \\
11 / 13 / 87\end{array}$ & 580.00 \\
\hline $\begin{array}{l}68 / 20 / 87 \\
11 / 13 / 87\end{array}$ & $\begin{array}{l}1334.06 \\
1626.16\end{array}$ \\
\hline $\begin{array}{l}68 / 28 / 87 \\
11 / 13 / 87\end{array}$ & $\begin{array}{l}7.00 \\
7.20\end{array}$ \\
\hline $\begin{array}{l}06 / 20 / 87 \\
11 / 13 / 87\end{array}$ & $\begin{array}{l}4380.01 \\
4866.80\end{array}$ \\
\hline $\begin{array}{l}08 / 26 / 87 \\
11 / 13 / 87\end{array}$ & $\left\{\begin{array}{l}\quad .25 \\
: 17\}\end{array}\right.$ \\
\hline $\begin{array}{l}98 / 2 \theta / 87 \\
11 / 13 / 87\end{array}$ & $\begin{array}{l}22386.00 \\
23868.00\end{array}$ \\
\hline $\begin{array}{l}08 / 20 / 87 \\
11 / 13 / 87\end{array}$ & $\begin{array}{l}15808.00 \\
14200.00\end{array}$ \\
\hline $11 / 13 / 87$ & 24.80 \\
\hline $\begin{array}{l}68 / 28 / 07 \\
11 / 13 / 07\end{array}$ & 8.00 \\
\hline $\begin{array}{l}68 / 20 / 87 \\
11 / 13 / 87\end{array}$ & $\begin{array}{r}5.60 \\
32.60\end{array}$ \\
\hline
\end{tabular}

\begin{tabular}{|c|c|}
\hline $\begin{array}{l}\text { SAYPLE } \\
\text { DATE }\end{array}$ & $3-1-9$ \\
\hline & NR \\
\hline $68 / 18 / 87$ & ${ }_{\mathrm{NR}}^{65.16}$ \\
\hline $68 / 18 / 87$ & NR \\
\hline $68 / 18 / 87$ & NR \\
\hline $68 / 18 / 87$ & $\begin{array}{l}7.21 \\
\mathrm{MR}\end{array}$ \\
\hline $00 / 18 / 07$ & 5586.61 \\
\hline $08 / 18 / 07$ & MR \\
\hline $80 / 18 / 87$ & 54306.00 \\
\hline $00 / 18 / 87$ & ${ }_{\mathrm{NR}}^{868.61}$ \\
\hline - & NR \\
\hline $88 / 18 / 87$ & NR \\
\hline $08 / 18 / 87$ & 7.19 \\
\hline
\end{tabular}

- - value exceeds privary drinking tater standard.

- - VALUE EXCEEDS PROPOSED PRIMARY DRIMKING tater STANDARD.

- - yalue exceeds screening leyel for furTher inyestigation.

- DETECTION LiUIt us nOt avaILABLE for couparison

NR - ANALYSIS NOT REQUESTED DR NDT YET REPORTED

YALUES IN \{ $\}$ ARE CDUNTING ERRIRS FOR RADIONUCLIDES

IATER STANDARD(S) IN PARENTHESES ARE PROPDSED ONLY 
TABLE C.5. (contd)

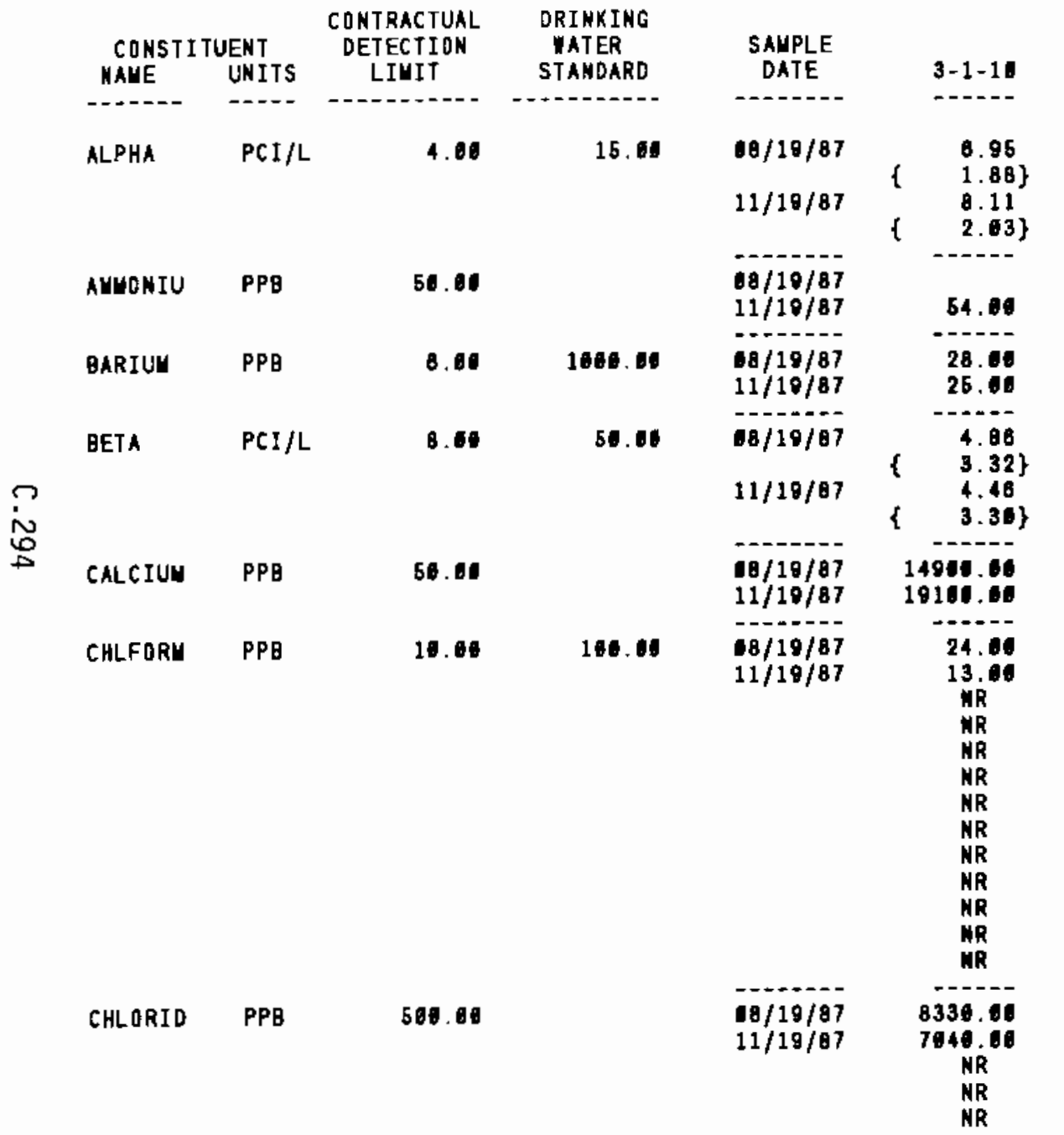

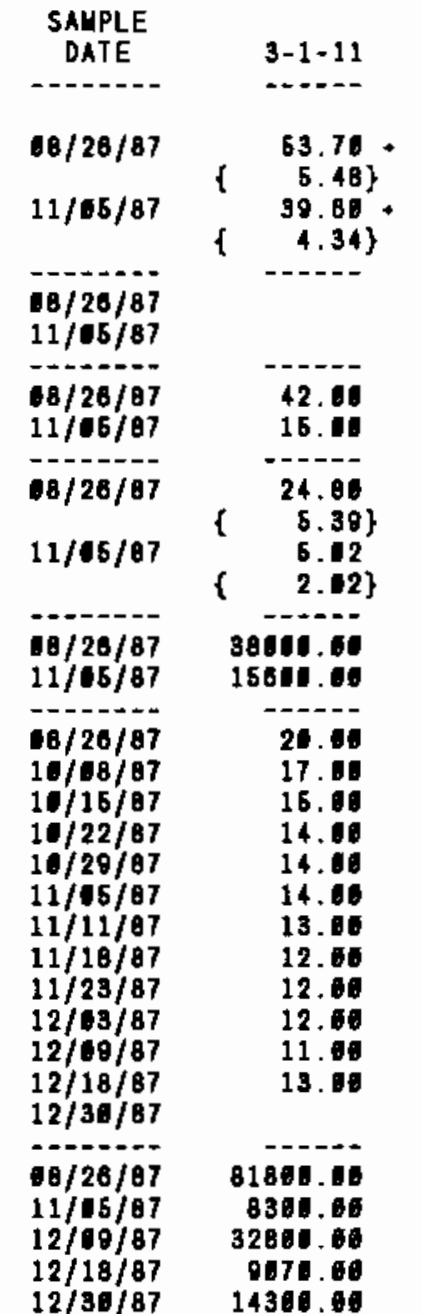

\begin{tabular}{|c|c|}
\hline $\begin{array}{l}\text { SAHPLE } \\
\text { DATE }\end{array}$ & $9-1-12$ \\
\hline$+\cdots----$ & ---- \\
\hline $68 / 20 / 87$ & $32.20+$ \\
\hline $11 / 11 / 87$ & $\begin{array}{c}1 \\
\{\quad 4.80 \\
4\end{array}$ \\
\hline $\begin{array}{l}08 / 20 / B 7 \\
11 / 11 / 87\end{array}$ & -...... \\
\hline $\begin{array}{l}08 / 24 / 07 \\
11 / 11 / 87\end{array}$ & 22.6 \\
\hline $08 / 20 / 87$ & 12.86 \\
\hline $11 / 11 / 87$ & 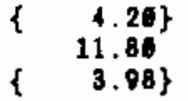 \\
\hline $\begin{array}{l}18 / 20 / 87 \\
11 / 11 / 87\end{array}$ & $\begin{array}{l}14380.08 \\
19106.01\end{array}$ \\
\hline $\begin{array}{l}08 / 20 / 87 \\
11 / 11 / 87\end{array}$ & 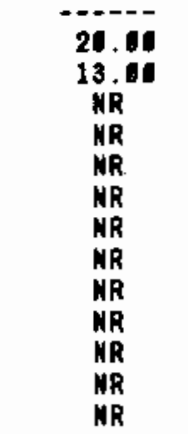 \\
\hline $\begin{array}{l}88 / 26 / 87 \\
11 / 11 / 87\end{array}$ & $\begin{array}{c}6184.00 \\
26860.00 \\
\text { NR } \\
\text { NR } \\
\text { NR }\end{array}$ \\
\hline
\end{tabular}

- - VALUE exceEdS PRIMARY oRINKING vater standard

- YALUE EXCEEDS PROPOSED PRIMARY DRINKING IATER STANDARD

+ - VALUE EXCEEDS SCREENING LEVEL FOR FURTHER INVESTIOATIDN.

- DETECTIDN LIMIT MAS NOT AYAILABLE FDR CDUPARISON

NR - ANALYSIS NDT REQUESTED OR NOT YET REPIRTED

YALUES IN \{\} ARE COUNTIMG ERRORS FDR RADIONUCLIDES

vater standaro(S) In parentheses aRE PRoposed only 
TABLE C.5. (contd)

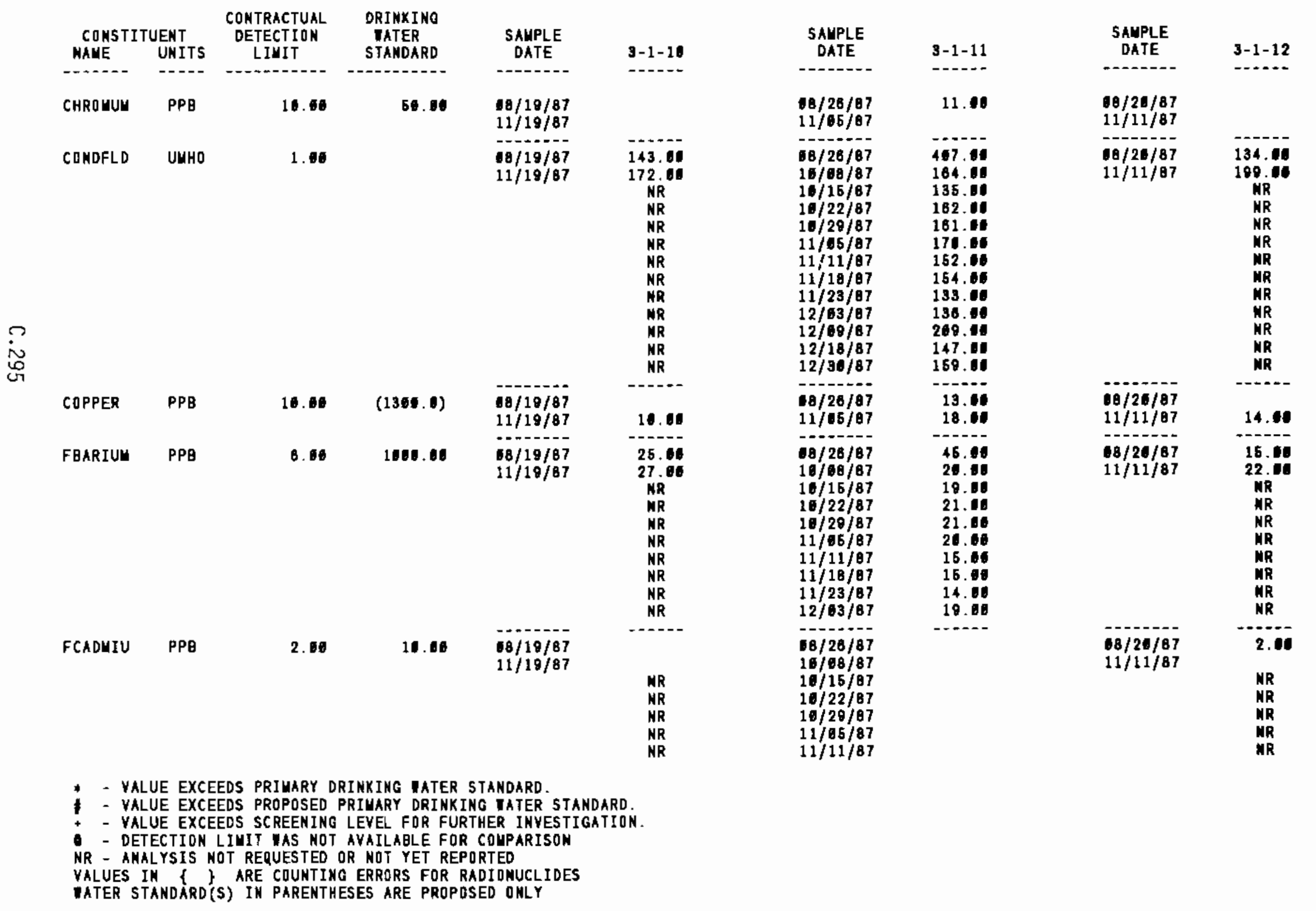


TABLE C.5. (contd)

\begin{tabular}{|c|c|c|c|c|c|}
\hline $\begin{array}{c}\text { CONST I } \\
\text { NALE }\end{array}$ & $\begin{array}{l}\text { UENT } \\
\text { UNITS }\end{array}$ & $\begin{array}{c}\text { CORTRACTUAL } \\
\text { DETECTIDN } \\
\text { LIUIT }\end{array}$ & $\begin{array}{l}\text { DRINKING } \\
\text { WATER } \\
\text { STAMDARD }\end{array}$ & $\begin{array}{c}\text { SAMPLE } \\
\text { DATE }\end{array}$ & $3-1-10$ \\
\hline FCADUIU & PPB & 2.00 & 18.06 & & $\begin{array}{l}\text { NR } \\
\text { NR } \\
\text { NR }\end{array}$ \\
\hline FCALCIU & PPB & 50.00 & & $\begin{array}{l}8 / 19 / 87 \\
11 / 19 / 87\end{array}$ & 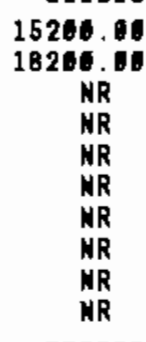 \\
\hline FCOPPER & PPB & 10.06 & $(1300.0)$ & $\begin{array}{l}8 / 10 / 87 \\
11 / 10 / 87\end{array}$ & $\begin{array}{l}\mathrm{NR} \\
\mathrm{NR} \\
\mathrm{NR} \\
\mathrm{NR} \\
\mathrm{NR} \\
\mathrm{NR} \\
\mathrm{NR} \\
\mathrm{NR}\end{array}$ \\
\hline FIRIN & PPB & 58.60 & & $\begin{array}{l}18 / 19 / 87 \\
11 / 19 / 87\end{array}$ & $\begin{array}{l}76.11 \\
\text { NR } \\
\text { NR } \\
\text { NR } \\
\text { NR } \\
\text { NR } \\
\text { NR } \\
\text { NR } \\
\text { NR }\end{array}$ \\
\hline FLUORID & PPB & 508.60 & 4080.00 & $\begin{array}{l}68 / 19 / 87 \\
11 / 19 / 87\end{array}$ & 515.06 \\
\hline
\end{tabular}

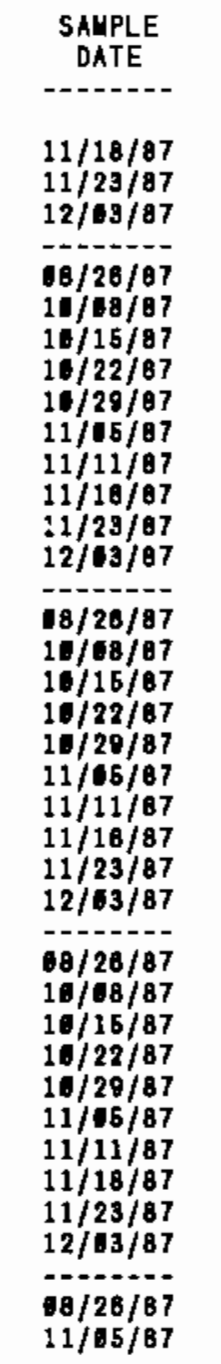

3-1-11

SAMPLE
DATE

(NR

-....

17200.00

18100.00

17101.00

17604.0

18799.

(18700.

18200.00

15795.00

16090.00

17706. 19

12.00

12.01

17. 19

19.00

19.08

28 . 10

26 .

24.06

B/21/87

$11 / 11 / 87$

NR
NR
$-\cdots-2$

(1)

$08 / 20 / 87$

$11 / 11 / 87$

$18 / 21 / 87$

$11 / 11 / 87$

16700.60

10065.06

MR

NR
NR
NR

NR
NR
NR

NR

NR

Nh

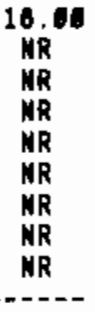

* - Value exceeds primary dRINKing iater standard.

- VALUE EXCEEDS PROPOSED PRIYARY DRINKING TATER STANDARD

- DETECTIDN LIUIT WAS HOT AYAILABLE FOR COMPARISON

NR - ANALYSIS NOT REQUESTED OR NOT YET REPORTED

YALUES IN I S ARE COUNTING ERRORS FOR RADIONUCLIDES

WATER STANDARD(S) IN PARENTHESES ARE PROPOSED ONLY 
TABLE C.5. (contd)

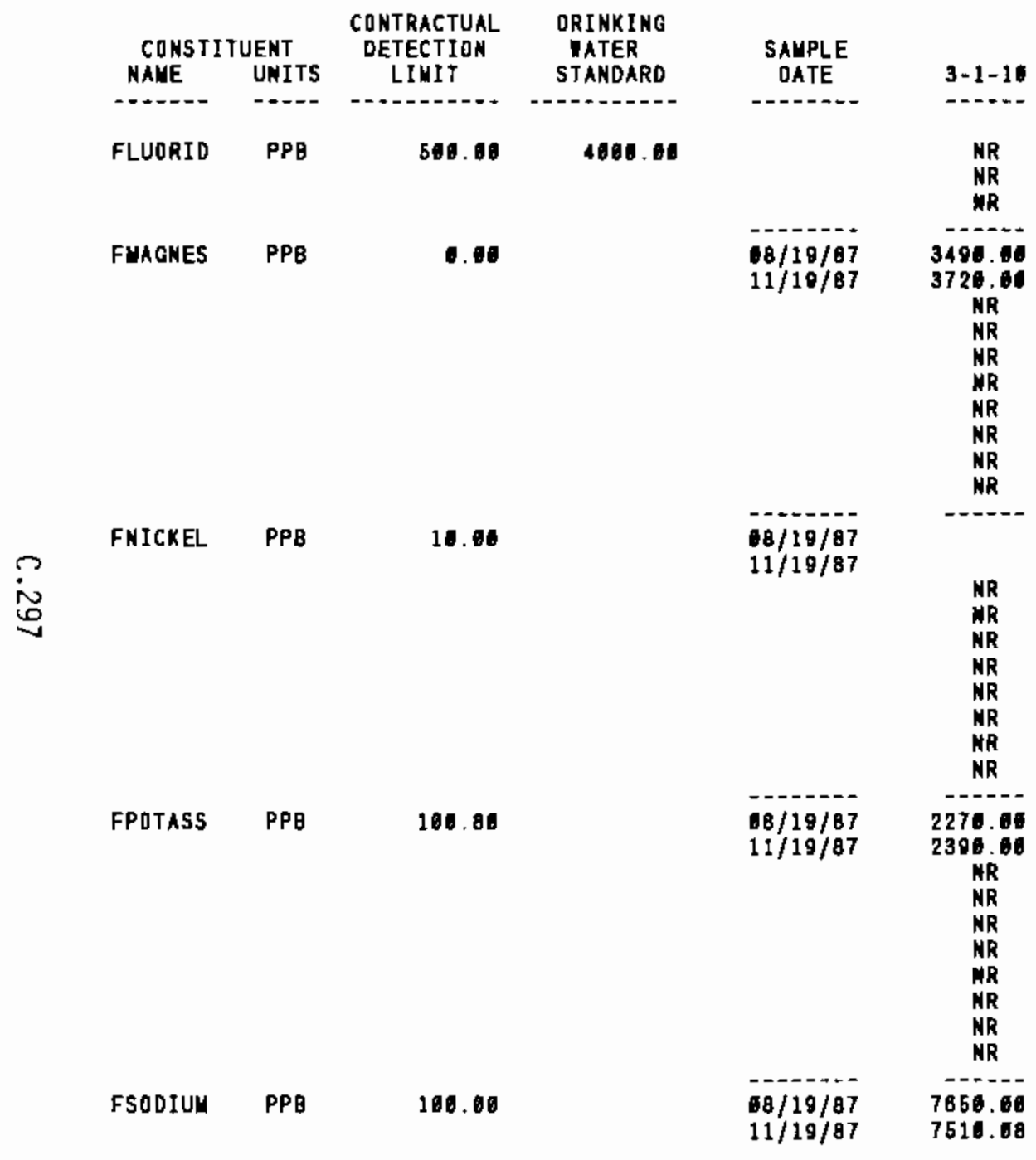

\begin{tabular}{|c|c|}
\hline $\begin{array}{c}\text { SAUPLE } \\
\text { DATE }\end{array}$ & $3-1-11$ \\
\hline $\begin{array}{l}12 / 99 / 87 \\
12 / 18 / 97 \\
12 / 3 E / 87\end{array}$ & \\
\hline $\begin{array}{l}08 / 28 / 87 \\
16 / 68 / 87 \\
10 / 15 / 87 \\
10 / 22 / 87 \\
16 / 29 / 87 \\
11 / 65 / 87 \\
11 / 11 / 87 \\
11 / 18 / 87 \\
11 / 23 / 87 \\
12 / 83 / 87\end{array}$ & 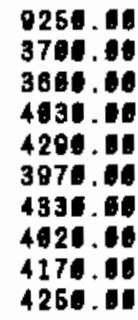 \\
\hline $\begin{array}{l}08 / 28 / 87 \\
16 / 98 / 87 \\
18 / 15 / 87 \\
16 / 22 / 87 \\
10 / 29 / 87 \\
11 / 85 / 87 \\
11 / 11 / 87 \\
11 / 18 / 87 \\
11 / 23 / 87 \\
12 / 03 / 87\end{array}$ & \\
\hline $\begin{array}{l}68 / 26 / 87 \\
16 / 98 / 87 \\
18 / 16 / 97 \\
16 / 22 / 87 \\
10 / 29 / 87 \\
11 / 05 / 87 \\
11 / 11 / 87 \\
11 / 18 / 87 \\
11 / 23 / 87 \\
12 / 69 / 87\end{array}$ & $\begin{array}{l}2840.60 \\
1540.00 \\
1409.00 \\
1430.00 \\
1530.00 \\
1470.00 \\
1520.06 \\
1510.00 \\
1380.00 \\
1360.00\end{array}$ \\
\hline $\begin{array}{l}88 / 28 / 87 \\
16 / 08 / 87\end{array}$ & $\begin{array}{r}19804.60 \\
7470.80\end{array}$ \\
\hline
\end{tabular}

\begin{tabular}{|c|c|}
\hline $\begin{array}{l}\text { SAMPLE } \\
\text { OATE }\end{array}$ & \begin{tabular}{c}
$3-1-12$ \\
\hdashline MR \\
NR \\
NR
\end{tabular} \\
\hline $\begin{array}{l}88 / 20 / 87 \\
11 / 11 / 87\end{array}$ & $\begin{array}{c}3736.60 \\
4340.00 \\
N R \\
N R \\
M R \\
\text { NR } \\
\text { NR } \\
\text { NR } \\
\text { NR } \\
\text { NR }\end{array}$ \\
\hline $\begin{array}{l}68 / 20 / 87 \\
11 / 11 / 87\end{array}$ & $\begin{array}{l}12.00 \\
\text { NR } \\
\text { MR } \\
\text { MR } \\
\text { WR } \\
\text { RR } \\
\text { RR } \\
\text { NR } \\
\text { NR }\end{array}$ \\
\hline $\begin{array}{l}68 / 20 / 87 \\
11 / 11 / 87\end{array}$ & $\begin{array}{c}2196.08 \\
2356 \\
\text { NR } \\
\text { NR } \\
\text { NR } \\
\text { NR } \\
\text { NR } \\
\text { NR } \\
\text { MR } \\
\text { NR }\end{array}$ \\
\hline $\begin{array}{l}88 / 28 / 87 \\
11 / 11 / 87\end{array}$ & $\begin{array}{r}9881.00 \\
15181.06\end{array}$ \\
\hline
\end{tabular}

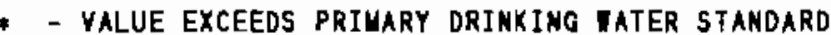

1 - YALUE EXCEEDS PROPOSED PRIMARY DRINKING TATER STANDARD.

- VALUE EXCEEDS SCREENING LEYEL fOR FURTHER IHVESTIGATION.

- Detectidn limit us not available for colparison

NR - ANALYSIS NOT REQUESTED OR NOT YET REPORTED

VALUES IN \{\} ARE CQUNTING ERRORS FOR RADIOHUCLIDES

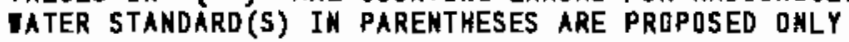


TABLE C.5. (contd)

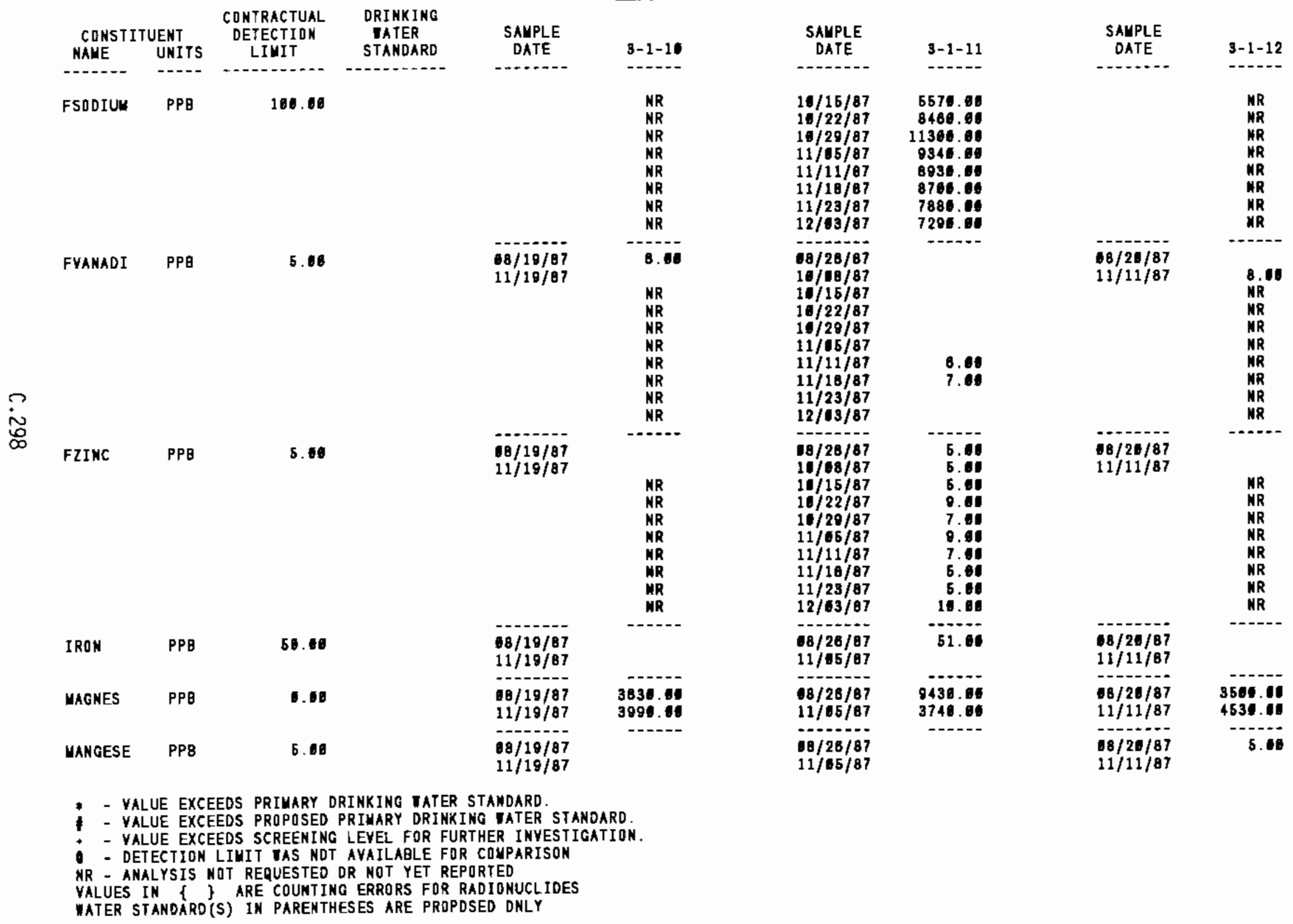


TACLE C.5. (contd)

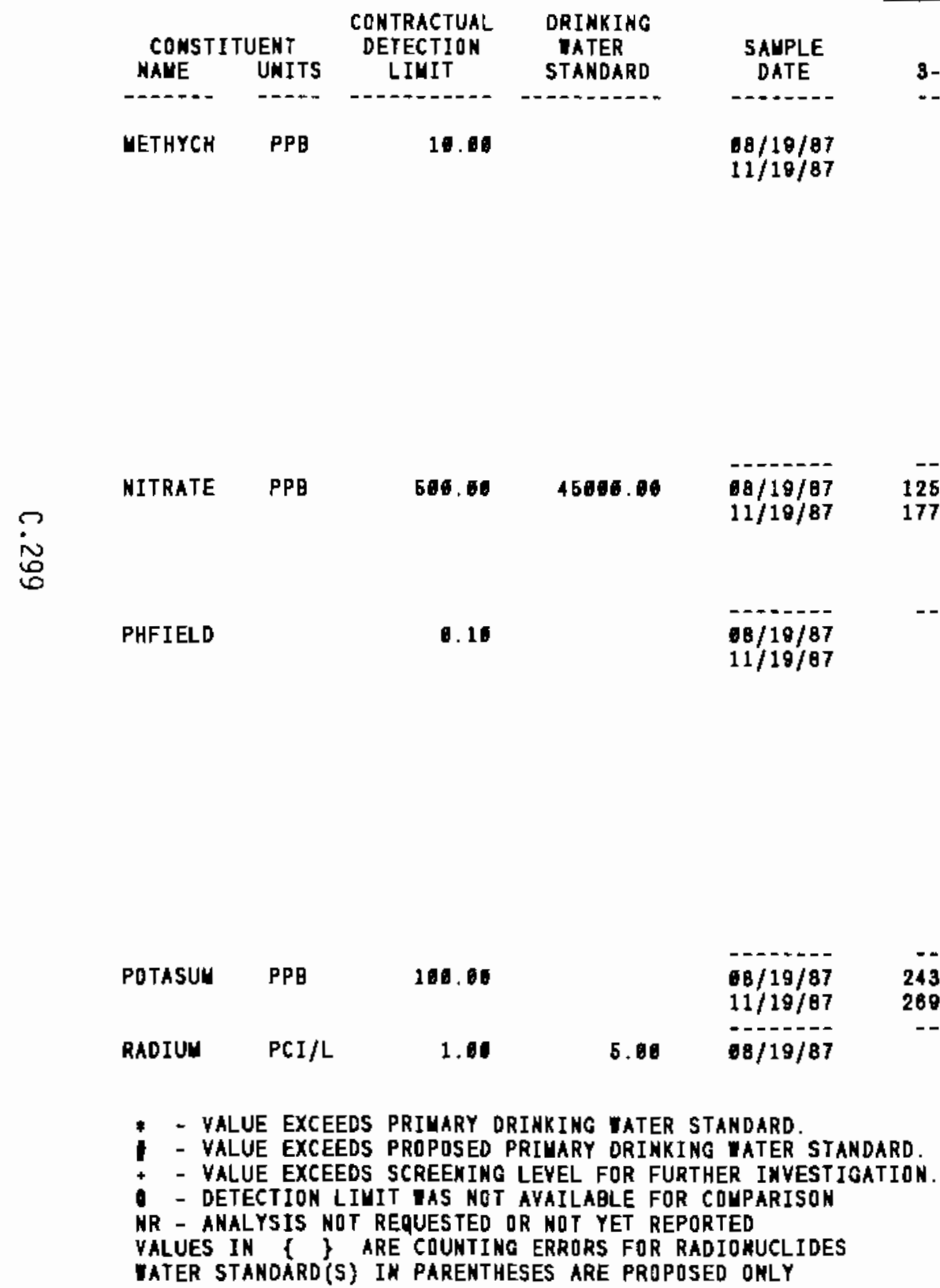

SAUPLE DATE

$-1-10$

SAMPLE

DATE

$3-1-12$

68/26/87

$16 / 08 / 97$

$19 / 16 / 87$

$16 / 29 / 87$

11/05/87

$11 / 06 / 97$

$11 / 11 / 87$

$11 / 18 / 87$

$11 / 23 / 87$

$12 / 03 / 67$

$12 / 80 / 87$

$12 / 18 / 87$

$12 / 31 / 87$

$10 / 26 / 87$

$11 / 65 / 87$

$11 / 65 / 87$

$12 / 69 / 87$

$12 / 18 / 87$

$12 / 30 / 87$

68/26/87

$10 / 08 / 87$

$10 / 15 / 87$

$10 / 22 / 97$

$1 B / 20 / B 7$

$11 / 65 / 87$

$11 / 11 / 87$

$11 / 18 / 87$

$11 / 23 / 87$

$12 / 63 / 87$

$12 / 98 / 87$

$12 / 18 / 87$

$12 / 30 / 87$

$-2-1-5$

$08 / 26 / 87$

$11 / 05 / 87$

$08 / 26 / 87$
18.64

0日/26/87

$11 / 11 / 87$

1336.

1336.0

1700.

2660.09

1000.00

6.60

7.00

6.90

6.3

6. 8

B. 91

7.1

7.34

7.10

6. 10

7.06

7.20

6.60

0.

2740.86

1356.6

-.-

0.14
o. 14$\}$

\begin{tabular}{|c|c|}
\hline & $\begin{array}{l}\mathrm{NR} \\
\mathrm{NR} \\
\mathrm{NR} \\
\mathrm{MR} \\
\mathrm{MR} \\
\mathrm{NR} \\
\mathrm{NR} \\
\mathrm{NR} \\
\mathrm{NR} \\
\mathrm{NR} \\
\mathrm{NR} \\
\mathrm{NR}\end{array}$ \\
\hline $\begin{array}{l}8 / 26 / 87 \\
1 / 11 / 87\end{array}$ & $\begin{array}{c}1380.00 \\
\text { NR } \\
\text { NR } \\
\text { NR }\end{array}$ \\
\hline $\begin{array}{l}88 / 28 / 87 \\
11 / 11 / 87\end{array}$ & $\begin{array}{l}6.40 \\
7.1 \\
\text { NR } \\
\text { NR } \\
\text { NR } \\
\text { NR } \\
\text { NR } \\
\text { NR } \\
\text { NR } \\
\text { MR } \\
\text { NR } \\
\text { NR } \\
\text { NR }\end{array}$ \\
\hline $\begin{array}{l}88 / 28 / 87 \\
11 / 11 / 87\end{array}$ & $\begin{array}{l}2150.00 \\
2506.06\end{array}$ \\
\hline
\end{tabular}


IABLE C.5. (contd)

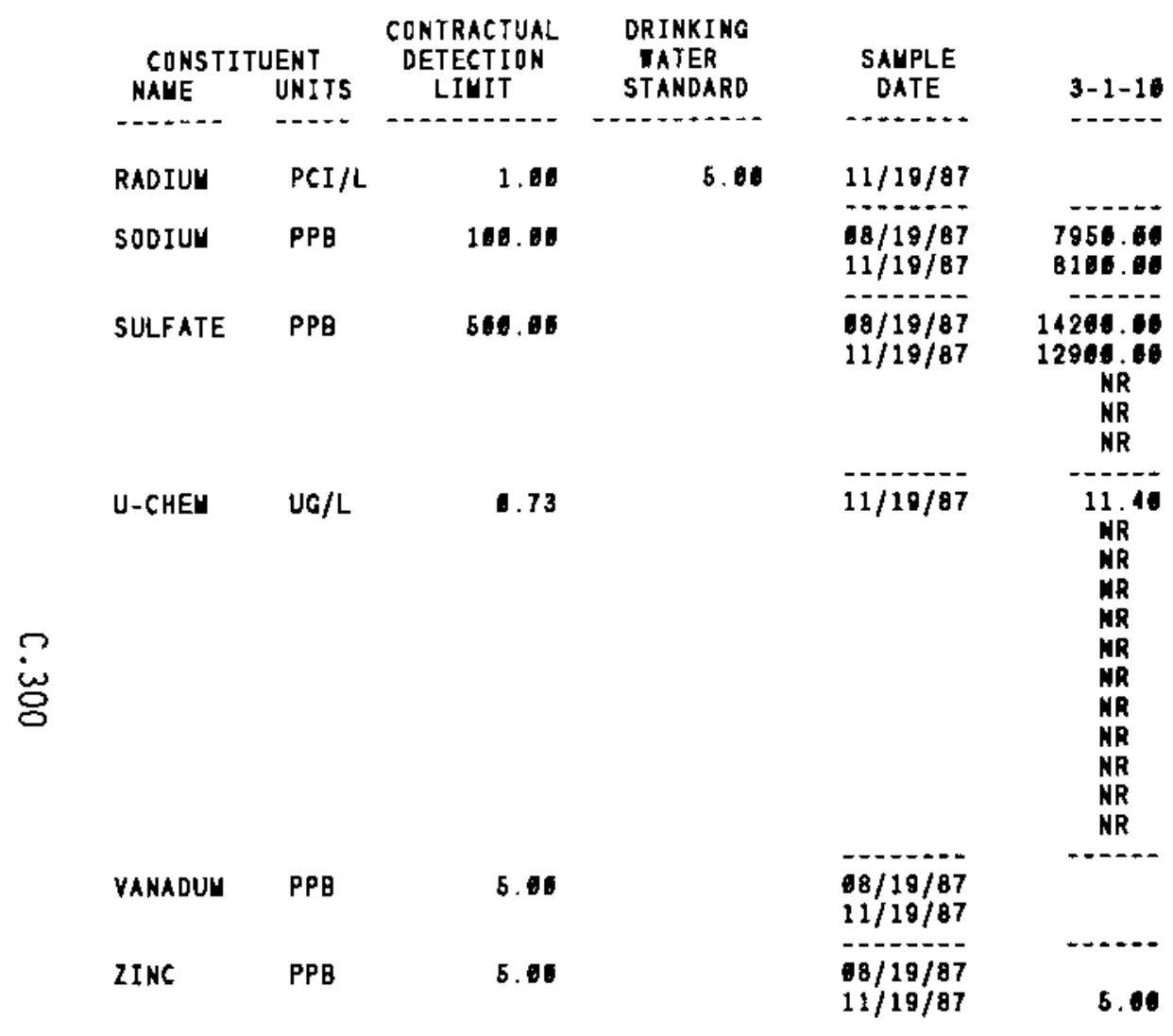

\begin{tabular}{|c|c|}
\hline $\begin{array}{c}\text { SAUPLE } \\
\text { DATE }\end{array}$ & $3-1-11$ \\
\hline$--1-0-0$ & ----- \\
\hline \multicolumn{2}{|l|}{$11 / 05 / 07$} \\
\hline $\begin{array}{l}18 / 26 / 87 \\
11 / 65 / 87\end{array}$ & $\begin{array}{r}20660.00 \\
8550.08\end{array}$ \\
\hline $\begin{array}{l}68 / 28 / 87 \\
11 / 05 / 87 \\
12 / 69 / 87 \\
12 / 18 / 87 \\
12 / 30 / 87\end{array}$ & $\begin{array}{l}13604.05 \\
13360.04 \\
16000.05 \\
13600.05 \\
13760.00\end{array}$ \\
\hline $\begin{array}{l}10 / 08 / 87 \\
10 / 16 / 87 \\
10 / 22 / 87 \\
10 / 20 / 87 \\
11 / 06 / 87 \\
11 / 11 / 87 \\
11 / 16 / 87 \\
11 / 23 / 87 \\
12 / 09 / 87 \\
12 / 09 / 87 \\
12 / 18 / 87 \\
12 / 91 / 87\end{array}$ & $\begin{array}{l}33.80 \\
46.10 \\
55.81 \\
44.81 \\
35.40 \\
46.50 \\
44.80 \\
48.10 \\
54.20 \\
61.80 \\
81.46 \\
78.81\end{array}$ \\
\hline $\begin{array}{l}68 / 28 / 87 \\
11 / 06 / 87\end{array}$ & 5.01 \\
\hline $\begin{array}{l}18 / 26 / 87 \\
11 / 06 / 87\end{array}$ & $\begin{array}{r}6.00 \\
25.00\end{array}$ \\
\hline
\end{tabular}

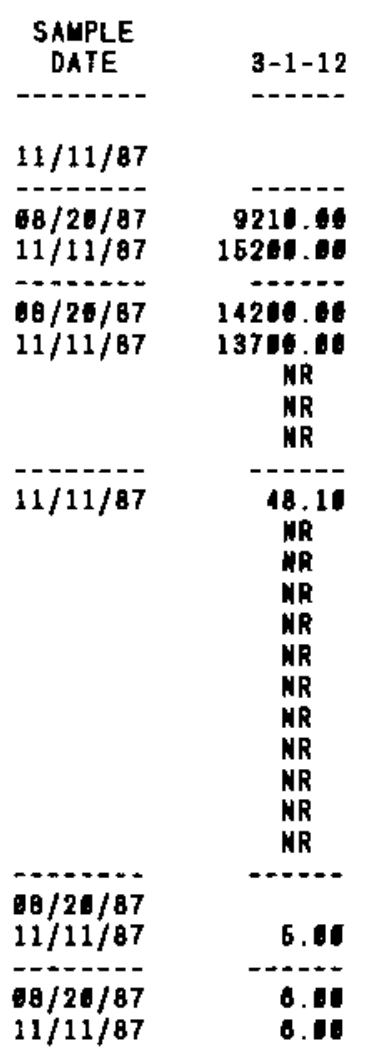

* - VAlUe eXCEedS PRIUARY dRINKIng tater STANDARD.

- - VALUE EXCEEDS PROPOSED PRIMARY DRINKING IATER STANDARD.

* - VALUE EXCEEDS SCREENING LEVEL FOR FURTHER INYESTIGATION.

- DETECTIOM LIUIT IAS NOT AVAILABLE FOR COHPARISON

NR - ANALYSIS NDT REQUESTED OR NOT YET REPDRTED

YALUES IN I I ARE COUNTING ERRORS FOR RADIDNUCLIDES

IATER STANDARD(S) IM PARENTHESES ARE PROPOSED ONLY 
TABLE C.5. (contd)

\begin{tabular}{|c|c|c|c|c|c|}
\hline $\begin{array}{c}\text { COMST I } \\
\text { NAME }\end{array}$ & $\begin{array}{l}\text { UENT } \\
\text { UNITS }\end{array}$ & $\begin{array}{l}\text { CONTRACTUAL } \\
\text { DETECTION } \\
\text { LIUIT }\end{array}$ & $\begin{array}{l}\text { DRINKING } \\
\text { TATER } \\
\text { STANDARO }\end{array}$ & $\begin{array}{c}\text { SAUPLE } \\
\text { DATE }\end{array}$ & $3-1-13$ \\
\hline ALPHA & $\mathrm{PCI} / \mathrm{L}$ & 4.66 & 15.00 & $\begin{array}{l}08 / 25 / 87 \\
08 / 25 / 87 \\
11 / 18 / 87 \\
11 / 18 / 87\end{array}$ & 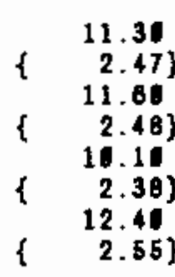 \\
\hline BARIUU & PPB & 8.10 & 1006.06 & $\begin{array}{l}68 / 25 / 87 \\
11 / 18 / 87\end{array}$ & $\begin{array}{l}32.11 \\
22.11\end{array}$ \\
\hline BETA & $\mathrm{PCI} / \mathrm{L}$ & 8. 60 & 50.00 & $\begin{array}{l}08 / 25 / 87 \\
88 / 25 / 87 \\
11 / 18 / 87 \\
11 / 18 / 87\end{array}$ & 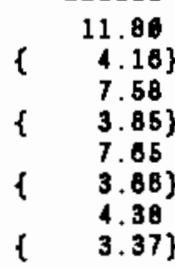 \\
\hline CADYIUH & PPB & 2.0 & 10.06 & $\begin{array}{l}68 / 25 / 87 \\
11 / 18 / 87\end{array}$ & 2.66 \\
\hline CALCIUU & PPB & 50.60 & & $\begin{array}{l}68 / 25 / 87 \\
11 / 18 / 87\end{array}$ & $\begin{array}{l}24300.01 \\
25100.00\end{array}$ \\
\hline CHLFORY & $\mathrm{PPB}$ & 10.00 & 106.00 & $\begin{array}{l}68 / 25 / 87 \\
68 / 25 / 87 \\
11 / 18 / 87 \\
11 / 18 / 87\end{array}$ & $\begin{array}{l}14.00 \\
14.00 \\
11.06 \\
11.06\end{array}$ \\
\hline CHLORID & PPB & 500.06 & & $\begin{array}{l}68 / 25 / 87 \\
68 / 25 / 87 \\
11 / 18 / 87 \\
11 / 18 / 87\end{array}$ & $\begin{array}{l}9739.60 \\
839.69 \\
8678.90 \\
8606.80\end{array}$ \\
\hline CHROYUM & PPB & 10.80 & 50.06 & $08 / 25 / 87$ & 12.08 \\
\hline
\end{tabular}

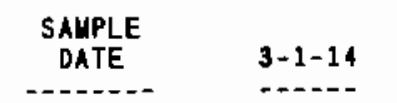

68/26/87

$11 / 18 / 87$

12.56
$\{\quad 2.38\}$
18.06
$\{\quad 2.28\}$

NR

NR
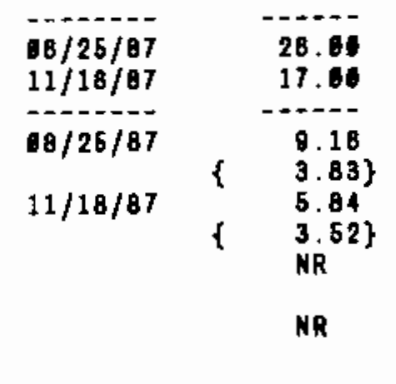

$08 / 25 / 87$

$11 / 18 / 87$

$08 / 25 / 87$

$11 / 18 / 87$

18100.06

21800.00

$08 / 25 / 87$

$11 / 18 / 87$

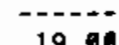

19.80
11.08
$N R$

NR

$88 / 25 / 87$

$11 / 18 / 87$

5720.08

6806.00

NR
NR

12.06

$68 / 25 / 67 \quad 13.60$

$8 B / 19 / 87$
18/19/87

$68 / 19 / 87$
$11 / 16 / 87$

$68 / 19 / 87$

$11 / 16 / 87$

$\begin{array}{cc}\text { SAYPLE } \\ \text { DATE }\end{array} \quad 3-1-15$

$88 / 19 / 87 \quad 6.32$

$\begin{array}{lll}11 / 16 / 87 & \left\{\begin{array}{l}2.27\} \\ 5.25\end{array}\right.\end{array}$

5.25
$1.76)$

NR

MR

-.-2.

46.11

22.60

13.10
$4.83\}$

$\{\quad 4.83\}$
$\quad 5.81$

5.81
$3.46\}$

NR

MR

88/19/67

$11 / 16 / 87$

11/18/87

35100.01

-

$08 / 19 / 87$

$11 / 16 / 87$

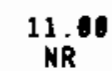

NR

NR

$09 / 19 / 87$

$11 / 16 / 87$

17960.06

7320.06

NR

* - Value exceeds primary drinking nater standard.

* - VALUE EXcEeds PROPDSED PRIMARY DRINKING taTER STANDARD.

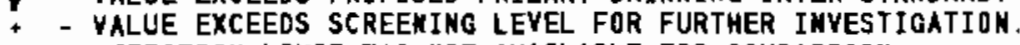

- Detection liUit Mas not availagle for couparison

NR - ANALYSIS NOT REQUESTED OR NDT YET REPORTED

VALUES IN $\{$ ARE COUNTING ERRORS FOR RADIONUCLIDES

IATER STANOARD'(S) IN PARENTHESES ARE PROPOSED ONLY 
TABLE C.5. (contd)

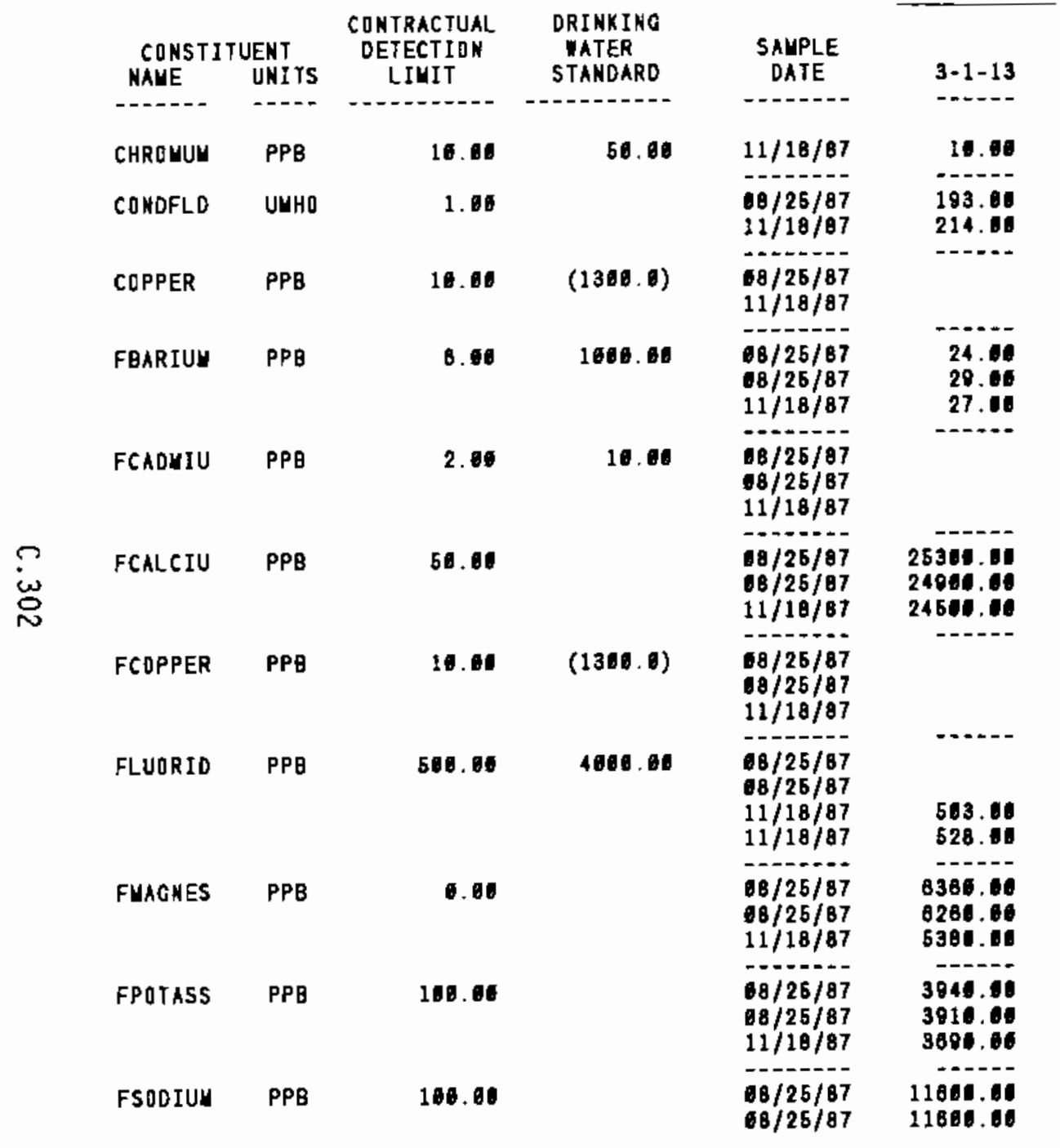

\begin{tabular}{|c|c|}
\hline $\begin{array}{l}\text { SAMPLE } \\
\text { DATE }\end{array}$ & $3-1-14$ \\
\hline $11 / 18 / 87$ & 16.71 \\
\hline $\begin{array}{l}68 / 25 / 87 \\
11 / 10 / 87\end{array}$ & $\begin{array}{l}154.80 \\
175.80\end{array}$ \\
\hline $\begin{array}{l}18 / 25 / 87 \\
11 / 18 / 87\end{array}$ & $\begin{array}{l}13.60 \\
10.86\end{array}$ \\
\hline $\begin{array}{l}88 / 25 / 87 \\
11 / 18 / 87\end{array}$ & $\begin{array}{l}24.00 \\
20.00 \\
N R\end{array}$ \\
\hline $\begin{array}{l}88 / 26 / 87 \\
11 / 18 / 87\end{array}$ & \\
\hline $\begin{array}{l}68 / 25 / 87 \\
11 / 18 / 87\end{array}$ & $\begin{array}{l}18000.00 \\
21004.00 \\
\text { MR }\end{array}$ \\
\hline $\begin{array}{l}08 / 25 / 87 \\
11 / 18 / 87\end{array}$ & 11.10 \\
\hline $\begin{array}{l}18 / 25 / 87 \\
11 / 18 / 87\end{array}$ & $\begin{array}{l}\text { NR } \\
\text { NR }\end{array}$ \\
\hline $\begin{array}{l}18 / 25 / 87 \\
11 / 18 / 87\end{array}$ & $\begin{array}{l}4116.00 \\
4980.00 \\
\mathrm{NR}\end{array}$ \\
\hline $\begin{array}{l}68 / 25 / 87 \\
11 / 18 / 87\end{array}$ & $\begin{array}{c}2780.60 \\
2790.06 \\
\text { NR }\end{array}$ \\
\hline $\begin{array}{l}08 / 25 / 87 \\
11 / 18 / 87\end{array}$ & $\begin{array}{l}8101.60 \\
7146.06\end{array}$ \\
\hline
\end{tabular}

SAYPLE

DATE $\quad 3-1-15$

$11 / 18 / 87$

$88 / 19 / 97$

$11 / 18 / 87$

$00 / 19 / 87$

$11 / 16 / 87$

$0 / 18 / 87$

$11 / 18 / 87$

$-\cdots \cdot 19 / 97$

$08 / 19 / 87$
$11 / 16 / 87$

-

a1.

180.10

......

42.06

22.06

NR

2.00

NR

$08 / 19 / 87 \quad 39200.61$

11/18/87 21406.06

NR

$-1 .-1$

$08 / 19 / 87$
$11 / 18 / 87$

-NR

- MR

$08 / 10 / 87$

$11 / 16 / 87$

NR

$08 / 19 / 87$

$11 / 18 / 87$

16860.06

52106.10 $\mathrm{NR}$

$68 / 19 / 97$

$11 / 18 / 97$

5420.00 1160.60

$\mathrm{MR}$

$0 / 10 / 87$

$11 / 10 / 87$

26508.01

11218.08

* - value exceeds privary drinking iater standard.

- - VALUE EXCEEDS PROPOSED PRIUARY ORINKING TATER STANDARD.

* - VALUE EXCEEDS SCREENING LEVEL FOR FURTHER INVESTIGATION.

- detectio* liUit uas not availagle for couparison

NR - AHALYSIS NOT REQUESTED OR NOT YET REPORTED

YALUES IN I ARE COUNTING ERRORS FOR RADIONUCLIDES

IATER STANDARD(S) IN PARENTHESES ARE PROPOSED ONLY 
TABLE C.5. (contd)

\begin{tabular}{|c|c|c|c|c|c|}
\hline \multicolumn{2}{|c|}{ CONSTITUENT } & $\begin{array}{l}\text { COYTRAC TUAL } \\
\text { DETECTIION }\end{array}$ & $\begin{array}{l}\text { DRINKING } \\
\text { IATER } \\
\text { STER }\end{array}$ & SAMPLE & $3-1-13$ \\
\hline --.....- & 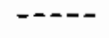 & $+\cdots$ & & -......-- & $-\cdots$ \\
\hline FSODIUY & PPB & 160.60 & & $11 / 10 / 87$ & 10300.00 \\
\hline FYANADI & PPB & 6.00 & & $\begin{array}{l}08 / 25 / 97 \\
18 / 25 / 87 \\
11 / 18 / 87\end{array}$ & $\begin{array}{l}8.60 \\
7.60\end{array}$ \\
\hline F2INC & PPB & 6.66 & & $\begin{array}{l}68 / 25 / 87 \\
68 / 25 / 87 \\
11 / 18 / 87\end{array}$ & $\begin{array}{r}44.69 \\
8.18\end{array}$ \\
\hline IRDN & PPB & 50.10 & & $\begin{array}{l}08 / 25 / 87 \\
11 / 18 / 87\end{array}$ & $\begin{array}{l}76.00 \\
88.00\end{array}$ \\
\hline HAGNES & PPB & 6.06 & & $\begin{array}{l}68 / 25 / 87 \\
11 / 18 / 87\end{array}$ & $\begin{array}{l}8698.80 \\
5450.86\end{array}$ \\
\hline UAMGESE & PPQ & 5.60 & & $\begin{array}{l}88 / 25 / 87 \\
11 / 18 / 87\end{array}$ & 5.00 \\
\hline VETHDME & PPB & 10.86 & & $\begin{array}{l}08 / 25 / 87 \\
08 / 25 / 87 \\
11 / 18 / 07 \\
11 / 18 / 87\end{array}$ & 18.00 \\
\hline METHYCH & $P P B$ & 10.00 & & $\begin{array}{l}88 / 25 / 87 \\
08 / 25 / 97 \\
11 / 18 / 87 \\
11 / 18 / 87\end{array}$ & \\
\hline NITRATE & PPB & 500.80 & 4500000 & $\begin{array}{l}08 / 25 / 87 \\
08 / 25 / 87 \\
11 / 18 / 87 \\
11 / 18 / 87\end{array}$ & $\begin{array}{l}8286.00 \\
8450.69 \\
3528.08 \\
3528.86\end{array}$ \\
\hline PHFIELD & & 0.18 & & $\begin{array}{l}08 / 25 / 87 \\
11 / 18 / 87\end{array}$ & $\begin{array}{l}7.38 \\
7.58\end{array}$ \\
\hline POTASUM & PPB & 100.00 & & $\begin{array}{l}88 / 25 / 87 \\
11 / 18 / 87\end{array}$ & $\begin{array}{l}3710.00 \\
3690.00\end{array}$ \\
\hline
\end{tabular}

\begin{tabular}{|c|c|}
\hline $\begin{array}{l}\text { SAMPLE } \\
\text { DATE }\end{array}$ & $3-1-14$ \\
\hline & MR \\
\hline $\begin{array}{l}68 / 25 / 87 \\
11 / 18 / 87\end{array}$ & 6.10 \\
\hline $\begin{array}{l}18 / 25 / 87 \\
11 / 18 / 87\end{array}$ & NR \\
\hline $\begin{array}{l}18 / 26 / 87 \\
11 / 18 / 87\end{array}$ & 67.00 \\
\hline $\begin{array}{l}68 / 25 / 87 \\
11 / 18 / 87\end{array}$ & $\begin{array}{l}4120.80 \\
4371.01\end{array}$ \\
\hline $\begin{array}{l}18 / 25 / 87 \\
11 / 18 / 87\end{array}$ & 5.00 \\
\hline $\begin{array}{l}00 / 25 / 87 \\
11 / 18 / 87\end{array}$ & $\begin{array}{l}\text { NR } \\
\text { NR }\end{array}$ \\
\hline $\begin{array}{l}80 / 25 / 87 \\
11 / 18 / 87\end{array}$ & $\underset{\text { NR }}{\mathrm{NR} .01}$ \\
\hline $\begin{array}{l}80 / 25 / 87 \\
11 / 18 / 87\end{array}$ & $\begin{array}{c}1870.80 \\
18 B 9.80 \\
\text { NR } \\
\text { NR }\end{array}$ \\
\hline $\begin{array}{l}08 / 25 / 87 \\
11 / 18 / 87\end{array}$ & 7.10 \\
\hline $\begin{array}{l}88 / 25 / 87 \\
11 / 18 / 87\end{array}$ & $\begin{array}{l}2830.80 \\
2749.86\end{array}$ \\
\hline
\end{tabular}

\begin{tabular}{|c|c|}
\hline $\begin{array}{l}\text { SAUPLE } \\
\text { DATE }\end{array}$ & $3-1-15$ \\
\hline & NR \\
\hline $\begin{array}{l}68 / 18 / 87 \\
11 / 16 / 87\end{array}$ & $\begin{array}{r}11.00 \\
7.11\end{array}$ \\
\hline $\begin{array}{l}18 / 19 / 87 \\
11 / 18 / 87\end{array}$ & \\
\hline & NR \\
\hline $\begin{array}{l}08 / 18 / 87 \\
11 / 18 / 87\end{array}$ & 06.18 \\
\hline $\begin{array}{l}68 / 19 / 87 \\
11 / 16 / 87\end{array}$ & $\begin{array}{r}10766.09 \\
4886.01\end{array}$ \\
\hline $\begin{array}{l}68 / 19 / 07 \\
11 / 18 / 87\end{array}$ & \\
\hline $\begin{array}{l}6 / 19 / 87 \\
11 / 16 / 87\end{array}$ & $\begin{array}{l}\text { NR } \\
\text { NR }\end{array}$ \\
\hline $\begin{array}{l}68 / 18 / 97 \\
11 / 18 / 87\end{array}$ & $\begin{array}{l}\text { MR } \\
\text { NR }\end{array}$ \\
\hline $\begin{array}{l}68 / 19 / 87 \\
11 / 18 / 87\end{array}$ & $\begin{array}{c}19365.00 \\
1870.00 \\
\text { NR } \\
\text { KR }\end{array}$ \\
\hline $\begin{array}{l}98 / 19 / 87 \\
11 / 16 / 87\end{array}$ & $\begin{array}{l}7.30 \\
7.71\end{array}$ \\
\hline $\begin{array}{l}88 / 19 / 87 \\
11 / 18 / 87\end{array}$ & $\begin{array}{l}5650.00 \\
4696.00\end{array}$ \\
\hline
\end{tabular}

* - yalue exceeds primary dRinking uater standaro

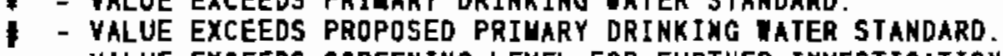

* - yalue exceeds SCREenino leVel for fuRTher inYestigation.

- Detection limit tas not ayailable for couparison

NR - ANALYSIS NOT REQUESTED OR NOT YET REPORTED

VALUES IN \{\} ARE COUNTINO ERRORS FOR RADIONUCLIDES

IATER STAMDARD(S) IN PAREMTHESES ARE PROPOSED ONLY 
TABLE C.5. (contd)

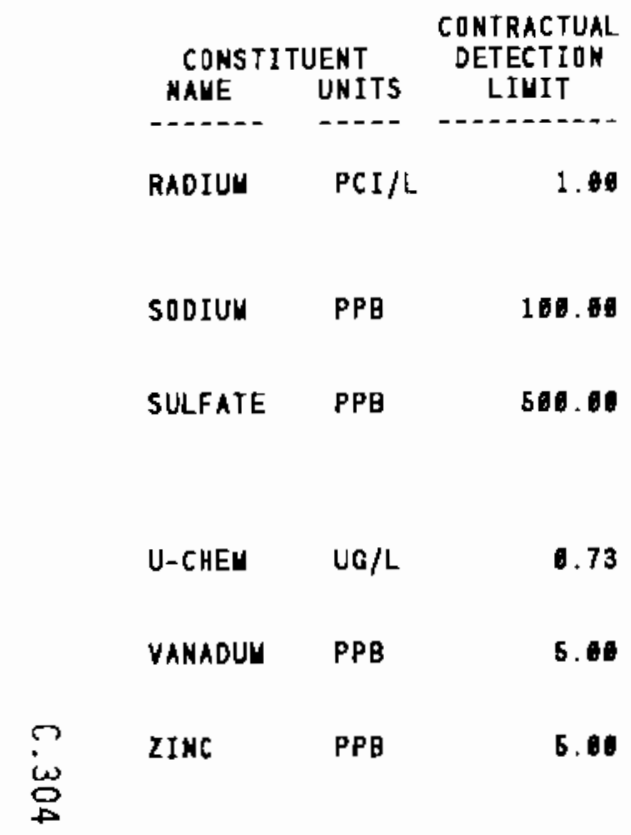

\begin{tabular}{|c|c|c|c|}
\hline $\begin{array}{l}\text { SAUPLE } \\
\text { DATE }\end{array}$ & $3-1-13$ & $\begin{array}{c}\text { SAHPLE } \\
\text { DATE }\end{array}$ & $3-1-14$ \\
\hline 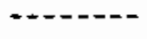 &.---- & 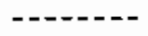 & $\cdots--$ \\
\hline $68 / 25 / 87$ & $\begin{array}{l}.27 \\
0.26\}\end{array}$ & $08 / 25 / 87$ & \\
\hline $11 / 18 / 67$ & & $11 / 18 / 87$ & \\
\hline $\begin{array}{l}68 / 25 / 87 \\
11 / 18 / 87\end{array}$ & $\begin{array}{l}11500.00 \\
16100.06\end{array}$ & $\begin{array}{l}18 / 25 / 87 \\
11 / 18 / 87\end{array}$ & $\begin{array}{l}8180.00 \\
7350.00\end{array}$ \\
\hline $\begin{array}{l}68 / 25 / 87 \\
68 / 26 / 87 \\
11 / 18 / 87 \\
11 / 18 / 87\end{array}$ & $\begin{array}{l}24200.06 \\
23300.06 \\
17506.66 \\
17360.06\end{array}$ & $\begin{array}{l}10 / 25 / 87 \\
11 / 18 / 87\end{array}$ & $\begin{array}{c}14600.00 \\
12006.06 \\
M R \\
\text { NR }\end{array}$ \\
\hline $\begin{array}{l}11 / 18 / 87 \\
11 / 18 / 87\end{array}$ & 13.10 & $11 / 18 / 87$ & 18,16 \\
\hline $\begin{array}{l}18 / 26 / 87 \\
11 / 18 / 87\end{array}$ & 9.01 & $\begin{array}{l}68 / 25 / 87 \\
11 / 10 / 87\end{array}$ & 8.00 \\
\hline $\begin{array}{l}88 / 25 / 87 \\
11 / 18 / 87\end{array}$ & B.6 & $\begin{array}{l}18 / 25 / 97 \\
11 / 18 / 87\end{array}$ & 16.16 \\
\hline
\end{tabular}

\begin{tabular}{|c|c|}
\hline $\begin{array}{l}\text { SAMPLE } \\
\text { DATE }\end{array}$ & $3-1-16$ \\
\hline------- & 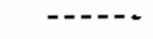 \\
\hline $08 / 19 / 87$ & \\
\hline $11 / 16 / 87$ & \\
\hline$\cdots$ & $-\ldots$ \\
\hline $\begin{array}{l}08 / 19 / 87 \\
11 / 16 / 87\end{array}$ & $\begin{array}{r}21160.06 \\
9780.40\end{array}$ \\
\hline $\begin{array}{l}98 / 10 / 87 \\
11 / 16 / 87\end{array}$ & $\begin{array}{c}45800 \\
14500.06 \\
\text { NR } \\
\text { YR }\end{array}$ \\
\hline $11 / 16 / 87$ & 7.87 \\
\hline $\begin{array}{l}18 / 18 / 87 \\
11 / 16 / 07\end{array}$ & $\begin{array}{r}0.06 \\
16.06\end{array}$ \\
\hline $\begin{array}{l}08 / 19 / 87 \\
11 / 16 / 87\end{array}$ & \\
\hline
\end{tabular}

- - VAlUE eXCEEDS PRIUARY dRIMKIng mater standard.

- - VALUE EXCEEDS PROPOSED PRIUARY DRINKING TATER STANDARD.

- - VALUE EXCEEOS SCREENING LEVEL FOR FURTHER INYESTIGATION

- DETECTIOH LIUIT IAS NDT AVAILABLE fOR COUPARISON

NR - ANALYSIS NOT REQUESTED OR NOT YET REPORTEO

VALUES IN \{ $\}$ ARE COUNTING ERRORS FOR RADIONUCLIDES

IATER STANDARD(S) IN PARENTHESES ARE PROPOSED ONLY 


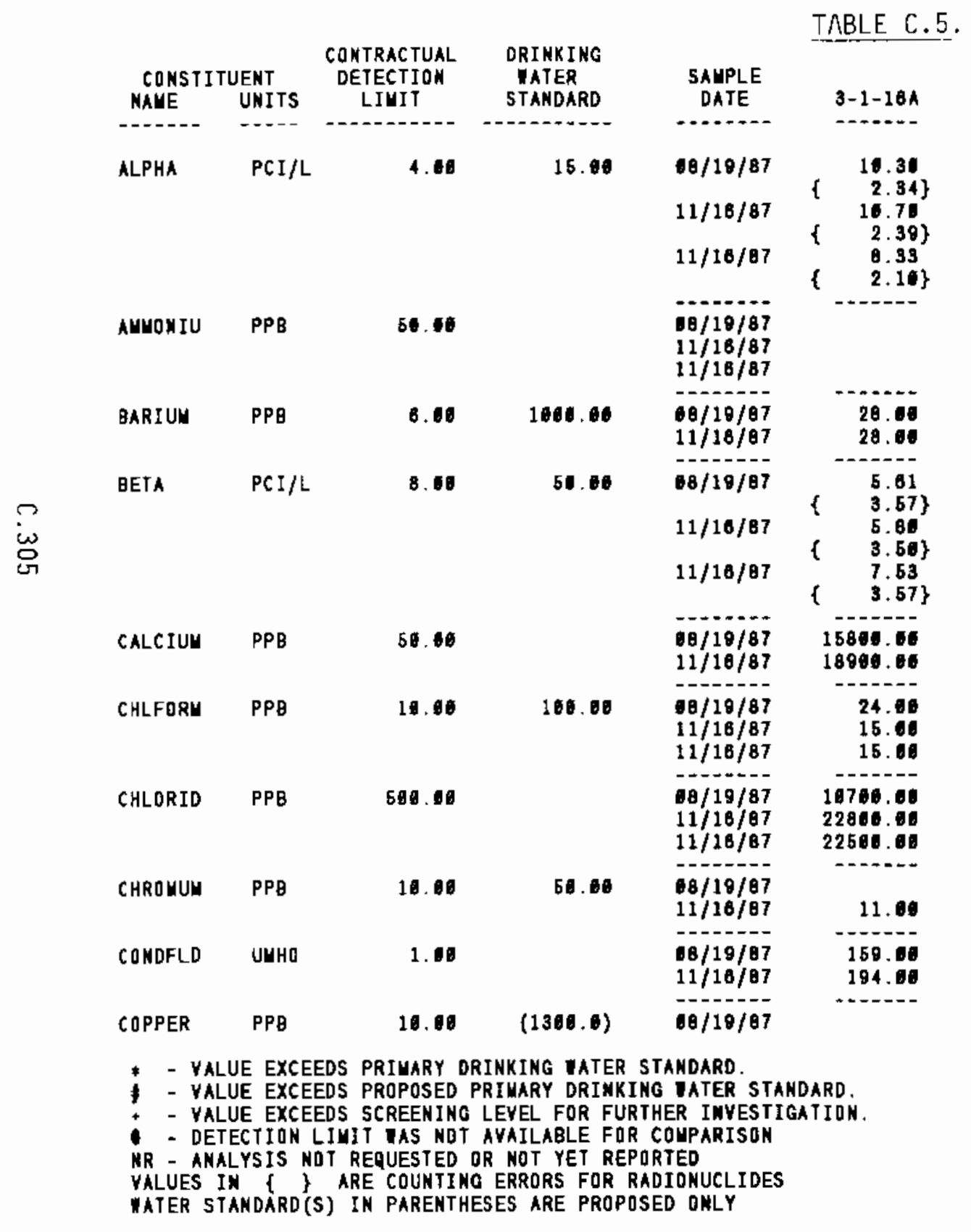

(contd)

\begin{tabular}{|c|c|c|c|}
\hline $\begin{array}{l}\text { SAMPLE } \\
\text { DATE }\end{array}$ & $3-1-168$ & $\begin{array}{c}\text { SAUPLE } \\
\text { DATE }\end{array}$ & $3-1-18 C$ \\
\hline $08 / 19 / 87$ & 2.18 & $08 / 19 / 87$ & \\
\hline $11 / 18 / 87$ & $\left.\begin{array}{ll}\{ & \begin{array}{l}1.55\} \\
\{\end{array} \\
\{ & 2.10 \\
& 1.55\}\end{array}\right\}$ & $11 / 16 / 87$ & MR \\
\hline $\begin{array}{l}6 / 119 / 87 \\
11 / 18 / 87\end{array}$ & $\begin{array}{c}97.60 \\
199.61 \\
\text { NR }\end{array}$ & $\begin{array}{l}68 / 19 / 97 \\
11 / 16 / 87\end{array}$ & $\begin{array}{c}135.06 \\
\text { BQ.06 } \\
\mathrm{MR}\end{array}$ \\
\hline $\begin{array}{l}0 / 19 / 87 \\
11 / 18 / 87\end{array}$ & 56.00 & $\begin{array}{l}68 / 19 / 87 \\
11 / 16 / 87\end{array}$ & B8. \\
\hline $88 / 19 / 87$ & $\begin{array}{ll}8.58 \\
4.88\end{array}$ & $18 / 10 / 87$ & $\begin{array}{l}0.26 \\
4.22\}\end{array}$ \\
\hline $11 / 18 / 87$ & $\begin{array}{ll}\left\{\begin{array}{l}4.43 \\
3.78\end{array}\right. \\
\mathrm{MR}\end{array}$ & $11 / 16 / 87$ & $\begin{array}{l}\text { 6. } 18 \\
3.86) \\
\text { NR }\end{array}$ \\
\hline $\begin{array}{l}68 / 19 / 87 \\
11 / 16 / 87\end{array}$ & $\begin{array}{l}16408.00 \\
16386.00\end{array}$ & $\begin{array}{l}18 / 10 / 87 \\
11 / 16 / 87\end{array}$ & $\begin{array}{l}11381.06 \\
14400.06\end{array}$ \\
\hline $\begin{array}{l}68 / 19 / 87 \\
11 / 16 / 87\end{array}$ & 40 & $\begin{array}{l}88 / 10 / 87 \\
11 / 16 / 87\end{array}$ & MR \\
\hline $\begin{array}{l}88 / 10 / 87 \\
11 / 16 / 07\end{array}$ & $\begin{array}{c}11200.66 \\
12106.68 \\
\text { NR }\end{array}$ & $\begin{array}{l}08 / 19 / 87 \\
11 / 16 / 87\end{array}$ & 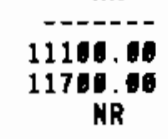 \\
\hline $\begin{array}{l}06 / 19 / 87 \\
11 / 18 / 87\end{array}$ & 18.06 & $\begin{array}{l}08 / 19 / 87 \\
11 / 16 / 87\end{array}$ & 20.00 \\
\hline $\begin{array}{l}88 / 19 / 87 \\
11 / 18 / 87\end{array}$ & $\begin{array}{l}288.68 \\
322.08\end{array}$ & $\begin{array}{l}08 / 10 / 87 \\
11 / 18 / 87\end{array}$ & $\begin{array}{l}331.18 \\
318.10\end{array}$ \\
\hline $08 / 18 / 87$ & & $08 / 19 / 87$ & \\
\hline
\end{tabular}


TABLE C.5. (contd)

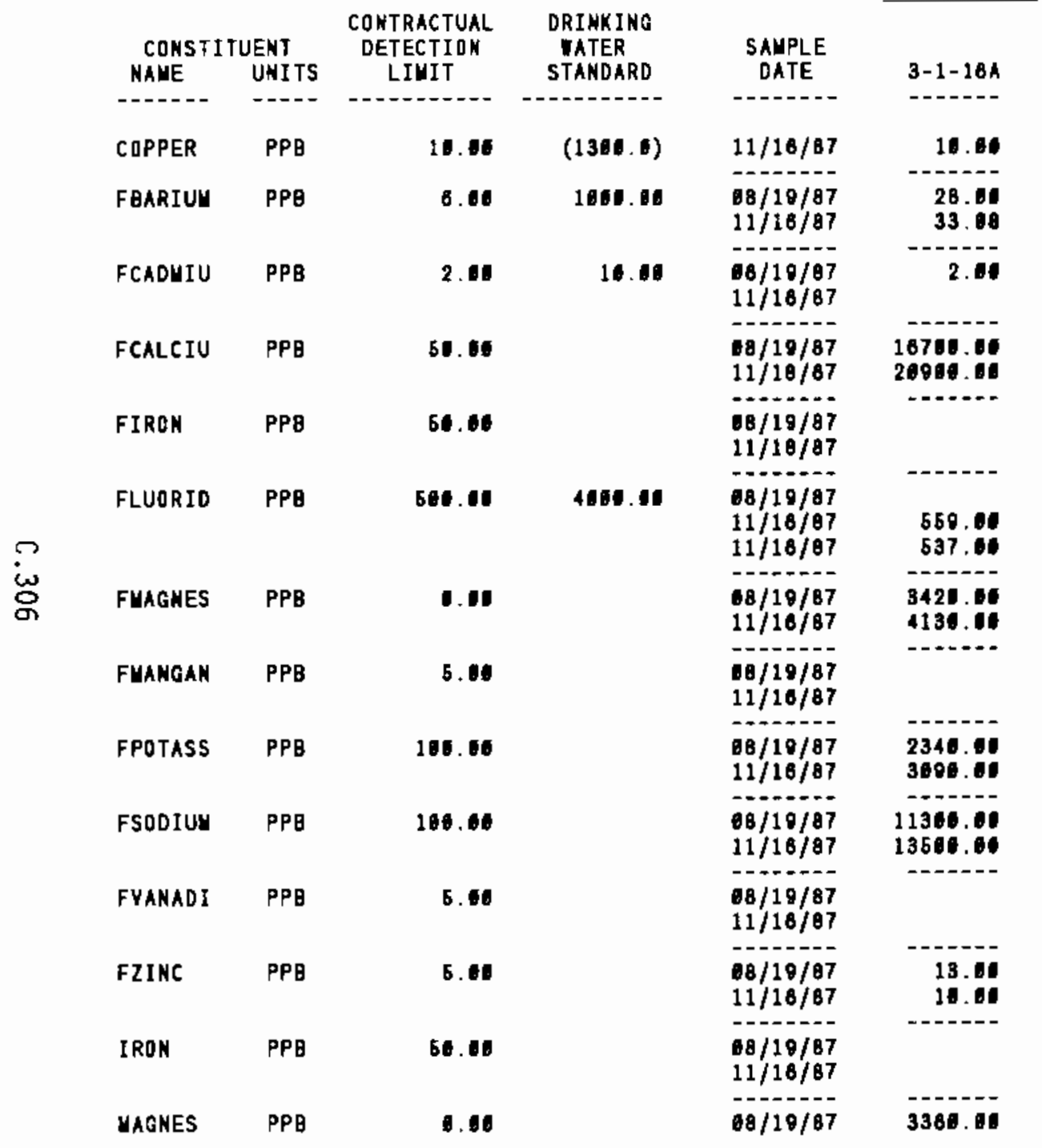

\begin{tabular}{|c|c|}
\hline $\begin{array}{c}\text { SAMPLE } \\
\text { DATE }\end{array}$ & $3-1-168$ \\
\hline - - & 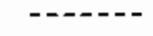 \\
\hline $11 / 16 / 87$ & \\
\hline $\begin{array}{l}08 / 19 / 87 \\
11 / 18 / 87\end{array}$ & $\begin{array}{l}56.00 \\
58.08\end{array}$ \\
\hline $\begin{array}{l}68 / 19 / 87 \\
11 / 16 / 87\end{array}$ & 2.00 \\
\hline $\begin{array}{l}18 / 10 / 97 \\
11 / 16 / 07\end{array}$ & $\begin{array}{l}17100.00 \\
25700.00\end{array}$ \\
\hline $\begin{array}{l}10 / 10 / 87 \\
1 \mathrm{i} / 16 / 87\end{array}$ & 85.09 \\
\hline $\begin{array}{l}6 / 10 / 87 \\
11 / 10 / 87\end{array}$ & $\begin{array}{c}1020.00 \\
1370.68\end{array}$ \\
\hline $\begin{array}{l}18 / 10 / 87 \\
11 / 16 / 87\end{array}$ & $\begin{array}{l}-1 .-1 .- \\
6380.01 \\
8860.41\end{array}$ \\
\hline $\begin{array}{l}10 / 10 / 97 \\
11 / 16 / 87\end{array}$ & $\begin{array}{l}86.00 \\
96.04\end{array}$ \\
\hline $\begin{array}{l}10 / 10 / 87 \\
11 / 18 / 87\end{array}$ & $\begin{array}{l}5170.00 \\
5865.00\end{array}$ \\
\hline $\begin{array}{l}8 / 19 / 87 \\
11 / 16 / 87\end{array}$ & $\begin{array}{l}51200.06 \\
54700.04\end{array}$ \\
\hline $\begin{array}{l}8 / 19 / 87 \\
11 / 16 / 87\end{array}$ & 8.6 \\
\hline $\begin{array}{l}80 / 10 / 87 \\
11 / 16 / 87\end{array}$ & 6.01 \\
\hline $\begin{array}{l}08 / 10 / 87 \\
11 / 18 / 07\end{array}$ & $\begin{array}{l}120.00 \\
118.00\end{array}$ \\
\hline $08 / 19 / 87$ & 6426.00 \\
\hline
\end{tabular}

\begin{tabular}{|c|c|}
\hline $\begin{array}{l}\text { SALPLE } \\
\text { DATE }\end{array}$ & $3-1-18 C$ \\
\hline - & \\
\hline $11 / 16 / 87$ & \\
\hline $\begin{array}{l}68 / 10 / 87 \\
11 / 18 / 87\end{array}$ & 87.00 \\
\hline $\begin{array}{l}68 / 19 / 87 \\
11 / 16 / 87\end{array}$ & \\
\hline $\begin{array}{l}08 / 10 / 87 \\
11 / 16 / 87\end{array}$ & $\begin{array}{l}11466.00 \\
14160.00\end{array}$ \\
\hline $\begin{array}{l}98 / 19 / 87 \\
11 / 18 / 87\end{array}$ & 02.06 \\
\hline $\begin{array}{l}18 / 10 / 87 \\
11 / 16 / 87\end{array}$ & $\begin{array}{l}1350.04 \\
1850.00\end{array}$ \\
\hline - & י-- - \\
\hline $\begin{array}{l}18 / 10 / 87 \\
11 / 16 / 87\end{array}$ & $\begin{array}{l}4000.04 \\
5120.01\end{array}$ \\
\hline $\begin{array}{l}08 / 10 / 87 \\
11 / 18 / 87\end{array}$ & 63.04 \\
\hline$-\cdots$ & \\
\hline $\begin{array}{l}08 / 19 / 67 \\
11 / 16 / 67\end{array}$ & $\begin{array}{l}6445.10 \\
8345.04\end{array}$ \\
\hline $\begin{array}{l}08 / 18 / 87 \\
11 / 16 / 87\end{array}$ & $\begin{array}{l}58901.00 \\
56201.00\end{array}$ \\
\hline $\begin{array}{l}68 / 10 / 87 \\
11 / 16 / 87\end{array}$ & \\
\hline $\begin{array}{l}68 / 10 / 87 \\
11 / 16 / 87\end{array}$ & 41.06 \\
\hline - & \\
\hline $\begin{array}{l}18 / 10 / 87 \\
11 / 16 / 87\end{array}$ & $\begin{array}{l}105.00 \\
108.00\end{array}$ \\
\hline $60 / 19 / 87$ & 5120.00 \\
\hline
\end{tabular}

* - value exceeds priuary drinking tater standard.

- - VALUE EXCEEDS PROPDSED PRIUARY DRINKING WATER STANDARD.

- - YALUE EXCEEDS SCREENING LEYEL FOR FURTHER INYESTIGATION.

- - DETECTION LIUIT TAS NOT AYAILABLE FOR COUPARISON

HR - ANALYSIS NOT REQUESTED OR NOT YET REPORTED

VALUES IN I \& ARE COUNTING ERRORS FOR RADIONUCLIDES

IATER STANDARD(S) IN PARENTHESES ARE PROPOSED ONLY 


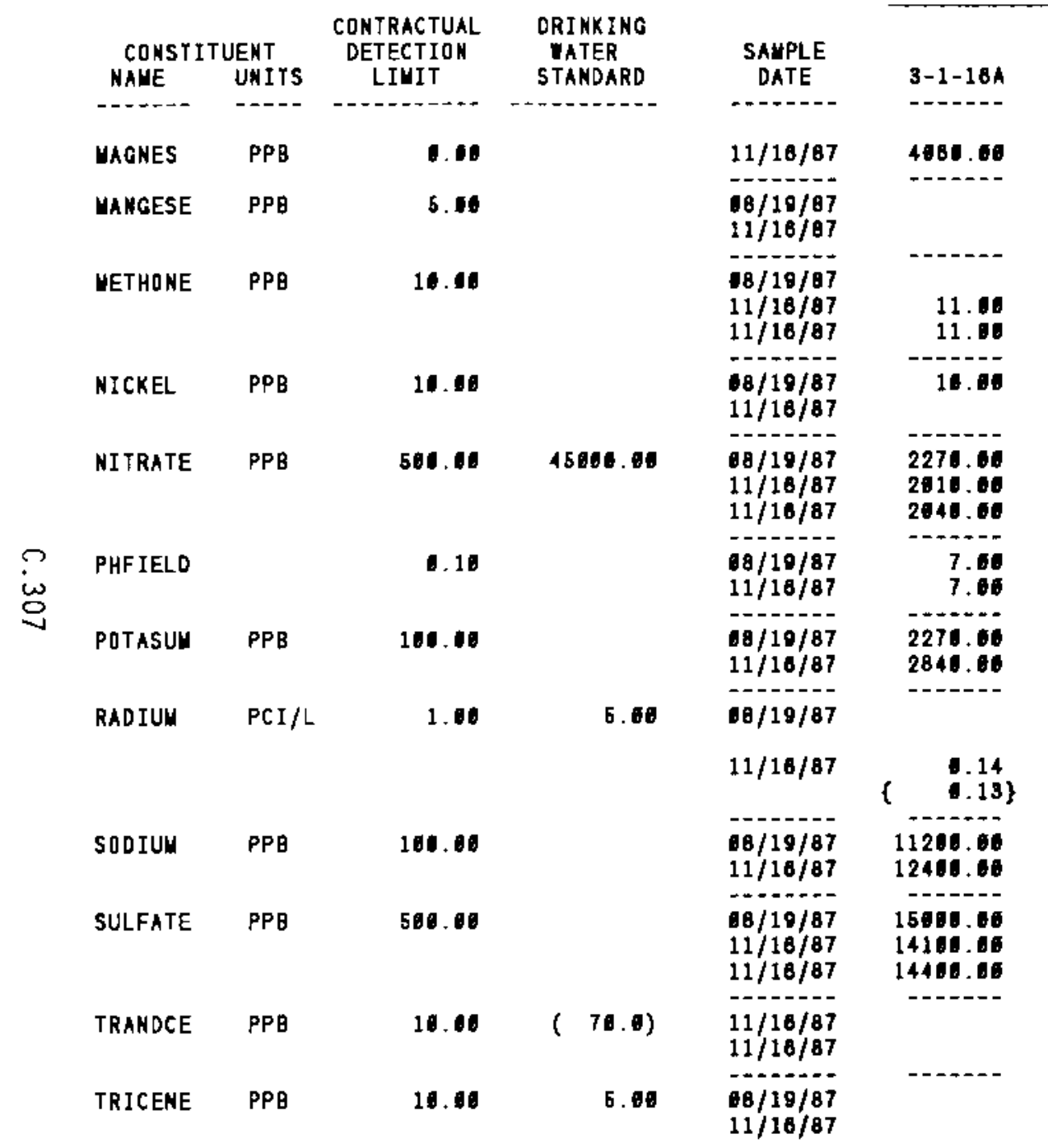

\begin{tabular}{|c|c|c|c|}
\hline $\begin{array}{l}\text { SAUPLE } \\
\text { DATE }\end{array}$ & $3-1-168$ & $\begin{array}{c}\text { SALPLE } \\
\text { DATE }\end{array}$ & $3-1-16 C$ \\
\hline$---\cdot---$ & ----- & ------ & -...-- \\
\hline $11 / 16 / 87$ & 6290.00 & $11 / 16 / 87$ & 6210.10 \\
\hline $\begin{array}{l}00 / 10 / 87 \\
11 / 16 / 87\end{array}$ & 80.00 & $\begin{array}{l}68 / 10 / 87 \\
11 / 16 / 87\end{array}$ & $\begin{array}{l}53.00 \\
33.00\end{array}$ \\
\hline $\begin{array}{l}68 / 19 / 87 \\
11 / 16 / 87\end{array}$ & 23.08 & $\begin{array}{l}68 / 10 / 87 \\
11 / 16 / 87\end{array}$ & $-\cdot-\cdot-$ \\
\hline $\begin{array}{l}18 / 10 / 97 \\
11 / 18 / 97\end{array}$ & 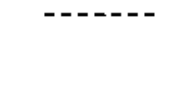 & $\begin{array}{l}00 / 19 / 87 \\
11 / 16 / 87\end{array}$ & \\
\hline $\begin{array}{l}18 / 19 / 97 \\
11 / 16 / 07\end{array}$ & ------ & $\begin{array}{l}18 / 19 / 87 \\
11 / 16 / 87\end{array}$ & -- \\
\hline & NR & & NR \\
\hline $\begin{array}{l}18 / 18 / 87 \\
11 / 18 / 87\end{array}$ & $\begin{array}{r}7.66 \\
8.36\end{array}$ & $\begin{array}{l}68 / 19 / 87 \\
11 / 16 / 87\end{array}$ & 7.60 \\
\hline $\begin{array}{l}08 / 10 / 87 \\
11 / 18 / 87\end{array}$ & $\begin{array}{l}--1--\overline{-} \\
5200.06 \\
5280.06\end{array}$ & $\begin{array}{l}08 / 18 / 87 \\
11 / 18 / 67\end{array}$ & $\begin{array}{l}5689.00 \\
6869.00\end{array}$ \\
\hline $86 / 19 / 87$ & 9.17 & $88 / 18 / 87$ & - \\
\hline $11 / 18 / 07$ & $\begin{array}{l}\left\{\begin{array}{l}15 \\
\end{array}\right. \\
\{\quad 0.22 \\
\{.15\}\end{array}$ & $11 / 16 / 87$ & \\
\hline $\begin{array}{l}06 / 18 / 87 \\
11 / 16 / 87\end{array}$ & $\begin{array}{l}52800.00 \\
56300.00\end{array}$ & $\begin{array}{l}18 / 10 / 87 \\
11 / 16 / 87\end{array}$ & $\begin{array}{l}52100.60 \\
58300.66\end{array}$ \\
\hline $\begin{array}{l}06 / 10 / 87 \\
11 / 16 / 97\end{array}$ & $\begin{array}{c}\text { E861.00 } \\
\text { B710.00 } \\
\text { NR }\end{array}$ & $\begin{array}{l}18 / 19 / 87 \\
11 / 16 / 87\end{array}$ & 4970.06 \\
\hline - - - & $-\cdots$ & - - - - & - \\
\hline $\begin{array}{l}16 / 10 / 87 \\
11 / 16 / 87\end{array}$ & $\begin{array}{l}63.00 \\
63.00\end{array}$ & $11 / 16 / 87$ & NR \\
\hline $\begin{array}{l}06 / 10 / 87 \\
11 / 16 / 87\end{array}$ & $19.00 *$ & $\begin{array}{l}00 / 19 / 87 \\
11 / 16 / 87\end{array}$ & \\
\hline
\end{tabular}

* - value exceeds priuary dRinxing vater standard.

- VALUE EXCEEDS PROPOSEO PRIUARY DRINKING TATER STANDARD.

- YALUE EXCEEDS SCREENIMG LEVEL FoR FURTHER INYESTIGATION.

- DETECTION LIUIT TAS HOT AYAILABLE FOR COUPARISON

NR - ANALYSIS NOT REQUESTED OR NOT YET REPDRTED

YALUES IN \& \& ARE COUNTING ERRORS FDR RADIONUCLIDES

IATER STANDARD(S) IN PARENTHESES ARE PROPOSED ONLY

ABLE C.5. (contd) 
TABLE C.5. (contd)

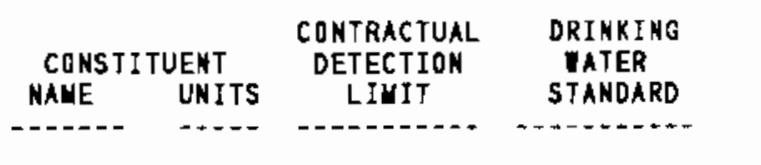

\begin{tabular}{|c|c|}
\hline $\begin{array}{l}\text { SAHPLE } \\
\text { DATE }\end{array}$ & $3-1-16 A$ \\
\hline & ---- \\
\hline $11 / 18 / 87$ & \\
\hline $\begin{array}{l}11 / 16 / 87 \\
11 / 16 / 87\end{array}$ & $\begin{array}{l}12.90 \\
13.20\end{array}$ \\
\hline $\begin{array}{l}18 / 19 / 87 \\
11 / 16 / 87\end{array}$ & 8.8 \\
\hline $\begin{array}{l}18 / 18 / 67 \\
11 / 16 / 07\end{array}$ & $\begin{array}{l}13.06 \\
23.06\end{array}$ \\
\hline
\end{tabular}

\begin{tabular}{|c|c|}
\hline $\begin{array}{l}\text { SAUPLE } \\
\text { DATE }\end{array}$ & $3-1-18 \theta$ \\
\hline & - - \\
\hline & NR \\
\hline יד & $-x-1,-\overline{1}$ \\
\hline $11 / 16 / 87$ & $\mathrm{NR}^{12}$ \\
\hline $\begin{array}{l}08 / 19 / 87 \\
11 / 16 / 87\end{array}$ & $-\cdots$ \\
\hline $\begin{array}{l}18 / 19 / 87 \\
11 / 16 / 87\end{array}$ & 40.00 \\
\hline
\end{tabular}

\begin{tabular}{|c|c|}
\hline $\begin{array}{l}\text { SALPLE } \\
\text { DATE }\end{array}$ & $3-1-1 B C$ \\
\hline$---1--$ & - - \\
\hline & $M R$ \\
\hline $11 / 16 / 87$ & 2.61 \\
\hline & $\mathrm{NR}$ \\
\hline $68 / 19 / 87$ & 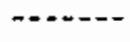 \\
\hline $\begin{array}{l}68 / 19 / 87 \\
11 / 16 / 87\end{array}$ & \\
\hline 9. & $-\infty=---$ \\
\hline $\begin{array}{l}98 / 19 / 87 \\
11 / 18 / 87\end{array}$ & 64.51 \\
\hline
\end{tabular}

$\varliminf_{\substack{\infty \\ \infty}}$

\footnotetext{
- value exceEds primary drinking tater stakdard.

7 - VALUE EXCEEDS PRDPDSED PRIMARY DRINKING iater STANDARD.

- VALUE EXCEEDS SCREENING LEYEL FOR FURTHER IMVESTIGATION.

- DETECTION LIUIT ins NOT aVAILAgLE for couparison

NR - ANALYSIS NOT REQUESTED OR NOT YET REPORTED

VALUES IN \{ ARE COUNTING ERRORS FOR RADIONUCLIDES

TATER STANDARD(S) IN PAREKTHESES ARE PROPOSED ONLY
} 
TABLE C.5. (contd)

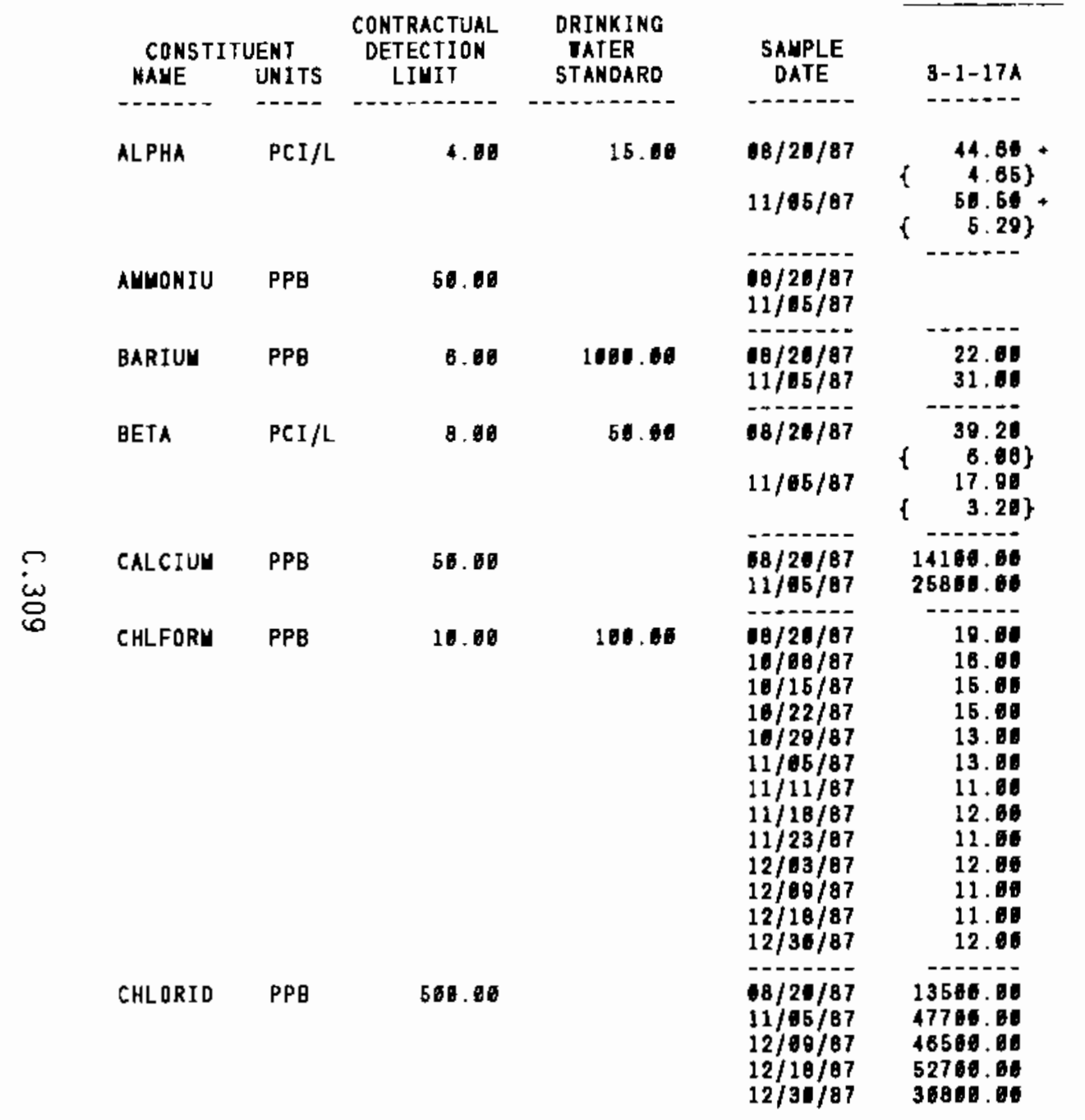

\begin{tabular}{|c|c|c|c|}
\hline $\begin{array}{l}\text { SAMPLE } \\
\text { DATE }\end{array}$ & $3-1-17 \theta$ & $\begin{array}{l}\text { SAUPLE } \\
\text { DATE }\end{array}$ & $3-1-17 C$ \\
\hline $68 / 20 / 87$ & & $18 / 25 / 87$ & \\
\hline $11 / 05 / 87$ & & $11 / 06 / 07$ & \\
\hline $\begin{array}{l}68 / 20 / 87 \\
11 / 05 / 87\end{array}$ & $\begin{array}{r}125.66 \\
85.60\end{array}$ & $\begin{array}{l}88 / 25 / 87 \\
11 / 08 / 07\end{array}$ & $\begin{array}{l}98.11 \\
86.01\end{array}$ \\
\hline $\begin{array}{l}08 / 20 / 87 \\
11 / 05 / 87\end{array}$ & $\begin{array}{l}83.60 \\
80.60\end{array}$ & $\begin{array}{l}0 B / 25 / 87 \\
11 / 06 / 87\end{array}$ & $\begin{array}{l}84.06 \\
89.06\end{array}$ \\
\hline $\begin{array}{l}18 / 21 / 87 \\
11 / 05 / 87\end{array}$ & 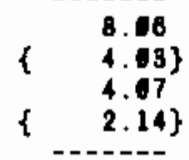 & $\begin{array}{l}18 / 25 / 87 \\
11 / 06 / 87\end{array}$ & 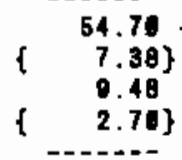 \\
\hline $\begin{array}{l}08 / 20 / 87 \\
11 / 05 / 87\end{array}$ & $\begin{array}{l}14608.08 \\
16404.01\end{array}$ & $\begin{array}{l}18 / 25 / 87 \\
11 / 00 / 67\end{array}$ & $\begin{array}{l}8984.66 \\
0496.06\end{array}$ \\
\hline $\begin{array}{l}08 / 28 / 87 \\
11 / 05 / 87\end{array}$ & $\begin{array}{l}\text { NR } \\
\text { NR } \\
\text { NR } \\
\text { NR } \\
\text { NR } \\
\text { NR } \\
\text { NR } \\
\text { MR } \\
\text { NR } \\
\text { NR } \\
\text { NR }\end{array}$ & $\begin{array}{l}18 / 26 / 87 \\
11 / 68 / 87\end{array}$ & $\begin{array}{l}\text { MR } \\
\text { MR } \\
\text { MR } \\
\text { NR } \\
\text { MR } \\
\text { MR } \\
\text { MR } \\
\text { MR } \\
\text { NR } \\
\text { NR } \\
\text { NR }\end{array}$ \\
\hline $\begin{array}{l}68 / 20 / 87 \\
11 / 65 / 87\end{array}$ & $\begin{array}{c}9210.6 \\
9840.60 \\
\text { NR } \\
\text { NR } \\
\text { NR }\end{array}$ & $\begin{array}{l}08 / 26 / 87 \\
11 / 06 / 87\end{array}$ & $\begin{array}{c}19 B 04.6 \\
10909.60 \\
\text { NR } \\
\text { NR } \\
\text { NR }\end{array}$ \\
\hline
\end{tabular}

- VALUE EXCEEDS PRIMARY dRINXING Mater STAMoard.

- VALUE EXCEEDS PROPOSED PRIUARY DRINKIMG IATER STANDARD.

- - yalue exceeds screening leVel for further inYestigatidn.

- Detection linit uas not available for couparison

NR - ANALYSIS NDT REQUESTED OR NOT YET REPORTED

VALUES IM \{\} ARE COUNTING ERRORS FOR RADIONUCLIDES

IATER STAMDARD'(S) IN PARENTHESES ARE PROPOSED OHLY 


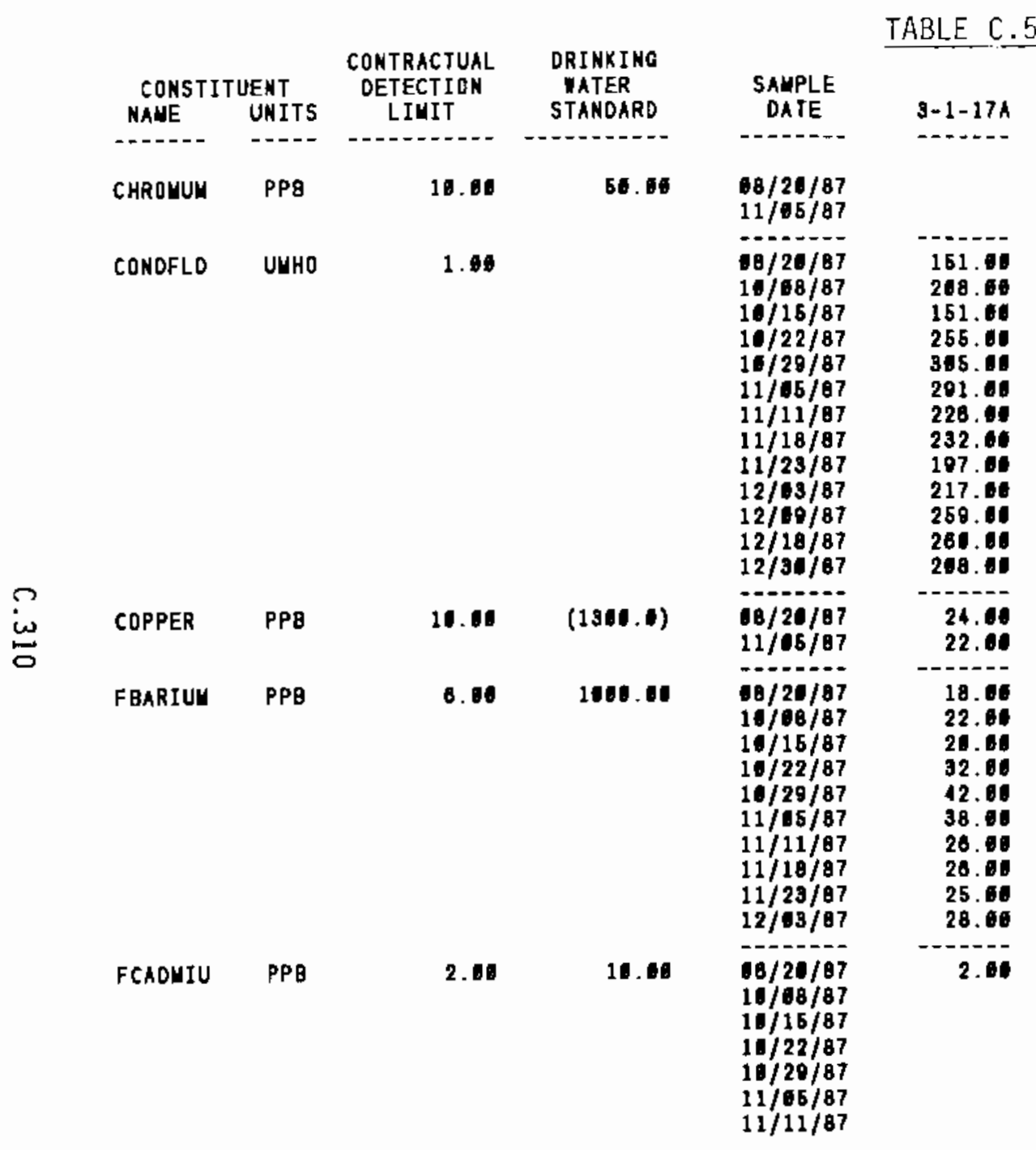

(contd)

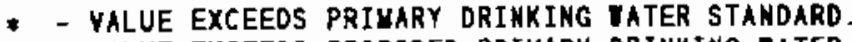

* - VALUE EXCEEOS PROPOSEd PRIUARY dRINKING JaTER STAHoARd.

+ - VALUE EXCEEDS SCREENING LEVEL FOR FURTHER INYESTIGATION.

- - DETECTIOH LIUIT IAS NOT AVAILABLE FOR COUPARISON

NR - ANALYSIS NOT REQUESTED OR NOT YET REPORTED

VALUES IN \& \& ARE CQUNTING ERRORS FOR RADIONUCLIDES

WATER STANDARO'(S) IN PARENTHESES ARE PROPOSED ONLY

\begin{tabular}{|c|c|}
\hline $\begin{array}{c}\text { SAUPLE } \\
\text { DATE }\end{array}$ & $3-1-17 B$ \\
\hline - - - - & ----- \\
\hline $\begin{array}{l}68 / 26 / 87 \\
11 / 85 / 87\end{array}$ & $\begin{array}{l}14.06 \\
14.00\end{array}$ \\
\hline 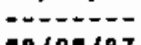 & $-\overline{905}$ \\
\hline $\begin{array}{l}08 / 20 / 87 \\
11 / 05 / 87\end{array}$ & $\begin{array}{c}295.00 \\
339.00 \\
\text { NR } \\
\text { NR } \\
\text { NR } \\
\text { NR } \\
\text { NR } \\
\text { NR } \\
\text { NR } \\
\text { NR } \\
\text { NR } \\
\text { NR } \\
\text { WR }\end{array}$ \\
\hline $\begin{array}{l}18 / 21 / 87 \\
11 / 05 / 87\end{array}$ & . \\
\hline $\begin{array}{l}08 / 20 / 87 \\
11 / 66 / 87\end{array}$ & $\begin{array}{l}59.00 \\
\text { WR. } \\
\text { NR } \\
\text { NR } \\
\text { NR } \\
\text { NR } \\
\text { NR } \\
\text { NR } \\
\text { NR } \\
\text { NR }\end{array}$ \\
\hline $18 / 20 / 87$ & 2.00 \\
\hline & $\begin{array}{l}\text { NR } \\
\text { NR } \\
\text { NR } \\
\text { NR } \\
\text { NR }\end{array}$ \\
\hline
\end{tabular}

\begin{tabular}{|c|c|}
\hline $\begin{array}{l}\text { SALPLE } \\
\text { DATE }\end{array}$ & $3-1-17 c$ \\
\hline $\begin{array}{l}08 / 26 / 87 \\
11 / 06 / 87\end{array}$ & \\
\hline $\begin{array}{l}68 / 25 / 97 \\
11 / 08 / 87\end{array}$ & $\begin{array}{c}319.06 \\
338.00 \\
\text { NR } \\
\text { NR } \\
\text { NR } \\
\text { NR } \\
\text { NR } \\
\text { NR } \\
\text { NR } \\
\text { NR } \\
\text { NR } \\
\text { MR } \\
\text { MR }\end{array}$ \\
\hline
\end{tabular}

$08 / 25 / 67$

$11 / 68 / 87$

$68 / 25 / 87$

$11 / 06 / 87$

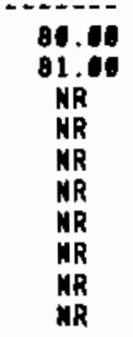

$18 / 25 / 8$

$11 / 106 / 87$

NR
MR
MR
NR
NR


IABLE C.5. (contd)

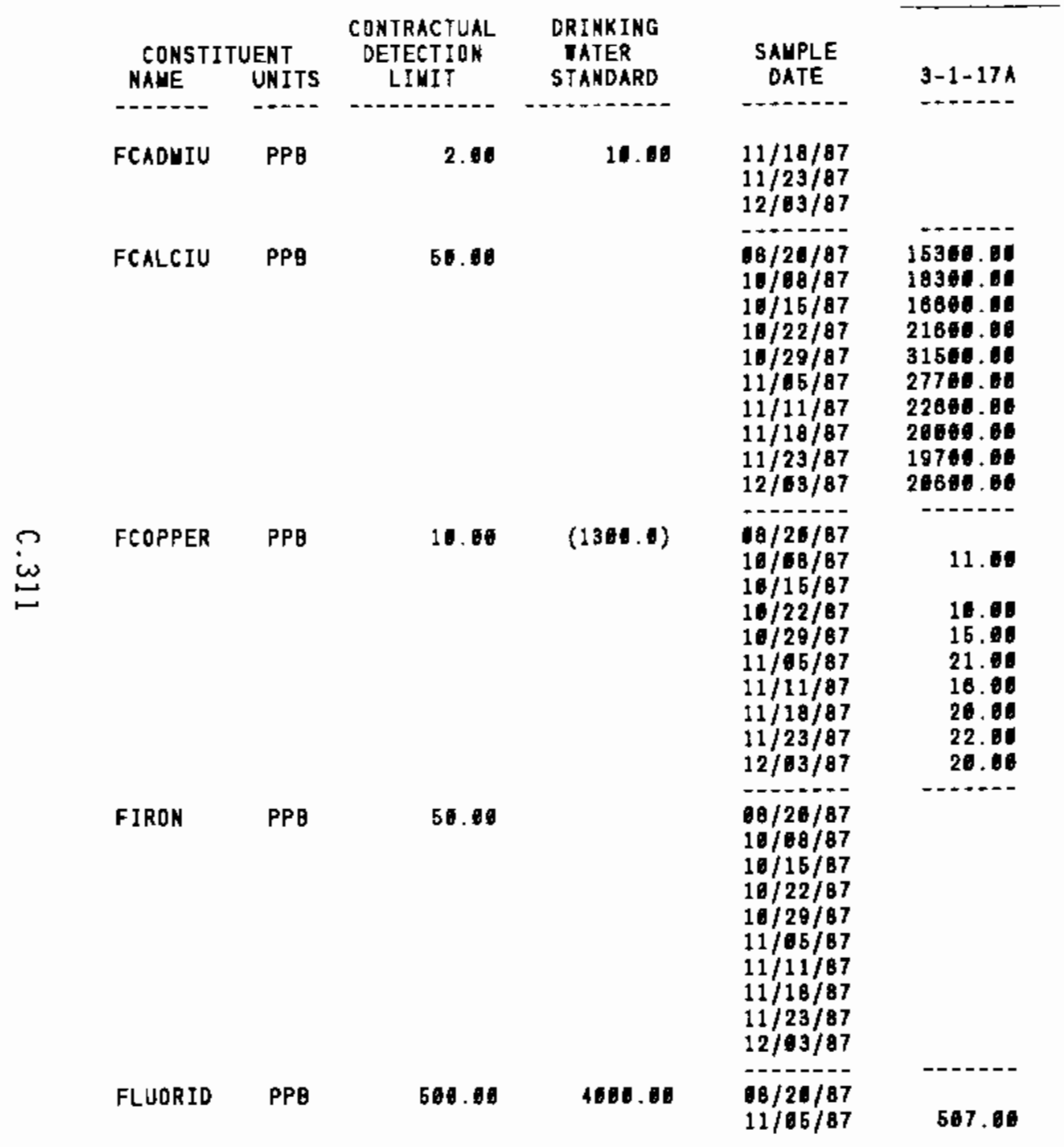

\begin{tabular}{|c|c|}
\hline $\begin{array}{l}\text { SALPLEE } \\
\text { DATEE }\end{array}$ & $3-1-178$ \\
\hline & $\begin{array}{l}\text { NR } \\
\text { NR } \\
\text { NR }\end{array}$ \\
\hline $\begin{array}{l}8 B / 20 / 87 \\
11 / 05 / 87\end{array}$ & $\begin{array}{c}16500.06 \\
18109.00 \\
\text { NR } \\
\text { NR } \\
\text { NR } \\
\text { NR } \\
\text { NR } \\
\text { NR } \\
\text { NR } \\
\text { MR }\end{array}$ \\
\hline $\begin{array}{l}6 / 20 / 87 \\
11 / 65 / 87\end{array}$ & $\begin{array}{l}\text { NR } \\
\text { NR } \\
\text { NR } \\
\text { NR } \\
\text { NR } \\
\text { NR } \\
\text { MR } \\
\text { NR }\end{array}$ \\
\hline $\begin{array}{l}68 / 26 / 87 \\
11 / 65 / 87\end{array}$ & $\begin{array}{c}131.80 \\
140.00 \\
\text { NR } \\
\text { NR } \\
\text { NR } \\
\text { NR } \\
\text { NR } \\
\text { NR } \\
\text { NR } \\
\text { NR }\end{array}$ \\
\hline $\begin{array}{l}88 / 20 / 20 \\
11 / 65 / B 7\end{array}$ & $\begin{array}{r}748.60 \\
1130.06\end{array}$ \\
\hline
\end{tabular}

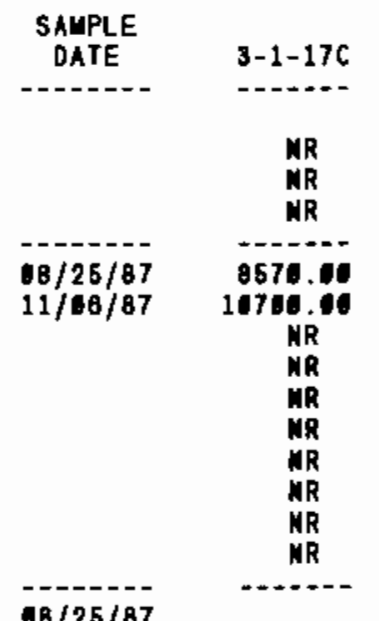

$08 / 25 / 87$

$11 / 86 / 87$

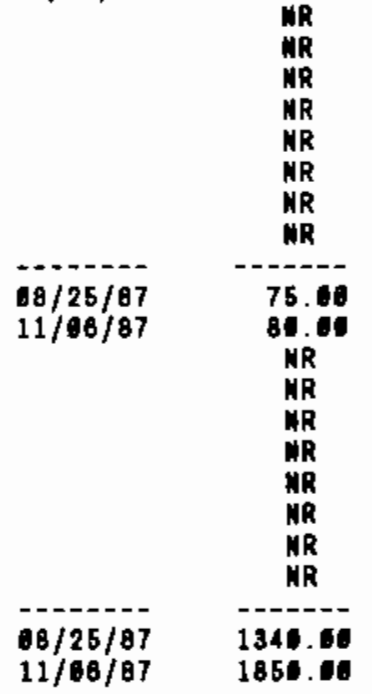

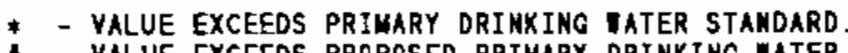

- - VALUE EXCEEDS PROPOSED PRIMARY DRINKING MATER STANDARD.

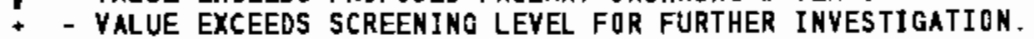

- DETECTIDN LIMIT IAS NOT AVAILABLE FOR COMPARISON

MR - ANALYSIS NOT REQUESTED OR NOT YET REPORTED

VALUES IN \& $\{$ ARE COUNTING ERRORS FOR RADIONUCLIDES

VATER STANDARD'S) IN PARENTHESES ARE PROPOSED ONLY 
TABLE C.5. (contd)

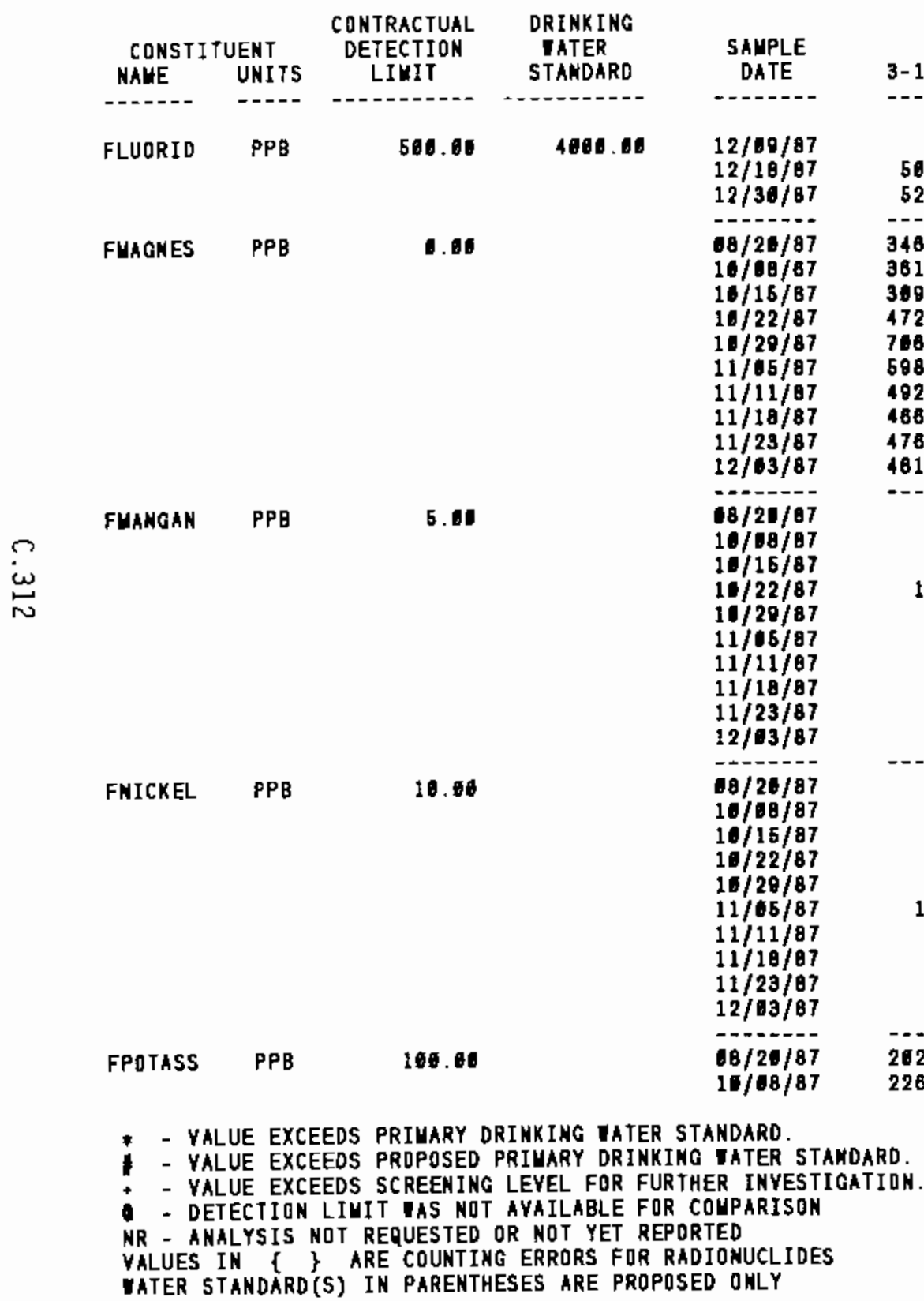

\begin{tabular}{|c|c|c|c|}
\hline $\begin{array}{c}\text { SAUPLE } \\
\text { DATE }\end{array}$ & $\begin{array}{c}3-1-178 \\
\text { NR } \\
\text { NR } \\
\text { NR }\end{array}$ & $\begin{array}{c}\text { SAMPLE } \\
\text { DATE }\end{array}$ & $\begin{array}{c}3-1-17 C \\
M R \\
M R \\
M R\end{array}$ \\
\hline $\begin{array}{l}08 / 26 / 87 \\
11 / 05 / 87\end{array}$ & $\begin{array}{c}6480.01 \\
6520.00 \\
\text { NR } \\
\text { NR } \\
\text { NR } \\
\text { NR } \\
\text { NR } \\
\text { MR } \\
\text { NR } \\
\text { NR }\end{array}$ & $\begin{array}{l}68 / 25 / 87 \\
11 / 08 / 87\end{array}$ & $\begin{array}{c}4486.16 \\
4050.00 \\
N R \\
N R \\
N R \\
N R \\
N R \\
N R \\
N R \\
N R\end{array}$ \\
\hline $\begin{array}{l}08 / 26 / 87 \\
11 / 65 / 87\end{array}$ & $\begin{array}{l}86.01 \\
83.00 \\
\text { NR } \\
\text { NR } \\
\text { NR } \\
\text { NR } \\
\text { NR } \\
\text { MR } \\
\text { NR } \\
\text { NR }\end{array}$ & $\begin{array}{l}68 / 25 / 87 \\
11 / 08 / 87\end{array}$ & $\begin{array}{c}22.00 \\
24.00 \\
M R \\
M R \\
M R \\
M R \\
M R \\
M R \\
M R \\
M R\end{array}$ \\
\hline $\begin{array}{l}08 / 20 / 87 \\
11 / 05 / 87\end{array}$ & $\begin{array}{l}\text { NR } \\
\text { NR } \\
\text { NR } \\
\text { NR } \\
\text { NR } \\
\text { NR } \\
\text { NR } \\
\text { KR }\end{array}$ & $\begin{array}{l}08 / 25 / 87 \\
11 / 08 / 87\end{array}$ & $\begin{array}{l}\text { NR } \\
\text { NR } \\
\text { MR } \\
\text { WR } \\
\text { NR } \\
\text { NR } \\
\text { NR } \\
\text { NR }\end{array}$ \\
\hline $\begin{array}{l}08 / 20 / 87 \\
11 / 05 / 87\end{array}$ & $\begin{array}{l}5820.00 \\
6080.00\end{array}$ & $\begin{array}{l}18 / 25 / 87 \\
11 / 108 / 87\end{array}$ & $\begin{array}{l}16760.60 \\
11186.68\end{array}$ \\
\hline
\end{tabular}

$3-1-17 \AA$

507.60

(5).0

68.00

6

4720.60

7680.00

5986. 06

920.60

886.60

4780.00

4610.00
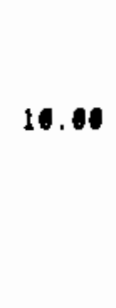

10.65

2620

2260.00

$11 / 05 / 87$

6980.00

$11 / 106 / 87$

11104.10

- DETECTION LIUIT HAS NOT AYAILABLE FOR COUPARISON

VATER STANDARD (S) IN PARENTHESES ARE PROPOSED OHLY 
TABLE C.5. (contd)

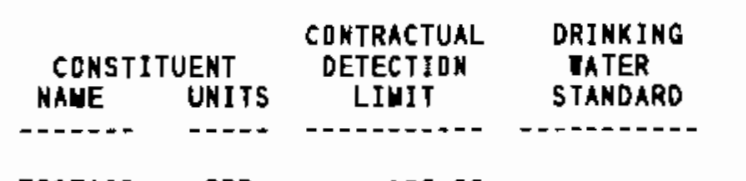

SAUPLE
DATE
$-\cdots--$
$16 / 15 / 87$
$16 / 22 / 87$
$16 / 29 / 87$
$11 / 85 / 87$
$11 / 11 / 97$
$11 / 18 / 87$
$11 / 23 / 87$
$12 / 63 / 87$

$3-1-174$

SAMPLE

DATE

DATE

$-1-.-1$

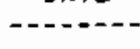

$3-1-17$

$3-1-17 C$

FPOTASS

100.60

2050.00

2280.00

2794.10

2464.01

241.00

2416.06

FSODIUN PPB 100.60

$98 / 26 / 87$

$10 / 08 / 87$

$10 / 15 / 87$

$18 / 29 / 87$

$11 / 65 / 87$

$11 / 11 / 87$

$11 / 18 / 87$

$11 / 23 / 87$

-..-

岕

12200.80

12506.06

$131 \% 6.06$

18460.00

17800.06

15704. .6

17368.60

17466.68

$08 / 20 / 8750$

FYANADI PPB

5.10

19/8B/87

$10 / 15 / 87$

$10 / 22 / 87$

$16 / 29 / 87$

6.68

$11 / 05 / 87$

$11 / 11 / 87$

$11 / 18 / 87$

$11 / 23 / 87$

$12 / 03 / 87$

B $/ 20 / 87$

$10 / 68 / 87$

$10 / 15 / 87$

$16 / 22 / 87$

$10 / 29 / 87$

$11 / 65 / 87$

$11 / 11 / 87$
$11 / 18 / 87$

\begin{tabular}{|c|c|}
\hline & $\begin{array}{l}\text { NR } \\
\text { NR } \\
M R \\
M R \\
M R \\
N R \\
N R \\
N R \\
N R\end{array}$ \\
\hline $\begin{array}{l}08 / 20 / 87 \\
11 / 05 / 87\end{array}$ & $\begin{array}{c}48181.06 \\
49006.04 \\
\text { NR } \\
\text { NR } \\
\text { NR } \\
\text { NR } \\
\text { NR } \\
\text { NR } \\
\text { NR } \\
\text { WR }\end{array}$ \\
\hline $\begin{array}{l}88 / 28 / 87 \\
11 / 05 / 87\end{array}$ & $\begin{array}{l}\text { NR } \\
\text { NR } \\
\text { NR } \\
\text { NR } \\
\text { NR } \\
\text { NR } \\
\text { NR } \\
\text { NR }\end{array}$ \\
\hline $\begin{array}{l}18 / 26 / 87 \\
11 / 65 / 87\end{array}$ & $\begin{array}{l}\text { NR } \\
\text { NR } \\
\text { MR } \\
\text { NR } \\
\text { NR } \\
\text { NR }\end{array}$ \\
\hline
\end{tabular}

NR

NR

NR

MR

NR

NR

$68 / 25 / 87$

$68 / 25 / 87$
$11 / 68 / 87$

o42as

71464.01

NR

NR

NR

NR

NR

7

7.69

6.06

B. 10

-..-.-

9.06

-...-

$11 / 06 / 87$

$N R$
$N R$
$N R$
$N R$
$N R$
$N R$
$N R$
$N R$

$11 / 60 / 87$

\begin{tabular}{c} 
NR \\
NR \\
NR \\
NR \\
NR \\
NR \\
NR \\
NR \\
\hdashline
\end{tabular}

$08 / 25 / 87$

$11 / 66 / 87$

NR
NR
NR
NR
NR
NR

* - VALUE EXCEEDS PRIMARY DRINKING YATER STANDARD.

- YALUE EXCEEDS PROPOSED PRIMARY DRINXING IATER STAKDARD.

- - yalue EXCEEds SCREening Leyel for further inyestigation.

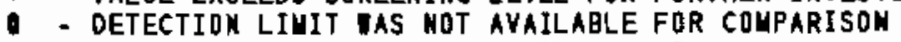

WR - ANALYSIS MOT REQUESTED OR NOT YET REPORTED

VALUES IN $f$ A ARE COUNTING ERRORS FOR RADIONUCLIDES

WATER STANOARD (S) IN PARENTHESES ARE PROPOSED ONLY 


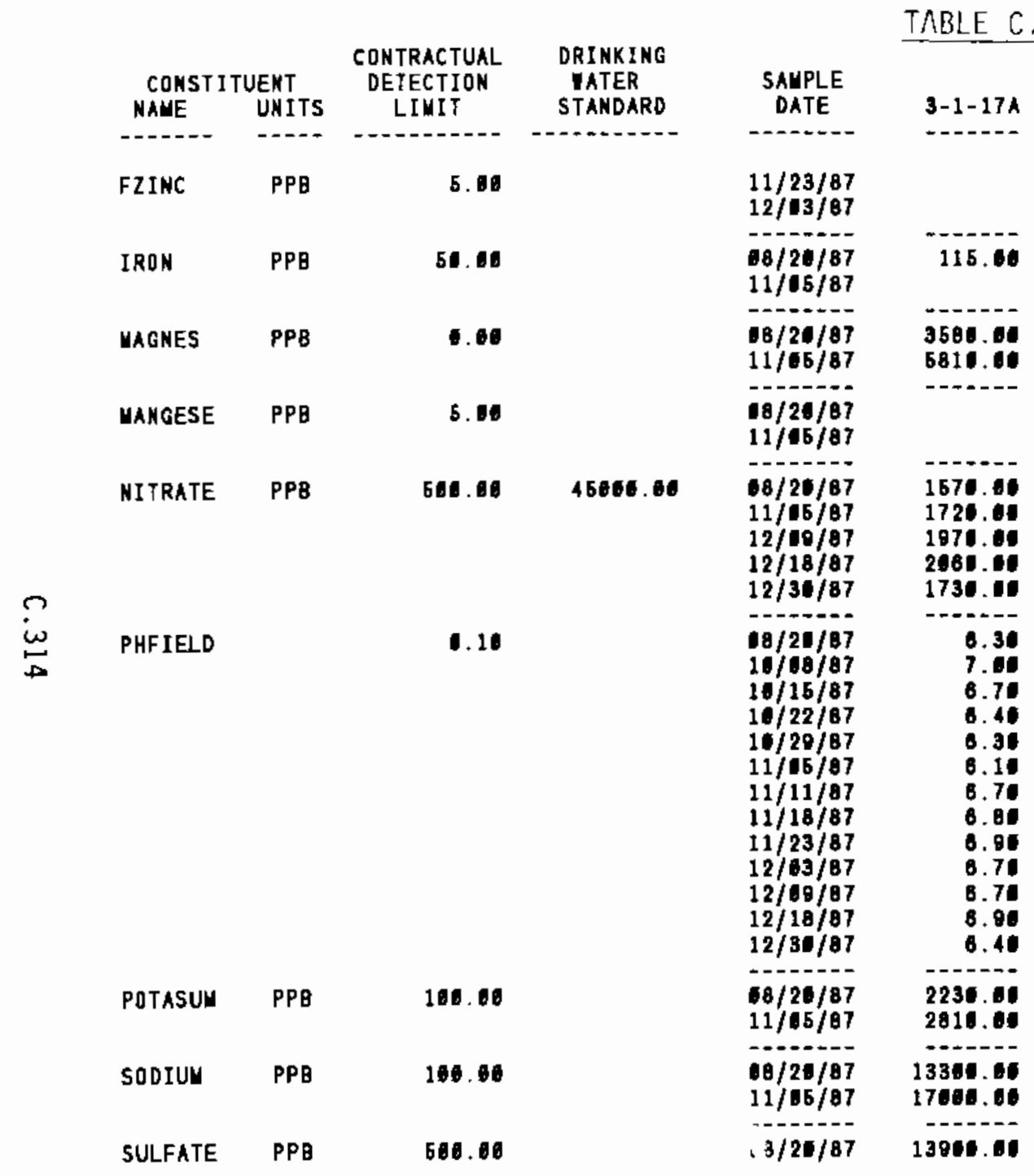

\begin{tabular}{|c|c|c|c|}
\hline $\begin{array}{l}\text { SAMPLE } \\
\text { DATE }\end{array}$ & $3-1-17 \theta$ & $\begin{array}{c}\text { SAUPLE } \\
\text { DATE }\end{array}$ & $3-1-17 c$ \\
\hline & $\begin{array}{l}\text { MR } \\
\text { NR }\end{array}$ & & $\begin{array}{l}\text { NR } \\
\text { MR }\end{array}$ \\
\hline $\begin{array}{l}98 / 20 / 87 \\
11 / 05 / 87\end{array}$ & $\begin{array}{l}154.00 \\
173.01\end{array}$ & $\begin{array}{l}08 / 25 / 87 \\
11 / 66 / 87\end{array}$ & $\begin{array}{l}84.10 \\
87.60\end{array}$ \\
\hline $\begin{array}{l}18 / 20 / 87 \\
11 / 05 / 87\end{array}$ & $\begin{array}{l}8250.60 \\
8450.60\end{array}$ & $\begin{array}{l}09 / 25 / 87 \\
11 / 16 / 87\end{array}$ & $\begin{array}{l}4860.10 \\
477.10\end{array}$ \\
\hline $\begin{array}{l}18 / 21 / 87 \\
11 / 05 / 87\end{array}$ & $\begin{array}{l}50.10 \\
82.01\end{array}$ & $\begin{array}{l}18 / 26 / 87 \\
11 / 06 / 87\end{array}$ & $\begin{array}{l}23.60 \\
24.06\end{array}$ \\
\hline $\begin{array}{l}08 / 20 / 87 \\
11 / 05 / 87\end{array}$ & $\begin{array}{l}\text { NR } \\
\text { NR } \\
\text { NR }\end{array}$ & $\begin{array}{l}68 / 25 / 87 \\
11 / 06 / 87\end{array}$ & $\begin{array}{l}\text { MR } \\
\text { NR } \\
\text { NR }\end{array}$ \\
\hline $\begin{array}{l}18 / 20 / 97 \\
11 / 65 / 87\end{array}$ & $\begin{array}{l}7.44 \\
5.30 \\
\text { NR } \\
\text { MR } \\
\text { NR } \\
\text { NR } \\
\text { NR } \\
\text { MR } \\
\text { KR } \\
\text { NR } \\
\text { NR } \\
\text { NR } \\
\text { MR }\end{array}$ & $\begin{array}{l}18 / 25 / 87 \\
11 / 06 / 97\end{array}$ & $\begin{array}{l}8.10 \\
7.96 \\
\text { MR } \\
\text { NR } \\
\text { NR } \\
\text { MR } \\
\text { MR } \\
\text { NR } \\
\text { NR } \\
\text { NR } \\
\text { NR } \\
\text { MR } \\
\text { NR }\end{array}$ \\
\hline $\begin{array}{l}88 / 26 / 87 \\
11 / 65 / 87\end{array}$ & $\begin{array}{l}5904.11 \\
8530.01\end{array}$ & $\begin{array}{l}68 / 25 / 87 \\
11 / 10 / 87\end{array}$ & $\begin{array}{l}11200.00 \\
11300.08\end{array}$ \\
\hline $\begin{array}{l}08 / 20 / 87 \\
11 / 05 / 87\end{array}$ & $\begin{array}{l}43706.60 \\
50806.60\end{array}$ & $\begin{array}{l}88 / 26 / 87 \\
11 / 66 / 87\end{array}$ & $\begin{array}{l}67296.00 \\
68306.10\end{array}$ \\
\hline $08 / 20 / 87$ & 4340.60 & $08 / 25 / 87$ & \\
\hline
\end{tabular}

- - Value exceeds prinary dRINKing vater standard

- VALUE EXCEDS PROPOSED PRIUARY DRINKING VATER STANDaRo.

- Y VALUE EXCEEDS SCREENING leVEL FOR FURTHER INYESTIGATION.

- DETECTITN LIMIT UAS NOT AVAILABLE for COUPARISDN

MR - ANALYSIS NOT RERUESTED OR HDT YET REPORTED

VALUES IN i f ARE COUNTING ERRORS FOR RADIONUCLIDES

WATER STANDARD(S) IN PARENTHESES ARE PROPOSED ONLY 
TABLE C.5. (contd)

\begin{tabular}{|c|c|c|c|c|c|}
\hline $\begin{array}{c}\text { CONSTI } \\
\text { HAME }\end{array}$ & $\begin{array}{l}\text { UENT } \\
\text { UNI TS }\end{array}$ & $\begin{array}{c}\text { CONTRACTUAL } \\
\text { DETECTION } \\
\text { LIMIT }\end{array}$ & $\begin{array}{l}\text { DRINKING } \\
\text { IATER } \\
\text { STANDARD }\end{array}$ & $\begin{array}{l}\text { SAUPLE } \\
\text { DATE }\end{array}$ & $9-1-17 A$ \\
\hline SULFATE & PPQ & 500.80 & & $\begin{array}{l}11 / 65 / 87 \\
12 / 90 / 87 \\
12 / 18 / 87 \\
12 / 30 / 87\end{array}$ & $\begin{array}{l}14460.60 \\
18366.00 \\
14606.00 \\
13860.01\end{array}$ \\
\hline TRANDCE & PPE & 18.06 & $(70.0)$ & $11 / 65 / 87$ & NR \\
\hline U-CHEW & UG/L & 0.73 & & $\begin{array}{l}10 / 88 / 87 \\
10 / 15 / 87 \\
16 / 22 / 87 \\
1 \% / 28 / 87 \\
11 / 95 / 87 \\
11 / 11 / 87 \\
11 / 18 / 87 \\
11 / 23 / 87 \\
12 / 93 / 87 \\
12 / 89 / 87 \\
12 / 18 / 87 \\
12 / 36 / 87\end{array}$ & $\begin{array}{r}44.58 \\
42.16 \\
43.26 \\
57.76 \\
47.36 \\
58.48 \\
69.88 \\
84.16 \\
68.78 \\
59.18 \\
183.16 \\
88.26\end{array}$ \\
\hline ZINC & PPB & 5.80 & & $\begin{array}{l}08 / 20 / 87 \\
11 / 65 / 87\end{array}$ & 11.8 \\
\hline
\end{tabular}

\begin{tabular}{|c|c|}
\hline $\begin{array}{l}\text { SAUPLEE } \\
\text { DATEE }\end{array}$ & $3-1-178$ \\
\hline $11 / 65 / 87$ & $\begin{array}{c}2980.68 \\
\text { NR } \\
\text { RR } \\
\text { MR }\end{array}$ \\
\hline \multirow[t]{2}{*}{$\begin{array}{l}88 / 20 / 87 \\
11 / 06 / 87\end{array}$} & $\begin{array}{l}28.60 \\
17.60\end{array}$ \\
\hline & $\begin{array}{l}\text { NR } \\
\text { NR } \\
\text { NR } \\
\text { MR } \\
\text { NR } \\
\text { NR } \\
\text { NR } \\
\text { MR } \\
\text { NR } \\
\text { NR } \\
\text { MR } \\
\text { NR }\end{array}$ \\
\hline $68 / 20 / 87$ & 9.60 \\
\hline
\end{tabular}

SAMPLE

DATE $\quad 3-1-17 C$

$11 / 88 / 87$

$-\cdot-\cdot--$

$11 / 05 / 87$

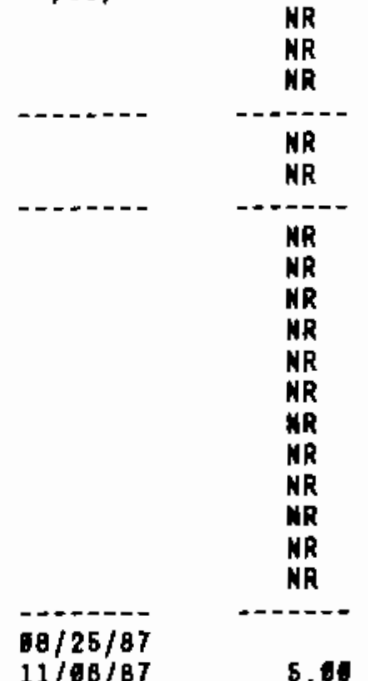

- - Value exceEds priuary dRINKINg tater standard.

- - VALUE EXCEEDS PROPOSED PRIUARY DRIMKINo MATER STANDARD.

- - VALUE EXCEEDS SCREENING LEVEL FOR FURTHER INYESTIGATIOA.

- DETECTION LIUIT TAS MOT AYAILABLE FOR COMPARISON

NR - ANALYSIS NOT REQUESTED DR HOT YET REPORTED

VALUES IN \{ $\}$ ARE COUNTINO ERRORS FOR RADIDNUCLIDES

IATER STANOARD(S) IN PARENTHESES ARE PROPOSED OHLY 


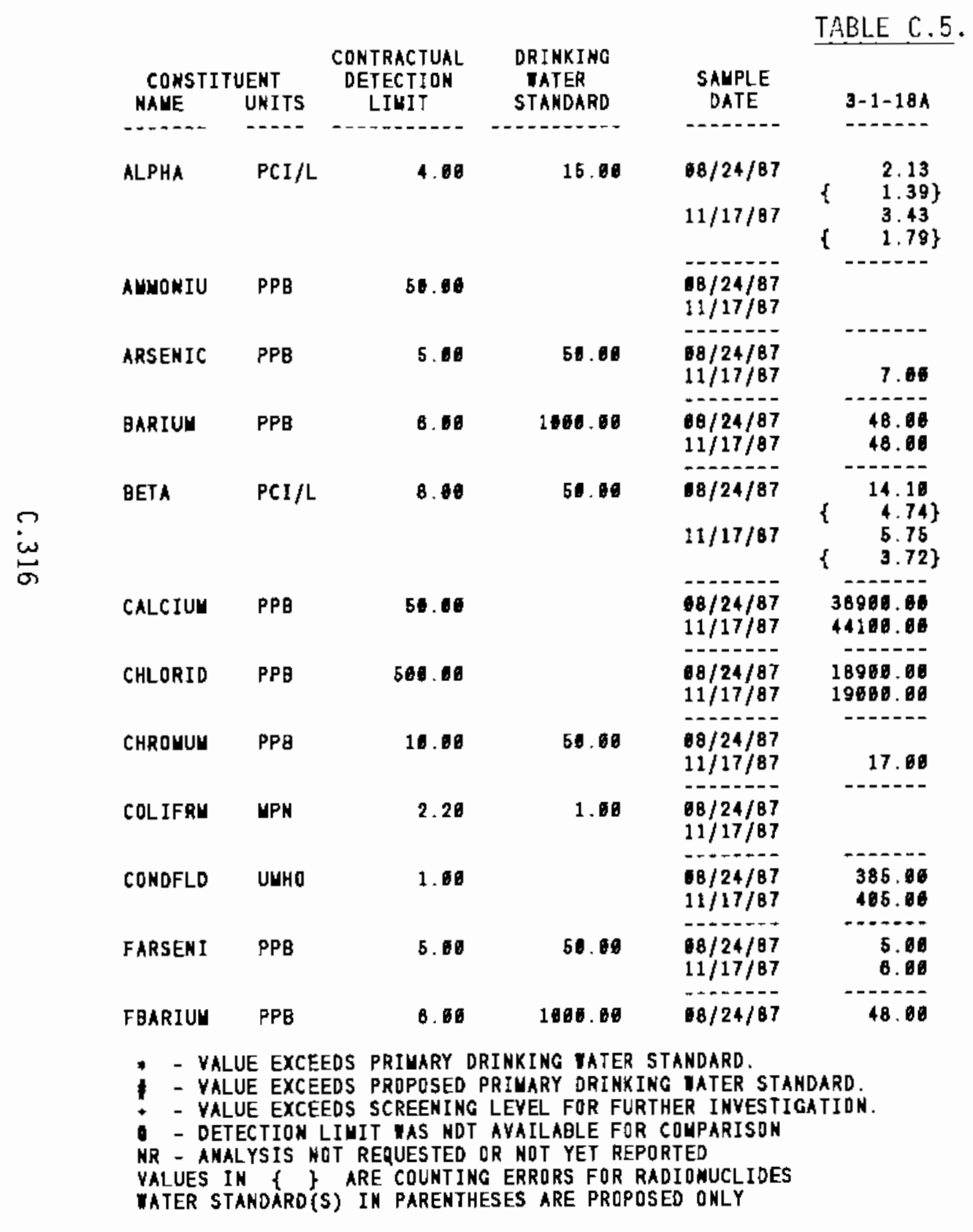

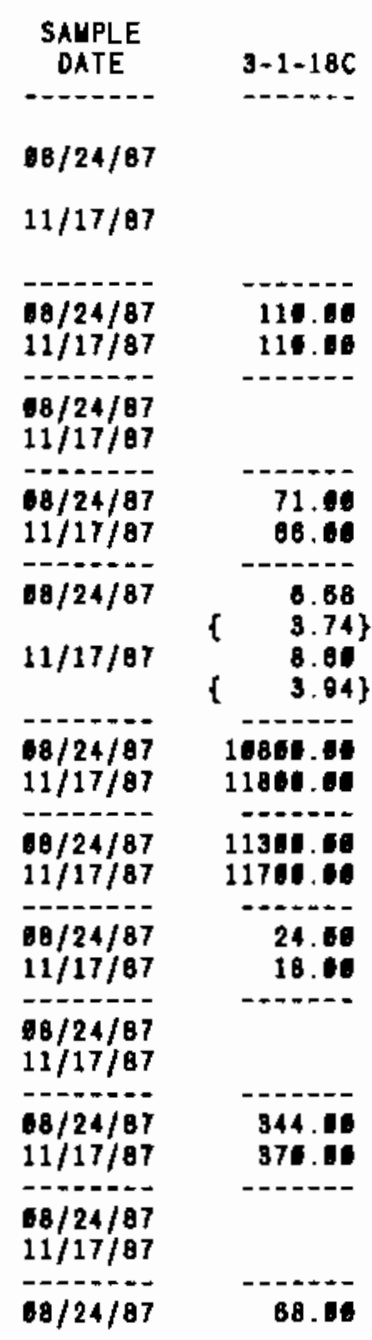




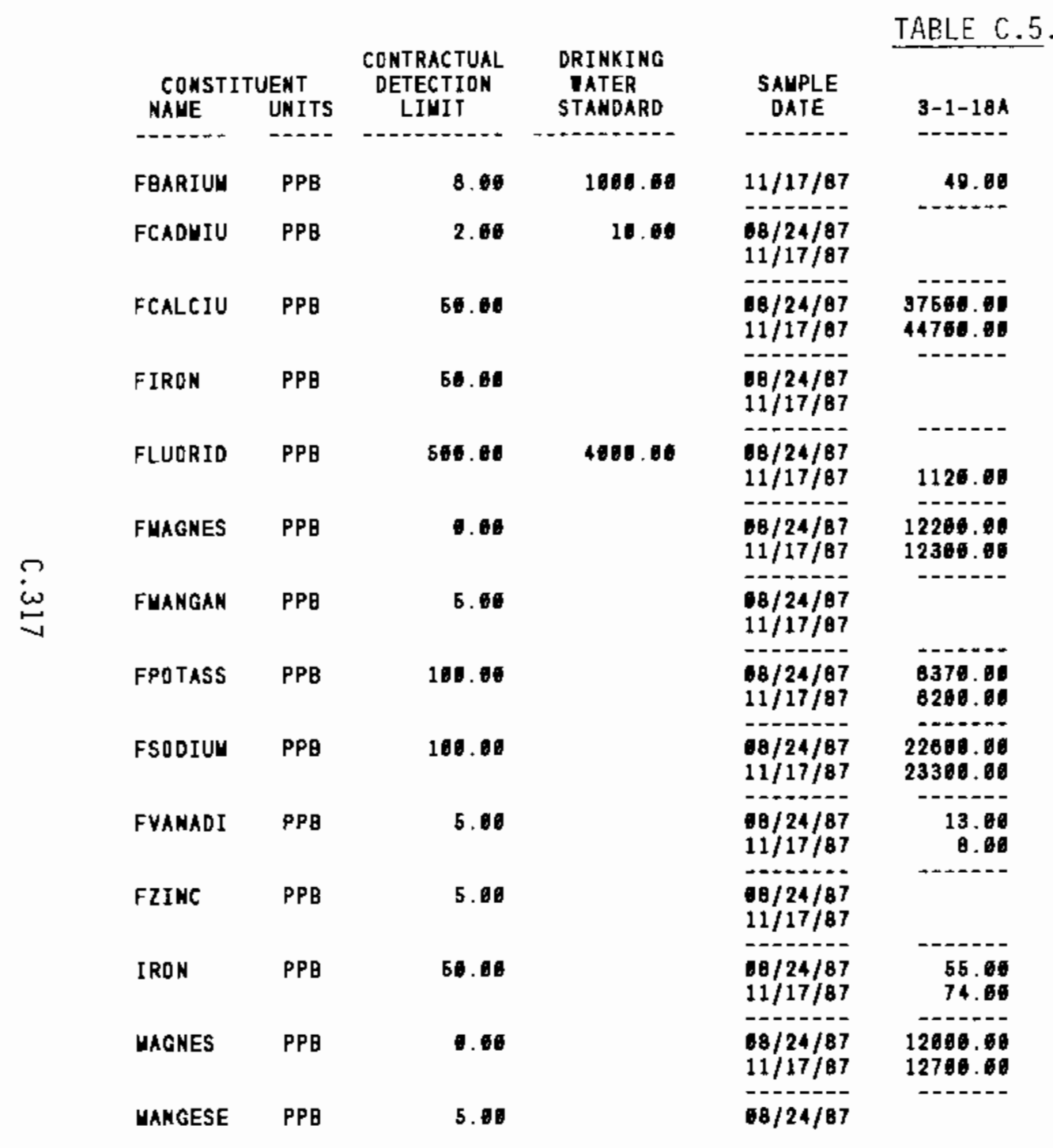

(contd)

* - Value exceeds prinary drinking tater standard.

- - VALUE EXCEEDS PROPOSED PRIUARY DRINKIMG UATER STANDARD.

- - VALUE EXCEEDS SCREENING LEVEL FOR FURTHER INYESTIGATION.

- - DETECTION LiUIT IAS nOt aVALABLE for COUPARISON

NR - ANALYSIS NOT REQUESTED OR NOT YET REPORTED

VALUES IN \{ \} ARE COUNTING ERRORS FOR RADIONUCLIDES

WATER STANDARD(S) IN PARENTHESES ARE PROPDSED ONLY

\begin{tabular}{|c|c|}
\hline $\begin{array}{l}\text { SALPLE } \\
\text { DATE }\end{array}$ & $3-1-18 \theta$ \\
\hline ---- & - - \\
\hline $11 / 17 / 87$ & 43.00 \\
\hline $\begin{array}{l}00 / 24 / 87 \\
11 / 17 / 87\end{array}$ & 2.06 \\
\hline - & - - - \\
\hline $\begin{array}{l}10 / 24 / 87 \\
11 / 17 / 87\end{array}$ & $\begin{array}{l}16100.10 \\
12600.08\end{array}$ \\
\hline $\begin{array}{l}08 / 24 / 87 \\
11 / 17 / 87\end{array}$ & $\begin{array}{l}173.16 \\
155.10\end{array}$ \\
\hline $\begin{array}{l}98 / 24 / 87 \\
11 / 17 / 87\end{array}$ & 1210.00 \\
\hline $\begin{array}{l}08 / 24 / 87 \\
11 / 17 / 87\end{array}$ & $\begin{array}{l}5000.60 \\
5440.66\end{array}$ \\
\hline - & -.... \\
\hline $\begin{array}{l}68 / 24 / 87 \\
11 / 17 / 87\end{array}$ & $\begin{array}{l}43.60 \\
40.60\end{array}$ \\
\hline $\begin{array}{l}68 / 24 / 87 \\
11 / 17 / 87\end{array}$ & $\begin{array}{l}8560 \\
6849\end{array}$ \\
\hline $\begin{array}{l}18 / 24 / 87 \\
11 / 17 / 87\end{array}$ & $\begin{array}{l}83200.00 \\
68300.10\end{array}$ \\
\hline $\begin{array}{l}18 / 24 / 87 \\
11 / 17 / 87\end{array}$ & \\
\hline $\begin{array}{l}08 / 24 / 87 \\
11 / 17 / 87\end{array}$ & 5.00 \\
\hline $\begin{array}{l}68 / 24 / 87 \\
11 / 17 / 87\end{array}$ & 371.60 \\
\hline $\begin{array}{l}68 / 24 / 87 \\
11 / 17 / 87\end{array}$ & $\begin{array}{l}5690.06 \\
5310.60\end{array}$ \\
\hline $8 / 24 / 87$ & 51.00 \\
\hline
\end{tabular}

\begin{tabular}{|c|c|}
\hline $\begin{array}{l}\text { SAUPLE } \\
\text { DATE }\end{array}$ & $3-1-18 C$ \\
\hline 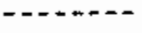 & \\
\hline $11 / 17 / 87$ & 71.00 \\
\hline $\begin{array}{l}08 / 24 / 87 \\
11 / 17 / 87\end{array}$ & \\
\hline $\begin{array}{l}0 / 24 / 87 \\
11 / 17 / 87\end{array}$ & $\begin{array}{l}10200.04 \\
12880.06\end{array}$ \\
\hline $\begin{array}{l}68 / 24 / 87 \\
11 / 17 / 87\end{array}$ & $\begin{array}{r}86.60 \\
85.60\end{array}$ \\
\hline $\begin{array}{l}06 / 24 / 87 \\
11 / 17 / 87\end{array}$ & 1239.00 \\
\hline $\begin{array}{l}18 / 24 / 87 \\
11 / 17 / 87\end{array}$ & $\begin{array}{l}5070.00 \\
6281.00\end{array}$ \\
\hline $\begin{array}{l}18 / 24 / 87 \\
11 / 17 / 87\end{array}$ & 46.5 \\
\hline $\begin{array}{l}08 / 24 / 67 \\
11 / 17 / 67\end{array}$ & $\begin{array}{l}8581.00 \\
8770.00\end{array}$ \\
\hline $\begin{array}{l}08 / 24 / 87 \\
11 / 17 / 87\end{array}$ & 84601.00 \\
\hline $\begin{array}{l}08 / 24 / 87 \\
11 / 17 / 67\end{array}$ & \\
\hline $\begin{array}{l}08 / 24 / 87 \\
11 / 17 / 87\end{array}$ & \\
\hline $\begin{array}{l}08 / 24 / 87 \\
11 / 17 / 87\end{array}$ & 188.16 \\
\hline $\begin{array}{l}98 / 24 / 87 \\
11 / 17 / 87\end{array}$ & 5290.10 \\
\hline $08 / 24 / 87$ & 51.08 \\
\hline
\end{tabular}




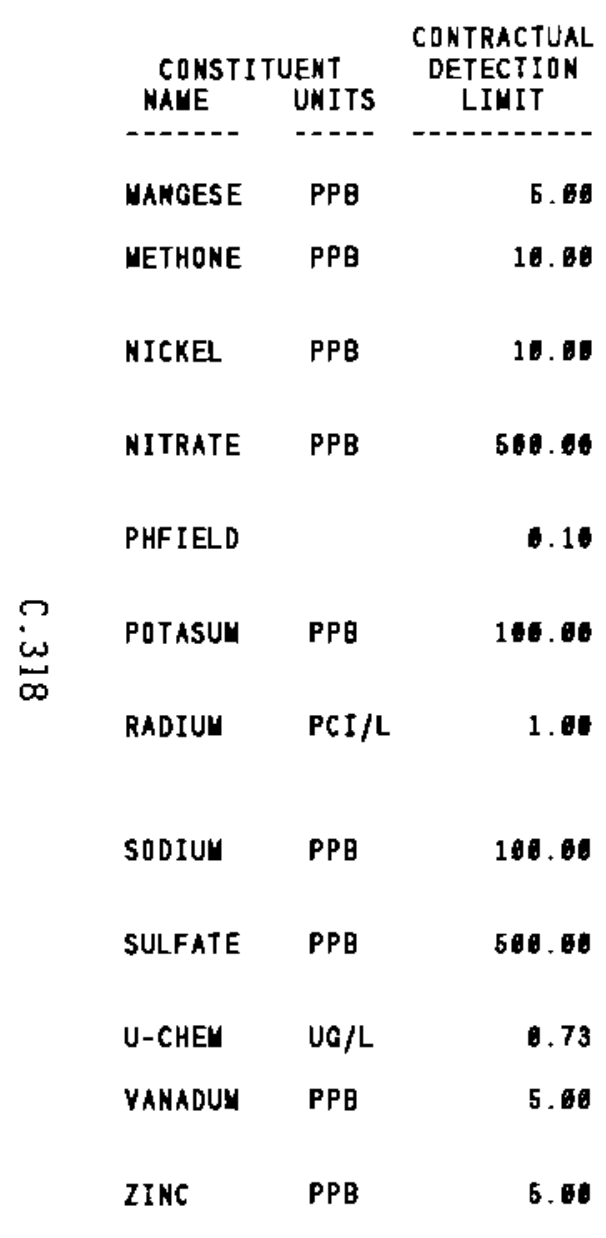

TABLE C.5. (contd)

DRINKING

WATER STAMDARD

SAUPLE DATE - DATE

$11 / 17 / 87$

68/24/87 $11 / 17 / 87$ $18 / 24 / 87$ $11 / 17 / 87$

$08 / 24 / 87$

$11 / 17 / 87$

68/24/87

$68 / 24 / 87$
$11 / 17 / 87$

$8 / 24 / 87$

$11 / 17 / 87$

$-2124107$

5. 10

$11 / 17 / 07$

18/24/87

$11 / 17 / 87$

$08 / 24 / 87$

$11 / 17 / 87$

$11 / 17 / 87$

$8 / 24 / 87$

$11 / 17 / 87$

08/24/87

$11 / 17 / 87$

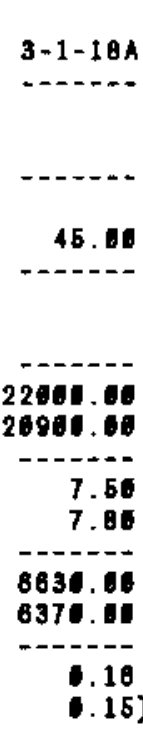

23280.00

2410100

48100.60

46200.00

4.28

14.

14.06

0.00

7.00
SALPLE

DATE

$11 / 17 / 87$

$-----$

$88 / 24 / 97$
$11 / 17 / 87$

68/24/87

$11 / 17 / 87$

-

$11 / 17 / 87$

$08 / 24 / 87$

$11 / 17 / 87$

68/24/07

$11 / 17 / 87$

$--2-1 / 24$

$11 / 17 / 87$

$60 / 24 / 87$

$11 / 17 / 87$

9/24/87

$11 / 17 / 87$

-11/17/87

-

98/24/87

$11 / 17 / 87$

68/24/87

$11 / 17 / 87$
3-1-189

51.00

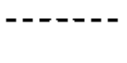

21.0

16.0

16.00

10.--

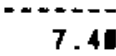

7.41

7460.10

6800.

-

$-1--$

64806.00

........
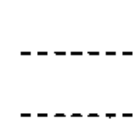

13.48
$11 / 17 / 87$

SAUPLE DATE

$11 / 17 / 87$

$68 / 24 / 07$

$11 / 17 / 87$

18/24/87

$11 / 17 / 87$

$68 / 24 / 87$

$11 / 17 / 87$

$18 / 24 / 87$

$11 / 17 / 87$

18/24/87

$11 / 17 / 87$

$08 / 24 / 67$

$11 / 17 / 87$

$08 / 24 / 87$

$11 / 17 / 87$

$11 / 17 / 87$

-124187

68/24/87

$11 / 17 / 87$

98/24/87

$11 / 17 / 87$
.....--

55. 06

21.10

$-\cdot--$

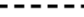

7.7

7.70
0.40

707.

6820.010

68749.09

66200.00

2180

2010.

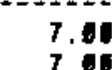

* - yalue exceEds privary dRINKIMg tater stanoARd

- VALUE EXCEEDS PROPOSED PRIUARY DRINKING tater STANDARD.

- VALUE EXCEEDS SCREENING LEVEL FOR FURTHER INYESTIGATIOR.

- DETECTION LIMIT IAS NOT AYAILABLE FOR COUPARISON

MR - AMALYSIS NOT REQUESTED DR NOT YET REPDRTED

VALUES IW \{ $\}$ ARE COUNTING ERRORS FOR RADIONUCLIDES

WATER STANDARD'(S) IN PARENTHESES ARE PROPOSED ONLY 


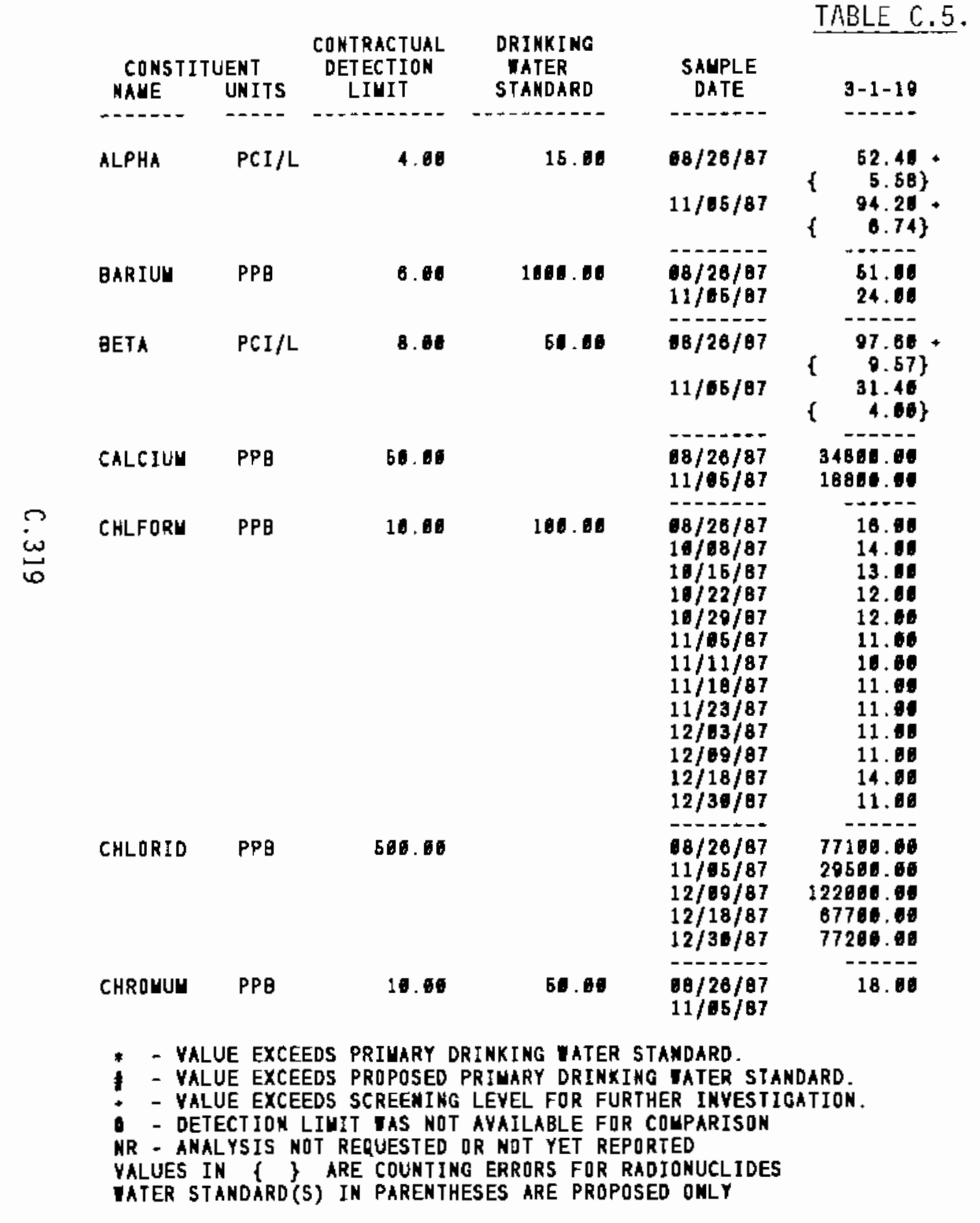

\begin{tabular}{|c|c|}
\hline $\begin{array}{l}\text { SAUPLE } \\
\text { DATE }\end{array}$ & $3-2-1$ \\
\hline $08 / 14 / 87$ & 8.77 \\
\hline $11 / 1 \theta / 87$ & $\begin{array}{r}11.70 \\
\{\quad 2.67\}\end{array}$ \\
\hline $\begin{array}{l}68 / 14 / 87 \\
11 / 10 / 87\end{array}$ & $\begin{array}{l}44.08 \\
63.08\end{array}$ \\
\hline $68 / 14 / 87$ & $\begin{array}{r}14.18 \\
4.377\end{array}$ \\
\hline $11 / 10 / 87$ & $\left.\begin{array}{r}13.80 \\
\{\quad 4.26\end{array}\right\}$ \\
\hline $\begin{array}{l}89 / 14 / 87 \\
11 / 16 / 87\end{array}$ & $\begin{array}{l}17486.60 \\
21586.00\end{array}$ \\
\hline $\begin{array}{l}68 / 14 / 87 \\
11 / 10 / 87\end{array}$ & $\begin{array}{c}13.08 \\
13.06 \\
\text { NR } \\
\text { NR } \\
\text { MR } \\
\text { MR } \\
\text { NR } \\
\text { NR } \\
\text { NR } \\
\text { NR } \\
\text { NR } \\
\text { MR } \\
\text { MR }\end{array}$ \\
\hline $\begin{array}{l}68 / 14 / 87 \\
11 / 1 \% / 87\end{array}$ & $\begin{array}{c}9570.08 \\
11900.00 \\
\text { NR } \\
\text { NR } \\
\text { NR }\end{array}$ \\
\hline $\begin{array}{l}0.78 / 1487 \\
11 / 10 / 87\end{array}$ & \\
\hline
\end{tabular}

\begin{tabular}{|c|c|c|}
\hline $\begin{array}{l}\text { SAUPLE } \\
\text { DATE }\end{array}$ & & 3-3-7 \\
\hline $98 / 13 / 87$ & & 9.56 \\
\hline $11 / 09 / 87$ & i & $\begin{array}{l}2.02\} \\
8.89 \\
2.11\}\end{array}$ \\
\hline $\begin{array}{l}68 / 13 / 87 \\
11 / 08 / 87\end{array}$ & & 43.11 \\
\hline $88 / 13 / 87$ & 1 & 14.26 \\
\hline $11 / 89 / 87$ & \{ & $\begin{array}{l}9.48 \\
3.97\}\end{array}$ \\
\hline $\begin{array}{l}88 / 13 / 87 \\
11 / 09 / 87\end{array}$ & & 180.06 \\
\hline $\begin{array}{l}88 / 13 / 87 \\
11 / 09 / 87\end{array}$ & & $\begin{array}{c}28.60 \\
\text { NR } \\
\text { NR } \\
\text { NR } \\
\text { NR } \\
\text { NR } \\
\text { NR } \\
\text { NR } \\
\text { NR } \\
\text { NR } \\
\text { NR } \\
\text { NR }\end{array}$ \\
\hline $\begin{array}{l}8 B / 13 / 87 \\
11 / 89 / 87\end{array}$ & & $\begin{array}{l}100.09 \\
\text { NR } \\
\text { NR } \\
\text { NR }\end{array}$ \\
\hline $\begin{array}{l}08 / 13 / 87 \\
11 / 09 / 87\end{array}$ & & \\
\hline
\end{tabular}


TABLE C.5. (contd)

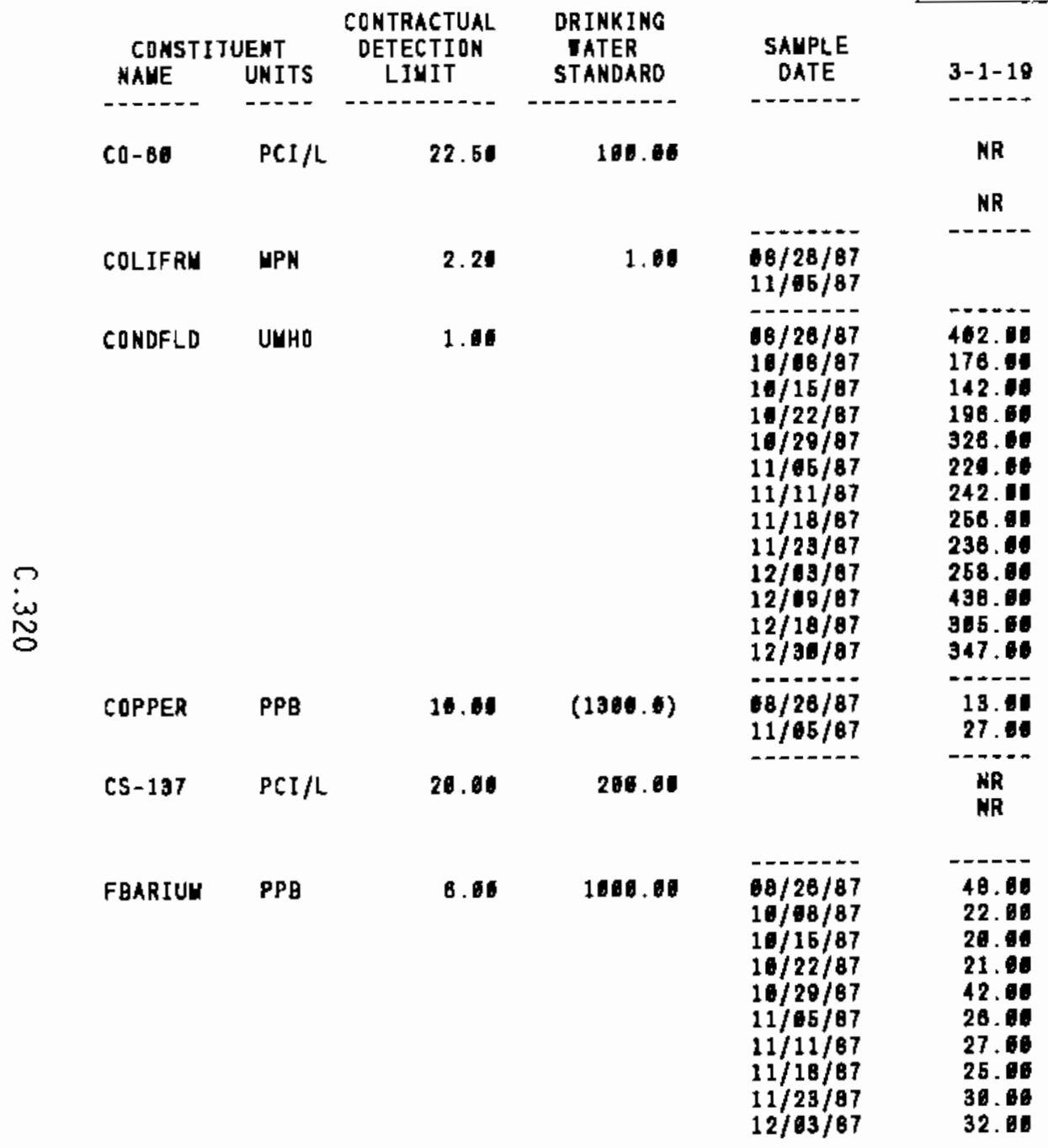

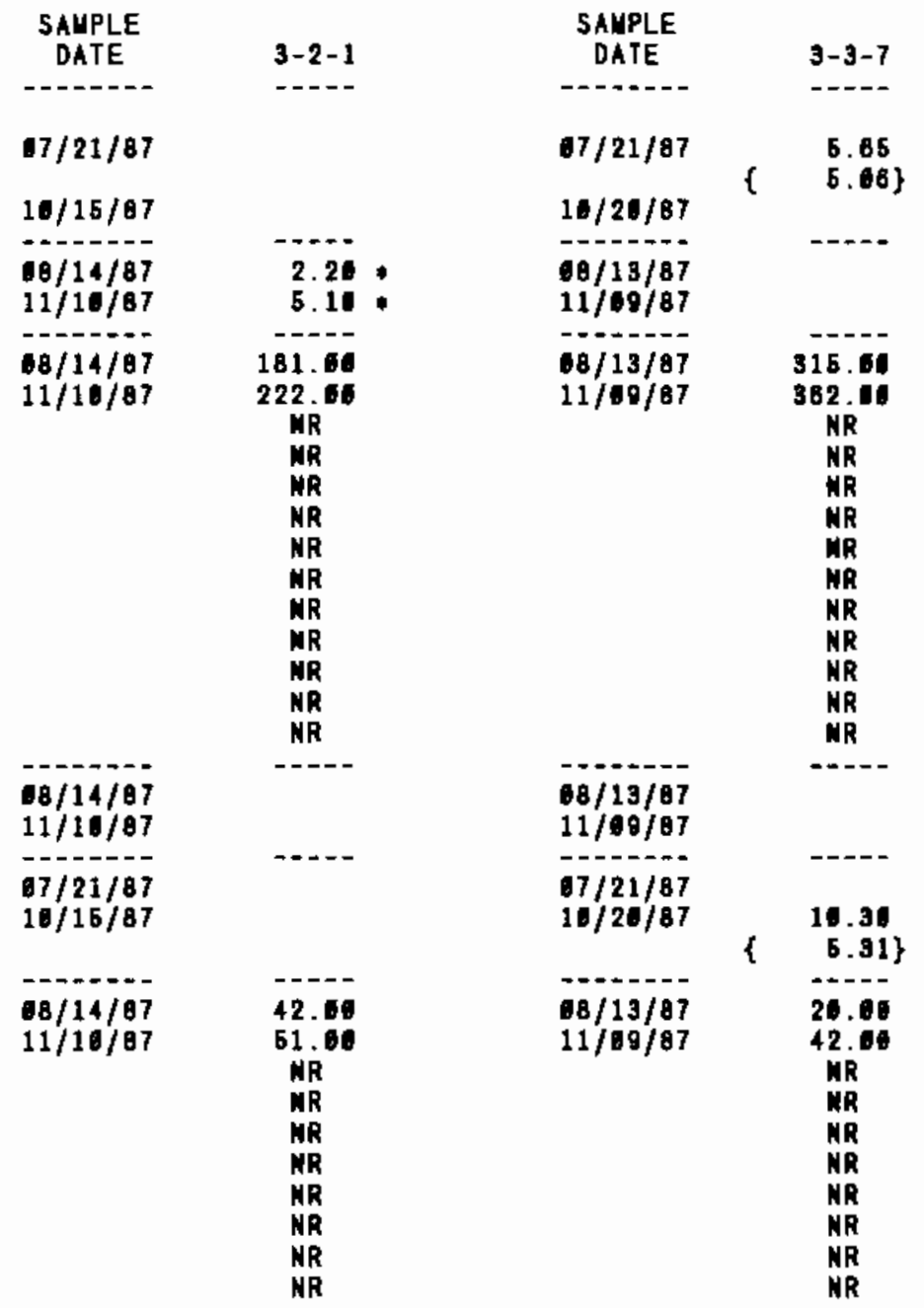

* - ValUe exceEds priuary orinking tater standard.

- VALUE EXCEEDS PROPOSED PRIMARY ORINKING TATER STANDARO.

- YALUE EXCEEDS SCREEMING LEYEL FOR FURTHER INVESTIOATION.

- oetection livit ias not available for couparisón

NR - ANALYSIS NOT REQUESTED OR MOT YET REPORTED

VALUES IN i $\}$ ARE COUNTING ERRORS FOR RADIONUCLIOES

IATER STANDARD (S) IN PARENTHESES ARE PROPOSED OKLY 
TABLE C.5. (contd)

\begin{tabular}{|c|c|c|c|c|}
\hline $\begin{array}{c}\text { CONST } \\
\text { NAME }\end{array}$ & $\begin{array}{l}\text { TUENT } \\
\text { UNITS }\end{array}$ & $\begin{array}{c}\text { CONTRACIUAL } \\
\text { DETECTION } \\
\text { LIUIT }\end{array}$ & $\begin{array}{l}\text { DRINKING } \\
\text { WATER } \\
\text { STANDARD }\end{array}$ & $\begin{array}{c}\text { SAMPLE } \\
\text { DATE }\end{array}$ \\
\hline FCALCIU & PPB & 56.06 & & $\begin{array}{l}08 / 28 / 87 \\
10 / 68 / 87 \\
10 / 15 / 87 \\
10 / 22 / 87 \\
10 / 29 / 87 \\
11 / 15 / 87 \\
11 / 11 / 87 \\
11 / 18 / 87 \\
11 / 23 / 87 \\
12 / 03 / 87\end{array}$ \\
\hline FCOPPER & $\mathrm{PPQ}$ & 16.60 & $(1306.0)$ & $\begin{array}{l}60 / 20 / 87 \\
10 / 18 / 87 \\
10 / 15 / 87 \\
10 / 22 / 87 \\
10 / 29 / 87 \\
11 / 65 / 87 \\
11 / 11 / 87 \\
11 / 18 / 87 \\
11 / 23 / 87 \\
12 / 03 / 87\end{array}$ \\
\hline FIRON & $\mathrm{PPB}$ & 60.80 & & $\begin{array}{l}08 / 28 / 87 \\
10 / 08 / 87 \\
10 / 15 / 87 \\
10 / 22 / 87 \\
10 / 29 / 87 \\
11 / 15 / 87 \\
11 / 11 / 87 \\
11 / 18 / 87 \\
11 / 23 / 87 \\
12 / 63 / 87\end{array}$ \\
\hline FLUORID & PPB & 500.00 & 4000.00 & $\begin{array}{l}08 / 26 / 07 \\
11 / 05 / 87 \\
12 / 09 / 87 \\
12 / 18 / 87 \\
12 / 36 / 87\end{array}$ \\
\hline
\end{tabular}

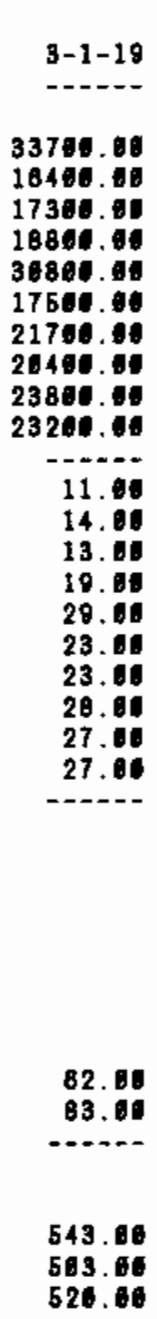

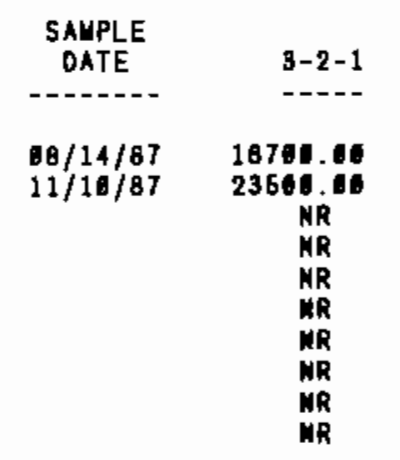

SAUPLE
DATE

3-3-7 -...----

-.--

90/14/87

$11 / 1 \% / 87$

68/13/87

$11 / 69 / 87$

19401.86

NR

IR

MR

IR

MR

\begin{tabular}{ll}
$11 / 1 \% / 87$ & NR \\
& NR \\
& NR \\
& NR \\
& NR \\
& NR \\
& NR \\
& NR \\
& \\
& $-\cdots$ \\
& \\
& \\
\hline $08 / 14 / 87$ &
\end{tabular}

$08 / 14 / 87$

$11 / 16 / 87$

-1.-1

$11 / 09 / 87$

$08 / 13 / 87$

$11 / 00 / 87$

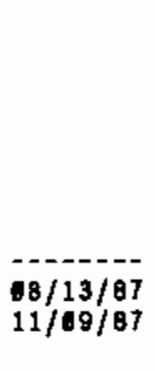

NR
NR
NR
NR
NR
NR
NR
NR
-
-

$08 / 14 / 87$

$11 / 16 / 87$

588.1.
NR
NR
NR

$68 / 13 / 87$

$11 / 09 / 87$

NR
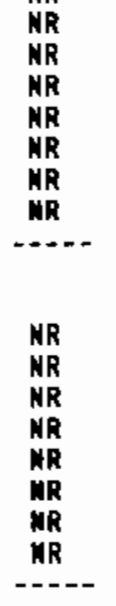

* - value exceeds priuary drinking tajer stamdard.

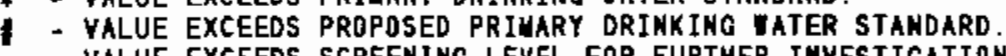

- - YALUE EXCEEDS SCREENINg LEVEL FOR FURTHER IMVESTIGaTION.

- DETECTION Linit ias not available for comparison

NR - AMALYSIS NOT REQUESTED OR NOT YET REPDRTED

VALUES IN ( ) ARE COUNTING ERRORS FOR RADIOMUCLIDES

IATER STANDARD(S) IN ParentheSES aRe PROPOSED ONLY 


$\begin{array}{cccc}\text { CDNSTITUENT } & \begin{array}{c}\text { CONTRACTUAL } \\ \text { DETECTION }\end{array} & \begin{array}{c}\text { DRINKING } \\ \text { IATER }\end{array} \\ \text { NAHE } & \text { UNITS } & \text { LIMIT } & \text { STANDARO } \\ -0- & - & \end{array}$

FUAGNES PPB

0. 00

FMANGAN PPB

6.00

N

TABLE C.5. (contd)

\begin{tabular}{|c|c|}
\hline $\begin{array}{l}\text { SAHPLE } \\
\text { OATE }\end{array}$ & $3-1-18$ \\
\hline - & \\
\hline $\begin{array}{l}08 / 26 / 87 \\
10 / 68 / 87 \\
10 / 16 / 87 \\
16 / 22 / 87 \\
16 / 29 / 87 \\
11 / 65 / 87 \\
11 / 11 / 87 \\
11 / 18 / 87 \\
11 / 23 / 87 \\
12 / 63 / 87\end{array}$ & $\begin{array}{l}8520.00 \\
3440.00 \\
3420.00 \\
4350.00 \\
7370.00 \\
4120.00 \\
5680.00 \\
5860.00 \\
6220.00 \\
5586.00\end{array}$ \\
\hline $\begin{array}{l}08 / 28 / 87 \\
10 / 08 / 87 \\
10 / 15 / 87 \\
10 / 22 / 87 \\
16 / 20 / 87 \\
11 / 65 / 87 \\
11 / 11 / 87 \\
11 / 18 / 87 \\
11 / 23 / 87 \\
12 / 63 / 87\end{array}$ & $\begin{array}{r}-10.00 \\
5.00 \\
0.06 \\
6.06 \\
8.06 \\
5.06\end{array}$ \\
\hline
\end{tabular}

FNICKEL PPB 11.06

$0 / 26 / 87$

$10 / 68 / 87$

$10 / 15 / 87$

$10 / 22 / 67$

$10 / 29 / 87$

$11 / 05 / 87$

$11 / 11 / 87$

$11 / 18 / 87$

$11 / 23 / 87$

$12 / 03 / 87$

FPDTASS PPB

100.60

$18 / 26 / 87$

$10 / 88 / 87$

10/15/87

$10 / 22 / 87$

11.0

3076.00

1900.60

1020.06

1940.00

2400.00

SALPLE

DATE 3-2-1

SAMPLE

DATE

3-3-7

$8 / 14 / 87$

$11 / 10 / 87$

4280.60

5140.00

NR

$\underset{N R}{N R}$

NR

MR

MR

WR

$8 / 14 / 87$

$11 / 10 / 87$

NR
NR
NR
NR
NR
NR
NR
NR

\section{8/14/87}

$11 / 10 / 87$
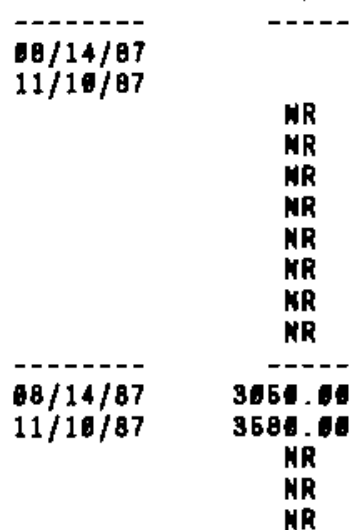

$68 / 13 / 87$ 4686.00

$11 / 69 / 87$ 8986

MR

NR

NR

NR
MR

MR
MR

AR

18/13/87

$11 / 00 / 87$

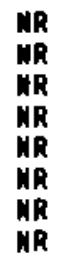

$18 / 13 / 87$

$11 / 09 / 87$

- - yalue exceeds primary dRIMKing vater standard.

- VALUE EXCEEDS PROPOSED PRINARY DRINKING TATER STANDARD.

- VALUE EXCEEDS SCREENING LEVEL FOR FURTHER INVESTIGAT

- DETECTION LIUIT TAS NOT AVAILABLE FOR COUPARISON

NR - AHALYSIS NOT REQUESTED OR MOT YET REPORTED

YALUES IN \{\} ARE COUNTING ERRORS FOR RADIONUCLIDES

TATER STAMDARD(S) IN PARENTHESES ARE PROPDSED ONLY 


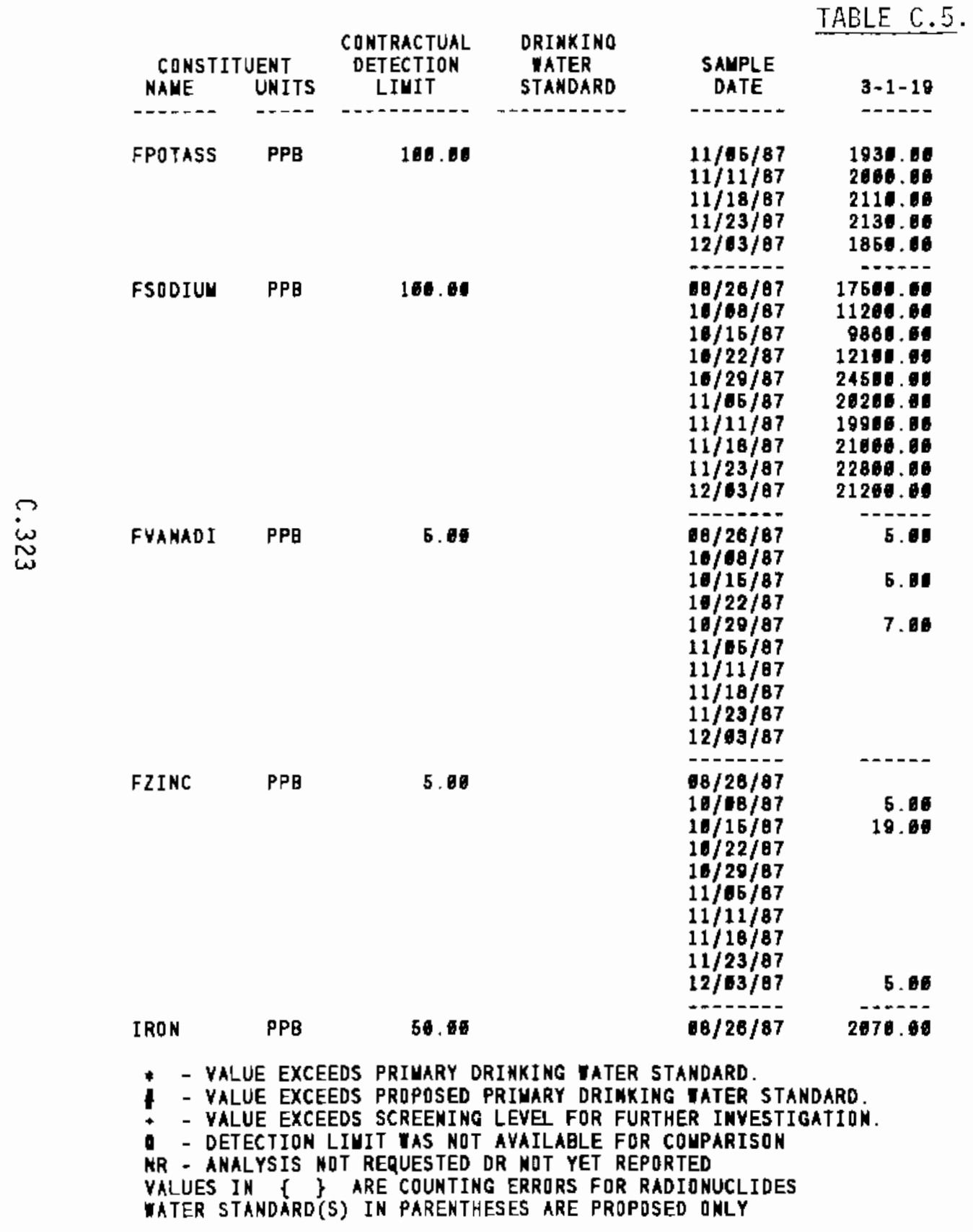

\begin{tabular}{|c|c|}
\hline $\begin{array}{c}\text { SAUPLE } \\
\text { DATE }\end{array}$ & $\begin{array}{c}3-2-1 \\
\cdots \\
M R \\
M R \\
\text { MR } \\
\text { NR } \\
\text { NR }\end{array}$ \\
\hline $\begin{array}{l}8 / 14 / 87 \\
11 / 16 / 87\end{array}$ & $\begin{array}{c}13300.0 \\
14200 \\
N R \\
\text { NR } \\
\text { NR } \\
\text { NR } \\
\text { NR } \\
\text { NR } \\
\text { NR } \\
\text { NR }\end{array}$ \\
\hline $\begin{array}{l}08 / 14 / 87 \\
11 / 16 / 87\end{array}$ & $\begin{array}{l}\text { NR } \\
N R \\
N R \\
N R \\
N R \\
N R \\
N R \\
N R \\
N R\end{array}$ \\
\hline $\begin{array}{l}60 / 14 / 87 \\
11 / 10 / 87\end{array}$ & $\begin{array}{l}\text { NR } \\
\text { NR } \\
\text { NR } \\
\text { NR } \\
\text { NR } \\
\text { NR } \\
\text { NR } \\
\text { NR }\end{array}$ \\
\hline $88 / 14 / 87$ & 94.00 \\
\hline
\end{tabular}

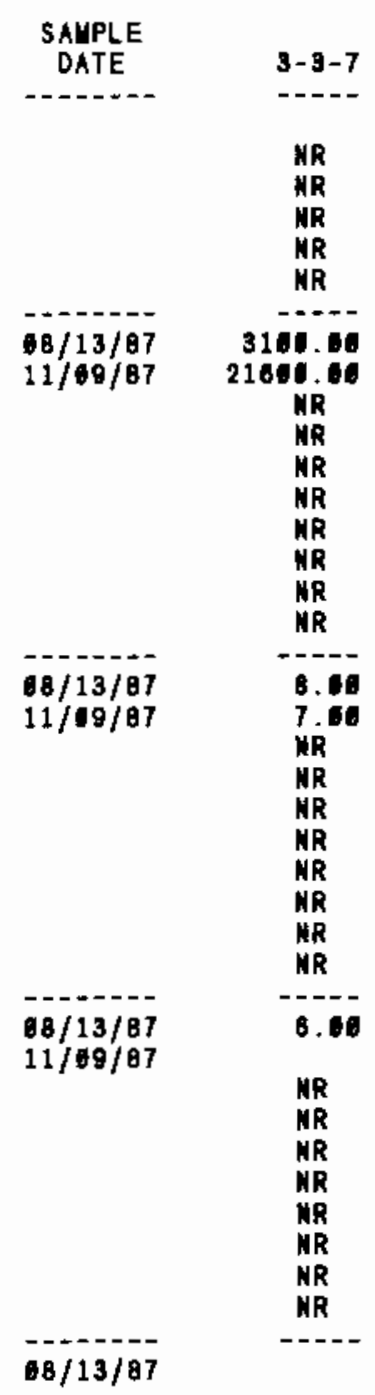


TABLE C.5. (contd)

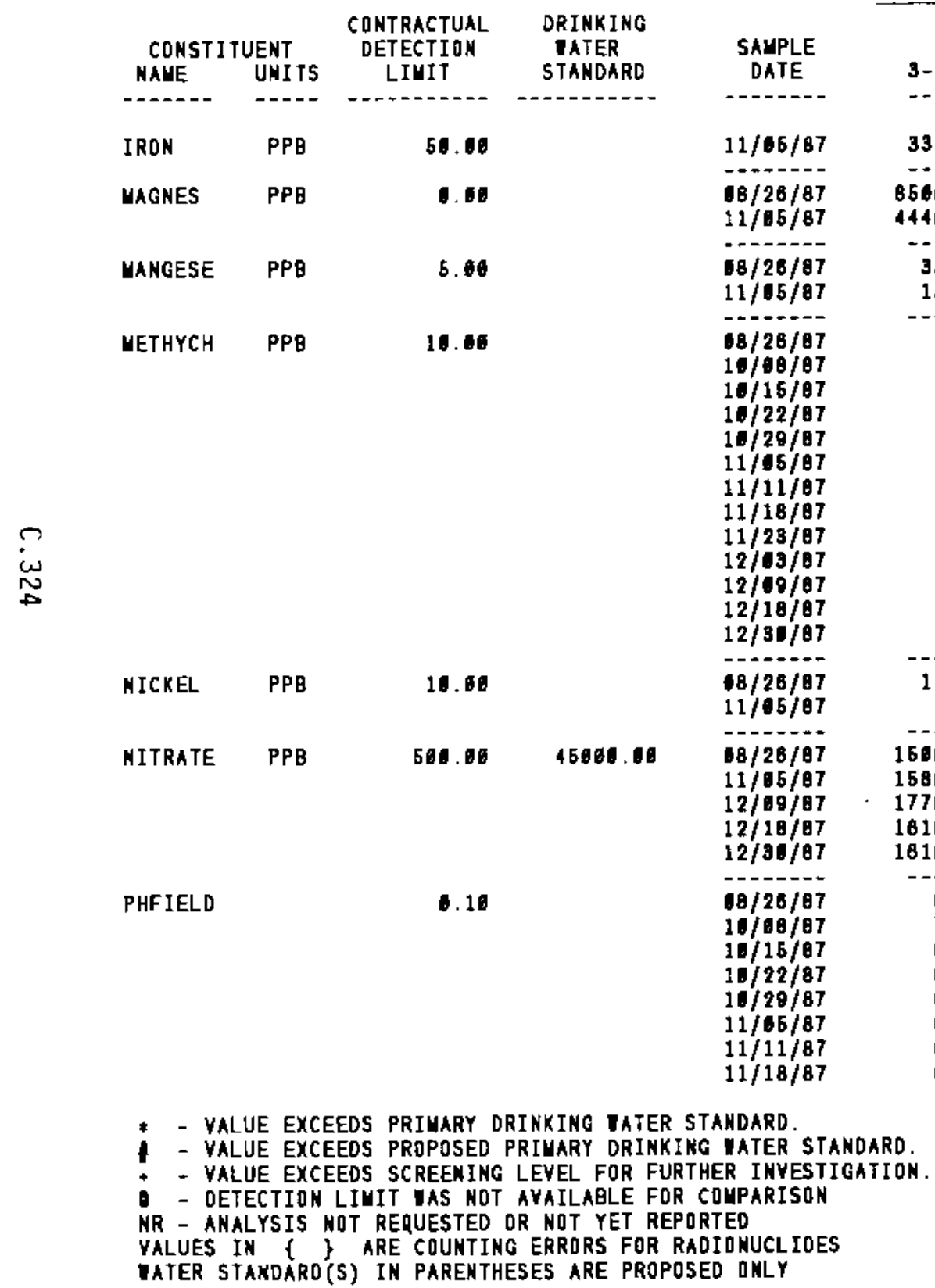

\begin{tabular}{|c|c|c|c|}
\hline $\begin{array}{c}\text { SAUPLE } \\
\text { DATE }\end{array}$ & $3-2+1$ & $\begin{array}{c}\text { SAWPLE } \\
\text { DATE }\end{array}$ & $3-3-7$ \\
\hline $11 / 10 / 87$ & 52.00 & $11 / 69 / 87$ & 930.00 \\
\hline $\begin{array}{l}88 / 14 / 87 \\
11 / 18 / 87\end{array}$ & $\begin{array}{l}4220.60 \\
5181.60\end{array}$ & $\begin{array}{l}98 / 13 / 87 \\
11 / 08 / 87\end{array}$ & $\begin{array}{l}8650.11 \\
\text { B540.00 }\end{array}$ \\
\hline $\begin{array}{l}88 / 14 / 87 \\
11 / 16 / 87\end{array}$ & & $\begin{array}{l}08 / 13 / 87 \\
11 / 09 / 87\end{array}$ & 24.00 \\
\hline \multirow[t]{2}{*}{$\begin{array}{l}68 / 14 / 87 \\
11 / 10 / 87\end{array}$} & 22.10 & $\begin{array}{l}08 / 13 / 87 \\
11 / 09 / 87\end{array}$ & \\
\hline & $\begin{array}{l}\text { NR } \\
\text { NR } \\
\text { NR } \\
\text { NR } \\
\text { NR } \\
\text { NR } \\
\text { NR } \\
\text { NR } \\
\text { NR } \\
\text { NR } \\
\text { NR }\end{array}$ & & $\begin{array}{l}\text { MR } \\
\text { MR } \\
\text { MR } \\
\text { MR } \\
\text { MR } \\
\text { MR } \\
\text { MR } \\
\text { MR } \\
\text { MR } \\
\text { MR } \\
\text { MR }\end{array}$ \\
\hline $\begin{array}{l}08 / 14 / 87 \\
11 / 10 / 87\end{array}$ & & $\begin{array}{l}68 / 13 / 87 \\
11 / 09 / 87\end{array}$ & 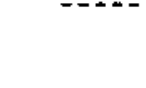 \\
\hline $\begin{array}{l}08 / 14 / 87 \\
11 / 10 / 87\end{array}$ & $\begin{array}{c}8876.01 \\
13406.01 \\
\text { NR } \\
\text { NR } \\
\text { NR }\end{array}$ & $\begin{array}{l}08 / 13 / 87 \\
11 / 69 / 87\end{array}$ & $\begin{array}{c}14865.00 \\
14780.04 \\
\text { NR } \\
\text { NR } \\
\text { NR }\end{array}$ \\
\hline $\begin{array}{l}08 / 14 / 87 \\
11 / 10 / 87\end{array}$ & $\begin{array}{l}\text { 6.58 } \\
\text { 6.45 } \\
\text { NR } \\
\text { NR } \\
\text { NR } \\
\text { NR } \\
\text { NR } \\
\text { NR }\end{array}$ & $\begin{array}{l}98 / 13 / 87 \\
11 / 09 / 87\end{array}$ & $\begin{array}{l}7.29 \\
7.31 \\
\mathrm{NR} \\
\mathrm{NR} \\
\mathrm{NR} \\
\mathrm{NR} \\
\mathrm{NR} \\
\mathrm{MR}\end{array}$ \\
\hline
\end{tabular}

11.00

15060

1586.00

770.06

1816.00

B. 50

8. 50

8.60

6. 20

6.60

6.90
6.80

NR

$-+-$

.11

$---$

NP

IR

RR

MR

CEEDS PRIUARY DRINKING JATER STANDARD .

- OEIECTION LIUIT HAS NOT AYAILAOLE FOR COUPARISON

YALUES IN

IATER STAKDARO'S) IN PARENTHESES ARE PROPOSED DHLY 


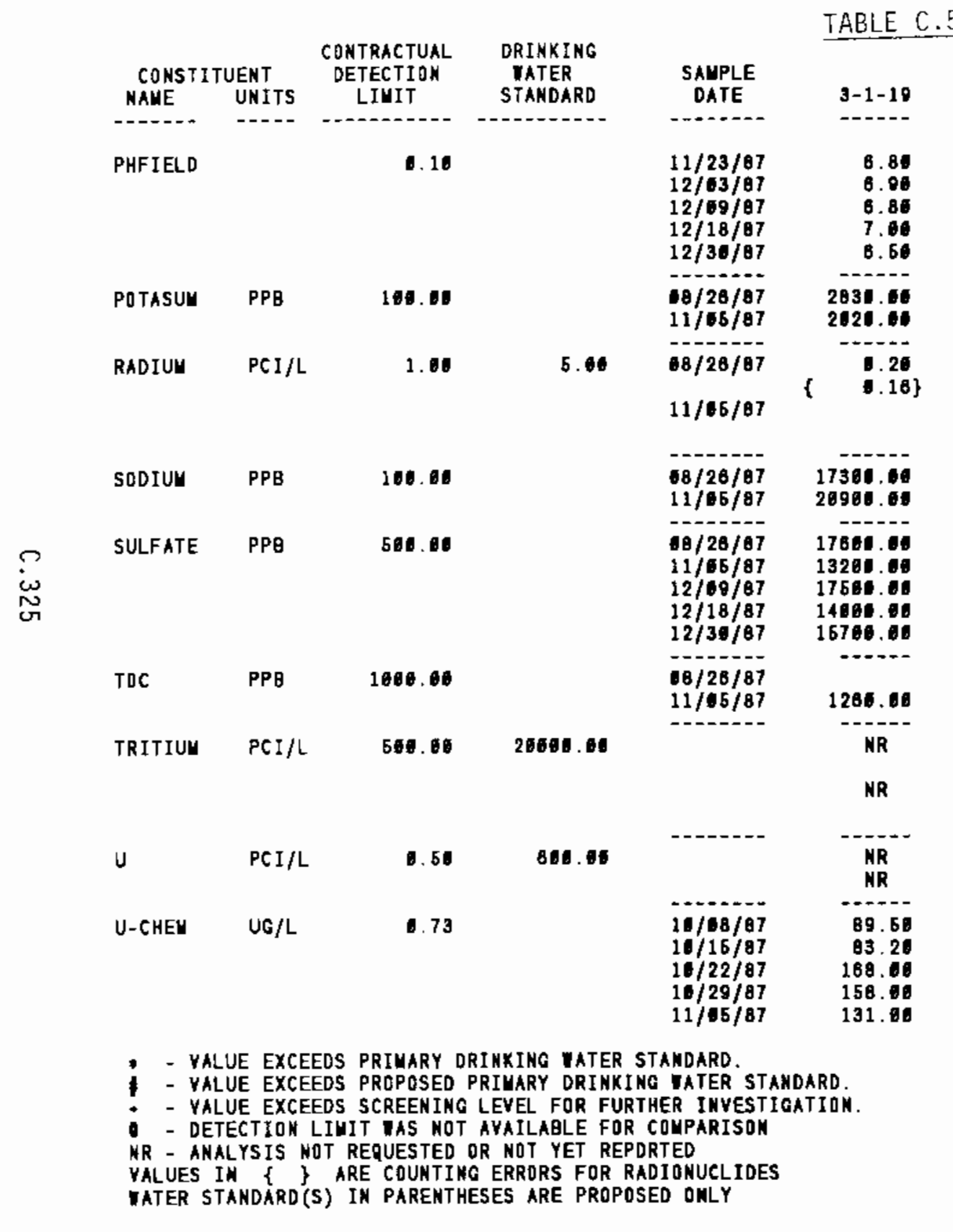

\begin{tabular}{|c|c|c|c|}
\hline $\begin{array}{l}\text { SAMPLE } \\
\text { DATE }\end{array}$ & $3-2+1$ & $\begin{array}{c}\text { SAMPLE } \\
\text { DATE }\end{array}$ & $5-3-7$ \\
\hline & $\begin{array}{l}\text { NR } \\
\text { NR } \\
\text { NR } \\
\text { NR } \\
\text { NR }\end{array}$ & & $\begin{array}{c}\text { MR } \\
M R \\
M R \\
M R \\
M R\end{array}$ \\
\hline $\begin{array}{l}88 / 14 / 87 \\
11 / 16 / 87\end{array}$ & $\begin{array}{l}2011.08 \\
3780.01\end{array}$ & $\begin{array}{l}68 / 13 / 87 \\
11 / 69 / 87\end{array}$ & $\begin{array}{l}4880.00 \\
5434.00\end{array}$ \\
\hline $88 / 14 / 87$ & & $18 / 13 / 87$ & \\
\hline $11 / 10 / 87$ & & $11 / 69 / 87$ & $\{\quad: .21\}$ \\
\hline $\begin{array}{l}88 / 14 / 97 \\
11 / 16 / 87\end{array}$ & $\begin{array}{l}12406.04 \\
14866.86\end{array}$ & $\begin{array}{l}08 / 13 / 87 \\
11 / 09 / 87\end{array}$ & $\begin{array}{l}20280.60 \\
21200.06\end{array}$ \\
\hline $\begin{array}{l}68 / 14 / 87 \\
11 / 16 / 87\end{array}$ & $\begin{array}{c}18804.04 \\
16200.05 \\
\text { NR } \\
\text { NR } \\
\text { NR }\end{array}$ & $\begin{array}{l}18 / 19 / 87 \\
11 / 69 / 87\end{array}$ & $\begin{array}{c}35604.00 \\
33701.00 \\
\text { NR } \\
\text { NR }\end{array}$ \\
\hline $\begin{array}{l}88 / 14 / 87 \\
11 / 10 / 87\end{array}$ & & $\begin{array}{l}18 / 13 / 87 \\
11 / 69 / 87\end{array}$ & \\
\hline $07 / 21 / 87$ & $\begin{array}{r}286.64 \\
\{167.66\}\end{array}$ & $67 / 21 / 87$ & $\begin{array}{r}1296.06 \\
186.66\end{array}$ \\
\hline $10 / 15 / 87$ & & $10 / 20 / 87$ & $\left(\begin{array}{r}1710.00 \\
258.00\end{array}\right\}$ \\
\hline $\begin{array}{l}67 / 21 / 87 \\
16 / 15 / 87\end{array}$ & $\begin{array}{l}7.27 \\
\text { B.89 }\end{array}$ & $\begin{array}{l}17 / 21 / 87 \\
16 / 26 / 87\end{array}$ & $\begin{array}{l}11.40 \\
18.70\end{array}$ \\
\hline $11 / 10 / 87$ & $\begin{array}{l}16.39 \\
\text { NR } \\
\text { NR } \\
\text { NR } \\
\text { NR }\end{array}$ & $11 / 60 / 87$ & $\begin{array}{l}12.10 \\
\text { NR } \\
\text { NR } \\
\text { NR } \\
\text { NR }\end{array}$ \\
\hline
\end{tabular}




\begin{tabular}{|c|c|c|}
\hline $\begin{array}{l}\text { CONST } \\
\text { NAME }\end{array}$ & $\begin{array}{l}\text { UENT } \\
\text { UNITS }\end{array}$ & $\begin{array}{c}\text { CONTRACTUAL } \\
\text { DETECTION } \\
\text { LIMIT }\end{array}$ \\
\hline $\mathrm{U}$-CHEU & UG $/ \mathrm{L}$ & 0.73 \\
\hline VANADUU & PPB & 5.80 \\
\hline ZINC & PPB & 5.01 \\
\hline
\end{tabular}

TABLE C.5. (contd)

DRINKING
WATER
STAKDARD

SAMPLE

DATE

3-1-10

SAMPLE

DATE 3-2-1

SAUPLE

$11 / 11 / 87 \quad 157.68$

$11 / 18 / 87$

$11 / 23 / 87 \quad 181.08$

$12 / 83 / 87$
$12 / 09 / 87$

$12 / 18 / 87$ 228.11

$12 / 30 / 87 \quad 448.06$

8/20/87 7.0

$11 / 65 / 87$

7. 09

$8 / 28 / 87$

--.--

$11 / 05 / 87$

6.60

\begin{tabular}{|c|c|}
\hline & $\begin{array}{l}\text { NR } \\
\text { NR } \\
\text { NR } \\
\text { NR } \\
\text { NR } \\
\text { NR } \\
\text { NR }\end{array}$ \\
\hline $\begin{array}{l}08 / 14 / 87 \\
11 / 10 / 87\end{array}$ & $\begin{array}{l}0.60 \\
5.00\end{array}$ \\
\hline $\begin{array}{l}88 / 14 / 87 \\
11 / 10 / 87\end{array}$ & $\begin{array}{l}10.06 \\
10.60\end{array}$ \\
\hline
\end{tabular}

NR
NR

NR

MR

MR

NR

$8 / 13 / 87$

$11 / 00 / 87$

10.60

--.--

$08 / 13 / 87$

11.

$11 / 00 / 87$

11.06

* - value exceeds privary dRInking tater standard.

1 - VALUE EXCEEDS PROPDSED PRIMARY DRINKING VATER STANDARD.

- VALUE EXCEEDS SCREEening LEVEL FOR FURTHER INYESTIGation.

- - DETECTIDH liHit nas not available for couparison

MR - AHALYSIS NDT REQUESTED OR NOT YET REPORTED

VALUES IN S A ARE COUNTING ERRORS FOR RADIONUCLIDES

IATER STANDARO'(S) IN PARENTHESES ARE PROPOSED ONLY 
TABLE C.5. (contd)

\begin{tabular}{|c|c|c|c|c|c|c|c|c|c|}
\hline $\begin{array}{l}\text { CONSTI } \\
\text { NANE }\end{array}$ & $\begin{array}{l}\text { UENT } \\
\text { UNITS }\end{array}$ & $\begin{array}{c}\text { CONTRACTUAL } \\
\text { DETECTION } \\
\text { LINIT }\end{array}$ & $\begin{array}{l}\text { DRINKING } \\
\text { WATER } \\
\text { STANDARD }\end{array}$ & $\begin{array}{c}\text { SAMPLE } \\
\text { DAJE }\end{array}$ & $3-3-16$ & $\begin{array}{c}\text { SAMPLE } \\
\text { DATE }\end{array}$ & $3-4-1$ & $\begin{array}{c}\text { SAMPLE } \\
\text { DATE }\end{array}$ & $3-4-7$ \\
\hline ALPHA & $\mathrm{PCI} / \mathrm{L}$ & 4.00 & 16.00 & $\begin{array}{l}18 / 13 / 87 \\
11 / 26 / 87\end{array}$ & 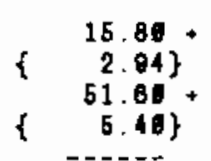 & $\begin{array}{l}88 / 13 / 07 \\
11 / 12 / 07\end{array}$ & $\begin{array}{c}12.76 \\
\{\quad 2.72\} \\
11.38 \\
\{\quad 2.76\}\end{array}$ & $\begin{array}{l}88 / 13 / 87 \\
11 / 88 / 87\end{array}$ & $\begin{array}{c}32.71 \\
4.45\} \\
38.86 \\
4.63\} \\
\ldots . .\end{array}$ \\
\hline ARSENIC & PPB & 5.46 & 58.60 & $\begin{array}{l}18 / 13 / 87 \\
11 / 20 / 87\end{array}$ & $-\cdots$ & $\begin{array}{l}68 / 13 / 87 \\
11 / 12 / 87\end{array}$ & 5.80 & $\begin{array}{l}68 / 13 / 87 \\
11 / 66 / 87\end{array}$ & \\
\hline BARIUM & PPB & B. 00 & 1006.08 & $\begin{array}{l}88 / 13 / 87 \\
11 / 26 / 87\end{array}$ & $\begin{array}{l}44.00 \\
54.00\end{array}$ & $\begin{array}{l}18 / 13 / 87 \\
11 / 12 / 87\end{array}$ & $\begin{array}{l}43.06 \\
38.66\end{array}$ & $\begin{array}{l}68 / 13 / 87 \\
11 / 06 / 87\end{array}$ & $\begin{array}{l}45.16 \\
48.01\end{array}$ \\
\hline BETA & PCI/L & B. 60 & 50.80 & $\begin{array}{l}08 / 13 / 87 \\
11 / 20 / 87\end{array}$ & 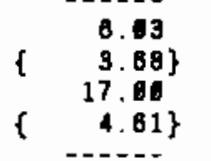 & $\begin{array}{l}68 / 13 / 87 \\
11 / 12 / 87\end{array}$ & $\begin{array}{l}9.98 \\
3.97\} \\
7.55 \\
3.76\}\end{array}$ & $\begin{array}{l}10 / 13 / 87 \\
11 / 98 / 87\end{array}$ & 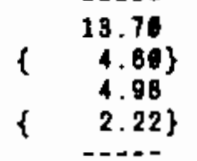 \\
\hline CALCIUM & PPB & 50.00 & & $\begin{array}{l}68 / 13 / 97 \\
11 / 26 / 97\end{array}$ & $\begin{array}{l}23600.08 \\
39106.00\end{array}$ & $\begin{array}{l}88 / 13 / 87 \\
11 / 12 / 87\end{array}$ & $\begin{array}{l}37786.60 \\
38606.60\end{array}$ & $\begin{array}{l}08 / 13 / 87 \\
11 / 06 / 87\end{array}$ & $\begin{array}{l}32809.66 \\
36519.60\end{array}$ \\
\hline CHLFORU & PPB & 10.00 & 100.08 & $\begin{array}{l}88 / 13 / 87 \\
11 / 20 / 87\end{array}$ & 12.06 & $\begin{array}{l}08 / 13 / 87 \\
11 / 12 / 87\end{array}$ & & $\begin{array}{l}68 / 13 / 87 \\
11 / 68 / 87\end{array}$ & \\
\hline CHLORID & PPB & 560.08 & & $\begin{array}{l}68 / 13 / 87 \\
11 / 26 / 87\end{array}$ & $\begin{array}{l}7448.60 \\
7006.80\end{array}$ & $\begin{array}{l}68 / 13 / 87 \\
11 / 12 / 87\end{array}$ & $\begin{array}{r}11760.08 \\
9016.08 \\
\end{array}$ & $\begin{array}{l}68 / 13 / 87 \\
11 / 66 / 87\end{array}$ & $\begin{array}{l}13106.60 \\
13606.01\end{array}$ \\
\hline COLIFRY & UPN & 2.20 & 1.68 & $\begin{array}{l}68 / 13 / 87 \\
11 / 28 / 87\end{array}$ & & $\begin{array}{l}88 / 13 / 87 \\
11 / 12 / 87 \\
\ldots\end{array}$ & $5.10 *$ & $\begin{array}{l}00 / 13 / 87 \\
11 / 06 / 67 \\
\end{array}$ & \\
\hline CONDFLD & UMHO & 1.00 & & $\begin{array}{l}08 / 13 / 87 \\
11 / 20 / 87 \\
-\end{array}$ & $\begin{array}{l}282.08 \\
292.86\end{array}$ & $\begin{array}{l}88 / 13 / 87 \\
11 / 12 / 87\end{array}$ & $\begin{array}{l}278.60 \\
317.60 \\
\end{array}$ & $\begin{array}{l}68 / 13 / 87 \\
11 / 86 / 87\end{array}$ & $\begin{array}{l}291.00 \\
351.00\end{array}$ \\
\hline CS-137 & $\mathrm{PCI} / \mathrm{h}$ & 20.06 & 200.00 & $\begin{array}{l}87 / 21 / 87 \\
10 / 19 / 87\end{array}$ & & $\begin{array}{l}67 / 23 / 87 \\
18 / 19 / 87\end{array}$ & $\begin{array}{l}8.55 \\
\{\quad 5.41\}\end{array}$ & $\begin{array}{l}07 / 21 / 87 \\
18 / 19 / 87\end{array}$ & \\
\hline FARSENI & PPB & 5.60 & 50.80 & $\begin{array}{l}08 / 19 / 87 \\
11 / 26 / 87\end{array}$ & - & $\begin{array}{l}68 / 13 / 87 \\
11 / 12 / 87\end{array}$ & 6.00 & $\begin{array}{l}08 / 13 / 87 \\
11 / 68 / 87\end{array}$ & 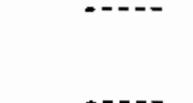 \\
\hline FBARIUU & PPB & 8.06 & 1008.08 & $08 / 13 / 87$ & 49.08 & $O B / 13 / B 7$ & 41.00 & $08 / 13 / 87$ & 45.00 \\
\hline
\end{tabular}

* - yalue exceEds privary dRinking tater standard.

- VALUE EXCEEDS PROPOSED PRIMARY DRINKING uATER STANDARD.

- VALUE EXCEEDS SCREEMIMG LEYEl for FURTHER IMYESTIGATION.

- DETECTION liUit uas not available for coyparison

NR - ANALYSIS NOT REQUESTED OR NOT YET REPORTED

VALUES IN $f$ ARE CDUNTING ERRDRS FOR RADIONUCLIDES

TATER STANOARD(S) IN PARENTHESES ARE PROPOSED ONLY 
TABLE C.5. (contd)

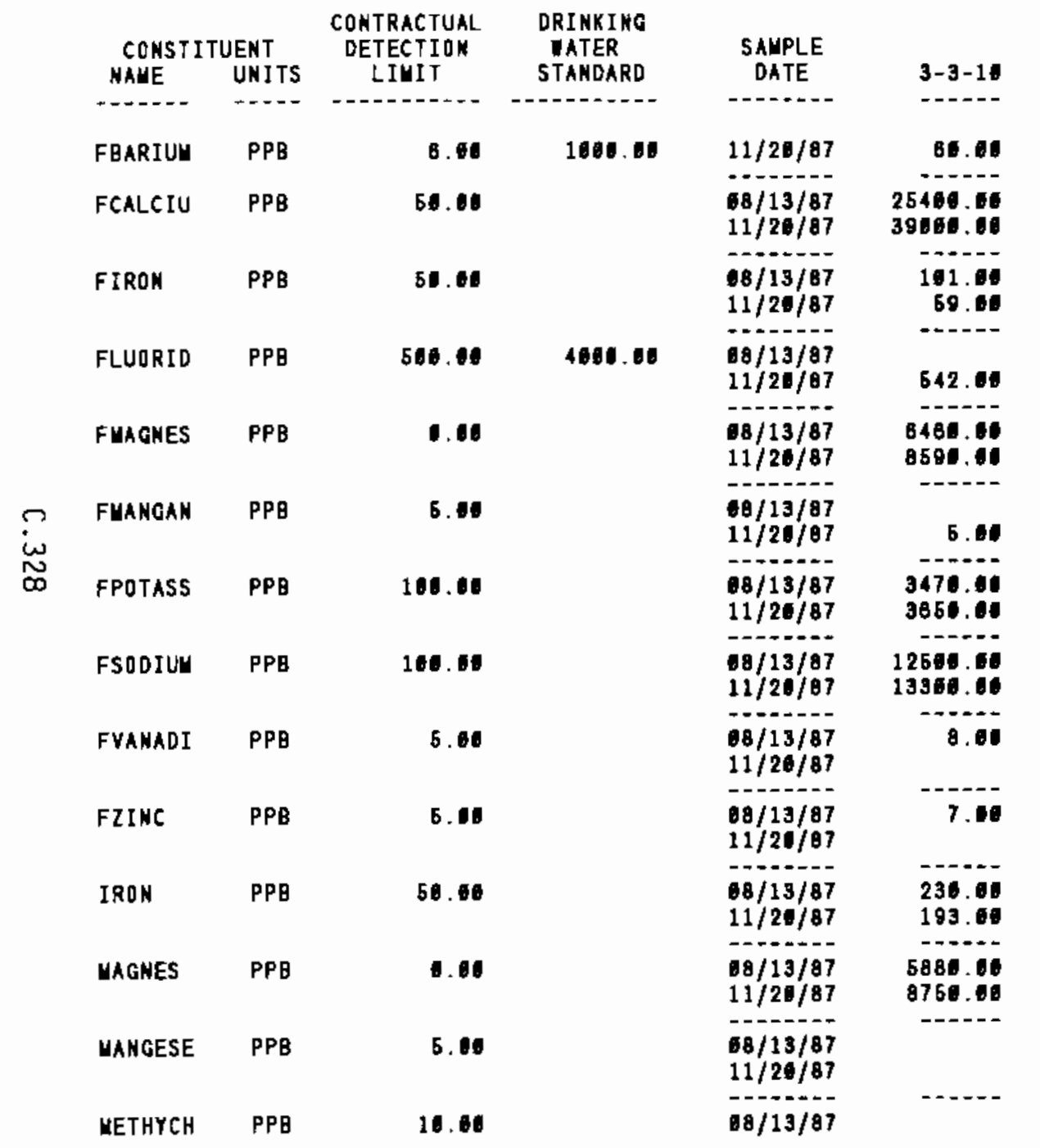

\begin{tabular}{|c|c|c|c|}
\hline $\begin{array}{l}\text { SALPLE } \\
\text { DATE }\end{array}$ & $3-4-1$ & $\begin{array}{c}\text { SAUPLE } \\
\text { OATE }\end{array}$ & $3-4-7$ \\
\hline $11 / 12 / 87$ & 38.00 & $11 / 08 / 87$ & 38.00 \\
\hline $\begin{array}{l}16 / 13 / 87 \\
11 / 12 / 87\end{array}$ & $\begin{array}{l}34200.60 \\
38600.60\end{array}$ & $\begin{array}{l}98 / 13 / 87 \\
11 / 16 / 87\end{array}$ & $\begin{array}{l}32804.10 \\
39260.01\end{array}$ \\
\hline $\begin{array}{l}18 / 13 / 87 \\
11 / 12 / 87\end{array}$ & & $\begin{array}{l}68 / 13 / 87 \\
11 / 08 / 87\end{array}$ & \\
\hline $\begin{array}{l}08 / 13 / 87 \\
11 / 12 / 87\end{array}$ & - & $\begin{array}{l}68 / 13 / 87 \\
11 / 06 / 87\end{array}$ & $58 B .06$ \\
\hline $\begin{array}{l}18 / 13 / 87 \\
11 / 12 / 87\end{array}$ & $\begin{array}{l}8010.60 \\
7890.66\end{array}$ & $\begin{array}{l}08 / 13 / 97 \\
11 / 06 / 87\end{array}$ & $\begin{array}{l}7726.10 \\
7790.16\end{array}$ \\
\hline $\begin{array}{l}68 / 13 / 87 \\
11 / 12 / 87\end{array}$ & & $\begin{array}{l}68 / 13 / 87 \\
11 / 86 / 87\end{array}$ & \\
\hline $\begin{array}{l}18 / 13 / 87 \\
11 / 12 / 87\end{array}$ & $\begin{array}{l}4526.06 \\
4910.60\end{array}$ & $\begin{array}{l}58 / 13 / 87 \\
11 / 06 / 87\end{array}$ & $\begin{array}{l}4330.06 \\
4460.10\end{array}$ \\
\hline $\begin{array}{l}60 / 13 / 87 \\
11 / 12 / 87\end{array}$ & $\begin{array}{l}177100.60 \\
17806.01\end{array}$ & $\begin{array}{l}68 / 19 / 87 \\
11 / 06 / 87\end{array}$ & $\begin{array}{l}18946.04 \\
18501.00\end{array}$ \\
\hline $\begin{array}{l}18 / 13 / 97 \\
11 / 12 / 87\end{array}$ & $\begin{array}{l}8.66 \\
5.64\end{array}$ & $\begin{array}{l}08 / 13 / 87 \\
11 / 86 / 87\end{array}$ & $\begin{array}{l}6.10 \\
5.10\end{array}$ \\
\hline $\begin{array}{l}68 / 13 / 87 \\
11 / 12 / 87\end{array}$ & 8.80 & $\begin{array}{l}98 / 13 / 87 \\
11 / 08 / 87\end{array}$ & 5.00 \\
\hline $\begin{array}{l}88 / 13 / 87 \\
11 / 12 / 87\end{array}$ & 388.86 & $\begin{array}{l}68 / 13 / 87 \\
11 / 06 / 87\end{array}$ & \\
\hline $\begin{array}{l}88 / 13 / 87 \\
11 / 12 / 87\end{array}$ & $\begin{array}{l}8650.01 \\
7680.06\end{array}$ & $\begin{array}{l}68 / 13 / 87 \\
11 / 08 / 87\end{array}$ & $\begin{array}{l}7586.00 \\
8381.01\end{array}$ \\
\hline $\begin{array}{l}18 / 13 / 87 \\
11 / 12 / 87\end{array}$ & 10.6 & $\begin{array}{l}08 / 13 / 87 \\
11 / 06 / 87\end{array}$ & \\
\hline $18 / 13 / 87$ & 138.01 & $08 / 13 / 07$ & 13.01 \\
\hline
\end{tabular}

* - VALUE EXCEEDS PRIUARY DRINKING IATER STANDARD

- VALUE EXCEEDS PROPOSED PRIUARY DRINKING TATER 5TANDARD

- VALUE EXCEEDS SCREENING LEVEL FOR FURTHER INVESTIGATION

- DETECTION LIUIT WAS NOT AVAILABLE FOR CDUPARISON

NR - ANALYSIS NOT REQUESTED OR NDT YET REPORTED

VALUES IN $f$ I ARE COUNTING ERRORS FOR RADIONUCLIDES

WATER STAMDARD(S) IN PARENTHESES ARE PROPOSED ONLY 
TABLE C.5. (contd)

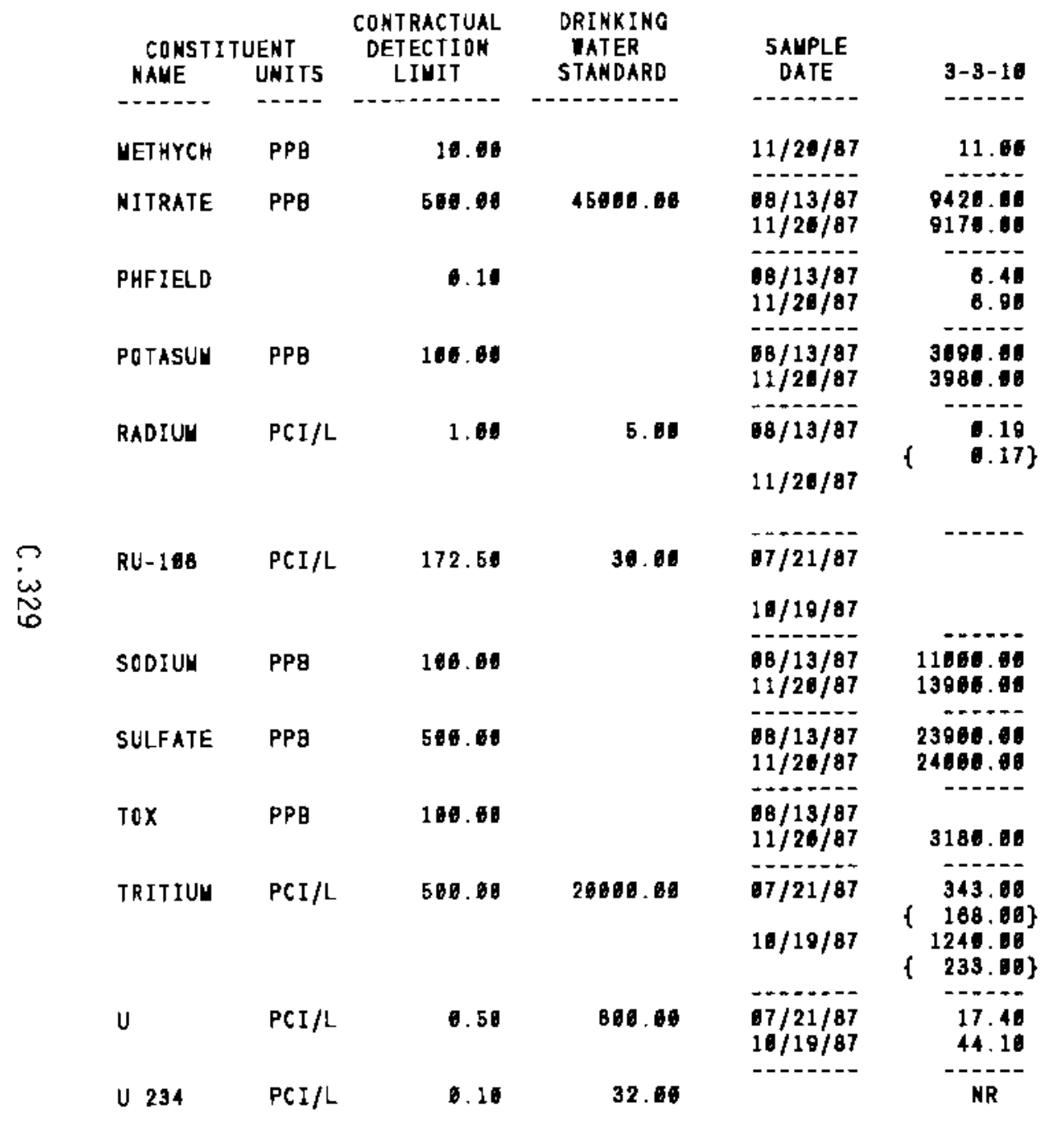

\begin{tabular}{|c|c|}
\hline $\begin{array}{c}\text { SAMPLE } \\
\text { DATE }\end{array}$ & $3-4-1$ \\
\hline ------- & ---- \\
\hline \multicolumn{2}{|l|}{$11 / 12 / 87$} \\
\hline------- & $n=-\infty$ \\
\hline $\begin{array}{l}68 / 13 / 87 \\
11 / 12 / 87\end{array}$ & $\begin{array}{l}15800.00 \\
15400.00\end{array}$ \\
\hline $\begin{array}{l}18 / 13 / 97 \\
11 / 12 / 87\end{array}$ & $\begin{array}{l}7.00 \\
7.40\end{array}$ \\
\hline----- & -- \\
\hline $\begin{array}{l}68 / 13 / 87 \\
11 / 12 / 87\end{array}$ & $\begin{array}{l}4826.00 \\
4890.00\end{array}$ \\
\hline \multicolumn{2}{|l|}{$08 / 13 / 87$} \\
\hline $11 / 12 / 87$ & $\left\{\begin{array}{l}.18 \\
\{.15\}\end{array}\right.$ \\
\hline 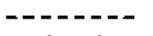 & ---- \\
\hline $07 / 23 / 87$ & $\left\{\begin{array}{l}68.20 \\
\{\quad 41.20\}\end{array}\right.$ \\
\hline \multicolumn{2}{|l|}{$1 \% / 1 \% / 87$} \\
\hline $\begin{array}{l}18 / 13 / 87 \\
11 / 12 / 87\end{array}$ & $\begin{array}{l}17700.00 \\
17300.00\end{array}$ \\
\hline $\begin{array}{l}68 / 13 / 87 \\
11 / 12 / 87\end{array}$ & $\begin{array}{l}29900.00 \\
28000.01\end{array}$ \\
\hline \multicolumn{2}{|l|}{$\begin{array}{l}68 / 13 / 87 \\
11 / 12 / 87\end{array}$} \\
\hline $7 / 23 / 87$ & 720.00 \\
\hline \multicolumn{2}{|l|}{$10 / 19 / 87$} \\
\hline 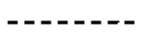 & ---- \\
\hline $\begin{array}{l}67 / 23 / 87 \\
1 \% / 19 / 87\end{array}$ & $\begin{array}{l}14.20 \\
13.20\end{array}$ \\
\hline$-\cdots-1$ & NR \\
\hline
\end{tabular}

\begin{tabular}{|c|c|}
\hline $\begin{array}{c}\text { SAHPLE } \\
\text { DATE }\end{array}$ & $3-4-7$ \\
\hline 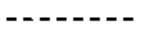 & $\cdots-$ \\
\hline $11 / 06 / 87$ & \\
\hline $\begin{array}{l}88 / 13 / 87 \\
11 / 66 / 87\end{array}$ & $\begin{array}{l}14900.06 \\
14360.06\end{array}$ \\
\hline $\begin{array}{l}88 / 13 / 87 \\
11 / 68 / 87\end{array}$ & $\begin{array}{r}7.16 \\
7.46\end{array}$ \\
\hline $\begin{array}{l}68 / 13 / 87 \\
11 / 68 / 87\end{array}$ & $\begin{array}{r}4340.00 \\
5260.00\end{array}$ \\
\hline $08 / 13 / 87$ & $\begin{array}{l}0.15 \\
0.13\}\end{array}$ \\
\hline $07 / 21 / 87$ & $\ldots$ \\
\hline $10 / 18 / 07$ & \\
\hline $\begin{array}{l}08 / 13 / 87 \\
11 / 06 / 87\end{array}$ & $\begin{array}{l}18300.60 \\
21380.64\end{array}$ \\
\hline $\begin{array}{l}68 / 13 / 87 \\
11 / 60 / 87\end{array}$ & $\begin{array}{l}32106.00 \\
32906.00\end{array}$ \\
\hline $\begin{array}{l}68 / 13 / 87 \\
11 / 06 / 87\end{array}$ & ---- \\
\hline $67 / 21 / 87$ & 1440.00 \\
\hline $10 / 19 / 87$ & $\begin{array}{r}1820.06 \\
\{\quad 250.66\}\end{array}$ \\
\hline $\begin{array}{l}97 / 21 / 87 \\
16 / 19 / 87\end{array}$ & $\begin{array}{l}31.81 \\
38.20\end{array}$ \\
\hline $7 / 21 / 87$ & 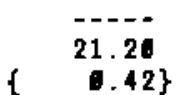 \\
\hline
\end{tabular}

* - YALUE EXCEEDS PRIUARY DRINKINg tater STANDARD.

- - VALUE EXCEEDS PROPOSED PRIUARY DRINKING IATER STANDARD.

- - VALUE EXCEedS SCREEHING LEVEL FDR FURTHER INYESTIgation.

- DETECTIOH LIUIT TAS NDT AVAILABLE FDR COUPARISON

NR - ANALYSIS NOT REQUESTED DR HOT YET REPORTED

VALUES IN \{\} ARE COUNTING ERRORS FOR RADIONUCLIDES

IAFER 5TANDARD'(S) IN PARENTHESES ARE PROPOSED DNLY 
TABLE C.5. (contd)

\begin{tabular}{|c|c|c|c|c|c|c|c|c|c|c|}
\hline $\begin{array}{c}\text { CONSTI } \\
\text { MAYE }\end{array}$ & $\begin{array}{l}\text { FUENI } \\
\text { UNITS }\end{array}$ & $\begin{array}{l}\text { CONTRACTUAL } \\
\text { DEEECTION } \\
\text { LIMIT }\end{array}$ & $\begin{array}{c}\text { DRINKING } \\
\text { INTER } \\
\text { STANDARD }\end{array}$ & $\begin{array}{c}\text { SAMPLE } \\
\text { DATE }\end{array}$ & 3-3-18 & $\begin{array}{c}\text { SAMPLE } \\
\text { DATE }\end{array}$ & $3-4-1$ & $\begin{array}{c}\text { SAMPLE } \\
\text { DATE }\end{array}$ & & $3-4-7$ \\
\hline U 235 & PCI/L & 6.10 & 32.80 & & NR & & MR & $67 / 21 / 87$ & $\mathfrak{t}$ & $\begin{array}{l}0.84 \\
8.83\}\end{array}$ \\
\hline U 23B & $\mathrm{PCI} / \mathrm{L}$ & 0.16 & 4.80 & & NR & & NR & $67 / 21 / 87$ & $\mathfrak{f}$ & $\begin{array}{l}20.80 \\
0.41\end{array}$ \\
\hline U-CHEM & UG/L & 0.73 & & $11 / 28 / 87$ & 76.71 & $11 / 12 / 87$ & 16.60 & & & NR \\
\hline VANADUY & PPB & 5.80 & & $\begin{array}{l}88 / 13 / 87 \\
11 / 26 / 87\end{array}$ & & $\begin{array}{l}88 / 13 / 87 \\
11 / 12 / 87\end{array}$ & $\begin{array}{l}16.81 \\
16.80\end{array}$ & $\begin{array}{l}88 / 13 / 87 \\
11 / 106 / 87\end{array}$ & & 7.80 \\
\hline ZINC & PPB & 5.80 & & $\begin{array}{l}8 / 13 / 87 \\
11 / 20 / 87\end{array}$ & $\begin{array}{r}11.60 \\
B .86\end{array}$ & $\begin{array}{l}88 / 13 / 87 \\
11 / 12 / 87\end{array}$ & 13.00 & $\begin{array}{l}18 / 13 / 87 \\
11 / 108 / 87\end{array}$ & & \\
\hline
\end{tabular}

$\overbrace{\tilde{\omega}}^{\omega}$

* - yalue eXCeEds PRIMARY dRINKing "ater standard.

- VALUE EXCEEDS PROPOSED PRIMARY DRINKING IATER STANDARD.

- VALUE EXCEEDS SCREEning LEVEL for fuRTHER inYestigation.

- DETECTION LINIT IAS NOT AVAILABLE for couparison

NR - ANALYSIS NOT REQUESTED OR NOT YET REPORTED

VALUES IN \{\} ARE COUNTINO ERRDRS FOR RADIONUCLIDES

IATER STANDARD(S) IN PARENTHESES ARE PROPOSED ONLY 


\begin{tabular}{|c|c|c|c|c|c|}
\hline \multicolumn{2}{|c|}{ CONSTITUENT } & $\begin{array}{c}\text { CONTRACTUAL } \\
\text { OETECTIDN } \\
\text { LINIT }\end{array}$ & $\begin{array}{l}\text { DRINKING } \\
\text { WATER } \\
\text { STANDARD }\end{array}$ & $\begin{array}{c}\text { SAHPLE } \\
\text { DATE }\end{array}$ & $3-4 \cdot 11$ \\
\hline -..--- & $-\ldots+$ & - & $+\cdots-\infty-\infty$ & $\ldots-\ldots$ & -....- \\
\hline $1,1,1-\mathrm{T}$ & PPB & 10.00 & 280.60 & $\begin{array}{l}98 / 28 / 87 \\
11 / 11 / 87\end{array}$ & \\
\hline ALPHA & $\mathrm{PCI} / \mathrm{L}$ & 4.00 & 15.06 & $\begin{array}{l}08 / 28 / 87 \\
11 / 11 / 87\end{array}$ & $\left\{\begin{array}{c}18.16 \\
\left\{\begin{array}{c}3.96\} \\
9.84 \\
\{\quad 2.48\}\end{array}\right\}\end{array}\right.$ \\
\hline ARSENIC & PPB & 5.60 & 50.06 & $\begin{array}{l}08 / 26 / 87 \\
11 / 11 / 87\end{array}$ & 5.00 \\
\hline BARIUU & PPB & 6.60 & 1060.00 & $\begin{array}{l}08 / 26 / 87 \\
11 / 11 / 87\end{array}$ & $\begin{array}{l}46.00 \\
43.06\end{array}$ \\
\hline BETA & $\mathrm{PCI} / \mathrm{L}$ & B. 00 & 50.00 & $\begin{array}{l}68 / 26 / 87 \\
11 / 11 / 87\end{array}$ & $\begin{array}{r}\quad 8.87 \\
4.02\} \\
11.89 \\
\{\quad 4.18\}\end{array}$ \\
\hline CALCIUY & PPB & 50.00 & & $\begin{array}{l}08 / 26 / 87 \\
11 / 11 / 87\end{array}$ & $\begin{array}{l}34606.00 \\
42560.00\end{array}$ \\
\hline CHLORID & PPB & 500.00 & & $\begin{array}{l}08 / 26 / 87 \\
11 / 11 / 87\end{array}$ & $\begin{array}{l}10000.06 \\
10906.06\end{array}$ \\
\hline CHROUUH & PPB & 10.00 & 50.00 & $\begin{array}{l}68 / 26 / 87 \\
11 / 11 / 87\end{array}$ & 23.00 \\
\hline COHDFLD & UUHO & 1.00 & & $\begin{array}{l}08 / 28 / 87 \\
11 / 11 / 87\end{array}$ & $\begin{array}{l}286.00 \\
330.00\end{array}$ \\
\hline FARSENI & PPB & 5.00 & 50.00 & $\begin{array}{l}68 / 28 / 87 \\
11 / 11 / 87\end{array}$ & 5. 0 \\
\hline FEARIUU & PPB & 8.00 & 1060.60 & $\begin{array}{l}08 / 26 / 67 \\
11 / 11 / 67\end{array}$ & $\begin{array}{l}41.06 \\
38.06\end{array}$ \\
\hline FCADUIU & PPB & 2.00 & 10.00 & $68 / 28 / 67$ & \\
\hline
\end{tabular}

contd)

* - value exceeds prinary drimking mater standaro.

1 - VALUE EXCEEDS PROPOSED PRIUARY DRINKING WATER STANDARD.

+ - VALUE EXCEEDS SCREENING LEVEL FOR FURTHER INVESTIOATION.

- DETECTION LIMIT WAS NOT AVAILABLE FDR COMPARISDN

NR - ANALYSIS MOT REQUESTED OR NOT YET REPQRTED

VALUES IN $\{j$ ARE COUNTING ERRORS FOR RADIONUCLIDES

WATER STANDARD'(S) IN PARENTHESES ARE PROPOSED ONLY

\begin{tabular}{|c|c|}
\hline $\begin{array}{c}\text { SAHPLE } \\
\text { DATE }\end{array}$ & $3-8-1$ \\
\hline 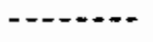 & $-\ldots$ \\
\hline $11 / 10 / 87$ & NR \\
\hline - & ---- \\
\hline $11 / 18 / 87$ & 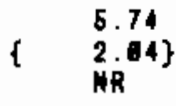 \\
\hline - & --- \\
\hline $11 / 19 / 87$ & $\begin{array}{l}7.00 \\
\text { MR }\end{array}$ \\
\hline - - & $\cdots$ \\
\hline $11 / 18 / 87$ & $\begin{array}{l}33.60 \\
\mathrm{NR}\end{array}$ \\
\hline $11 / 19 / 87$ & 5.21 \\
\hline $11 / 18 / 67$ & $\begin{array}{l}\text { J. } \\
\text { NR }\end{array}$ \\
\hline - & $\ldots$ \\
\hline $11 / 19 / 87$ & $\begin{array}{c}37400.06 \\
\text { NR }\end{array}$ \\
\hline - & -- \\
\hline $11 / 19 / 87$ & $\begin{array}{c}8830.06 \\
\text { NR }\end{array}$ \\
\hline $11 / 19 / 87$ & ----- \\
\hline & NR \\
\hline 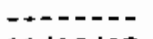 & $\cdots$ \\
\hline $11 / 19 / 87$ & $\begin{array}{c}368.60 \\
N R\end{array}$ \\
\hline 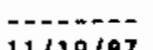 & $\overline{7}$ \\
\hline $11 / 28 / 87$ & $\begin{array}{l}7.00 \\
\mathrm{NR}\end{array}$ \\
\hline $11 / 10 / 87$ & 38.08 \\
\hline $11 / 19 / 87$ & $-\cdots-$ \\
\hline
\end{tabular}

\begin{tabular}{|c|c|}
\hline $\begin{array}{c}\text { SALPLE } \\
\text { DATE }\end{array}$ & $3-8-2$ \\
\hline 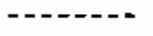 & ---- \\
\hline $\begin{array}{l}00 / 18 / 87 \\
11 / 13 / 87\end{array}$ & 19.00 \\
\hline $6 / 18 / 87$ & 1.16 \\
\hline $11 / 13 / 87$ & $\begin{array}{l}1.06\} \\
2.86 \\
1.56\}\end{array}$ \\
\hline $\begin{array}{l}68 / 18 / 87 \\
11 / 13 / 87\end{array}$ & 7.00 \\
\hline $\begin{array}{l}0 / 18 / 87 \\
11 / 13 / 87\end{array}$ & $\begin{array}{l}---2 . \\
45.06 \\
43.04\end{array}$ \\
\hline $8 / 18 / 87$ & 8.69 \\
\hline $11 / 13 / 87$ & 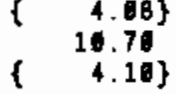 \\
\hline $\begin{array}{l}00 / 18 / 87 \\
11 / 13 / 87\end{array}$ & $\begin{array}{l}38309.05 \\
41806.06\end{array}$ \\
\hline $\begin{array}{l}68 / 18 / 87 \\
11 / 13 / 87\end{array}$ & $\begin{array}{l}10500.00 \\
11800.00\end{array}$ \\
\hline $\begin{array}{l}98 / 18 / 87 \\
11 / 13 / 87\end{array}$ & --- \\
\hline $\begin{array}{l}18 / 18 / 87 \\
11 / 13 / 87\end{array}$ & 266.80 \\
\hline $\begin{array}{l}68 / 18 / 87 \\
11 / 13 / 87\end{array}$ & 5.06 \\
\hline $\begin{array}{l}08 / 18 / 87 \\
11 / 13 / 87\end{array}$ & 42.00 \\
\hline $68 / 18 / 87$ & 2.00 \\
\hline
\end{tabular}




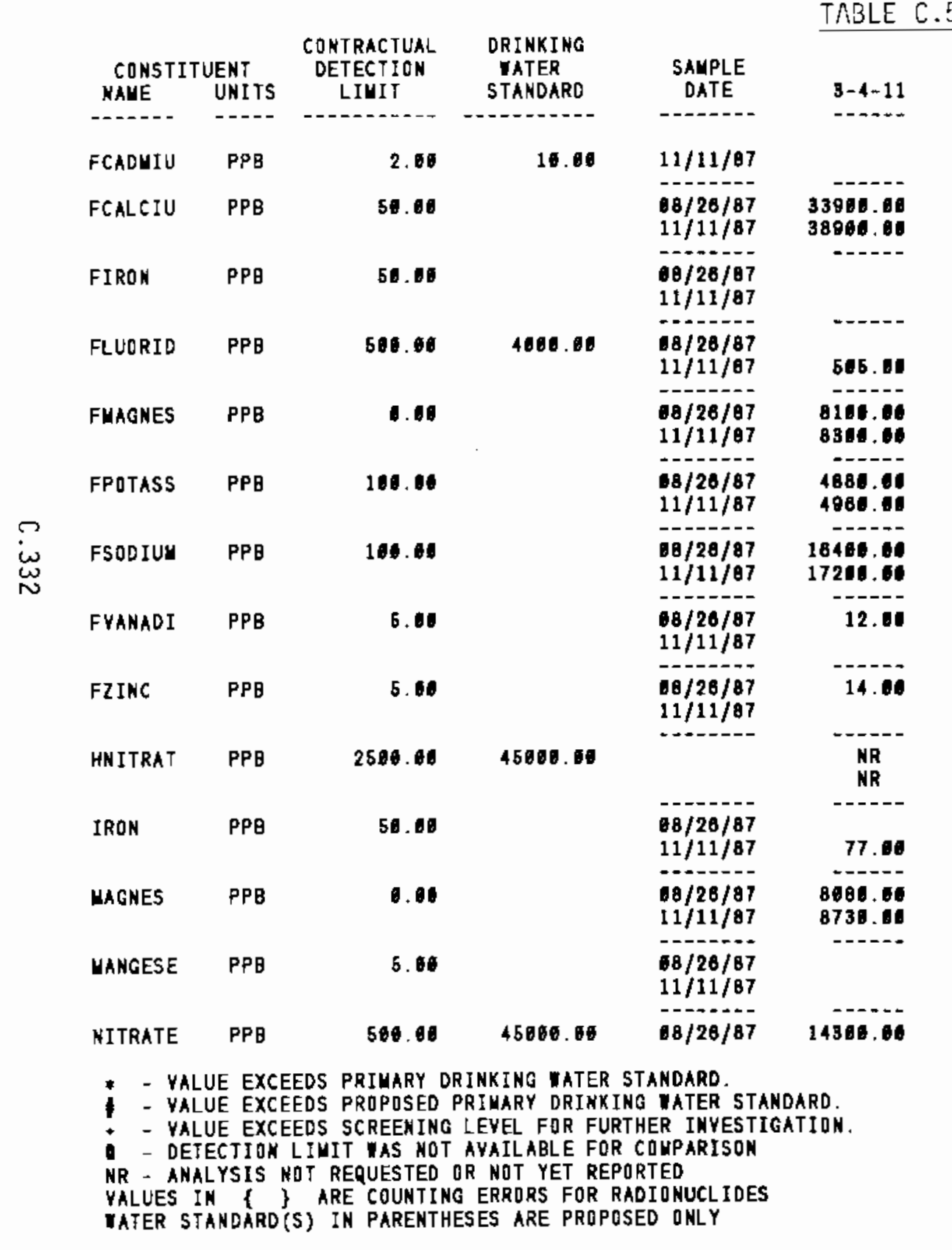


TABLE C.5. (contd)

\begin{tabular}{|c|c|c|c|c|c|}
\hline $\begin{array}{c}\text { CONSTI } \\
\text { NAWE }\end{array}$ & $\begin{array}{l}\text { UENT } \\
\text { UNI IS }\end{array}$ & $\begin{array}{l}\text { CONTRACTUAL } \\
\text { DETECTID\# } \\
\text { LIUIT }\end{array}$ & $\begin{array}{l}\text { DRINAING } \\
\text { WATER } \\
\text { STANDARO }\end{array}$ & $\begin{array}{c}\text { SAMPLE } \\
\text { DATEE }\end{array}$ & $3-4-11$ \\
\hline NITRATE & PPB & 508.80 & 45080.60 & $11 / 11 / 87$ & 18900.06 \\
\hline PHFIELD & & 0.10 & & $\begin{array}{l}8 B / 28 / 87 \\
11 / 11 / 87\end{array}$ & $\begin{array}{l}7.10 \\
7.80\end{array}$ \\
\hline POTASUM & $\mathrm{PPB}$ & 108.06 & & $\begin{array}{l}18 / 28 / 87 \\
11 / 11 / 87\end{array}$ & $\begin{array}{l}4760.00 \\
5356.00\end{array}$ \\
\hline SODIUU & $\mathrm{PPB}$ & 100.06 & & $\begin{array}{l}18 / 28 / 87 \\
11 / 11 / 87\end{array}$ & $\begin{array}{r}17160.06 \\
18266.00 \\
\end{array}$ \\
\hline SULFATE & PPB & 500.00 & & $\begin{array}{l}08 / 28 / 87 \\
11 / 11 / 87\end{array}$ & $\begin{array}{l}27160.00 \\
29260.00\end{array}$ \\
\hline $\mathrm{u}$ & $\mathrm{PCI} / \mathrm{L}$ & 6.50 & 806.60 & & $\begin{array}{l}\text { MR } \\
\text { NR }\end{array}$ \\
\hline U-CHEY & UO/L & 0.73 & & $11 / 11 / 87$ & 16.81 \\
\hline YAMADUL & $\mathrm{PPB}$ & 5.06 & & $\begin{array}{l}18 / 28 / 87 \\
11 / 11 / 87\end{array}$ & $\begin{array}{r}11.00 \\
13.06\end{array}$ \\
\hline ZINC & PPB & 5.66 & & $\begin{array}{l}88 / 28 / 87 \\
11 / 11 / 87\end{array}$ & 178.16 \\
\hline
\end{tabular}

\begin{tabular}{|c|c|}
\hline $\begin{array}{l}\text { SAUPLE } \\
\text { DATE }\end{array}$ & $3-8-1$ \\
\hline & MR \\
\hline $11 / 19 / 87$ & ${ }_{\mathrm{MR}}^{7.70}$ \\
\hline $11 / 19 / 87$ & $\underset{\mathrm{NR}}{4081.06}$ \\
\hline $11 / 19 / 87$ & 13000.06 \\
\hline $11 / 18 / 87$ & 20400.06 \\
\hline $\begin{array}{l}07 / 22 / 87 \\
10 / 10 / 87\end{array}$ & $\begin{array}{l}3.54 \\
3.33\end{array}$ \\
\hline $11 / 10 / 87$ & 6. 11 \\
\hline $11 / 19 / 87$ & ${ }_{\mathrm{NR}}^{9.60}$ \\
\hline $11 / 19 / 87$ & NR \\
\hline
\end{tabular}

SAHPLE

3-8-2

DIID)

$11 / 13 / 87 \quad 24681.04$

$88 / 18 / 87 \quad 7.01$

$11 / 13 / 87 \quad 7.61$

$08 / 18 / 87$ 6120.08

$11 / 13 / 87 \quad 5570.01$

$68 / 18 / 87 \quad 18606.01$

$11 / 13 / 87$ 19466.16

$68 / 18 / 87 \quad 29986.66$

$11 / 13 / 87$ 30100.66

$07 / 22 / 87 \quad 1.78$

$10 / 10 / 87 \quad 1.86$

10/19/87 -

$\overline{11 / 13 / 87} \quad 2.75$

$18 / 18 / 87 \quad 18.01$

$11 / 13 / 87 \quad 15.91$

$09 / 18 / 87 \quad 0.01$

$11 / 13 / 87$

* - VAlUe eXceEos privary dRinking water standaro.

- - VALUE EXCEEDS PROPDSED PRIYARY DRINKING TATER STANDARD.

+ - VALUE EXCEEDS SCREENING LEVEL FDR FURTHER INVESTIGATION.

- DETECTIDN LIUIT UAS NOT AVAILABLE FOR COUPARISON

NR - ANALYSIS NDT REQUESTED OR NOT YET REPORTED

VALUES IN \{ $\}$ ARE COUNTING ERRDRS FOR RADIONUCLIDES

WATER STANOARO(S) IN PARENTHESES ARE PROPOSED ONLY 
IABLE C.5. (contd)

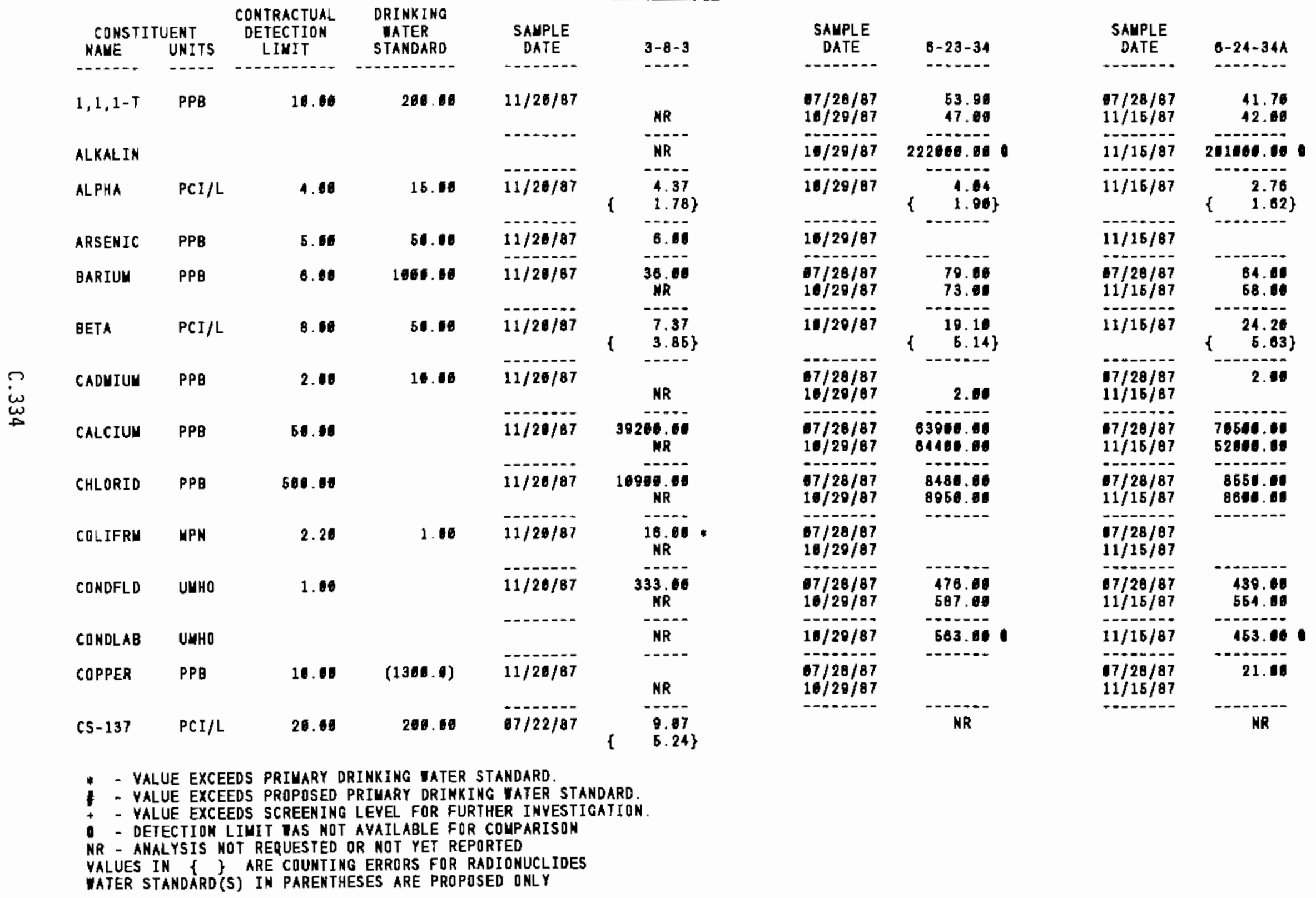


TABLE C. 5. (contd)

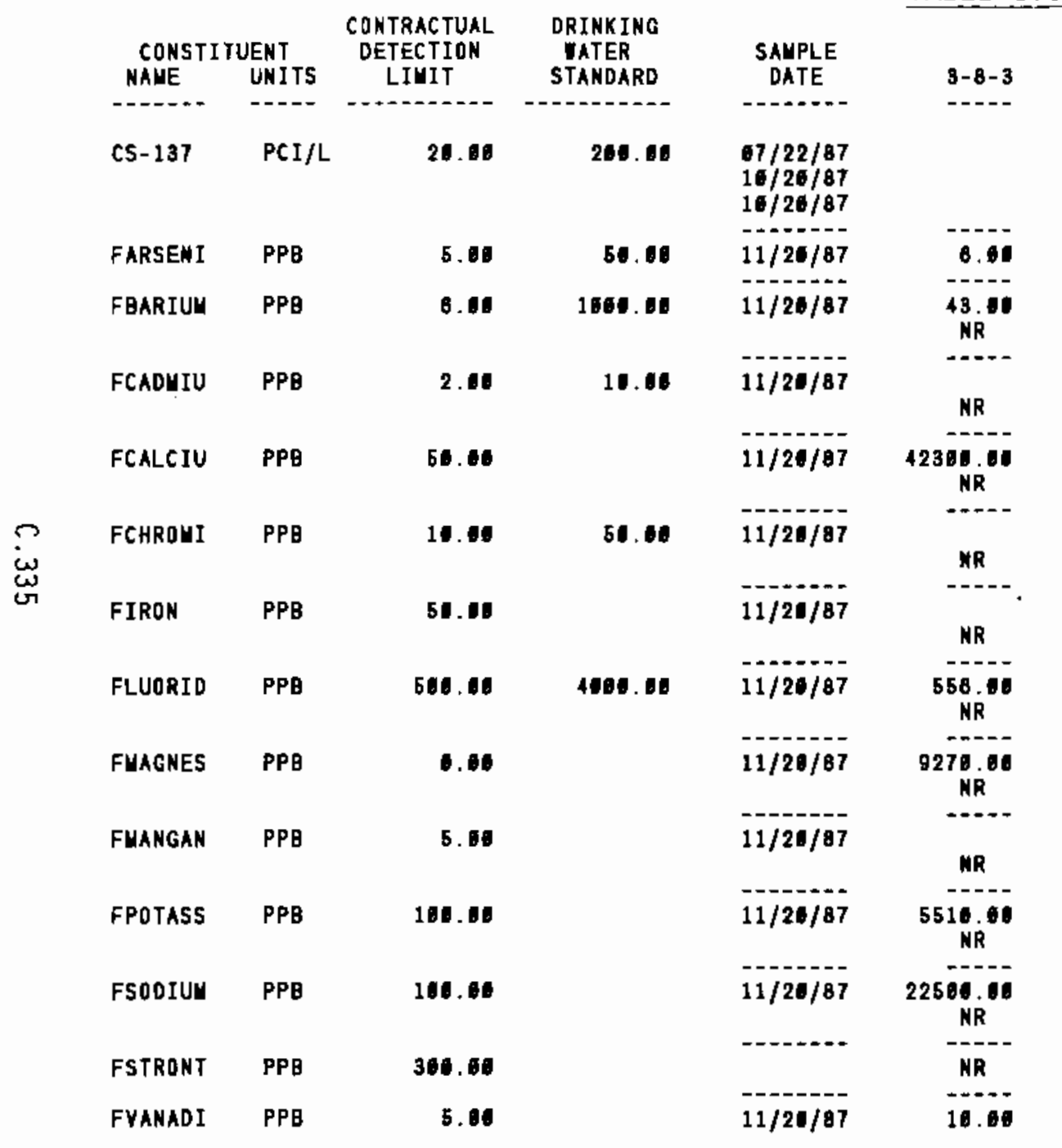

\begin{tabular}{|c|c|}
\hline $\begin{array}{c}\text { SAMPLE } \\
\text { DATE }\end{array}$ & $6-23-34$ \\
\hline & $\begin{array}{l}\text { MR } \\
\text { MR } \\
\text { MR }\end{array}$ \\
\hline $10 / 29 / 87$ & \\
\hline $\begin{array}{l}07 / 28 / 87 \\
10 / 29 / 87\end{array}$ & $\begin{array}{l}80.08 \\
83.00\end{array}$ \\
\hline $\begin{array}{l}07 / 28 / 87 \\
18 / 29 / 87\end{array}$ & 2.00 \\
\hline $\begin{array}{l}17 / 28 / 87 \\
10 / 29 / 87\end{array}$ & $\begin{array}{l}53910.00 \\
70406.00\end{array}$ \\
\hline $\begin{array}{l}07 / 28 / 87 \\
10 / 29 / 87\end{array}$ & 11.00 \\
\hline $\begin{array}{l}07 / 28 / 87 \\
10 / 20 / 87\end{array}$ & \\
\hline $\begin{array}{l}17 / 28 / 87 \\
16 / 29 / 87\end{array}$ & $\begin{array}{l}658.06 \\
737.00\end{array}$ \\
\hline $\begin{array}{l}67 / 28 / 97 \\
10 / 29 / 87\end{array}$ & $\begin{array}{l}15160.00 \\
17400.00\end{array}$ \\
\hline $\begin{array}{l}97 / 28 / 87 \\
10 / 29 / 87\end{array}$ & 6.80 \\
\hline $\begin{array}{l}07 / 28 / 87 \\
10 / 29 / 87\end{array}$ & $\begin{array}{l}7316.06 \\
8906.00\end{array}$ \\
\hline $\begin{array}{l}67 / 28 / 87 \\
10 / 29 / 87\end{array}$ & $\begin{array}{l}22500.80 \\
25600.86\end{array}$ \\
\hline $10 / 29 / 87$ & 317.00 \\
\hline $07 / 28 / 87$ & 17.00 \\
\hline
\end{tabular}

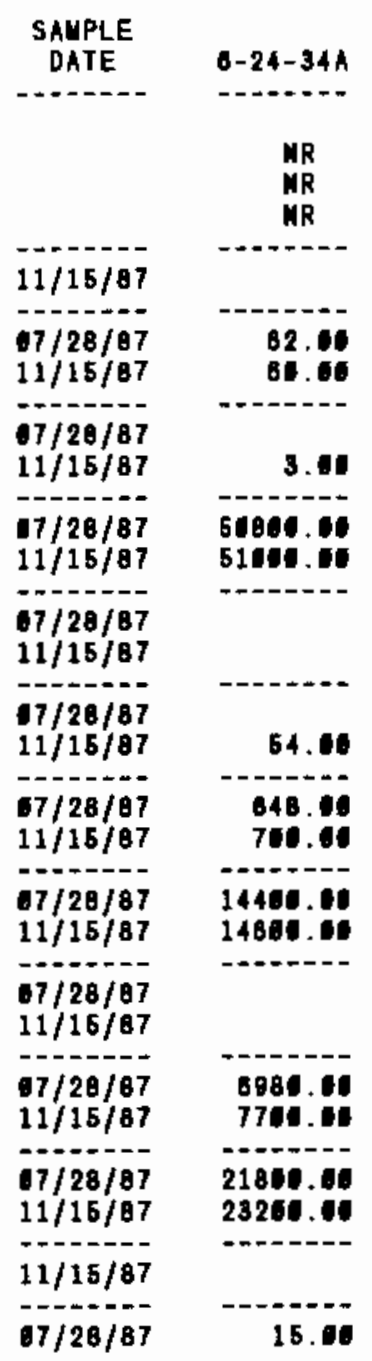

* - Value exceeds privary drinking mater standaro

- - VALUE EXCEEDS PROPOSED PRIUARY DRINKING iATER STANDARD.

- - YALUE EXCEEDS SCREEMING LEYEL FOR FURTHER IMYESTIGATION.

- detection liuit ias ndt ayailable for comparison

NR - ANALYSIS NOT REQUESTED DR NOT YET REPORTED

VALUES IN I I ARE COUNTINO ERRORS FOR RADIONUCLIDES

IATER STANDARD(S) IN PARENTHESES ARE PROPOSED ONLY 
TABLE C.5. (contd)

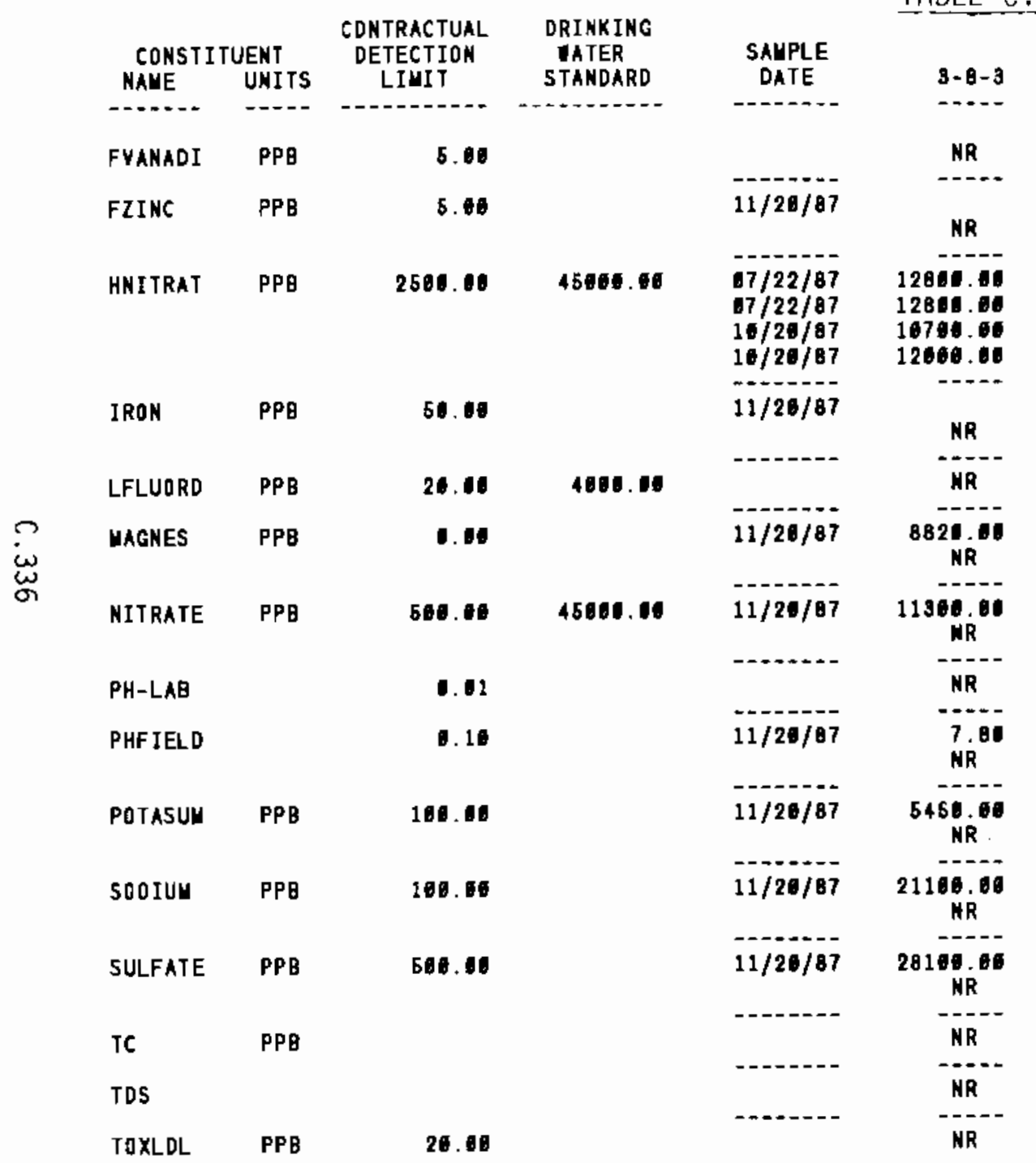

\begin{tabular}{|c|c|}
\hline $\begin{array}{l}\text { SAMPLE } \\
\text { DATE }\end{array}$ & $8-23-34$ \\
\hline $10 / 28 / 87$ & 15.80 \\
\hline \multirow{2}{*}{$\begin{array}{l}07 / 28 / 87 \\
11 / 29 / 67 \\
-\end{array}$} & $\begin{array}{l}37.06 \\
35.16\end{array}$ \\
\hline & $\begin{array}{l}\text { NR } \\
\text { WR } \\
\text { NR } \\
\text { NR }\end{array}$ \\
\hline $\begin{array}{l}17 / 28 / 87 \\
10 / 29 / 87\end{array}$ & $\begin{array}{l}481.00 \\
488.00\end{array}$ \\
\hline $10 / 28 / 87$ & 440.11 \\
\hline $\begin{array}{l}07 / 28 / 87 \\
10 / 29 / 87\end{array}$ & $\begin{array}{l}18206.01 \\
15200.15\end{array}$ \\
\hline $\begin{array}{l}7 / 28 / 87 \\
10 / 20 / 87\end{array}$ & $\begin{array}{l}10204.01 \\
22100.01\end{array}$ \\
\hline $10 / 29 / 87$ & 7.10 \\
\hline $\begin{array}{l}07 / 28 / 87 \\
16 / 29 / 67\end{array}$ & $\begin{array}{l}0.10 \\
5.10\end{array}$ \\
\hline $\begin{array}{l}87 / 28 / 87 \\
16 / 29 / 87\end{array}$ & $\begin{array}{l}7860.06 \\
8860.1\end{array}$ \\
\hline $\begin{array}{l}67 / 28 / 87 \\
16 / 20 / 87\end{array}$ & $\begin{array}{l}23250.00 \\
28416.00\end{array}$ \\
\hline $\begin{array}{l}07 / 28 / 87 \\
10 / 29 / 87\end{array}$ & $\begin{array}{l}48000.14 \\
51204.00\end{array}$ \\
\hline $10 / 29 / 87$ & 59500.00 \\
\hline $10 / 28 / 87$ & 344800.66 \\
\hline $10 / 29 / 87$ & 49.58 \\
\hline
\end{tabular}

\begin{tabular}{|c|c|}
\hline $\begin{array}{l}\text { SAMPLEE } \\
\text { DATE }\end{array}$ & $0-24-34 A$ \\
\hline $11 / 15 / 87$ & 18.11 \\
\hline \multirow[t]{2}{*}{$\begin{array}{l}67 / 28 / 87 \\
11 / 16 / 87 \\
-\end{array}$} & $\begin{array}{l}36.06 \\
23.61\end{array}$ \\
\hline & $\begin{array}{l}\text { NR } \\
\text { NR } \\
\text { NR } \\
\text { WR }\end{array}$ \\
\hline $\begin{array}{l}07 / 28 / 87 \\
11 / 15 / 87\end{array}$ & $\begin{array}{l}365.66 \\
447.66\end{array}$ \\
\hline $11 / 15 / 87$ & 471. 16 \\
\hline $\begin{array}{l}07 / 28 / 87 \\
11 / 15 / 87\end{array}$ & $\begin{array}{l}15480.06 \\
14700.04\end{array}$ \\
\hline $\begin{array}{l}17 / 28 / 87 \\
11 / 15 / 87\end{array}$ & $\begin{array}{l}10400.00 \\
24408.00\end{array}$ \\
\hline $11 / 16 / 87$ & 7.23 \\
\hline $\begin{array}{l}07 / 28 / 87 \\
11 / 15 / 87\end{array}$ & $\begin{array}{l}0.20 \\
6.20\end{array}$ \\
\hline $\begin{array}{l}67 / 28 / 87 \\
11 / 16 / 87\end{array}$ & 7856.60 \\
\hline $\begin{array}{l}07 / 28 / 87 \\
11 / 15 / 87\end{array}$ & $\begin{array}{l}23504.00 \\
23000.00\end{array}$ \\
\hline $\begin{array}{l}67 / 28 / 87 \\
11 / 15 / 87\end{array}$ & 45706.00 \\
\hline $11 / 15 / 87$ & 62480.60 \\
\hline $11 / 15 / 67$ & 337000.01 \\
\hline $11 / 15 / 87$ & 46.80 \\
\hline
\end{tabular}

* - yalue exceeds primary dRinking mater standard.

- VALUE EXCEEDS PROPOSED PRIMARY DRINKING taTER STANOARD.

- YALUE EXCEEDS SCREENING LEYEL FOR FURTHER INYESTIGATION.

- DEtection livit tas not available for comparison

NR - AMALYSIS NOT REQUESTED OR NOT YET REPORTED

YALUES IN \{\} ARE COUNTING ERRORS FOR RADIONUCLIDES

WATER STANDARD(5) IN PARENTHESES ARE PRDPDSEO GNLY 


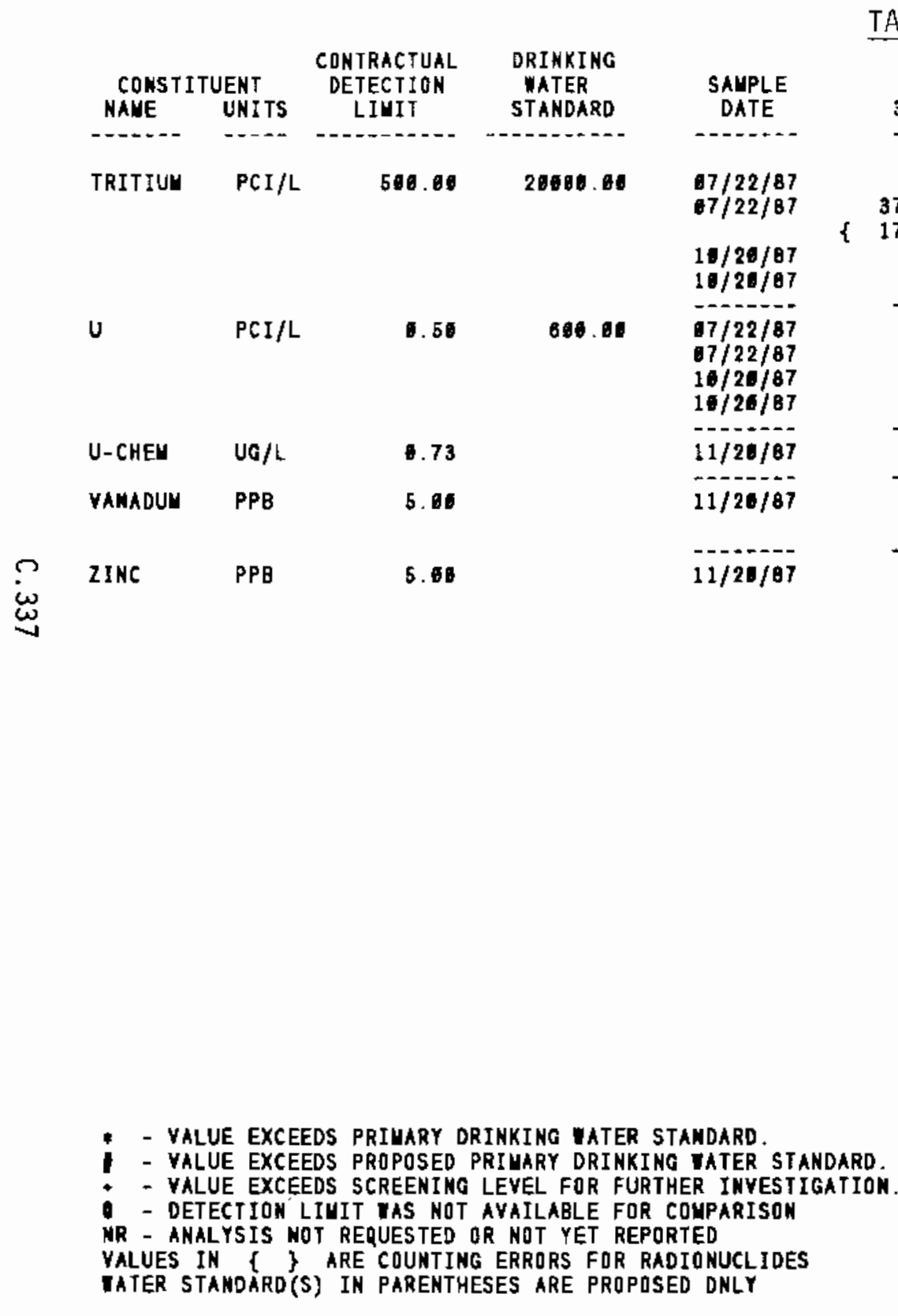

TABLE C.5. (contd)

$3-8-3$

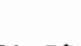

$170.00\}$

$-2 .-1$

4.33
3. 47

3. 33

3. 71

5.20

9.00

NR

NR

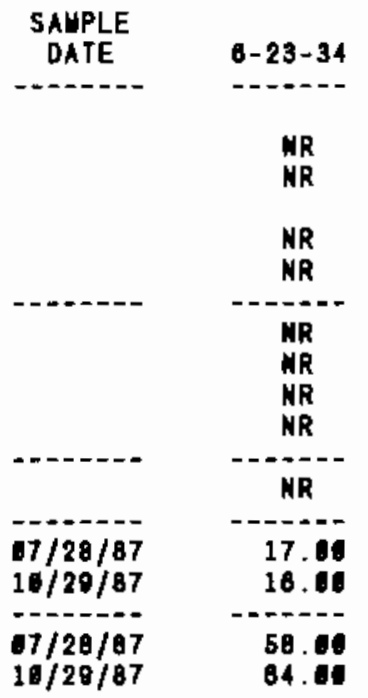

SAMPLE DATE

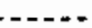

$6-24-34 A$

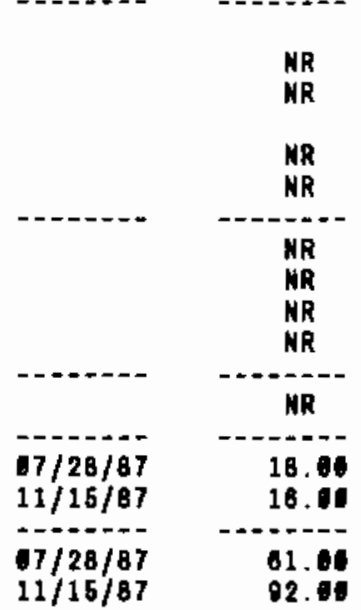

- - Value exceeds priuary drinking vater standard.

VALUE EXCEEDS PROPOSED PRIUARY DRINKING TATER STANDARD.

- DETECTION LIUIT IAS NOT AVAILABLE FOR COMPARISOH

ANALYSIS NOT REQUESTED OR NOT YET REPORTED

VALUES IN \{ $\}$ ARE COUNTING ERRORS FOR RADIONUCLIDES 
TABLE C.5. (contd)

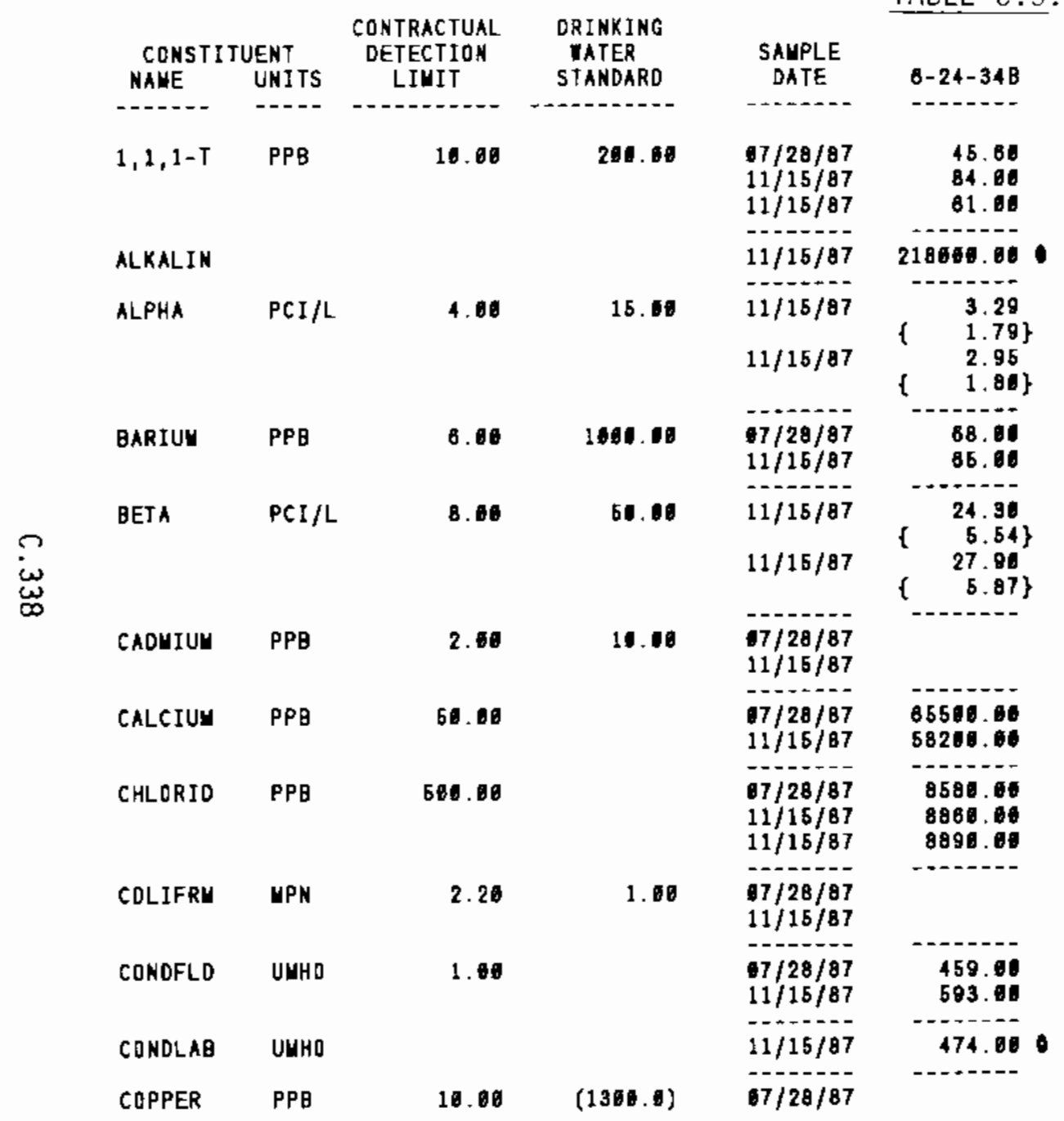

\begin{tabular}{|c|c|}
\hline $\begin{array}{c}\text { SAHPLE } \\
\text { DATE }\end{array}$ & $8-24-34 C$ \\
\hline - & 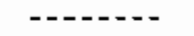 \\
\hline $\begin{array}{l}07 / 27 / 87 \\
11 / 16 / 87\end{array}$ & $\begin{array}{c}27.50 \\
35.00 \\
N R\end{array}$ \\
\hline$\overline{11 / 16 / 87}$ & 208006.00 \\
\hline $11 / 16 / 87$ & $\begin{array}{ll}2.15 \\
1.52\} \\
N R\end{array}$ \\
\hline $\begin{array}{l}-1 / 27 / 87 \\
11 / 16 / 87\end{array}$ & $\begin{array}{l}51.00 \\
48.00\end{array}$ \\
\hline $11 / 16 / 87$ & $\left\{\begin{array}{c}18.09 \\
5.00 \\
\text { NR }\end{array}\right\}$ \\
\hline $\begin{array}{l}97 / 27 / 97 \\
11 / 15 / 87\end{array}$ & - - \\
\hline $\begin{array}{l}07 / 27 / 97 \\
11 / 15 / 07\end{array}$ & 58960.00 \\
\hline $\begin{array}{l}1 / 27 / 87 \\
11 / 16 / 87\end{array}$ & $\begin{array}{c}0820.66 \\
2200.06\end{array}$ \\
\hline $\begin{array}{l}-1 / 27 / 87 \\
11 / 16 / 87\end{array}$ & ------ \\
\hline $\begin{array}{l}17 / 27 / 87 \\
11 / 16 / 87\end{array}$ & $\begin{array}{r}427.00 \\
686.06\end{array}$ \\
\hline $11 / 16 / 87$ & 458.00 \\
\hline $97 / 27 / 87$ & \\
\hline
\end{tabular}

\begin{tabular}{|c|c|}
\hline $\begin{array}{c}\text { SANPLE } \\
\text { DATE }\end{array}$ & $8-24-35$ \\
\hline 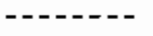 & 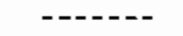 \\
\hline \multicolumn{2}{|l|}{$\begin{array}{l}87 / 27 / 87 \\
16 / 28 / 87\end{array}$} \\
\hline & NR \\
\hline $10 / 29 / 87$ & 132000.00 \\
\hline $11 / 28 / 87$ & $\begin{cases}4.10 \\
1.72\} \\
\text { NR }\end{cases}$ \\
\hline$\ldots$ & n \\
\hline $\begin{array}{l}07 / 27 / 87 \\
10 / 28 / 87\end{array}$ & $\begin{array}{l}43.06 \\
43.00\end{array}$ \\
\hline $1 / / 29 / 87$ & $\left\{\begin{array}{c}16.20 \\
4.02\} \\
\mathrm{NR}\end{array}\right.$ \\
\hline 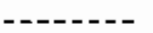 & ------- \\
\hline $\begin{array}{l}07 / 27 / 87 \\
10 / 20 / 87\end{array}$ & $\begin{array}{l}3.10 \\
2.10\end{array}$ \\
\hline $\begin{array}{l}07 / 27 / 87 \\
10 / 29 / 87\end{array}$ & 40100.46 \\
\hline------ & $\ldots .$. \\
\hline $\begin{array}{l}67 / 27 / 87 \\
16 / 29 / 87\end{array}$ & $\begin{array}{c}8489.00 \\
8689.00 \\
\text { NR }\end{array}$ \\
\hline $\begin{array}{l}67 / 27 / 87 \\
10 / 28 / 87\end{array}$ & 2.20 \\
\hline 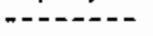 & ------- \\
\hline $\begin{array}{l}67 / 27 / 87 \\
10 / 28 / 87\end{array}$ & $\begin{array}{l}941.00 \\
416.00\end{array}$ \\
\hline $10 / 29 / 87$ & 417.4 \\
\hline $7 / 27 / 87$ & B1.00 \\
\hline
\end{tabular}

* - value eXCEEDS PRiyary dRINKING tater stahdaRd.

- VALUE EXCEEDS PROPOSED PRIUARY DRINKING WATER STANDARD.

+ - VALUE EXCEEDS SCREEMING LEVEL FGR FURTHER INYESTIGATION.

- - DETECYION LIMIT WAS RDT AVAILABLE FDR COUPARISON

NR - ANALYSIS NOT REQUESTED OR NOT YET REPORTED

VALUES IN \{ \} ARE COUNTING ERRORS FOR RADIONUCLIDES

IATER STANOARD'S) IN PARENTHESES ARE PRDPOSED ONLY 
TABLE C.5. (contd)

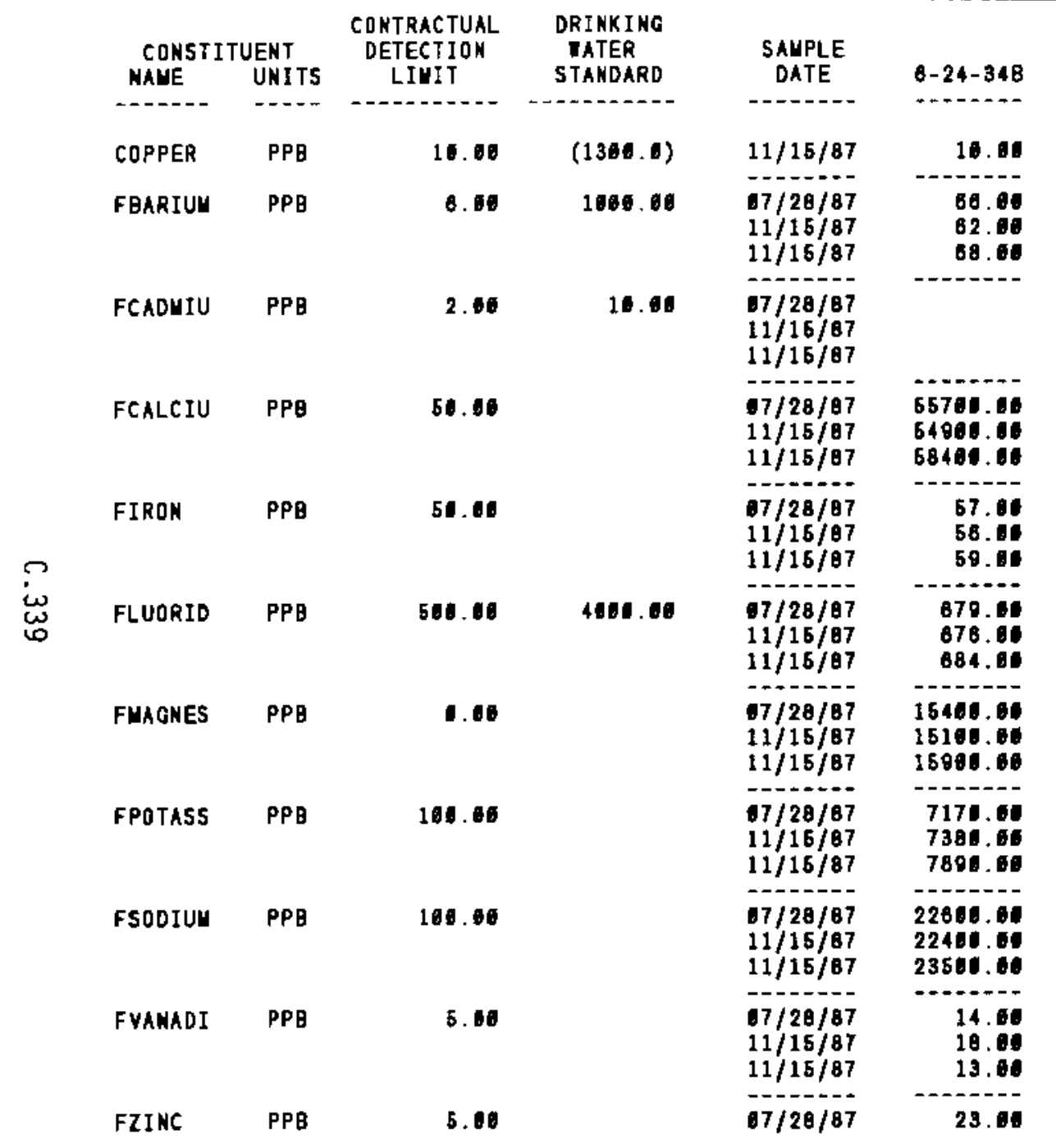

\begin{tabular}{|c|c|}
\hline $\begin{array}{c}\text { SAUPLE } \\
\text { DATE }\end{array}$ & $6-24-34 C$ \\
\hline $11 / 16 / 87$ & \\
\hline $\begin{array}{l}07 / 27 / 87 \\
11 / 16 / 87\end{array}$ & $\begin{array}{l}61.66 \\
49.66 \\
\text { RR }\end{array}$ \\
\hline $\begin{array}{l}07 / 27 / 87 \\
11 / 15 / 87\end{array}$ & R \\
\hline $\begin{array}{l}07 / 27 / 87 \\
11 / 15 / 87\end{array}$ & $\begin{array}{c}51200.00 \\
54109.06 \\
\mathrm{KR}\end{array}$ \\
\hline $\begin{array}{l}07 / 27 / 87 \\
11 / 15 / 87\end{array}$ & $\begin{array}{l}69.66 \\
\text { MR }\end{array}$ \\
\hline $\begin{array}{l}07 / 27 / 87 \\
11 / 15 / 87\end{array}$ & $\begin{array}{l}635.00 \\
643.66 \\
\text { NR }\end{array}$ \\
\hline $\begin{array}{l}07 / 27 / 87 \\
11 / 15 / 87\end{array}$ & $\begin{array}{c}14809.00 \\
16400.00 \\
\text { NR }\end{array}$ \\
\hline $\begin{array}{l}07 / 27 / 87 \\
11 / 15 / 87\end{array}$ & $\begin{array}{c}7008.00 \\
758.61 \\
\text { NR }\end{array}$ \\
\hline $\begin{array}{l}07 / 27 / 87 \\
11 / 16 / 87\end{array}$ & $\begin{array}{c}22289.09 \\
23300.00 \\
\text { NR }\end{array}$ \\
\hline $\begin{array}{l}67 / 27 / 87 \\
11 / 15 / 87\end{array}$ & $\begin{array}{l}14.66 \\
18.66 \\
\text { NR }\end{array}$ \\
\hline $97 / 27 / 87$ & 38.60 \\
\hline
\end{tabular}

\begin{tabular}{|c|c|}
\hline $\begin{array}{l}\text { SAYPLE } \\
\text { DATE }\end{array}$ & $6-24-35$ \\
\hline $10 / 29 / 87$ & \\
\hline $\begin{array}{l}97 / 27 / 87 \\
16 / 29 / 87\end{array}$ & $\begin{array}{l}43.08 \\
48.98 \\
\text { NR }\end{array}$ \\
\hline $\begin{array}{l}67 / 27 / 87 \\
10 / 29 / 87\end{array}$ & 2.00 \\
\hline $\begin{array}{l}67 / 27 / 87 \\
10 / 20 / 87\end{array}$ & $\begin{array}{c}34161.06 \\
42366\end{array}$ \\
\hline $\begin{array}{l}67 / 27 / 87 \\
10 / 29 / 87\end{array}$ & $\begin{array}{l}56.08 \\
\text { NR }\end{array}$ \\
\hline $\begin{array}{l}07 / 27 / 87 \\
10 / 29 / 87\end{array}$ & $\begin{array}{c}883.10 \\
754.10 \\
\text { NR }\end{array}$ \\
\hline $\begin{array}{l}07 / 27 / 87 \\
16 / 28 / 87\end{array}$ & $\begin{array}{c}16711.00 \\
12064.18 \\
\text { MR }\end{array}$ \\
\hline $\begin{array}{l}67 / 27 / 87 \\
16 / 20 / 87\end{array}$ & $\begin{array}{c}6790.18 \\
7916.06 \\
\text { NR }\end{array}$ \\
\hline $\begin{array}{l}07 / 27 / 87 \\
10 / 29 / 87\end{array}$ & $\begin{array}{l}22304.60 \\
24411.60 \\
\text { MR }\end{array}$ \\
\hline $\begin{array}{l}07 / 27 / 87 \\
10 / 29 / 87\end{array}$ & $\begin{array}{l}19.90 \\
21.06\end{array}$ \\
\hline $07 / 27 / 87$ & 16.16 \\
\hline
\end{tabular}

* - VAlue eXCEeds PRIVARY dRINKIng tater standard

1 - VALUE EXCEEDS PROPOSED PRIUARY DRINKING YATER STANDARD.

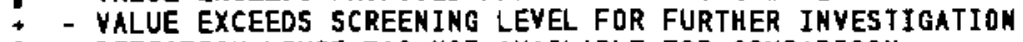

- DETECTION LiMIT ias not available for couparison

NR - AMALYSIS NOT REQUESTED OR NOT YET REPORTED

VALUES IN \{\} ARE COUNTING ERRORS FOR RADIONUCLIDES

mater Standard(S) In Parentheses aRe proposed only 


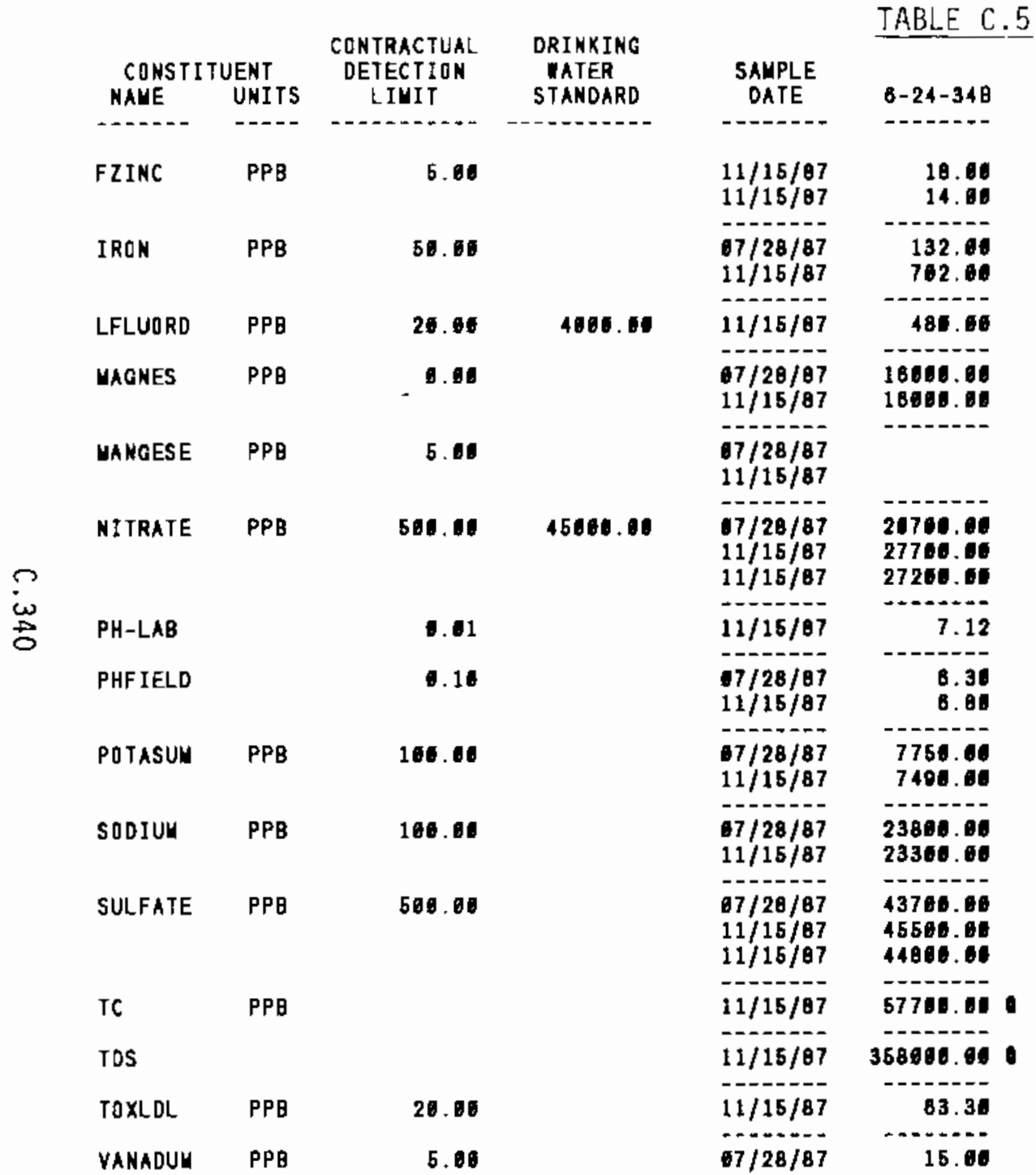

\begin{tabular}{|c|c|}
\hline $\begin{array}{c}\text { SAUPLE } \\
\text { DATE }\end{array}$ & $6-24-34 C$ \\
\hline 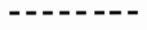 & $----n$ \\
\hline $11 / 15 / 87$ & $\begin{array}{l}46.66 \\
\text { NR }\end{array}$ \\
\hline $\begin{array}{l}07 / 27 / 87 \\
11 / 25 / 87\end{array}$ & $\begin{array}{r}81.06 \\
128.06\end{array}$ \\
\hline $11 / 15 / 87$ & 516.00 \\
\hline $\begin{array}{l}7 / 27 / 87 \\
11 / 16 / 87\end{array}$ & $\begin{array}{l}-1400.00 \\
14800.00 \\
15500.00\end{array}$ \\
\hline $\begin{array}{l}07 / 27 / 87 \\
11 / 15 / 87\end{array}$ & 6.66 \\
\hline $\begin{array}{l}67 / 27 / 67 \\
11 / 15 / 67\end{array}$ & $\begin{array}{c}21706.04 \\
28000.00 \\
\text { NR }\end{array}$ \\
\hline $11 / 15 / 87$ & 7.88 \\
\hline $\begin{array}{l}07 / 27 / 87 \\
11 / 15 / 87\end{array}$ & $\begin{array}{r}0.58 \\
0.86\end{array}$ \\
\hline $\begin{array}{l}07 / 27 / 87 \\
11 / 15 / 87\end{array}$ & $\begin{array}{l}6960.00 \\
7390.00\end{array}$ \\
\hline $17 / 27 / 87$ & 21860.00 \\
\hline $\begin{array}{l}97 / 27 / 87 \\
11 / 16 / 87\end{array}$ & $\begin{array}{c}43980.00 \\
4460.00 \\
\mathrm{HR}\end{array}$ \\
\hline $11 / 15 / 87$ & 51200.00 \\
\hline $11 / 15 / 87$ & 342006.00 \\
\hline $11 / 15 / 87$ & $-----\overline{0}$ \\
\hline $07 / 27 / 87$ & 16.00 \\
\hline
\end{tabular}

\begin{tabular}{|c|c|}
\hline $\begin{array}{l}\text { SAUPLE } \\
\text { DATE }\end{array}$ & $6-24-35$ \\
\hline --.--- & -----0 \\
\hline $10 / 29 / 87$ & $\begin{array}{l}27.00 \\
\text { NR }\end{array}$ \\
\hline $\begin{array}{l}17 / 27 / 87 \\
10 / 29 / 87\end{array}$ & $\begin{array}{l}142.06 \\
100.06\end{array}$ \\
\hline $10 / 20 / 87$ & 525.06 \\
\hline $\begin{array}{l}67 / 27 / 87 \\
10 / 29 / 87\end{array}$ & $\begin{array}{l}10800.00 \\
16980.00\end{array}$ \\
\hline $\begin{array}{l}67 / 27 / 87 \\
16 / 29 / 87\end{array}$ & -+--- \\
\hline $\begin{array}{l}07 / 27 / 87 \\
16 / 29 / 67\end{array}$ & $\begin{array}{c}18704 \\
21860 \\
\mathrm{MR}\end{array}$ \\
\hline $10 / 20 / 87$ & $\begin{array}{r}7.75 \\
\end{array}$ \\
\hline $\begin{array}{l}67 / 27 / 87 \\
16 / 20 / 87\end{array}$ & $\begin{array}{l}7.20 \\
5.50\end{array}$ \\
\hline $\begin{array}{l}67 / 27 / 87 \\
10 / 29 / 87\end{array}$ & $\begin{array}{l}-5600.00 \\
8580.00\end{array}$ \\
\hline $\begin{array}{l}17 / 27 / 87 \\
18 / 29 / 87\end{array}$ & 21304.66 \\
\hline $\begin{array}{l}9 / 27 / 97 \\
16 / 29 / 97\end{array}$ & $\begin{array}{c}47600.06 \\
61600.00 \\
\text { NR }\end{array}$ \\
\hline $10 / 28 / 87$ & 30700.00 \\
\hline $18 / 29 / 87$ & 269000.00 \\
\hline $10 / 28 / 87$ & \\
\hline $67 / 27 / 87$ & 22.00 \\
\hline
\end{tabular}

* - value exceEds primary dRIMKing tater standard

* - VAlUE EXCEEDS PRGPOSED PRIUARY DRINKIHG Uater STAKDARO

+ - VALUE EXCEEDS SCREENING LEVEL FOR FURTHER INYESTIGATION.

- DETECTION LIUIT IAS NOT AVAILABLE FOR COUPARISON

NR - ANALYSIS NOT REQUESTED OR NOT YET REPORTED

VALUES IN I I ARE CDUNTING ERRORS FOR RADIONUCLIDES

WATER STANDARD(S) IN PARENTHESES ARE PROPOSED OHLY 


\begin{tabular}{|c|c|c|c|c|c|c|c|c|c|}
\hline $\begin{array}{l}\text { CONSTI } \\
\text { NANE }\end{array}$ & $\begin{array}{l}\text { UENT } \\
\text { UNITS }\end{array}$ & $\begin{array}{c}\text { CONTRACTUAL } \\
\text { DETECTION } \\
\text { LIUIT }\end{array}$ & $\begin{array}{l}\text { DRINKING } \\
\text { TATER } \\
\text { STAMOARO }\end{array}$ & $\begin{array}{l}\text { SAHPLE } \\
\text { DATE }\end{array}$ & TABLE C & $\begin{array}{l}\text { SAMPLE } \\
\text { DATE }\end{array}$ & $6-24-34 C$ & $\begin{array}{c}\text { SAUPLE } \\
\text { DATE }\end{array}$ & 6-24-36 \\
\hline VAFADUU & PPB & 6.80 & & $11 / 16 / 87$ & 17.00 & $11 / 15 / 87$ & 15.66 & $10 / 29 / 87$ & 19.01 \\
\hline ZINC & PPB & 6.66 & & $\begin{array}{l}87 / 28 / 87 \\
11 / 15 / 87\end{array}$ & $\begin{array}{l}31.08 \\
82.00\end{array}$ & $\begin{array}{l}07 / 27 / 87 \\
11 / 16 / 87\end{array}$ & $\begin{array}{l}37.60 \\
71.60\end{array}$ & $\begin{array}{l}07 / 27 / 87 \\
11 / 29 / 87\end{array}$ & $\begin{array}{l}43.01 \\
11.06\end{array}$ \\
\hline
\end{tabular}

* - VALUE EXCEEDS PRIYARY DRINKING IATER STANDARO.

- VALUE EXCEEDS PROPOSED PRIUARY dRINKING IaTER STANDARD.

- VALUE EXCEEDS SCREENING LEVEL FOR FURTHER INVESTIGATION

- DETECTION linit tas nOt available for couparison

NR - ANALYSIS NOT REQUESTED OR NOT YET REPORTED

VALUES IN \{\} ARE COUNTING ERRORS FDR RADIORUCLIDES

IATER STANDARD(S) IN PARENTHESES aRE PROPGSED ONLY 
IABLE C.5. (contd)

\begin{tabular}{|c|c|c|c|c|c|}
\hline $\begin{array}{l}\text { CONSTI } \\
\text { NALE }\end{array}$ & $\begin{array}{l}\text { UENT } \\
\text { UNITS }\end{array}$ & $\begin{array}{l}\text { CONTRACTUAL } \\
\text { DETECTIOK } \\
\text { LIUIT }\end{array}$ & $\begin{array}{l}\text { DRINKING } \\
\text { WATER } \\
\text { STANDARO }\end{array}$ & $\begin{array}{c}\text { SAMPLE } \\
\text { DATE }\end{array}$ & $B-25-33 A$ \\
\hline ALKALIN & & & & $11 / 15 / 87$ & 124006.00 \\
\hline ALPHA & $\mathrm{PCI} / \mathrm{L}$ & 4.00 & 15.88 & $\begin{array}{l}67 / 29 / 87 \\
11 / 16 / 87\end{array}$ & $\begin{array}{ll} & 2.64 \\
\{ & 1.42\} \\
\{\quad 3.44 \\
\{\quad 1.87\}\end{array}$ \\
\hline ARSENIC & PPQ & 6.60 & 58.60 & $\begin{array}{l}07 / 29 / 87 \\
11 / 16 / 87\end{array}$ & \\
\hline BARIUM & PPB & 6.06 & 1086.00 & $\begin{array}{l}07 / 29 / 87 \\
11 / 15 / 87\end{array}$ & $\begin{array}{l}23.60 \\
24.66\end{array}$ \\
\hline BETA & $\mathrm{PCI} / \mathrm{L}$ & 8.00 & 50.60 & $\begin{array}{l}1 / 28 / 87 \\
11 / 15 / 87\end{array}$ & 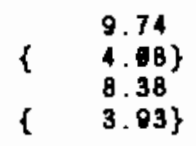 \\
\hline CALCIUN & $\mathrm{PPB}$ & 58.80 & & $\begin{array}{l}67 / 28 / 87 \\
11 / 16 / 87\end{array}$ & $\begin{array}{l}38866.00 \\
29866.08\end{array}$ \\
\hline CHLORID & $\mathrm{PPB}$ & 508.60 & & $\begin{array}{l}07 / 29 / 87 \\
11 / 15 / 87\end{array}$ & $\begin{array}{l}8554.60 \\
8850.86\end{array}$ \\
\hline CONDFLD & UUHO & 1.06 & & $\begin{array}{l}07 / 28 / 87 \\
11 / 15 / 87\end{array}$ & $\begin{array}{l}371.00 \\
413.00\end{array}$ \\
\hline CONDLAB & UNHO & & & $11 / 15 / 87$ & 365.60 \\
\hline FARSEN I & PPB & 5.68 & 50.00 & $\begin{array}{l}17 / 29 / 87 \\
11 / 15 / 87\end{array}$ & \\
\hline FEARIUU & $\mathrm{PPB}$ & 6.00 & 1000.00 & $\begin{array}{l}67 / 29 / 87 \\
11 / 16 / 87\end{array}$ & $\begin{array}{l}28.80 \\
30.00\end{array}$ \\
\hline FCALCIU & PPB & 50.00 & & $\begin{array}{l}07 / 29 / 87 \\
11 / 15 / 87\end{array}$ & $\begin{array}{l}28200.06 \\
28500.00\end{array}$ \\
\hline FLUQRID & PPB & 500.00 & 4808.66 & $07 / 28 / 87$ & 638.00 \\
\hline
\end{tabular}

\begin{tabular}{|c|c|}
\hline $\begin{array}{l}\text { SAMPLE } \\
\text { DATE }\end{array}$ & $B-26-34 A$ \\
\hline $10 / 29 / 87$ & 134060.60 \\
\hline $67 / 29 / 87$ & $\begin{array}{l}2.06 \\
1.29\}\end{array}$ \\
\hline $18 / 29 / 87$ & $\begin{array}{l}1.31 \\
\{\quad 1.22\}\end{array}$ \\
\hline $\begin{array}{l}67 / 29 / 87 \\
10 / 29 / 87\end{array}$ & 6.06 \\
\hline $\begin{array}{l}07 / 29 / 87 \\
10 / 29 / 87\end{array}$ & $\begin{array}{l}38.69 \\
37.66\end{array}$ \\
\hline $67 / 29 / 87$ & 21.60 \\
\hline $10 / 29 / 87$ & $\begin{array}{r}22.96 \\
\{\quad 5.26\}\end{array}$ \\
\hline $\begin{array}{l}07 / 29 / 87 \\
10 / 29 / 87\end{array}$ & $\begin{array}{l}36389.60 \\
37960.60\end{array}$ \\
\hline $\begin{array}{l}67 / 29 / 87 \\
16 / 29 / 87\end{array}$ & $\begin{array}{l}7831.00 \\
8316.00\end{array}$ \\
\hline $\begin{array}{l}07 / 29 / 87 \\
10 / 29 / 87\end{array}$ & $\begin{array}{l}347.60 \\
471.06\end{array}$ \\
\hline $10 / 29 / 87$ & 417.10 \\
\hline $\begin{array}{l}07 / 29 / 87 \\
10 / 29 / 87\end{array}$ & \\
\hline $\begin{array}{l}67 / 29 / 87 \\
10 / 29 / 87\end{array}$ & $\begin{array}{l}37.68 \\
46.06\end{array}$ \\
\hline $\begin{array}{l}07 / 29 / 87 \\
10 / 20 / 87\end{array}$ & $\begin{array}{l}30808.00 \\
42486.08\end{array}$ \\
\hline $67 / 29 / 87$ & 873.00 \\
\hline
\end{tabular}

\begin{tabular}{|c|c|}
\hline $\begin{array}{l}\text { SALPLE } \\
\text { OATE }\end{array}$ & $8-26-348$ \\
\hline $16 / 29 / 87$ & 122000.06 \\
\hline $37 / 29 / 87$ & $\begin{array}{l}1.94 \\
1.35\end{array}$ \\
\hline $10 / 29 / 87$ & $\left\{\begin{array}{l}2.60 \\
\{\quad 1.36\end{array}\right\}$ \\
\hline $\begin{array}{l}07 / 29 / 87 \\
10 / 28 / 87\end{array}$ & \\
\hline $\begin{array}{l}07 / 29 / 87 \\
10 / 28 / 87\end{array}$ & 34.00 \\
\hline & $\begin{array}{c}26.28 \\
\{\quad 6.47\}\end{array}$ \\
\hline $10 / 28 / 87$ & $\left\{\begin{array}{r}22.20 \\
5.20\end{array}\right.$ \\
\hline $\begin{array}{l}97 / 29 / 87 \\
10 / 29 / 87\end{array}$ & $\begin{array}{l}34240.06 \\
36410.80\end{array}$ \\
\hline $\begin{array}{l}67 / 29 / 87 \\
10 / 29 / 87\end{array}$ & $\begin{array}{l}7510.00 \\
7656.00\end{array}$ \\
\hline $\begin{array}{l}07 / 28 / 87 \\
10 / 29 / 87\end{array}$ & $\begin{array}{r}357.66 \\
437.66\end{array}$ \\
\hline $10 / 29 / 87$ & 496.66 \\
\hline $\begin{array}{l}67 / 29 / 87 \\
16 / 29 / 87\end{array}$ & 6.91 \\
\hline $\begin{array}{l}07 / 29 / 87 \\
10 / 28 / 87\end{array}$ & $\begin{array}{l}34.00 \\
34.60\end{array}$ \\
\hline $\begin{array}{l}67 / 29 / 87 \\
10 / 20 / 87\end{array}$ & $\begin{array}{l}30406.60 \\
36146.06\end{array}$ \\
\hline $07 / 29 / 87$ & 886.00 \\
\hline
\end{tabular}

- - Value exceeds priuary dRinking inter standard

- - YALUE EXCEEDS PROPOSED PRIUARY DRINKING IATER STANDARD.

- YALUE EXCEEDS SCREEKING LEVEL FDR FURTHER IMVESTIGATION.

- - detection linit tas not available for couparisom

NR - ANALYSIS NOT REQUESTED OR NOT YET REPORTED

YALUES IN \{ \} ARE COUNTING ERRORS FOR RADIONUCLIDES

IATER STANDARD(S) IN PARENTHESES ARE PROPOSED ONLY 
TABLE C.5. (contd)

\begin{tabular}{|c|c|c|c|c|c|}
\hline$\underset{\text { MAME }}{\text { CONSTI }}$ & $\begin{array}{l}\text { UENT } \\
\text { UNITS }\end{array}$ & $\begin{array}{l}\text { CONTRACTUAL } \\
\text { DETECTION } \\
\text { LINIT }\end{array}$ & $\begin{array}{l}\text { ORINKING } \\
\text { WATER } \\
\text { STANDARD }\end{array}$ & $\begin{array}{c}\text { SALPLE } \\
\text { DATE }\end{array}$ & $8-25-33 A$ \\
\hline FLUORID & PPB & 500.60 & 4000.60 & $11 / 15 / 87$ & 585.06 \\
\hline FMAGNES & PPB & 0.80 & & $\begin{array}{l}07 / 29 / 87 \\
11 / 15 / 87\end{array}$ & $\begin{array}{l}8810.60 \\
8350.01\end{array}$ \\
\hline FUANGAN & PPB & 5.68 & & $\begin{array}{l}67 / 28 / 87 \\
11 / 15 / 87\end{array}$ & $\begin{array}{l}6.116 \\
5.16\end{array}$ \\
\hline FPOTASS & PPB & 160.60 & & $\begin{array}{l}07 / 29 / 87 \\
11 / 15 / 87\end{array}$ & $\begin{array}{l}5780.60 \\
8886.00\end{array}$ \\
\hline FSODIUU & $\rho P \theta$ & 100.68 & & $\begin{array}{l}67 / 29 / 87 \\
11 / 15 / 87\end{array}$ & $\begin{array}{l}37360.18 \\
38168.60\end{array}$ \\
\hline FYANADI & PPB & 5.60 & & $\begin{array}{l}17 / 29 / 87 \\
11 / 15 / 87\end{array}$ & $\begin{array}{l}11.06 \\
10.00\end{array}$ \\
\hline FZINC & PPB & 5.60 & & $\begin{array}{l}07 / 29 / 87 \\
11 / 15 / 07\end{array}$ & 5.60 \\
\hline IRON & PPB & 50.60 & & $\begin{array}{l}67 / 29 / 87 \\
11 / 15 / 87\end{array}$ & $\begin{array}{l}88.06 \\
89.06\end{array}$ \\
\hline LFLUORD & PPB & 20.00 & 4000.00 & $11 / 15 / 07$ & 400.00 \\
\hline HAGNES & PPB & 0.08 & & $\begin{array}{l}97 / 29 / 87 \\
11 / 15 / 87\end{array}$ & $\begin{array}{l}0450.00 \\
8416.00\end{array}$ \\
\hline WANGESE & PPB & 5.80 & & $\begin{array}{l}67 / 29 / 87 \\
11 / 15 / 87\end{array}$ & $\begin{array}{l}8.06 \\
8.66\end{array}$ \\
\hline NICKEI & PPB & 16.69 & & $\begin{array}{l}07 / 29 / 87 \\
11 / 15 / 87\end{array}$ & 16.00 \\
\hline NITRATE & PPB & 596.00 & 45606.00 & $\begin{array}{l}07 / 29 / 87 \\
11 / 15 / 87\end{array}$ & $\begin{array}{l}5758.60 \\
8546.08\end{array}$ \\
\hline PH-LAB & & 0.81 & & $11 / 15 / 87$ & 8. 26 \\
\hline PHFIELD & & 0.18 & & $07 / 29 / 87$ & 7.80 \\
\hline
\end{tabular}

\begin{tabular}{|c|c|}
\hline $\begin{array}{l}\text { SAUPLE } \\
\text { DATE }\end{array}$ & $B-25-34 A$ \\
\hline $10 / 28 / 87$ & 780.06 \\
\hline $\begin{array}{l}07 / 29 / 87 \\
16 / 29 / 87\end{array}$ & $\begin{array}{l}10460.80 \\
12760.80\end{array}$ \\
\hline $\begin{array}{l}07 / 29 / 87 \\
10 / 29 / 87\end{array}$ & \\
\hline $\begin{array}{l}67 / 29 / 87 \\
10 / 29 / 87\end{array}$ & $\begin{array}{l}5910.80 \\
7380.60\end{array}$ \\
\hline $\begin{array}{l}07 / 20 / 87 \\
10 / 29 / 87\end{array}$ & $\begin{array}{l}22780.89 \\
24380.10\end{array}$ \\
\hline $\begin{array}{l}97 / 29 / 87 \\
18 / 29 / 87\end{array}$ & $\begin{array}{l}23.06 \\
18.4\end{array}$ \\
\hline $\begin{array}{l}67 / 29 / 87 \\
10 / 29 / 87\end{array}$ & $\begin{array}{r}5.60 \\
49.60\end{array}$ \\
\hline $\begin{array}{l}07 / 29 / 87 \\
10 / 29 / 87\end{array}$ & 287.00 \\
\hline $10 / 20 / 87$ & 580.00 \\
\hline $\begin{array}{l}67 / 29 / 87 \\
10 / 29 / 87\end{array}$ & $\begin{array}{l}10400.00 \\
11806.01\end{array}$ \\
\hline $\begin{array}{l}07 / 29 / 87 \\
10 / 29 / 87\end{array}$ & \\
\hline $\begin{array}{l}07 / 29 / 87 \\
10 / 20 / 87\end{array}$ & \\
\hline $\begin{array}{l}97 / 29 / 87 \\
10 / 20 / 87\end{array}$ & $\begin{array}{l}24160.80 \\
27368.86\end{array}$ \\
\hline $16 / 29 / 87$ & 7.72 \\
\hline $07 / 29 / 87$ & 7.40 \\
\hline
\end{tabular}

\begin{tabular}{|c|c|}
\hline $\begin{array}{l}\text { SAUPLE } \\
\text { DATE }\end{array}$ & $B-25-34 B$ \\
\hline $18 / 29 / 87$ & 758.60 \\
\hline $\begin{array}{l}87 / 29 / 87 \\
16 / 29 / 87\end{array}$ & $\begin{array}{l}10390.00 \\
11160.06\end{array}$ \\
\hline $\begin{array}{l}07 / 28 / 87 \\
10 / 29 / 87\end{array}$ & \\
\hline $\begin{array}{l}17 / 29 / 87 \\
10 / 29 / 87\end{array}$ & $\begin{array}{l}5766.68 \\
8868.06\end{array}$ \\
\hline $\begin{array}{l}17 / 29 / 87 \\
10 / 20 / 87\end{array}$ & 21701.06 \\
\hline $\begin{array}{l}17 / 29 / 87 \\
10 / 29 / 87\end{array}$ & $\begin{array}{l}23.18 \\
26.81\end{array}$ \\
\hline $\begin{array}{l}97 / 20 / 87 \\
16 / 20 / 87\end{array}$ & 7.10 \\
\hline $\begin{array}{l}67 / 29 / 87 \\
19 / 29 / 87\end{array}$ & \\
\hline $10 / 20 / 07$ & 580.00 \\
\hline $\begin{array}{l}07 / 29 / 87 \\
10 / 29 / 87\end{array}$ & $\begin{array}{l}10460.61 \\
11300.06\end{array}$ \\
\hline $\begin{array}{l}07 / 29 / 87 \\
16 / 29 / 87\end{array}$ & \\
\hline $\begin{array}{l}57 / 20 / 87 \\
10 / 29 / 87\end{array}$ & \\
\hline $\begin{array}{l}67 / 20 / 87 \\
16 / 29 / 87\end{array}$ & $\begin{array}{l}23916.06 \\
28066.06\end{array}$ \\
\hline $16 / 29 / 87$ & 7.82 \\
\hline $7 / 29 / 87$ & 7.46 \\
\hline
\end{tabular}

* - Value exceeds primary dRINKING tater standard.

- VALUE EXCEEDS PRDPDSED PRIMARY dRINKING vater STANDard.

- - YALUE EXCEEDS SCREENING LEVEl FOR FURTHER INYESTIGATION.

- Detection liUit as nat available for coMparison

NR - ANALYSIS NOT REQUESTEO OR NOT YET REPQRTED

VALUES IN \{\} ARE COUNTING ERRORS FOR RADIONUCLIDES

TATER STANDARO'S) IN PARENTHESES ARE PROPOSED ONLY 
TABLE C.5. (contd)

\begin{tabular}{|c|c|c|}
\hline $\begin{array}{l}\text { CONSTI } \\
\text { NAUE }\end{array}$ & $\begin{array}{l}\text { UENT } \\
\text { UNITS }\end{array}$ & $\begin{array}{c}\text { CONTRACTUAL } \\
\text { DETECTION } \\
\text { LIUIT }\end{array}$ \\
\hline PHFIELD & & 0.10 \\
\hline POTASUI & PP9 & 100.00 \\
\hline SDDIUY & PPB & 106.00 \\
\hline SULFATE & PPB & 508.00 \\
\hline TC & PPB & \\
\hline TDS & & \\
\hline TOC & PPB & 1000.00 \\
\hline VAMADUE & PPB & 5.60 \\
\hline ZINC & PPB & 5.00 \\
\hline
\end{tabular}

DRINKING

VATER STANDARD

\begin{tabular}{|c|}
\hline $\begin{array}{l}\text { SALPLE } \\
\text { OATE }\end{array}$ \\
\hline $11 / 15 / 87$ \\
\hline $\begin{array}{l}87 / 29 / 87 \\
11 / 16 / 87\end{array}$ \\
\hline $\begin{array}{l}7 / 29 / 87 \\
1 / 16 / 87\end{array}$ \\
\hline $\begin{array}{l}7 / 29 / 87 \\
11 / 15 / 87\end{array}$ \\
\hline $\begin{array}{l}7 / 20 / 87 \\
1 / 15 / 87\end{array}$ \\
\hline $11 / 15 / 87$ \\
\hline $\begin{array}{l}07 / 29 / 87 \\
11 / 15 / 87\end{array}$ \\
\hline $\begin{array}{l}77 / 29 / 87 \\
11 / 15 / 87\end{array}$ \\
\hline $\begin{array}{l}87 / 29 / 87 \\
11 / 15 / 87\end{array}$ \\
\hline
\end{tabular}

$0-25 \cdot 33 A$

-........

6.00

5770.06

5790.06

38006.0

38360.06

63710.

07306.06

29310.00

28216.06

284000 .

-

$\begin{array}{r}1326.66 \\ \hdashline .2 .65\end{array}$

11.06
8.06

9.86

15.80

\begin{tabular}{|c|c|}
\hline $\begin{array}{l}\text { SALPLE } \\
\text { DATE }\end{array}$ & $B-25-34 A$ \\
\hline $10 / 28 / 87$ & 8.80 \\
\hline $\begin{array}{l}07 / 29 / 87 \\
10 / 29 / 87\end{array}$ & $\begin{array}{l}8180.06 \\
8090.00\end{array}$ \\
\hline $\begin{array}{l}17 / 29 / 87 \\
16 / 29 / 87\end{array}$ & $\begin{array}{l}23500.60 \\
21600.06\end{array}$ \\
\hline $\begin{array}{l}07 / 29 / 87 \\
10 / 20 / 87\end{array}$ & $\begin{array}{l}40100.00 \\
45500.00\end{array}$ \\
\hline $\begin{array}{l}07 / 29 / 87 \\
10 / 29 / 87\end{array}$ & $\begin{array}{l}27800.08 \\
31700.08\end{array}$ \\
\hline $10 / 28 / 87$ & 257060.06 \\
\hline $\begin{array}{l}07 / 29 / 87 \\
10 / 29 / 87\end{array}$ & \\
\hline $\begin{array}{l}67 / 29 / 87 \\
10 / 29 / 87\end{array}$ & $\begin{array}{l}30.60 \\
18.00\end{array}$ \\
\hline $\begin{array}{l}17 / 29 / 87 \\
16 / 29 / 87\end{array}$ & $\begin{array}{r}7.60 \\
53.66\end{array}$ \\
\hline
\end{tabular}

53.68
SAYPLE

18/29/87

$17-12--1$

67/29/87

$10 / 29 / 87$

67/29/87

$10 / 29 / 87$

$07 / 29 / 87$

$10 / 29 / 87$

$-7-129 / 97$

97/29/87

$10 / 29 / 87$

$10 / 29 / 87$

$67 / 29 / 87$

16/29/87

$07 / 29 / 87$

$16 / 29 / 87$

-7/29/87

$67 / 29 / 87$
$16 / 20 / 87$
6-25-34

8.16

0350

B140.0

23606.

23186.06

30000.08

427.9.61

28300.00

28100.00

255600.00.

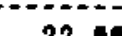

22.60

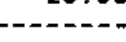

9.66

- - Value exceeds priuary orinking mater standard.

- YALUE EXCEEDS PROPOSED PRIMARY DRINKING IATER STANDARD.

- - Value exceeds screening level for further inYestigation.

- - detection linit ias ndt avallable for coupariso*

NR - ANALYSIS NOT RERUESTED OR NOT YET REPORTED

VALUES IN I ARE COUNTIHG ERRORS FOR RADIOMUCLIDES

WATER STANDARD(S) IN PARENTHESES ARE PROPDSED ONLY 


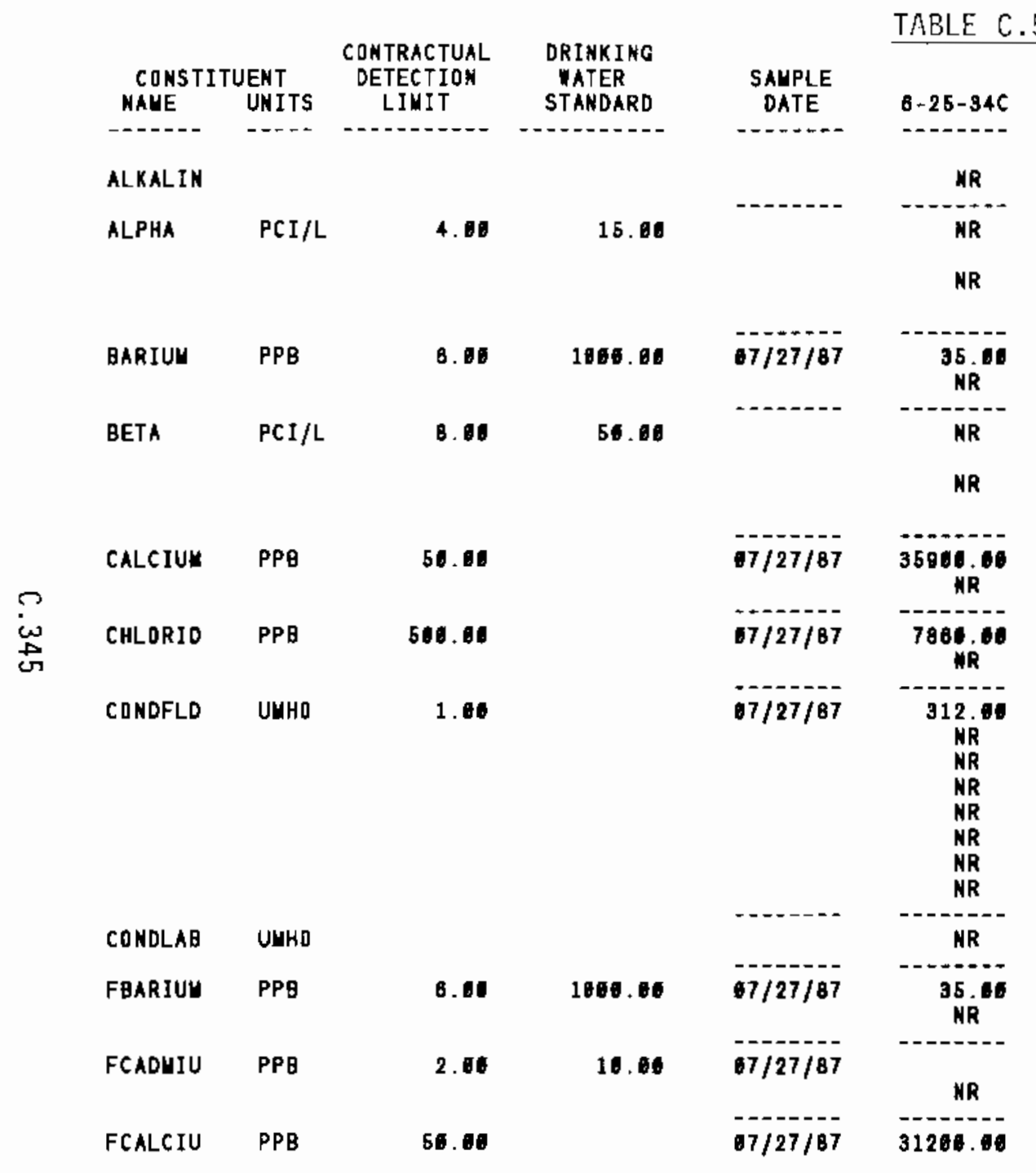

(contd)

- - VALUE eXCEEDS PRIWARY dRINKING MaTER STANOARD.

* - YALUE EXCEEDS SCREENING LEVEL FOR FURTHER INYESTIGATIDN.

- DETECTIDN LIUIT NAS MOT AVAILABLE FOR CDUPARISON

NR - ANALYSIS NOT REQUESTED OR NDT YET REPORTED

VALUES IN \{ \} ARE COUNTIMO ERRORS FOR RADIONUCLIDES

IATER STANDARD(S) IN PARENTHESES ARE PROPOSED ONLY

\begin{tabular}{|c|c|}
\hline $\begin{array}{l}\text { SALPLE } \\
\text { DATE }\end{array}$ & $6-2 B-34$ \\
\hline $11 / 15 / 87$ & 111006.60 \\
\hline $07 / 30 / 87$ & $\begin{array}{l}2.75 \\
1.30\}\end{array}$ \\
\hline $11 / 15 / 87$ & $\begin{array}{l}3.46 \\
1.86\}\end{array}$ \\
\hline $\begin{array}{l}07 / 38 / 87 \\
11 / 15 / 87\end{array}$ & $\begin{array}{l}34.11 \\
27.18\end{array}$ \\
\hline $07 / 30 / 87$ & $\begin{array}{r}21.38 \\
5.68\}\end{array}$ \\
\hline $11 / 15 / 87$ & $\left\{\begin{array}{r}31.30 \\
\{\quad 5.81\}\end{array}\right.$ \\
\hline $\begin{array}{l}07 / 30 / 87 \\
11 / 16 / 87\end{array}$ & $\begin{array}{l}28564.00 \\
20360.00\end{array}$ \\
\hline $\begin{array}{l}67 / 36 / 87 \\
11 / 15 / 87\end{array}$ & 8850.01 \\
\hline $\begin{array}{l}97 / 36 / 87 \\
97 / 30 / 87 \\
67 / 30 / 87 \\
07 / 30 / 87 \\
11 / 15 / 87 \\
11 / 15 / 87 \\
11 / 15 / 87 \\
11 / 15 / 87\end{array}$ & $\begin{array}{l}288.60 \\
270.60 \\
270.60 \\
280.80 \\
361.81 \\
358.11 \\
358.68 \\
358.06\end{array}$ \\
\hline $11 / 16 / 87$ & 333.00 \\
\hline $\begin{array}{l}07 / 30 / 87 \\
11 / 15 / 87\end{array}$ & $\begin{array}{l}28.11 \\
32.11\end{array}$ \\
\hline $\begin{array}{l}07 / 36 / 87 \\
11 / 15 / 87\end{array}$ & 2.00 \\
\hline $7 / 36 / 87$ & 28800.00 \\
\hline
\end{tabular}


TABLE C.5. (contd)

\begin{tabular}{|c|c|c|c|c|c|}
\hline $\begin{array}{l}\text { CONSTI } \\
\text { NAUE }\end{array}$ & $\begin{array}{l}\text { UENT } \\
\text { UNITS }\end{array}$ & $\begin{array}{l}\text { CONTRACTUAL } \\
\text { DETECTION } \\
\text { LIUIT }\end{array}$ & $\begin{array}{l}\text { DRINKING } \\
\text { NATER } \\
\text { STANDARD }\end{array}$ & $\begin{array}{c}\text { SAUPLE } \\
\text { DATE }\end{array}$ & $6-25-34 C$ \\
\hline FCALCIU & PPB & 50.00 & & & NR \\
\hline FLUORID & PPB & 500.08 & 4060.86 & $67 / 27 / 87$ & $\begin{array}{c}682.60 \\
W R\end{array}$ \\
\hline FUAGNES & PPB & 0.00 & & $07 / 27 / 87$ & 10800.00 \\
\hline FPDTASS & PPB & 100.00 & & $07 / 27 / 87$ & $\underset{\mathrm{NR}}{8120.08}$ \\
\hline FSODIUY & $P P B$ & 160.01 & & $67 / 27 / 87$ & 22766.00 \\
\hline FVANADI & PPB & 5.86 & & $67 / 27 / 87$ & ${ }_{\mathrm{HR}}^{23.66}$ \\
\hline FZINC & PPB & 5.01 & & $87 / 27 / 87$ & NR \\
\hline IRDN & PPB & 58.00 & & $87 / 27 / 87$ & ${ }_{\mathrm{NR}}^{88.01}$ \\
\hline LFLUDRD & PPB & 20.00 & 4666.68 & & NR \\
\hline HAGNES & PPB & 0.06 & & $07 / 27 / 87$ & 10700.00 \\
\hline NITRATE & PPB & 500.06 & 45000.60 & $07 / 27 / 87$ & $\underset{\mathrm{NR}}{24806.00}$ \\
\hline$P H-L A B$ & & D. 01 & & 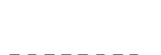 & $\# R$ \\
\hline PHFIELD & & 0.10 & & $07 / 27 / 87$ & $\begin{array}{l}7,40 \\
\text { KR } \\
\text { NR } \\
\text { NR } \\
\text { NR } \\
\text { NR }\end{array}$ \\
\hline
\end{tabular}

\begin{tabular}{|c|c|}
\hline $\begin{array}{l}\text { SALPLE } \\
\text { DATE }\end{array}$ & $6-26-33$ \\
\hline $10 / 29 / 87$ & 32400.06 \\
\hline $\begin{array}{l}67 / 29 / 87 \\
16 / 29 / 87\end{array}$ & $\begin{array}{l}893.00 \\
801.00\end{array}$ \\
\hline $\begin{array}{l}07 / 29 / 87 \\
10 / 29 / 87\end{array}$ & $\begin{array}{r}8380.60 \\
18390.66\end{array}$ \\
\hline $\begin{array}{l}07 / 29 / 87 \\
10 / 29 / 87\end{array}$ & $\begin{array}{l}6540.10 \\
8508.00\end{array}$ \\
\hline $\begin{array}{l}07 / 28 / 87 \\
10 / 29 / 87\end{array}$ & $\begin{array}{l}21760.00 \\
23760.00\end{array}$ \\
\hline $\begin{array}{l}67 / 29 / 87 \\
16 / 29 / 87\end{array}$ & $\begin{array}{l}29.00 \\
24.00\end{array}$ \\
\hline $\begin{array}{l}17 / 28 / 97 \\
11 / 20 / 97\end{array}$ & 7.00 \\
\hline $\begin{array}{l}17 / 28 / 87 \\
10 / 29 / 87\end{array}$ & \\
\hline $10 / 28 / 87$ & 690.08 \\
\hline $\begin{array}{l}107 / 29 / 87 \\
10 / 29 / 87\end{array}$ & $\begin{array}{r}10600.86 \\
9690.86\end{array}$ \\
\hline $\begin{array}{l}97 / 29 / 87 \\
16 / 29 / 87\end{array}$ & $\begin{array}{l}24860.90 \\
29186.00\end{array}$ \\
\hline $10 / 29 / 87$ & 7.98 \\
\hline $\begin{array}{l}67 / 29 / 87 \\
16 / 29 / 87\end{array}$ & $\begin{array}{l}7.50 \\
7.10 \\
\text { MR } \\
\text { MR } \\
\text { NR } \\
\text { NR }\end{array}$ \\
\hline
\end{tabular}

SAUPLE DATE

8-28-34

11/15/87 29860.

$07 / 38 / 87 \quad 784.80$

$11 / 15 / 87 \quad 594.06$

$07 / 3187 \quad 9370.60$

$11 / 15 / 87 \quad 10300.00$

$07 / 30 / 87$ 5590.68

$11 / 15 / 87 \quad 6270.06$

$67 / 36 / 87 \quad 21800$.

$11 / 15 / 87 \quad 23766.06$

$07 / 30 / 87 \quad 25.06$

$07 / 38687$
$11 / 16 / 87$

07/30/B7

$11 / 16 / 87$

-.......

5.60

$07 / 30 / 87$

$11 / 15 / 87$

$11 / 15 / 87$

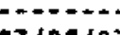

$07 / 30 / 87$
$11 / 15 / 87$

67/38/87

$11 / 15 / 87$

i11.....

$67 / 36 / 87$

$07 / 36 / 87$

$67 / 36 / 87$
$67 / 36 / 87$

$67 / 36 / 87$

$11 / 16 / 87$

$11 / 15 / 87$

28. 10

620.00

9890.00

9980.08

24809

30800.00

8.

8.04
-6.90
7.66
7.80
7.96
6.78
6.78

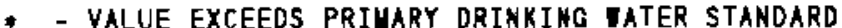

- - YALUE EXCEEDS PROPOSED PRIUARY DRINKING vaTER STANDARD.

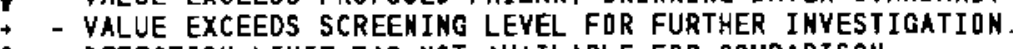

- - DETECTION LINIT IAS NOT AVAILABLE FDR COUPARISON

NR - ANALYSIS NOT REQUESTED DR NOT YET REPORTED

VALUES IN \& $\}$ ARE COUNTING ERRORS FOR RADIONUCLIDES

WATER STANDARD(S) IN PARENTHESES ARE PROPOSED ONLY 


\begin{tabular}{|c|c|c|c|c|c|}
\hline$\underbrace{\text { CDNSTI }}_{\text {NAME }}$ & $\begin{array}{l}\text { UENT } \\
\text { UNITS }\end{array}$ & $\begin{array}{c}\text { CORTRACTUAL } \\
\text { DETECIION } \\
\text { LIUIT }\end{array}$ & $\begin{array}{l}\text { DRINKING } \\
\text { ITATER } \\
\text { STAKDARD }\end{array}$ & $\begin{array}{c}\text { SANPLE } \\
\text { DATE }\end{array}$ & $6-25-34 C$ \\
\hline PHF IELD & & 8.10 & & & $\begin{array}{l}\text { NR } \\
\text { NR }\end{array}$ \\
\hline POTASUY & PPB & 100.00 & & $07 / 27 / 87$ & ${ }_{\mathrm{KR}}^{8231.8 \mathrm{O}}$ \\
\hline SDOIUU & PPB & 106.60 & & $87 / 27 / 87$ & ${ }_{\mathrm{NR}}^{23106.60}$ \\
\hline SULFATE & PPB & 600.00 & & $07 / 27 / 87$ & ${ }_{\mathrm{NR}}^{38000.60}$ \\
\hline TC & PPB & & & & $\begin{array}{l}\text { NR } \\
\text { MR }\end{array}$ \\
\hline TDS & & & & & NR \\
\hline TDC & PPB & 1060.60 & & $67 / 27 / 87$ & $\begin{array}{l}\text { NR } \\
N R \\
\text { NR } \\
\text { NR } \\
\text { NR } \\
\text { NR } \\
\text { NR }\end{array}$ \\
\hline YAMADUU & PPB & 5.01 & & $07 / 27 / 87$ & ${ }_{\mathrm{NR}}^{27.106}$ \\
\hline ZINE & PPB & 5.61 & & $07 / 27 / 87$ & $\begin{array}{l}8.08 \\
\text { RR }\end{array}$ \\
\hline
\end{tabular}

SALPLE

DATE

DATE - $-28-33$

NR

6820.06

5760.00

$07 / 29 / 87$

$10 / 29 / 87$

$67 / 29 / 87$

$16 / 29 / 87$

$67 / 28 / 87$

$16 / 29 / 87$

07/29/87

$16 / 29 / 87$

$10 / 20 / 67$

7)/29/87

$10 / 29 / 87$

23260.00

22366.00

-

41000.00

28500.06

25260.00

236000.01

-......

1296.06

NR
MR
NR

NR

NR

NR

$07 / 29 / 87$

$10 / 29 / 87$

-7/20/87

$10 / 29 / 87$

30.00

21.00

9.00
SAYPLE

DATE

$8-28-34$

$11 / 15 / 87$

$11 / 15 / 87$

-7/30/87

97/30/07
$11 / 15 / 07$

$07 / 30 / 87$

$11 / 15 / 87$

-7/39/87

$11 / 15 / 87$

-7/96/87

$7 / 86 / 87$

$11 / 16 / 87$

$11 / 15 / 97$

17/30/87

67/36/87

$07 / 36 / 87$

7) 13487

$7 / 30 / 87$
$11 / 15 / 87$

$11 / 15 / 87$

$11 / 15 / 87$

$11 / 16 / 87$

$11 / 16 / 87$

1/31/87

$11 / 15 / 87$

24.60

$--1-2-7$

$11 / 15 / 87$

6. 68

B.

5740.6

5930.00

22506

22564.60

37800.

38590.06

28106.01

25496 .61

244000.00

28.6

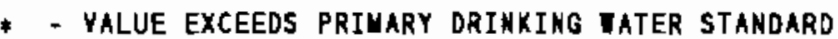

- YALUE EXCEEDS PROPOSED PRIUARY DRINKING IATER STANDARD.

- VALUE EXCEEDS SCREENING LEYEL FOR FURTHER INYESTIGATION.

- DETECTION LIUIT IAS NOT AYALABLE FOR COUPARISON

NR - ANALYSIS NOT REQUESTEO OR NOT YET REPORTED

YALUES IN \{\} ARE COUNTING ERRORS FOR RADIONUCLIDES

IATER STANDARD(S) IN PARENTHESES ARE PROPDSED ONLY 
TABLE C.5. (contd)

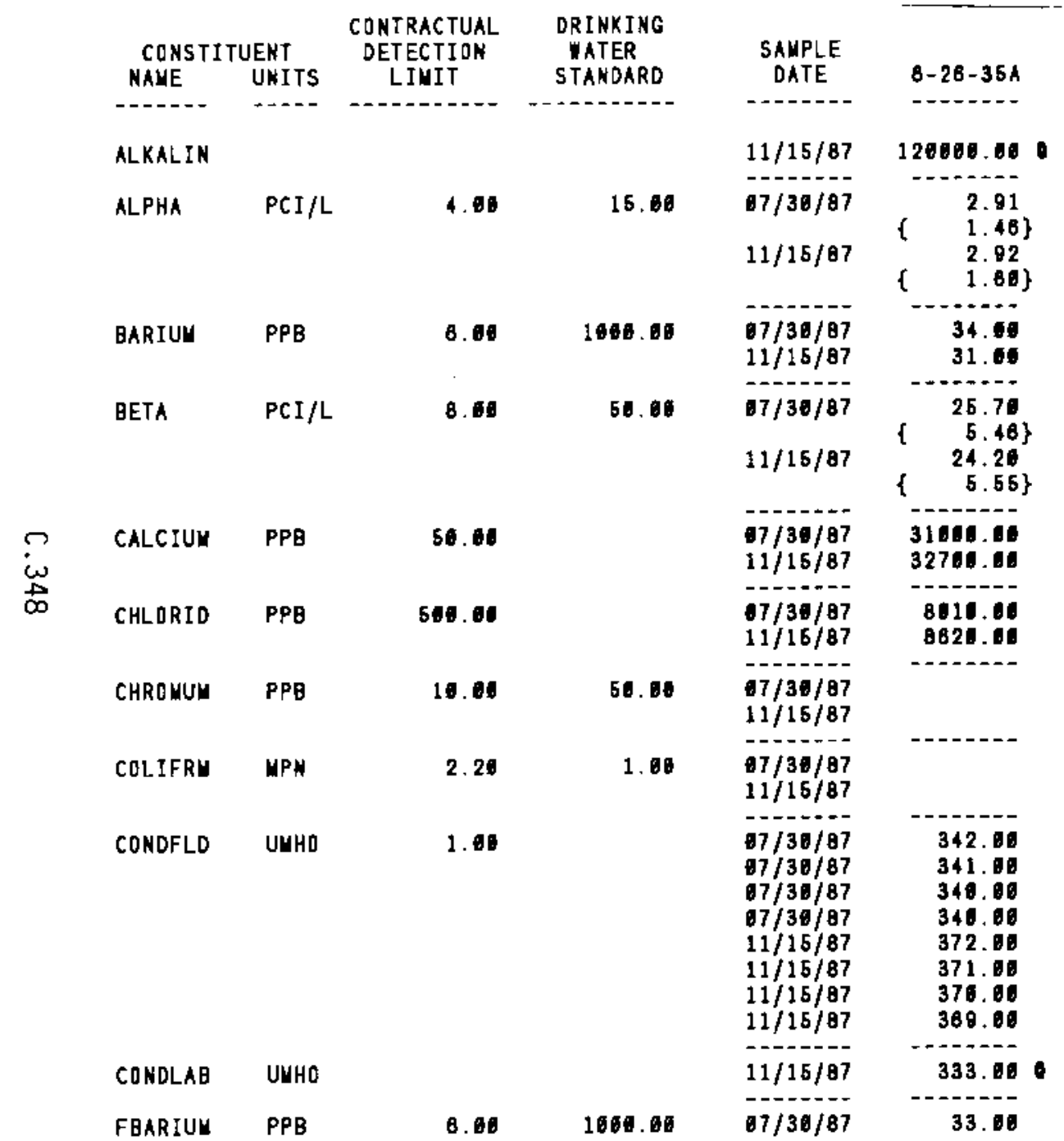

\begin{tabular}{|c|c|c|c|}
\hline $\begin{array}{l}\text { SAMPLE } \\
\text { DATE }\end{array}$ & $6-28-35 C$ & $\begin{array}{l}\text { SAUPLE } \\
\text { DATE }\end{array}$ & $8-81-68$ \\
\hline $11 / 15 / 87$ & 123060.010 & $12 / 13 / 87$ & 93200.00 \\
\hline $\begin{array}{l}17 / 30 / 87 \\
11 / 15 / 87\end{array}$ & $\left.\begin{array}{ll}\{ & 1.21 \\
\{ & 1.14\} \\
\{ & 2.54 \\
1.50\end{array}\right\}$ & $12 / 13 / 87$ & $\left\{\begin{array}{l}1.31 \\
\{.95\} \\
N R\end{array}\right.$ \\
\hline $\begin{array}{l}17 / 31 / 87 \\
11 / 15 / 87\end{array}$ & $\begin{array}{l}55.66 \\
51.66\end{array}$ & & $\begin{array}{l}\text { NR } \\
\text { NR }\end{array}$ \\
\hline $67 / 30 / 87$ & $\left\{\begin{array}{l}16.50 \\
4.75\}\end{array}\right.$ & $12 / 13 / 87$ & \\
\hline $11 / 15 / 87$ & $\begin{array}{c}23.10 \\
\quad 5.27\}\end{array}$ & & NR \\
\hline $\begin{array}{l}67 / 36 / 87 \\
11 / 15 / 87\end{array}$ & $\begin{array}{l}38560.00 \\
39660.00\end{array}$ & & $\begin{array}{l}\text { NR } \\
\text { MR }\end{array}$ \\
\hline $\begin{array}{l}07 / 31 / 87 \\
11 / 15 / 87\end{array}$ & $\begin{array}{l}9490.11 \\
8926.01\end{array}$ & $12 / 13 / 87$ & $\underset{* R}{1276.66}$ \\
\hline $\begin{array}{l}67 / 38 / 87 \\
11 / 15 / 87\end{array}$ & 10.01 & & $\begin{array}{l}\text { NR } \\
\text { NR }\end{array}$ \\
\hline $\begin{array}{l}67 / 38 / 87 \\
11 / 15 / 87\end{array}$ & $\begin{array}{l}18.01 \% \\
18.60 *\end{array}$ & & $\begin{array}{l}\text { NR } \\
\text { NR }\end{array}$ \\
\hline $\begin{array}{l}67 / 38 / 87 \\
67 / 30 / 87 \\
67 / 30 / 87 \\
97 / 38 / 87 \\
11 / 15 / 87 \\
11 / 155 / 87 \\
11 / 158 / 87 \\
11 / 15 / 87\end{array}$ & $\begin{array}{l}359.00 \\
357.00 \\
358.00 \\
357.00 \\
424.00 \\
424.00 \\
424.00 \\
423.06\end{array}$ & $12 / 13 / 87$ & $\begin{array}{c}173.88 \\
\text { NR } \\
\text { NR } \\
\text { NR } \\
\text { KR } \\
\text { NR } \\
\text { MR } \\
\text { NR }\end{array}$ \\
\hline $11 / 15 / 87$ & 375.000 & & MR \\
\hline $87 / 38 / 87$ & 54.00 & $12 / 13 / 87$ & 17.06 \\
\hline
\end{tabular}

* - Value exceeds priuary drinking water standard.

- VALUE EXCEEDS PROPOSED PRIUARY DRINKING iater STANDARD.

* VALUE EXCEEDS SCREENING LEVEL FOR FURTHER INVESTIGATIDN

- DETECTION Linit ias NDT AVAILABLE for COUPARISON

HR - AMALYSIS NDT REQUESTED OR NOT YET REPORTED

VALUES IN $\{$ ARE COUNTING ERRORS FOR RADIOMUCLIDES

WATER STANDARD(S) IN PARENTHESES ARE PROPOSED ONLY 
TABLE C.5. (contd)

\begin{tabular}{|c|c|c|c|c|c|}
\hline \multicolumn{2}{|c|}{ CONSTI TUENT } & $\begin{array}{c}\text { CONTRACTUAL } \\
\text { DETECTION } \\
\text { LIUIT }\end{array}$ & $\begin{array}{l}\text { DRINKING } \\
\text { NATER } \\
\text { STANDARD }\end{array}$ & $\begin{array}{c}\text { SAHPLE } \\
\text { DATE }\end{array}$ & $6-26-35 A$ \\
\hline FBARIUU & PPB & 8.08 & 1008.80 & $11 / 15 / 87$ & 35.00 \\
\hline FCALCIU & PPB & 68.00 & & $\begin{array}{l}07 / 30 / 87 \\
11 / 15 / 87\end{array}$ & $\begin{array}{l}30960.06 \\
31780.66\end{array}$ \\
\hline FCHROUI & $\mathrm{PPB}$ & 10.06 & 68.00 & $\begin{array}{l}67 / 39 / 87 \\
11 / 15 / 87\end{array}$ & \\
\hline FLUDRID & PPB & 500.06 & 4800.00 & $\begin{array}{l}97 / 30 / 87 \\
11 / 16 / 87\end{array}$ & $\begin{array}{l}674.00 \\
646.06\end{array}$ \\
\hline FUAGMES & PPB & 0.00 & & $\begin{array}{l}17 / 36 / 87 \\
11 / 16 / 87\end{array}$ & $\begin{array}{l}10000.00 \\
10900.00\end{array}$ \\
\hline FUANGAN & PPB & 5.00 & & $\begin{array}{l}17 / 36 / 87 \\
11 / 16 / 87\end{array}$ & \\
\hline FPDTASS & PPQ & 108.08 & & $\begin{array}{l}07 / 38 / 87 \\
11 / 16 / 87\end{array}$ & $\begin{array}{l}5984.08 \\
6451.09\end{array}$ \\
\hline FSDOIUU & PPB & 100.00 & & $\begin{array}{l}07 / 38 / 87 \\
11 / 15 / 87\end{array}$ & $\begin{array}{l}22860.00 \\
23100.00\end{array}$ \\
\hline FVANADI & PPB & 6.00 & & $\begin{array}{l}07 / 38 / 87 \\
11 / 15 / 87\end{array}$ & $\begin{array}{l}23.60 \\
27.60\end{array}$ \\
\hline IRON & PPB & 56.00 & & $\begin{array}{l}07 / 38 / 87 \\
11 / 15 / 87\end{array}$ & $\begin{array}{l}245.00 \\
496.00\end{array}$ \\
\hline LFLUORD & $\mathrm{PPB}$ & 20.00 & 4600.08 & $11 / 15 / 87$ & 590.00 \\
\hline MAGNES & PPB & 0.00 & & $\begin{array}{l}17 / 38 / 87 \\
11 / 15 / 87\end{array}$ & $\begin{array}{l}10500.60 \\
10900.00\end{array}$ \\
\hline WANGESE & PPB & 5.00 & & $\begin{array}{l}07 / 38 / 87 \\
11 / 15 / 87\end{array}$ & 5.60 \\
\hline WETHYCH & PPB & 16.66 & & $\begin{array}{l}07 / 30 / 87 \\
11 / 15 / 87\end{array}$ & \\
\hline
\end{tabular}

\begin{tabular}{|c|c|}
\hline $\begin{array}{l}\text { SAUPLE } \\
\text { DATE }\end{array}$ & $8-26-35 C$ \\
\hline $11 / 15 / 87$ & \\
\hline $\begin{array}{l}97 / 36 / 87 \\
11 / 15 / 87\end{array}$ & 37006.00 \\
\hline $\begin{array}{l}97 / 36 / 87 \\
11 / 16 / 87\end{array}$ & \\
\hline $\begin{array}{l}67 / 39 / 87 \\
11 / 15 / 87\end{array}$ & 655.66 \\
\hline $\begin{array}{l}07 / 31 / 87 \\
11 / 15 / 87\end{array}$ & 11980.66 \\
\hline $\begin{array}{l}07 / 30 / 87 \\
11 / 15 / 97\end{array}$ & 101.60 \\
\hline $\begin{array}{l}7 / 31 / 87 \\
11 / 15 / 87\end{array}$ & 5941.06 \\
\hline $\begin{array}{l}07 / 30 / 87 \\
11 / 15 / 87\end{array}$ & 19960.60 \\
\hline $\begin{array}{l}67 / 30 / 97 \\
11 / 15 / 87\end{array}$ & 7.60 \\
\hline $\begin{array}{l}07 / 30 / 87 \\
11 / 15 / 87\end{array}$ & \\
\hline $11 / 15 / 87$ & 426.00 \\
\hline $\begin{array}{l}07 / 30 / 87 \\
11 / 15 / 87\end{array}$ & $\begin{array}{l}12068.00 \\
12306.01\end{array}$ \\
\hline $\begin{array}{l}07 / 36 / 87 \\
11 / 15 / 87\end{array}$ & $\begin{array}{l}103.00 \\
104.00\end{array}$ \\
\hline $\begin{array}{l}07 / 36 / 87 \\
11 / 15 / 87\end{array}$ & 360.11 \\
\hline
\end{tabular}

\begin{tabular}{|c|c|}
\hline $\begin{array}{l}\text { SAMPLE } \\
\text { DATE }\end{array}$ & $8-81-68$ \\
\hline & NR \\
\hline $12 / 13 / 87$ & $3046 \mathrm{HR}_{\mathrm{KR}}$ \\
\hline $12 / 13 / 87$ & ${ }_{\mathrm{NR}}^{17.110}$ \\
\hline $12 / 13 / 87$ & \\
\hline $12 / 13 / 87$ & O035. 10 \\
\hline $12 / 13 / 87$ & \\
\hline $12 / 13 / 07$ & $\begin{array}{c}2368.66 \\
\text { MR }\end{array}$ \\
\hline $12 / 13 / 87$ & ${ }_{\mathrm{NR}}^{400}$ \\
\hline $12 / 13 / 87$ & NR \\
\hline & $\begin{array}{l}\mathrm{NR} \\
\mathrm{kR}\end{array}$ \\
\hline $12 / 13 / 87$ & 186.06 \\
\hline & $\begin{array}{l}\text { MR } \\
\text { MR }\end{array}$ \\
\hline & $\begin{array}{l}\text { NR } \\
\text { NR }\end{array}$ \\
\hline $12 / 13 / 87$ & vo \\
\hline
\end{tabular}

* - Value exceEds privary dRINking tater standard.

1 - VALUE EXCEEDS PROPOSED PRIMARY DRINKING IATER STAMDARD.

+ - VALUE EXCEEDS SCREENING LEVEL FOR FURTHER INYESTIGATION.

- detection linit tas not available for cDiparison

NR - AMALYSIS NOT REQUESTED OR NOT YET REPORTED

VALUES IN \{\} ARE CDUNTINO ERRORS FOR RADIONUCLIDES

IATER STANDARD(S) IN PARENTHESES ARE PROPOSED ONLY 
TABLE C.5. (contd)

\begin{tabular}{|c|c|c|c|c|c|}
\hline $\begin{array}{c}\text { CONST: } \\
\text { NAHE }\end{array}$ & $\begin{array}{l}\text { UENT } \\
\text { UNITS }\end{array}$ & $\begin{array}{c}\text { CONTRACTUAL } \\
\text { DETECTION } \\
\text { LIMIT }\end{array}$ & $\begin{array}{l}\text { DRINKING } \\
\text { IATER } \\
\text { STANDARO }\end{array}$ & $\begin{array}{c}\text { SAYPLE } \\
\text { DATE }\end{array}$ & $B-28-35 A$ \\
\hline NITRATE & $\mathrm{PPB}$ & 500.00 & 45000.00 & $\begin{array}{l}67 / 30 / 87 \\
11 / 15 / 87\end{array}$ & $\begin{array}{l}22800.01 \\
28500.00\end{array}$ \\
\hline PH-LAB & & 0.01 & & $11 / 15 / 87$ & 7.97 \\
\hline PHF IELD & & (1) & & $\begin{array}{l}67 / 38 / 87 \\
67 / 38 / 87 \\
67 / 38 / 87 \\
67 / 90 / 87 \\
11 / 15 / 87 \\
11 / 15 / 87 \\
11 / 15 / 87 \\
11 / 15 / 87\end{array}$ & $\begin{array}{l}7.40 \\
7.50 \\
7.58 \\
7.58 \\
8.98 \\
6.80 \\
6.88 \\
6.80\end{array}$ \\
\hline POTASUY & PPB & 190.00 & & $\begin{array}{l}97 / 96 / 87 \\
11 / 16 / 87\end{array}$ & $\begin{array}{l}5940.86 \\
8108.06\end{array}$ \\
\hline SODIUU & PPB & 100.06 & & $\begin{array}{l}07 / 36 / 87 \\
11 / 15 / 87\end{array}$ & $\begin{array}{l}22100.606 \\
22300.66\end{array}$ \\
\hline SULFATE & PPB & 608.00 & & $\begin{array}{l}17 / 30 / 87 \\
11 / 16 / 87\end{array}$ & $\begin{array}{l}42100.60 \\
43000.60\end{array}$ \\
\hline TC & PPB & & & $\begin{array}{l}1 / 90 / 87 \\
11 / 16 / 87\end{array}$ & $\begin{array}{l}27908.00 \\
28596.00\end{array}$ \\
\hline TOS & & & & $11 / 15 / 87$ & 256000.00 \\
\hline TOC & PPB & 1090.00 & & $\begin{array}{l}67 / 38 / 87 \\
67 / 30 / 87 \\
67 / 30 / 87 \\
07 / 30 / 87 \\
11 / 15 / 87 \\
11 / 16 / 87 \\
11 / 16 / 87 \\
11 / 15 / 87\end{array}$ & 3736.68 \\
\hline TOX & PPB & 100.00 & & $67 / 30 / 87$ & \\
\hline \multicolumn{6}{|c|}{ 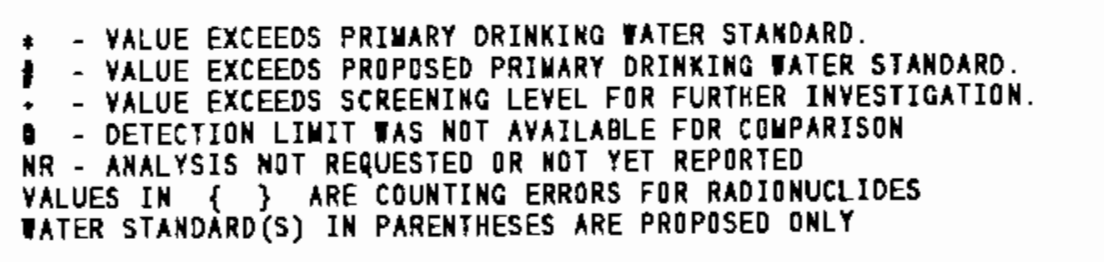 } \\
\hline
\end{tabular}

\begin{tabular}{|c|c|c|c|}
\hline $\begin{array}{l}\text { SAHPLE } \\
\text { DATE }\end{array}$ & $8-26-35 C$ & $\begin{array}{l}\text { SAUPLE } \\
\text { DATE }\end{array}$ & $8-81-58$ \\
\hline $\begin{array}{l}07 / 36 / 87 \\
11 / 15 / 87\end{array}$ & $\begin{array}{l}19400.06 \\
21860.06\end{array}$ & $12 / 13 / 87$ & $\underset{\mathrm{NR}}{2106}$ \\
\hline $11 / 15 / 87$ & 8.98 & $12 / 13 / 87$ & 8.05 \\
\hline $\begin{array}{l}17 / 31 / 87 \\
17 / 30 / 87 \\
97 / 30 / 87 \\
07 / 30 / 87 \\
11 / 15 / 87 \\
11 / 15 / 87 \\
11 / 15 / 87 \\
11 / 15 / 87\end{array}$ & $\begin{array}{l}7.10 \\
7.06 \\
7.16 \\
7.16 \\
4.86 \\
4.86 \\
4.56 \\
4.46\end{array}$ & $12 / 13 / 87$ & $\begin{array}{l}7.60 \\
\text { NR } \\
\text { NR } \\
\text { NR } \\
\text { NR } \\
\text { NR } \\
\text { NR } \\
\text { NR }\end{array}$ \\
\hline $\begin{array}{l}07 / 30 / 87 \\
11 / 16 / 87\end{array}$ & $\begin{array}{l}5810.00 \\
0250.06\end{array}$ & & $\begin{array}{l}\text { NR } \\
\text { NR }\end{array}$ \\
\hline $\begin{array}{l}17 / 38 / 87 \\
11 / 16 / 87\end{array}$ & $\begin{array}{l}19700.66 \\
21200.86\end{array}$ & & $\begin{array}{l}\text { NR } \\
\text { NR }\end{array}$ \\
\hline $\begin{array}{l}17 / 36 / 87 \\
11 / 15 / 87\end{array}$ & $\begin{array}{l}74400.00 \\
69800.00\end{array}$ & $12 / 13 / 87$ & 15300.60 \\
\hline $\begin{array}{l}67 / 30 / 87 \\
11 / 16 / 87\end{array}$ & 28500.608 & $12 / 13 / 87$ & 22006.00 \\
\hline $11 / 15 / 87$ & 273000.000 & & NR \\
\hline $\begin{array}{l}67 / 30 / 87 \\
67 / 30 / 87 \\
97 / 39 / 87 \\
67 / 39 / 87 \\
11 / 15 / 87 \\
11 / 15 / 87 \\
11 / 15 / 87\end{array}$ & NR & $12 / 13 / 87$ & $\begin{array}{l}\text { NR } \\
\text { NR } \\
\text { NR } \\
\text { NR } \\
\text { NR } \\
\text { NR } \\
\text { RR }\end{array}$ \\
\hline & & & MR \\
\hline
\end{tabular}

- yalUe EXCEEDS PRIYary DRINKIKG yater STAKDaro.

TOT AYATLABLE FDR COUPARISOH

VALUES IN \{ $\}$ ARE COUNTING ERRORS FOR RADIONUCLIDES

MATER STANDARD (S) IN PARENTHESES ARE PROPOSED ONLY 
TABLE C.5. (contd)

\begin{tabular}{|c|c|c|c|c|c|c|c|c|c|}
\hline $\begin{array}{l}\text { CONSTI } \\
\text { NAUE }\end{array}$ & $\begin{array}{l}\text { UENT } \\
\text { UNITS }\end{array}$ & $\begin{array}{c}\text { CONTRACTUAL } \\
\text { DETECTION } \\
\text { LINIT }\end{array}$ & $\begin{array}{l}\text { DRINKING } \\
\text { VATER } \\
\text { STANDARD }\end{array}$ & $\begin{array}{c}\text { SAMPLE } \\
\text { DATE }\end{array}$ & $6-26-35 A$ & $\begin{array}{c}\text { SAUPLE } \\
\text { DATE }\end{array}$ & $6-26-35 C$ & $\begin{array}{c}\text { SAUPLE } \\
\text { DATE }\end{array}$ & $6-81-58$ \\
\hline$\cdots+\cdots$ & $\ldots$ & - & 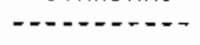 & ----- & $\ldots+\cdots--$ & -----1 & $---1+\cdots$ & $-\cdots+-1$ & --1- \\
\hline$T 0 X$ & PPB & 100.00 & & $\begin{array}{l}67 / 38 / 87 \\
67 / 36 / 87 \\
67 / 36 / 87\end{array}$ & & $\begin{array}{l}67 / 30 / 87 \\
67 / 30 / 87 \\
67 / 30 / 87\end{array}$ & $\begin{array}{l}236.00 \\
230.00 \\
228.00\end{array}$ & & $\begin{array}{l}\text { NR } \\
\text { NR } \\
\text { NR }\end{array}$ \\
\hline VANADUL & PPQ & 5.00 & & $\begin{array}{l}97 / 30 / 87 \\
11 / 16 / 87\end{array}$ & 23.60 & $\begin{array}{l}67 / 38 / 87 \\
11 / 15 / 87\end{array}$ & $\begin{array}{r}9.00 \\
8.00\end{array}$ & 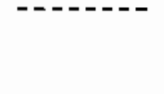 & NR \\
\hline ZINC & PPE & 5.60 & & $\begin{array}{l}67 / 36 / 87 \\
11 / 15 / 87\end{array}$ & $\begin{array}{r}6.00 \\
12.00\end{array}$ & $\begin{array}{l}67 / 30 / 87 \\
11 / 15 / 87\end{array}$ & 26.00 & 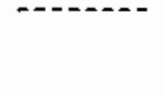 & NR \\
\hline
\end{tabular}

$\stackrel{\varrho}{\omega}$

* - VALUE EXCEEDS PRIMARY DRINKING MATER STANDARD.

| - VALUE EXCEEDS PROPOSED PRIMARY DRINKING TATER STANDARO.

+ - VALUE EXCEEDS SCREENING LEVEL FOR FURTHER INYESTIGATIDN.

- DETECTION LIUIT UAS NOT AVAILABLE FOR COUPARISON

NR - ANALYSIS NOT REQUESTED OR NOT YET REPORTED

VALUES IN \{ ? ARE COUNTING ERRORS FOR RADIONUCLIDES

WATER STANDARD(S) IN PARENTHESES ARE PROPOSED ONLY 
IABLE C.5. (contd)

\begin{tabular}{|c|c|c|c|c|c|}
\hline $\begin{array}{l}\text { CONST } \\
\text { NAUE }\end{array}$ & $\begin{array}{l}\text { UENT } \\
\text { UNITS }\end{array}$ & $\begin{array}{c}\text { CONTRACTUAL } \\
\text { DEETECTION } \\
\text { LIMIT }\end{array}$ & $\begin{array}{l}\text { DRINK ING } \\
\text { TATER } \\
\text { STAKOARD }\end{array}$ & $\begin{array}{c}\text { SALPLE } \\
\text { DATE }\end{array}$ & $6-S 19-E 13$ \\
\hline ALPHA & $\mathrm{PCI} / \mathrm{L}$ & 4.60 & 15.90 & $\begin{array}{l}68 / 27 / 87 \\
11 / 19 / 87\end{array}$ & $\begin{array}{ll} & 2.46 \\
\{ & 1.45\} \\
\{ & 2.34 \\
\{ & 1.72\}\end{array}$ \\
\hline BARIUU & PPB & 8.00 & 1080.00 & $\begin{array}{l}0 B / 27 / 87 \\
11 / 18 / 87\end{array}$ & $\begin{array}{l}52.66 \\
54.68\end{array}$ \\
\hline BETA & $\mathrm{PCI} / \mathrm{L}$ & 8.00 & 58.60 & $\begin{array}{l}18 / 27 / 87 \\
11 / 19 / 87\end{array}$ & 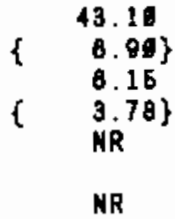 \\
\hline CALCIUH & PPB & 50.80 & & $\begin{array}{l}08 / 27 / 87 \\
11 / 19 / 87\end{array}$ & $\begin{array}{l}37206.60 \\
82085.80\end{array}$ \\
\hline CHLORID & PPB & 500.00 & & $\begin{array}{l}68 / 27 / 87 \\
11 / 19 / 87\end{array}$ & $\begin{array}{r}18800.04 \\
5250.00\end{array}$ \\
\hline CONDFLO & UMHO & 1.06 & & $\begin{array}{l}68 / 27 / 87 \\
11 / 19 / 87\end{array}$ & $\begin{array}{l}291.06 \\
465.06\end{array}$ \\
\hline$C S+137$ & $P C I / L$ & 28.08 & 200.60 & $\begin{array}{l}87 / 99 / 87 \\
68 / 27 / 87 \\
18 / 21 / 87\end{array}$ & $\begin{array}{l}4.51 \\
\{\quad 3.68\}\end{array}$ \\
\hline FARSENI & PPB & 5.00 & 58.00 & $\begin{array}{l}68 / 27 / 87 \\
11 / 19 / 87\end{array}$ & B. 10 \\
\hline FBARIUU & PPB & B.66 & 1006.60 & $\begin{array}{l}08 / 27 / 87 \\
11 / 19 / 87\end{array}$ & $\begin{array}{l}49.00 \\
58.00\end{array}$ \\
\hline FCALCIU & PPB & 50.08 & & $88 / 27 / 87$ & 36864.08 \\
\hline
\end{tabular}

\begin{tabular}{|c|c|}
\hline $\begin{array}{l}\text { SAUPLE } \\
\text { DATE }\end{array}$ & $B-S 30-E 15 A$ \\
\hline $88 / 17 / 87$ & \\
\hline $11 / 26 / 87$ & $\begin{array}{l}2.83 \\
1.4 B)\end{array}$ \\
\hline $\begin{array}{l}08 / 17 / 87 \\
11 / 26 / 87\end{array}$ & $\begin{array}{l}81.80 \\
68.80\end{array}$ \\
\hline $97 / 69 / 87$ & 4.33 \\
\hline $18 / 17 / 87$ & 5.37 \\
\hline $18 / 21 / 87$ & 4.65 \\
\hline $11 / 21 / 87$ & $\begin{array}{l}1.81 \\
9.10 \\
4.88\end{array}$ \\
\hline $\begin{array}{l}8 / 17 / 87 \\
11 / 26 / 87\end{array}$ & $\begin{array}{l}52208.01 \\
67060.01\end{array}$ \\
\hline $\begin{array}{l}08 / 17 / 87 \\
11 / 20 / 87\end{array}$ & $\begin{array}{l}6851.06 \\
4848.60\end{array}$ \\
\hline $\begin{array}{l}86 / 17 / 87 \\
11 / 28 / 87\end{array}$ & $\begin{array}{l}374.60 \\
398.06\end{array}$ \\
\hline & $\begin{array}{l}\text { NR } \\
\text { NR } \\
\text { NR }\end{array}$ \\
\hline $\begin{array}{l}98 / 17 / 87 \\
11 / 26 / 87\end{array}$ & \\
\hline $\begin{array}{l}68 / 17 / 87 \\
11 / 26 / 87\end{array}$ & $\begin{array}{l}53.66 \\
58.60\end{array}$ \\
\hline $08 / 17 / 87$ & 50100.00 \\
\hline
\end{tabular}

* - value exceeds primary orinking mater standard.

* - VALUE EXCEEDS PROPOSED PRIMARY DRINKIHG MaTER STANDARD.

- - VALUE EXCEEDS SCREENING LEVEL For FURTHER INVESTIgation.

- detection limit ias not availagle for couparison

NR - ANALYSIS NDT RERUESTED OR NOT YET REPORTED

YALUES IN I ARE COUNTING ERRORS FOR RAOIONUCLIOES

TATER STANDARD(S) IN PARENTHESES ARE PROPOSED OKLY 
TABLE C.5. (contd)

\begin{tabular}{|c|c|c|c|c|c|}
\hline \multicolumn{2}{|c|}{$\begin{array}{l}\text { CONSTITUENT } \\
\text { MANE UNIST }\end{array}$} & $\begin{array}{l}\text { CONTRACTUAL } \\
\text { DETECTION } \\
\text { LIUIT }\end{array}$ & $\begin{array}{l}\text { DRINKING } \\
\text { WATER } \\
\text { STAMDARD }\end{array}$ & $\begin{array}{l}\text { SAMPLE } \\
\text { DATE }\end{array}$ & $6-S 18-E 13$ \\
\hline FCALCIU & PPB & 56.68 & & $11 / 10 / 87$ & 82000.86 \\
\hline FIRON & PPB & 50.00 & & $\begin{array}{l}88 / 27 / 87 \\
11 / 10 / 87\end{array}$ & 181.00 \\
\hline FWAGNES & PPB & 0.00 & & $\begin{array}{l}68 / 27 / 87 \\
11 / 19 / 87\end{array}$ & $\begin{array}{l}12460.09 \\
11860.08\end{array}$ \\
\hline FPOTASS & PPB & 108.06 & & $\begin{array}{l}68 / 27 / 87 \\
11 / 19 / 87\end{array}$ & $\begin{array}{l}\text { 6230. } \\
\text { 5070.4 }\end{array}$ \\
\hline FSDDIUU & PPB & 100.60 & & $\begin{array}{l}68 / 27 / 87 \\
11 / 18 / 87\end{array}$ & $\begin{array}{l}21880.60 \\
13196.60\end{array}$ \\
\hline FVAMADI & PPB & 5.80 & & $\begin{array}{l}18 / 27 / 87 \\
11 / 19 / 87\end{array}$ & $\begin{aligned} 16.08 \\
5.01\end{aligned}$ \\
\hline FZINC & PPB & 6.60 & & $\begin{array}{l}68 / 27 / 87 \\
11 / 10 / 87\end{array}$ & 192.06 \\
\hline IROM & PPB & 50.01 & & $\begin{array}{l}88 / 27 / 87 \\
11 / 10 / 87\end{array}$ & 114.00 \\
\hline MAGNES & PPB & 0.09 & & $\begin{array}{l}88 / 27 / 97 \\
11 / 19 / 97\end{array}$ & $\begin{array}{l}12600.01 \\
12001.06\end{array}$ \\
\hline WETHYCH & PPB & 18.00 & & $\begin{array}{l}18 / 27 / 87 \\
11 / 19 / 87\end{array}$ & 236.00 \\
\hline NITRATE & PPB & 608.68 & 45800.00 & $\begin{array}{l}68 / 27 / 87 \\
11 / 19 / 87\end{array}$ & $\begin{array}{l}22100.00 \\
11200.00\end{array}$ \\
\hline PHF IELD & & 0.10 & & $\begin{array}{l}08 / 27 / 87 \\
11 / 10 / 87\end{array}$ & $\begin{array}{l}7.80 \\
7.80\end{array}$ \\
\hline POTASUI & $P P B$ & 166.06 & & $\begin{array}{l}88 / 27 / 97 \\
11 / 19 / 97\end{array}$ & $\begin{array}{l}8310.80 \\
8100.80\end{array}$ \\
\hline RU-10B & $\mathrm{PCI} / \mathrm{L}$ & $172.5 \mathrm{t}$ & 38. 80 & $07 / 69 / 87$ & \\
\hline
\end{tabular}

\begin{tabular}{|c|c|}
\hline $\begin{array}{l}\text { SAMPLE } \\
\text { DATE }\end{array}$ & B-S30-E1 5A \\
\hline $11 / 20 / 87$ & 85500.00 \\
\hline $\begin{array}{l}68 / 17 / 87 \\
11 / 26 / 87\end{array}$ & \\
\hline $\begin{array}{l}08 / 17 / 87 \\
11 / 20 / 87\end{array}$ & $\begin{array}{r}11606.06 \\
12206.10\end{array}$ \\
\hline $\begin{array}{l}00 / 17 / 87 \\
11 / 26 / 87\end{array}$ & $\begin{array}{l}5721.40 \\
5931.46\end{array}$ \\
\hline $\begin{array}{l}08 / 17 / 87 \\
11 / 26 / 87\end{array}$ & $\begin{array}{l}12900.06 \\
13060.00\end{array}$ \\
\hline $\begin{array}{l}08 / 17 / 87 \\
11 / 20 / 87\end{array}$ & 5.60 \\
\hline $\begin{array}{l}98 / 17 / 87 \\
11 / 26 / 87\end{array}$ & $\begin{array}{r}61.06 \\
112.00\end{array}$ \\
\hline $\begin{array}{l}60 / 17 / 87 \\
11 / 28 / 87\end{array}$ & 61.00 \\
\hline $\begin{array}{l}08 / 17 / 87 \\
11 / 20 / 87\end{array}$ & $\begin{array}{l}11600.10 \\
11901.06\end{array}$ \\
\hline $\begin{array}{l}00 / 17 / 87 \\
11 / 29 / 87\end{array}$ & \\
\hline $\begin{array}{l}08 / 17 / 87 \\
11 / 20 / 87\end{array}$ & $\begin{array}{l}15600.60 \\
11190.00\end{array}$ \\
\hline \begin{tabular}{l}
$19 / 11 / 87$ \\
$11 / 20 / 87$ \\
\hdashline
\end{tabular} & $\begin{array}{l}7.06 \\
7.76\end{array}$ \\
\hline $\begin{array}{l}88 / 17 / 87 \\
11 / 26 / 87\end{array}$ & $\begin{array}{l}5780.80 \\
5451.06\end{array}$ \\
\hline
\end{tabular}

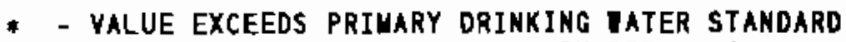

- - YALUE EXCEEDS PROPOSED PRIUARY DRINKING IATER STANDARD.

- yALUE EXCEEDS SCREENINg LEVEL FDR fURTHER INYESTIGATION.

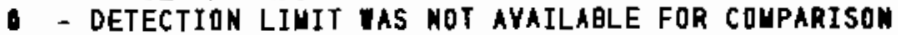

HR - AMALYSIS NOT REQUESTED OR NOT YET REPORTED

YALUES IN $($ I ARE COUNTING ERRORS FOR RADIONUCLIDES

IATER STANDARD(S) IN PARENTHESES ARE PROPOSED ONLY 
TABLE C.5. (contd)

\begin{tabular}{|c|c|c|c|c|c|c|c|}
\hline $\begin{array}{l}\text { CONSTI } \\
\text { NAME }\end{array}$ & $\begin{array}{l}\text { UENT } \\
\text { UNITS }\end{array}$ & $\begin{array}{c}\text { CONTRACTUAL } \\
\text { DETECTION } \\
\text { LIUIT }\end{array}$ & $\begin{array}{l}\text { DRINKING } \\
\text { WATER } \\
\text { STAMDARD }\end{array}$ & $\begin{array}{c}\text { SAMPLE } \\
\text { DATE }\end{array}$ & $6.519-E 13$ & $\begin{array}{c}\text { SALPLE } \\
\text { DATE }\end{array}$ & $8-538-E 15 A$ \\
\hline$R U-10 B$ & $\mathrm{PCI} / \mathrm{L}$ & 172.50 & 30.06 & $\begin{array}{l}08 / 27 / 87 \\
16 / 21 / 87\end{array}$ & $\begin{array}{l}88.70 \\
\{\quad 60.90\}\end{array}$ & & $\begin{array}{l}\text { KR } \\
\text { NR }\end{array}$ \\
\hline SODIUN & PP8 & 180.08 & & $\begin{array}{l}00 / 27 / 87 \\
11 / 10 / 87\end{array}$ & $\begin{array}{l}22360.66 \\
1280600\end{array}$ & $\begin{array}{l}68 / 17 / 87 \\
11 / 20 / 87\end{array}$ & $\begin{array}{l}12706.06 \\
10704.60\end{array}$ \\
\hline SULFATE & $P P B$ & 500.00 & & $\begin{array}{l}10 / 27 / 87 \\
11 / 19 / 87\end{array}$ & $\begin{array}{l}52400.60 \\
18660.06\end{array}$ & $\begin{array}{l}68 / 17 / 87 \\
11 / 21 / 87\end{array}$ & $\begin{array}{l}18710.00 \\
16301.06\end{array}$ \\
\hline TOC & PP8 & 1000.00 & & $\begin{array}{l}68 / 27 / 87 \\
11 / 10 / 87\end{array}$ & & $\begin{array}{l}08 / 17 / 87 \\
11 / 26 / 87\end{array}$ & 1584.64 \\
\hline TOX & PPB & 106.86 & & $\begin{array}{l}08 / 27 / 87 \\
11 / 19 / 87\end{array}$ & 135.00 & $\begin{array}{l}88 / 17 / 87 \\
11 / 20 / 87\end{array}$ & \\
\hline TRITIUN & $\mathrm{PCI} / \mathrm{L}$ & 500.00 & 20800.00 & $\begin{array}{l}07 / 60 / 87 \\
10 / 21 / 87\end{array}$ & $\begin{array}{r}6280.60 \\
2298.86\} \\
8456.06 \\
397.06\}\end{array}$ & $\begin{array}{l}07 / 60 / 87 \\
10 / 21 / 87\end{array}$ & $\left.\begin{array}{r}270.00 \\
185.06\end{array}\right\}$ \\
\hline U & $\mathrm{PCI} / \mathrm{L}$ & 1. 68 & 886.00 & $\begin{array}{l}17 / 69 / 87 \\
10 / 21 / 67\end{array}$ & $\begin{array}{l}2.85 \\
3.11\end{array}$ & $\begin{array}{l}07 / 69 / 87 \\
10 / 21 / 87\end{array}$ & $\begin{array}{l}1.44 \\
1.46\end{array}$ \\
\hline U-CHEN & UG/L & 6.73 & & $\begin{array}{l}88 / 27 / 87 \\
11 / 19 / 87\end{array}$ & $\begin{array}{l}4.73 \\
1.82\end{array}$ & $11 / 21 / 87$ & 2.13 \\
\hline VAKADUU & PPB & 5.00 & & $\begin{array}{l}69 / 27 / 87 \\
11 / 18 / 87\end{array}$ & $\begin{array}{r}14.06 \\
8.81\end{array}$ & $\begin{array}{l}68 / 17 / 87 \\
11 / 20 / 87\end{array}$ & $\begin{array}{l}5.06 \\
8.60\end{array}$ \\
\hline ZINC & PPB & 5.60 & & $\begin{array}{l}88 / 27 / 87 \\
11 / 19 / 87\end{array}$ & 51.60 & $\begin{array}{l}08 / 17 / 87 \\
11 / 20 / 87\end{array}$ & $\begin{array}{l}38.00 \\
32.00\end{array}$ \\
\hline
\end{tabular}

\footnotetext{
- - VALUE EXCEEDS PRIMARY dRINKING Mater STANDaRd.

- - VALUE EXCEEDS PROPOSED PRIUARY DRINKING VATER STAMDARD.

- - VALUE EXCEEDS SCREENING LEVEL FOR FURTHER INYESTIGATION.

- - DETECTION LIUIT WAS NOT AVAILABLE FOR COMPARISON

NR - ANALYSIS NOT RERUESTED OR NOT YET REPORTED

YALUES IN \{ \} ARE COUNTING ERRORS FDR RADIONUCLIDES

WATER STANDARD(S) IN PARENTHESES ARE PROPOSED ONLY
} 
IABLE C.6. Constituents Analyzed for in Ground-Water Samples from Site-Wide Radiological (plus Nitrate) Monitoring Wells outside the SiteWide Chemical or Compliance Monitoring Networks

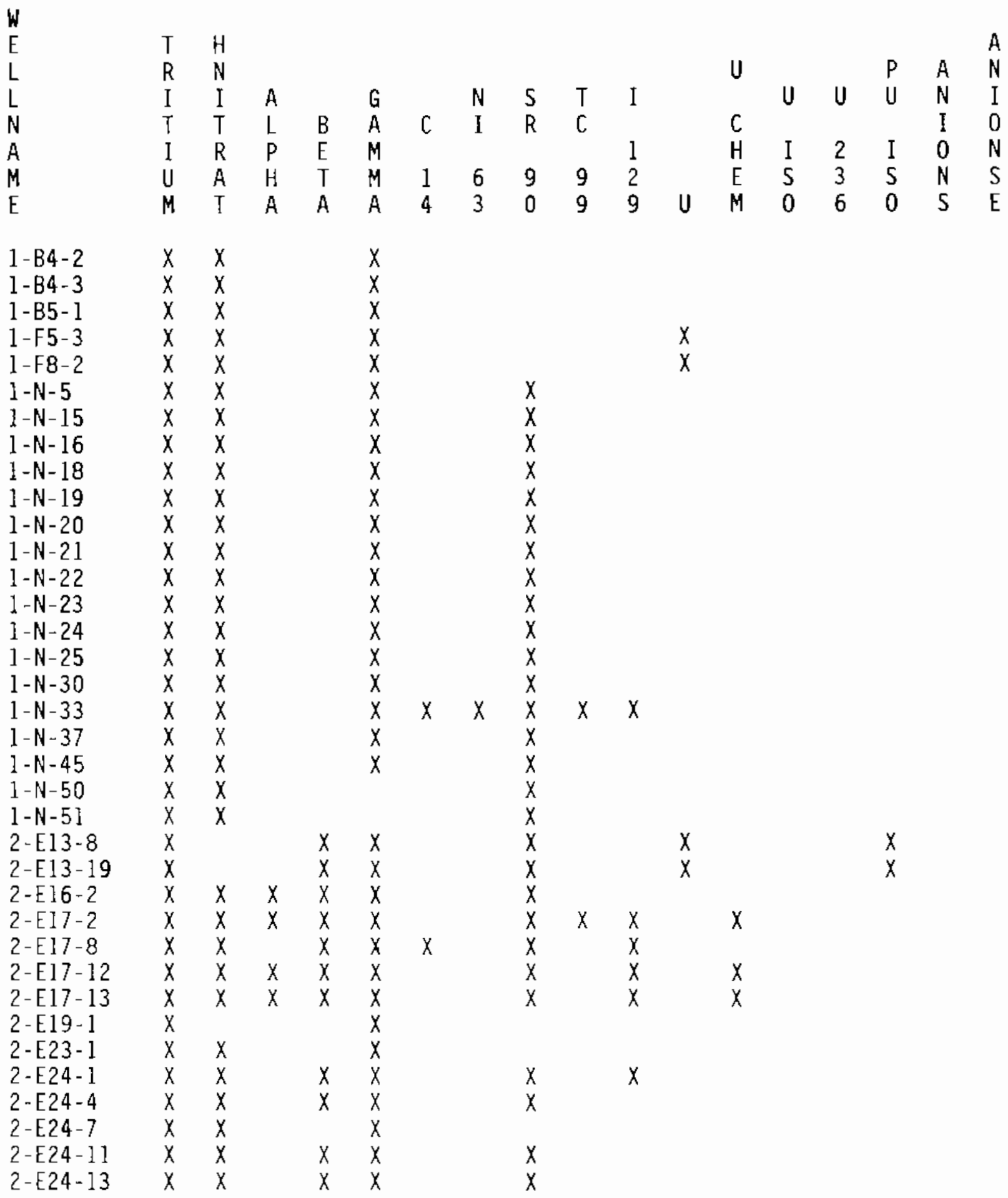


IABLE C.6. (contd)

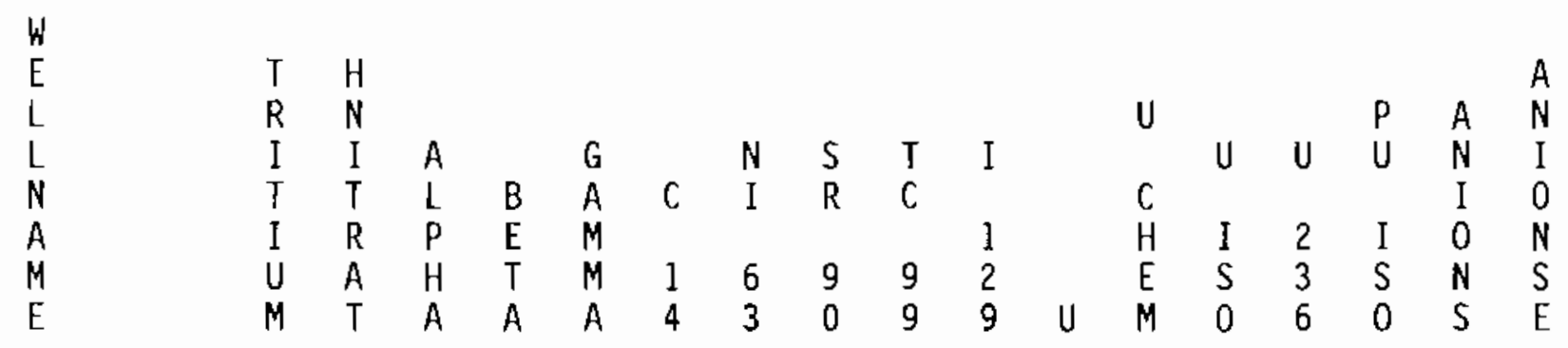

\begin{tabular}{|c|c|c|c|c|c|c|c|c|c|c|c|}
\hline $2-E 25-2$ & $x$ & $x$ & & $x$ & $x$ & $x$ & & & & & \\
\hline $2-E 25-3$ & $x$ & & & $x$ & $x$ & $x$ & & & & & \\
\hline $2-E 25-6$ & $x$ & $x$ & $x$ & $x$ & $x$ & $x$ & & & & & \\
\hline $2-E 25-9$ & $x$ & $\ddot{x}$ & $\ddot{x}$ & $x$ & $x$ & $\ddot{x}$ & & & & & \\
\hline $2-E 25-10$ & & & $\ddot{x}$ & $x$ & $x$ & & & & $x$ & & \\
\hline $\begin{array}{l}2-E 25-11 \\
2-E 25-13\end{array}$ & $x$ & $\begin{array}{l}x \\
x\end{array}$ & $x$ & $\begin{array}{l}x \\
x\end{array}$ & $x$ & $x$ & & & & & \\
\hline 2-E25-17 & $x$ & $\hat{x}$ & $x$ & $\hat{x}$ & $x$ & $x$ & & & & & \\
\hline 2-E26-1 & $x$ & & & & $x$ & & & & & & \\
\hline $2-E 26-2$ & $x$ & $x$ & & $x$ & $x$ & $x$ & & & & & \\
\hline $2-E 26-3$ & $x$ & $x$ & & & $x$ & $x$ & & & & & \\
\hline $2-E 26-4$ & $\dot{x}$ & $x$ & & $x$ & $x$ & $x$ & & & & & \\
\hline $2-E 26-6$ & $x$ & $x$ & $x$ & $x$ & $x$ & $x$ & & & & & \\
\hline $2-E 26-8$ & $x$ & $x$ & & & & & & & & & \\
\hline $2-E 27-1$ & $x$ & $x$ & & & $x$ & $x$ & & $x$ & & & $x$ \\
\hline $2-E 27-7$ & & $x$ & $x$ & $x$ & & & & & & & \\
\hline $2-E 28-1$ & $x$ & $x$ & & & $x$ & $x$ & & & & & $x$ \\
\hline $2-E 28-5$ & $x$ & $x$ & & & $x$ & & & & & & \\
\hline $2-E 28-7$ & $x$ & & $x$ & $x$ & $x$ & $x$ & & & $x$ & $x$ & $x$ \\
\hline 2-E28-9 & & & $x$ & $\ddot{x}$ & & & & & $x$ & & \\
\hline $2-E 28-12$ & $x$ & & & $x$ & $x$ & & & & & & \\
\hline $2-E 28-13$ & $x$ & & & $x$ & $x$ & & & & & & \\
\hline $2-E 28-16$ & & & $x$ & $\dot{x}$ & & & & & $x$ & & \\
\hline $2-E 28-17$ & & & $x$ & & & & & & $x$ & & \\
\hline $2-E 28-18$ & $x$ & $x$ & $x$ & $x$ & $x$ & $x$ & & & $x$ & $x$ & \\
\hline 2-E28-23 & $\hat{x}$ & $\hat{x}$ & $\hat{x}$ & $\hat{x}$ & $\hat{x}$ & $\hat{x}$ & & & $x$ & $x$ & $x$ \\
\hline $2-E 28-24$ & & & $\hat{x}$ & $x$ & $\ddot{x}$ & $\hat{x}$ & & & $\hat{x}$ & $x$ & $\mathrm{x}$ \\
\hline $2-E 28-25$ & & & $x$ & $x$ & $x$ & $\dot{x}$ & & & $x$ & $x$ & $\ddot{x}$ \\
\hline $2-E 33-7$ & $x$ & & & $x$ & $x$ & $x$ & $x$ & $x$ & & & $x$ \\
\hline $2-E 33-9$ & $x$ & $x$ & & $x$ & $x$ & $x$ & $x$ & $x$ & & & $x$ \\
\hline $2-E 33-12$ & $x$ & $x$ & & & $x$ & $x$ & & $x$ & & & $x$ \\
\hline $2-E 33-14$ & $x$ & $x$ & & & $x$ & $x$ & & $x$ & & & $x$ \\
\hline $2-E 33-20$ & $x$ & $x$ & & $x$ & $x$ & $x$ & & $x$ & & & $x$ \\
\hline $2-E 33-26$ & & & & $\ddot{x}$ & $\hat{x}$ & $\hat{x}$ & $x$ & & & & \\
\hline $2-w 6-1$ & $x$ & $x$ & & & $x$ & & & & & & \\
\hline $2-W 10-1$ & $x$ & & & $x$ & $x$ & $x$ & & $x$ & & & $x$ \\
\hline
\end{tabular}


IABLE C.6. (contd)

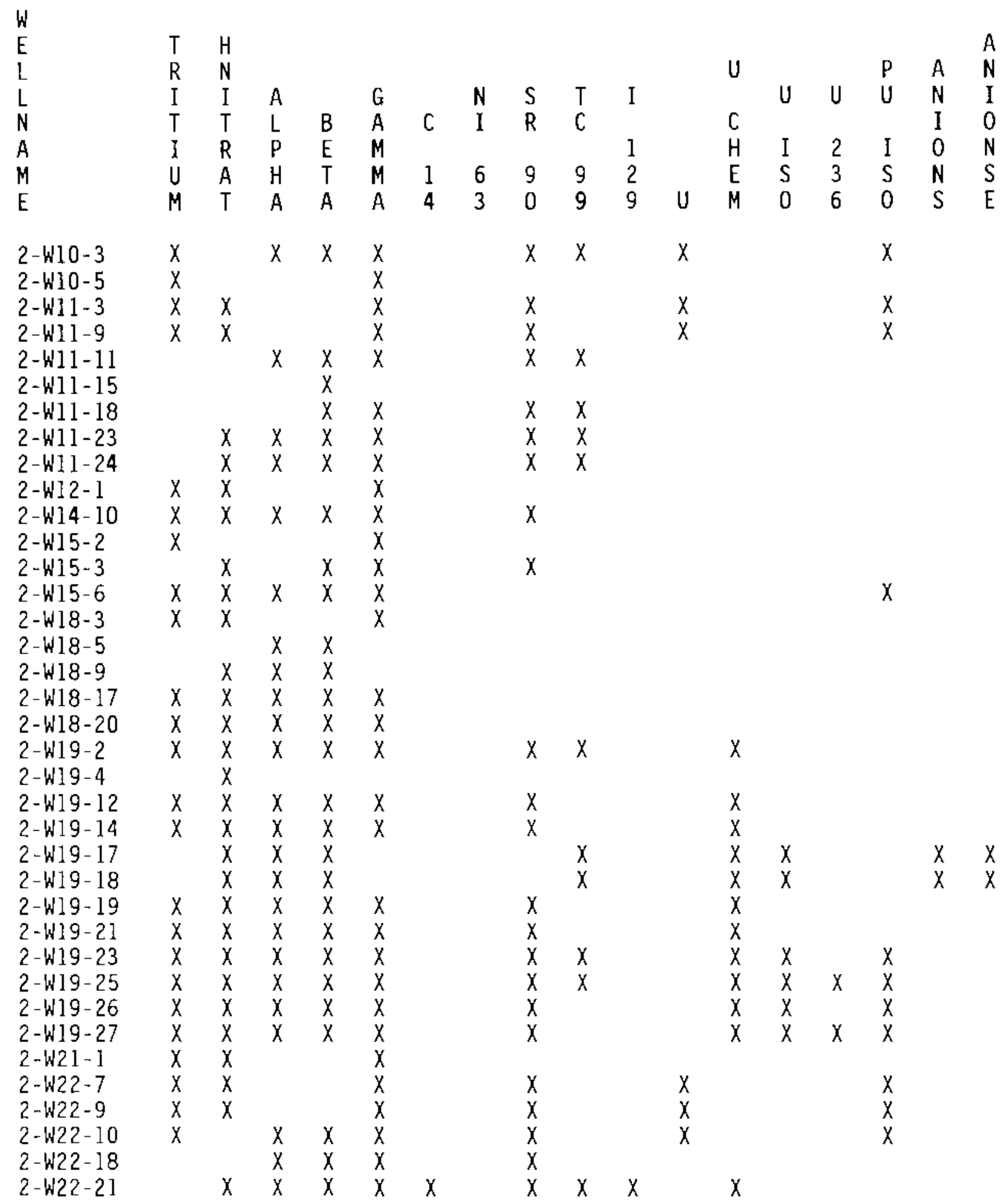


IABLE C.6. (contd)

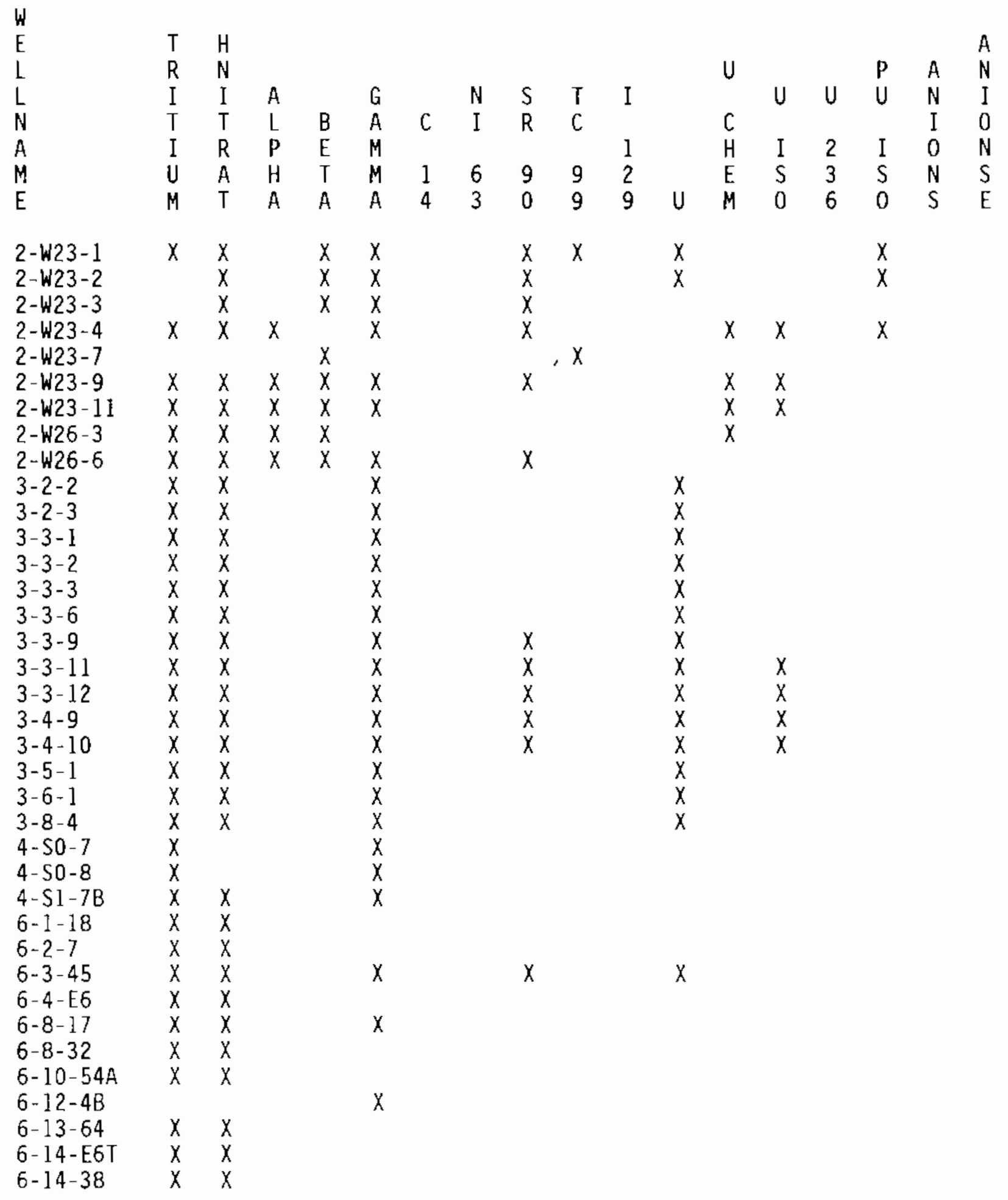


TABLE C.6. (contd)

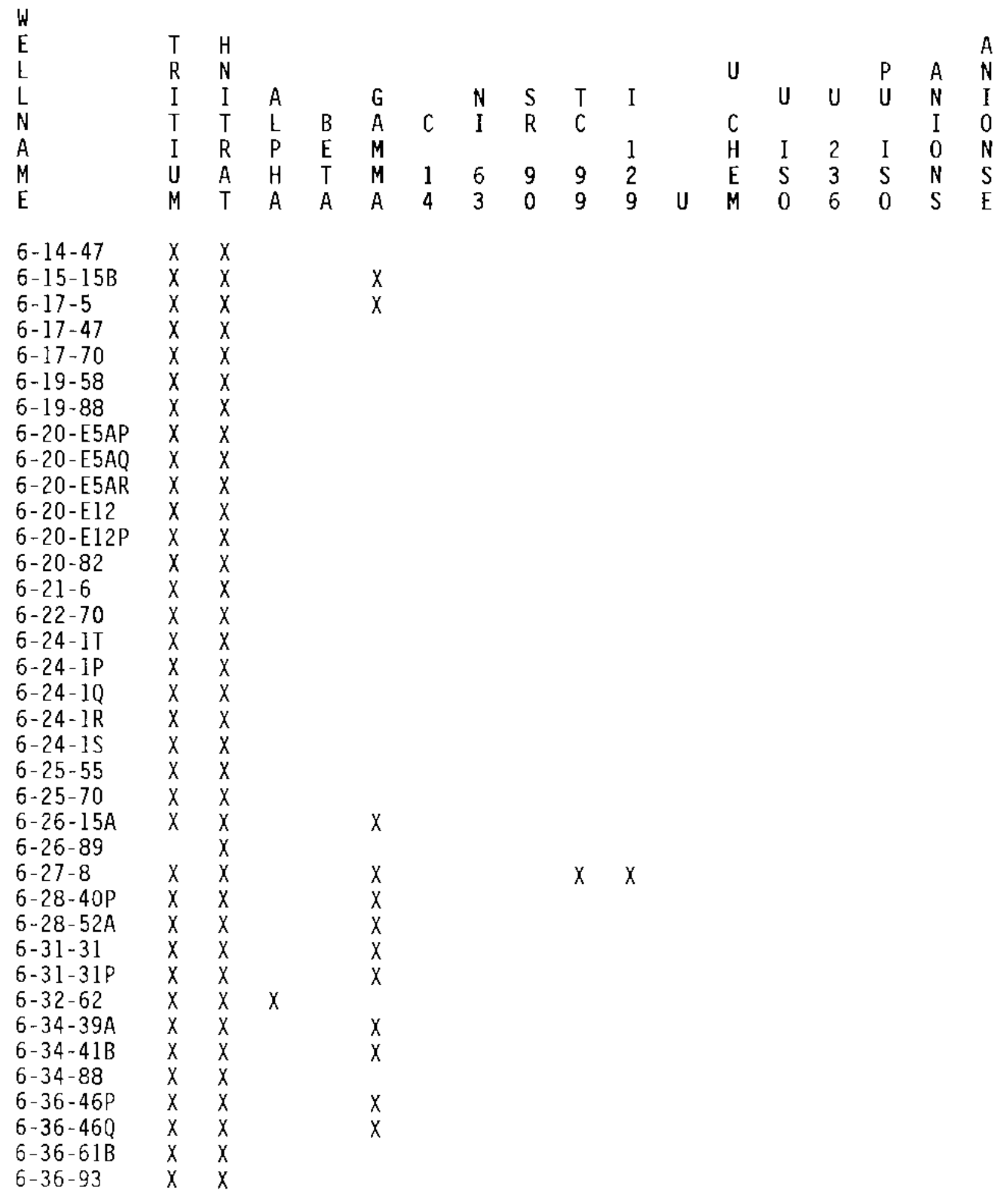


TABLE C.6. (contd)

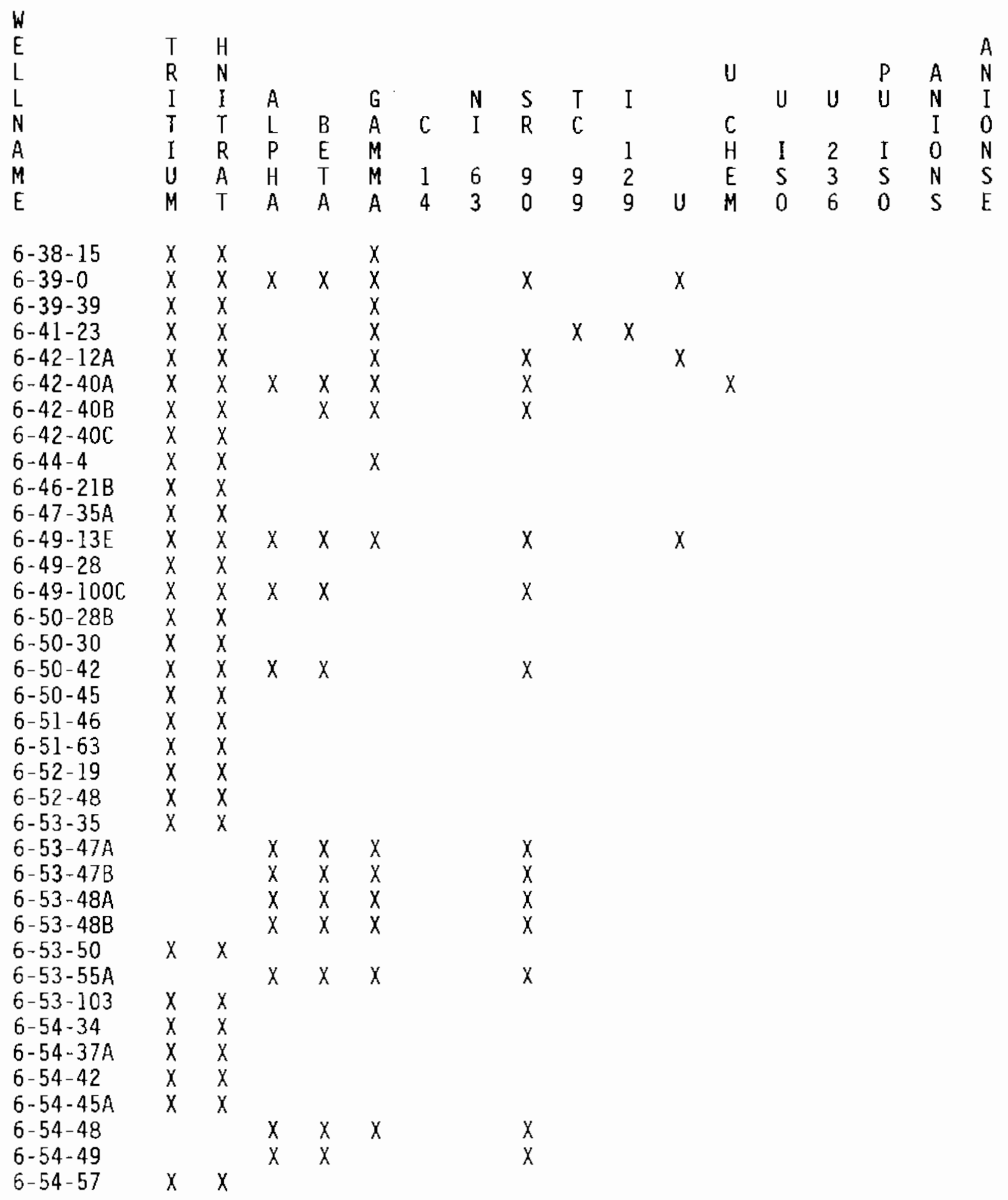


IABLE C.6. (contd)

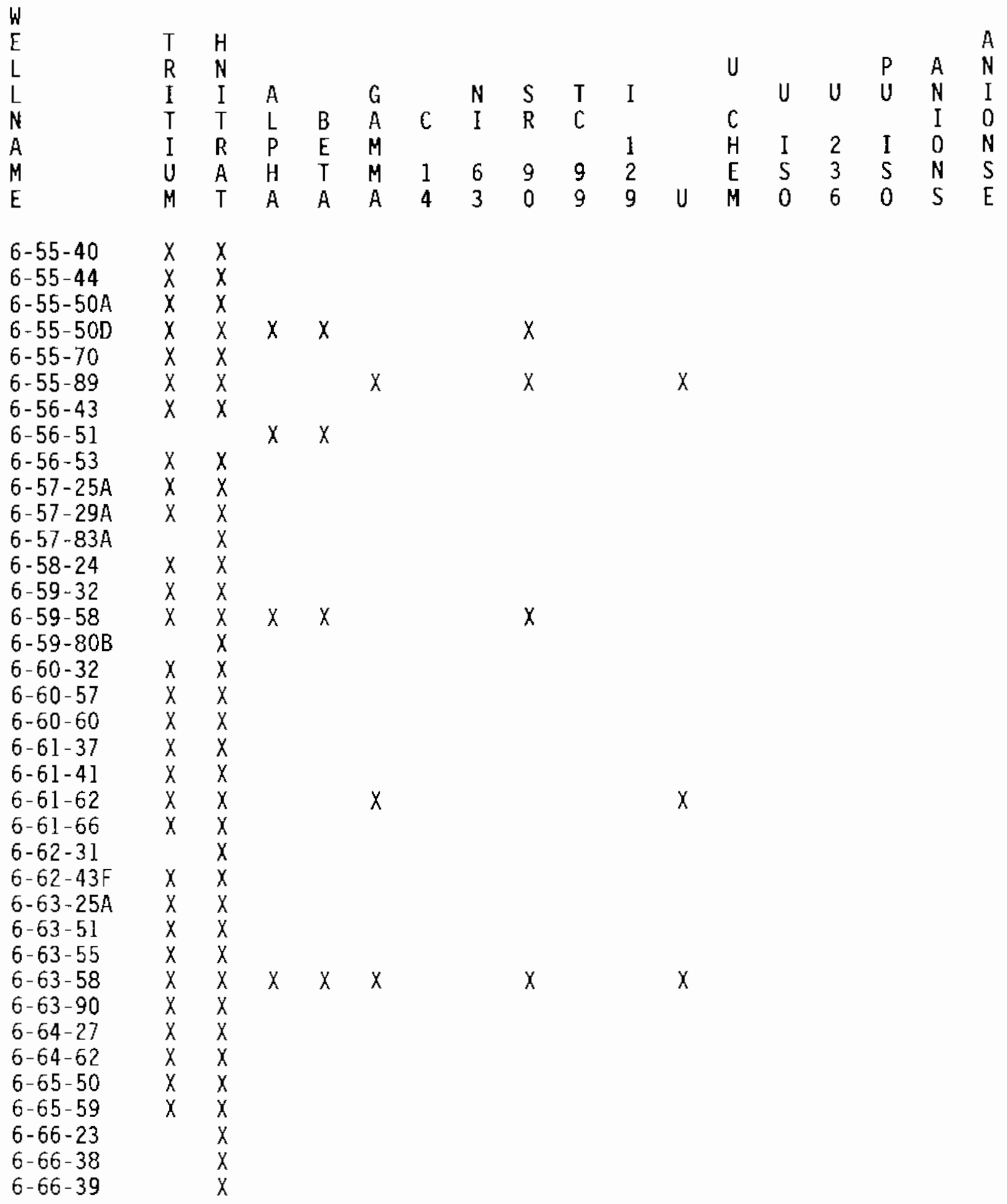


IABLE C.6. (contd)

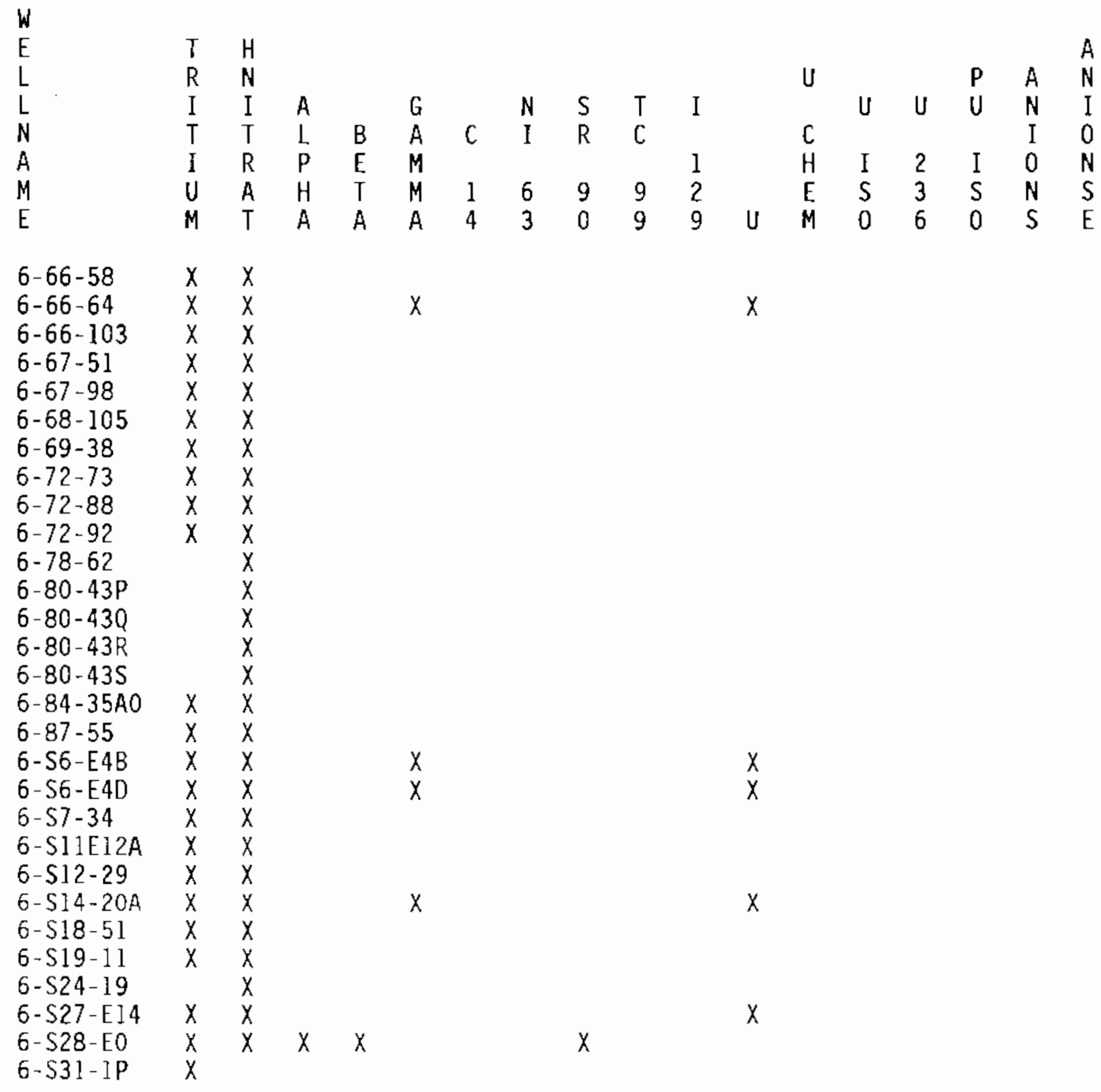


TABLE C.7. Results for Site-Wide Radiological (plus Nitrate) Monitoring Wells outside the Site-Wide Chemical or Compliance Monitoring Networks

\begin{tabular}{|c|c|c|c|c|c|}
\hline \multicolumn{2}{|c|}{ CONST I TUENT } & $\begin{array}{l}\text { CDNTRACTUAL } \\
\text { DETECTION } \\
\text { LIUIT }\end{array}$ & $\begin{array}{l}\text { DRINKING } \\
\text { SATER } \\
\text { STANDARD }\end{array}$ & $\begin{array}{l}\text { SAMPLE } \\
\text { DATE }\end{array}$ & $1-84-2$ \\
\hline---- & $-\ldots$ & - & - & $\cdots$ & $--\ldots$ \\
\hline$C O-66$ & $\mathrm{PCI} / \mathrm{L}$ & 22.50 & 160.00 & $\begin{array}{l}67 / 02 / 87 \\
10 / 19 / 87\end{array}$ & $\begin{array}{l}5.07 \\
4.54\}\end{array}$ \\
\hline HNITRAT & PPB & 2500.00 & 45000.00 & $\begin{array}{l}67 / 62 / 87 \\
10 / 19 / 87\end{array}$ & $\begin{array}{r}11200.00 \\
9590.00\end{array}$ \\
\hline TRITIUH & $\mathrm{PCI} / \mathrm{L}$ & 580.00 & 20000.00 & $107 / 62 / 87$ & $\begin{array}{r}2590.00 \\
228.00\} \\
4180.00 \\
311.08\}\end{array}$ \\
\hline
\end{tabular}

\begin{tabular}{|c|c|}
\hline $\begin{array}{c}\text { SAMPLE } \\
\text { DATE }\end{array}$ & $1-84-3$ \\
\hline - - - & ---- \\
\hline $07 / 82 / 87$ & $\begin{array}{c}12.88 \\
8.43\}\end{array}$ \\
\hline $10 / 19 / 87$ & \\
\hline $\begin{array}{l}07 / 02 / 87 \\
10 / 19 / 87\end{array}$ & $\begin{array}{l}18200.00 \\
26700.00\end{array}$ \\
\hline $7 / 62 / 87$ & $\begin{array}{c}6060.60 \\
331.60\}\end{array}$ \\
\hline $10 / 19 / 87$ & $\begin{array}{c}40700.00 \\
\{\quad 781.00\}\end{array}$ \\
\hline
\end{tabular}

SAMPLE

0. $102 / 87$

$07 / 62 / 87$

$18 / 19 / 87$

97/02/87

9470.06

$10 / 19 / 87$

1360.00

136.09
198.00

( 311.00$\}$

781.00 .

1626.00

* - yalue eXCEEDS PRIMARY dRINKINg Iater stakDaro.

- - YALUE EXCEEDS PROPOSED PRIMARY DRINKING TATER STANDARD.

$\rightarrow$ - VALUE EXCEEDS SCREENING LEVEL FOR FURTHER INYESTIGATION

- DETECTION LIUIT UAS NOT AYAILABLE FOR COMPARISOM

NR - ANALYSIS NDT REQUESTED OR NOT YET REPORTED

VALUES IN \{\} ARE COUNTING ERRORS FOR RADIONUCLIDES

WATER STANDARD(S) IN PARENTHESES ARE PROPOSED ONLY 


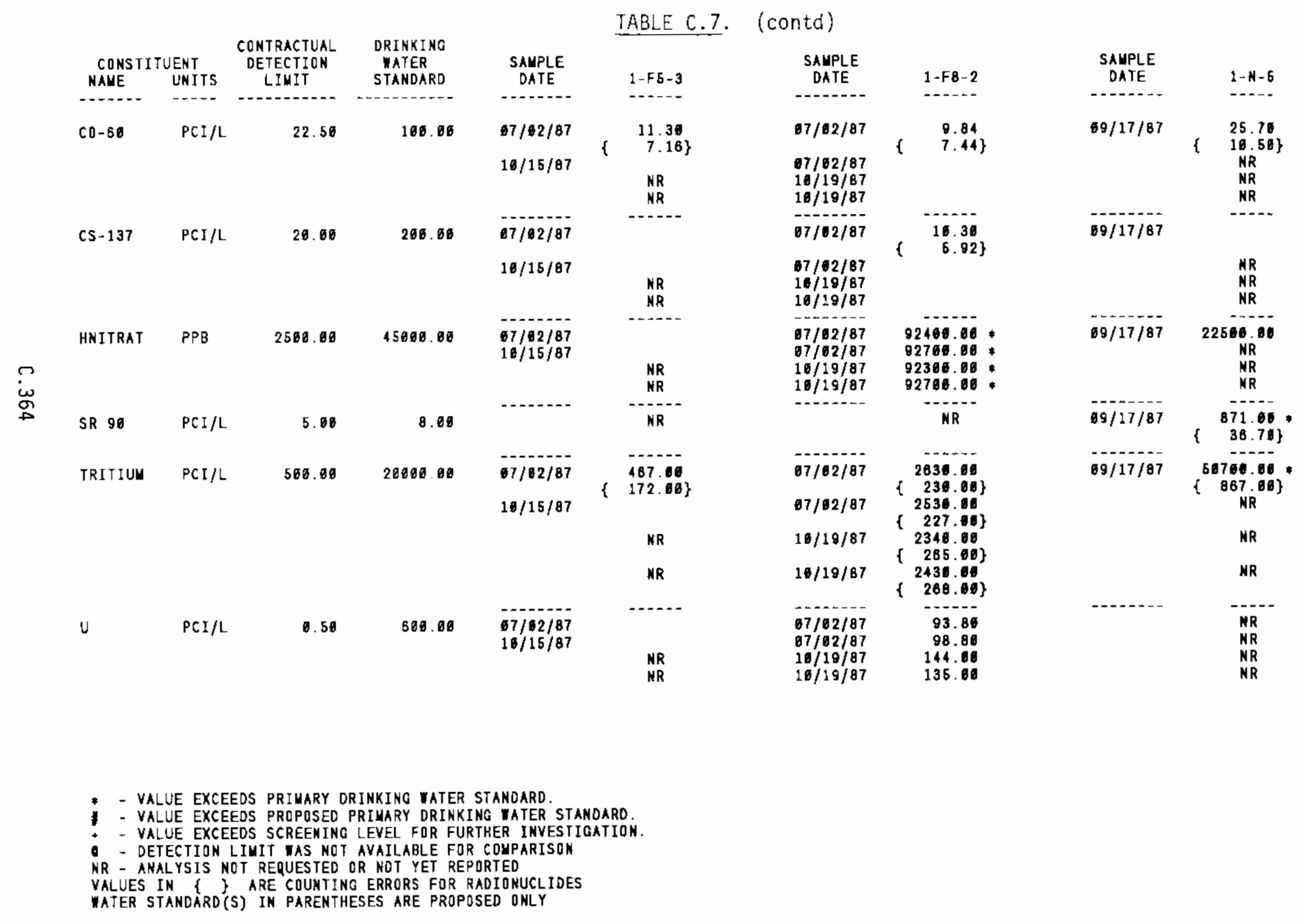


TABLE C.7. (contd)

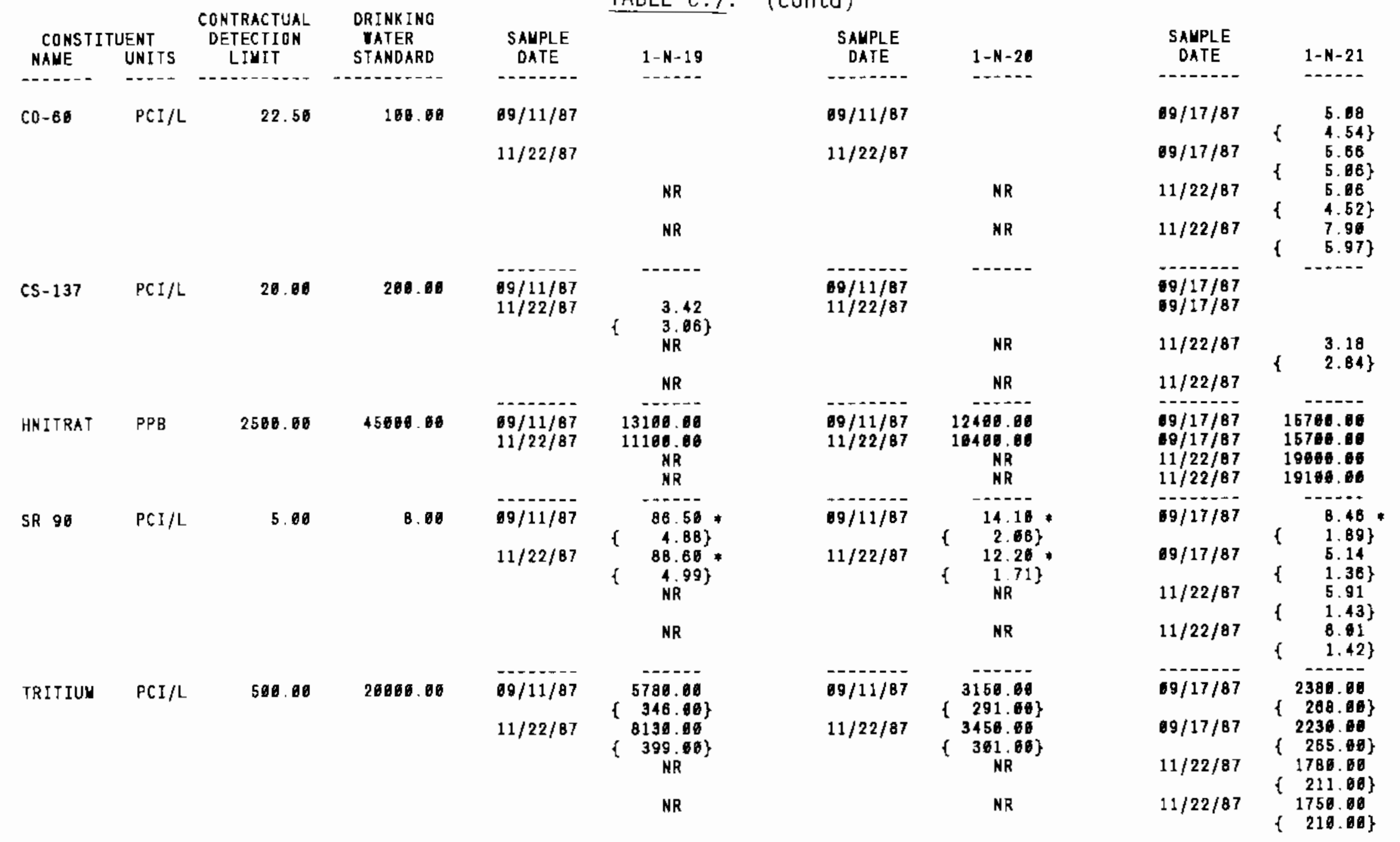

* - Value exceeds primary orinking tater stahoard.

* - VALUE EXCEEDS PROPOSED PRIMARY DRINKING WATER STANDARD.

+ - YALUE EXCEEDS SCREENING LEVEL FOR FURTHER INYESTIGATIOK.

- DETECTION LIMIT WAS NOT AVAILABLE FOR COMPARISON

NR - ANALYSIS NQT RERUESTED OR NOT YET REPORTED

YALUES IN $\{$ \& ARE CDUNTING ERRORS FOR RADIONUCLIDES

VATER STANDARD'(S) IN PARENTHESES ARE PROPOSED ONLY 
TABLE C.7. (contd)

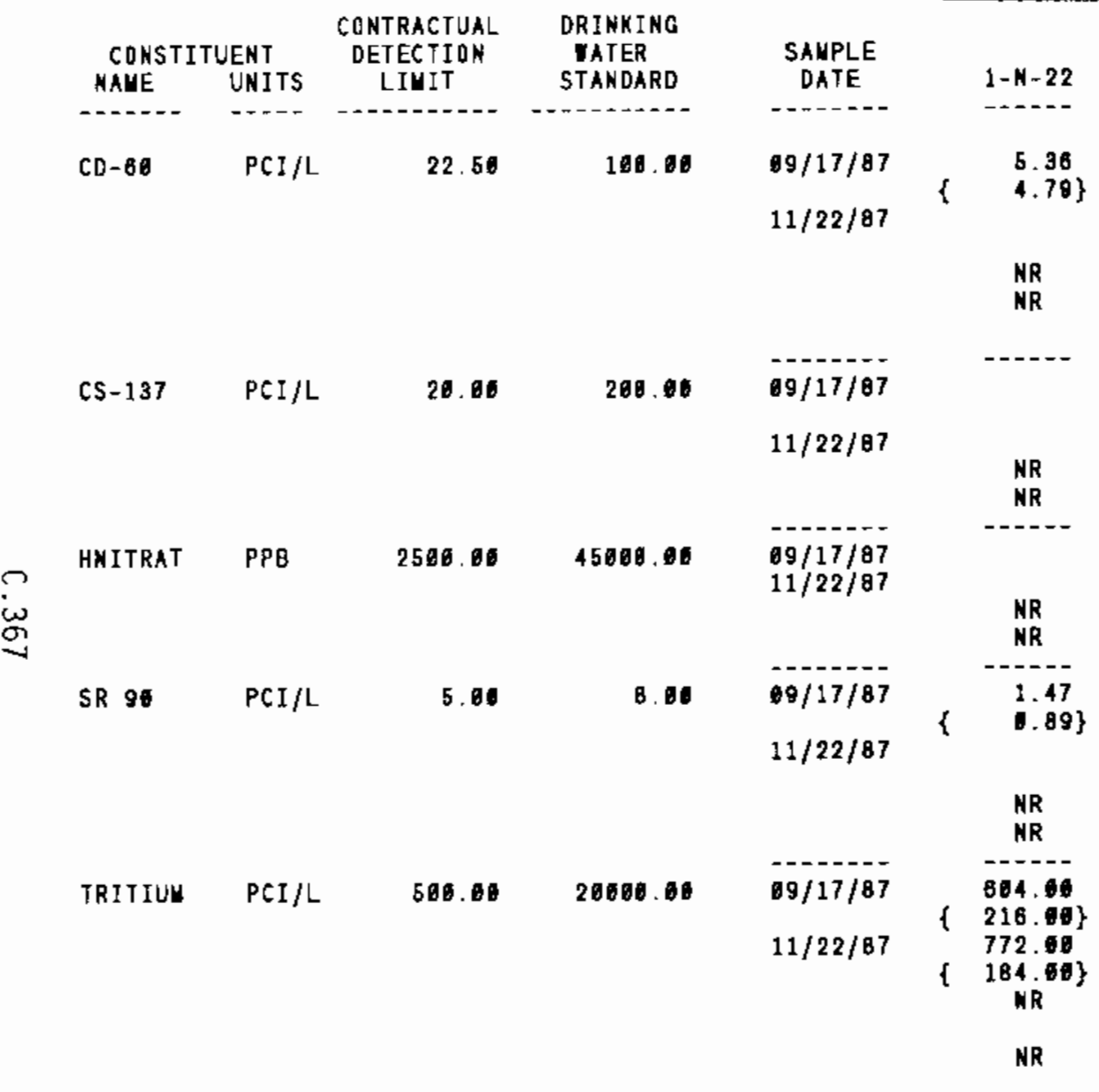

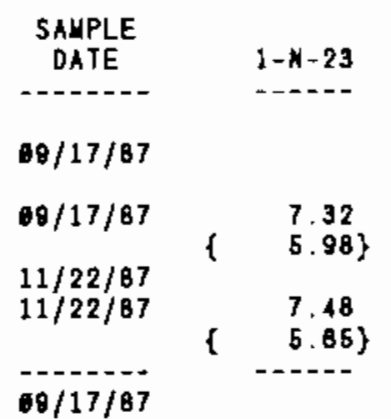

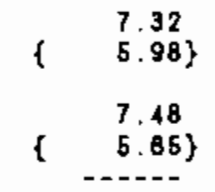

g9/17/87

$11 / 22 / 87$
$11 / 22 / 87$

-

$09 / 17 / 87$
$99 / 17 / 87$

$11 / 22 / 87$

$11 / 22 / 87$

$-10-17 / 87$

8790

8796.

6950.80

8816.8

09/17/87

$11 / 22 / 87$

$68 / 17 / 87$

$69 / 17 / 87$

$11 / 22 / 87$

$11 / 22 / 87$

-.-.--
1266.00

(237.86\}

1456.06

\{ 243.00$\}$

8200.00

\{ 936.00$\}$

7910.60
339.86
$11 / 22 / 87$

SAMPLE

DATE 1-N-24

69/17/87

$11 / 22 / 87$

NR
I-H-24

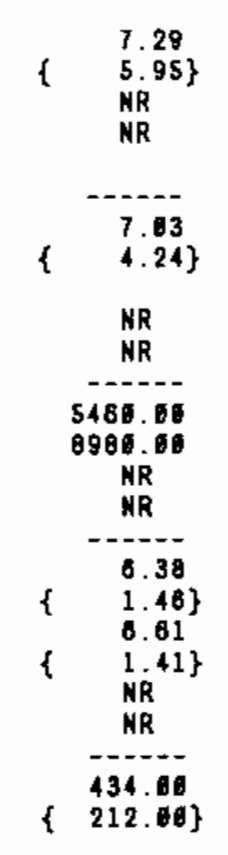

NR

NR

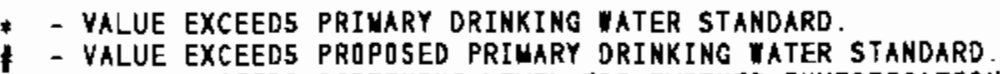

- YALUE EXCEEDS SCREENING LEYEL FoR FURTHER INYESTIGATION.

- DETECTION LIMIT VAS NOT AYAILABLE FOR COUPARISON

NR - ANALYSIS NOT REQUESTED OR NOT YET REPORTED

VALUES IN \{\} ARE COUNTING ERRORS FOR RADIDHUCLIDES

IATER STANDARD(S) IN PARENTHESES ARE PROPOSED ONLY 
TARLE C.7. (contd)

\begin{tabular}{|c|c|c|c|c|}
\hline $\begin{array}{l}\text { CONST } \\
\text { NAME }\end{array}$ & $\begin{array}{l}\text { TEENT } \\
\text { UNITS }\end{array}$ & $\begin{array}{c}\text { CONTRACTUAL } \\
\text { DETECTION } \\
\text { LIUIT }\end{array}$ & $\begin{array}{l}\text { DRINKING } \\
\text { WATER } \\
\text { STANDARD }\end{array}$ & $\begin{array}{c}\text { SAUPLE } \\
\text { DATE }\end{array}$ \\
\hline C 14 & $\mathrm{PCI} / \mathrm{L}$ & & & \\
\hline $00-80$ & $\mathrm{PCI} / \mathrm{L}$ & 22.50 & 100.00 & $\begin{array}{l}09 / 17 / 87 \\
11 / 22 / 87\end{array}$ \\
\hline
\end{tabular}

HNITRAT PP

2500.00

45000.06

506.80

$\sum_{\substack{\infty \\ \infty}}$

I-129 PCI/L

10.60

50.06

NI-63 PCI/L

172.60

30.00

RU-1DB PCI/L

05.50
$11 / 22 / 87$

SB $125 \mathrm{PCI} / \mathrm{L}$

SR 90

PCI/L

5.06

$$
8.00 \quad 09 / 17 / 87 \quad\left\{\begin{array}{c}
1.21 \\
1.06\}
\end{array}\right.
$$

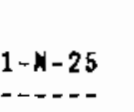

NR

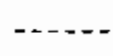

NR

NR

$09 / 17 / 87$

$11 / 22 / 87$

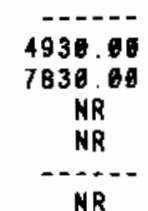

$\begin{array}{cc}\text { NR } \\ \text { NR } \\ \cdots & \text { NR }\end{array}$

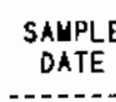

1-N-30

NR

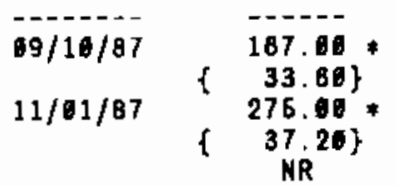

NR

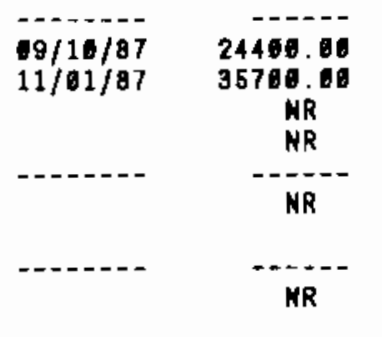

99/10/B?

$11 / 01 / 87$

NR

NR

\section{$117.00 *$}

NR

NR

NR

NR

KR

$09 / 10 / 87$

* - Value eXCEEDS priuary dRINKINg mater standard.

* - VALUE EXCEEDS PROPOSED PRIMARY DRINKING MaTER STANDARD.

* - VALUE EXCEEDS SCREENING LEVEL FaR FURTHER INVESTIGATION.

- DETECTIDN LIMIT IAS NDT AVAILABLE FOR COUPARISON

NR - ANALYSIS NOT RERUESTED OR NOT YET REPORTED

VALUES IN \{ $\}$ ARE COUNTING ERRORS FOR RADIONUCLIDES

WTER STANDARD(S) IN PARENTHESES ARE PROPOSED DNLY 


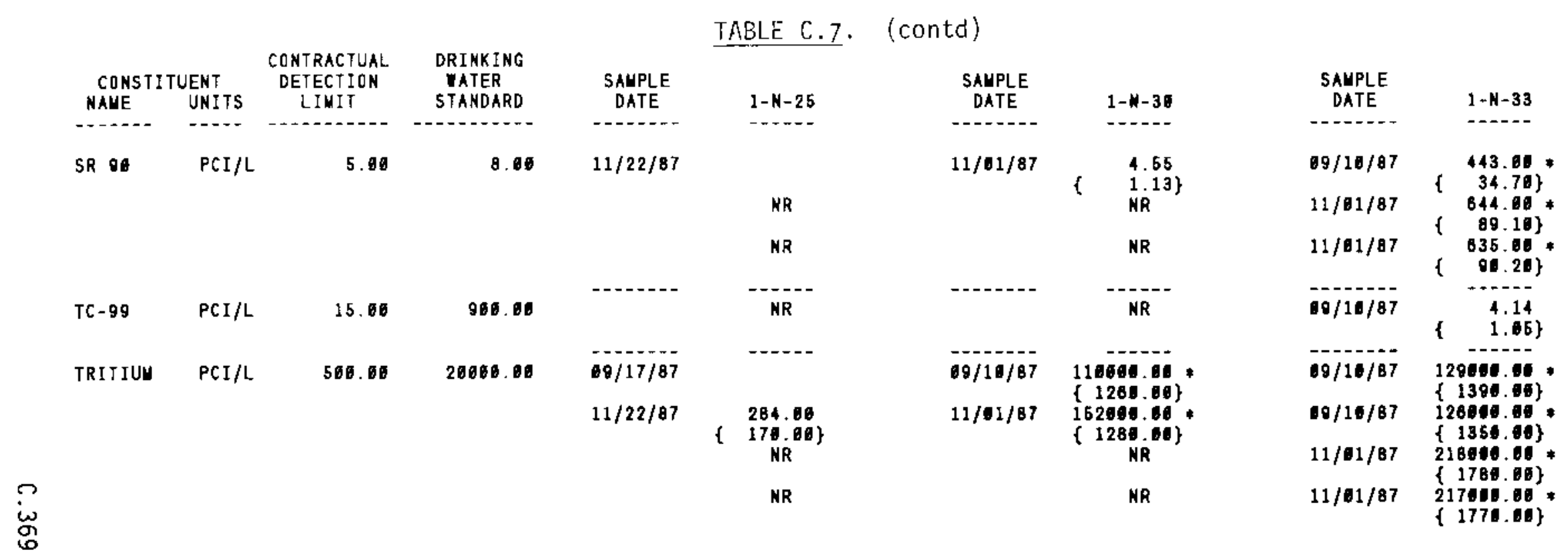

* - VAlUe exceEds privary dRinXing vater standard

- VALUE EXCEEDS PROPOSED PRIUARY DRINKING TATER STANDARD

- VALUE EXCEEDS SCREENING LEVEL FOR FURTHER INYESTIGATIDN.

- DETECTION LiUIT TAS NOT AVALABLE for COMPARISON

NR - ANALYSIS NDT REQUESTED DR NOT YET REPORTED

VALUES IN I ? ARE COUNTING ERRORS FOR RADIONUCLIDES

WATER STAKDARO(S) IN PARENTHESES ARE PROPOSED ONLY 


\begin{tabular}{|c|c|c|c|c|c|}
\hline $\begin{array}{l}\text { CONST } \\
\text { NAYE }\end{array}$ & $\begin{array}{l}\text { UENT } \\
\text { UNITS }\end{array}$ & $\begin{array}{c}\text { CONTRACTUAL } \\
\text { DETECTIDN } \\
\text { LIUIT }\end{array}$ & $\begin{array}{l}\text { DRINKING } \\
\text { WATER } \\
\text { STANDARO }\end{array}$ & $\begin{array}{c}\text { SAYPLE } \\
\text { DATE }\end{array}$ & $1-N-37$ \\
\hline$C O-60$ & $\mathrm{PCI} / \mathrm{L}$ & 22.50 & 106.60 & $11 / 81 / 87$ & $\left\{\begin{array}{c}389.06 \\
45.46\end{array}\right\}^{N R}$ \\
\hline HNI TRAT & PPB & 2500.00 & 45000.08 & $11 / 01 / 87$ & 34426.06 \\
\hline$R U-168$ & PCI/L & 172.50 & 30.08 & $11 / 01 / 87$ & NR \\
\hline 58125 & $\mathrm{PCI} / \mathrm{L}$ & & & $11 / 01 / 87$ & $\left\{\begin{array}{r}155.00 \\
39.30\end{array}\right\}$ \\
\hline SR 96 & PCI $/ \mathrm{L}$ & 5.60 & 8.00 & $11 / 01 / 87$ & $\left\{\begin{array}{c}36,30 \\
4.38\} \\
N R\end{array}\right.$ \\
\hline YRITIUU & $\mathrm{PCI} / \mathrm{L}$ & 500.00 & 20080.08 & $11 / 81 / 87$ & $\left.\begin{array}{c}215000.00 \\
\{1779.80\end{array}\right\}$ \\
\hline
\end{tabular}

* - VALUe exceEds priuary dRINKing Mater standard.

* - YALUE EXCEEDS PROPOSED PRIUARY DRINKING IATER STANDARD.

- VALUE EXCEEDS SCREENING LEVEL FOR FURTHER INYESTIGATION.

- DETECTION LIMIT TAS NOT AVAILABLE FUR COMPARISON

NR - ANALYSIS NOT REQUESTED OR NOT YET REPORTED

YALUES IN \{ ARE CDUNTING ERRDRS FOR RADIONUCLIDES

IATER STANDARD(S) IN PARENTHESES ARE PROPOSED ONLY

\begin{tabular}{|c|c|c|c|}
\hline $\begin{array}{l}\text { SAUPLE } \\
\text { DATE }\end{array}$ & $1-N-45$ & $\begin{array}{l}\text { SAMPLE } \\
\text { DATE }\end{array}$ & $1-N-50$ \\
\hline $87 / 23 / 87$ & $\begin{array}{r}279.60 \\
\quad 37.46\}\end{array}$ & & NR \\
\hline $11 / 22 / 87$ & $\left\{\begin{array}{c}211.00 \\
29.86\end{array}\right.$ & & NR \\
\hline $\begin{array}{l}77 / 23 / 87 \\
11 / 22 / 87\end{array}$ & $\begin{array}{l}38866.00 \\
26689.00\end{array}$ & $\begin{array}{l}07 / 23 / 87 \\
11 / 01 / 87\end{array}$ & $\begin{array}{l}33806.80 \\
48200.08\end{array}$ \\
\hline $\begin{array}{l}07 / 23 / 87 \\
11 / 22 / 87\end{array}$ & $\left\{\begin{array}{r}125.66 \\
\{\quad 82.46\end{array}\right.$ & & $\begin{array}{l}\text { NR } \\
\text { NR }\end{array}$ \\
\hline $11 / 22 / 87$ & $\left\{\begin{array}{c}143.00 \\
32.78\}\end{array}\right.$ & & HR \\
\hline $07 / 23 / 87$ & 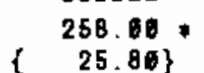 & $07 / 23 / 87$ & \\
\hline $11 / 22 / 87$ & $\left.\begin{array}{c}3160.80 \\
91.80\end{array}\right\}^{*}$ & $11 / 61 / 87$ & \\
\hline 67/23/87 & $\begin{array}{l}119000.08 \\
\{1290.00\}\end{array}$ & $67 / 23 / 87$ & $\begin{array}{c}96500.60 \\
\{1020.06\}\end{array}$ \\
\hline $11 / 22 / 87$ & $\begin{array}{c}79368.60 \\
\{1079.06\}\end{array}$ & $11 / 81 / 87$ & $\begin{array}{l}121660.06 \\
\{1320.00\}\end{array}$ \\
\hline
\end{tabular}


TABLE C.7. (contd)

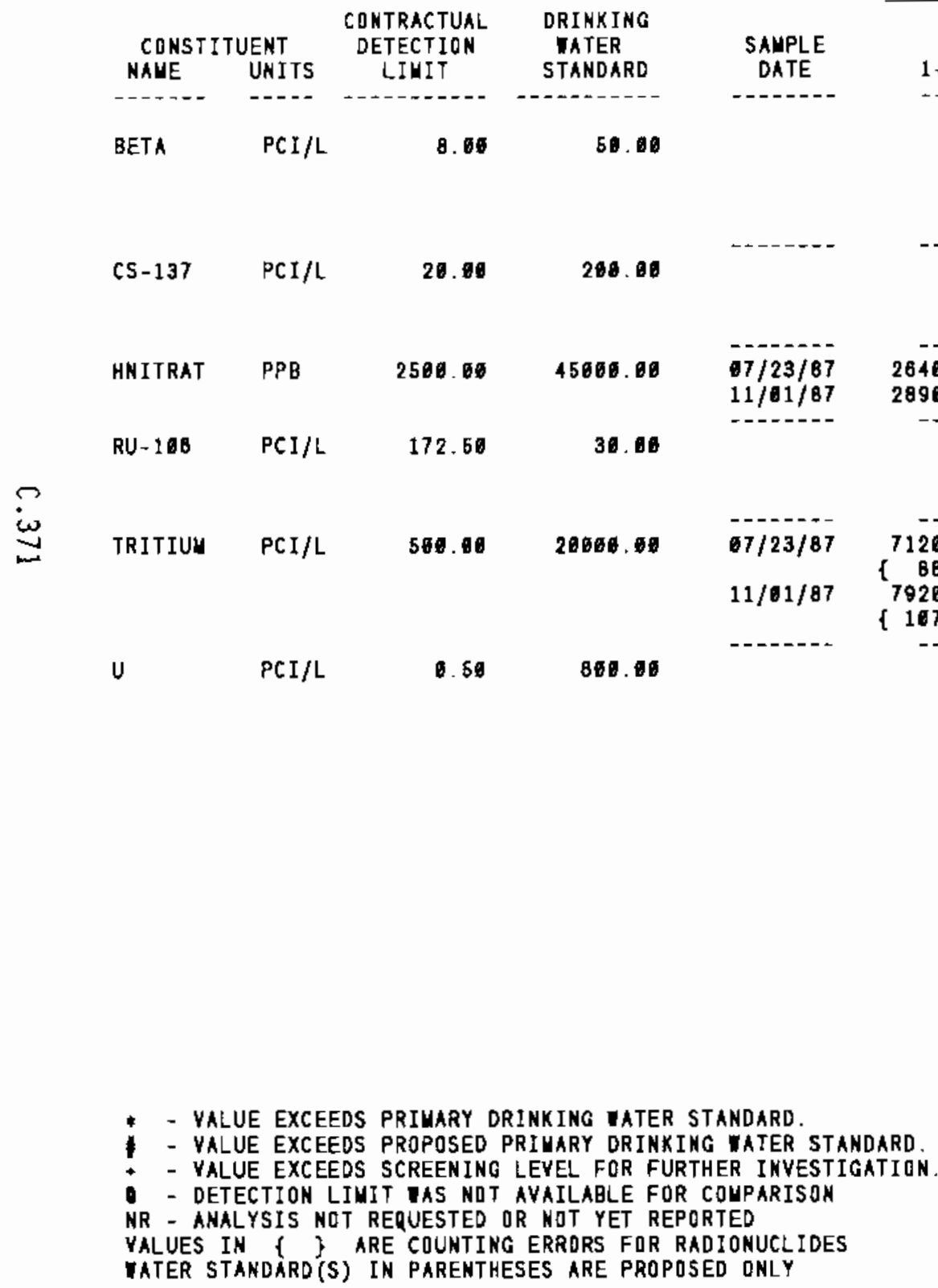

\begin{tabular}{|c|c|c|c|}
\hline $\begin{array}{l}\text { SAUPLE } \\
\text { DATE }\end{array}$ & $2-E 13-8$ & $\begin{array}{l}\text { SAUPLE } \\
\text { DATE }\end{array}$ & $2-E 13-19$ \\
\hline $16 / 28 / 87$ & 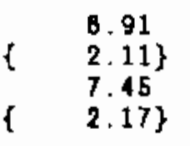 & $16 / 28 / 87$ & $\begin{array}{l}5.88 \\
1.96) \\
3.85 \\
1.76\}\end{array}$ \\
\hline $18 / 03 / 87$ & $\begin{array}{l}3.44 \\
3.00\}\end{array}$ & $08 / 03 / 87$ & $\begin{array}{l}4.13 \\
3.37\} \\
\mathrm{NR}\end{array}$ \\
\hline & $\begin{array}{l}\text { NR } \\
\text { NR }\end{array}$ & & $\begin{array}{l}\text { NR } \\
\text { NR }\end{array}$ \\
\hline $68 / 03 / 87$ & $\left\{\begin{array}{l}47.10^{*} \\
(33.30\end{array}\right\}^{*}$ & $08 / 03 / 87$ & \\
\hline $10 / 28 / 87$ & & & NR \\
\hline B / 83/87 & & $08 / 03 / 87$ & \\
\hline & NR & & NR \\
\hline $68 / 03 / 87$ & 1.96 & $B B / 03 / 87$ & 2.31 \\
\hline
\end{tabular}

* - YALUE eXCEEDS pRIUARY dRINKINg taTER STANDARD.

XCEDS PROPOSED PRIUARY

AETECTION LIMIT TAS NDT AVAILABLE FOR COUPARISOA

DALYSIS NOT RERUESTED OR MOT YET REPORTED 


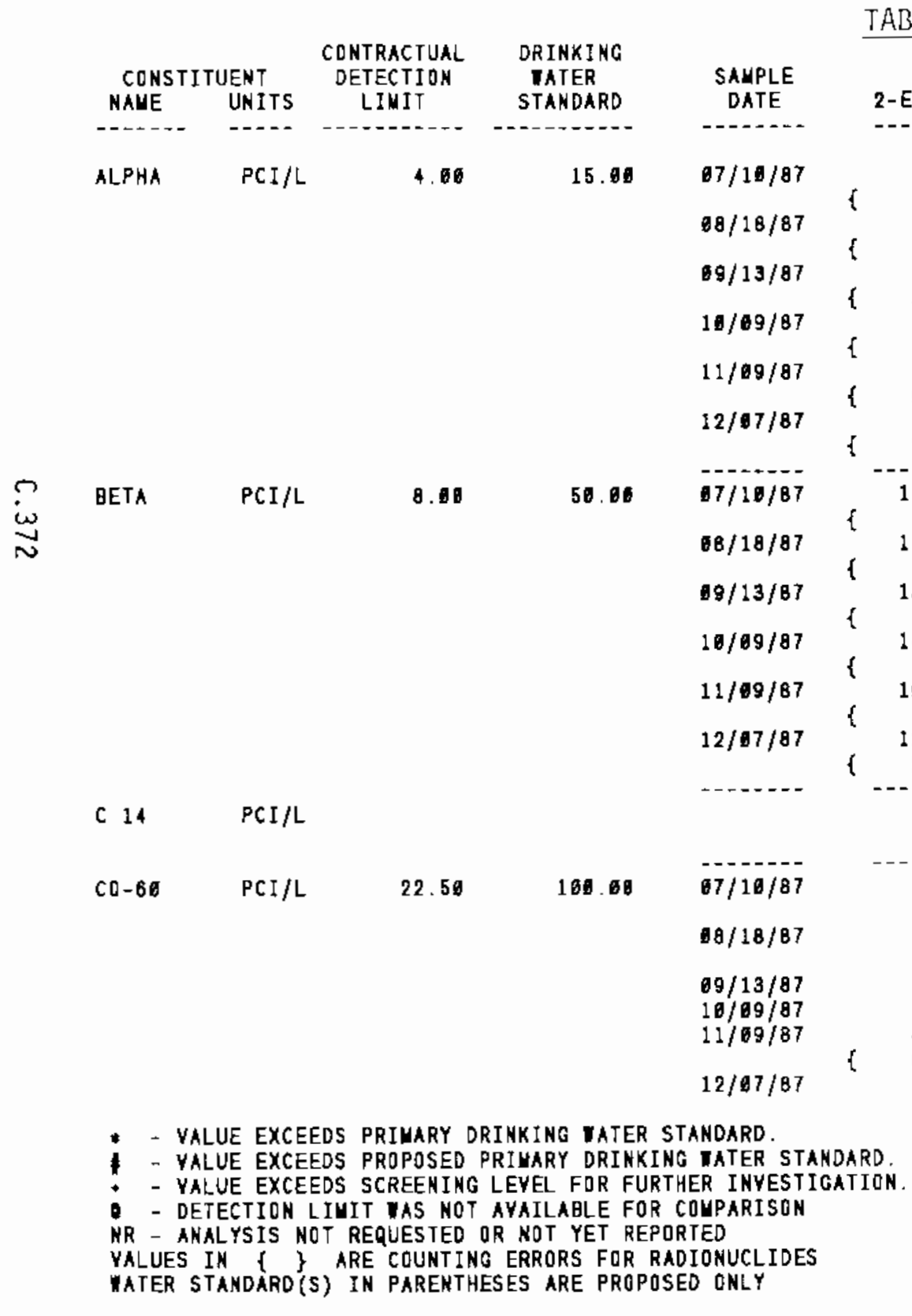

ABLE C.7. (contd)

E16-2

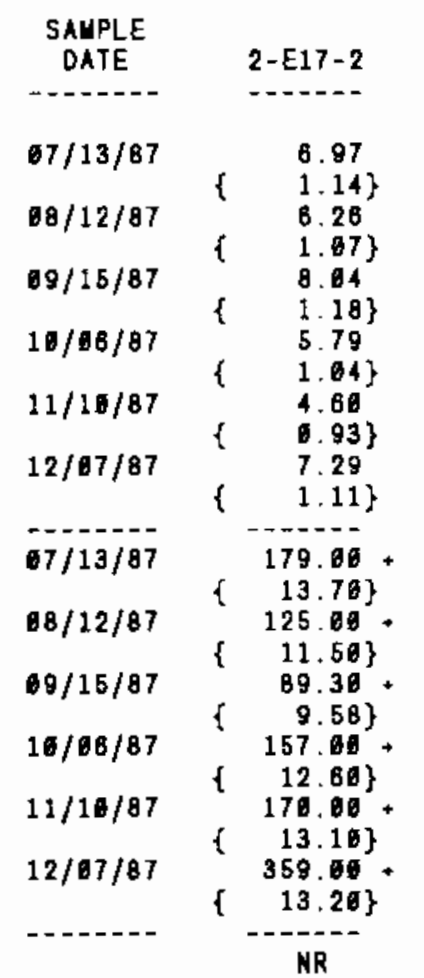

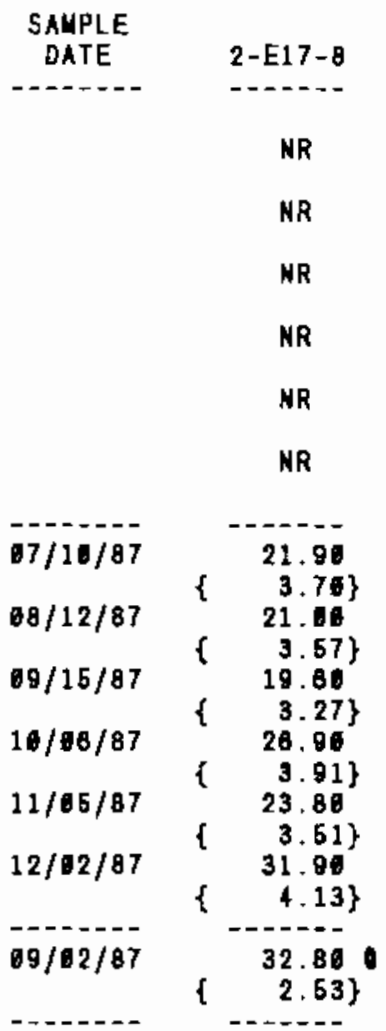

$07 / 13 / 87 \quad 39.80 \quad 07 / 10 / 87$

$16 / 66 / 87$

$10 / 66 / 87$

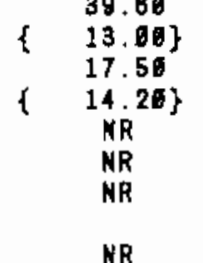

NR
NR
NR
NR

- VALUE EXCEEDS PRIHARY DRINKING VATER STANDARD.

- VALUE EXCEEDS PROPOSED PRIMARY DRIHKING TATER STANDARD

- YALUE EXCEEDS SCREENING LEYEL FDR FURTHER INYESTIGATION

R - AKALYSIS NOT REQUESTED OR NDT YET REPORTED

MATER STANDARO(S) IN PARENTHESES ARE PROPOSEO ONLY 


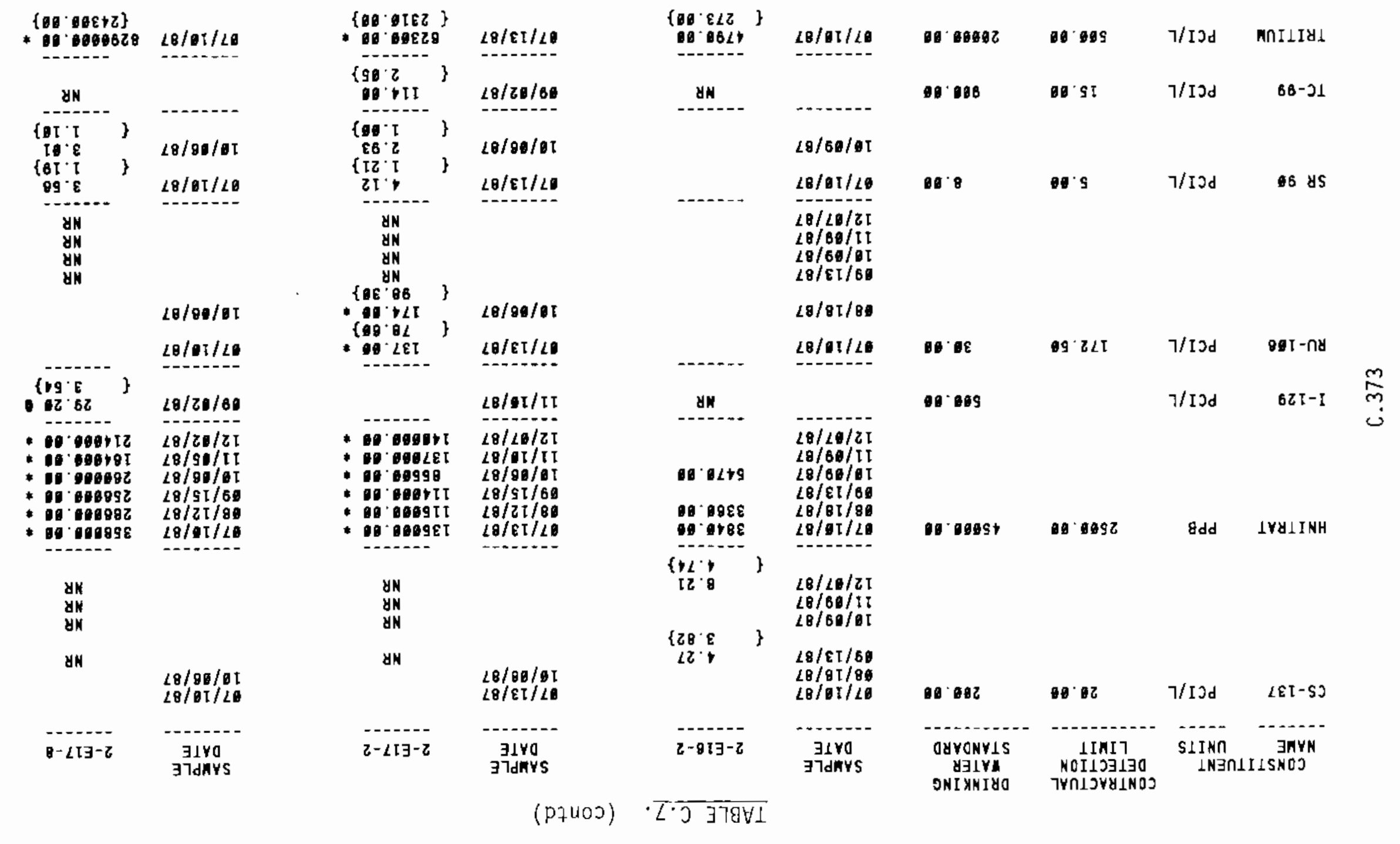

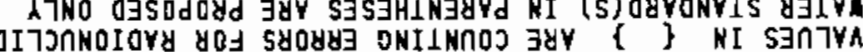
NoI YoI -

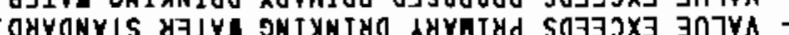


TABLE C.7. (contd)

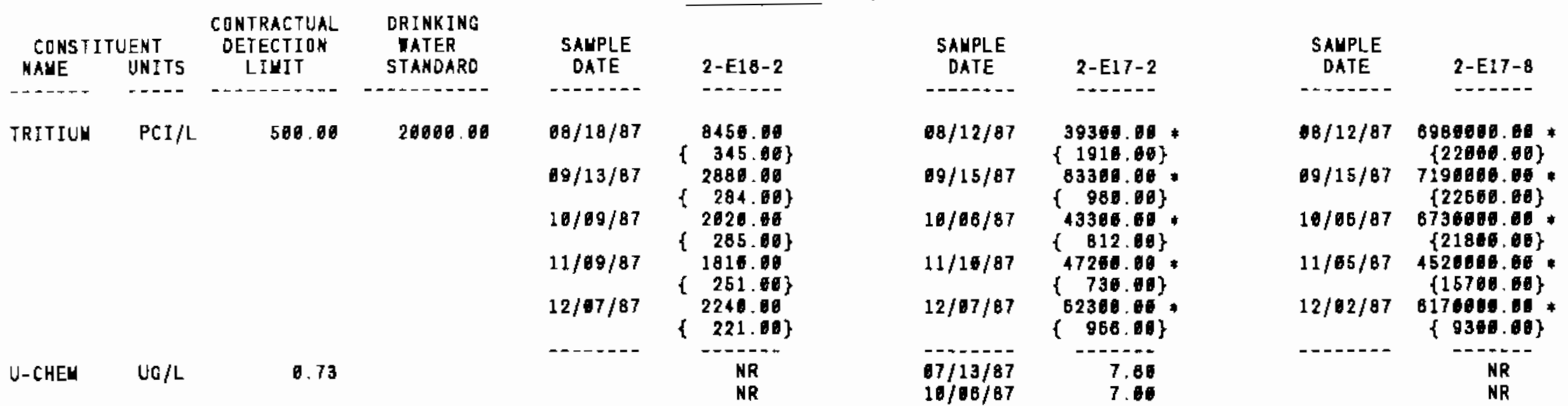

$\stackrel{?}{w}$

* - value exceeds primary dRinking tater stahdaro

- - VALUE EXCEEDS PROPOSED PRIYARY DRINKING WATER STANDARD

* - VALUE EXCEEDS SCREENING LEVEL FDR FURTHER INYESTIGATION

- DETECTION LIMIT IAS NDT AVAILABLE FOR CDMPARISON

NR - ANALYSIS NOT RERUESTEO OR NOT YET REPORTED

VALUES IN \{ $\}$ ARE COUNTING ERRORS FOR RAOIOHUCLIDES

WATER STAHDARD(S) IN PARENTHESES ARE PRDPOSED ONLY 


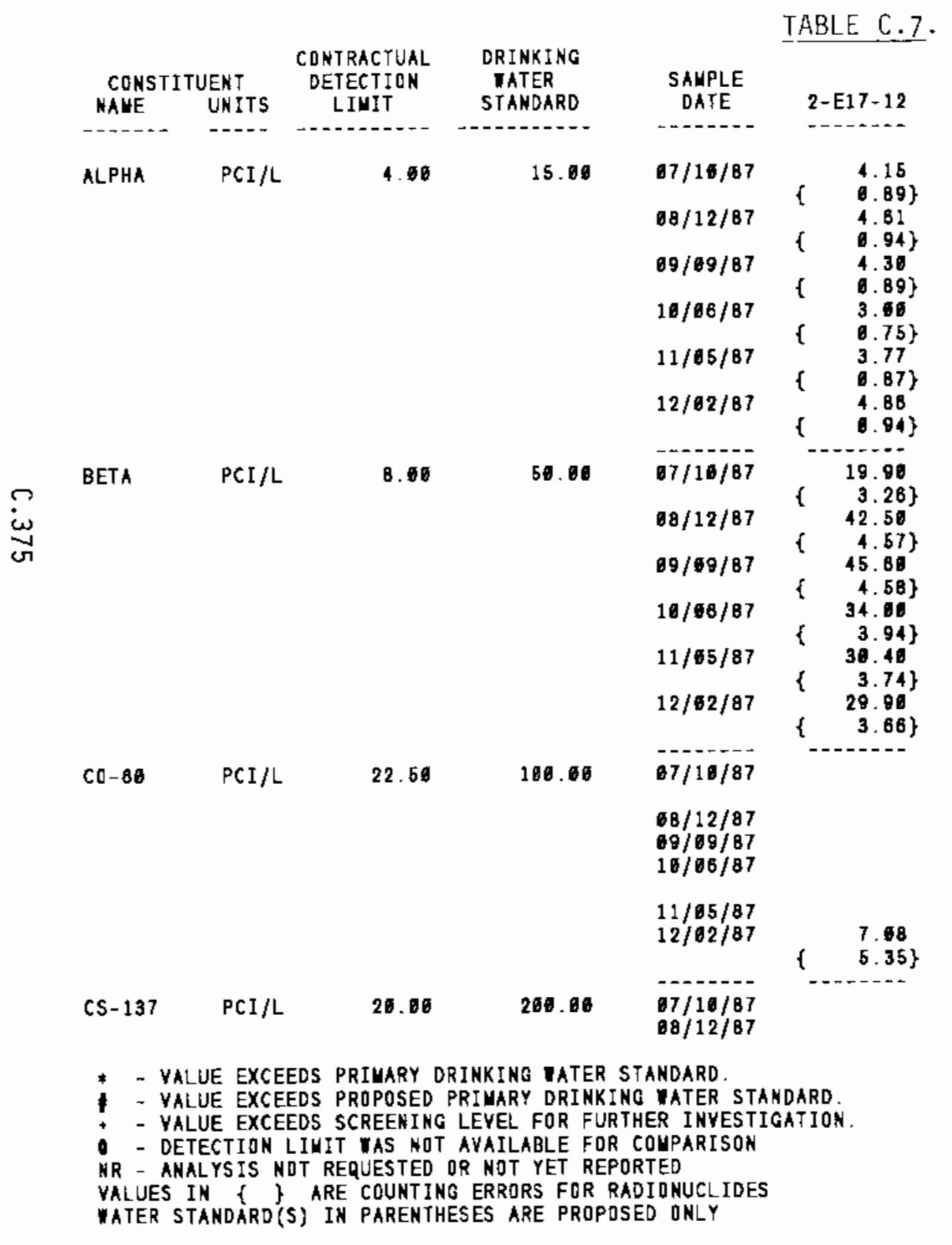

(contd)

\begin{tabular}{|c|c|c|c|}
\hline $\begin{array}{l}\text { SAMPLE } \\
\text { DATE }\end{array}$ & $2-E 17-13$ & $\begin{array}{c}\text { SAMPLE } \\
\text { DATE }\end{array}$ & $2-E 19-1$ \\
\hline $07 / 20 / 87$ & 3. 72 & & NR \\
\hline $88 / 12 / 87$ & 4.26 & & NR \\
\hline 09/69/87 & $\begin{array}{l}8.803 \\
4.38\end{array}$ & & NR \\
\hline $16 / 06 / 87$ & $\begin{array}{l}3.91\} \\
3.15\end{array}$ & & NR \\
\hline & $0.75\}$ & & \\
\hline $11 / 65 / 87$ & $\begin{array}{l}3.88 \\
\text { e. } 86\}\end{array}$ & & NR \\
\hline $12 / 02 / 87$ & $\begin{array}{l}\left.\quad \begin{array}{l}4.98 \\
\quad 0.98\end{array}\right\}\end{array}$ & & NR \\
\hline $07 / 10 / 87$ & $\begin{array}{r}38.90 \\
\{\quad 4.39\}\end{array}$ & & NR \\
\hline $68 / 12 / 87$ & $\begin{array}{r}35.20^{\circ} \\
\quad 4.15 \\
\end{array}$ & & NR \\
\hline $69 / 09 / 87$ & $\begin{array}{r}36.46 \\
\{\quad 4.61\}\end{array}$ & & NR \\
\hline $10 / 06 / 87$ & $\begin{array}{c}22.36 \\
3.23\}\end{array}$ & & NR \\
\hline $11 / 65 / 87$ & 35.60 & & MR \\
\hline $12 / 82 / 87$ & $\begin{array}{r}\quad 37.48 \\
\{\quad 4.12\}\end{array}$ & & NR \\
\hline $07 / 10 / 87$ & $\left.\begin{array}{ll} & 5.88 \\
\{\quad 5.80\end{array}\right\}$ & $68 / 10 / 87$ & \\
\hline $\begin{array}{l}08 / 12 / 87 \\
69 / 09 / 87 \\
16 / 08 / 87\end{array}$ & & & $\begin{array}{l}\text { NR } \\
\text { KR }\end{array}$ \\
\hline & $\begin{array}{l}(5.89 \\
(\quad 5.44\}\end{array}$ & & NR \\
\hline $\begin{array}{l}11 / 95 / 87 \\
12 / 02 / 87\end{array}$ & & & $\begin{array}{l}\mathrm{NR} \\
\mathrm{NR}\end{array}$ \\
\hline $\begin{array}{l}67 / 10 / 87 \\
68 / 12 / 87\end{array}$ & & $08 / 10 / 87$ & NR \\
\hline
\end{tabular}


TABLE C.7. (contd)

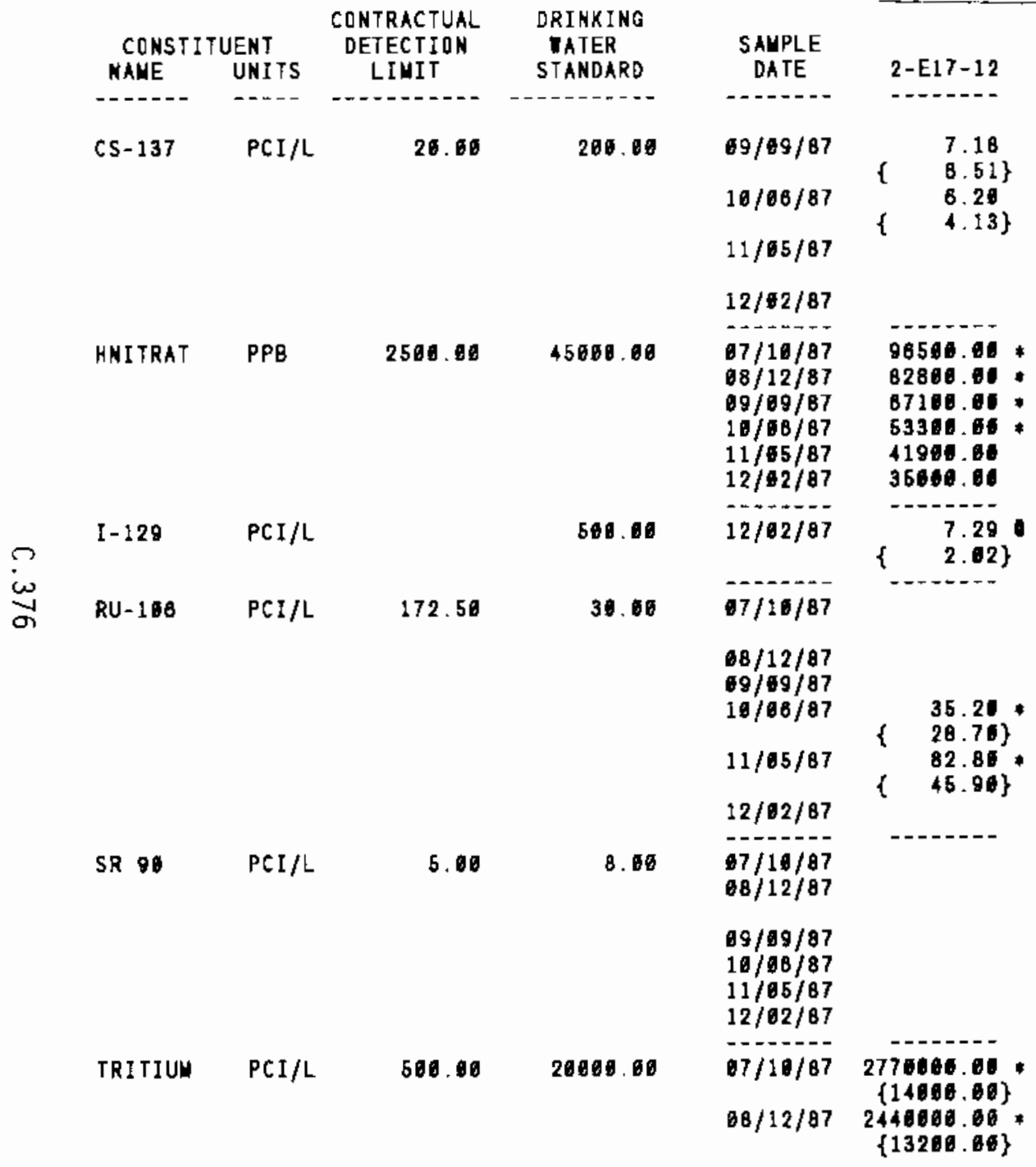

\begin{tabular}{|c|c|c|}
\hline $\begin{array}{l}\text { SAlYLLE } \\
\text { DATEE }\end{array}$ & \multicolumn{2}{|c|}{$2-E 17-13$} \\
\hline $89 / 69 / 87$ & & \multirow{4}{*}{$\begin{array}{l}8.13 \\
8.40\}\end{array}$} \\
\hline $10 / 86 / 87$ & & \\
\hline $11 / 05 / 87$ & & \\
\hline $12 / 02 / 87$ & & \\
\hline $\begin{array}{l}67 / 10 / 87 \\
88 / 12 / 87 \\
69 / 00 / 87 \\
10 / 96 / 87 \\
11 / 65 / 87 \\
12 / 82 / 87\end{array}$ & \multicolumn{2}{|c|}{ 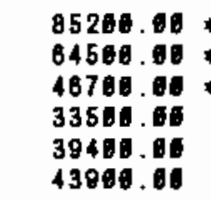 } \\
\hline $12 / 82 / 87$ & $\left\{\begin{array}{r}10 \\
1\end{array}\right.$ & $\begin{array}{l}0.18 \\
1.95\}\end{array}$ \\
\hline $07 / 10 / 87$ & \multirow{2}{*}{\multicolumn{2}{|c|}{$\begin{array}{l}59.16 \\
\{\quad 37.46\}\end{array}$}} \\
\hline $\begin{array}{l}00 / 12 / 87 \\
09 / 68 / 87 \\
10 / 86 / 87\end{array}$ & & \\
\hline $11 / 05 / 87$ & \multirow{4}{*}{\multicolumn{2}{|c|}{$\left.\begin{array}{ll} & 6.71 \\
\{\quad & 0.62\end{array}\right\}$}} \\
\hline $12 / 62 / 87$ & & \\
\hline $\begin{array}{l}07 / 16 / 87 \\
68 / 12 / 87\end{array}$ & & \\
\hline $\begin{array}{l}69 / 09 / 87 \\
16 / 98 / 87 \\
11 / 65 / 87 \\
12 / 62 / 87\end{array}$ & & \\
\hline $07 / 16 / 87$ & $\begin{array}{r}2736800 \\
\{13800\end{array}$ & $\begin{array}{l}0.86 \\
0.86\}\end{array}$ \\
\hline $88 / 12 / 87$ & $\begin{array}{r}2156068 \\
\{12200\end{array}$ & 6. \\
\hline
\end{tabular}

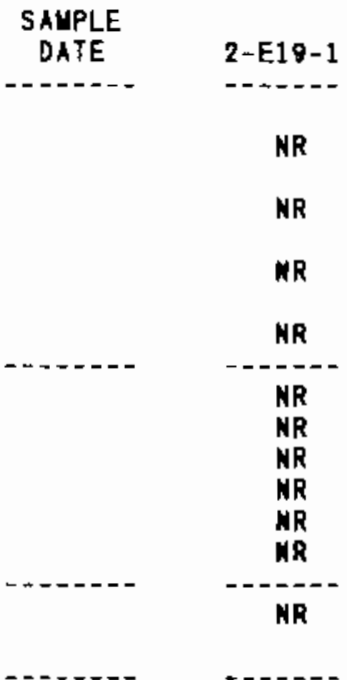

$98 / 10 / 87$

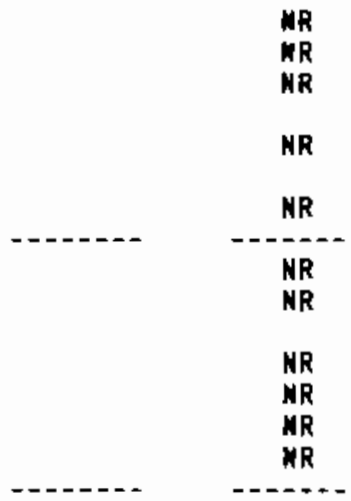

NR

* - value exceeds primary drinking tater standard

- - VALUE EXCEEDS PROPOSED PRIUARY dRINKING Mater STANDARD.

+ - YALUE EXCEEDS SCREENING LEYEL FOR FURTHER INYESTIGATION.

- DETECTION LIUIT nS NOT AVAILABLE For COUPARISON

NR - AKALYSIS NOT REQUESTED OR NOT YET REPORTED

VALUES IN \{ $\}$ ARE COUNTING ERRORS FOR RADIONUCLIDES

WATER STANDARD(S) IN PARENTHESES ARE PROPOSED ONLY 
TABLE C.7. (contd)

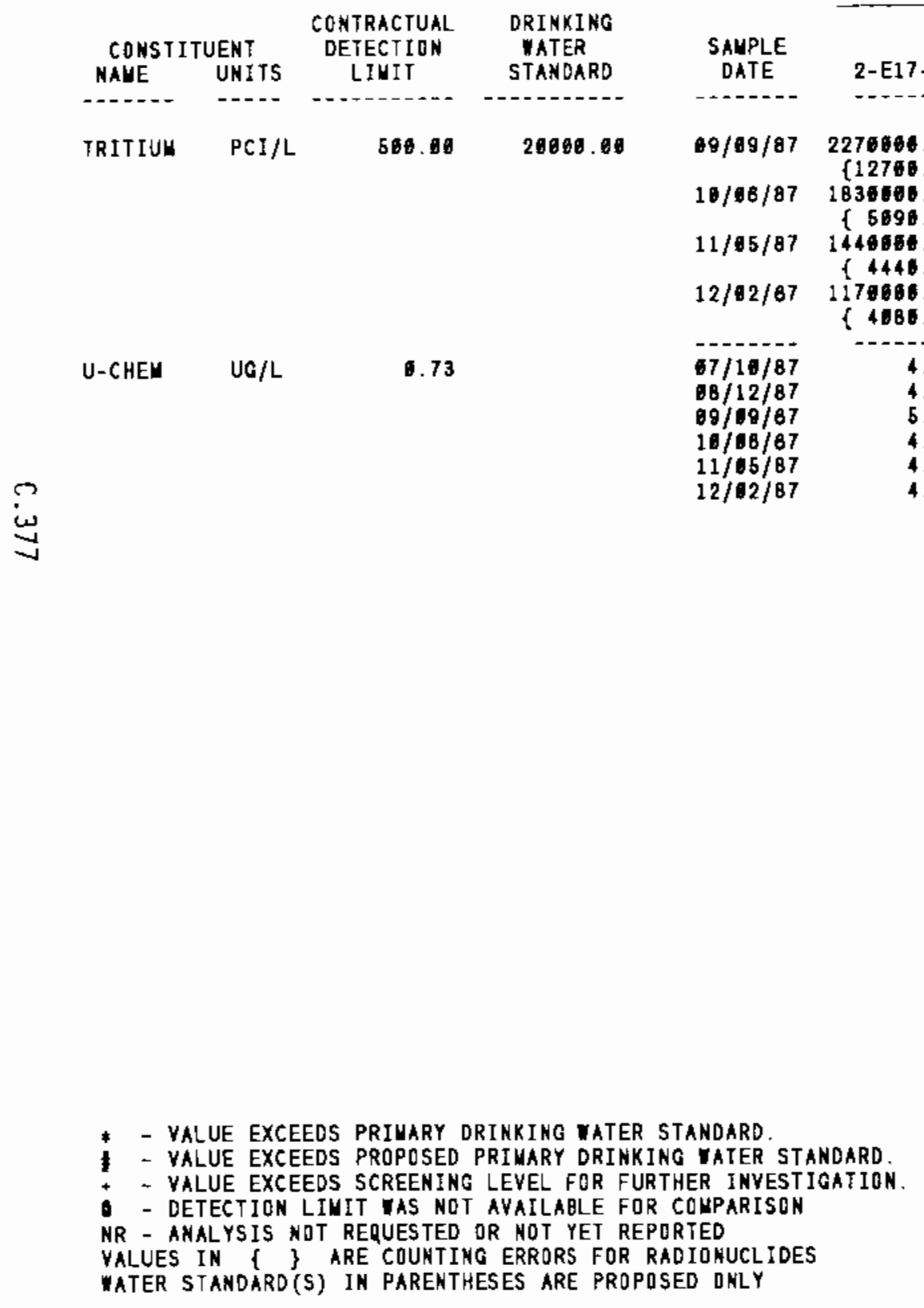

\begin{tabular}{|c|c|c|c|}
\hline $\begin{array}{l}\text { SAUPLE } \\
\text { DATE }\end{array}$ & $2-E 17-13$ & $\begin{array}{l}\text { SAYPLE } \\
\text { DATE }\end{array}$ & $2-E 10-1$ \\
\hline$----\infty$ & 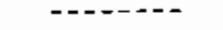 & $---x-\infty$ & $--\cdots--0$ \\
\hline $09 / 09 / 87$ & $\begin{array}{c}1710000.00 * \\
\{4890.00\}\end{array}$ & & NR \\
\hline $10 / 06 / 87$ & $\begin{array}{c}1140068.00 \\
\{3906.00\}\end{array}$ & & NR \\
\hline $11 / 05 / 87$ & $\begin{array}{c}1500000.06 \\
\{4600.06\}\end{array}$ & & NR \\
\hline $12 / 02 / B 7$ & $\begin{array}{c}1638606.06 \\
\{4700.06\}\end{array}$ & & NR \\
\hline $\begin{array}{l}-7 / 16 / 87 \\
68 / 12 / 87 \\
60 / 09 / 87 \\
10 / 06 / 87 \\
11 / 95 / 87 \\
12 / 02 / 87\end{array}$ & $\begin{array}{r}6.22 \\
4.65 \\
4.88 \\
4.70 \\
5.93 \\
4.50\end{array}$ & 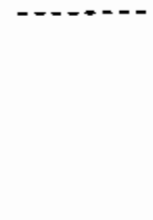 & $\begin{array}{c}\text { NR } \\
\text { MR } \\
\text { MR } \\
\text { WR } \\
\text { NR } \\
\text { NR } \\
\text { NR }\end{array}$ \\
\hline
\end{tabular}

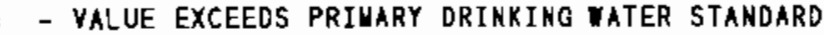

- VALUE EXCEEdS PROPOSED PRIMARY DRINKING TATER STANDARD.

TECTION LIUIT UAS NOT AYALLABE FOR CQUPAR

WATER STANDARD(S) IN PARENTHESES ARE PROPOSED DNLY 


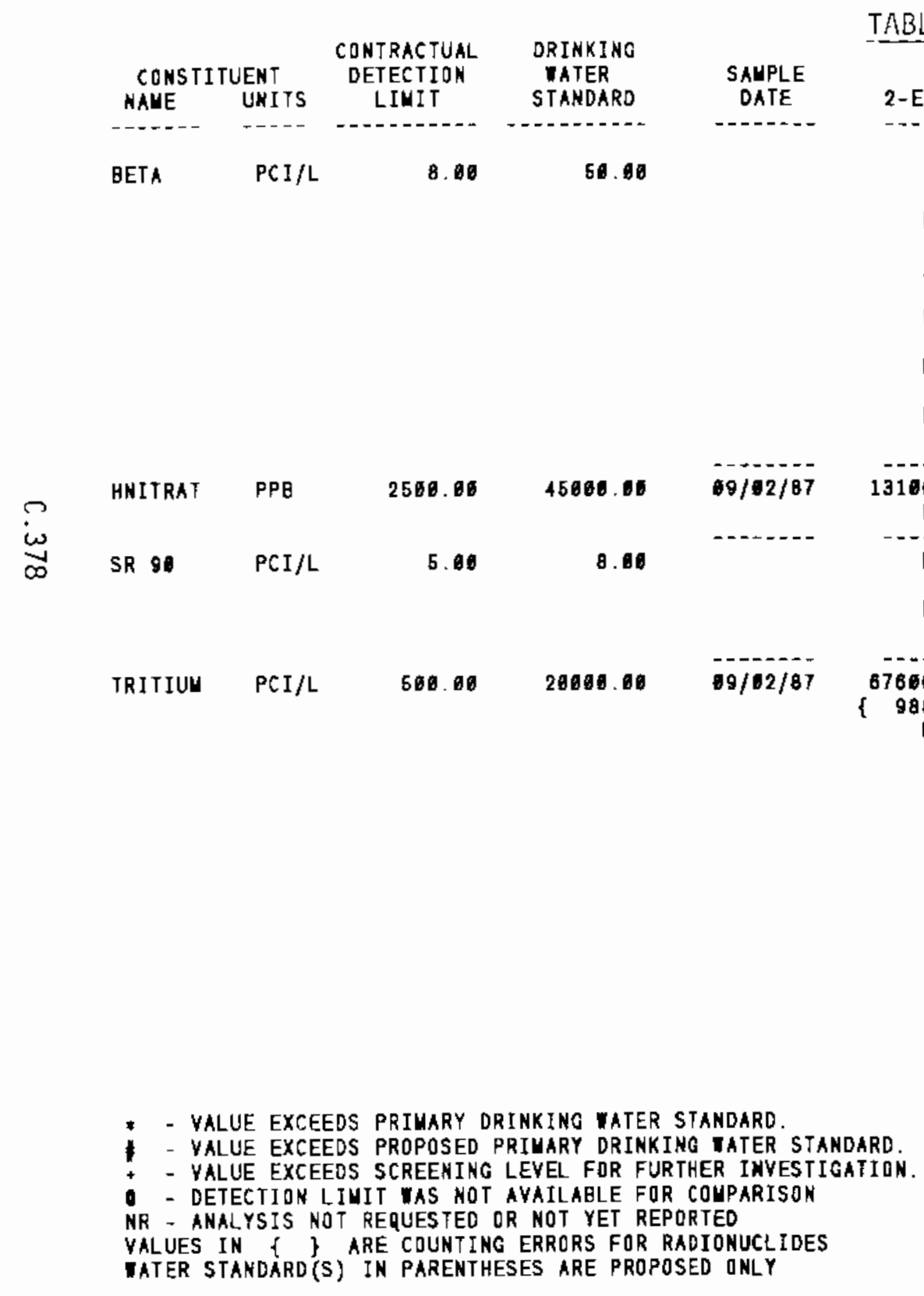

ABLE C.7. (contd)

\begin{tabular}{|c|c|c|}
\hline E24-7 & $\begin{array}{l}\text { SAUPLE } \\
\text { DATE }\end{array}$ & $2-E 24-11$ \\
\hline & - & - \\
\hline NR & $07 / 13 / 87$ & 24.40 \\
\hline NR & $68 / 12 / 87$ & $\begin{array}{r}14.96 \\
3.06\}\end{array}$ \\
\hline NR & $69 / 15 / 07$ & $\begin{array}{r}18.46 \\
2.98\end{array}$ \\
\hline NR & $10 / 06 / 87$ & $\begin{array}{r}23.36 \\
\{\quad 3.68\}\end{array}$ \\
\hline NR & $11 / 10 / 87$ & $\begin{array}{r}39.50 \\
\{\quad 4.54\}\end{array}$ \\
\hline NR & $12 / 07 / 87$ & $\begin{array}{r}18.26 \\
\{\quad 3.37\}\end{array}$ \\
\hline NR & $\begin{array}{l}07 / 13 / 87 \\
10 / 06 / 87\end{array}$ & $\begin{array}{l}228000.06 \\
327000.06\end{array}$ \\
\hline $\mathrm{NR}$ & $07 / 13 / 87$ & $\left\{\begin{array}{r}0.98 \\
\quad 0.91\}\end{array}\right.$ \\
\hline MR & $10 / 86 / 87$ & $\begin{array}{l}1.10 \\
\{\quad B . B 6\}\end{array}$ \\
\hline $\begin{array}{l}00.00 \\
\mathrm{NR}\end{array}$ & $16 / 86 / 87$ & $\begin{array}{c}B 270000.06 * \\
\{29800.08\} \\
9110000.00 * \\
\{25100.00\}\end{array}$ \\
\hline
\end{tabular}

SAUPLE

DATE

2-E24-13

$9 / 02 / 87 \quad 9.44$

$\{2.21\}$

8.35

2.10$$
\text { NR }
$$

NR

NR

NR

$90 / 02 / 8$

$10 / 28 / 87$

2840.

2840.00

2750.00

$09 / 02 / 87$

NR

$69 / 02 / 67$

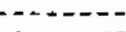

8436.00

\{ 395.00$\}$

VALUE EXCEEDS PRIMARY DRINKING WATER STANDARD.

YALE EXCEEDS SCREEMING LEYEL FOR FURTHER IUYESTIOATION.

TATER STANOARD(S) IN PARENTHESES ARE PROPOSED ONLY 


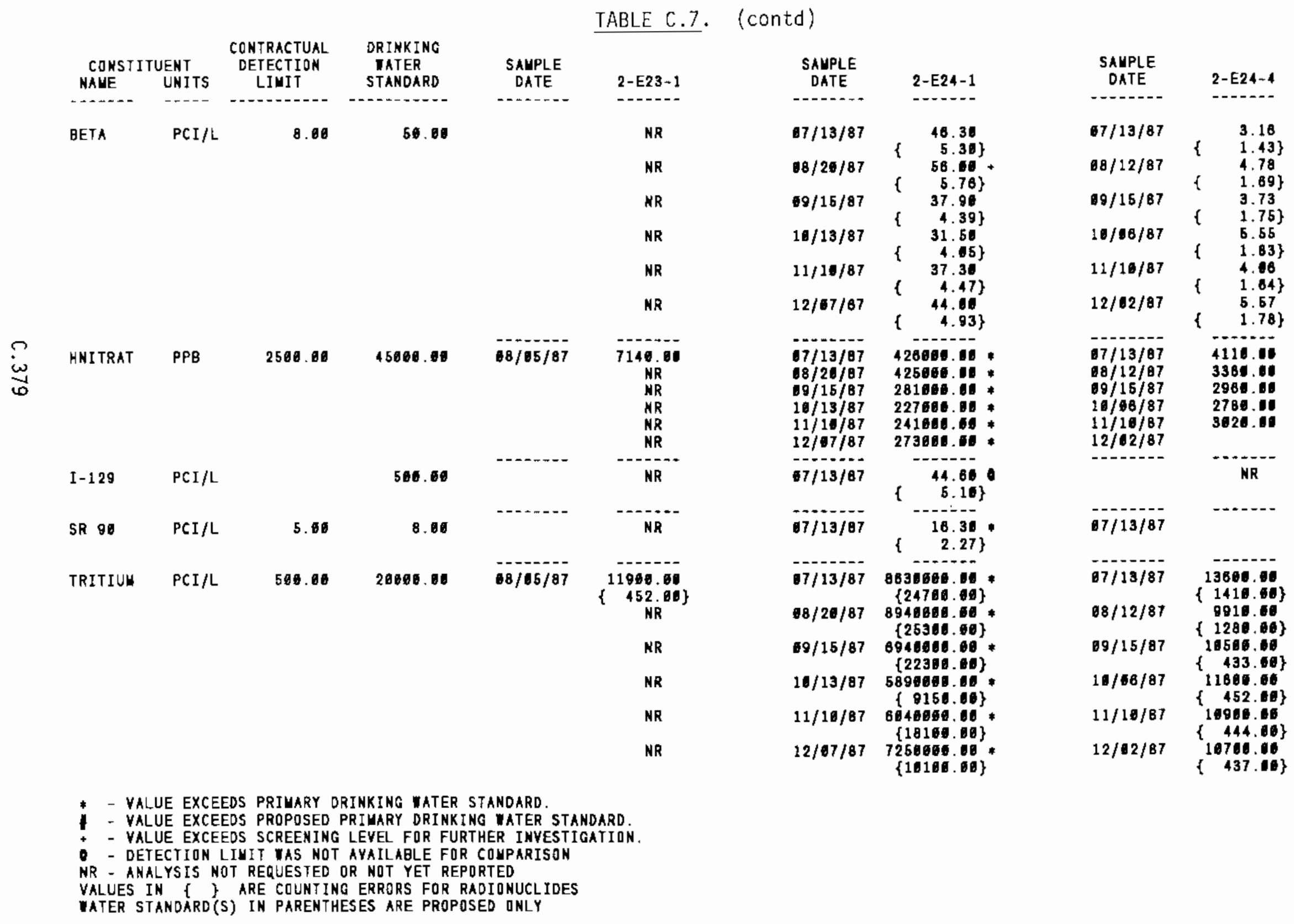




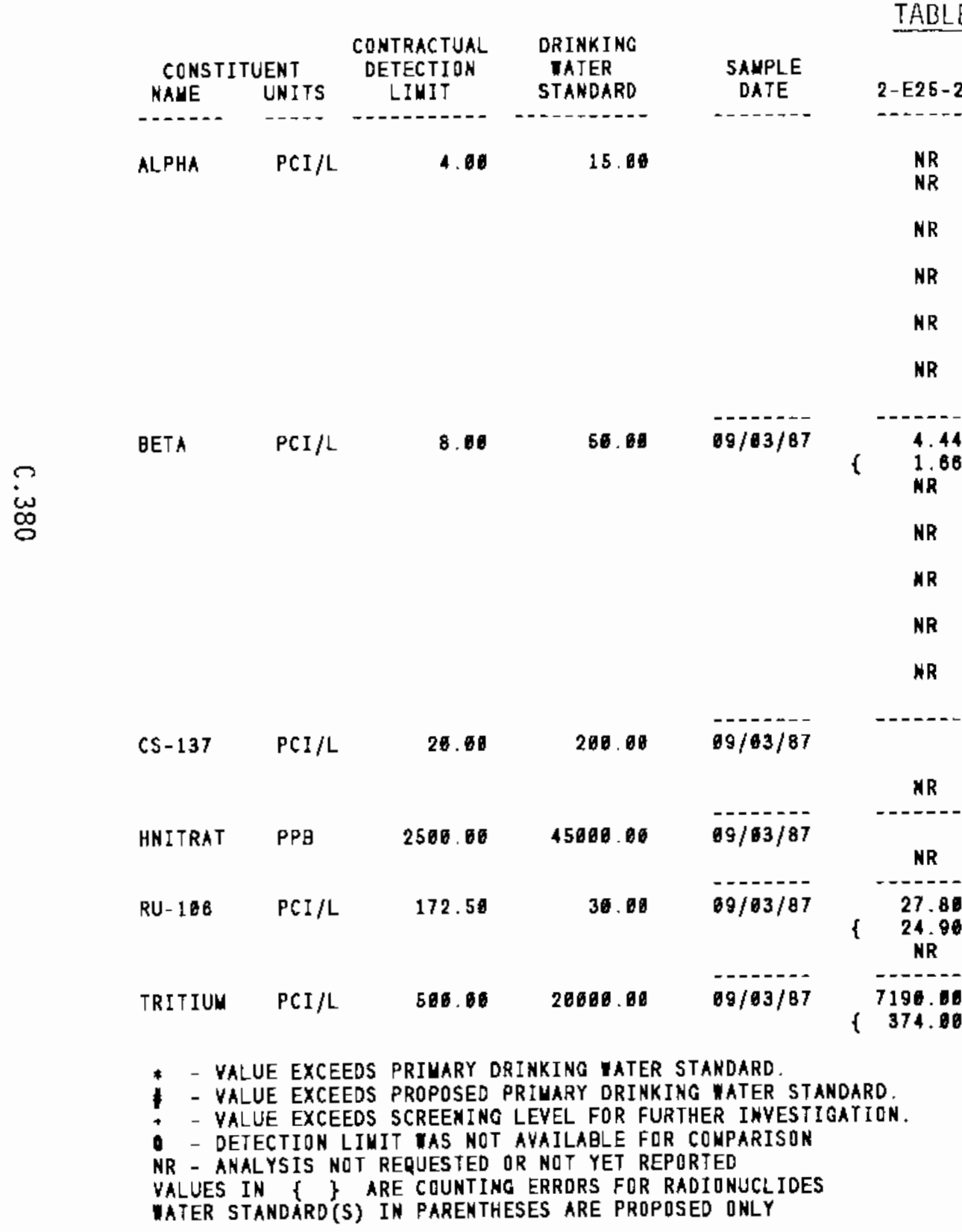

ABLE C.7. (contd)

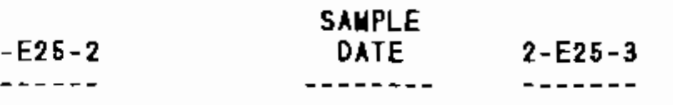
SAYPLE

-.-.....

\begin{tabular}{|c|c|}
\hline & $\begin{array}{l}\text { NR } \\
\text { NR }\end{array}$ \\
\hline & $N R$ \\
\hline & NR \\
\hline & NR \\
\hline & NR \\
\hline - & $--\ldots-$ \\
\hline $10 / 03 / 87$ & $\begin{array}{r}6.24 \\
1 \quad 1.73\end{array}$ \\
\hline $10 / 28 / 87$ & $\begin{array}{ll} & \begin{array}{l}4.42 \\
1.64 \\
N R\end{array}\end{array}$ \\
\hline & NR \\
\hline & NR \\
\hline & $R R$ \\
\hline$--1, \ldots$ & -..-- \\
\hline $09 / 03 / 67$ & $\left\{\begin{array}{l}6.40 \\
4.5 \\
N R\end{array}\right\}$ \\
\hline 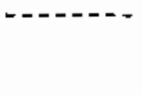 & $\begin{array}{l}\text { NR } \\
\text { NR }\end{array}$ \\
\hline $69 / 63 / 87$ & $--x-1-0$ \\
\hline & NR \\
\hline $69 / 93 / 87$ & $\begin{array}{r}3110.00 \\
\{\quad 288.00\end{array}$ \\
\hline
\end{tabular}

\begin{tabular}{|c|c|}
\hline $\begin{array}{l}\theta 7 / 10 / B 7 \\
8 B / 18 / 87\end{array}$ & 0.73 \\
\hline $09 / 13 / 87$ & $\begin{array}{l}0.42\} \\
0.95\end{array}$ \\
\hline $10 / 09 / 87$ & $\begin{array}{l}0.47\} \\
1.28\end{array}$ \\
\hline $11 / 16 / 87$ & $\begin{array}{l}0.52\} \\
1.45\end{array}$ \\
\hline $12 / 07 / 87$ & $\begin{array}{l}.55 \\
1.17 \\
0.51\}\end{array}$ \\
\hline $97 / 16 / 87$ & 4.81 \\
\hline $68 / 18 / 87$ & $\begin{array}{l}1.72\} \\
4.03 \\
1.643\end{array}$ \\
\hline $10 / 13 / 87$ & $\begin{array}{l}4.54 \\
1.85\end{array}$ \\
\hline $10 / 09 / 87$ & $\begin{array}{l}5.23 \\
1.77\end{array}$ \\
\hline $11 / 10 / 87$ & $\begin{array}{r}4.93 \\
\{\quad 1.72\end{array}$ \\
\hline $12 / 07 / 87$ & $\begin{array}{r}4.32 \\
\{\quad 1.87\}\end{array}$ \\
\hline $07 / 1 / 87$ & $-\cdots$ \\
\hline $10 / 09 / 87$ & \\
\hline $\begin{array}{l}07 / 10 / 87 \\
10 / 69 / 87\end{array}$ & 3290.00 \\
\hline $67 / 10 / 87$ & \\
\hline $16 / 69 / 87$ & \\
\hline $67 / 10 / 87$ & $\left\{\begin{array}{r}4830.06 \\
1158.00\}\end{array}\right.$ \\
\hline
\end{tabular}

NALUES IN $\{$ \& ARE CGUNTING ERRORS FO 
TABLE C.7. (contd)

\begin{tabular}{|c|c|c|c|c|c|c|c|c|c|}
\hline $\begin{array}{l}\text { CONSTI } \\
\text { NAME }\end{array}$ & $\begin{array}{l}\text { JENT } \\
\text { UNITS }\end{array}$ & $\begin{array}{c}\text { CONTRACTUAL } \\
\text { DEIECTION } \\
\text { LIMIT }\end{array}$ & $\begin{array}{l}\text { DRINKING } \\
\text { WFER } \\
\text { STANDARD }\end{array}$ & $\begin{array}{c}\text { SAMPLE } \\
\text { DATE }\end{array}$ & $2-E 25-2$ & $\begin{array}{l}\text { SAUPLE } \\
\text { DATE }\end{array}$ & $2-E 25-3$ & $\begin{array}{l}\text { SAUPLEE } \\
\text { DATEE }\end{array}$ & $2-E 25-8$ \\
\hline \multirow[t]{5}{*}{ TRITIUU } & $\mathrm{PCI} / \mathrm{L}$ & 589.60 & 206000.00 & & NR & & NR & $68 / 18 / 87$ & \multirow{5}{*}{ 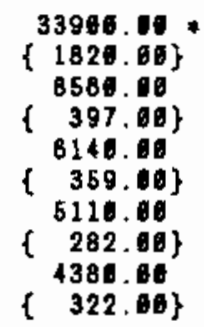 } \\
\hline & & & & & NR & & NR & $09 / 13 / 87$ & \\
\hline & & & & & NR & & NR & $10 / 09 / 87$ & \\
\hline & & & & & NR & & NR & $11 / 18 / 87$ & \\
\hline & & & & & NR & & NR & $12 / 07 / 87$ & \\
\hline
\end{tabular}

* - Value exceEds primary drinking mater standard.

- VALUE EXCEEDS PROPOSED PRIMARY DRTNKING YATER STANDARD

- VALUE EXCEEDS SCREENING LEYEL fOR FURTHER INYESTIGATION.

- - detection liuit nas not available for couparison

NR - ANALYSIS NOT REQUESTED OR NOT YET REPORTED

VALUES IN \{\} ARE CDUNTING ERRORS FOR RADIONUCLIDES

TATER STANDARD(S) IN PARENTHESES ARE PROPOSED ONLY 
TABLE C.7. (contd)

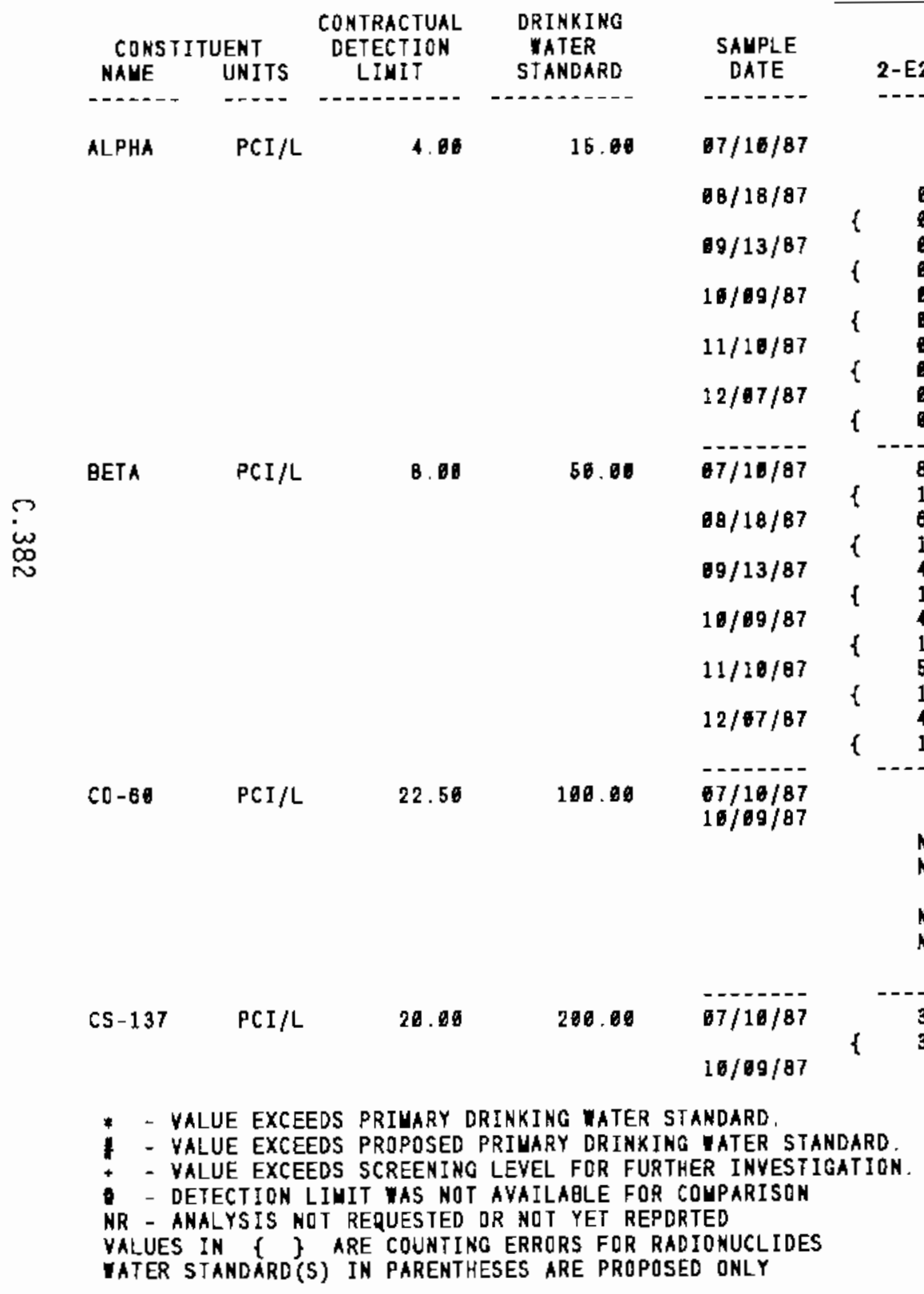

\begin{tabular}{|c|c|}
\hline $\begin{array}{l}\text { SAMPLE } \\
\text { DATE }\end{array}$ & $2-E 25-11$ \\
\hline $1 / 10 / 87$ & 1. 80 \\
\hline $88 / 18 / 87$ & $\begin{array}{l}\text { a. } 49\} \\
\text { B. } 51\end{array}$ \\
\hline 89/13/87 & $\begin{array}{l}0.38 \\
0.79\end{array}$ \\
\hline $16 / 68 / 87$ & 1.41 \\
\hline $11 / 09 / 87$ & $\begin{array}{l}0.64 \\
8.89 \\
8.41\end{array}$ \\
\hline $12 / 87 / 87$ & $\begin{array}{r}0.06 \\
(\quad 0.45)\end{array}$ \\
\hline $97 / 10 / 87$ & 8.77 \\
\hline $08 / 18 / 87$ & $\begin{array}{l}2.21 \\
9.50\end{array}$ \\
\hline $69 / 13 / 87$ & 11.50 \\
\hline $10 / 08 / 87$ & $\begin{array}{r}2.44 \\
16.90\end{array}$ \\
\hline $11 / 89 / 87$ & $\begin{array}{r}2.333 \\
15.86\end{array}$ \\
\hline $12 / 67 / 87$ & $\left.\begin{array}{r}2.72 \\
100 \\
\{\quad 2.40\end{array}\right\}$ \\
\hline $\begin{array}{l}07 / 10 / 87 \\
08 / 18 / 87 \\
68 / 13 / 87 \\
16 / 96 / 87\end{array}$ & \\
\hline $\begin{array}{l}11 / 69 / 87 \\
12 / 67 / 87\end{array}$ & $\left\{\begin{array}{r}5.05 \\
4.51\end{array}\right.$ \\
\hline & \\
\hline
\end{tabular}

$08 / 18 / 87$ 
TABLE C.7. (contd)

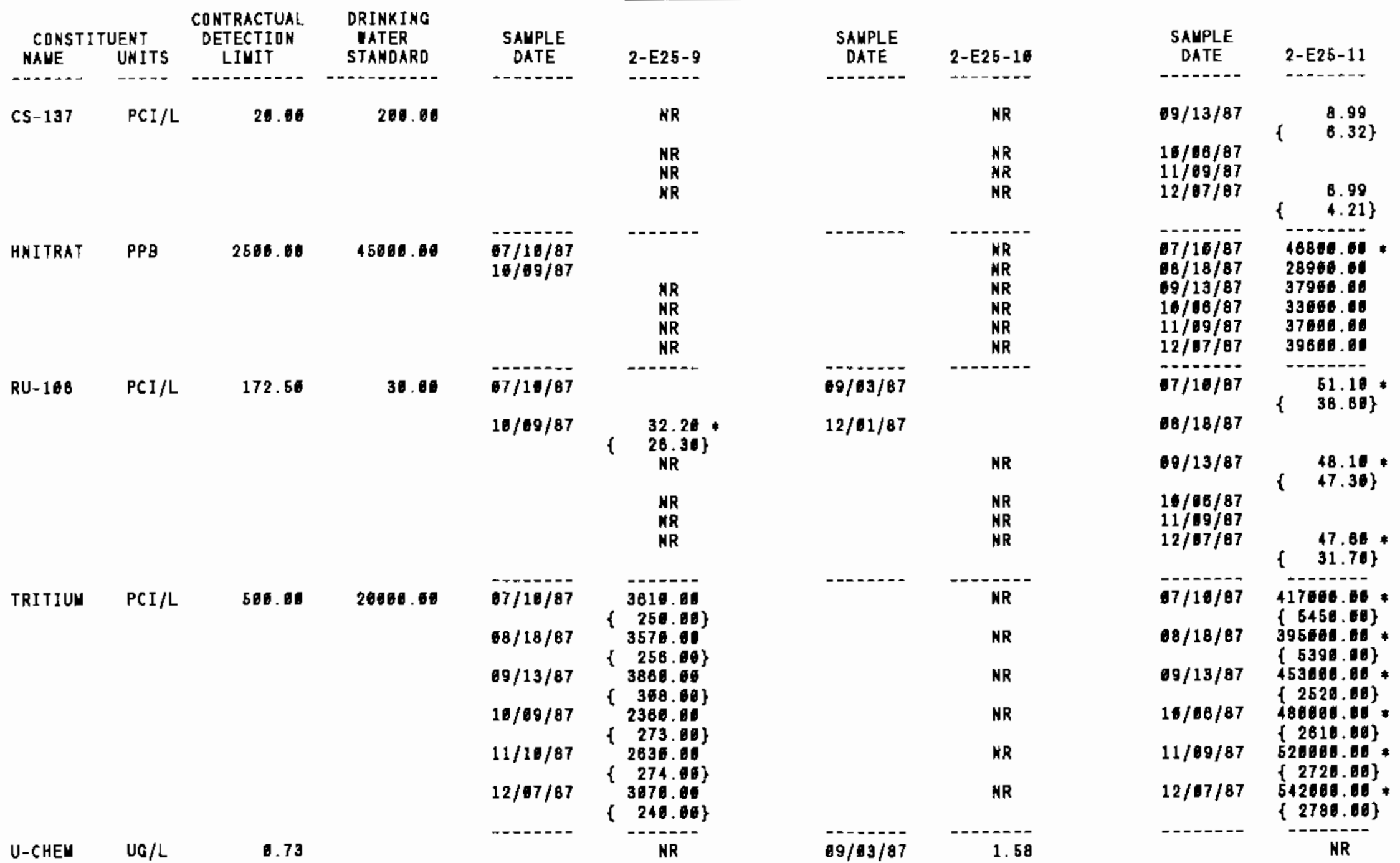

* - value exceeds primary drinking mater standard.

- YALUE EXcEeds PROPOSED PRIUARY DRINKING tater STANDARD.

- - YALUE EXCEEDS SCREEnING LEVEL FOR FURTHER INYESTIGation.

- DETECTIDN LIUIT WAS NOT AYAILABLE FOR COMPARISON

NR - ANALYSIS NDT REQUESTED OR NDT YET REPORTED

VALUES IN \{\} ARE COUNTIMG ERRORS FOR RADIDNUCLIDES

IATER STANDARD'(S) IN PARENTHESES ARE PROPOSED ONLY 
TABLE C.7. (contd)

\begin{tabular}{|c|c|c|c|c|c|c|c|c|c|}
\hline $\begin{array}{c}\text { CONST } \\
\text { NAUE }\end{array}$ & $\begin{array}{l}\text { UENT } \\
\text { UNI TS }\end{array}$ & $\begin{array}{c}\text { CONTRACTUAL } \\
\text { DETECTION } \\
\text { LINIT }\end{array}$ & $\begin{array}{l}\text { DRINKING } \\
\text { WATER } \\
\text { STANDARD }\end{array}$ & $\begin{array}{c}\text { SALPLE } \\
\text { DATE }\end{array}$ & $2-E 25-9$ & $\begin{array}{l}\text { SAUPLE } \\
\text { DATE }\end{array}$ & $2-E 25-16$ & $\begin{array}{c}\text { SAMPLE } \\
\text { DATE }\end{array}$ & $2-E 25-11$ \\
\hline$-\ldots-n$ & ---- & U & - & $-\cdots$ & $--n--$ & 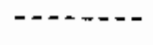 & $\cdots+-1$ & 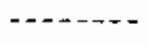 & $-\cdots--.-$ \\
\hline $\mathrm{U}-\mathrm{CHEH}$ & $U G / L$ & 6.73 & & & NR & $12 / 01 / 87$ & 1.52 & & NR \\
\hline
\end{tabular}

* - value exceEds primary dRinking tater standard

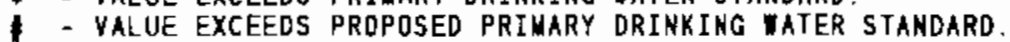

- VALUE EXCEEDS SCREENING LEVEL FOR FURTHER INVESTIGATION.

- - DETECTION LINIT WAS NDT AVAILABLE FOR COUPARISON

NR - ANALYSIS NDT REQUESTED DR NDT YET REPORTED

VALUES IN \{\} ARE COUNTING ERRORS FOR RADIONUCLIDES

WATER STANDARD(S) IN PARENTHESES ARE PROPOSED ONLY 
TABLE C.7. (contd)

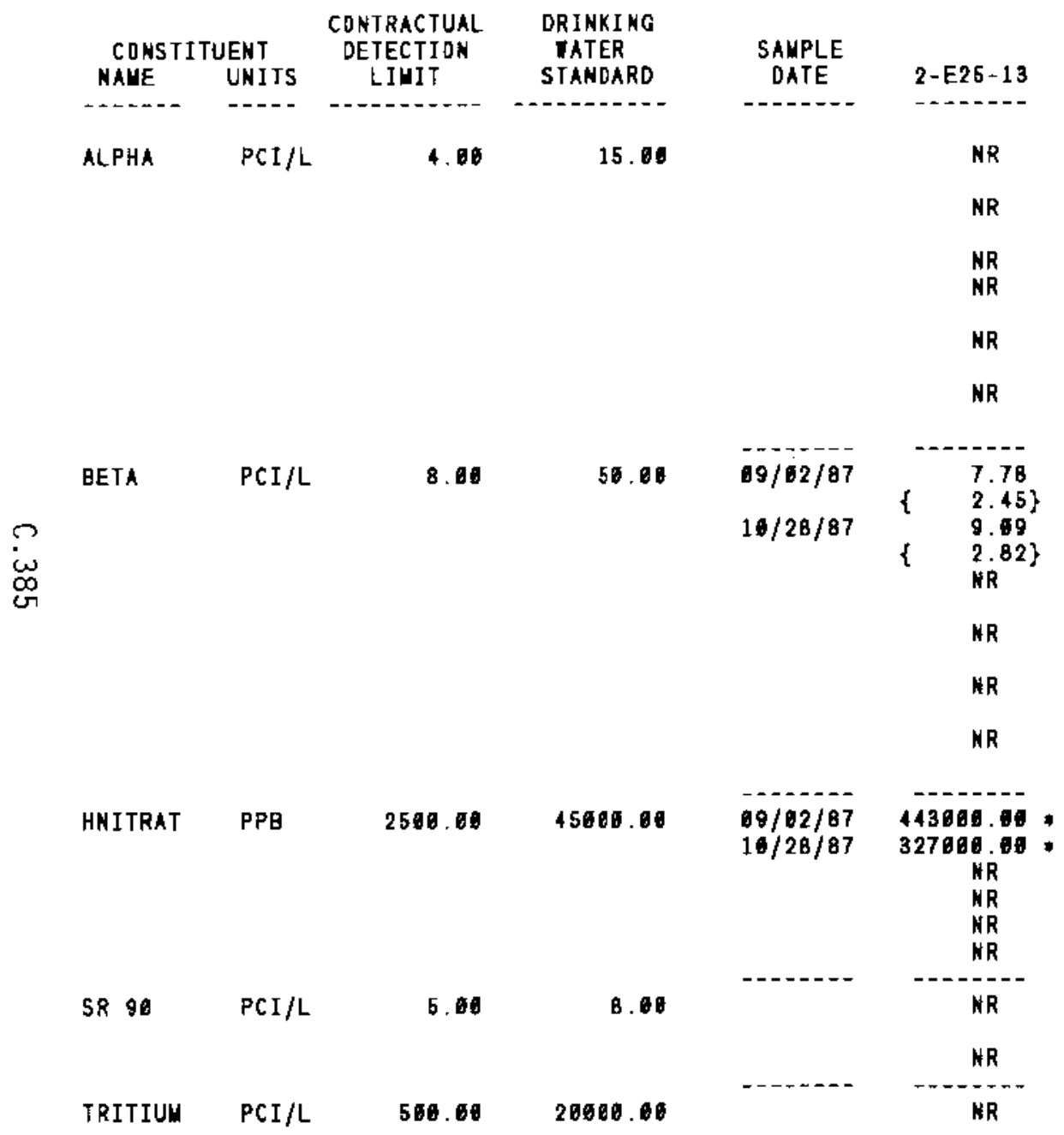

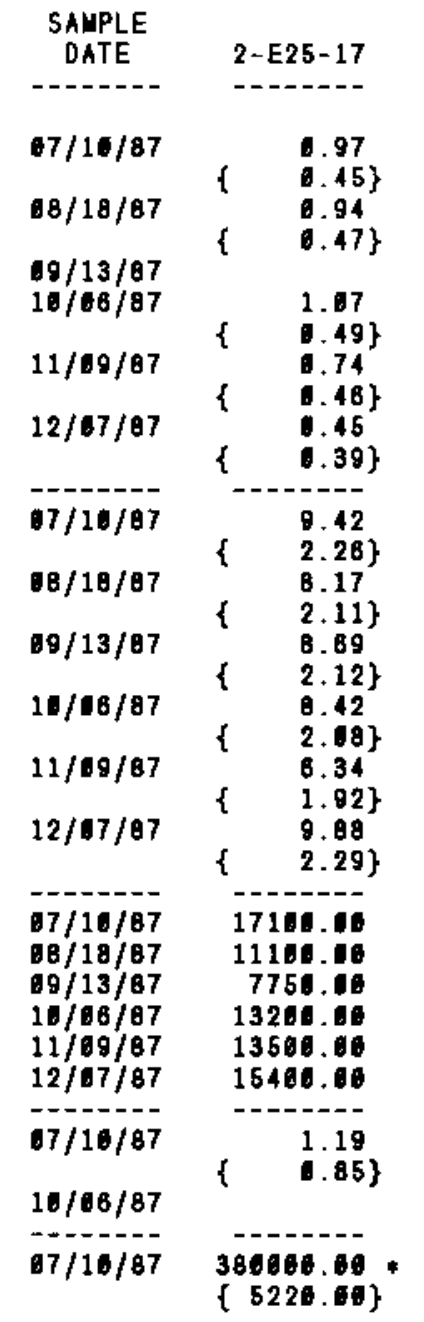

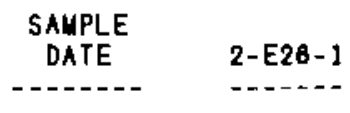

NR

NR

NR

NR

NR

NR

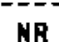

NR

WR

MR

NR

NR

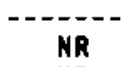

NR

NR

NR

NR

NR

NR

20

$528.66\}$

* - VALUE EXCEEDS PRIMARY DRINKING WATER STANDARD.

* - VALUE EXCEEDS PROPOSED PRIUARY DRINKING YATER STAMDARD.

+ - VALUE EXCEEDS SCREENING LEYEL FDR FURTHER INYESTIGATIDN.

- - detection limit us not available for comparison

NR - ANALYSIS NOT REQUESTED OR NOT YET REPORTED

VALUES IN $(?)$ ARE COUNTING ERRORS FOR RADIQNUCLIDES

WATER STAHDARD'S) IN PARENTHESES ARE PRDPOSED DNLY 
TABLE C.7. (contd)

\begin{tabular}{|c|c|c|c|c|c|}
\hline $\begin{array}{l}\text { CONSTIT } \\
\text { NAHE }\end{array}$ & $\begin{array}{l}\text { UENT } \\
\text { UHI ITS }\end{array}$ & $\begin{array}{c}\text { CONTRACTUAL } \\
\text { DETECTION } \\
\text { LIUIT }\end{array}$ & $\begin{array}{l}\text { DRINKING } \\
\text { MATER } \\
\text { STANDARD }\end{array}$ & $\begin{array}{l}\text { SAMPLE } \\
\text { DATE }\end{array}$ & $2-E 25-13$ \\
\hline \multirow[t]{5}{*}{ TRITIUN } & $\mathrm{PCI} / \mathrm{L}$ & & & & NR \\
\hline & & & & & NR \\
\hline & & & & & NR \\
\hline & & & & & NR \\
\hline & & & & & \\
\hline
\end{tabular}

\begin{tabular}{|c|c|}
\hline $\begin{array}{l}\text { SAAPLE } \\
\text { DATE }\end{array}$ & 2-E25-17 \\
\hline$/ 18 / 87$ & \\
\hline $69 / 13 / 87$ & $\begin{array}{l}14046.60\} \\
179060.00\end{array}$ \\
\hline $10 / 98 / 87$ & $\begin{array}{l}281006.00 \\
\{2000.00\}\end{array}$ \\
\hline $11 / 68 / 87$ & 274008.00 \\
\hline $12 / 07 / 87$ & $\begin{array}{l}382690.60 \\
\{2300.60\}\end{array}$ \\
\hline
\end{tabular}

SANPLE

2-E28-1
$00 / 18 / 87 \quad 223600.00 \%$
$179000.00 *$$$
\text { DATE }
$$

NR

HR

MR

NR

NR

- - value exceeds primary drinxing vater standard.

- - VALUE EXCEEDS PROPOSED PRIUARY ORINKING UATER STANDARO

+ - VALUE EXCEEDS SCREENIRg LEVEL FOR FURTHER INYESTIGATION.

- DETECTION LIUIT WAS NDT AVAILABLE FOR COUPARISON

NR - ANALYSIS NOT REQUESTED OR NOT YET REPORTED

VALUES IN \{\} ARE COUNTING ERRORS FOR RADIONUCLIDES

WATER STANDARD(S) IN PARENTHESES ARE PROPOSED GNLY 
TABLE C.7. (contd)

\begin{tabular}{|c|c|c|c|c|c|c|c|c|c|}
\hline $\begin{array}{l}\text { CONSTI } \\
\text { NAUE }\end{array}$ & $\begin{array}{l}\text { UENT } \\
\text { UNI TS }\end{array}$ & $\begin{array}{c}\text { CONTRACTUAL } \\
\text { DETECTION } \\
\text { LIMIT }\end{array}$ & $\begin{array}{l}\text { DRINKING } \\
\text { TATER } \\
\text { STANDARD }\end{array}$ & $\begin{array}{c}\text { SAMPLE } \\
\text { DATE }\end{array}$ & $2-E 26-2$ & $\begin{array}{c}\text { SANPLEE } \\
\text { DATE }\end{array}$ & $2-E 26-3$ & $\begin{array}{l}\text { SAUPLEE } \\
\text { DATEE }\end{array}$ & $2-E 28-4$ \\
\hline BETA & PCI/L & 8.68 & 50.80 & $\begin{array}{l}69 / 93 / 87 \\
12 / 01 / 87\end{array}$ & 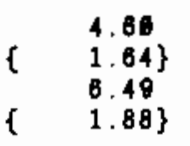 & & $\begin{array}{l}\text { NR } \\
\text { NR }\end{array}$ & $\begin{array}{l}69 / 63 / 87 \\
12 / 01 / 87\end{array}$ & 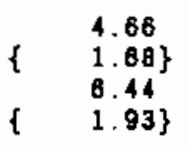 \\
\hline TRITIUN & PCI $/ \mathrm{L}$ & 590.09 & 20000.00 & $\begin{array}{l}69 / 03 / 87 \\
12 / 01 / 87\end{array}$ & $\begin{array}{r}2470.06 \\
271.06\} \\
2848.86 \\
281.06\}\end{array}$ & $08 / 03 / 87$ & $\left\{\begin{array}{c}3356.00 \\
293.06\} \\
N R\end{array}\right\}$ & $\begin{array}{l}60 / 03 / 87 \\
12 / 01 / 87\end{array}$ & $\left.\begin{array}{c}38866.06 \\
\left\{\begin{array}{c}344.06 \\
38106.08\end{array}\right. \\
\{787.86\end{array}\right\}$ \\
\hline
\end{tabular}

$\underset{\omega}{\omega}$

* - value exceeds privary dRinking mater standard

- VALUE EXCEEDS PROPOSED PRI PARY DRI HKING IATER STANDARD.

- - YALUE EXCEEDS SCREENING LEVEL for fuRTHER InVESTIGATION.

- DETECTION LIUIT IAS NOT AVAILABLE FoR COUPARISON

NR - ANALYSIS ROT REQUESTED OR NOT YET REPORTED

YALUES IN \{ $\}$ ARE COUNTING ERRORS FOR RADIONUCLIDES

WATER STAKDARD(S) IN PARENTHESES ARE PROPOSED ONLY 


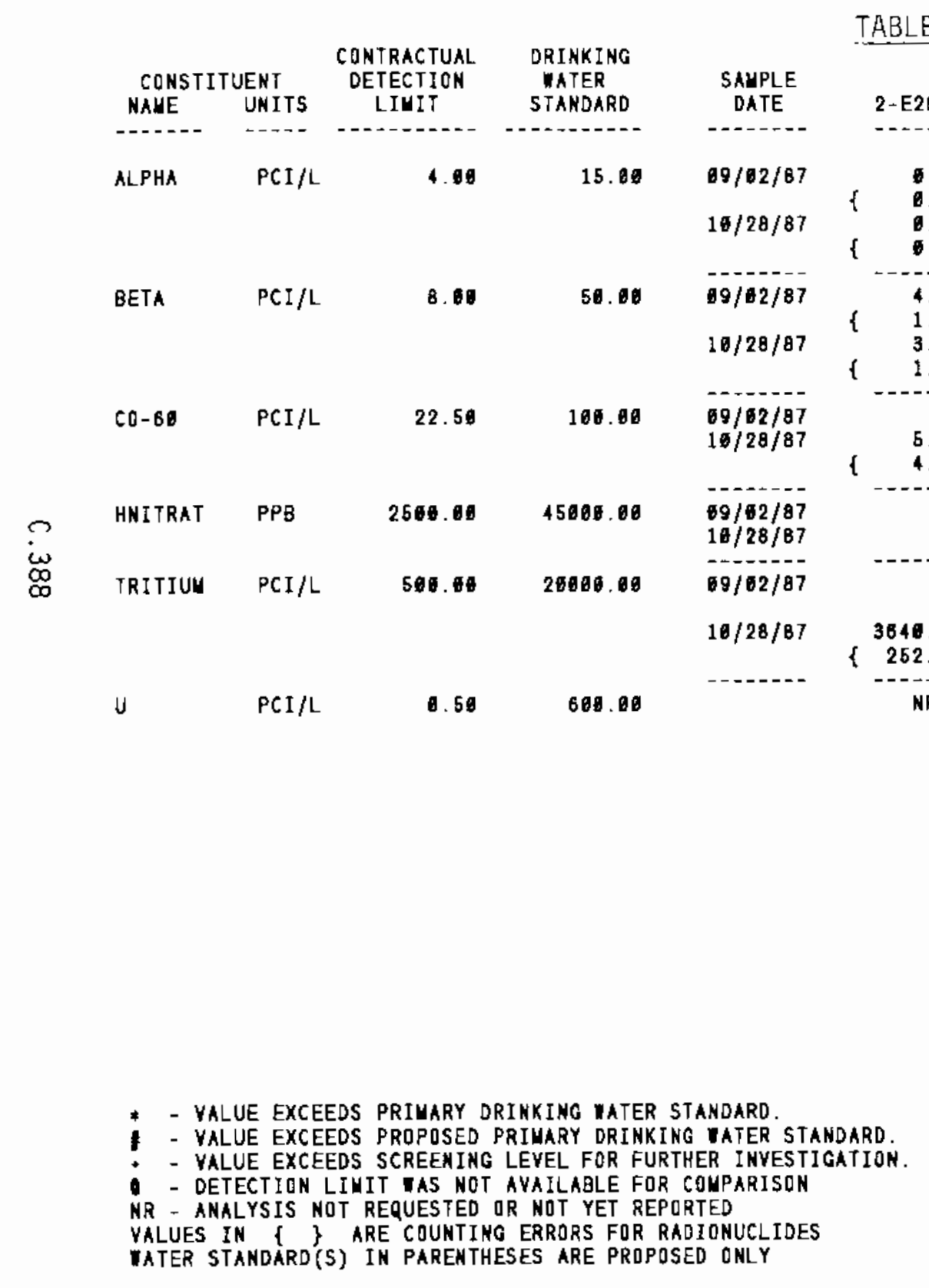




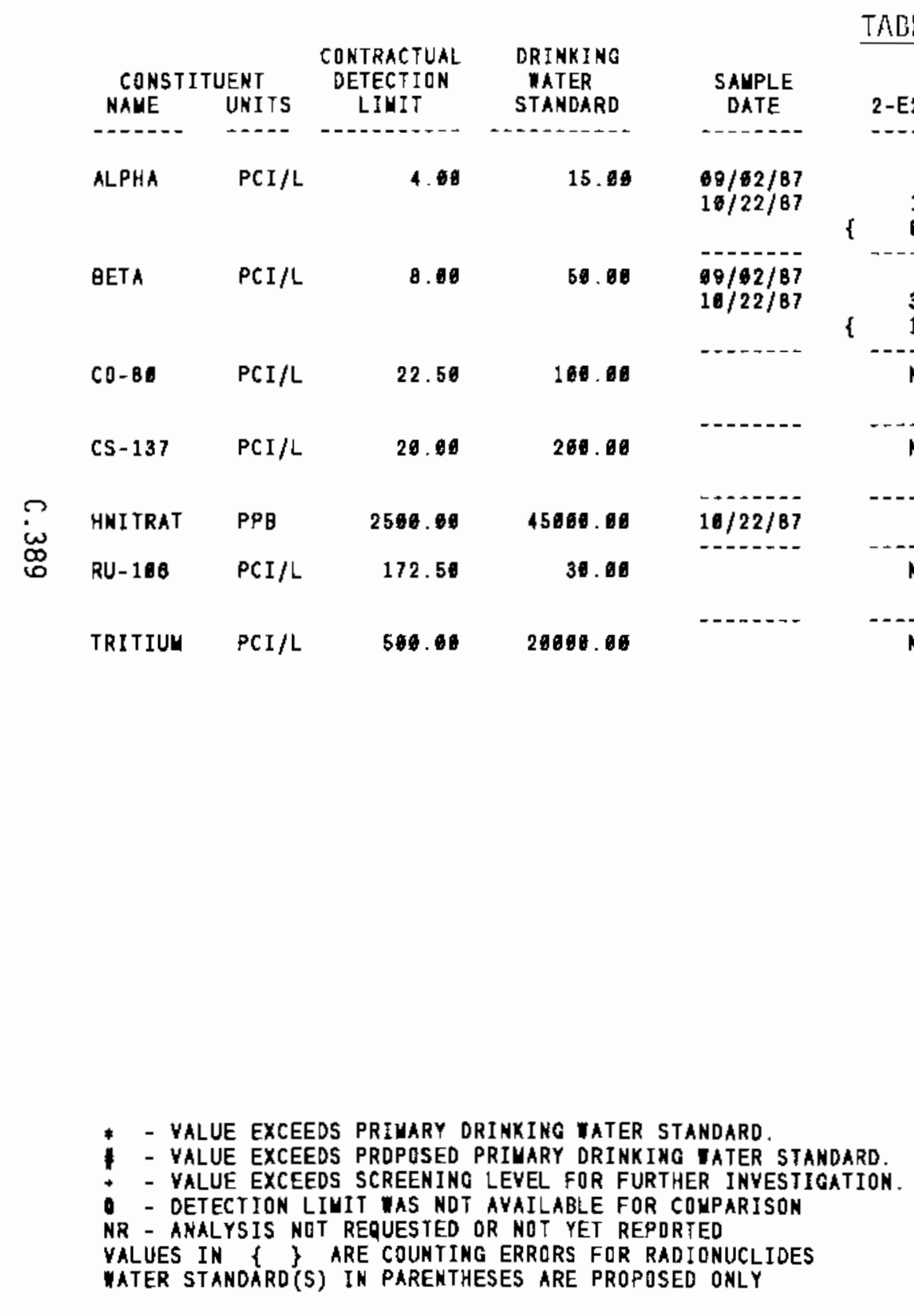

ADLE C.7. (contd)

\begin{tabular}{|c|c|c|c|c|}
\hline$-E 27-7$ & $\begin{array}{c}\text { SAMPLE } \\
\text { DATE }\end{array}$ & $2-E 28-1$ & $\begin{array}{l}\text { SAUPLE } \\
\text { DATE }\end{array}$ & $2-E 2 B-5$ \\
\hline$---n$ & ------ & ----- & $-+\cdots+\cdots$ & $-\cdots-\cdots$ \\
\hline $\begin{array}{l}1.69 \\
0.58\}\end{array}$ & & $\begin{array}{l}\text { NR } \\
\text { NR }\end{array}$ & & $\begin{array}{l}\text { NR } \\
\text { NR }\end{array}$ \\
\hline $\begin{array}{l}3 . B 8 \\
1.5 B\}\end{array}$ & - & $\begin{array}{l}\text { NR } \\
\text { NR }\end{array}$ & $-\ldots, \ldots$ & NR \\
\hline NR & $08 / 81 / 87$ & $\begin{array}{r}7.36 \\
8.01\}\end{array}$ & $69 / 61 / 87$ & 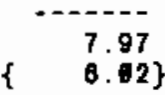 \\
\hline NR & $69 / 01 / 87$ & ---1 & $99 / 01 / B 7$ & $\left\{\begin{array}{r}4.83 \\
3.85\end{array}\right\}$ \\
\hline---- & $69 / 01 / 87$ & 4850.66 & $69 / 01 / 87$ & 2000.06 \\
\hline NR & $09 / 01 / 67$ & $\left\{\begin{array}{c}59.60 \\
39.76\}\end{array}\right.$ & $19 / 01 / 87$ & $--\infty-\infty$ \\
\hline NR & $09 / 01 / 87$ & $\begin{array}{r}7176.00 \\
375.00\}\end{array}$ & $09 / 81 / 87$ & $\left\{\begin{array}{r}2540.00 \\
274.00\}\end{array}\right.$ \\
\hline
\end{tabular}




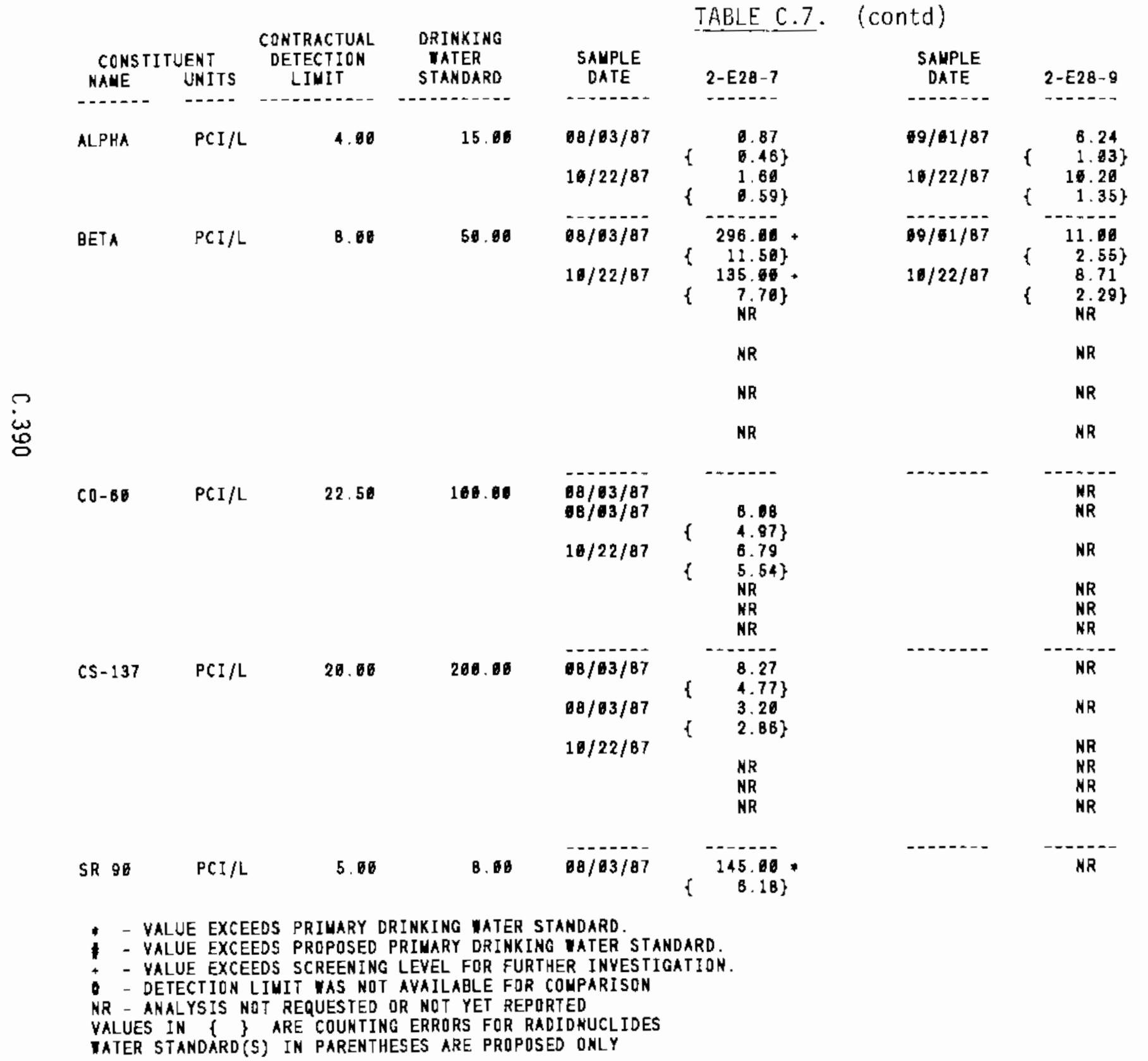




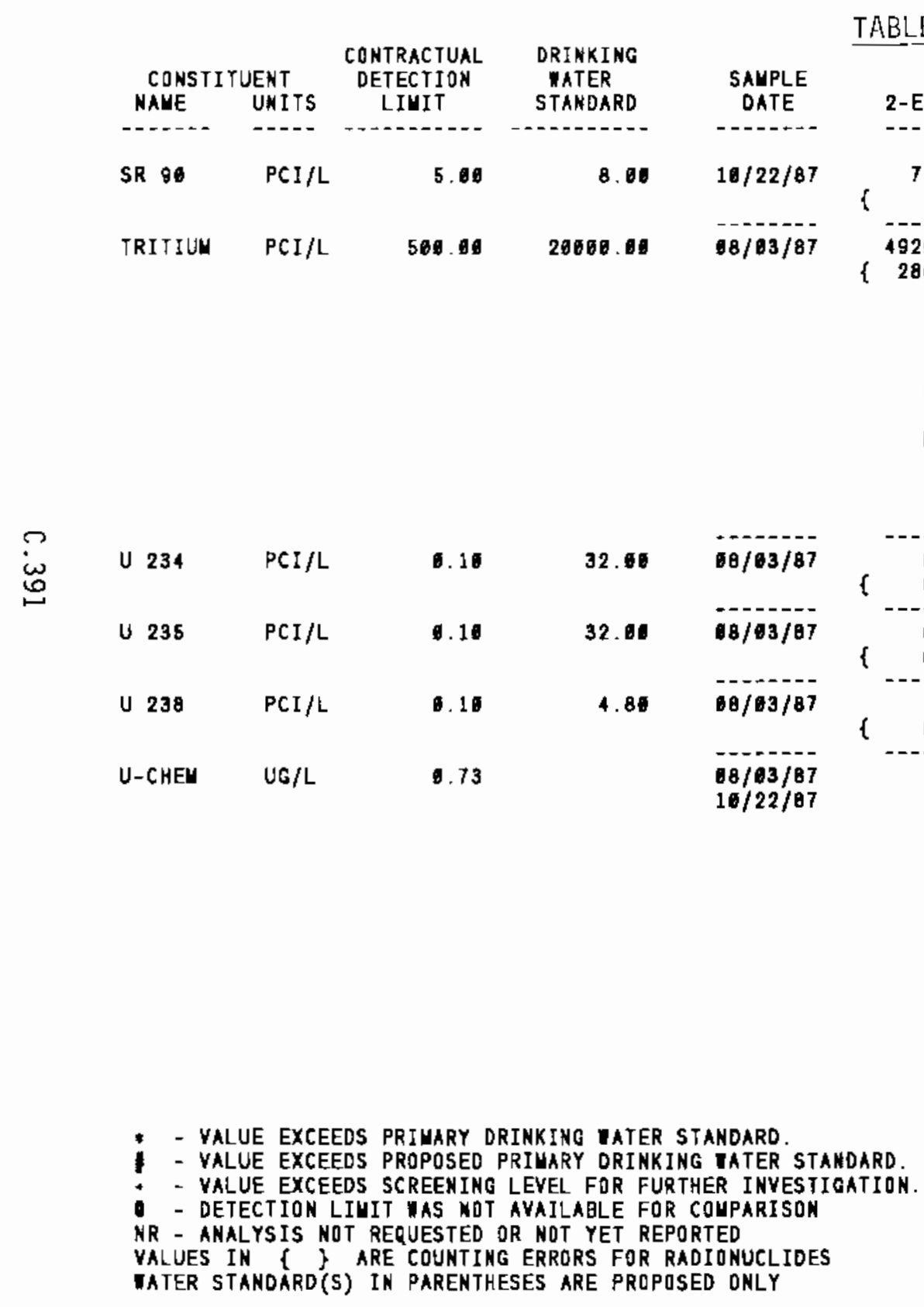


TABLE C.7. (contd)

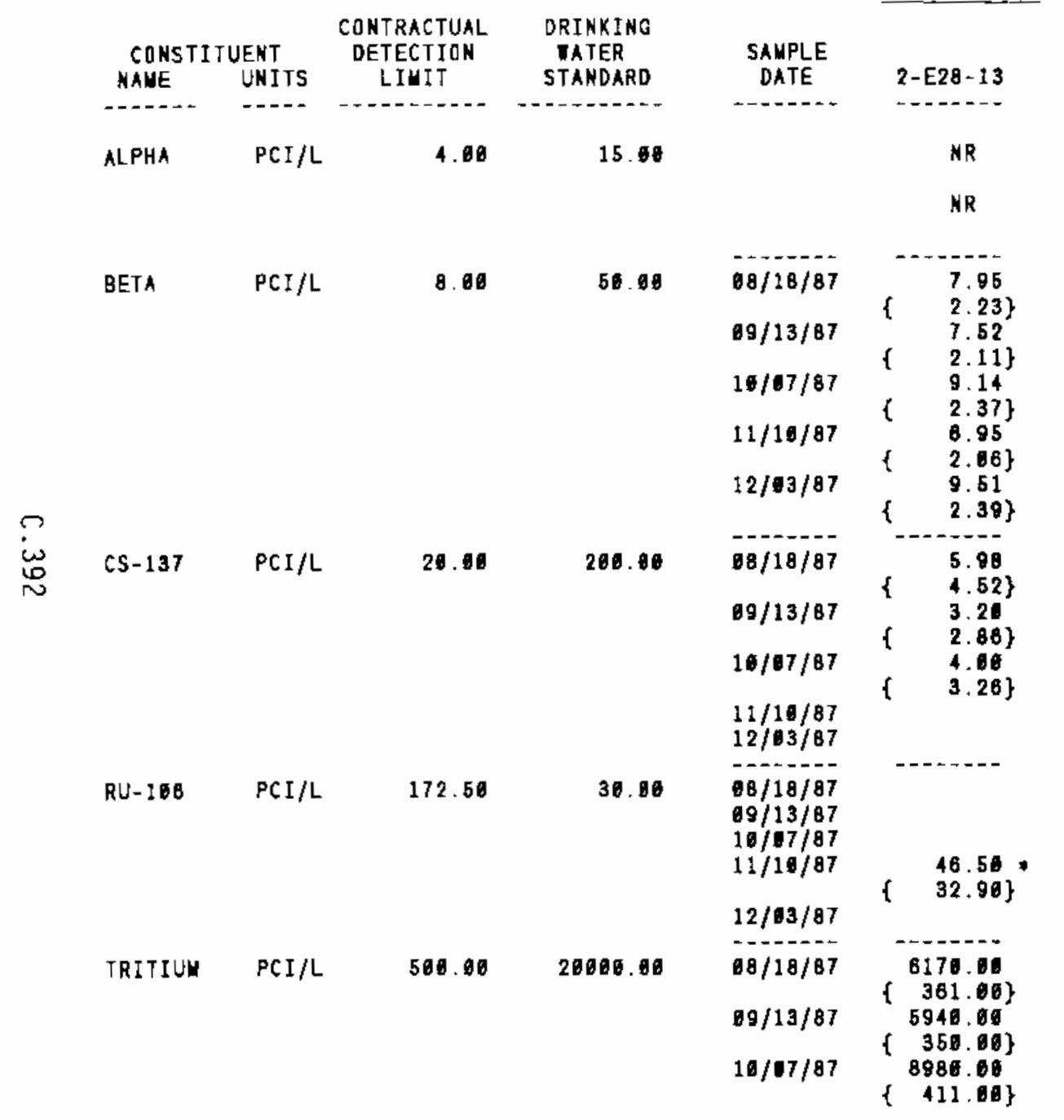

\begin{tabular}{|c|c|}
\hline $\begin{array}{l}\text { SAYPLE } \\
\text { DATE }\end{array}$ & $2-E 28-18$ \\
\hline $69 / 01 / 87$ & 6. 37 \\
\hline $16 / 22 / 87$ & $\begin{array}{r}7.14 \\
\{\quad 1.14\}\end{array}$ \\
\hline $09 / 01 / 87$ & 0.86 \\
\hline $10 / 22 / 87$ & $\left\{\begin{array}{l}7.54 \\
\{\quad 2.10\}\end{array}\right.$ \\
\hline & NR \\
\hline & NR \\
\hline - & MR \\
\hline & NR \\
\hline & NR \\
\hline & $\begin{array}{l}\text { NR } \\
\text { NR }\end{array}$ \\
\hline & $\begin{array}{l}\text { NR } \\
\text { NR } \\
\text { NR } \\
\text { NR }\end{array}$ \\
\hline & NR \\
\hline & NR \\
\hline & NR \\
\hline & NR \\
\hline
\end{tabular}

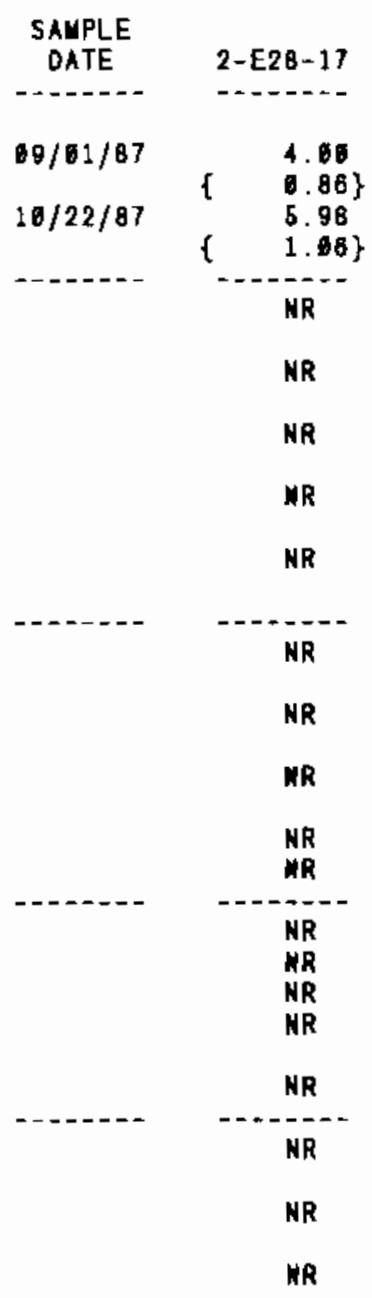

* - value exceeds primary orinking tater standard

1 - VALUE EXCEEDS PROPOSED PRIUARY dRINKIMG YaTER STANDARD

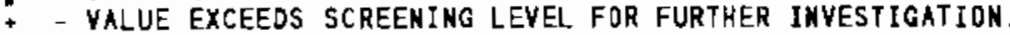

- detection limit as not ayailable for couparison

NR - ANALYSIS NOT REQUESTED OR NOT YET REPORTED

VALUES IN \{\} ARE COUNTING ERRORS FOR RADIONUCLIDES

WATER STAKOARD(S) IN PARENTHESES aRE PRDPOSED OMLY 
TABLE C.7. (contd)

\begin{tabular}{|c|c|c|c|c|c|c|c|c|c|}
\hline $\begin{array}{l}\text { CONSTI } \\
\text { NAUE }\end{array}$ & $\begin{array}{l}\text { UENT } \\
\text { UNITS }\end{array}$ & $\begin{array}{c}\text { CONTRACTUAL } \\
\text { DETECTION } \\
\text { LIUIT }\end{array}$ & $\begin{array}{l}\text { DRINKING } \\
\text { WATER } \\
\text { STANDARD }\end{array}$ & $\begin{array}{l}\text { SAUPLE } \\
\text { OATE }\end{array}$ & $2-E 28-13$ & $\begin{array}{l}\text { SAHPLE } \\
\text { DATE }\end{array}$ & 2-E28-16 & $\begin{array}{c}\text { SAUPLE } \\
\text { DATE }\end{array}$ & $2-E 28-17$ \\
\hline------- & $\ldots \ldots$ & $-\ldots$ & 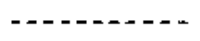 & 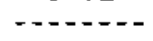 & ----- & $-\ldots-1$ & $---1--$ & ------ & ------ \\
\hline TRITIUH & PCI /L & 500.00 & 20000.00 & & $\begin{array}{r}7100.00 \\
\{\quad 382.06\}\end{array}$ & & NR & & NR \\
\hline & & & & $12 / 03 / B 7$ & $\begin{array}{r}5300.00 \\
\quad 340.00\}\end{array}$ & & NR & & NR \\
\hline U-CHEH & $U G / L$ & 0.73 & & 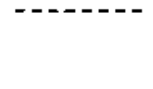 & $\begin{array}{l}\text { NR } \\
\text { NR }\end{array}$ & $\begin{array}{l}09 / 01 / 97 \\
16 / 22 / 97\end{array}$ & $\begin{array}{r}11.60 \\
9.21\end{array}$ & $\begin{array}{l}69 / 61 / 87 \\
16 / 22 / 87\end{array}$ & $\begin{array}{r}7.78 \\
5.92\end{array}$ \\
\hline
\end{tabular}

* - VALUE EXCEEDS PRIMARY DRINKING MaTER STANDARD.

- VALUE EXCEEDS PROPOSED PRIMARY DRINKIHG MATER STANDARD

+ VALUE EXCEEDS SCREENING LEVEL FOR FURTHER INVESTIGATION.

- DETECTION LIMIT TAS NOT AVAILABLE FOR COUPARISON

NR - ANALYSIS NOT REQUESTED OR NOT YET REPORTED

VALUES IN \{ $\}$ ARE COUNTING ERRORS FOR RADIONUCLIDES

WATER STANDARD(S) IN PARENTHESES ARE PRDPOSED ONLY 
TABLE C.7. (contd)

\begin{tabular}{|c|c|c|c|c|c|}
\hline $\begin{array}{c}\text { CONST } \\
\text { NAWE }\end{array}$ & $\begin{array}{l}\text { UENT } \\
\text { UNITS }\end{array}$ & $\begin{array}{l}\text { CONTRACTUAL } \\
\text { DETECTION } \\
\text { LINIT }\end{array}$ & $\begin{array}{l}\text { DRINKING } \\
\text { VATER } \\
\text { STANDARD }\end{array}$ & $\begin{array}{c}\text { SAUPLE } \\
\text { DATE }\end{array}$ & $2-E 28-18$ \\
\hline ALPHA & $\mathrm{PCI} / \mathrm{L}$ & 4.88 & 15.86 & $\begin{array}{l}87 / 17 / 87 \\
88 / 13 / 87 \\
69 / 13 / 87 \\
16 / 87 / 87 \\
11 / 18 / 87 \\
12 / 83 / 87\end{array}$ & 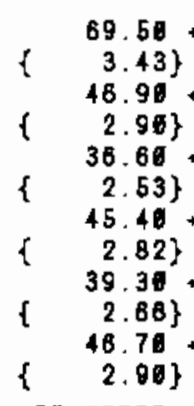 \\
\hline BETA & $P \in I / L$ & 8.00 & 58.86 & $\begin{array}{l}87 / 17 / 87 \\
88 / 13 / 87 \\
69 / 13 / 87 \\
16 / 87 / 87 \\
11 / 16 / 87 \\
12 / 03 / 87\end{array}$ & 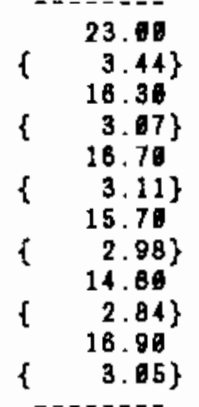 \\
\hline CO-68 & PCI/L & 22.58 & 190.46 & $\begin{array}{l}97 / 17 / 87 \\
80 / 13 / 87 \\
89 / 13 / 87 \\
16 / 97 / 87 \\
11 / 16 / 87 \\
12 / 83 / 87\end{array}$ & 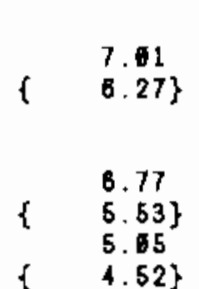 \\
\hline
\end{tabular}

\begin{tabular}{|c|c|}
\hline $\begin{array}{l}\text { SALPLE } \\
\text { DATE }\end{array}$ & $2-E 28-23$ \\
\hline $69 / 61 / 87$ & $23.36+$ \\
\hline \multirow[t]{4}{*}{$10 / 22 / 87$} & $\left\{\begin{array}{c}37.09 \\
5.69 \\
\quad R R\end{array}\right.$ \\
\hline & NR \\
\hline & NR \\
\hline & NR \\
\hline $69 / 81 / 87$ & $\begin{array}{c}11800.06 \\
\{\quad 70.50\}\end{array}$ \\
\hline \multirow[t]{4}{*}{$16 / 22 / 87$} & $\begin{array}{c}9146.00 \\
382.08\} \\
N R\end{array}$ \\
\hline & NR \\
\hline & HR \\
\hline & NR \\
\hline \multirow[t]{3}{*}{$\begin{array}{l}99 / 61 / 87 \\
10 / 22 / 87\end{array}$} & --- \\
\hline & $\begin{array}{l}\text { NR } \\
\text { NR } \\
\text { NR }\end{array}$ \\
\hline & NR \\
\hline 69/01/87 & $\left\{\begin{array}{c}1580.00 \\
\left\{\begin{array}{c}73.56\}\end{array}\right.\end{array}\right.$ \\
\hline
\end{tabular}

SAMPLE

DATE 2-E28-24

$69 / 61 / 87$

$18 / 22 / 87$

$(-\cdots-1-1$

$\begin{array}{llll}\text { CS-137 PCI/L } & 20.60 & 298.06 & 07 / 17 / 87\end{array}$

* - value exceeds primary drinking tater standard

1 - VALUE EXCEEDS PROPOSED PRIUARY DRINKING IATER STANDARD.

- - VAlue eXCeEds SCREening level for further inYestigation.

- DETECTION LIMIT UAS NOT AVAILAGLE FoR COUPARISON

NR - ANALYSIS NOT REQUESTED OR NOT YET REPORTED

VALUES IN I I ARE COUNTING ERRORS FOR RADIONUCLIDES

MATER STANDARD'S) IN PARENTHESES ARE PROPLSED DNLY 
IABLE C.7. (contd)

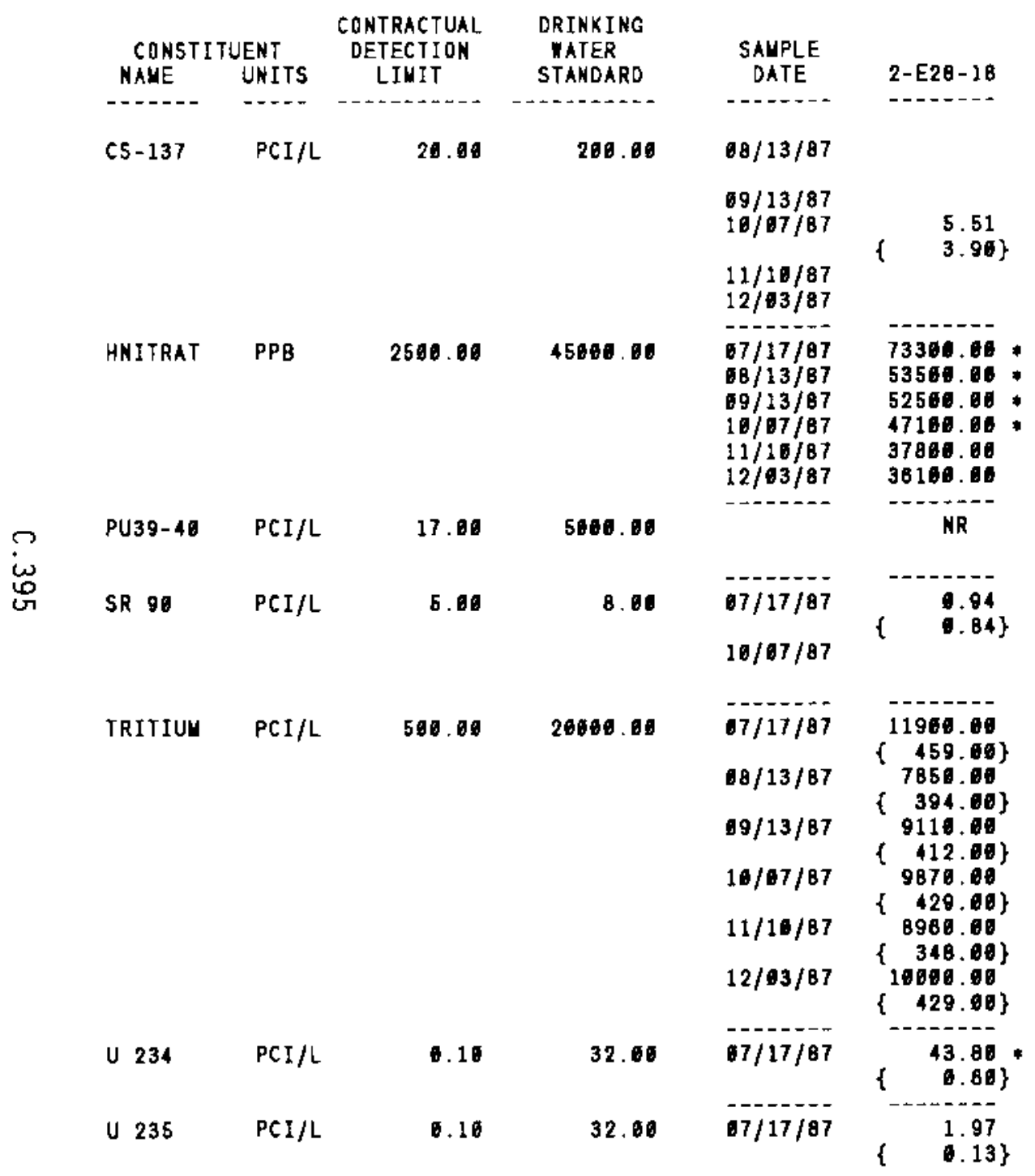
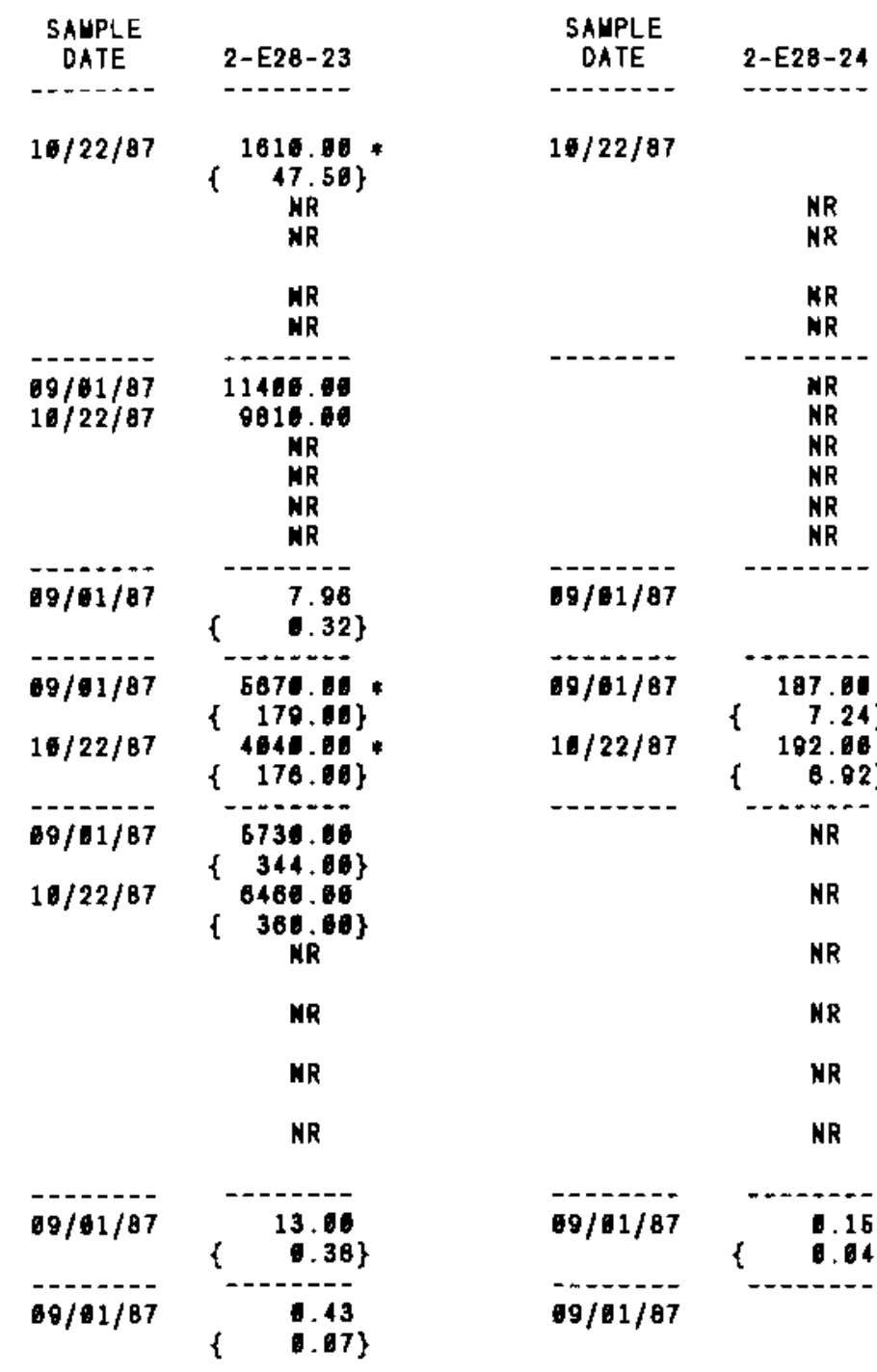

$10 / 22 / 87$

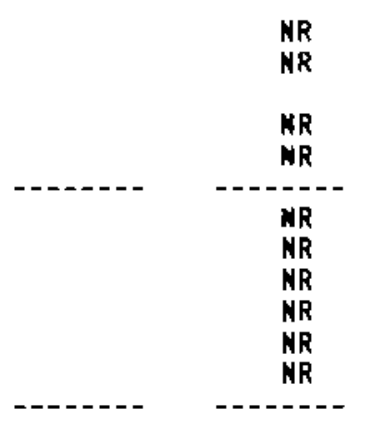

19/01/87

$90 / 61 / 87$

$187.00 *$

$10 / 22 / 87$

$\{7.24\}$

182.06
$\quad 6.82\}$

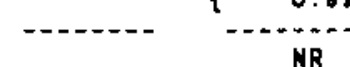

NR

NR

NR

MR

NR

NR

- VALUE EXCEEDS PROPOSED PRIHARY DRINKING WATER STAMDARO

- - VALUE EXCEEDS SCREENING LEVEL FoR FURTHER IHVESTIGATION.

- - DETECTION LIUIT NAS NOT AVAILABLE FOR COUPARISON

NR - ANALYSIS MDT REQUESTED OR NOT YET REPORTED

VALUES IN \{\} ARE COUNTING ERRORS FDR RADIONUCLIDES

YATER STANDARD(S) IN PARENTHESES ARE PROPOSED OKLY 


\begin{tabular}{ccc} 
CONSTITUENT & $\begin{array}{c}\text { CONTRACTUAL } \\
\text { DETECTION } \\
\text { LIMIT }\end{array}$ & $\begin{array}{c}\text { DRINKING } \\
\text { NATER } \\
\text { STANDARD }\end{array}$ \\
\hline & UNITS & -
\end{tabular}

$\begin{array}{llll}\text { U } 238 & \text { PCI/L } & 0.10 & 4.80 \\ \text { U-CHEY } & \text { UG/L } & 1.73\end{array}$

is
TABLE C.7. (contd)

\begin{tabular}{|c|c|}
\hline $\begin{array}{l}\text { SAUPLE } \\
\text { DATE }\end{array}$ & $2-E 28-18$ \\
\hline - - - - & - - - - - \\
\hline $67 / 17 / 87$ & $\begin{array}{r}48.70 \\
\{\quad 0.62\}\end{array}$ \\
\hline $\begin{array}{l}07 / 17 / 87 \\
68 / 13 / 87 \\
09 / 13 / 87 \\
16 / 07 / 87 \\
11 / 16 / 87 \\
12 / 63 / 87\end{array}$ & $\begin{array}{r}121.00 \\
57.80 \\
55.20 \\
83.90 \\
57.06 \\
51.30\end{array}$ \\
\hline
\end{tabular}

$11 / 10 / 87 / 87 \quad 51.30$

\section{2-E28-24}

DATE - - - - -
19/01/87

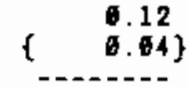

$08 / 81 / 87$

$10 / 22 / 67$
NR
NR
NR
NR

NR

NR

NR
NR

* - value exceeds primary drinking tater stanoard

* - VALUE EXCEEDS PROPOSED PRIUARY DRINKING WATER STANDARD.

+ - VALUE EXCEEDS SCREENING LEVEL FOR FURTHER INVESTIGATION

- DETECTION LIMIT UAS NDT AVAILABLE FOR COUPARISDN

NR - ANALYSIS NOT REQUESTED OR NDT YET REPORTEO

VALUES IN \& $\}$ ARE COUNTING ERRORS FOR RADIONUCLIDES

WATER STANDARO(S) IN PARENTHESES ARE PROPOSED DNLY 
TABLE C.7. (contd)

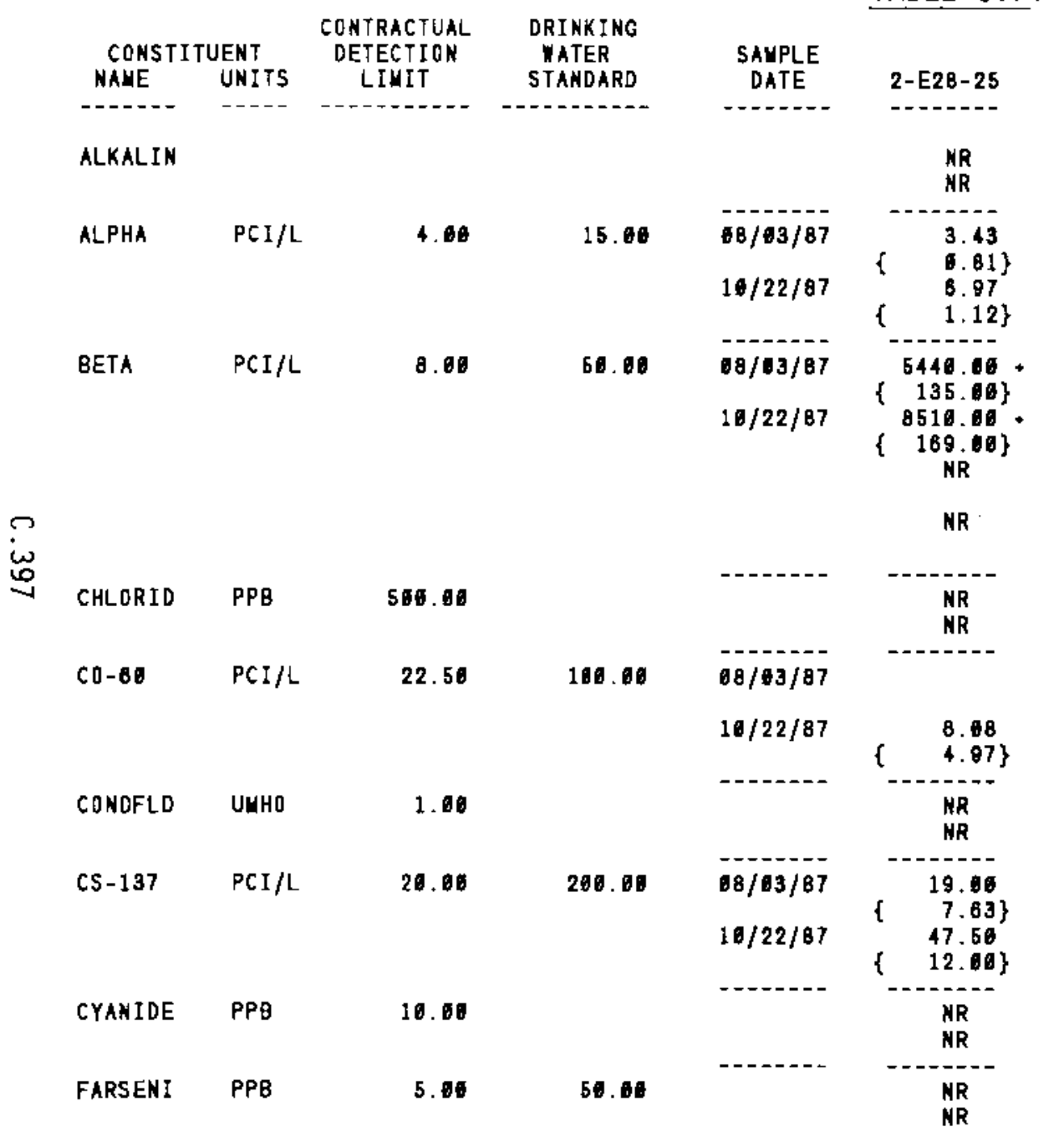

\begin{tabular}{|c|c|}
\hline $\begin{array}{l}\text { SAUPLE } \\
\text { DATE }\end{array}$ & $2-E 33-3$ \\
\hline - & 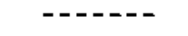 \\
\hline $\begin{array}{l}68 / 06 / 87 \\
12 / 68 / 87\end{array}$ & $\begin{array}{l}96300.00: \\
99409.00:\end{array}$ \\
\hline 9月/16/87 & $-1--\overline{24}$ \\
\hline ה & $\{\quad 1.07\}$ \\
\hline $12 / 06 / 87$ & $\left\{\begin{array}{l}1.98 \\
\{\quad 1.32\}\end{array}\right.$ \\
\hline $08 / 08 / 07$ & 253.06 \\
\hline $68 / 08 / 07$ & 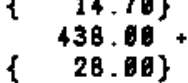 \\
\hline $12 / 68 / 87$ & $\begin{array}{r}27.76 \\
\{\quad 5.56\}\end{array}$ \\
\hline $12 / 08 / B 7$ & $\begin{array}{r}29.80 \\
\{\quad 3.78\}\end{array}$ \\
\hline $\begin{array}{l}68 / 66 / 87 \\
12 / 68 / 87\end{array}$ & $\begin{array}{l}3636.00 \\
3746.00\end{array}$ \\
\hline $68 / 68 / 87$ & 24.16 \\
\hline $12 / 06 / 87$ & 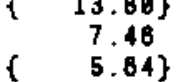 \\
\hline $\begin{array}{l}68 / 66 / 87 \\
12 / 68 / 87\end{array}$ & $\begin{array}{r}-156.00 \\
963.00\end{array}$ \\
\hline $68 / 66 / 87$ & 5.11 \\
\hline $12 / 66 / 87$ & \\
\hline - - & ------ \\
\hline $\begin{array}{l}68 / 66 / 87 \\
12 / 68 / 87\end{array}$ & 38.70 \\
\hline $\begin{array}{l}98 / 68 / 87 \\
12 / 66 / 87\end{array}$ & $\begin{array}{l}12.00 \\
13.00\end{array}$ \\
\hline
\end{tabular}

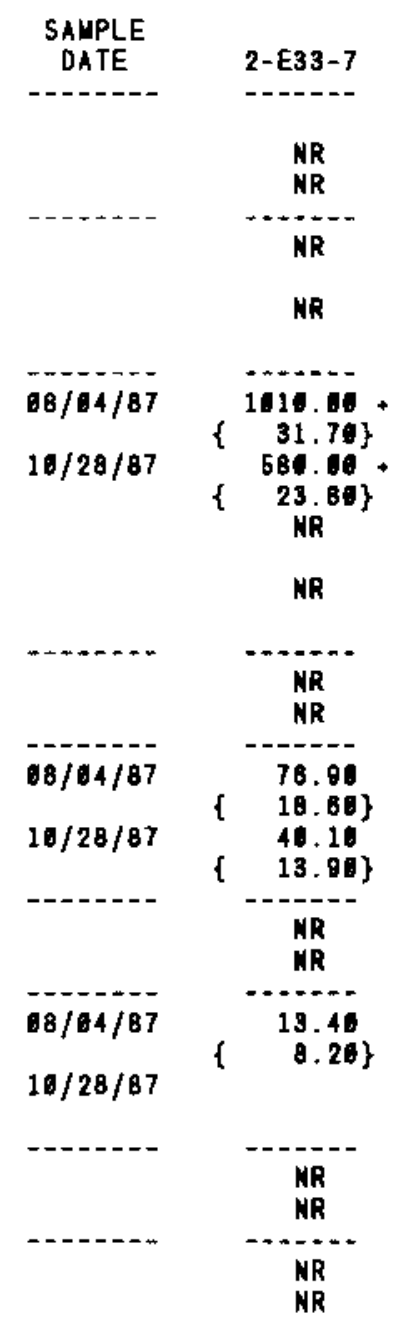

* - value exceEds primary dRinking tater standard.

| - VALUE EXCEEDS PROPOSED PRIMARY DRIMKING TATER STANDARO

- YALUE EXCEEDS SCREENIMG LEVEL FOR FURTHER INYESTIGATION.

- DETECTION LIUIT TAS NDT AVAILABLE FOR COUPARISON

NR - ANALYSIS NOT REQUESTED OR NOT YET REPORTED

VALUES IN \{ \} ARE COUNTING ERRORS FOR RADIONUCLIOES

IATER STANOARO(S) IN PARENTHESES ARE PROPOSED ONLY 
TABLE C.7. (contd)

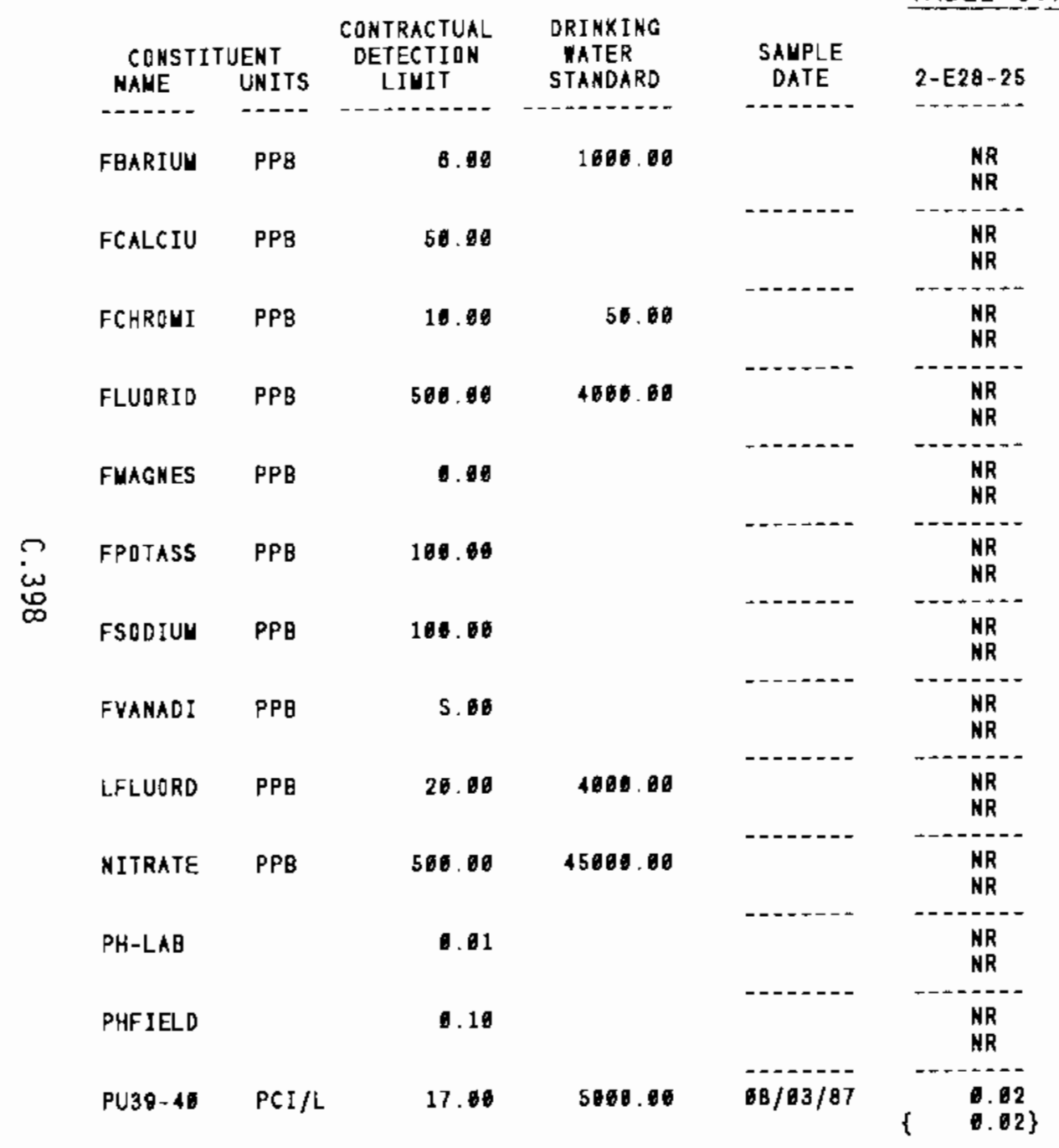

\begin{tabular}{|c|c|c|c|}
\hline $\begin{array}{l}\text { SALPLE } \\
\text { DATE }\end{array}$ & $2-E 33-3$ & $\begin{array}{c}\text { SAMPLE } \\
\text { DATE }\end{array}$ & $2-E 33-7$ \\
\hline $\begin{array}{l}80 / 08 / 87 \\
12 / 86 / 87\end{array}$ & $\begin{array}{l}19.60 \\
28.06\end{array}$ & & $\begin{array}{l}\text { NR } \\
\text { NR }\end{array}$ \\
\hline $\begin{array}{l}9 B / 06 / B 7 \\
12 / 6 B / B 7\end{array}$ & $\begin{array}{l}25500.00 \\
29260.80\end{array}$ & & $\begin{array}{l}\text { NR } \\
\text { NR }\end{array}$ \\
\hline $\begin{array}{l}08 / 08 / 87 \\
12 / 06 / 87\end{array}$ & $\begin{array}{l}10.00 \\
13.00\end{array}$ & & $\begin{array}{l}\text { NR } \\
\text { NR }\end{array}$ \\
\hline $\begin{array}{l}68 / 68 / 87 \\
12 / 06 / 87\end{array}$ & $\begin{array}{l}781.00 \\
799.00\end{array}$ & & $\begin{array}{l}\text { NR } \\
\text { NR }\end{array}$ \\
\hline $\begin{array}{l}68 / 08 / 87 \\
12 / 06 / 87\end{array}$ & $\begin{array}{l}8240.09 \\
8190.98\end{array}$ & & $\begin{array}{l}\text { NR } \\
\text { NR }\end{array}$ \\
\hline $\begin{array}{l}88 / 06 / 87 \\
12 / 06 / 87\end{array}$ & $\begin{array}{l}5059.68 \\
4670.80\end{array}$ & & $\begin{array}{l}\text { NR } \\
\text { NR }\end{array}$ \\
\hline $\begin{array}{l}68 / 06 / 87 \\
12 / 06 / 87\end{array}$ & $\begin{array}{l}32800.60 \\
26800.80\end{array}$ & & $\begin{array}{l}\text { MR } \\
\text { NR }\end{array}$ \\
\hline $\begin{array}{l}68 / 66 / 87 \\
12 / 06 / 87\end{array}$ & $\begin{array}{l}28.60 \\
30.00\end{array}$ & & $\begin{array}{l}\text { MR } \\
\text { MR }\end{array}$ \\
\hline $\begin{array}{l}88 / 06 / 87 \\
12 / 08 / 87\end{array}$ & $\begin{array}{l}805.60 \\
860.60\end{array}$ & & $\begin{array}{l}\text { NR } \\
\text { MR }\end{array}$ \\
\hline $\begin{array}{l}06 / 06 / 87 \\
12 / 06 / 87\end{array}$ & $\begin{array}{l}66800.00 \\
37808.00\end{array}$ & & $\begin{array}{l}\text { MR } \\
M R\end{array}$ \\
\hline $\begin{array}{l}68 / 06 / 87 \\
12 / 08 / 87\end{array}$ & $\begin{array}{l}7.72 \\
B .10\end{array}$ & & $\begin{array}{l}\text { NR } \\
\text { NR }\end{array}$ \\
\hline $\begin{array}{l}08 / 68 / 87 \\
12 / 66 / 87\end{array}$ & $\begin{array}{l}7.56 \\
7.50\end{array}$ & & $\begin{array}{l}\text { NR } \\
\text { NR }\end{array}$ \\
\hline $0 B / 08 / B 7$ & & $08 / 64 / 07$ & \\
\hline
\end{tabular}

* - value exceeds privary drinking unter standard.

- VALUE EXCEEDS PROPOSED PRIUARY DRINKING YATER STANDARD.

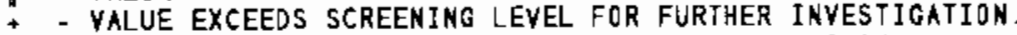

- DETECTION LIMIT IAS NOT AVAILABLE fOR COUPARISON

NR - ANALYSIS NOT REQUESTED OR NOT YET REPORTED

VALUES IN \{\} ARE COUNTING ERRORS FOR RADIONUCLIDES

-ATER STANDARD (S) IN PARENTHESES ARE PROPOSED DNLY 


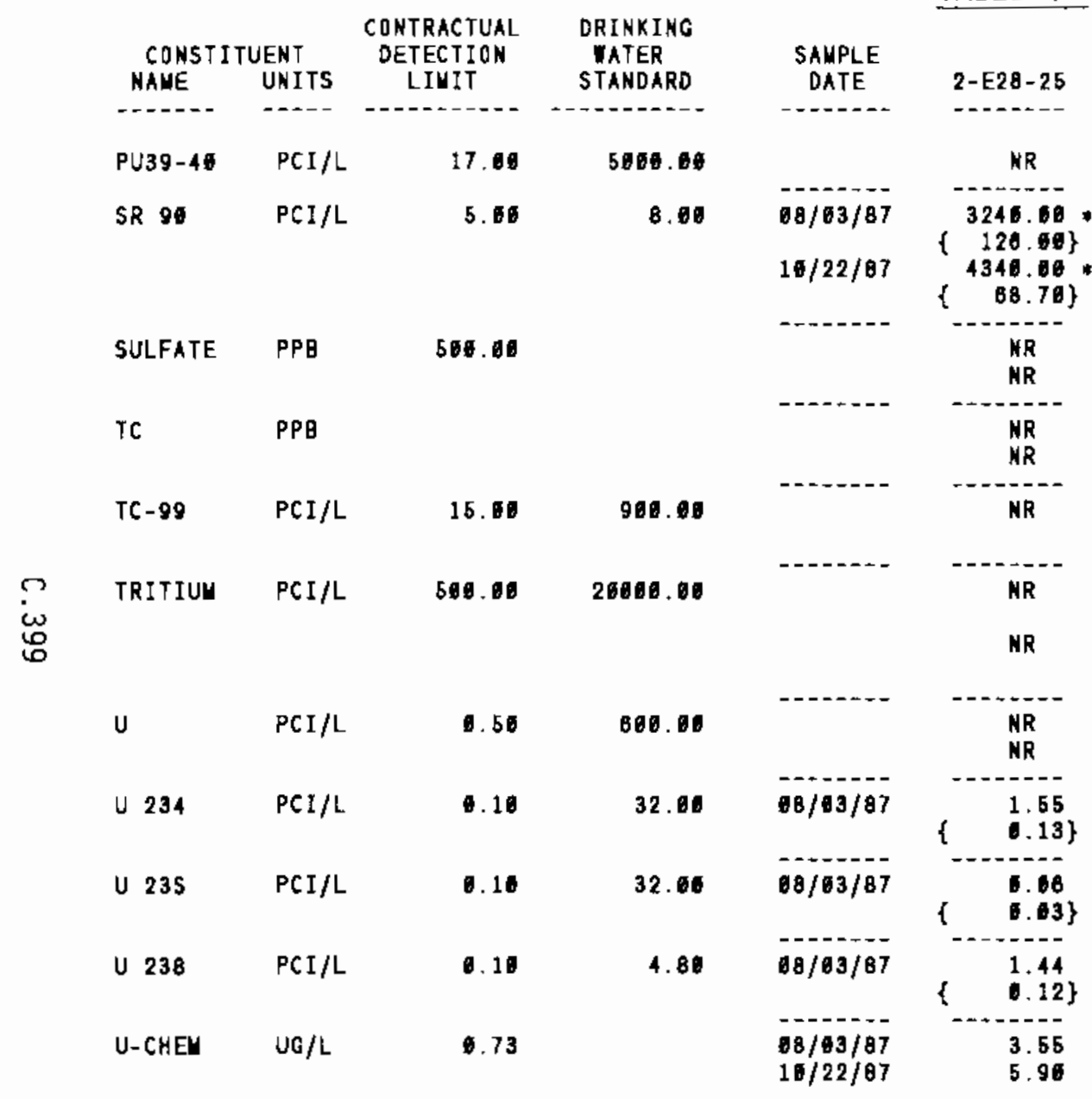

\begin{tabular}{|c|c|}
\hline $\begin{array}{l}\text { SAMPLE } \\
\text { DATE }\end{array}$ & $2-E 33-3$ \\
\hline 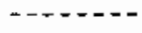 & 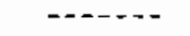 \\
\hline \multicolumn{2}{|l|}{$98 / 08 / 87$} \\
\hline $08 / 08 / 87$ & ------ \\
\hline & NR \\
\hline - & - - \\
\hline $\begin{array}{l}08 / 06 / 87 \\
12 / 08 / 87\end{array}$ & $\begin{array}{l}34400.00 \\
33500.00\end{array}$ \\
\hline $\begin{array}{l}06 / 06 / 87 \\
12 / 06 / 87\end{array}$ & $\begin{array}{l}22500.00 \\
22600.00\end{array}$ \\
\hline $68 / 66 / 87$ & $\left\{\begin{array}{r}1406.00 \\
6.35\}\end{array}\right.$ \\
\hline OB/0B/87 & $\begin{array}{l}858.06 \\
\quad 191.00\}\end{array}$ \\
\hline $12 / 06 / 87$ & $\begin{array}{r}1696.00 \\
250.09\}\end{array}$ \\
\hline $\begin{array}{l}08 / 06 / 87 \\
08 / 66 / 87\end{array}$ & $\begin{array}{l}1.17 \\
1.73\end{array}$ \\
\hline$-\cdots+-\infty$ & NR \\
\hline 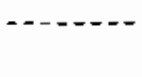 & NR \\
\hline מ & NR \\
\hline - & $\begin{array}{l}\text { NR } \\
\text { NR }\end{array}$ \\
\hline
\end{tabular}

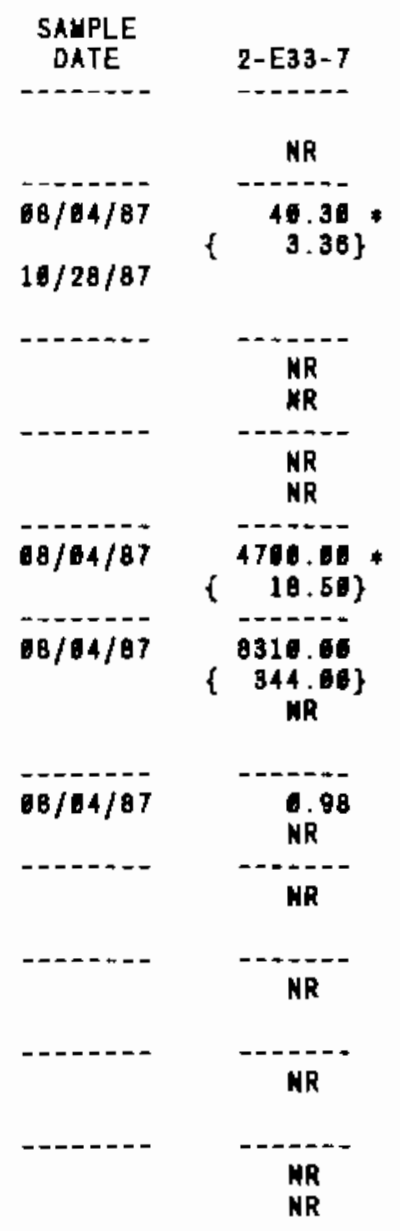

* - yalue exceeds priHary dRINKINg water standaro

* - VAlUe EXCEEDS PROPOSED PRIUARY dRINKING TATER STANDARD

+ - VALUE EXCEEDS SCREENIHG LEYEL FOR FURTHER INYESTIGAFION.

- DETECTION LIUIT WAS NOT AYAILABLE FOR COMPARISON

NR - ANALYSIS NOT REQUESTED OR NOT YET REPORTED

VALUES IN $($ ) ARE COUNTING ERRORS FOR RADIDNUCLIDES

TATER STAMDARD(S) IN PARENTHESES ARE PROPOSED DNLY

TABLE C.7. (contd) 
TABLE C.7. (contd)

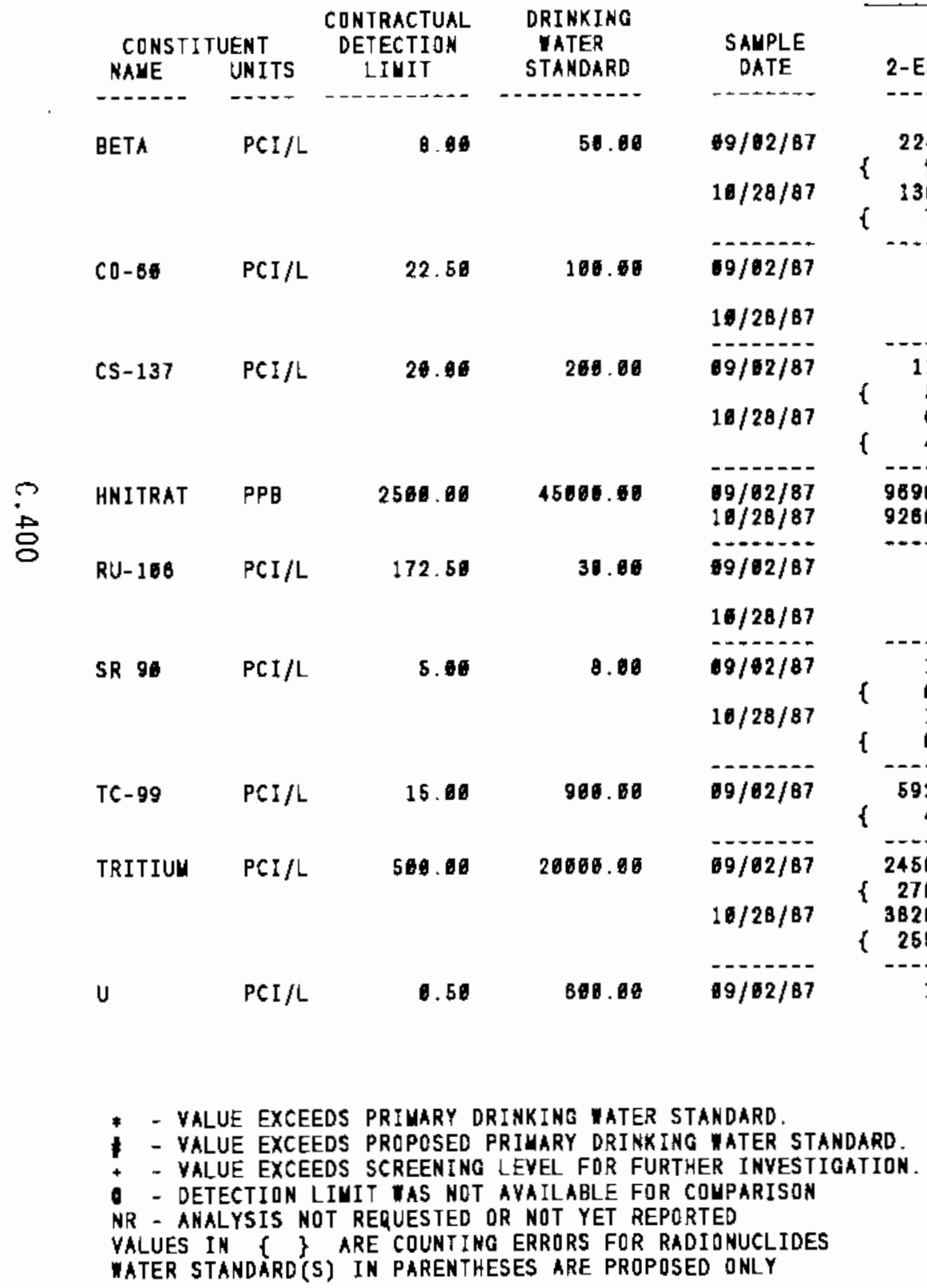

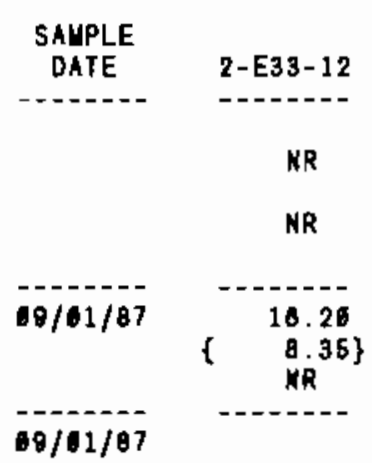

NR

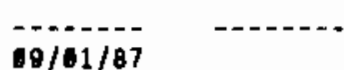

MR

$--10-2$

$10 / 01 / 87$

-

$\left\{\begin{array}{c}111.06 \\ 51.06\end{array}\right.$

NR

$68 / 11 / 87$

NR

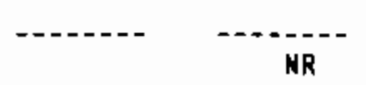

$09 / 01 / 87 \quad \begin{array}{ll}385.60 \\ 212.60\end{array}$

NR

$89 / 01 / 87$

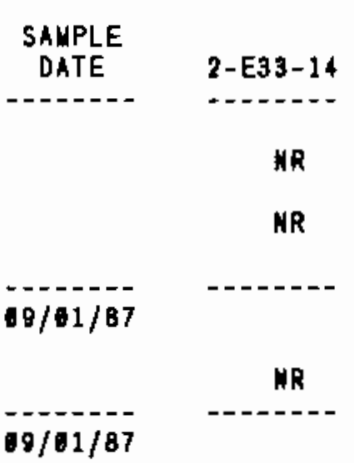

NR

$69 / 01 / 87 \quad 12400.10$

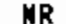

n...... - -

$69 / 01 / 87$

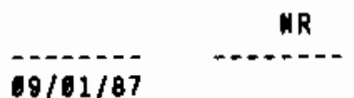

NR

NR

$69 / 01 / 97$

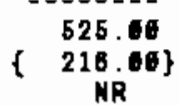

NR

$00 / 01 / 87$

$\overline{1.22}$

VALUES IN \& \& ARE COUNTING ERRORS FOR RADIDNUCLIDES
WATER STANDARD(S) IN PARENTHESES ARE PROPOSED ONLY 


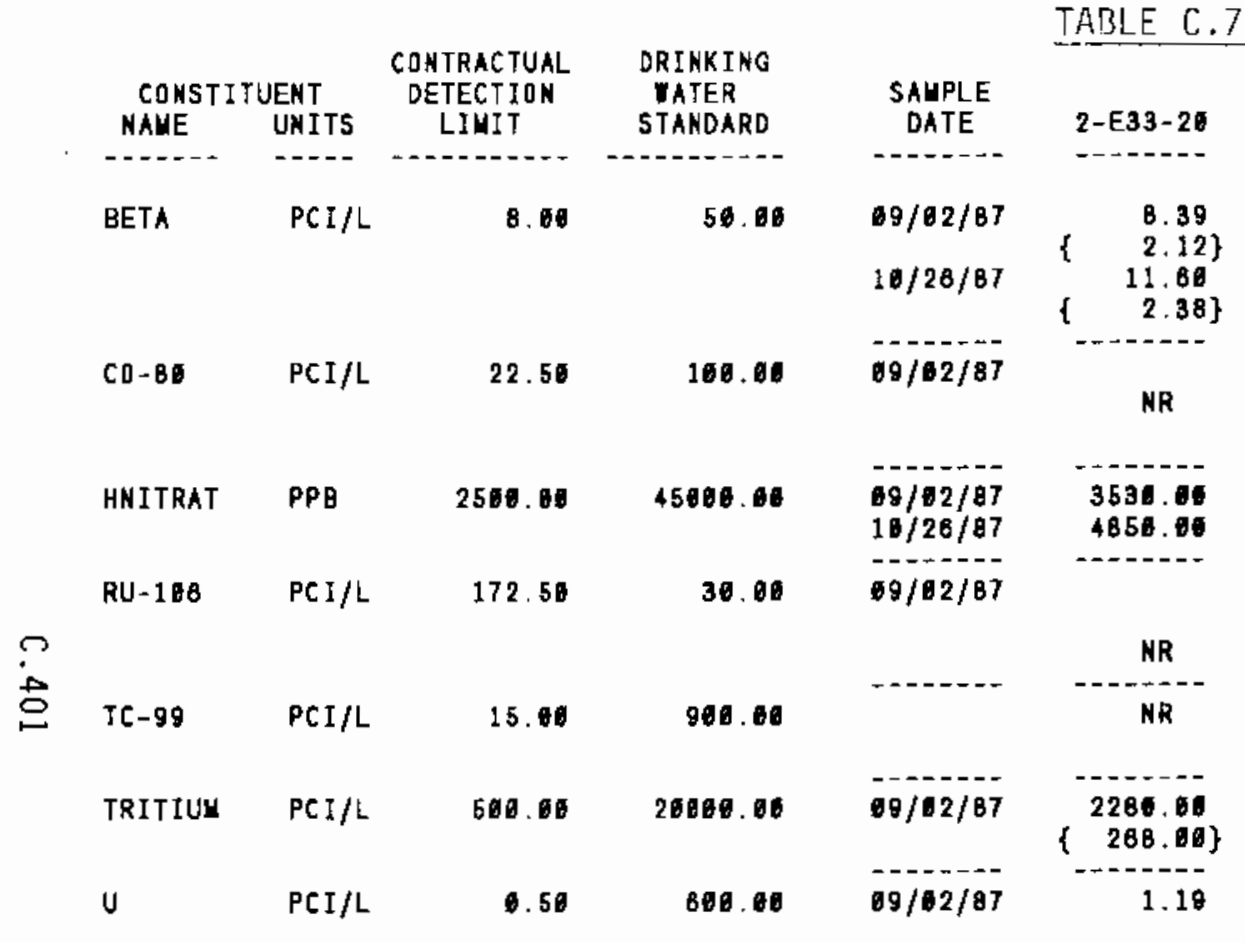

(contd) 


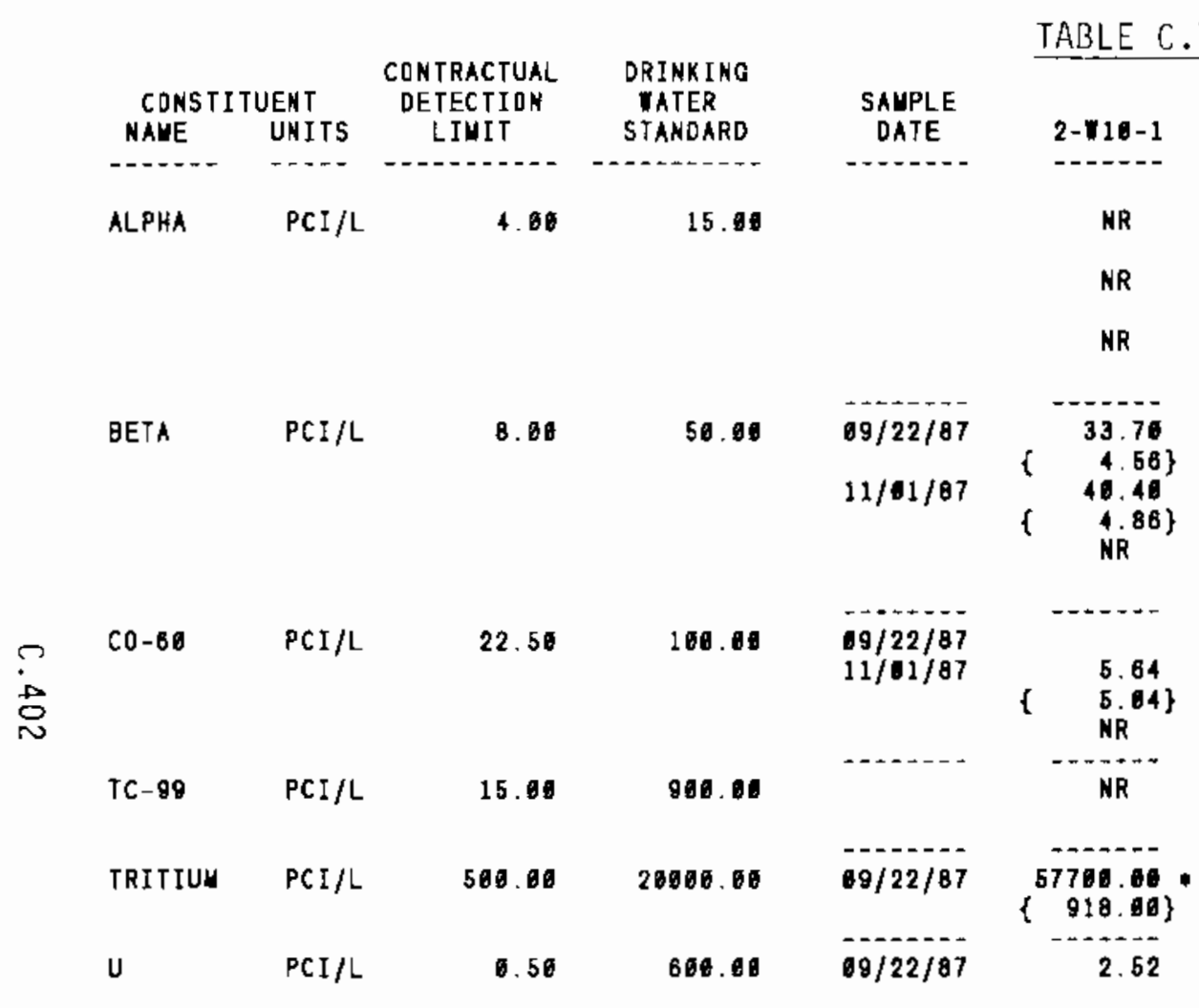

\section{(contd)}




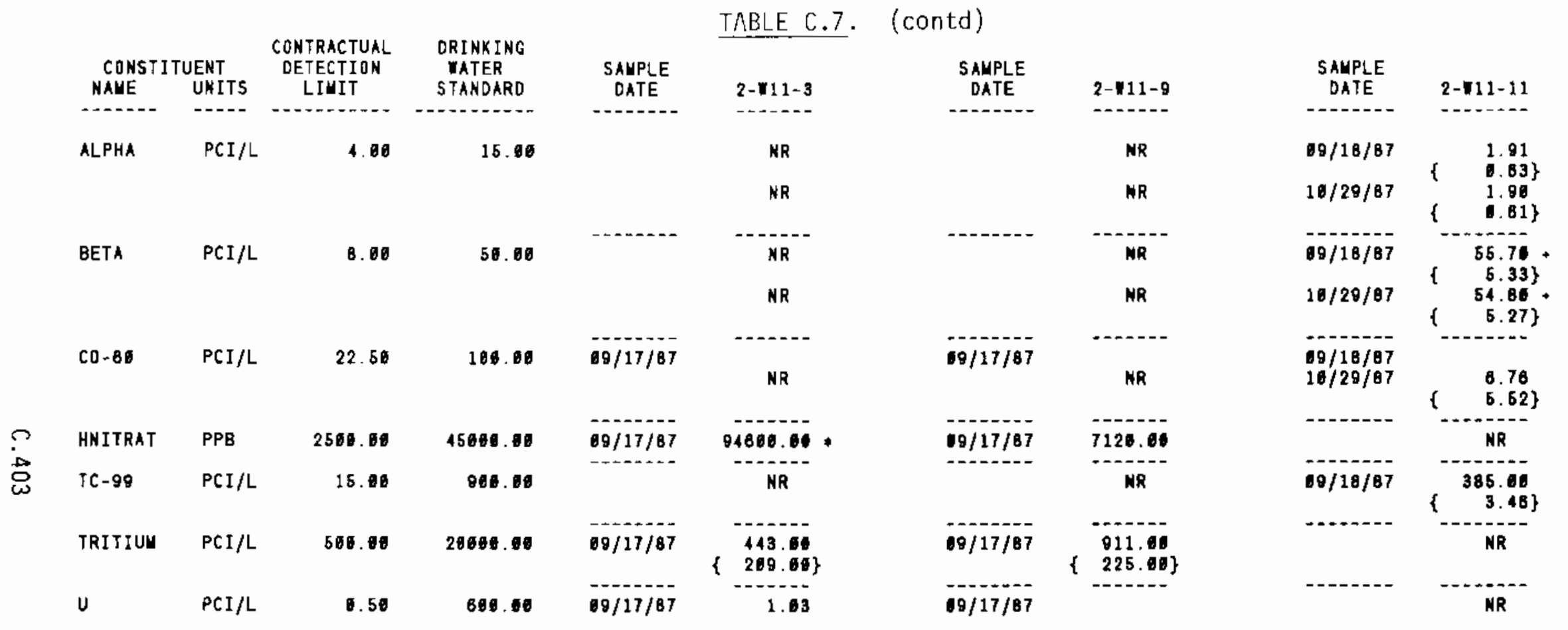

* - VALUE EXCEEOS PRIUARY DRINKING mater STANoARD.

- VALUE EXCEEDS PROPDSEO PRIUARY ORIMKIHo IATER STANDARD.

* YALUE EXCEEDS SCREENING LEVEL FOR FURTHER INYESTIGATION.

- DETECTION LIUIT WAS NOT AVAILABLE FOR COUPARISON

NR - ANALYSIS NOT REQUESTED OR NOT YET REPORTED

VALUES IN \{\} ARE COUNTING ERRORS FOR RAOIDHUCLIDES

WATER STANDARO(S) IN PARENTHESES ARE PROPOSED ONLY 


\begin{tabular}{|c|c|c|c|c|c|c|c|c|c|c|}
\hline & CONS T I TUENT & $\begin{array}{l}\text { UENT } \\
\text { UNI IS }\end{array}$ & $\begin{array}{l}\text { CONTRACTUAL } \\
\text { DETECTION } \\
\text { LINIT }\end{array}$ & $\begin{array}{l}\text { DRINKING } \\
\text { WATER } \\
\text { STANDARD }\end{array}$ & $\begin{array}{c}\text { SAHPLE } \\
\text { DATE }\end{array}$ & TABLE C & $\begin{array}{l}\text { SAYPLE } \\
\text { DATE }\end{array}$ & $2-\pi 11-18$ & $\begin{array}{c}\text { SAHPLE } \\
\text { DATE }\end{array}$ & $2-11-23$ \\
\hline & $-\cdots---$ & ---- & nanconos & 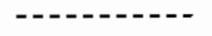 & $----n$ & 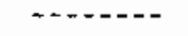 & $-\ldots-1$ & ------ & $++\infty+\infty$ & 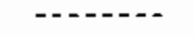 \\
\hline & ALPHA & $\mathrm{PCI} / \mathrm{L}$ & 4.00 & 15.00 & & $\begin{array}{l}\text { NR } \\
\text { NR }\end{array}$ & & $\begin{array}{l}\text { NR } \\
\text { NR }\end{array}$ & $\begin{array}{l}09 / 18 / 87 \\
10 / 29 / 87\end{array}$ & $\begin{array}{ll}\{\quad 0.88 \\
\{\quad 0.46\} \\
\{\quad 0.16 \\
\{\quad 04\}\end{array}$ \\
\hline & BETA & $\mathrm{PCI} / \mathrm{L}$ & 8.60 & 50.60 & $\begin{array}{l}09 / 18 / 87 \\
10 / 29 / 87\end{array}$ & $\begin{array}{c}21.40 \\
\{\quad 3.24\} \\
\{3.70 \\
\{\quad 3.17\}\end{array}$ & $\begin{array}{l}08 / 18 / 87 \\
10 / 29 / 87\end{array}$ & $\begin{array}{c}52.20 \\
5.36\} \\
55.20 \\
5.54\}\end{array}$ & $10 / 29 / 87$ & $\begin{array}{cc} & 36.40 \\
& 4.77\} \\
& 20.56 \\
\{\quad 3.85\}\end{array}$ \\
\hline & $c 0-66$ & $\mathrm{PCI} / \mathrm{L}$ & 22.60 & 100.00 & & $\begin{array}{l}\text { MR } \\
\text { NR }\end{array}$ & $\begin{array}{l}60 / 18 / 87 \\
10 / 28 / 87\end{array}$ & $\begin{array}{c}8.47 \\
\quad 8.91\} \\
12.18 \\
\{\quad 7.88\}\end{array}$ & $\begin{array}{l}09 / 18 / 87 \\
10 / 29 / 87\end{array}$ & $\left\{\begin{array}{c}11.80 \\
7.14\} \\
13.90 \\
\{\quad 7.70\}\end{array}\right.$ \\
\hline 8 & HNITRAT & PPB & 2500.00 & 45000.00 & - n. & $\begin{array}{c}\text { NR } \\
\text { NR }\end{array}$ & $---1-1--$ & $\begin{array}{l}\text { NR } \\
\text { NR }\end{array}$ & $\begin{array}{l}99 / 18 / 87 \\
18 / 29 / 87\end{array}$ & $\begin{array}{l}42000.00 \\
436006.06\end{array}$ \\
\hline & $T C-9 \theta$ & $\mathrm{PCI} / \mathrm{L}$ & 15.00 & 900.00 & $-\cdots+\infty+\infty$ & MR & $69 / 18 / 87$ & $\left\{\begin{array}{r}510.69 \\
3.94\}\end{array}\right.$ & $99 / 18 / 87$ & $\begin{array}{r}270.06 \\
\{\quad 2.97\}\end{array}$ \\
\hline
\end{tabular}

- - VALUE EXCEEDS PRIUARY DRINKING mater STAKDARD.

- VALUE eXCEEDS PROPDSEd PRIUARY dRINKING Mater standoard.

- VALUE EXCEEDS SCREENIHG LEVEL. FOR FURFHER IHVESTIGATION.

- DETECTION LiMIT TAS NDT aVAILABLE FDR COUPARISON

NR - ANALYSIS NDT REQUESTED OR NOT YET REPORTED

VALUES IN \{ $\}$ ARE COUNTING ERRORS FOR RADIONUCLIOES

IATER STANDARO'S) IN PARENTHESES ARE PROPOSED ONLY 


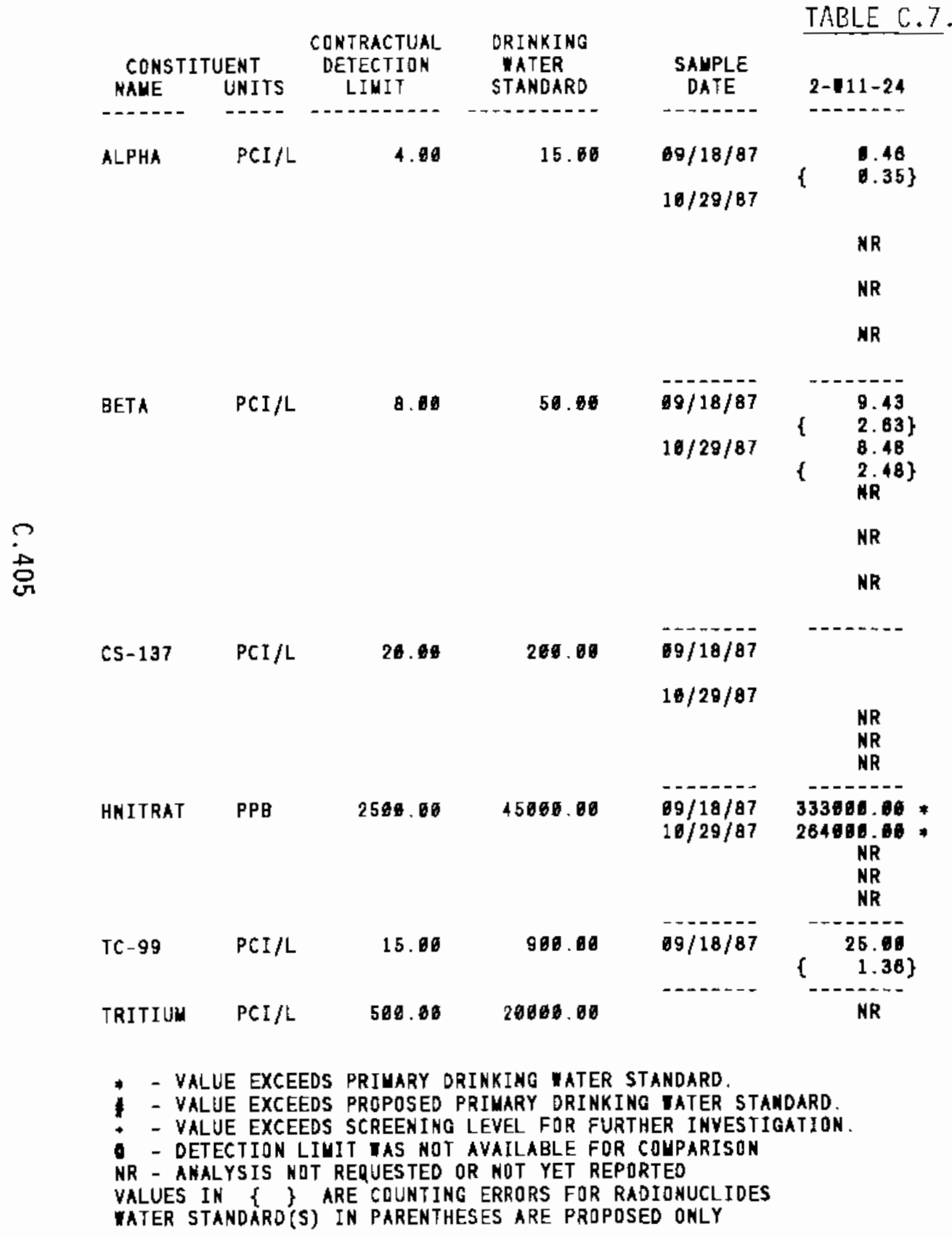

\section{(contd)}




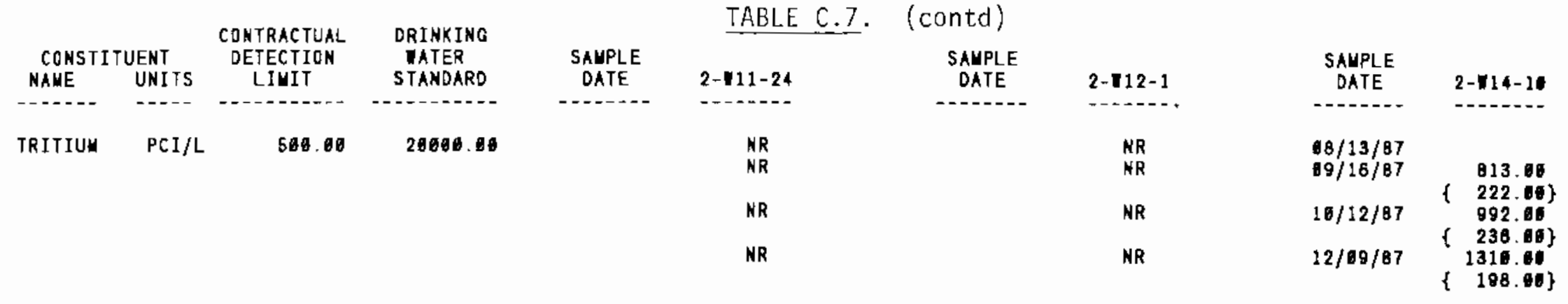

$\stackrel{\text { के }}{\circ}$

* - VALUE EXCEEDS PRIMARY DRINKING TATER STANDARD.

* - VALUE EXCEEDS PROPOSED PRIMARY DRINKING MaTER STANDARO.

* - VALUE EXCEEDS SCREENING LEVEL FDR FURTHER INYESTIGATION.

- DETECTION LIMIT MAS NOT AYAILABLE FOR COUPARISON

NR - ANALYSIS NOT REQUESTED OR NOT YET REPORTED

VALUES IN \{ \} ARE COUNTING ERRORS FOR RADYONUCLIDES

WATER STANDARD'S) IN PARENTHESES ARE PROPDSED ONLY 


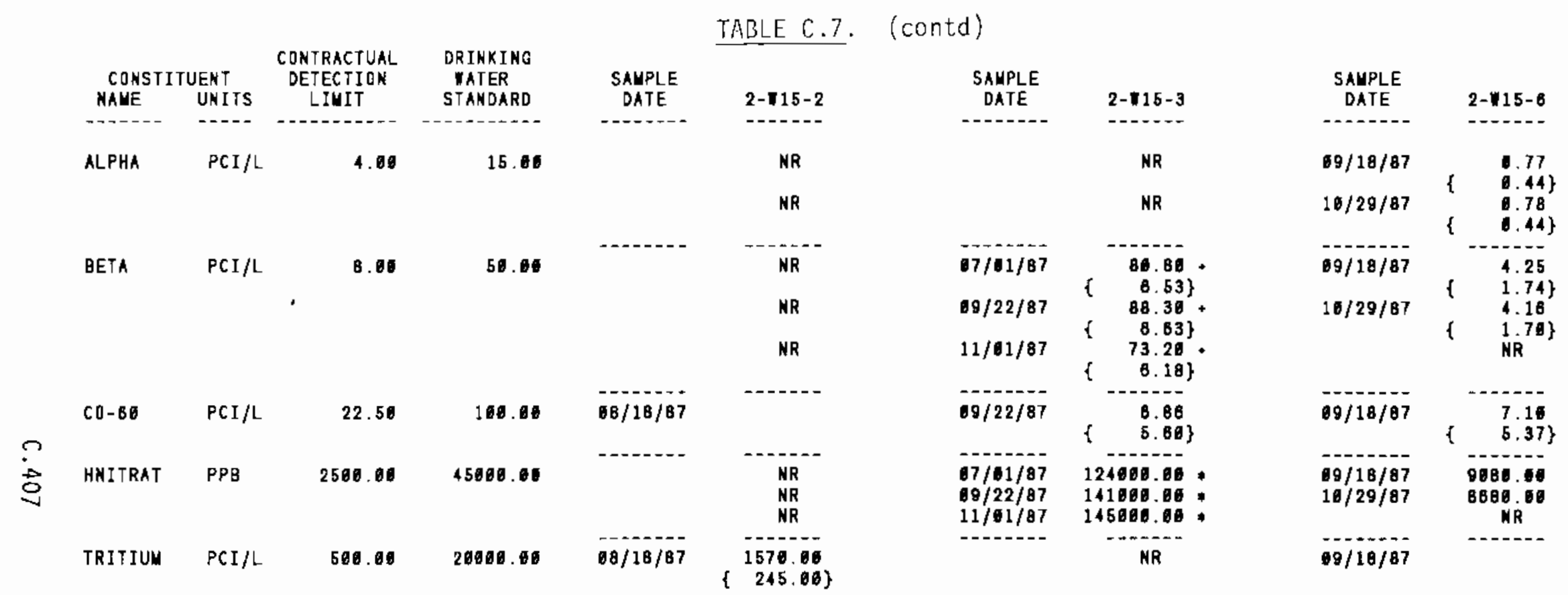

- - VAlUe exceeds priUary drinking mater standard

\# - VALUE EXCEEDS PRDPDSED PRIUARY DRINKING WATER STANDARD.

+ - VALUE EXCEEDS SCREENING LEVEL FOR FURTHER INVESTIGATION.

- DETECTION LIUIT IAS NOT AVAILABLE FOR COMPARISON

NR - ANALYSIS NDT REQUESTED QR NDT YET REPORTED

VALUES IN \{\} ARE COUNTING ERRORS FDR RADIONUCLIDES

WATER STANDARD'(S) IN PARENTHESES ARE PROPOSED DNLY 


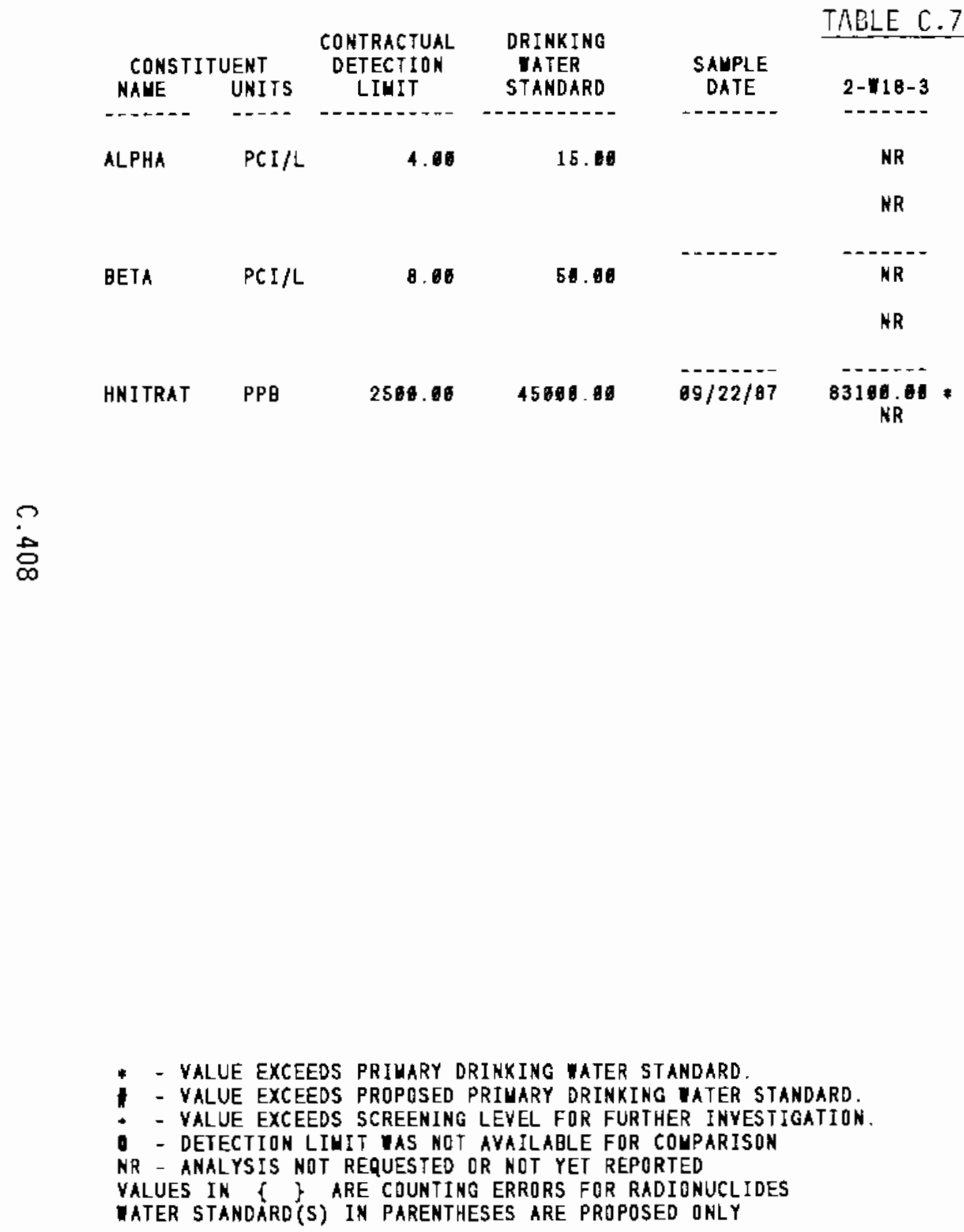

(contd)

SAUPLE DATE

2-118-5

$69 / 17 / 87$

6.77
$+\quad .42]$

$0 / 29 / 87$

$10 / 29 / 87$

$-0 / 17 / 87$

$\quad .83$
$\quad .44\}$

$10 / 29 / 87$

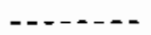

SAUPLE

DATE

2-718-9

--D.--

$68 / 19 / 97$

$11 / 01 / 87$

$08 / 18 / 87$

$11 / 01 / 87$

---2---

$08 / 19 / 87$

$11 / 61 / 87$

$\left\{\begin{array}{r}-1.59 \\ 3.83\} \\ 1.84 \\ 1.27 \\ -5930.01 \\ 5261.00\end{array}\right.$

VALUE EXCEEDS PRIUARY DRINXING NTER STANDARD.

VALUES IN ARE COUNTING ERRORS FQR RADIONUCLIDE 


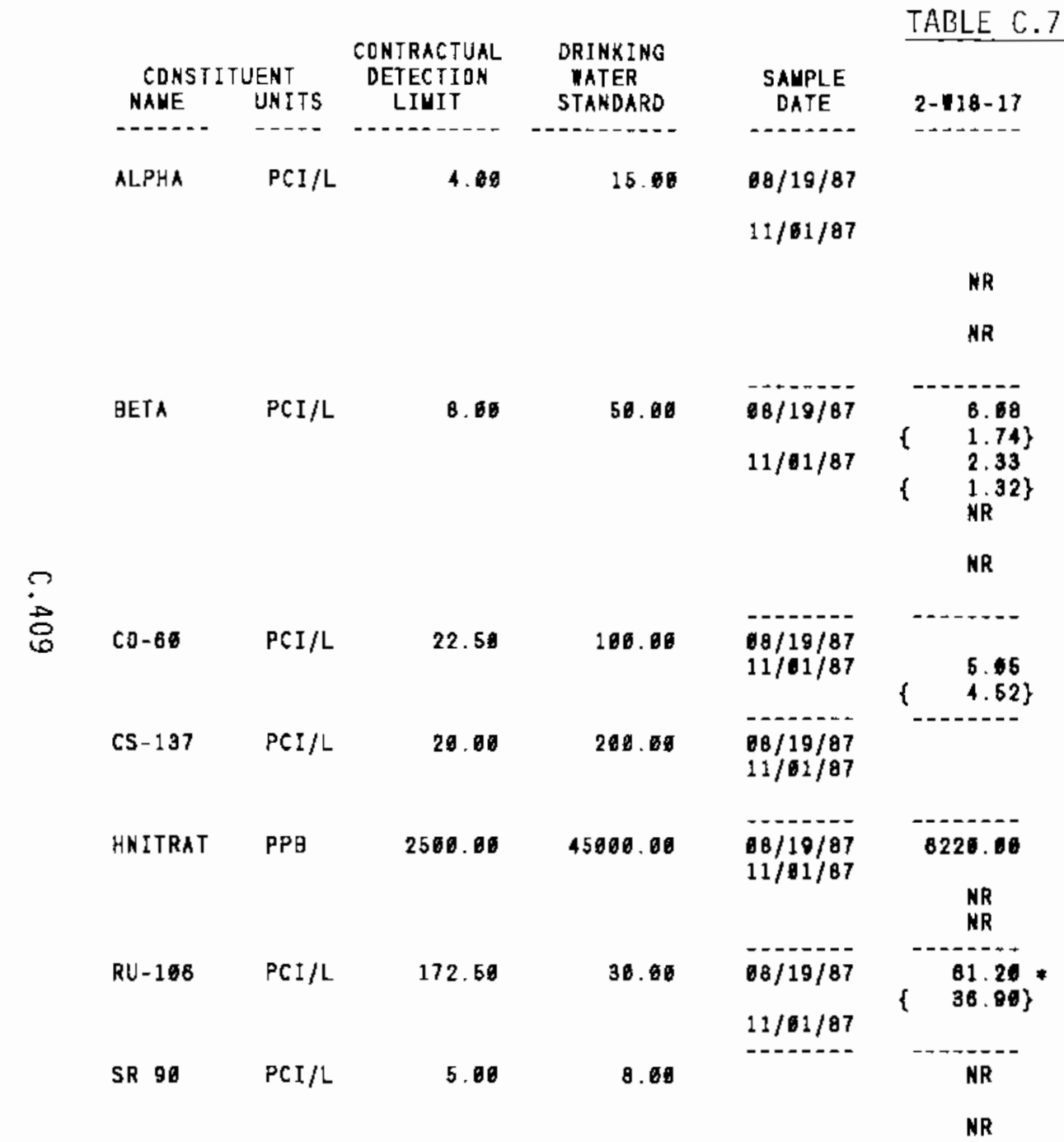

\section{(contd)}

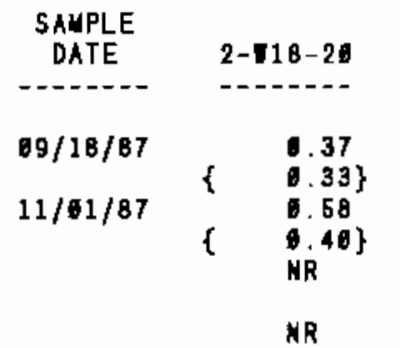

$\begin{array}{lll}00 / 18 / 87 & & 3.71 \\ 11 / 01 / 87 & \{ & 1.51\} \\ & \{ & 8.11 \\ & 1.79 \\ & & \\ & & \\ & & \end{array}$

$09 / 18 / 87$

$11 / 61 / 87$

69/18/87

$11 / 81 / 87$

$99 / 18 / 87$

11/01/87

11)01/07

99/18/87

$11 / 61 / 87$

NR

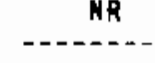

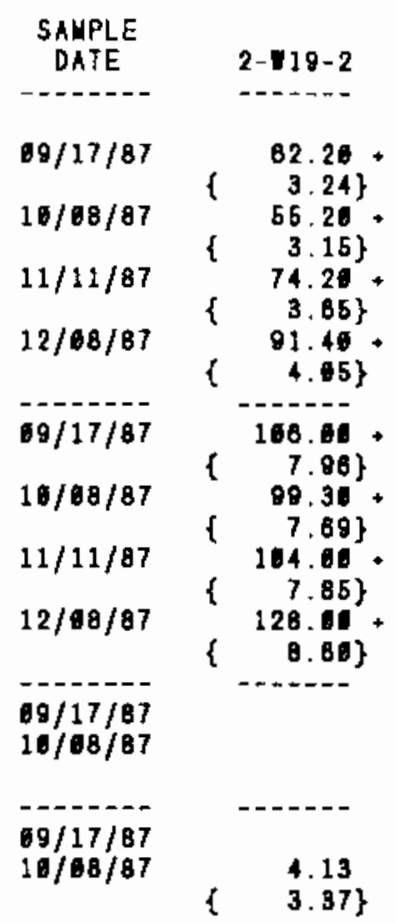

$69 / 17 / 87$

$10 / 68 / 87$

$11 / 11 / 87$

$88 / 17 / 87$

$10 / 08 / 87$

$09 / 17 / 87$

$18 / 88 / 87$
477600.06

585860.00 :

$323060.00 *$

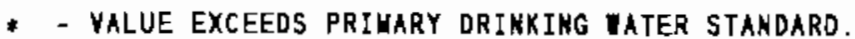

- VALUE EXCEEDS PROPOSED PRIUARY DRINKING IATER STANDARD.

+ - VALUE EXCEEDS SCREENING LEVEL FOR FURTHER INYESTIGATIDK.

- DETECTIDN LIMIT WAS NOT AVAILABLE FOR COMPARISON

NR - ANALYSIS NOT REQUESTED OR NOT YET REPORTED

VALUES IN \{ \} ARE CDUNTING ERRDRS FOR RADIONUCLIDES

WATER STANDARD(S) IN PARENTHESES ARE PROPOSED ONLY 


\begin{tabular}{|c|c|c|c|c|c|c|c|c|c|}
\hline$\underset{\text { MAME }}{\text { CONSTI }}$ & $\begin{array}{l}\text { TUENT } \\
\text { UNITS }\end{array}$ & $\begin{array}{l}\text { CONTRACTUAL } \\
\text { DETECTIION } \\
\text { LIUIT }\end{array}$ & $\begin{array}{l}\text { DRINKING } \\
\text { SATER } \\
\text { STANDARD }\end{array}$ & $\begin{array}{c}\text { SALPLE } \\
\text { DATE }\end{array}$ & TABLE C. & $\begin{array}{l}\text { ntd) } \\
\text { SAMPLE } \\
\text { DATE }\end{array}$ & $2-w_{18-28}$ & $\begin{array}{c}\text { SAMPLE } \\
\text { DATE }\end{array}$ & $2-119-2$ \\
\hline TC- 99 & $P C I / L$ & 15.80 & 980.80 & & NR & & NR & $69 / 17 / 87$ & $\left\{\begin{array}{r}581.06 \\
\{.22\}\end{array}\right.$ \\
\hline TRITIUV & PCI/L & 580.68 & 20008.00 & $\begin{array}{l}88 / 19 / 87 \\
11 / 61 / 87\end{array}$ & 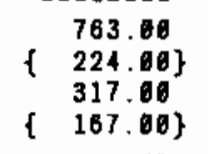 & $\begin{array}{l}89 / 18 / 87 \\
11 / 81 / 87\end{array}$ & $\left.\begin{array}{r}1690.60 \\
\{\quad 206.40\end{array}\right\}$ & $\begin{array}{l}89 / 17 / 87 \\
16 / 68 / 87\end{array}$ & 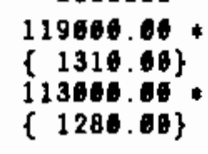 \\
\hline U-CHEM & UG/L & 8.73 & & & $\begin{array}{l}\mathrm{NR} \\
\mathrm{NR} \\
\mathrm{NR} \\
\mathrm{NR}\end{array}$ & & $\begin{array}{l}N R \\
N R \\
N R \\
N R\end{array}$ & $\begin{array}{l}19 / 17 / 87 \\
11 / 68 / 87 \\
11 / 11 / 87 \\
12 / 68 / 87\end{array}$ & $\begin{array}{l}73.56 \\
69.36 \\
76.16 \\
94.56\end{array}$ \\
\hline
\end{tabular}

* - VALUE eXCEEDS PRIUARY dRINKING tater standard

- VALUE EXCEEDS PROPOSED PRIMARY DRINKING WATER STAMDARD.

- - VALUE EXCEEDS SCREENING LEVEL FOR FURTHER INYESTIGATION.

- DETECTION LINIT MAS NOT AVAILABLE FDR COUPARISON

NR - ANALYSIS NOT REQUESTED OR NOY YET REPORTEO

VALUES IN \{ $\}$ ARE COUNTING ERRORS FOR RADIONUCLIDES

WATER STANDARD'S) IN PARENTHESES ARE PROPOSED ONLY 


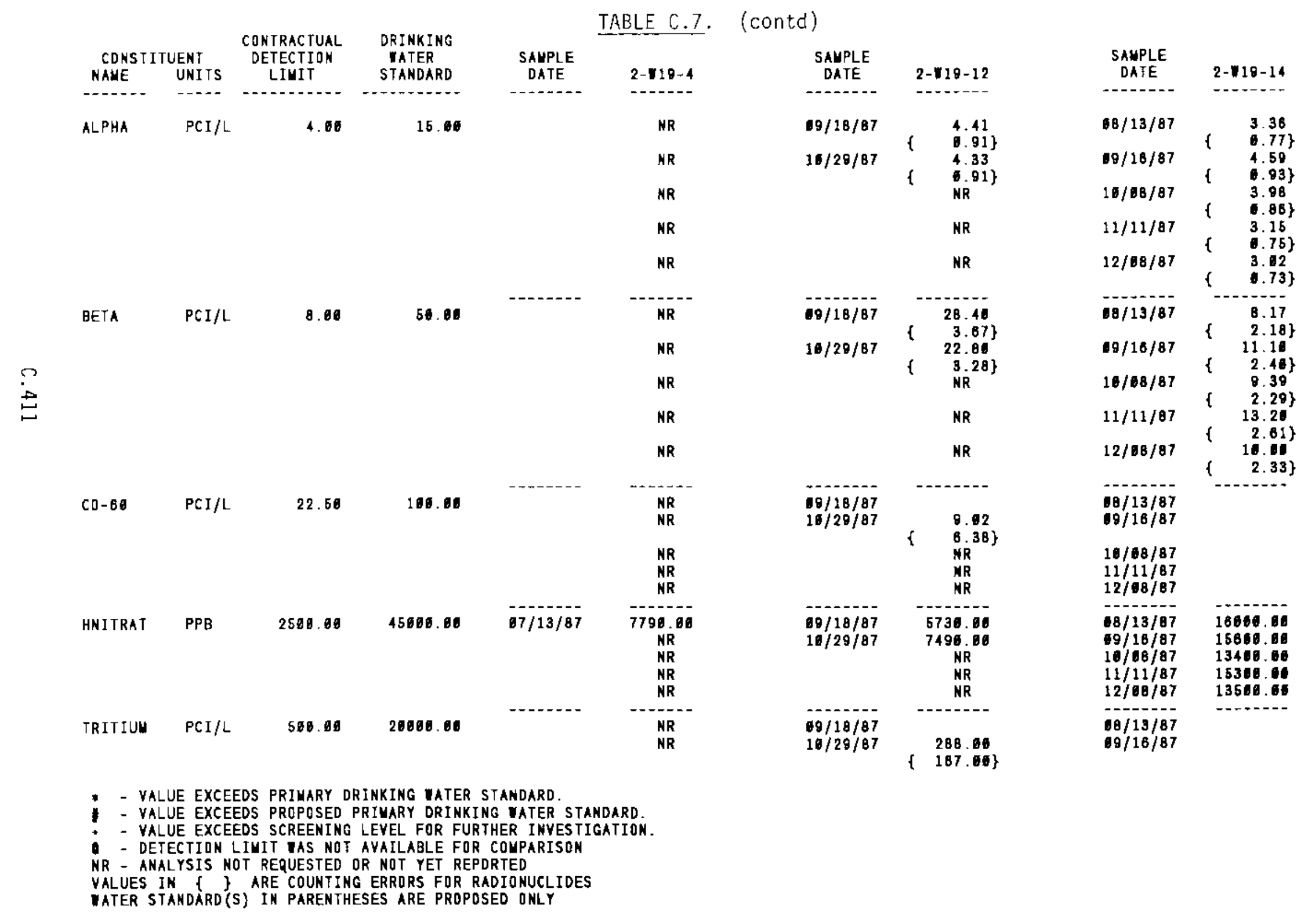




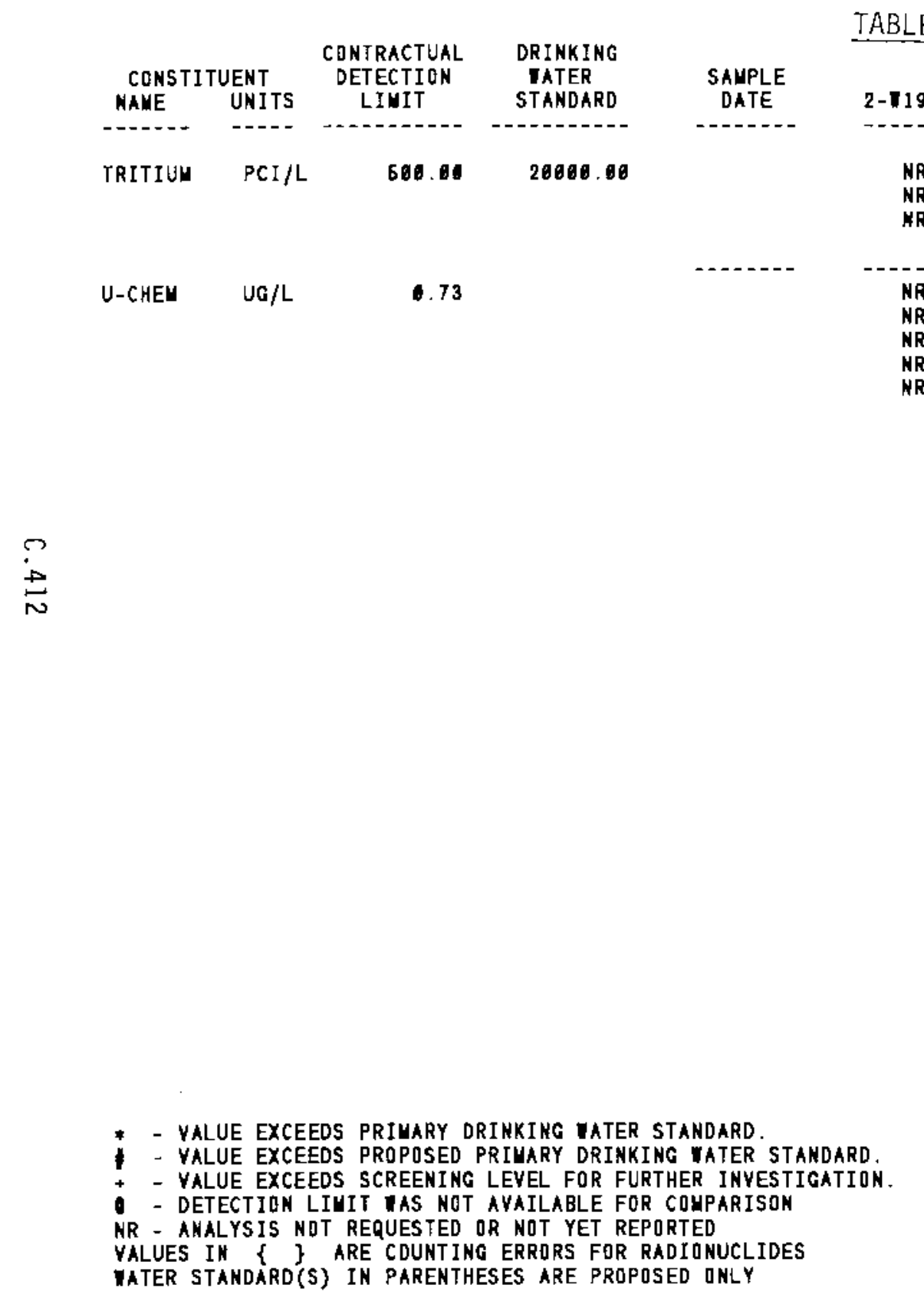

TABLE C.7. (contd)

\begin{tabular}{|c|c|c|c|c|c|c|c|c|c|}
\hline $\begin{array}{l}\text { CONSTI } \\
\text { MAME }\end{array}$ & $\begin{array}{l}\text { UENT } \\
\text { UNITS }\end{array}$ & $\begin{array}{l}\text { DETECTION } \\
\text { LINIT }\end{array}$ & $\begin{array}{l}\text { IATER } \\
\text { STANDARD }\end{array}$ & $\begin{array}{c}\text { SAMPLE } \\
\text { DATE }\end{array}$ & $2-719-4$ & $\begin{array}{l}\text { SALPLE } \\
\text { DATE }\end{array}$ & $2-\mp 19-12$ & $\begin{array}{l}\text { SAUPLE } \\
\text { DATE }\end{array}$ & $2-119-14$ \\
\hline----- & ---- & n....-- & - - & ----- & $----\cdot$ & 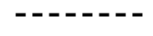 & 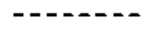 & $\cdots-1-1$ & 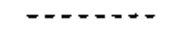 \\
\hline TRITIUH & PCI/L & 600.00 & 20000.00 & & $\begin{array}{l}\text { NR } \\
\text { NR } \\
\text { KR }\end{array}$ & & $\begin{array}{l}\text { NR } \\
\text { NR } \\
\text { NR }\end{array}$ & $\begin{array}{l}10 / 08 / 87 \\
11 / 11 / 87 \\
12 / 60 / 87\end{array}$ & $\begin{array}{r}305.00 \\
\{\quad 180.00\}\end{array}$ \\
\hline U-CHEU & UG/L & 0.73 & & $\cdots+2-0-$ & $\begin{array}{l}\text { NR } \\
\text { NR } \\
\text { NR } \\
\text { NR } \\
\text { NR }\end{array}$ & $\begin{array}{l}60 / 18 / 87 \\
16 / 29 / 87\end{array}$ & $\begin{array}{l}5.12 \\
4.81 \\
\text { NR } \\
\text { NR } \\
\text { NR }\end{array}$ & $\begin{array}{l}-10 / 13 / 87 \\
60 / 16 / 87 \\
18 / 08 / 87 \\
11 / 11 / 87 \\
12 / 08 / 87\end{array}$ & $\begin{array}{r}3.75 \\
4.74 \\
3.78 \\
4.28 \\
4.23\end{array}$ \\
\hline
\end{tabular}

* - yalue exceeds privary dRINKIRg vater standard.

VALUE EXCEEDS PROPOSED PRIUARY DRINKING MATER STANDARD.

NR - ANALYSIS NDT REQUESTED OR NOT YET REPORTED

INANDARD(S) IN PARENTHESES ARE PROPOSED ONLY 


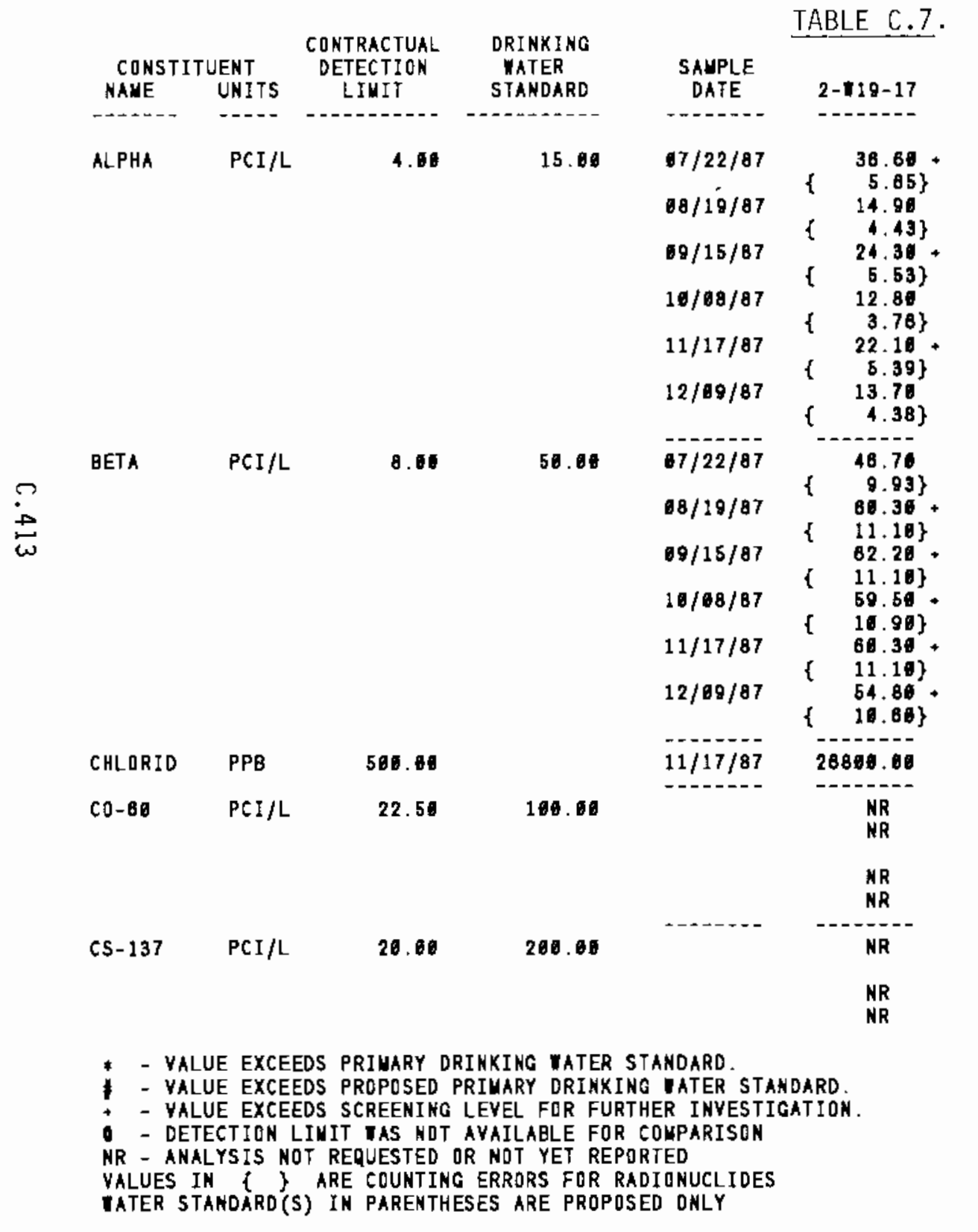

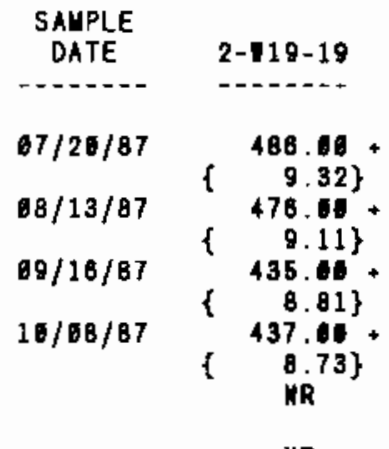

NR

$\{539.06\}$

8290.06.

$\left\{\begin{array}{r}567.08\} \\ 78360\end{array}\right.$

(557.06)

680600.

\{ 528.06\}

$11 / 17 / 87 \quad 23460.00$

NR

NR

NR
NR

HR

NR
NR
67/20/87

18/13/87

99/16/87

$16 / 68 / 87$

518.08.

$\{\quad 18.40\}$

476.00 .

$\{17.86\}$

$333.65+$

$\{14.00\}$

266.08.

1.68

MR

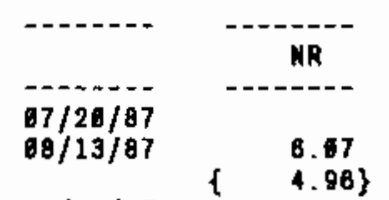

09/18/87

$10 / 00 / 87$

$07 / 20 / 87$

$08 / 13 / 87$

$69 / 16 / 87$ 


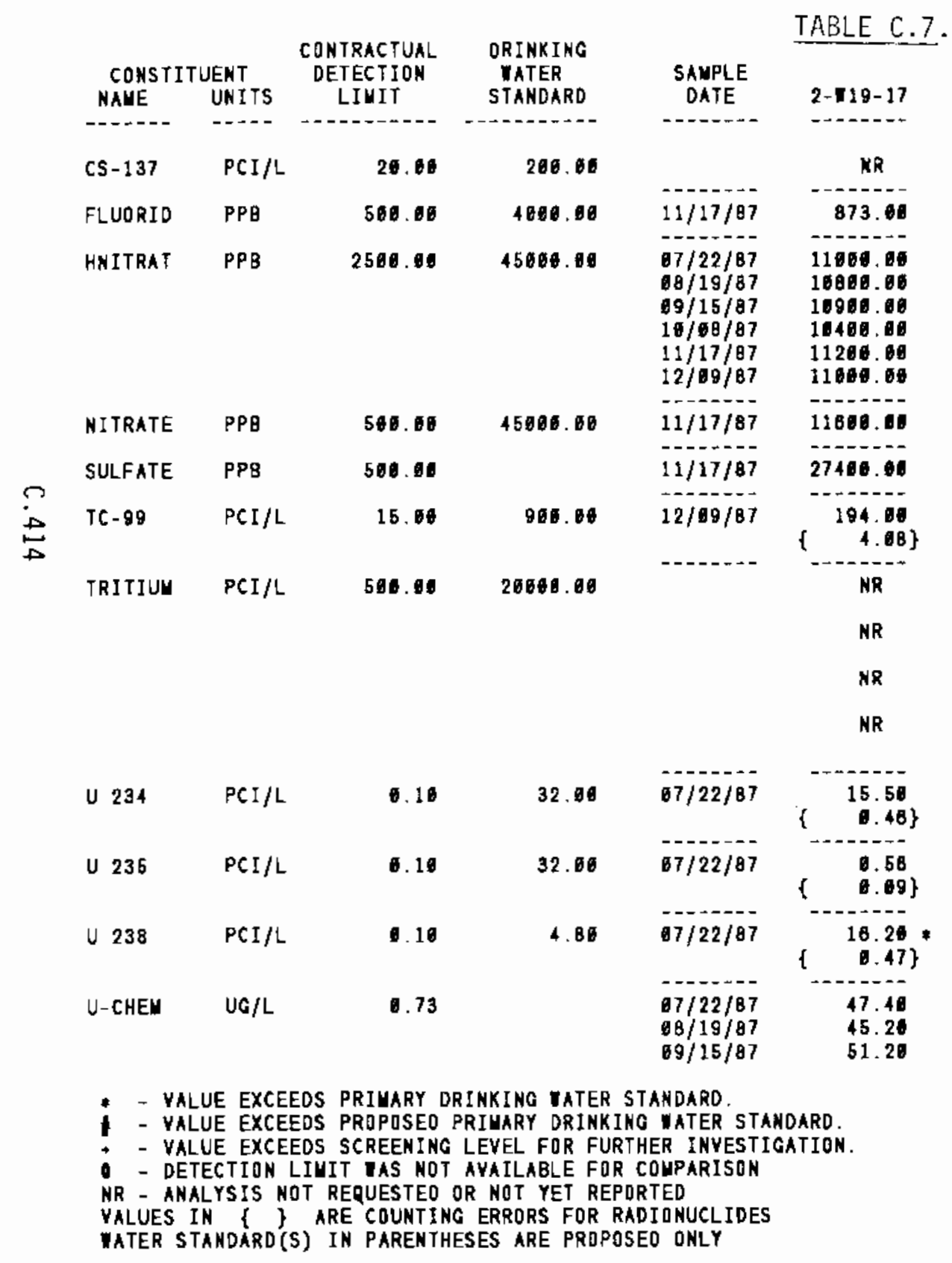

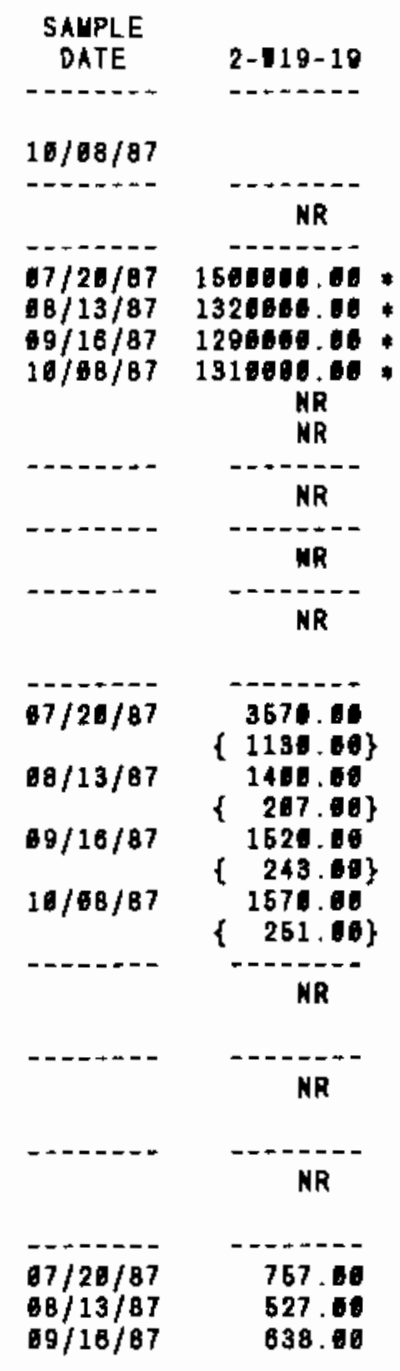


TABLE C.7. (contd)

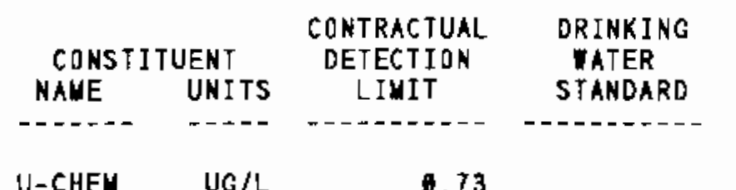

\begin{tabular}{|c|c|c|c|}
\hline $\begin{array}{l}\text { SAUPLE } \\
\text { DATE }\end{array}$ & $2-10-17$ & $\begin{array}{l}\text { SAUPLE } \\
\text { DATE }\end{array}$ & $2-18-18$ \\
\hline - - - - & - & $\cdots$ & - \\
\hline $\begin{array}{l}10 / 68 / 87 \\
11 / 17 / 87 \\
12 / 69 / 87\end{array}$ & $\begin{array}{l}31.30 \\
40.10 \\
46.90\end{array}$ & $\begin{array}{l}19 / 08 / 87 \\
11 / 17 / 87 \\
12 / 08 / 87\end{array}$ & $\begin{array}{l}6156.00 \\
7436.00 \\
6380.00\end{array}$ \\
\hline
\end{tabular}

SAUPLE

DATE

- DATE-...

U-CHEU

UG/L

t. 73

46. 90

$12 / 08 / 87$

6380.00

$16 / 68 / 87$

590.00

NR

$\overbrace{0}^{\infty}$

* - Value exceeds primary drinking tater standaro.

- - VALUE EXCEEDS PROPOSED PRIMARY dRINKIMG taTeR STANDARD.

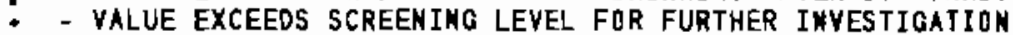

- DETECTION LIUIT tas NDT AVAILABLE For COMPARISON

NR - AMALYSIS NOT REQUESTED OR NOT YET REPORTED

VALUES IN \{ $\}$ ARE COUMTINO ERRORS FOR RADIONUCLIDES

VATER STANDARD(S) IN PARENTHESES ARE PRDPOSED ONLY 


\section{TABLE C.7. (contd)}

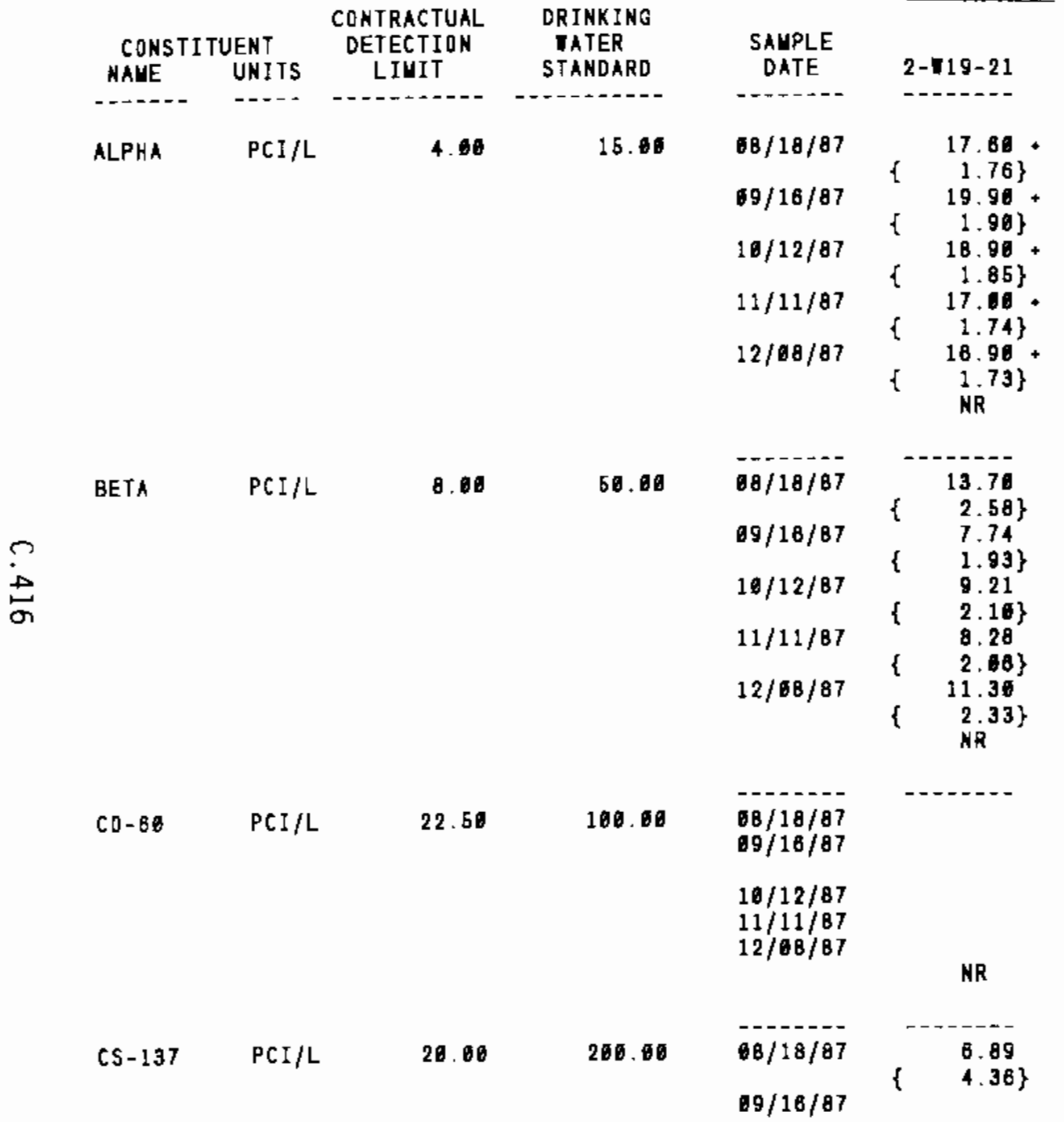

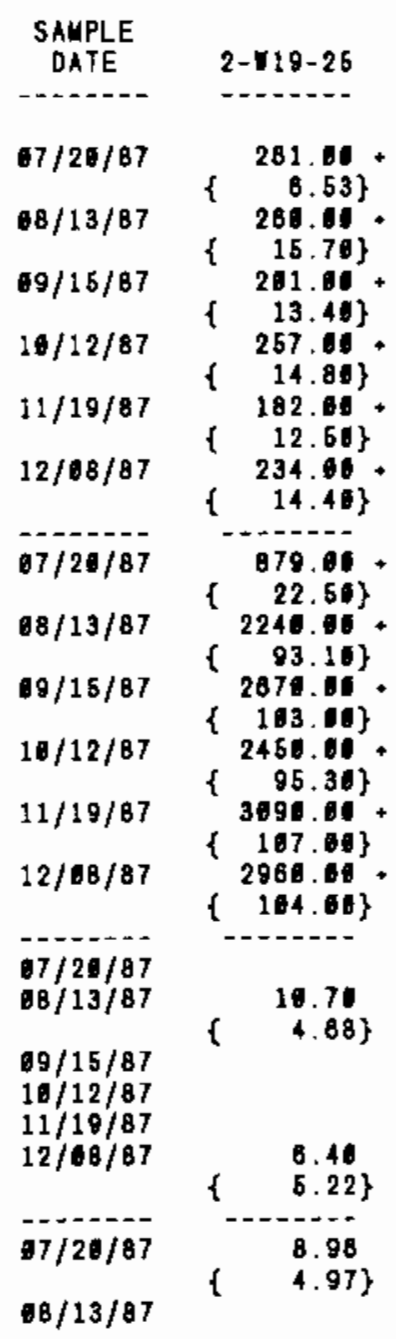

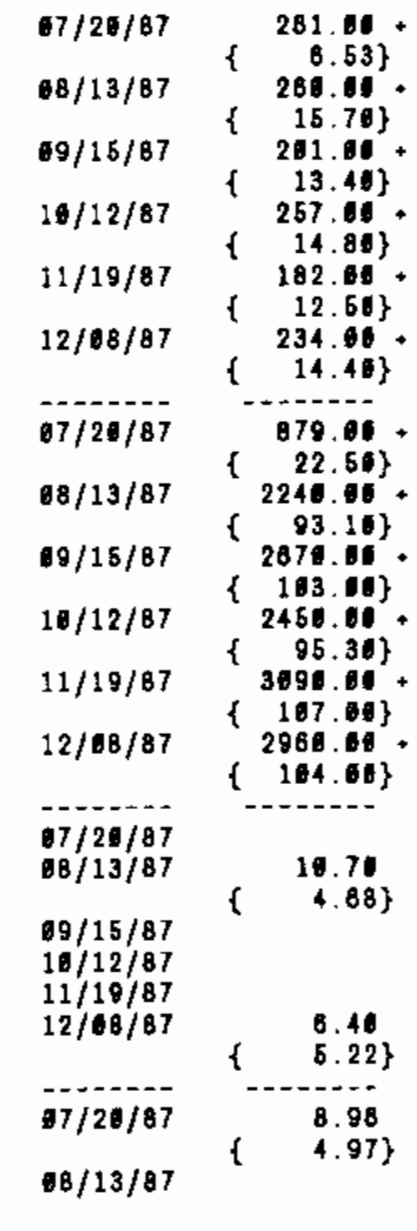

- - VALUE eXCEeds PRIVARY dRINKING MATER STANDARD.

- - VALUE EXCEEDS PROPOSED PRIMARY DRINKING WATER STANDARD

- VALUE EXCEEDS SCREENING LEYEL FOR FURTHER INYESTIGATION.

- DETECTION LINIT TAS NOT AYALABLE FOR COUPARISON

NR - ANALYSIS NOT RERUESTED OR NOT YET REPORTED

YALUES IN \{ $\}$ ARE COUNTING ERRORS FOR RADIONUCLIDES

TATER STANDARD(S) IN PARENTHESES ARE PROPOSED ONLY

\begin{tabular}{|c|c|}
\hline $\begin{array}{l}\text { SAYPLE } \\
\text { DATE }\end{array}$ & $2-19-23$ \\
\hline $67 / 20 / 87$ & 171.06. \\
\hline $68 / 13 / 87$ & $\begin{array}{l}15.53\} \\
158.08\end{array}$ \\
\hline $68 / 18 / 87$ & $\begin{array}{c}133.66\} \\
11.16\}\end{array}$ \\
\hline $10 / 12 / 87$ & $\begin{array}{c}142.60 \\
10.86\}\end{array}$ \\
\hline $11 / 18 / 87$ & 134.06 \\
\hline $12 / B 8 / B 7$ & $\left\{\begin{array}{l}140.00 \\
\{11.30\}\end{array}\right.$ \\
\hline $67 / 28 / 87$ & $\begin{array}{r}128.60 \\
\quad 8.41\}\end{array}$ \\
\hline $68 / 13 / 87$ & 284.09 \\
\hline $69 / 16 / 87$ & 367.60 \\
\hline $10 / 12 / 87$ & $\begin{array}{r}35.18 \\
250.60\end{array}$ \\
\hline $11 / 19 / 87$ & $\left\{\begin{array}{l}31.10 \\
345.80\end{array}\right.$ \\
\hline $12 / 08 / 87$ & $\left\{\begin{array}{c}338.00 \\
\quad 35.78\}\end{array}\right.$ \\
\hline $\begin{array}{l}67 / 20 / 87 \\
68 / 13 / 87\end{array}$ & \\
\hline $\begin{array}{l}69 / 16 / 87 \\
16 / 12 / 87 \\
11 / 19 / 87 \\
12 / 88 / 87\end{array}$ & \\
\hline $67 / 28 / 87$ & \\
\hline $68 / 13 / 87$ & $\begin{array}{l}3.58 \\
\quad 2.41\}\end{array}$ \\
\hline
\end{tabular}


TABLE C.7. (contd)

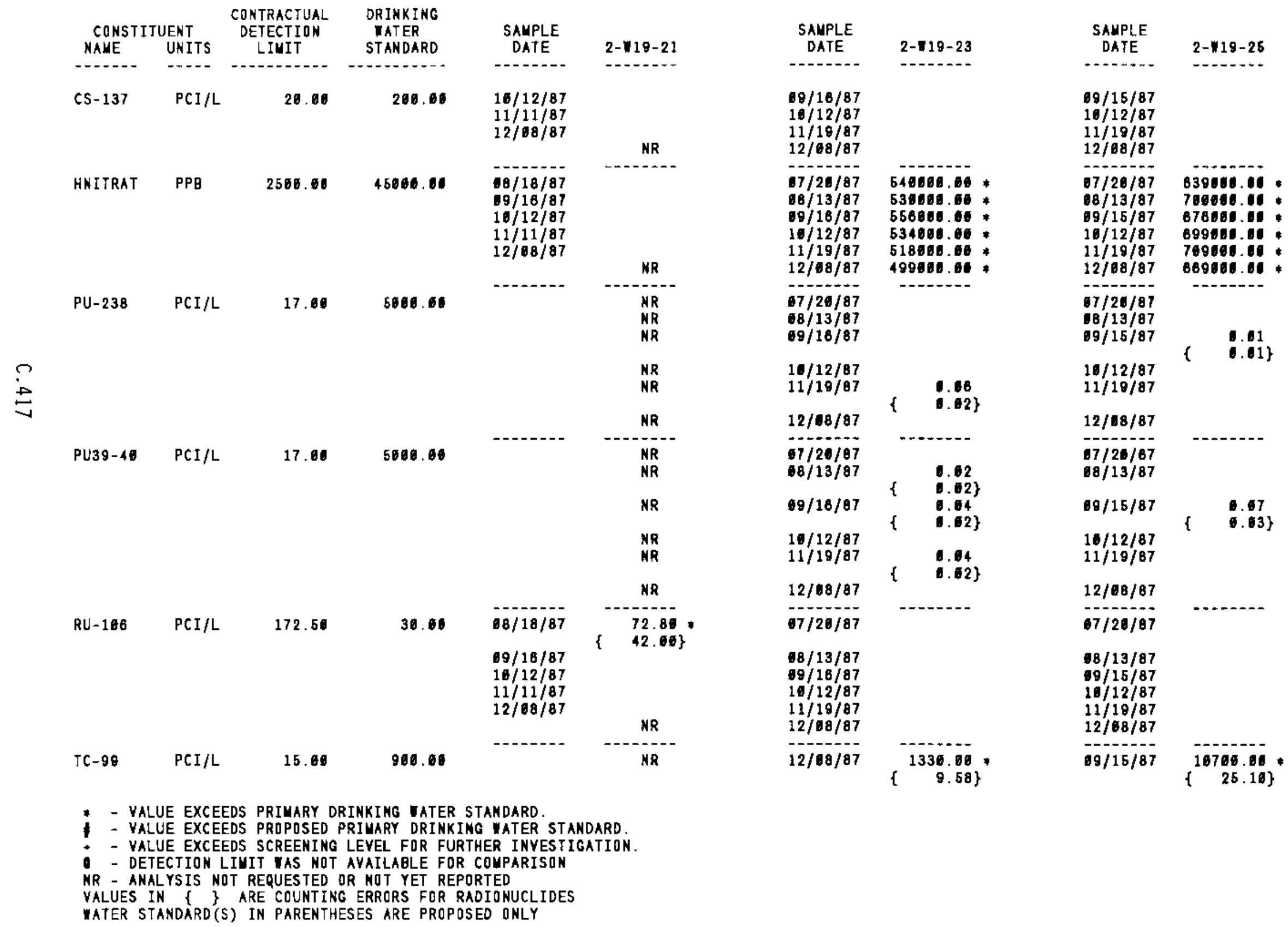


IABLE C.7. (contd)

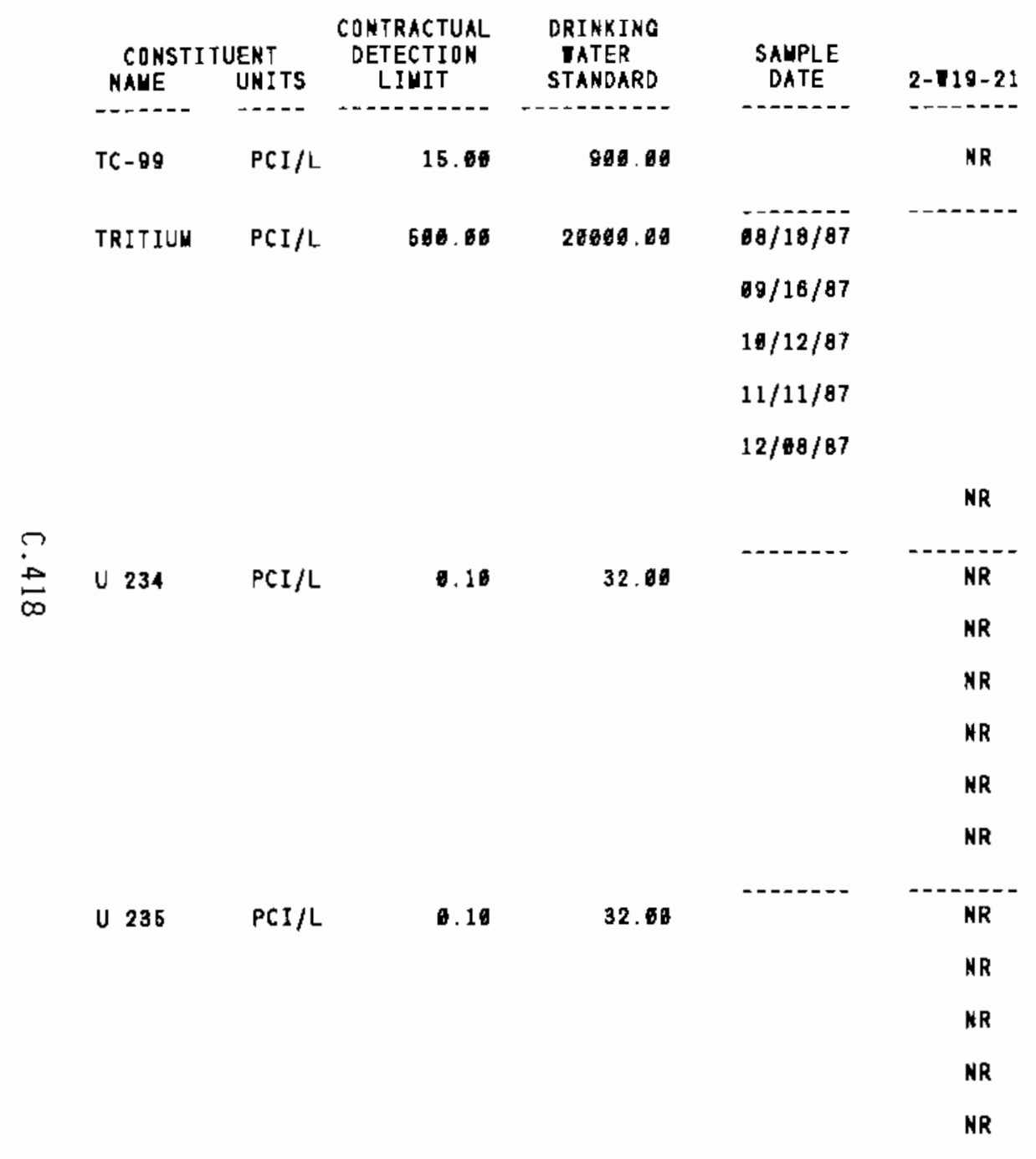

\begin{tabular}{|c|c|}
\hline \multirow[t]{2}{*}{$\begin{array}{c}\text { SAUPLE } \\
\text { DATE }\end{array}$} & $2-119-23$ \\
\hline & NR \\
\hline $87 / 20 / 87$ & 1170.00 \\
\hline $88 / 13 / 87$ & $\begin{array}{r}228.68\} \\
1890.60\end{array}$ \\
\hline $89 / 18 / 87$ & $\left\{\begin{array}{r}243.60\} \\
1690.00\end{array}\right.$ \\
\hline & $\{235.00\}$ \\
\hline $18 / 12 / 87$ & 847.64 \\
\hline $11 / 19 / 87$ & $\left\{\begin{array}{r}228.00\} \\
1010.00\end{array}\right.$ \\
\hline & $\left\{\begin{array}{l}190.06\} \\
857.00\end{array}\right.$ \\
\hline & \\
\hline $07 / 20 / 87$ & $74.76 *$ \\
\hline $98 / 13 / 87$ & $87.66 *$ \\
\hline $89 / 18 / 87$ & $73.60^{\circ}$ \\
\hline $10 / 12 / 87$ & $82.10^{\circ}$ \\
\hline $11 / 19 / 87$ & 68.58, \\
\hline $12 / 0 B / B 7$ & $\begin{array}{r}28.26 \\
\{\quad 2.56\}\end{array}$ \\
\hline $07 / 20 / 67$ & 3.67 \\
\hline $88 / 13 / 97$ & 1.83 \\
\hline $89 / 16 / 87$ & $\begin{array}{l}1.10\} \\
2.34\end{array}$ \\
\hline $18 / 12 / 87$ & (6.43) \\
\hline & $0.77\}$ \\
\hline $11 / 19 / 87$ & $\begin{array}{l}3.71 \\
\cdot .67\}\end{array}$ \\
\hline
\end{tabular}

\begin{tabular}{|c|c|}
\hline $\begin{array}{l}\text { SAUPLE } \\
\text { DATE }\end{array}$ & $2-719-25$ \\
\hline $12 / 08 / 87$ & $\left\{\begin{array}{c}13886.06 \\
\{\quad 29.80\end{array}\right.$ \\
\hline $67 / 20 / 87$ & $\left.\begin{array}{c}1720.10 \\
242.00\end{array}\right\}$ \\
\hline $68 / 13 / 87$ & $\begin{array}{r}1530.06 \\
25 B .60\}\end{array}$ \\
\hline $99 / 16 / 87$ & 1850.00 \\
\hline $18 / 12 / 87$ & $\begin{array}{r}1700.00 \\
\{\quad 251.00\}\end{array}$ \\
\hline $11 / 19 / 87$ & 1576.01 \\
\hline $12 / 08 / 87$ & $\begin{array}{r}256.00\} \\
2568.80 \\
\{\quad 228.86\}\end{array}$ \\
\hline $67 / 28 / 87$ & $\left\{\begin{array}{r}135.60 \\
\{3.26\end{array}\right.$ \\
\hline $68 / 13 / 87$ & $\left\{\begin{array}{r}124.06^{*} \\
9.04\}\end{array}\right.$ \\
\hline $69 / 15 / 87$ & $\begin{array}{r}126.00^{\circ} \\
\end{array}$ \\
\hline $10 / 12 / 87$ & $118.00^{\circ}$ \\
\hline $11 / 19 / 87$ & 109. \\
\hline $12 / 88 / 87$ & $\begin{array}{c}132.06{ }^{*} \\
\{\quad 3.95\}\end{array}$ \\
\hline $87 / 20 / 87$ & 5. 71 \\
\hline $88 / 13 / 87$ & \\
\hline $09 / 15 / 87$ & $\begin{array}{l}2.47\} \\
5.01\end{array}$ \\
\hline $10 / 12 / 87$ & $\begin{array}{l}6.87\} \\
4.29\end{array}$ \\
\hline & $\begin{array}{l}0.58\} \\
8.85\end{array}$ \\
\hline & $0.76\}$ \\
\hline
\end{tabular}

- - value exceeds primary drinking nater standard.

- VALUE EXCEEDS PROPDSED PRIUARY DRI INING MATER STANOARD.

* - Value eXceEds SCREening leVel for further inVestigation.

- Detection limit tas not available for coUparison

NR - ANALYSIS NOT RERUESTED OR NDT YET REPORTED

VALUES IN \{\} ARE COUNTING ERRORS FOR RADIONUCLIOES

VATER STANDARD(S) IN PARENTHESES aRE PROPOSED ONLY 
TABLE C.7. (contd)

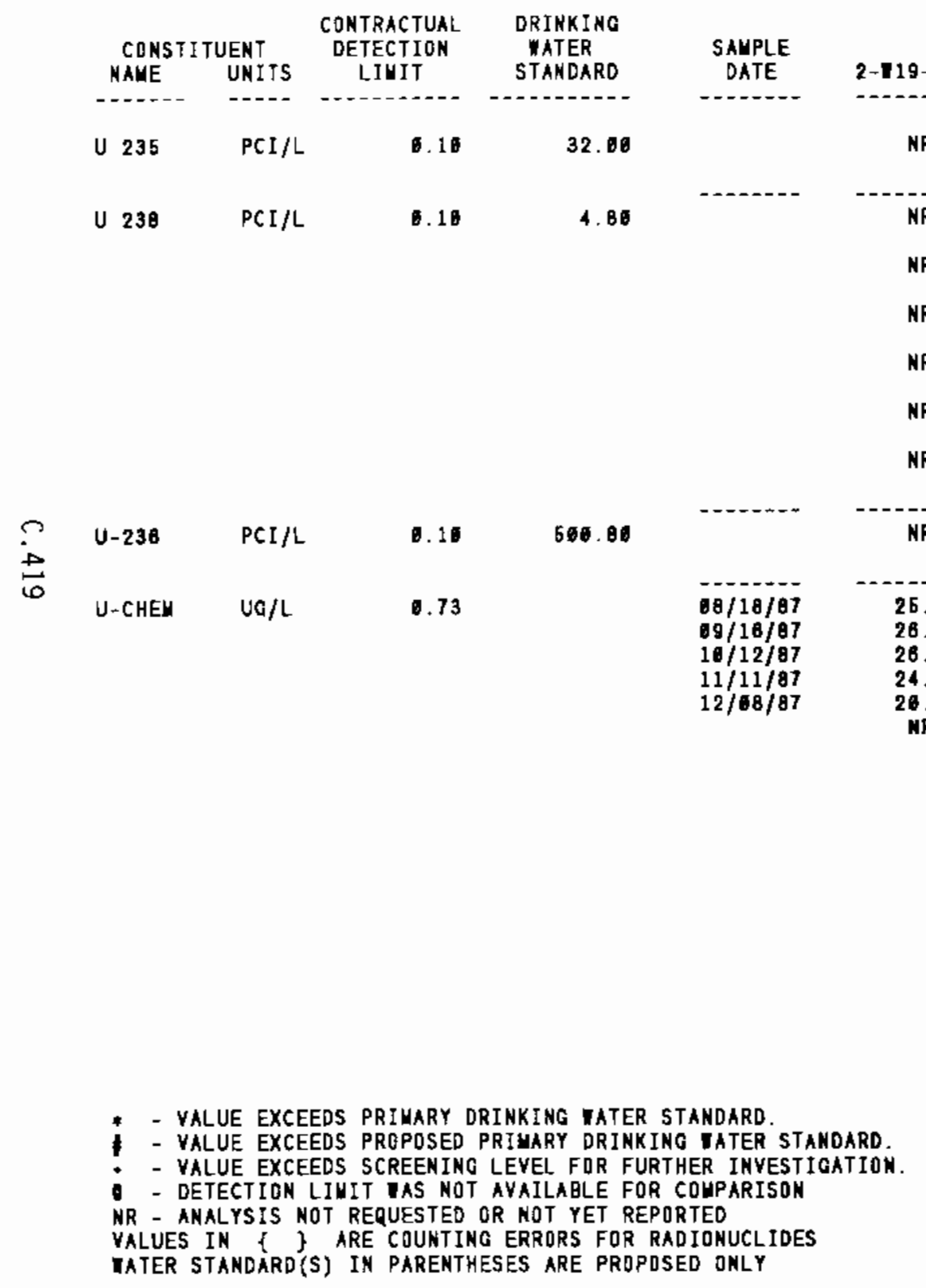

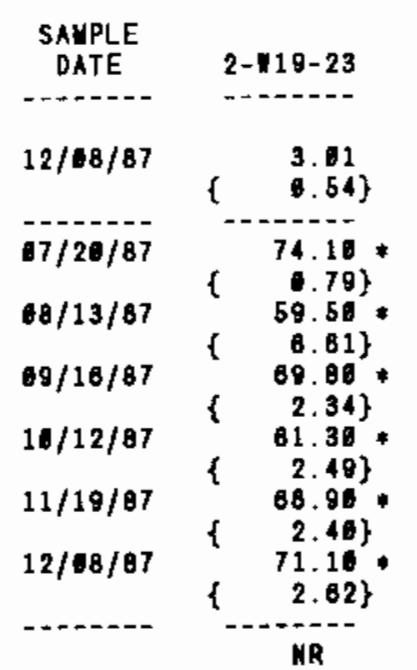

$07 / 26 / 87$

$07 / 26 / 87$

$6 / 13 / 87$
$69 / 16 / 87$

$10 / 12 / 87$

$11 / 19 / 87$

$12 / 08 / 87$

186.96

186.96

185.00

212.00

208.06

217.06

198.60
SALPLE

DATE

$12 / 08 / 87$

$2-119-26$

$12 / 08 / 87 \quad 6.37$

$67 / 26 / 87$

$68 / 13 / 87$

$69 / 15 / 87$

$10 / 12 / 87$

$11 / 10 / 87$

$12 / 08 / 87$

$69 / 16 / 87$

$6.87\}$
.-137.09

137.00

$\left\{\begin{array}{r}3.24\} \\ 133.08\end{array}\right.$

$(\quad 0.05\}$

123.09.

(3.15)

$\{3.6\}$

116.65

$\{3.13\}$

$\left\{\begin{array}{r}138.64 \\ 4.04\end{array}\right.$

1.78

$67 / 20 / 87$

98/13/87

99/15/87

$10 / 12 / 87$

$11 / 18 / 87$

$12 / 68 / 87$

1. 78

-

381.8

384.65

360.00

411.00

374.00

375.00

DALUE EXCEDS SCREENING LEVEL FOR FURTHER INYESTC

VALUES IN I ARE COUNTING ERRORS FOR RADIONUCLIDES

IATER STANDARD(S) IN PARENTHESES ARE PRDPDSED ONLY 
TABLE C.7. (contd)

\begin{tabular}{|c|c|c|c|c|c|}
\hline $\begin{array}{c}\text { CONST } \\
\text { NAUE }\end{array}$ & $\begin{array}{l}\text { UENT } \\
\text { UNITS }\end{array}$ & $\begin{array}{l}\text { CONTRACTUAL } \\
\text { DETECTION } \\
\text { LIUIT }\end{array}$ & $\begin{array}{l}\text { DRINKING } \\
\text { WATER } \\
\text { STANDARD }\end{array}$ & $\begin{array}{l}\text { SALPLE } \\
\text { DATEE }\end{array}$ & $2-19-2 B$ \\
\hline ALPHA & $\mathrm{PCI} / \mathrm{L}$ & 4.08 & 15.06 & $\begin{array}{l}07 / 20 / 87 \\
08 / 13 / 87 \\
11 / 19 / 87 \\
12 / 08 / 87\end{array}$ & 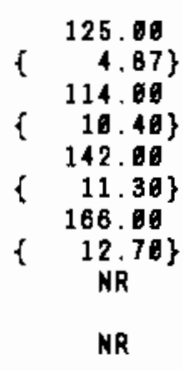 \\
\hline BETA & PCI/L & 8.86 & 58.08 & $\begin{array}{l}07 / 20 / 87 \\
08 / 13 / 87 \\
11 / 19 / 87 \\
12 / 08 / 87\end{array}$ & 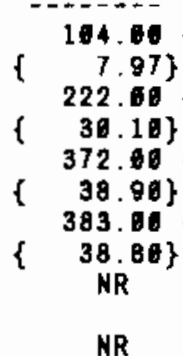 \\
\hline$C 0-60$ & PCI/L & 22.58 & 100.80 & $\begin{array}{l}07 / 28 / 87 \\
08 / 13 / 87 \\
11 / 19 / 87 \\
12 / 08 / 87\end{array}$ & 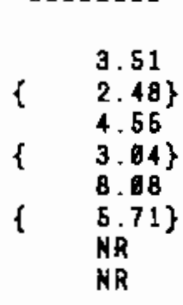 \\
\hline CS -137 & PCI $/ \mathrm{L}$ & 29.80 & 200.06 & $\begin{array}{l}87 / 26 / 87 \\
68 / 13 / 87\end{array}$ & \\
\hline
\end{tabular}

\begin{tabular}{|c|c|c|c|}
\hline $\begin{array}{c}\text { SANPLE } \\
\text { DATE }\end{array}$ & $2-19-27$ & $\begin{array}{c}\text { SALPLE } \\
\text { DATE }\end{array}$ & $2-\pi 21-1$ \\
\hline $07 / 01 / 87$ & 8. 51 & & NR \\
\hline$B B / 1 B / B 7$ & 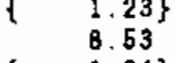 & & NR \\
\hline $09 / 18 / 87$ & $\left\{\begin{array}{r}1.24\} \\
10.49\end{array}\right.$ & & NR \\
\hline & $\{\quad 1.34\}$ & & \\
\hline $18 / 12 / 87$ & $\begin{array}{r}0.81 \\
\{\quad 1.22\}\end{array}$ & & NR \\
\hline $11 / 11 / 87$ & 9.54 & & NR \\
\hline $12 / 68 / 87$ & $\left\{\begin{array}{l}9.14 \\
\quad 1.28\end{array}\right.$ & & NR \\
\hline $07 / 01 / 87$ & $\begin{array}{l}15.26 \\
2.79\}\end{array}$ & & WR \\
\hline $8 B / 18 / 87$ & 10.20 & & MR \\
\hline $09 / 18 / 87$ & 3.81\} & & MR \\
\hline $08 / 10 / 01$ & $\begin{array}{r}11.34\} \\
2.34\end{array}$ & & Nn \\
\hline $10 / 12 / 87$ & $17.70^{\circ}$ & & NR \\
\hline & $\left\{\begin{array}{r}2.83\} \\
13.06\end{array}\right\}$ & & NR \\
\hline $12 / 88 / 87$ & 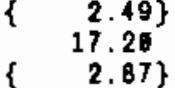 & & MR \\
\hline $\begin{array}{l}07 / 91 / 87 \\
68 / 18 / 87\end{array}$ & & $98 / 17 / 87$ & NR \\
\hline $69 / 18 / 87$ & & & NR \\
\hline $16 / 12 / 87$ & & & NR \\
\hline $\begin{array}{l}11 / 11 / 87 \\
12 / 08 / 87\end{array}$ & & & $\begin{array}{l}\text { NR } \\
\text { NR }\end{array}$ \\
\hline $\begin{array}{l}07 / 01 / 87 \\
08 / 18 / 87\end{array}$ & & $89 / 17 / 07$ & NR \\
\hline
\end{tabular}

- Value exceeds primary drinking mater stanoaro.

- YALUE EXCEEDS PROPOSED PRIYARY DRINKING MATER STANDARO.

- VALUE EXCEEDS SCREENING LEVEL FOR FURTHER INYESTIGATIDN.

- DETECTION LIMIT US NDT AVAILABLE for couparisoy

NR - ANALYSIS NGT REQUESTED OR NOT YET REPORTED

VALUES IN \{\} ARE COUNTING ERRORS FOR RADIONUCLIDES

ATER STANDARD(S) IN PARENTHESES ARE PRDPOSED DNLY 
TABLE C.7. (contd)

\begin{tabular}{|c|c|c|c|c|c|}
\hline $\begin{array}{l}\text { CONSTI } \\
\text { NAME }\end{array}$ & $\begin{array}{l}\text { UENT } \\
\text { UNITS }\end{array}$ & $\begin{array}{c}\text { CONTRACTUAL } \\
\text { DETECTION } \\
\text { LIUIT }\end{array}$ & $\begin{array}{l}\text { DRINKING } \\
\text { WATER } \\
\text { STANOARD }\end{array}$ & $\begin{array}{l}\text { SALPLE } \\
\text { OATE }\end{array}$ & $2-119-28$ \\
\hline----- & ---- & - - n- & - - n- & $-\ldots-n$ & $-\cdots+\infty$ \\
\hline$C 5-137$ & $\mathrm{PCI} / \mathrm{L}$ & 20.00 & 200.00 & $\begin{array}{l}11 / 18 / 87 \\
12 / 68 / 87\end{array}$ & 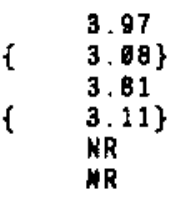 \\
\hline
\end{tabular}

$2500.00-450090000$ $\begin{array}{ll}07 / 13 / 87 & 717000.00 * \\ 11 / 18 / 87 & 927000.06\end{array}$ $\begin{array}{rr}11 / 18 / 87 & 927000.00 * \\ 12 / 08 / 87 & 1290000.08 *\end{array}$ NR NR

$\begin{array}{llll}\mathrm{RU}-106 & \mathrm{PCI} / \mathrm{L} & 172.50 & 30.60 \quad 07 / 20 / 87 \\ 08 / 13 / 87\end{array}$

$11 / 19 / 87$

$D$
$\mathbb{N}$

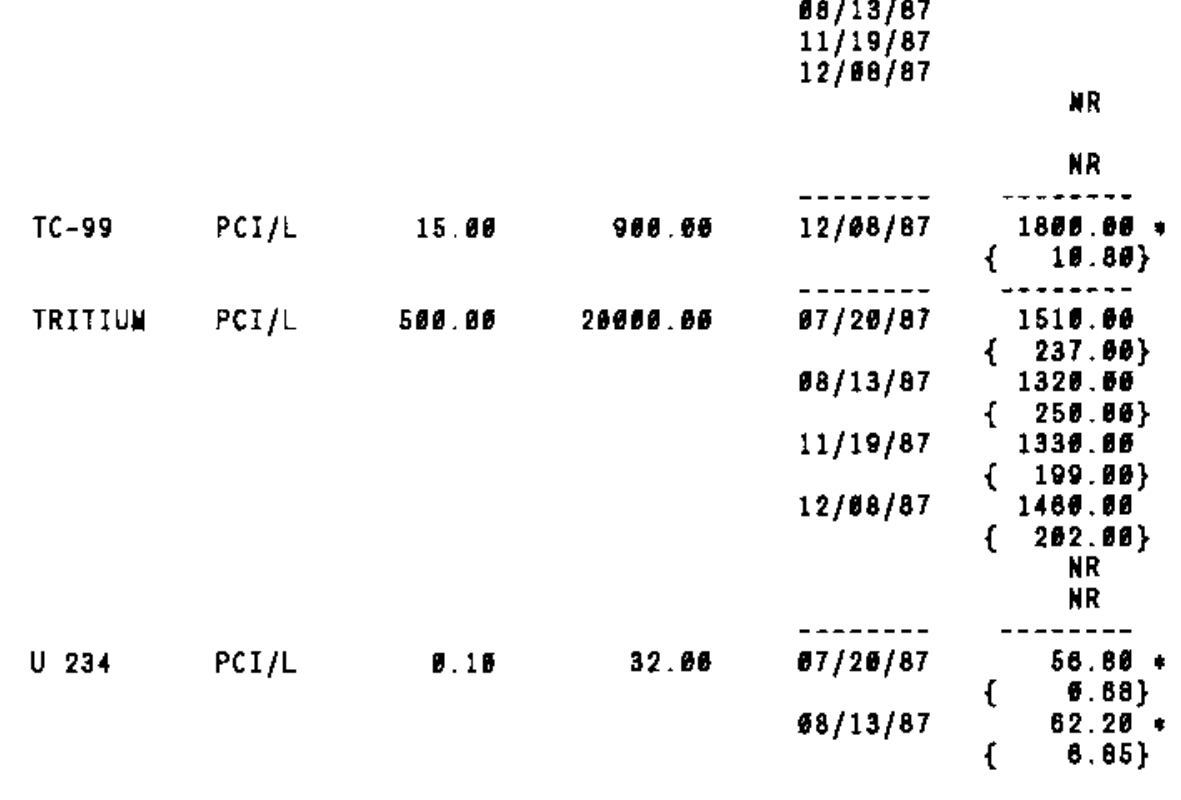

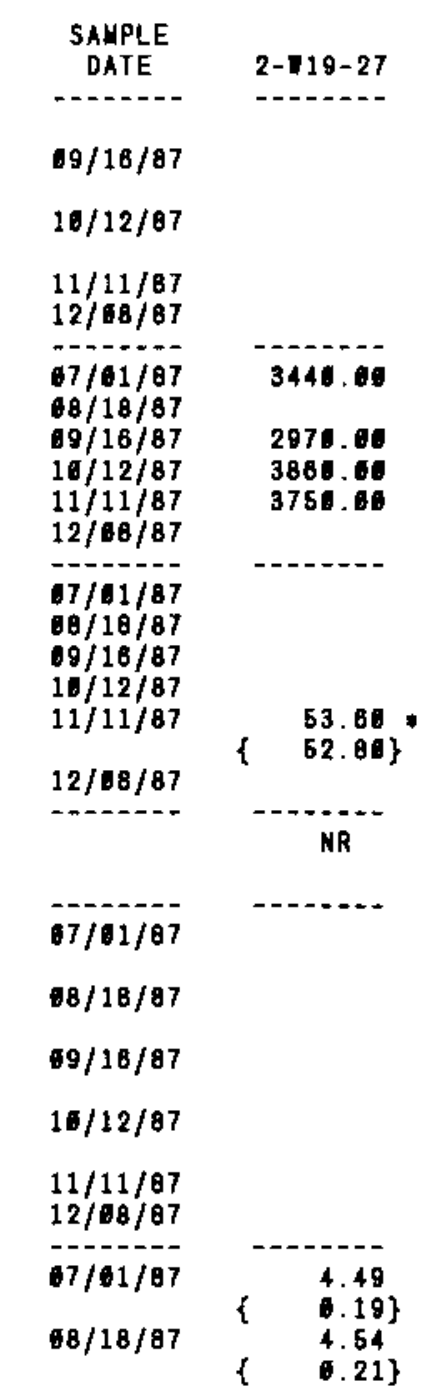

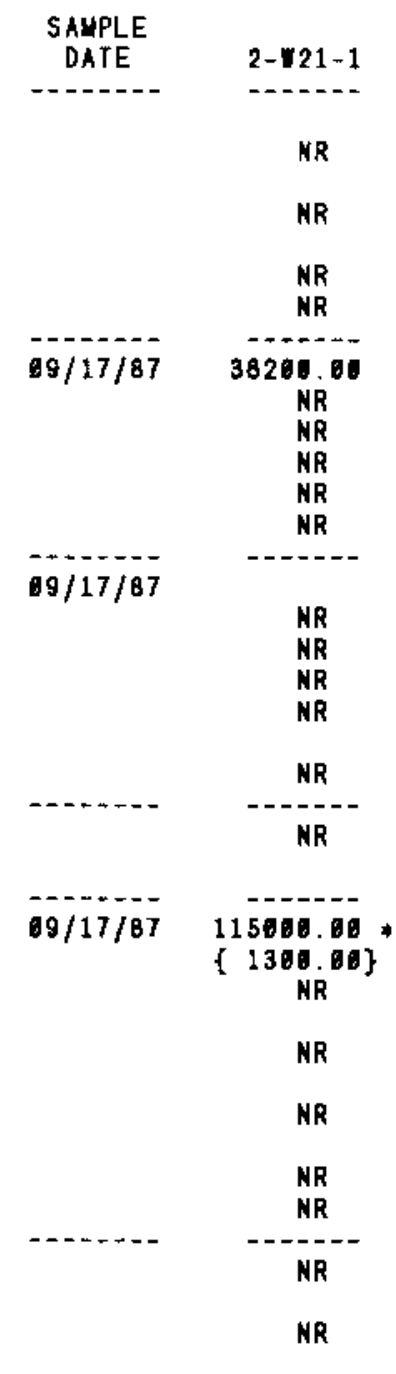

* - Value exceeds primary drinking mater standard.

| - VALUE EXCEEDS PROPOSED PRIUARY ORINKING MATER STANDARD.

- VALUE EXCEEDS SCREENing LEVEL FOR FURTHER InVESTIgation.

- DETECTION LIUIT WAS NDT AVAILABLE FOR COUPARISUN

NR - ANALYSIS NDT REQUESTED OR NOT YET REPORTEO

VALUES IN \{\} ARE COUNTING ERRORS FOR RADIONUCLIDES

TATER STANDARD(S) IN PARENTHESES ARE PROPOSED ONLY 
TABLE C.7. (contd)

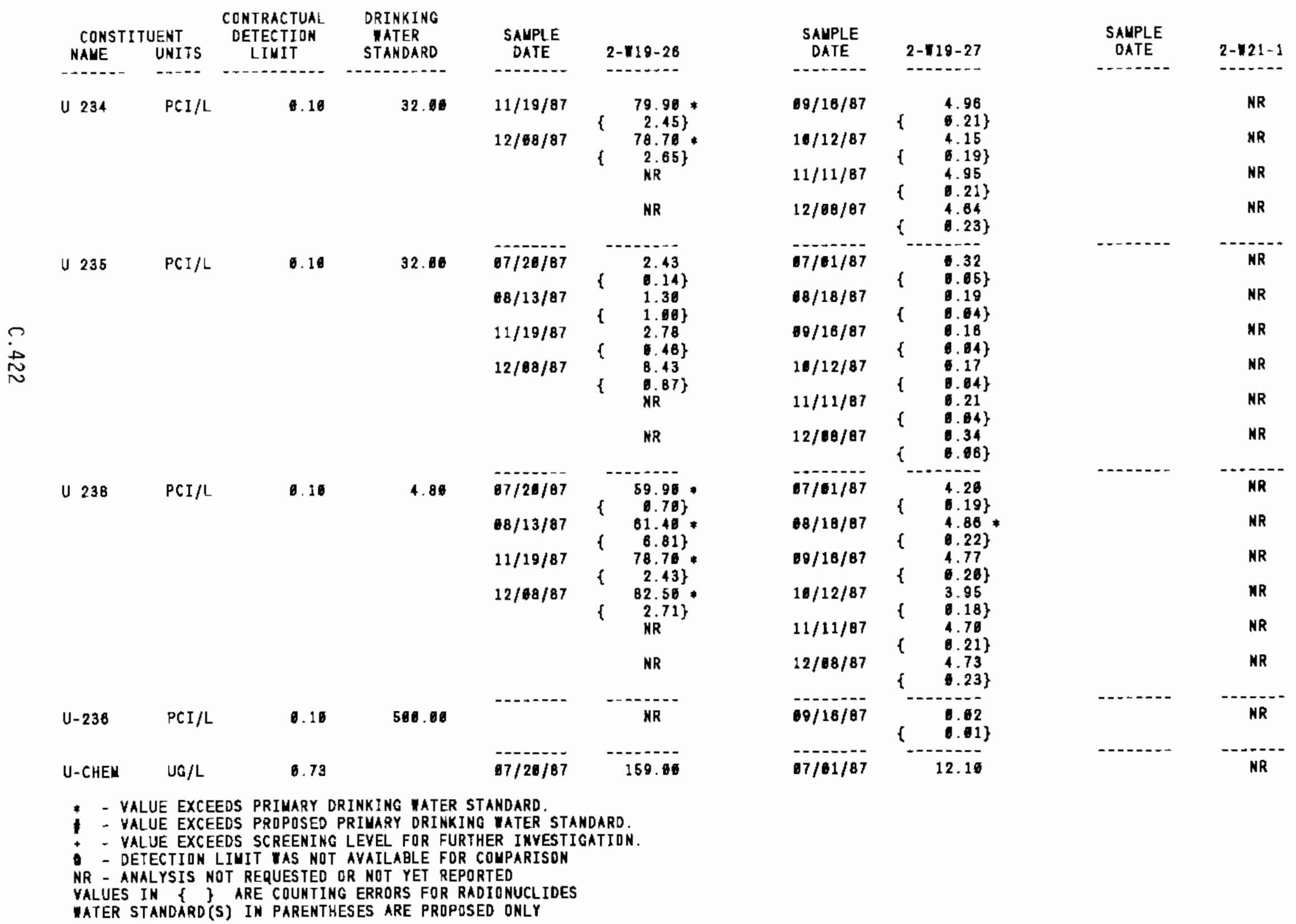


TABLE C.7. (contd)

\begin{tabular}{|c|c|c|c|c|c|c|c|c|c|}
\hline $\begin{array}{l}\text { CONSTI } \\
\text { NAUE }\end{array}$ & $\begin{array}{l}\text { UENT } \\
\text { UNITS }\end{array}$ & $\begin{array}{c}\text { CONTRACTUAL } \\
\text { DETECTION } \\
\text { LIMIT }\end{array}$ & $\begin{array}{l}\text { DRINKING } \\
\text { WATER } \\
\text { STANDARD }\end{array}$ & $\begin{array}{c}\text { 5AMPLE } \\
\text { OATE }\end{array}$ & $2-718-28$ & $\begin{array}{l}\text { SAUPLE } \\
\text { DATE }\end{array}$ & $2-19-27$ & $\begin{array}{c}\text { SAUPLE } \\
\text { DATE }\end{array}$ & $2-21-1$ \\
\hline----- & $\cdots-$ & $---\infty-n-$ & $\cdots--\infty \cdots$ & $-----\infty$ & $-\infty-\infty-$ & ------ & ------ & $\cdots--1--$ & ------- \\
\hline U-CHEM & $\mathrm{UG} / \mathrm{L}$ & 6.73 & & $\begin{array}{l}6 B / 13 / 87 \\
11 / 19 / 87 \\
12 / 68 / 87\end{array}$ & $\begin{array}{c}196.00 \\
245.00 \\
222.00 \\
\text { MR } \\
\text { MR }\end{array}$ & $\begin{array}{l}68 / 18 / 87 \\
69 / 16 / 87 \\
10 / 12 / 87 \\
11 / 11 / 87 \\
12 / 08 / 87\end{array}$ & $\begin{array}{l}11.60 \\
13.00 \\
12.60 \\
12.00 \\
16.00\end{array}$ & & $\begin{array}{l}\text { NR } \\
\text { NR } \\
\text { NR } \\
\text { NR } \\
\text { NR }\end{array}$ \\
\hline
\end{tabular}

* - VAlUe eXCEeds primary dRIHKing vater standard.

* VALUE EXCEEDS PROPDSED PRIMARY DRINKING IATER STANDARD.

- VALUE EXCEEDS SCREENING LEVEL FOR FURTHER INYESTIGATION.

- DETECTION LIMIT IAS MOT AVAILABLE FOR COUPARISUN

HR - ANALYSIS NOT REQUESTED OR NOT YET REPDRTED

VALUES IN \{\} ARE COUNTING ERRORS FOR RADIONUCLIDES

WATER STANDARO'(S) IN PARENTHESES ARE PROPOSED ONLY 
TABLE C.7. (contd)

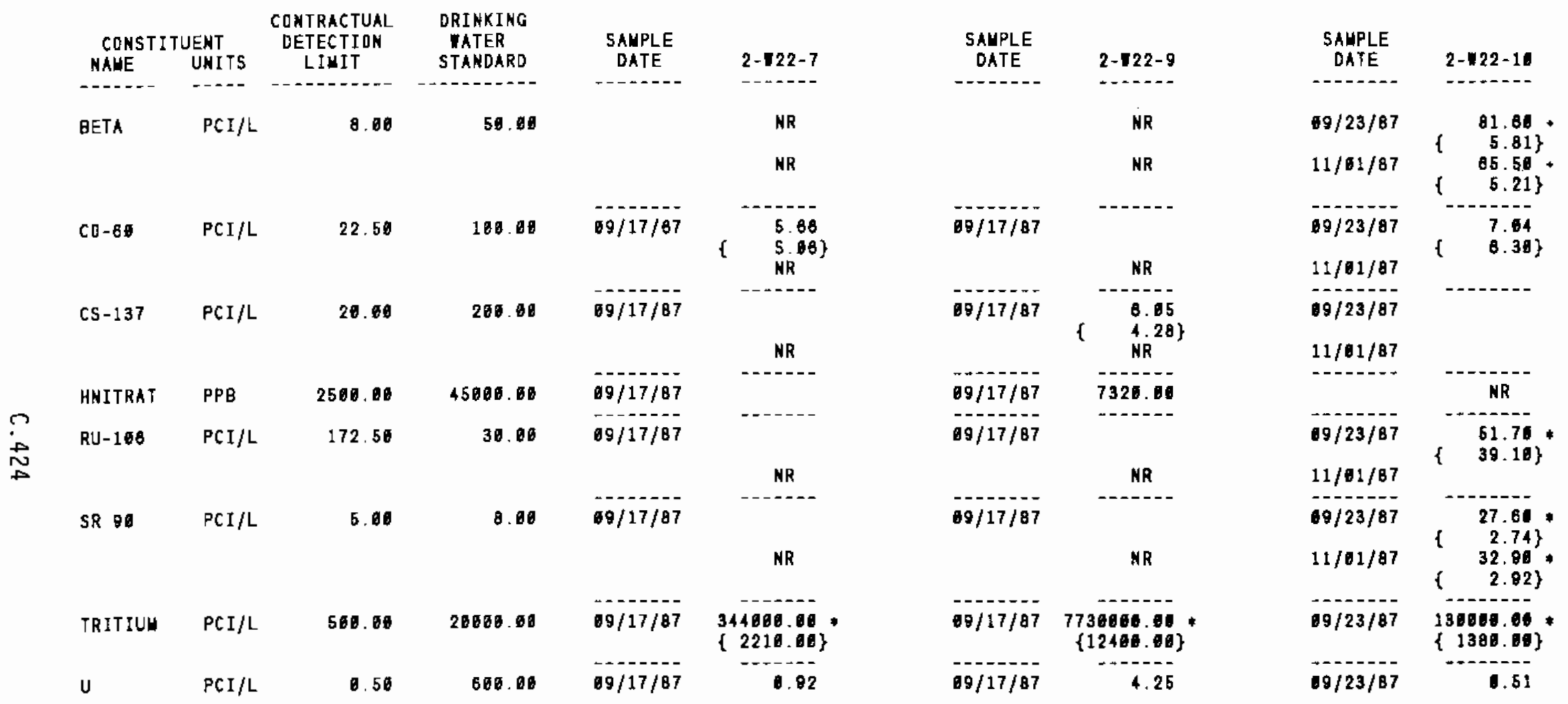

* - VALUE exceEdS PRIMARY dRINKING water STANDARD.

- - VALUE EXCEEDS PROPOSED PRIUARY DRINKING WATER STANDARD.

+ - VALUE EXCEEOS SCREENING LEVEL FOR FURTHER INVESTIGATION.

- DETECTION LiMIT MAS NOT aVAILABLE FOR CDLPARISOH

NR - ANALYSIS NOT REQUESTED OR NOT YET REPORTED

YALUES IN \& \& ARE COUNTING ERRORS FOR RAOIDNUCLIDES

WATER STANDARD(S) IN PARENTHESES ARE PROPOSED ONLY 
TABLE C.7. (contd)

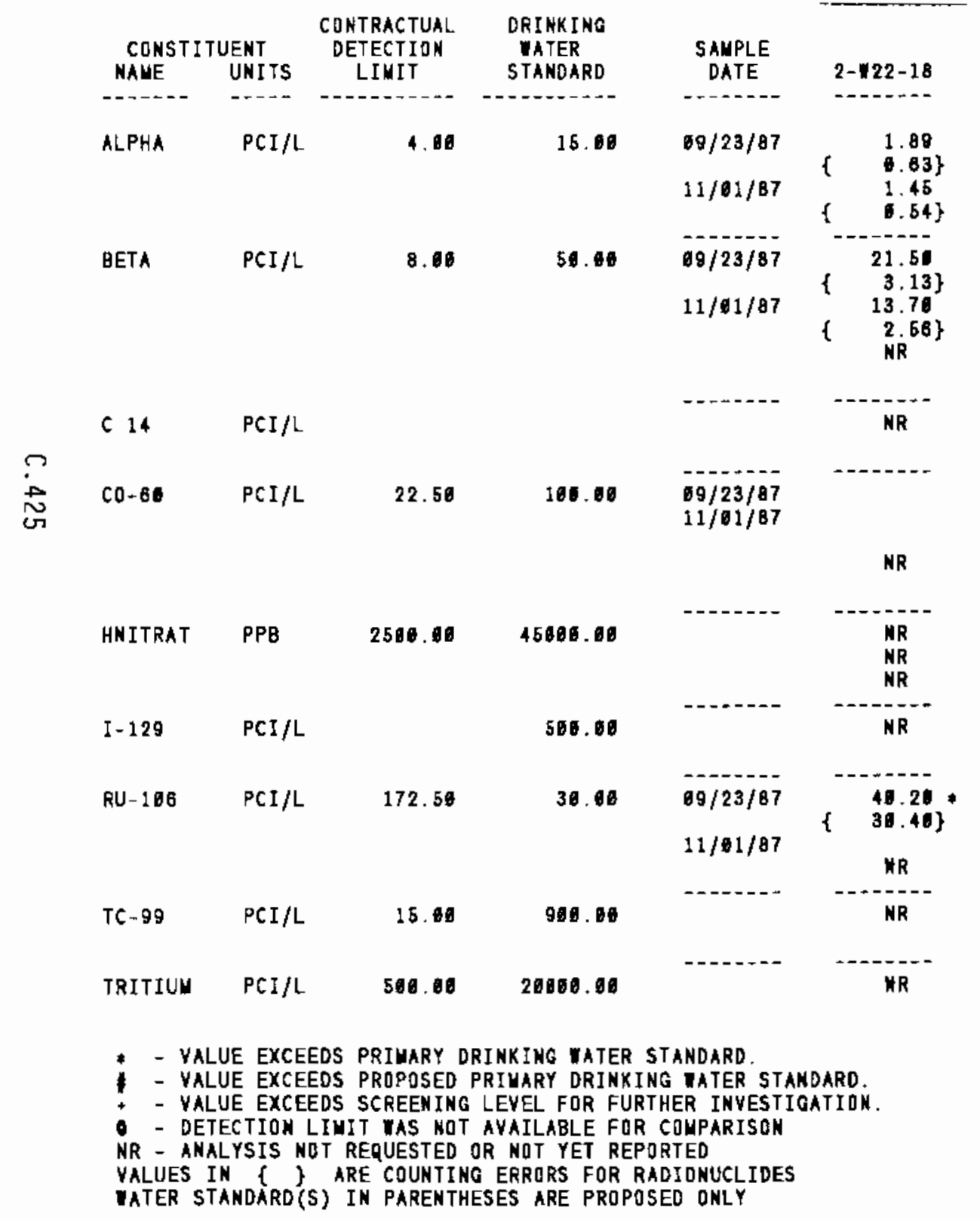

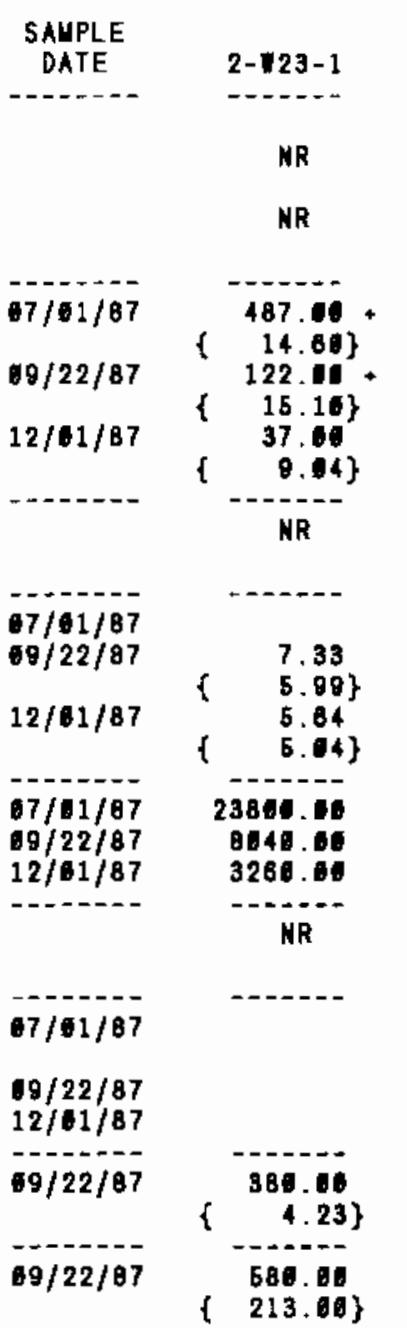


TABLE C.7. (contd)

\begin{tabular}{ccc}
\multicolumn{2}{c}{ CONSTITUENT } & $\begin{array}{c}\text { CONTRACTUAL } \\
\text { DETECTION } \\
\text { NANE }\end{array}$ UNITS \\
LIMIT
\end{tabular}
DRINKING
TAFER
STANDARD

U

0.50

600.00

U-CHEM UG/L

๑. 73

$\begin{array}{cc}\text { SAUPLE } & \\ \text { DATE } & 2-122-18 \\ & \text { NR } \\ & \text { NR } \\ & \text { NR }\end{array}$

SAMPLE

DATE

+....-.-

2-122-21

SAMPLE

DATE

2-123-1

NR

$99 / 17 / 87$

NR
20.20
23.20

$69 / 22 / 87$

7.14
$-N R$
NR

* - Value exceeds priuary drinKing mater standard

- - VALUE EXCEEDS PRQPOSED PRIMARY ORINKING WATER STANOARD.

- - VALUE EXCEEDS SCREENING LEVEL FOR FURTHER INVESTIGATION.

- - DETECTION LiUit was not aVAILABLE for COUPARISON

NR - ANALYSIS NOT REQUESTED OR NOT YET REPDRTEO

VALUES IN \{ $\}$ ARE COUNTING ERRORS FDR RADIONUCLIOES
WTER STANDARD(S) IN PARENTHESES ARE PROPOSEO ONLY 
TABLE C.7. (contd)

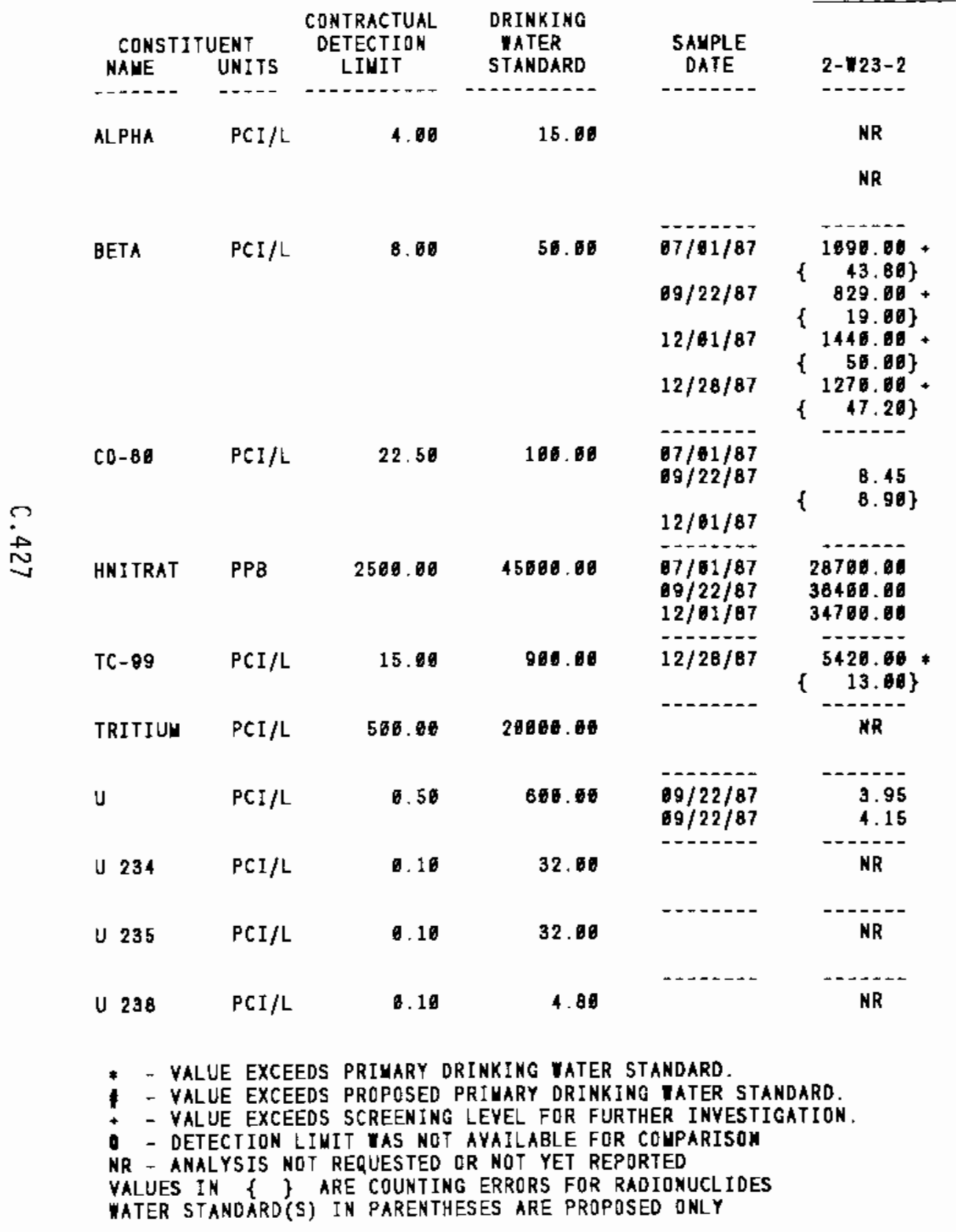

SALPLE DATE DATE

$2-\div 23-4$

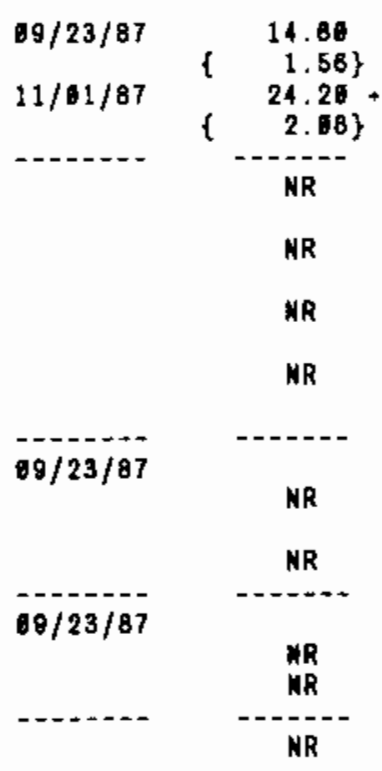

$09 / 23 / 87$

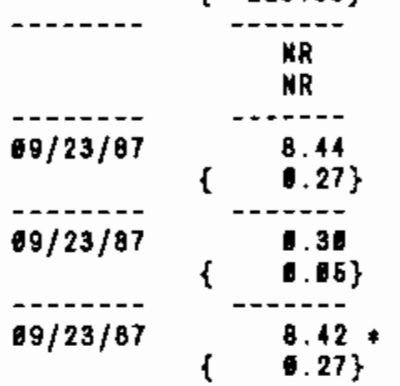

780 $\{220.00\}$

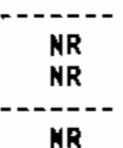

MR

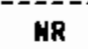

$----$

* - VALUE EXCEEDS PROPOSED PRIMARY DRINKING TATER STANDARD.

- DETECIION LIUIT UAS NOT AYAILABLE FOR COUPARISOA

VALUES IN \{ \} ARE COUNTING ERRORS FOR RADIONUCLIDES

WATER STANOARD'S) IN PARENTHESES ARE PROPOSED ONLY 
TABLE C.7. (contd)

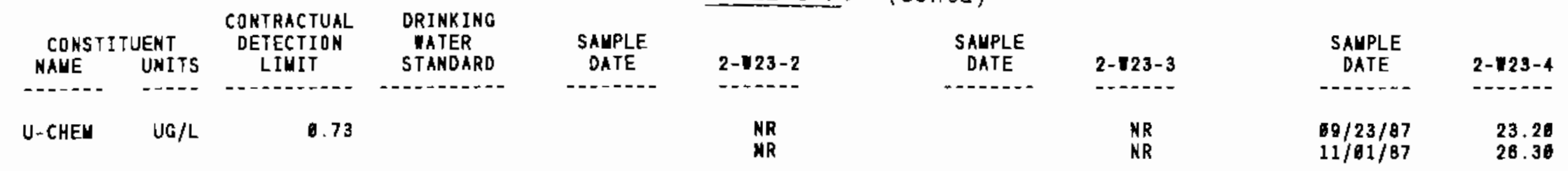

* - value exceeds primary drinking mater standard.

1 - VALUE EXCEEDS PROPOSED PRIMARY DRINKING IATER STANDARD.

- VALUE EXCEEDS SCREENING LEYEL FOR FURTHER INVESTIGATION.

- detection liUit ins not availagle for couparison

NR - ANALYSIS NOT REQUESTED OR NOT YET REPORTED

VALUES IN \& I ARE COUNTING ERRQRS FOR RADIOHUCLIDES

IATER STAKDARD (S) IN PARENTHESES ARE PROPOSED ONLY 
TABLE C.7. (contd)

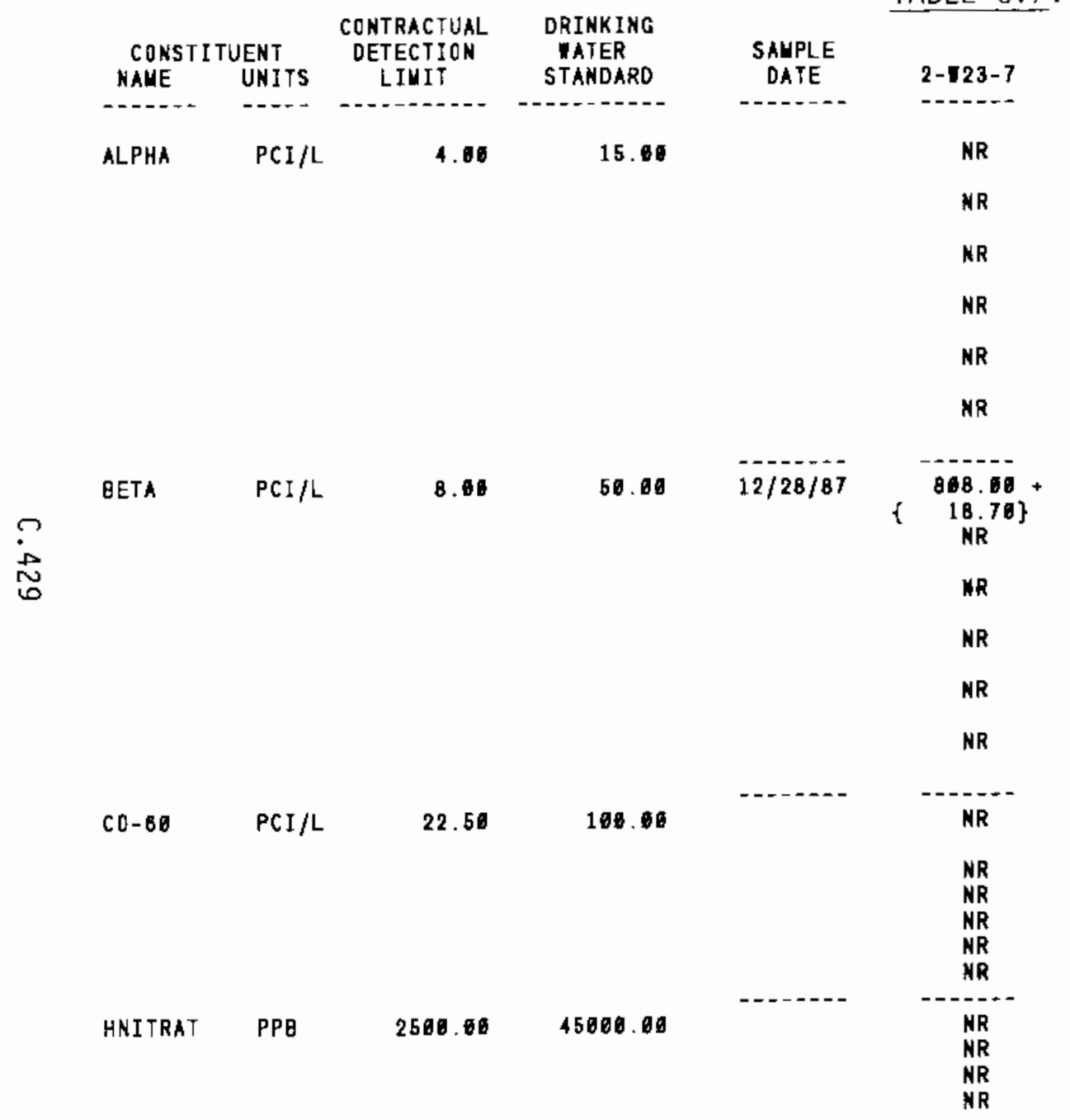

\begin{tabular}{|c|c|}
\hline $\begin{array}{l}\text { SAUPLE } \\
\text { DATE }\end{array}$ & $2-123-9$ \\
\hline$--1+x$ & $-\cdots-1$ \\
\hline $07 / 24 / 87$ & $27.00+$ \\
\hline $68 / 19 / 87$ & $\begin{array}{c}2.21\} \\
14.39 \\
1.59\}\end{array}$ \\
\hline $08 / 16 / 87$ & $\begin{array}{c}24.16 \\
1.99\end{array}$ \\
\hline $16 / 13 / 87$ & $\begin{array}{c}19.00^{\circ} \\
1.83\}\end{array}$ \\
\hline $11 / 17 / 87$ & $\left\{\quad \begin{array}{r}17.40 \\
1.76\}\end{array}\right.$ \\
\hline $12 / 11 / 87$ & $\left\{\begin{array}{c}22.28+ \\
2.00\end{array}\right\}$ \\
\hline $67 / 24 / 87$ & 12.58 \\
\hline $08 / 19 / 87$ & $\begin{array}{l}2.80\} \\
8.94\end{array}$ \\
\hline $69 / 16 / 87$ & 11.503 \\
\hline $10 / 13 / 87$ & $\begin{array}{l}8.05 \\
2.27\}\end{array}$ \\
\hline $11 / 17 / 87$ & $\begin{array}{r}9.72 \\
\{\quad 2.21\}\end{array}$ \\
\hline $12 / 11 / 87$ & $\left\{\begin{array}{r}10.20 \\
\quad 2.32\}\end{array}\right.$ \\
\hline $07 / 24 / 87$ & $\left\{\begin{array}{l}0.11 \\
5.47\end{array}\right\}$ \\
\hline $\begin{array}{l}8 / 18 / 87 \\
09 / 18 / 87 \\
10 / 13 / 87 \\
11 / 17 / 87 \\
12 / 11 / 87\end{array}$ & \\
\hline $\begin{array}{l}97 / 24 / 87 \\
08 / 19 / 87 \\
69 / 18 / 87 \\
16 / 13 / 87\end{array}$ & $\begin{array}{l}469000.00 * \\
285000.00 * \\
282060.00 * \\
142000.00\end{array}$ \\
\hline
\end{tabular}

\begin{tabular}{|c|c|}
\hline $\begin{array}{l}\text { SAMPLE } \\
\text { DATE }\end{array}$ & $2-723-11$ \\
\hline --...-- & - \\
\hline $07 / 24 / 87$ & $\begin{array}{r}18.26 \\
1.76\}\end{array}$ \\
\hline $68 / 18 / 87$ & $18.90+$ \\
\hline $68 / 18 / 87$ & $21.5 \theta^{2}+$ \\
\hline $16 / 13 / 87$ & $26.40+$ \\
\hline $11 / 17 / 87$ & $20.69+$ \\
\hline $12 / 68 / 87$ & $\left\{\begin{array}{c}22.10 \\
2.09\end{array}\right\}$ \\
\hline $67 / 24 / 87$ & 9.63 \\
\hline $08 / 18 / 87$ & $\begin{array}{l}2.15\} \\
8.69\end{array}$ \\
\hline $69 / 18 / 87$ & $\begin{array}{l}2.10\} \\
7.94\end{array}$ \\
\hline $10 / 13 / 87$ & $\begin{array}{l}2.64\} \\
8.56\end{array}$ \\
\hline $11 / 17 / 87$ & $\begin{array}{l}2.08\} \\
7.02\end{array}$ \\
\hline & $1.97\}$ \\
\hline $12 / 68 / 87$ & $\begin{array}{r}7.74 \\
\{\quad 2.09\}\end{array}$ \\
\hline $7 / 24 / 87$ & 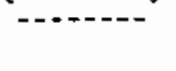 \\
\hline $10 / 13 / 87$ & \\
\hline & $\begin{array}{l}\text { NR } \\
\text { NR } \\
\text { MR } \\
\text { NR }\end{array}$ \\
\hline $\begin{array}{l}7 / 24 / 87 \\
08 / 18 / 87 \\
60 / 16 / 87 \\
10 / 13 / 87\end{array}$ & 2751.09 \\
\hline
\end{tabular}

* - Value exceeds priuary drinking mater standard.

- VALUE EXCEEDS PROPOSEO PRIMARY DRINKING WATER STANDARD.

+ - VALUE EXCEEDS SCREENING LEVEL FOR FURTHER INYESTIGATION.

- DETECTION LIUIT TAS NOT AVALABLE FOR COUPARISON

NR - ANALYSIS NOT REQUESTED OR NOT YET REPDRTED

VALUES IN I ? ARE COUNTING ERRDRS FQR RADIONUCLIDES

WATER STANDARD'(S) IN PARENTHESES ARE PROPOSEO ONLY 


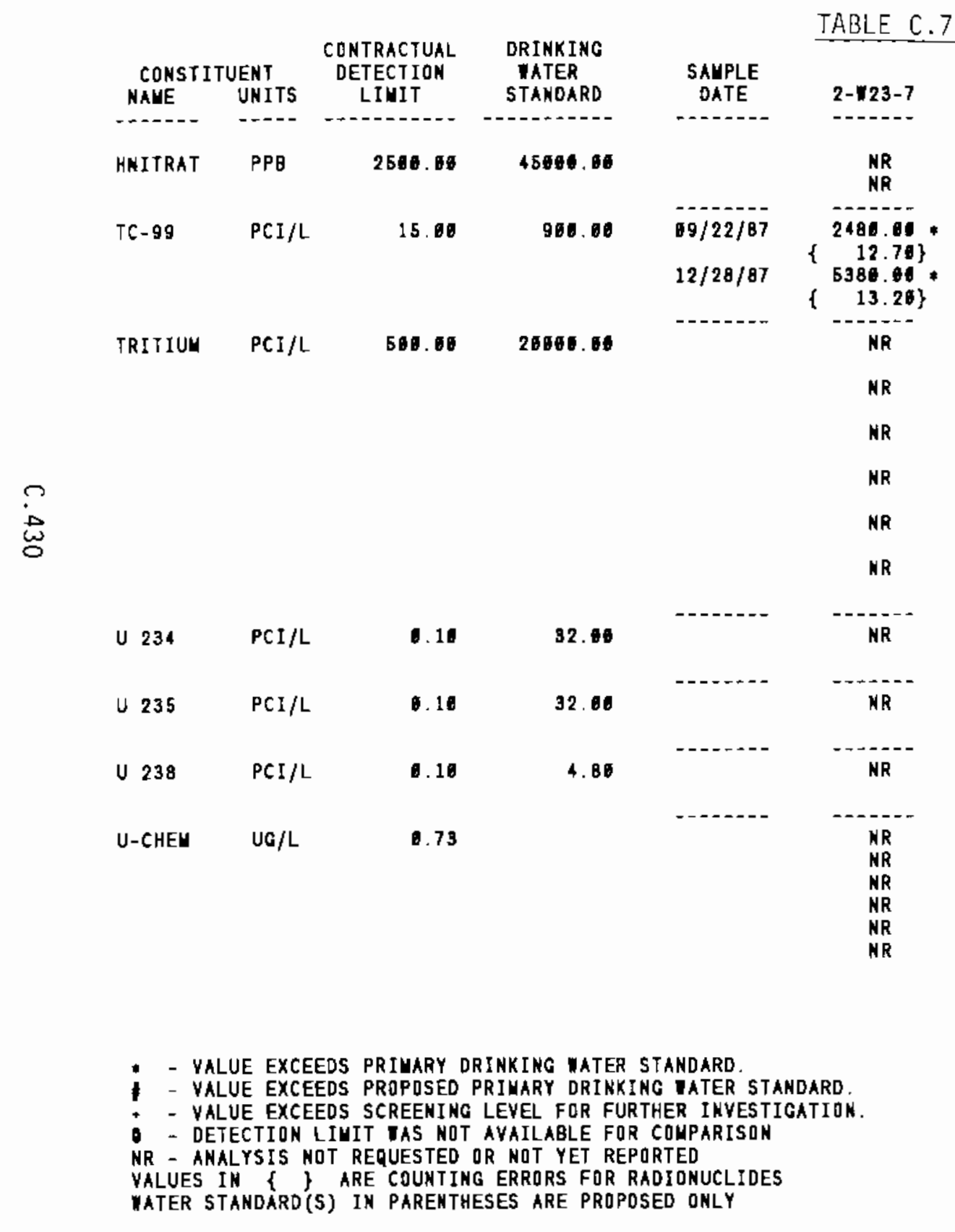

(contd) 


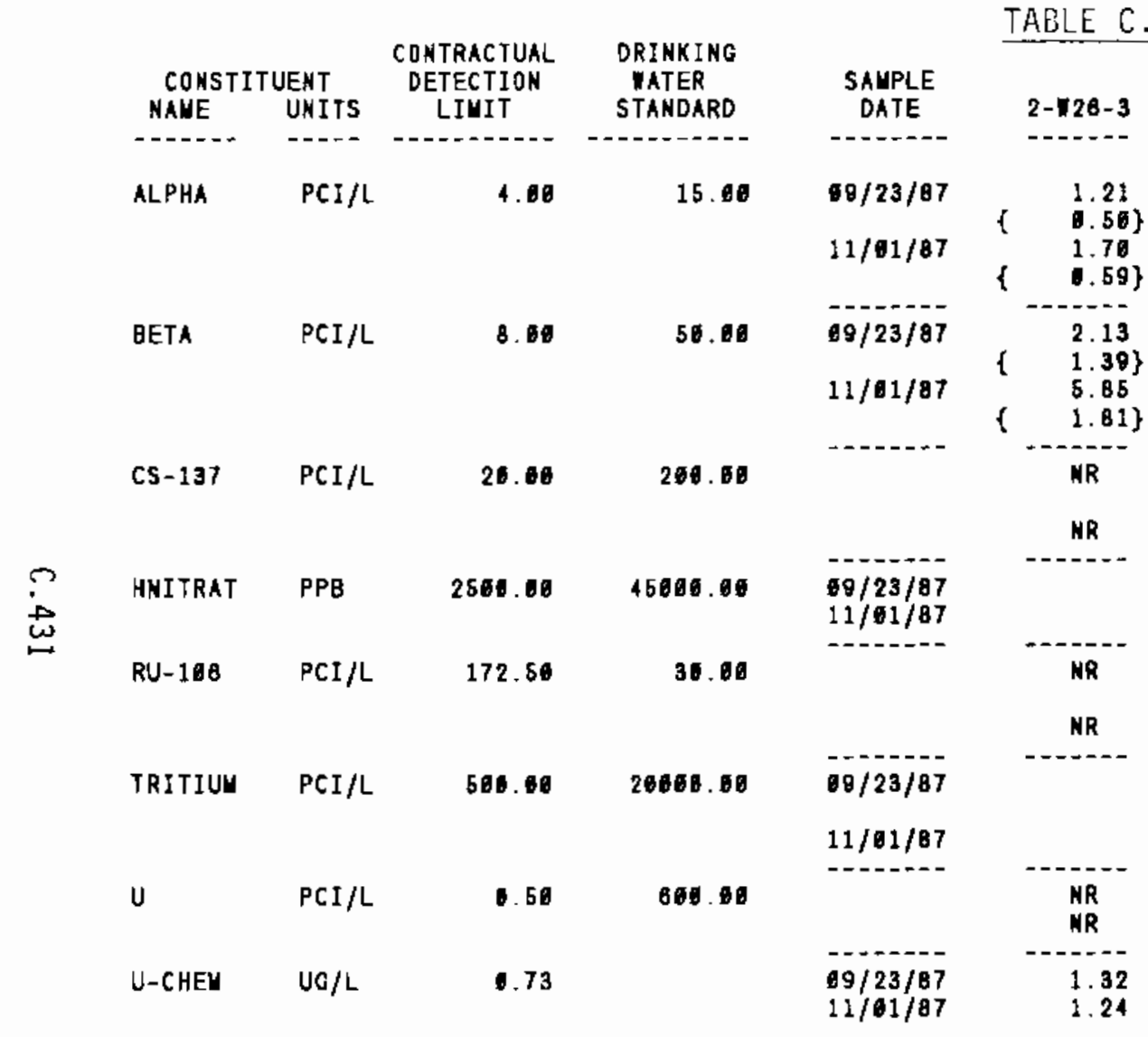

(contd) 


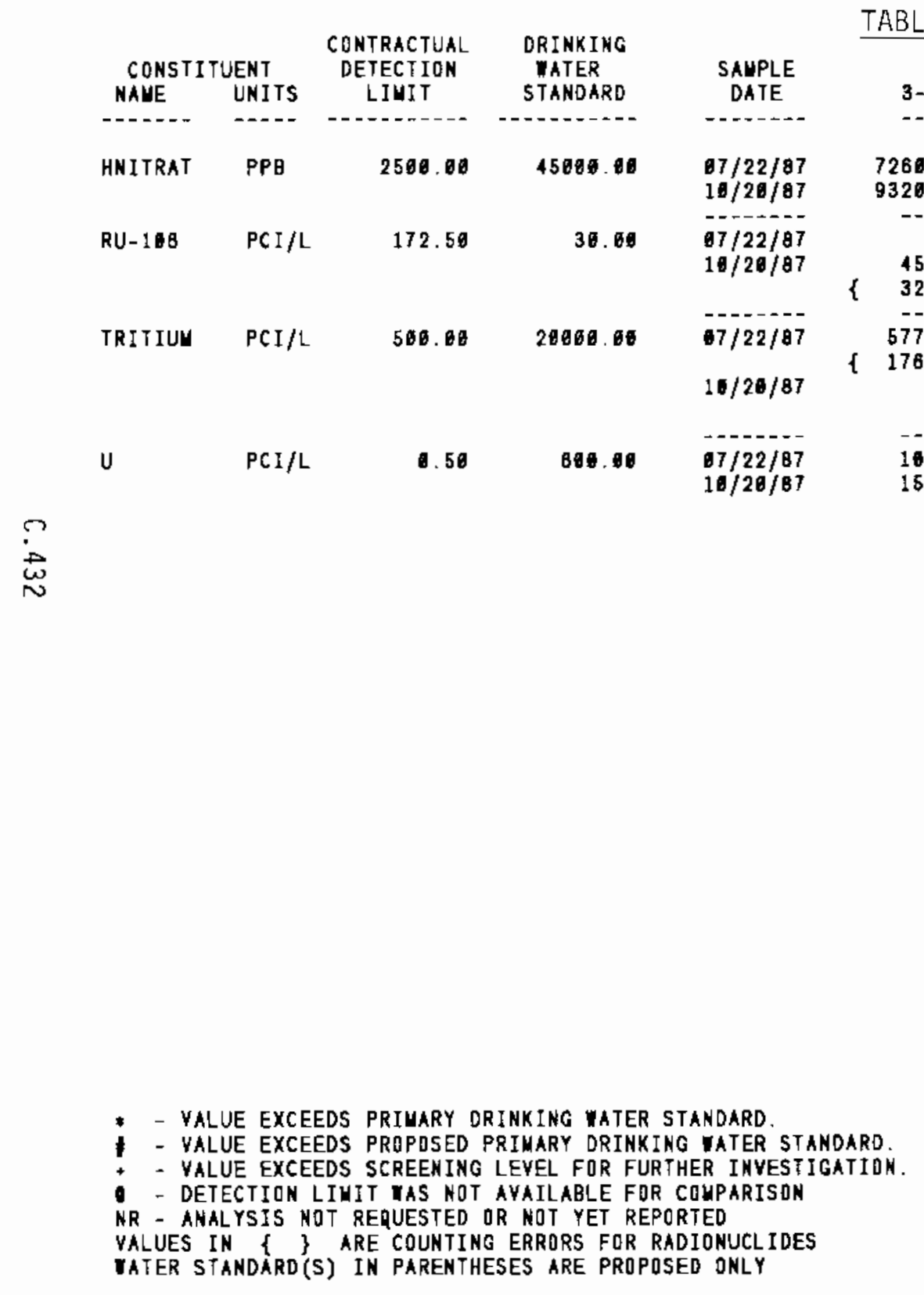

ABLE C.7. (contd)

SAMPLE

SAMPLE
DATE

SAUPLE

DATE

3-3-2

07/21/87

11700.00

10100.00

$16 / 15 / 87$

…-

$07 / 21 / 87$

$10 / 20 / 87$

1390.00

-

$07 / 21 / 87$

12160.00

$45.50 * 10 / 16 / 87$

$07 / 21 / 87$

$10 / 28 / 87$

----

$32.26\}$

577.08

07/21/87

$18 / 15 / 87$

4.

403.00
$170.00\}$

-........

$07 / 21 / 87$

$15 / 26 / 87$

16.8

15.80

$-7 / 21 / 87$

18/15/87

226.06)

7.00

07/21/87

$10 / 20 / 87$

8.41

10187

7. 
TABLE C.7. (contd)

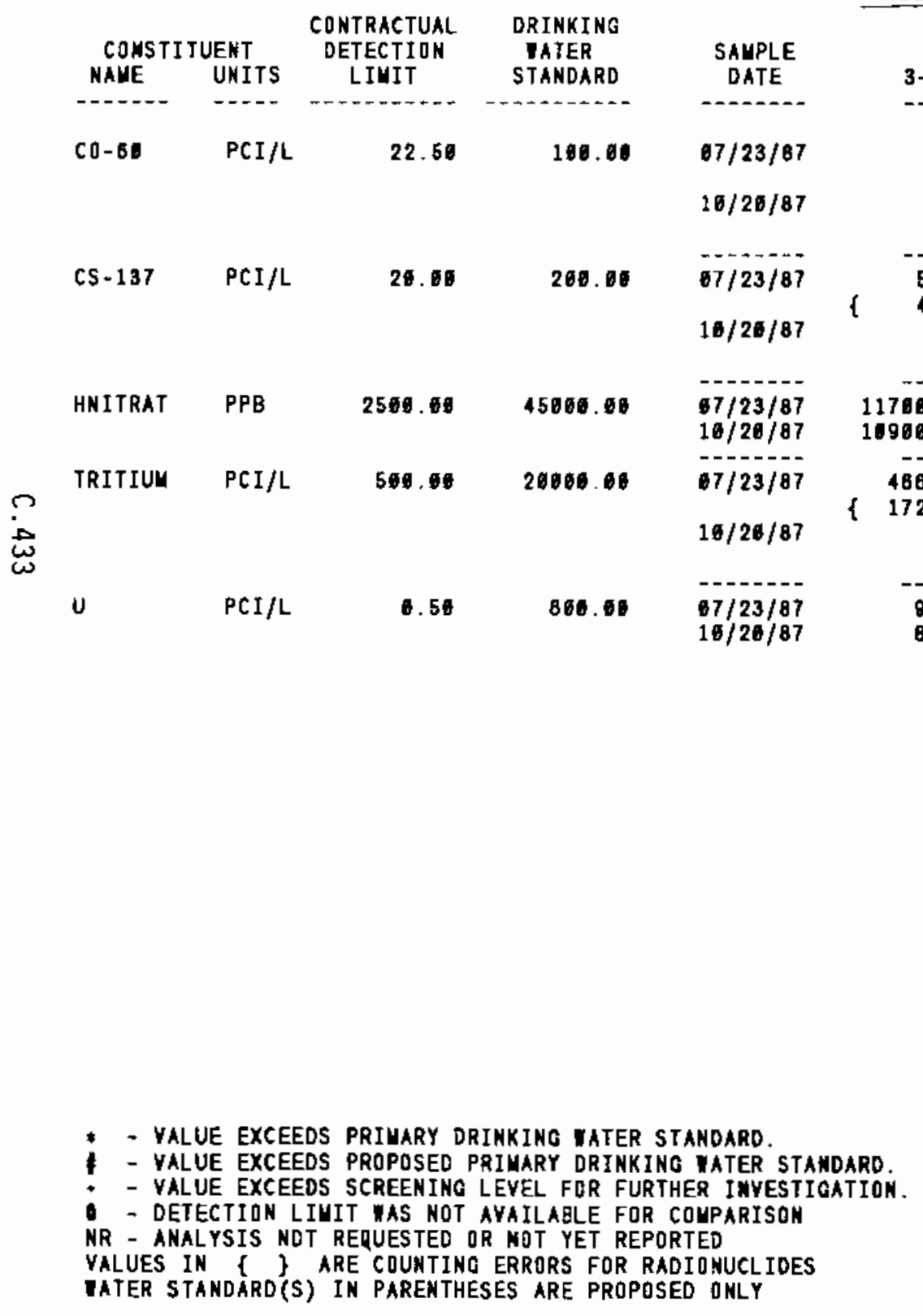

\begin{tabular}{|c|c|c|c|}
\hline $\begin{array}{l}\text { SAMPLE } \\
\text { DATE }\end{array}$ & 3-3-8 & $\begin{array}{c}\text { SAUPLE } \\
\text { DATE }\end{array}$ & $3-3-0$ \\
\hline $67 / 21 / 87$ & 7.62 & $07 / 21 / 87$ & \\
\hline $19 / 20 / 87$ & 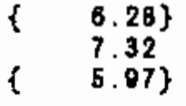 & $16 / 19 / 87$ & \\
\hline $67 / 21 / 87$ & & $17 / 21 / 87$ & \\
\hline $10 / 20 / 87$ & $\left.\begin{array}{ll} & 8.27 \\
\{\quad 4.89\end{array}\right\}$ & $10 / 10 / 87$ & \\
\hline $\begin{array}{l}07 / 21 / 87 \\
10 / 20 / 87\end{array}$ & $\begin{array}{r}15300.66 \\
15980.60\end{array}$ & $\begin{array}{l}07 / 21 / 87 \\
16 / 19 / 87\end{array}$ & $\begin{array}{l}10600.00 \\
10700.00\end{array}$ \\
\hline $07 / 21 / 87$ & $\begin{array}{l}342.00 \\
\{\quad 188.00\}\end{array}$ & $67 / 21 / 87$ & \\
\hline $10 / 28 / 87$ & 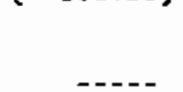 & $10 / 19 / 87$ & $\left\{\begin{array}{l}722.06 \\
217.06\end{array}\right\}$ \\
\hline $\begin{array}{l}07 / 21 / 87 \\
10 / 20 / 87\end{array}$ & $\begin{array}{r}13.80 \\
8.98\end{array}$ & $\begin{array}{l}07 / 21 / 87 \\
10 / 10 / 87\end{array}$ & $\begin{array}{l}13.30 \\
17.40\end{array}$ \\
\hline
\end{tabular}

TATER STANDARD(S) IN PARENTHESES ARE PROPOSED DNLY 


\section{TABLE C.7. (contd)}

\begin{tabular}{|c|c|c|}
\hline CQNSTITUENT & $\begin{array}{l}\text { CONTRACTUAL } \\
\text { DETECTION }\end{array}$ & \\
\hline NAME UNITS & LIUIT & STANDARD \\
\hline
\end{tabular}

\section{SAUPLE}

DATE

$67 / 23 / 87$

CD-60 PCI/L

22.56

160.00

$10 / 20 / 87$

$\begin{array}{ll} & 5.76 \\ \{\quad 8.10\}\end{array}$

HNITRAT PPB

2500.00

45000.00

$67 / 23 / 87$

$18 / 29 / 87$

RU-166 PCI/L $\quad 172.58$

36.60

67/23/87

$10 / 20 / 87$

$67 / 23 / 87$

$16 / 20 / 87$

$\underset{⿱ ㇒}{\infty}$

SR 90 PCI/L

8.60

$67 / 23 / 87$

$10 / 2 \theta / 87$

g) $123 / 87$

$18 / 20 / 87$

$07 / 23 / 87$

U $234 \quad \mathrm{PCI} / \mathrm{L}$

6. 10

32.0

$07 / 23 / 87$

U $235 \quad \mathrm{PCI} / \mathrm{L}$

0.10

32.98

$97 / 23 / 87$

U $238 \quad \mathrm{PCI} / \mathrm{L}$

0.10
4. $80 \quad 07 / 23 / 87$
3-3-11

11600.00

13806.00

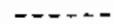

$\left\{\begin{array}{r}79.46 \\ 4.40\}\end{array}\right.$
SAMPLE

DATE

$67 / 21 / 87$

$16 / 19 / 87$

1/21/87

$18 / 19 / 87$

$7 / 21 / 97$

$16 / 19 / 87$

$67 / 21 / 87$

$10 / 19 / 87$

1/21/87

$10 / 19 / 87$

$7 / 21 / 87$

$10 / 19 / 87$

$7 / 21 / 87$

$7 / 21 / 8$

$7 / 21 / 87$

$7 / 21 / 87$

\section{3-3-12}

-...--

12600.00

18200.00

$-\cdots$
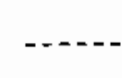

$10 .-2$

1840.60
$213.66\}$

3106.08

287.06

26.50

29.20

14.10

$\left\{\begin{array}{c}13.99 \\ \quad \quad .33\}\end{array}\right.$

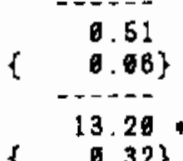

$\left\{\begin{array}{c}14.34\} \\ -. .2 .5\end{array}\right.$

$\left\{\begin{array}{r}0.50 \\ \quad 0.06\end{array}\right.$

$\{\quad 0.32\}$
SAMPLE

DATE 3-4-9

97/21/87 9.82

$16 / 19 / 87$

$7 / 21 / 87$

$10 / 19 / 87$

67/21/87

$10 / 19 / 87$

67/21/87

$16 / 19 / 87$

$07 / 21 / 87$

$16 / 18 / 87$

7/21/B

18/19/87

$\{\quad 7.42\}$

-7/21/87

$07 / 21 / 87$

D7/21/87

$67 / 21 / 87$

\subsection{0}

13700.00

43.5

$\left\{\begin{array}{l}43.30 \\ 35.40\end{array}\right\}$

13.16

\subsection{9}

$1810.00\}$

\{ 245.00$\}$

25.96

28.86

15.

$\{\quad .36\}$

0.73
$1 \quad 0.98$

( 0.08 )

$\left\{\begin{array}{c}15.70 \\ \{\quad .38\}\end{array}\right.$

* - VALUE eXCEEDS PRIMARY dRINKING Mater STANDARD.

* - VAlUE EXCEEDS PROPOSED PRIMARY DRINKING MATER STANDARD.

* - VALUE EXCEEDS SCREEHING LEVEL FOR FURTHER INYESTIGATION.

- DETECTIDN LIUIT WAS NOT AVAILABLE FOR COYPARISON

NR - AHALYSIS NDT REQUESTED OR NOT YET REPORTED

VALUES IN \{ \} ARE COUNTING ERRORS FDR RADIONUCLIDES

WATER STANDARD(S) IN PARENTHESES ARE PROPOSED ONLY 


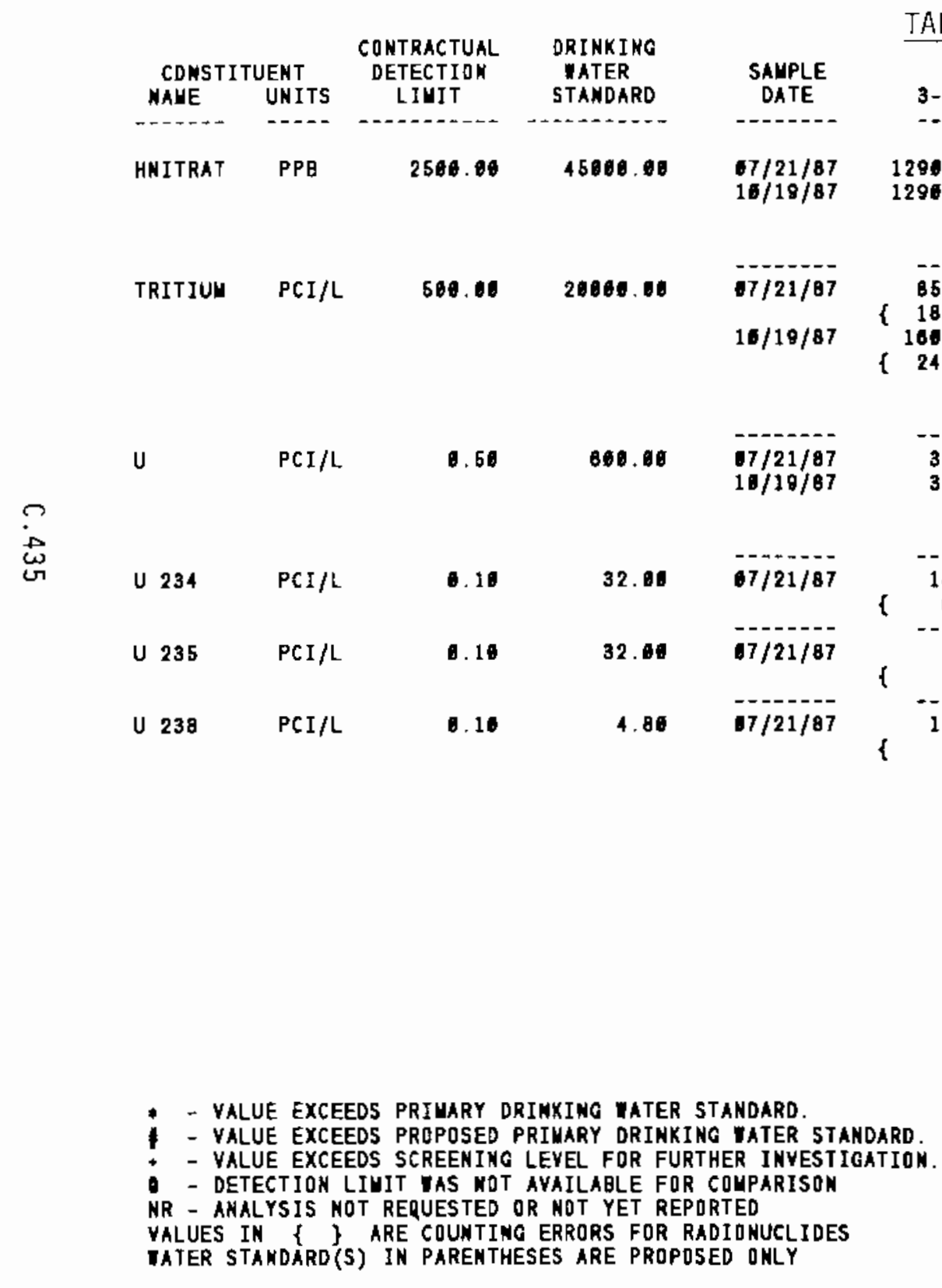

TABLE C.7. (contd)

DATE 3-5-1

DATE

3-8-1

--D-n

$10 / 20 / 87$

47500.00

17/22/87

67/22/87

NR

$10 / 26 / 87$

$10 / 26 / 87$

NR

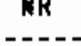

$07 / 22 / 87$

67/22/87

$16 / 26 / 87$

$16 / 20 / 87$

184.06

$07 / 23 / 87$

(n)

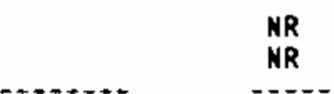

MR

$07 / 23 / 87$

30.70

MR

4. 17

NR

NR

NR

MR

$16 / 20 / 87$

67/22/87

$67 / 22 / 87$

$16 / 21 / 87$

1./20/87

18.76

5.48

NR

$---$

- - - -

nR

6.08)

NR

MR

NR

.

9216.

18.50

$0.39\}$

UE EXCEEDS PRIUARY DRINKING UATER STANOARD.

- VALUE EXCEEDS PREPOSED PRIMARY DRINKING YATER STANDARD

- DETECTION LIUIT TAS MOT AVAILABLE FOR COMPARISON

YALUES IN \{ \} ARE COUNTING ERRORS FOR RADIONUCLIDES

TATER STAMDARD(S) IN PARENTHESES ARE PROPOSED ONLY 


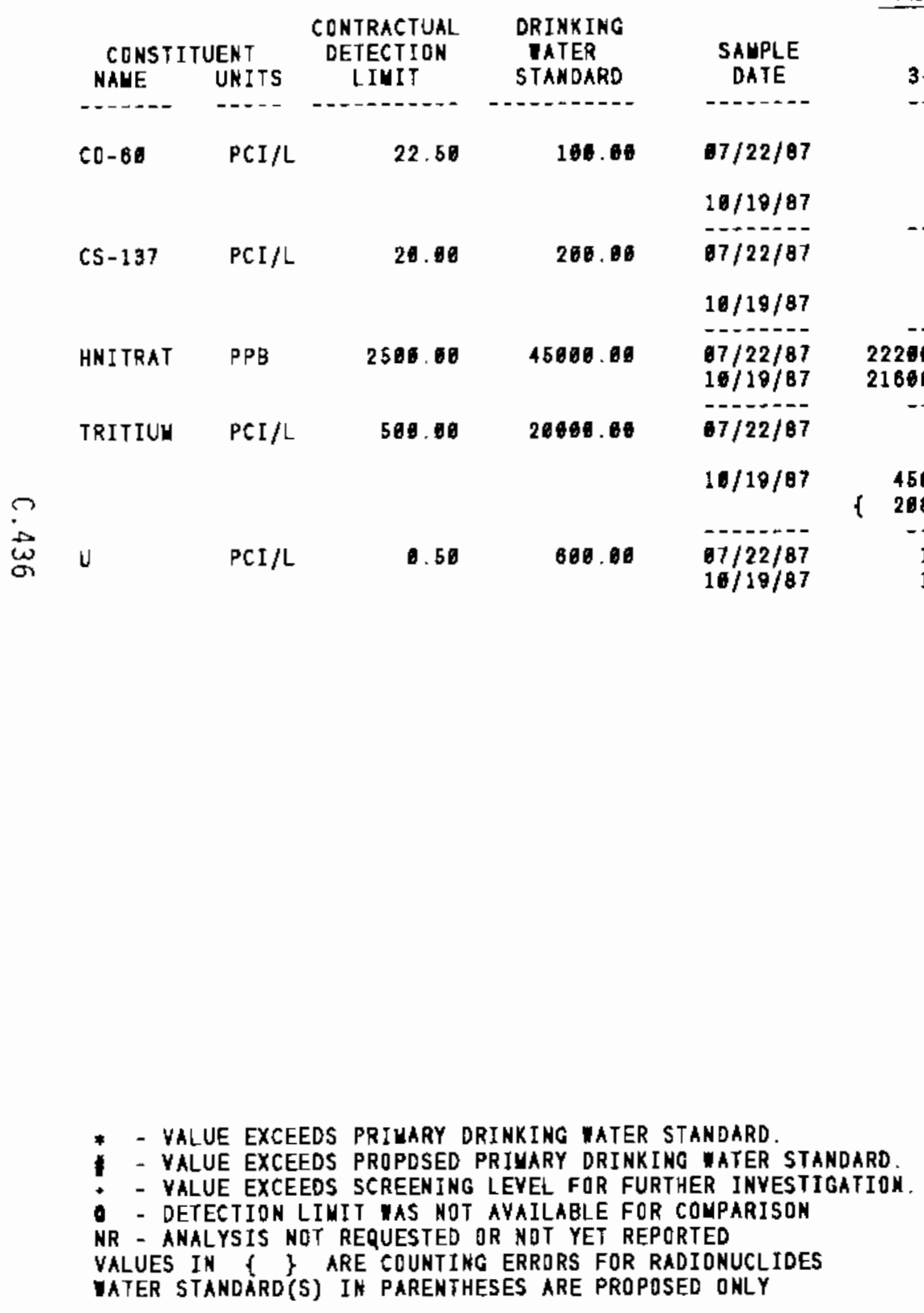

ABLE C.7. (contd)

\begin{tabular}{|c|c|c|c|c|}
\hline $3-8-4$ & $\begin{array}{c}\text { SAMPLE } \\
\text { DATE }\end{array}$ & 4-S6-7 & $\begin{array}{c}\text { SAUPLE } \\
\text { DATE }\end{array}$ & $4-56-8$ \\
\hline$-\cdots$ & $-\cdots-1-1$ & $\cdots--$ & - - - & $-\ldots$ \\
\hline & $7 / 28 / 87$ & \multirow{2}{*}{$5.44\}$} & $07 / 28 / 87$ & \\
\hline \multirow{3}{*}{----} & $11 / 20 / 87$ & & $11 / 20 / 87$ & \\
\hline & $07 / 28 / 87$ & \multirow{2}{*}{$\begin{array}{l}8.89 \\
4.54\}\end{array}$} & $07 / 28 / 87$ & $--\cdots$ \\
\hline & & & & \\
\hline 60.00 & -no-n & MR & $----n$ & NR \\
\hline & $67 / 28 / 87$ & $\left\{\begin{array}{r}14100.00 \\
487.00\}\end{array}\right.$ & $67 / 28 / 87$ & $\begin{array}{r}41006.00 \\
\{677.00\}\end{array}$ \\
\hline $\begin{array}{l}50.60 \\
88.60\}\end{array}$ & $11 / 20 / 87$ & $\left\{\begin{array}{c}37800.80 \\
\{757.06\}\end{array}\right.$ & $11 / 20 / 87$ & $\left\{\begin{array}{c}38566.00 \\
\{\quad 687.09\}\end{array}\right.$ \\
\hline $\begin{array}{l}1.09 \\
1.96\end{array}$ & $+=-1,-\infty$ & $\begin{array}{l}\text { NR } \\
\text { NR }\end{array}$ & - & $\begin{array}{l}\text { NR } \\
\text { NR }\end{array}$ \\
\hline
\end{tabular}

- YALUE EXCEEDS PRIUARY ORINKTHG IATER STANDARD.

* YALUE EXCEEDS PROPDSED PRIYARY DRINKIMg MATER STANDARD.

ALUES IN $(Y$ ARE COUNTIKG ERRDRS FOR RADIONUCLIDES

ATER STANDARD(S) IH PARENTHESES ARE PROPOSED ONLY 
TABLE C.7. (contd)

\begin{tabular}{|c|c|c|c|c|c|c|c|c|c|}
\hline $\begin{array}{l}\text { CONSTI } \\
\text { NAUE }\end{array}$ & $\begin{array}{l}\text { UENT } \\
\text { UNITS }\end{array}$ & $\begin{array}{l}\text { CONTRACTUAL } \\
\text { DETECTION } \\
\text { LIMIT }\end{array}$ & $\begin{array}{l}\text { DRINKING } \\
\text { WATER } \\
\text { STANDARD }\end{array}$ & $\begin{array}{l}\text { SAUPLE } \\
\text { DATE }\end{array}$ & 4-51-7日 & $\begin{array}{c}\text { SAMPLE } \\
\text { DATE }\end{array}$ & $8-1-18$ & $\begin{array}{c}\text { SAMPLE } \\
\text { DATE }\end{array}$ & $8-2-7$ \\
\hline$\ldots-\ldots$ & $\cdots-$ & 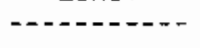 & $\cdots$ & $-\cdots-\cdots$ & ----- &.----1 & $-\ldots$ & $-\ldots-\ldots$ & $\ldots-$ \\
\hline HNITRAT & PPB & 2500.60 & 45000.00 & $\begin{array}{l}07 / 23 / 87 \\
11 / 26 / 87\end{array}$ & & $\begin{array}{l}07 / 24 / 87 \\
16 / 25 / 87\end{array}$ & $\begin{array}{l}18860.00 \\
26460.06\end{array}$ & $\begin{array}{l}07 / 24 / 87 \\
10 / 21 / 87\end{array}$ & $\begin{array}{l}32860.00 \\
4886600\end{array}$ \\
\hline TRITIUM & $\mathrm{PCI} / \mathrm{L}$ & 500.00 & 20000.00 & $\begin{array}{l}07 / 23 / 87 \\
11 / 26 / 87\end{array}$ & $\begin{array}{c}58300.06 \\
\{918.06\} \\
50200.68 \\
\{818.06\}\end{array}$ & $\begin{array}{l}07 / 24 / 87 \\
10 / 26 / 87\end{array}$ & $\begin{array}{c}49406.00 \\
\left\{\begin{array}{c}850.65 \\
50900.00\end{array}\right. \\
\left\{\begin{array}{c}888.00\}\end{array}\right.\end{array}$ & $\begin{array}{l}07 / 24 / 87 \\
10 / 21 / 87\end{array}$ & $\left\{\begin{array}{r}10700.00 \\
371.00\} \\
13300.00 \\
\{\quad 468.00\}\end{array}\right.$ \\
\hline
\end{tabular}

* - value exceeds privary drinking mater standard.

* - VALUE EXCEEDS PROPDSED PRIMARY DRINXING YATER STANOARO.

- VRLUE EXCEES SCREEN ING LEVE For FURTHER INVESTIOATION.

NR - DETECTION LIUIT WAS NOT AVAILABLE FDR COUPARISON

VALUES IN \{\} ARE CDUNTING ERRORS FOR RADIDNUCLIDES

IATER STANOARO(S) IN PARENTHESES ARE PROPISED ONLY 


\begin{tabular}{|c|c|c|c|c|c|c|c|c|c|}
\hline $\begin{array}{c}\text { CONSTI } \\
\text { NAUE }\end{array}$ & $\begin{array}{l}\text { UENT } \\
\text { UNITS }\end{array}$ & $\begin{array}{c}\text { CONTRACTUAL } \\
\text { DETECTIDN } \\
\text { LIUIT }\end{array}$ & $\begin{array}{l}\text { DRINKING } \\
\text { IATER } \\
\text { STANDARD }\end{array}$ & $\begin{array}{c}\text { SAYPLE } \\
\text { DATE }\end{array}$ & $8-3-45$ & $\begin{array}{c}\text { SAMPLE } \\
\text { DATE }\end{array}$ & $8-4-E 6$ & $\begin{array}{l}\text { SAUPLE } \\
\text { DATE }\end{array}$ & $8-8-17$ \\
\hline$C O-6 \theta$ & $\mathrm{PCI} / \mathrm{L}$ & 22.50 & 100.00 & $08 / 06 / 87$ & NR & & NR & $10 / 25 / 87$ & $\begin{array}{l}9.73 \\
8.88\}\end{array}$ \\
\hline CS -137 & PCI/L & 20.00 & 200.06 & $68 / 68 / 87$ & $\left\{\begin{array}{c}12.10 \\
B .08 \\
\mathrm{NR}\end{array}\right\}$ & & $\begin{array}{l}\text { NR } \\
\text { NR }\end{array}$ & $\begin{array}{l}67 / 29 / 87 \\
18 / 25 / 87\end{array}$ & $\begin{array}{l}7.94 \\
8.88\}\end{array}$ \\
\hline HNITRAT & PPB & 2500.00 & 45000.000 & $\begin{array}{l}08 / 60 / 87 \\
10 / 28 / 87\end{array}$ & 7620.80 & $\begin{array}{l}07 / 29 / 87 \\
10 / 21 / 87\end{array}$ & $\begin{array}{l}12600.60 \\
12800.00\end{array}$ & $\begin{array}{l}67 / 29 / 87 \\
16 / 25 / 87\end{array}$ & $\begin{array}{l}31400.00 \\
35000.00\end{array}$ \\
\hline \multirow[t]{2}{*}{ RU- $1 \$ 8$} & \multirow[t]{2}{*}{$\mathrm{PCI} / \mathrm{L}$} & \multirow[t]{2}{*}{172.56} & \multirow[t]{2}{*}{30.00} & \multirow[t]{2}{*}{$68 / 66 / 87$} & & & NR & $07 / 29 / 87$ & \multirow[t]{2}{*}{$\begin{array}{r}69.96 \\
\{\quad 42.16\}\end{array}$} \\
\hline & & & & & NR & & NR & $10 / 25 / 87$ & \\
\hline TRITIUU & $\mathrm{PCI} / \mathrm{L}$ & 500.00 & 26900.06 & $68 / 08 / 87$ & & $67 / 29 / 87$ & & $07 / 29 / 87$ & 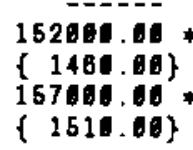 \\
\hline
\end{tabular}

* - Value exceEds priuary drinking vater standard.

- - VALUE EXCEEDS PROPOSED PRIUARY DRINKING TATER STAMDARD

+ - VALUE EXCEEDS SCREENING LEVEL FOR FURTHER INVESTIGATIDN.

- DETECTION LIUIt IAS NOT AVAILABLE fOR COMPARISOM

NR - AHALYSIS NOT REQUESTED OR NOT YET REPORTED

VALUES IN \{ $\}$ ARE COUNTING ERRORS FOR RADIONUCLIDES

WATER STANDARD'(S) IN PARENTHESES ARE PROPOSED ONLY 
TABLE C.7. (contd)

\begin{tabular}{|c|c|c|c|c|c|c|c|c|c|}
\hline $\begin{array}{l}\text { CONSTI } \\
\text { NANE }\end{array}$ & $\begin{array}{l}\text { UENT } \\
\text { UNI TS }\end{array}$ & $\begin{array}{c}\text { CONTRACTUAL } \\
\text { DETECTIOH } \\
\text { LIMIT }\end{array}$ & $\begin{array}{l}\text { DRINKING } \\
\text { IATER } \\
\text { STANDARD }\end{array}$ & $\begin{array}{c}\text { SAHPLE } \\
\text { DATE }\end{array}$ & $6-8-32$ & $\begin{array}{c}\text { SAHPLE } \\
\text { DATE }\end{array}$ & $6-10-54 A$ & $\begin{array}{c}\text { SAUPLE } \\
\text { DATE }\end{array}$ & $6-12-4 B$ \\
\hline------ & ---- & - - & $--\ldots+-1-1$ & $\cdots+-\cdots$ & 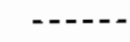 & - - & $--1-1$ & - & $-\cdots-1$ \\
\hline HNITRAT & PPB & 2560.08 & 45000.00 & $\begin{array}{l}67 / 29 / 87 \\
10 / 25 / 87\end{array}$ & $\begin{array}{l}4990.00 \\
3610.60\end{array}$ & $\begin{array}{l}08 / 05 / 87 \\
10 / 29 / 87\end{array}$ & $\begin{array}{l}12800.00 \\
13500.00\end{array}$ & & $\begin{array}{l}\text { NR } \\
\text { NR }\end{array}$ \\
\hline TRITIUH & $\mathrm{PCI} / \mathrm{L}$ & 500.60 & 20000.00 & $\begin{array}{l}07 / 29 / 87 \\
10 / 25 / 87\end{array}$ & - & $\begin{array}{l}08 / 85 / 87 \\
10 / 28 / 87\end{array}$ & $\begin{array}{l}302.06 \\
\{\quad 170.00\}\end{array}$ & 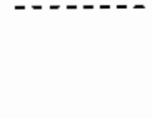 & NR \\
\hline
\end{tabular}

- VALUE EXCEEDS PROPOSED PRIMARY DRINKING WaTER STANDARD.

- - YALUE EXCEEDS SCREENING LEYEL FQR FURTHER INYESTIGATION.

- - DETECTION LIUIT UAS NOT AVAILABLE fOR COMPaRisON

NR - ANALYSIS NDT REQUESTED OR NOT YET REPDRTED

VALUES IN \{\} ARE COUNTING ERRDRS FDR RADIOHUCLIDES

IATER STANDARD'(S) IH PARENTHESES ARE PROPOSED OHLY 


\section{TABLE C.7. (contd)}

\begin{tabular}{|c|c|c|c|c|c|c|c|c|c|}
\hline $\begin{array}{c}\text { CONSTI } \\
\text { RAME }\end{array}$ & $\begin{array}{l}\text { UENT } \\
\text { UNITS }\end{array}$ & $\begin{array}{c}\text { CONTRACTUAL } \\
\text { DETECTION } \\
\text { LIUIT }\end{array}$ & $\begin{array}{l}\text { DRINKING } \\
\text { WATER } \\
\text { STAHDARD }\end{array}$ & $\begin{array}{l}\text { SAMPLE } \\
\text { DATE }\end{array}$ & $6-13-84$ & $\begin{array}{c}\text { SAHPLE } \\
\text { DATE }\end{array}$ & $0-14-E B T$ & $\begin{array}{l}\text { SAUPLE } \\
\text { DRTE }\end{array}$ & $8-14-38$ \\
\hline HNITRAT & PPB & 2500.00 & 460000.06 & $\begin{array}{l}08 / 08 / 87 \\
10 / 28 / 87\end{array}$ & & $10 / 25 / 87$ & 21106 & $88 / 27 / 87$ & $\begin{array}{l}3326.06 \\
\text { RR }\end{array}$ \\
\hline TRITIUU & $\mathrm{PCI} / \mathrm{L}$ & 500.00 & 20000.00 & $\begin{array}{l}68 / 86 / 87 \\
16 / 28 / 87\end{array}$ & & $10 / 25 / 87$ & $\left\{\begin{array}{c}47808.00 \\
732.00 \\
\text { NR }\end{array}\right.$ & $68 / 27 / 87$ & NR \\
\hline
\end{tabular}

- - Value exceEds primary dRinking tater standaro

- VALUE EXCEEDS PRDPDSED PRIMARY DRIMKING IATER STANDARD.

+ - VALUE EXCEEDS SCREENING LEVEL FoR FURTHER INYESTIGATION.

- DETECTION LIUIT WAS NOT AVAILABLE for COUPARISOH

NR - ANALYSIS NOT REQUESTEO OR NDT YEI REPORTED

VALUES IM \{ ? ARE CDUHTING ERRORS FOR RADIDNUCLIDES

- 
TABLE C.7. (contd)

\begin{tabular}{|c|c|c|c|c|c|c|c|c|c|}
\hline $\begin{array}{l}\text { CONSTI } \\
\text { NAYE }\end{array}$ & $\begin{array}{l}\text { UENT } \\
\text { UNITS }\end{array}$ & $\begin{array}{c}\text { CONTRACTUAL } \\
\text { DETECTION } \\
\text { LINIT }\end{array}$ & $\begin{array}{l}\text { DRINKING } \\
\text { TATER } \\
\text { STANDARD }\end{array}$ & $\begin{array}{l}\text { SAHPLE } \\
\text { DATE }\end{array}$ & $6-14-47$ & $\begin{array}{c}\text { SAUPLE } \\
\text { DATE }\end{array}$ & $6-15-15 B$ & $\begin{array}{c}\text { SALPLE } \\
\text { DATE }\end{array}$ & $6-17-5$ \\
\hline 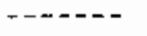 & $-\cdots$ & - - & 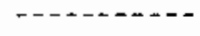 & -----1 & ----- & $---\infty$ & ------- & $--1-1$ & ...... \\
\hline \multirow[t]{2}{*}{$C O-60$} & $\mathrm{PCI} / \mathrm{L}$ & 22.50 & 100.00 & & NR & $07 / 29 / 87$ & $\begin{aligned} & 6.68 \\
&\{\quad 5.44\}\end{aligned}$ & $08 / 19 / 87$ & \\
\hline & & & & & NR & $10 / 21 / 87$ & & $10 / 21 / 87$ & $\begin{array}{l}8.67 \\
6.66\}\end{array}$ \\
\hline HNITRAT & $\mathrm{PPB}$ & 2500.00 & 45000.00 & $\begin{array}{l}08 / 65 / 87 \\
10 / 26 / 87\end{array}$ & -8 & $\begin{array}{l}07 / 29 / 87 \\
18 / 21 / 87\end{array}$ & $\begin{array}{l}18700.06 \\
19900.00\end{array}$ & $\begin{array}{l}68 / 19 / 87 \\
10 / 21 / 87\end{array}$ & $\begin{array}{l}63300.05 \\
67600.00\end{array}$ \\
\hline RU-106 & $\mathrm{PCI} / \mathrm{L}$ & 172.50 & 36.60 & ----- & $\begin{array}{l}\text { NR } \\
\text { NR }\end{array}$ & $\begin{array}{l}07 / 28 / 87 \\
10 / 21 / 87\end{array}$ & 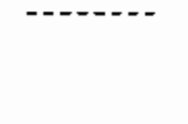 & $\begin{array}{l}68 / 19 / 97 \\
16 / 21 / 87\end{array}$ & $\begin{array}{l}48.20 \\
47.50\}\end{array}$ \\
\hline
\end{tabular}

\# - VALUE EXCEEDS PROPOSED PRIUARY DRINKING TATER STANDARD.

- - VALUE EXCEEDS SCREENING LEVEL FOR FURTHER INYESTIGATION.

- DETECTION LIUIT WAS NOT AVAILABLE FOR COUPARISON

NR - ANALYSIS MOT REQUESTED DR NOT YET REPDRTED

VALUES IN \& ARE COUNTING ERRDRS FOR RADIDNUCLIDES

WATER STANDARD'(S) IN PARENTHESES ARE PROPOSEO ONLY 
TABLE C.7. (contd)

\begin{tabular}{|c|c|c|c|c|c|c|c|c|c|}
\hline $\begin{array}{l}\text { CONSTI } \\
\text { NALE }\end{array}$ & $\begin{array}{l}\text { UENT } \\
\text { UNITS }\end{array}$ & $\begin{array}{c}\text { CONTRACTUAL } \\
\text { DETECTION } \\
\text { LIUIT }\end{array}$ & $\begin{array}{l}\text { DRINKING } \\
\text { ATER } \\
\text { STANDARD }\end{array}$ & $\begin{array}{c}\text { SANPLE } \\
\text { DATE }\end{array}$ & $6-17-47$ & $\begin{array}{l}\text { SAMPLE } \\
\text { DATE }\end{array}$ & $8-17-7$ & $\begin{array}{c}\text { SAUPLE } \\
\text { DATE }\end{array}$ & $8-18-58$ \\
\hline$\ldots+\cdots$ & $\cdots$ & 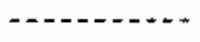 & $---1--10$ & ------ & ------ & $-\ldots . .-1$ &.---- & $-\ldots .$. & $\ldots .$. \\
\hline HNITRAT & PPQ & 2506.60 & 45006.00 & $\begin{array}{l}68 / 65 / 87 \\
10 / 26 / 87\end{array}$ & & $\begin{array}{l}18 / 08 / 87 \\
10 / 28 / 87\end{array}$ & $\begin{array}{l}42200.60 \\
44500.00\end{array}$ & $\begin{array}{l}18 / 05 / 87 \\
11 / 13 / 87\end{array}$ & \\
\hline TRITIUU & $\mathrm{PCI} / \mathrm{L}$ & 580.60 & 20006.00 & $18 / 26 / 87$ & 206.08 & 18/06/87 & $\cdots+\infty$ & $18 / 05 / 87$ & $\cdots$ \\
\hline
\end{tabular}

* - value exceeds primary drinking tater standard.

- VALUE EXCEEDS PROPOSED PRIVARY DRINKING IATER STANDARD.

- - VALUE EXCEEOS SCREENING LEVEL FOR FURTHER INVESTIGATION.

- DETECTIDN LIUIT WAS NDT AYAILABLE FOR COUPARISON

NR - ANALYSIS NOT REQUESTED OR NDT YET REPJRTED

VALUES IN \{ $\}$ ARE CDUNTING ERRORS FOR RADIONUCLIDES

IATER STANDARO(S) IN PARENTHESES ARE PROPOSED DNLY 
TABLE C.7. (contd)

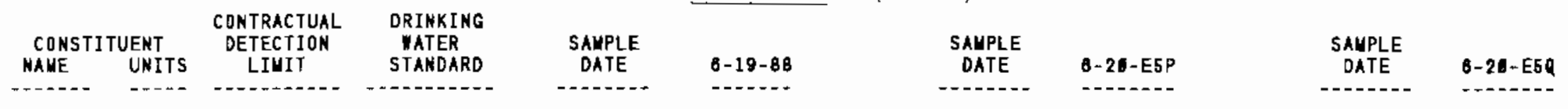

- - Value exceeds privary drinking vater standard.

- VALUE EXCEEDS PROPOSED PRIUARY DRINKING IATER STANDARD

+ - YALUE EXCEEDS SCREEHING LEVEL FOR FURTHER INVESTIGATION.

- DETECTION LIWIT IAS NQT AVAILABLE FDR COUPARISON

NR - ANALYSIS NOT REQUESTED OR NOT YET REPORTED

VALUES IN \{ \} ARE COUNTING ERRORS FOR RADIONUCLIDES

IATER STANDARD(S) IN PARENTHESES ARE PRDPOSED ONLY 
TABLE C.7. (contd)

\begin{tabular}{|c|c|c|c|c|c|c|c|c|c|}
\hline $\begin{array}{l}\text { CQNSTI } \\
\text { MAME }\end{array}$ & $\begin{array}{l}\text { UENT } \\
\text { UNITS }\end{array}$ & $\begin{array}{c}\text { CONTRACTUAL } \\
\text { DETECTION } \\
\text { LIUIT }\end{array}$ & $\begin{array}{l}\text { DRINKING } \\
\text { IATER } \\
\text { STANDARD }\end{array}$ & $\begin{array}{c}\text { SAUPLE } \\
\text { DATE }\end{array}$ & $B-2 \theta-E 5 R$ & $\begin{array}{c}\text { SAYPLE } \\
\text { DATE }\end{array}$ & $8-20-E 12$ & $\begin{array}{c}\text { SAMPLE } \\
\text { DATE }\end{array}$ & $8-28-E 12 P$ \\
\hline HNITRAT & PPB & 2560.06 & 45600.00 & $69 / 17 / 87$ & NR & $\begin{array}{l}88 / 19 / 87 \\
16 / 25 / 87\end{array}$ & 35800.06 & $09 / 17 / 87$ & NR \\
\hline TRITIUN & $\mathrm{PCI} / \mathrm{L}$ & 506.06 & 20080.00 & $09 / 17 / 87$ & NR & $\begin{array}{l}08 / 18 / 87 \\
18 / 25 / 87\end{array}$ & $\left\{\begin{array}{l}657.08 \\
218.89\end{array}\right\}$ & $99 / 17 / 87$ & NR \\
\hline
\end{tabular}

- yalue exceeds privary drinking tater standaro.

- VALUE EXCEEDS PROPDSED PRIMARY DRINKING ta TER STANDARD.

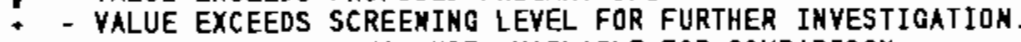

- DETECTION LIUIT ias nOt aVAILABLE for couparison

NR - ANALYSIS NIT REQUESTED DR NOT YET REPORTED

VALUES IN \{\} ARE COUNTING ERRORS FOR RADIDNUCLIDES

itTer STANDARD(S) IN PARENTHESES aRe PROPOSED ONLY 
TABLE C.7. (contd)

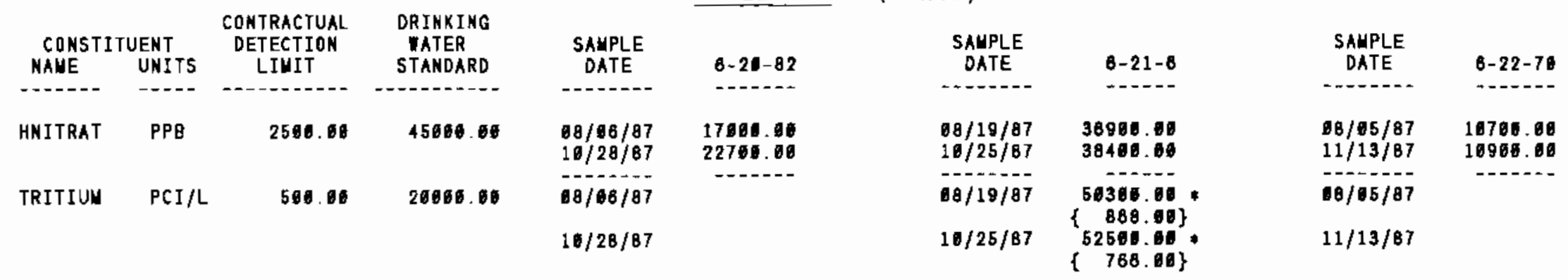

B

* - Value exceeds pRIMARY dRIAKIng vater standard.

- VALUE EXCEEDS PROPOSED PRIUARY DRINKING YATER STANDARD.

- YALUE EXCEEDS SCREEMING LEVEL FDR FURTHER INYESTIGATIOH.

- DETECTION LIUIT IAS NOT AVAILABLE FOR COUPARISON

NR - ANALYSIS NOT REQUESTED OR NOT YET REPORTED

VALUES IN \{\} ARE COUNTING ERRORS FOR RADIONUCLIDES

IATER STANDARD(S) IM PaRENTHESES aRE PROPOSED ONLY 


\section{TABLE C.7. (contd)}

\begin{tabular}{|c|c|c|c|c|c|c|c|c|c|}
\hline CONSTI & IUENT & $\begin{array}{l}\text { CONTRACTUAL, } \\
\text { DETECTION }\end{array}$ & $\begin{array}{l}\text { DRINKING } \\
\text { IATER }\end{array}$ & SAMPLE & & SAUPLE & $094-10$ & SAMPLE & $=04-10$ \\
\hline NAME & UNITS & & & DATE & $8-24-1 T$ & & $\begin{array}{l}B-24-1 P \\
\cdots\end{array}$ & & $6-24-1 Q$ \\
\hline TRITIUU & $\mathrm{PCI} / \mathrm{L}$ & 50000 & 28006.60 & $10 / 25 / 87$ & 13790.66 & $99 / 17 / 87$ & & $89 / 17 / 87$ & \\
\hline
\end{tabular}

* - value exceeds privary drinking tater standard.

* - VALUE EXCEEDS PRDPDSED PRIMARY DRINKING TATER STANDARD.

- - VALUE EXCEEDS SCREENING LEVEL FOR FURTHER INYESTIGATION.

- DETECTIDN LIUIT Was nOt hyatLable for coyparison

NR - ANALYSIS NOT REQUESTED OR NOT YET REPORTED

YALUES IN \{\} ARE COUNTING ERRORS FOR RADIONUCLIDES

WATER STANDARD(S) IN PARENTHESES ARE PROPOSED ONLY 
TABLE C.7. (contd)

\begin{tabular}{|c|c|c|c|c|c|c|c|c|c|}
\hline \multirow{2}{*}{\multicolumn{2}{|c|}{ CONST I TUENT }} & \multirow[b]{2}{*}{$\begin{array}{c}\text { CONTRACTUAL } \\
\text { DETECTION } \\
\text { LIMIT }\end{array}$} & \multirow[b]{2}{*}{$\begin{array}{l}\text { DRINKING } \\
\text { IATER } \\
\text { STANDARD }\end{array}$} & \multirow[b]{2}{*}{$\begin{array}{c}\text { SAUPLE } \\
\text { DATE }\end{array}$} & \multirow[b]{2}{*}{$8-24-1 R$} & \multirow[b]{2}{*}{$\begin{array}{c}\text { SAUPLE } \\
\text { DATE }\end{array}$} & \multirow[b]{2}{*}{$6-24-15$} & \multirow[b]{2}{*}{$\begin{array}{l}\text { SAUPLE } \\
\text { DATE }\end{array}$} & \multirow[b]{2}{*}{$6-25-55$} \\
\hline & & & & & & & & & \\
\hline----- & $-\ldots$ & 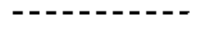 & 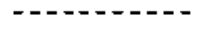 & ---n--- & $---\infty$ & $---1--1$ & $-\ldots+\cdots$ & 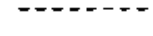 & 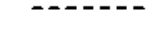 \\
\hline HN I TRAT & PPB & 2500.00 & 45000.60 & $69 / 17 / 87$ & NR & $69 / 17 / 87$ & NR & $\begin{array}{l}68 / 19 / 87 \\
11 / 13 / 87\end{array}$ & $\begin{array}{l}14000.00 \\
14600.00\end{array}$ \\
\hline
\end{tabular}

* - yalue exceeds priuary drinking tater standaro

- VALUE EXCEEDS PROPOSED PRIMARY DRINKING IATER STANDARD.

- VALUE EXCEEDS SCREENING LEYEL FOR FURTHER IMYESTIGATION.

- detection livit us not ayailable for comparison

NR - ANALYSIS NOT REQUESTED OR NOT YET REPORTED

VALUES IN \{ \} ARE COUNTING ERRORS FOR RADIONUCLIDES

IATER STANDARD(S) IN PARENTHESES ARE PROPGSED ONLY 


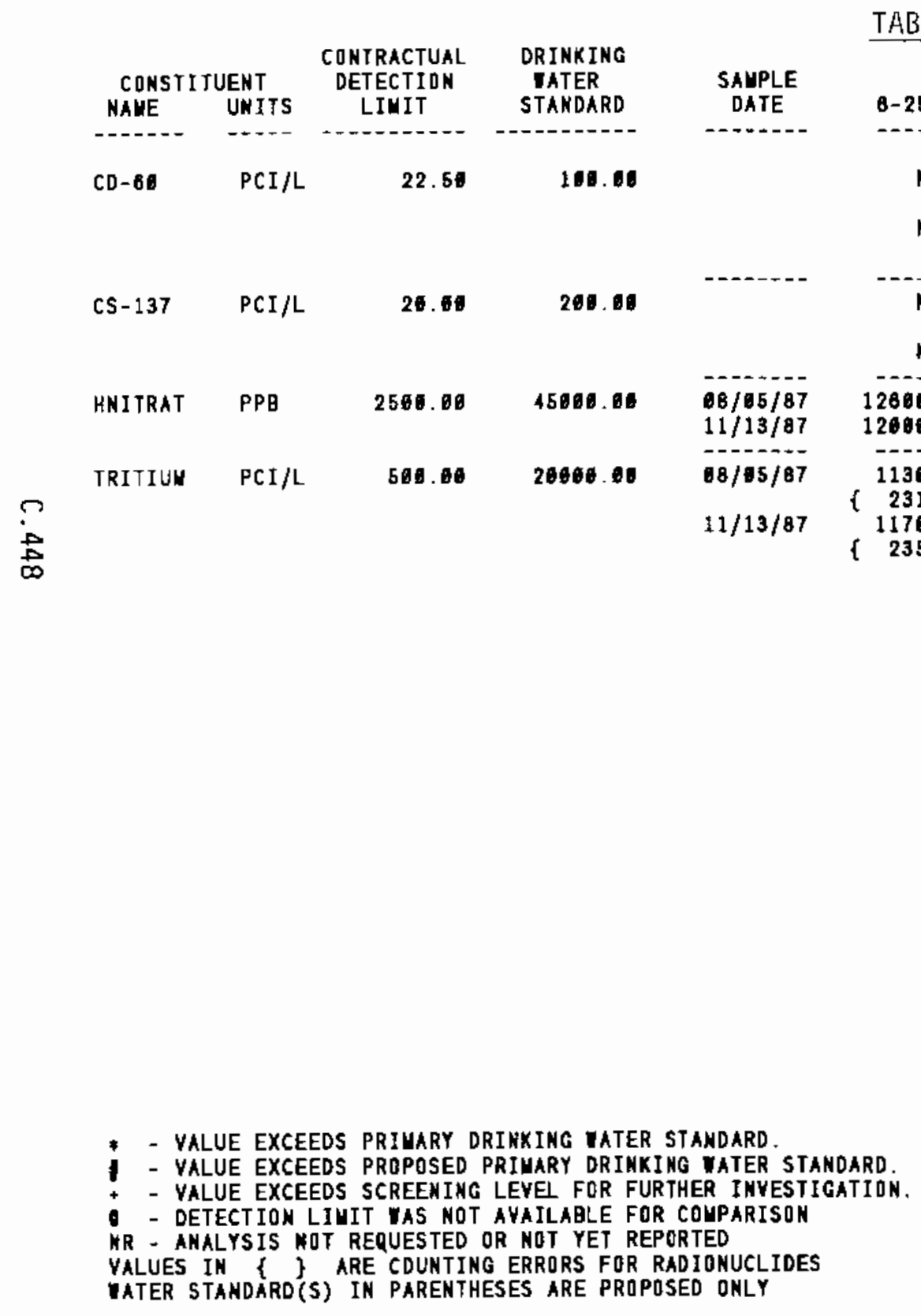

ABLE C.7. (contd)

\begin{tabular}{l} 
NR \\
NR \\
NR \\
NR \\
NR \\
\hdashline 600.00 \\
2000.00 \\
130.09 \\
$231.00\}$ \\
170.00 \\
$235.06\}$
\end{tabular}

\begin{tabular}{|c|c|}
\hline $\begin{array}{c}\text { SANPLE } \\
\text { DATE }\end{array}$ & 6-26-15A \\
\hline$---1, \ldots$ & ---n-- \\
\hline $19 / 20 / 87$ & $\begin{array}{l}9.86 \\
7.48\}\end{array}$ \\
\hline $10 / 25 / 87$ & $\begin{array}{r}15.00 \\
\{\quad 8.11\}\end{array}$ \\
\hline $88 / 20 / 87$ & $\left\{\begin{array}{l}6.84 \\
4.84\}\end{array}\right.$ \\
\hline $10 / 25 / 87$ & \\
\hline $\begin{array}{l}08 / 24 / 87 \\
10 / 25 / 87\end{array}$ & 37060.06 \\
\hline 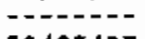 & - - \\
\hline $68 / 20 / 87$ & $\begin{array}{l}347600.00 \\
(1950.06)\end{array}$ \\
\hline $16 / 25 / 87$ & $\begin{array}{l}307080.00 \\
\{21180.00\}\end{array}$ \\
\hline
\end{tabular}

\begin{tabular}{cc}
$\begin{array}{c}\text { SAUPLE } \\
\text { DATE }\end{array}$ & $6-26-89$ \\
& NR \\
& NR \\
& NR \\
& NR \\
\hline $69 / 23 / 87$ & NR \\
& NR \\
& NR
\end{tabular}

- VALUE EXCEEDS PRIUARY DRINXIMG IATER STANDARD

- VALUE EXCEEDS PRBPOSED PRIUARY DRINKING VATER STANDARD

- DETECTION LIUIT UAS NOT AYAILABLE FOR COUPARISON

DETECTSIS NOT REQUESTED OR NOT YET REPORTED

IATER STANDARD'(S) IN PARENTHESES ARE PROPOSED ONLY 


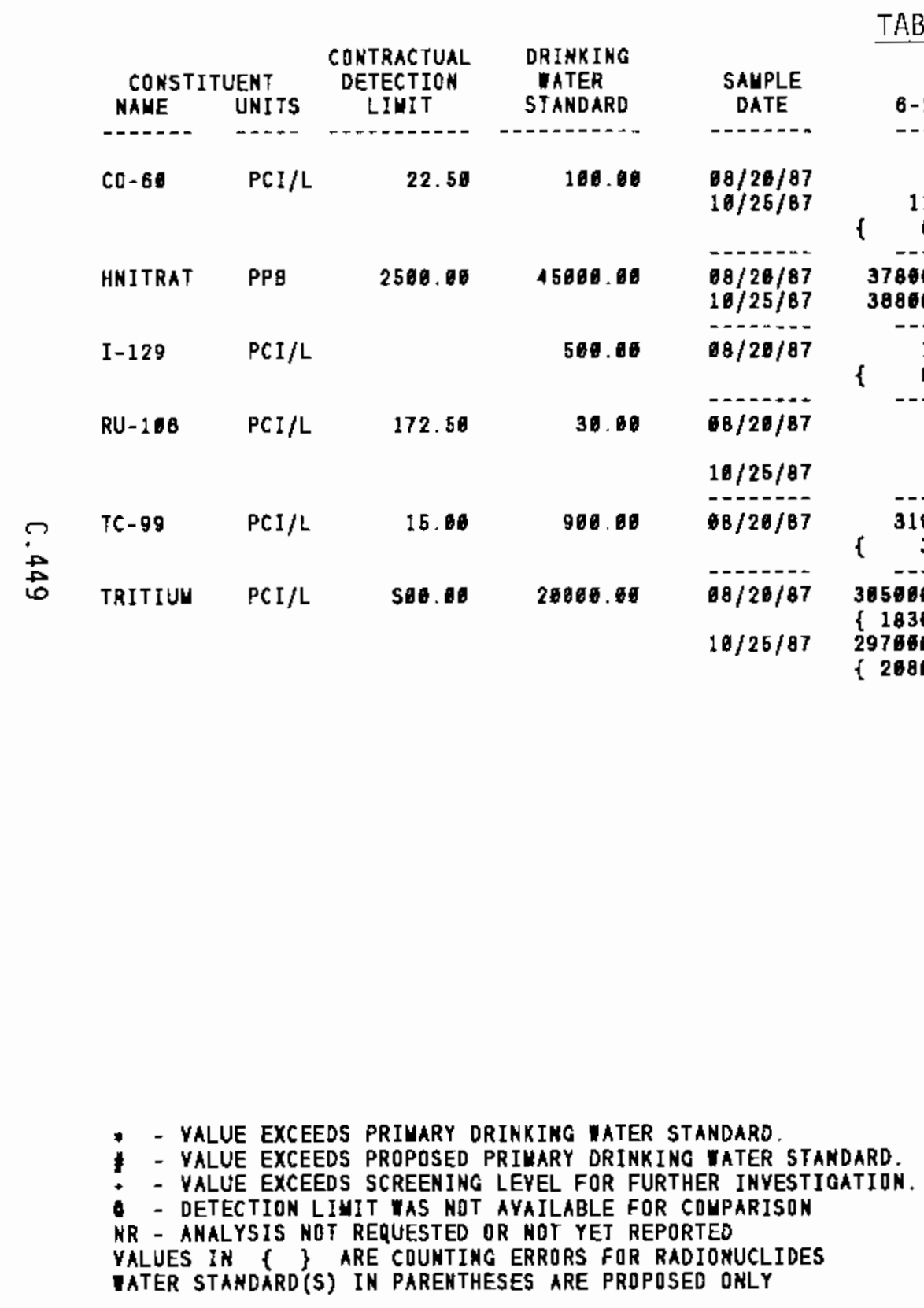

ABLE C.7. (contd)

\begin{tabular}{|c|c|c|c|c|}
\hline$-27-8$ & $\begin{array}{l}\text { SAUPLE } \\
\text { DATE }\end{array}$ & $6-28-46 P$ & $\begin{array}{c}\text { SAUPLE } \\
\text { DATE }\end{array}$ & $8-28-52 A$ \\
\hline--- & $\cdots+-1$ & 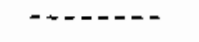 & $----1-$ & $----\cdots$ \\
\hline $\begin{array}{l}1.20 \\
6.72\}\end{array}$ & $99 / 17 / 87$ & NR & $\begin{array}{l}98 / 18 / 87 \\
11 / 13 / 87\end{array}$ & \\
\hline .00 & $9 / 17 / 87$ & NR & $\begin{array}{l}68 / 19 / 87 \\
11 / 13 / 87\end{array}$ & 10800.00 \\
\hline $\begin{array}{l}1.85 \\
.15\}\end{array}$ & - - - & NR & - & NR \\
\hline-- & $69 / 17 / 87$ & $\left\{\begin{array}{c}98.50 \\
49.86 \\
\text { NR }\end{array}\right.$ & $11 / 13 / 87$ & ------ \\
\hline .00 & - - & NR & --ne- & NR \\
\hline $\begin{array}{l}0.00 \\
0.00\} \\
0.00 *\end{array}$ & $69 / 17 / 87$ & $-\cdots-1$ & $\begin{array}{l}08 / 19 / 67 \\
11 / 13 / 87\end{array}$ & (22900.09 \\
\hline
\end{tabular}

- YALUE EXCEEDS PRIMARY DRINKIMG UATER STANDARD.

- VALUE EXCEEDS PROPOSED PRIUARY DRINKING TATER STAKDARD.

- DETECTION LIHIT WAS NDT AVAILABLE FOR COUPARISON

IATER STANDARD(S) IN PAREHTHESES ARE PROPOSED ONLY 
TABLE C.7. (contd)

\begin{tabular}{|c|c|c|c|c|c|c|c|c|c|}
\hline$\underset{\text { MAUE }}{\text { CONSTI }}$ & $\begin{array}{l}\text { UENT } \\
\text { UNITS }\end{array}$ & $\begin{array}{l}\text { CONTRACTUAL } \\
\text { DETECTION } \\
\text { LIUIT }\end{array}$ & $\begin{array}{l}\text { DRINKING } \\
\text { DATER } \\
\text { STANDARD }\end{array}$ & $\begin{array}{l}\text { SAUPLE } \\
\text { DATE }\end{array}$ & $6-31-31$ & $\begin{array}{l}\text { SAYPLE } \\
\text { DATE }\end{array}$ & $6-31-31 P$ & $\begin{array}{c}\text { SALPLE } \\
\text { DATE }\end{array}$ & $6-32-62$ \\
\hline ALPHA & $\mathrm{PCI} / \mathrm{L}$ & 4.00 & 16.00 & & $\begin{array}{l}\text { MR } \\
\text { MR }\end{array}$ & & $\begin{array}{l}\text { NR } \\
\text { NR }\end{array}$ & $\begin{array}{l}16 / 27 / 87 \\
12 / 88 / 87\end{array}$ & $\begin{array}{l}2.16 \\
0.84 \\
2.16 \\
1.84\end{array}$ \\
\hline HNITRAT & $\mathrm{PPB}$ & 2500.00 & 45080.06 & $\begin{array}{l}97 / 29 / 87 \\
10 / 28 / 87\end{array}$ & $\begin{array}{l}7659.08 \\
8190.00\end{array}$ & $69 / 17 / 87$ & KR & $\begin{array}{l}68 / 27 / 87 \\
12 / 88 / 87\end{array}$ & 28500.66 \\
\hline TRITIUN & $P C I / L$ & 500.00 & 20000 & $\begin{array}{l}07 / 29 / 87 \\
10 / 26 / 87\end{array}$ & 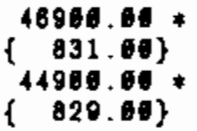 & $69 / 17 / 87$ & NR & $\begin{array}{l}68 / 27 / 87 \\
12 / 08 / 87\end{array}$ & $\left\{\begin{array}{r}1861.06 \\
259.06 \\
2929.66 \\
214.06\end{array}\right\}$ \\
\hline
\end{tabular}

* - value exceeds primary drinking mater standard

- VALUE EXCEEDS PROPOSED PRIUARY DRINKING IATER STAMDARD.

- VALUE EXCEEDS SCREENIHg LEVEL gOR FURTHER INVESTIGATION.

- DETECTIDN LIUIT WAS NOT AVAILABLE FOR COUPARISON

NR - ANALYSIS NOT REQUESTED OR NOT YET REPORTED

VALUES IN $f$ ) ARE COUNTING ERRORS FOR RADIONUCLIDES

IATER STANDARD(S) IN PARENTHESES ARE PROPOSED DKLY 
TABLE C.7. (contd)

\begin{tabular}{|c|c|c|c|c|c|c|c|c|c|}
\hline \multicolumn{2}{|c|}{ CONSTITUENT } & $\begin{array}{l}\text { CONTRACTUAL } \\
\text { DETECTION } \\
\text { LIUIT }\end{array}$ & $\begin{array}{l}\text { DRINKING } \\
\text { WATER } \\
\text { STANDARD }\end{array}$ & $\begin{array}{l}\text { SAUPLE } \\
\text { DATE }\end{array}$ & $6-34-39 A$ & $\begin{array}{l}\text { SAUPLE } \\
\text { DATE }\end{array}$ & $6-34-41 B$ & $\begin{array}{l}\text { SALPLE } \\
\text { DATE }\end{array}$ & $6-34-88$ \\
\hline------- & ---- & - - & $---1-1-2$ & $---1-0$ & 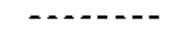 & ------ & n...... & ----- & ----- \\
\hline$C S-137$ & $\mathrm{PCI} / \mathrm{L}$ & 20.00 & 200.00 & $\begin{array}{l}07 / 36 / 87 \\
10 / 25 / 87\end{array}$ & $\begin{array}{ll} & 5.12 \\
\{\quad 3.82\}\end{array}$ & $\begin{array}{l}07 / 30 / 87 \\
10 / 25 / 87\end{array}$ & & & $\begin{array}{l}\text { MR } \\
\text { MR }\end{array}$ \\
\hline HNITRAT & PPB & 2500.00 & 45000.80 & $\begin{array}{l}07 / 39 / 87 \\
16 / 25 / 87\end{array}$ & 2980.60 & $\begin{array}{l}07 / 30 / 87 \\
10 / 25 / 87\end{array}$ & $\begin{array}{l}11860.00 \\
10100.00\end{array}$ & $\begin{array}{l}68 / 05 / 97 \\
12 / 98 / 87\end{array}$ & $\begin{array}{l}19200.06 \\
19360.06\end{array}$ \\
\hline TRITIUM & $P C I / L$ & 560.00 & 20000.00 & $10 / 25 / 87$ & 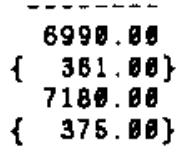 & $\begin{array}{l}07 / 30 / 87 \\
10 / 25 / 87\end{array}$ & 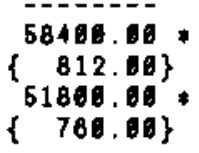 & $\begin{array}{l}18 / 05 / 87 \\
12 / 68 / 87\end{array}$ & \\
\hline
\end{tabular}

* - Value exceEds primary drinking mater standard.

- - VALUE EXCEEDS PROPOSED PRIMARY DRINKING TATER STANDARD.

+ - YALUE EXCEEDS SCREENING LEYEL FOR FURTHER INYESTIGATION.

- DETECTION LIUIT TAS NOT AVAILABLE FOR CONPARISON

NR - ANALYSIS NOT REQUESTED OR NOT YET REPORTED

VALUES IN \& $\}$ ARE COUNTING ERRORS FOR RADIONUCLIDES

WATER STANDARD'S) IN PARENTHESES ARE PRDPOSED ONLY 
TABLE C.7. (contd)

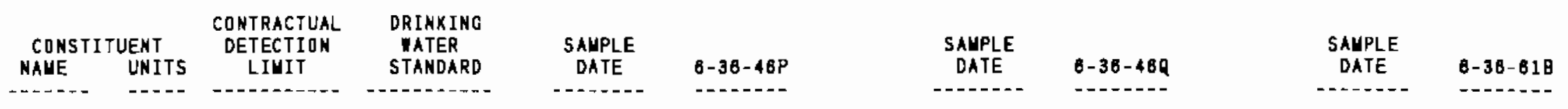

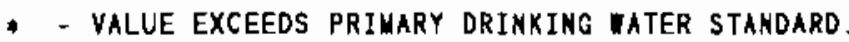

1 - VALUE EXCEEDS PROPQSED PRIUARY DRINKING IATER STANDARD.

* - VALUE EXCEEDS SCREENIHG LEVEL fOR FURTHER INVESTIGATION.

- DETECTION LIUIT IAS ROT aVAILABLE fOR COHParison

NR - ANALYSIS NOT REQUESTED OR NOT YET REPORTED

VALUES IN I I ARE COUNTING ERRORS FOR RADIONUCLIDES

WATER STANDARD(S) IN PARENTHESES ARE PROPOSED OKLY 
IABLE C.7. (contd)

\begin{tabular}{|c|c|c|c|c|c|}
\hline \multicolumn{2}{|c|}{ CONSTITUENT } & $\begin{array}{c}\text { CONTRACTUAL } \\
\text { DETECTION } \\
\text { LIUIT }\end{array}$ & $\begin{array}{l}\text { DRINKING } \\
\text { WATER } \\
\text { STANDARD }\end{array}$ & $\begin{array}{c}\text { SAYPLE } \\
\text { DATE }\end{array}$ & $B-36-83$ \\
\hline----- & ---- & $-\cdots--\infty$ & 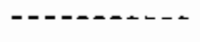 & ------ & 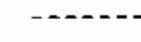 \\
\hline ALPHA & PCI $/ L$ & 4.00 & 15.00 & & NR \\
\hline BETA & $\mathrm{PCI} / \mathrm{L}$ & 8.60 & 50.00 & ------ & NR \\
\hline \multirow[t]{2}{*}{$C 0-60$} & $\mathrm{PCI} / \mathrm{L}$ & 22.50 & 100.00 & ------ & NR \\
\hline & & & & & NR \\
\hline \multirow[t]{2}{*}{$C S-137$} & $\mathrm{PCI} / \mathrm{L}$ & 20.00 & 290.60 & & HR \\
\hline & & & & & $\begin{array}{l}N R \\
N R\end{array}$ \\
\hline HAITRAT & PPB & 2560.60 & 45000.00 & $\begin{array}{l}67 / 61 / 87 \\
69 / 23 / 87\end{array}$ & $\begin{array}{c}34606.00 \\
37706.00 \\
\text { NR }\end{array}$ \\
\hline \multirow[t]{3}{*}{$R U-188$} & $\mathrm{PCI} / \mathrm{L}$ & 172.60 & 36.00 & & NR \\
\hline & & & & & NR \\
\hline & & & & $-1-1-1$ & NR \\
\hline \multirow[t]{3}{*}{ TRITIUH } & $\mathrm{PCI} / \mathrm{L}$ & 500.00 & 20600.00 & $07 / 01 / 07$ & \\
\hline & & & & $09 / 23 / 87$ & \\
\hline & & & & & NR \\
\hline U & $\mathrm{PCI} / \mathrm{L}$ & E. 50 & 600.86 & & NR \\
\hline
\end{tabular}

\begin{tabular}{|c|c|}
\hline $\begin{array}{l}\text { SAMPLE } \\
\text { DATE }\end{array}$ & $6-36-16$ \\
\hline & NR \\
\hline 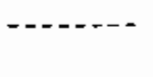 & NR \\
\hline 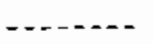 & -... \\
\hline $\begin{array}{l}97 / 31 / 87 \\
11 / 62 / 87\end{array}$ & $\begin{array}{l}13.66 \\
\left\{\quad \begin{array}{l}7.86 \\
N R\end{array}\right\}\end{array}$ \\
\hline $67 / 31 / 87$ & ----- \\
\hline $11 / 62 / 87$ & MR \\
\hline - - - - - - & ----- \\
\hline $\begin{array}{l}67 / 31 / 87 \\
11 / 62 / 87\end{array}$ & $\begin{array}{c}53700.08 * \\
54268.60 * \\
\text { NR }\end{array}$ \\
\hline $1 / 31 / 87$ & 54.50 \\
\hline $11 / 02 / 87$ & 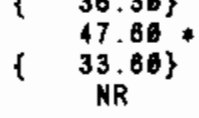 \\
\hline $67 / 31 / 87$ & $528000.00 *$ \\
\hline $11 / 82 / 87$ & $\begin{array}{c}\{89000.00 \\
\{2280.00\} \\
\text { FR }\end{array}$ \\
\hline - & NR \\
\hline
\end{tabular}

\begin{tabular}{|c|c|}
\hline $\begin{array}{l}\text { SAUPLE } \\
\text { DATE }\end{array}$ & $6-39-0$ \\
\hline 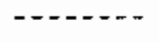 & ---- \\
\hline $08 / 07 / 87$ & $\begin{array}{l}3.82 \\
\text { D. } 84\}\end{array}$ \\
\hline 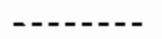 & - n. \\
\hline $08 / 97 / 87$ & $\left\{\begin{array}{c}68.60 \\
\{\quad 5.81\end{array}\right.$ \\
\hline $\begin{array}{l}-8 / 31 / 87 \\
68 / 67 / 87\end{array}$ & 11.26 \\
\hline $16 / 27 / 87$ & $6.78\}$ \\
\hline $07 / 31 / 87$ & 3.45 \\
\hline $\begin{array}{l}68 / 67 / 87 \\
16 / 27 / 87\end{array}$ & $3.98\}$ \\
\hline $\begin{array}{l}07 / 31 / 87 \\
8 / 67 / 87 \\
18 / 27 / 87\end{array}$ & $\begin{array}{l}--1--- \\
35900.08 \\
38960.08 \\
39700.08\end{array}$ \\
\hline $7 / 31 / 87$ & $-\cdots$ \\
\hline $08 / 07 / 87$ & $\begin{array}{l}56.20 \\
35.50\end{array}$ \\
\hline $10 / 27 / 87$ & \\
\hline $07 / 31 / 87$ & $\begin{array}{l}228000.00 \\
\{1770.06\}\end{array}$ \\
\hline $98 / 07 / 87$ & $\begin{array}{l}243006.00 \\
\{1640.00\}\end{array}$ \\
\hline $10 / 27 / 87$ & $\begin{array}{l}251600.00 \\
\{1900.06\}\end{array}$ \\
\hline $68 / 67 / 87$ & 3.12 \\
\hline
\end{tabular}

- VALUE eXCEEDS PRIUARY dRINKINg vater STANDARD.

- - VALUE EXCEEDS PROPOSED PRIUARY DRINKING TATER STANDARD.

+ - VALUE EXCEEDS SCREENING LEVEL FDR FURTHER INVESTIGATIDN.

- DETECTION LIUIT UAS NOT AVAILABLE FOR COUPARISON

NR - ANALYSIS NDT REQUESTED OR NDT YET REPDRTED

YALUES IN \{ $\}$ ARE COUNTING ERRORS FUR RADIONUCLIDES

TATER STANDARD(S) IN PARENTHESES ARE PROPOSED ONLY 


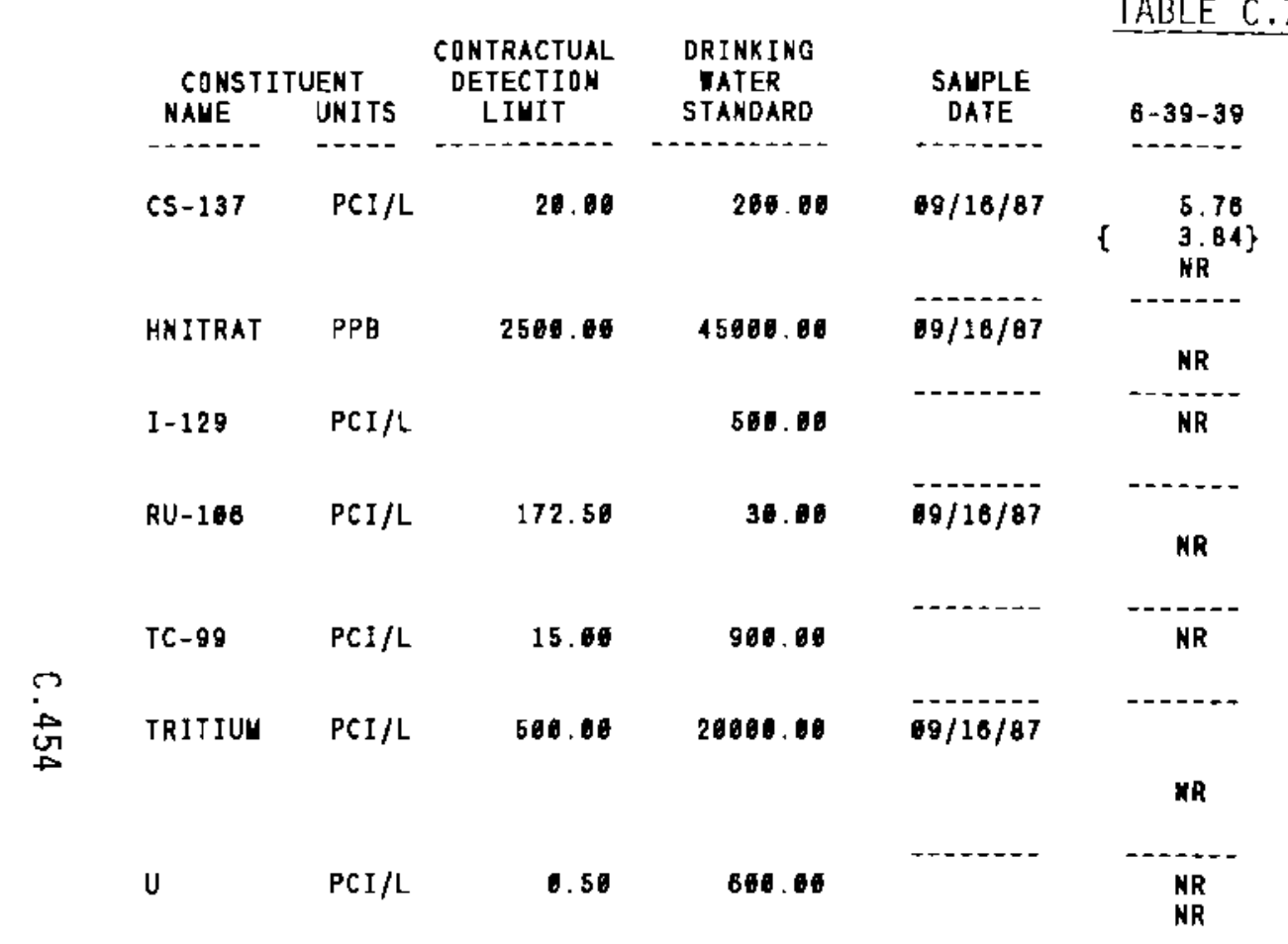

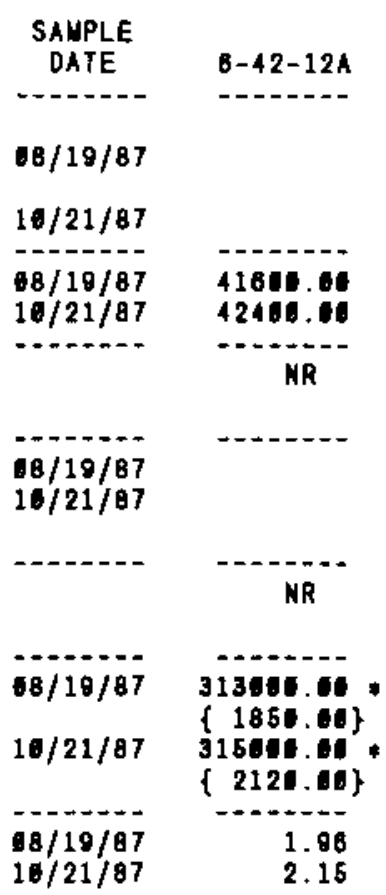

\footnotetext{
- - value exceeds priuary dRINking vater standard.

- - VAlUE EXCEEDS PROPOSED PRIUARY DRINKING TATER STANDARD

- VALUE EXCEEDS SCREENING LEVEL FOR FURTHER INYESTIGATION

- DETECTION LIUIT IAS NOT AVALABLE FOR COUPARISON

NR - ANALYSIS NOT REQUESTED OR NOT YET REPORTED

VALUES IN \{ \} ARE COUNTING ERRORS FOR RADIONUCLIDES

TATER STANDARD(S) IN PARENTHESES ARE PROPOSED DNLY
}

ABLE C.7. (contd) 
TABLE C.7. (contd)

\begin{tabular}{|c|c|c|c|c|c|}
\hline $\begin{array}{l}\text { CONSTI } \\
\text { NAWE }\end{array}$ & $\begin{array}{l}\text { TUENT } \\
\text { UNITS }\end{array}$ & $\begin{array}{c}\text { CONTRACTUAL } \\
\text { DETECTION } \\
\text { LIUIT }\end{array}$ & $\begin{array}{l}\text { DRINKING } \\
\text { WATER } \\
\text { STANDARD }\end{array}$ & $\begin{array}{l}\text { SAUPLE } \\
\text { DATE }\end{array}$ & $B-42-46 A$ \\
\hline$-\ldots--$ & ---- & m...---- & - & $------\infty$ & $----1--$ \\
\hline ALPHA & $\mathrm{PCI} / \mathrm{L}$ & 4.00 & 15.00 & $\begin{array}{l}67 / 09 / 87 \\
68 / 11 / 87 \\
69 / 09 / 87 \\
10 / 06 / 87 \\
11 / 04 / 87 \\
12 / 01 / 87\end{array}$ & $\begin{array}{ll}\{ & 0.72 \\
\{ & .48 \\
\{ & .78 \\
\{ & .43 \\
\{ & .81 \\
\{ & .44\}\end{array}$ \\
\hline $\begin{array}{l}c \\
0 \\
G \\
0\end{array}$ & $\mathrm{PCI} / \mathrm{L}$ & 8.00 & 50.00 & $\begin{array}{l}07 / 09 / 87 \\
68 / 11 / 87 \\
09 / 09 / 87 \\
10 / 08 / 87 \\
11 / 04 / 87 \\
12 / 01 / 87\end{array}$ & 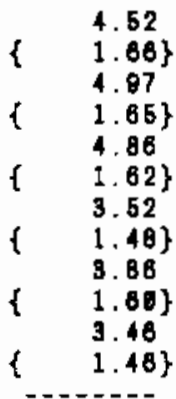 \\
\hline$C 0-60$ & PCI/L & 22.50 & 100.00 & $\begin{array}{l}07 / 09 / 87 \\
08 / 11 / 87 \\
09 / 69 / 87 \\
16 / 06 / 87 \\
11 / 64 / 87 \\
12 / 01 / 87 \\
-\end{array}$ & $\begin{array}{r}5.07 \\
\{\quad 5.43\}\end{array}$ \\
\hline CS-137 & $\mathrm{PCI} / \mathrm{L}$ & 20.00 & 200.00 & $\begin{array}{l}07 / 09 / 87 \\
08 / 11 / 87 \\
\\
09 / 09 / 87 \\
16 / 06 / 87 \\
11 / 64 / 87\end{array}$ & $\begin{array}{l}4.4 B \\
\{\quad 3.3 B\}\end{array}$ \\
\hline
\end{tabular}

\begin{tabular}{|c|c|c|c|}
\hline $\begin{array}{c}\text { SAMPLE } \\
\text { DATE }\end{array}$ & $B-42-48 B$ & $\begin{array}{c}\text { SAMPLE } \\
\text { DATE }\end{array}$ & $6-42-48 C$ \\
\hline & $\begin{array}{l}\text { NR } \\
\text { NR } \\
\text { NR } \\
\text { NR }\end{array}$ & & $\begin{array}{l}N R \\
K R \\
K R \\
N R\end{array}$ \\
\hline & NR & & NR \\
\hline & NR & & NR \\
\hline $97 / 69 / 87$ & 3.46 & & NR \\
\hline $98 / 11 / 87$ & $\left.\begin{array}{ll}\{ & 1.53 \\
& 4.48\end{array}\right\}$ & & NR \\
\hline 09/89/87 & $\left\{\begin{array}{l}1.58\} \\
5.89\end{array}\right\}$ & & MR \\
\hline $18 / 08 / 87$ & $\left\{\begin{array}{l}\{.19 \\
\quad 37\end{array}\right.$ & & Mr \\
\hline & $\{\quad 1.56\}$ & & $\mathrm{nn}$ \\
\hline $11 / 94 / 87$ & $\begin{array}{ll}8.96 \\
\{\quad 1.85\}\end{array}$ & & $F R$ \\
\hline $12 / 107 / 87$ & $\left\{\begin{array}{l}4.04 \\
\{1.62\}\end{array}\right.$ & & NR \\
\hline $\begin{array}{l}87 / 69 / 87 \\
98 / 11 / 87\end{array}$ & 5.85 & & $\begin{array}{l}\text { NR } \\
\text { NR }\end{array}$ \\
\hline $\begin{array}{l}09 / 89 / 87 \\
19 / 06 / 87 \\
11 / 84 / 87 \\
12 / 07 / 87\end{array}$ & & & $\begin{array}{l}\mathrm{NR} \\
\mathrm{NR} \\
\mathrm{NR} \\
\mathrm{NR}\end{array}$ \\
\hline $\begin{array}{l}87 / 69 / 87 \\
68 / 11 / 87\end{array}$ & & & $\begin{array}{l}\mathrm{NR} \\
\mathrm{NR}\end{array}$ \\
\hline $\begin{array}{l}89 / 69 / 87 \\
16 / 608 \\
11 / 64 / 87\end{array}$ & & & $\begin{array}{l}\text { NR } \\
N R \\
N R\end{array}$ \\
\hline
\end{tabular}

* - VAlUe eXCEeds primary dRinking Mater standard.

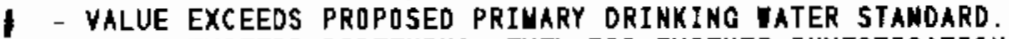

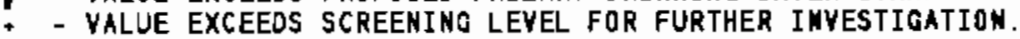

- DETECTIDN liUit as not ayailable for couparison

WR - ANALYSIS NOT REQUESTED OR NDT YET REPORTED

VALUES IN \{ \} ARE COUNTING ERRORS FOR RAOIONUCLIDES

WATER STANOARD(S) IN PARENTHESES ARE PROPOSED ONLY 


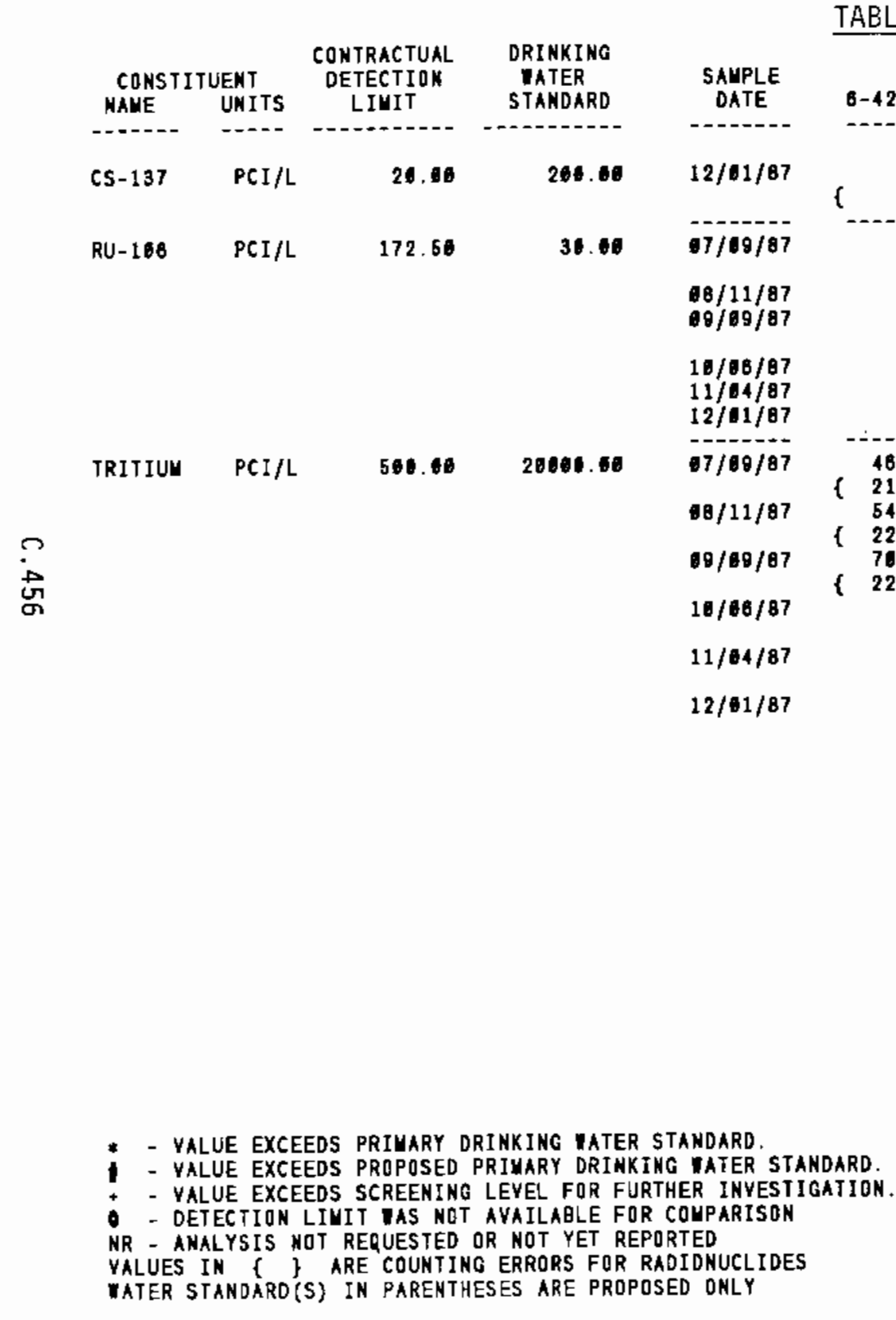

ABLE C.7. (contd)

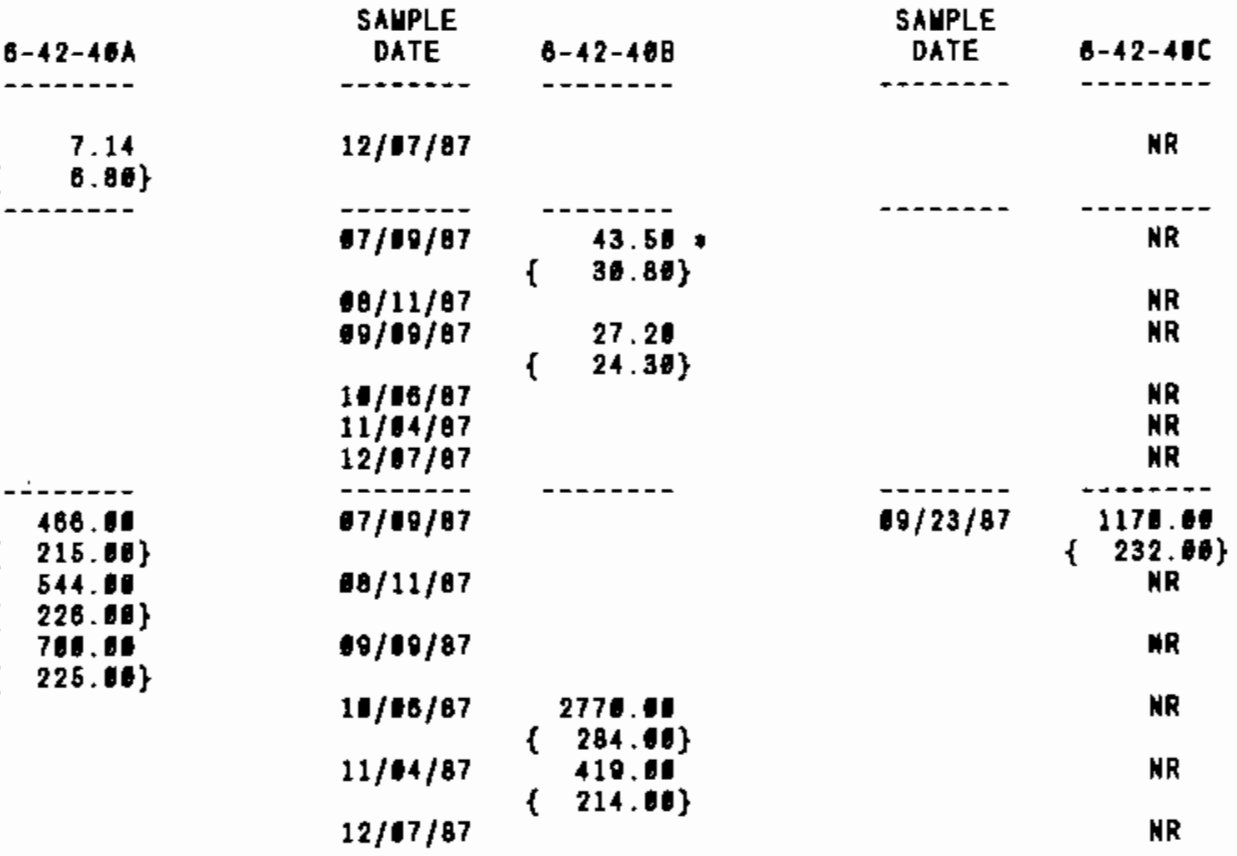

YALUE EXCEEDS PRIUARY DRINKING MATER STANDARD.

- YALUE EXCEEDS PROPOSED PRIMARY DRINKING MATER STANDARD.

WATER STANDARO(S) IN PARENTHESES ARE PROPOSED ONLY 
TABLE C.7. (contd)

\begin{tabular}{|c|c|c|c|c|c|c|c|c|c|}
\hline $\begin{array}{l}\text { CONSTI } \\
\text { NAME }\end{array}$ & $\begin{array}{l}\text { UENT } \\
\text { UNITS }\end{array}$ & $\begin{array}{c}\text { CONTRACTUAL } \\
\text { DEIECIION } \\
\text { LIUIT }\end{array}$ & $\begin{array}{l}\text { DRINKING } \\
\text { WATER } \\
\text { STANDARD }\end{array}$ & $\begin{array}{l}\text { SAMPLE } \\
\text { DATE }\end{array}$ & $6-44-4$ & $\begin{array}{l}\text { SALPLE } \\
\text { DATE }\end{array}$ & $6-40-218$ & $\begin{array}{l}\text { SAYPLE } \\
\text { DATE }\end{array}$ & $8-47-35 A$ \\
\hline----- & $\ldots$ & $-\cdots+\cdots$ & 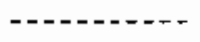 & $---1-n$ & ----- & $---1-1$ & $\cdots-\cdots$ & $\cdots-\cdots$ & $\cdots$ \\
\hline HNITRAT & PPB & 2560.68 & 45000.00 & $\begin{array}{l}07 / 31 / 87 \\
10 / 27 / 87\end{array}$ & 5030.00 & $\begin{array}{l}08 / 04 / 87 \\
10 / 25 / 87\end{array}$ & $\begin{array}{l}15000.00 \\
16100.00\end{array}$ & $\begin{array}{l}08 / 64 / 87 \\
10 / 25 / 87\end{array}$ & $\begin{array}{l}11000.00 \\
15900.00\end{array}$ \\
\hline TRITIUM & $\mathrm{PCI} / \mathrm{L}$ & 560.68 & 20000.00 & $10 / 27 / 87$ & $\begin{array}{l}129006.00 * \\
\{1340.00\} \\
145000.00 \\
\{1440.06\}\end{array}$ & $\begin{array}{l}08 / 04 / 87 \\
10 / 25 / 87\end{array}$ & $\begin{array}{c}48000.00 \\
\left\{\begin{array}{c}742.06\} \\
48200.00\end{array}\right. \\
\{\quad 718.06\}\end{array}$ & $\begin{array}{l}68 / 64 / 87 \\
10 / 25 / 87\end{array}$ & $-\ldots+\ldots$ \\
\hline
\end{tabular}

* - VALUE EXCEEDS PRIMARY DRINKING WaTER STANDARD

| - VALUE EXCEEDS PRDPOSED PRIUARY DRINXING TATER STANDARD.

- - VALUE EXCEEDS SCREENIMG LEVEL FDR FURTHER IHVESTIGATION.

- - DETECTIDN LIMIT WAS NOT AVAILABLE FRR COMPARISON

NR - ANALYSIS NOT REQUESTED OR NOT YET REPORTED

VALUES IN \{ \} ARE COUNTING ERRORS FOR RADIONUCLIDES

WATER STANDARO'S) IN PARENTHESES ARE PRDPOSED ONLY 
TABLE C.7. (contd)

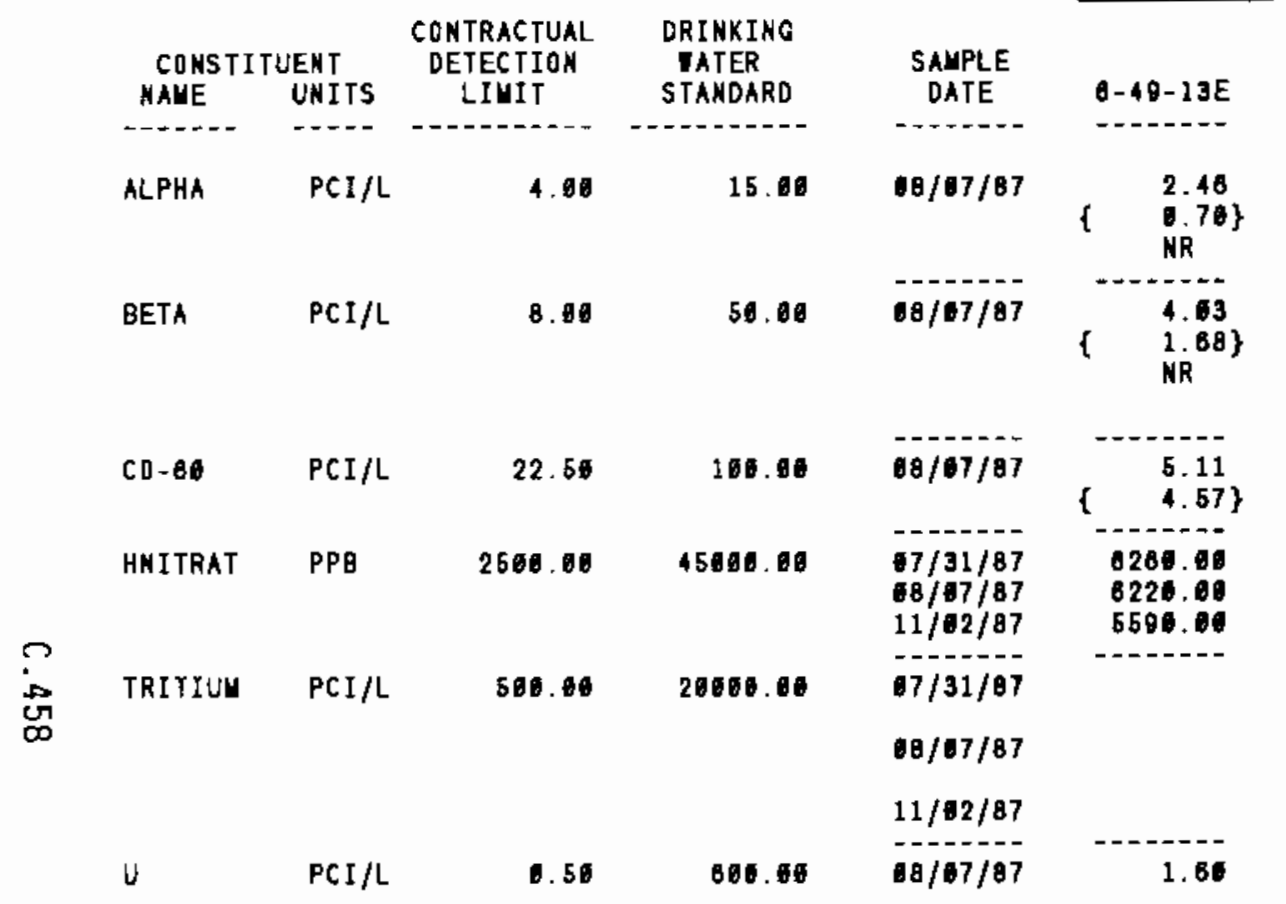
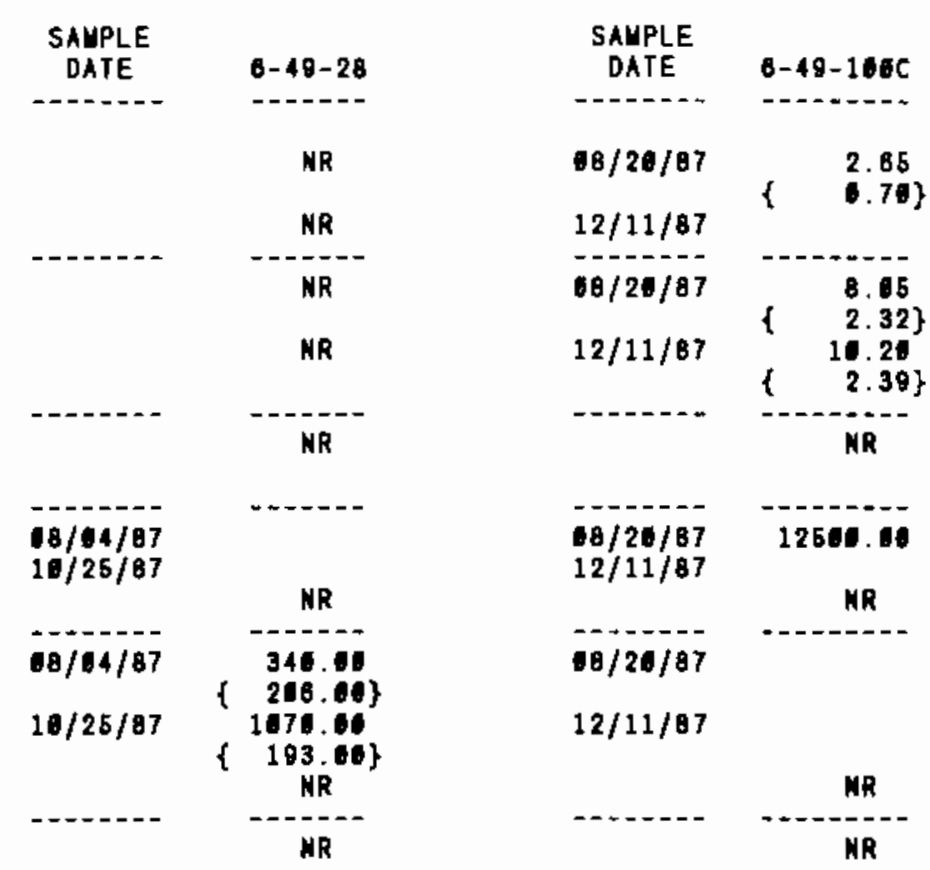

* - value exceeds priuhry drinxing vater standaro.

- - VALUE EXCEEDS PROPOSEO PRIUARY DRINXING WaTER STANDARD.

+ - VALUE EXCEEDS SCREENING LEVEL FDR FURTHER INYESTIGATION.

- DETECTIDN LIUIT TAS NOT AVAILABLE FOR COMPARISON

NR - ANALYSIS NOT REQUESTED OR NQT YET REPORTED

VALUES IN \{\} ARE COUNTING ERRDRS FOR RADIDNUCLIDES

YATER STANDARO(S) IN PARENTHESES ARE PROPOSED ONLY 
TABLE C.7. (contd)

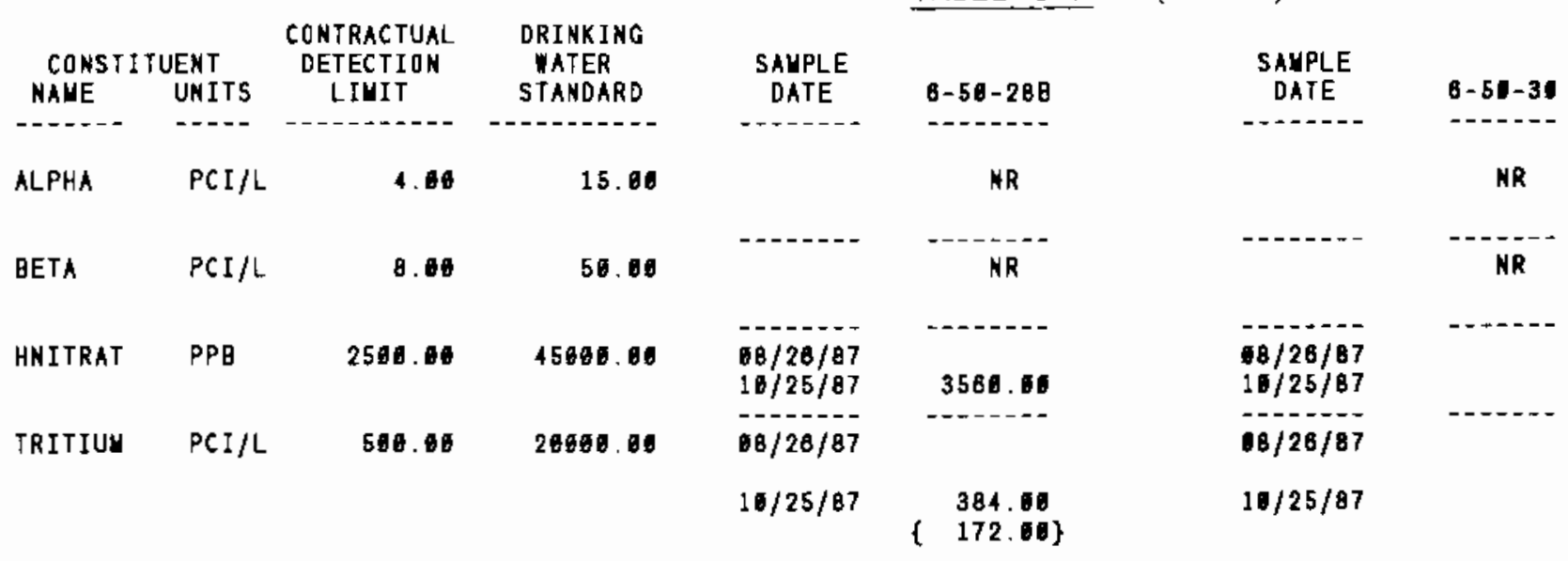

点

* - value exceeds priuary drinking water standard

- VALUE EXCEEDS PROPOSED PRIUARY DRINKING MATER STANDARD.

- - VALUE EXCEEDS SCREENING LEVEL FOR FURTHER INVESTIGATION

- DETECTION LIUIT WAS NDT AVALLABLE FOR COUPARISON

NR - ANALYSIS NOT REQUESTED OR NOT YET REPORTED

YALUES IN \{ $\}$ ARE COUNTING ERRORS FOR RADIONUCLIDES

vaTER STANDARD(S) IN PARENTHESES ARE PROPOSED ONLY 
TABLE C.7. (contd)

\begin{tabular}{|c|c|c|c|c|c|c|c|c|c|}
\hline $\begin{array}{l}\text { CONSTI } \\
\text { NAUE }\end{array}$ & $\begin{array}{l}\text { UENT } \\
\text { UNI IS }\end{array}$ & $\begin{array}{c}\text { CONTRACTUAL } \\
\text { DETECTIDN } \\
\text { LIUIT }\end{array}$ & $\begin{array}{l}\text { DRINKING } \\
\text { ATER } \\
\text { STANDARD }\end{array}$ & $\begin{array}{l}\text { SAUPLE } \\
\text { DATE }\end{array}$ & $6-50-45$ & $\begin{array}{l}\text { SALPLE } \\
\text { DATE }\end{array}$ & $8-51-48$ & $\begin{array}{l}\text { SALPLE } \\
\text { DATE }\end{array}$ & $8-51-63$ \\
\hline$-\cdots---$ & $-\cdots$ & 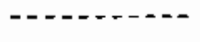 & - & $---\infty+\infty$ & $-+\cdots+\cdots$ & $\ldots+\infty$ & $----n$ & 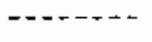 & - \\
\hline HNITRAT & PPB & 2500.00 & 45000.68 & $99 / 18 / 87$ & NR & $00 / 16 / 87$ & NR & $\begin{array}{l}18 / 20 / 87 \\
12 / 08 / 87\end{array}$ & $\begin{array}{l}16168.00 \\
15908.08\end{array}$ \\
\hline
\end{tabular}

- - yALUE EXCEEDS PRIUARY DRINKIMG MATER STAMDARD.

| - YalUE EXCEEDS PROPDSEO PRIUARY DRINKING MaTER STANDARD.

- - VALUE EXCEEDS SCREEAING LEVEL FOR FURTHER INYESTIGATION.

- DETECTION LIUIT WAS NOT AVAILABLE FOR COLPARISOM

NR - ANALYSIS NOT REQUESTED OR NOT YET REPORTED

YALUES IN \{\} ARE COUNTING ERRORS FOR RADIONUCLIDES

MATER STANDARO'S) IN PARENTHESES ARE PROPOSED ONLY 
TABLE C.7. (contd)

\begin{tabular}{|c|c|c|c|c|c|c|c|c|c|}
\hline $\begin{array}{l}\text { CONSTI } \\
\text { NAME }\end{array}$ & $\begin{array}{l}\text { UENT } \\
\text { UNI TS }\end{array}$ & $\begin{array}{l}\text { CONTRACTUAL } \\
\text { DETECTION } \\
\text { LIUIT }\end{array}$ & $\begin{array}{l}\text { DRINKING } \\
\text { WATER } \\
\text { STAKDARD }\end{array}$ & $\begin{array}{c}\text { SAUPLE } \\
\text { DATE }\end{array}$ & $6-52-19$ & $\begin{array}{c}\text { SAHPLE } \\
\text { DATE }\end{array}$ & $6-62-48$ & $\begin{array}{c}\text { SAUPLE } \\
\text { DATE }\end{array}$ & $6-53-35$ \\
\hline------ & $\ldots$ & $---\infty+-\infty$ & - - & $\cdots$ & $-\cdots+\infty$ & 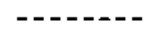 & - - & $-\ldots+\ldots$ & -non \\
\hline HNITRAT & $\mathrm{PPB}$ & 2500.00 & 45000.06 & $\begin{array}{l}06 / 23 / 87 \\
11 / 04 / 87\end{array}$ & $\begin{array}{l}4410.00 \\
4740.00\end{array}$ & $69 / 16 / 87$ & NR & $\begin{array}{l}18 / 26 / 87 \\
12 / 67 / 87\end{array}$ & 50200.06 \\
\hline
\end{tabular}

* - VALUE eXCEEDS PRIMARY dRINKING tater standaro.

1 - VALUE EXCEEDS PROPOSED PRIMARY DRINKIMG WATER STAMDARD.

+ - VALUE EXCEEDS SCREENING LEVEL FOR FURTRER INVESTIGATION

- DETECTION LIUIT TAS NOT AVAILABLE FOR COUPARISON

NR - ANALYSIS NOT REQUESTED OR NOT YET REPDRTED

VALUES IN \{\} ARE COUNTING ERRDRS FQR RADIONUCLIDES

WATER STANDARD(S) IN PARENTHESES ARE PROPOSED ONLY 
TABLE C.7. (contd)

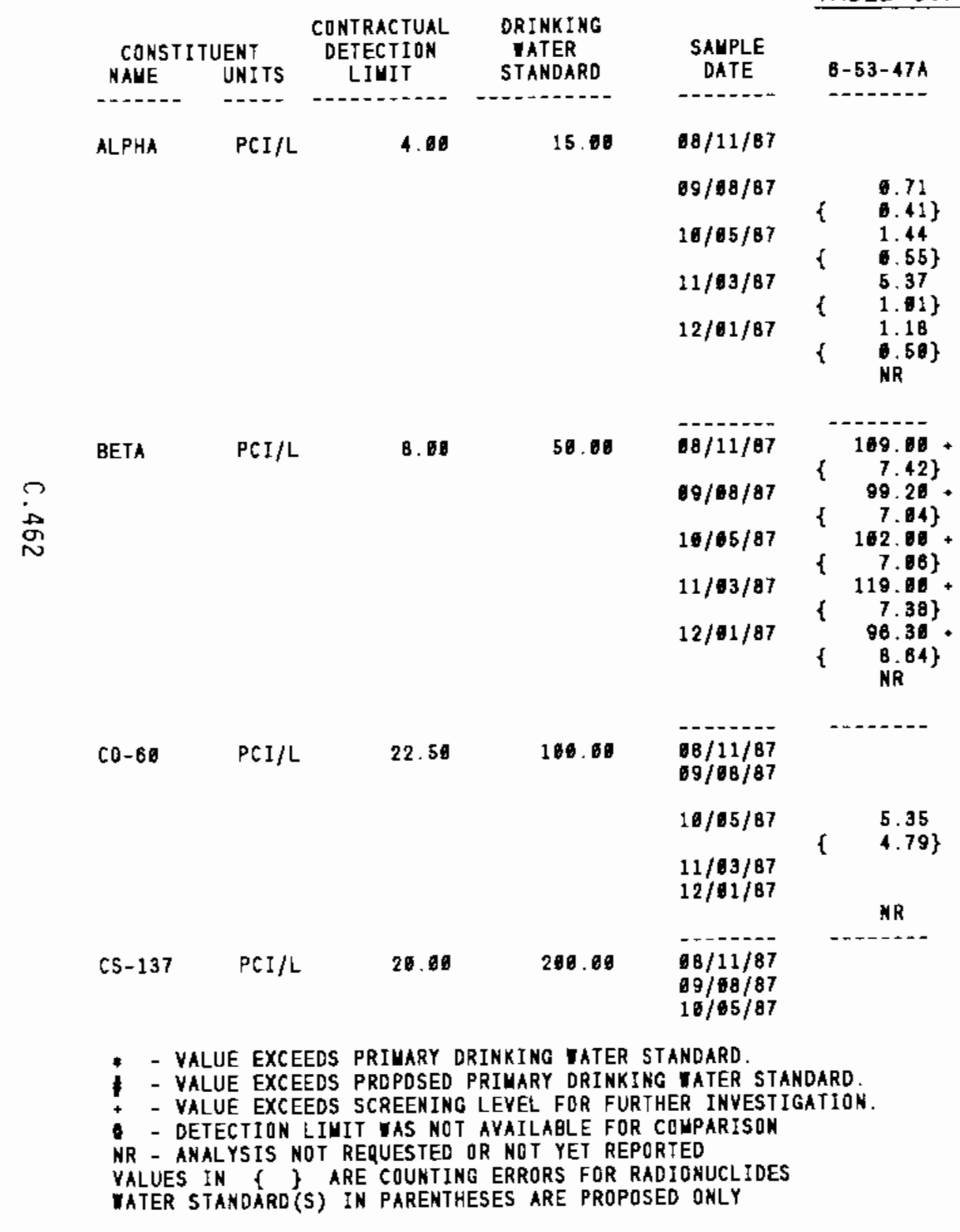

\begin{tabular}{|c|c|}
\hline $\begin{array}{l}\text { SAUPLE } \\
\text { DATE }\end{array}$ & $8-53-47 B$ \\
\hline $67 / 88 / 87$ & 3.49 \\
\hline $88 / 11 / 87$ & $\begin{array}{l}0.81\} \\
2.98\end{array}$ \\
\hline $19 / 68 / 87$ & $\begin{array}{l}0.75\} \\
3.63 \\
9.734\end{array}$ \\
\hline $10 / 65 / 87$ & $\begin{array}{l}8.73\} \\
2.94\end{array}$ \\
\hline $11 / 83 / 87$ & $\begin{array}{l}9.78\} \\
3.59\end{array}$ \\
\hline & B. 83$\}$ \\
\hline $12 / 81 / 87$ & $\begin{array}{l}3.48 \\
\{\quad 0.81\}\end{array}$ \\
\hline $07 / 68 / 87$ & $\begin{array}{r}135.00 \\
\{\quad 7.79\}\end{array}$ \\
\hline $68 / 11 / 87$ & $\begin{array}{r}157.60^{\circ} \\
\end{array}$ \\
\hline $09 / 08 / 87$ & $153.60^{\circ}$ \\
\hline $16 / 65 / 87$ & 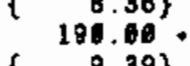 \\
\hline $11 / 63 / 87$ & $\left\{\begin{array}{c}9.39\} \\
173.60\end{array}\right.$ \\
\hline $12 / 61 / 87$ & $\begin{array}{r}8.98\} \\
182.68 \\
\left(\begin{array}{rl}8.57\}\end{array}\right.\end{array}$ \\
\hline $\begin{array}{l}67 / 68 / 87 \\
98 / 11 / 87\end{array}$ & 5.85 \\
\hline $09 / 88 / 87$ & \\
\hline $\begin{array}{l}10 / 65 / 87 \\
11 / 63 / 87 \\
12 / 01 / 87\end{array}$ & \\
\hline $\begin{array}{l}07 / 88 / 87 \\
68 / 11 / 87 \\
69 / 68 / 87\end{array}$ & \\
\hline
\end{tabular}

\begin{tabular}{|c|c|}
\hline $\begin{array}{l}\text { SAMPLE } \\
\text { DATE }\end{array}$ & $6-53-48 A$ \\
\hline $17 / 68 / 87$ & 18.80 \\
\hline $88 / 11 / 87$ & $\begin{array}{l}1.82\} \\
1.25 \\
1.593\end{array}$ \\
\hline $89 / 88 / 87$ & $\begin{array}{l}.88 \\
0.44\}\end{array}$ \\
\hline $10 / 05 / 87$ & $\begin{array}{l}2.54 \\
.88\}\end{array}$ \\
\hline $11 / 83 / 87$ & $\begin{array}{l}3.82 \\
8.82\end{array}$ \\
\hline $12 / \$ 1 / 87$ & $\begin{array}{r}5.24 \\
\{\quad 1.06\}\end{array}$ \\
\hline $67 / 68 / 87$ & $\begin{array}{r}12.06 \\
\{\quad 4.28\}\end{array}$ \\
\hline $68 / 11 / 87$ & $\left\{\begin{array}{r}12.58 \\
4.28\}\end{array}\right.$ \\
\hline $69 / 68 / 87$ & \\
\hline $16 / 85 / 87$ & $\begin{array}{l}0.41 \\
3.96\}\end{array}$ \\
\hline $11 / 03 / 87$ & $\begin{array}{l}8.21 \\
2.18\}\end{array}$ \\
\hline $12 / 81 / 87$ & $\begin{array}{r}\quad 7.00 \\
\{\quad 3.59\}\end{array}$ \\
\hline $\begin{array}{l}07 / 08 / 87 \\
88 / 11 / 87\end{array}$ & \\
\hline $69 / 68 / 87$ & \\
\hline $\begin{array}{l}10 / 05 / 87 \\
11 / 63 / 87 \\
12 / 61 / 87\end{array}$ & \\
\hline $\begin{array}{l}67 / 68 / 87 \\
88 / 11 / 87 \\
89 / 68 / 87\end{array}$ & \\
\hline
\end{tabular}

- - VALUE EXCEEDS PRDPDSED PRIMARY DRINKING IATER STANDARD

+ - VALUE EXCEEDS SCREENING LEVEL FOR FURTHER INVESTIGATION.

NR - ANALYSIS NO

YALUES IN \& ARE COUKTING ERRORS FOR RADIONUCLIDES 
TABLE C.7. (contd)

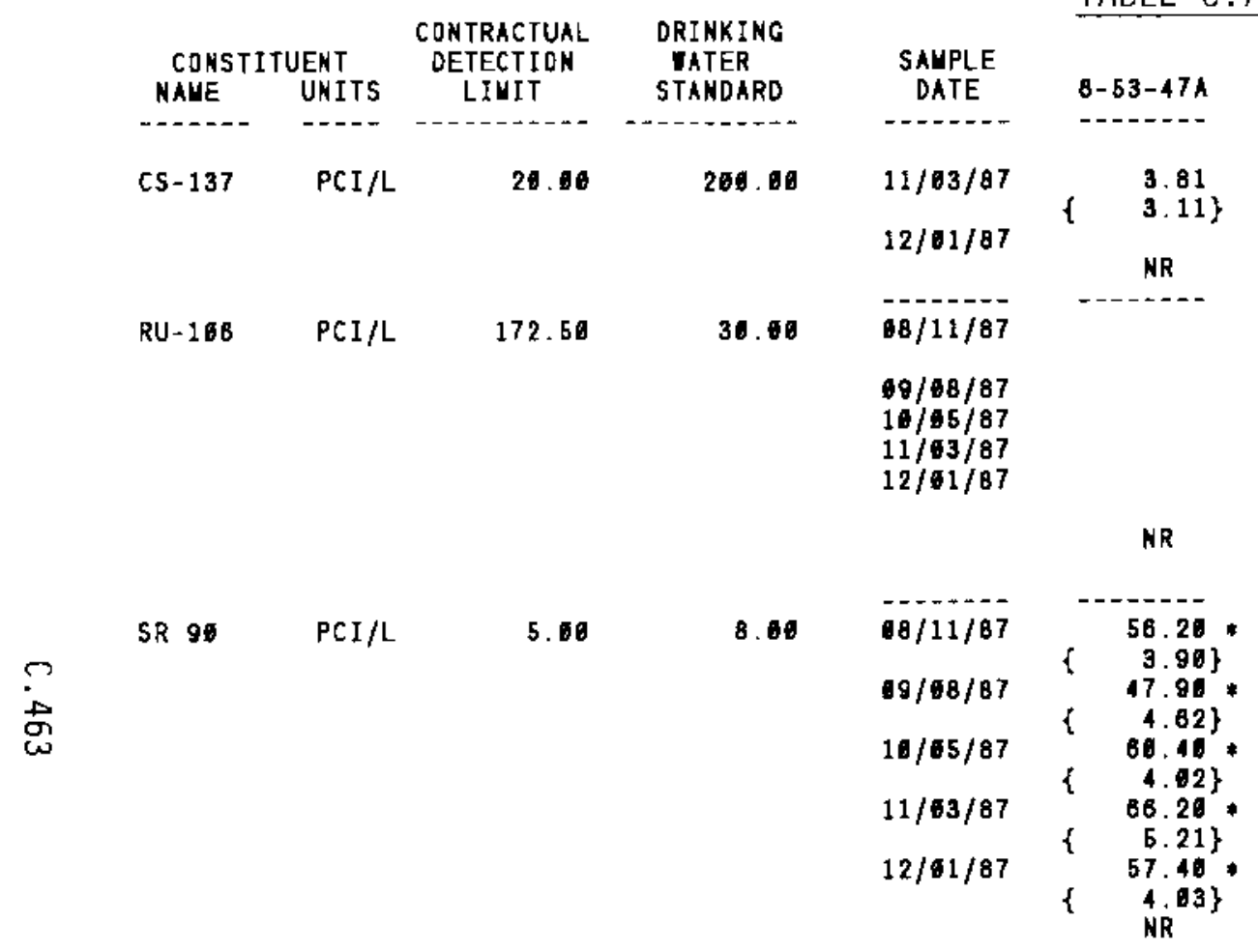

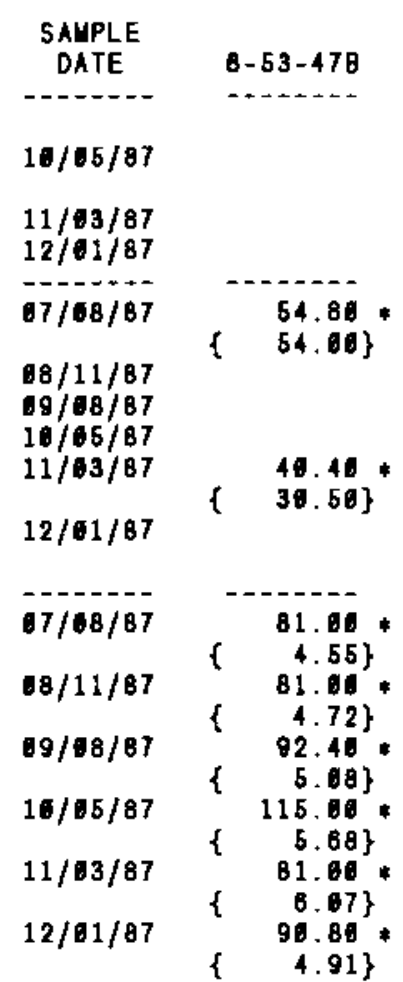

SAMPLE

DATE

$10 / 65 / 87$

$11 / 03 / 87$
$12 / 01 / 87$

67/08/87

$58 / 11 / 87$

$99 / 08 / 87$

$10 / 05 / 87$

$11 / 63 / 87$

$12 / 01 / 87$

$07 / 08 / 87$

$98 / 11 / 87$

09/08/87

$10 / 85 / 87$

$11 / 63 / 87$

$12 / 61 / 87$

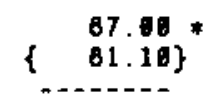

81.10\}

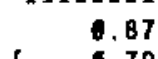

$\{\quad .87$
$\quad .79$
97

$\{\quad .05\}$

$\left\{\begin{array}{l}1.82 \\ 1.17\}\end{array}\right.$

$43\}$

$4.91\}^{*}$

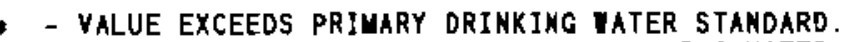

- VALJE EXCEEDS PROPOSED PRIMARY DRINKING WATER STANDARD.

* - VALUE EXCEEDS SCREENINg LEVEL FoR FURTHER INVESTIGATION.

- DETECTION LIUIT IAS NOT AVAILABLE FOR COUPARISON

NR - ANALYSIS NDT REQUESTED OR NOT YET REPORTED

VALUES IN \{\} ARE CDUNTING ERRORS FOR RADIONUCLIDES

WATER STANDARD(S) IN PARENTHESES ARE PROPOSED OKLY 


\section{TABLE C.7. (contd)}

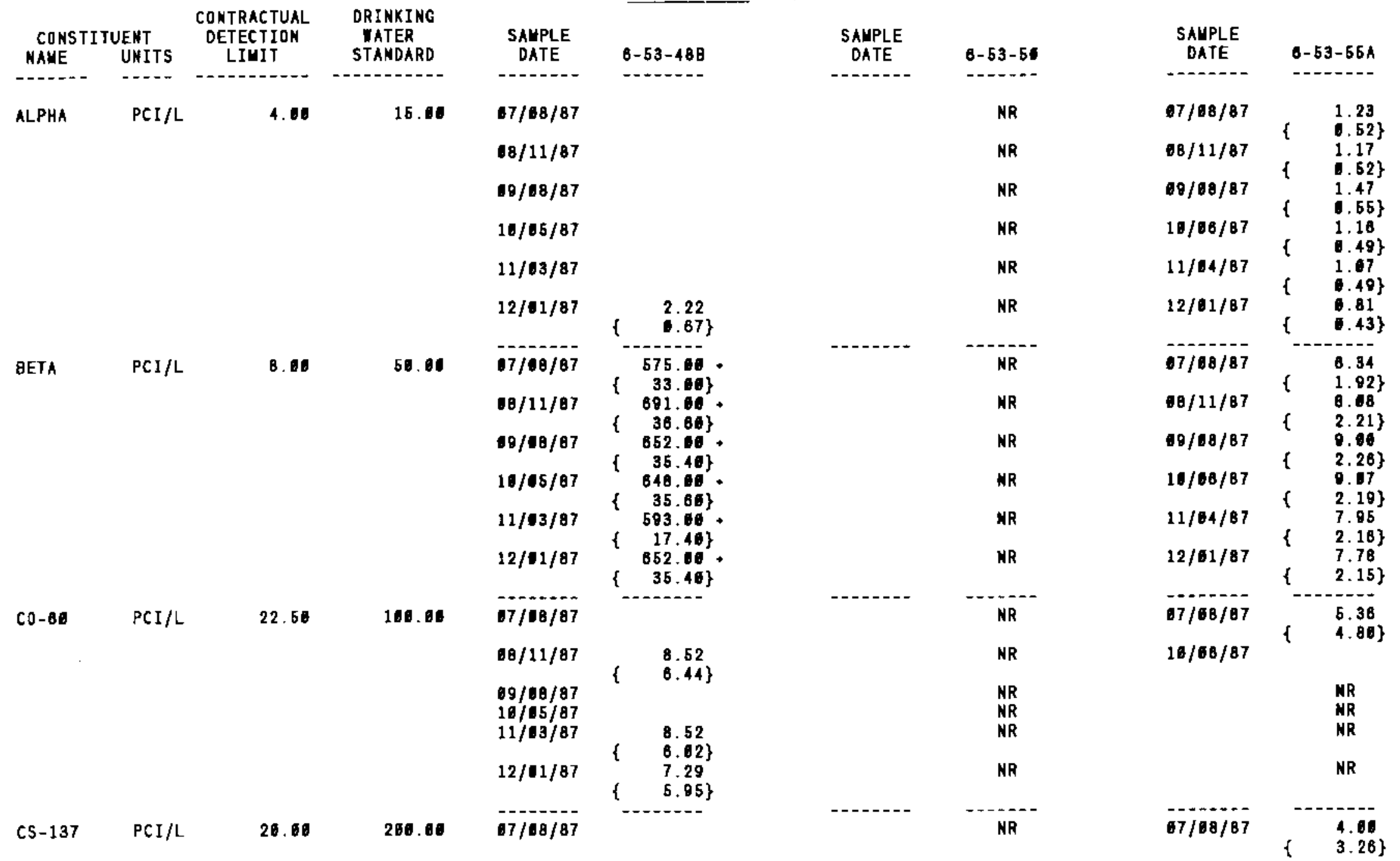

* - value exceeds primary orinking mater stakdaro.

1 - VALUE EXCEEOS PROPOSED PRIUARY DRINKING TATER STAMOARO.

* - VALUE EXCEEDS SCREENING LEVEL FOR FURTHER INYESTIGATION.

- DETECTION LIMIT UAS NOT AVAILABLE FOR CDUPARISON

NR - ANALYSIS NOT REQUESTED OR NDT YET REPORTED

YALUES IN \& ARE COUNTING ERRORS FOR RADIONUCLIDES

IATER STANDARD(S) IN PARENTHESES ARE PROPOSED ONLY 


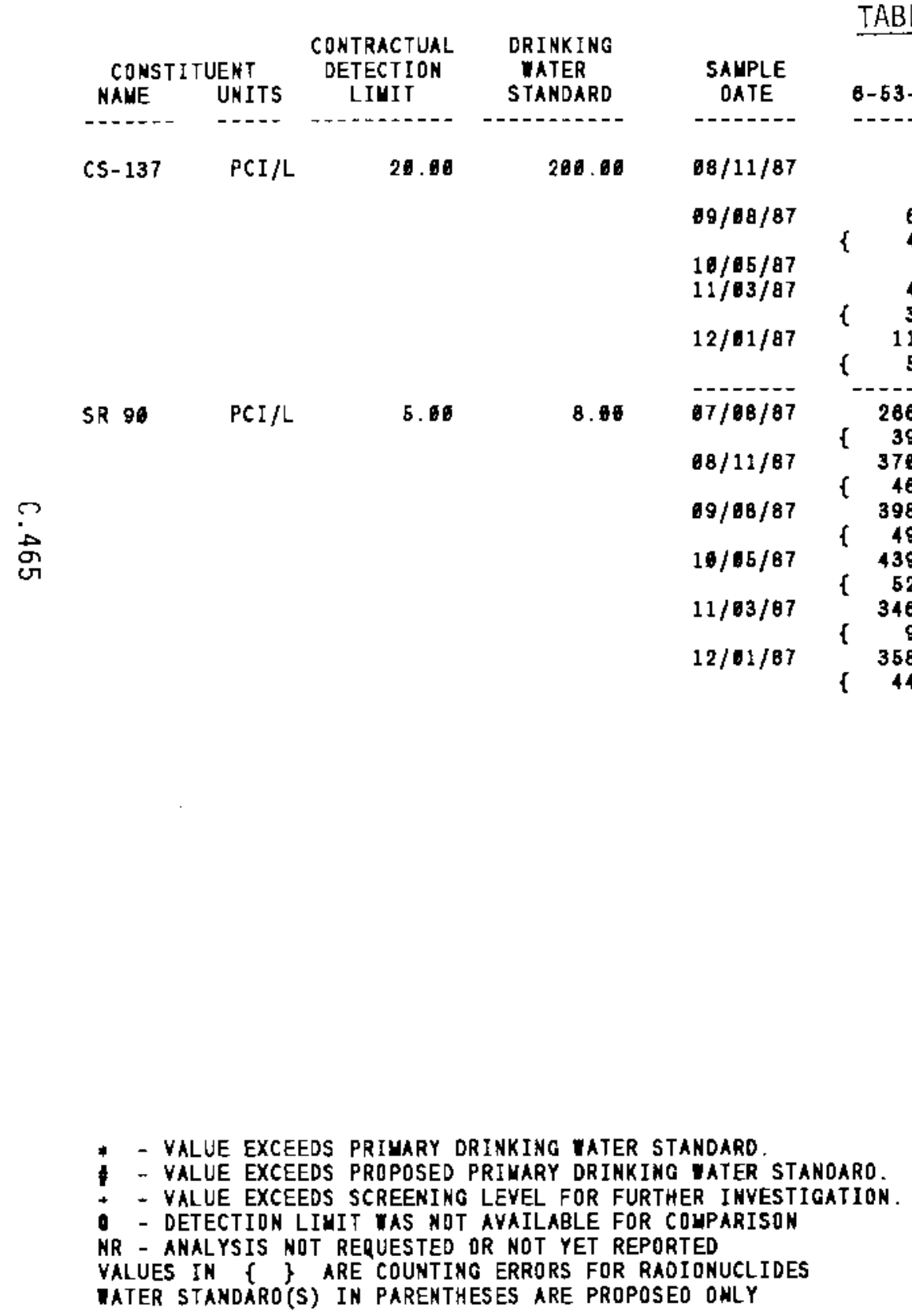

TABLE C.7. (contd)

$10 / 68 / 87$

$$
\begin{aligned}
& \begin{array}{l}
7.70 \\
5.13\}
\end{array} \\
& \text { NR }
\end{aligned}
$$

NR

$97 / 68 / 87$

10/08/87

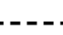

66.00 $39.06\}$

NR

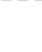

NR

46. 49.

NR

NR

NR

NR - ANALYSIS NDT RERUESTED DR NOT YET REPORTED

VALUES IN \{\} ARE COUNTING ERRORS FOR RAOIONUCLIDES

WATER STANDARO(S) IN PARENTHESES ARE PROPOSEO OHLY 
IABLE C.7. (contd)

\begin{tabular}{|c|c|c|c|c|c|c|c|c|c|}
\hline $\begin{array}{c}\text { CONSTI } \\
\text { NALE }\end{array}$ & $\begin{array}{l}\text { UENT } \\
\text { UNITS }\end{array}$ & $\begin{array}{c}\text { CONTRACTUAL } \\
\text { DETECTION } \\
\text { LIUIT }\end{array}$ & $\begin{array}{l}\text { DRINKING } \\
\text { WATER } \\
\text { STANDARD }\end{array}$ & $\begin{array}{c}\text { SALPLE } \\
\text { DATE }\end{array}$ & $6-53-103$ & $\begin{array}{l}\text { SAHPLE } \\
\text { DATE }\end{array}$ & $B-54-34$ & $\begin{array}{c}\text { SAMPLE } \\
\text { DATE }\end{array}$ & $6-54-37 A$ \\
\hline------ & ---- & 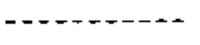 & - & $\cdots+\cdots$ & ----- & ------ & ------ & ----- & $\ldots \ldots$ \\
\hline HNITRAT & PPG & 2500.00 & 45000.00 & $69 / 23 / 87$ & NR & $\begin{array}{l}88 / 28 / 87 \\
12 / 16 / 87\end{array}$ & $\begin{array}{l}10700.00 \\
10100.00\end{array}$ & $\begin{array}{l}18 / 26 / 87 \\
12 / 10 / 87\end{array}$ & \\
\hline TRIJIUU & $\mathrm{PCI} / \mathrm{L}$ & 500.00 & 20080.00 & 69/23/87 & $\begin{array}{c}346.00 \\
206.00\} \\
N R\end{array}$ & $\begin{array}{l}68 / 2 B / 87 \\
12 / 10 / 87\end{array}$ & ----- & $\begin{array}{l}68 / 28 / 87 \\
12 / 10 / 87\end{array}$ & \\
\hline
\end{tabular}

* - value exceeds priuary drinking inter standard.

- VALUE EXCEEDS PRDPOSED PRIUARY DRINKING TATER STANDARD.

- VALUE EXCEEDS SCREENING LEVEL FOR FURTHER INVESTIGATION

- DETECTION LIUIT MAS NOT AVAILABLE FOR COUPARISON

NR - ANALYSIS NOT REQUESTED OR NOT YET REPORTED

VALUES IN \& \& ARE COUNTING ERRORS FOR RADIDNUCLIDES

WATER STANDARD(S) IN PARENTHESES ARE PROPOSED DNLY 
TABLE C.7. (contd)

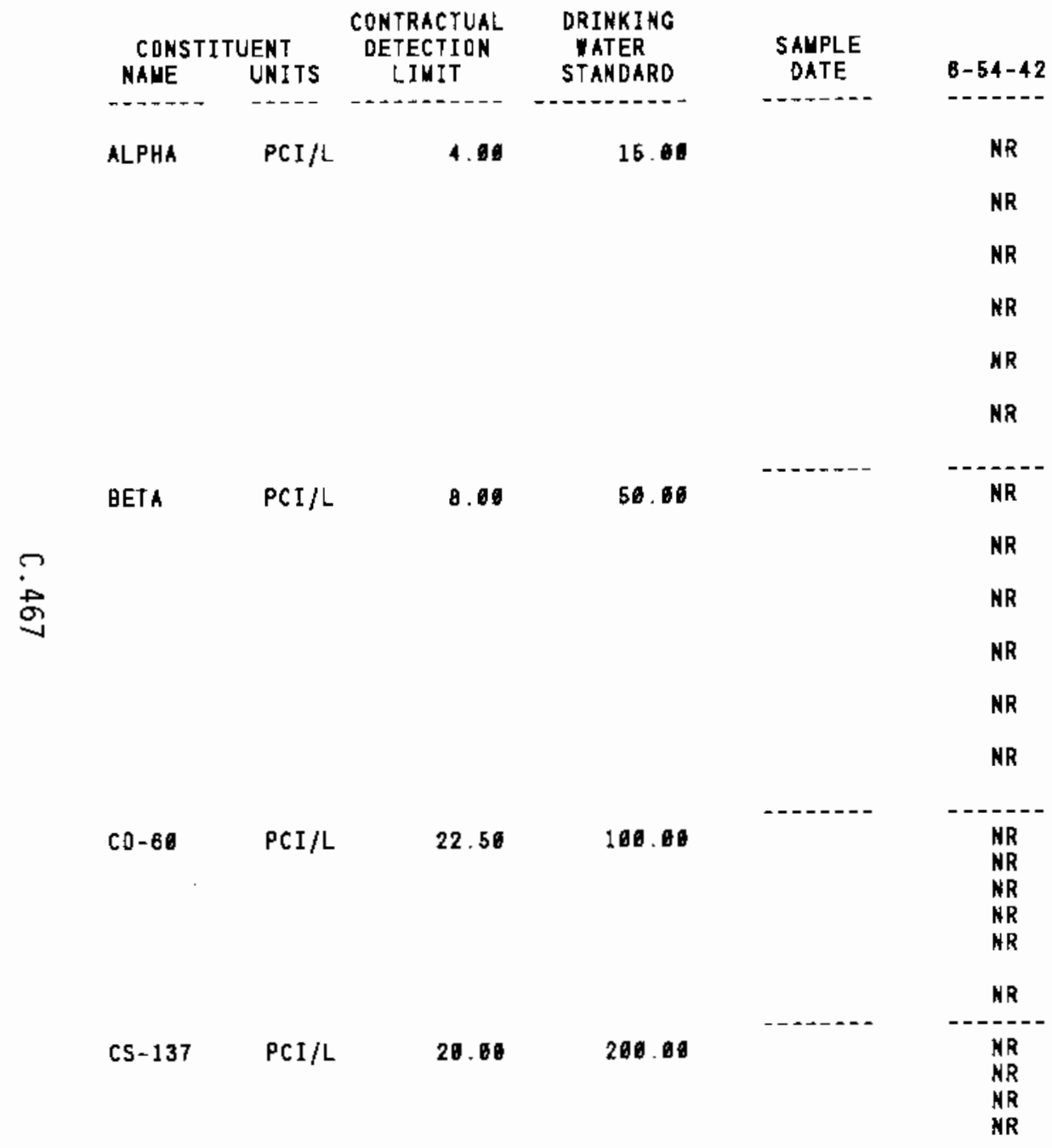

$\left.\begin{array}{cc}\text { SAUPLE } \\ \text { DATE }\end{array}\right)$ B-54-45A

SAUPLE

DATE

$6-54-48$

$(-\cdots+--$

\begin{tabular}{|c|c|}
\hline & \\
\hline $1 / 08 / 87$ & $\begin{array}{l}1.13 \\
6.5\end{array}$ \\
\hline $18 / 11 / 87$ & 1.38 \\
\hline $39 / 69 / 87$ & $\begin{array}{l}0.06\} \\
1.98\end{array}$ \\
\hline $18 / 66 / 87$ & 6.61\} \\
\hline $10 / 60 / 65$ & $0.58\}$ \\
\hline $11 / 83 / 87$ & $\begin{array}{l}0.81 \\
. .47\}\end{array}$ \\
\hline $12 / 01 / 87$ & $\begin{array}{l}2.24 \\
\{\quad 0.85\}\end{array}$ \\
\hline $07 / 08 / 87$ & $\begin{array}{r}108.00 \\
\{\quad 7.13\}\end{array}$ \\
\hline $88 / 11 / 87$ & 113.00 \\
\hline 08/89/87 & 106.08 \\
\hline $18 / 08 / 87$ & $\begin{array}{r}7.94\} \\
111.06\end{array}$ \\
\hline $11 / 03 / 87$ & 105.00 \\
\hline $12 / 01 / 87$ & $\left\{\begin{array}{r}111,00 \\
7,22\}\end{array}\right.$ \\
\hline $\begin{array}{l}67 / 08 / 87 \\
68 / 11 / 87 \\
69 / 69 / 87 \\
10 / 88 / 87 \\
11 / 03 / 87\end{array}$ & \\
\hline $12 / 01 / 87$ & \\
\hline $\begin{array}{l}67 / 68 / 87 \\
68 / 11 / 87 \\
69 / 68 / 87 \\
10 / 66 / 87\end{array}$ & $\begin{array}{l}\begin{array}{l}4.98 \\
3.77\}\end{array}\end{array}$ \\
\hline
\end{tabular}

- - Value exceeds primary drinking tater standard.

- VALUE EXCEEDS PROPOSED PRIMARY DRINKING IATER STAMDARD.

- VALUE EXCEEDS SCREENING LEYEL FOR FURTHER INYESTIGation.

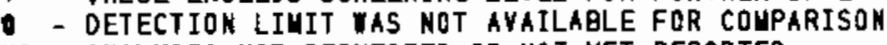

NR - ANALYSIS NOT REQUESTED OR NOT YET REPORTED

VALUES IN \{ \} ARE COUNTING ERRORS FOR RADIONUCLIDES

MATER STANDARD(S) IN PARENTHESES ARE PROPOSED ONLY 
TABLE C.7. (contd)

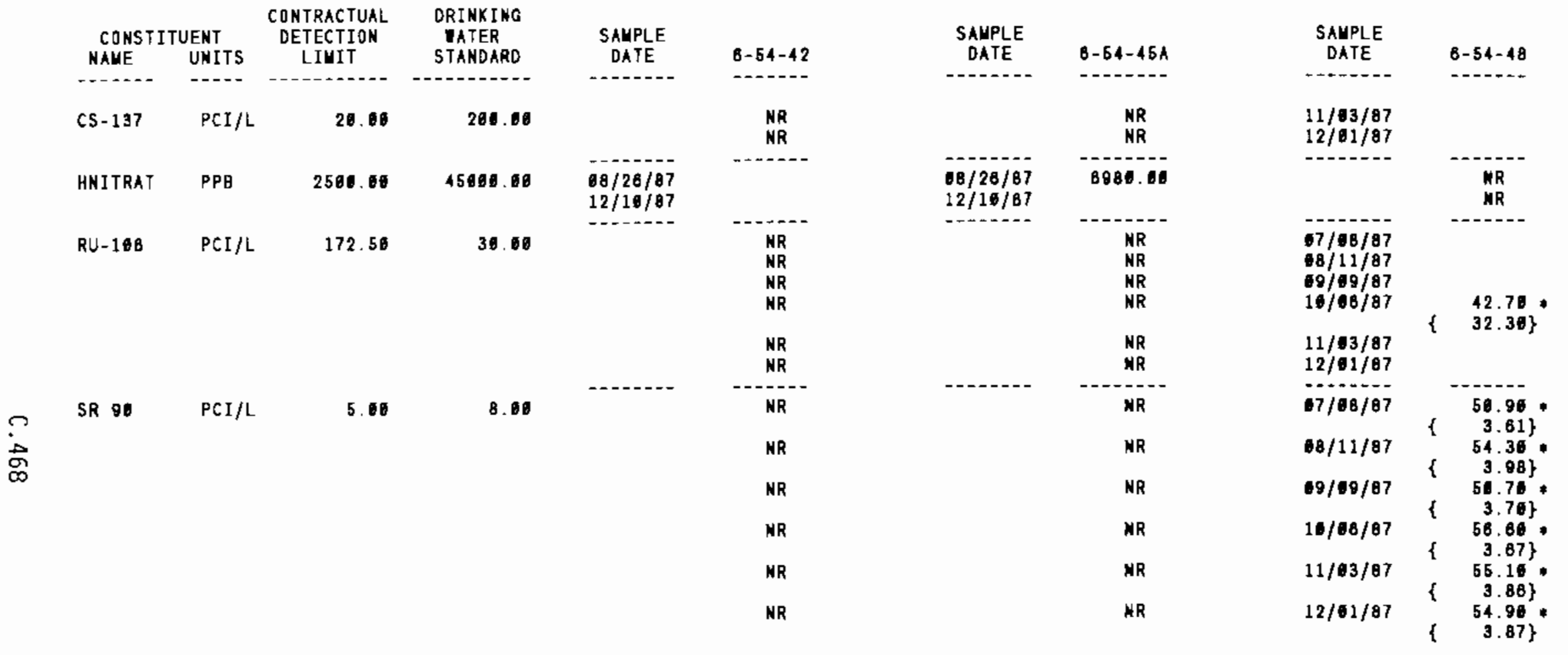

* - VALUE eXCEeds priuary dRinking mater standaro.

* - VALUE EXCEEDS PROPOSED PRIMARY DRINXING IATER STANDARD.

- - VALUE EXCEEDS SCREENING LEVEL FOR FURTHER INVESTIGATION

- DETECTION LIMIT TAS NOT AVAILABLE FOR CONPARISON

NR - ANALYSIS NDT REQUESTED OR NOT YET REPORTED

VALUES IN \{ \} ARE COUNTING ERRORS FOR RADIONUCLIDES

TATER STANDARD(S) IN PARENTHESES ARE PROPOSED ONLY 


\section{TABLE C.7. (contd)}

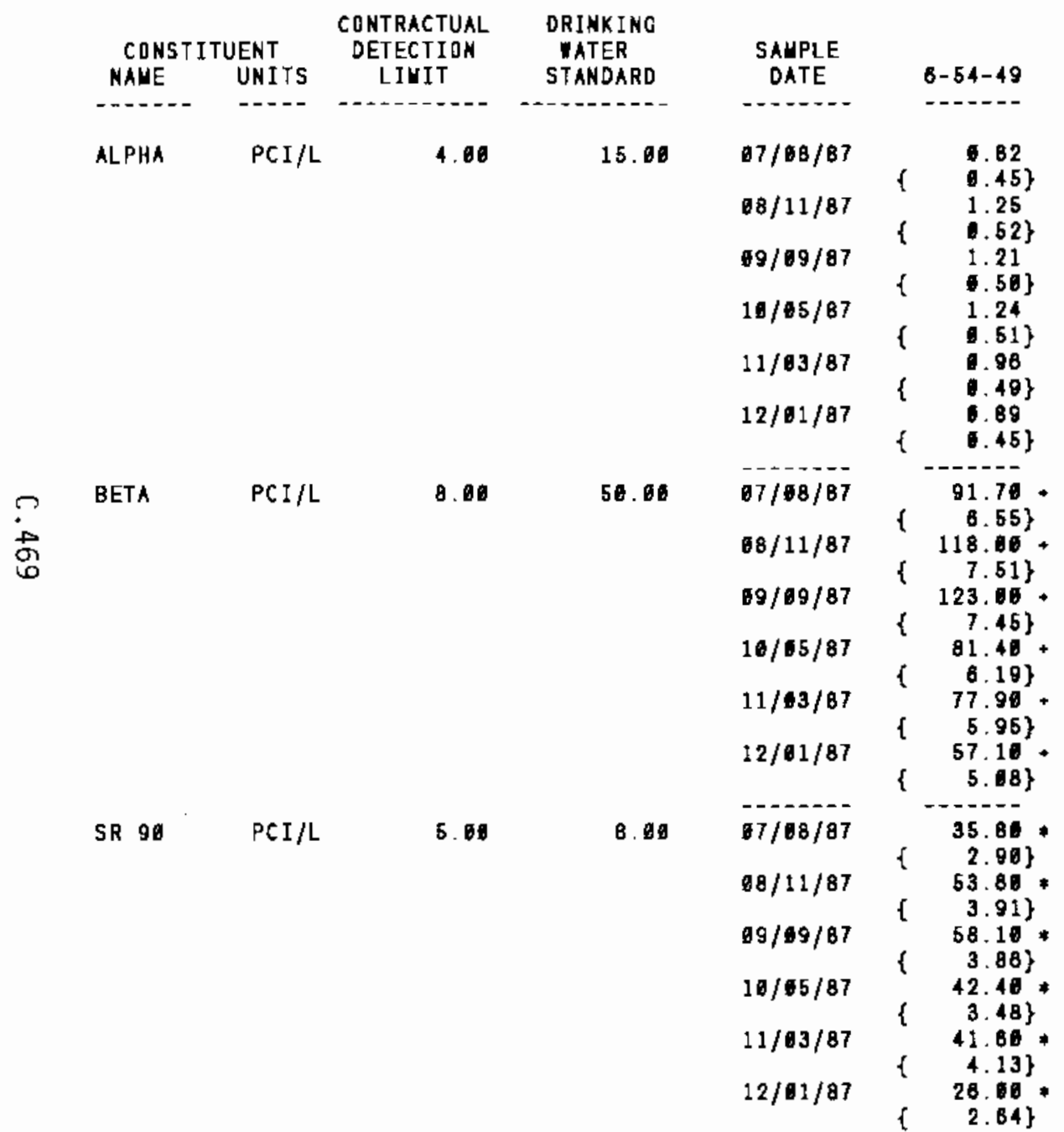

\begin{tabular}{|c|c|c|c|}
\hline \multirow{8}{*}{$\begin{array}{l}\text { SAMPLE } \\
\text { DATE }\end{array}$} & $6-54-57$ & $\begin{array}{c}\text { SAUPLE } \\
\text { DATE }\end{array}$ & $6-55-46$ \\
\hline & $--\cdots-1$ & n........ & -..--- \\
\hline & NR & & NR \\
\hline & $M R$ & & NR \\
\hline & NR & & NR \\
\hline & NR & & NR \\
\hline & NR & & MR \\
\hline & NR & & NR \\
\hline \multirow[t]{6}{*}{------} & NR & -----1 & NR \\
\hline & NR & & NR \\
\hline & NR & & NR \\
\hline & $\mathbf{R R}$ & & NR \\
\hline & NR & & NR \\
\hline & NR & & YR \\
\hline \multirow[t]{6}{*}{ 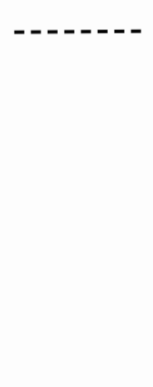 } & NR & 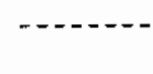 & NR \\
\hline & NR & & MR \\
\hline & NR & & HR \\
\hline & KR & & NR \\
\hline & NR & & NR \\
\hline & NR & & NR \\
\hline
\end{tabular}

* - YALUE EXCEEDS PRIMARY ORINKING MATER STAKDARD.

- VALUE EXCEEDS PROPOSED PRIYARY DRINKING WATER STANDARD.

- VALUE EXCEEDS SCREENIMG LEVEL FOR FURTHER INYESTIGATION.

- DETECTION LIUIT WAS ROT AVAILABLE FOR COUPARISON

HR - ANALYSIS NOT RERUESTEO OR HOT YET REPORTED

YALUES IH \{ $\{$ ARE CDUNTING ERRORS FOR RADIONUCLIDES

IATER STANDARD(S) IN PARENTHESES ARE PROPOSED ONLY 
TABLE C.7. (contd)

\begin{tabular}{|c|c|c|c|c|c|}
\hline $\begin{array}{l}\text { CONSTI } \\
\text { WAUE }\end{array}$ & $\begin{array}{l}\text { UENT } \\
\text { UNITS }\end{array}$ & $\begin{array}{c}\text { CONTRACTUAL } \\
\text { DETECTION } \\
\text { LIHIY }\end{array}$ & $\begin{array}{l}\text { DRINKING } \\
\text { WATER } \\
\text { STANDARD }\end{array}$ & $\begin{array}{c}\text { SAYPLE } \\
\text { DATE }\end{array}$ & $8-55-44$ \\
\hline -....... & $-\cdots-$ & 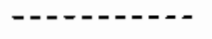 & $-\infty-1-0-1$ & ----- & $\cdots+\ldots$ \\
\hline \multirow[t]{3}{*}{ BETA } & $\mathrm{PCI} / \mathrm{L}$ & 8.00 & 50.00 & & NR \\
\hline & & & & & NR \\
\hline & & & & $-\ldots$ & - \\
\hline HNITRAT & PPB & 2560.00 & 45600.00 & $\begin{array}{l}06 / 26 / 87 \\
12 / 10 / 87\end{array}$ & \\
\hline \multirow[t]{2}{*}{ SR 90} & $\mathrm{PCI} / \mathrm{L}$ & 5.00 & 0.00 & 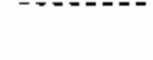 & NR \\
\hline & & & & & $N R$ \\
\hline TRITIUN & $P C I / L$ & 560.00 & 20000.00 & $\begin{array}{l}66 / 26 / 87 \\
12 / 18 / 87\end{array}$ & 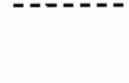 \\
\hline
\end{tabular}

\begin{tabular}{|c|c|}
\hline $\begin{array}{l}\text { SAUPLE } \\
\text { DATE }\end{array}$ & $6-55-56 A$ \\
\hline \multirow[t]{3}{*}{ 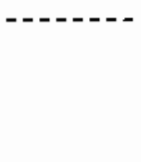 } & 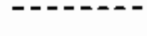 \\
\hline & NR \\
\hline & NR \\
\hline - - - - - - & ------ \\
\hline $\begin{array}{l}\theta 6 / 26 / 87 \\
11 / 25 / 87\end{array}$ & $\begin{array}{l}3680.00 \\
2600\end{array}$ \\
\hline \multirow[t]{2}{*}{------} & NR \\
\hline & NR \\
\hline $\begin{array}{l}16 / 26 / 87 \\
11 / 25 / 87\end{array}$ & \\
\hline
\end{tabular}

\begin{tabular}{|c|c|}
\hline $\begin{array}{l}\text { SAUPLE } \\
\text { DATE }\end{array}$ & $8-55-50 D$ \\
\hline - & 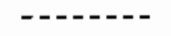 \\
\hline $6 B / 27 / B 7$ & 44.66 \\
\hline $11 / 25 / 87$ & $\begin{array}{r}45.68 \\
4.64 \text { ? }\end{array}$ \\
\hline $\begin{array}{l}6 \mathrm{~B} / 27 / 87 \\
11 / 25 / 87\end{array}$ & \\
\hline $08 / 27 / 87$ & $\left\{\begin{array}{l}1.81 \\
\{.74\}\end{array}\right.$ \\
\hline $11 / 25 / 87$ & \\
\hline $\begin{array}{l}8 / 27 / 87 \\
11 / 26 / 87\end{array}$ & $\begin{array}{l}365.00 \\
\{\quad 173.06\}\end{array}$ \\
\hline
\end{tabular}

* - value exceeds priuary drinking mater standard.

- - VALUE EXCEEDS PROPOSED PRIMARY DRINXING TATER STAHDaRd.

- - VALUE EXCEEDS SCREENING LEVEL FOR FURTHER INVESTIGATION.

- DETECTION LIUIT WAS NOT AVAILABLE FOR COMPARISON

NR - ANALYSIS NDT REQUESTED OR NOT YET REPORTED

VALUES IN \{ \} ARE COUNTING ERRQRS FOR RADIONUCLIDES

WATER STANDARD'S) IN PARENTHESES ARE PROPOSED ONLY 
TABLE C.7. (contd)

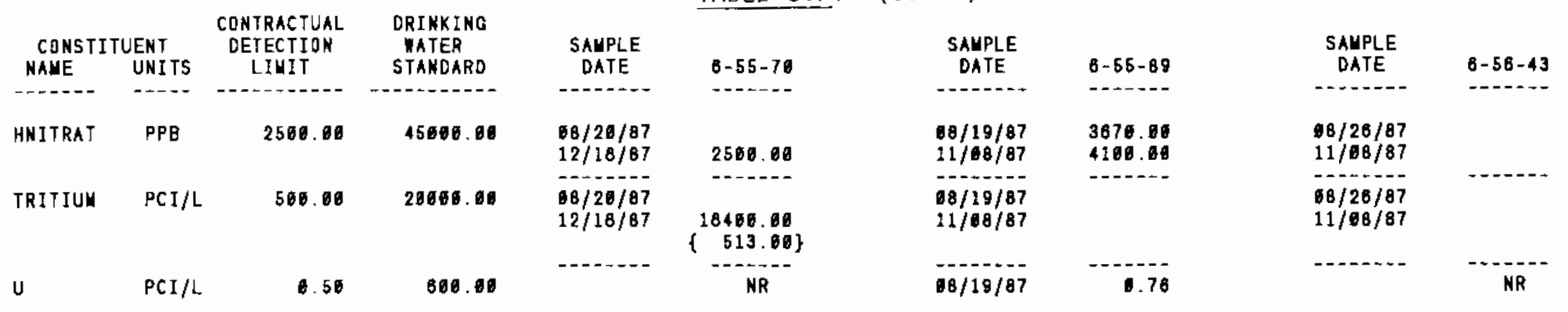

- - VALUE EXCEEDS PROPOSED PRIUARY DRINKING WATER STANOARD.

- VALUE EXCEEDS SCREENING LEYEL FOR FURTHER INYESTIGATION.

- DETECTION LIMIT WAS NOT AVAILABLE FOR COUPARISON

NR - ANALYSIS NOT REQUESTEO OR NOT YET REPORTED

VALUES IN \& \& ARE COUNTING ERRORS FOR RADIONUCLIDES

TATER STANDARD(S) IN PARENTHESES ARE PROPOSED ONLY 


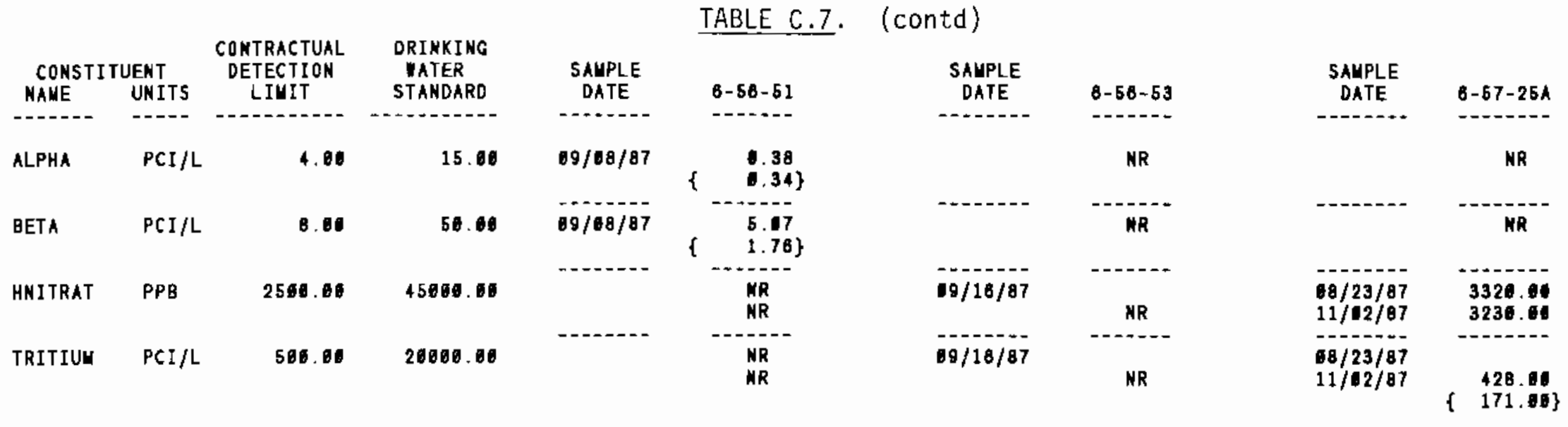

* - value eXceEds primary dRINKING mater standard

- - VALUE EXCEEDS PROPOSED PRIMARY DRINKING maTER STANDARD.

- - YALUE EXCEEDS SCREENIMG LEVEL FOR FURTHER INYESTIGATION

- DETECTION LINIT MAS NDT AVAILABLE fOR COUPARISON

NR - ARALYSIS NDT REQUESTED OR NOT YET REPORTED

YALUES IH \& I ARE COUNTING ERRORS FOR RADIONUCLIDES

TATER STANDARD(S) IN PARENTHESES ARE PROPOSED ONLY 
TABLE C.7. (contd)

\begin{tabular}{|c|c|c|}
\hline $\begin{array}{l}\text { CONSTI I TUENT } \\
\text { NALE UNITS }\end{array}$ & $\begin{array}{c}\text { CONTRACTUAL } \\
\text { OETECTION } \\
\text { LINIT }\end{array}$ & $\begin{array}{l}\text { DRINKING } \\
\text { WATER } \\
\text { STANDARD }\end{array}$ \\
\hline - & - & - - - - - - - - \\
\hline
\end{tabular}

\begin{tabular}{|c|c|}
\hline $\begin{array}{c}\text { SAMPLE } \\
\text { DATE }\end{array}$ & $B-57-29 A$ \\
\hline 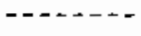 & 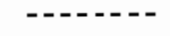 \\
\hline $\begin{array}{l}08 / 23 / 87 \\
11 / 02 / 87\end{array}$ & $\begin{array}{l}2960.00 \\
2840.00\end{array}$ \\
\hline $68 / 23 / 87$ & 746.00 \\
\hline $11 / 02 / 87$ & $\begin{array}{l}\left\{\begin{array}{l}223.00 \\
679.06 \\
\{\quad 178.00\end{array}\right\}\end{array}$ \\
\hline
\end{tabular}

SALPLE

DATE

$8-57-83 A$

D...... -

HAITRAT PPB 2509.00 45606.00

$12 / 22 / 87$

5930.06

TRITIUN PCI/L

589.90

20000.00

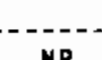

SAUPLE

DATE

$68 / 23 / 87$

$11 / 08 / 87$

$68 / 23 / 87$

$11 / 08 / 87$

0 - 58-24

3296.06 4460.00

* - value exceeos priuary drinking tater standaro.

- - VALUE EXCEEDS PROPOSED PRIMARY DRINXING WATER STANDARD.

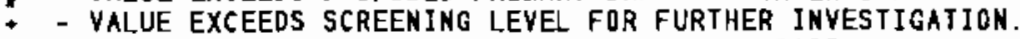

- DEIECTIDN LIUIT NAS NOT AVAILABLE FOR COMPARISON

NR - ANALYSIS NOT REQUESTED OR NOT YET REPORTED

VALUES IN I I ARE CDUNTING ERRORS FOR RADIONUCLIDES

WATER STANDARDO(S) IN PARENTHESES ARE PROPQSED ONLY 
TABLE C.7. (contd)

\begin{tabular}{|c|c|c|c|c|c|}
\hline \multicolumn{2}{|c|}{ CONSTITUENT } & $\begin{array}{l}\text { CONTRACTUAL } \\
\text { DETECTION } \\
\text { LIUIT }\end{array}$ & $\begin{array}{l}\text { DRINKING } \\
\text { TATER } \\
\text { STANDARD }\end{array}$ & $\begin{array}{l}\text { SAUPLE } \\
\text { DATE }\end{array}$ & $6-58-32$ \\
\hline------ & ---- & ---n-c-ce & 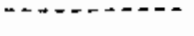 & $\cdots+-2-\infty$ & $+-\infty+--$ \\
\hline ALPHA & $\mathrm{PCI} / \mathrm{L}$ & 4.00 & 15.00 & & NR \\
\hline BETA & $\mathrm{PCI} / \mathrm{L}$ & 8.00 & 50.05 & $\cdots+-\cdots$ & NR \\
\hline HNITRAT & PPQ & 2560.00 & 45090.00 & $\begin{array}{l}-10-1 / 87 \\
11 / 23 / 87 \\
11 / 687\end{array}$ & 4000 \\
\hline TRITIUM & $\mathrm{PCI} / \mathrm{L}$ & 590.00 & 2006000 & $18 / 23 / 87$ & $\begin{array}{l}594.00 \\
217.00\} \\
810.00 \\
222.00\}\end{array}$ \\
\hline
\end{tabular}

\begin{tabular}{|c|c|}
\hline $\begin{array}{c}\text { SAUPLE } \\
\text { DATE }\end{array}$ & $8-59-58$ \\
\hline 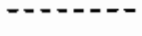 & $\cdots$ \\
\hline $67 / 69 / 87$ & $\begin{array}{l}1.24 \\
\{\quad 9.51\}\end{array}$ \\
\hline $07 / 69 / 87$ & $\left\{\begin{array}{l}5.3 \\
1.77\end{array}\right.$ \\
\hline $\begin{array}{l}07 / 09 / 87 \\
12 / 21 / 87\end{array}$ & ( \\
\hline $\begin{array}{l}12 / 08 / 87 \\
12 / 21 / 87\end{array}$ & $\left\{\begin{array}{l}786.00 \\
182.00\} \\
852.80 \\
269.06\}\end{array}\right.$ \\
\hline
\end{tabular}

SAHPLE

DATE

- D.+-

(--1--

NR

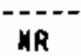

MR

$68 / 18 / 97$

$12 / 13 / 67$

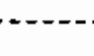

MR

NR

* - value exceeds primary dRinking vater standard.

- VALUE EXCEEOS PRDPQSED PRIMARY DRINXING MATER STAMDARD.

- VALUE EXCEEDS SCREENING LEVEL FDR FURTHER IMVESTIGATIDX.

- DETECTION LIUIT IAS NDT AVAILABLE FOR COUPARISOH

NR - ANALYSIS NOT REQUESTED OR NOT YET REPORTED

VALUES IN \& ARE COUNTING ERRQRS FOR RADIONUCLIDES

WATER STANDARD(S) IN PARENTHESES ARE PROPOSED OHLY 
TABLE C.7. (contd)

\begin{tabular}{|c|c|c|c|c|c|c|c|c|c|}
\hline $\begin{array}{l}\text { CONSTI } \\
\text { WAHE }\end{array}$ & $\begin{array}{l}\text { UENT } \\
\text { UNITS }\end{array}$ & $\begin{array}{l}\text { CONTRACTUAL } \\
\text { DETECTION } \\
\text { LIMIT }\end{array}$ & $\begin{array}{l}\text { DRINKING } \\
\text { IAIER } \\
\text { STANDARD }\end{array}$ & $\begin{array}{c}\text { SALPLE } \\
\text { DATE }\end{array}$ & $8-66-32$ & $\begin{array}{c}\text { SAUPLE } \\
\text { DATE }\end{array}$ & $6-68-57$ & $\begin{array}{c}\text { SAMPLE } \\
\text { DATE }\end{array}$ & $0-86-80$ \\
\hline HNITRAT & PPB & 2500.00 & 45060.00 & $\begin{array}{l}08 / 23 / 87 \\
11 / 68 / 87\end{array}$ & $\begin{array}{l}5968.00 \\
5580.80\end{array}$ & $\begin{array}{l}68 / 23 / 87 \\
11 / 08 / 87\end{array}$ & & $\begin{array}{l}68 / 23 / 87 \\
11 / 08 / 87\end{array}$ & \\
\hline TRITIUU & PCI $/ \mathrm{L}$ & 500.00 & 2000900 & $\begin{array}{l}68 / 23 / 87 \\
11 / 08 / 87\end{array}$ & $\begin{array}{l}975.60 \\
229.06\} \\
621.00 \\
228.06\}\end{array}$ & $\begin{array}{l}68 / 23 / 87 \\
11 / 88 / 87\end{array}$ & 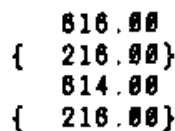 & $\begin{array}{l}68 / 23 / 87 \\
11 / 08 / 87\end{array}$ & 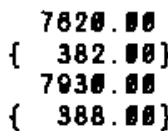 \\
\hline
\end{tabular}

- - Value eXceeds primary dRINKINo tater standard.

- YALUE EXCEEDS PROPOSED PRIMARY DRINKING WATER STANDARD.

- - VALUE EXCEEDS SCREENINg LEYEL FOR FURTHER INYESTIGATIDN.

- DETECTION LIUIT MAS NDT AVALABLE FOR COUPARISDN

NR - ANALYSIS NOT REQUESTED OR NDT YET REPORTED

YALUES IN \{\} ARE CDUNTING ERRORS FOR RADIONUCLIDES

WATER STANDARD(S) IN PARENTHESES ARE PROPOSED DHLY 
TABLE C.7. (contd)

\begin{tabular}{|c|c|c|c|c|c|c|c|c|c|}
\hline $\begin{array}{c}\text { CONSTI } \\
\text { NAWE }\end{array}$ & $\begin{array}{l}\text { UENT } \\
\text { UNITS }\end{array}$ & $\begin{array}{c}\text { CONTRACTUAL } \\
\text { DETECTIDA } \\
\text { LIUIT }\end{array}$ & $\begin{array}{l}\text { DRINKING } \\
\text { ATER } \\
\text { STAKDARO }\end{array}$ & $\begin{array}{c}\text { SAUPLE } \\
\text { DATE }\end{array}$ & $8-61-37$ & $\begin{array}{l}\text { SAYPLE } \\
\text { DATE }\end{array}$ & $8-61-41$ & $\begin{array}{l}\text { SAUPLE } \\
\text { DATE }\end{array}$ & $8-61-62$ \\
\hline $\mathrm{CO}-60$ & PCI $/ L$ & 22.68 & 106.00 & & KR & & NR & $18 / 20 / 87$ & $\begin{array}{l}15.80 \\
\{\quad 11.36\}\end{array}$ \\
\hline HNITRAT & PPB & 2506.06 & 45000.00 & $\begin{array}{l}68 / 23 / 87 \\
11 / 08 / 97\end{array}$ & $\begin{array}{l}4646.19 \\
4528.00\end{array}$ & $\begin{array}{l}08 / 23 / 87 \\
11 / 68 / 87\end{array}$ & $\begin{array}{l}3286.80 \\
3126.80\end{array}$ & $\begin{array}{l}08 / 20 / 87 \\
11 / 08 / 87\end{array}$ & $\begin{array}{l}46589.00 \\
51700.00\end{array}$ \\
\hline TRITIUW & PCI/L & 580.00 & 20000.00 & $\begin{array}{l}08 / 23 / 87 \\
11 / 68 / 87\end{array}$ & 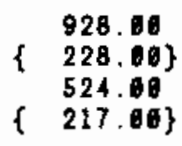 & $\begin{array}{l}88 / 23 / 87 \\
11 / 68 / 87\end{array}$ & $\left\{\begin{array}{l}338.66 \\
\{\quad 268.66\}\end{array}\right.$ & $\begin{array}{l}18 / 26 / 87 \\
11 / 08 / 87\end{array}$ & 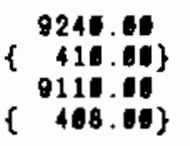 \\
\hline u & $\mathrm{PCI} / \mathrm{L}$ & 0.50 & 800.00 & & NR & & NR & $68 / 26 / 87$ & 1.00 \\
\hline
\end{tabular}

- - VALUE EXCEEDS PROPOSED PRIUARY DRINKING UATER STANDARD

- VALUE EXCEEDS SCREENING LEVEL FDR FURTHER INYESTIGATION.

- DETECTION LIUIT IAS NOT AVAILABLE FOR COUPARISD

NR - ANALYSIS NOT REQUESTED DR NOT YET REPDRTED

VALUES IN \{\} ARE COUNTING ERRORS FDR RADIOHUCLIDES

WATER STANDARO(S) IN PARENTHESES ARE PROPOSED ONLY 
TABLE C.7. (contd)

\begin{tabular}{|c|c|c|c|c|c|c|c|c|c|}
\hline $\begin{array}{l}\text { CONSTI } \\
\text { NAME }\end{array}$ & $\begin{array}{l}\text { UENT } \\
\text { UNITS }\end{array}$ & $\begin{array}{c}\text { CONTRACTUAL } \\
\text { DETECTION } \\
\text { LIUIT }\end{array}$ & $\begin{array}{c}\text { DRINKING } \\
\text { WATER } \\
\text { STANDARD }\end{array}$ & $\begin{array}{c}\text { SAUPLE } \\
\text { DATE }\end{array}$ & $8-61-66$ & $\begin{array}{c}\text { SAHPLE } \\
\text { DATE }\end{array}$ & $6-62-31$ & $\begin{array}{c}\text { SAUPLE } \\
\text { DATE }\end{array}$ & $6-62-43 F$ \\
\hline----- & ---- & 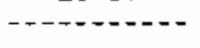 & - - - & $\cdots-\cdots$ & ----- & $-\ldots$ & $-\cdots$ & $+\cdots+\cdots$ & $-\cdots-$ \\
\hline HNITRAT & PPE & 2560.00 & 45000.00 & $\begin{array}{l}B 8 / 28 / 87 \\
11 / 68 / B 7\end{array}$ & $\begin{array}{l}4800.00 \\
5230.60\end{array}$ & $\begin{array}{l}68 / 23 / 87 \\
11 / 68 / 87\end{array}$ & $\begin{array}{l}37906.00 \\
41860.00\end{array}$ & $\begin{array}{l}18 / 23 / 87 \\
11 / 98 / 87\end{array}$ & $\begin{array}{l}3480.66 \\
3730.66\end{array}$ \\
\hline TRITIUU & $\mathrm{PCI} / \mathrm{L}$ & 500.00 & 20000.00 & $\begin{array}{l}08 / 26 / 87 \\
11 / 08 / 87\end{array}$ & -...-- & - n-n & $\begin{array}{c}\text { NR } \\
\text { NR }\end{array}$ & $\begin{array}{l}68 / 23 / 87 \\
11 / 88 / 87\end{array}$ & $\begin{array}{r}623.00 \\
217.00 \\
674.01 \\
221.08\}\end{array}$ \\
\hline
\end{tabular}

* - value exceeds privary drinking tater standaro.

- VALUE EXCEEDS PROPOSED PRIMARY DRIMKING WATER STANDARD.

- - VALUE EXCEEDS SCREENING LEVEL FOR FURTHER INVESTIGATION.

- DETECTIDN LIUIT WAS NOT AVATLAELE FOR COMPARISON

NR - ANALYSIS NOT REQUESTED OR NOT YET REPDRTED

VALUES IN \{ ? ARE COUNTING ERRORS FDR RADIGNUCLIDES

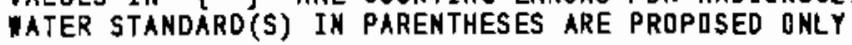


TABLE C.7. (contd)

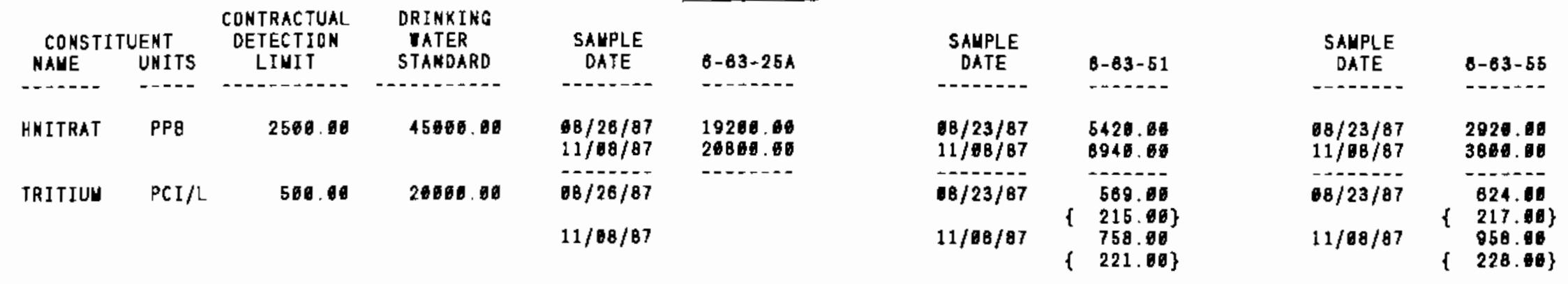

* - Value exceeds primary dRINKINg mater stakdard.

- VALUE EXCEEDS PROPOSED PRIMARY DRINKING iater STANDARD.

- VALUE EXCEEDS SCREENING LEVEL FDR FURTHER INVESTIGATION.

- DETECTION LIMIT NAS NDT AVAILABLE FOR COMPARISON

NR - ANALYSIS NOT REQUESTED OR NOT YET REPORIED

VALUES IN \{ $\}$ ARE CQUNTING ERRDRS FOR RADIDNUCLIDES

IATER STANDARD(S) IN PARENTHESES ARE PROPOSED ONLY 


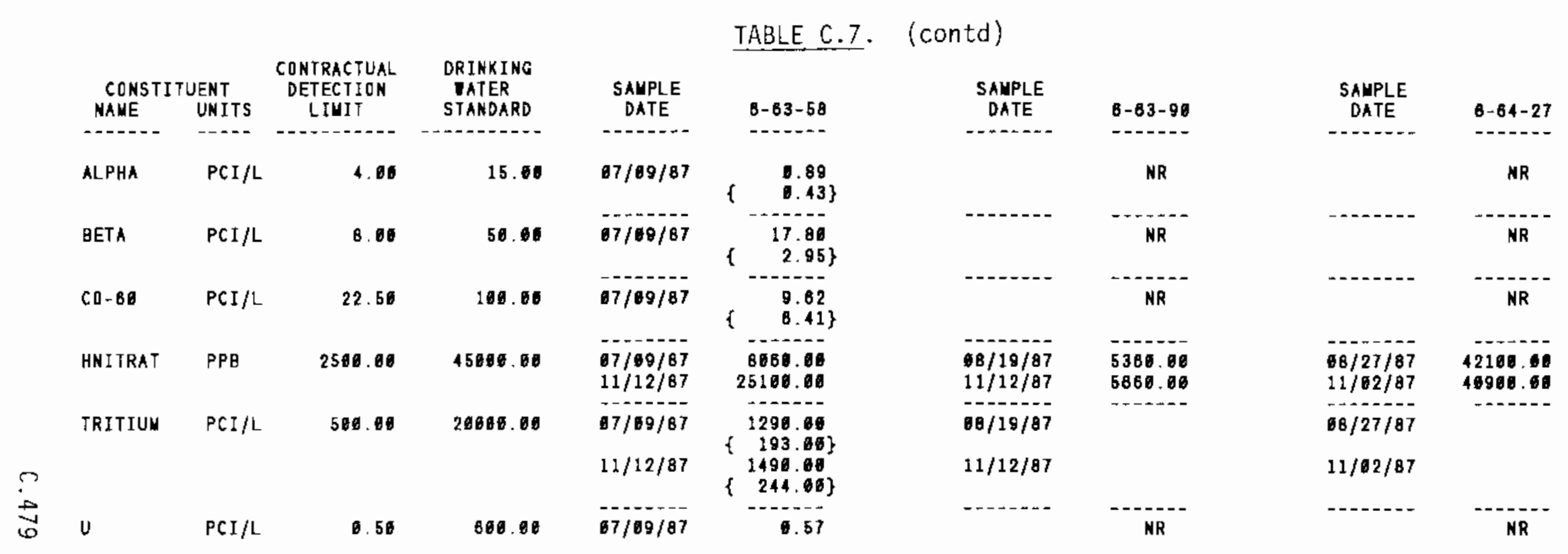

* - Value EXCEEDS priuary drinxing water standaro.

| - VALUE EXCEEDS PROPESED PRIUARY DRINKING WATER STANDARD.

- - VALUE EXCEEDS SCREENIHG LEVEL FOR FURTHER INVESTIGATION.

- DETECTION LIUIT MAS NOT AVAILABLE FOR CDUPARISUN

NR - ANALYSIS NOT RERUESTED OR NOT YET REPORTED

VALUES IN \{ \} ARE CDUNTING ERRORS FOR RADIONUCLIDES

WATER STANDARD(S) IN PARENTHESES ARE PROPOSED ONLY 
TABLE C.7. (contd)

\begin{tabular}{|c|c|c|c|c|c|c|c|c|c|}
\hline $\begin{array}{l}\text { CONSTI } \\
\text { NAUE }\end{array}$ & $\begin{array}{l}\text { UENT } \\
\text { UNITS }\end{array}$ & $\begin{array}{c}\text { CONTRACTUAL } \\
\text { DETECTION } \\
\text { LINIT }\end{array}$ & $\begin{array}{l}\text { DRIKKING } \\
\text { WATER } \\
\text { STANDARD }\end{array}$ & $\begin{array}{l}\text { SAUPLE } \\
\text { DATE }\end{array}$ & $0-64-82$ & $\begin{array}{l}\text { SAUPLE } \\
\text { DATE }\end{array}$ & $6-65-50$ & $\begin{array}{l}\text { SAMPLE } \\
\text { DATE }\end{array}$ & $B-65-59 A$ \\
\hline - no. & $\cdots$ & 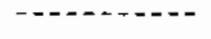 & $--1, \ldots+\infty+\infty$ & ------ & $\cdots+--$ & - - - & - - - n & 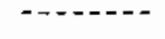 & -......- \\
\hline HNITRAT & $P P B$ & 2500.00 & 45006.80 & $\begin{array}{l}08 / 27 / 87 \\
11 / 68 / 87\end{array}$ & $\begin{array}{l}28808.00 \\
29600.00\end{array}$ & $\begin{array}{l}66 / 23 / 87 \\
11 / 08 / 87\end{array}$ & 2600.00 & $\begin{array}{l}68 / 23 / 87 \\
11 / 68 / 87\end{array}$ & $\begin{array}{l}2750.00 \\
3820.00\end{array}$ \\
\hline TRITIUM & $\mathrm{PCI} / \mathrm{L}$ & 500.00 & 20000.00 & $18 / 27 / 87$ & $\left\{\begin{array}{c}9380.00 \\
418.06\} \\
9250.60 \\
\{\quad 408.06\}\end{array}\right.$ & $\begin{array}{l}66 / 23 / 87 \\
11 / 08 / 87\end{array}$ & $\left\{\begin{array}{l}687.00 \\
228.00\} \\
492.00 \\
210.08\}\end{array}\right.$ & $11 / 08 / 87$ & 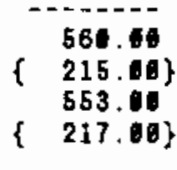 \\
\hline
\end{tabular}

- - yalue exceEds priuary dRINKing mater stahdard.

- YALUE EXCEEDS PROPOSED PRIVARY DRINKING WATER STANDARD.

- - yALUE EXCEEDS SCREENIMG LEYEL FOR FURTHER INYESTIGATIEN.

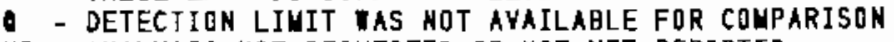

NR - ANALYSIS NOT REQUESTED OR NOT YET REPORTED

YALUES IN \{ \} ARE COUNTING ERRORS FOR RADIONUCLIDES

IATER STANDARD(S) IN PARENTHESES ARE PROPOSED ONLY 
TABLE C.7. (contd)

\begin{tabular}{|c|c|c|c|c|c|c|c|c|c|}
\hline $\begin{array}{l}\text { CONSTI } \\
\text { NAHE }\end{array}$ & $\begin{array}{l}\text { UENT } \\
\text { UNITS }\end{array}$ & $\begin{array}{l}\text { CONTRACTUAL } \\
\text { DETECTION } \\
\text { LIHIT }\end{array}$ & $\begin{array}{l}\text { DRINKING } \\
\text { WATER } \\
\text { STANDARD }\end{array}$ & $\begin{array}{l}\text { SAUPLE } \\
\text { DATE }\end{array}$ & $6-68-23$ & $\begin{array}{c}\text { SAMPLE } \\
\text { DATE }\end{array}$ & $6-66-38$ & $\begin{array}{c}\text { SALPLE } \\
\text { DATE }\end{array}$ & $6+66 \sim 38$ \\
\hline----- & ---- & - - - & - & $--\cdots-1$ & $-\cdots$ & $-\ldots-n$ & 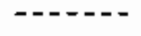 & --no-n & 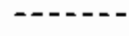 \\
\hline HNITRAT & $P P B$ & 2580.00 & 45000.68 & $\begin{array}{l}08 / 27 / 87 \\
11 / 02 / 87\end{array}$ & $\begin{array}{l}43800.00 \\
43200.00\end{array}$ & $\begin{array}{l}68 / 23 / 87 \\
11 / 08 / 87\end{array}$ & & $\begin{array}{l}18 / 23 / 87 \\
11 / 08 / 87\end{array}$ & \\
\hline
\end{tabular}

* - value exceeds priuary drinking mater standard.

- - VALUE EXCEEDS PROPOSED PRIUARY DRINKING WATER STAMDARD.

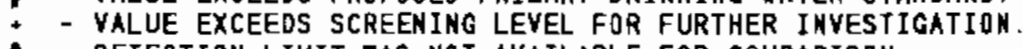

- DETECTION LIUIT TAS NOT AVAILABLE FOR COUPARISUK

NR - ANALYSIS NOT REQUESTED OR NOT YET REPORTED

VALUES IN \{ $\}$ ARE COUNTING ERRORS FOR RADIONUCLIDES

WATER STANDARO(S) IN PARENTHESES ARE PROPOSED DNLY 
TABLE C.7. (contd)

\begin{tabular}{|c|c|c|c|c|c|c|c|c|c|}
\hline $\begin{array}{l}\text { CONSII } \\
\text { NAUE }\end{array}$ & $\begin{array}{l}\text { JENT } \\
\text { UNITS }\end{array}$ & $\begin{array}{l}\text { CONTRACTUAL } \\
\text { DETECTION } \\
\text { LIHIT }\end{array}$ & $\begin{array}{l}\text { DRINKING } \\
\text { IATER } \\
\text { STANDARD }\end{array}$ & $\begin{array}{l}\text { SALPLE } \\
\text { DATE }\end{array}$ & $6-66-58$ & $\begin{array}{l}\text { SAMPLE } \\
\text { DATE }\end{array}$ & $6-86-64$ & $\begin{array}{c}\text { SAUPLE } \\
\text { DATE }\end{array}$ & $6-6 B-193$ \\
\hline$---n$ & ---- & -------- & $--+-1---$ & -+---- & 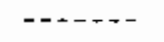 & $---1-\ldots$ & ------ & $----\cdots$ & - - - \\
\hline HNITRAT & PPB & 2560.00 & 45000.60 & $\begin{array}{l}68 / 23 / 87 \\
11 / 68 / 87\end{array}$ & 3520.80 & $\begin{array}{l}08 / 26 / 87 \\
11 / 08 / 87\end{array}$ & $\begin{array}{l}26500.60 \\
15500.00\end{array}$ & $\begin{array}{l}68 / 19 / 87 \\
12 / 17 / 87\end{array}$ & \\
\hline TRITIUU & $\mathrm{PCI} / \mathrm{L}$ & 500.60 & 20006.00 & $11 / 68 / 87$ & $\begin{array}{l}484.00 \\
213.66\} \\
460.00 \\
211.08\end{array}$ & $\begin{array}{l}08 / 26 / 87 \\
11 / 08 / 87\end{array}$ & $\begin{array}{c}8360.00 \\
416.09\} \\
8490.00 \\
368.00\}\end{array}$ & $12 / 17 / 87$ & \\
\hline U & $\mathrm{PCI} / \mathrm{L}$ & 0.56 & 800.00 & $\mu-\log$ & NR & $8 / 26 / 87$ & 1.42 & & NR \\
\hline
\end{tabular}

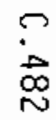

* - value exceeds priuary drinking tater standard.

- - VALUE EXCEEDS PROPOSEd PRIMARY DRINKINO UATER STANDARD

- - VALUE EXCEEDS SCREENING LEVEL FOR FURTHER INYESTIGATION.

- DETECTIQN LIUIT IAS NOT AVAILABLE FDR COUPARISON

NR - ANALYSIS NDT REQUESTED OR NOT YET REPORTED

VALUES IN 1 I ARE COUHTING FRRDRS FOR RADIONUCLIDES

IATER STANDARD'(S) IN PARENTHESES ARE PROPOSED ONLY 
TABLE C.7. (contd)

\begin{tabular}{|c|c|c|c|c|c|c|c|c|c|}
\hline $\begin{array}{l}\text { CONSTI } \\
\text { NAME }\end{array}$ & $\begin{array}{l}\text { UENT } \\
\text { UNITS }\end{array}$ & $\begin{array}{c}\text { CONTRACTUAL } \\
\text { DETECTION } \\
\text { LIUIT }\end{array}$ & $\begin{array}{l}\text { DRINKING } \\
\text { TATER } \\
\text { STANDARD }\end{array}$ & $\begin{array}{l}\text { SAMPLE } \\
\text { DATE }\end{array}$ & $6-87-51$ & $\begin{array}{c}\text { SAMPLE } \\
\text { DATE }\end{array}$ & $6-87-98$ & $\begin{array}{l}\text { SAUPLE } \\
\text { DATE }\end{array}$ & $8-88-165$ \\
\hline HNITRAT & $P P B$ & 2500.06 & 45080.60 & $\begin{array}{l}08 / 25 / 87 \\
12 / 13 / 87\end{array}$ & & $\begin{array}{l}68 / 19 / 87 \\
11 / 12 / 87\end{array}$ & $\begin{array}{l}5396.08 \\
5348.86\end{array}$ & $\begin{array}{l}68 / 19 / 87 \\
12 / 17 / 87\end{array}$ & \\
\hline TRITIUU & $\mathrm{PCI} / \mathrm{L}$ & 560.89 & 20000.00 & $\begin{array}{l}B 8 / 25 / 87 \\
12 / 13 / 87\end{array}$ & $\begin{array}{l}511.08 \\
217.86\} \\
579.86 \\
211.96\}\end{array}$ & $\begin{array}{l}88 / 19 / 87 \\
11 / 12 / 87\end{array}$ & & $\begin{array}{l}68 / 18 / 87 \\
12 / 17 / 87\end{array}$ & $\left.\begin{array}{r}338.06 \\
\{\quad 286.68\end{array}\right\}$ \\
\hline
\end{tabular}

* - yalue eXceEds priuary oRinking tater standard

- - YaLUE EXCEEDS PRDPDSED PRIUARY DRINKING WATER STANDARD.

- - YALUE EXCEEDS SCREENING LEVEL FOR FURTHER INVESTIGATIDN.

- DETECTION LIUIT WAS NOT AVAILABLE FOR COUPARISON

NR - ANALYSIS NOT RERUESTED OR NOT YET REPORTEO

VALUES IN \& ? ARE COUNTING ERRORS FOR RADIONUCLIOES

WATER STANDARD(S) IN PAREMTHESES ARE PROPOSED OHLY 
TABLE C.7. (contd)

\begin{tabular}{|c|c|c|c|c|c|c|c|c|c|}
\hline $\begin{array}{l}\text { CONSTI } \\
\text { NAUE }\end{array}$ & $\begin{array}{l}\text { UENT } \\
\text { UNITS }\end{array}$ & $\begin{array}{c}\text { CONTRACTUAL } \\
\text { DETECIION } \\
\text { LIUIT }\end{array}$ & $\begin{array}{c}\text { DRINKING } \\
\text { NATER } \\
\text { STANDARD }\end{array}$ & $\begin{array}{c}\text { SAHPLE } \\
\text { DATE }\end{array}$ & B- $69-38$ & $\begin{array}{c}\text { SAUPLE } \\
\text { OATE }\end{array}$ & $6-72-73$ & $\begin{array}{c}\text { SAUPLE } \\
\text { DATE }\end{array}$ & $6-72-88$ \\
\hline$---1-\infty$ & ---- & 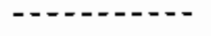 & - - - & $\cdots+--\infty$ & --.--- & $-\ldots-1$ & ----- & - & ----- \\
\hline HNITRAT & PPB & 2500.00 & 45000.00 & $\begin{array}{l}68 / 23 / 87 \\
11 / 08 / 87\end{array}$ & & $68 / 20 / 87$ & $\frac{3900.00}{\mathrm{NR}}$ & $\begin{array}{l}68 / 19 / 87 \\
11 / 12 / 87\end{array}$ & $\begin{array}{l}9530.00 \\
7850.00\end{array}$ \\
\hline TRITIUM & $\mathrm{PCI} / \mathrm{L}$ & 500.00 & 20600.00 & $\begin{array}{l}6 B / 23 / 87 \\
11 / 08 / 87\end{array}$ & $\left\{\begin{array}{l}383.00 \\
211.06\}\end{array}\right.$ & $68 / 20 / 87$ & $\left\{\begin{array}{c}2356.00 \\
267.00\} \\
N R\end{array}\right.$ & $\begin{array}{l}08 / 19 / 87 \\
11 / 12 / 87\end{array}$ & $\begin{array}{r}3660.00 \\
288.06\} \\
3356.06 \\
292.00\}\end{array}$ \\
\hline
\end{tabular}

* - value exceeds priuary drinking mater stakdard.

* - VALUE EXCEEDS PRDPDSED PRIUARY DRINKING WATER STANDARD.

- - VAlUE EXCEEDS SCREERING LEVEL FOR fuRTHER INVESTIGATION.

- - DETECTION LIMIT AS RDT AVAILABLE FOR COUPARISON

HR - ANALYSIS NOT REQUESTED OR NOT YET REPORTED

VALUES IN \{ \} ARE COUNTING ERRORS FOR RADIONUCLIDES

AATER STANDARD(S) IN PARENTHESES ARE PRDPOSED ONLY 
IABLE C.7. (contd)

\begin{tabular}{ccc}
\multicolumn{2}{c}{ CONSTITUENT } & \multicolumn{2}{c}{$\begin{array}{c}\text { CONTRACTUAL } \\
\text { DETECTION }\end{array}$} \\
NAUE UNITS & LIUIT \\
\hline
\end{tabular}

HNITRAT PPB

TRITIUN PCI/L
DRINKING

MATER

STAMDARD

2500.00
500.00

45000.00

20600.00
SAUPLE

DATE

DATE

$08 / 19 / 87$

$68 / 19 / 87$
6-72-92

8730.01

2180.00

$\left\{\begin{array}{r}281.00\} \\ \text { ind }\end{array}\right.$

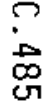

\author{
* - value exceeds primary drinxing nater standard \\ * - VALUE EXCEEDS PROPOSED PRIMARY DRINKING WATER STANDARD. \\ - VALUE EXCEEOS SCREENING LEVEL FOR FURTHER INVESTIGATION. \\ - DETECTION LIUIT NAS NOT AVAILABLE FDR COLPARISON \\ NR - ANALYSIS NOT REQUESTED OR NOT YET REPORTED \\ VALUES IN \{ $\}$ ARE COUNTING ERRORS FOR RADIONUCLIDES \\ WATER STANDARD(S) IN PARENTHESES ARE PROPOSED ONLY
}


TABLE C.7. (contd)

\begin{tabular}{|c|c|c|c|c|c|c|c|c|c|}
\hline $\begin{array}{l}\text { CONS } \\
\text { NAVE }\end{array}$ & $\begin{array}{l}\text { UENT } \\
\text { UNITS }\end{array}$ & $\begin{array}{l}\text { CONIRACTUAL } \\
\text { DETECTION } \\
\text { LIUIT }\end{array}$ & $\begin{array}{l}\text { DRINKING } \\
\text { IATER } \\
\text { STANDARD }\end{array}$ & $\begin{array}{l}\text { SAUPLE } \\
\text { DATE }\end{array}$ & $8-8 b-43 a$ & $\begin{array}{l}\text { SAUPLE } \\
\text { DATE }\end{array}$ & $8-80-43 R$ & $\begin{array}{l}\text { SAUPLE } \\
\text { DATE }\end{array}$ & $6-80-435$ \\
\hline HNITRA & PPB & 2500.00 & 45000.08 & $09 / 23 / 87$ & 2500.60 & $09 / 23 / 87$ & & $69 / 23 / 87$ & 9096.00 \\
\hline
\end{tabular}

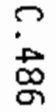

* - VALUE eXCEEDS PRIMARY dRINKING mater STANDARD

1 - VALUE EXCEEDS PROPOSED PRIHARY ORINXING TATER STANDARD

* - VALUE EXCEEDS SCREENING LEVEL FOR FURTHER INVESTIGATION.

- DETECTION LIHIT MS NOT AVAILABLE FOR COMPARISON

NR - ANALYSIS NOT RERUESTED OR NOT YET REPORTED

VALUES IN \& ARE COUNTING ERRORS FOR RADIDNUCLIDES

TATER STANDARO(S) IN PARENTHESES ARE PROPOSED ONLY 
TABLE C.7. (contd)

\begin{tabular}{|c|c|c|c|c|c|}
\hline \multicolumn{2}{|c|}{ CONSTITUENT } & $\begin{array}{l}\text { CONTRACTUAL } \\
\text { DETECTION } \\
\text { LIHIT }\end{array}$ & $\begin{array}{l}\text { DRINKING } \\
\text { WATER } \\
\text { STANDARO }\end{array}$ & $\begin{array}{l}\text { SAUPLE } \\
\text { DATE }\end{array}$ & $8-84-3540$ \\
\hline 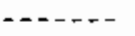 & $-\ldots$ & $--1-1--1$ & - n-nen- & $\ldots$ & $\ldots \ldots$ \\
\hline HN I TRAT & PPB & 2500.00 & 45000.00 & $69 / 23 / B 7$ & $\begin{array}{c}4600.00 \\
\mathrm{KR}\end{array}$ \\
\hline TRITIUH & $\mathrm{PCI} / \mathrm{L}$ & 500.00 & 20000.00 & $99 / 23 / 87$ & \\
\hline & & & & & NR \\
\hline U & $\mathrm{PCI} / \mathrm{L}$ & 0.58 & 800.00 & & $\begin{array}{l}\text { NR } \\
\text { NR }\end{array}$ \\
\hline
\end{tabular}

\begin{tabular}{|c|c|}
\hline $\begin{array}{l}\text { SAUPLE } \\
\text { DATE }\end{array}$ & $6-87-56$ \\
\hline$\cdots+\cdots+\infty$ & $\ldots . . .+$ \\
\hline $12 / 13 / 87$ & 21280.00 \\
\hline OB/28/87 & 53100.06 \\
\hline $12 / 13 / 87$ & $\begin{array}{c}\left\{\begin{array}{r}781.60\} \\
50509.06\end{array}\right. \\
\{743.06\}\end{array}$ \\
\hline--- & KR \\
\hline
\end{tabular}

\begin{tabular}{|c|c|}
\hline $\begin{array}{c}\text { SAHPLE } \\
\text { DATE }\end{array}$ & $B-S 6-E 4 B$ \\
\hline - - - & --10-c- \\
\hline $\begin{array}{l}07 / 24 / 87 \\
10 / 28 / 87\end{array}$ & $\begin{array}{l}18700.00 \\
17900.00\end{array}$ \\
\hline $67 / 24 / 87$ & 27200.60 \\
\hline $16 / 26 / 87$ & $\left\{\begin{array}{r}27460.09 \\
\{656.00\}\end{array}\right.$ \\
\hline $\begin{array}{l}07 / 24 / 87 \\
16 / 28 / 87\end{array}$ & $\begin{array}{l}2.70 \\
3.11\end{array}$ \\
\hline
\end{tabular}

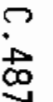

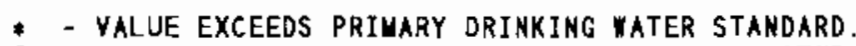

1 - VALUE EXCEEDS PRDPOSED PRIHARY DRINKING WATER STANDARD.

+ - VALUE EXCEEDS SCREENING LEYEL FDR FURTHER INYESTIGATION.

- - DETECTIOX liUit Mas nOt aVAilable for conparison

NR - ANALYSIS NOT REQUESTED OR NOT YET REPORTED

VALUES IN \{ $\}$ ARE COUNTING ERRORS FOR RADIONUCLIDES

MATER STANDARD(S) IN PARENTHESES ARE PROPOSED ONLY 


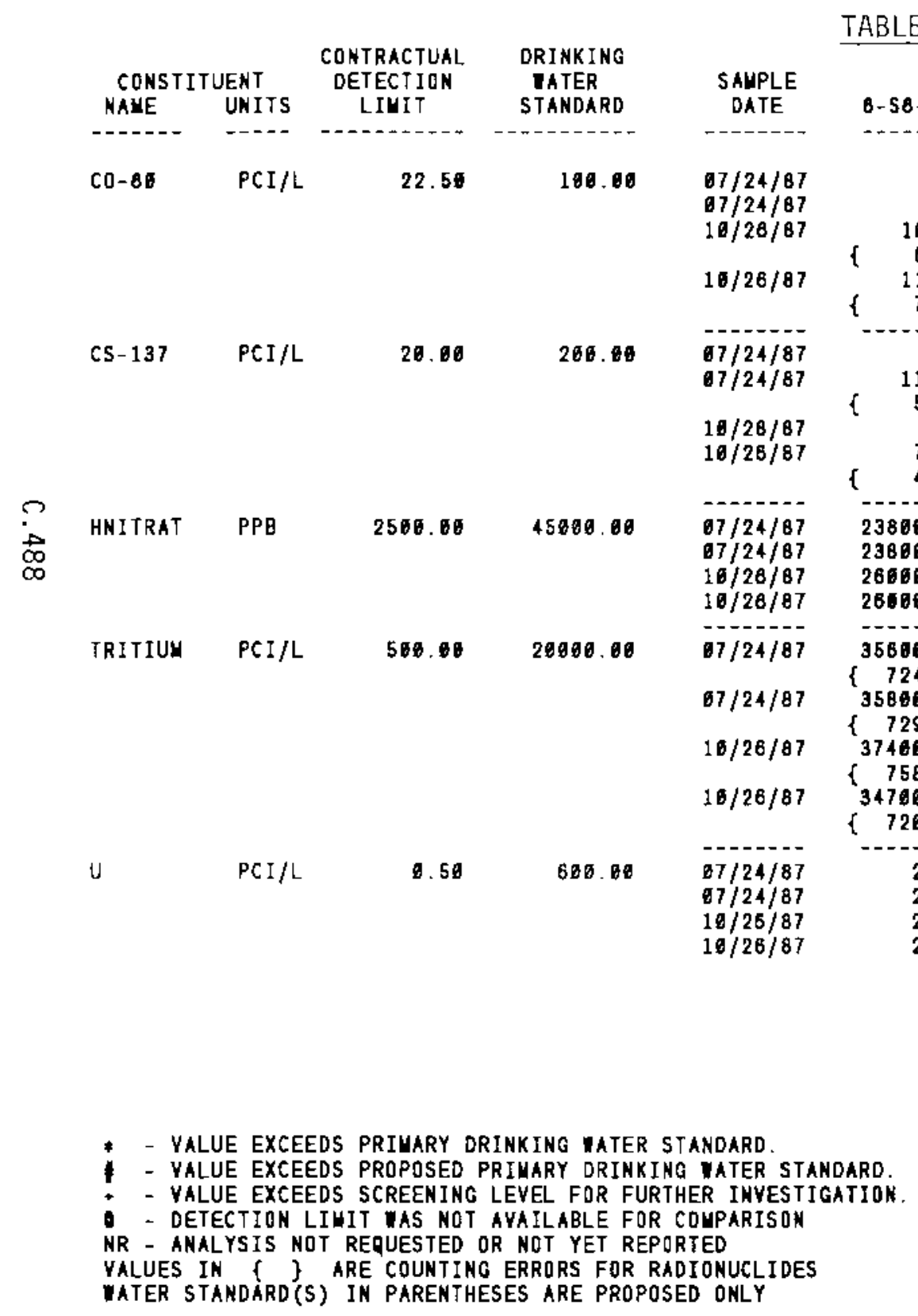

SAMPLE

DATE B-S11-E12A

DATE 6-\$7-34

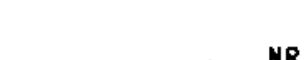

NR

NR

NR

NR

NR

NR

(1.78

1.00
$7.38\}$
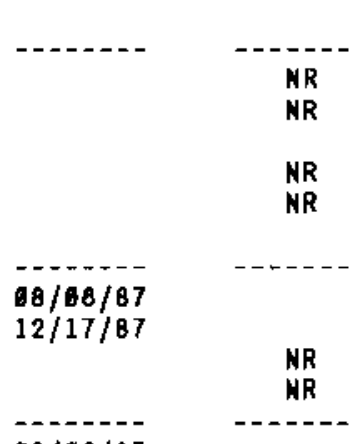

$08 / 06 / 87$

$12 / 17 / 87$

$724.00\}$

$\left\{\begin{array}{c}35860.00 \\ 729.00\}\end{array}\right.$

$37460.00 *$

$758.00\}$

$720.60\}$

- VALUE EXCEEDS PRIUARY DRINKING MATER STANDARD

- YALUE EXCEEDS PROPOSED PRIMARY DRINKING vaTER STANDARD.

- DETECTION LIWIT WAS NOT AYAILABLE FOR COMPARISON

YALUES IN $S$ I ARE COUNTING ERRORS FOR RADIONUCLIDES

ATER STANDARD(S) IN PARENTHESES ARE PROPOSED ONLY 
TABLE C.7. (contd)

\begin{tabular}{|c|c|c|c|c|c|c|c|c|c|}
\hline $\begin{array}{l}\text { CONSTI } \\
\text { NAUE }\end{array}$ & $\begin{array}{l}\text { UENT } \\
\text { UNITS }\end{array}$ & $\begin{array}{l}\text { CONTRACTUAL } \\
\text { DETECTION } \\
\text { LIYIT }\end{array}$ & $\begin{array}{l}\text { DRINKING } \\
\text { VATER } \\
\text { STANDARD }\end{array}$ & $\begin{array}{c}\text { SAMPLE } \\
\text { DATE }\end{array}$ & $6-512-28$ & $\begin{array}{c}\text { SAMPLE } \\
\text { DATE }\end{array}$ & $8-514-26 A$ & $\begin{array}{l}\text { SAUPLE } \\
\text { DATE }\end{array}$ & $6-518-51$ \\
\hline -..... & $\cdots$ & $\cdots-\cdots$ & - & ---- & $\cdots$ & $\cdots+\cdots$ & 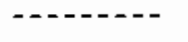 & ------- & ------- \\
\hline$C 0-60$ & $\mathrm{PCI} / \mathrm{L}$ & 22.50 & 100.00 & & NR & $07 / 24 / 87$ & $\left.\begin{array}{ll} & 7.56 \\
\{\quad 5.87\end{array}\right\}$ & & HR \\
\hline HNITRAT & PPB & 2500.00 & 45000.00 & $\begin{array}{l}68 / 60 / 87 \\
10 / 28 / 87\end{array}$ & $\begin{array}{l}17600.06 \\
16600.00\end{array}$ & $\begin{array}{l}07 / 24 / 87 \\
10 / 25 / 87\end{array}$ & $\begin{array}{l}4290.00 \\
3250.00\end{array}$ & $\begin{array}{l}07 / 24 / 87 \\
10 / 25 / 87\end{array}$ & 328000 \\
\hline TRITIUU & $\mathrm{PCI} / \mathrm{L}$ & 500.00 & 20600.06 & $\begin{array}{l}08 / 06 / 87 \\
10 / 28 / 87\end{array}$ & & $\begin{array}{l}07 / 24 / 87 \\
10 / 25 / 87\end{array}$ & & $\begin{array}{l}07 / 24 / 87 \\
10 / 25 / 87\end{array}$ & $\begin{array}{r}306.00 \\
\{\quad 170.00\end{array}$ \\
\hline
\end{tabular}

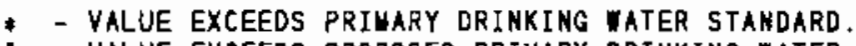

- - VALUE EXCEEDS PROPOSED PRIHARY DRINKING WATER STANDARD.

* - VALUE EXCEEDS SCREENINg LEYEL FOR FURTHER INYESTIGATION.

- DETECTION LIUIT IAS NOT AVAILABLE FOR COUPARISON

NR - ANALYSIS NOT REQUESTED OR NOT YET REPORTED

VALUES IN \{ \} ARE COUNTING ERRORS FOR RADIONUCLIDES

WATER STANDARD(S) IN PARENTHESES ARE PRDPOSED ONLY 


\begin{tabular}{|c|c|c|c|c|c|c|c|c|c|}
\hline $\begin{array}{l}\text { CENSTI } \\
\text { NAUE }\end{array}$ & $\begin{array}{l}\text { UENT } \\
\text { UNITS }\end{array}$ & $\begin{array}{c}\text { CONTRACTUAL } \\
\text { DETECTION } \\
\text { LIUIT }\end{array}$ & $\begin{array}{l}\text { DRINKING } \\
\text { WATER } \\
\text { STAHDARD }\end{array}$ & $\begin{array}{c}\text { SAUPLE } \\
\text { DATE }\end{array}$ & $8-518-11$ & $\begin{array}{c}\text { SAMPLE } \\
\text { DATE }\end{array}$ & $6-524-19$ & $\begin{array}{c}\text { SAMPLE } \\
\text { DATE }\end{array}$ & $0-527-E 14$ \\
\hline$-\ldots .--$ & ---- & $-\ldots \ldots \ldots$ & 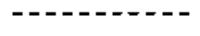 & 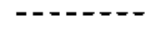 & $\ldots \ldots$ & $----\cdots$ & $\ldots . . .$. & $-\ldots-1--$ & $-\ldots . .-1$ \\
\hline HNITRAT & PPB & 2580.00 & 45000.00 & $69 / 23 / 87$ & $\begin{array}{c}9139,00 \\
\text { NR } \\
\text { NR } \\
\text { NR } \\
\text { NR } \\
\text { NR }\end{array}$ & $19 / 23 / 87$ & $\begin{array}{l}\text { NR } \\
\text { NR } \\
\text { NR } \\
\text { NR } \\
\text { NR }\end{array}$ & $\begin{array}{l}07 / 09 / 87 \\
68 / 27 / 87 \\
09 / 68 / 87 \\
16 / 66 / 87 \\
11 / 63 / 87 \\
12 / 68 / 07\end{array}$ & $\begin{array}{l}27060.00 \\
25360.00 \\
23100.00 \\
21800.00 \\
20800.00 \\
20000.00\end{array}$ \\
\hline U & $\mathrm{PCI} / \mathrm{L}$ & b. 50 & 600.06 & & $\begin{array}{l}\text { NR } \\
\text { NR } \\
\text { NR } \\
\text { NR } \\
\text { NR } \\
\text { NR }\end{array}$ & 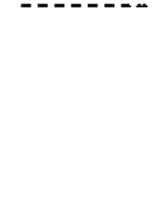 & $\begin{array}{l}\text { NR } \\
\text { NR } \\
\text { NR } \\
\text { NR } \\
\text { NR } \\
\text { NR }\end{array}$ & $\begin{array}{l}07 / 13 / 87 \\
68 / 27 / 87 \\
69 / 68 / 87 \\
10 / 06 / 87 \\
11 / 83 / 87 \\
12 / 68 / 87\end{array}$ & $\begin{array}{r}4.16 \\
4.58 \\
4.20 \\
4.25 \\
4.12 \\
3.97\end{array}$ \\
\hline
\end{tabular}

* - VALUE EXCEEDS PRIMARY DRINKING WATER STANDARD.

* - VALUE EXCEEDS PROPOSED PRIMARY DRINKIKG MaTER STANDARD.

- - VALUE EXCEEDS SCREENINg LEVEl FoR FURTHER INYESTIGATION.

- DETECTION LINIT UAS NOT AVAILABLE FOR COMPARISOM

NR - ANALYSIS NOT REQUESTED OR NOT YET REPORTED

VALUES IN $\{$ \& ARE COUNTING ERRORS FOR RADIDNUCLIDES

WATER STANDARD(S) IN PARENTHESES ARE PROPOSED ONLY 


\section{TABLE C.7. (contd)}

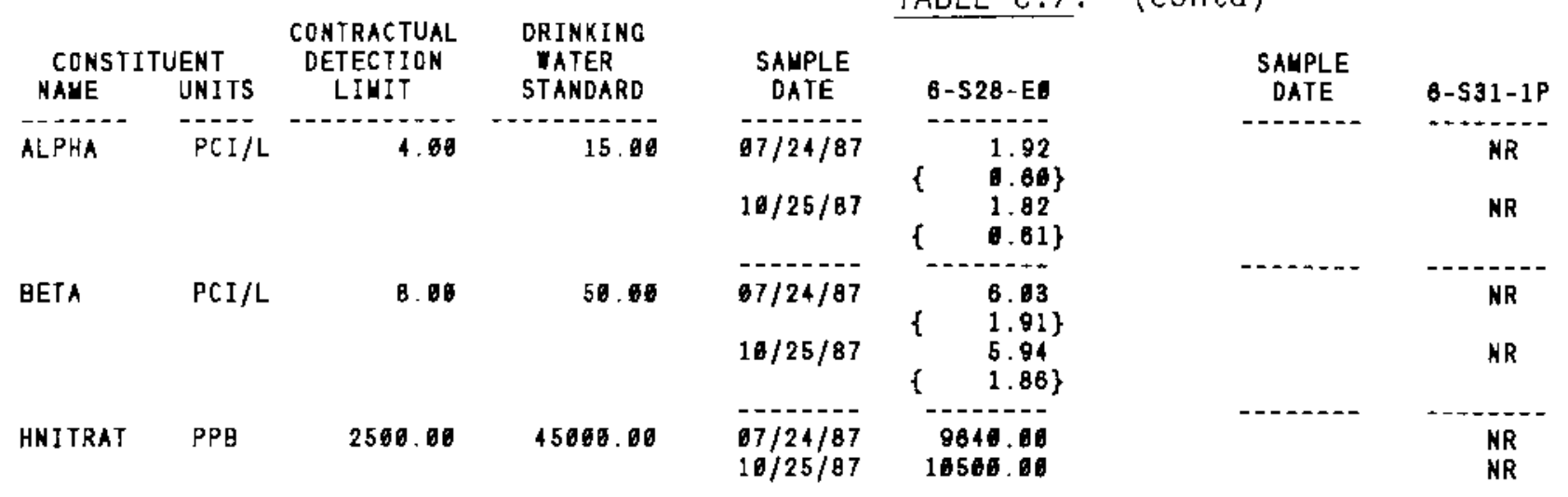

$\stackrel{?}{\leftrightarrow}$

* - value exceeds pritary dRinking Mater standard

* - VALUE EXCEEDS PROPOSED PRIUARY DRINKING Yater STANDARD.

- VALUE EXCEEDS SCREENING LEVEL FDR FURTHER INVESTIGATION.

- DETECTION LiMIT WAS NOT AVAILABLE FOR COUPARISON

KR - ANALYSIS NOT REQUESTED OR NOT YET REPORTED

VALUES IN \{\} ARE COUNTING ERRORS FOR RADIONUCLIDES

WATER STANDARO(S) IN PARENTHESES ARE PROPBSED ONLY 



\section{APPENDIX D}

MAXIMUM CONTAMINANT LEVELS AND

DERIVED CONCENTRATION GUIDELINES 


\title{
APPENDIX D
}

\author{
MAXIMUM CONTAMINANT LEVELS AND \\ DERIVED CONCENTRATION GUIDELINES
}

IABLE D.1. Radiological Maximum Contaminant Leve1s [from U.S. Environmental Protection Agency (OFR 1986) and State of Washington Public Water Supplies (WDSHS 1983]

Contaminant

Gross alpha (excluding uranium)

Combined radium- 226 and radium-228

Radium-226 (State of Washington on 1y) Gross beta and gamma radioactivity from manmade radionuclides
Limit

The following list provides the annual average concentrations that yield an annual dose of $4 \mathrm{mi} 17$ irem to the indicated organ assuming a $2-L$ daily intake. Data are taken from EPA (1976).

\begin{tabular}{|c|c|c|}
\hline Radionuclide & Critical Organ & Concentration, $\mathrm{pCi} / \mathrm{L}$ \\
\hline Antimony-125 & GI (LLI) & 300 \\
\hline Carbon-14 & Fat & 2,000 \\
\hline Cesium- 137 & Whole body & 200 \\
\hline Cobalt-60 & GI (LLI) & 100 \\
\hline Iodine-129 & Thyroid & 1 \\
\hline Nickel-63 & Bone & 50 \\
\hline Ruthen ium- 103 & GI (LLI) & 200 \\
\hline Rutheni um- 106 & GI (LLI) & 30 \\
\hline Strontium-90 & Bone marrow & 8 \\
\hline Technetium-99 & GI (LLI) & 900 \\
\hline
\end{tabular}


TABLE D.2. Chemical Maximum Contaminant Levels [from U.S. Environmenta] Protection Agency (OFR 1986) and State of Washington Public Water Supplies (WDSHS 1983)]

\section{Chemical Constituent}

\section{Concentration}

Arsenic

Barium

Cadmi um

Carbon tetrachloride

Ch]oroform

Chromium

Copper

Fluorine

Lead

Mercury

Nitrate ion

Selenium

Trans-1,2-

Dichloroethene

Trichloroethene

$1,1,1$-Trichloroethane

$\begin{array}{rl}50 & \mathrm{ppb}^{(\mathrm{a})} \\ 1 & \mathrm{ppm} \\ 10 & \mathrm{ppb} \\ 5 & \mathrm{ppb} \\ 100 & \mathrm{ppb} \\ 50 & \mathrm{ppb} \\ 1.3 & \mathrm{ppm} \\ 2 & \mathrm{ppm} \\ 50 & \mathrm{ppb} \\ 2 & \mathrm{ppb} \\ 45 & \mathrm{ppm} \\ 10 & \mathrm{ppb}(\mathrm{b}) \\ 70 & \mathrm{ppb} \\ & \\ 5 & \mathrm{ppb} \\ 200 & \mathrm{ppb}\end{array}$

(a) $\mathrm{ppb}=\mathrm{ppm} / 1000$.

(b) Proposed recommended maximum contaminant level. 
IABLE D. 3. Proposed Derived Concentration Guides (a)

Radionuclide

Americium-241

Ant imony-125

Cesium-137

Cobalt-60

Iodine-129

Nickel-63

Plutoni um-238

Pluton i um-239,240

Radium-226

Ruthen ium- 103

Ruthen ium-106

Stront i um-89

Stront ium-90

Technet ium-99

Tritium

Uranium-234

Uranium-235

Uranium-236

Uranium-238
Concentration, $\mathrm{pCi} / \mathrm{L}$

30

60,000

3,000

5,000

500

300,000

400

300

100

50,000

6,000

20,000

1,000

100,000

$2,000,000$

500

600

500

600

(a) Concentrations of radionuclides in water that could be continuously consumed and not exceed an effective dose equivalent of $100 \mathrm{mrem} / \mathrm{yr}$. Consumption is assumed to be $730 \mathrm{~L}$ of drinking water per year. 


\section{REFERENCES}

DOE Order 5480.1A. "Environmental Protection, Safety, and Health Protection Program for DOE Operations." U.S. Department of Energy, Washington, D.C.

OFR (Office of the Federa] Register). 1986. Code of Federal Regulations, "Protection of Environment." 40 CFR Parts 100 to 149.

EPA. 1976. National Interim Primary Drinking Water Regulations. EPA-570/9-76-003, Office of Water Supply, Washington, D.C.

Washington State Department of Social and Health Services (WDSHS). August 1983 (Revised). "Public Water Supplies". Washington Administration Code, Chapter 248-54, 01 ympia, Washington. 
PNL-6315-2

UC- 11,41

\section{DISTRIBUTION}

No. of

Copies

OFFSITE

10 DOE/Office of Scientific and Technical Information

B. Blake

133 1st Ave. North

Minneapolis, MN 55401

E. A. Jacobson

Desert Research Institute University of Nevada System

Reno, NV

T. L. Traub

DOE Public Reading Room

Federa] Building, Room 157

Richland, WA 99352

\section{ONSITE}

7 DOE Richland Operations Office
M. J. Anthony
J. D. Goodenough
P. J. Krupin
R. J. Nevarez
M. W. Shupe
M. W. Tiernan
M. Vargas

25 Westinghouse Hanford Company

M. R. Adams

L. C. Brown

G. D. Carpenter

M. A. Christie

M. L. Cramer

L. P. Diedicker

J. J. Dorian

K. R. Fecht

K. A. Gano

K. A. Gasper

E. M. Greagor

R. L. Jackson

M. J. Lauterbach

A. G. Law

D. L. Lund
No. of

Copies
A. C. Mckarns
S. M. McKinney
P. S. Peacock
K. M. Probasko
P. S. Schaus
J. A. Serkowski
L. L. Swanson
D. M. TuTburg
R. E. Wheeler
S. A. Wiegman

63 Pacific Northwest Laboratory

D. J. Bates

R. W. Bryce (12)

L. J. Criscenti

C. E. Elderkin

J. C. Evans (10)

F. W. Falco

M. D. Freshley

J. S. Fruchter

R. M. Fruland

R. E. Gephart

M. J. Graham

R. H. Gray

S. H. Hall

J. M. Hales

M. S. Hanson (2)

P. C. Hays

N. W. Hinman

K. M. Krupka

W. W. Laity

P. E. Long

S. P. Luttre11

R. E. Lundgren

P. J. Mitche11

R. W. Nelson

J. T. Rieger

R. Schalla

D. R. Sherwood (5)

C. S. Simmons

R. L. Skaggs

R. M. Smith

J. A. Stottlemyre

G. P. Streile

R. E. Wildung

Publishing Coordination

Technical Report Files (5) 


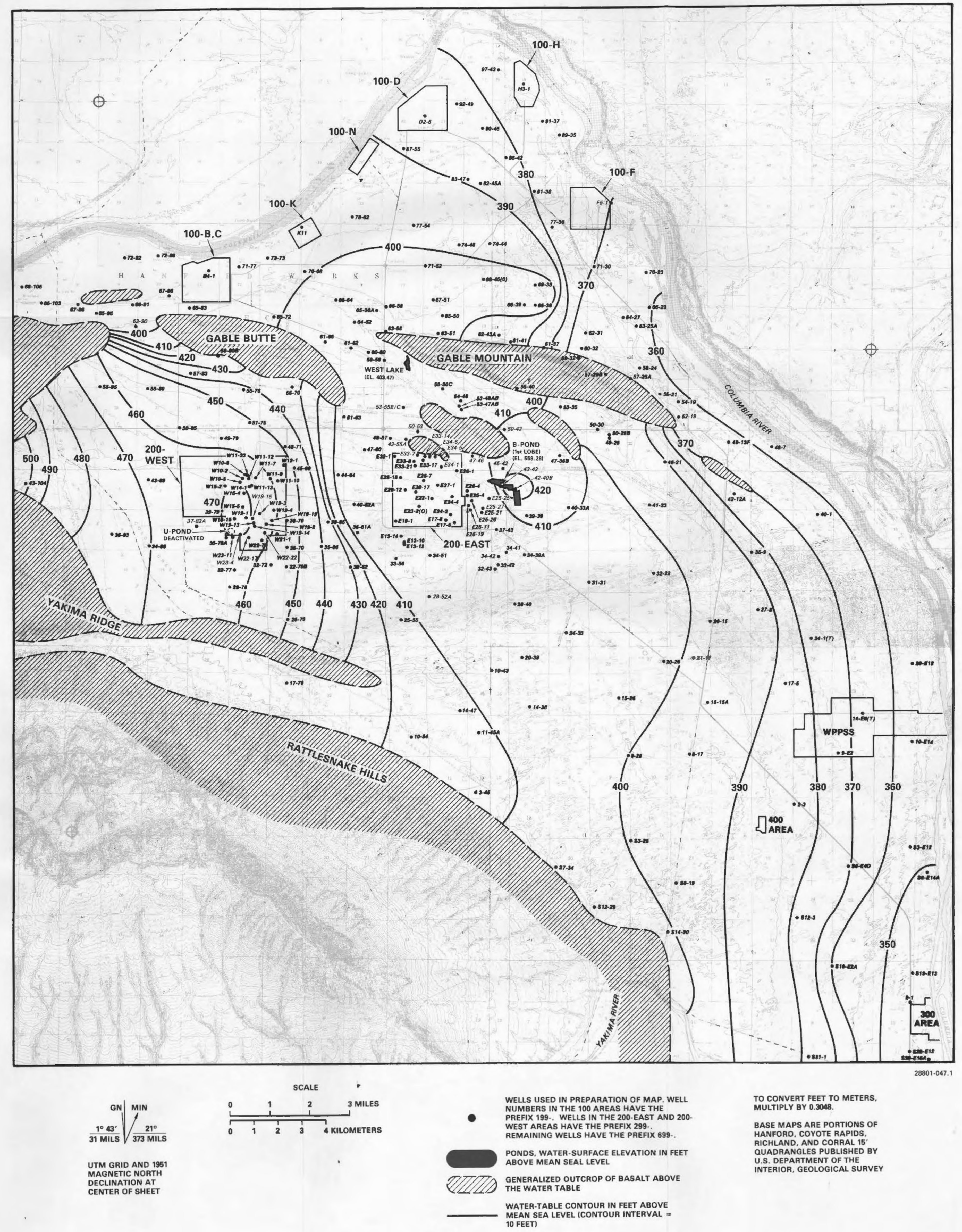

TO CONVERT FEET TO METERS, MULTIPLY BY 0.3048.

BASE MAPS ARE PORTIONS OF RICHLAND, AND CORRAL 15 , QUADRANGLES PUBLISHED BY U.S. DEPARLS PUBLSHE BY 